\title{
NREL Photovoltaic Program FY 1997 Annual Report
}

R.D. McConnell, PV Communications Leader

A. Hansen, Communications Coordinator S. Smoller, Technical Editor

\section{DISTRIBUTION OF THIS DOCUMENT IS UNLINMTED}

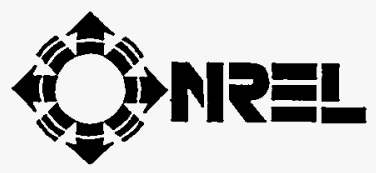

National Renewable Energy Laboratory 1617 Cole Boulevard

Golden, Colorado 80401-3393

A national laboratory of the U.S. Department of Energy

Managed by Midwest Research Institute for the U.S. Department of Energy under Contract No. DE-AC36-83CH10093

June 1998 


\section{NOTICE}

This report was prepared as an account of work sponsored by an agency of the United States government. Neither the United States government nor any agency thereof, nor any of their employees, makes any warranty, express or implied, or assumes any legal liability or responsibility for the accuracy, completeness, or usefulness of any information, apparatus, product, or process disclosed, or represents that its use would not infringe privately owned rights. Reference herein to any specific commercial product, process, or service by trade name, trademark, manufacturer, or otherwise does not necessarily constitute or imply its endorsement, recommendation, or favoring by the United States govemment or any agency thereof. The views and opinions of authors expressed herein do not necessarily state or reflect those of the United States government or any agency thereof.

Available to DOE and DOE contractors from:

Office of Scientific and Technical Information (OSTI)

P.O. Box 62

Oak Ridge, TN 37831

Prices available by calling (423) $576-8401$

Available to the public from:

National Technical Information Service (NTIS)

U.S. Department of Commerce

5285 Port Royal Road

Springfield, VA 22161

(703) $487-4650$ 


\section{DISCLAIMER}

Portions of this document may be illegible electronic image products. Images are produced from the best available original document. 


\section{PREFACE}

This report summarizes the in-house and subcontracted research and development (R\&D) activities under the NREL PV Program from October 1, 1996, through September 30, 1997 (fiscal year [FY] 1997). The NREL PV Program is part of the U.S. Department of Energy's (DOE's) National Photovoltaics Program, as described in the DOE National Photovoltaics Program Plan for 19962000. The mission of the DOE National Photovoltaics Program is to make PV a significant part of the domestic economy -as an industry and an energy resource. The two primary goals of the national program are to (1) maintain the U.S. industry's world leadership in research and technology development, and (2) help the U.S. industry remain a major, profitable force in the world market. The NREL PV Program provides leadership and support to the national program toward achieving its mission and goals.

The FY 1997 budget authority for carrying out the NREL PV Program was $\$ 39.3$ million in operating funds and $\$ 0.4$ million in capital equipment funds. Subcontract activities represent a major part of the NREL PV Program, with $\$ 21.8$ million (55\% of PV funds) going to some 84 subcontractors. Cost sharing by industry added over almost $\$ 8.8$ million to the subcontract $R \& D$ activities with industry.

The activities of NREL's PV Program are to: conduct basic, applied, and engineering research; manage subcontracted R\&D projects; perform research complementary to subcontracted work; develop and maintain state-of-the-art measurement and device capabilities; develop PV manufacturing technology and modules; transfer results to industry; and evolve viable partnerships for PV systems and market development. The research activities under the program are summarized under the eight project areas: PV Fundamental and Exploratory Research, PV Electronic Materials and Devices, PV Measurements and Characterization, Thin Film PV Partnership, PVMaT Technology, PV Engineering and Reliability, PV Domestic Applications and Markets, and PV International Applications and Markets.

\section{Approved for the NATIONAL RENEWABLE ENERGY LABORATORY}

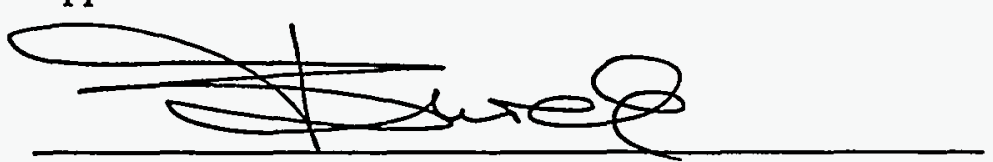

Thomas Surek

Technology Manager, Photovoltaics 



\section{TABLE OF CONTENTS}

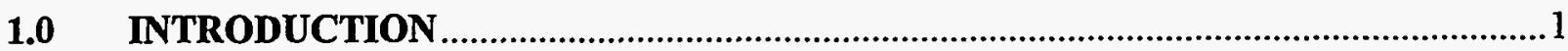

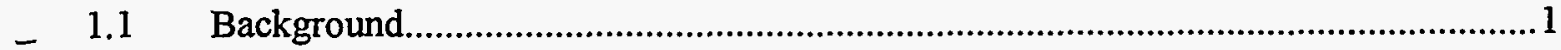

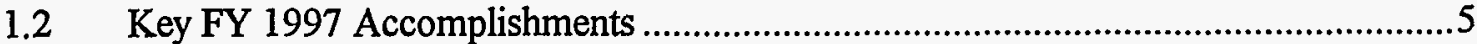

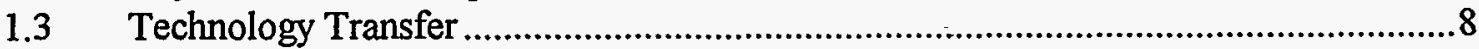

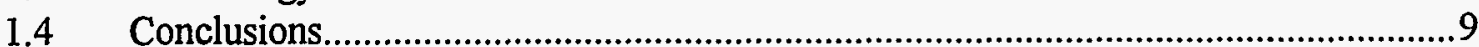

\subsection{PV FUNDAMENTAL AND EXPLORATORY RESEARCH}

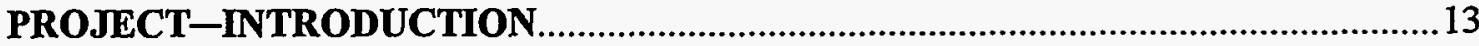

\section{Crystalline Silicon In-House Research}

Crystalline Silicon Materials Research

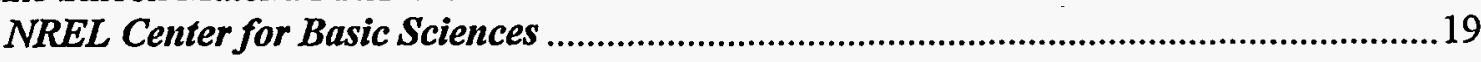

\section{Crystalline Silicon Subcontracts}

Investigation of Gettering Mechanisms in Crystalline Silicon

Duke University

Fundamental Research on Post-Growth Quality Enhancement Techniques

Georgia Institute of Technology

Defect Simulators for Materials Specification and Silicon Solar Cell Processing

Massachusetts Institute of Technology.

Detailed Non-Contact Electrical and Structural Characterization of Photovoltaic

Silicon Substrates

North Carolina State University.

Theoretical Analysis of Hydrogen Passivation of Impurities and Defects

Texas Tech University

Recombination Properties of Copper Precipitates in Silicon

University of California at Berkeley

Optimization of Gettering Processes for Photovoltaic Silicon

University of South Florida.

Exploratory Materials and Devices In-House Research

EE/ER Collaborative Research: Photochemical Solar Cell Development and Optimization NREL Center for Basic Sciences 
Solid State Spectroscopy

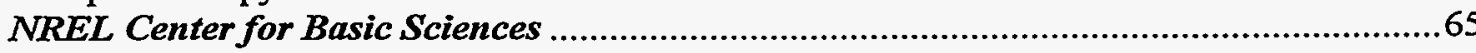

Solid State Theory of Novel Photovoltaic Materials and Devices

NREL Center for Basic Sciences

Exploratory Materials and Devices Subcontracts

Novel Low-Temperature Substrate Technology for Compound Semiconductor Solar Cells on LowCost Substrates

California Institute of Technology

Solar Energy Conversion at Dye-Sensitized Nanostructured Electrodes Fabricated by

Sol-Gel Processing

Johns Hopkins University

20\% (AM1.5) Efficiency GaAs Solar Cells on Sub-mm Grain-Size Poly-Ge and Its

Transition to Low-Cost Substrates

Research Triangle Institute

Vapor Phase Epitaxial Liftoff of GaAs and Silicon Single Crystal Films

University of California at Los Angeles.

Fundamental Studies of the Effect of Crystal Defects on CuInSe $2 / \mathrm{CdS}$ Heterojunction Behavior University of Illinois.

Electronic Processes in Thin-Film PV Materials

University of Utah.

Electrochemical Fabrication of Dye-Sensitized Heterojunctions

University of Washington

\section{Historically Black Colleges and Universities Subcontracts}

Photovoltaic Applications in Developing Countries-Marketing Renewable Energy Devices Central State University.

PV Application in Building Design

Hampton University

Historically Black College and University Photovoltaic (PV) Associates Program

Mississippi Valley State University.

Photovoltaic Research Activity and Training of Students-1996-97

Southern University A\&M College

Historically Black Colleges and Universities Photovoltaics Research Associate Program

Texas Southern University 


\subsection{PV ELECTRONIC MATERIALS AND DEVICES}

PROJECT-INTRODUCTION.

PV Electronic Materials and Devices Project In-House Research

Development of Polycrystalline $\mathrm{Cu}(\mathrm{In}, \mathrm{Ga}) \mathrm{Se}_{2}$ Thin Films and Devices NREL Center for Photovoltaics and Electronic Materials

High-Efficiency Concepts and Concentrators

NREL Center for Photovoltaics and Electronic Materials

Hydrogenated Amorphous Silicon Device Research

NREL Center for Photovoltaics and Electronic Materials 155

Nanoparticles and New Ideas for Photovoltaics

NREL Center for Photovoltaics and Electronic Materials

NREL Clean Room/Device Fabrication Team Activities

NREL Center for Photovoltaics and Electronic Materials

Thin-Film CdS/CdTe Solar Cell Development NREL Center for Photovoltaics and Electronic Materials

\subsection{PV MEASUREMENTS AND CHARACTERIZATION} PROJECT-INTRODUCTION

PV Measurements and Characterization Project In-House Research

Analytical Microscopy Characterization of PV Devices NREL Center for Measurements and Characterization

Electrooptical Characterization NREL Center for Measurements and Characterization

PV Efficiency Measurements-Standard Reporting Conditions NREL Center for Measurements and Characterization

Surface and Interface Characterization

NREL Center for Measurements and Characterization .

5.0 THIN-FILM PV PARTNERSHIP PROJECT-INTRODUCTION

Thin-Film PV Partnership Subcontracts

Development of Monolithic Interconnected, Silicon-Film ${ }^{\mathrm{TM}}$ Modules AstroPower, Inc. 
Nanostructure of Amorphous-Silicon-Based Solar Cell Materials by Small-Angle

$\mathrm{X}$-Ray Scattering

Colorado School of Mines.

Polycrystalline Thin-Film Cadmium Telluride Solar Cells Fabricated by Electrodeposition Colorado School of Mines.

Device Physics of Thin-Film Polycrystalline Cells and Modules

Colorado State University.

Development of High, Stable-Efficiency Triple-Junction a-Si Alloy Solar Cells

Energy Conversion Devices, Inc.

CIS Photovoltaic Technology

Energy Photovoltaics, Inc.

CdTe Module Testing and Study of Transients and Irreversible Effects in CdTe

Thin-Film Solar Cells

Florida Solar Energy Center. 243

Deposition of $\mathrm{CuIn}_{1-\mathrm{x}} \mathrm{Ga}_{\mathrm{x}} \mathrm{Se}_{2}$ Thin-Films Using a Two-Stage Process

Florida Solar Energy Center.

Optimization of Transparent and Reflecting Electrodes for Amorphous Silicon Solar Cells

Harvard University

Application of CIS to High Efficiency PV Module Fabrication

International Solar Electric Technology

Research on Amorphous Silicon and Alloy Solar Cells

Iowa State University.

Atomic-Scale Characterization of Hydrogenated Amorphous Silicon Films and Devices

National Institute of Standards and Techinology.

Wide Band Gap Amorphous Silicon-Based Solar Cells with High Stabilized Performance

Pennsylvania State University.

Development and Application of a Computer Model for CdTe and CuInSe 2 Based Solar Cells

Purdue University.

Thin-Film Photovoltaic Partnership Program CIS-Based Thin Film PV Technology

Siemens Solar Industries.

CdTe Polycrystalline Thin-Film Photovoltaic Module Development

Solar Cells, Inc. 
Amorphous Silicon Research

Solarex.

Research on Wide Bandgap, Amorphous-Silicon-Based Solar Cells

Syracuse University

Thin Film Amorphous Silicon Alloy Research

United Solar Systems Corporation

Photocharge Transport and Recombination Measurements in Amorphous Silicon

Films and Solar Cells by Photoconductive Frequency Mixing

University of California at Los Angeles.

Processing and Modeling Issues for Thin-Film Solar Cells

University of Delaware, Institute of Energy Conversion

Processing of CuInSe $\mathrm{C}_{2}$-Based Solar Cells: Characterization of Deposition Processes

in Terms of Chemical Reaction Analysis

University of Florida

Experimental Study of Photodegradation in a-Si:H Solar Cells

University of North Carolina

Microscopic Origins of Metastable Effects in a-Si:H and Deep Defect Characterization in a-Si,Ge:H Alloys

University of Oregon

Advanced Processing Technology for High-Efficiency Thin-Film CuIn $\mathrm{Ga}_{1-\mathrm{x}} \mathrm{Se}_{2}$ and

CdTe Solar Cells; Heterojunction Development and Optimization in Thin-Film Compound

Semiconductor Solar Cells

University of South Florida

High-Efficiency Thin-Film Cadmium Telluride Photovoltaic Cells

University of Toledo

Optimization of Laser Scribing for Thin-Film PV Modules

University of Toledo

Alternative Window Schemes for CuInSe 2 -Based Solar Cells

Washington State University/Tri-Cities.

6.0 PHOTOVOLTAIC MANUFACTURING TECHNOLOGY (PVMAT)

PROJECT-INTRODUCTION.

Photovoltaic Manufacturing Technology (PVMaT) Subcontracts

Next Generation Three-Phase Inverters

Advanced Energy Systems, Inc. 
Manufacture of an AC Photovoltaic Module

Ascension Technology, Inc.

Market-Driven EFG Modules

ASE Americas.

Large-Area Silicon-Film ${ }^{\mathrm{TM}}$ Manufacturing

AstroPower, Inc.

PVMaT Work at Evergreen Solar

Evergreen Solar, Inc.

Monolithic Amorphous Silicon Modules on Continuous Polymer Substrates

Iowa Thin Films Technologies, Inc.

Three-Phase Power Conversion System for Utility Interconnected PV Applications

Ominion Power Engineering Corporation.

PVMaT Improvements in the Manufacturing of the PVI Powergrid ${ }^{\mathrm{M}}$, Year-Two

Annual Report

Photovoltaics International, $L L C$

Photovoltaic $\mathrm{Cz}$ Silicon Module Improvements

Siemens Solar Industries.

High-Throughput Manufacturing of Thin-Film CdTe PV Modules

Solar Cells, Inc.

Development of Standardized, Low-Cost AC PV Systems

Solar Design Associates, Inc.

Design, Fabrication, and Certification of Advanced Modular PV Power Systems

Solar Electric Specialties Company

Cast Polycrystalline Silicon Photovoltaic Module Manufacturing Technology Improvements

Solarex

Full-Scale Testing of Modules Prepared with Experimental EVA-Based Encapsulants STR (formerly Springborn Laboratories, Inc.)

Development of a Modular, Bi-Directional Power Inverter for Photovoltaic Applications

Trace Engineering Company, Inc.

Development of a Low-Cost Integrated $20 \mathrm{~kW}$ A.C. Solar Tracking Sub-Array

for Grid-Connected PV Power System Applications

Utility Power Group. 399 


\section{PV Engineering and Reliability In-House Research}

Improving the Stability/Durability of Encapsulated PV Cells and Minimodules

NREL Center for PV Engineering and Reliability

Module Performance, Testing, and Technology Validation

NREL Center for PV Engineering and Reliability

Photovoltaic Solar Radiometric Measurements and Evaluation

NREL Center for PV Engineering and Reliability

Photovoltaic System Performance and Standards

NREL Center for PV Engineering and Reliability

Solar Resource Characterization

NREL Solar Resource Assessment Team

\section{PV Engineering and Reliability Subcontracts}

Photovoltaic Module Energy Rating Procedure

Endecon Engineering.

Development of Test Methods and Procedures for Evaluation of PV Systems

NEOS Corporation

PV Certification and Accreditation Management Support

PowerMark Corporation

Mánagement and Administration of the IEC/TC82

Solar Energy Industries Assoc.

The Design and Construction of a Custom-Made PV Encapsulant and Solar Cell

Degradation Monitor

University of Colorado at Denver

\subsection{PV DOMESTIC APPLICATIONS AND MARKETS PROJECT-INTRODUCTION.}

\section{PV Domestic Applications and Markets Subcontracts}

Computer Modeling of Building Integrated Photovoltaic Systems and its Application to the Million Solar Roofs Initiative

McNeil Technologies.

Renewable Energy Analysis and Economic Applications for Electric Power

Pacific Energy Group. 
Solar Resource Utility Load Matching Assessment University at Albany.

Evaluation of Photovoltaic Peak-Shaving Applications in the U.S. Buildings Sector

University of Delaware.

\subsection{INTERNATIONAL APPLICATIONS AND MARKETS} PROJECT-INTRODUCTION.

International Applications and Markets Subcontracts

Support for Commercial Project Development in China Institute of Policy and Management, Chinese Academy of Sciences.

Photovoltaics for Rural Energy in Gansu Province in the People's Republic of China Solar Electric Light Fund

Evaluation of Intermediate Applications for Photovoltaics in the United States and Developing Countries

University of Delaware .503

10.0 LIST OF ACTIVE SUBCONTRACTS .509

11.0 NREL PHOTOVOLTAIC PROGRAM FY 1997 BIBLIOGRAPHY .521 


\subsection{INTRODUCTION}

This report summarizes the in-house and subcontracted research and development (R\&D) activities under the NREL PV Program from October 1, 1996, through September 30, 1997 (FY 1997). The NREL PV Program is part of the U.S. Department of Energy's (DOE's) National Photovoltaics Program, as described in the DOE National Photovoltaics Program Plan for 1996-2000. The FY 1997 budget authority for carrying out the NREL PV Program was $\$ 39.3$ million in operating funds and \$0.4 million in capital equipment funds. Subcontract activities represent a major part of the NREL PV Program, with $\$ 21.8$ million (55\% of PV funds) going to some 84 subcontractors. Cost sharing by industry added almost $\$ 8.8$ million to the subcontract R\&D activities with industry.

\subsection{Background}

The NREL PV Program is part of the DOE National Photovoltaics Program managed by the Office of Photovoltaic and Wind Technologies. This office is under the Office of Utility Technologies in DOE's Office of Energy Efficiency and Renewable Energy. Major program thrusts in FY 1997 continued to be implemented based on DOE's National Photovoltaics Program Plan for 1996-2000. The program mission is to:

Work in partnership with U.S. industry to develop and deploy photovoltaic technology for generating economically competitive electric power, making photovoltaics an important contributor to the nation's and the world's energy use and environmental improvement.

The two primary goals of the national program are to (1) maintain the U.S. industry's world leadership in research and technology development, and (2) help the U.S. industry remain a major, profitable force in the world market. The NREL PV Program provides leadership and support to the national program toward achieving its mission and goals.

The DOE strategy over the next few years will be to lay the groundwork for a growing U.S. PV technology and industrial base, with increased emphasis on market and project development activities with industry. To accomplish this, the national program embraces three relatively equal-priority activities: (1) technology development and validation, (2) market conditioning, and (3) project venturing. The new strategy continues a strong technology development program, but emphasizes R\&D for the technologies and companies that are positioned to substantially penetrate the market, reduce prices, and scale up manufacturing. Program activities include continuing efforts to form partnerships with manufacturers and utilities (the ultimate benefactors and users), with universities, and with federal and state agencies.

Under the DOE National Photovoltaics Program, the NREL PV Program supports long-term fundamental and mid-term applied $R \& D$, near-term manufacturing development, and near-term systems and market development in PV energy technology. The project also provides services to industry, electric utilities, and other users, and provides overall programmatic support for the National Photovoltaics Program. The NREL subcontract program is responsible for most of the R\&D, manufacturing technology development, and some of the systems and market development task areas 
under the National Photovoltaics Program. It is implemented via competitive public solicitations. One of the most important subcontracting mechanisms is government/industry partnership, with industry sharing the cost of research with DOE/NREL.

Closer work with industry, utilities, and other end users on PV manufacturing technology, systems, and market needs is being increasingly emphasized. Approaches for this emphasis include mitigating barriers to PV adoption in the utility and international marketplaces, and project venturing with decision makers and organizations representing domestic and international market sectors for PV. As appropriate for the system and market development areas, NREL is supporting activities such as education; technical assistance and training; market, economic, and financial analysis; technology characterizations; regulatory, rate, and value analysis; codes and standards assessment and development; working with customers in project development activities; and co-financing demonstration projects. Under project venturing, DOE is placing particular emphasis on supporting and strengthening programs already in place, such as PV:BONUS (Photovoltaics Building Opportunities in the United States) and the Utility PhotoVoltaics Group (UPVG).

NREL's PV activities include laboratory research and subcontract project management. We also provide technical support to efforts contracted from the DOE Golden Field Office (GO), such as PV:BONUS and UPVG. The primary research activities are conducted in advanced PV material technologies, including amorphous silicon (a-Si) thin-film materials; polycrystalline thin films, such as copper indium diselenide (CIS), cadmium telluride (CdTe), and their alloys; and high-efficiency crystalline cells, including silicon ( $\mathrm{Si}$ ) and gallium arsenide and their alloys. These activities are conducted in-house and through subcontracts. with industry (mostly cost shared) and universities. The research activities are closely coordinated through several NREL/industry/university "team" efforts. Improving PV device manufacturing is vital. We are pursuing two complementary approaches:

-

Government/industry partnerships, such as the PV Manufacturing Technology-or PVMaT - project (which focuses on improving manufacturing processes and products, accelerating manufacturing cost reduction, and laying the foundation for increased production capacity).

- Module development research to evaluate modules (and module performance) and suggest solutions to manufacturers' module problems.

System and market development rounds out the balanced approach pursued. The objective is to create an environment in which system technology, user acceptance, and the PV industry can accommodate the continued expansion of PV into large applications and markets. NREL subcontracts also support research on future-generation PV technologies for highly qualified research teams to expand the current limits of PV technology. Transferring research results into commercial products and applications in a timely and effective manner is another major activity of NREL's PV Program.

Subcontracted R\&D is a significant part of the NREL PV Program; typically more than $50 \%$ of the program's budget is allocated yearly to subcontracts. Table 1.1-1 shows the history of distribution of both subcontract and in-house budgets. From FY 1978 through FY 1997, we awarded almost 
1300 subcontracts totaling nearly $\$ 378$ million out of the total operating budget of $\$ 601$ million. In FY 1997, we awarded some 84 subcontracts with a total funding of $\$ 21.8$ million. Cost sharing by industry subcontractors added more than $\$ 8.8$ million to the $\$ 16.3$ million in NREL funding to 47 industry subcontractors. NREL also subcontracted with 37 universities (not counting Sunrayce schools), with a total funding of $\$ 5.5$ million. Figure 1.1-1 shows the industry-university-government distribution of subcontract funds by categories in DOE's National Photovoltaics Program Plan for 1996-2000. Table 1.1-2 shows the contacts for the various NREL PV Program areas.

Table 1.1-1 Budget History of NREL Photovoltaics Program.

\begin{tabular}{|l|c|c|c|c|c|}
\hline Fiscal Year & $1978-1993$ & 1994 & 1995 & 1996 & 1997 \\
& $\$ M$ & $\$ M$ & $\$ M$ & $\$ M$ & $\$ M$ \\
\hline
\end{tabular}

Task Area
\begin{tabular}{|l|c|c|c|c|c|}
\hline In-House R\&D & 124.8 & 16.4 & 18.8 & 17.6 & 17.5 \\
\hline Capital Equipment & 22.9 & 3.3 & 0.8 & 0.3 & 0.4 \\
\hline Subtotal (in-house) & 147.7 & 19.7 & 19.6 & 17.9 & 17.9 \\
\hline
\end{tabular}

\section{Subcontracted R\&D}

\begin{tabular}{|l|c|c|c|c|c|}
\hline Amorphous Silicon Thin Films & \multicolumn{1}{|c|}{83.4} & 4.3 & 4.2 & 3.4 & 3.5 \\
\hline Polycrystalline Thin Films & 60.5 & 6.7 & 8.2 & 6.6 & 6.6 \\
\hline High-Efficiency Concepts & 37.7 & 0.6 & 0.3 & 0.4 & 0.5 \\
\hline Crystalline Silicon & 25.9 & 1.2 & 1.0 & 0.9 & 0.9 \\
\hline New Ideas & 19.2 & 0.2 & 0.0 & 0.0 & 0.0 \\
\hline University Participation & 8.4 & 0.8 & 0.8 & 0.5 & 0.6 \\
\hline Subtotal (R\&D subcontracts) & 235.2 & 13.8 & 14.5 & 11.8 & 12.1 \\
\hline
\end{tabular}

Manufacturing Technology Development and Systems and Market Development Subcontracts

\begin{tabular}{|l|c|c|c|c|c|}
\hline & 29.2 & 16.6 & 10.7 & 8.4 & 7.9 \\
\hline $\begin{array}{l}\text { PVMaT Project } \\
\text { Engineering Project }\end{array}$ & 2.6 & 0.6 & 0.6 & 0.3 & 0.1 \\
\hline $\begin{array}{l}\text { PV Analysis and Applications } \\
\text { Development Project }\end{array}$ & 3.2 & 2.9 & 3.3 & 2.0 & 1.7 \\
\hline Subtotal (other subcontracts) & 35.0 & 20.1 & 14.6 & 10.7 & 9.7 \\
\hline Subtotal (subcontracts) & 270.2 & 33.9 & 29.1 & 22.5 & 21.8 \\
\hline TOTAL NREL PV PROGRAM & 417.9 & 53.6 & 48.7 & 40.4 & 39.7 \\
\hline
\end{tabular}

${ }^{1}$ Includes $15 \%$ to $20 \%$ for technical program management, fees, etc.

${ }^{2}$ Includes $\$ 9$ million for photoelectrochemical cell research

${ }^{3}$ Significant subcontract effort initiated in FY 1991

${ }^{4}$ Significant subcontract effort initiated in FY 1992 


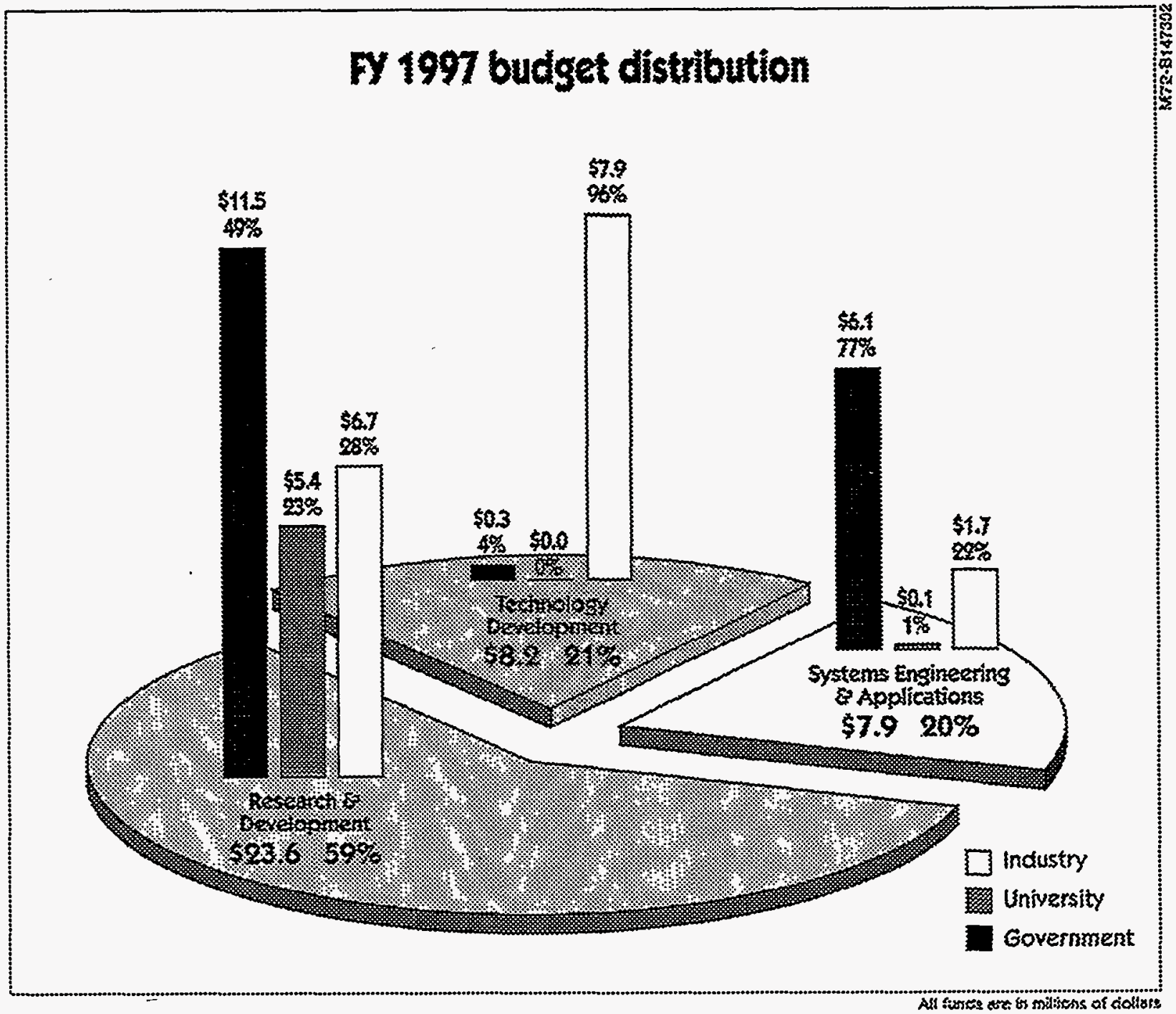

Figure 1.1-1. FY 1997 budget distribution, with total funding of $\$ 39.7$ million.

Table 1.1-2. Program Contacts by Project Area

\begin{tabular}{|l|l|c|}
\hline \multicolumn{1}{|c|}{ Project } & \multicolumn{1}{c|}{ Leader } & Telephone \\
\hline PV Program Management & Thomas Surek & (303) 384-6471 \\
\hline $\begin{array}{l}\text { PV Fundamental and } \\
\text { Exploratory Research }\end{array}$ & Robert D. McConnell & (303) 384-6419 \\
\hline $\begin{array}{l}\text { PV Electronic Materials and } \\
\text { Devices }\end{array}$ & John P. Benner & (303) 384-6496 \\
\hline $\begin{array}{l}\text { PV Measurements and } \\
\text { Characterization }\end{array}$ & Lawrence L. Kazmerski & (303) 384-6600 \\
\hline Thin Film PV Partnership & Ken Zweibel & (303) 384-6441 \\
\hline PVMaT & C. Edwin Witt & (303) 384-6402 \\
\hline
\end{tabular}




\begin{tabular}{|l|l|c|}
\hline \multicolumn{1}{|c|}{ Project } & \multicolumn{1}{c|}{ Leader } & Telephone \\
\hline $\begin{array}{l}\text { PV Engineering and } \\
\text { Reliability }\end{array}$ & Roland L. Hulstrom & (303) 384-6420 \\
\hline $\begin{array}{l}\text { PV Domestic Applications } \\
\text { and Markets }\end{array}$ & John Thornton & (303) 384-6469 \\
\hline $\begin{array}{l}\text { PV International } \\
\text { Applications and Markets }\end{array}$ & Roger Taylor & (303) 384-7389 \\
\hline
\end{tabular}

\subsection{Key FY 1997 Accomplishments}

Table 1.2-1 describes some key achievements in the NREL PV Program during FY 1997. The accomplishments are grouped by the eight main project areas of the program.

\section{Table 1.2-1. Key Achievements}

PV Fundamental and Exploratory Research Project

\begin{tabular}{|l|l|}
\hline NREL & $\begin{array}{l}\text { Received R\&D } 100 \text { award for PV Optics, a software package for optical } \\
\text { design of solar cells and modules. }\end{array}$ \\
\hline NREL & $\begin{array}{l}\text { Performed first-principles theoretical calculations to study the electronic } \\
\text { structure of CuInSe } 2 \text {, CuGaSe } \\
\text { higher-efficiency, more-stable solar cells. }\end{array}$ \\
\hline NREL & Investigated four novel growth methods for thin silicon films or filaments. \\
\hline NREL & $\begin{array}{l}\text { In collaboration with Dr. M. Grätzel, NREL researchers fabricated a dye- } \\
\text { sensitized photochemical cell with 10\% efficiency (NREL-verified). }\end{array}$ \\
\hline NREL & $\begin{array}{l}\text { NREL studies in solid-state spectroscopy provided strong evidence of Te } \\
\text { precipitates in the microstructure of CdTe films. This work could lead to } \\
\text { more-stable or -efficient CdTe solar cells. }\end{array}$ \\
\hline $\begin{array}{l}\text { University } \\
\text { of } \\
\text { Washington }\end{array}$ & $\begin{array}{l}\text { Researchers developed a solid hole conductor to replace the liquid } \\
\text { electrolyte for accepting injected holes from the oxidized dye in a dye- } \\
\text { sensitized photochemical cell. }\end{array}$ \\
\hline $\begin{array}{l}\text { Texas } \\
\text { Southern } \\
\text { University }\end{array}$ & $\begin{array}{l}\text { TSU developed a highly successful four-week Renewable Energy and } \\
\text { Environmental Protection Academy for inner-city high school students, } \\
\text { including an exchange visit with Port Elizabeth Technikon in Port Elizabeth, } \\
\text { South Africa, for honors high-school students. }\end{array}$ \\
\hline $\begin{array}{l}\text { Research } \\
\text { Triangle } \\
\text { Institute }\end{array}$ & $\begin{array}{l}\text { Completed their successful demonstration of high-efficiency GaAs cells on } \\
\text { sub-mm grain-size Ge by exploring the use of lower-cost substrates such as } \\
\text { glass and molybdenum foils. }\end{array}$ \\
\hline
\end{tabular}

PV Electronic Materials and Devices Project

\begin{tabular}{|l|l|}
\hline NREL & $\begin{array}{l}\text { Developed an electrodeposition process for } \mathrm{CuInSe}_{2} \text {, yielding cells with } \\
\text { efficiencies of } 14.1 \% .\end{array}$ \\
\hline NREL & $\begin{array}{l}\text { Developed a model for the Staebler-Wronski metastability that accounts for } \\
\text { nearly all experimental results. }\end{array}$ \\
\hline
\end{tabular}




\begin{tabular}{|l|l|}
\hline NREL & $\begin{array}{l}\text { Explored dry processes to replace chemical-bath deposition of CdS or } \\
\mathrm{CdCl}_{2} \text { treatment of CdTe. }\end{array}$ \\
\hline NREL & $\begin{array}{l}\text { Explored properties and processing of nanoparticle precursors in inks for } \\
\text { preparation of semiconductors, conducting oxides, and solar-cell contacts. }\end{array}$ \\
\hline NREL & Continued development of high-rate, hot-wire deposition of silicon films. \\
\hline
\end{tabular}

PV Measurements and Characterization Project

\begin{tabular}{|l|l|}
\hline NREL & $\begin{array}{l}\text { Performed more than 40,000 measurements on some 15,000 samples } \\
\text { representing every photovoltaic technology. }\end{array}$ \\
\hline NREL & $\begin{array}{l}\text { Produced } 65 \text { journal, conference proceeding, and book publications with } \\
\text { some 70\% of these done in collaboration with other NREL and subcontract } \\
\text { researchers. }\end{array}$ \\
\hline NREL & $\begin{array}{l}\text { Developed large-area, continuous, solar simulator for the evaluation of cells } \\
\text { and modules under standard conditions for flat-plate and concentrator } \\
\text { technologies. }\end{array}$ \\
\hline NREL & $\begin{array}{l}\text { Brought on-line a time-of-flight secondary-ion mass spectrometer for high- } \\
\text { resolution determination of impurities in cell materials. }\end{array}$ \\
\hline NREL & Developed a large-area laser scanner for versatile module diagnostics. \\
\hline NREL & $\begin{array}{l}\text { Developed a scanning micro-FTIR system for examination of insulators } \\
\text { through conductors. }\end{array}$ \\
\hline NREL & $\begin{array}{l}\text { Developed a near-field scanning optical microscope for nanoscale imaging } \\
\text { and spectroscopic studies of semiconductors. }\end{array}$ \\
\hline
\end{tabular}

Thin Film PV Partnership Project

\begin{tabular}{|l|l|}
$\begin{array}{l}\text { Siemens } \\
\text { Solar } \\
\text { Industries }\end{array}$ & $\begin{array}{l}\text { Produced the first, commercially available CuInSe } \\
\text { CIS modules (up to 1 square foot) were measured at NREL to be about } \\
10 \% \text { efficient. }\end{array}$ \\
\hline $\begin{array}{l}\text { Solar Cells } \\
\text { Inc. }\end{array}$ & $\begin{array}{l}\text { Developed a modified process for depositing CdS/CdTe at high controllable } \\
\text { rates, opening up the possibility of large-scale, low-cost manufacturing of } \\
\text { CdTe products. }\end{array}$ \\
\hline $\begin{array}{l}\text { CdTe } \\
\text { National } \\
\text { R\&D Team }\end{array}$ & $\begin{array}{l}\text { Focused on determining the key issues of CdTe device degradation under } \\
\text { accelerated testing. }\end{array}$ \\
\hline $\begin{array}{l}\text { United } \\
\text { Solar }\end{array}$ & $\begin{array}{l}\text { Built and made fully operational the first, multimegawatt (5 MW) } \\
\text { amorphous silicon production plant in the world. }\end{array}$ \\
\hline Solarex & $\begin{array}{l}\text { Built and nearly completed the shakedown of the largest (10 MW) } \\
\text { amorphous silicon manufacturing facility in the world. }\end{array}$ \\
\hline NREL & $\begin{array}{l}\text { Re-competed the entire Thin Film Program in a national competition } \\
\text { yielding over 60 proposals from companies and universities, leading to } \\
\text { awards to 36 organizations. }\end{array}$ \\
\hline
\end{tabular}

PVMaT Project

\begin{tabular}{|l|l|}
\hline NREL & $\begin{array}{l}\text { Issued the Phase 5A Request for Proposals with somewhat more emphasis } \\
\text { on specific problems amenable to manufacturing R\&D solutions. }\end{array}$ \\
\hline
\end{tabular}




\begin{tabular}{|l|l|}
\hline $\begin{array}{l}\text { PVMaT } \\
\text { industrial } \\
\text { participants }\end{array}$ & $\begin{array}{l}\text { "Average" module manufacturing costs have been reduced 32\% and total } \\
\text { manufacturing capacity for 12 PVMaT industrial participants has more than } \\
\text { doubled in the last three years. }\end{array}$ \\
\hline
\end{tabular}

\section{PV Engineering and Reliability Project}

\begin{tabular}{|l|l|}
\hline NREL & $\begin{array}{l}\text { Completed and implemented several new and expanded testing capabilities, } \\
\text { including the Outdoor Accelerated Test System, a High-Voltage Stress test, } \\
\text { the Performance and Energy Ratings Testbed, the Large-Scale Matrix } \\
\text { Experiment, the High-Flux Solar Furnace Accelerated Exposure Testbed, } \\
\text { the PV System Performance Characterization Flexible Testbed, and the } \\
\text { Multipyranometer Array. }\end{array}$ \\
\hline NREL & Over 72 PV modules were tested both indoors and outdoors. \\
\hline NREL & $\begin{array}{l}\text { A set of NREL-developed formulations for PV module encapsulants were } \\
\text { shown to have superior photo-thermal stability and adhesion properties. }\end{array}$ \\
\hline NREL & $\begin{array}{l}\text { Accelerated sun exposure tests of cerium-containing glass revealed it } \\
\text { develops improved UV-filtering protection properties. }\end{array}$ \\
\hline NREL & $\begin{array}{l}\text { Outdoor performance monitoring of 8 PV systems was continued and 16 } \\
\text { professional engineering test reports were provided to 7 PV manufacturers. }\end{array}$ \\
\hline NREL & $\begin{array}{l}\text { Conducted the annual PV Performance and Reliability Workshop, with } \\
\text { more than 125 participants. }\end{array}$ \\
\hline NREL & $\begin{array}{l}\text { Published a 150-page "Radiometric Measurements Guide for Photovoltaic } \\
\text { Performance Measurements." }\end{array}$ \\
\hline NREL & $\begin{array}{l}\text { Prepared color and monochrome contour maps of annual global horizontal } \\
\text { and direct-normal solar irradiance for each state of the United States. }\end{array}$ \\
\hline
\end{tabular}

PV Domestic Applications and Markets Project

\begin{tabular}{|l|l|}
\hline NREL & $\begin{array}{l}\text { Developed consumer economics and policy analysis as a foundation for the } \\
\text { Million Solar Roofs Initiative. }\end{array}$ \\
\hline NREL & $\begin{array}{l}\text { Provided analysis and market development support to all potential Million Solar } \\
\text { Roofs partners. }\end{array}$ \\
\hline NREL & $\begin{array}{l}\text { Provided training and support to federal, state, and local organizations in the } \\
\text { selection and application of photovoltaics. }\end{array}$ \\
\hline NREL & $\begin{array}{l}\text { NREL-cost-shared exhibits were on display at events whose attendance totaled } \\
\text { more than 750,000 in FY 1997. }\end{array}$ \\
\hline $\begin{array}{l}\text { Sunrayce } \\
\text { participants }\end{array}$ & $\begin{array}{l}\text { Over 1,200 college students participated directly with 36 solar cars in this event } \\
\text { starting in Indianapolis, Indiana, and finishing in Colorado Springs, Colorado. } \\
\text { Hundreds of thousands of people saw or heard of this event. }\end{array}$ \\
\hline NREL & $\begin{array}{l}\text { Supported the Corporation for Solar Technology in its efforts to develop the } \\
\text { Solar Enterprise Zone at the Nuclear Weapons Test Site near Las Vegas. }\end{array}$ \\
\hline
\end{tabular}

PV International Applications and Markets Project

\begin{tabular}{|l|l|}
\hline Brazil & $\begin{array}{l}\text { Completed two 50-kW hybrid power systems as part of a joint project between } \\
\text { Brazil's utilities and DOE/NREL. }\end{array}$ \\
\hline China & $\begin{array}{l}\text { The Gansu solar home system project in westem China, supported by the U.S. } \\
\text { DOE and the Chinese Ministry of Agriculture, installed } 300 \text { household systems, } \\
\text { including 10 school systems. }\end{array}$ \\
\hline
\end{tabular}




\begin{tabular}{|l|l|}
\hline China & $\begin{array}{l}\text { NREL provided technical assistance to the Beijing Solar Energy Research } \\
\text { Institute, the Institute of Electrical Engineering of the Chinese Academy of } \\
\text { Sciences, and the Chinese Ministry of Agriculture. }\end{array}$ \\
\hline India & $\begin{array}{l}\text { The Ramakrishna Mission Project, jointly supported by the U.S. DOE and the } \\
\text { Ministry of Non-Conventional Energy Sources, procured } 30 \mathrm{~kW} \text { of PV systems } \\
\text { for } 300 \text { domestic home-lighting units, common-area lighting. vaccine } \\
\text { refrigeration, battery charging, a large water pump, electrification of a weaving } \\
\text { center, several youth centers, and a clinic facility. }\end{array}$ \\
\hline $\begin{array}{l}\text { South } \\
\text { Africa }\end{array}$ & $\begin{array}{l}\text { NREL provided program expertise to U.S.-South Africa Binational } \\
\text { Commission. }\end{array}$ \\
\hline NREL & $\begin{array}{l}\text { Provided support to the U.S. DOE Office of Utility Technologies for technical } \\
\text { assistance projects in India, Indonesia, Philippines, Nepal, and Zambia. }\end{array}$ \\
\hline NREL & $\begin{array}{l}\text { Initiated and completed successful new interagency agreement with USAID, } \\
\text { including assignment of NREL personnel to AID global offices. }\end{array}$ \\
\hline
\end{tabular}

\subsection{Technology Transfer}

Consistent with DOE policy, technology transfer within NREL's PV Program is defined as collaborative R\&D with industry to help industry commercialize products or services. An underlying theme of NREL technology transfer activities is the joint work accomplished by industry and NREL researchers focused on a common R\&D objective. Among government laboratories, seven principal tools affect technology transfer: subcontracted $R \& D$, cooperative $R \& D$, industry-sponsored $R \& D$, NREL user facilities, technology licensees, researcher exchanges, and information dissemination. NREL's PV Program conducts its technology transfer primarily through subcontracts, cooperative $R \& D$, and information dissemination.

\section{Subcontracts with Industry}

About $55 \%$ of the 84 subcontracts placed in FY 1997 were with the U.S. PV industry. The NREL funding of $\$ 16.3$ million to industry was supplemented by an additional $\$ 8.8$ million (estimated) of cost sharing by the industry partners. The majority of industry funding (and cost sharing) was in the PVMaT project (See Figure1.1-1). Technically knowledgeable NREL research managers participate in defining, evaluating, awarding, and negotiating statements of work submitted by industry researchers in competitive solicitations. Following subcontract awards, NREL subcontract managers direct and evaluate research progress by visiting subcontractor sites and evaluating subcontractor deliverables in the NREL laboratories.

\section{Cooperative R\&D}

NREL in-house researchers frequently perform informal cooperative R\&D with their industrial counterparts working under NREL subcontracts. These interactions have been ongoing since PV research started in 1977 at the Solar Energy Research Institute (now NREL). Most involve performance measurements and materials analyses performed with NREL's large and unique set of capabilities for PV efficiency and materials analysis. Informal cooperative R\&D, as distinguished from formal cooperative research and development agreements (CRADAs), is a natural complement 
to NREL's subcontracted PV program. Informal cooperative R\&D during FY 1997 included more than 40,000 measurements on some 15,000 PV material samples, devices, and modules on properties ranging from composition and microstructure to cell and module performance. More than 150 organizations from the worldwide PV community worked with NREL researchers in this fashion.

We performed research under 11 CRADAs during FY 1997: Solarex (a-Si scale-up), DayStar Technologies (CIS module), Golden Photon (CdTe nanoparticles), Ebara Solar (Si ribbon), Energy Conversion Devices (module characterization), Crystal Systems (Si materials processing), UniSil (Si technology support), Lockheed Missiles and Space (III-V solar cells), Spectrolab (III$\mathrm{V}$ tandem cells), Lockheed Martin (CIS modules), and a company wanting confidentiality for its CRADA on III-V solar cells.

\section{Information Dissemination}

Effective traditional ways of transferring technology are to report R\&D results to the technical community by publishing in scientific journals and by presenting at technical conferences and meetings. Section 8.0 contains a bibliography of FY 1997 publications, including subcontractor reports. During FY 1997, PV program staff helped organize several conferences and workshops, including the NREL/SNL Photovoltaics Program Review, the Seventh Annual NREL Workshop on Silicon Defects and Impurities, the NREL/SNL Photovoltaic Performance and Reliability Workshop, the NREL Photovoltaics Standards and Codes Forum, and NREL's First Conference on Future Generation Photovoltaic Technologies. These gatherings provide important opportunities for industry researchers to exchange technical information with NREL and university researchers.

NREL's program also distributes its quarterly newsletter, NREL PV Working With Industry, to approximately 1000 addresses. Adding to traditional information dissemination routes are several PV web sites. These include:

- NREL Photovoltaics (http://www.nrel.gov/pv),

- the DOE PV Program (http://www.eren.doe.gov/pv),

- the National Center for Photovoltaics (http://www.nrel.gov/ncpv),

- Measurements and Characterization (http://www.nrel.gov/measurements),

- Basic Sciences and Materials (http://www.nrel.gov/basic_sciences),

- $\quad$ the Renewable Resource Data Center (http://rredc.nrel.gov).

Notable in FY 1997 was NREL's rapid response to the President's Million Solar Roofs initiative by creating the Million Solar Roofs web site (http://www.MillionSolarRoofs.org).

\subsection{Conclusions}

This report reviews the in-house and subcontracted R\&D activities under the NREL PV Program in FY 1997. Major PV program thrusts during FY 1997 continued to be implemented based on DOE's National Photovoltaics Program Plan for 1996-2000.

In summary, the activities of the PV Program are to: conduct basic, applied, and engineering research; 
manage subcontracted R\&D projects; perform research complementary to subcontracted work; develop and maintain state-of-the-art measurement and device capabilities; develop PV manufacturing technology and modules; transfer results to industry; and evolve viable partnerships for PV systems and market development.

This report describes the in-house and subcontracted R\&D activities, many of which encompass close collaborations between NREL and outside researchers.

The research activities under the program are summarized under the eight project areas in the following sections: PV Fundamental and Exploratory Research, PV Electronic Materials and Devices, PV Measurements and Characterization, Thin Film PV Partnership, PVMaT, PV Engineering and Reliability, PV Domestic Applications and Markets, and PV International Applications and Markets. The sections include a brief overview that includes the objectives, approaches, and some key developments. Following that are technical summaries of the in-house and subcontract activities. The subcontract sections were provided by the subcontractors themselves. Section 7.0 provides a list of FY 1997 subcontracts and Section 8.0 lists major research publications and subcontractor reports. 
2.0 PV Fundamental and Exploratory Research Project 


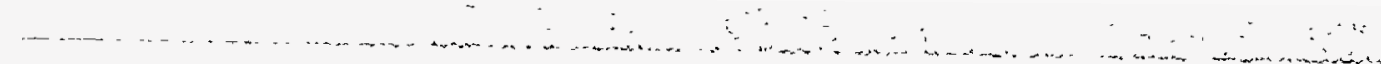




\title{
2.0 PV FUNDAMENTAL AND EXPLORATORY RESEARCH-Introduction
}

\author{
Robert McConnell
}

The Photovoltaic (PV) Fundamental and Exploratory Research Project supports long-term research and development (R\&D) needed to keep the pipeline full of leading-edge technologies for the U.S. PV industry, to develop future-generation PV technologies, and to achieve the longterm goals of the DOE Photovoltaics Program. This project develops and maintains NREL capabilities in solid-state theory of novel PV materials and devices, photochemical solar cell development, crystalline silicon materials research, and solid-state spectroscopy. It also supports the Historically Black Colleges and Universities program. During fiscal year 1997, several subcontracts ended which had been awarded under earlier DOE programs directed towards university participation in PV R\&D, novel PV ideas, and high-efficiency solar cells based on GaAs and GaAs alloys. The following summaries present the highlights of these NREL and subcontracted R\&D activities.

Because earlier subcontract activities in these project areas approached completion in 1997, NREL held its first conference on Future-Generation Photovoltaic Technologies in March 1997 to identify possible research topics and capabilities for a potential solicitation of research proposals in fiscal year 1998. The emphasis at the conference was on technical discussion and assessment of recent achievements for PV technologies that have a long-term potential to simultaneously achieve high performance and very low cost. These future-generation PV technologies were those not in production and not expected to enter production soon because additional long-term R\&D and innovation were needed. The conference proceedings, published by the American Institute of Physics, includes articles from many of the world's best PV researchers on such topics as dye-sensitized photochemical cells, low-cost substrates for single-crystal films, new thermophotovoltaic concepts, innovative concentrator approaches, the use of nanocrystalline materials in solar cells, organic semiconductor solar cells, new PV materials, and a fresh look at some old PV concepts. Many of the presentations referenced a long history for their ideas; new technologies and discoveries might now make some of these concepts feasible. The Conference on Future-Generation Photovoltaic Technologies demonstrated that there are many highly capable researchers interested in the long-term $R \& D$ needed to develop future-generation PV technologies.

This project also helps coordinate and integrate the fundamental and exploratory research sponsored by the DOE PV Program, with relevant R\&D sponsored by the DOE Office of Energy Research. A high-efficiency PV project was formalized within DOE's Center of Excellence for the Synthesis and Processing of Advanced Materials. The project focuses on two areas: 1)†silicon-based thin films, in which key scientific and technological problems involving amorphous and polycrystalline silicon thin films are addressed and 2) next-generation thin-film photovoltaics, which is concerned with advances in materials and physics of nonsilicon-based materials for solar cells. Project meetings are held during the year to share research and encourage collaboration among 20 research projects involving national laboratories and universities funded by either of the DOE programs. 
Highlights of the fiscal year 1997 research activities for this project include the following:

- PV Optics, a software package for optical design of solar cells and modules, was developed. This easy-to-use and versatile package is suitable for the design of thin cells (such as amorphous silicon and multijunction devices) as well as thick cells such as those based on silicon wafers. The software calculates parameters such as reflectance, transmittance, and absorbance in each semiconductor layer, as well as Maximum Achievable Current Density and optical loss in metallic reflectors or contacts. This software received the R\&D 100 Award in 1997.

- The subcontract program in Silicon Materials Research ended, giving way to a new competitive procurement. This five-year program produced some of the most valuable results in impurity gettering and hydrogen passivation of impurities and defects. Some of the processes developed under this program have led to the highest laboratory cell efficiencies on commercial PV silicon and are being adopted by the PV industry to improve commercial cell efficiencies

- NREL solid-state theorists performed first-principles theoretical calculations to study the electronic structure of $\mathrm{CuInSe}_{2}$ (CIS), $\mathrm{CuGaSe}_{2}$ (CGS), and their alloys (CIGS). Their theoretical results await experimental testing and could lead to higher efficiency, more stable, CIGS solar cells.

- NREL crystalline silicon researchers investigated four novel growth methods for thin silicon films or filaments. In addition to estimating throughputs (in $\mathrm{cm}^{2} / \mathrm{min}$ ), they performed exploratory experiments for each method.

- NREL silicon device modelers made progress in understanding the mechanism of hydrogen diffusion and passivation in silicon. They also hosted a workshop focusing on defects and impurities in silicon.

- NREL photochemical scientists, in collaboration with Dr. M. Grätzel, fabricated a dyesensitized photochemical cell with $10 \%$ efficiency (NREL-verified) for conversion of sunlight to. electricity.

- NREL studies in solid-state spectroscopy provided strong evidence of Te precipitates in the microstructure of $\mathrm{CdTe}$ films. This work could lead to more stable or more efficient $\mathrm{CdTe}$ solar cells.

- University of Washington researchers made progress in their efforts to improve the stability and efficiency of dye-sensitized PV cells by replacing the liquid electrolyte with a solid hole conductor, which can accept injected holes from the oxidized dye.

- Texas Southern University (TSU) leveraged its undergraduate PV associates program by obtaining support from the Electric Power Research Institute and Houston Lighting and Power to install a 4-kilowatt PV array, including batteries, inverters, and a variety of DC and AC appliances. TSU also developed a highly successful four-week Renewable Energy and Environmental Protection Academy for inner-city high school students, including an exchange 
visit with Port Elizabeth Technikon in Port Elizabeth, South Africa, for honors high school students.

- Research Triangle Institute closed out its successful demonstration of high-efficiency GaAs cells on sub-millimeter, grain-size Ge by exploring the use of lower-cost substrates such as glass and molybdenum foils. Se-passivation of grain boundaries and higher growth temperatures led to improved lifetimes in GaAs thin films on molybdenum. 


\section{Crystalline Silicon In-House Research}


Title:

Organization:

Contributors:
Crystalline Silicon Materials Research

Basic Sciences Center, Crystal Growth and Devices Team

National Renewable Energy Laboratory, Golden, CO

\section{Introduction}

Our research on crystalline silicon (c-Si) materials was in two task areas. Task 1 emphasizes research on innovative approaches to $\mathrm{Si}$ crystal growth methods, which complements work in industry that examines next-generation technologies. These new crystal growth methods have potentially superior throughput, reduced energy and/or materials cost, and/or improved conversion efficiency compared to existing approaches [1].

Task 2 seeks to develop a fundamental understanding of the role of defects and impurities in c-Si materials and device processing [2]. The research in this task helps ameliorate the deleterious effects of defects and impurities in c-Si solar cells, which is an important objective with high industry relevance because the concentrations of defects and impurities are frequently increased by the use of lower-cost Si feedstock and c-Si growth methods. Major activities in this task include generation and characterization of samples with controlled concentrations of dopants, impurities, and defects, and research on methods to getter impurities and passivate defects.

Task 1: Novel c-Si Growth Methods (Milestone: Compare three novel c-Si growth methods)

Four novel growth methods were investigated in FY 1997. These include: (1) thin-layer Si liquidphase epitaxy (LPE), (2) thin-layer Si electrodeposition (ED), (3) silicon filament (SiFi) growth, and (4) silicon thin-layer growth by plasma-enhanced chemical vapor transport (PECVT) with a novel transport agent. These were compared with each other and also against the benchmark of existing c-Si technology and thin-film technology. The most relevant parameter for gigawatt (GW)-scale future photovoltaic (PV) manufacturing plants is throughput (and, of course, cost). In this regard, approximate throughputs of existing technologies are shown in Table 1 (per unit of equipment, not including equipment turn-around time and downtime).

LPE on Metallurgical Grade (MG)-Si [3,4]

Our main recent focus was on understanding the limitations of the $\mathrm{MG}-\mathrm{Si}$ substrate. Through diagnostic solar cell studies, we verified that residual $\mathrm{SiC}$ particles in the $\mathrm{MG}-\mathrm{Si}$ are acting as shunts and limiting performance relative to what we obtained for single-crystal Si substrates. We made a few attempts to reduce the SiC density but have not yet made diagnostic cells on LPE layers grown on the new material. We see two viable ways to directly form MG-Si sheet substrates from melted MG-Si. One is expected to have $\sim 600 \mathrm{~cm}^{2} / \mathrm{min}$ ultimate throughput capability/unit and a high probability of reduced or eliminated $\mathrm{SiC}$ particles. The other is expected
Table 1. Approximate Throughputs for Various Growth

Methods

\begin{tabular}{|l|r|}
\hline Method & $\begin{array}{r}\text { Throughput } \\
\left(\mathrm{cm}^{2} / \mathrm{min}\right)\end{array}$ \\
\hline Czochralski Pulling & 600 \\
Float Zoning & 1,500 \\
Casting/Directional Solidification & 1,700 \\
Electromagnetic Continuous Casting & 3,000 \\
Wire Sawing & 1,200 \\
Web, ESP, String Ribbon & 20 \\
EFG Nonagons/Cylinders & 200 \\
Silicon Film@, Product 1 & 3,000 \\
Thin-Film Amorphous Si & $30-600$ \\
Thin-Film CdTe & $100-800$ \\
Thin-Film CIS & $60-130$ \\
\hline
\end{tabular}


to have $3000 \mathrm{~cm}^{2} / \mathrm{min}$ ultimate throughput capability/unit, but with no clear way to eliminate the SiC. Either would require extensive development, which is not planned at this time with currently available resources. The LPE process itself is expected to be capable of $\sim 600 \mathrm{~cm}^{2} / \mathrm{min}$ ultimate throughput/unit at our demonstrated growth rate of $1 \mathrm{micron} / \mathrm{min}$ and temperature of $850^{\circ}-900^{\circ} \mathrm{C}$. Non-silicon substrates are problematic for LPE, but we have begun to explore a glass-composite substrate formed by screen printing and firing that we are developing in our diagnostic device activity. Exploration of this substrate will continue in FY 1998. We have the screen printer in place, and have identified a frit formulation. This substrate will also be explored with our other thin-layer growth approaches. Only low emphasis will be placed on LPE growth in FY 1998 (T.H. Wang, T.F. Ciszek, Y.S. Tsuo, and M. Landry).

\section{Electrodeposition [5]}

We showed thin-layer Si electrodeposition to be capable of growing single-crystal layers on singlecrystal substrates, epitaxial layers (replicating the grains) on MG-Si substrates, and polycrystalline layers with grain size on the order of a few microns on sheet metal (Ag) substrates. Our fused-salt mixture of $\mathrm{KF}$ and $\mathrm{LiF}$ solvent with $\mathrm{K}_{2} \mathrm{SiF}_{6}$ solute, in conjunction with silicon electrodes, showed evidence (via secondary ion mass spectroscopy [SIMS] analysis) of self-purification with the lowpurity $(99 \%)$ chemicals used. However, $\mathrm{Li}$ doping is present and tends to make the grown layers $\mathrm{n}$ type with $\sim 0.3 \mathrm{ohm}-\mathrm{cm}$ resistivity. We achieved higher growth rates ( 45 microns $/ \mathrm{h}$ ) than those previously reported, at a growth temperature of $\sim 850^{\circ} \mathrm{C}$. Future work should focus on the purification effect, use of low-grade input silicon feedstock, control (elimination) of unwanted n-type doping, alternate fused-salt compositions for use with lower-temperature substrates, material analysis, and diagnostic device fabrication and analysis. We believe the ultimate throughput of this growth method to be on the order of several thousand $\mathrm{cm}^{2} / \mathrm{min}$. The work was done largely through an Association of Western Universities fellowship supporting a graduate student (J. Moore, T.H. Wang, M. Heben, and T.F. Ciszek).

\section{Silicon Filaments (SiFis) [G]}

The silicon filament is an interesting form of silicon with many potential applications, such as infrared light guides, semiconductor delay lines, and linear-concentrator solar cell arrays. We grew high-purity, dislocation-free, single-crystal filaments in diameters of 0.3 to $2 \mathrm{~mm}$ by a micro float-zone pedestal (FZP) method, at growth rates of as much as $30 \mathrm{~mm} / \mathrm{min}$. These filaments had minority charge carrier lifetimes of as much as $600 \mu \mathrm{sec}$. Si dendrite filaments (flat ribbons) with widths of 0.5 to $2 \mathrm{~mm}$ were also grown. These have a single-crystal (111) face, but with some (111) internal twins and dislocations. Maximum lifetimes here were about $50 \mu \mathrm{sec}$. The advantage of the dendrites is high growth rate (estimated to be on the order of 15 meters/h). Finally, we grew small, hollow Si tubular filaments from a capillary shaping die. The multicrystalline tubes had lifetimes of as much as $40 \mu \mathrm{sec}$. Their advantage is access to the internal surface, e.g., for rear contacts to concentrator solar cells. Ultimate linear growth rates for the non-dendritic filaments are estimated to be on the order of 3 meters/h, and throughput may be a problem. The throughput of the dendrite filaments may be viable for PV use, depending on the concentration factor (Ciszek and Wang).

\section{PECVT}

An experimental PECVT apparatus for silicon thin-layer growth was built, tested, and debugged. Studies in the 70s and early 80 s showed a potentially very fast growth rate of silicon by chemical vapor transport in a close-spaced configuration. The growth rate is mainly determined by dissociation of silicon-containing species on a substrate surface. We are investigating the use of a plasma to enhance this dissociation process, using a novel gas species with low breakdown potential (in a plasma) to see if 
high growth rate is achievable. Only preliminary, exploratory experiments have been conducted so far, in which a thin and uniform film of silicon on a single-crystal substrate was obtained. It is too early to estimate a throughput for this approach (Wang, Ciszek, and Landry).

\section{Task 2: Defect and Impurity Studies in Crystalline Silicon}

Iron-Dopant Pair Defects [7] (Milestone: Generate, characterize, and fabricate devices on Fe-doped crystals with various p-type resistivities)

Both single-crystal, dislocation-free, and multicrystalline silicon ingots co-doped with $\mathrm{Fe}$ and $\mathrm{Ga}$ were grown. The ranges of $\mathrm{Fe}$ and $\mathrm{Ga}$ uniform dopant concentrations produced by pill doping were $4 \times 10^{11}$ $\mathrm{cm}^{-3}$ to $4 \times 10^{15} \mathrm{~cm}^{-3}$ and $3 \times 10^{15} \mathrm{~cm}^{-3}$ to $5 \times 10^{16} \mathrm{~cm}^{-3}$, respectively. Float zoning was used to produce controlled samples. Sample wafers from these ingots were distributed to a number of universities for study, including University of California at Berkeley, Massachusetts Institute of Technology (MIT), and Georgia Institute of Technology (GIT). Interest has been expressed by other universities as well. In addition, we have made some definitive characterization studies of these materials at NREL. At the 26th Institute of Electrical and Electronics Engineers (IEEE) PV conference, we reported the lifetime degradation effect of Fe-Ga defect pairs (see Table 2), and the mechanisms for it [5]. We compared these defects to the more thoroughly studied $\mathrm{Fe}-\mathrm{B}$ defect pairs and contrasted their behavioral response. We also, in collaboration with GIT, refined the energy levels and captured cross sections of the Fe-Ga pair defect. We provided the first experimental estimates of binding energies for the defects (Ciszek and Wang).

$\mathrm{Ga}$ is an important dopant for obtaining uniform dopant distributions in continuous growth

Table 2. Minority-Carrier Lifetime of Dislocation-free $<100>$ FZ Single Crystals Doped With Fe and $\mathrm{Ga}$

\begin{tabular}{|l|c|c|c|c|}
\hline Crystal & $\begin{array}{c}\text { Resistivity } \\
(\Omega-\mathrm{cm})\end{array}$ & $\begin{array}{c}\mathrm{Ga} \\
\left(\mathrm{cm}^{-3}\right)\end{array}$ & $\begin{array}{c}\text { Fe Target } \\
\left(\mathrm{cm}^{-3}\right)\end{array}$ & $\begin{array}{c}\text { Lifetime } \\
(\mu \mathrm{s})\end{array}$ \\
\hline $41121 \mathrm{a}$ & 3.5 & $3.8 \times 10^{15}$ & 0 & $>1400$ \\
\hline $51129-1$ & 24,000 & 0 & $1.2 \times 10^{14}$ & 12 \\
\hline 70515 & 6 & $2.2 \times 10^{15}$ & $4.8 \times 10^{11}$ & 2.2 \\
\hline $51212-\mathrm{la}$ & 4.1 & $3.3 \times 10^{15}$ & $1.3 \times 10^{14}$ & 0.40 \\
\hline $51212-\mathrm{lb}$ & 1.4 & $1 \times 10^{16}$ & $1.3 \times 10^{14}$ & 0.34 \\
\hline
\end{tabular}
processes such as ribbon crystallization, float-zoning, or electromagnetic continuous casting. It also does not exhibit light degradation of PV performance such as that reported for boron. Fe is a common impurity in $\mathrm{Si}$, so an understanding of the interactions between it and $\mathrm{Ga}$ is important. We also began diagnostic-device studies to document the effects of Fe-Ga pairs on solar cell parameters (Tsuo).

In related work [8,9], we made significant advances in finite element modeling (FEM) of minoritycarrier lifetime measurements in various $\mathrm{Si}$ sample geometries and crystal structures (such as grain boundaries), and with different light sources. This work pointed out large discrepancies between the widely used asymptotic approximation and the FEM simulations, and was useful in defining regimes in which meaningful lifetime values can be measured. Lifetime is the major tool we use to gauge the effects of defects and impurities in the controlled Si samples we fabricate (Wang and Ciszek).

Defect Gettering [10] (Milestone: Demonstrate that regions with a high density of defect clusters in multicrystalline silicon can be gettered by optical processing)

Defect clusters are agglomerates of dislocation networks, stacking faults, and (in some cases) grain boundaries. They are a major bottleneck in improving performance of solar cells fabricated on commercial substrates because they are sites for impurity precipitation during cooldown of an ingot and are difficult to getter and/or passivate. Our network analysis has shown that, in a solar cell, the 
defect clusters shunt the device and act as "sinks" that dissipate power internally within the cell. Thus, the bad regions exhibit very low minority-carrier diffusion lengths. The objective of our work is to improve the gettering ability of these defect clusters by dissolving the precipitates before or during gettering. However, dissolution of such precipitates in a conventional manner requires several hours at temperatures exceeding $1000^{\circ} \mathrm{C}$. However, by invoking nonequilibrium process conditions such optical processing (OP), impurity dissolution might be greatly enhanced. We intend to use such an approach for the dissolution and gettering of impurities.

Preliminary work performed this year has shown that $O P$ can enhance the diffusion length of all regions that have very low starting diffusion. A procedure that involves simultaneous use of $\mathrm{OP}$ and $\mathrm{Al}$ gettering has resulted in an increase in the average diffusion length from 7 to $30 \mu \mathrm{m}$. We also obtained similarly encouraging results by including hydrogen passivation as a part of gettering; the minoritycarrier diffusion length of typical samples increased from about 10 to $70 \mu \mathrm{m}$ (B.L. Sopori and J.M. Gee).

In related work [11], progress was made in understanding the mechanism of hydrogen diffusion and passivation in silicon. Experiments with damage-mediated diffusion of hydrogen in silicon from a molecular gas showed that a small amount of surface damage can act as a catalyst to facilitate deep diffusion of hydrogen (B.L. Sopori and M. Symko).

\section{Other Activities}

Our program hosts a workshop on crystalline-silicon PV technology, with a focus on defects and impurities. We also conduct funds-in projects for several c-Si PV companies to help improve crystal growth processes and develop $\mathrm{Si}$ ingot lifetime test instruments, and are involved in modeling of crystal growth, optical properties, and lifetime characterization.

\section{References}

1. T.F. Ciszek and J.M Gee, "Crystalline Silicon R\&D at the U.S. National Center for Photovoltaics." In Proceedings of the 14th European Photovoltaic Solar Energy Conference, Barcelona, Spain, June 30-July 4, 1997 (to be published).

2. T.F. Ciszek and T.H. Wang, "Silicon Defect and Impurity Studies Using Controlled Samples." In Proceedings of the 14th European Photovoltaic Solar Energy Conference, Barcelona, Spain, June 30-July 4, 1997 (to be published).

3. T.F. Ciszek and T.H. Wang, "Crystallization from High-Temperature Solutions of Si in Cu/Al Solvent." U.S. Patent No. 5,544,616 (1996).

4. T.H. Wang, T.F. Ciszek, C.R. Schwertfeger, H. Moutinho, and R. Matson, "Growth of Silicon Thin Layers on Cast MG-Si from Metal Solution for Solar Cells." Solar Energy Materials and Solar Cells; 41/42, pp. 19-30, (1996).

5. J.T. Moore, T.H. Wang, M.J. Heben, K. Douglas, and T.F. Ciszek, "Fused-Salt Electrodeposition of Thin-Layer Silicon." In 26th IEEE Photovaltaic Specialists Conference Record, Anaheim, California, 1997 (to be published, IEEE, New Jersey).

6. T.F. Ciszek and T.H. Wang, "Growth and Properties of Silicon Filaments for Photovoltaic Applications." In 26th IEEE Photovoltaic Specialists Conference Record, Anaheim, Califormia, 1997 (to be published, IEEE, New Jersey).

7. T.F. Ciszek, T.H. Wang, W.A. Doolittle, and A. Rohatgi, "Minority-Carrier Lifetime Degradation in Silicon Co-Doped with Iron and Gallium." In 26th IEEE Photovoltaic Specialists Conference Record, Anaheim, California, 1997 (to be published, IEEE, New Jersey). 
8. T.H. Wang and T.F. Ciszek, "Numerical Simulations of Transient Photoconductance Decay." In 26th IEEE Photovoltaic Specialists Conference Record, Anaheim, California, 1997 (to be published, IEEE, New Jersey).

9. T.H. Wang and T.F. Ciszek, "Effects of Sample Inhomogeneity and Geometry on Photoconductivity Decay." In Silicon Recombination Lifetime Characterization Methods, ASTM STP 1340, D.C. Gupta, F. Bacher, and W.H. Hughes, eds., American Society for Testing and Materials (to be published, 1998).

10. J.M. Gee and B.I. Sopori, "The Effect of Gettering on Areal Inhomogeneities in Large-Area Multicrystalline-Silicon Sólar Cells." In 26th IEEE Photovoltaic Specialists Conference Record, Anaheim, California, 1997 (to be published, IEEE, New Jersey).

11. B.L. Sopori, M. Symko, R. Reedy, K. Jones, and R. Matson, "Mechanisms of Hydrogen Diffusion in Silicon Solar Cells During Forming Gas Anneal." In 26th IEEE Photovoltaic Specialists Conference Record, Anaheim, California, 1997 (to be published, IEEE, New Jersey). 



\section{Crystalline Silicon Subcontracts}




\section{Title: $\quad$ Investigation of gettering mechanisms in crystalline silicon}

\section{Qrganization: Department of Mechanical Engineering and Materials Science,}

Duke University, Durham, North Carolina 27708

Contributors: T. Y. Tan and U. M. Gösele, principal investigators;

S. M. Joshi and P. S. Plekhanov, graduate students

\section{Objective}

The objectives of this program are to develop accurate physical models for gettering and apply the models to design efficient gettering processes for crystalline silicon materials. In this work, outstanding qualitative predictions of earlier simulations for aluminum gettering of gold in silicon have been confirmed. Furthermore, simulations in accordance with the present experimental conditions have been performed for the purpose of seeking quantitative fittings of the gettering model to experimental results. This is expected to provide information on silicon self-diffusion parameters, which will aid in further modeling efforts. In addition, modeling of void formation from vacancies during silicon crystal growth has been performed using the enthalpy of vacancy formation as a parameter, and comparison with experimental results from literature gives a bound for this parameter. The exact value of this parameter is undetermined in the literature, and a more accurate knowledge of this and other silicon self-diffusion parameters is sought for our modeling efforts.

\section{Aluminum gettering of gold in silicon}

Previous modeling [1] of $\mathrm{Al}$ gettering of $\mathrm{Au}$ in Si indicated that even if the Al gettering layer was present on only one surface of a Si wafer, Au would be gettered from near both wafer surfaces to progressively greater depth. In other words, proximity to a Si surface would control the gettering process rather than proximity to the gettering layer. Experiments were conducted [2] to test this prediction by first deliberately contaminating Si samples with $\mathrm{Au}$ and then performing Al gettering. Comparison of Au profiles before and after contamination and then after gettering has been performed using the Spreading Resistance Profiling (SRP) technique [3].

$125 \mathrm{~nm}$ of $\mathrm{Au}$ was evaporated onto single crystal p-type B-doped 10-40 $\Omega$-cm resistivity $530 \mu \mathrm{m}$ thick FZ Si wafers and then annealed at $950^{\circ} \mathrm{C}$ for $16 \mathrm{hr}$ and air-cooled. $15 \mu \mathrm{m}$ was etched off from both surfaces in two steps with aqua regia and RCA treatments at each step to remove the surface Au and leave Si to be $\sim 500 \mu \mathrm{m}$ thick. SRP samples were prepared from both as-received, and Audiffused and etched samples. From Au-diffused and etched samples, four pieces were selected and on three of these an Al layer of $1 \mu \mathrm{m}$ thickness was evaporated on one side. All four were sealed in evacuated quartz ampoules and annealed at $1000^{\circ} \mathrm{C}$ and water-quenched. The three samples with $\mathrm{Al}$ were annealed for $30 \mathrm{~min}, 2 \mathrm{hr}$ and $8 \mathrm{hr}$ respectively while the sample without Al, serving as a control, was annealed for $8 \mathrm{hr}$.

A comparison of SRP profiles of as-received sample to that after Au diffusion and etching clearly shows the U-shaped Au diffusion profile obtained, Fig. 1(a). The profile for the control sample, Fig. 1(b), shows that in the absence of the Al layer, there is no outdiffusion of Au from the Si and there is only flattening of the U-shaped profile. The profile for the $30 \mathrm{~min}$ Al gettered sample, Fig. 1(b), clearly shows that even though there is an Al layer on only one surface, Au is gettered from near both surfaces, but not from the interior. The profile for the $2 \mathrm{hr}$ Al gettered sample, Fig. 1(b), becomes quite close to the original sample profile, indicating that the Au concentration is now below the detection limit of the SRP technique at all depths.

Modeling based on our experimental conditions was performed by considering that Au diffused in $\mathrm{Si}$ via the kickout mechanism [4], which required three coupled differential equations for substitutional and interstitial $\mathrm{Au}, \mathrm{Au}_{\mathrm{s}}$ and $\mathrm{Au}_{\mathrm{i}}$, respectively, and $\mathrm{Si}$ self-interstitials, $I$. An $\mathrm{Al}$ gettering layer on one surface could be represented by a boundary condition at the Si/Al interface that the Au concentration on the Si side was in dynamic equilibrium with the Au concentration on the Al side. The model was implemented using the partial differential equation solver ZOMBIE [5]. First, the indiffusion of Au at $950^{\circ} \mathrm{C}$ was simulated and then, after taking into account the etching of $15 \mu \mathrm{m}$ from both surfaces, the subsequent $\mathrm{Al}$ gettering of $\mathrm{Au}$ from the same sample at $1000^{\circ} \mathrm{C}$ was simulated. 
The resulting profiles for $\mathrm{Au}_{\mathrm{s}}$ and $I$ are shown in Fig. 2(a) and Fig. 2(b) respectively. According to the kickout mechanism, $\mathrm{Au}$ primarily exists as $\mathrm{Au}_{\mathrm{s}}$ but diffuses primarily as $A u_{i}$, and the interchange between the two requires interaction with 1 . Thus during indiffusion, the changeover of $A u_{s}$ to $A_{i}$ creates a supersaturation of $I$, as seen in the simulation results for $\mathrm{Au}$ indiffusion at $950^{\circ} \mathrm{C}$. Since this supersaturation can only be relieved at the two surfaces in the absence of dislocations or grain boundaries, the characteristic $U$-shaped indiffusion profile of $\mathrm{Au}$ is obtained even when there is an $\mathrm{Au}$ source on only one wafer surface. On the other hand, during outdiffusion of Au to the gettering layer, the changeover of $\mathrm{Au}_{\mathrm{s}}$ to $\mathrm{Au}_{\mathrm{i}}$ creates an undersaturation of $I$ which can only be relieved at the surfaces. Therefore, Au can be quickly gettered from near both surfaces, but only slowly to greater depths from the sunfaces. This gives rise to the simulated and experimentally observed phenomenon that even though the $\mathrm{Al}$ gettering layer is present on only one surface, the gettering of $\mathrm{Au}$ is controlled by proximity to both surfaces. Conversion of SRP profiles to Au profiles followed by quantitative fitting of the simulations to the experiments is required to get an estimate of the selfinterstitial contribution to silicon self-diffusivity.

\section{Void nucleation and growth during silicon crystal growth}

Voids in $\mathrm{Si}$, formed during Si crystal growth, have been posing an ever increasing problem. If a void is located just below the gate of an MOS transistor, it can significantly reduce gate oxide breakdown strength [6]. This becomes especially crucial as, with the development of new technology, the dimensions of individual transistors decrease to those comparable with the sizes of the voids themselves. Further, voids were found to be more prone to formation in silicon crystals of large diameters $[7,8]$. Taking into account the modern trend towards using Si wafers of larger and larger diameters, an understanding of the mechanism of void formation becomes an important problem. Besides, the investigation into it provides us with new data about point defects in Si, namely their diffusivities and thermal equilibrium concentrations, the parameters that are very important for the modeling of many processes in Si, e.g. diffusion and precipitation of impurities, defect formation, etc. The existing data are very inconsistent which require further investigations.

During CZ crystal growth, the concentration of vacancies at the crystal growth front is at its thermal equilibrium value corresponding to the melting temperature. As the ingot is pulled up with its temperature decreased, the vacancies become frozen-in and supersaturated. This supersaturation can be relaxed by the formation of extended defects: voids or vacancy-type dislocation loops. We have calculated attainable supersaturation values and corresponding nucleation barriers for these extended defects and shown that only void can nucleate, but not dislocation loops. This is consistent with experimental observations.

The nucleation rate of voids has been calculated as function of temperature using enthalpy of vacancy formation as a parameter. Comparison of the calculated results with the experimental data provides us with the nucleation temperature range. The void growth process has also been modeled by numerically solving a system of coupled partial differential equations describing the void size and vacancy concentration profiles [9]. Comparison of the results of nucleation and growth modeling allowed us to narrow down the field of unknown parameters. We found that the experimentally observed void concentrations and sizes may be achieved if the enthalpy of vacancy formation is within the range 2.9 to $3.6 \mathrm{eV}$. The corresponding void nucleation temperature range is $980^{\circ}$ to $1080^{\circ} \mathrm{C}$.

\section{Conclusions}

Experiments of $\mathrm{Al}$ gettering of $\mathrm{Au}$ in $\mathrm{Si}$ have been performed that validate previous simulation predictions and allow for further refinement of the gettering model. Modeling of void nucleation and growth during the Si crystal growth process has been performed that, in combination with experimental data, allows to establish bounds on the enthalpy of vacancy formation.

\section{References}

1. R. Gafiteanu, U. M. Gösele and T. Y. Tan, in Defect and Impurity Engineered Semiconductors and Devices, edited by S. Ashok, J. Chevallier, I. Akasaki, N.M. Johnson, and B.L. Sopori (Mater. Res. Soc. Proc. 378, Pittsburgh, PA, 1995) p.297. 
2. S. M. Joshi, U. M. Gösele and T. Y. Tan, in Semiconductor Process and Device Performance Modeling, to be published as MRS Fall 97 Proceedings.

3. N. A. Stolwijk, J. Hölzl, W. Frank, E. R. Weber and H. Mehrer, Appl. Phys. A 39, 37 (1986).

4. U. Gösele, Wv. Frank and A. Seeger, Appl. Phys. 23, 361 (1980).

5. W. Jüngling, P. Pichler, S. Selberherr, E. Guerrero, and H. W. Pötzl, IEEE Trans. Electron, Dev. ED-32, 156 (1985).

6. J.-G. Park, H. Kirk, K.-C. Cho, H.-K. Lee, C.-S. Lee, and G. A. Rozgonyi, in Semiconductor Silicon 1994, edited by H. R. Huff, W. Bergholz, and K. Sumino (The Electrochem. Soc., Pennington, NJ, 1994) p.370.

7. M. Kato, Y. Ikida, and Y. Kitagawa, Jpn. J. Appl. Phys. 35, 5597 (1996).

8. T. Ueki, M. Itsumi, and T. Takada, Appl. Phys. Lett. 70, 1248 (1997).

9. P. S. Plekhanov, U. M. Gösele and T. Y. Tan, in Semiconductor Process and Device Performance Modeling, to be published as MRS Fall 97 Proceedings.

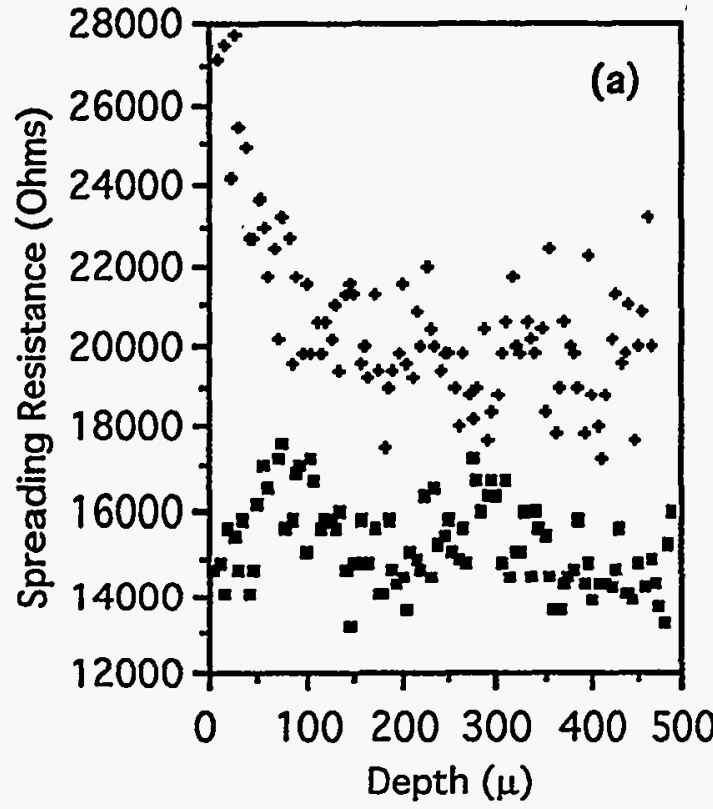

- Original wafer profile

+ Au-indiffused, $950^{\circ} \mathrm{C} / 16 \mathrm{hr}$

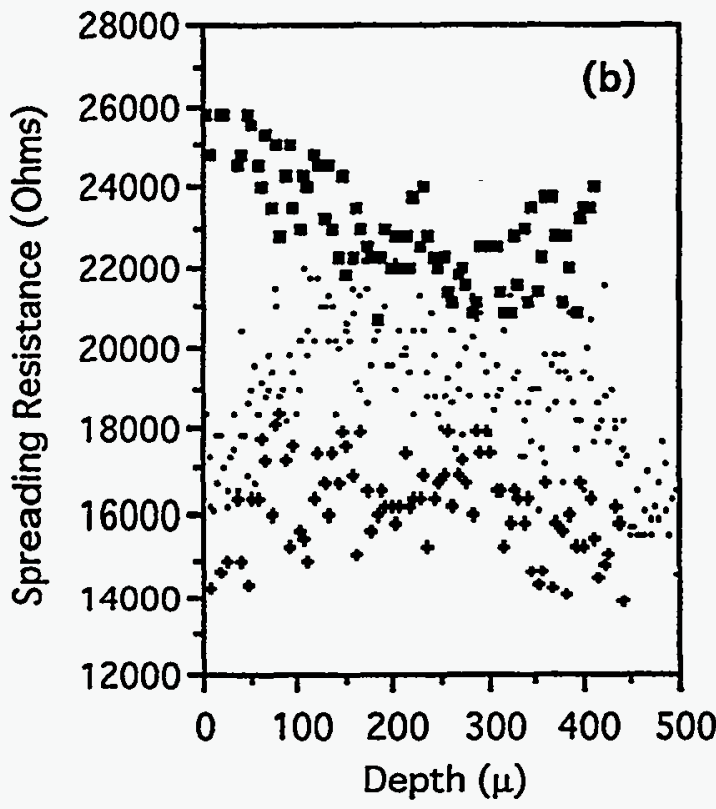

- Control, $1000^{\circ} \mathrm{C} / 8 \mathrm{hr}$

- Al-gettered, $1000^{\circ} \mathrm{C} / 30 \mathrm{~min}$

- Al-gettered, $1000^{\circ} \mathrm{C} / 2 \mathrm{hr}$

Fig. 1. Spreading resistance depth profiles of Si samples (a) from original wafer and after Au indiffusion at $950^{\circ} \mathrm{C}$ for $16 \mathrm{hr}$, (b) control annealed at $1000^{\circ} \mathrm{C}$ for $8 \mathrm{hr}$ and after Al gettering at $1000^{\circ} \mathrm{C}$ for $30 \mathrm{~min}$ and for $2 \mathrm{hr}$ respectively. All profiles except one were measured from the unpolished wafer surface (at $0 \mu \mathrm{m}$ depth). For the $30 \mathrm{~min} \mathrm{Al}$ gettering, two samples were taken from adjacent locations and profiles measured from both surfaces (i.e., from $0 \mu \mathrm{m}$ and $500 \mu \mathrm{m}$ ). The profile shown above is a composite of the two. For Au indiffusion as well as Al gettering, the metal films were deposited on the unpolished surface, i.e., on the left side of the profiles shown. 

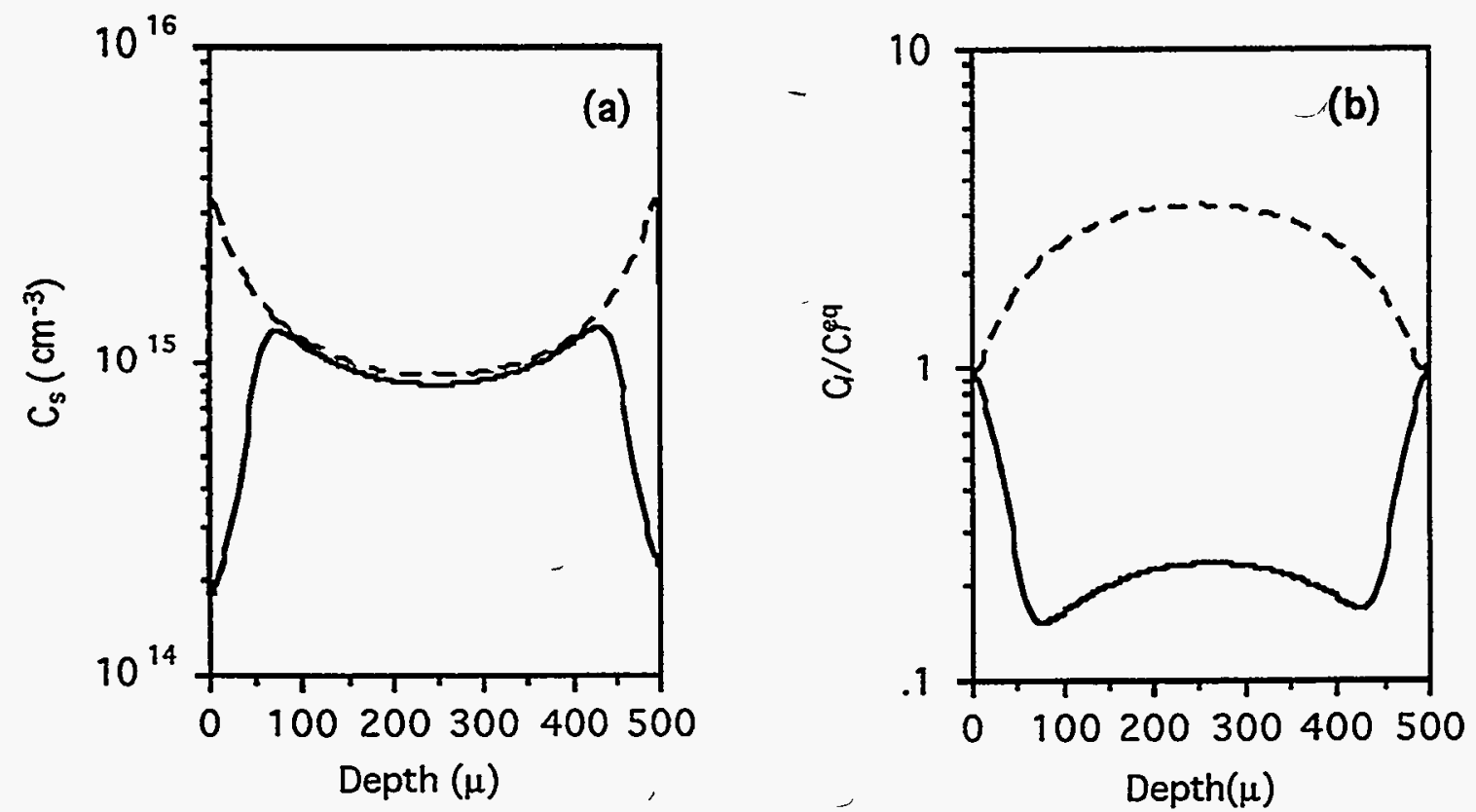

Au-indiffused, $950^{\circ} \mathrm{C} / 16 \mathrm{hr}$ - Al gettered, $1000^{\circ} \mathrm{C} / 2 \mathrm{hr}$

Fig. 2 Simulated profiles of (a) $\mathrm{Au}_{\mathrm{S}}$ and (b) $I$, after $950^{\circ} \mathrm{C} / 16 \mathrm{hr}$ anneal followed by removal of 15 $\mu \mathrm{m}$ from both surfaces and then after $1000^{\circ} \mathrm{C}, 2 \mathrm{hr}$ Al gettering following the etching. $\mathrm{C}_{I}$ is normalized by the thermal equilibrium $I$ concentration at $1000^{\circ} \mathrm{C}$. The $\mathrm{Al}$ gettering layer was on the left surface. 
Title:

Fundamental Research on Post-Growth Quality Enhancement Techniques

Organization:

School of Electrical and Computer Engineering Georgia Institute of Technology, Atlanta, Georgia 30332-0250

\author{
Contributors: $\quad$ A. Rohatgi, Director; P. Doshi, Research Engineer; \\ S. Narasimha and Jens Moschner; Graduate Research Assistants
}

\title{
Objective
}

The objective of this program is to investigate and optimize quality enhancement techniques, such as PECVD SiN deposition for surface and bulk defect passivation, phosphorus and aluminum and RTP treatments for defect and impurity gettering/passivation and integrate the best gettering and passivation techniques to achieve high-efficiency multicrystalline silicon (mc-Si) solar cell.

\section{Pre-Getter Anneal (PGA) Treatments for Impurity Release and Precipitate Dissolution}

The gettering process is comprised of three steps: 1) impurity release from the active area of a device, 2) impurity diffusion out of the active area, and 3) impurity capture in a region of the device where its effects are not detrimental to device performance [1]. In regions of mc-Si wafers where the defect density is high, the gettering process is release-limited. It has been suggested that the response of such regions can be improved by incorporating a pre-getter anneal (PGA) treatment prior to the conventional gettering step [2]. If the PGA is intense enough, it can provide the energy needed to release impurities from defective regions in the device. The released impurities can then be removed from the active area by the ensuing gettering step. It is important to note, however, that each step in this two step process must be designed for compatibility. For example, if the gettering step following the PGA is of insufficient intensity (in temperature and time), there will be a net accumulation of released impurities in previously unaffected regions of the device. On the other hand, if the PGA treatment is of insufficient intensity, the impurity release from defective regions of the material will not occur. The above concepts are depicted in the response surface model of Fig.1.

Table 2 shows the results of an RPGA experiment on HEM mc-Si. Four HEM samples in Table 1 were used. Samples were subjected to a rapid PGA (RPGA) treatment in an RTP unit for 2 minutes at $1050^{\circ} \mathrm{C}$ in an $\mathrm{N}_{2}$ ambient. At the end of the 2 minute period, the samples were quenched from $1050^{\circ} \mathrm{C}$ to $750^{\circ} \mathrm{C}$ in 10 seconds by turning off the tungsten halogen lamps in the RTP unit. The ensuing phosphorus gettering treatment consisted of a short diffusion $\left(840^{\circ} \mathrm{C}\right.$ for 20 minutes) using phosphorus solid sources. Matched samples were included at this stage in the process in order to establish the response of the baseline (non-RPGA) process. Each parameter in this table represents the average value taken from 12 solar cells across the sample.

Table 2. Results of Process I (RPGA followed by light phosphorus getter. Solar cells were measured without AR coatings.)

\begin{tabular}{l|cccc|cccc}
\hline & \multicolumn{3}{|c|}{ Without RPGA Treatment } & \multicolumn{4}{c}{ With RPGA Treatment } \\
$\begin{array}{l}\text { Sample/ } \\
\text { Ingot ID }\end{array}$ & $\begin{array}{c}\text { Voc } \\
(\mathbf{m V})\end{array}$ & $\begin{array}{c}\text { Jsc } \\
\left(\mathbf{m A} / \mathbf{c m}^{2}\right)\end{array}$ & FF & $\begin{array}{c}\text { Efficiency } \\
(\%)\end{array}$ & $\begin{array}{c}\text { Voc } \\
(\mathbf{m V})\end{array}$ & $\begin{array}{c}\text { Jsc } \\
\left(\mathbf{m A} / \mathbf{c m}^{2}\right)\end{array}$ & FF & $\begin{array}{c}\text { Efficiency } \\
(\%)\end{array}$ \\
\hline HEM 1HS7 & 577 & 20.3 & .781 & 9.1 & 584 & $\mathbf{2 1 . 9}$ & .792 & 10.1 \\
\hline HEM 1HS4 & 578 & 20.0 & .770 & 8.9 & 580 & 21.9 & .744 & 9.4 \\
\hline HEM 1HS8 & 559 & 21.6 & .560 & 6.8 & 563 & 20.4 & .613 & 7.0 \\
\hline HEM 50-1 & 574 & 22.2 & .752 & 9.6 & 570 & 21.1 & .784 & 9.4 \\
\hline
\end{tabular}

Two altogether different results are indicated by the measured data. In the case of samples from ingots 1HS7 and IHS4, the average $I_{s c}$ response increases dramatically as a result of the RPGA treatment, which leads to a 
substantial increase in cell performance. LBIC analysis of a representative matched pair of devices from ingot 1HS4 (Fig.2) reveals that the intragrain response is considerably higher for cells undergoing the RPGA treatment. This is either due to the dissolution of precipitates in the intragrain regions, or impurity release from dislocation clusters within grains. For these samples, the light phosphorus diffusion removes the impurities released by the RPGA treatment.

In the case of samples from ingots IHS8 and 50-1, the average $J_{s c}$ response falls dramatically as a result of the RPGA treatment. LBIC maps of a representative pair from ingot 50-1 (Fig. 3) show that the collection efficiency in the intragrain regions is reduced as a result of the RPGA treatment. For samples which do not undergo the RPGA, LBIC maps reveal denuded zones around structural defects, which indicates that impurities have segregated to the defect sites during the ingot growth stage or possibly during cell processing. However, in matched devices undergoing the RPGA treatment, the denuded zones vanish and the regions in the immediate vicinity of structural defects exhibit reduced collection efficiency. This shows that the RPGA treatment does enhance the release of impurities away from defect sites and into the surrounding bulk silicon. However, in the present case, the ensuing light phosphorus gettering treatment does not effectively remove the released impurities. Thus, the overall $J_{s e}$ response is reduced.

\section{Comparison of Front and Back Surface Passivation Schemes for Silicon Solar Cells}

The objective of this work is to provide a comprehensive and systematic investigation of front and back surface passivation schemes available for laboratory and industrial (screen-printed) silicon solar cells.

Front surface passivation was investigated on $40 \Omega /$ sq. and $90 \Omega /$ sq. n-type emitters on both planar and textured substrates. Based upon $\mathrm{J}_{\text {se }}$ measurements by the PCD technique, standard $\mathrm{TiO}_{2}$ films were unable to provide any measure of surface passivation. The introduction of a thin RTO film rectuced $\mathrm{J}_{0 c}$ on planar surfaces by a factor of three on $40 \Omega / \mathrm{sq}$. and ten on $90 \Omega / \mathrm{sq}$. On textured emitters, $J_{n e}$ (initially $>1 \mathrm{pA} / \mathrm{cm}^{2}$ ) was reduced by a factor of three in both cases. SiN deposition on the same $40 \Omega /$ sq. emitters gave comparable passivation regardless of the deposition technique (remote or direct). However, on $90 \Omega / s q$., remote SiN passivation gave considerably lower $\mathrm{J}_{0 e}$ than direct $\mathrm{SiN}$, and was comparable or better than RTO. Double layers of PECVD SiN on top of RTO resulted in little or no improvement over the RTO passivation alone on the $40 \Omega / s q$. emitters. On $90 \Omega / s q$. , this $\mathrm{RTO} / \mathrm{SiN}$ combination was found to be the best passivation scheme, achieving $\mathrm{J}_{n_{e}}$ values below $50 \mathrm{fA} / \mathrm{cm}^{2}$ (Fig 4.).

On the back of the solar cell, surface passivation is not usually employed by industry. Since most cells are limited by recombination in the base and $\mathrm{J}_{0 b}$, back surface passivation may provide the opportunity to enhance the performance considerably. Without any passivation, the $S_{\text {hack }}$ is $\gg 10^{4} \mathrm{~cm} / \mathrm{s}$, however, an available option is to employ an Al back surface field (BSF), which has achieved $S_{\text {beck }}$ values as low as $200 \mathrm{~cm} / \mathrm{s}$. As on emitters, $\mathrm{TiO}_{2}$ did not give any passivation. In contrast, thin RTO resulted in an $S_{\text {hack }}$ value of $100 \mathrm{~cm} / \mathrm{s}$ on $1.3 \Omega \mathrm{cm}$ p-type Si, provided a FGA is performed. This RTO can be grown simultaneously with the formation of the BSF or the emitter. To avoid high temperature passivation steps, PECVD SiN can be used as an alternative. In this case, the $S_{\text {back }}$ value depends strongly on the deposition method. The remote SiN gave considerably better passivation than both direct nitrides, as well as RTO. Both the direct nitrides were inferior to RTO, whereas the combination of RTO and SiN was found to be the best passivation scheme on the flat surface. In fact, the combination of RTO and $\mathrm{SiN}$, followed by a short contact firing cycle, reduced $S_{\text {eff }}$ to excellent values of less than $30 \mathrm{~cm} / \mathrm{s}$, regardless of the source of PECVD SiN (Fig. 5).

\section{Fabrication of High Efficiency Cells on Various Promising PV Grade mc-Si.}

Phosphorus and Al gettering treatments we optimized for mc-Si cell processing. In addition forming gas anneal was used for bulk defect passivation. Attempts were made to fabricate high-efficiency multicrystalline solar cells using the optimized gettering conditions, oxide passivation, and evaporated double-layer $\mathrm{ZnS} / \mathrm{MgF}_{2}$ 
antireflection coating. The emitter region was formed by $930^{\circ} \mathrm{C} / 25 \mathrm{~min}$ phosphorus diffusion followed by an etchback to $80 \Omega / \square$. Then, as explained in Section II-A, the $850^{\circ} \mathrm{C}$ oxide passivation and Al drive-in were performed. The front grid contact was formed by evaporation of titanium/silver $(600 \AA / 600 \AA)$ and the lift-off technique. Back contact was formed by evaporating $500 \AA \mathrm{Ti} / 500 \AA \mathrm{Ag}$ on the top of the Al Back Surface Field (BSF) contact region. Contacts were annealed in the forming gas ambient for $30 \mathrm{~min}$ at $400 \circ \mathrm{C}$. About $5-\mu \mathrm{m}$-thick silver was plated on the front grid pattern to recuce the series resistance. Finally, a 550- $\AA \mathrm{ZnS} / 1100-\AA \mathrm{MgF}_{2}$ double-layer AR coating was deposited by thermal evaporation on the cells.

Using the above process, which integrates several gettering and passivation techniques, cell were fabricated on various promising cast and ribbon mc-Si. Fig 6 shows that we were above to achieve efficiencies of:

- $18.6 \%$ on HEM mc-Si

- $17.8 \%$ on OTC mc-Si

- $17.1 \%$ on Solarex cost on mc-Si

- $17.2 \%$ on Bayer mc-Si

- $17.3 \%$ on dendritic web Si ribbon

- $16 \%$ on EFG sheet silicon

- $15.3 \%$ on Evergreen string ribbon

\section{References}

[1] J.S. Kang and D.K. Schroder, "Gettering in Silicon," J. Appl. Phys., 26, pp. 1039-1051, 1983. [2] S.A. McHugo, H. Hieslmair, and E.R. Weber, "Gettering of Metallic impurities in photovoltaic silicon," Appl. Phys. A, 64, pp. 127-137, 1997. 

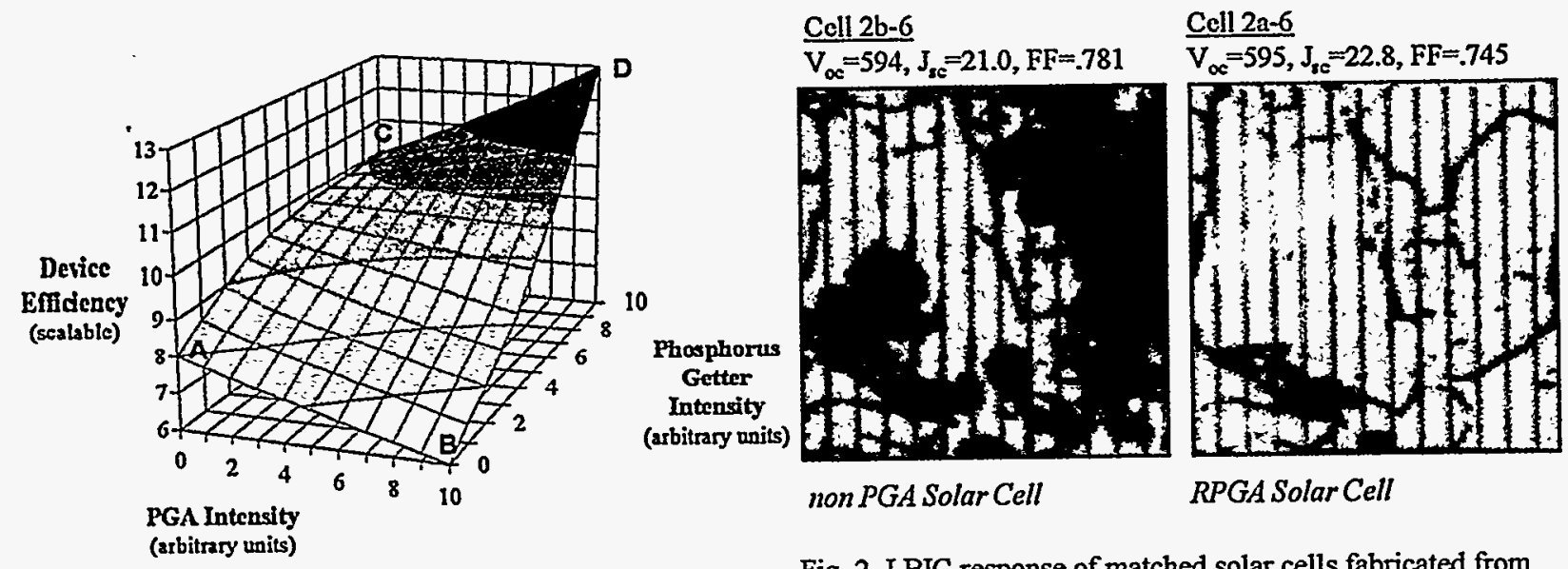

Fig. 1. Response surface model which shows the conceptual dependence of mc-Si solar cell performance on 1) the PGA intensity and 2) the ensuing phosphorus gettering efficiency.
Fig. 2. LBIC response of matched solar cells fabricated from overall collection efficiency. wafers from ingot 1HS4. The RPGA has improved the

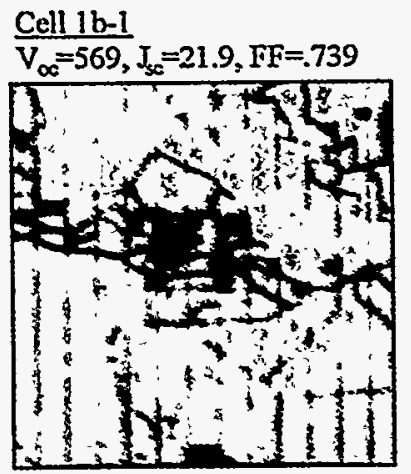

non PGA Solar Cell

Fig. 3. LBIC response of matched solar cells fabricated from wafers from ingot 50-1. The RPGA has reduced the collection efficiency in the vicinity of the defect centers.

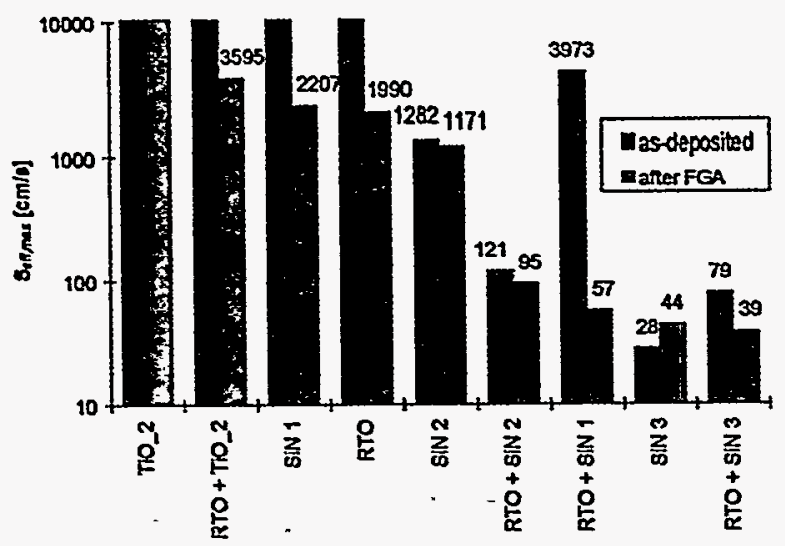

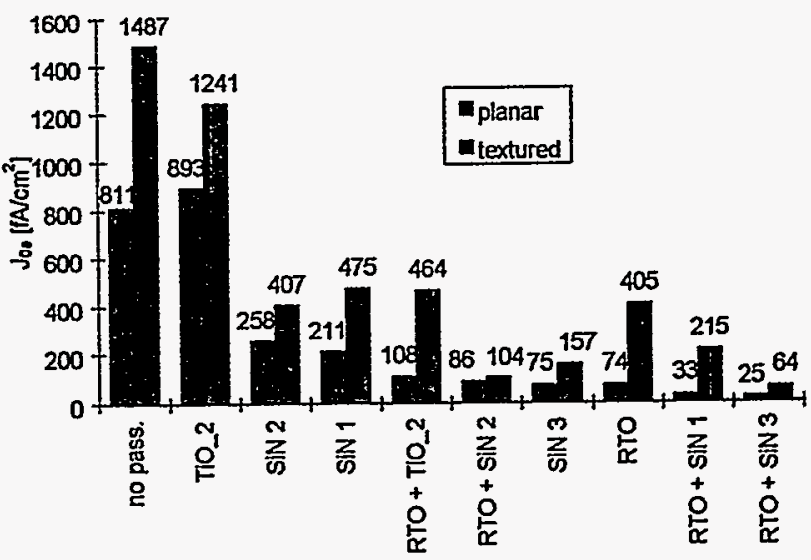

Fig. 4. Emitter saturation current densities for $90 \Omega / s q$. RTP emitters.

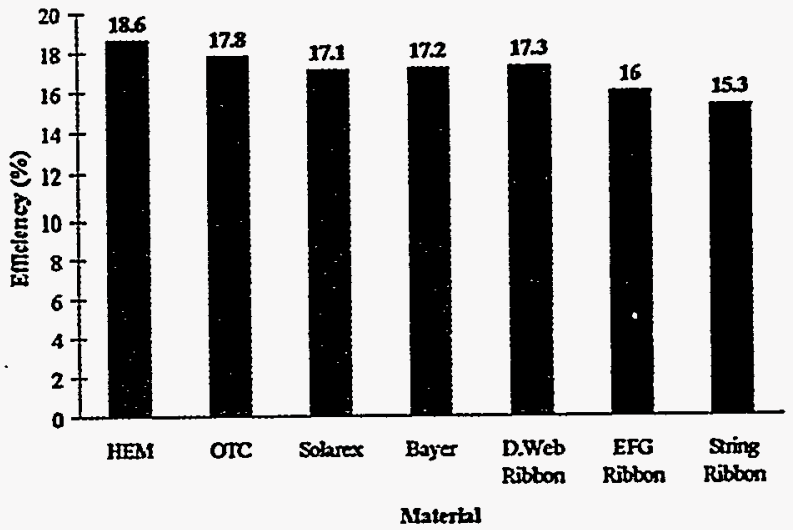

Fig. 6. High efficiency solar cell processing technologies developed at Georgia Tech.

Fig. 5. Surface recombination velocities for planar samples after contact firing. 
Title:

Organization: $\quad$ Department of Materials Science \& Engineering

Massachusetts Institute of Technology, Cambridge, MA 02139

Contributors: $\quad$ L. C. Kimerling, Principle Investigator; A. L. Smith, S. Zhao, S. H. Ahn, M. T. Platero, L. Chalfoun

\section{Introduction}

Our research effort continues to focus on the structure, stability, and kinetics of lifetime degrading defects in Si for the optimization of solar cells[1,2]. We have applied the knowledge base created by this research in the form of two process simulators. A simulator of Fe gettering during the $\mathrm{Al}$ back surface contact anneal incorporates our understanding of dopant enhanced solubility and our results from Al gettering experiments. The second simulator treats defect reaction kinetics. An extensive set of defect reaction equations describes the role of defect, dopant concentrations and processing conditions. The process simulators provide a means of optimization of materials specifications and processes to achieve a desired minority carrier diffusion length. Last year we developed a rapid thermal ramp rate process for contact formation to yield optimal back surface field (BSF) uniformity. This method has been applied to the processing of completed cells by the Rohatgi group at Georgia Tech with notable enhancement in cell efficiency [3].

\section{Gettering Simulator}

We have constructed a gettering simulator that quantifies the design and effectiveness of gettering treatments [4]. The simulation includes the competing gettering mechanisms of internal gettering (IG), segregation to molten $\mathrm{Al}$ and $\mathrm{Al}$-dopant enhanced solubility. The contamination, processing timetemperature profile of the gettering step, the material specifications, and the device structure are simulator inputs. The resulting contamination profile of the wafer is the output that determines the minority carrier diffusion length.

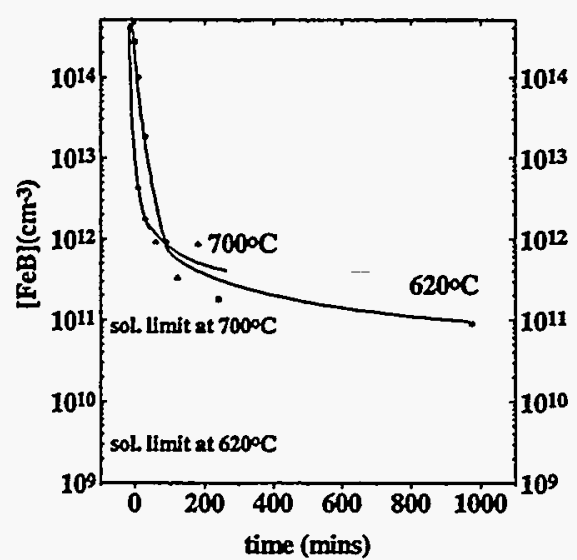

Figure $1 \mathrm{FeB}$ pair concentration as a function of annealing time and temperature from experiment.

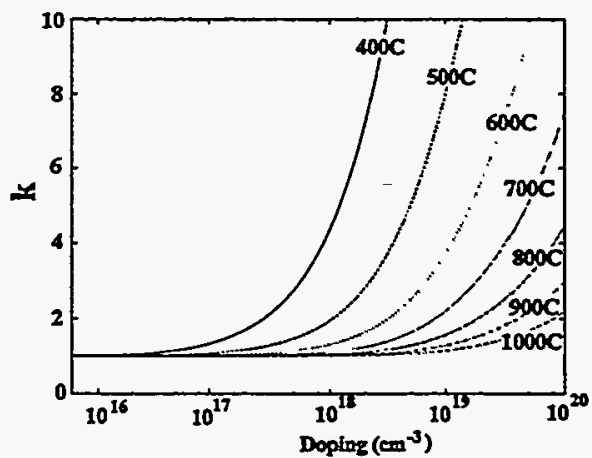

Figure 2 Segregation coefficient, $k$, for various temperatures as a function of Doping level in the Heavily doped region.

Physical Basis

The treatment of IG and segregation follows the work of Tan et al where silicide formation at precipitates is approximated to be diffusion limited and is calculated by a mean field approximation [5]. We have 
produced experimental results to verify this model. Gettering experiments by Al back surface contact formation were performed at various annealing temperatures using a quench to study the instantaneous contamination levels. Figure 1 plots the Fe concentration in solid solution, measured in the center of a wafer by DLTS, following saturation at $1000 \mathrm{C}$, quench in water, and annealing at $620 \mathrm{C}$ and $700 \mathrm{C}$ [6]. The data, analyzed according to the model, gives values of precipitate density and size that are consistent with the $\mathrm{Cz}$ silicon used. As shown in Figure 1, the Fe concentration in solution decays asymptotically toward the Fe solubility at the heat treatment temperature, and the rate of decay increases with increasing temperature.

Dopant enhanced solubility effects are considered in the model, since $\mathrm{Al}$ is a p-type dopant and can enhance $\mathrm{Fe}$ solubility at the $\mathrm{Si}-\mathrm{Al}$ interface [7]. We calculate a segregation coefficient, $\mathrm{k}$, for $\mathrm{Fe}$ in $\mathrm{Si}$ between a highly doped region and a lightly doped region defined as the ratio of the respective $\mathrm{Fe}$ solubility limits. Figure 2 shows $\mathrm{k}$ as a function of doping in the highly doped region for various temperatures. These results demonstrate that $\mathrm{k}$ is on the order of unity at temperatures high enough for $\mathrm{Fe}$ to be significantly mobile. This result constrains the expectations for $\mathrm{p}+$ gettering to be much more modest than some had hoped.

\section{Simulation Results}

The gettering simulator was applied to several cases including a constant anneal to determine if the $\mathrm{p}+$ layer could be responsible for gettering in the absence of precipitates. Fig. 3 shows the cell structure used and the resulting $\mathrm{Fe}$ concentration for a constant anneal at $800 \mathrm{C}$. The plot includes concentrations at the front surface of the cell (labeled FS) and at the back surface in the p+layer for both the case with an Al back surface contact and without the Al layer. The simulation demonstrates that little gettering can be accomplished by solubility enhancement alone. Another case testing the role of cooling rates from the gettering temperature is presented in Figure 4. The gettering experiments of Figure 1 show results for quenched samples to isolate basic process parameters. A solar cell gettering process will inevitably end with a slow cool to room temperature. For this reason, it is necessary to consider the effect of this timetemperature profile on Fe gettering. Diffusivity decreases with decreasing temperature, however solubility enhancement increases as temperature drops. The results show that a slower cool can yield two orders of magnitude improvement in Fe removal.

We focus on $\mathrm{Fe}$ due to its ubiquitous nature and its strong potential for minority carrier lifetime degradation. Since Fe has an intermediate value of diffusivity, it is easily introduced at high temperatures and trapped in solution at concentrations above the room temperature solubility limit. For these reasons, $\mathrm{Fe}$ is seen as a lifetime limiting defect and understanding its removal is of primary importance. The principles of the simulator can be easily applied to other transition metal contaminants.

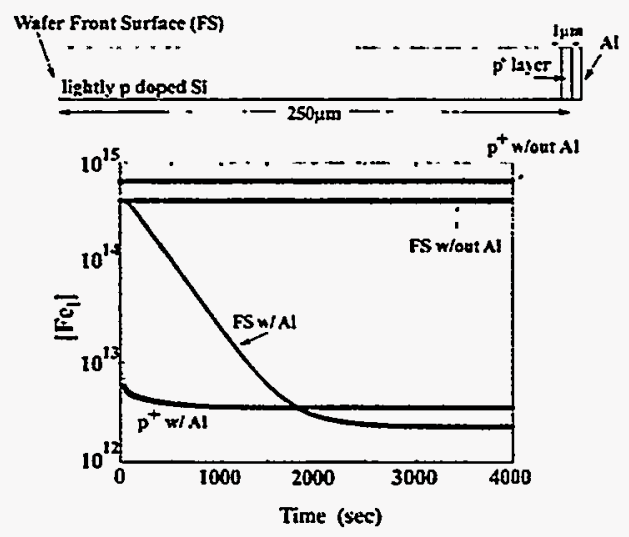

Figure 3 Interstitial Fe concentration as a function of annealing time for the front surface at highly doped back surface with an without an $\mathrm{Al}$ sink.

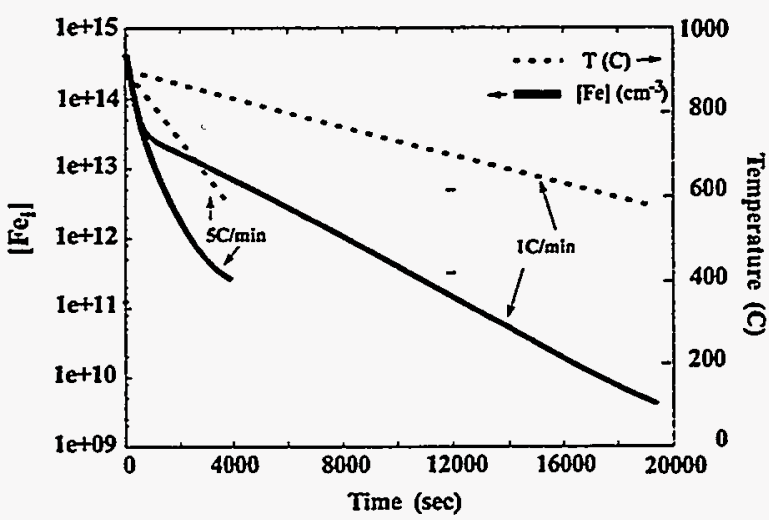

Figure 4 Results of simulation of a cool on Fe concentration in a solar cell structure for cools of $5^{\circ} \mathrm{C} / \mathrm{min}$ and $1^{\circ} \mathrm{C} / \mathrm{min}$. 


\section{Point Defect Reaction Simulator}

The point defect reaction simulator allows for the analysis of the interaction of point defects to determine material specifications required for a desired minority carrier lifetime.

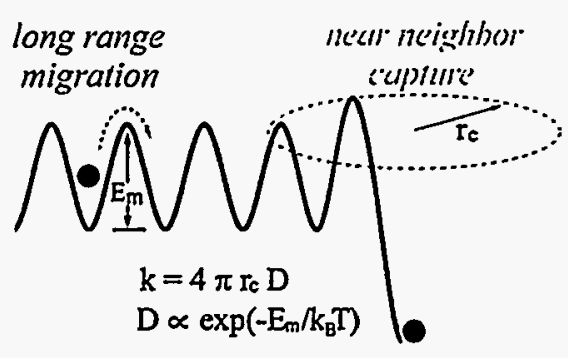

Figure 5 The schematic diagram of migration and capture of interstitials. $E_{m}$ is the interstitial migration energy.
The interactions among self-interstitials $\left(\mathrm{Si}_{\mathrm{i}}\right)$, vacancies $(\mathrm{V})$, impurities $(\mathrm{C}, \mathrm{O})$, and dopants $(\mathrm{B}, \mathrm{P})$ in silicon not only lead to the formation of undesirable point defect products which affect device operation, but they also generate defect associates that control processes such as diffusion of dopants. Electron beam irradiation was used to generate $\mathrm{Si}_{\mathrm{i}}$ and $\mathrm{V}$ to initiate defect reactions. DLTS measurement was used to explore the hierarchy and paths of defect reactions involving $\mathrm{Si}_{\mathrm{i}}, \mathrm{V}, \mathrm{O}, \mathrm{C}, \mathrm{B}$, and $\mathrm{P}$ in silicon. We model the defect reactions as a three step process: (i) the displacement reaction for the generation of $\mathrm{Si}_{\mathbf{i}}$ and V; (ii) the Watkins replacement reaction for the generation of $B$ and $\bar{C}$ interstitials $\left(B_{i}\right.$ and $\left.C_{i}\right)$; (iii) the diffusion limited reaction for the formation of defect pairs and high order defect

complexes (e.g., clusters). Within the framework of reaction kinetics, for the first time, we have simulated the defect reaction process [8,9].

A kinetic model (coupled ordinary differential equations) has been constructed to simulate the interstitial defect reactions in Si. The interstitial diffusion coefficient and the pair formation capture radius are two parameters used in the model to describe long range migration and near neighbor capture of interstitials. First order diffusion limited pairing reactions are the predominant processes in the defect reactions. The reaction kinetics are determined by the interstitial diffusivity, pairing capture radius, and background dopant and impurity concentrations ([B], [P], [O], and [C]). The model is consistent with the DLTS defect assignments. In addition to the interstitial related pairing reactions, the model also covers $\mathrm{Si}_{\mathrm{i}}-\mathrm{V}$, $\mathrm{B}_{\mathrm{i}}-\mathrm{V}, \mathrm{C}_{\mathrm{i}}-\mathrm{V}$ recombination processes, $\mathrm{V}$-related defects $\left(\mathrm{VV}, \mathrm{VO}_{\mathrm{i}}\right.$, and $\left.\mathrm{VP}_{\mathrm{s}}\right)$, and small clusters such as $\left(\mathrm{C}_{\mathrm{i}}\right)_{2} \mathrm{C}_{\mathrm{s}}$ and $\left(\mathrm{B}_{\mathrm{i}}\right)_{2} \mathrm{~B}_{\mathrm{s}}$. The model can be improved by including pair dissociation reactions and higher order kinetic processes such as large $\mathrm{B}$ - and $\mathrm{C}$ - related clusters and $\mathrm{Si}_{\mathrm{i}}$-clusters on $\mathrm{C}$ - and $\mathrm{B}$-complexes.

Comprehensive knowledge of defect interaction mechanisms and a complete defect interaction parameter base are essential for reliable processing of data from the measurements. The mathematical model describing the defect reaction system is closed and self-consistent, and sufficient initial conditions are known. The solution (i.e. the defect evolution) exists and is singular, and no ambiguity is contained in the evolution of the defect system. The simulator can be used to construct the interstitial defect reaction hierarchy diagram, to predict defect behavior in Si cell processing and to characterize background dopant and impurity concentrations (e.g., [B], [P], [O], and [C]) in Si by combined electron irradiation and DLTS measurement techniques.

\section{Processing Benchmark}

Due to an availability of controlled data, the simulator has been applied to the case of a reactive ion etch (RIE), a process which induces point defect reactions [10]. RIE causes substrate surface contamination, substrate damage, and induces defect reactions to produce recombination centers at depths into the micrometer range. We have developed a model describing the indiffusion of interstitials and the subsequent reactions in the defect reaction region to predict the defect depth profiles. We formulate the reaction kinetics as a series of 1-D coupled interstitial diffusion-reaction partial differential equations (PDEs) with a moving boundary. The predicted defect depth profiles are consistent with those measured in the photoluminescence (PL) experiments. We conclude that the defect depth profiles are determined by interstitial diffusion coefficient $\left(D_{i}\right)$, etch rate $\left(v_{E}\right)$, etch time $\left(t_{E}\right)$, defect reaction rate $(K)$, and background dopant and impurity concentrations ([C], [B], and [O]) in the Si substrate. The micrometer 
range defect depth profiles result from: (i) fast diffusing $\mathrm{Si}$ self-interstitial ( $\mathrm{Si}_{\mathrm{i}}$ ) indiffusion to a micrometer depth range of $\left(D_{i} / K\right)^{1 / 2}$ limited by $[C]$ and $[B]$; (ii) the generation of carbon and boron interstitials $\left(C_{i}\right.$ and $\left.B_{i}\right)$ through the Watkins replacement reactions, and (iii) the formation of $C_{i}$ and $B_{i}$ related defect pairs through diffusion limited pairing reactions. $C_{i}$ and $B_{i}$ indiffusion are limited by $\nu_{E}$ and extremely shallow $\left(D_{i} / v_{E}\right)$ during a typical RIE process with $v_{E} \sim 2000 \AA / m i n$ and $t_{E} \sim 10 \mathrm{~min}$. The indiffusion of vacancies $(V)$, contamination from the etching plasma and enhanced diffusion effects are also considered in the model.

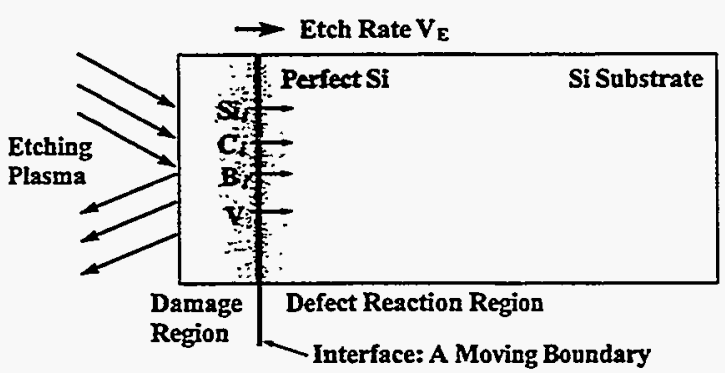

Figure 6 Schematic of the structure of the reactive ion etch for application to the defect reaction simulator.

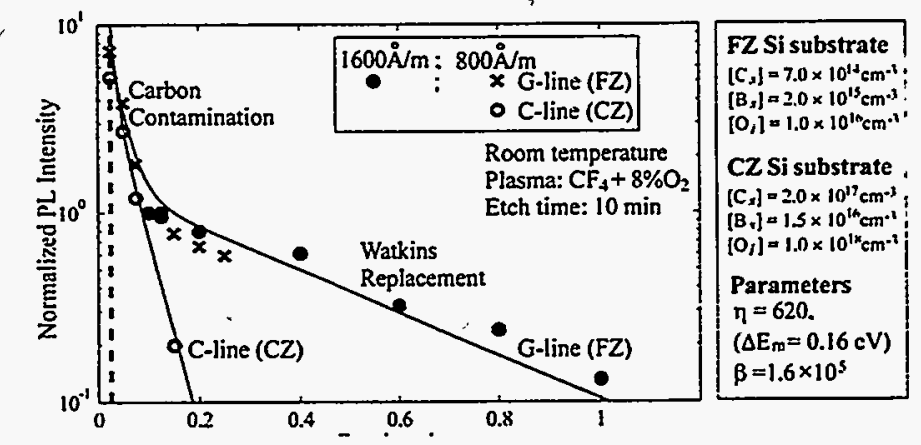

Figure 7 Dots show defect concentrations as a function of depth from processed surface. Solid line shows simulator results. $\eta$ is the diffusion enhancement parameter and $\beta$ is the ratio of $\mathrm{C}_{\mathrm{i}}$ to $\mathrm{Si}_{\mathrm{i}}$.

\section{Conclusion}

This program addresses the limiting factors in cell performance with low cost manufacturing. The understanding and development of robust process technologies are critical to the development of PV. These process simulators represent two major milestones in the program where fundamental knowledge generated by research has been incorporated into predictive design tools.

\section{References}

1 L.L. Chalfoun, A.L. Smith, S. Zhao, and L.C. Kimerling, "Process Optimization of Alloyed Aluminum Backside Contacts for Silicon Solar Cells," poster at the 6th National Renewable Energy Laboratory (NREL) Workshop of the Role of Impurities and Defects in Semiconductors, August 12-14, 1996, Snowmass Village, Colorado 2 L.C. Kimerling, "The Role of Dislocations and Associated Defects in Determining Minority Carrier Lifetime", 7th National Renewable Energy Laboratory (NREL) Workshop of the Role of Impurities and Defects in Semiconductors, August 1996, Vail, Colorado

3 S. Narasimha, A. Rohatgi, 7th National Renewable Energy Laboratory (NREL) Workshop of the Role of Impurities and Defects in Semiconductors, August 1996, Vail, Colorado

4 A.L. Smith, S. Zhao, Sang H. Ahn, Gerd Norga, L.L. Chalfoun, and L.C. Kimerling, "The Reaction Kinetics of Fe at Si Surfaces and Interfaces in Si Solar Cells," poster at the 7th National Renewable Energy Laboratory (NREL) Workshop of the Role of Impurities and Defects in Semiconductors, August 1996, Vail, Colorado 5 T.Y.Tan, R. Gafiteanu, and U.Goesele, Proc. 6th NREL Workshop on the Role of Impurities and Defects in Si Dev. Proc., 110 (1996).

6 S.H. Ahn, S.Zhao, A.L.Smith, L.L.Chalfoun, H.Nakashima, M.T.Platero, and L.C.Kimerling, Mater.Res.Soc. Symp. Proc., (442), 169 (1997).

7 L.L. Chalfoun,"Process Optimization of Alloyed Aluminum Backside Contacts for Silicon Solar Cells",Master's thesis at MIT, June 1996

8 S. Zhao, L.V.C. Assali, and L.C. Kimerling, Mater. Sci. Forum, (196-201), 1333 (1995).

9 S. Zhao, A.M. Agarwal, J.L. Benton, G.H. Gilmer, and L.C. Kimerling, Mater. Res. Soc. Symp., (442), 231 (1997).

10 S.Zhao, L.C. Kimerling “Defect Reactions Induced By Reactive Ion Etching” Fall MRS 1997, to be publ. 

Title:
Detailed Non-Contact Electrical and Structural Characterization of Photovoltaic Silicon Substrates
Organizations: $\quad$ Department of Materials Science and Engineering North Carolina State University
Raleigh, NC 27695-7916.
Contributions: $\quad$ B. Sopori, Program Manager; G. A. Rozgonyi, Principal Investigator; A. Romanowski and A. Karoui

\section{Introduction}

The objectives of this work are (i) the measurement and the separation of surface recombination velocity and recombination/trapping lifetimes, using a contactless frequency resolved photoconductance technique, and (ii) an investigation of the multiple external gettering by $\mathrm{Al}$ and $\mathrm{Ti}$ thin films.

\section{Frequency-Resolved Microwave Reflection Photoconductance: Surface Recombination Velocity and Recombination and Trapping Lifetime.}

In modern silicon materials, oxygen related defects $[1,2]$ and metal impurities affect carrier recombination lifetime. To evaluate recombination properties the Microwave Photo Conductance Decay ( $\mu-\mathrm{PCD}$ ) is used. Commercially available $\mu-\mathrm{PCD}$ tools use relatively high excitation levels. Since recombination lifetime and surface recombination velocity are injection level dependent, the reduction of injection levels was the main goal of this work (our R\&D project). Free carrier kinetics depend on recombination processes as well as trapping. The recombination process is described by the surface recombination velocity and bulk recombination lifetime; whereas the trapping process is described by the lifetime of trapped carriers. In previous work we have demonstrated that the presence of the trapping processes and surface recombination results in a deformation of the Nyquist plot [3,4], and this has proved to be a powerful indicator/tool/method for the analysis of the carrier kinetics.

Theoretical analysis has shown that the generation-recombination process is described by twolevel carrier transitions. The nature of the main level is the recombination center while the other is the trapping center. The transitions are characterized by three time constants: (1) recombination lifetime $\tau$ associated with the recombination center, (2) carrier capture lifetime $\tau_{c}$ and (3) emission lifetimes $\tau_{e}$ of the trapping center expressed as follows:

$$
\frac{1}{\tau_{c}}=c_{t} N_{t}\left(1-f_{t}\right), \quad \frac{1}{\tau_{\mathrm{c}}}=\mathrm{c}_{\mathrm{t}}\left(\mathrm{n}_{\mathrm{dc}}+\mathrm{n}_{2}\right)
$$

Here $f_{t}$ is the filling factor of the traps, $N_{t}$ the trap concentration, $c_{t}$ the trapping efficiency, $n_{d c}$ the $\mathrm{dc}$ minority carrier concentration, and $n_{2}$ is a parameter related to the activation energy of trapping centers. In previous work a semi-infinite semiconductor was analyzed. Since carrier diffusion lengths in modern silicon technology are comparable to the typical wafer thickness, both front and back surface recombination effects were included in the present approach.

Generally the Nyquist plot in the complex plan is represented with two non-axial semi- circles, correlated with recombination and trapping centers. In the case where only the recombination center exist (i.e. no traps), the figure reduces to a single semi-circle, which can be described by three parameters $f_{m}, x_{m}$ and $y_{m}$, [3]. As the surface recombination velocity $v_{s}$ increases, the semi-circle is 
deformed on both sides. The evaluation procedure for material properties - recombination/trapping lifetimes and surface recombination velocity, is based on the determination of the phase shift function and a subsequent minimization of the deviation between the measured phase $\Psi$ and $\Phi$ its theoretical value, which depends on the frequency. The nonlinear method used for the fitting procedure is described in Ref. [4]. The integrity of the fitting algorithm and its accuracy were checked using simulated experimental data, which had been generated by the theoretical model for which the primary parameters were altered by noise.

Based on the described simulation, the following conclusions can be drawn:

- lifetimes related to recombination $(\tau)$ and trapping ( $\tau_{c}$ : capture, $\tau_{e}$ :emission) are retrieved with acceptable fitting confidence, even in the presence of high measurement noise. Both trap related lifetimes $\tau_{c}$ and $\tau_{e}$ are; however, better recovered if the trap emission lifetime $\tau_{e}$ is low (e. i. high emission rate). The higher the trap emission lifetimes (500 $\mu$ s versus $5 \mathrm{~ms})$ the larger the relative evaluation errors $\delta \tau_{d} \tau_{c}$ and $\delta \tau_{e} / \tau_{e}$.

- surface recombination velocity may not be properly recovered with high confidence when the $v_{s}$ is low (i.e. passivated surface). Large evaluation errors resulting from low surface recombination velocity or large emission lifetime are due to weak free carrier modulation via the existing trapping centers. In such cases, the free carrier concentration is only controlled by the bulk recombination centers. As a result deformation of the Nyquist plot is weak. The evaluation procedure described is therefore most suitable for samples with large surface recombination velocities. This indicates that in practice, no special surface preparation is required.

In order to examine the described approach, p-type silicon wafers were cut from the same portion of an ingot doped with boron at concentration about $10^{15} \mathrm{~cm}^{-3}$ and measured using the FR-PC technique. One group of the wafers was annealed in order to annihilate the thermal donors (TD) present in the material, while the second group was not annealed. It was expected that preventing TD annihilation would result in an increase of the number of trapping centers. Figures $1 \mathrm{a}$ and $\mathrm{lb}$ illustrate the experimental data, together with the simulated curves of the normalized spectra $\operatorname{Im}\{R(\omega)\} / Y_{m}$ in the frequency band $\left[75 \mathrm{~Hz}, 100 \mathrm{kHz}\right.$ ], for an "as grown" (no TDA) wafer measured at $100^{\circ} \mathrm{C}$ and $220^{\circ} \mathrm{C}$, respectively. $R(\omega)$ is the measured microwave reflectance of the sample at the modulation frequency $\omega$. Due to the free carrier trapping effects, the low frequency portion of the graph in Fig. la deviates from the Lorentzian shape. Notice that this part of the spectrum disappears at elevated temperatures (Fig. 1b). Additional information is obtained from the corresponding Nyquist plots presented in Figs. 2a and $2 \mathrm{~b}$, for the same sample and the same temperature. The disappearance of the low frequency arch at $220^{\circ} \mathrm{C}$ indicates that the recombination process alone (no trapping) at this temperature controls the excess carrier concentration. Lifetimes evaluated by three different methodologies are illustrated in Fig. 3. The effective lifetime $\tau_{e f f}$ is evaluated in time domain using the conventional $\mu \mathrm{PCD}$ technique, while the two other lifetimes are determined by the FR-PC in conjunction with TVF, a two-variable-fitting (referred to $\tau_{2}$ ) and FVF, a four-variable-fitting (referred to $\tau_{4}$ ). The fitting vectors used are $\stackrel{a}{a}=\left[v_{s}, \tau\right]$ and $G=\left[v_{s}, \tau, \tau_{c}, \tau_{e}\right]$, where $v_{s}$ is the recombination velocity at the front surface. The lifetime $\tau_{4}$ in Fig. $3 \mathrm{a}$ falls rapidly at a temperature of about $180^{\circ} \mathrm{C}$, and the fitting algorithm becomes unstable (the error function $\chi$ converges slowly). Therefore, at high temperature range lifetime is evaluated by a Two-Variable-Fitting procedure. Below $180^{\circ} \mathrm{C}$, it is clear that the $\tau_{2}$ lifetime matches the $\tau_{4}$ (obtained by FVF) well. It is also in a good agreement with the effective lifetime $\tau_{e f f}$, particularly above $180^{\circ} \mathrm{C}$. At high temperatures the emission rate of trapping centers are so high that trapping process can not effect the carrier density, consequently $\tau_{4}$ loses its physical meaning and the FVF is substituted by TVF. Figure $3 \mathrm{~b}$ illustrates the behavior of frequency and time-resolved lifetimes as a function of temperature for the TD annealed wafer. Good agreement is observed between $\tau_{2}$, $\tau_{4}$ and $\tau_{\text {eff }}$ in the temperature range 
24 to $100^{\circ} \mathrm{C}$. Above $100^{\circ} \mathrm{C}$, the FVF procedure becomes unstable again, as observed earlier for asgrown wafers, but at higher temperature. The TVF procedure has therefore been used for lifetime evaluation. At room temperature the surface recombination velocity given by both TVF and FVF procedures are about the same, and lie in the region of $1000 \mathrm{~cm} / \mathrm{s}$. They become very low above $60^{\circ} \mathrm{C}$. This significant change is not yet fully understood; the most likely explanation is that both surface and bulk become active and generate the large variation. The dependency of capture and emission trapping lifetimes, $\tau_{c}$ and $\tau_{e}$, on temperature is shown in Fig. 4. Both emission and capture trapping lifetimes initially increase with temperature. Then, for the as-grown samples, after reaching a maximum at a temperature of about $70^{\circ} \mathrm{C}$ the $\tau_{c}$ and $\tau_{e}$ lifetimes decrease. In the case of TDA samples either $\tau_{c}$ or $\tau_{e}$ lifetimes increase and disappear at elevated temperature.

\section{Conclusions}

Acceptable agreement is found between the FR-PC and $\mu-P C D$ techniques as far as the recombination lifetime is concemed. Easy visualization and separation of trapping versus recombination processes by a Nyquist plot has been demonstrated. The FR-PC technique along with the TVF and FVF based on nonlinear simplex fitting procedure has been successfully used for the determination of the recombination and trapping lifetime properties in CZ silicon. The trapping centers associated with thermal donors were studied in as-grown wafers without a thermal donor annihilation process, and the emission and capture characteristic properties were determined. It was also demonstrated that low temperature TD annealing resulted in traps being annihilated.

\section{Ti Thin Film External Gettering}

To better understand the thin film $\mathrm{Al}$ and Ti gettering processes previously presented a second set of $\mathrm{CZ}$ p-type samples were intentionally contaminated with $\mathrm{Ni}$ and $\mathrm{Fe}$ impurities by an aqueous solution containing metallic impurities $\left(\mathrm{FeCl}_{3}\right.$ for iron contamination and or $\mathrm{Ni}\left(\mathrm{NO}_{3}\right)_{2}$ for nickel contamination). It is well known that $850^{\circ} \mathrm{C}$ is the optimal temperature for $\mathrm{Al}$ gettering (release capture limited gettering process $[5,6,7])$, and the temperature of $860^{\circ} \mathrm{C}$ is the lowest eutectic point for Ti-Si alloys. Therefore, three temperatures were used: two high around the titanium eutectic point, 800 and $900^{\circ} \mathrm{C}$, and a low one, $500^{\circ} \mathrm{C}$, for $120 \mathrm{~min}$ in $\mathrm{N}_{2}$ atmosphere. For comparative purpose $\mathrm{Al}$ gettering was tested in the same conditions.

For the purpose of post-gettering electrical measurements, the upper layers, which were saturated by the getter metal, were removed in order to avoid the microwave screening and to be able to make Schottky diodes. About $3 \mu \mathrm{m}$ of the Ti metal and the Ti-Si silicide layers were removed by dipping the samples into the CP-4 solution. One sample was also beveled mechanically and etched using a Secco solution.

The histograms in Fig. 5 present the effective lifetime measured by $\mu$-PCD. Globally a lifetime enhancement subsequent to either $\mathrm{Al}$ or Ti gettering is obtained for wafers contaminated with $\mathrm{Ni}$.

After.low temperature annealing $\left(500^{\circ} \mathrm{C}\right)$ :

- a lifetime improvement is obtained for Ni contaminated samples,

- lifetime degradation is clearly seen for Fe contaminated samples, and

- the lifetime is higher for samples gettered with Ti than those with Al.

These properties are reversed for high temperature annealing. The lifetime becomes higher for $\mathrm{Al}$ gettering than for $\mathrm{Ti}$, particularly for $\mathrm{Fe}$ contaminated samples. The highest overall measured lifetime was for a $\mathrm{Ni}$ contaminated samples gettered by $\mathrm{Al}$ at $800^{\circ} \mathrm{C}$. In the case of $\mathrm{Fe}$ contaminated samples, the best results were obtained for $\mathrm{Al}$ gettering at $900^{\circ} \mathrm{C}$.

In order to check the gettering efficiency, Al Schottky barriers of $1 \mathrm{~mm}$ diameter were 
evaporated and ohmic contacts were made on the back surface using InGa eutectic. Before $\mathrm{Al}$ evaporation the samples were RCA cleaned and dipped in BOE. Unfortunately, instead of producing rectifying barriers, good ohmic contacts were created, indicating that chemical processes and the RTA treatment had converted the samples from p- to n-type material. The above hypothesis was confirmed by the I-V characteristic of a Ti (new) based Schottky diode made by a fresh Ti dot, after a stripping of the previous layer. Unfortunately, the high leakage current of $400 \mu \mathrm{A}$ did not allow DLTS measurement to be made.

\section{Conclusions}

Lifetime data show that a $\mathrm{Ti}$ thin film can be used for selective impurity gettering. At low temperature, thin $\mathrm{Ti}$ film gettering efficiency is better than $\mathrm{Al}$. For high temperature annealing the opposite is true, in this case $\mathrm{Al}$ seems to be a better external getter than $\mathrm{Ti}$. For samples containing $\mathrm{Ni}$ the best lifetime enhancement was achieved for an anneal at $800^{\circ} \mathrm{C}$, whereas for $\mathrm{Fe}$ contaminated $\mathrm{CZ}$ silicon, the best lifetime was obtained for samples annealed at $900^{\circ} \mathrm{C}$.

It is useful to recall the main concept that led to the new technique for external gettering, that is, the growth of a titanium oxide thin layer simultaneously with the temperature cycling of the metal external getter at $500^{\circ} \mathrm{C}$. This thin oxide layer provides a good antireflective coating for photovoltaic cells. This layer is; however, not formed on samples annealed at high temperatures, which is consistent with a low temperature gettering for Ti. Moreover, PV manufacturing is more favorable to low budget processing. Double gettering ( $\mathrm{Ti}$ at front and $\mathrm{Al}$ at back) can be efficient for simultaneous and complementary getterings, cost effective process for PV technology, and provide supplementary

functions for the used layers.

\section{References,}

1. Kitagawara Y., Yoshida T., Hamaguchi T., and Takenaka T., J. Electrochemical Soci. 142, 3505 (1995).

2. Borghesi A., Pivac B., Sassella A., and Stella A., J. Appl. Phys. 77, 4169 (1995).

3. Romanowski A., Buczkowski A., Karoui A., and G. Rozgonyi, Frequency-Resolved Microwave Reflection Photoconductance: Surface Recombination Velocity and Recombination and Trapping Lifetime, submitted to JAP.

4. Romanowski A., Buczkowski A., Karoui A., and G. Rozgonyi, Silicon Recombination Lifetime Characterization Methodds, ASTM STP 1340 (Edts. D. Gupta, F. Bacher and W: Hughes), 1998.

5. Joshi S.M., Gosele U.M., and Tan T.Y., J. Appl.Phys. 77, 3858 (1995). :

6. Martinuzzi S., Perichaud I., and Simon J.J., Appl. Phys-Lett. 70, 2744 (1997).

7. Rohatgi A., Weber E.R., and Kimerling L.C., J. Electronic Materials 22, 65 (1993). 


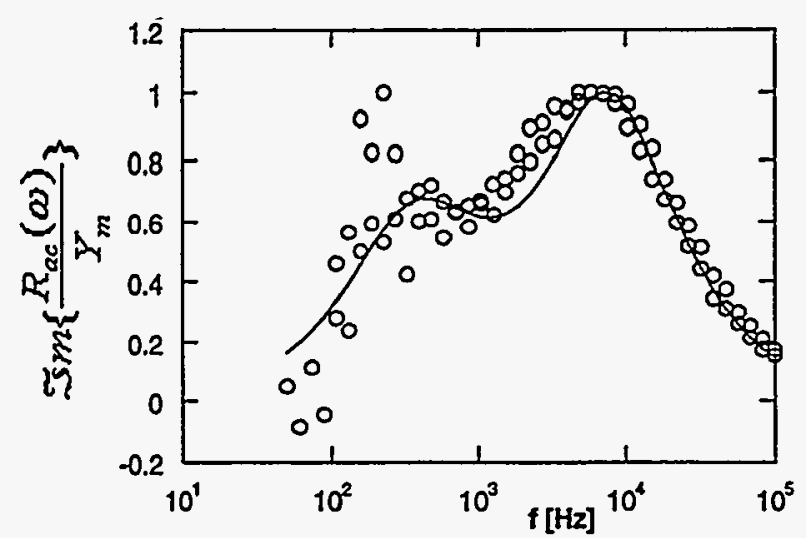

(a) $100^{\circ} \mathrm{C}$

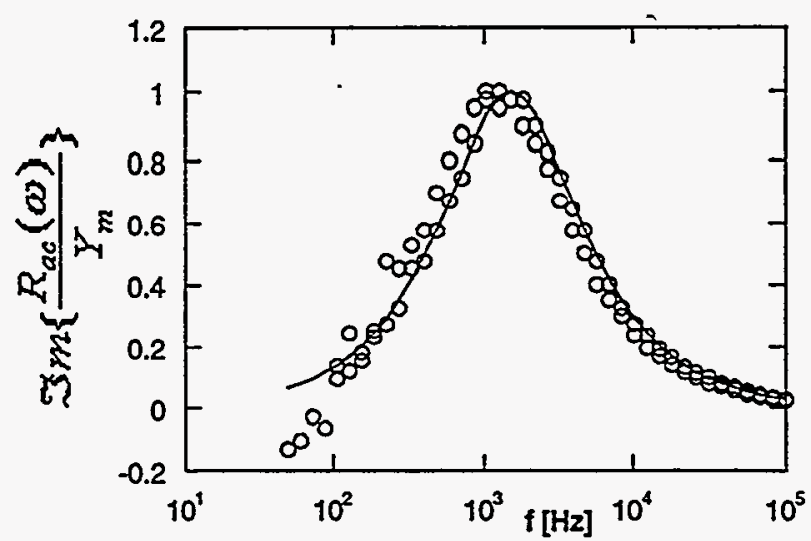

(b) $220^{\circ} \mathrm{C}$

Fig. 1. Normalized experimental and theoretical spectra $\Im m\left\{\frac{R_{a c}(\omega)}{Y_{m}}\right\}$ measured at $100^{\circ} \mathrm{C}$ and $220^{\circ} \mathrm{C}$ for as-grown wafer.

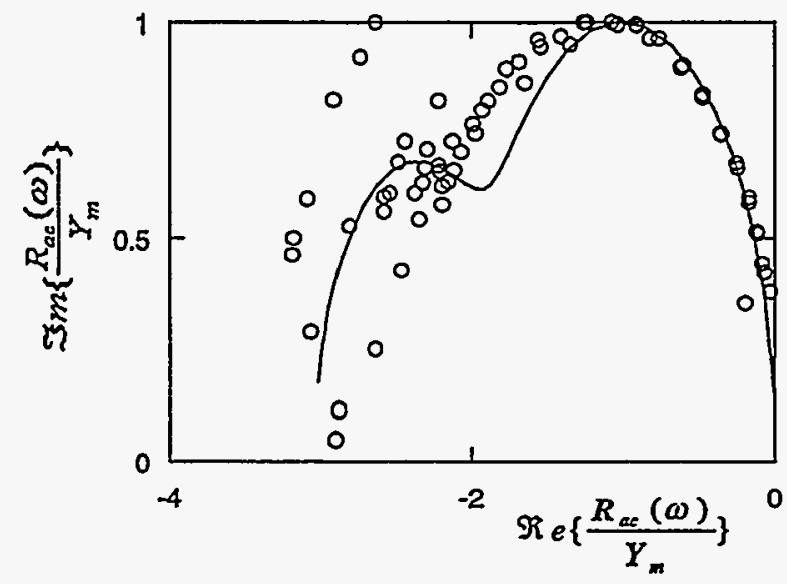

(a) $100^{\circ} \mathrm{C}$

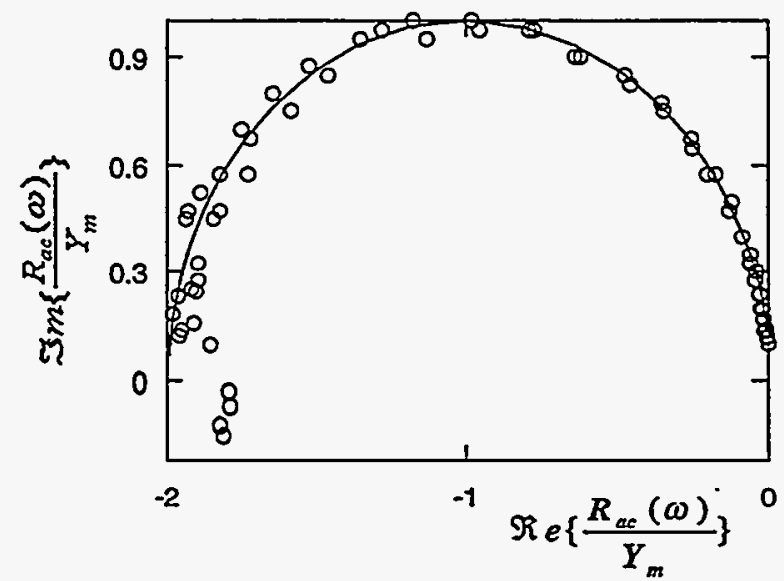

(b) $220^{\circ} \mathrm{C}$

Fig. 2. Normalized experimental and theoretical Nyquist plots measured at $100^{\circ} \mathrm{C} 220^{\circ} \mathrm{C}$ for as grown wafer.

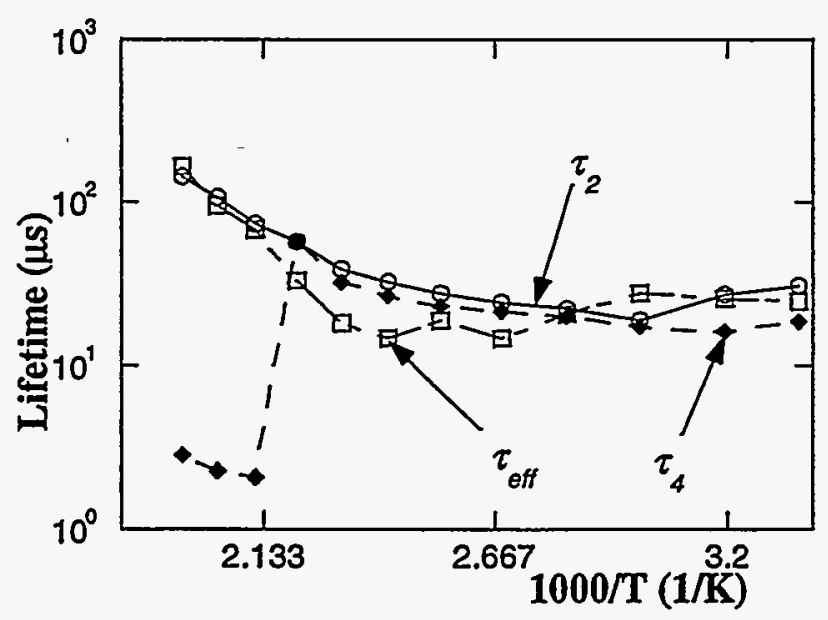

(a) as grown wafers

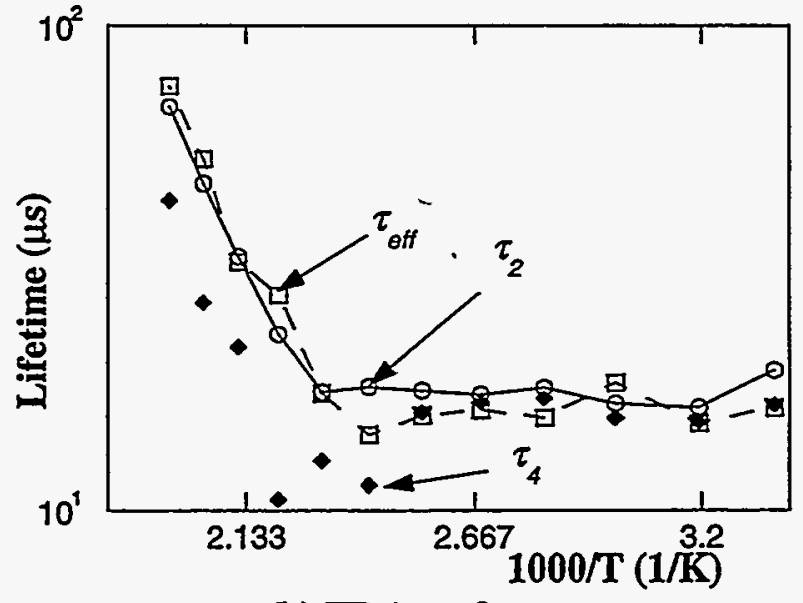

(b) TDA wafers

Fig. 3. FR lifetimes $\tau_{2}, \tau_{4}$, evaluated using "two - and four - variable fitting" procedures and effective lifetime $\tau_{e f f}$ measured by $\mu-\mathrm{PCD}$ technique for two different types of wafers. 


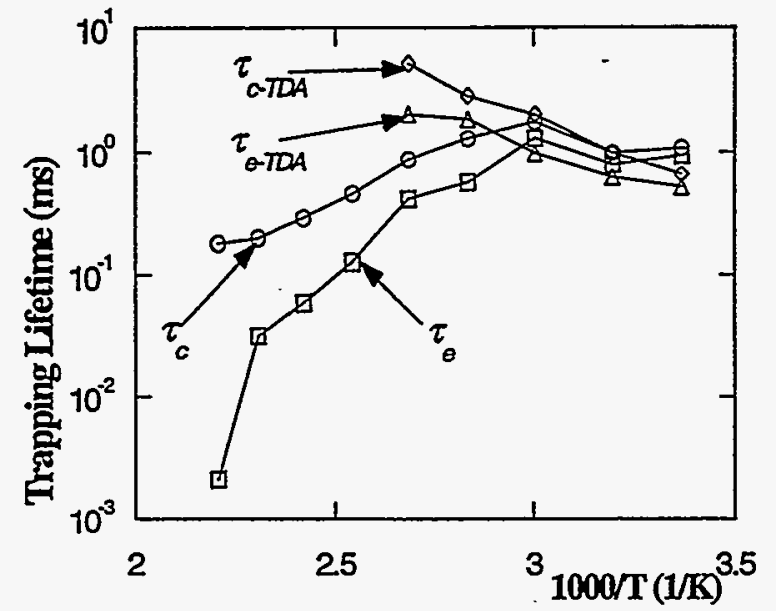

Fig.4. Evaluated trapping lifetimes: capture $\tau_{c}$ and emission $\tau_{e}$ using "fourparameters" fitting procedure vs. temperature for as-grown and TDA wafers.

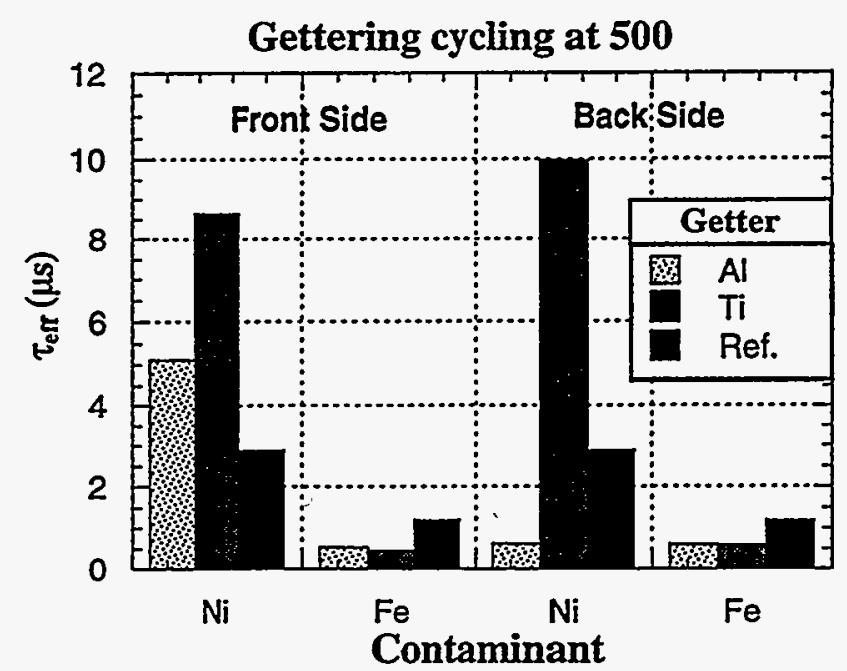

Fig.5a. Lifetime histogram for wafers annealed at $500^{\circ} \mathrm{C}$.

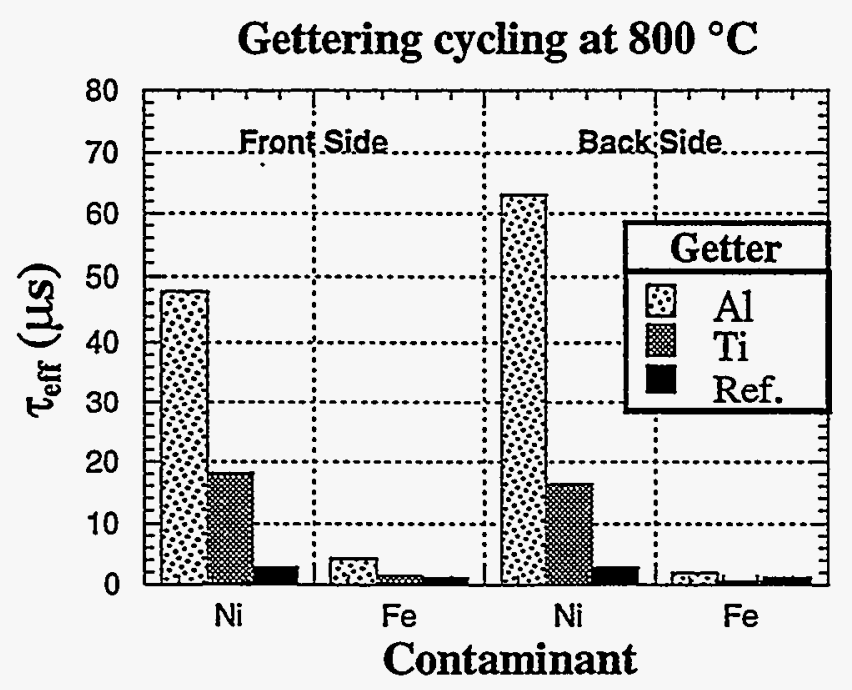

Fig.5b: Lifetime bar histogram for wafers annealed at $800^{\circ} \mathrm{C}$.
Gettering cycling at $900{ }^{\circ} \mathrm{C}$

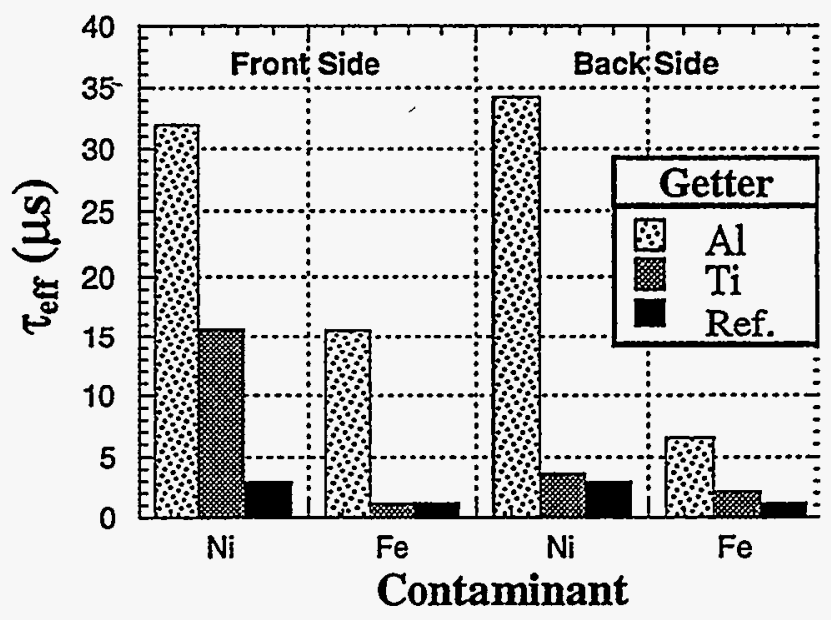

Fig.5c: Lifetime bar histogram for wafers annealed at $900^{\circ} \mathrm{C}$. 
Title:

Organization:

Contributors:
Theoretical Analysis of Hydrogen Passivation Of Impurities and Defects

\author{
Physics Department, Texas Tech University
}

Lubbock, TX 79409-1051

Stefan K. Estreicher, principal investigator

Jeffrey L. Hastings, graduate student

\section{Objectives}

Poly-Si solar cells often benefit substantially from the passivation[1,2] by $\mathrm{H}$ of dangling bonds at vacancies, dislocations, grain boundaries, and other defects. However, recent theoretical work has shown that not all vacancy-related defects are electrically active and therefore benefit from hydrogenation. [3,4] It is remarkable that clean Si surfaces cannot be hydrogenated from a forming gas (except at very high temperatures). The surface must be damaged in some way in order for $\mathrm{H}_{2}$ to dissociate and penetrate into the bulk. In addition, the complexes containing transition metals such as $\mathrm{Cu}$ of $\mathrm{Fe}$ are poorly understood and there little experimental or theoretical information about their microscopic structure or properties.

The objectives of this research is to investigate these issues theoretically at or near the ab-initio level, and provide new insights into (a) the properties of vacancy clusters and their interactions with hydrogen, (b) the role of surface damage in the hydrogenation process, (c) the equilibrium configurations of some transition metals and related complexes, and (d) their interactions with hydrogen.

\section{Theoretical Approach}

The equilibrium geometries, electronic configurations, diffusion paths, and potential energy surfaces are calculated at various levels of ab-initio Hartree-Fock (HF) theory in molecular clusters. The outputs provide precious chemical information on the details of the rebonding around a complex. This approach is particularly useful regarding $3 d$ transition metals because neither semiempirical techniques nor ab-initio, plane-wave-based, density functional methods can handle these elements.

The dynamics of complex formation and dissociation, vibrational frequencies, and diffusion properties are obtained with the ab-initio tight-binding molecular dynamics (MD) method developed by Sankey et al. [5,6] In the MD approach, the host crystal is represented by periodic supercells of 64 atoms. For details about the theoretical techniques and the cluster and supercells approximations, see Refs. [1] and [7].

\section{Vacancy Aggregates and Hydrogen}

One results of last year's subcontract was a detailed study of the formation and stability of small vacancy aggregates. $[3,4]$ The key results were that (1) vacancy-vacancy interactions lead to the formation of stable aggregates, and (2) the most stable of these aggregates is 
the ring-hexavacancy $\left(\mathrm{V}_{6}\right)$. Theory predicts that this 'missing ring' reconstructs so well that it has no electrical or optical activity. Yet, it is a large void and could be a gettering center for a range of impurities. The stability of $V_{6}$ has recently been confirmed by another theoretical group,[8] but direct experimental proof is still not available.

Ongoing calculations show that interstitial $\mathrm{H}$ is attracted to all vacancy clusters, where it traps with binding energies of the order of $3 \mathrm{eV}$. We do not know at this point if the vacancy clusters are passivated, partially passivated, or activated by the trapping of one $\mathrm{H}$ interstitial.

FIGURE 1: Energy gained by forming the $\left\{\mathrm{V}_{\mathrm{n}}, \mathrm{H}\right\}$ complexes starting with isolated $\mathrm{V}_{\mathrm{n}}$ and bond-centered hydrogen. Some data on the penta- and hexa-vacancies are missing. Different binding energies correspond to different trapping sites for $\mathrm{H}$ in a given vacancy aggregate.

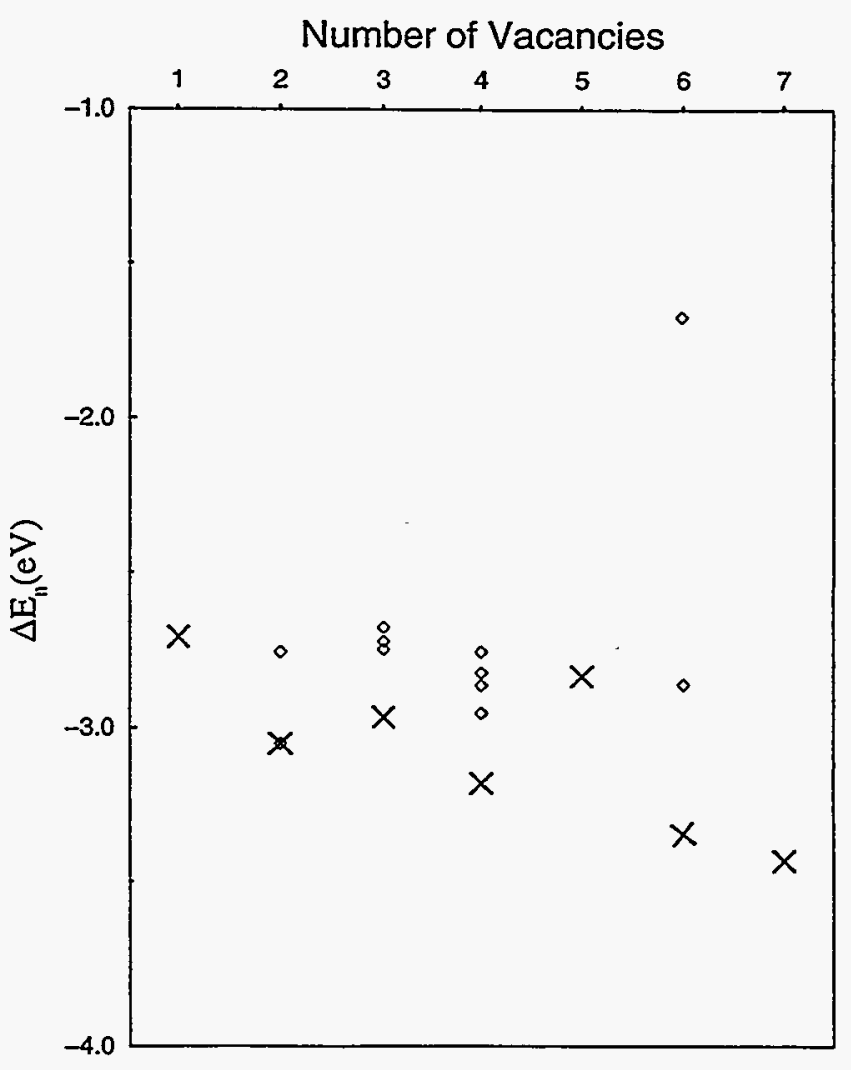

\section{Molecular Hydrogen Dissociation by Defects}

Ongoing MD simulations show that the $\mathrm{H}_{2}$ molecule is unstable in the vicinity of stretched, distorted, or imperfectly reconstructed $\mathrm{Si}-\mathrm{Si}$ bonds. At the same level of theory, $\mathrm{H}_{2}$ is stable at the tetrahedral interstitial site in Si. High- and low-temperature simulations show that if $\mathrm{H}_{2}$ is inside a vacancy or a hexavacancy, it spontaneously dissociates into two $\mathrm{H}$ atoms which then replace one reconstructed $\mathrm{Si}-\mathrm{Si}$ bond by strong two $\mathrm{Si}-\mathrm{H}$ bonds. In the case of the monovacancy, the gain in energy for this process is substantial: over $4 \mathrm{eV}$. We found no indication of a potential energy barrier for this process.

Further, if interstitial $\mathrm{H}_{2}$ is located not inside a vacancy aggregate but in the immediate vicinity of such a defect, the $\mathrm{H}-\mathrm{H}$ bond weakens and the molecule dissociates as well. This also seems to occur if $\mathrm{H}_{2}$ is near a Si self-interstitial (I). Although these results are still very preliminary, the picture which emerges is that of defect-induced dissociation of $\mathrm{H}_{2}$ in $\mathrm{Si}$. Vacancies and self-interstitials diffusing through the bulk efficiently dissociate interstitial $\mathrm{H}_{2}$ into two interstitial $\mathrm{H}$ which may diffuse away or be trapped by the defect which dissociated the molecule. This process provides a straightforward explanation as to why 
isolated $\mathrm{H}_{2}$ has never been detected in $\mathrm{c}-\mathrm{Si}$, and why proton implantation results in the formation of defects such as $\{\mathrm{I}, \mathrm{H}, \mathrm{H}\}$ while $\{\mathrm{I}, \mathrm{H}\}$ has not been identified.

These results strongly suggest that the exposure of a damaged Si surface to an $\mathrm{H}_{2}$ gas would result in the spontaneous dissociation of $\mathrm{H}_{2}$ into atomic $\mathrm{H}$. The latter binds to the surface layer which becomes super-saturated with hydrogen, providing a reservoir from where $\mathrm{H}$ diffuses into the bulk. However, the same gas would not interact with a perfectly reconstructed surface (no strained $\mathrm{Si}-\mathrm{Si}$ bonds). Hydrogen would remain in molecular form, and fail to bind to the surface.

\section{Transition Metals}

With the exception of our work on $\mathrm{Ti},[9]$ there is no published theoretical work on transition metals in Si that would include total energy calculations with lattice relaxations and distortions. All the work published to date assumed an equilibrium site for the transition metal and no lattice relaxation.

Ongoing calculations deal with copper in Si. They are performed at the approximate ab-initio Hartree-Fock level, and aim at determining the equilibrium sites and configurations. Single-point ab-initio calculations in the optimized geometries will provide more reliable energetics and electronic structures than the approximate calculations. The defects of interest are interstitial $\mathrm{Cu}(0$ and +1 charge states), $\mathrm{Cu}$-vacancy and $\mathrm{Cu}$-hexavacancy interactions, $\mathrm{Cu}$-acceptor pairs (B, $\mathrm{Al}$, and $\mathrm{Ga}$ will be considered), and $\mathrm{Cu}-\mathrm{Cu}$ interactions such as interstitial/substitutional pairs. The energy eigenvalues will indicate if these

various defects are electrically active. Finally, the interactions of selected complexes with hydrogen will be investigated. Similar work with Fe is planned.

\section{Recent publications which acknowledge NREL support}

S.K. Estreicher and P.A. Fedders: Molecular-Dynamics Studies of Defects and Impurities in Bulk Semiconductors, in 'Computational Studies of New Materials', ed. D.A. Jelski and T.F. George (World Scientific, Singapore, in print).

S.K. Estreicher and D.E. Boucher: Theoretical Studies in GaN, in GaN and Related Materials, ed. S.J. Pearton (Gordon and Breach, New-York, 1997), p.171.

S.K. Estreicher and P.A. Fedders: Molecular-Dynamics Modeling of Microscopic defects in Silicon, 14th NREL/SNL PV Program Review, ed. C.E. Witt, M. Al-Jassin, and J.M. Gee (AIP, New-York, 1997), p.729.

S.K. Estreicher, J.L. Hastings, and P.A. Fedders: The Ring-Hexavacancy in Silicon: $A$ stable and Inactive Defect, Appl. Phys. Lett. 70, 432 (1997).

J.I. Hastings, S.K. Estreicher, and P.A. Fedders: Vacancy Aggregates in Silicon, Phys. Rev. B 56, 10215 (1997).

S.K. Estreicher, J. Weber, A. Derecskei-Kovacs, and D.S. Marynick: Noble-Gas-Related Defects in Silicon and the Origin of the 1018 meV Photoluminescence Line, Phys. Rev. B 55, 5037 (1997). 


\section{References}

1. S.K. Estreicher, Mat. Sci. Engr. R 14, 319-412 (1995).

2. B.L. Sopori, X.J. Deng, J.P. Benner, A. Rohatgi, P. Sana, S.K. Estreicher, Y.K. Park, and M.A. Roberson, Sol. En. Mat. \& Sol. Cells 41/42, 159 (1996).

3. S.K. Estreicher, J.L. Hastings, and P.A. Fedders, Appl. Phys. Lett. 70, 432 (1997).

4. J.L. Hastings, S.K. Estreicher, and P.A. Fedders, Phys. Rev. B 56, 10215 (1997).

5. O.F. Sankey and D.J. Niklewski, Phys. Rev. B 40, 3979 (1989).

6. O.F. Sankey, D.J. Niklewski, D.A. Drabold, J.D. Dow, Phys. Rev. B 41, 12750 (1990).

7. S.K. Estreicher and P.A. Fedders in 'Computational Studies of New Materials', ed. D.A. Jelski and T.F. George (World Scientific, Singapore, in print).

8. A. Bongiorno, L. Colombo, and T. Diaz de La Rubia, private communication.

9. D.E. Woon, D.S. Marynick, and S.K. Estreicher, Phys. Rev. B 45, 13383 (1992). 
Title:

Organization:

Contributors:
Recombination Properties of Copper Precipitates in Silicon

University of California at Berkeley, Berkeley, CA

E.R.Weber, Principal Investigator; C.Flink, T.Heiser,

H.Hieslmair, A.A.Istratov, H.Hedemann, M.Seibt,

W.Seifert and M.Kittler

\section{Introduction}

It is well established that defect clusters are main lifetime-reducing defects in PV silicon. Comparing different grains of the same EFG silicon ribbon, the iron precipitation rate was found to correlate directly with the carrier diffusion length [1]. X-ray microanalysis mapping revealed high concentrations of transition metals in localized spots on grain boundaries and in even higher concentrations inside of the grains [2]. Gettering studies showed that these precipitates of metals can not be effectively dissolved and gettered using standard gettering procedures [3]. Recently, Werner et al. reported first TEM images of bulk microdefects [4]. In this report we summarize the results of studies of recombination activity of copper microprecipitates and show that precipitates of copper have substantially different electrical properties and much higher recombination activity than the interstitial copper and thus can be dangerous lifetime-reducing defects.

\section{Sample preparation}

For Transmission Electron Microscopy (TEM) and Deep Level Transient Spectroscopy (DLTS) studies of copper-silicide precipitates, copper was diffused from a metal layer into (100)-oriented phosphorus-doped n-type and boron-doped p-type $\mathrm{FZ}$ silicon samples. The free electron density, determined from capacitancevoltage characteristics (CV) at room temperature in as-grown samples, was (1 to 2) $\times 10^{15} \mathrm{~cm}^{-3}$. Copper diffusion at $850^{\circ} \mathrm{C}$ in a vertical furnace was followed by a quench in $10 \% \mathrm{NaOH}$ solution (estimated quenching rate $2000 \mathrm{~K} / \mathrm{s}$ ). Schottky diodes were fabricated by thermal evaporation of gold on chemically etched surfaces of n-type samples and of aluminum on p-type samples. Backside ohmic contacts were prepared using $\mathrm{GaAl}$ eutectics.

\section{TEM studies of copper-silicide precipitates}

TEM foils in (110) orientation were prepared from the bulk of the samples by standard techniques involving mechanical thinning followed by ion-milling. Electron micrographs were obtained at $200 \mathrm{kV}$ in a Philips CM200-UT-FEG. TEM investigations revealed plate-shaped precipitates, mostly parallel to $\mathrm{Si}(111)$ planes as is shown in Fig.1. Some of the precipitates had s-shaped form which besides the (111) habit plane showed a (113) orientation in their central part. The diameter of the precipitates was about $25 \mathrm{~nm}$, thickness did not exceed 2-3 $\mathrm{Si}(111)$ layers. A dislocation loop bounding the platelets was detected from the rigid shift introduced in the silicon matrix by the precipitate. Strong lattice strains due to large volume expansion during the formation of copper silicide were detected as contrasts around the precipitates.

DLTS spectra of copper-silicide precipitates obtained after a rapid quench and their comparison with simulations.

DLTS spectra obtained on $\mathrm{Cu}$-diffused samples after quenching in $\mathrm{NaOH}$ consist of a single peak of an unusual, almost rectangular shape (Fig. 2). DLTS measurements using different correlation frequencies revealed that the peak shifts along the temperature axis as a whole (Fig. $2 \mathrm{~b}$ ). No change in its shape was detected as would be expected if the peak were due to a superposition of independent point defects. Hence, we 
conclude that the peak stems from one single defect. Spectra obtained for different pulse widths (Fig. 2a) demonstrated a non-exponential capture behavior also pointing to an extended defect as a source of the spectra. Neither the shape of the spectra nor the capture kinetics could be satisfactorily explained by a simple Gaussian distribution of point defect levels as was proposed in the model of Omling et al. [5]. It follows from Fig.2, that the shape and temperature position of the high-temperature side of the peak is independent of the DLTS filling pulse width, whereas the low-temperature side changes as the pulse width increases. It has been recently shown by simulations $[6,7]$ and analytical calculations $[8,9]$, that this kind of behavior is a fingerprint of bandlike states.

In the first study of band-like states [6] it was assumed that the precipitates were neutral when empty and became negative when filled with electrons, i.e., that the precipitates created acceptor-like states in the band gap. However, the quantitative description of spectra obtained from nickel-silicide precipitates was successful only when the defects were assumed amphoteric, i.e., the defect occupation $F^{(\mathbb{N})}$ when it is neutral was set to a non-zero value [10]. For $\mathrm{NiSi}_{2}$ precipitates $\mathrm{F}^{(\mathrm{M})}$ was found to be between 0.3 to 0.5 . Simulations with qualitative agreement with the DLTS data for copper silicide precipitates in n-type material (Fig. 4) have only been reached with $F^{(M)}=0.8$ to 1.0 .

The simulated spectra are presented in Fig. 3 . The position of the defect band assumed for the simulations was between $\mathrm{E}_{\mathrm{C}}-0.15 \mathrm{eV}$ and $\mathrm{E}_{\mathrm{C}^{-}}-0.35 \mathrm{eV}$. The shape of the peaks and their dependence on the correlation frequencies and pulse width reproduce the main features of the experimental curves. The exact parameters of the defect band can be established only from a numerical fit. Such a fit requires an enormous amount of involved calculations (about $10^{9}$ rate equations have to be solved) and could not be accomplished at the time when this report was prepared.

\section{Recombination activity of the precipitated copper.}

The recombination activity of precipitated copper was studied comparing the minority carrier diffusion length of the samples, characterized by TEM and DLTS above. The diffusion lengths of minority carriers, determined by EBIC from the dependence of collection efficiency versus accelerating voltage [11], are presented in Table 1 together with the value obtained earlier for the interstitial copper [12].

As follows from Table 1, copper-silicide precipitates are extremely active recombination centers. The diffusion length $L_{D}$ in samples containing precipitated copper is only slightly larger than the average distance $l$ between the precipitates. In contrast, the ratio $L_{D} /$ for the interstitial copper is about 300 times higher. This means that the same concentration of copper is far more recombinative active when it is precipitated than when it is in the interstitial state, and that the recombination activity of copper-silicide precipitates can not be obtained by just adding the recombinative properties of single copper atoms. In our opinion, there are two mechanisms playing a decisive role in the recombination properties of copper-silicide precipitates. The first mechanism is the attraction of the charge carriers by space charge regions around the precipitates. This model was suggested by Kittler et al. $[13,14,15]$ to explain the unusually high recombination activity of nickel-silicide precipitates in silicon. The second important recombination mechanism is the recombination via the bandlike states of copper precipitates. As it follows from Shockley-Read-Hall statistics, the highest recombination activity is expected from defects which have a level close to the mid-gap. Copper-

Table 1. Minority carrier diffusion length $\mathrm{L}_{D}$, determined for the samples after different quench, and its ratio to the average distance between precipitates $l$, determined from the precipitates density $\mathrm{N}_{\mathrm{p}}$.

\begin{tabular}{|c|c|c|c|c|}
\hline type of recombination centers & $\mathrm{N}_{\mathrm{P}} \mathrm{cm}^{-3}$ & $l, \mu \mathrm{m}$ & $\mathrm{L}_{\mathrm{D},} \mu \mathrm{m}$ & $\begin{array}{c}\text { ratio } \\
\mathrm{L}_{\mathrm{D}} / l\end{array}$ \\
\hline $\mathrm{NaOH}$, precipitates, $\mathrm{n}-\mathrm{Si}, \mathrm{p}-\mathrm{Si}$ & $4.3 \times 10^{12}$ & 0.6 & $<2.2$ & 3.6 \\
\hline interstitial copper, $\mathrm{p}-\mathrm{Si}$ & $10^{15}$ & 0.1 & 110 & 1100 \\
\hline
\end{tabular}



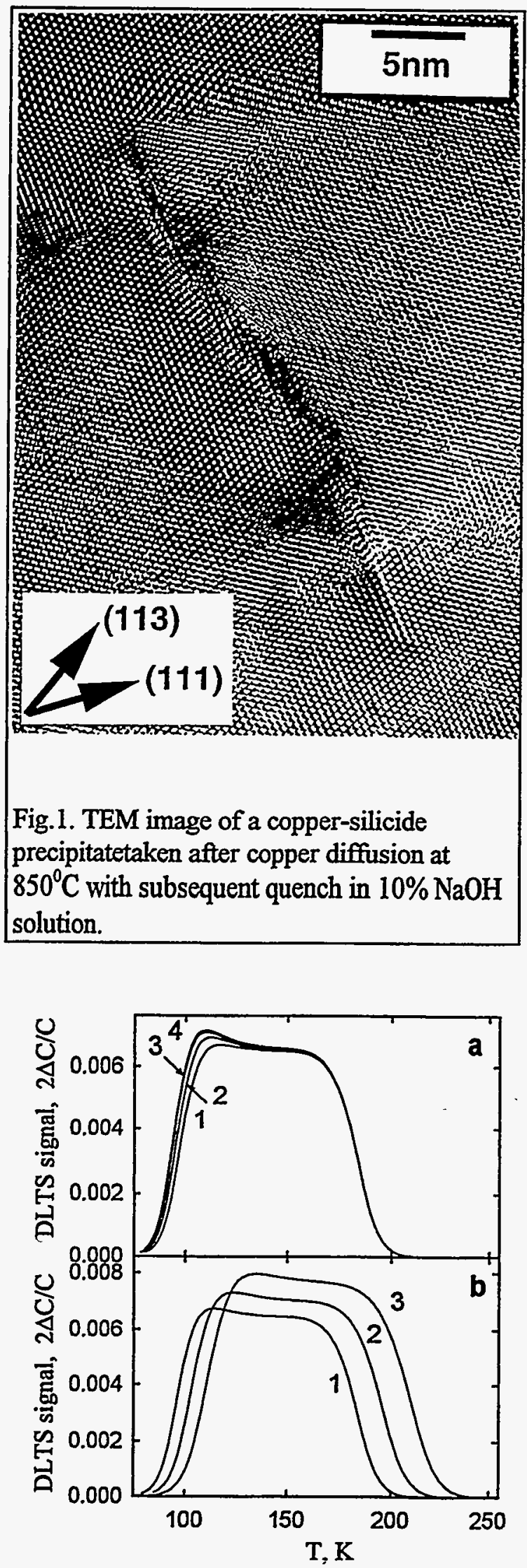

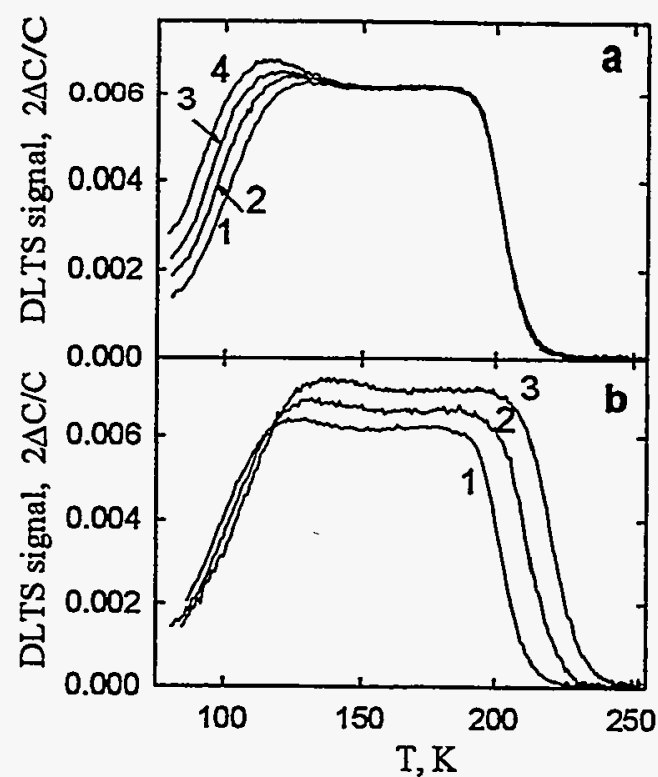

Fig. 2. Experimental DLTS spectra of the coppersilicide precipitates obtained after a fast quench of $n-$ type silicon samples: a) filling pulse width variation, $t_{\mathrm{p}}$ $=30 \mu$ s (curve 1), $300 \mu$ s (curve 2), 3 ms (curve 3) and $30 \mathrm{~ms}$ (curve 4); the spectra were taken with the sinusoidal lock-in correlation frequency $f=1.907 \mathrm{~Hz}$. b) sinusoidal lock-in correlation frequency variation: curve $1-1.907 \mathrm{~Hz}$, curve $2-7.63 \mathrm{~Hz}$, curve $3-30.52$ $\mathrm{Hz}$; filling pulse width $\mathrm{t}_{\mathrm{p}}=100 \mu \mathrm{s}$.
Fig.3. Simulated pulse width and correlation frequency variations for a band-like defect with a box-like distribution of levels between $\mathrm{E}_{\mathrm{C}}-0.15$ $\mathrm{eV}$ and $\mathrm{E}_{\mathrm{C}}-0.35 \mathrm{eV}, \mathrm{F}_{\mathrm{N}}=0.91$, $\alpha=0.53 \mathrm{eV}$, capture cross section $\sigma=1.0 \times 10^{-17} \mathrm{~cm}^{-2}$ and a total concentration of deep levels of $2.0 \times 10^{14}$ $\mathrm{cm}^{-3}$. Further values of parameters are assumed as in the experimental spectra in Fig. 2. 
silicide precipitates form a defect band with a position quite close to the mid-gap. Therefore, the band can provide an excellent recombination channel for minority carriers, attracted by the electric field of charged precipitates.

The deleterious role of microdefects on the lifetime of minority carrier in solar cells has been discussed for a long time (see for example [16]). Correia et al. [17] found that in $\mathrm{Cu}$-contaminated samples the diffusion length of the minority carriers dropped by more than an order of magnitude due to defects in the form of platelets in (111) planes with a diameter of about $12 \mathrm{~nm}$. These defects were surrounded by strong deformation fields, typical for $\mathrm{Cu}$-precipitates. This suggests that copper precipitates may be one of the defects responsible for the lifetime reduction in PV silicon.

As discussed above, the density of defect states can be determined only from an elaborate numerical fit, and the apparent defect density determined from the amplitude of the DLTS peak is much less than the real value. In our experiments, the samples quenched into $10 \% \mathrm{NaOH}$ after $850^{\circ} \mathrm{C}$ anneal (equilibrium $\mathrm{Cu}$ solubility was about $10^{17} \mathrm{~cm}^{-3}$ ) had a diffusion length less than $2 \mu \mathrm{m}$ and the apparent amplitude of the DLTS signal was about $2 \Delta \mathrm{C} / \mathrm{C} \sim 6 \times 10^{-3}$. If the density of precipitates decreased by a factor of 500 , then the DLTS signal amplitude would drop below the detection limit $\left(2 \Delta \mathrm{C} / \mathrm{C} \sim 10^{-5}\right.$ for an average DLTS setup); however, using the Shockley-Read-Hall statistics, the diffusion length can be estimated to remain below $45 \mu \mathrm{m}$. This may explain why in PV silicon no deep levels in significant concentration have been found by DLTS in spite of the low minority carrier diffusion length.

\section{Acknowledgments}

The use of the Lawrence Berkeley National Laboratory experimental facilities which are funded through DOE is acknowledged.

\section{References}

1. J.Bailey and E.R.Weber, phys.stat.sol.(a) 137, 515 (1993).

2. M.Werner, E.R.Weber, S.McHugo and K.L.Chapman, Solid State Phenomena 51-52, 81 (1996).

3. S.A.McHugo, J.Bailey, H.Hieslmair and E.R.Weber, in: IEEE $1^{\text {st }}$ World Conference on Photovoltaic Energy Conversion, Hawaii, 1994, vol.II, p.1607.

4. M.Werner, H.J.Möller and E.Wolf, MRS Symp.Proc. 469, 89 (1997).

5. P. Omling, E.R.Weber, L.Montelius and H.Alexander, Phys.Rev.B 32, 6571, (1985)

6. W.Schröter, J.Kronewitz, U.Gnauert, F.Riedel and M.Seibt, Phys.Rev.B 52, 13726 (1995).

7. H.Hedemann and W.Schröter, Journal de Physique III, 7, 1389 (1997).

8. H. Hedemann, Ph.D. thesis, Cuvillier-Verlag Göttingen, ISBN 3-89588-377-8 (1995).

9. H. Hedemann and W. Schröter, submitted to Phys.Rev.B

10. F.Riedel, J.Kronewitz, U.Gnauert, M.Seibt and W.Schröter, Solid State Phenomena, 47-48, 359 (1995).

11. A.A.Istratov, H.Hieslmair, T.Heiser, C.Flink, E.R.Weber, W.Seifert and M.Kittler, in: VIIth Workshop on the Role of Impurities and Defects in Silicon Device Processing, B.L.Sopori, Editor, p.158, NREL, Golden, Colorado (1997).

12. A.A.Istratov, C.Flink, H.Hieslmair, T.Heiser and E.R.Weber, Appl.Phys.Lett. 71, 2121 (1997).

13. M. Kittler, J. Lärz, W. Seifert, M. Seibt and W. Schröter, Appl. Phys. Lett. 58, 911 (1991)

14. M. Kittler and W. Seifert phys. stat. sol. (a) 150, 463 (1995)

15. M. Kittler and W. Seifert, Mater. Sci. Forum 196-201, 1123 (1995)

16. J.Bailey and E.R.Weber, phys.stat.sol.(a) 137, 515 (1993).

17. A.Correia, D.Ballutaud, A.Boutry-Forveille and J.L.Marice, J.Appl.Phys. 78, 6543 (1995). 
Organization: Center of Microelectronics Research,

$$
\text { University of South Florida, Tampa, Florida } 33620
$$

Contributors:

\author{
B. Sopori, program manager \\ L. Jastrzebski, principal investigator, \\ S. Ostapenko, and Y. Koshka
}

\title{
Objectives
}

Effort was focused on developing a new diagnostic approach to defect characterization in bulk polycrystalline silicon (poly-Si) wafers and solar cells using room-temperature photoluminescence (RTPL). Luminescence mapping is recognized as a sensitive non-contact and non-destructive technique used to monitor crystalline Si quality. As previously reported [1], RTPL mapping applied to PV poly-Si is comparable to surface photovoltage possessing, with some additional advantages. We performed a quantitative correlation of RTPL intensity and minority carrier diffusion length, $\mathrm{L}$, in strongly inhomogeneous poly-Si wafers. As a unique feature, RTPL offers a new possibility of a separate defect diagnostics in $\mathrm{p} / \mathrm{n}$ junction and bulk of solar cells.

\section{Apparatus and samples}

RTPL spectra were analyzed using a SPEX 500M grating spectrometer coupled with liquid nitrogen cooled Ge detector. An $\mathrm{Ar}^{+}$- laser $514 \mathrm{~nm}$ line with power from 10 to $80 \mathrm{~mW}$ was used as the excitation source. PL spectra were corrected to a set-up spectral response. RTPL mapping was performed by placing a wafer at a programmable $x-y$ stage. Mapping spatial resolution was controlled by the Ar laser spot size, which can range from a couple of millimeters down to $100 \mu \mathrm{m}$. For bias experiments, PL intensity was measured under dc voltages from $-1.5 \mathrm{~V}$ to $1.5 \mathrm{~V}$ applied to solar cell. Minority carrier diffusion length $(\mathrm{L})$ was measured with a commercial SPV tool (CMS-II). To perform point-by-point comparison of the RTPL intensity and diffusion length, spatial precautions were undertaken with regard to a sample positioning and holding. Poly-Si $10 \mathrm{~cm} \times 10 \mathrm{~cm}$ wafers and solar cells were grown by the EFG technique. PL and SPV data was collected after three consecutive solar cell processing, including Pdiffusion, hydrogen passivation, and $\mathrm{Al}$ back-side alloying.

\section{(a) RTPL versus minority carrier diffusion length}

Band-to band photoluminescence with maximum at $1.09 \mathrm{eV}$ and "defect" PL band $\left(\mathrm{h} v_{\max }=0.8 \mathrm{eV}\right)$ are observed on EFG wafers at room temperature (Figure1). As previously reported [1], band-to-band PL intensity shows a dramatic increase by a factor of two orders of magnitude after three solar-cell processing steps. It was also observed that upgrading PL intensity reflected a positive correlation with improvement of minority carrier diffusion length measured by SPV [2]. RTPL mapping was compared with identical mapping of the diffusion length (same wafer regions and space resolution). Point-by-point dependence is presented in Figure 2, which shows a clear trend: increasing of $L$ is accompanied by increase of band-to-band RTPL intensity. The best fit of data-points using power functions is shown as a solid line, which reveals the quadratic dependence. This observation is a consequence that non-radiative recombination dominates in silicon at $300 \mathrm{~K}$. In fact, $\mathrm{RTPL}$ intensity, $\mathrm{I}_{\mathrm{b}-\mathrm{b}}$, can be expressed versus $\mathrm{L}$,

$$
I_{b-b} \sim P_{b-b} /\left(P_{b-b}+P_{d e f}+P_{n o n-r a d}\right) \sim P_{b-b} / P_{\text {non-rad }} \sim \tau_{\text {non-rad }} \sim \mathrm{L}^{2}
$$


where $P_{b-b}, P_{d e f}$, and $P_{n o n-r a d}$ are probabilities of band-to-band PL, "defect" PL and non-radiative recombination.

We can suggest that at high laser excitation (above $10 \mathrm{~mW}$ ) the mapping is similar to SPV mapping and reflects distribution of the bulk diffusion length. This is different at low laser excitation $(10 \mathrm{~mW}$ and less) where the recombination in $\mathrm{p} / \mathrm{n}$ junction dominates.

\section{(b) RTPL in bulk and in $\mathbf{p} / \mathbf{n}$ junction}

In order to separate bulk and p/n junction quality, RTPL was measured under dc bias. We observed two effects: (a) PL intensity is strongly increased when forward bias is applied to solar cell, and (b) PL intensity line scans show a noticeable difference under reverse and forward bias. This is shown in Figure 3.

Bias dependence is explained as a different contribution to RTPL of bulk and $\mathrm{p} / \mathrm{n}$ junction. As illustrated in Fig.4. Generally, two solar cell regions contribute to PL intensity. Light excited carriers can recombine in the $\mathrm{p} / \mathrm{n}$ junction where they are generated due to strong absorption of $514 \mathrm{~nm}$ laser light. This process will be observed as the $\mathrm{p} / \mathrm{n}$ junction photoluminescence. Penetration of minority carriers into the bulk is a source of the bulk PL. Depletion region electric field influences a fraction of $\mathrm{p} / \mathrm{n}$ junction PL to bulk PL restricting diffusion of minority carriers into the bulk. The forward bias causes lowering of the junction barrier for minority carriers (Fig. 4a). The minority carriers (electrons) diffuse into the bulk p-region and recombine with majority carriers increasing the bulk PL component. The situation changes when reverse bias is applied: increased height of the barrier prevents electrons to be injected into the bulk (Fig. 4b). Separation of electron-hole pairs by barrier field causes electrons and holes to be injected to the regions where they increase concentration of majority carriers (electrons in nregion and holes in p-region). Thus, these separated carriers are lost for PL. The only contribution to PL occurs from carriers that recombine directly in $\mathrm{p} / \mathrm{n}$ junction. Consequently, a separate monitoring of $\mathrm{p} / \mathrm{n}$ junction and bulk is available by RTPL mapping under reverse and forward bias respectively.

Figure 3 shows PL line scans measured at different biases. In addition to the increase of PL intensity with forward bias, dramatic change of the line scan profile is observed. At reverse bias PL intensity distribution is flat due to homogeneous properties of $\mathrm{p} / \mathrm{n}$ junction. With increasing of forward bias, a bulk recombination contributes to RTPL intensity. PL variation across the line scan at $0.8 \mathrm{~V}$ forward bias is apparently due to non-homogeneity of bulk properties.

An interesting observation was made by individual mapping of band-to-band and "defect" RTPL, and plotting them as $\mathrm{I}_{\mathrm{b}-\mathrm{b}}$ versus $\mathrm{I}_{\text {def. }}$ Generally, data points show a strong scattering across the entire $10 \mathrm{~cm} \times 10 \mathrm{~cm}$ EFG wafer. However, taking data locally from different areas of a few $\mathrm{cm}^{2}$, we observed a clear reverse dependence as illustrated in Fig.5. It proves that areas with high intensity of "defect" PL contain increased concentration of non-radiative centers that reduce diffusion length and band-to-band PL intensity. PL mapping of the "defect" band can assess monitoring of "bad" regions in PV poly-Si and control their elimination using defect gettering and passivation.

\section{Conclusions}

Room temperature PL mapping is a reliable tool for mónitoring quality upgrading in PV polycrystalline silicon. It is a preparation free technique, possessing a high throughput, and offers additional advantages by selective monitoring of bulk and $\mathrm{p} / \mathrm{n}$ junction in solar cells. 


\section{References}

1. Annual report, Photovoltaic Program, FY 1996. NREL/BK-210-21966 (August 1997) , p. 49

2. Bailey, J., Kalejs, J.P., and Keaveny, C. (1994), “Material Electronic Quality Specification factors for Poly-Si Wafers", IEEE Conference on Photovoltaic energy Conversion -1994; Waikoloa, Hawaii, pp. $1356-1359$.

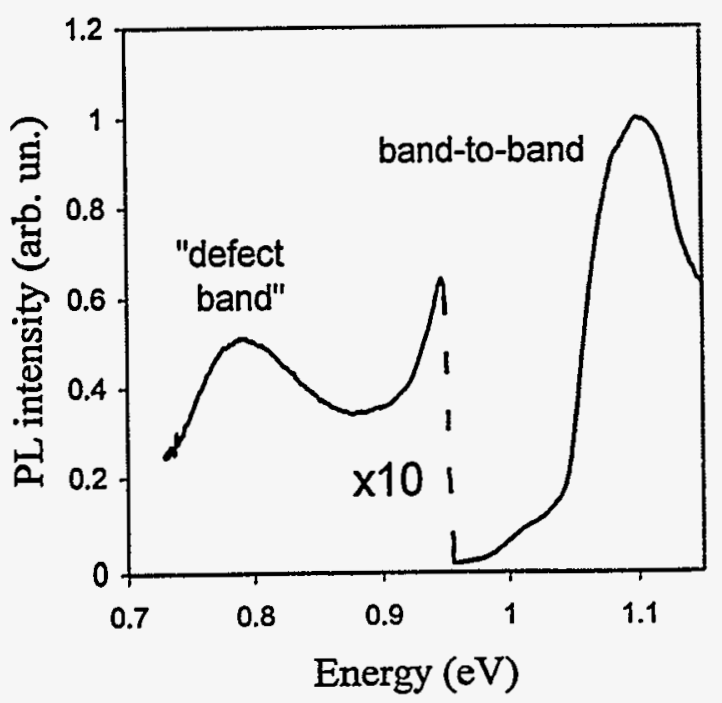

Fig. 1 Photoluminescence in EFG poly-Si at room temperature; excitation $514 \mathrm{~nm}, 40 \mathrm{~mW}$

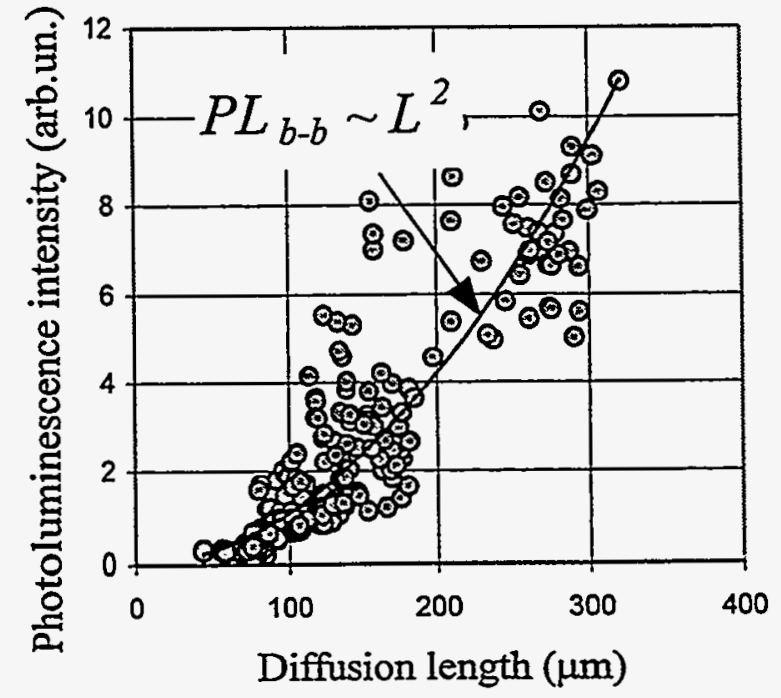

Fig. 2 Band-to-band PL intensity measured across the entire $10 \times 10 \mathrm{~cm}$ EFG wafer reveals the power-law dependence versus minority carrier diffusion length.

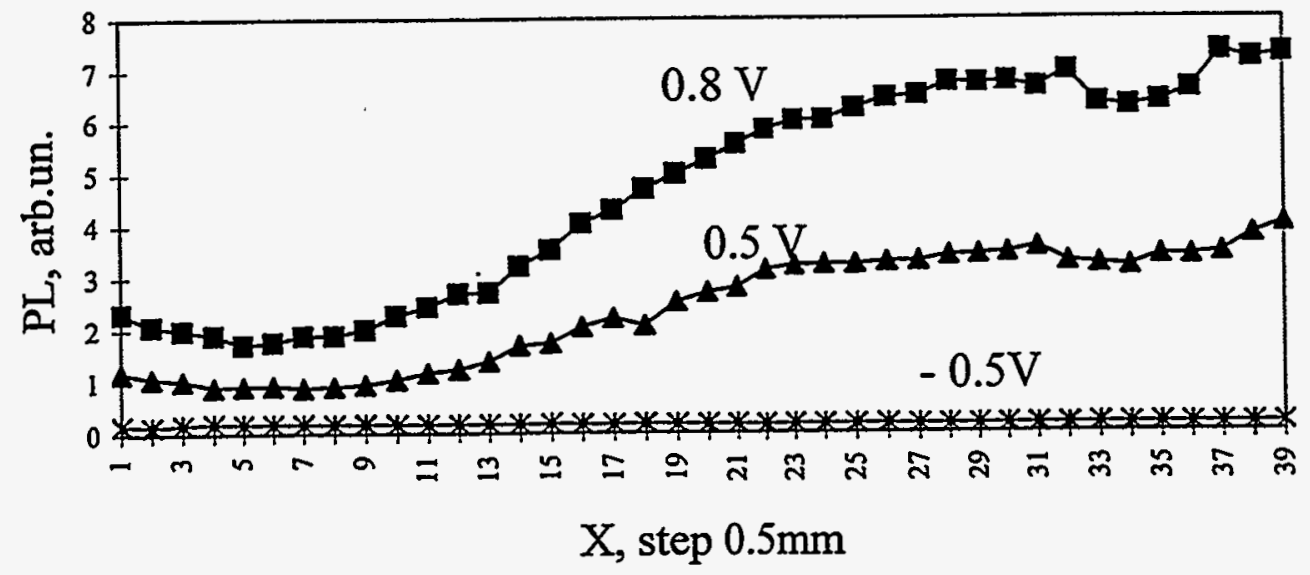

Fig. 3 Band-to-band RTPL line scans are changed under applied bias. 
a)

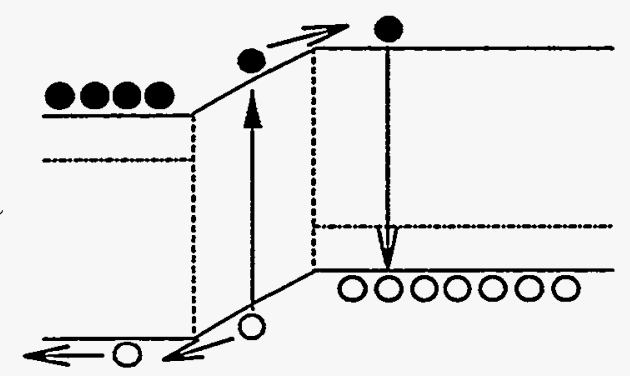

b)

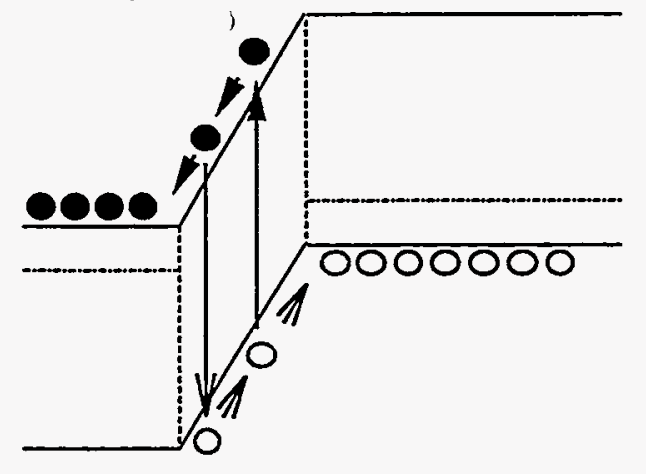

Fig. 4 The model of a bias influence on the PL intensity from solar cell: (a) at forward bias, light-exited electrons are injected in the bulk p-region where they recombine with majority holes giving rise to the bulk $\mathrm{PL}$; (b) at reverse bias, recombination occurs in $\mathrm{p} / \mathrm{n}$ junction.

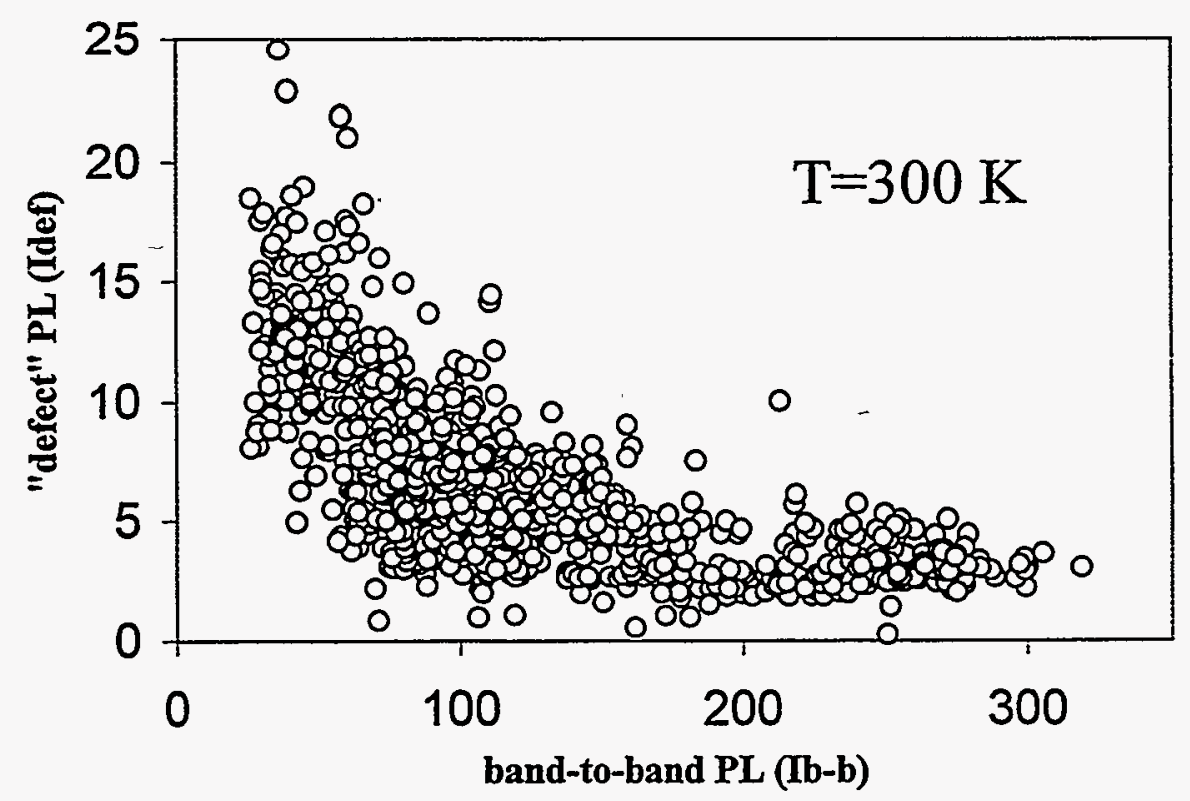

Fig. 5 Reverse dependence between band-to-band and defect photoluminescence measured at a $15 \times 15 \mathrm{~mm}$ wafer region 


\title{
Exploratory Materials and Devices
}

\author{
In-House Research
}


Title: $\quad \therefore \quad$ EE/ER Collaborative Research: Photochemical Solar Cell Development
and Optimization

Organization: $\quad$ Center for Basic Sciences, Chemical Sciences Team

National Renewable Energy Laboratory, Golden, CO

Contributors: $\quad$ A.J. Frank (Senior Scientist); B.A. Gregg (Senior Scientist); N.-G. Park (Postdoctoral Associate); A. Zaban (Postdoctoral Associate); S.-Y. Huang (Postdoctoral Associate); B. O'Regan (Graduate Student, subcontract to University of Washington); M. Grätzel (EPFL Collaborator); A.J. Nozik (Team Leader)

\section{Objectives}

The objective of this work is to develop very-low-cost, durable, and efficient $(>10 \%)$ photochemical solar cells; this new type of solar cell is based on the dye-sensitization of nanocrystalline films of titanium dioxide $\left(\mathrm{TiO}_{2}\right)$. The research focuses on defining and solving the problems that limit the efficiency and commercial viability of dye-sensitized nanocrystalline $\mathrm{TiO}_{2}$ photochemical solar -cells.

\section{Technical Approach}

This project is part of an integrated program of basic and applied research and development jointly funded by the Office of Energy Research (ER)/Basic Energy Sciences (BES)/Chemical Sciences Division and the Office of Energy Efficiency and Renewable Energy (EE) PV program to explore, understand, and develop photochemical solar cells. This new.type of cell offers the possibility of very inexpensive manufacturing and materials cost, with projected module costs of $\$ 55 / \mathrm{m}^{2}$ and $\$ 0.60 / \mathrm{Wp}$.

The cell is formed upon adsorbing a monolayer of an appropriate dye molecule on a $10-30 \mu \mathrm{m}$ nanocrystalline film of $\mathrm{TiO}_{2}$ particles (30-nm diameter); which is supported on transparent conducting oxide glass, filling the void space between the particles in the thin nanocrystalline film with a nonaqueous electrolyte containing iodine and iodide ions, installing a counter-electrode, and sealing the edges of the cell to contain all the components. The program is designed so that basic research on the mechanisms and fundamental properties supported by the ER/BES/Chemical Sciences program will support and underpin the cell development program supported by the EE PV program.

\section{Results}

(1) The conversion efficiency of an NREL-produced closed (sealed) cell; without an antireflection coating and with minimal optimization of cell parameters, was increased during the past year from $5.0 \%$ to $8.6 \%$. A short-term stability test, covering a three-day period, showed that the cell efficiency did not change. Photocurrent densities at the $16 \mathrm{~mA} / \mathrm{cm}^{2}$ level (AM 1.5) are now reproducible. Several cells have shown short-circuit current density $\left(J_{\mathrm{sc}}\right)$ as high as $18 \mathrm{~mA} / \mathrm{cm}^{2}$, which matches the 
previous best reported value for $\mathrm{TiO}_{2}$ solar cells, using the same sensitizing dye. Adding an antireflection coating is expected to bring the photocurrent density above $19 \mathrm{~mA} / \mathrm{cm}^{2}$. This approaches the highest reported value of $20.4 \mathrm{~mA} / \mathrm{cm}^{2}$ for cells containing a new-generation dye system. In part, the improvements in cell performance were the result of our research activities under the ER/Chemical Sciences program.

(2) In collaboration with Dr. M. Grätzel, a 10\% dye cell was fabricated and measured at NREL's standard laboratory to be $10 \%$ efficient.

(3) Varying the redox electrolyte composition and the dye has a dramatic effect on cell performance. Preliminary results suggest that $\mathrm{J}_{\mathrm{sc}}$ increases with decreasing size of the iodide cation. The highest $\mathrm{J}_{\mathrm{sc}}$ was achieved with $\mathrm{Li}^{+}$ions. The composition of the redox electrolyte was also found to strongly influence $\mathrm{V}_{\mathrm{oc}}$, ff, eff, and the choice of sensitizing dye.

(4) We developed a new technique for probing the kinetics and energetics of charge transport and charge transfer in nanocrystalline semiconductor electrodes. We refer to this technique as intensitymodulated photovoltage spectroscopy (IMVS). With IMVS, we have investigated a number of important processes that govern the performance of dye-sensitized nanocrystalline $\mathrm{TiO}_{2}$ solar cells. Among the critical issues addressed were the underlying cause for the photovoltage and the improved performance resulting from chemically modifying the $\mathrm{TiO}_{2}$ surface, the mechanism and kinetics of charge recombination at the solid/redox electrolyte interface, and the effect of the nanoporous structure on the mass transport of redox species in the solution phase. From the determination of the order of the recombination reaction, the identity of the iodide species involved in the back electron transfer was inferred.

(5) A comparison of the time constants for charge recombination and the charge-collection efficiency, obtained from preliminary IMVS and intensity-modulated photocurrent spectroscopy (IMPS) measurements, shows that the charge-collection efficiency is $90 \%-97 \%$ for solar cells, fabricated with $8-\mu \mathrm{m}$-thick $\mathrm{TiO}_{2}$ films and illuminated with $680-\mathrm{nm}$ monochromatic light. Furthermore, the charge collection efficiency was found to depend on surface treatment of the $\mathrm{TiO}_{2}$ films. Films treated with TBP (4-tert-butylpyridine) displayed a higher charge-collection efficiency than those treated with $\mathrm{NH}_{3}$ (ammonia). Additional study is planned to determine whether the higher charge-collection efficiency of the TBP-treated films accounts for its higher photocurrent density compared with that of $\mathrm{NH}_{3}$-treated samples. This is an important issue because $\mathrm{NH}_{3}$-treated samples show a higher open-circuit voltage $\left(\mathrm{V}_{o c}\right)$ than TBP-treated films.

(6) Treatment of the surface of $\mathrm{TiO}_{2}$ films with $\mathrm{TiCl}_{4}$ is found to have a significant effect on the $\mathrm{J}_{\mathrm{sc}}$ $\mathrm{V}_{o c}$, fill factor (ff), and conversion efficiency eff. During a three-day period, increased exposure of the films to $\mathrm{TiCl}_{4}$ led to higher $\mathrm{J}_{\mathrm{sc}}$ and eff and lower $\mathrm{V}_{\mathrm{oc}}$ and ff. The period for optimizing the cell parameters was determined. The improved $\mathrm{J}_{\mathrm{sc}}$ is presumed to arise from the hydrolysis of $\mathrm{TiCl}_{4}$ to form $\mathrm{TiO}_{2}$ particles, resulting in additional dye adsorption sites. IMPS and IMVS measurements of the cells indicate that the time constant for both electron diffusion through $\mathrm{TiO}_{2}$ films and back reaction with redox species at the $\mathrm{TiO}_{2}$ /solution interface increased with exposure time.

(7) IMPS measurements indicate that exchanging a small cation $\left(\mathrm{Li}^{+}\right)$for a large cation (tetrabutylammonium ion) has no effect on the time constant (in the millisecond regime) for electron 
transport across the $\mathrm{TiO}_{2}$ film. This result strongly suggests that the interaction between cations and accumulated electrons in the $\mathrm{TiO}_{2}$ film is weak.

(8) The potential distribution in dye-sensitized photochemical solar cells has been established for the first time using impedance spectroscopy. It was found that ions in the electrolyte diffuse freely into the film and neutralize applied fields over very short distances from the back contact; no electric fields exist beyond $10 \mathrm{~nm}$. Thus, photoinduced charge transport occurs by diffusion and is unassisted by electric fields. These results indicate that it will be difficult to replace the liquid electrolyte with a solid electrolyte unless the latter is designed to contain mobile-charged species.

(9) Our study of the relative energetics at the semiconductor/sensitizing dye/electrolyte interface led to the important conclusion that the redox potential of the sensitizing dye is not fixed relative to either the potential of the semiconductor or the potential of the solution. It can be made to approximately follow the semiconductor potential by using large electrolyte ions, or it can be made almost independent of the semiconductor potential by using very small ions. The $\mathrm{V}_{\mathrm{oc}}$-limiting kinetic step will be different in the two cases.

(10) It has been shown that the oxidation-reduction potential of the dye molecules becomes $\mathrm{pH}$ dependent when it is adsorbed on the $\mathrm{TiO}_{2}$ nanoparticles; in solution the oxidation potential is independent of $\mathrm{pH}$. This result is critical for understanding the details of the cell operation and why the charge-transfer reaction and the recombination rate are independent of $\mathrm{pH}$. Understanding these factors is important for optimizing the photovoltage and photocurrent of the cell.

(11) Some perylene dyes have been shown to be efficient sensitizers of high-surface-area tin oxide electrodes. The perylenes are very stable and have absorption coefficients 5-10 times higher than those of the ruthenium complexes normally used. This may allow us to make much thinner cells with reduced mass-transfer limitations, an important criterion for an eventual solid-state cell.

(12) We developed a laser-scanning system, with $50-\mu \mathrm{m}$ optical resolution, to map the local PV characteristics across the $\mathrm{TiO}_{2}$ electrode.

\section{Technology Transfer}

Several companies have expressed interest in photochemical solar cells and have visited NREL for discussions:

- Informal discussions on photochemical solar cells were held with researchers and research managers from Monsanto, duPont, Exxon, and Kodak. These companies will probably begin research and development programs in this area.

- We met with Ed Funk, president of Superconductive Components, Inc., to discuss a possible cooperative research and development agreement (CRADA) to move the photochemical solar cells toward commercialization. He said he would make a decision in a month or two about whether to proceed. 
- Two researchers from Monsanto visitéd NREL for two days in September to learn some experimental techniques for improving the performance of the photochemical solar cells produced at Monsanto. They have made a proposal to Monsanto management to begin a major multimillion-dollar program that would include funding for NREL participation at about $\$ 200 \mathrm{~K}-\$ 300 \mathrm{~K}$.

- NREL received further contact from Superconductive Technologies Company concerning another possible CRADA for a total of $\$ 600 \mathrm{~K}$ over three years to help develop commercially viable photochemical solar cells. We may have to choose between these two organizations.

- A.J. Frank, B.A. Gregg, AJ. Nozik, and D. Christensen visited Monsanto in St. Louis to discuss their activities in dye-sensitized solar cells, and to help establish a major Monsanto research effort in the area. A "funds-in" CRADA may evolve from this interaction.

\section{Conclusions}

Photochemical solar cells have the potential to develop into a new low-cost and commercially viable PV alternative. After a little over two years of research, the efficiency of NREL-produced devices is approaching 9\%; cells produced at EPFL with our collaborator, Dr. M. Grätzel, have been measured by NREL at $10 \%$. The ultimate attainable efficiency for photochemical solar cells is unknown. NREL research is focused on improving the cell efficiency, developing cascade-type cells, developing interconnect strategies to produce large-area modules, developing solid ionic conductors to replace the liquid electrolyte and characterizing and improving cell lifetime. Discussions with several industrial organizations about possible CRADAs and other cooperative agreements are continuing. A potentially important near-term spin-off application from the EE/ER Photochemical Solar Cell Project in the form of a photoelectrochromic window has been discovered, and industrial relationships to develop and market this new invention are in progress.

\section{Publications}

1. "Charge Recombination in Dye-Sensitized Nanocrystalline $\mathrm{TiO}_{2}$ Solar Cells," Huang, S.Y.; Schlichthörl, G.; Nozik, A.J.; Grätzel, M.; Frank, A.J. J. Phys. Chem. B 1997, 101, 2576.

2. "Band Edge Movement and Recombination Kinetics in Dye-Sensitized Nanocrystalline $\mathrm{TiO}_{2}$ Solar Cells: A Study by Intensity Modulated Photovoltage Spectroscopy," Schlichthörl, G.; Huang S.Y.; Sprague, J.; Frank, A.J. J. Phys Chem. B 1997, 101, 8139.

3. "pH-dependent Redox Potential Induced in a Sensitizing Dye by Adsorption onto $\mathrm{TiO}_{2}$," Zaban, A.; Ferrere, S.; Sprague, J.; Gregg, B.A. J. Phys. Chem. 1997, 101, 55.

4. "Dye Sensitization of Nanocrystalline Tin Oxide by Perylene Derivatives," Ferrere, S.; Zaban, A.; Gregg, B.A. J. Phys. Chem. B 1997, 23, 4490.

5. "Impedance Spectroscopy of High Surface Area TiO ${ }_{2}$ Electrodes," Zaban, A.; Meier, A.; Nozik, A.J.; Gregg, B.A Proc. Symp. Electrode Materials and Processes for Energy Conversion and Storage IV 1997, in press. 
6. "Electric Potential Distribution and Short-Range Screening in Nanoporous $\mathrm{TiO}_{2}$ Electrodes," Zaban, A.; Meier, A.; Gregg, B.A. J. Phys. Chem. B 1997, 101, 7985.

7. "Photochemical Solar Cells Based on Dye-Sensitization of Nanocrystalline $\mathrm{TiO}_{2}$ " Frank, A.J.; Gregg, B.A.; Nozik, A.J.; Zaban, A.; Ferrere, S.; Schlichthörl, G.; Huang S.Y. In Future Generation Photovoltaic Technologies: First NREL Conference; McConnell, R.D., Ed.; AIP Conference Proceedings 404; American Institute of Physics: Woodbury, NY; 1997, p.145.

8. "Photochemical Solar Cells Based on Dye-Sensitization of Nanocrystalline $\mathrm{TiO}_{2}$," Deb, S.; Ferrere, S.; Frank, A.J.; Gregg, B.A.; Huang S.Y.; Nozik, A.J.; Schlichthörl; G; Zaban, A. Proc. 26th IEEE Photovoltaic Specialists Conference, in press.

9. "Dye-Sensitized Nanocrystalline $\mathrm{TiO}_{2}$ Solar Cells," Schlichthörl, G.; Huang, S.Y.; Nozik, A.J.; Frank, AJ. In Proc. 6th CMC Int. Symposium on Photochemical Electron Transfer: Molecular Approach to Photocatalysis; Center for Molecular Catalysis, Seoul National University: Seoul, South Korea; 1997, pp.23-28.

10. "Photosensitization of Semiconductors by $\left[\mathrm{Fe}^{\mathrm{I}}(2,2 \text { '-bipyridine-4,4'-dicarboxylic acid })_{2}\left(\mathrm{CN}_{2}\right)\right]$ : Band Selective Electron Injection from Ultra Short Lived Excited States," Ferrere, S.; Gregg, B.A. J. Am. Chem. Soc., in press.

11. "Relative Energetics at the Semiconductor/Sensitizing Dye/Electrolyte Interface," Zaban, A.; Ferrere, S.; Gregg, B. J. Phys. Chem. B., in press.

\section{Presentations}

NREL hosted a workshop entitled "Dye Sensitization of Semiconductors" in Golden, Colorado, on March 10-11. The workshop was by invitation only. Attendees included 7 researchers from universities, 1 from Brookhaven National Laboratory, 4 from industry, 2 from DOE/BES, and 10 from NREL. A.J. Nozik, A.J. Frank, and B.A. Gregg made presentations on the NREL Photochemical Solar Cell Project.

A.J. Nozik, AJ. Frank, and B.A Gregg made a joint presentation on the NREL Photochemical Solar Cell Project at the Future-Generation Photovoltaic Technologies: First NREL Conference in Denver, Colorado, March 24-26, 1997.

A. Zaban (with A. Meier, A.J. Nozik, and B.A. Gregg) presented a seminar entitled "Impedance Spectroscopy of High-Surface-Area $\mathrm{TiO}_{2}$ Electrodes" at the Electrochemical Society Symposium on Electrode Materials and Processes for Energy Conversion and Storage IV, Montreal, Canada, May 4-9, 1997.

A.J. Frank (with coauthors G. Schlichthörl, S.Y. Huang, J. Sprague, A.J. Nozik, and M. Grätzel) presented a lecture entitled "Investigation of Fundamental Processes Controlling the Operation of Dye-Sensitized Nanocrystalline $\mathrm{TiO}_{2}$ Solar Cells" at the Third International Meeting in New Trends in Photoelectrochemistry held in Estes Park, Colorado, May 11-14, 1997. 
G. Schlichthörl (with coauthors S.Y. Huang, J. Sprague, and A.J. Frank) presented a poster entitled "Intensity Modulated Photovoltage Spectroscopy: ANew Tool to Study the Recombination Kinetics in Dye-Sensitized $\mathrm{TiO}_{2}$ Cells" at the Third International Meeting in New Trends in Photoelectrochemistry held in Estes Park, Colorado, May 11-14, 1997.

A.J. Frank (with coauthor G. Schlichthörl) presented an invited lecture entitled "Interfacial Photochemical Processes: Dye-Sensitized Nanocrystalline Semiconductor Electrodes" at the Twenty-First DOE Solar Photochemistry Research Conference held at Copper Mountain, Colorado, June 7-11, 1997.

G. Schlichthörl (with coauthors S.Y. Huang, J. Sprague, and A.J. Frank) presented a poster entitled "Intensity-Modulated Photovoltage Spectroscopy: A New Tool to Study the Recombination Kinetics in Dye-Sensitized $\mathrm{TiO}_{2}$ Cells" at the Twenty-First DOE Solar Photochemistry Research Conference held at Copper Mountain, Colorado, June 7-11, 1997.

B.A. Gregg (with coauthors C. Bechinger and R. Pitts) presented a seminar entitled "Photoelectrochromic Smart Windows" at the SPIE meeting in San Diego, California, July 27August 1, 1997.

A. Zaban (with coauthor B.A. Gregg) presented a seminar entitled "Dye-Sensitized Solar Cells" at Case Western University in Cleveland, Ohio, August 8, 1997.

AJ. Frank (with coauthors G. Schlichthörl, S.Y. Huang, and A.J. Nozik) presented an invited lecture entitled "Dye-Sensitized Nanocrystalline $\mathrm{TiO}_{2}$ Solar Cells" at the 6th Center for Molecular Catalysis International Symposium on Photochemical Electron Transfer: Molecular Approach to Photocatalysis, Chungnam National University, Taejon, South Korea, December 4, 1997.

A.J. Frank (with coauthors G. Schlichthörl, S.Y. Huang, and A.J. Nozik) presented an invited lecture entitled "Dye-Sensitized Nanocrystalline $\mathrm{TiO}_{2}$ Solar Cells" at the Joint Seminar of the Chemistry Department and the Materials Science and Engineering Department, Korea University, Seoul, South Korea, December 5, 1997. 
Title:

Organization:

Contributors:

\section{Solid State Spectroscopy}

NREL Center for Basic Sciences

\section{Objectives}

This project involves an attempt to understand the way in which intrinsic and extrinsic features of semiconductor materials used for photovoltaic applications influence the performance of solar cell devices. The intrinsic features involve parameters such as critical points in the joint interband density of electronic states and the position and slope of absorption edges associated with them, radiative recombination efficiences and lifetimes, lattice structural properties associated with long and short range order, and thermal conductivity. The extrinsic features involve defects such as grain boundaries, disclocations, impurities and their effects on radiative recombination. The techniques used for these investigations are: photoluminescence(PL) spectroscopy, PL excitation spectroscopy, modulated reflectance, pump and probe spectroscopy, Raman spectroscopy, and near-field scanning optical microscopy (NSOM).

\section{Technical Approach}

To identify the specific effects of defects such as grain boundaries and dislocations on radiative recombination in polycrystalline thin films of CdTe and to study the passivation of these defects by chemical treatment, a systematic micro-photoluminescence study at liquid Helium temperatures of a set of carefully prepared samples of CdTe large grain thin films was conducted. Samples with average grain size of $8 \mu \mathrm{m}$ were studied with an average spatial resolution of better than $2 \mu \mathrm{m}$. The first part of this effort consisted of constructing the setup capable of doing these measurements. The setup comprised of a special liquid Helium microstat, a very long working distance microscope objective for excitation and collection, and a spectrograph based CCD for rapid acquisition of PL spectra. The second part of this effort comprised of studying various samples of CdTe large grain thin films which had been subjected to various types of treatments. The third part of this effort involved conducting micro-Raman studies to examine whether it was posible to identify precipitates in the microstructure of the thin films.

\section{Results}

Preliminary micro-PL studies of large grain polycrystalline CdTe films subjected to postdeposition treatment revealed that the PL was highly spatially anisotropic. Later these measurements were performed at $4.2 \mathrm{~K}$ on samples subjected to different post deposition treatments: 1) as deposited, 2) $400^{\circ} \mathrm{C}$ annealed, and 3) $400^{\circ} \mathrm{C}$ annealed in $\mathrm{CdCl}_{2}$. The as deposited film showed relatively spatially uniform (but low) PL intensity, the film that was annealed showed showed slightly higher PL efficiency, but this efficiency varied dramatically with the spatial scan. The film that was annealed with $\mathrm{CdCl}_{2}$ showed better than an order of magnitude enhancement of PL efficiency and spatial homogeneity. All the above measurements were 
conducted on the back surface of $\mathrm{CdTe} / \mathrm{CdS}$ solar cells since this was a relatively straight forward measurement.

Since the front surface $\mathrm{CdTe}$ regions of the solar cells are of technological interest, we later extended the low temperature Photoluminescence(PL) measurement studies on CdTe films grown at NREL by directly investigating the more interesting front surface CdTe region of the solar cell. Films of CdTe grown on glass substrates were lifted off from the substrates and flipped over making the front surface region of the solar cell amenable to PL mapping. The results are shown in Figs. 1 and 2. Because the average grain size is roughly $8 \mu \mathrm{m}$ the PL intensity line scan shown in Fig. 1 clearly indicates that at the grain boundaries the PL efficiency falls by a factor of 3 . The PL spectra shown as a function of position in Fig 2. Clearly indicate that in certain regions there is a red shift of the peak of the PL which occurs due to alloying of the CdTe with CdS.

The micro-Raman studies on large grain polycrystalline CdTe films revealed (see Fig.3) that in addition to the phonons that are expected in the Raman spectra of CdTe there was a clear signature of phonons that are only expected from elemental Te. These data provided strong evidence for the evidence of Te precipitates in the microstructure of the CdTe films.

\section{Publications:}

R. Dhere, D. Rose, D. Albin; S. Asher, M. Al-Jassim, H. Cheong, A. Swartzlander, H. Moutinho, T. Coutts, and P. Sheldon, "Influence of CdS/CdTe interface properties on device properties." IEEE 1997 Photovoltaic Specialist Conf.

\section{Conclusions}

The low-temperature micro-PL measurement technique has been shown to be a powerful method of directly mapping out the effects of various post-deposition treatments on the radiative recombination efficiency of polycrystalline solar cell devices. The micro-raman measurements indicate that it is possible to probe the microstructure of these thin films for elemental precipitates. 
Title: Solid State Theory Of Novel Photovoltaic Materials And Devices

Organization: $\quad$ National Renewable Energy Laboratory, Golden, CO

Basic Sciences Center

Contributors: $\quad$ A. Zunger, Research Fellow

S.-H. Wei, Senior Scientist

S.B. Zhang, Senior Scientist

This report provides a summary of the FY 1997 PV research activities of the Solid State Theory Group:

1. Defect Physics of CuInSe : Effects of Ga Additions

2. Transparent Conductors

3. Dopability of PV Semiconductors

More details can be found in the journal articles cited at the end of this report.

\section{Defect Physics of CuInSe 2 : Effects of Ga Additions [1-4]}

Because $\mathrm{CuInSe}_{2}$ has a band gap of only $\sim 1 \mathrm{eV}$, i.e., lower than the ideal value for photovoltaic (PV) solar cells, it has been suggested that $\mathrm{Ga}$ addition will raise the gap, thus increasing the open-circuit voltage. Currently, the best $\mathrm{CuInSe}_{2}$ solar cells are indeed made with $30 \% \mathrm{Ga}$. To further improve the efficiency and stability of $\mathrm{CuInGaSe}_{2}$ (CIGS)-based solar cells, we need to understand the basic physics of $\mathrm{Ga}$ addition and its effect on the properties of $\mathrm{CuInSe}_{2}$ (CIS). We performed first-principles theoretical calculations to study the electronic structures of CIS, $\mathrm{CuGaSe}_{2}$ (CGS), and their alloys (CIGS). We also extended our previous study of defect formation in CIS to CGS and performed parallel calculations to study the formation energies and structural properties of various defect complexes and ordered defect compounds of $\mathrm{CuGaSe}_{2}$. We found that:

(i) The band gap of CIGS increases according to $E_{g}(x)=(1-x) E_{g}(C I S)+x E_{g}(C G S)-b x(1-x)$. Our calculated bowing coefficient is $b=0.21 \mathrm{eV}$. The most recently measured value is $0.24 \mathrm{eV}$.

(ii) The valence band offset between CIS and CGS is only $0.04 \mathrm{eV}$. Thus, the difference in band gaps $(0.64 \mathrm{eV})$ appears mostly in the conduction band.

(iii) The formation energies of single acceptor defects $\left(V_{C u}, V_{G a}\right.$, and $\left.C u_{G a}\right)$ in $\mathrm{CuGaSe}_{2}$ are similar to those of their counterparts in CuInSe $e_{2}$. However, the formation energies of single donor defects $\left.\left(\mathrm{Ga}_{\mathrm{Cu}}, \mathrm{Cu}\right)_{i}\right)$ in $\mathrm{CuGaSe}_{2}$ are larger than those of their counterparts in $\mathrm{CuInSe}$. The 
large formation energy of the donor defects in CGS suggests fewer donors at equilibrium, due to the latter's larger band gap (as compared to that of CIS).

(iv) As in CIS, the formation of charge-compensated defect pairs or arrays (e.g., $G a_{C u}^{2+}+$ $2 V_{C_{u}}^{-}$) greatly reduces the formation energy. However, the formation energy of $\mathrm{Ga}_{C_{u}}^{2+}+2 V_{C_{u}}^{-}$in $\mathrm{CuGaSe}_{2}$ is about $0.3 \mathrm{eV} / \mathrm{pair}$ higher than the formation energy of $\mathrm{In}_{\mathrm{Cu}}^{2+}+2 V_{\mathrm{Cu}_{u}}^{-}$in $\mathrm{CuInSe}_{2}$, suggesting that the stability of the chalcopyrite 1:1:2 phase relative to that of the ordered defect compound (ODC) phase in CGS is larger than in CIS (i.e., it is more difficult to form ODC in $\mathrm{CuGaSe}_{2}$ than in $\mathrm{CuInSe}_{2}$ ).

(v) The acceptor levels [e.g., $V_{C u}(-/ 0)$ ] in CGS are slightly shallower than those in CIS, suggesting higher hole density. However, the $G a_{C u}$ donor levels in CGS are much deeper than the donor levels of $I n_{C u}$ in CIS, suggesting less electron density. The reasons for this are twofold: (a) $\mathrm{Ga}$ is smaller than $\mathrm{In}$, so lattice compression in $\mathrm{CuGaSe}_{2}$ pushes up the conduction band minimum (CBM) energy level of $\mathrm{CuGaSe}_{2}$ (the $\mathrm{CBM}$ of $\mathrm{CuGaSe}_{2}$ is $0.6 \mathrm{eV}$ higher than that of $\mathrm{CuInSe}_{2}$ ) and (b) the wave function of this $\mathrm{Ga}_{\mathrm{Cu}}$ antisite defect has $s$ character and is localized on the group III atom. The $\mathrm{Ga} 4 s$ orbital energy is about $0.7 \mathrm{eV}$ lower than the In $5 s$ orbital energy. Thus, insofar as III-on-I antisites contribute to n-typeness, CGS will be less n-type than CIS.

These results await experimental testing.

\section{Transparent Conductors}

Application of transparent conducting oxides (TCOs), which are used extensively in PV device applications, is limited by the lack of knowledge of the basic properties of the TCOs and the lack of understanding of the mechanism that results in the above-noted peculiar physical properties. Indeed, there is almost no effort in the literature to explain the microscopic mechanism creating simultanious transparency and conductivity. In FY 1997, we began studying the TCOs to determine what constitutes the common ground for the transparent-conducting behavior among only a handful of known TCO materials $-\mathrm{ZnO}, \mathrm{SnO}_{2}, \mathrm{Cd}_{2} \mathrm{SnO}_{4}$, and $\mathrm{In}_{2} \mathrm{O}_{3}$. Concerning the crystal structures, it is not known if $\mathrm{Cd}_{2} \mathrm{SnO}_{4}$ assumes a normal or inverse spinel structure in thermoequilibrium. We calculated the total energies for normal versus inverse spinel structures of $\mathrm{Cd}_{2} \mathrm{SnO}_{4}$. Although the difference in lattice parameters of the two is tiny $(\sim 0.4 \%)$, the total energy difference between normal and inverse spinel is large ( $>1.3 \mathrm{eV}$ per 14-atom cell). The inverse spinel is much more stable than the normal spinel. We find strong dependence of the physical properties of $\mathrm{Cd}_{2} \mathrm{SnO}_{4}$ on the atomic structures. Often, electron effective masses are comparable for two different crystal structures when the electron Fermi energy is near the CBM. However, to achieve good conductivity, the Fermi energy in $\mathrm{Cd}_{2} \mathrm{SnO}_{4}$ needs to be high in the 
conduction band. The effective masses at such limits can differ by as much as $500 \%$ from those of the CBM. For a long time, it was thought that the good carrier transport properties of TCOs were the result of exceptionally small electron effective masses in these materials. Recently, Mulligan and Coutts showed that this is not the case. Our preliminary results support their findings. We also calculated the band structures for $\mathrm{ZnO}$ and $\mathrm{Cd}_{2} \mathrm{SnO}_{4}$. We found that $\mathrm{Cd}_{2} \mathrm{SnO}_{4}$ has a direct band gap. The bottom of the conduction band of $\mathrm{Cd}_{2} \mathrm{SnO}_{4}$ is distinct from that of $\mathrm{ZnO}$ as well as $\mathrm{SnO}_{2}$ as it is equally weighted among the $\mathrm{Cd}$ and $\mathrm{Sn}$ atoms. We are currently (FY 1998) studying defect-related properties in TCO to identify the physical origin of the charge carriers in these materials. We need to determine whether oxygen vacancies, or other defects, lead to conductivity.

\section{Dopability of PV Semiconductors [5]}

Applications of PV semiconductors depend critically on the ability to dope them. For different materials the maximal doping limits vary by more than 5 orders of magnitude. There are noticeable, but unexplained systematic trends showing asymmetry of $\mathrm{n}$ versus $\mathrm{p}$ dopability: e.g., $\mathrm{ZnO}, \mathrm{ZnS}, \mathrm{CdS}$, and CdTe are good n-type conductors, whereas $\mathrm{ZnTe}$ and $\mathrm{CuInTe}_{2}$ are good ptype conductors. On the other hand, $\mathrm{ZnO}$ and $\mathrm{ZnS}$ cannot be made p-type and $\mathrm{CuAlSe}_{2}$ and $\mathrm{CuGaSe}_{2}$ cannot be made n-type. CuInSe $\mathrm{C}_{2}$ can be doped both p-type and n-type. As we add $\mathrm{Ga}$ to form the $\mathrm{Cu}\left(\mathrm{In}_{l_{-x}} G a_{x}\right) S e_{2}$ alloy, the system can no longer be doped n-type for large $\mathrm{x}$. Also, despite the fact that $\mathrm{ZnO}$ has the band gap typical of an insulator, it can be doped strongly n-type (but not p-type).

We analyzed the systematics underlying the experimental doping limits data, using the phenomenological "doping pinning rule." The rule states that the Fermi level $E_{F}$ of any given semiconductor is pinned to within a range of several $\mathrm{eV}$ by the formation of native defects. We derive universal p-type pinning energy $E_{\text {pin }}^{(p)}$ (= the lower bound for $E_{F}$ ) and n-type pinning energy $E_{\text {pin }}^{(n)}$ (= the upper bound for $E_{F}$ ) for all II-VI and separately for all I-III-VI $\mathrm{I}_{2}$ semiconductors, which not only systematize the known trends, but also predict dopability where it is unknown. The central insight of the model is that inability to dope a given material and the $p$ versus $n$ asymmetry in dopability are not simply related to the existence of a wide gap, as assumed previously, but reflect the absolute position of the valence band maximum (VBM) and the CBM with respect to some fixed energy (e.g., the vacuum). 
Our results can be summarized as follows:

(i) There exist a set of intrinsic, vacuum-referred Fermi level pinning positions, $E_{p i n}^{(n / p)}$ for semiconductors. The $E_{p i n}^{(n / p)}$ values derived from experimental data for a given type of semiconductors (e.g., III-V) tend to line up around the intrinsic $E_{p i n}^{(n / p)}$. For different types of semiconductors (i.e., between II-VI and I- $\mathrm{III}_{\mathrm{V}} \mathrm{VI}_{2}$ ), the intrinsic pinning positions are, however, different.

(ii) We can phrase the "doping limit rule" as "Materials in which the CBM is much higher than the lined-up $E_{\text {pin }}^{(n)}$ value are difficult to dope n-type. Materials in which the VBM is much lower than the lined-up $E_{p i n}^{(p)}$ value are difficult to dope p-type." (We have the numerical value of the pinning energies [5].) In other words, a good p-type conductor must have a sufficiently small work function, whereas a good n-type conductor must have a sufficiently large (positive) electron affinity.

(iii) $\mathrm{ZnO}$ can be doped highly n-type, making it a transparent conductor but not p-type. The explanation for the asymmetry resides on the relative position of the band edges (the CBM and the VBM) with respect to the respective $E_{p i n}^{(n / p)}$ values: despite its large band gap, the CBM of $\mathrm{ZnO}$ is significantly lower than $E_{\text {pin }}^{(n)}$. This induces heavy n-type dopability. The large band gap of $\mathrm{ZnO}$, however, pushes its VBM much below $E_{p i n}^{(p)}$, thus prohibiting p-type dopability.

(iv) Our $E_{p i n}^{(n i p)}$ values show the following trends:

(1) Materials whose CBMs are too high above $E_{p i n}^{(n)}$ cannot be doped n-type. These include $\mathrm{CuGaSe}_{2}, \mathrm{CuAlSe}_{2}$, and $\mathrm{CuInTe}_{2}$.

(2) Materials whose VBM are too deep below $E_{p i n}^{(p)}$ cannot be doped p-type. These include $\mathrm{ZnO}$ and $\mathrm{ZnS}$.

(3) Materials with CBM significantly lower than $E_{p i n}^{(n)}$ can be doped heavily n-type. These include $\mathrm{ZnO}, \mathrm{CdS}, \mathrm{CdSe}$, and $\mathrm{CdTe}$.

(4) Materials with VBM significantly higher than $E_{p i n}^{(p)}$ can be doped heavily p-type. These include CuInTe $\mathrm{C}_{2}$ and $\mathrm{ZnTe}$.

(v) The II-VI compounds in general are better n-type conductors than the corresponding $\mathrm{Cu}$ $\mathrm{III}-\mathrm{VI}_{2}$ compounds. This is mostly due to the much higher $E_{p i n}^{(n)}$ for II-VIs than $E_{p i n}^{(n)}$ for Cu-III$\mathrm{VI}_{2} \mathrm{~s}$. 
(vi) The $\mathrm{Cu}-\mathrm{III}-\mathrm{VI}_{2}$ compounds are in general better p-type conductors than the corresponding II-VI (Zn-VI or Cd-VI) compounds, because the VBMs of the chalcopyrites are high due to $\mathrm{p}-\mathrm{d}$ repulsion with $\mathrm{Cu} \mathrm{d}$. Thus, strong $\mathrm{p}-\mathrm{d}$ coupling seems to encourage $\mathrm{p}$ dopability.

(vii) For $\mathrm{Cu}\left(G a_{x} I n_{1-x}\right) S e_{2}$ alloy, our calculation shows that this material has good p-type conductivity for all $\mathrm{x}$ value although it is a good $\mathrm{n}$-type conductor only for $\mathrm{x}<0.2$, beyond which n-type conductivity is difficult.

These predictions await experimental testing.

\section{References}

1. S.-H. Wei, S. B. Zhang, and A. Zunger, "Why is heavily-defected $\mathrm{CuInSe}_{2}$ a good optoelectronic material: Defect physics in CuInSe," in Proceedings of the 11th Inter. Conf. of Ternary and Multinary Compounds, edited by R. D. Tomlinson AIP, New York, (1998) (in press).

2. A. Zunger, S. B. Zhang, and S.-H. Wei, "Revisiting the defect physics in $\mathrm{CuInSe}_{2}$ and $\mathrm{CuGaSe}_{2}$," in Proceedings of the 26th IEEE PV Specialist Conf., edited by P. A. Basore AIP, New York, p. 313 (1998).

3. S. B. Zhang, S.-H. Wei, A. Zunger, and H. Katayama-Yoshida, "Defects physics of the CuInSe $e_{2}$ chalcopyrite semiconductor," Phys. Rev. B, 57, 9642 (1998).

4. S.-H. Wei, S. B. Zhang, and A. Zunger, "The effects of $\mathrm{Ga}$ addition to $\mathrm{CuInSe}_{2}$ on its electronic, structural and point defect properties,"Appl. Phys. Lett., (in press).

5. S. B. Zhang, S.-H. Wei and A. Zunger, "A phenomenological model for systematization and prediction of doping limits in II-VI and I-III-VI $\mathrm{I}_{2}$ compounds," J. Appl. Phys., 83, 3192 (1998). 
, 


\section{Exploratory Materials and Devices \\ Subcontracts}


Title: $\quad$ Novel Low-Temperature Substrate Technology for Compound Semiconductor Solar Cells on Low-Cost Substrates

Organization: Thomas J. Watson Laboratory of Applied Physics, California Institute of Technology, Pasadena, CA 91125

Contributors: Harry A.Atwater, Principal Investigator, Claudine M. Chen, Steve Na

\section{Objectives}

The objectives of this program are to develop low-temperature $\left(\mathrm{T}<450^{\circ} \mathrm{C}\right)$ solid phase crystallization approaches to obtaining large-grained $(20-100 \mu \mathrm{m})$ crystalline Ge films on low-cost amorphous (e.g., soda-lime glass) substrates, which can serve as templates for $\mathrm{GaAs}$ growth for thin film GaAs solar cells.

\section{Approach}

Figure 1 depicts the approach for fabrication of large-grained $\mathrm{Ge}$ films by selective nucleation and solid phase epitaxy (SNSPE) of crystalline Ge seeds in amorphous $\mathrm{Ge}$ thin films [1,2]. Selective nucleation results from reactions between a patterned array of deposited In islands and amorphous Ge to yield large (20-30 $\mu \mathrm{m})$ grains in the fully crystallized film at temperatures below $450^{\circ} \mathrm{C}$. These large-grained polycrystalline $\mathrm{Ge}$ thin films are being used as templates for epitaxial growth of $\mathrm{GaAs}$, in collaboration with the Research Triangle Institute and the National Renewable Energy Laboratory.

Define Nuclestion Sitas In Amorphous Go on Low-Cost Substrito

1st Anneal: Selective Nucleation

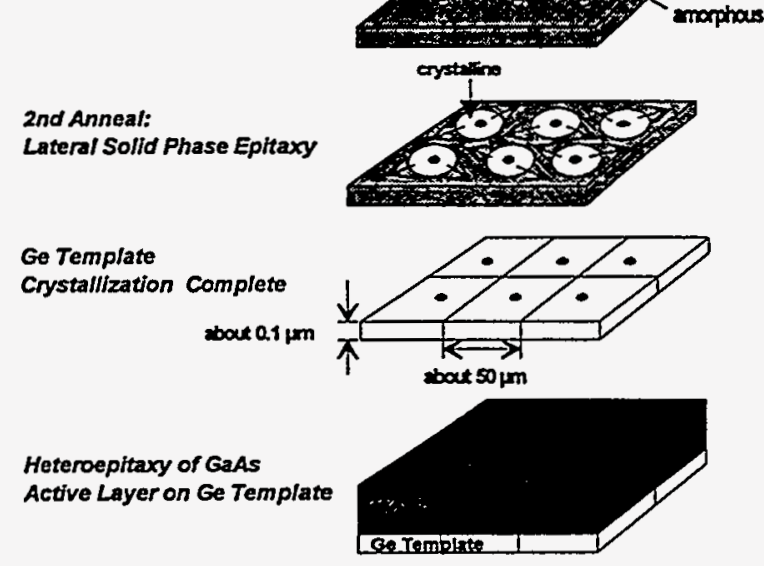

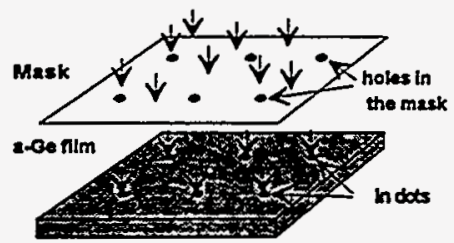

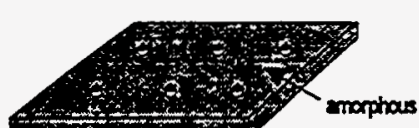

Figure 1. A selective nucleation and solid phase epitaxy process for largegrained polycrystalline GaAs films on low-cost glass substrates is depicted schematically. Indium islands are deposited in a patterned array onto amorphous $\mathrm{Ge}$ films. The first anneal promotes selective nucleation and the second anneal enables large grain size to be achieved by lateral solid phase epitaxy. The large-grained Ge film then serves as an epitaxial template for growth of GaAs to form the photovoltaic device active region. 


\section{Results}

Selective Nucleation

Selective nucleation is accomplished by patterning a periodic array of $5 \mu \mathrm{m}$ diameter In islands by mechanically masked evaporation on top of an amorphous Ge film. Array periods range from $20-100 \mu \mathrm{m}$. Post-deposition annealing leads to heterogeneous nucleation of Ge crystals under the In pattern but not elsewhere in the amorphous $\mathrm{Ge}$ film. Further annealing causes the nucleated Ge crystals to seed growth of large (20-30 $\mu \mathrm{m}) \mathrm{Ge}$ grains. By contrast, random crystallization in an amorphous Ge film typically yields average grain sizes of $0.25-1 \mu \mathrm{m}$. A variety of materials other than In (e.g., Sn, Ni, $\mathrm{Co}, \mathrm{Pd}, \mathrm{Cu}$ and $\mathrm{Al}$ ) have also been used for selective nucleation. To date, the island seed regions produced by selective nucleation are polycrystalline, rather than a single grain per island. Efforts to produce a single grain in each seed region are underway.

\section{Dopant Enhanced Solid Phase Epitaxy of Ge}

The kinetics of lateral solid phase epitaxy closely resembles those reported previously for Ge solid phase epitaxy in amorphous layers on single-crystal Ge substrates[3]. The apparent activation energy for lateral solid phase epitaxy in polycrystalline films is identical, to within experimental error, to the activation energy for vertical solid phase epitaxy of single crystal Ge, as indicated in Fig. 3(a). However the absolute solid phase epitaxial growth rate in polycrystalline films is approximately five times lower than that for solid phase epitaxy of (100)-oriented single crystal Ge. Doping of the amorphous Ge starting material with electronic dopants significantly enhances the solid phase epitaxy rate [5]. Doping of a-Ge was performed by ion implantation of $B$ and $P$ to yield peak concentrations in the range of 0.3-1.4 at.\%. Doping with phosphorus in the range of 0.61.4 at. $\%$ enabled lateral solid phase epitaxy at rates of $1-3 \mu \mathrm{m} / \mathrm{hr}$ at $\mathrm{T}=400{ }^{\circ} \mathrm{C}$. This corresponds to an increase in the solid phase epitaxy rate by a factor of 5-15 relative to undoped films, enabling Ge grain sizes as large as 20-30 $\mu \mathrm{m}$, as illustrated in Fig. 2. In samples co-doped with $B$ and $P$, a compensation effect is observed leading to a reduction of the solid phase epitaxial growth rate enhancement with $P$ doping only; i.e., equal amounts of $B$ and $P$ yield no enhancement in the growth rate.

\section{Limits to Achievable Grain Size}

We have continued our research into producing the largest possible grain size in Ge thin films via metal-induced selective nucleation, followed by lateral solid phase epitaxy. The limitation of grain size at the present time is due to randomly nucleated grains impeding the growth of selectively nucleated grains. Therefore we investigated the dependence of the random nucleation rate on $\mathrm{Ge}$ film thickness. For $\mathrm{Si}$ thin films on $\mathrm{SiO}_{2}$, it has been demonstrated that the number of nucleated $\mathrm{Si}$ crystals per unit area increases linearly with Si film thickness, as would be expected for a volumetrically uniform homogeneous nucleation process. If this were also true for $\mathrm{Ge}$ films on $\mathrm{SiO}_{2}$, then the Ge film thickness should be reduced as much as possible to minimize the number of randomly nucleated grains. For Ge films of 20,50 , and $80 \mathrm{~nm}$ thickness annealed in high vacuum at $475{ }^{\circ} \mathrm{C}$ for $10 \mathrm{~min}$, all three film thicknesses showed about the same density of crystals, about 0.1 
crystal per $\mu \mathrm{m}^{2}$, as observed by transmission electron microscopy. This result suggests that unlike the case for $\mathrm{Si}$, the random nucleation is heterogeneous, and probably originates from either the $\mathrm{Ge} / \mathrm{SiO}_{2}$ interface or the $\mathrm{Ge}$ film surface, rather than within the interior of the thin film. Similar results were obtained for Ge films on soda-lime glass substrates as well [4].

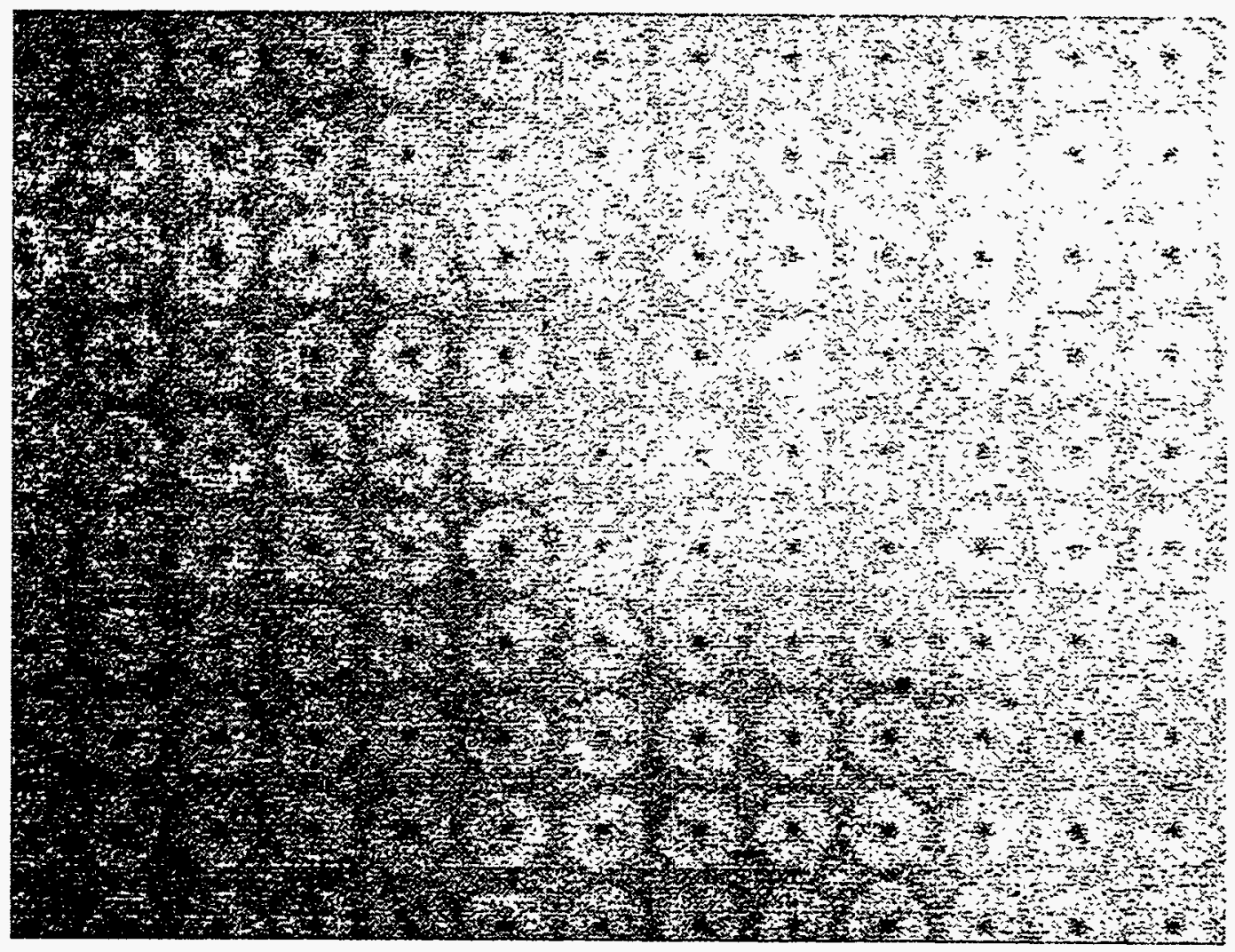

$100 \mu \mathrm{m}$

Figure 2. Plan-view optical micrograph of partially-crystallized $100 \mathrm{~nm}$ Ge film on soda lime glass. Lightly-shaded gray circles are Ge grains; dark points in the grain centers are the selective nucleation sites. The gray background is the remaining uncrystallized amorphous $G e$. The final grain size upon complete crystallization is approximately 30 ram.

At present, each predefined selective nucleation site in the Ge films produces not one, but multiple crystals, due to the structure of the multiple In islands formed at each site by mechanical masking, resulting in a polycrystalline microstructure with wedge-shaped grains emanating from the selective nucleation site. Several other approaches to reducing the nucleation density per site to a single grain are deemed promising. These include: i) reduction of the In island size by deposition of ultrafine nanocrystal In particles onto the amorphous Ge starting material, with each nanocrystal seeding a single Ge grain, and ii) partial etchback of the deposited In islands to reduce their size and to reduce the density of $\mathrm{Ge}$ grains at the In island edges. If each of these elements of the selective nucleation 
process is optimized, Ge grain sizes in the $100-1000 \mu \mathrm{m}$ range may be possible. Beyond this, the ultimate limit to suppression of random nucleation and increase in grain size may be substrate surface cleaning.

\section{Summary}

Selective nucleation and solid phase epitaxy leads to large grained Ge films on soda-lime glass [4]. The SNSPE process works as well as with glass substrates as previously demonstrated for a similar process using $\mathrm{SiO}_{2} / \mathrm{Si}$ substrates $[1,2]$. The fact that the Ge-onsoda-lime-glass crystallization kinetics and film microstructure are very similar to those previously observed for Ge-on-thermally-oxidized-Si is encouraging since it implies that the random nucleation rate is unaffected by impurities in the glass or the microstructure of the Ge/glass interface. Ge grain sizes of $20-30 \mu \mathrm{m}$ have been achieved. Current work is focused on achievement of a single grain per nucleation site, and to further enhancement of grain size, as well as studies of heteroepitaxial $\mathrm{GaAs}$ growth on $\mathrm{Ge} / \mathrm{SiO}_{2}$ and on Ge/glass, in conjunction with NREL and the Research Triangle Institute. Future work will be aimed at exploitation of the large-grained Ge films on glass as templates for heteroepitaxy of GaAs on glass, and development of similar approaches for thin film polycrystalline $\mathrm{Si}$ films on glass substrates [5].

\section{References}

1. C.M. Yang and H.A. Atwater, Mat. Res. Soc. Symp. Proc. 403, 113 (1996).

2. C.M. Yang and H.A. Atwater, Appl. Phys. Lett., 68, 3392 (1996).

3. I. Suni, G. Goltz, M-A Nicolet and S.S. Lau, Thin Solid Films, 93, 171 (1982); I. Suni, G. Goltz, M.G. Grimaldi, M-A Nicolet and S.S. Lau, , Appl. Phys. Lett., 40, 269 (1982).

4. Proceedings of $1^{\text {st }}$ NREL Conference on Future Generation Photovoltaic Technologies, Denver CO, March 24 $4^{\text {th }}-27^{\text {th }}$, 1997, American Institute of Physics Conf. Proc. Vol. 404, pp 345-353.

5. Presentation at Silicon Thin Film Team/High Efficiency Photovoltaic Project Meeting, Vail CO., 8//11/97. 
Title: Solar Energy Conversion at Dye Sensitized Nanostructured Electrodes Fabricated by Sol-

\section{Gel Processing}

Organization: The Johns Hopkins University, Baltimore, MD 21218

Contributors: $\quad$ P.C. Searson and G.J. Meyer, Principle Investigators

F. Cao and J.M. Stipkala

\section{Objectives}

We are exploring the use of nanostructured materials as substrates for dye-mediated light-toelectricity conversion in a photoelectrochemical cell. The objective is to develop new materials, electrolytes, and molecular sensitizers which increase efficiency and stability. Materials processing and synthesis is carried out in conjunction with characterization of cell performance, investigation of operating mechanisms, and modeling of charge transfer and transport in these systems.

\section{Results}

\section{Charge Transport Under Open Circuit Conditions}

The photocurrent transient response of dye sensitized $\mathrm{TiO}_{2}$ photoelectrochemical cells is relatively slow with time constants on the order of milliseconds to seconds. In contrast, the rate of electron injection from the excited states of the dye molecules on the order of picoseconds or smaller. In previous work [1-4] we have shown that the transient photocurrent response in these films is controlled by electron transport in the particle network where the electron mobility is dependent on the electron concentration in the film. Electron transport processes are also responsible for establishing equilibrium at the $\mathrm{TiO}_{2}$ /electrolyte interface under open circuit conditions.

The photovoltage transients exhibit a single exponential rise at light intensities up to $50 \mathrm{~mW} \mathrm{~cm}-2$. Figure 1 shows an example of the photovoltage rise at $0.2 \mathrm{~mW} \mathrm{~cm}$-2 for front and backside illumination. The rise times are in the range $10 \mathrm{~ms}-10 \mathrm{~s}$ and exhibit a power law dependence on light intensity with a slope of -0.60 to -0.66 , as seen in Figure 2. The rise time is significantly longer for front side illumination due to the longer diffusion length to the back side contact. At high light intensity, a small deviation from a single time constant is observed with the emergence of a fast component at the beginning of the photovoltage rise.

Carrier transport in the semiconductor film can be described by the continuity equation [4]:

$$
\frac{\partial\left(D_{0} \frac{n(x, t)}{n_{0}} \frac{\partial(n(x, t))}{\partial x}\right)}{\partial x}-\frac{\partial(n(x, t))}{\partial t}-\frac{n(x, t)-n_{0}}{\tau_{0}}+\Gamma \alpha \exp (-\alpha x)=0
$$

where $\mathrm{n}$ is the electron density under illumination, $\mathrm{n}_{0}$ is the electron density in the dark, $\mathrm{D}_{0}$ is the electron diffusion coefficient in the dark, $\tau_{0}$ is the electron lifetime (assuming first order 
recombination kinetics), $\Gamma$ is the photon flux, and $\alpha$ is the absorption coefficient of the film.

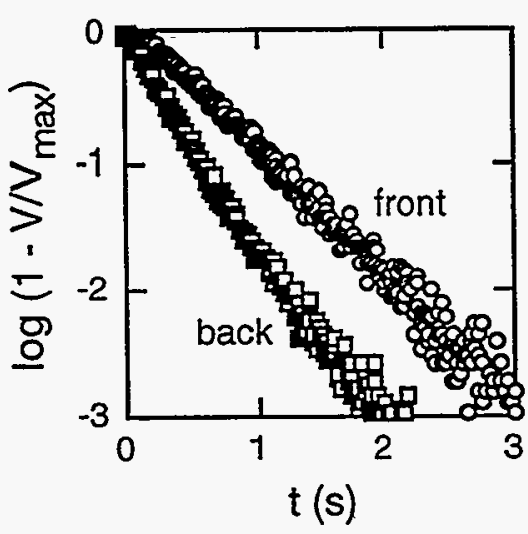

Figure 1. Semi-logarithmic plot of photovoltage transients under monochromatic $(\lambda=514 \mathrm{~nm})$ illumination at $0.2 \mathrm{~mW} \mathrm{~cm}^{-2}$.

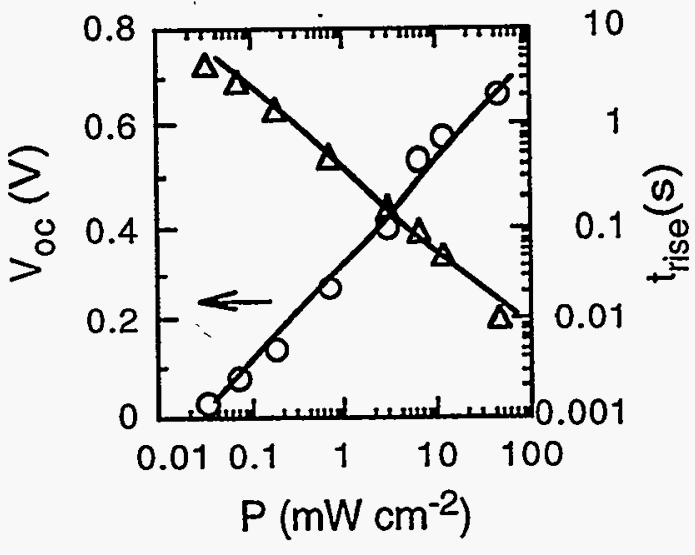

Figure $2 . \mathrm{V}_{\mathrm{oc}}$ and $\mathrm{t}_{1 / 2}$ versus light intensity for backside illumination. (514 nm)

Under short circuit conditions, no photovoltage is generated and the electron concentration at the $\mathrm{TiO}_{2} /$ tin oxide junction is equal to the dark concentration at all times. In contrast, under open circuit conditions, the electron density at the $\mathrm{TiO}_{2} /$ tin oxide junction is not fixed and determines the Fermi level and hence the photovoltage [5]. Figure 3 shows the evolution of the electron density profiles for backside illumination, calculated from equation $\{1\}$, in terms of dimensionless light intensity $\left(\beta=\Gamma \alpha d^{2} / n_{0} D_{0}\right)$ and recombination rate $\left(\sigma=d^{2} / \tau_{0} D_{0}\right)$. Initially, the increase in the electron density is more pronounced at $\mathrm{TiO}_{2}$ /tin oxide interface whereas in the latter part of the transient, the density in the outer portion of the film builds up to the steady state profile. The shape of the concentration profile depends on the absorption and recombination characteristics of the $\mathrm{TiO}_{2}$ film.

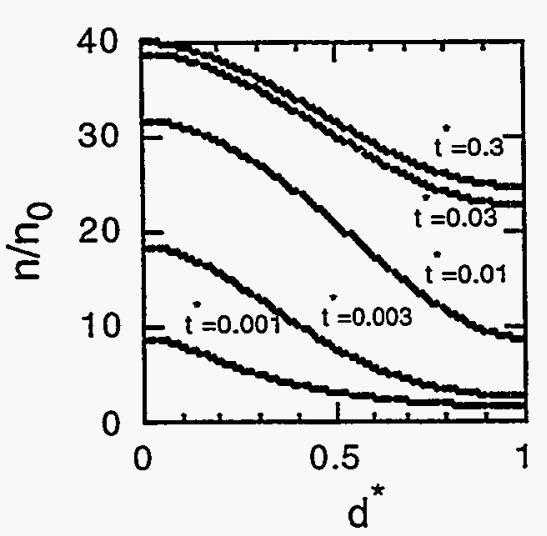

Figure 3. Calculated electron concentration for $\alpha d=3, \beta=1000$ and $\rho=10$.

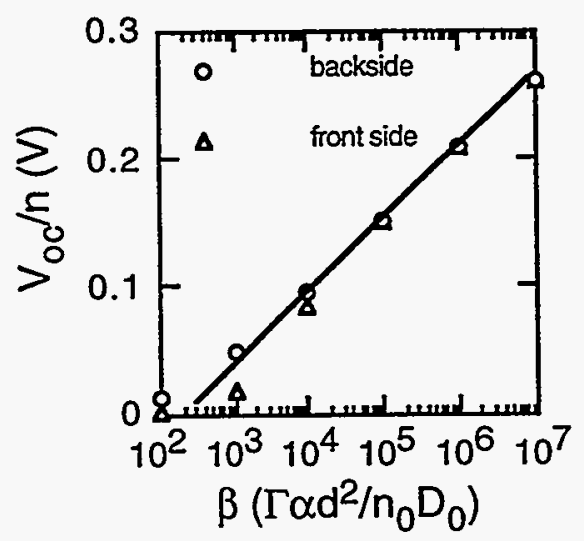

Figure 4. Open circuit photovoltage calculated from the diode equation for $\alpha \mathrm{d}=3$ and $\rho=10$.

$\mathrm{V}_{\mathrm{oc}}$ can be derived from the calculated electron density at the $\mathrm{TiO}_{2} /$ tin oxide interface since the electron density determines the Fermi level at the interface and hence the photovoltage. Figure 4 shows the calculated $V_{o c}$ (assuming Boltzmann statistics) versus light intensity $(\beta)$ showing that $V_{o c}$ is 
linear with the logarithm of the light intensity. For front side illumination, $\mathrm{V}_{\mathrm{oc}}$ becomes much smaller at low light intensity due to the long carrier transit time through the film. The experimental data correspond to the theoretical model with introduction of an ideality factor of about 2 .

\section{Molecular Dye Electron Transfer Processes}

In previous work, we have shown that dye molecules anchored to colloidal anatase films efficiently convert light into electricity despite the fact that an insulating propylene spacer separates the dye from the semiconductor surface [6]. This led to some speculation that the chromophoric ligands need not be directly bound to the semiconductor surface [7,8]. In more recent work, we have shown conclusively that this is the case [9]. With bimetallic $\mathrm{Ru}-(\mathrm{CN})-\mathrm{Re}$ dye molecules, we have observed monochromatic absorbed-photon-to-current efficiencies of about 0.7 . In these sensitizers, the facial geometry about $\operatorname{Re}(\mathrm{I})$ functions to anchor the dye molecule to the semiconductor surface and the ambidendate $\mathrm{CN}$ ligand orientates a visible light absorbing $\mathrm{Ru}(\mathrm{bpy})$ group remote to the semiconductor surface. We have quantified the kinetic rate constants for 'remote' interfacial electron transfer for the first time. Average rate constants for interfacial charge recombination to yield ground state products are $3.1 \times 10^{5} \mathrm{~s}^{-1}$ for $\mathrm{Re}-\mathrm{NC}-\mathrm{Ru} / \mathrm{TiO}_{2}$ and $2.5 \times 10^{5} \mathrm{~s}^{-1}$ for $\mathrm{Ru}-\mathrm{NC}-\mathrm{Re} / \mathrm{TiO} \mathrm{O}_{2}$. Significantly, the rate of charge recombination is about a factor of two slower than that measured for $\left(4,4^{\prime}-\left(\mathrm{CO}_{2} \mathrm{H}\right)_{2}-2,2 \text {-bipyridine }\right)_{2} \mathrm{Ru}(\mathrm{CN})_{2}$ measured under the same conditions. Unfortunately, the difference in rate constants is not sufficiently large to manifest itself in a larger power output from the solar cell. However, the high photocurrent efficiency and decreased recombination rates observed for these bimetallic dye molecules strongly suggest that an as of yet undetermined optimal sensitizersurface orientation exists wherein quantitative electron injection occurs, but the back reaction to ground state products is further inhibited. We note that preliminary results with related bimetallic sensitizers, based on NCS as the bridging and/or non-chromophoric ligands, are in complete agreement with these conclusions. Furthermore, the weaker field NCS ligands result in enhanced absorption in the red part of the visible spectrum and hence a larger solar energy conversion efficiency than those recently reported [9].

In previous work we have shown that rapid intramolecular "hole" transfer results in remarkably long lived interfacial charge separation [10]. Specifically, light excitation of $\left[\mathrm{Ru}\left(4,4^{\prime}-\left(\mathrm{CO}_{2} \mathrm{H}\right)_{2}-2,2-\right.\right.$ bipyridine $)_{2}\left(4-\mathrm{CH}_{3}, 4^{\prime}-\mathrm{CH}_{2}-\mathrm{PTZ} \text {,-bpy) }\right]^{2+}$, where $\mathrm{PTZ}$ is phenothiazine, results in rapid, $<10 \mathrm{~ns}$, intramolecular and interfacial electron transfer that ultimately produces a charge separated state where the hole is located on the PTZ group and the electron is in $\mathrm{TiO}_{2}$. This charge separated state has a lifetime of $\sim 300$ microseconds which is about $10^{3}$ times slower than a model compound without the PTZ group measured under the same conditions. The increased lifetime of the charge separated pair results in a larger $V_{\text {oc }}$. Furthermore, the measured interfacial kinetics applied to the diode equation predict the open circuit photovoltage difference. In more recent studies, we have shown that this is true over five order of magnitude change in incident irradiance. Open circuit photovoltages measured in the presence of iodide are also well described by the diode equation, however an ideality factor of two is required [11]. 
To test whether long-lived charge separated states and larger open circuit photovoltages could be realized by intermolecular electron transfer, we explored light induced electron transfer with a family of phenothiazine donors. Visible light excitation of $\mathrm{Ru}\left(4,4^{\prime}-\left(\mathrm{CO}_{2} \mathrm{H}\right)_{2}-2,2\right.$-bipyridine $)(\text { bpy })_{2} / \mathrm{TiO}_{2}$ rapidly forms the interfacial charge separated state. Phenothiazine donors efficiently intercept the charge separated state and reduce the oxidized sensitizer in propylene carbonate electrolyte. The bimolecular electron transfer rate was measured to be $\mathrm{k}=3.2 \times 10^{8} \mathrm{M}^{-1} \mathrm{~s}^{-1}$. Significantly, at high phenothiazine concentrations all the oxidized sensitizers in the nanocrystalline film could be accessed by the donors within experimental error. Recombination of the electron in $\mathrm{TiO}_{2}$ with the oxidized phenothiazine followed a second-order equal concentration kinetic model under the same conditions. Uncertainties in the appropriate path length lead to a systematic error in calculated rates. However, the bimolecular rate constant was approximately $10^{7}-10^{8} \mathrm{M}^{-1} \mathrm{~s}^{-1}$, consistent with colloidal solution studies where the path length was better defined. Unfortunately, the recombination process is very efficient and oxidized phenothiazines are unable to escape the sensitized $\mathrm{TiO}_{2}$ network. Therefore, the use of phenothiazine donors in regenerative solar cells results in negligible photocurrent production.

\section{References}

1. Cao, F.; Oskam, H.; Searson, P.C.; Stipkala, J.M.; Heimer, T.A.; Farzhad, F.; Meyer, G.J. "The Electronic and Optical Properties of Nanostructured $\mathrm{TiO}_{2}$ Films" J. Phys. Chem. 1995, 99, 11974. 2. Cao, F.; Oskam, G.; Searson, P.C. "A Solid State, Dye Sensitized Photoelectrochemical Cell", J. Phys. Chem. 1995, 99, 17071.

3. Oskam, G.; Cao, F.; Searson, P.C. "Charge Transport in Nanostructured $\mathrm{TiO}_{2}$ Films", in Nanostructured Materials in Electrochemistry, eds. P.C. Searson and G.J. Meyer, Electrochemical Society, Pennington, NJ (1995), p.98.

4. Cao, F.; Oskam, G.; Meyer, G.J.; Searson, P.C. "Electron Transport in Porous Nanocrystalline $\mathrm{TiO}_{2}$ Photoelectrochemical Cells", J. Phys. Chem. 1996, 100, 17021.

5. Cao, F.; Searson, P.C. "Open Circuit Photovoltage in Nanocrystalline $\mathrm{TiO}_{2}$ Films",in preparation.

6. Heimer, T.A.; D'Arcangelis, S.T.; Farzhad, F.; Stipkala, J.M.; Meyer, G.J. "An AcetylacetonateBased Semiconductor-Surface Linkage", Inorg. Chem. 1996, 35, 5319.

7. Meyer, G.J. "Efficient Light-to-Electrical Energy Conversion: Nanocrystalline $\mathrm{TiO}_{2}$ Films Modified with Inorganic Sensitizers", J. Chem. Ed. 1997, 74, 652.

8. Stipkala, J.M.; Heimer, T.A.; Kelly, C.A.; Livi, K.J.T.; Meyer, G.J. "Light Induced Charge Separation at Sol-gel Processed Seconductors", Chem. Mater. 1997, 2, 2341.

9. Argazzi, R.A.; Bignozzi, C.A.; Heimer, T.A.; Meyer, G.J. "Remote Interfacial Electron Transfer from Supramolecular Sensitizers", Inorg. Chem. 1997, 36, 2.

10. Argazzi, R.A.; Bignozzi, C.A.; Heimer, T.A.; Castellano, F.N.; Meyer, G.J. "Long-Lived Charge Separation Across Nanocystalline TiO2 Interfaces", J. Am. Chem. Soc. 1995, 117, 11815. 11. Argazzi, R.A.; Bignozzi, C.A.; Heimer, T.A.; Castellano, F.N.; Meyer, G.J. "Light Induced Charge Separation Across Nanocrystalline $\mathrm{TiO}_{2}$ Interfaces with Phenothiazine Donors", J. Phys. Chem. B $1997, \underline{101}, 2591$. 
Title:

20\% (AM1.5) Efficiency GaAs Solar Cells on Sub-mm Grain-Size Poly-Ge and Its Transition to Low-Cost Substrates

\author{
Organization: Research Triangle Institute, RTP, NC 27709
}

Contributors: R. Venkatasubramanian, Principal Investigator; B.C. O’Quinn, and E. Siivola

\begin{abstract}
Material and device issues related to the development of GaAs solar cells on poly-Ge substrates are discussed. Device-structure optimization studies have led us to achieve an AM1.5 efficiency of $\sim 20 \%$ for a 4-cm ${ }^{2}$-area GaAs cell on sub-mm grain-size poly-Ge and an efficiency of $\sim 21 \%$ for a 0.25 - $\mathrm{cm}^{2}$-area cell. The successful demonstration of high-efficiency GaAs cells on sub-mm grain-size poly-Ge substrates have motivated us to develop high-quality GaAs materials on lower-cost substrates such as glass and moly foils. To date, we have achieved a best minority-carrier lifetime of $0.41 \mathrm{nsec}$ in an nGaAs thin-film on moly. The role of Group-VI dopant in the possible passivation of grain-boundaries in poly-GaAs is discussed. Development of PV-quality GaAs material, with minority-carrier lifetime of 1 to $2 \mathrm{nsec}$, on low-cost moly foils can significantly impact both the terrestrial PV applications.
\end{abstract}

\title{
Introduction
}

Single-junction $\mathrm{GaAs}$ and multi-junction $\mathrm{GaInP}_{2} / \mathrm{GaAs}$ solar cells grown on single-crystal $\mathrm{GaAs}$ substrates have achieved record efficiencies of 25.7\% [1] and 29.5\% [2], respectively, under AM1.5 illumination. Similar efficiencies have been demonstrated on single-crystal Ge substrates [3]. Even so, a significant cost reduction is needed for application of these solar cells to terrestrial photovoltaic systems. Large-area polycrystalline Ge substrates can potentially reduce cell substrate-cost as well as cell processing-cost, on a unit-area basis. Development of GaAs solar cells on large-grain poly-Ge substrates appears to be a good first step towards the desirable transitioning of these cells onto other low-cost substrates, through understanding and control of the various loss mechanisms.

Our original demonstration of a small-area $15.8 \%$ efficient $\mathrm{GaAs}$ solar cell [4] and later a $18.2 \%$ efficient large-area GaAs cell [5] on cast, optical-grade, large-grain polycrystalline Ge substrates indicated the feasibility to develop on these substrates. In this paper, first, we discuss some of the key material and device issues related to the development of $\mathrm{GaAs}$ solar cells on polycrystalline Ge substrates, including the dark-current reduction mechanism with an undoped spacer at the $\mathrm{p}^{+}-\mathrm{n}$ depletion layer. Second, we present device-structure studies, such as the optimization of the spacer thickness and the emitter thickness, that have led us to achieve an open-circuit voltage $\left(\mathrm{V}_{o c}\right)$ exceeding 1 Volt and $A M I .5$ efficiency of $20 \%$ for $4-\mathrm{cm}^{2}$-area GaAs cells on sub-mm grain-size poly-Ge. We have also measured an efficiency of $\sim 21 \%$ for a $0.25-\mathrm{cm}^{2}$-area cell on a similar poly-Ge substrate. Third, we describe our current focus in applying these material and device concepts to the development of GaAs solar cells on molybdenum foils.

\section{GaAs Cell Development On Poly-Ge}

Shown in Figure. 1, is a schematic of the GaAs solar cell device structure that we have developed [4] for use on polycrystalline Ge substrates. Note the undoped spacer between the base and the emitter. The undoped spacer at the depletion layer of the $\mathrm{p}^{+}-\mathrm{n}$ junction reduces the dark-current and improves the $V_{o c}$, fill-factor, and the efficiency of cells [4]. While the spacer has no effect in dark-current reduction in $\mathrm{GaAs}$ cells on single-crystal Ge substrates, there is a 40 -fold reduction in the saturation dark current in $\mathrm{GaAs}$ cells with the spacer on poly-Ge substrates, as shown in Figure 2.

The reduction in dark current has shown to be associated with the reduction of tunneling currents near the depletion-layer of the $\mathrm{p}^{+}-\mathrm{n}$ junction in polycrystalline materials. Study of dark currents in $\mathrm{p}^{+}-\mathrm{n}$ GaAs junctions as a function of temperature (77K to $288 \mathrm{~K}$ ) support this hypothesis [5]. The band diagram of a p-n junction near the vicinity of a grain boundary, due to possible n-type Se-dopant accumulation at 


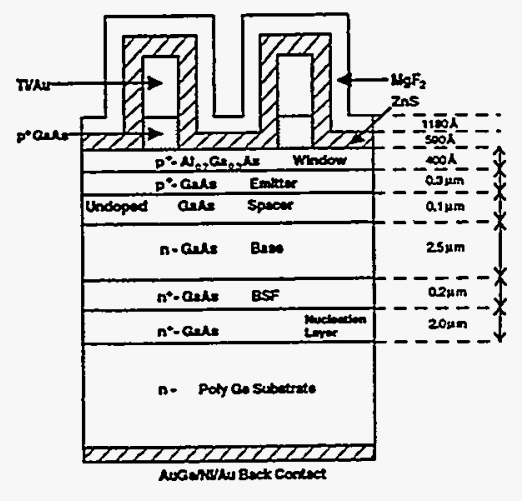

Fig. 1. Schematic of a $\mathrm{p}^{+} \mathrm{n}$ GaAs solar cell device structure on a poly-Ge substrate.

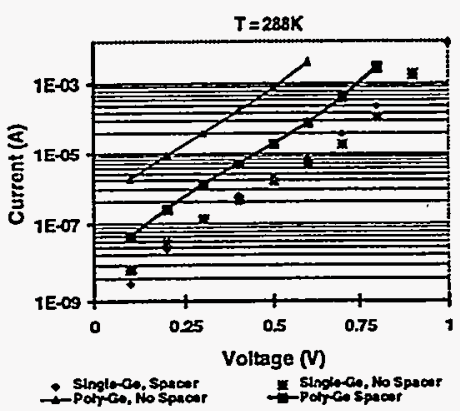

Fig. 2. Effect of an undoped spacer on dark current reduction in GaAs cells; note the effect of spacer on single-crystal Ge versus poly-Ge substrates.

grain-boundaries, is expected to resemble that of a p-n junction (Fig. 3 ). This would lead to electron tunneling (dark) current from the p-emitter to the $\mathrm{n}^{+}$-base, depending on the empty states below the Fermi-energy $\left(\mathrm{E}_{\mathrm{f}}\right)$ in the $\mathrm{n}^{+}$-region. Such a model fits the temperature dependence and we suggest that the reduction of these tunneling dark-currents may be a key to $V_{o c}$ enhancement in many of the III- $V$ p-n junction cells on polycrystalline substrates.

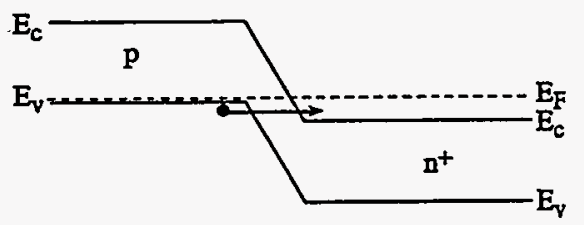

Fig. 3 Schematic of electron tunneling from pGaAs to the heavily-doped $n^{+}-\mathrm{GaAs}$

In order to improve the efficiency of the $\mathrm{p}^{+}-\mathrm{n}$ GaAs cells on poly-Ge, from our previous best of $\sim 18.2 \%$ with a $\mathrm{J}_{\mathrm{sc}}$ of $\sim 23 \mathrm{~mA} / \mathrm{cm}^{2}$ [5], we have recently investigated several approaches to improve the blueresponse of the cells Reducing the thickness of the $\mathrm{Al}_{\mathrm{x}} \mathrm{Ga}_{1-\mathrm{x}} \mathrm{As}$ window, the use of an optimal emitter thickness, an optimized AR-coat, and a reduction of the emitter grid-metallization coverage, have all led to an AM1.5 $\mathrm{J}_{\mathrm{sc}}$ of as much as $\sim 27 \mathrm{~mA} / \mathrm{cm}^{2}$. Towards the improvement of $\mathrm{V}_{\mathrm{oc}}$ values, we have considered a study of the optimum spacer thickness. We investigated the effect of varying the spacer thickness on cell $\mathrm{V}_{\mathrm{oc}}$, for approximately similar grain-size in the GaAs cells. A typical set of data is shown in Table 1. We conclude that as long as the spacer is thick enough to avoid tunneling of carriers between the heavily-doped $\mathrm{p}-\mathrm{GaAs}$ emitter and the heavily-doped $\mathrm{n}-\mathrm{GaAs}$ base regions, formed near the grain-boundaries, a thinner spacer could be advantageous for lowering the dark current. Under optimal spacer thickness, we have also considered the effect of the emitter thickness and the amount of emittergrid metallization coverage on the $\mathrm{V}_{o c}$ of cells. The data shown in Table 2, suggest that a significant component to dark-current generation may also be from the emitter-contact regions or from the $\mathrm{Al}_{\mathrm{x}} \mathrm{Ga}_{1-\mathrm{x}} \mathrm{As}$ window-GaAs cap hetero-interface in these poly-GaAs solar cells with an optimal spacer.

These material and device optimization studies have enabled us to achieve a significant improvement in large-area GaAs cell efficiencies on $\mathrm{mm}$ and sub-mm grain-size poly-Ge substrates. The typical grain structures and other crystalline features in the substrates are as small as $400 \mu \mathrm{m}$. The time-line of progress of cell efficiencies shown in Table 3 indicate that the recent cell results are in spite of smaller, sub-mm grain-sizes in addition to higher resistivity of the starting poly-Ge substrates. The data in Table 3 clearly suggest that large-area GaAs cell efficiencies in excess of $20 \%$ are achievable on mm and sub$\mathrm{mm}$ grain-size poly-Ge substrates. We observe that in the small-area, $21 \%$-efficient cell, a $V_{o c}$ of as much as 1.04 Volt and $\mathrm{a} \mathrm{J}_{\mathrm{sc}} \sim 27 \mathrm{~mA} / \mathrm{cm}^{2}$ have been observed, thereby approaching some of the best single-junction $\mathrm{GaAs}$ cell results on single-crystal GaAs substrates [1]. 
Table 1. Effect of Spacer Thickness on $\mathrm{V}_{\text {oc }}$ of GaAs Cells on mm-Grain-Size Poly$\mathrm{Ge}$; Cell Area $\sim 4 \mathrm{~cm}^{2}$.

\begin{tabular}{|c|c|c|}
\hline Cell \# & $\begin{array}{c}\text { Spacer Thickness } \\
(\mu \mathrm{m})\end{array}$ & $\mathrm{V}_{\text {oc }}(\mathrm{V})$ \\
\hline $1-2766$ & 0.22 & 0.90 \\
\hdashline $1-2763$ & 0.14 & 0.96 \\
\hdashline $1-2782$ & 0.14 & 0.96 \\
\hdashline $1-2786$ & 0.10 & 1.00 \\
\hline
\end{tabular}

Table 2. Effect of Emitter Thickness

Plus Emitter Grid-Coverage on $\mathrm{V}_{\mathrm{oc}}$ of GaAs Cells on mm-Grain-Size Poly-Ge Substrates; Cell Area $\sim 4 \mathrm{~cm}^{2}$

\begin{tabular}{|c|c|c|c|}
\hline Cell \# & $\begin{array}{c}\text { Emitter Thickness } \\
(\mu \mathrm{m})\end{array}$ & $\begin{array}{c}\text { Grid } \\
\text { Coverage } \\
(\%)\end{array}$ & $\begin{array}{c}\mathrm{V}_{\mathrm{nc}} \\
(\mathrm{V})\end{array}$ \\
\hline $1-2786$ & 0.14 & 9 & 1.00 \\
\hline $1-2804$ & 0.22 & 9 & 1.01 \\
\hline $1-2809$ & 0.28 & 9 & 1.01 \\
\hline $1-2840$ & 0.28 & 5 & 1.03 \\
\hline
\end{tabular}

Table 3. Progress of GaAs Cell Efficiency on Poly-Ge Substrates.

\begin{tabular}{|c|c|c|c|}
\hline Ref. & Grain-Size(cm) & Cell Area $\left(\mathrm{cm}^{2}\right)$ & AM1.5 Eff. (\%) \\
\hline PVSC 1993 [4] & 0.5 & 1 & 15.8 \\
\hline PVSC 1996 [5] & 0.5 & 4 & 18.2 \\
\hline Current state-of-art & $0.04-0.1$ & 4 & $-1 . \cdots$ \\
\hline & $0.04-0.1$ & 0.25 & $-1 . \cdots$ \\
\hline
\end{tabular}

\section{PV-Quality GaAs On Moly Foils}

The successful transition of high-efficiency GaAs cells on to sub-mm grain-size poly-Ge substrates have motivated us to consider the development of high-efficiency GaAs solar cells on significantly lower-cost substrates such as glass and moly foils. Thin moly-foils are attractive due to the thermal expansion match between moly and $\mathrm{GaAs}$, the ease of cell fabrication with minimal or no backside contact processing, the mechanical strength of moly for manufacturing, and for possible use in flexible, lightweight solar arrays.

Minority-carrier lifetime is an important material parameter that directly affects the potential efficiency achievable in GaAs solar cells. Figure 4 indicates the dependence of 1-sun AM1.5 solar cell efficiency as a function of the minority-carrier lifetime. This model is very similar to the work of Ref. 6 . In this figure, we also indicate the GaAs solar cell efficiencies, as a function of lifetime, in two less-than perfect $\mathrm{GaAs}$ materials, GaAs-on-Si [7] and GaAs-on-poly Ge [8]. Given the significant dependence of cell efficiency on the minority-carrier lifetime, we have focused our initial efforts on improving this parameter in GaAs thin-films on moly. An important understanding resulting from our development of high-efficiency GaAs solar cells on poly-Ge was that, most likely, there is an accumulation of Se-dopants at the grain boundaries, similar to a proposal on an earlier work at the grain boundaries can lead to $\mathrm{n} / \mathrm{n}^{*}$ minority-carriers for holes in n-GaAs. Thus we anticipate improved minority-carrier lifetime with increased Se-doping in poly-GaAs on moly. The dependence of minority carrier lifetime as a function of Se-doping level in GaAs grown on single-crystal GaAs substrates (i.e., no grain boundaries) and that of

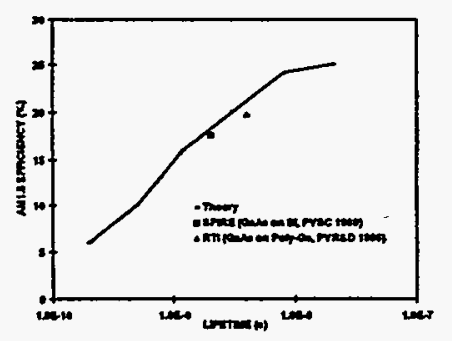

Fig. 4. Dependence of GaAs solar cell efficiency on lifetime and some benchmark $\mathrm{GaAs}$ cell efficiencies on lessthan-perfect substrates 
GaAs grown on moly are compared in Fig. 5. While the lifetime falls continuously with increased Sedoping level in the case of single-crystal GaAs films, primarily due to the reduction in the radiative lifetime, the poly-GaAs films on moly show a significant increase in lifetime with Se-doping up to a certain level. The moderate increase in lifetime in these GaAs films, in spite of the anticipated decrease in radiative lifetime, gives clear evidence for the Se-passivation of grain-boundaries.

In addition to Se-passivation of the grain boundaries, higher growth temperatures lead to improved lifetimes in GaAs thin-films on moly. The improvement in lifetime with higher growth temperature is thought to be due to a reduction of intra-grain dislocation densities. The effect of improved lifetimes with higher growth temperature was also evident in the higher $\mathrm{J}_{\mathrm{sc}}$ levels obtained in GaAs solar cells on moly (Fig. 6). Work is in progress to enhance $V_{o c}$ and fill-factor values in GaAs solar cells on moly foils.
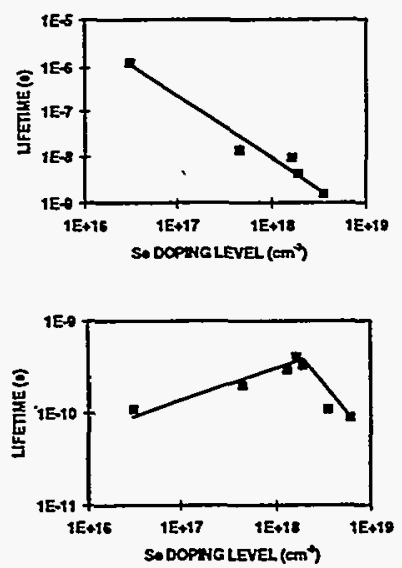

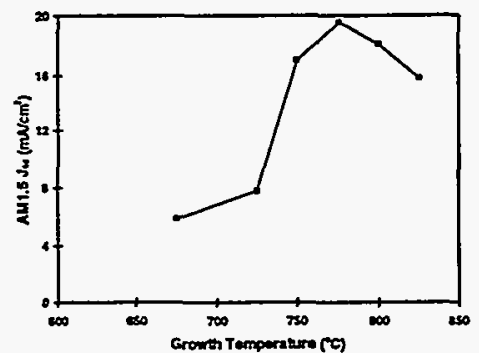

Fig. 6. Dependence of $J_{s c}$ on growth temperature in Se-passivated $\mathrm{p}^{+}-\mathrm{n}$ GaAs solar cells on moly foils -

Fig. 5. Dependence of lifetime on Se-doping level in GaAs films (a) on single-crystal GaAs substrates and (b) on moly foils

\section{Acknowledgements}

This work has been performed under Subcontract No. YAL-3-1-3357-03 from the National Renewable Energy Laboratory, with Dr. Robert McConnell as the Technical Monitor.

\section{References}

1. S.R. Kurtz, J.M. Olson, and A. Kibbler, Proc. of 2Ist IEEE PVSC (IEEE Press, NY, 1990); pp.138.

2. J.M. Olson, S.R. Kurtz, A.G. Kibbler, and P. Faine, Proc. of the 21st IEEE PVSC (IEEE Press, NY, 1990); pp.24.

3. M. Green and K. Emery, Progress in Photovoltaics, 2, (1994); pp. 231-234.

4. R. Venkatasubramanian, M.L. Timmons, P.R. Sharps, and J.A. Hutchby, Proc. of the 23rd IEEE PVSC (IEEE Press, NY, 1993); pp.691.

5. R. Venkatasubramanian, B.C. O'Quinn, J.S. Hills, P.R. Sharps, M.L. Timmons, and J.A. Hutchby, Proc. of the 25th IEEE PVSC (IEEE Press, NY, 1996); pp.31.

6. M. Yamaguchi, C. Amano, Y. Itoh, R.K. Ahrenkiel, and M.M. Al-Jassim, Proc. of 20th IEEE PVSC (IEEE Press, NY, 1988); pp.749.

7. S.M. Vernon, S.P. Tobin, V.E. Haven, C. Bajgar, T.M. Dixon, M. Al-Jassim, R.K. Ahrenkiel, and K. Emery, Proc. of the 20th IEEE PVSC (IEEE Press, NY, 1988); pp.481.

8. R. Venkatasubramanian, B.C. O'Quinn, and E. Siivola, Proc. of the NRELSNL PVR\&D Review, (AIP Press, NY, 1996); pp.259.

9. K.P. Pande, D. H. Reep, S.K. Shastry, A.S. Weiner, J.M. Borrego and S.K. Ghandhi, IEEE Trans. on Electron Devices, Vol. ED-27, (1980); pp. 635 
Title:

Organization :

Contributors :

\title{
Vapor Phase Epitaxial Liftoff of GaAs and Silicon Single Crystal Films
}

\author{
University of California, Los Angeles \\ Electrical Engineering Department \\ 420 Westwood Plaza \\ Los Angeles, California, 90095-1594
}

E. Yablonovitch, Principal Investigator; W. Chang, C. P. Kao, G. A. Pike and J. A. Slone

\section{Introduction:}

The opportunity exists to make high-quality solar cells at low cost by epitaxial liftoff (ELO) of thin films from single crystal substrates, and then re-using the substrates repetitively. In this way the substrate expense would drop out of the cost formula. Furthermore, the ultra-thin cells do have the potential of higher performance than conventional thick solar cells, due to a better tradeoff between a higher operating voltage and the light-trapping assisted current.

In this article, we will present the potential improvements in the epitaxial liftoff process by using a vapor phase etch to undercut the thin films.

\section{Large Area Epitaxial Liftoff:}

During this past year, the UCLA group has shifted its attention from producing small area demonstration solar cells, to the practical development of large area epitaxial liftoff. Of course, large areas will be needed for producing significant amounts of photo-voltaic energy. The major problem in the epitaxial liftoff process as applied to solar cells is the scale-up to large area crack-free sheets. Indeed large sheets of many small discrete devices do not present insurmountable ELO problems. But in large área devices such as solar cells, the failure mode turns out to be the risk of cleavage cracks being present in the lifted off thin films. The cleavage cracks are an open circuit to current flow, and leave the solar cells opencircuited or at least very resistive.

There have been a great many tests of the effect of the epitaxial liftoff process on the lifted off devices [1]. The outcome of all this testing is, that as a general nule, electronic devices are unharmed by the liftoff process. However there is some susceptibility to pinholes in the epitaxial films, and in large area devices such as solar cells, there is a significant risk of forming cleavage cracks. The normal cleavage planes in III-V semiconductors are $<110>$ oriented. As free standing thin films, GaAs sheets have very little mechanical strength, and need to be mechanically supported at each stage of the liftoff process. It is generally accepted that a strain as small as $0.1 \%$ can produce cleavage cracks in GaAs.

The mechanical support can be helpful by supplying compressive stress on the thin GaAs films, or it can be harmful by causing the film to stretch. The films are strong in compression, but weak in tension. During the liftoff, some parts of the film are still attached while other parts are already free. The nonuniformity of these effects tends to put undesirable tensile strains on the films during the liftoff process.

It is now generally accepted that cleavage cracks are the main problem in the application of Epitaxial Liftoff to solar cells. There is some recent work [2] from the Netherlands which addresses some of these problems. They introduced some significant new innovations:

They cover the GaAs liftoff film with $2-3 \mu \mathrm{m}$ of evaporated Copper metal. This provides both mechanical strength and a favorable thermal expansion co-efficient. Copper contracts more when being cooled from temperatures above ambient, and therefore provides desirable compression on the GaAs film. This allows 
some tensile stress to be applied during liftoff, which apparently expedites the etch rate. Under-cutting rates on the order of $1 \mathrm{~mm} /$ hour were demonstrated, which is faster than the normal etch rate $\approx 0.3 \mathrm{~mm} /$ hour. We presume that this might be attributed to stress-enhanced corrosion, which is a known effect in metallurgy. A single droplet of HF acid applied to the wafer edge provided the under-cut etch in the work by van Geelen et al [2].

\section{The Vapor Phase Liftoff Process:}

In this article we will present experimental parametric studies on using acid vapor rather than an aqueous acid for implementing the liftoff process. This permits a cleaner liftoff process, with process simplifications that may assist in addressing the cleavage crack issue. A similar vapor epitaxial liftoff process is presented here for both $\mathrm{GaAs} / \mathrm{AlAs}$, as well as $\mathrm{Si} / \mathrm{SiO}_{2}$ Silicon-on-insulator wafers.

There have been a number of previous studies of using HF acid vapor for micro-electro-mechanical micro-fabrication and processing steps $[3,4]$. In addition there have been studies using anhydrous HF vapor [5]. Here we address the effects of vapor partial pressure, and its influence on under-cutting speed in comparison to aqueous HF acid.

We have chosen to work with the vapor of concentrated hydrofluoric acid (49\%), since it is close to the azeotropic composition, which is where the chemical composition in the vapor matches [6] that in the liquid. A diagram of our undercut etching apparatus is as follows:

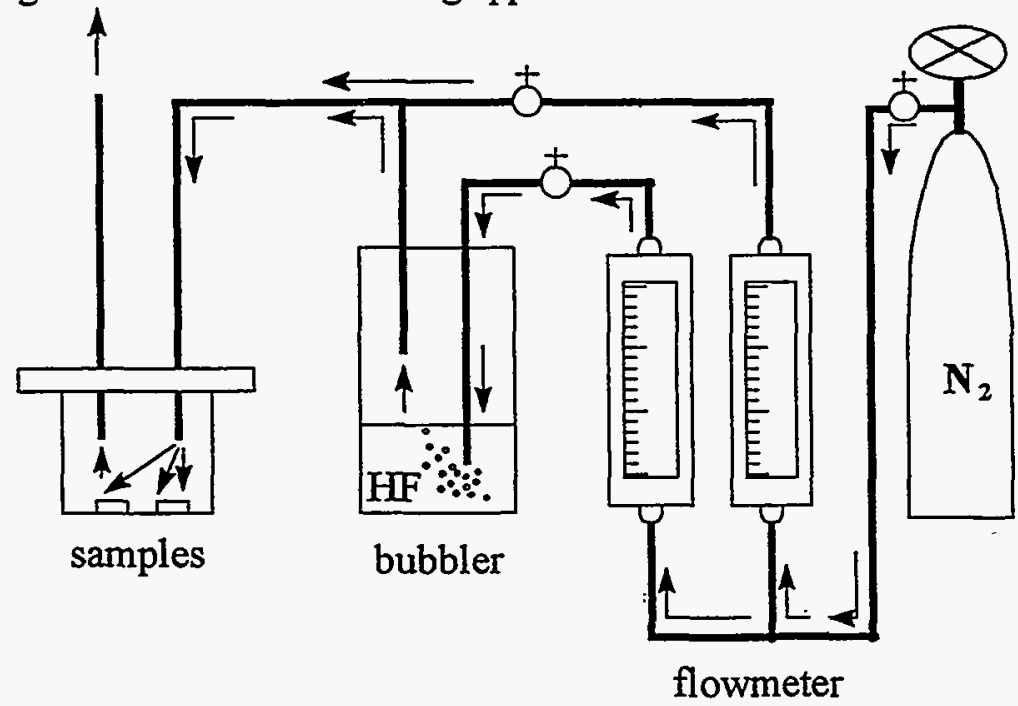

Figure 1

The symbols $\circlearrowleft$ represent flow control valves which blend the saturated vapor from the bubbler with dry $\mathrm{N}_{2}$ to adjust the acid vapor pressure.

We plot the lateral under-cutting rates versus the percentage of saturated vapor, a kind of relative humidity for the acid vapor. One hundred percent of saturation could lead to condensation. Less than $100 \%$ of saturation is dry in principle, but the condensation conditions could be quite different in a narrow slot where surface energies influence the condensation equilibrium. Furthermore, since the acid re-acts with the AlAs or $\mathrm{SiO}_{2}$ surface, the reaction products may have a smaller vapor pressure and remain condensed. Therefore the process cannot be regarded as completely dry, even when the vapor pressure is $<100 \%$ of saturation. We can assume that sub-microscopic amounts of aqueous acid may be present in the etching slot, but that the mass transport conditions might be different in gas phase vapor etching than in aqueous bath etching.

Figures 2(b) shows the initial etch rate of $50 \mathrm{~nm}$ thick AlAs slots. The vapor etch rate is noticeably slower than the aqueous solution point marked on the right hand axis. The scatter is due to non-uniformity 
in the etch rate. The etch rate is maximum at saturation, but unsaturated vapors were almost equally effective. These etch rates are all slower than the stress-induced etch speedup measured by van Geelen et al [2]. Stress is a double edged sword, and we were trying to avoid the risk of cleavage crack formation.

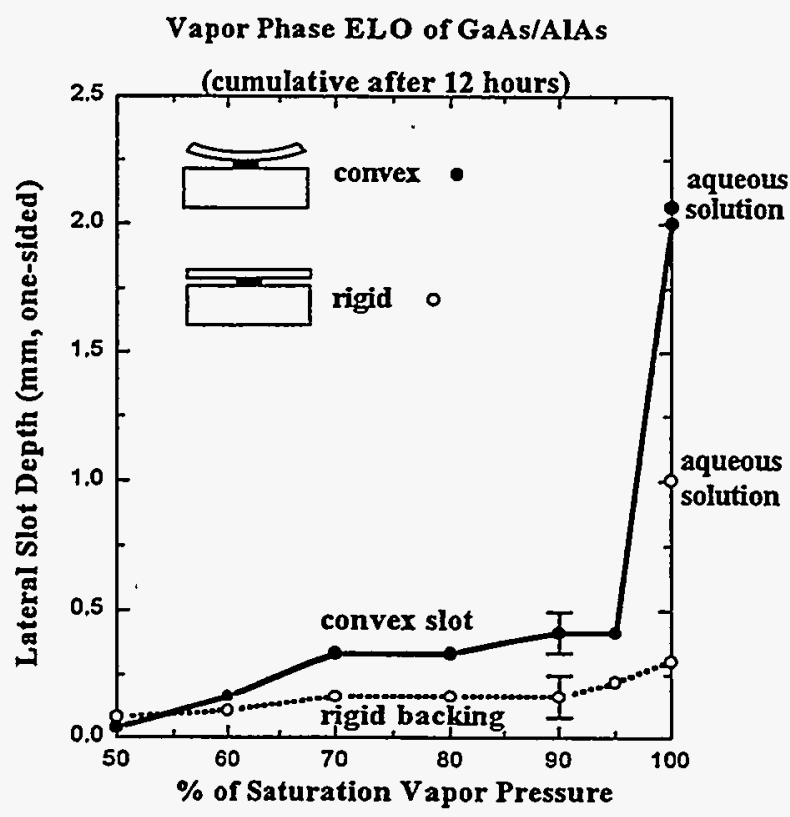

Figure 2(a)

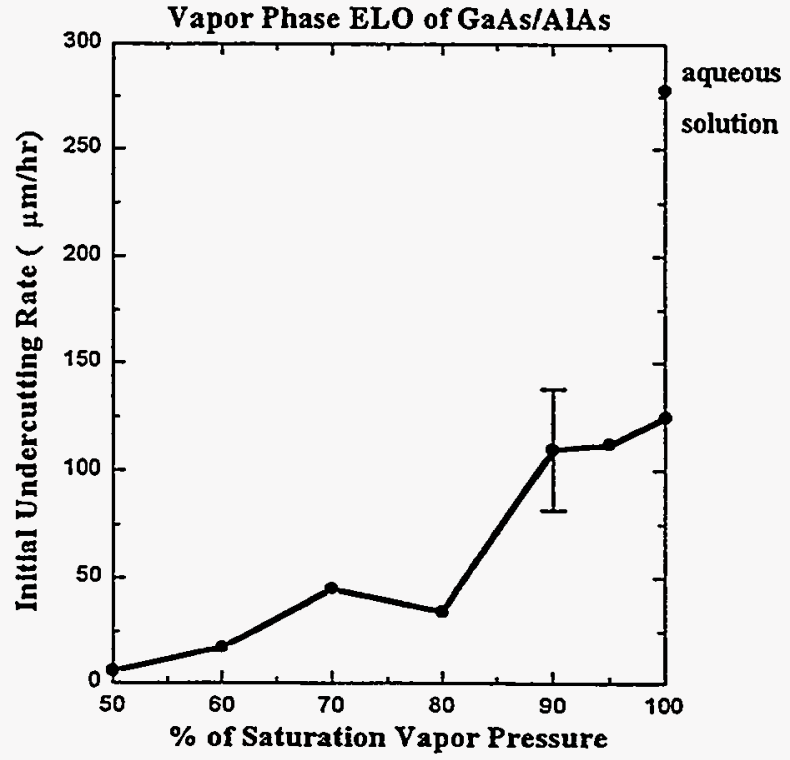

Figure 2(b) 
Vapor Phase ELO of $\mathrm{SiSiO}_{2}$ (SOI)
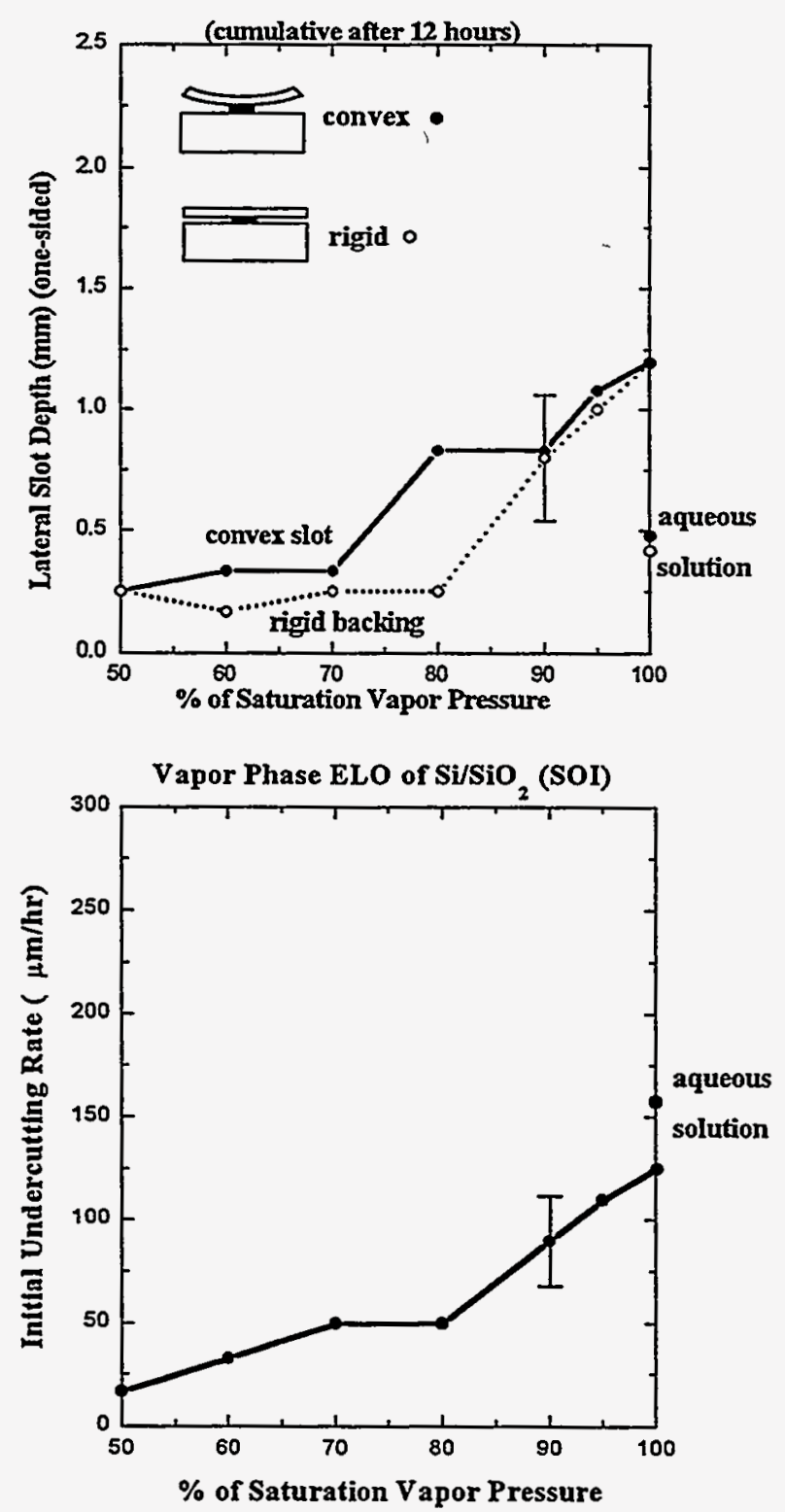

Figure 3(a)

Figure 3(b)

In Figure 2(a) the cumulative etch rate after 12 hours is given. Generally the etching slows down in a deeper slot owing to the difficulty of mass transport of reactants and reaction products into and out of the slot. Mass transport is somewhat easier in the convex geometry as originally discussed in [7]. Nevertheless the plane parallel slot labeled as rigid, allows a $2 \mathrm{~mm}$ wide stripe to be undercut in 12 hours. This is surprisingly good in view of the mass transport issues. A large area array consisting of $2 \mathrm{~mm}$ stripes would be ideal for solar cells, so this is very promising. As mentioned in the introduction however, the problem is to prevent cracking in such stripe arrays.

We have also performed vapor ELO measurements on SOI films, as shown in Fig. 3(b). The initial vapor undercutting rate is slightly slower than in aqueous solution. However the cumulative rate in aqueous etch comes to a halt at about a 500 $\mu$ m slot depth, while the vapor etch continues to etch almost linearly with time and doesn't slow down. The mass transport issues for $\mathrm{SiO}_{2}$ slots are analyzed in [8], and 
the high solubility of the $\mathrm{SiF}_{4}$ reaction products should permit the aqueous etching to continue with little slowdown in slots deeper than 500um. Therefore the stopping of the aqueous etching in Fig. 3(a) are puzzling to us, but gratifyingly the vapor etch seems to not have that problem. Again arrays of $2 \mathrm{~mm}$ wide stripes should be available from SOI wafers. If these could be manufactured and the substrates re-used at low cost then this would also be a possible thin film solar cell option.

We have demonstrated that HF acid vapor epitaxial liftoff is a viable option for separating thin film solar cell material from a parent substrate and is comparable or superior to aqueous acid liftoff. As in all epitaxial liftoff, the separated substrate is in excellent condition and ready to be re-used. The vapor process is essentially dry, except that sub-microscopic amounts of liquid acid might actually be present inside the under-cutting slots.

Large area arrays of 1 or $2 \mathrm{~mm}$ stripes can be lifted off, but they generally include an unacceptable number of cleavage cracks. Crack formation is a stubborn issue that may require new approaches such as those introduced by the Netherlands group [2].

\section{References:}

1. W. K. Chan and E. Yablonovitch, "Epitaxial Liftoff and Related Techniques", Chap. 8 on p. 297 in Integrated Optoelectronics, ed. by M. Dagenais, R. F. Leheny and J. Crow, (Academic Press, New York, 1995).

2. A. van Geelen, P. R. Hageman, G. J. Bauhuis, P. C. van Rijsingen, P. Schmidt, and L. J. Giling, "Epitaxial Liftoff GaAs Solar Cell from a Reusable GaAs Substrate", Mat'ls. Sci. and Eng'g. B45, (1997) pp. 162-171.

3. T. A. Lober and R. T. Howe, "Surface Micromaching Processes for Electrostatic Microactuator Fabrication", Technical Digest of IEEE Solid-State Sensor and Actuator Workshop, cat. No. 88TH0215-4, (1988).

4. K. R. Williams and R. S. Muller, "Etch Rates for Micromachining Processing", J. Microelec.-mech. Systems, 5 (1996) pp. 256-269.

5. Y. I. Lee, K. H. Park, J. Lee, C. S. Lee, H. J. Yoo and C. J. Kim, "Dry Release for Surface Micromachining with HF Vapor Phase Etching", J. Microelec.-mech. Systems, 6, (1997) pp. 226-233.

6. R. A. Smith in Kirk-Othmer Encyclopedia of Chemical Technology Vol. 11, 4th Ed., ed. by M. HoweGrant (John Wiley New York 1991) p. 361

7. E. Yablonovitch, T. J. Gmitter, J. P. Harbison and R. Bhat, "Extreme Selectivity in the Lift-Off of Epitaxial GaAs Films", App. Phys. Lett. 51, (1987), p. 2222.

8. D. J. Monk, D. S. Soane, and Roger T. Howe, "Hydrofluoric Acid Etching of Silicon Dioxide Sacrificial Layers", J. Electrochem. Soc. 141 (1994), pp. 270-274. 
Title:

Organization:

Contributors:
Fundamental Studies of the Effect of Crystal Defects on $\mathrm{CuInSe}_{2} / \mathrm{CdS}$ Heterojunction Behavior

\author{
Department of Materials Science and Engineering
} University of Illinois, Urbana, $\mathbb{Z}$

R. McConnell, program manager; A. Rockett, principal investigator; D. Schroeder (graduated: Ph.D. August, 1997), G. Berry, John Belanger (graduated: M.S. October, 1997), Shawn O'Conner (new), Dongxiang Liao (new), graduate research assistants; Benjamin French, Terrence Yeoh, and Jessica Nelson, undergraduate research assistants.

\section{Objectives}

The project seeks to determine the source of sample-to-sample variability in $\mathrm{CuIn}_{1-\mathrm{x}} \mathrm{Ga}_{\mathrm{x}} \mathrm{Se}_{2}$ (CIGS)-based solar cell performances and the mechanisms restricting carrier collection at large voltages in CIGS/CdS heterojunctions. The detailed objectives involve determining the relative importance of structural and chemical defects in the material and how they affect electronic properties and hence device performances. A byproduct of the project is fundamental data on the properties of CIGS alloys as a function of processing conditions and composition. This is important to improving device modelling. Finally, as a university research program the goals include the training of graduate and undergraduate students and the exposure of as many individuals as possible to renewable energy research (see contributors list).

\section{Approach}

The basic approach is to grow the highest quality CIGS under a variety of conditions and to measure the morphology, microstructure, and properties of the resulting layers. Electrical properties have been measured extensively this year for correlation with compositional and structural data. Except as noted, the results described were recorded for CIGS deposited in a single step from magnetron sputtered $\mathrm{Cu}$ and In fluxes and evaporated molecular $\mathrm{Se}$. The single crystal $\mathrm{CuIn}_{1-\mathrm{x}} \mathrm{Ga}_{\mathrm{x}} \mathrm{Se}_{2}$ layers were deposited directly on "epi-ready" GaAs (001) or (111) As wafers. Deposition temperatures were $\sim 650^{\circ} \mathrm{C}$.

Microstructural studies were carried out by X-ray diffaction and by scanning electron microscopy. Composition analyses used secondary ion mass spectrometry (SIMS), and energydispersive spectroscopy. Hall-effect measurements were obtained as a function of temperature using gold contacts in a van der Pauw pattern. Electroluminescence measurements were made on solar cells from the University of Delaware, the National Renewable Energy Laboratory (NREL), and Siemens Solar Industries. Emission from forward-biased solar cells was studied as total intensity and as a function of wavelength and bias current. Spectral response was also measured for each device in the same instrument. Characterization of Na transport in CIGS deposited at several industrial laboratories has been carried out using SIMS as part of the NREL CIS team effort. 
Results (10/1/96 - 9/30/97)

\section{Characterization of hole transport in p-CIGS}

The major focus of our research during the past year has been to extended our previous characterization of hole transport in CIGS to include a study as a function of Ga content in the alloys.[1] The data for high Ga-content alloys and pure $\mathrm{CuGaSe}_{2}$ show that addition of $\mathrm{Ga}$ causes the acceptor concentration to increase to values closer to the number of majority point defects in the material as compared to the values in pure $\mathrm{CuInSe}_{2}$. Overall, the data shows a generally exponential increase in hole state density with increasing Ga content. [See Figure 1.] However, it is also possible or even likely (data are insufficient to date to say for sure) that the hole concentration increases gradually for concentrations below $\sim 25 \% \mathrm{Ga}$ with an abrupt three order of magnitude rise in the $30-70 \% \mathrm{Ga}$ range. We have not obtained sufficient data yet to clarify the exact behavior. The depth of the acceptor states does not change significantly except as would be expected from broadening of the defect band as acceptor concentration rises. This is significant as it indicates that no fundamental change in the defects giving rise to the acceptor states occurred. Finally, and equally remarkably, the hole mobility remained constant or may have actually increased as the $\mathrm{Ga}$ content increased in spite of the massive increase in hole concentration. The general behavior near room temperature was dominated by phonon scattering for all $\mathrm{Ga}$ contents studied. Low temperature behavior was controlled by impurity band hopping conduction. There was no change in any of these results for samples with a $\mathrm{Ga}$ concentration gradient as compared with samples of similar composition without a $\mathrm{Ga}$ gradient.

These results imply that the addition of $\mathrm{Ga}$ increases acceptor density by changing either the energy associated with the formation of metal vacancies or the ease with which defects are able to cluster and form complexes which push their states out of the bandgap. We believe that the most probable explanation is due to changes in the clustering of defects. Theoretical calculations of the binding of these defects in $\mathrm{CuInSe}_{2}$ and $\mathrm{CuGaSe}_{2}$ are consistent with the observed changes in acceptor concentration. The observation of a relation between acceptor density and $\mathrm{Ga}$ content may also explain observation of temperature dependence of acceptor concentration in epitaxial $\mathrm{CuInSe}_{2}$ on;GaAs by Neumann et al.[2] which may have resulted from increasing diffusion of $\mathrm{Ga}$ into the epitaxial layer. Most important, these results may explain the relatively poor performance in high Ga-content photovoltaic devices. Modeling of these devices has indicated an increased carrier concentration. It has been unclear up to this point why such an increase should occur. The current study clarifies this point and suggests that it may be an intrinsic feature of $\mathrm{CuGaSe}_{2}$.

Finally, the results of this study suggest that the mobility of majority and minority carriers in CIGS are likely to be substantially different. Majority carriers measured in this work clearly have excellent mobilities for all film compositions. On the other hand, minority carriers are likely to be dominated by motion in defect bands, especially in heavily compensated materials. Reduction in compensation by the addition of $\mathrm{Na}$ could significantly increase this mobility with consequent improvements in device performances.

\section{The Effect of Impurities on CIGS}

During this year we completed work on the effect of $\mathrm{Na}$ on hole transport in single crystals of CIGS. Na was added by diffusion at 550 or $600^{\circ} \mathrm{C}$ from a surface covered with either $\mathrm{Na}_{2} \mathrm{Se}$ (by 
evaporation) or $\mathrm{NaOH}$ (by drying from solution) as described in Ref. [3]. Residual surface $\mathrm{Na}$ in both forms was then removed by rinsing with deionized water. A drive-in diffusion was then used to produce a uniform $\mathrm{Na}$ level in the film. This level was increased by a factor of $\square 80$ (checked by SIMS) with respect to the as-grown films. No significant oxygen contamination was found in the films doped from $\mathrm{NaOH}$. Characterization of the films and modeling of the results showed a dramatic reduction in compensation with no other apparent changes in state densities or depths. This behavior was independent of $\mathrm{Cu} / \mathrm{In}$ or $\mathrm{Ga} /(\mathrm{In}+\mathrm{Ga})$ ratios. DC photoconductivity measurements on the samples with and without Na showed no change in trapping rate as a result of $\mathrm{Na}$ addition. The results were the same for all samples studied. We conclude that $\mathrm{Na}$ contained within CIGS grains acts to reduce compensation.

Now that the basic characterization of CIGS is complete and the most important impurities, Ga and $\mathrm{Na}$, have been studied, an additional effort has been begun to examine the effects of other impurities in CIGS. We have carried out a preliminary analysis of the effect of ion implanted $\mathrm{Cr}$, $\mathrm{Se}$ (as a control), O, $\mathrm{Al}$, and $\mathrm{Zn}$. The majority of the effect seems to be related to the ion implantation damage, as demonstrated by the Se implant. The dose in this case was too low to affect significantly the Se concentration in the material. Hence, all damage must have been the result of ion bombardment. In as-implanted samples the carrier concentration was found be higher than in as-deposited samples and to increase with decreasing temperature at low temperatures, suggesting a hopping conduction mechanism. Virtually all of the apparent damage was removed by a $550^{\circ} \mathrm{C}$ anneal for 25 minutes. In the case of $\mathrm{Cr}$, a nearly identical behavior was observed but a type conversion in as implanted samples was also found as samples were cooled. Again, nearly all damage was apparently removed upon annealing. Residual changes included a reduction in acceptor concentration and an increase in neutral impurity scattering. In the $\mathrm{Al}, \mathrm{Zn}$ and $O$ implanted samples the as-implanted mobilities were $<10 \mathrm{~cm}^{2} / \mathrm{Vs}$ at $300 \mathrm{~K}$ and hopping conduction dominated behavior. These values did not recover significantly during annealing $\left(550^{\circ} \mathrm{C}, 25 \mathrm{~min}\right) . \mathrm{DC}$ photoconductivity measurements showed that after annealing of the $\mathrm{Cr}$ and Se implanted samples, no change in recombination rate had occurred, further indicating that $\mathrm{Cr}$ is not a recombination center in CIGS.

The implications of these results are primarily that the impurities have relatively modest effects with most of the changes resulting from implantation damage. This is good news in that these impurities which are some of the species likely to be introduced during device processing appear to have little or no impact on hole transport in CIGS.

\section{Electroluminescence from CIGS Solar Cells}

Observations of electroluminescence from forward biased solar cells, begun in 1995 were continued during 1997.[4] Results obtained during this year indicate that all solar cells examined showed four to five (depending upon peak fits) luminescence lines. The relative intensities of these luminescence features changed with temperature and $\mathrm{Ga}$ content but the locations of the lines did not change substantially. All features were apparently subgap transitions (defect state to defect state). The lack of shift in wavelength with $\mathrm{Ga}$ content is surprising and suggests that there may be small areas of relatively Ga-free material phase separated in CIGS polycrystals which collect and recombine carriers radiatively. Further study of this phenomenon is currently underway. We are attempting to obtain devices based on pure $\mathrm{CuGaSe}_{2}$ for comparison. 


\section{CIS Team Activities}

This year we have participated actively in the NREL CIGS Team working on the properties of Mo back contacts for CIGS solar cells. Our responsibility has been primarily SIMS analysis of samples prepared at Energy Photovoltaics, International Solar Electric Technologies, and Martin Marietta. Analyses have shown correlations between the microstructure of the CIGS and the concentration of impurities, indicating that the impurities may be present primarily in the grain boundaries of the material, in agreement with the results reported previously for $\mathrm{Na}$ in CIGS.[5]

\section{Conclusions}

The results obtained this year have provided details concerning several major effects critical to operation of CuIn $\mathrm{Cu}_{1-\mathrm{x}} \mathrm{Ga}_{\mathbf{x}} \mathrm{Se}_{2}$-based solar cells. Data on the concentration effect of $\mathrm{Ga}$ has provided information which explains why solar cells containing large amounts of Ga have not been as successful as had been hoped. The results suggest that modeling of device performance requires differences in the mobilities of majority and minority carriers in heavily compensated CIGS. Preliminary data on impurities indicates that most common impurities should not have detrimental effects on the performance of CIGS and in some cases, such as for $\mathrm{Na}$, substantial improvements may result. Electroluminescence data for operating devices continue to be unexpected with less temperature dependence than would be expected for normal semiconductors under the observed conditions. Future work is expected to complete the investigations outlined above and to extend the work to sulfur alloys in addition to $\mathrm{Ga}$ alloys.

\section{References:}

1. David J. Schroeder, Jose Luis Hernandez, Gene D. Berry and Angus A. Rockett, "Hole Transport and Doping States in Epitaxial CuIn 1-x Gax Se2", J. Appl. Phys. in press.

2. H. Neumann, E. Nowak, B. Schumann, and G. Kühn, Thin Solid Films, 74, p. 197, 1980.

3. David J. Schroeder and Angus A. Rockett, "Electronic Effects of Sodium in Epitaxial CuIn1${ }_{x} \mathrm{GaxSe}_{2}$ ", J. Appl. Phys., in press 11/15/97 issue.

4. John Belanger, "Temperature Dependent Junction Electroluminescence of CuIn 1-x $\mathrm{Ga}_{\mathrm{x}} \mathrm{Se}_{2}$ Photovoltaic Devices", M.S. Thesis, University of Illinois, 1997.

5. Angus Rockett, $M$ Bodegård, Karin Granath and Lars Stolt, M. Marudachalam, H. Hichri, R. W. Birkmire, J. M. Schultz,A. B. Swartzlander, and M. M. Al-Jassim, Proceedings of the 25th IEEE Photovoltaic Specialists Conference, 1996 (IEEE, New York, 1996), p. 985.

\section{Figure:}




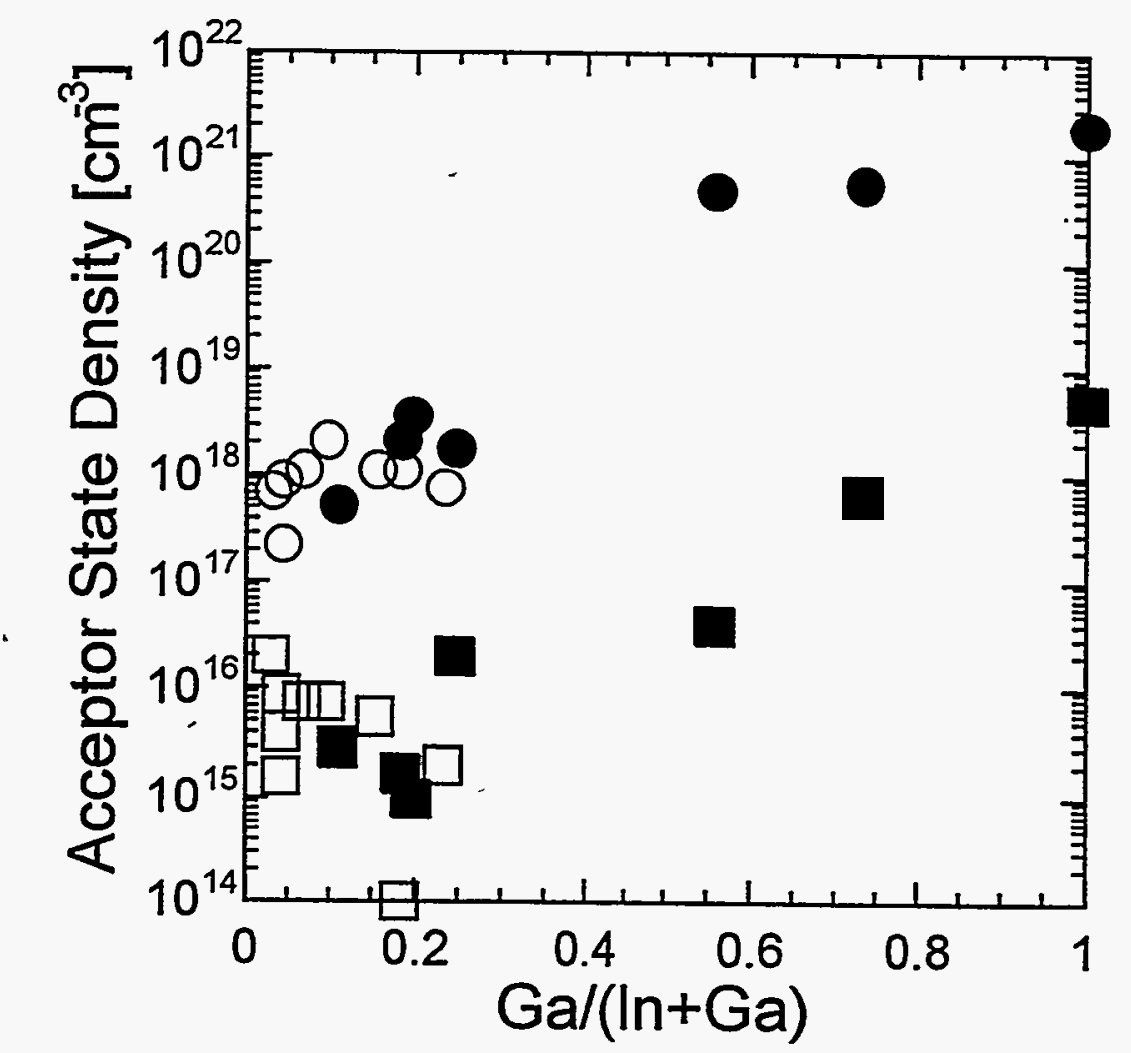

Figure 1: Hall-effect results showing the state density as a function of $\mathrm{Ga}$ content in $\mathrm{CuIn}_{1-\mathrm{x}} \mathrm{Ga}_{\mathrm{x}} \mathrm{Se}_{2}$ epitaxial layers. Higher concentration deep acceptor state values are circles, shallower state values are squares. Open points indicate samples containing a significant $\mathrm{Ga}$ gradient. 


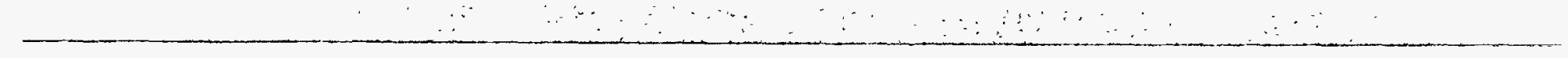

(


Organization:

Department of Physics, University of Utah, Salt Lake City, Utah 84112

Contributors:

P.C. Taylor, principal investigator; G.A. Williams, W.D. Ohlsen, J. M. Viner, S. L. Chen, T. Su

\section{Objectives}

The major objectives of this subcontract are (1) to identify metastabilities caused by carrier recombination or by frozen-in departures from equilibrium, and to determine if the metastabilities that plague devices are "intrinsic" and therefore not correctable or "extrinsic" and therefore capable of being eliminated in future devices, (2) to characterize the important defects and impurities in the bulk and at surfaces and interfaces because interfaces are becoming more important with the advent of tandem cells with several heterojunctions, and (3)to measure the motion of hydrogen in a-Si:H and related alloys on a microscopic scale because the motion of hydrogen is thought to play an important role in many of the metastable effects that occur in a-Si:H and related alloys and the microscopic mechanism for this motion is not known.

\section{Approaches}

Three new approaches, that have not been employed in the past, have been emphasized: (1) below-gap spectroscopy, such as optical absorption, photoluminescence (PL), PL excitation, electron spin resonance and other spectroscopies using primarily a tunable Ti sapphire laser system to excite carriers well below the optical gap (down to $\sim 1.1 \mathrm{eV}$ ). (2) novel muclear magnetic resonance (NMR) techniques such as measurements of the spin-lattice relaxation in a dipolar field $\left(T_{1 D}\right)$, to probe local hydrogen motion in doped and intrinsic a-Si:H and selected alloys. (3) doping with group VI elements that introduce new metastabilities into a-Si:H and related alloys.

\section{Research Results}

The subcontract is divided into several tasks. One task is the growth and characterization of doped and undoped a-Si:H and related alloys. Alloys with concentrations of group VI elements, such as sulfur or selenium, have been found to exhibit very inefficient, n-type doping, and in the sulfur system the potential of these alloys for photovoltaic applications has been investigated. ${ }^{1}$ These films are thermally very stable. We have continued our characterization of films of $\mu \mathrm{c}-\mathrm{Si}: \mathrm{H}$, that were grown at different plasma deposition frequencies. In these films the hydrogen is incorporated in both clustered and dilute phases, just as in a$\mathrm{Si}: \mathrm{H}$. The plasma assisted chemical vapor deposition (PECVD) system is being up-graded to a threechamber system capable of making state-of-the-art devices.

A second task concerns below gap spectroscopy in a-Si:H and related alloys. We have been using Photoluminescence (PL) excitation spectroscopy (PLE), time resolved PL, optically detected magnetic resonance (ODMR), and spin-dependent photoinduced absorption (PADMR) to probe defects which produce absorption below the gap in a-Si:H. In particular, PL, photoinduced absorption (PA), and PADMR in a-SiS $: \mathrm{H}$ have been investigated from 80 to $300 \mathrm{~K}$. The PADMR spectrum is composed of three contributions: a narrow resonance at $\mathrm{g} \approx 2$, a broad resonance at $\mathrm{g} \approx 2$, and a narrow, asymmetric resonance at $g \approx 4$. The excitation energy dependence of the PADMR shows that the broad $g \approx 2$ resonance is associated with the $\mathrm{g} \approx 4$ resonance and that each is due to trapped electron-hole pairs in the triplet $\operatorname{spin}(I=1)$ configuration. 


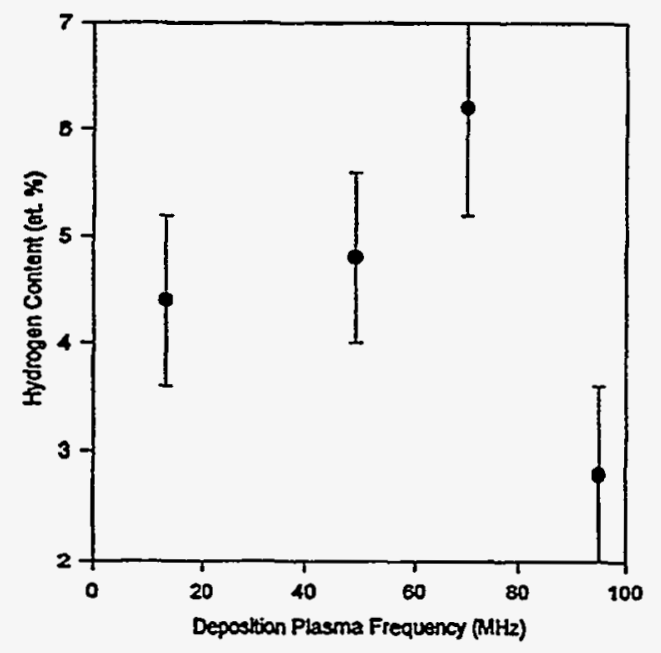

Figure 1 Hydrogen content in four $\mu c-S i: H$ samples ( 3 at. \% silane concentration) measured from ${ }^{1} \mathrm{H} N \mathrm{NM}$ as a function of plasma deposition frequency.

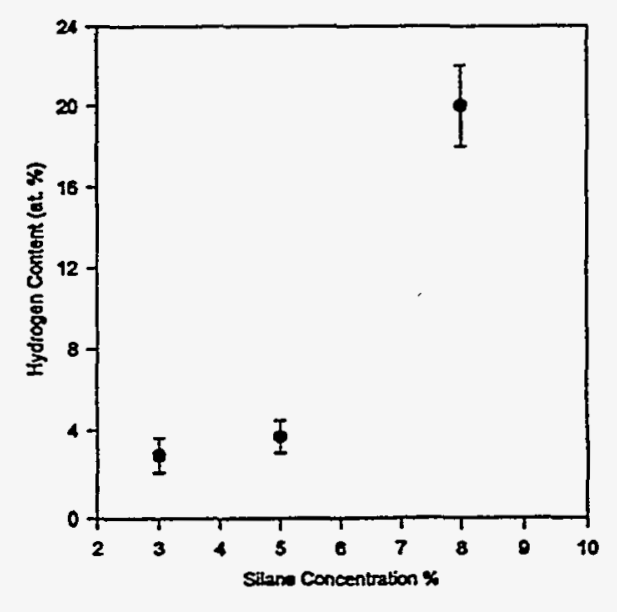

Fig. 2. The hydrogen content of three samples of $\mu \mathrm{c}-\mathrm{Si}: \mathrm{H}$ at $95 \mathrm{MHz}$ plasma deposition frequency as a function of silane concentration.

A third task involves studies of hydrogen bonding and local diffusion of hydrogen in a-Si:H using NMR techniques. We have measured the role of $\mathrm{SiH}_{2}$ in the microcrystalline silicon ( $\left.\mu \mathrm{c}-\mathrm{Si}: \mathrm{H}\right)$ structure using ${ }^{1} \mathrm{H}$ NMR. The degree of crystallinity increases with the deposition frequency of the PECVD, but the hydrogen concentration is essentially constant (for constant silane partial pressure) for frequencies up to about 100 MHz as shown in Fig. 1. These results agree with infrared absorption measurements. In Fig. 2 are shown the hydrogen concentrations for samples made at $95 \mathrm{MHz}$ at different silane partial pressures. It is clear from Fig. 2 that the hydrogen concentration increases as the silane concentration increases. This result in consistent with transmission electron microscopy (TEM) results that show the volume fraction of crystallinity decreasing with increasing silane partial pressure.

A fourth task involves the study of interfaces, defects and impurities in a-Si:H and related alloys, including the metastabilities that are related to such interfaces, defects and impurities. Measurements in the past year have concentrated on a-Si:H samples that have been alloyed with sulfur. ${ }^{4,5,7}$ For example, sulfur alloying reduces the degradation in both the dark- and photo-conductivity. ${ }^{4}$ ESR studies of silicon-sulfur alloys have shown that the ESR and LESR are very similar to those observed in a-Si:H for sulfur concentration less than about 1 at. \%, but that for larger sulfur concentrations significant changes occur. These changes are probably related to the occurrence of persistent photoconductivity at larger sulfur concentrations. Using light-induced ESR as an example, ${ }^{5}$ this situation is shown for various sulfur concentrations in Fig. 3. There is also a change in the conduction processes in silicon-sulfur alloys as the films are rapidly cooled from elevated temperatures. ${ }^{6}$ In the rapidly-cooled state the conductivity. follows the thermally activated process that dominates at higher temperatures, but when slowly cooled the conductivity is dominated by a hopping process. This behavior is very different from that observed in phosphorus-doped a-Si:H. Finally, silicon-sulfur alloys with larger concentrations of sulfur (exceeding 1 at. \%) do not show any appreciable metastable, optically-induced ESR on light soaking. ${ }^{7}$ In addition to the results on the silicon-sulfur alloys, we have also studied defects and metastabilities in silicon-nitrogen alloys. In particular, the measurement of PL and ESR in nitrogen-rich hydrogenated amorphous silicon nitride (a-SiN ${ }_{1.6}: \mathrm{H}$ ) films has provided a probe of optically-induced metastabilities in these films. ${ }^{8}$ 


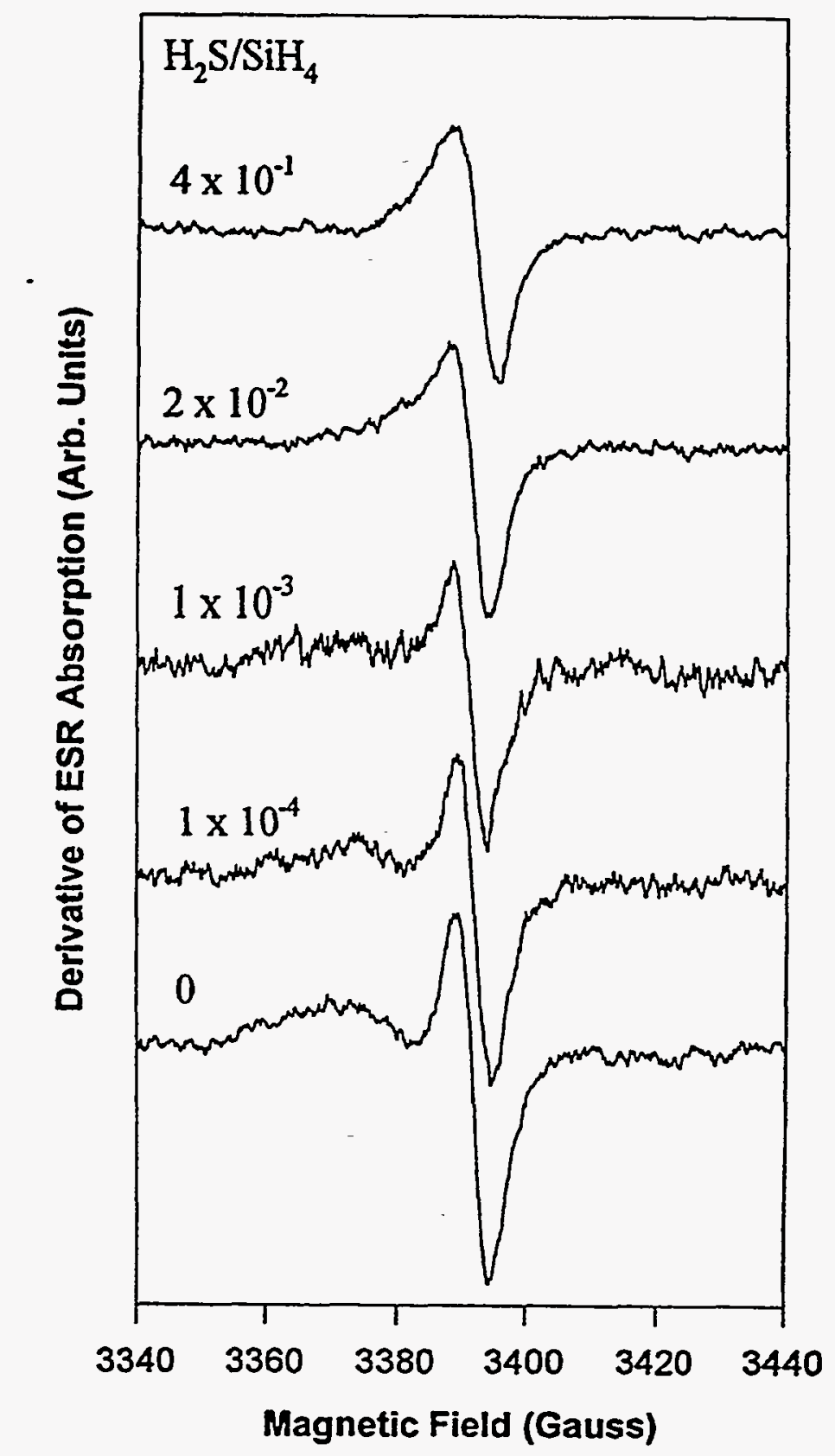

Fig. 3. LESR spectra of a-Si:H as a function of $\mathrm{S}$ doping level. A spectrum of undoped a-Si:H is shown for comparison. These data were taken at $40 \mathrm{~K}$ with $20 \mu \mathrm{W}$ microwave power. The dark ESR spectra have been subtracted out.

\section{Conclusions}

Major accomplishments of the previous year include (1) an evaluation of the potential usefulness of aSiS $: H$ alloys in photovoltaic applications, (2) an identification of the optically-induced absorption due to triplet (spin one) electron-hole pairs in a-Si:H, (3) an understanding of the PECVD frequency dependence 
of hydrogen incorporation in $\mu \mathrm{c}-\mathrm{Si}: \mathrm{H},{ }^{\prime}(4)$ an understanding of the defects and the important metastabilities that occur in silicon-sulfur alloys as a function of sulfur concentration, and (5) an understanding of the important optically-induced metastabilities in $a-\mathrm{Si}_{x} \mathrm{~N}_{1-x}: \mathrm{H}$ alloys. Future directions for the research include (1) measurement of local hydrogen motion in hot-wire samples of a-Si:H and (2) further investigations of the metastabilities introduced into a-Si:H by the presence of group VI impurities (sulfur and selenium).

\section{References}

1. "The Potential of Hydrogenated Amorphous Silicon-Chalcogen Alloys for Photovoltaic Applications: The Role of Persistent Photoconductivity" (S.L. Wang, J.M. Viner, P.C. Taylor, T. Itoh and S. Nitta), NREL/SNL Photovoltaics Program Review, edited by C.E. Witt, A. Al-Jassim and J.M. Gee (AIP Press, New York, 1997), Vol 394, p. 557.

2. "Spin Dependence of Photoinduced Absorption in a-Si:H" (N. Schultz, Z.V. Vardeny and P.C. Taylor), in Amorphous and Microcrystalline Silcion Technology - 1997, edited by S. Wagner, M. Hack, E.A. Schiff, R. Schropp and I. Shimizu (Materials Research Society, Pittsburgh, 1997), Vol. 467, p. 179.

3. "Role of $\mathrm{SiH}_{2}$ in ${ }^{1} \mathrm{H} N \mathrm{MR}$ of $\mu \mathrm{c}-\mathrm{Si}: \mathrm{H}$ Deposited with Different Plasma Excitation Frequencies and Silane Concentrations" (P. Hari, P.C. Taylor and F. Finger), in Advances in Microcrystalline and Nanocrystalline Semiconductors-1996, edited by P.M. Fauchet, R.W. Collins, J.-C. Vial, I. Shimizu, P.A. Alivisatos and T. Shimada (Materials Research Society, Pittsburgh, 1997), Vol. 452.

4. "Light-Induced Effects in a-Si:H Films Alloyed with Sulfur" (Jong-Hwan Yoon, P.C. Taylor, B. Yan, and Czang-Ho Lee), in Amorphous and Microcrystalline Silcion Technology - 1997, edited by S. Wagner, M. Hack, E.A. Shiff, R. Schropp and I. Shimizu (Materials Research Society, Pittsburgh, 1997), Vol. 467, p. 97.

5. "Electron Spin Resonance of Hydrogenated Amorphous Silicon Alloyed with Sulfur" (B. Yan and P.C. Taylor), in Amorphous and Microcrystalline Silcion Technology - 1997, edited by S. Wagner, M. Hack, E.A. Shiff, R. Schropp and I. Shimizu (Materials Research Society, Pittsburgh, 1997), Vol. 467, p. 103.

6. "Relaxation to Hopping Conductivity in Sulfur-Doped Hydrogenated Amorphous Silicon" (J.-H. Yoon and P.C. Taylor), J. Non-Cryst. Solids (1997), in press.

7. "Light-Induced Recombination Centers in Hydrogenated Amorphous Silicon-Sulfur Alloys" (J.-H. Yoon, P.C. Taylor and Czang-Ho Lee), J. Non-Cryst. Solids (1997), in press.

8. "Defect Structure in Nitrogen-Rich Amorphous Silicon Nitride Films" (B. Yan, J.H. Dias da Silva and P.C. Taylor), J. Non-Cryst. Solids (1997), in press. 
Title:

Electrochemical Fabrication of Dye-Sensitized Heterojunctions

Organization: $\quad$ Department of Chemical Engineering, University of Washington, Seattle, WA.

Contributors: $\quad$ Daniel T Schwartz, Principal Investigator, Brian O'Regan, Graduate Assistant.

\section{Objectives:}

Our research is focused on the development of dye-sensitized wide-band-gap heterojunctions as photovoltaic devices. Our aim is to bring together the cost advantages of dye-sensitized cells with the stability and packaging advantages of solid materials. Dye-sensitized photovoltaics are "majority carrier devices" in that the hole and the electron are separated into different materials within a picosecond after a photon is adsorbed. Because of this, there are no minority carriers available for bulk recombination. When bulk recombination is eliminated as a loss route, materials purity requirements can be relaxed and lower costs should result.

To fabricate dye-sensitized heterojunctions (DSHs) we have focused on ruthenium polypyridyl dyes at the interface between $\mathrm{n}-\mathrm{TiO}_{2}$ and $\mathrm{p}-\mathrm{CuSCN}$. $\mathrm{TiO}_{2}$ is the most commonly used substrate for dye sensitization and CuSCN is a little studied wide-band-gap semiconductor $(\sim 3.5 \mathrm{eV})$ with a low work function. In earlier work we have shown that $\mathrm{CuSCN}$ is a good substrate for dye sensitized hole injection, and that $\mathrm{CuSCN}$ can be electrodeposited onto dye sensitized $\mathrm{TiO}_{2}$ surfaces. 4,5 We have found that simple (planar) low surface area $\mathrm{TiO}_{2} /$ dye/CuSCN DSHs show a high efficiency charge separation when photons are absorbed by the dye. ${ }^{3}$ We have shown that the low surface DSHs are stable to long periods of illumination with visible light. We have also examined dye-sensitized heterojunctions using $\mathrm{ZnO}, \mathrm{SnO}_{2}$, and $\mathrm{Nb}_{2} \mathrm{O}_{5}{ }^{1}$

\section{Recent Results}

This past year has seen a major milestone in the project. We have successfully fabricated a dye sensitized heterojunction starting with high surface area $\mathrm{TiO}_{2}$. This is important because only high surface area devices can contain enough dye molecules to absorb a large fraction of the incident sunlight. Figure 1 shows a physical schematic of the high surface area DSH. ${ }^{2}$ For the high surface area DSHs, the as fabricated Incident Photon to Current Efficiency (IPCE) is very low, usually $0.3 \%$. The key discovery of this year has been that a subsequent treatment, consisting of several hours of UV irradiation, can cause a 20 to 50 fold increase in the photocurrent efficiency. Our record IPCE so far is $17 \%$, which corresponds to . an Absorbed Photon to Current Efficiencies (APCE, also known as the internal quantum efficiency) of $60 \%$. The action spectra of a high surface area DSH is shown in Figure 2. Open circuit voltages as high as $550 \mathrm{mV}$ have been recorded in full sun.

Figure 3 shows the short circuit photocurrent as a function of time for three different DSHs illuminated with white light containing some near UV $(\lambda>340 \mathrm{~nm})$. Because of the importance of understanding the "UV effect" in order to improve the efficiency further, a major portion of the effort of the last year has gone into understanding the changes caused by UV irradiation. Similar effects occur in photoelectrochemical cells based on the same $\mathrm{TiO}_{2}$ and dye. The increase in efficiency caused by UV illumination of a $\mathrm{TiO}_{2} /$ dye electrode in ethanol/Lil is shown in figure $4((\Delta))$. A faster increase occurs during visible light irradiation in acetonitrile/KSCN (figure 4, (o/). Most recently we have found that soaking the completed DSH in ethanol solutions of the oxidized form of thiocyanate, $(\mathrm{SCN})_{3}{ }^{-}$, can also cause large increases in the photocurrent efficiency. The increase in photocurrent efficiency as a function of cumulative exposure to $\mathrm{K}(\mathrm{SCN})_{3}$ is also shown in figure $4((\rightarrow))$.

\section{Conclusions}

On the basis of these, and related, results we have concluded that the increase in efficiency under UV illumination occurs by the following mechanism: 
a) UV photons absorbed by the $\mathrm{TiO}_{2}$ create an electron/hole pair.

b) The hole diffuses to the $\mathrm{TiO}_{2} / \mathrm{CuSCN}$ interface and oxidizes an adsorbed $\mathrm{SCN}^{-}$

c) The oxidized thiocyanate radicals form $(\mathrm{SCN})_{3}{ }^{-}$and perhaps polymerize to $(\mathrm{SCN})_{\mathrm{X}}$.

d) The $(\mathrm{SCN})_{3}{ }^{-}$or $(\mathrm{SCN})_{\mathrm{x}}$ at the interface acts as a catalyst for hole injection from the oxidized dye into the valence band of CuSCN.

Step $d$ is the key to the increased efficiency. Without this catalytic interaction, the hole injection appears to be too slow to compete with the recombination of the oxidized dye with an electron from $\mathrm{TiO}_{2}$.

In summary, we have shown, for the first time, that it is possible to construct a solid-state dye-sensitized device that absorbs significant fraction of the incident visible light and converts the absorbed photons to current with a reasonable efficiency. Although this device is far short of achieving a commercially viable efficiency our results show that further research is justified. It is also our belief that fundamental studies of dye sensitized hole injection into a variety of p-type transparent conductors (including conductive polymers and molecular hole conductors) might well pay dividends in knowledge that could be used to improve dye sensitized liquid junction cells as well as solid state cells. This work could easily be accomplished by depositing these materials onto the types of dye sensitized metal oxide substrates that we have characterized in our work with CuSCN.

\section{References}

1. B. O'Regan and D. T. Schwartz, accepted for publication in Chem. Mater. 1998

2. B. O'Regan and D. T. Schwartz, to be submitted to Advanced Materials.

3. B. O'Regan and D. T. Schwart, Chem. Mater. 7, 1349 (1995).

4. B. O'Regan and D. T. Schwartz, J. Appl. Phys 80,4749 (1996).

5. B. O'Regan and D. T. Schwartz, in Symposium on Nanostructured Materials in Electrochemistry; edited by G. J. Meters and P.C. Season (Electrochemical Society, Penning ton, NJ, 1995), p. 208. 


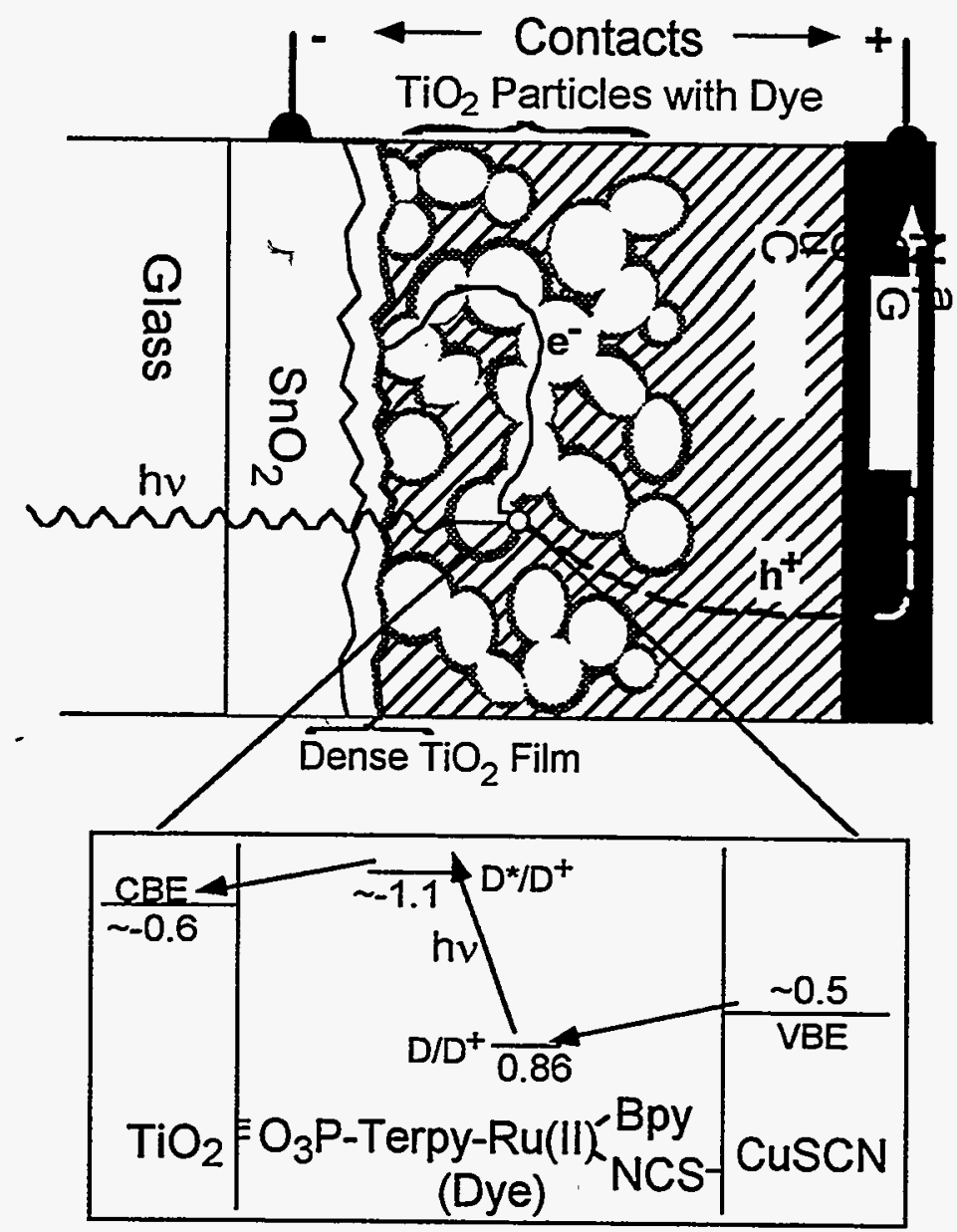

Figure 1. Upper: Physical schematic of the DSH. Layers, left to right: Commercial conductive $\mathrm{SnO}_{2}$ glass, dense $\mathrm{TiO}_{2}$ (50-100 $\mathrm{nm})$, porous $\mathrm{TiO}_{2}(1.5 \mu \mathrm{m})$, dye, $\mathrm{CuSCN}(3-5 \mu \mathrm{m})$, and pressed graphite powder $(0.3 \mathrm{~mm})$. The porous $\mathrm{TiO}_{2}$ layer has many more layers of particles than shown (particle size 10-40 nm). The dark areas around each $\mathrm{TiO}_{2}$ particle represent the monolayer of dye. Lower: Magnified view of the $\mathrm{TiO}_{2} /$ dye/CuSCN interface and an energy diagram. All potentials vs SCE. $\mathrm{D} / \mathrm{D}^{+}$is the oxidation potential of the dye in solution. CBE and VBE indicate the conduction and valence band edges. The $\mathrm{Ru}$ dye binds to the $\mathrm{TiO}_{2}$ via the phosphonate group. 


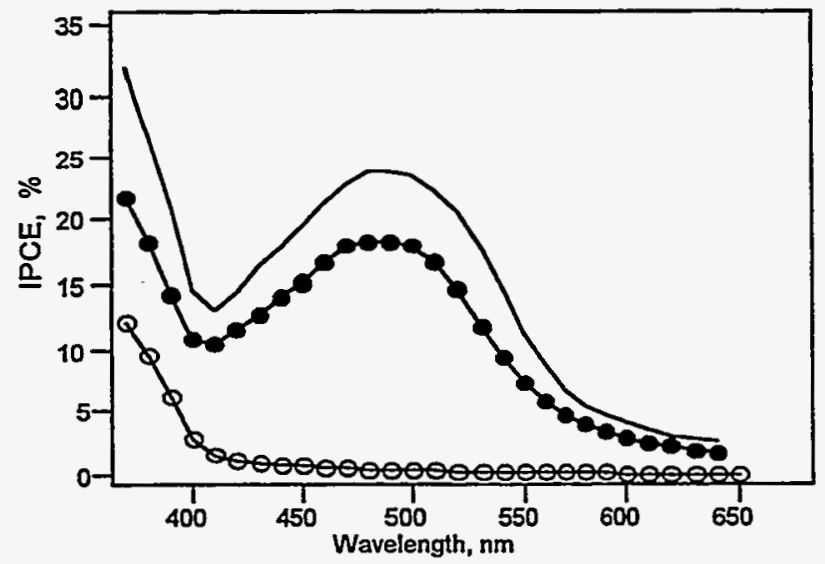

Figure 2. The photocurrent action spectrum of a porous-

$\mathrm{TiO}_{2} /$ Dye/CuSCN heterojunction after UV treatment $(\rightarrow \rightarrow-)$ compared to the action spectrum of the same heterojunction without dye, after the same UV treatment (-O-O-). Also shown is the action spectrum of an identical $\mathrm{TiO}_{2}$ film (with dye) in a photoelectrochemical cell (-).

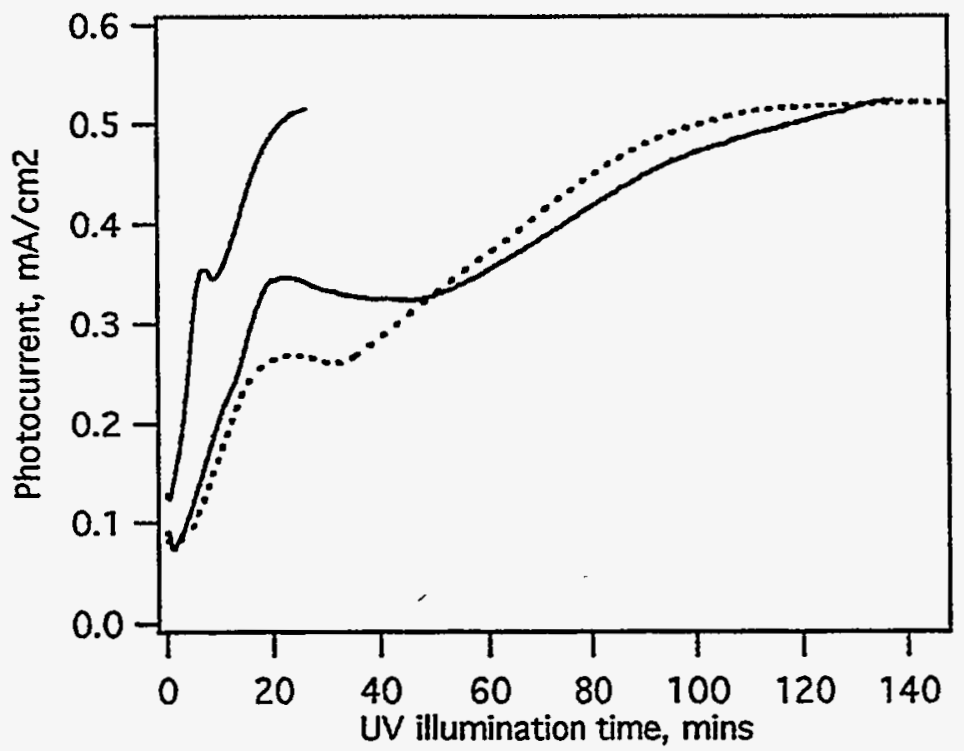

Figure 3. Photocurrent vs time for three porous $\mathrm{TiO}_{2} \mathrm{DSHs}$ illuminated with a Xenon arc lamp filtered through glass optics. 


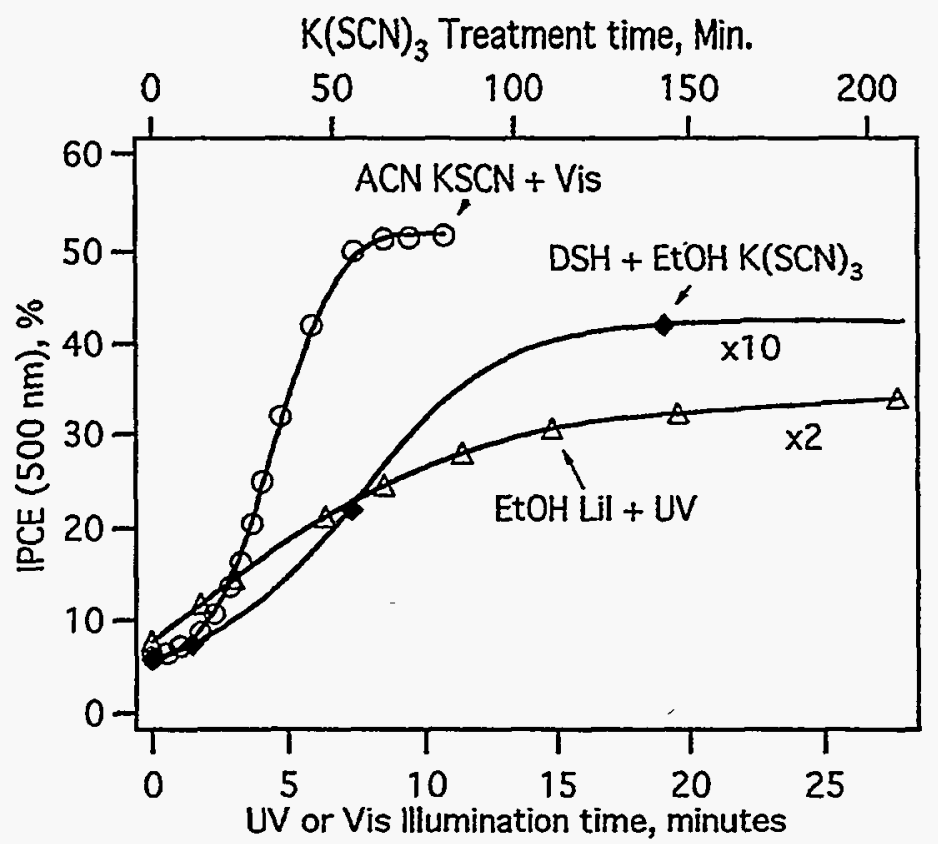

Figure 4. Dye sensitized IPCE vs illumination time for two dye sensitized $\mathrm{TiO}_{2}$ photoelectrochemical cells, and vs cumulative exposure to oxidized thiocyanate for $\mathrm{a} \mathrm{TiO}_{2} /$ dye/CuSCN DSH. 


\section{Historically Black Colleges and Universities Subcontracts}


Title:

Photovoltaic Applications in Developing Countries-Marketing Renewable Energy Devices

Organization: Office of Sponsored Research, Central State University, Wilberforce, Ohio

Contributors: C.W. Fuller, Principal Investigator

H. K. Evans, Student Research Associate

\section{Background}

The National Renewable Energy Laboratory's Historically Black Colleges and Universities (HBCU) Photovoltaic Associates Program provides undergraduate support for photovoltaic energy research and education at HBCU's. The aim is to attract qualified science, engineering and business students toward pursuing a career in these areas with emphasis in photovoltaic technology. Central State University currently has one student participating in a three year program entitled, "Photovoltaic Applications in Developing Countries". The following data includes the results of research conducted by the student rersearch associate during Year Two.

\section{Objectives}

The overall goal for Year Two was to investigate the blossoming renewable energy market that exists in the impoverished rural communities of West Africa. Also, the feasibility of a simple hybrid renewable energy system was investigated as a carry over from Year One.

\section{Approach}

\section{Year One}

During Year One, it was observed that some Aeromotor windmills operating deep within the interior of Senegal did not receive enough wind to operate during the dry season that normally lasts from September through May. This scenario is potentially disastrous since the villages in question often rely on the Aeromotor windmills as their main method of water procurement. To augment water supply during times of low wind, a hybrid system could be produced using PV panels as a back up power supply.

At CSU's wind energy test site, an amorphous silicon PV module (no data available for the module because it was government surplus) was mounted onto an already in place Windseeker II $250 \mathrm{~W}$ wind turbine. The wind energy test site combines a wind turbine with a Solarjack SDS series DC submersible pump for the purpose of simulating waterpumping conditions (Fig. 1). The wind turbine along with the PV panel keep the battery bank charged at an optimal level, approximately $12 \mathrm{~V}$. An anemometer was used to collect windspeed so that the amount of water produced per period of time could be contrasted with windspeed. The diagnostic tests were carried out to determine the rate of water production at low wind speeds as opposed to power production.

\section{Year Two}

Year Two activities examined factors affecting the market governing laws of supply and demand in relation to renewable energy device procurement in poor, rural communities abroad. Activities included: using the Internet as a primary information resource, visiting a PV module manufacturer and a PV 
module developer, gathering information from agencies concerned with the dissemination of renewable energy, and attending the annual ASES conference. By taking a holistic approach in the examination of the PV industry, from manufacturer to end user, the factors hindering the dissemination of PV to the impoverished areas of developing countries were defined.

\section{Results}

Year One

Year One data (Fig. 2)focuses on water pumping rates as a function of windspeed; the combined voltage output of the PV panel and wind turbine are not considered, as they are responsible for recharging the battery bank to an optimal level of $11 \mathrm{~V}$ to $13 \mathrm{~V}$. Water production for twenty minutes of pumping time is recorded. The data suggests that the maximum water production will peak at 14 to 15 gallons per 20 minutes of pumping time.

\section{Year Two}

Observations of the PV market in West Africa determined that the major obstacles to the wide scale dissemination of PV devices include a lack of capital and an unwillingness to break away from tradition.

Developing countries have the need for PV, however, many households lack the disposable income to benefit from PV technology. Local affordability of PV devices is a major concern. A few productive agricultural communities sell surplus crops to provide down payments on PV systems, however, many communities lack food security and must store all surplus to survive the dry season. Infusions of capital are therefore necessary to provide the villagers with the means to take advantage of PV power.

In many cases, funds are made available through local banks that work in conjunction with international relief agencies and other NGO's. These agencies provide the initial capital which finance the low interest, long term, revolving credit funds. Monthly payments on the loans are locally affordable, as they are approximately equal to a month's supply of batteries or fuel.

Typically, small PV installations in rural communities abroad are used for illumination purposes. Systems fabricated for illumination by Senegalese installers cost approximately $\$ 1000$ (US currency). A basic system consists of a $50 \mathrm{~W}$ panel, four $10 \mathrm{~W}$ neon lights, a regulator, a deep cycle battery, distilled water for topping off the electrolyte level and the related cable, wiring, and fuses. Systems fabricated for water pumping are more expensive as the depth of the water, the quality of equipment, and village location influence the system price.

A degree of technical know- how at the village level is required to avoid the non-functioning system scenario. This is accomplished through technology transfer and in-country training of village personnel.

Development of the PV market abroad is vitally important to the long term success of domestic PV manufacturers and the ecology of the village areas abroad. Currently, module manufacturers like Solarex export as much as $70 \%$ of their production output. The number of panels exported would be greatly diminished if funding strategies were not in place to subsidize the purchase of PV modules and systems. 
PV installations in the village areas simultaneously provide cheap, clean power and reduce environmental degradation. Previous surveys of the surrounding countryside of rural communities in West Africa conducted during Year One vividly demonstrated the rampant effects of deforestation. Deforestation continues unchecked because villagers use timber as cooking fuel and construction material and the foliage as animal feed. Small PV systems produce light, curtailing the combustion of wood and kerosene and their related health and environmental risks. Where timber is already scarce, a program similar to the one observed at Port Elizabeth Technikon (Port Elizabeth, South Africa) can be developed using local soil in combination with cement mixtures to augment the villages construction needs while keeping costs low.

\section{Summary}

The data from Year One shows that a simple hybrid renewable energy system can provide adequate volumes of water at low wind speeds. Further testing in West Africa would be needed in order to verify the hybrid system under Sub-Saharan conditions. (It is important to note that the depth of water in West Africa would be a limiting factor to water procurement). Research from Year Two greatly increased the student researcher's understanding and appreciation for the global economy and how it functions. The opportunity to work with local companies in the start-up phase helped the student researcher visualize what actions are necessary to be successful in the renewable energy business. Collaboration with relief agencies abroad afforded me a rare glimpse at work being done using renewable energy to combat the spread of diseases and to increase food security. In impoverished areas of the developing world, renewable energy is all but a panacea for environmental ills, high fuel costs, and limited fuel availability. Most renewable energies of interests to villages are prohibitively expensive and methods of making them locally affordable should be continually studied since renewable energy is one of the developing world's last hopes at achieving sustainability. The popularity of renewable energy technology will be the ultimate factor in determining local affordability for developing countries, and when supply finally outstrips demand, prices will fall.

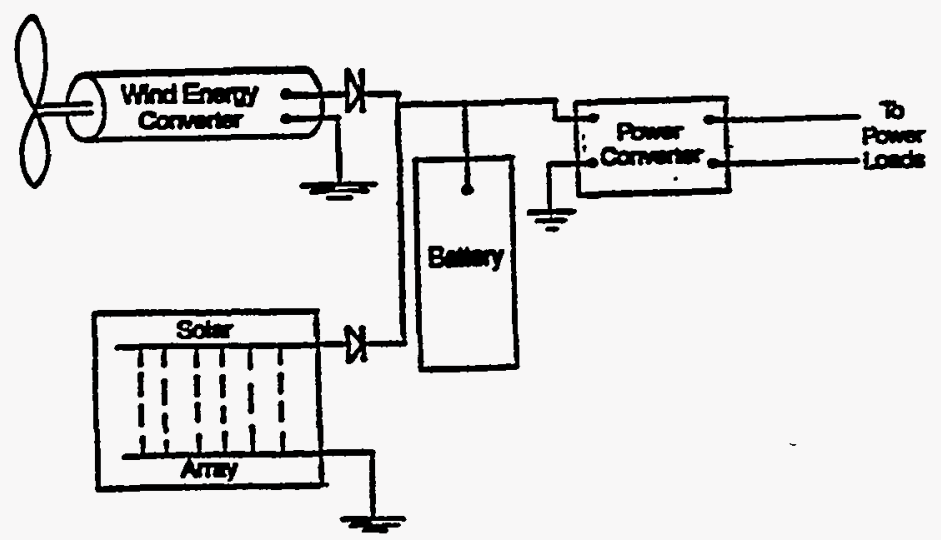

Figure 1. Schematic of hybrid system

\section{Pumping Rates}

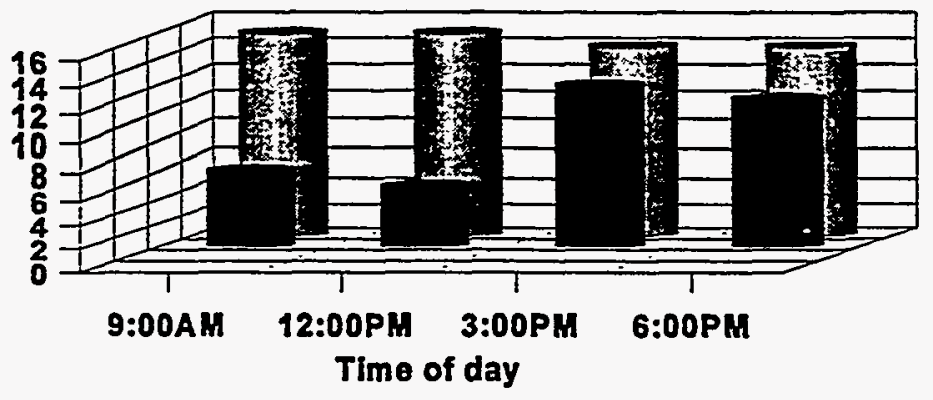
Gallons per $\mathbf{2 0}$ minutes
Wind speed
*Data from the week
of $10 / 6 / 97$

Figure 2. Plot of windspeed vs. water production 
1 
'Title: $\quad$ PV Application in Building Design

Orizanization: Department of Architecture

Hampton University

Contributors: $\quad$ Sallie A. Hood, Ronald Sakal, Norma Sellman

Faculty Investigators (No longer at Hampton University)

Santora McKinney and Toren Williams

Student Researchers

Our focus on the environmental design and architectural applications of photovoltaic (PV) technologies enabled our two senior 5 th year students to have their terminal design/thesis projects incorporate photovoltaic systems, with the total architectural program and final project.

The senior 5th year architecture students completed their thesis projects of exploring the possibilities of integrating appropriate sound solar design techniques into building design. Specifically each student explored the effects of integrating photovoltaic technology into building design with the expressed goals of:

- Showing that photovoltaics can improve building aesthetics and can integrate with the buildings material components.

- Showing the use of photovoltaic technology as a viable means of decreasing a building's energy consumption and can be used as a sustainable energy source for the 21 st century.

- A cost-effective means of improving a buildings energy efficiency rating.

The lower division students who interned at NREL during the past summer with NREL building researchers continue to serve as role models to the other students and helped influenced the student's architecture design projects by the use of the photovoltaic technology.

\section{PROJECT STATEMENT}

As a vehicle to further explore concepts in photovoltaics, I will design a new School of Marine Science to replace Hampton University's current Marine Science Laboratory. The purpose of this school is to educate students in the field of marine biology and environmental sciences. In addition to this, the school will provide a platform to promote public awareness and disseminate information regarding environmental issues concerning the Chesapeake Bay and its tributaries.

The components of the school include: a boat dock, a laboratory, an auditorium, a library, classrooms, administrative areas, circulation and other functional areas as required. Two unique components of this school will be the laboratory and the library. The laboratory will be designed as a micro-aquatic environment to provide students the opportunity to study and interact with living specimens. The library will be a fully on-line multimedia library, thereby eliminating certain structural limitation and reducing the need to allocate space for housing volumes of books.

The theme of the facility will be one that reflects the essence of the sea. By architecturally manipulating light, form, color, texture and sound the school will attempt to capture the ambiance of the sea. 
In the design of this museum my primary objective was to explore the possibilities of incorporating Photovoltaics as a sculptural element while providing enough energy to fully light the building. This approach mean that the south face had to be unobstructed to maximize the full potential of the suns, rays, in order for the PV to be most efficient. The structure of the building also must respond to the integration of PV. in a harmonious manner, creating a solution that does not conflict with the overall part of the design.

In the solution the issues that were concentrated on were: ground/sky interface; structure; orientation; response to existing context; and most important, the integration of PV The design was separated into three main areas; the auditorium; the galleries; and the lobby. The galleries were designed to respond to the street while also creating a defined space as one travels down Emancipation Dr.; which is the street directly facing the museum. Also the form was curvilinear to follow the path of the sun and create a natural ventilating system in which the atrium played an important role.

The designs maintain its original concept of creating a sculptural expression through the use of PV elements while providing enough energy to full light the building.

\section{The Amount of Watts/Day Needed}

Revised Thesis:

1. Galleries

2. Auditorium/Admin. 10925

3. Lobby/Gift shop 9100

\section{TOTAL}

1000 L (32925 SQ. Ft. $)=32925000 \mathrm{~L} / \mathrm{Sq} . \mathrm{Ft}$.

3292500OL/Sq.FT.(I W/220L)=149659.09W/Sq.Ft.

Average recommended lumens provided by Architectural Lighting Design by Gary R. Steffy.

\section{The Amount of Watts/Day Produced by PV}

Revised Thesis:

3300 Sq.Ft. of PV(1M3.28Ft.) $=943 \mathrm{M}$ of PV

(IM)(SE*-.)(Insolation) $=\mathrm{KWH} /$ Day

$943 \mathrm{M}(24)(3.983)=90129.6 \mathrm{KWH} /$ Day

$=90129600 \mathrm{WHs} /$ Day

Conclusion:

These calculations are designed for the winter months which proves there is enough energy produced by the amount of PV used to efficiently light the building during the course of the year. The surplus energy created can be used to operate other remaining systems that require electrical power. 


\section{BIBLIOGRAPHY}

AIA Foundation. Energy in Design Applications. The American Institute of Architects 1981

Barnett, Dianna. A Premier on Sustainable Building. Rocky Mountain Institute 1995

Callendar, John. Time-Saver Standards. McGraw-Hill Inc. 1966.

Cook, Gary. Photovoltaic Foundamentals. U.S. Department of Energy 1993.

Crosbie, Michael. Green Architecture. Rockport Publishers Inc. New York 1994

Davis, Douglas. The Museum Transformed. Abbeville Press Publishers New York 1990.

Montaner, Josep. New Museums. Princeton Architectural Press, New York 1990.

Steele, James. Museum Builders. Academy editions 1994

Steffy, Gary. Architectural Lighting Design. Van Nostrand Reinhold Co. New York 1990. 


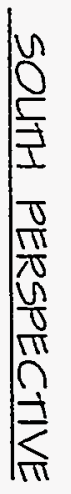

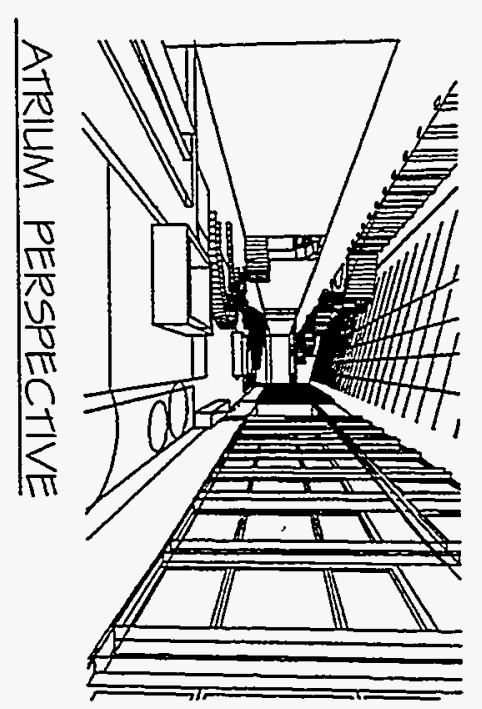

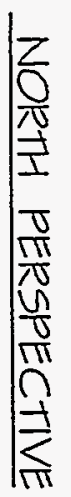

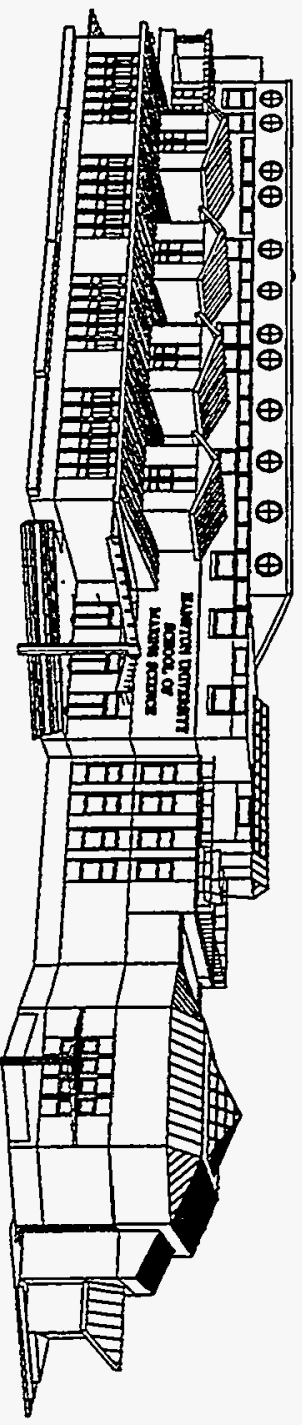

IHAMMIPTON UNIVIEIRSITYY SCHOOOL OIF MARINIE SCULNCE SANTORA MCIKINNNEY 

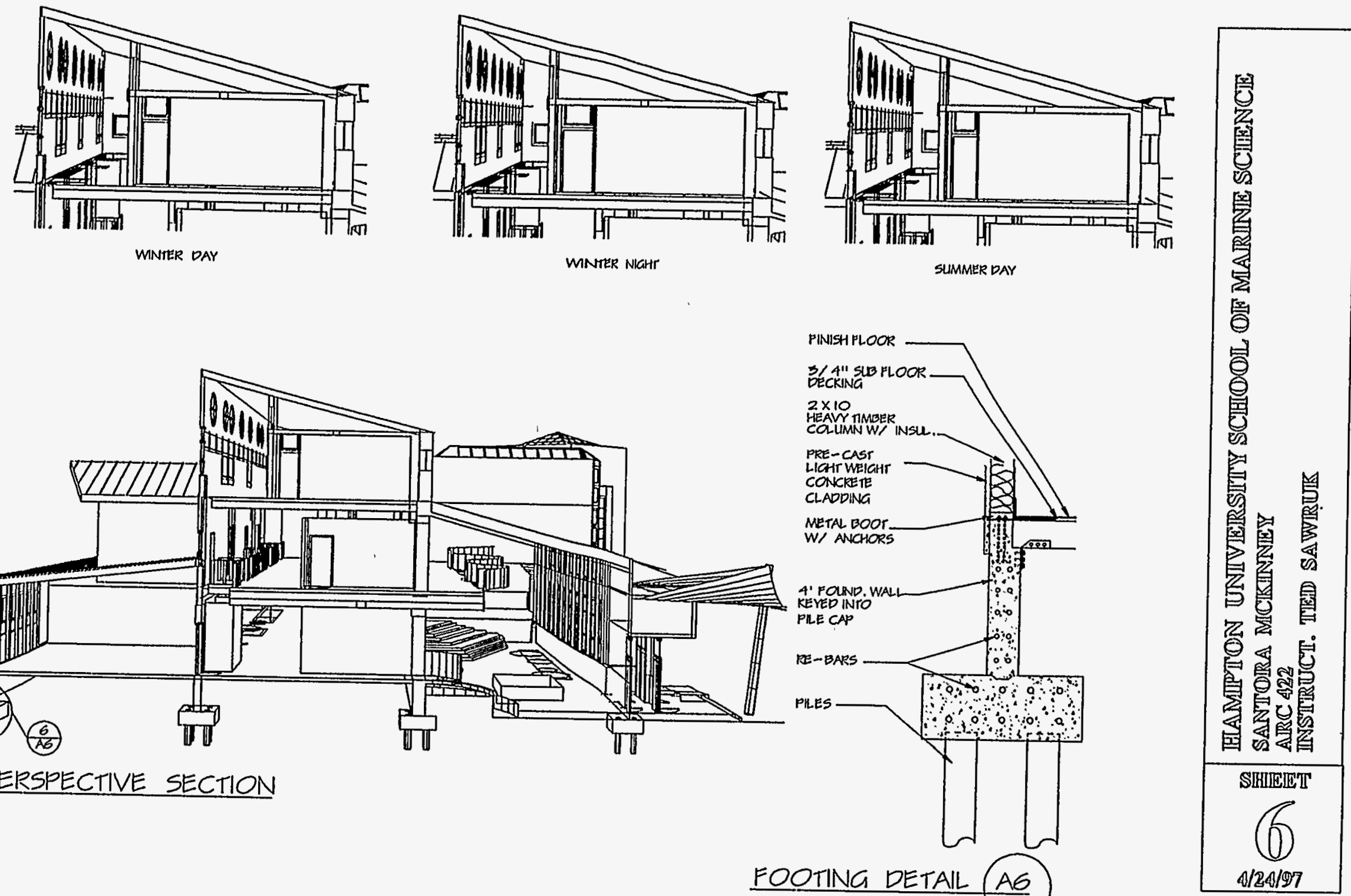

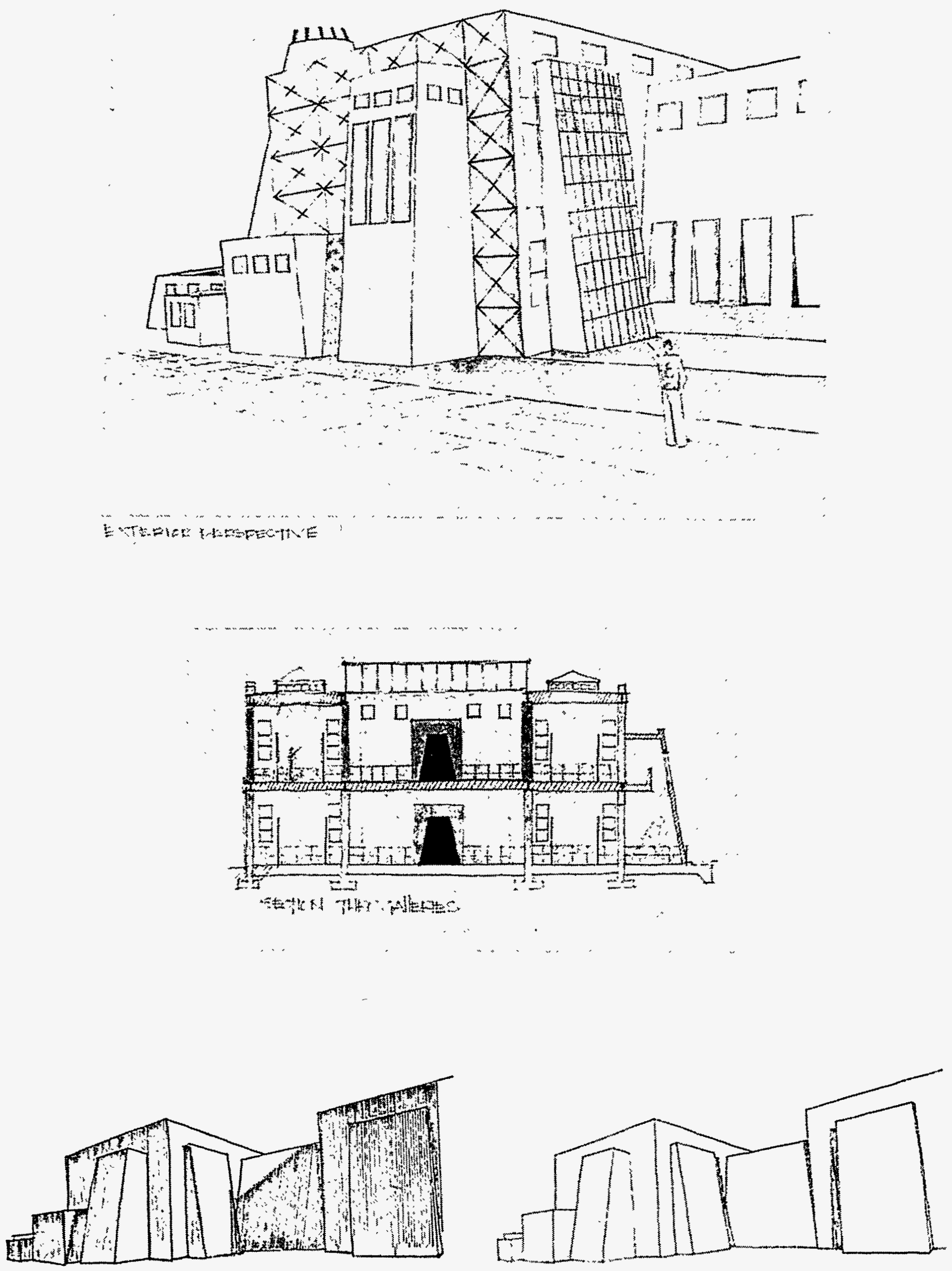
Title:

Organization: $\quad$ Mississippi Valley State University

Itta Bena, Mississippi

Contributors: $\quad$ B.S. Balam, Director; T.P. Pillai, Principal Investigator

Historically Black College and University

Photovoltaic (PV) Associates Program

\section{Objectives}

In the first phase of the project our major objectives were (1) to expose to and involve minority students to Photovoltaic (PV) energy as the energy of the future (2) to train them to acquire in-depth knowledge of the fundamental scientific principles, technology of manufacturing, and operation of PV cells and panels, to produce energy on a smaller scale to suit individual or special needs [1-3] and that on a large scale [4-5] to supply like a public utility system (3) to study the cost effectiveness of use of $\mathrm{PV}$ at present and in future.

In the second phase of the project our objectives are:

(1) hands on experience with PV panels and installing them to produce Direct Current (DC) and storing in storage devices

(2) to invert DC into Alternating Current $(\mathrm{AC})$ to operate household electric appliances

(3) to disseminate the knowledge of the future renewable and pollution free energy source to the general public through seminars and community outreach activities.

\section{Approach}

Three highly motivated and qualified science majors [student associates] were selected to work with the PV program at MVSU. These students were selected based on their interest and future career objectives. These individuals were required to take all fundamental science courses and then do a literature search to have an in-depth knowledge of PV fundamentals and technology. Individual students made their literature search from various sources - books, publications $[6,7,8]$ and Internet. Discussions have been carried out five hours, every week. Group discussions were conducted to strengthen the understanding and review the findings relevant to the Photovoltaics. PV panels were bought and set up. All three students have participated in intensively testing the PV panels. The panels were fixed to frames. The DC generated has been tested for stability of voltage. DC appliances connected to the panes run smoothly. At the present time more storage batteries are needed to store DC. We are in the process of additional electrical wiring. At the present time there are four PV panels[2] in operation. Four more PV panels[2] are in our order list so that more useful energy can be produced and large power can be produced. A study and tabulation of most PV materials were made for their effectiveness to stability, reliability and cost effectiveness and usefulness. From our studies it is our understanding that Silicon-based PV panels are highly reliable under most working conditions. Our task at the present time is to produce a larger AC generation from more PV panels and invert AC from DC. One of the students is working on portable PV panels 
and how he can demonstrate the use of PV energy to general public. Four seminars were conducted to enlighten students and general audience in different aspects of the future, renewable, and "Unlimited source and Pollution free Energy," and "Energy of the new Millennium" [9].

$\underline{\text { Results }}$

The supply of energy may appear somewhat balanced with the present level of demand. Nevertheless, the demand for more energy in the future is more than ever before. More energy cannot be produced without endangering the environment and risk taking. While energy conservation is one aspect of the solution, it is not going to fully solve the problem. The major aspect of solving the greater demand for the energy is to find low cost renewable and nonpolluting sources of energy. That is none other than PV energy. Hence, PV energy, if it can be produced at a lower cost per $\mathrm{kWh}$, is the solution to the energy demand of the twenty-first century.

While the energy related problems of the future are real, ordinary citizens cannot solve these problems unless they are given clear directions by the Scientists and Technologists, to alternate sources of energies, followed by manufacturers to meet the demands of the alternate sources of energy. Technology is always ahead of the comprehension of the ordinary citizens; this is especially true for poor and Black-American communities of the Mississippi Delta which Mississippi Valley State University (MVSU) primarily serves academically. Hence a special approach and effort must be made to expose the relatively new PV technology to minority citizens. Our next task is to disseminate knowledge of PV energy for everyday life.

\section{References}

1. Brazil 50-Watt system for home electricity for fluorescent lighting, Cacimbas, Ceara, Brazil.

2. Siemens, model Pro4, 4650 Adler Lane, Camerilo, CA.

3. Enersol's solar based rural electrification in Honduras, supported by SNL.

4. The 290 kilowatt PV hybrid system generator for a military operation in China Lake, CA.

5. 500 kilowatt solar plant, Pacific Gas \& Electric Co, Kerman, CA.

6. NREL Photovoltaic Program FY 1995 Annual Report. June 1996. NREL/TP -410-211017.

7. NREL/SNL Photovoltaics Program Review 14 th Conference Edited by Edwin Witt etal, 1996.

8. IEEE Twenty-five, Edited by Don Gwinner, NREL/TP-410-21091, May 1995.

9. Department of Energy, DOE /GO- 10095-241, May 1996. 
Title:

Photovoltaic Research Activity and Training of Students-1996-97

Organization: $\quad$ Electrical Engineering Department and Department of Physics, Southern University A\&M College, Baton Rouge, LA 70813.

Project Director: Rambabu Bobba, Department of Physics, rambabu@stark.phys.subr.edu

Contributors: $\quad$ P. K. Bhattacharya and one student, and Rambabu Bobba, Tameka Page, and Leady Shamona

\section{Introduction}

A great responsibility and liability is involved when students are trained to achieve certain goals in the workplace-or the undergraduate laboratory of a department. In our department, we are seeking to develop the scientific and technical know-how to carry out photovoltaics (PV) research and train highly qualified scientific and engineering personnel to meet the needs of the United States. Our project is focused on creating a state-of-the-art learning environment in a rapidly advancing technological world of photovoltaics for a new generation of engineering and science undergraduates.

\section{Approach}

The students are best motivated to perform research in an environment in which their team spirit and creative nature is allowed full play. Although, as advisors, we have not received any major funding, the students and professors of Southern University have worked to develop process technology, characterization, and quality testing of various types of materials used for solar cells and fuel cells. They have collaborated with NREL scientists to carry out these programs with the help of student trainees at NREL. NREL funded Southern University students work for approximately 40 hours/week, on programs at Colorado. They evaluated X-ray absorption near- edge structures (XANES) and measured and tested new PV modules and materials. They also wrote three to four funded proposals and we worked on two. Tameka Page and Leady Shamona published a paper entitled "A Study of Electrochemical and Structural Properties of Carbon Supported Electrodes, New Catalysts and Membranes" in the Journal of Solid State Ionics. One of the PV students attended the 1 th International Conference on Solid State Ionics with the assistance of a Takahasi Fellowship from ASSSI.

As a requirement of the program, the students also worked on establishing the laboratory for energy conversion and storage and devices such as fuel cells and batteries; characterized doped and undoped $\mathrm{CdTe}$ and $\mathrm{ZnTe}$ samples for solar cells; and designed and fabricated thin-film microsensors that can be used to measure corrosion rate in energy systems such as central heating, dishwashers, turbines, windmills, and car air conditioners.

To elucidate cathodic and anodic oxidation of methanol crossover events, i.e., $\mathrm{CO}$ poisoning in the direct oxidation of methanol fuel cells (DMFCs), cyclic voltammetry and X-ray Absorption FineStructure Spectroscopy (XANES) measurements were carried out on carbon-supported electrodes and some perfluorinated ionomeric membranes. The transition metal alloy catalysts were evaluated as low-cost alternatives to DMFCs and compared with generally accepted noble metal and alloy catalysts. The alloy catalyst $\mathrm{Pt}-\mathrm{Co} / \mathrm{C}$ was to be a better catalyst for methanol electro-oxidation 
compared to all other VIIlb transition metals. The observed current oscillations in $\mathrm{Pt}-\mathrm{Cu} / \mathrm{C}$ in lowand medium-temperature regions were due to the dual role played by the surface reaction between $\mathrm{MOH}$ and $\mathrm{PtCO}$. Pt/C/Nafion showed the highest resistance compared to Pt-Ru/C. For the first time, the sulfonate group in Nafion was characterized by sulfur K-XANES using a synchrotron radiation source (see Figures 1-3). Thermal treatment of the membranes resulted in changes in the spectra, demonstrating the good thermal stability of Nafion films and their use as a popular PEM in PEFCs. Membranes treated with phosphatic acid exhibited structural changes in the surface region, which could be due to an enhanced proton conductance.

We have explored the use of X-ray absorption spectroscopy for the characterization of CdTe and $\mathrm{ZnTe}$ thin-film samples. (Two sets of samples were provided by NREL scientists.) In recent years, photovoltaic cells equipped with CdTe absorber layers have achieved noteworthy efficiencies. However, they were dependent on the amount of oxygen present in the $\mathrm{He} / \mathrm{O} 2$ ambient in which they were grown (using the technique of close sublimation). Figures 4 and 5 show the X-ray absorption spectra of samples grown in a pure He atmosphere; one was grown in an ambient with an oxygen partial pressure of 8 torr at the $\mathrm{L} 111$ edge of $\mathrm{Cd}$ and $\mathrm{Te}$. We found that, at least at a partial pressure of 8 torr, microscopic environment and band structure of the investigated compound remain unchanged. Measurements need to be made in the fluorescence mode, which offers higher sensitivity to small changes; such studies are in progress. Another problem with using 'CdTe thin films in solar cells is providing ohmic contacts for these films. NREL recently developed $\mathrm{Cu}$-doped $\mathrm{ZnTe}$ films as a back contact layer. Two processes denoted as $A$ and $B$ employed to produce these films lead to completely different conductivities of the materials. Figures 6 and 7 show the XAS of the samples. One proposed explanation for this was the different tendency towards the forming of CuTe and related phases. At the $\mathrm{Zn}-\mathrm{K}$ edge, a very slight difference at the very limit of the detection may be visible. But at the Cu-K-edge an interesting effect evolves. One of the samples (UC272) shows but with reduced density of free states in the $\mathrm{Cu}-3 \mathrm{~d}$ band and a shift of the energy positions of the remaining edge structures towards lower energies. A possible explanation could be that, in UC272, copper is present in small clusters, whereas in UC300 single copper atoms or even small clusters may be embedded into the $\mathrm{ZnTe}$ environment. This would also explain why doping the material with $3 \%-10 \%$ copper shows no effect on the other edges, as if the copper atoms neighbor mostly with other copper atoms. Also, this study has provided a reasonable explanation for the low effectivity of dopants in these thin-film samples.

In the field of sensors, Bhattacharyas group studied thin films and explored two types of designs, linear and circular. The PV students were given new insight into the testing of thin-film sensors. Two feasible methods are electrodeposition and sputtering. The linear-film type of sensor has as many as seven electrodes; each can be made in different metallic thin films and multilayers. Typically the two outermost electrodes have a width of 3175.5 microns, and a length of 19040.9 microns; the pairs of inner electrodes have a width of 3175.5 microns and a length 15866.4 microns; and the innermost central electrode width is in the shape of a T with similar upper and lower widths of about 3175.5 microns (see Figure 8). In addition, a serpentine geometry resistive thermal device (RTD) electrode with three or four I/O pads is situated on the top. This gives an average temperature of the whole substrate over the entire length and is made of thin $\sim 2000 \AA$ gold film. On the left is a thermocouple situated inside the outermost electrode, which can be of a similar thickness and width as the RTD electrode; however, two different metal layers have been designed (one can be copper and the other 
constantan, or a chrome alumel type of thermocouple). On the inside of the right outermost electrode, we designed an electrode that could be used as a chloride-sensitive or a $\mathrm{pH}$ electrode. This will remove all possibilities of hybrid construction of the total atmospheric corrosion, although in most of them, relative humidity is a standard parameter that is measured extrinsically. The circular, film-type sensors are very condensed (see Figure 9), with a circular radius covering a larger area and uniform field region between each other.

There are seven possible different electrodes placed in three semicircles. In these semicircles, different elements can be evaporated or deposited for testing. Testing of these sensors for controls and storage-related problems is a great application. If manufactured using deep-level X-ray lithography (at the Center for Advanced Microstructures and Devices), these high-aspect ratio electrochemical sensors will be cheap and adaptable for smarter appliances. These features will be integrated into the technical aspects of atmospheric-corrosion detection. We also completed installation of LABVIEW software and peripherals to measure process parameters in semiconductor processing. A newly set-up printshop is working and diffusion and annealing furnaces for our process-control needs are functioning. Since we did not have a UHP water system, we installed Millipore -RO and $\mathrm{Q}$ systems in cascade to get highest resistivity water of $18 \mathrm{M} \Omega-\mathrm{cm}$; better wafer cleaning and surface preparation.

\section{Student Accomplishments}

We submitted abstracts of the work proposed above in the forthcoming American Society for Engineering Education/GSW Conference at New Orleans, Louisiana. And our training has borne fruit: Tameka Page has received employment offers from Rockwell International, Lockheed-Martin, Schlumberger, and Lucent Technologies. Danisha Williams received a job offer from Motorola's Cellular Infrastructure Group and accepted. Earlier, Thai Tam had been offered a job at Texas Instruments and is now working there. The 1997 summer trainees, Brandon Mayo and Denisha Hawkins, are presently helping to set up the Scanning Tunneling Microscope in the Department of Physics.

We gave opportunities to all PV interns to attend either national or international conferences during 1997. We also exhibited posters in NSBP, DOE-FETC, NREL-PV, and LAS workshops and conferences. Two students attended a thermal-analysis training workshop in Memphis, Tennessee. In addition, we have emphasized the importance of graduate education and provided GRE preparation books to these students.

\section{Major Reports Published in FY 1997 ,}

"A Study of Electrochemical and Structural Properties of Carbon Supported Electrodes, New Catalysts and Ion Conducting Membranes," Y. Xu, Tameka Page, Leady Shamona, et al. and B. Rambabu. Accepted for publication in the Journal of Solid State Ionics, and presented at the 11th International Conference on Solid State Ionics, November 16-22, 1997, Honolulu, Hawaii.

"Electrical Conductivity Studies of Silverselenophosphate Glassy System: Applications to Solid State Primary Batteries", N. Satyanarayana and B. Rambabu. Accepted for publication in the Journal of 
Solid State, Ionics, and presented at the 11th International Conference on Solid State Ionics, November 16-22, 1997, Honolulu, Hawaii.

"AC Conductivity and Dielectric Loss Studies of Silverselenovanadate Glassy System," M. Venkateswarlu and B. Rambabu. Presented at the 11th International Conference on Solid State Ionics, November 16-22, 1997, Honolulu, Hawaii.

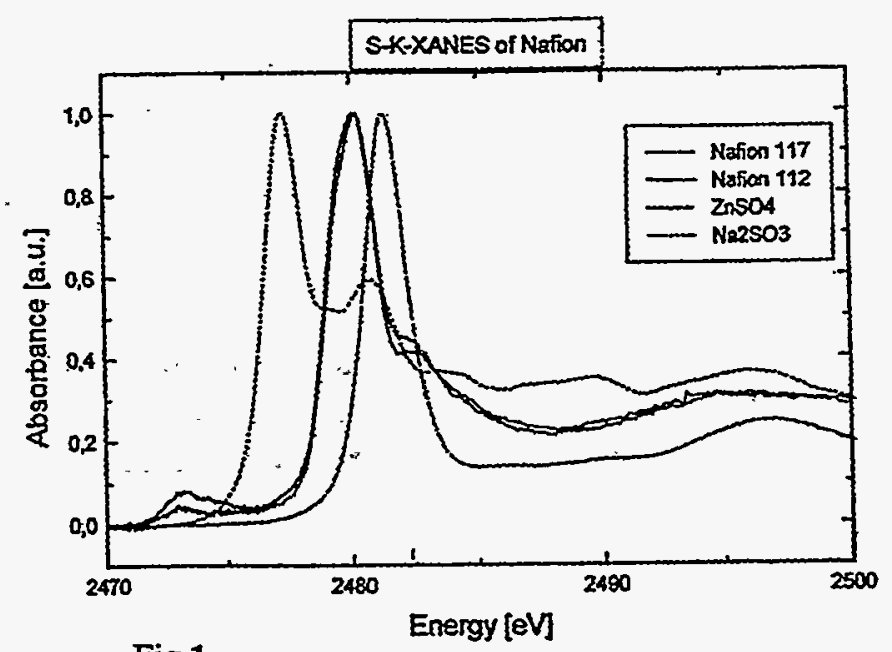

Fig.1

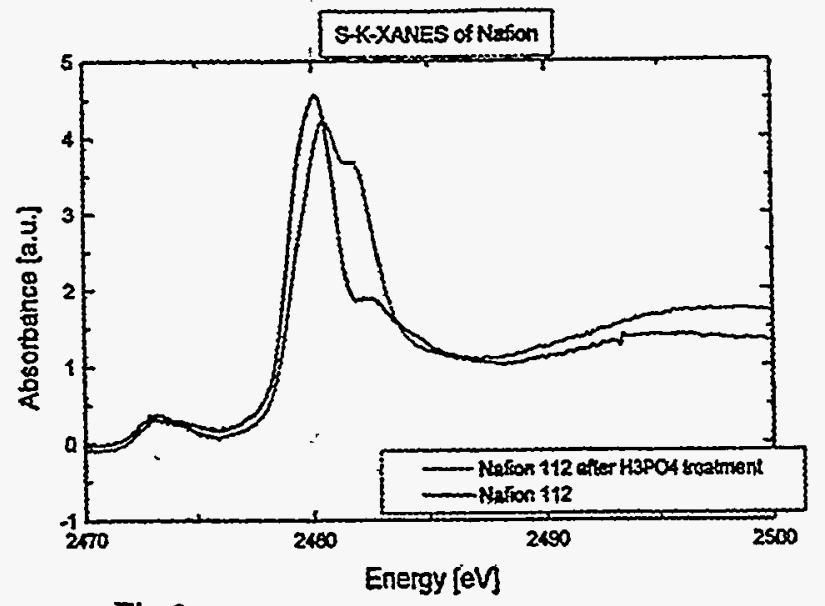

Fig.3

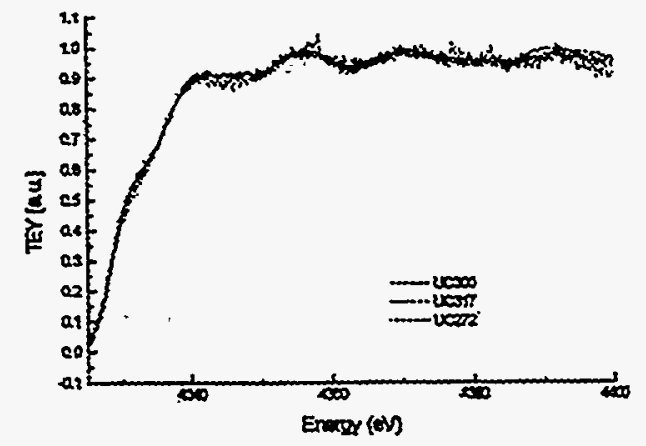

Fig.5: Te $\mathrm{L}_{\text {in }}$-Edge of ZnTE:Cu-films

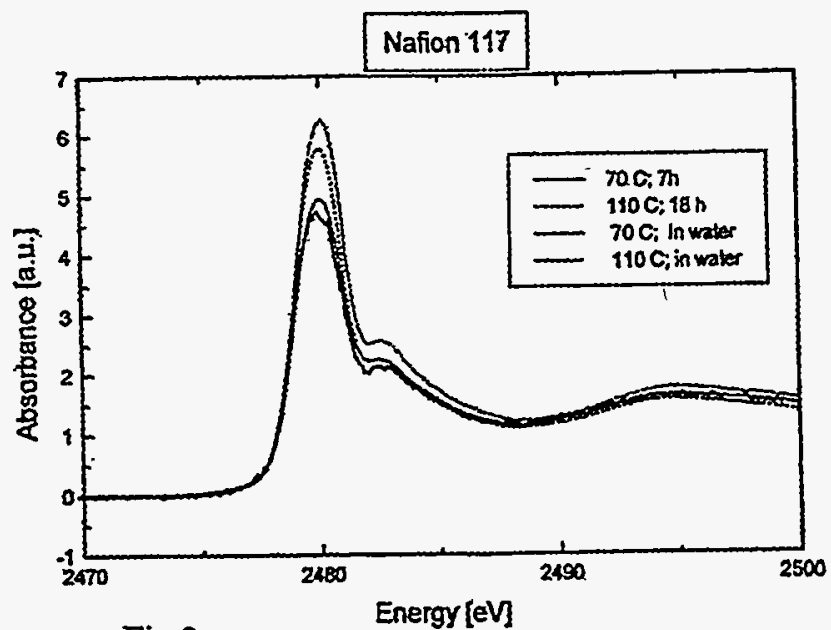

Fig.2

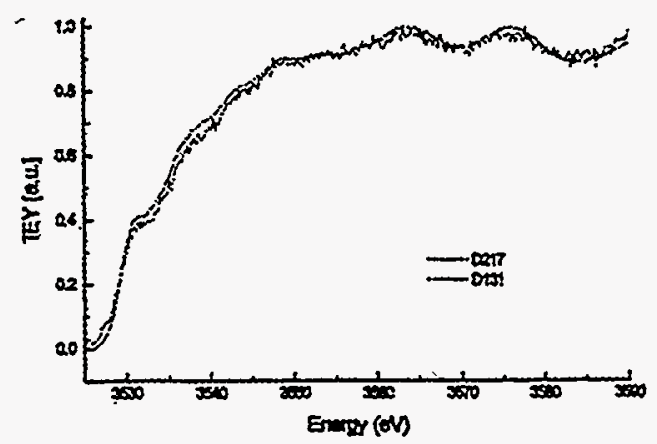

Fig.4: $\mathrm{Cd} \mathrm{l}_{\mathrm{m}}$-edge XANES of CdTe-Films grown in ambients with different amounts of oxygen

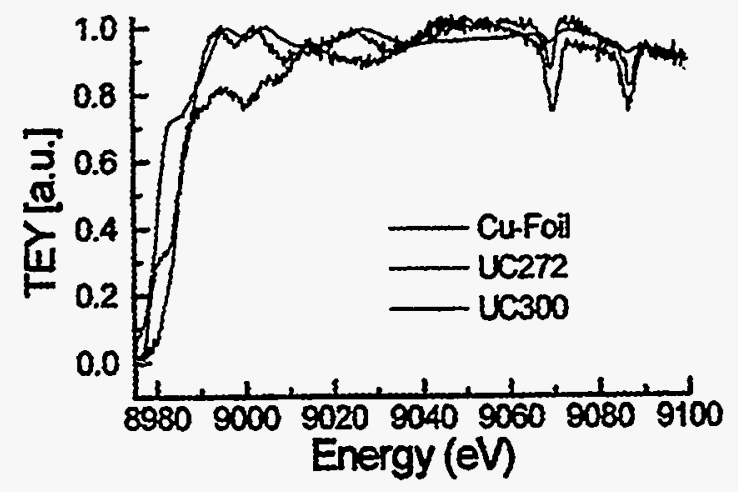

Fig.6: Cu K-edge of ZnTe:Cu-films 


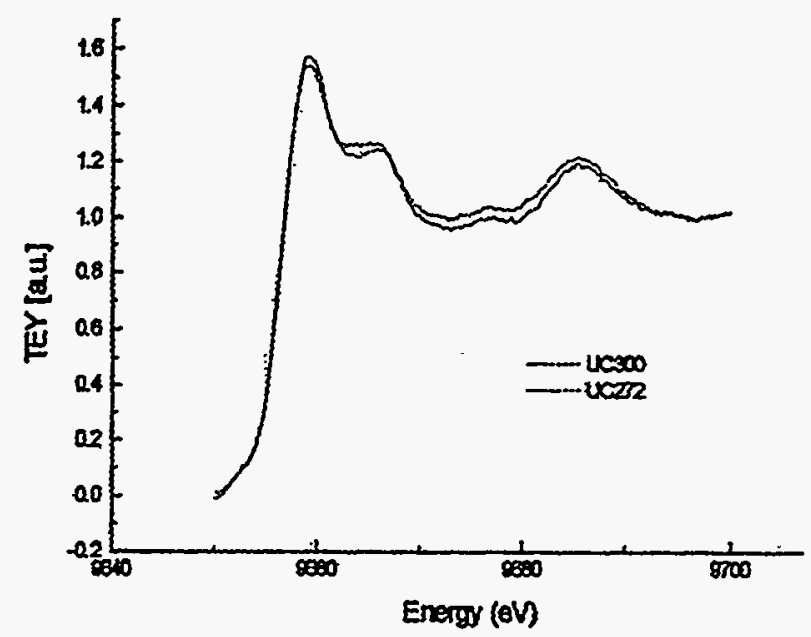

Fig.7: Zn L-edge of ZnTe:Cu-films

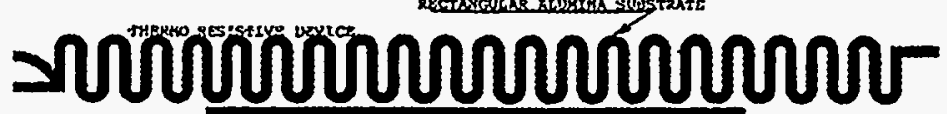

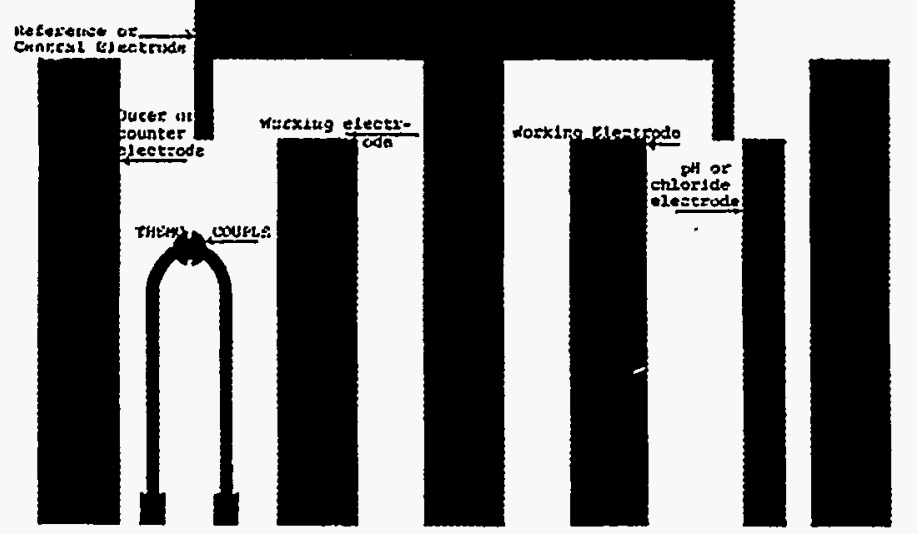

Fig.8

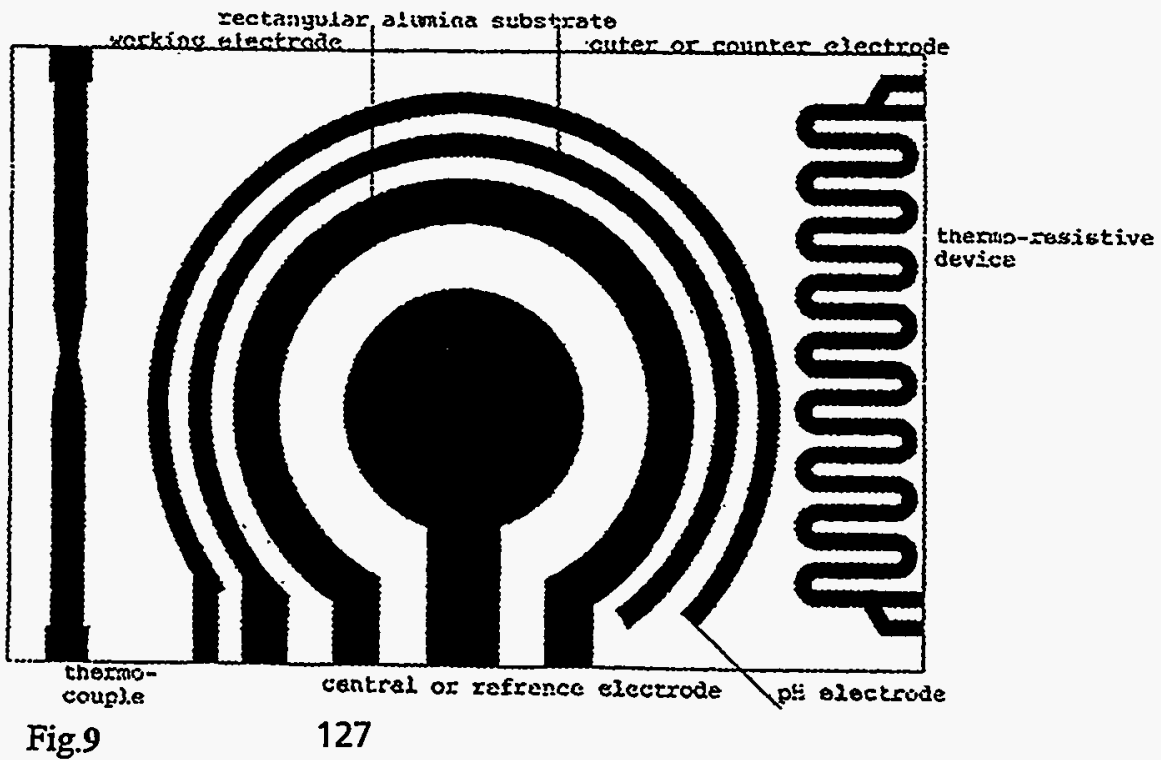


Title:

Organization:

Contributors:
Historically Black Colleges and Universities Photovoltaics Research Associate Program

Texas Southern University, Houston, Texas

School of Technology

J. Hill, Principal Investigator; O. LaFleur, J. Pichumani

\section{Introduction}

Texas Southern University (TSU) has demonstrated its commitment to the advancement of renewable energy technologies through the School of Technology Photovoltaic Research and Demonstration Laboratory. This lab has provided the structure for the School's participation in NREL's Historically Black Colleges and Universities (HBCU) PV Research Associates Program, as well as allowed the University to participate in research projects conducted by local institutions working in the field of photovoltaics. Education and research have been the primary focus of the School of Technology's involvement in photovoltaics and other renewable energy technologies at TSU.

\section{NREL HBCU PV Research Associates Program} Photovoltaic Industry Survey: Employment Outlook and Educational and Skills Needs Assessment

TSU's primary project with NREL under their HBCU PV Research Associates Program is to determine the photovoltaic employment outlook, while assessing the education and skills needed within the PV industry. This information will ultimately be used to provide guidance to universities to shape their training programs based on the needs of the PV industry. Surveys were distributed to 150 organizations within the PV technologies labor market both domestically and internationally including PV development, basic science, manufacturing, and final consumer products. Response has been positive to date. PV industry institutions were identified from various sources including the Solar Energy Industry Association (SEIA), related periodicals, and contacts with local, regional and international PV groups. Completed surveys are being returned on an on-going basis, and the responses are recorded and tabulated immediately.

Once a fair representation of the surveys has been returned, the resulting data will be analyzed and statistical conclusions will be drawn. The findings will be reported in its final format after review from appropriate peers. TSU is confident that the information gathered in this study will help shape college curricula to encourage students to pursue education and careers in photovoltaics. 


\section{PV Research and Demonstration Laboratory}

The PV Lab at TSU was installed in January 1997 as part of a project which was jointly funded by the Electric Power Research Institute (EPRI) and Houston Lighting and Power (HL\&P). The goal of the project is to demonstrate the technical potential of using commercially available photovoltaic equipment to generate electric power for off-the-grid applications in the small commercial sector.

The PV system itself consists of two 2-kilowatt arrays with battery storage for both AC and DC applications. The end-use equipment for the $120 \mathrm{~V}$ ac system includes 12 -volt incandescent lighting, small radio, some small kitchen appliances (coffee maker and blender) and will soon include a clothes washer and dryer. The end-use equipment for the $24 \mathrm{~V}$ dc system currently includes a vaccine refrigerator (with the heat pump whose performance is being tested for NASA). A 350 Watt wind turbine was installed adjacent to the PV array in October 1997 with the assistance of wind energy expertise from South Africa as part of a staff exchange program. The lab is in the process of designing and testing a data acquisition system to monitor the performance of the PV system and the wind turbine, and is hopeful to establish a meteorological database including solar insolation, temperature, wind speed, and wind direction for the downtown Houston area.

The PV lab and wind turbine have proven to be excellent educational tools for both high school and college students. Several TSU classes have held lectures in the lab describing the PV system and other aspects of photovoltaics. Students from the Technology department as well as computer science, physics and chemistry departments have expressed interest in or have already begun working in the lab.

\section{High School Educational Outreach}

The TSU School of Technology has held educational programs for two consecutive summers (1996 and 1997) called the Renewable Energy and Environmental Protection (REEP) Academy. By seeding financial and other support from the local utility, state energy office and national laboratories, the program accomplishes its objectives to increase knowledge of and promote interest in renewable energy and environmental issues for inner city high school students who will soon be entering college. The focus of the program is on current technologies and issues involved in renewable energy.

High school students from across the region are selected to participate in a series of seminars conducted by experts from across the country. In addition to classroom activities, the students go on field trips visiting local organizations involved in related subjects. In addition to the local field trips, students were divided into groups and traveled to the following locations: National Renewable Energy Laboratory (NREL) to view their involvement with photovoltaics, wind energy conversion, solar thermal and alternate fuels development; the Alternate Energy Institute at West Texas A\&M to visit their wind farms, and; Port Elizabeth, South Africa to observe their Rural School 
Electrification Program involving small-scale photovoltaic systems providing classroom lights.

The program was organized and facilitated by TSU staff and faculty. Six college students functioned as counselors for the REEP students. Three of these students previously had summer internships through NREL as part of the HBCU program in which they were able to spend summers in Senegal, Ghana and South Africa. These students shared their experiences with the REEP students in studying renewable energy applications in Africa. This exchange gave REEP students an excellent perspective of opportunities that may be available to them in studying renewable energies. Additionally, the NREL HBCU students' interpretations of renewable energy applications provided the REEP students with an alternate outlook to the scheduled lectures.

Classroom and laboratory activities included constructing solar cars; building solar cookers; a mock United Nations conference debating the ivory trade in Africa. In addition to these activities, TSU's PV Research and Demonstration Lab was available for the students to receive hands-on experience in testing the performance and output of PV panels. Students assisted the TSU staff with activities such as tilting the angle of the panels to receive maximum sunlight for the positioning of the summer sun. Low-wattage panels were available to the students to connect small devices using tool kits provided by the Academy. The TSU environmental labs were also used during the Academy to allow the students to test soil samples for trace elements and minerals and to conduct air filter experiments to test the pollen count. These activities in conjunction with the seminars conducted by experts were successful in improving the general knowledge of the students.

The students were administered a pre-test to assess the students' level of knowledge prior to exposure to the various discussions and activities involving renewable energy and environmental protection. The purpose of the pre-test was to establish a baseline for the group's background on energy concepts and attitude toward environmental protection. These results were compiled but not shared with the students. At the end of the program, the students were again administered a 30-question test which sought to measure the learning or achievement that had occurred during the four weeks of instruction, activities and field trips. Of the 67 students participating in the program, 40 test results from the 1997 Academy were analyzed and tabulated as shown in Table 1.

Overall, the students were given a comprehensive outlook of succeeding in college and in career for both technical (specifically science and engineering related) and non-technical interests. At the same time, the local groups (community, industry and state and local governments) were able to benefit by explaining the significance of their particular field as it relates locally, nationally and globally and the importance of giving back to the community. 


\begin{tabular}{|c|c|c|c|c|c|}
\hline 80 & on & re & $\mathrm{H}_{0.8}$ & 80.6 & \\
\hline Tes: & 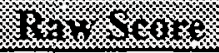 & $\%$ & 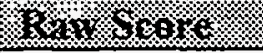 & $4 \%$ & Grans \\
\hline 118.: & 19 & $63 \%$ & 25 & $77 \%$ & $23 \%$ \\
\hline 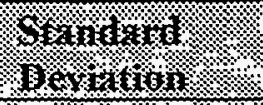 & 3.25 & & 3.50 & & \\
\hline Mullorome & 24 & $80 \%$ & 30 & $100 \%$ & \\
\hline
\end{tabular}

Table 1. Results of Pre- and Post-Test Scores for the REEP Academy 1997

\section{International Exchange Program}

TSU's School of Technology has established relationships with universities in South Africa who participate in similar photovoltaics research programs. Port Elizabeth (P.E.) Technikon staff in Port Elizabeth, South Africa assisted the School in designing and installing its PV Research and Demonstration Lab in January 1997. The Technikon also hosted the REEP group (15 REEP students, 5 college counselors, and 5 staff members) by explaining their involvement in South Africa's Rural School Electrification Program and allowing the REEP group to tour the schools receiving electricity from PV systems. In return, TSU will be hosting two South African college students during the Spring Semester of 1998. These students will join the TSU PV lab team consisting of TSU faculty, staff and students (including the NREL HBCU PV Research Associate) to participate in small-scale PV installation projects across campus and other lab activities. TSU and P.E. Technikon are confident that this collaboration will be beneficial to all participating and promote greater interest in photovoltaics for other students.

\section{Summary}

TSU's involvement in NREL's PV program has prompted further activities in photovoltaics which has received much support from local utilities, state energy and environmental groups, industry and other universities internationally. TSU's School of Technology looks forward to strengthening its PV program by continuing and expanding its collaborations with renewable energy institutions. 
3.0 PV Electronic Materials and Devices Project 



\title{
3.0 PV ELECTRONIC MATERIALS AND DEVICES PROJECT-Introduction
}

\author{
John P. Benner
}

\section{Objectives}

The Electronic Materials and Devices (EM\&D) Project will create a portfolio of photovoltaic (PV) electric power technologies and the relevant experience base to provide alternatives the world will need to flourish. The project carries out research and development at the Solar Energy Research Facility at NREL while maintaining close contact with research and production partners nationally to ensure fast and effective technology transfer.

\section{Technical Approach}

Primary tasks within the project include:

- Amorphous Silicon Research-better understanding of a-Si materials, devices, and deposition processes and further development of a-Si technology through improvements in each of these areas, with a key result being improvements in the stabilized efficiency of amorphous-silicon-based PV cells.

- Copper Indium Diselenide and its Alloys-address and resolve technical issues related to performance and reliability, process development, and manufacturability of CIS-based solar cells and submodules.

- Cadmium Telluride-develop reproducible processes for high-efficiency $\mathrm{CdS} / \mathrm{CdTe}$ devices and explore alternative processes which lend themselves to improved device performance, improved device understanding, improved reproducibility, lower cost and/or improved manufacturability.

- High-Efficiency III-V Alloys-establish III-Vs as a competitive terrestrial PV technology by developing innovative new technologies for lower cost, higher efficiency technologies and advancing related science and engineering.

- New Ideas in Photovoltaics-explore the properties and processing of nanoparticle precursors in inks for preparation of semiconductors, conducting oxides, and solar cell contacts.

- Device Fabrication and Clean Room-develop and maintain state-of-the-art semiconductor processing techniques related to PV solar cell development and effectively integrate photolithographic processing, vacuum metallization, and thermal processing.

In basic science, some of our contributions include examinations of cadmium diffusion, doping, and formation of homojunctions in copper indium diselenide, development of a model for Staebler-Wronski metastability that accounts for nearly all experimental results, and probing the 
alloying of $\mathrm{CdS}$ with $\mathrm{CdTe}$ with high spatial resolution photoluminescence and application of more conventional probes with free-standing films separated from the substrate.

We are developing new processes and materials that offer advantages in manufacturing while maintaining high performance of the resulting solar cells. Some of these described in the following task summaries include:

- Dry processes to replace chemical bath deposition of $\mathrm{CdS}$ or $\mathrm{CdCl}_{2}$ treatment of $\mathrm{CdTe}$

- Improved conducting oxides

- Electro-deposition of CIS-yielding cells with efficiencies of $14.1 \%$

- Atmospheric pressure and in-air processing technologies

- Hot-wire deposition of silicon films.

Through the National Teams and direct interactions, project scientists work directly with industry to assist in solving development and production problems. In several instances where companies are commercializing technologies developed at NREL, these interactions involve extensive travel for on-site collaborations. 


\section{PV Electronic Materials and Devices Project In-House Research}



Title: Development of Polycrystalline $\mathrm{Cu}(\mathrm{In}, \mathrm{Ga}) \mathrm{Se}_{2}$ Thin Films and Devices

Organization: $\quad$ National Center for Photovoltaics (NCPV) National Renewable Energy Laboratory, Golden, CO

Contributors:

Full Time

R. Noufi, Principal Scientist and Team Leader

K. Ramanathan, Senior Scientist

M. Contreras, Senior Scientist

F. Hasoon, Senior Scientist

J. Dolan, Master Technician

J. Keane, Master Technician

H. Wiesner, Graduate Student

\section{Objective:}

Part Time

S. Asher, Senior Scientist

R. Matson, Senior Scientist

D. Niles, Senior Scientist .

R. Bhattacharya, Senior Scientist

A. Franz, Assoc. Staff Scientist

A. Mason, Master Technician

J. Alleman, Master Technician

J. Webb, Senior Scientist

We seek to advance the development of CIS-based PV toward commercialization.

\section{Technical Approach:}

We addressed the following technical problems:

- Demonstrate progress in developing CIGS absorber processing for potential manufacturing. The processes we addressed with varying degrees of emphasis were electrodeposition (ED), sputtering, and sublimation.

- Alternative junction formation; i.e., the development of a transparent conducting oxide (TCO)/CIGS junction as an alternative to the use of a CdS buffer.

- The impact of the substrate/back contact on the CIGS material properties and hence the device performance.

- Partnership with industry to transfer the CIS technology and address technical issues of importance to the industry.

\section{Results:}

Device Development

Our best device efficiencies to date for different CIGS processing and alternate device structures are listed in Table 1.

Table 1. Highest Efficiencies for Different CIGS Processing and Alternate Device Structures

\begin{tabular}{|l|l|l|l|l|l|}
\hline $\begin{array}{l}\text { Process/Device } \\
\text { Structure }\end{array}$ & $\begin{array}{l}\mathbf{V}_{\text {oc }} \\
(\mathbf{m V})\end{array}$ & $\begin{array}{l}\mathbf{J}_{\text {sc }} \\
\left(\mathbf{m A} / \mathbf{c m}^{2}\right)\end{array}$ & $\begin{array}{l}\mathbf{F F} \\
(\%)\end{array}$ & $\begin{array}{l}\eta \\
(\%)\end{array}$ & Comments \\
\hline Evaporation & 674 & 34.0 & 77.2 & 17.7 & world record \\
\hline Rapid evaporation & 623 & 32.9 & 75.0 & 15.3 & $20 \mathrm{~min}$. vs. 45 $\mathrm{min}$. \\
\hline Electrodeposition & 656 & 29.0 & 74.1 & 14.1 & \\
\hline
\end{tabular}




\begin{tabular}{|l|l|l|l|l|l|}
\hline $\begin{array}{l}\text { Process/Device } \\
\text { Structure }\end{array}$ & $\begin{array}{l}\mathbf{V}_{\text {oc }} \\
(\mathbf{m V})\end{array}$ & $\begin{array}{l}\mathbf{J}_{\text {sc }} \\
\left(\mathbf{m A} / \mathbf{c m}^{2}\right)\end{array}$ & $\begin{array}{l}\mathbf{F F} \\
(\%)\end{array}$ & $\begin{array}{l}\eta \\
(\%)\end{array}$ & Comments \\
\hline Sputtering & 388 & 31.54 & 66.5 & 8.1 & compound targets \\
\hline Sublimation & 590 & 32.0 & 72.0 & 13.7 & simulated in a Bell jar \\
\hline Alternate junction & 527 & 36.0 & 71.0 & 13.5 & $\begin{array}{l}\text { ZnO/CIGS direct } \\
\text { junction }\end{array}$ \\
\hline Stainless-steel substrate & 673 & 30.0 & 77.3 & 15.6 & \\
\hline
\end{tabular}

\section{Alternative Process Development}

Electrodeposition. Co-deposition of Cu-In-Ga-Se (CIGS) by the electrodeposition process was performed from a bath containing $\mathrm{CuCl}_{2}, \mathrm{InCl}_{3}, \mathrm{H}_{2} \mathrm{SeO}_{3}, \mathrm{GaCl}_{3}$, and $\mathrm{LiCl}$ dissolved in deionized water. We electrodeposited precursor CIGS films by applying both direct-current (DC) voltage and an alternating-current (AC) voltage at high frequency (20 kilohertz [kHz]). As-deposited precursors are $\mathrm{Cu}$-rich films.

We added additional In, $\mathrm{Ga}$, and Se to ED films by physical vapor deposition (PVD) to adjust the final composition to CuIn $n_{1-x} \mathrm{Ga}_{x} \mathrm{Se}_{2}$ and then allowed it to crystallize at $550^{\circ} \mathrm{C}$ for 5 minutes. The substrate (precursor film) temperature during the PVD step was also $550^{\circ} \mathrm{C}$. We also selenized the films by exposing them to selenium vapor during the cooldown time $\left(-20^{\circ} \mathrm{C} / \mathrm{min}\right)$. In addition, we optimized the electrodeposition process to minimize or eliminate the PVD step.

We checked the compositional uniformity of the ED-precursor films by analyzing a $100-\mathrm{cm}^{2}$ substrate by electron probe micro-analysis (EPMA) and Inductively Coupled Plasma (ICP). The results show the uniformity is within the error for the two techniques.

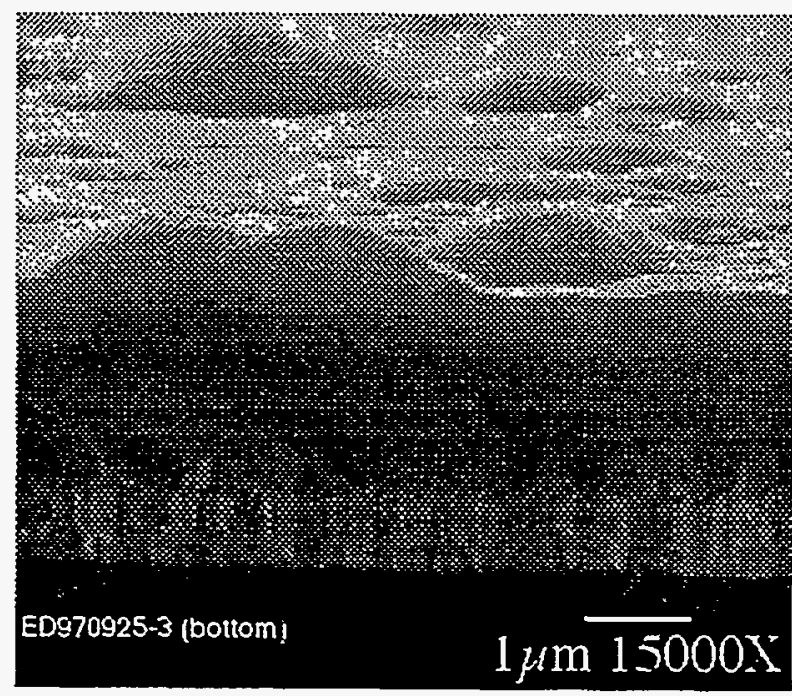

Fig. 1. SEM photograph of a representative ED precursor film

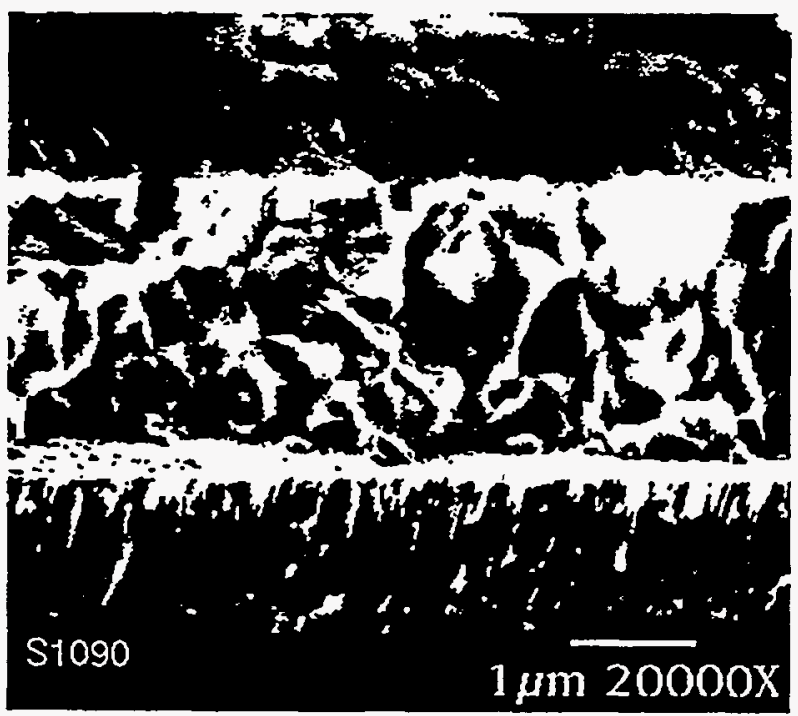

Fig. 2. SEM photograph of a processed film (from ED precursor) 
Figures 1 and 2 are scanning electron microscope (SEM) photographs of the representative CuIn-Ga-Se precursor and processed film (absorber layer), respectively. As the micrograph shows, the morphology of the as-deposited precursor film is densely packed and uniform.

Figures 3 and 4 show the $I-V$ and quantum efficiency (QE) curves prepared from ED precursors. The final configuration for all devices is $\mathrm{MgF}_{2} / \mathrm{ZnO} / \mathrm{CdS} / \mathrm{CuIn}_{1 \times x} \mathrm{Ga}_{x} \mathrm{Se}_{2} / \mathrm{Mo}$. The official efficiency measured at NREL for a device prepared from electrodeposited precursors is $14.1 \%$ (see Table 1 for I-V parameters).

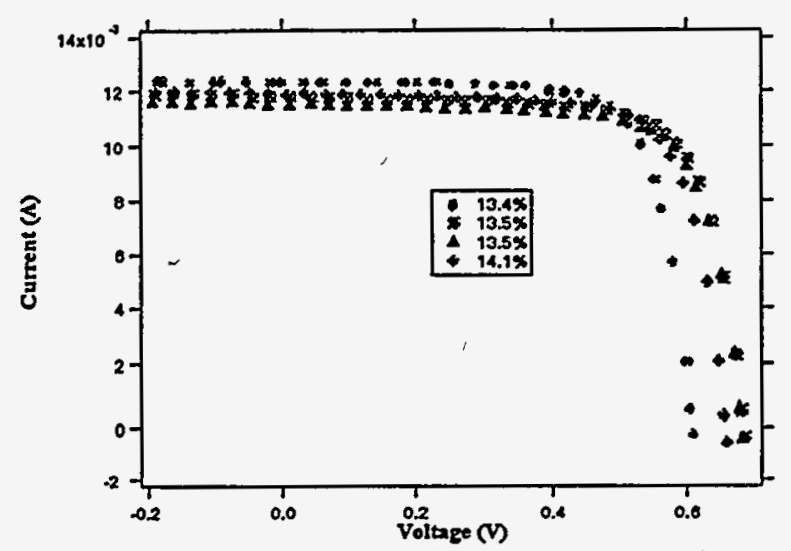

Fig. 3. I-V curves for devices prepared from electrodeposited precursor

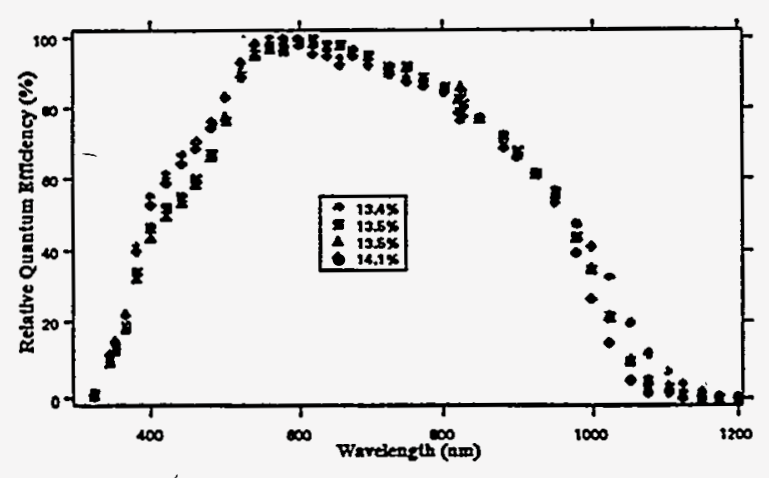

Fig. 4. QE curves for devices prepared from electrodeposited precursor

Sputtering from Compound Targets. This effort focused on developing sputtered precursors from compound (binary, ternary, and quaternary) targets. Such precursors are sputtered at low substrate temperatures $\left(<200^{\circ} \mathrm{C}\right)$ and are later recrystallized by selenization at high substrate temperatures $\left(-550^{\circ} \mathrm{C}\right)$. This is a highly effective and low-cost approach to delivering precursor materials. It presents excellent compositional uniformity and the potential for large-scale processing. Best results to date have been obtained from precursor films sputtered from a mixedphase target $\left(\mathrm{CIGS}+\mathrm{Cu}_{2} \mathrm{Se}\right)$. The addition of extra $\mathrm{Cu}_{2} \mathrm{Se}$ to the CIGS target increases its conductivity and allows the use of DC sputtering, which yields higher sputtering rates (as compared to radio frequency $[R F]$ sputtering). The resulting precursor film is polycrystalline with a fine-grain morphology and $\mathrm{Cu}$-rich composition, made up mostly of CIGS and $\mathrm{Cu}_{2-\mathrm{x}} \mathrm{Se}$ phases (according to X-ray diffaction [XRD] data analysis). After the selenization step in the treatment of such precursors, we observed that $\mathrm{Ga}$-containing materials incorporate $\mathrm{Ga}$ in a nonuniform fashion across the thickness of the resulting absorber film. This has been observed by $\mathrm{X}$-ray and depth-profiling techniques. Even though some recrystallization occurs after the selenization, the final morphology is still not optimum (grain size is much smaller than the thickness of the film) and a "double-layered" structure results.

The best devices made to date using the sputtering of precursors from compound targets show total-area efficiencies around $8 \%$. Device performance is limited because of poor fill factors (FF) and low open-circuit voltages (see Table I for I-V data). 


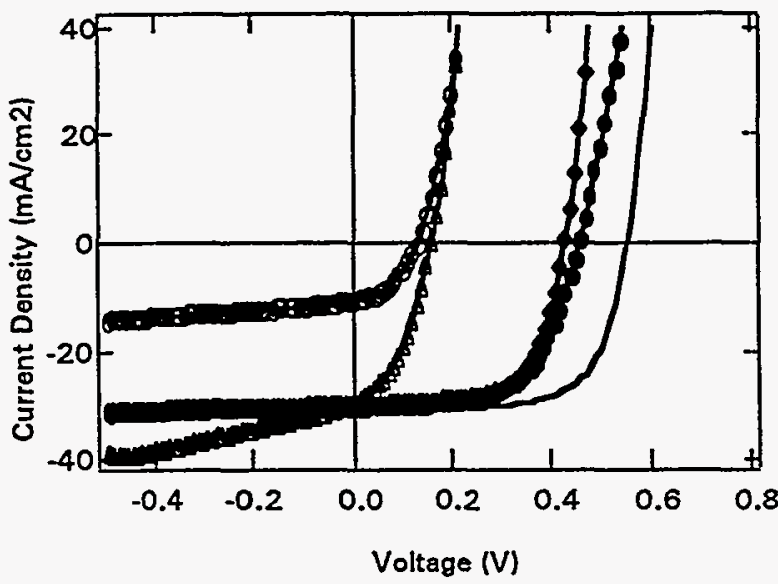

Fig. 5. Light I-V curves for CIGS films with various treatments

\section{Study of the CdS/CIGS Interface}

We considered the interaction between the chemical bath deposition (CBD) process and the CIGS films. We treated partial electrolyte -solutions to separate out the effects of $\mathrm{Cd}, \mathrm{S}$, and CdS. Figure 5 shows the I-V characteristics of five devices fabricated from identical CIGS films. When $\mathrm{ZnO}$ is sputtered directly (open circles) or when PVD CdS is used as the buffer layer (triangles), very poor characteristics are obtained. When the CIGS films are treated in Cd photoelectron (PE) before such depositions are made, a significant improvement occurs (diamonds and solid circles, respectively), window layers (solid line). It is clear that the Cd PE treatment modifies the CIGS surface favorably as evidenced by the increases in $\mathrm{V}_{\mathrm{oc}}, \mathrm{J}_{\mathrm{sc}}$, and fill factor. Chemical composition of the CIGS films was determined using X-ray photoelectron spectroscopy before and after the Cd PE treatment. About 20 at.\% Cd was detected, and it was found to trail off to a much lower value at a depth of 5-10 nm. The introduction of $\mathrm{Cd}$ can easily occur in the CIS. The $\mathrm{Cu}$-deficient nature of the surface makes for an ideal route for substituting $\mathrm{Cd}$ in the $\mathrm{Cu}$ vacancy sites. In fact, the ionic radius of $\mathrm{Cd}^{2+}$ is $0.97 \AA$ and that of $\mathrm{Cu}^{+}$is $0.96 \AA$ Our studies lead us to argue that the effects of the CBD process are two fold. In the first phase, the Cd-substituted CIGS is formed, resulting in an n-type region. In the second phase of the CBD, the CdS film grows slowly as the sulfur is slowly released by the hydrolysis of thiourea. The CdS layer can be viewed as a "buffer"; it provides a transition region as well as some shielding against sputter damage during the $\mathrm{ZnO}$ deposition.

\section{Alternative Junction Fabrication Process}

We used the insight and understanding gained from the studies described in the previous section to develop a set of criteria for forming junctions without $\mathrm{Cd}, \mathrm{CdS}$, or other extrinsic "buffer layers." Our goal is to eventually demonstrate an in-line process step that follows the absorber deposition in a seamless manner. Wet-chemical steps are not favored, and the process should be capable of rapid throughput. Atmospheric pressure or moderate vacuum conditions are preferred. Environmental concerns preclude the use of $\mathrm{Cd}$ and other heavy elements. Finally, the devices should not require a heat treatment or light soaking, and they must show good stability under light exposure and moderate heating. 
We have developed a method for fabricating CIS junctions that does not involve the use of Cd or other buffer layers. The details are the subject of a patent application. The CIGS bulk and surface compositions are found to be the important factors. At present, the junction formation is

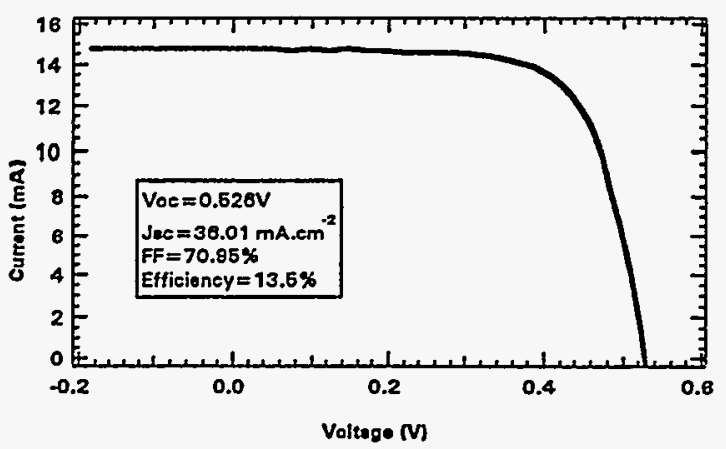

Fig. 6. Light I-V curve for a CdS-free $\mathrm{MgF}_{2} / \mathrm{ZnO} / \mathrm{CIGS}$ device. Measured at NREL with a XT 25 solar simulator. carried out ex situ, and in-line processing is being investigated. Following the junction formation step, a standard bilayer $\mathrm{ZnO}$ is deposited by RF sputtering in the usual manner; direct sputtering of the $\mathrm{ZnO}$ on CIGS typically yields only $2 \%-5 \%$ devices. Fig. 6 shows the I-V characteristics of the best device obtained so far. The device efficiency is $13.5 \%$ and it is somewhat limited by the low $\mathrm{Ga}$ content of the absorber. The current density is higher in the buffer-free device because of the elimination of CdS-related absorption losses. The device parameters did not show any degradation due to light soaking or short anneals at $200^{\circ} \mathrm{C}$. We noted slight improvements in $\mathrm{V}_{\mathrm{oc}}$ and FF. We believe that the junction formation can be accomplished in situ or ex situ using established techniques employed in other semiconductor industries.

\section{The Impact of Substrate/Back Contact on Device Performance}

By intentionally varying the amount of $\mathrm{Na}$ in the Mo and absorber layers of CIGS-based solar cells, we have shown to first order that the presence of $\mathrm{Na}$ has a beneficial effect on the performance of these devices. Positive trends are observed in open-circuit voltage, fill factor, efficiency, hole density, diode-quality factor, and series resistance with the addition of $\mathrm{Na}$. These trends imply improvements in junction quality and overall electronic performance as sodium concentration increases. We also observed that the presence of a sodium-diffusion barrier may induce a CIGS-Mo blocking barrier that is reduced or negated by the presence of sodium. What is the optimum concentration of $\mathrm{Na}$ in the film and what is the mechanism of the observed effects?

To date, we quantified the concentration to be about 0.1 atomic $\%$. We are in the process of measuring where it resides, i.e., in the bulk of the grains or at grain boundaries. Also, we presented two possible models of the mechanisms of the role of $\mathrm{Na}$ based on experimental measurements:

(1) Based on X-ray photoelectron spectroscopy (XPS) measurements, the most plausible interpretative of the data point to $\mathrm{Na}$ occupying In and/or $\mathrm{Ga}$ sites; i.e., $\mathrm{Na}_{\mathrm{In}}$ and/or $\mathrm{Na}_{\mathrm{Ga}}$. These point defects act as double acceptors or compensate donors. This mechanism is mostly active at grain boundaries and internal surfaces. As a result, the Fermi level should move closer to the CIGS valence band maximum.

(2) The incorporation of $\mathrm{Na}$ "annihilates" donor states stemming from the $\mathrm{In}_{\mathrm{Cu}}$ anti-site. During growth, $\mathrm{Na}$ and $\mathrm{In}$ (and/or $\mathrm{Ga}$ ) compete for a $\mathrm{Cu}$ site. $\mathrm{Na}$ is more likely to reside on the $\mathrm{Cu}$ site, 
therefore altering the number of defect pairs $\left(2 \mathrm{~V}_{\mathrm{Cu}}-\mathrm{In}_{\mathrm{Cu}}\right)$. This model is based on XRD and photoluminescence measurements.

Both models are consistent with the experimental observations and both may impact the point defect pairs that are considered most likely to affect the carrier concentration in CIGS, i.e., the [2V $\left.\mathrm{V}_{\mathrm{Cu}}-\mathrm{In}_{\mathrm{Cu}}\right]$ pair and the $\left(\mathrm{Cu}_{\mathrm{In}}-\mathrm{In}_{\mathrm{Cu}}\right)$ pair. We will attempt to narrow down the most likely mechanism.

\section{Technology Transfer/Industry Interaction:}

In 1997 we had three active interactions with industry designed to transfer processing know-how in CIS PV technology.

1. Optical Coating Laboratory (OCLI)

The following is a brief summary of the progress in the CIS technology transfer to OCLI:

- Using the NREL CIGS processing, OCLI is able to deposit high-quality, reproducible CIGS films on soda lime glass and on 8 mil. flexible borosilicate glass.

- NREL has confirmed 14.7\%-efficient devices on soda lime glass and 12\% on AF45 8 mil. Flexible glass.

- NREL has confirmed a submodule (8 cells) 2" $\times 2$ " substrate of $8.3 \%$ AM1.5.

- NASA has confirmed the above submodule at $7.8 \%$ AMO.

- OCLI is now focusing on all-laser scribing in vacuum.

\section{Lockheed-Martin Astronautics (LMA)}

The objective of this funds-in CRADA is to transfer the NREL CIS processing know-how to LMA's in-line system capable of handling $1-\mathrm{ft}^{2}$ substrate. Two types of substrates were used: the first was soda lime glass to qualify the machine and the second lightweight flexible substrate. The objective is to process CIS using roll-to-roll substrate. To date, we have met the first-phase milestone of demonstrating $7 \%$ device efficiency extracted from $1-\mathrm{ft}^{2}$ flexible substrate.

\section{DayStar Technologies}

The objective is to transfer the CIS technology to novel cell geometry/structure, which operates to under $20 \mathrm{X}$ concentration. The novel structure uses filaments of glass or metal as substrates. DayStar/NREL has demonstrated submodule efficiencies approaching $9 \%$.

\section{References:}

1. R. N. Bhattacharya, T. A. Berens, H. Wiesner, J. Keane, K. Ramanathan, R. J. Matson, A. Swartzlander, and R. N. Noufi, "12.3\% CuIn $\mathrm{Ga}_{1-x} \mathrm{Se}$ Device from Electrodeposited Precursor," J. Electrochem. Soc., Vol. 144, No. 4, April 1997.

2. R. N. Bhattacharya, F. Hasoon, H. Weisner, K. Ramanathan, H. Field, R. J. Matson, J. Keane, A. Swartzlander, A. Mason, and R. N. Noufi, "13.6\% Efficient $\mathrm{CuIn}_{1-x} \mathrm{Ga}_{\mathrm{x}} \mathrm{Se}-\mathrm{Based}$ Device 
from Electrodeposited Precursor," Electrochemical Society Proceedings, Vol. 97-13, pp. 298-305, 1997.

3. R. N. Bhattacharya, J. E. Granata, W. Bachelor, F. Hasoon, H. Wiesner, K. Ramanathan, J. Keane, R. N. Noufi, and J. R. Sites, "CuIn ${ }_{1-x} \mathrm{Ga}_{x} \mathrm{Se}_{2}$-based Photovoltaic Cells from Electrodeposited Precursor," Proceedings paper, SPIE, Vol. 3138, pp. 90-95, 1997.

4. R. N. Bhattacharya, W. Batchelor, J. E. Granata, F. Hasoon, H. Wiesner, K. Ramanathan, J. Keane, and R. N. Noufi, "CuIn ${ }_{1-x} \mathrm{Ga}_{x} \mathrm{Se}_{2}$-based Photovoltaic Cells from Electrodeposited and Chemical Bath Deposited Precursors," Proceedings Paper, International Symposium on New Materials for Hydrogen-Fuel Cell-Photovoltaic Systems-1, September 1-4, 1997 (to be published).

5. M. A. Contereas, H. Wiesner, J. Tuttle, K. Ramanathan, and R. Noufi, "Issues on the Chalcopyrite/Defect-Chalcopyrite Junction Model for High-Efficiency $\mathrm{Cu}(\mathrm{In}, \mathrm{Ga}) \mathrm{Se}_{2}$ Solar Cells," Solar Energy Materials and Solar Cells, Vol. 49, pp. 239-247,1997.

6. M. A. Contreras, T. J. Gillespie, C. H. Marshall, T. Berens, R. N. Bhattacharya, D. L. Schulz, C. J. Curtis, D. S. Ginley, K. Ramanathan, and R. Noufi, "Fabrication Methods of $\mathrm{Cu}(\mathrm{In}, \mathrm{Ga}) \mathrm{Se}_{2}$ Polycrystalline Materials and Devices Currently under Development at the National Renewable Energy Laboratory," Presented at the 14th European PVSC, Barcelona, Spain, 30 June-4 July 1997 (in press).

7. M. A. Contreras, B. Egaas, P. Dippo, J. Webb, J. Granata, K. Ramanathan, S. Asher, A. Swartzlander, and R. Noufi, "On the Role of $\mathrm{Na}$ and Modifications to $\mathrm{Cu}(\mathrm{In}, \mathrm{Ga}) \mathrm{Se}_{2}$ Absorber Materials using Thin-MF ( $M=N a, K, C s)$ Precursor Layers," Presented at the 26th IEEE PVSC, Anaheim, California, 29 September-3 October, 1997 (in press).

8. M. A. Contreras, B. Egaas, P. Dippo, J. Webb, S. Asher, A. Swartzlander, K. Ramanathan, F. S. Hasoon, and R. Noufi, "Changes to the Electrical and Structural Properties of Polycrystalline Thin-Film $\mathrm{Cu}(\mathrm{In}, \mathrm{Ga}) \mathrm{Se}_{2}$ Materials by the Use of Thin MF (M=Na, K, Cs) Precursor Layers," Presented at the ICTMC-11, Salford, UK, 8-12 September 1997 (in press).

9. K. Ramanathan, H. Wiesner, S. Asher, D. Niles, J. Webb, J. Keane, and R. Noufi, "A Study of the CBD CdS/CuIn, $\mathrm{GaSe}_{2}$ Interfaces." MRS Fall Meeting, Boston, Massachusetts, December 1997 (to be published).

10. K. Ramanathan, R. N. Bhattacharya, J. Granata, J. Webb, D. Niles, M. A Contreras, H. Wiesner, F. S. Hasoon, and R. Noufi, "Advances in the CIS Research at NREL." Presented at the 26th IEEE PVSC, Anaheim, California, 29 September-3 October, 1997 (in press).

11. K. Ramanathan, R. Noufi, J. Granata, J. Webb, and J. Keane, "Prospects for In-situ Junction Formation in CuInSe ${ }_{2}$ Based Solar Cells." To appear in Solar Energy Materials and Solar Cells (Conference, Cancun, Mexico). 

Title:

Organization:

Contributors:
High-Efficiency Concepts and Concentrators

\author{
National Renewable Energy Laboratory, Golden, CO
}

Dan Friedman, Alan Kibbler, Charlene Kramer, Sarah Kurtz, Jerry Olson.

Team members: Bob McConnell, Bill McMahon, Robert Reedy, Mark Wanlass, Kim Jones, Brian Keyes, Rick Matson, Alice Mason, John Geisz, Halden Field, Keith Emery, Pat Dippo, Scott Ward, Anna Duda, Dick Ahrenkiel, Mowafak Al-Jassim, Phil Ahrenkiel

\section{Objectives}

- To establish III-Vs as a competitive terrestrial photovoltaic (PV) technology by developing III-V photovoltaic technologies, advancing related science and engineering, coordinating the allied efforts with our partners, and facilitating commercialization.

- To develop III-V concentrator cells and support the transition of III-V concentrator-cell technology to industry for future U.S. utility market needs.

- To reduce the cost of III-V cells in order to address lower concentration systems and, ultimately, to be able to achieve flat-plate costs.

\section{Results}

\section{Toward Developing a Very-High-Efficiency 3- or 4-Junction Cell}

The GaInP/GaAs dual-junction technology is now well established on the production line. The first 10-kW commercial satellite was launched in August 1997. Further development of the dualjunction technology will provide small increases in the yield and efficiency of the space cells. Eventually, the cells may also be used in terrestrial concentrator systems. Although NREL research can aid in pushing the dual-junction technology to its limits, the most significant opportunities lie in adding more junctions to the structure. Figure 1 compares the theoretical and estimated ( $80 \%-90 \%$ of theoretical) practical efficiencies of four structures (1). This year we began exploring the $\mathrm{Ge}$ junction and options for the new material. The new material should have a band gap of about $1 \mathrm{eV}$. In order to assure high-quality crystal growth, it must also either be lattice-matched to GaAs or a scheme must be devised for bringing the appropriate layers together without compromising their quality. We have chosen to give priority to possibilities that will result in a monolithic stack grown in a single, high-temperature step, giving mechanical stacks and other less manufacturable schemes lower priority.

Reports of successful growth of III-V alloys using thallium have recently appeared in the literature (2). Although these alloys are predicted to be unstable (3), Asahi (4) reported a $\sim 0.9-\mathrm{eV}$ band gap for a GaTIP layer grown lattice-matched to GaAs by molecular beam epitaxy (MBE). Attempts to grow the thallium alloys by metal-organic chemical vapor deposition (MOCVD) have been unsuccessful, so we purchased a thallium source for our MBE system. This 
source was installed in summer 1997 and we did about 30 runs. We grew thallium droplets and numerous arsenide and phosphide layers. However, careful examination of the layers showed that the band gaps and lattice constants were very similar (within experimental uncertainty) with what we would have obtained without the thallium. Another group, led by C. Abernathy (5), has reported results similar to ours. Abernathy was able to duplicate the results of Asahi, except that she used secondary ion mass spectroscopy (SIMS) to measure the thallium concentration and found it to be much lower than Asahi reported. The lattice-constant shift that Asahi reported could be explained by other impurities and may not be related to thallium incorporation as he assumed. As far as we know, Asahi has not yet completed the SIMS measurement to confirm that he actually succeeded in incorporating thallium. Although there remains some evidence that it is possible to grow a subset of the thallium alloys, there is some doubt as to whether it is possible to grow the GaTIP alloy lattice-matched to GaAs. We observed that thallium may be an excellent surfactant-a surface layer of thallium can affect the structure of the growing surface without affecting the final composition of the film. We anticipate using it in the future as a surfactant while we remain open to the possibility of incorporating it into the bulk growth.

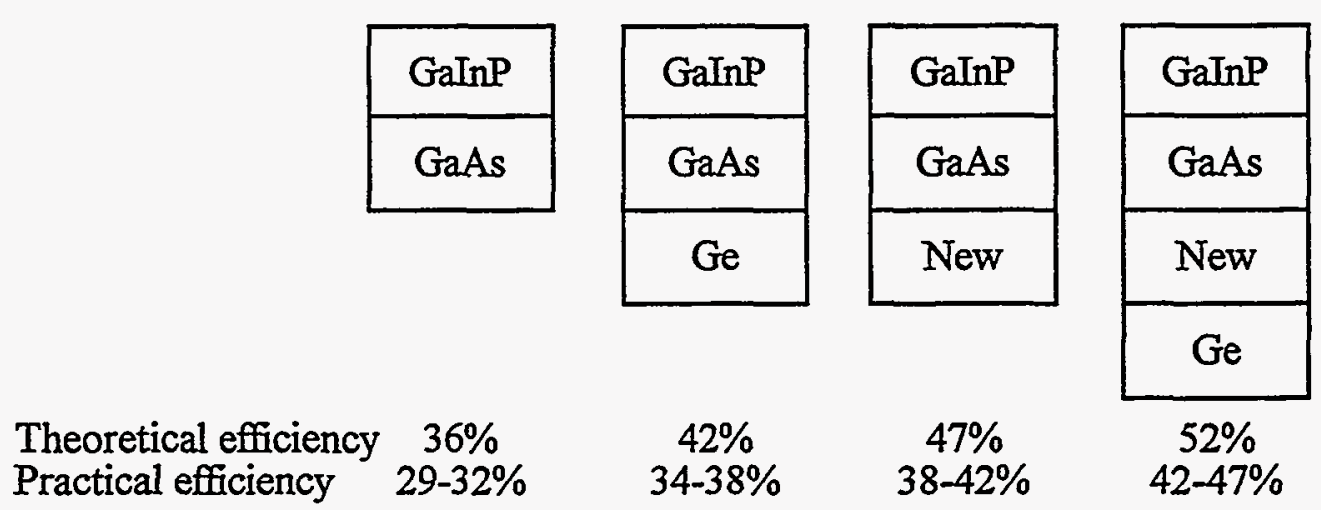

Figure 1. Theoretical and practical efficiencies of two-, three-, and four-junction concepts. The efficiencies assume 500 suns irradiance and an AM1.5 direct spectrum. The practical efficiencies are taken to be $80 \%-90 \%$ of the theoretical efficiencies. A 30\% efficiency has been achieved for the two-junction structure. The "new" material is discussed in the text.

In the meantime, reports have shown that incorporation of nitrogen into GaAs decreases the band gap instead of increasing it as one might expect. Furthermore, by using about $3 \%$ nitrogen and about $8 \%$ indium, it is possible to grow an alloy that is lattice-matched to GaAs, but with a band gap of about $1 \mathrm{eV}$ (6). Just like the thallium, the incorporation of nitrogen is not thermodynamically favorable, but there is a possibility of "freezing" it into the lattice by growing a layer that is not in equilibrium. Pursuing this direction, in September 1997 we installed a dimethylhydrazine (nitrogen) source on the low-pressure chemical vapor deposition system. The results were a dramatic contrast to those with thallium. In a very few runs we were able to measure a $1-\mathrm{eV}$ band gap for a layer lattice-matched to GaAs. Also, within the first month we grew a set of devices. The best of these showed an open-circuit voltage of $0.5 \mathrm{~V}$. Unfortunately, the short-circuit currents for these devices were only about $20 \%$ of what we would have liked, implying a problem with the quality of the material. This will be the subject of investigation in the 
coming year. In addition to the nitride alloy, we have a list of other possibilities for the new material. We will be investigating each of these until we find one that works to our satisfaction.

We also began working on germanium junctions, which are usually formed during growth of GaAs on the germanium. This gives little control over the details of the junction and usually results in a fairly low open-circuit voltage. By trying some innovative approaches, we were able to increase the voltage to as high as $230 \mathrm{mV}$. However, significant work will be required to incorporate this junction into a complete cell.

We investigated growth of tandem cells (and parts of tandem cells) without the selenium dopant (7). There are two reasons to do this: we are having a problem obtaining hydrogen selenide at the 700-ppm level and our industrial partners are sometimes interested in using $\mathrm{Si}$ instead of Se as a dopant. Si is known to have fewer problems with memory effect and dopant diffusion. However, switching between the two dopants is not as easy as one would expect. When using an n-on-p structure, one would expect that changing the n-type dopant in the window and emitter layers would change the blue response (response at the front of the cell). However, what we observe is that changing from $\mathrm{Se}$ to $\mathrm{Si}$ causes the red response to be degraded and the $\mathrm{V}_{\mathrm{Oc}}$ to drop as is expected when the quality of the passivation of the back of the cell is degraded. This is consistent with reports that the Japanese have published - that a highly doped GaInP layer will not work to passivate the back of the cell. We never had this problem until we switched to Si doping of the front of the cell-the same configuration that the Japanese use.

\section{Toward Reducing Cost of III-V Cells}

In an effort to exploit the potential high efficiency and low cost of a thin-film GaAs cell grown on a cheap substrate, we completed about 100 runs, surveying the effects of different types of substrates and growth conditions. We looked at the film optoelectronic properties, the statistics for the crystal orientation, grain size, and background impurity levels. For GaAs grown on glass a large fraction of the crystals are observed to nucleate with a (111) plane parallel to the substrate surface. The (111) texture increased when the arsenic overpressure was increased for growth by evaporation. However, the opposite trend was observed when the layers were grown by MOCVD. Isolated single crystals with grain sizes of up to $30 \mathrm{Om}$ were obtained when a selective epitaxy approach was used. Almost all of the GaAs-on-glass samples had minority-carrier lifetimes that were less than the system response $(17 \mathrm{psec})$, but one sample showed a lifetime of $0.25 \mathrm{nsec}$. Another sample showed a lifetime of $2.5 \mathrm{nsec}$, but the wavelength dependence indicated that the luminescence may have come from the glass itself.

Growth of GaAs directly on bare soda-lime glass could be a problem, especially at high temperature. Sodium contamination is a serious problem for single-crystal silicon, but there is emerging evidence that sodium is helpful in passivating copper indium diselenide grain boundaries. In a comparison of $\mathrm{GaAs}$ grown on soda-lime glass at temperatures as high as $750^{\circ} \mathrm{C}$, SIMS measurements showed that there was no measurable contamination in most of the film, but that sodium, potassium, and calcium-rich regions could be observed on the surface. The mechanism of the migration of these elements is not known. 
Based on a literature review, we explored the material and performance requirements for a $20 \%$, potentially low-cost, GaAs solar cell (8). An important issue limiting efficiency for GaAs cells is recombination through deep levels associated with the junction at the grain boundaries. The effect of this junction recombination on cell efficiency is shown as a function of grain size in Fig. 2. We concluded that to achieve over $20 \%$ efficiency, dislocation densities need to be reduced to less than $5 \times 10^{6} / \mathrm{cm}^{2}$ for very large-grain material. Assuming that the intragrain and surface (grain-boundary)-recombination properties are state of the art, grain sizes of 20-50 om are needed to reach $20 \%$.

One element of the high cost of III-V cells is the high cost of the precursors associated with MOCVD. Evaporation of the elements is expected, eventually, to give high-quality GaAs at a low cost. A number of companies are developing MBE production tools with epi-product costs competitive with the more traditional MOCVD systems. In light of this, we are investigating evaporation (MBE) of III-Vs (9). A critical step is learning to dope each of the layers. Carbon is a good p-type dopant in $\mathrm{GaAs}$ and we have had no trouble doping GaAs p-type using a carbon tetrabromide source. However, historically, there has been some difficulty with doping GaInP with carbon when using MOCVD. A study was completed (10) showing that it is possible to achieve a hole concentration of $3 \times 10^{18}$ ? $\mathrm{cm}^{3}$ using carbon tetrabromide in evaporated GaInP, as shown in Fig. 3. However, the conditions under which this can be achieved are fairly limited: a surface with a high A-type step density is required and the phosphorus pressure must be reduced as far as it can be without losing control of the surface. All of the layers studied showed high carbon concentrations, but only a few showed the high hole concentrations.

\section{Toward Supporting the Concentrator Industry}

A study of the current status of the concentrator industry showed that the key development is the entry of larger companies with significant financial resources (11). While previously the industry included only companies with a handful of employees, several efforts now have the backing of very large companies. BP Solar is currently installing a $0.5-\mathrm{MW}$ demonstration plant using a. reflective parabolic trough and silicon cells. Their target is a future large-scale utility market. Honda has purchased a minority share of SunPower, which is developing a hybrid product that will generate electricity and heat.

Photovoltaics International has significant financial resources, is completing a Photovoltaic Manufacturing Technology program, and will be marketing product soon. The technology developed by the other small companies may be more quickly introduced as more investors enter the PV industry. The industry is summarized in the map shown in Fig. 4.

Since the termination of funding for the concentrator initiative in the early 90 s, the concentrator industry has had little opportunity to come together to address common problems. At a workshop in March, members of the concentrator industry concluded that they could do better by working together. As a result of this, we now have a mailing list that is used to communicate with the concentrator industry and an effort is under way to develop standards for concentrator systems. The companies have been focusing on the standards development because the lack of concentrator standards is a key barrier to their competing in the PV industry. Bob McConnell is 


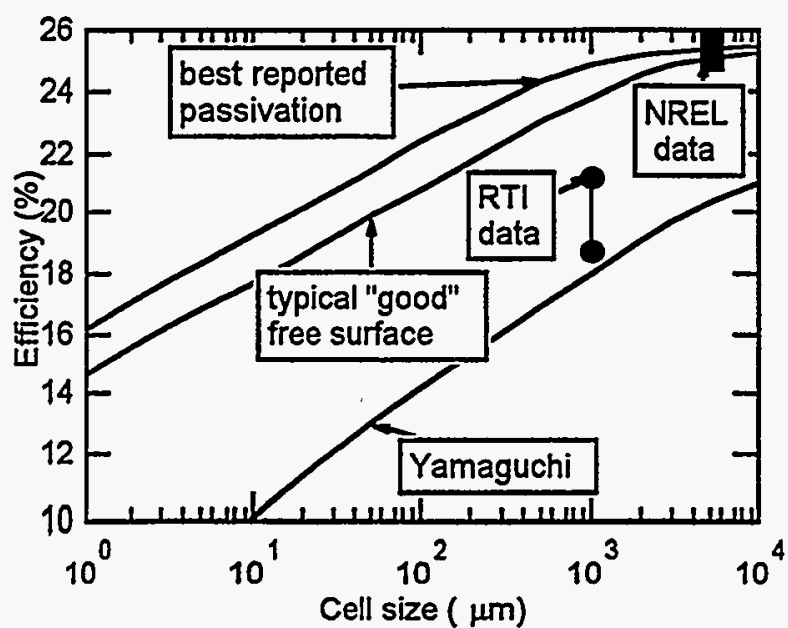

Figure 2. Efficiency that can be expected for a single-crystal GaAs cell based on data reported in the literature.

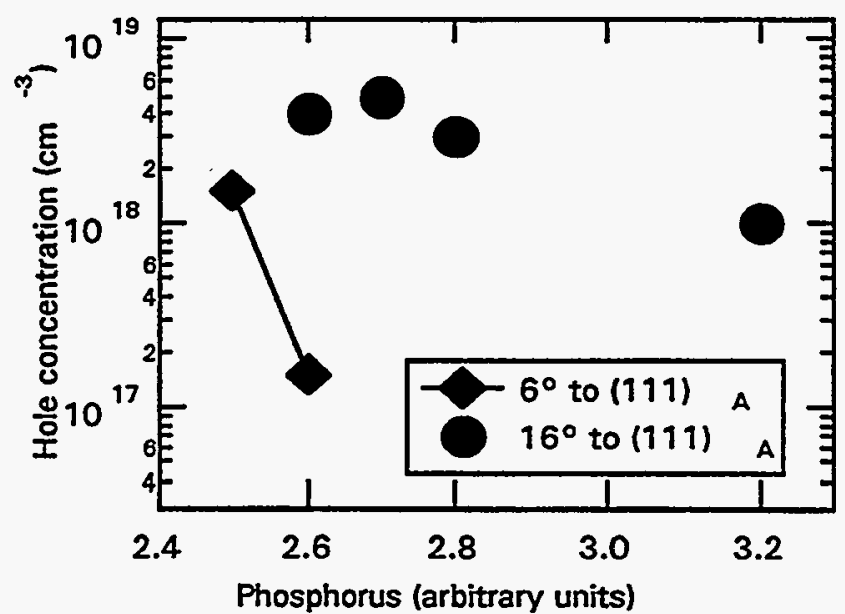

Figure 3. Hole concentration as a function of phosphorus pressure for carbon-doped GaInP.

leading the writing of the "Recommended Practice for Qualification of Photovoltaic (PV) Concentrator Modules." Alex Maish is leading a separate effort to develop a standard for trackers. The most important result of the March workshop is that an industry that was quite fragmented and that felt very neglected, now has a sense of unity and is feeling a little more included in the DOE program.

Although the III-V cells are very successful in the space industry, a large piece of work will be required to convert these to terrestrial concentrator cells and incorporate them into terrestrial concentrator systems. In order to fabricate concentrator cells that can perform well at high concentration ratios $(500 \mathrm{X})$, we have been developing evaporated grid structures. After many problems during the last year, we have finally succeeded in developing a process that works. It uses $\mathrm{Ag}$ grids and lift-off to produce narrow (as small as $5 \mu \mathrm{m}$ ) grid lines with respectable heights ( $7 \mu \mathrm{m}$ or more). We sent devices to Midway Labs and EDTEK for evaluation. EDTEK was very impressed with the high voltages and high fill factors. Midway did an analysis showing that they would be able to enter the water-pumping market with a $140-\mathrm{W}$ product if they could use the $\mathrm{GaInP} / \mathrm{GaAs}$ cells to give higher voltages (12). The economics for the commercial GaAs/GaInP cells are competitive with what they are currently paying for the $\mathrm{Si}$ concentrator cells. EDTEK is already using GaAs cells and is interested in using the higher efficiency and higher voltage dualjunction $\mathrm{GaInP} / \mathrm{GaAs}$ cells. A separate study by $\mathrm{R}$. Swanson comparing various approaches to concentrators concluded that, for large installations in sunny areas, a III-V very-high-efficiency cell in a dish or point-focus concentrator represents the cheapest option (compared with the other concentrators, silicon, and thin-film options.) 


\section{U. S. Concentrator Industry}

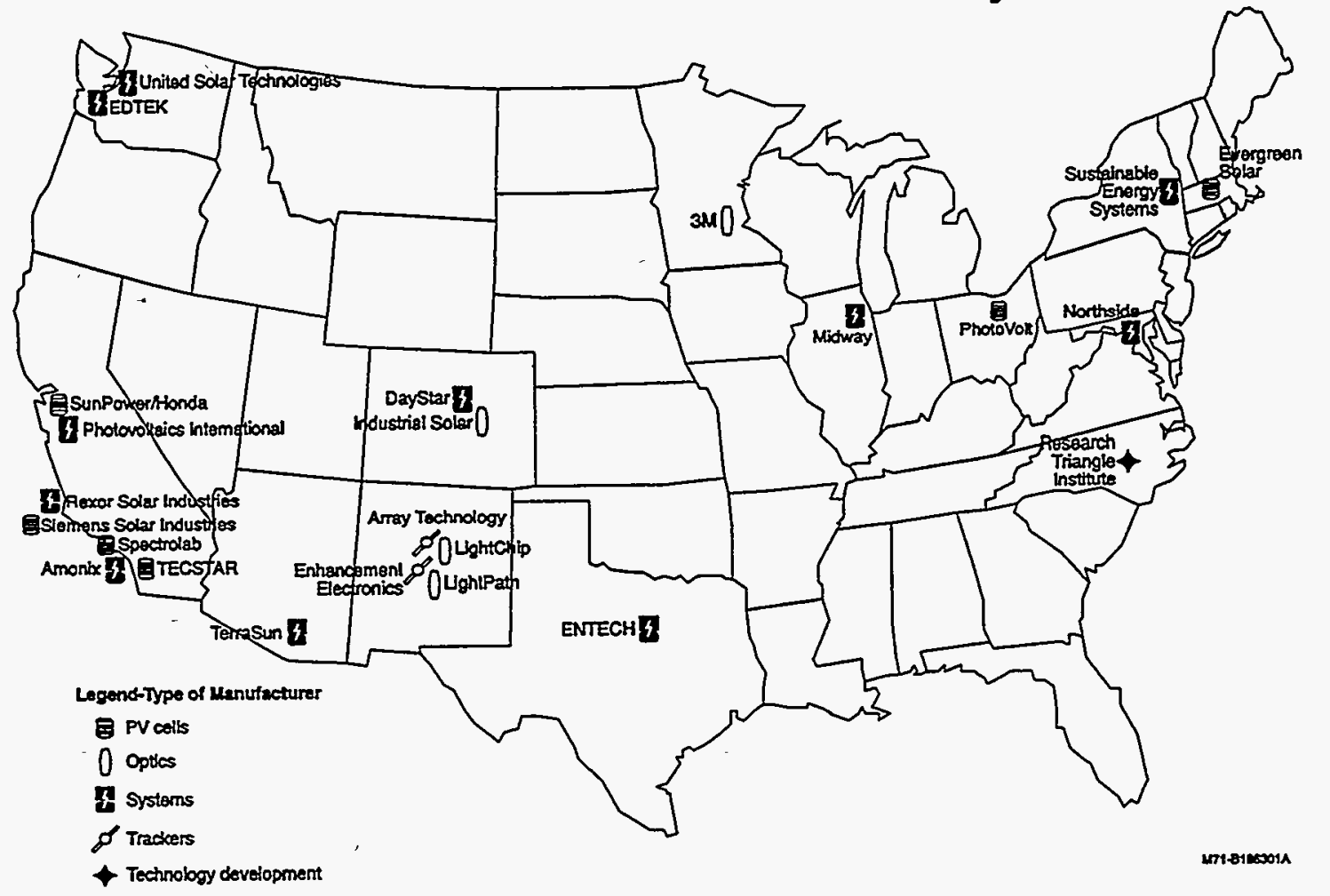

Figure 4. Locations of U.S. companies that are developing various concentrator products.

\section{References}

1. S. R. Kurtz, D. Myers and J. M. Olson, "Projected Performance of Three- and Four-Junction Devices using GaAs and GaInP," 26th IEEE Photovoltaic Specialists Conference;-Anaheim, 1997, (New York: IEEE).

2. H. Asahi, K. Yamamoto, K. Iwata, S. Gonda and K. Oe, "New III-V Compound Semiconductors TIInGaP for $0.9 \mathrm{mu} \mathrm{m}$ to over $10 \mathrm{mu} \mathrm{m}$ Wavelength Range Laser Diodes and their First Successful Growth," Jpn. J. Appl. Phys., 35 (1996), pp. L876-L879.

3. M. A. Berding, M. Van Schilfgaarde, A. Sher, M. J. Antonell and C. R. Abernathy, "Thermodynamical Properties of Thallium-Based MII-V Materials," J. Electron Mater., 26 (1997), pp. 683-687.

4. H. Asahi, M. Fushida, H. Koh, K. Yamamoto, K. Asami, S. Gonda, and K. Oe, "Gas Source MBE Growth of TIInGaP and TIInGaAs as New Materials for Long-Wavelength Applications," InP and Related Materials. Hyannis, MA: IEEE; (1997), pp. 448-451.

5. M. J. Antonell, C. R. Abernathy, A. Sher, M. Berding and M. Van Schilfgaarde, "Growth of Tl-Containing III-V Materials by Gas-Source Molecular Beam Epitaxy," InP and Related Materials. Hyannis, MA: IEEE; (1997), pp. 444-447. 
6. M. Kondow, K. Uomi, A. Niwa, T. Kitatani, S. Watahiki, and Y. Yazawa, "GaInNAs: A Novel Material for Long-Wavelength-Range Laser Diodes with Excellent High-Temperature Performance," Jpn. J. Appl. Phys., 35 (1996), pp.1273-1275.

7. S. R. Kurtz, J. M. Olson, D. J. Friedman, and R. Reedy, "Effect of Front-Surface Doping on Back-Surface Passivation in GaInP Cells," 26th IEEE Photovoltaic Specialists Conference; Anaheim, 1997, (New York: IEEE).

8. S. R. Kurtz and R. McConnell, "Requirements for a $20 \%$-Efficient Polycrystalline GaAs Solar Cell," Future-Generation Photovoltaic Technologies. Edited by R. McConnell. (AIP, Denver, CO, 1997), Vol. 404, pp. 191-206.

9. D. J. Friedman and A. E. Kibbler, "Real-Time Control of Group-V Flux in III-V MolecularBeam Epitaxy Using a Valved Cracker," J. Vac. Sci. Tech. (submitted).

10. D. J. Friedman, A. E. Kibbler and R. Reedy, "Selection of Substrate Orientation and Phosphorus Flux to Achieve p-type Carbon Doping of GA0.5In0.5P by Molecular Beam Epitaxy," Appl. Phys. Lett., 71 (1997), pp.1095-1097.

11. S. R. Kurtz and D. Friedman, "Recent Developments in Terrestrial Concentrator Photovoltaics," NREL/SNL Photovoltaics Program Review. Edited by A.-J. Witt and Gee. (AIP, Lakewood, CO, 1996), Vol. 394, pp. 247-258.

12. R. Hoffmann, J. O'Gallagher and R. Winston, "High Concentration Low Wattage Solar Arrays and Their Applications," NREL/SNL Photovoltaics Program Review. Edited by A.-J. Witt and Gee. (AIP, Lakewood, CO, 1996), Vol. 394, pp. 739-744. 
Title:

Organization:

Contributors:
Hydrogenated Amorphous Silicon Device Research

NREL In-House Amorphous Silicon Research Team

National Renewable Energy Laboratory, Golden, CO

H. M. Branz (Team Leader), R. S. Crandall, E. Iwaniczko, H. Mahan, B.P. Nelson, Q. Wang, Y. Xu

\section{Objectives}

The NREL Amorphous Silicon Team pursues breakthrough advances in materials and devices in order to improve the stabilized efficiency of amorphous-silicon-based photovoltaic (PV) cells and meet DOE PV cost goals. During FY 1997, our objectives were to understand and overcome the light-induced degradation of hydrogenated amorphous silicon (a-Si:H), and to develop hot-wire (HW) chemical vapor deposition (CVD) amorphous silicon for use as the mid-gap and low-gap cells of a triple-junction, amorphous-silicon-based solar cell. We collaborated closely with PV industry and academic institutions through participation in and direction of the National Amorphous Silicon Teams.

\section{Staebler-Wronski (SW) Metastability Model}

During FY 1997, we devised and published a promising new model of the light-induced metastability (Staebler-Wronski effect) in a-Si:H. ${ }^{1}$ Twenty years of study had not previously produced a viable model of light-induced metastable defect formation in a-Si:H. This undoubtedly contributed to the field's inability to eliminate the SW degradation of a-Si:H solar cells. Our new microscopic model of the a-Si:H metastability accounts quantitatively for the kinetics of defect creation and annealing by treating the light-induced emission and retrapping processes for mobile atomic $\mathrm{H}$. For the first time, nearly all experimental results concerning the SW effect can be understood within a single model. There are several novel predictions that we are presently investigating. Although validation of the model will take time, the new model is stimulating new attempts to solve the PV metastability problem.

In our new "H collision" model, ${ }^{1}$ dangling-bond (DB) defects are created when light-induced carriers stimulate emission of mobile $\mathrm{H}$ from a Si-H bond according to:

$$
\mathrm{Si}-\mathrm{H}--->\mathrm{DB}+\mathrm{Si}-\mathrm{H} / \mathrm{DB}
$$

The mobile $\mathrm{H}$, notated by Si-H/DB, is usually envisioned as a mobile Si-H-Si bond-centered $\mathrm{H}$ configuration, but it can be more useful to visualize it as a mobile $\mathrm{Si}-\mathrm{H}$ bond accompanied by a mobile DB. As the mobile $\mathrm{H}$ atom hops from site to site, each broken bond reforms behind it. This mobile state for $\mathrm{H}$ in $\mathrm{Si}-\mathrm{H}$ was proposed by Biswas et al., ${ }^{2}$ based tight-binding molecular dynamics simulations.

Mobile $\mathrm{H}$ retraps to Si-H through one of two mechanisms. Normal retrapping to an ordinary $\mathrm{DB}$ is given by

$$
\mathrm{Si}-\mathrm{H} / \mathrm{DB}+\mathrm{DB} \rightarrow \mathrm{Si}-\mathrm{H}
$$


the inverse process to Eqn. (1). No net DBs are created if $\mathrm{H}$ reconfigures only through the cyclical processes of Eqns. (1) and (2)-this is the "normal" creation and retrapping of mobile $\mathrm{H}$.

A second retrapping process

$$
\mathrm{Si}-\mathrm{H} / \mathrm{DB}+\mathrm{Si}-\mathrm{H} / \mathrm{DB} \quad-->\mathrm{M}(\mathrm{Si}-\mathrm{H})_{2}
$$

is less frequent than normal retrapping but is important because it is a key step of SW defect creation. Eqn. (3) represents collision of two mobile $\mathrm{H}$ atoms to form a metastable complex, $\mathrm{M}(\mathrm{Si}-\mathrm{H})_{2}$, containing two $\mathrm{Si}-\mathrm{H}$ bonds in close proximity. This reaction occurs when the two mobile DBs in Eqn. (3) come close enough together to form a Si-Si bond. The two mobile $\mathrm{H}$ are immediately immobilized in $\mathrm{M}(\mathrm{Si}-\mathrm{H})_{2}$. Bond-counting admits the possiblity of forming an $\mathrm{M}(\mathrm{Si}-\mathrm{H})_{2}$ complex by Eqn. (3) without any DB formation. Combining Eqn. (3) with Eqn. (1), the net reaction leaves $\mathrm{DB}$ defects at the sites of the original $\mathrm{H}$ excitation according to:

$$
2 \mathrm{Si}-\mathrm{H}--->2 \mathrm{DB}+2 \mathrm{Si}-\mathrm{H} / \mathrm{DB}--->>2 \mathrm{DB}+\mathrm{M}(\mathrm{Si}-\mathrm{H})_{2}
$$

The created SW defects appear on the right-hand side of Reaction (4).

Light-induced or thermal annealing of the SW effect is described by the back reaction of Eqn. (3). Emission of one mobile $\mathrm{H}$ from $\mathrm{M}(\mathrm{Si}-\mathrm{H})_{2}$ leaves a $\mathrm{DB}$ and a $\mathrm{Si}-\mathrm{H}$ bond in close proximity to one another. By pairing with the $\mathrm{DB}$, this $\mathrm{H}$ soon becomes mobile. Thus, $\mathrm{M}(\mathrm{Si}-\mathrm{H})_{2}$ is a negative$U$ hydrogen pair of the class described by Zafar and Schiff, ${ }^{3}$ although it may not take the microscopic form they proposed. Under most conditions, normal retrapping of mobile $\mathrm{H}$ to DBs as in Eqn. (2) will dominate the retrapping of any $\mathrm{H}$ emitted from $\mathrm{M}(\mathrm{Si}-\mathrm{H})_{2}$.

Fig. 1 depicts, schematically, the SW reaction of Eqn. (4). The Fig. 1 depiction of mobile $H$ is somewhat unrealistic: mobile $\mathrm{H}$ actually travels by a random walk through the $\mathrm{Si}$-Si bonds through distances much greater than those illustrated. Also, Fig. 1 illustrates only one of many conceivable structures of $\mathrm{M}(\mathrm{Si}-\mathrm{H})_{2}$.

If we assume that $\mathrm{H}$ emission to the mobile state is proportional to the electron-hole pair generation rate, $G$, the mathematics corresponding to Eqns. (1) - (4) solves ${ }^{1}$ to a time dependence of $\mathrm{DB}$ density

$$
N_{d b}(t)=\left(3 C_{s w}\right)^{1 / 3} G^{2 / 3} t^{1 / 3} .
$$

Here, $\mathrm{C}_{\mathrm{sw}}$ is a constant, depending on the constants for mobile $\mathrm{H}$ emission and trapping. This form for $\mathrm{N}_{\mathrm{db}}(\mathrm{t})$ during defect creation was first obtained by Stutzmann, Jackson, and Tsai and has been observed in many experiments. ${ }^{4,5}$ Their derivation assumed that $n p$ recombination creates metastable DB defects directly. However, derivation of Eqn. (5) with carrier recombination leads to kinetics inconsistent with recent experiments at $4.2 \mathrm{~K}^{5}$ and under laser illumination. ${ }^{6}$ In contrast, our new model is consistent with all the experiments and now provides the only quantitative explanation of Staebler-Wronski creation kinetics.

\section{Solarex CRADA Collaboration on Metastability}

We collaborated with Solarex Corporation on the study of light-induced metastability in a-Si:H devices under a cooperative research and development agreement (CRADA) signed in 1997. Light-induced metastability limits the efficiency of a-Si:H PV, so its understanding and 
eradication is a priority for NREL and our industry partners. Solarex has recently been studying the phenomenon of high-intensity light annealing of light-induced metastability in reverse-biased solar cells. ${ }^{7,8}$ As part of the NREL-Solarex CRADA, Solarex asked the NREL Amorphous Silicon Team to apply its metastability and measurement expertise to the problem.

Using both NREL- and Solarex-grown a-Si:H p-i-n solar cells, we successfully reproduced the effect in our laboratory and see a similar, remarkably rapid, recovery of photovoltaic response. By fitting to stretched exponential functions, we have calculated time constants for light-induced creation and annealing of the effect. We will next extend our study to examine the effect of different applied bias and light intensity and will look for any differences between Solarex and NREL devices. To make these measurements, we first designed, built, and tested a system in which we can duplicate the high-intensity light-soaking conditions, anneal samples, and perform capacitance and current measurements. The capacitance measurements will give important information about the spatial and energy distribution of electronic defects in PV devices.

\section{Hot-Wire n-i-p Device Fabrication}

We developed two capabilities that enabled ús to fabricate devices containing only HWCVD a-Si:H entirely within NREL. First, we modified one of the chambers in the multichamber deposition system to permit rapid heating and cooling of devices and to give better temperature uniformity and control. We have now demonstrated that our larger multichamber deposition system in the Solar Energy Research Facility building is capable of fabricating HWCVD material of the same quality we previously could make only in a smaller single-chamber system. In this way, we reduced P-contamination of the i-layer material. This advance doubles the number of systems in which we can optimize device parameters.

Second, we developed a good transparent top contact for the HWCVD substrate (n-i-p) solar cells. We deposited indium-tin oxide (ITO) by thermal evaporation of the metals in an oxygen atmosphere. Our ITO transmission is above $88 \%$ and the resistivity is less than $80 \mathrm{ohms}$ per square. This advance speeds our work by giving us the capability to complete devices in-house, rather than in collaboration with our industrial partner, United Solar Systems Corporation (USSC).

With these improvements completed, we began to optimize substrate devices fabricated entirely in-house. Our best devices incorporate the multichamber HWCVD a-Si:H, a plasmaenhanced CVD a-SiC:H p-layer and our new in-house ITO. Our best device had $\mathrm{V}_{\text {oc }}=0.86 \mathrm{~V}$, $\mathrm{J}_{\mathrm{sc}}=12.7 \mathrm{~mA}-\mathrm{cm}^{-2}$, and a fill factor $(\mathrm{FF})=0.62$. This open-circuit voltage is $0.02 \mathrm{~V}$ lower than we had obtained with earlier devices in which a microctystalline p-layer and ITO were deposited at USSC. Our $\mathrm{J}_{\mathrm{sc}}$ is nearly identical to the previous devices and the fill factor improved by 0.03 , most likely due to the in-house p-layer.

\section{Low-Gap and Mid-Gap Hot-Wire CVD a-SiGe:H Materials}

We deposited hot-wire CVD amorphous silicon germanium.(a-SiGe:H) films 20 to 40 times faster ( $40 \AA / \mathrm{sec}$ ) than the best conventional materials, but with comparable material quality.

A $1.45 \mathrm{eV}$ a-SiGe:H material is presently used in the low-gap component cell of triplejunction a-Si PV and $1.65 \mathrm{eV}$ a-SiGe:H is used in the mid-gap cell. The low deposition rates (1-2 $\mathrm{A} / \mathrm{s}$ ) of a-SiGe:H alloys deposited by the conventional plasma-enhanced (PE) CVD technique add significant cost to a-Si-based triple-junction PV cells. Increased deposition rates would reduce plant capital and labor costs by reducing the required number of deposition chambers, 
reducing the deposition time, and increasing the germane gas utilization fraction. Because germane costs ten times more than silane, germane utilization is a significant cost driver in a-Si triple cells. We have varied the band gaps of this rapidly grown hot-wire a-SiGe:H from 1.4 to $1.7 \mathrm{eV}$.

To optimize the HWCVD a-SiGe:H, we deposited more than 160 samples and characterized them with over 2,000 measurements especially designed to probe a wide range of filamentsubstrate spacings. These characterizations include several optoelectronic measures of film PV potential, $\mathrm{Ge}$ and $\mathrm{H}$ content, $\mathrm{H}$ bonding, thickness and microstructure. Figure 2 compares the photo-to-dark conductivities of our HWCVD a-SiGe:H to the best plasma-enhanced chemical vapor deposition a-SiGe:H for the full range of band gaps we have grown. Thus, our best hotwire-grown a-SiGe:H has optoelectronic properties equal to or better than those of conventional a-SiGe:H through the entire band-gap range from 1.4 to $1.7 \mathrm{eV}$. Our best-quality films can be deposited at rates equal to or above $40 \AA / \mathrm{s}$ at all these band gaps. We remain cautious about this material because we have not yet explored device-level issues such as hydrogen loss near interfaces. Our focus now turns to incorporating hot-wire a-SiGe:H into devices suitable for the low-gap and mid-gap cells of a triple-junction device.

\section{References}

1. H. M. Branz, Solid-State Commun. 105/6, 387 (1998).

2. R. Biswas, Q. Li, B. C. Pan, and Y. Yoon, Phys. Rev. B 57, 2253 (1998).

3. S. Zafar and E. A. Schiff, J. Non-Cryst. Solids 137, 323 (1991).

4. M. Stutzmann, W. B. Jackson, and C. C. Tsai, Phys. Rev. B 32, 23 (1985).

5. P. Stradins and H. Fritzsche, Phil. Mag. B 69, 121 (1994).

6. P. Tzanetakis, N. Kopidakis, M. Androulidaki, C. Kalpouzos, P. Stradins, and H. Fritzsche, J. Non-Cryst. Solids 198-200, 458 (1996).

7. A. E. Delahoy and T. Tonon, in Stability of Amorphous Silicon Materials and Devices, AIP Conf. Proc. 157, edited by B. L. Stafford and E. Sabisky (AIP, New York, 1987), p. 263.

8. D. E. Carlson and K. Rajan, Appl. Phys. Lett. 70, 2168 (1997). 


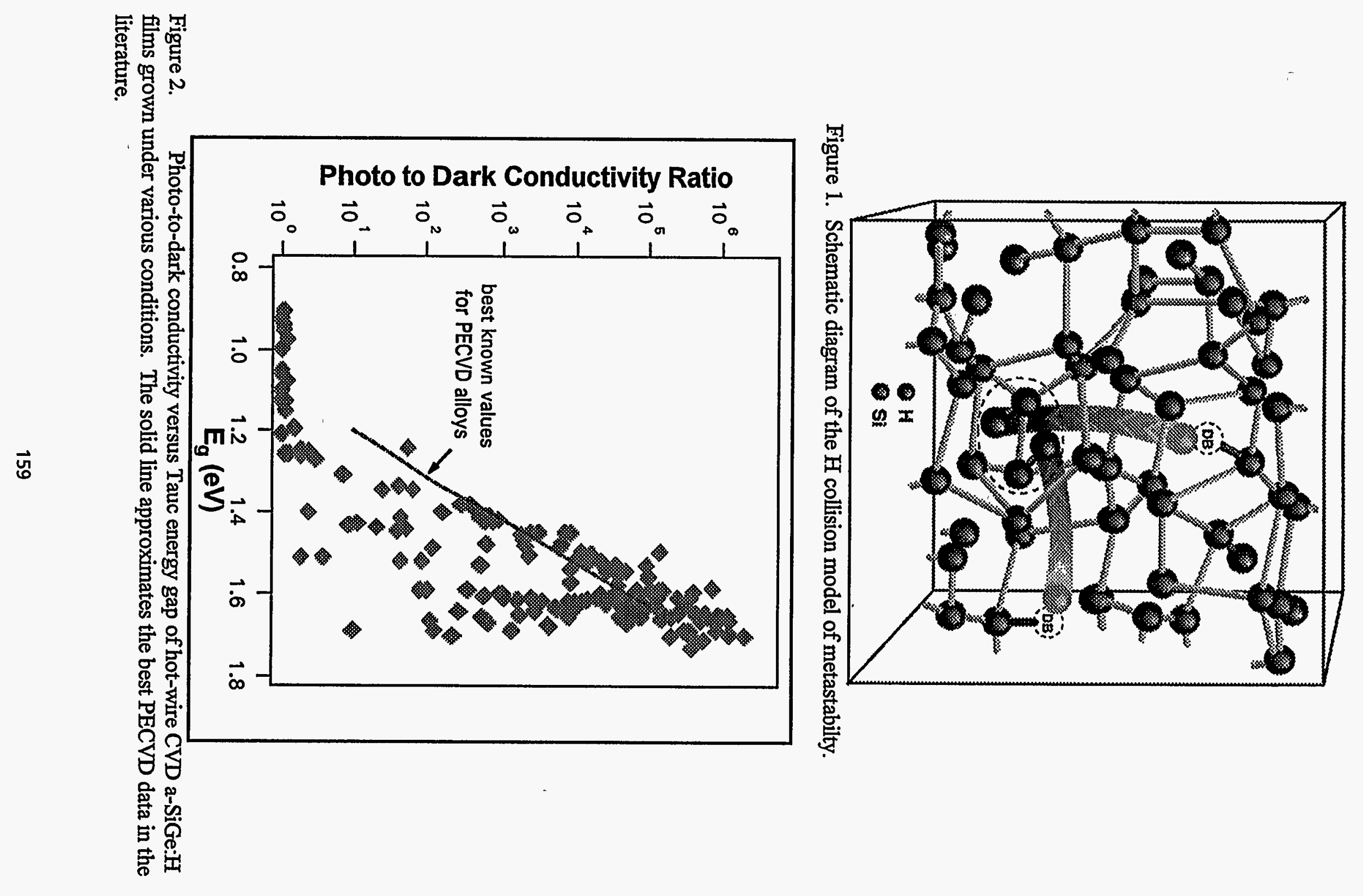


Title:

Organization:

Contributors:
Nanoparticles and New Ideas for Photovoltaics

New Ideas Team, Center for Photovoltaics and Electronic Materials National Renewable Energy Laboratory, Golden, CO

D.L. Schulz (Temporary Staff Scientist), C.J. Curtis (Senior Scientist),

J.D. Perkins (Temporary Staff Scientist), P.A. Parilla (Senior Scientist),

C. Carlson (Graduate Student), J. Bertelson (NTEP High School

Teacher/Summer Student), A. Cram (NTEP High School

Teacher/Summer Student), D.S. Ginley (Team Leader)

\section{Objectives}

The objectives of the team are two fold: 1) the continued exploration of nanoparticle precursors leading to low-cost, nonvacuum deposition technologies and 2) an exploration of new technological breakthroughs that could potentially impact photovoltaics (PV). Particular areas of interest include nanoparticle-derived absorbers, transparent conducting oxides (TCOs), and metal contacts for solar cell technologies in the former, and the development of textured buffer layers for low-cost substrates and the investigation of p-type TCOs in the latter.

\section{Technical Approach}

Nanoparticle Precursors: This project represents an integrated effort between the PV program and the Basic Energy Sciences' Chemical Sciences program to evaluate the potential of nanoparticle-based inks as precursors for PV devices and to develop an understanding of the mechanisms of nanoparticle interactions during the formation of electronic materials. The nanoparticle precursors could potentially impact PV absorbers, contacts, and TCOs. They have some key advantages versus standard particle-based systems that may include lower sintering temperatures and denser films with fewer pinholes. This nanoparticle ink approach is amenable to low-cost, nonvacuum particulate deposition technologies such as spray deposition, screen printing, or electrophoresis. We have expanded our investigations this year to include contacts and TCOs. Nanoparticle inks are being formulated for $\mathrm{CdTe}, \mathrm{Cu}-\mathrm{In}-\mathrm{Ga}-\mathrm{Se}, \mathrm{Cu}-\mathrm{Se}, \mathrm{In}-\mathrm{Se}, \mathrm{Al}, \mathrm{Ag}$, and $\mathrm{SnO}_{2}$. The general approach is to develop low-cost synthetic routes to the nanoparticles, protect them with capping agents, suspend them in an appropriate solvent with additives, and spray-deposit or screen-print them to form uniform layers or patterns. Subsequent thermal processing may be required to produce semiconductor-quality electronic materials.

Other Projects: Although there is very little budget for other work, we have been leveraging existing NREL projects (i.e., Superconductivity, Basic Energy Sciences Defective Oxides, and United States Industry Consortium [USIC]) to initiate small projects with potentially large impacts on the PV program. These include an investigation of textured buffer layers for low-cost substrates and growth of a p-type TCO.

\section{Results}

Nanoparticle Precursors for Electronic Materials: Introduction

The use of nanoparticles as precursors in low-cost, nonvacuum, particulate-based film growth technologies is presently being investigated. Nanoparticles possess enhanced reactivity 
owing to the higher surface energies in the smaller particles versus standard-sized particle precursors. Also, film thicknesses an order of magnitude less than conventional film growth can be realized, affording the ability to form thin films using traditional thick-film technologies (e.g., screen printing, electrophoresis, and spray deposition).

This year we have made substantial progress in developing several electronic materials of interest to the photovoltaics community. We have performed initial survey-type experiments in the areas of nanoparticle-derived CdTe absorbers, nanoparticle-derived $\mathrm{Cu}-\mathrm{In}-\mathrm{Ga}-\mathrm{Se}$ absorbers, nanoparticle-derived $\mathrm{SnO}_{2}$ transparent contacts, and nanoparticle-derived $\mathrm{Al}$ - and $\mathrm{Ag}$ - contacts to Si. All of these show promise for application given appropriate optimization.

\section{Nanoparticle CdTe Materials}

In the area of CdTe materials, we developed a novel route to CdTe nanoparticles while using volatile capping agents to stabilize the colloid. ${ }^{1,2}$ The metathesis reaction consists of reacting cadmium iodide in methanol with sodium telluride in methanol at low temperature, as follows:

$$
\mathrm{CdI}_{2}+\mathrm{Na}_{2} \mathrm{Te} \stackrel{\mathrm{CH}_{3} \mathrm{OH} \mathrm{I} \sim-78^{\circ} \mathrm{C}}{\longrightarrow} \text { methanol-capped } \mathrm{CdTe}+2 \mathrm{NaI}
$$

We spray-deposited $\mathrm{CdTe}$ nanoparticles onto $\mathrm{CdS} / \mathrm{SnO}_{2} /$ glass substrates and prepared solar cells after appropriate regrowth and contact formation. A comparison was made between nanoparticle-based CdTe films and standard ball-milled $\mathrm{CdTe}$ in collaboration with Golden Photon, Inc. In particular, the behavior of these two precursors in the presence of $\mathrm{CdCl}_{2}$ was probed. Figure 1 shows scanning electron microscopy (SEM) images of the product of a ballmilled precursor film (Fig. 1a) and a nanoparticle-derived precursor film (note scale difference in Fig. 1b). Both of these films were subjected to an almost identical $\mathrm{CdCl}_{2}$ process as instructed by Golden Photon. A notable difference in surface morphology is apparent. It seems that the nanoparticles process differently than the ball-milled particles when exposed to the $\mathrm{CdCl}_{2}$ treatment. Given appropriate optimization, it may be expected that denser CdTe films can be formed from nanoparticle precursors. One nanoparticle-derived CdTe device was finished into a solar cell with an efficiency of $6 \%$.
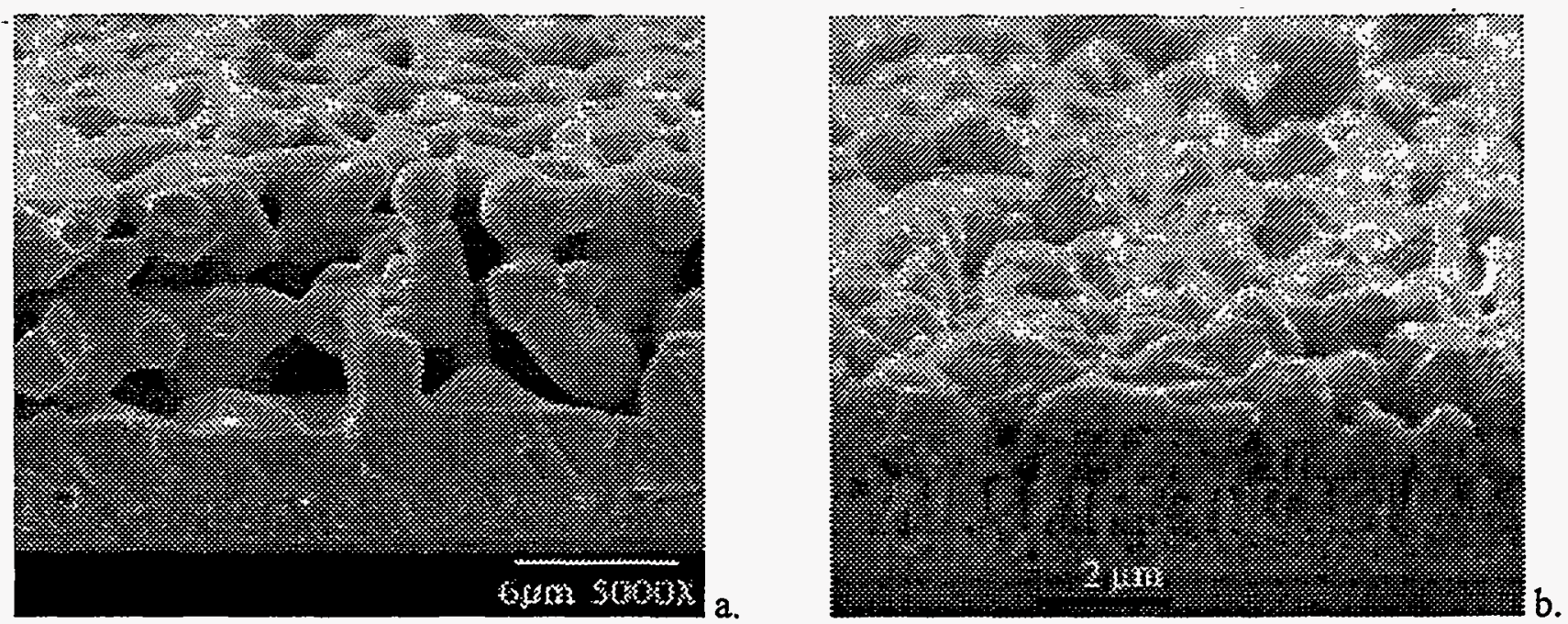

Figure 1. $\mathrm{CdCl}_{2}$-treated $\mathrm{CdTe}$ prepared from a (a) ball-milled precursor, and (b) nanoparticle precursor. 
Nanoparticle $\mathrm{Cu}-\mathrm{In}-\mathrm{Ga}-\mathrm{Se}$ Materials

Nanoparticle Synthesis: In the area of Cu-In-Ga-Se (CIGS) materials, we extended our simple metathesis reaction approach (Eq. 1) to the synthesis of CIGS nanoparticles. Although a metathesis reaction has been reported for the synthesis of colloidal CdTe, this was to our knowledge the first report for the solution synthesis of mixed-metal selenide nanoparticles. For example, $\mathrm{Cu}-\mathrm{In}$-Ga-Se nanoparticle colloids were prepared by reacting a mixture of $\mathrm{CuI}$, InI $\mathrm{I}_{3}$, and $\mathrm{GaI}_{3}$ with $\mathrm{Na}_{2} \mathrm{Se}$ as follows: ${ }^{3}$

$$
\begin{aligned}
& 1.10 \mathrm{CuI}+0.68 \mathrm{InI}_{3}+0.23 \mathrm{GaI}_{3}+1.91 \mathrm{Na}_{2} \mathrm{Se} \stackrel{\text { pyridine/methanol, }-3.82 \mathrm{NaI}}{\longrightarrow} \\
& \mathrm{Cu}-\mathrm{In}-\mathrm{Ga}-\mathrm{Se} \stackrel{400^{\circ} \mathrm{C}, \mathrm{N}_{2}, \mathrm{TGA}}{\longrightarrow} \mathrm{Cu} u_{1.10} \mathrm{In}_{0.68} \mathrm{Ga}_{0.23} \mathrm{Se}_{1.91}
\end{aligned}
$$

The overall size distribution for the resultant amorphous particles was estimated to be $10-30 \mathrm{~nm}^{3}$ Several other CIGS-related colloids including $\mathrm{Cu}_{2} \mathrm{Se}_{3} \mathrm{In}_{2} \mathrm{Se}_{3}, \mathrm{Ga}_{2} \mathrm{Se}_{3}, \mathrm{CuSe}$, and $\mathrm{CuInSe}_{2.5}$ were prepared via a similar low-temperature metathesis. ${ }^{3-5}$

These colloids were used as precursor inks for the spray deposition of CIGS thin films. It was determined that the stoichiometry of the films was nearly identical to that of the precursor colloid. For example, a Cu-In-Ga-Se colloid with a metal stoichiometry of $\mathrm{Cu}_{1.10} \mathrm{In}_{0.68} \mathrm{Ga}_{0.23}$ produced a $\mathrm{Cu}-\mathrm{In}$-Ga-Se precursor film with a metal stoichiometry of $\mathrm{Cu}_{1.13} \mathrm{In}_{0.64} \mathrm{Ga}_{0.23}$, a difference that is approximately equal to the error associated with the ICPAES measurement itself. The utility of this nanoparticle precursor method lies in the ability to control subsequent film stoichiometries via initial solution synthesis. Given limitations in both reactant and product solubility, we envision the extension of this metathesis-based approach to even the most complex materials compositions.

CIGS and CIS precursor films prepared by spraying nanoparticle colloids were thermally processed in an effort to promote crystallization and grain growth. We used several different approaches to produce large-grained films from small-grained precursors.

Approach \#1. Hybrid Spray Deposition and Evaporation: According to the hybrid approach, CIGS films with a Cu-rich stoichiometry were spray-deposited, transferred to an evaporator, and heated to $550^{\circ} \mathrm{C}$ under a selenium flux, soaked at that temperature for $10 \mathrm{~min}$, and then $\mathrm{Ga}$ and $\mathrm{In}$ were evaporated at $550^{\circ} \mathrm{C}$ as a means of bringing the composition of the $\mathrm{Cu}$ rich absorber back to near stoichiometric CIGS. SEM characterization showed that flux-assisted grain growth did not occur in the nanoparticle-derived $\mathrm{Cu}-\mathrm{In}-\mathrm{Ga}-\mathrm{Se}$ precursor layer. The best CIGS device produced by the hybrid process was $8.0 \%$ efficient. $^{3}$

Given the lack of large-grain development while collaborating with the CIS team at NREL, we set out with a new goal to form large-grained CIS from small-grained precursors according to a completely nonvacuum approach while attempting to access a CuSe liquid-assisted growth regime. ${ }^{4}$

Approach \#2. CuInSe .5 Mo Precursor Film: For this approach we used a precursor film with the nominal stoichiometry CuInSe 2.5 . This film contained $25 \%$ molar excess Se with respect to the previously used $\mathrm{CuInSe}_{2}$ precursor. The as-sprayed $\mathrm{CuInSe}$ 2.5 $_{2} / \mathrm{Mo}$ film morphology was a uniform nanoporous deposit. After annealing under static $\dot{\mathrm{N}}_{2}$ at $600^{\circ} \mathrm{C}$ for $20 \mathrm{~min}$, SEM characterization of the annealed film showed little evidence of liquid-assisted growth. XRD data showed $\mathrm{CuInSe}_{2}$ phase formation. ${ }^{5}$

Approach \#3. CuSe/In $2 \mathrm{Se}_{3} / \mathrm{Mo}$ Precursor Film: $\mathrm{CuSe} / \mathrm{In}_{2} \mathrm{Se}_{3} / \mathrm{Mo}$ composite films were used to probe whether the lack of evidence for liquid-assisted growth may be due to the binary 
selenides (i.e., $\mathrm{CuSe}$ and $\mathrm{In}_{2} \mathrm{Se}_{3}$ ) forming the product $\mathrm{CuInSe}_{2}$ prior to melting of the CuSe phase owing to intimate particle mixing in the $\mathrm{CuInSe}_{2.5}$ precursor films. The as-sprayed $\mathrm{CuSe} / \mathrm{In}_{2} \mathrm{Se}_{3} / \mathrm{Mo}$ film morphology was a uniform $\mathrm{In}_{2} \mathrm{Se}_{3}$ intermediate layer and a nonuniform CuSe top layer. After annealing under static $\mathrm{N}_{2}$ at $600^{\circ} \mathrm{C}$ for 20 minutes, little evidence of liquidassisted growth and CIS phase were observed by SEM and XRD, respectively.

Approach \#4. Annealing CuInSe $2.5 / \mathrm{Mo}$ and CuSe/In $2 \mathrm{Se}_{3} / \mathrm{Mo}$ Precursor Films in a Sealed Tube: In an effort to provide an environment with a substantial $\cdot \mathrm{P}_{\mathrm{Se}}$ and form liquid-phase $\mathrm{CuSe}$, CuInSe $.5 / \mathrm{Mo}$ and $\mathrm{CuSe} / \mathrm{In}_{2} \mathrm{Se}_{3} / \mathrm{Mo}$ precursor films were subjected to annealing in the presence of $\mathrm{Se}_{2}(\mathrm{~g})$ in a sealed tube. After annealing the precursor films at $537^{\circ} \mathrm{C}$ for $1 \mathrm{~h}$ in the sealed tube, SEM characterization showed no large-grain growth in the nanoparticle layer for the $\mathrm{CuSe} / \mathrm{In}_{2} \mathrm{Se}_{3} / \mathrm{Mo}$ film and only large crystals extending upward from the nanoparticle layer for the $\mathrm{CuInSe}_{2.5} / \mathrm{Mo}$ film. Also, a major MoSe 2 phase was observed, which limits this approach.

Approach \#5. Spray Deposition of CIGS on $\mathrm{CdS} / \mathrm{SnO}_{2} / \mathrm{Glass}$ : In an example of serendipity, we observed the formation of a dense layer upon spraying CIGS colloid onto a CdS film. Accordingly, a $\mathrm{Cu}_{1.10} \mathrm{In}_{0.68} \mathrm{Ga}_{0.23} \mathrm{Se}_{\mathrm{x}}$ colloid was spray-deposited onto a $\mathrm{CdS} / \mathrm{SnO}_{2} /$ glass substrate at $225^{\circ} \mathrm{C}$. Figure 2 shows SEM micrographs of one such film in which the CIGS layer appears to be very dense. XRD characterization of this as-sprayed film shows only peaks due to the substrate as determined in a control sample (i.e., $\mathrm{CdS} / \mathrm{SnO}_{2} /$ glass). After annealing at $550^{\circ} \mathrm{C} / 10$ min under $\mathrm{Ar}$, interdiffusion of $\mathrm{Cd}$ and $\mathrm{S}$ into the CIGS layer occurs with no CIGS phase formation. ${ }^{5}$ Although the goal of forming a dense film from a nanoparticle inks seems to have been reached by this example, the mechanism of this growth phenomenon is yet to be determined.
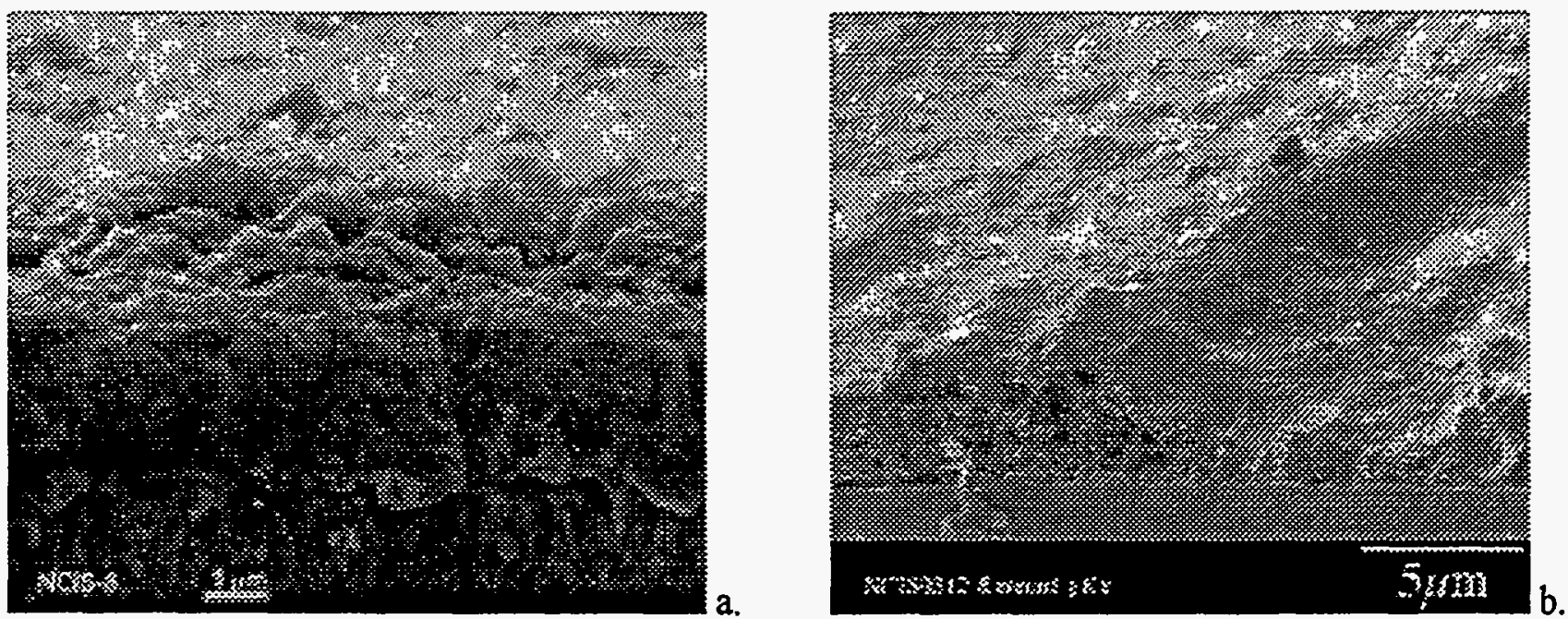

Figure 2. SEM photos of a CIGS/CdSSnO ${ }_{2}$ /glass film as-sprayed at $225^{\circ} \mathrm{C}$ at (a) hi mag and (b) low mag.

\section{Nanoparticle Contacts to Silicon Materials}

Nanoparticle $\mathrm{Al}$ and $\mathrm{Ag}$ prepared by the exploding-wire process (Argonide, Inc.) have been used for contacts to $\mathrm{p}$ - and $\mathrm{n}$-type $\mathrm{Si}$, respectively. Toward this end, nano- $\mathrm{Al}$ and nano- $\mathrm{Ag}$ were applied in slurry form to p-type and n-type Si films, respectively. After annealing these nano-Al/p-Si and nano-Ag/n-Si films above the eutectic temperatures, the metal contacts were characterized by nearly linear IV curves. 
Nanoparticle Spray Deposition of Transparent Conducting Oxides

Thin films of $\mathrm{SnO}_{2}$ were prepared by colloidal spray deposition. Aqueous $\mathrm{SnO}_{2}$ colloid, purchased commercially (Alfa), was diluted with water, various surfactants were added and the resulting ink was spray-deposited using an ultrasonic spray head. There was a correlation between the use of particular surfactants and film quality. Although several parameters such as deposition temperature, colloid delivery rate, surfactant type, etc. were varied, the UV-Vis spectra $(400-2000 \mathrm{~nm})$ were all very similar with $\% \mathrm{~T}>75 \%$.

Textured Buffer Layers for Low-cost Substrates/p-type TCOs

We have completed a unique deposition chamber specifically developed for deposition of textured layers that combines ion-beam assisted vapor deposition through the application of a soft ion gun and off-axis pulsed laser deposition. The chamber has been used for initial growth of the $\mathrm{LaNiO}_{3}$ layers as well as $\mathrm{SrTiO}_{3}, \mathrm{LaAlO}_{3}$, and $\mathrm{CeO}_{2}$. We obtained biaxially textured films for all of the materials, and are working on evaluating buffer layers suitable for PV materials and $\mathrm{CuAlO}_{2}$ as a p-type TCO.

\section{Industrial Interest}

UNISUN is a small photovoltaic company based in Newbury Park, California, which is interested in developing low-cost routes to $\mathrm{CuInSe}$ solar cells. Final cooperative research and development agreement negotiations are under way.

Argonide is a small Sanford, Florida-based company interested in developing nano-sized metal particles produced by the exploding-wire process. The Thrust II proposal to USIC is pending.

Spire Corporation has been in contact with the NREL New Ideas Subteam and the CdTe Team regarding a work-for-others contract. Spire is interested in thick semiconductor films for detector technologies.

Recently, the Basic Energy Sciences/Materials Science Division has provided a new source of funding for this research while the Chemical Sciences Division continues its support.

\section{Conclusions}

Most of our work was on the continuing development of nanoparticle precursors. Progress was made on CdTe, CIGS, and Al colloid inks. We observed that the enhanced reactivity of the nanoparticles dramatically changes the nature of the sintering processes. In the case of CIGS, phase formation occurs more rapidly than liquid-phase sintering. We are developing grain boundary engineering approaches to take advantage of this reactivity. The aluminum colloids appear to make ohmic contacts, potentially leading to an alternative approach to screen printing for contacts to silicon solar cells. We developed and tested the appropriate instrumentation to investigate textured growth and p-type TCOs:

\section{References (1997 Publications)}

(1) Schulz, D. L.; Pehnt, M.; Rose, D. H.; Urgiles, E.; Cahill, A. F.; Niles, D. W.; Jones, K. M.; Ellingson, R. J.; Curtis, C. J.; Ginley, D. S. Chem. Mater. 1997, 9, 889-900.

(2) Schulz, D. L.; Pehnt, M.; Urgiles, E.; Niles, D. W.; Jones, K. M.; Curtis, C. J.; Ginley, D. S. 25th IEEE Photovoltaics Specialists Conference Proceedings 1997, 929-932. 
(3) Schulz, D. L.; Curtis, C. J.; Flitton, R. A.; Wiesner, H.; Keane, J.; Matson, R. J.; Jones, K. M.; Parilla, P. A.; Noufi, R.; Ginley, D. S. J. Electron. Mater. (in press).

(4) Schulz, D. L.; Curtis, C. J.; Flitton, R. A.; Ginley, D. S. Mater. Res. Soc. Symp. Proc. (submitted).

(5) Schulz, D. L.; Curtis, C. J.; Cram, A.; Alleman, J. L.; Mason, A.; Matson, R. J.; Perkins, J. D.; Ginley, D. S. 26th IEEE Photovoltaic Specialists Conference Proceedings 1998, 483-486. 
Title:

Organization:

Contributors:

\section{NREL Clean Room/Device Fabrication Team Activities}

Center for Photovoltaics and Electronic Materials

National Renewable Energy Laboratory, Golden, CO

Team Members: Tim Gessert (Team Leader), M. Cudzinovic (Assist. 1/975/97), A. Szalaj (Assist. 6/97-), J. Alleman, C. Allman (Admin. Assistant), D. Bertram, A. Cahill, W. Chen, M. Contreras, A. Duda, D. Friedman, D Ginley, J. Keane, C. Kramer, S. Kurtz, M. Landry, X. Li, W. McMahon, K. Melerberg, A. Natarajan, J. Olson, M. Page, P. Parilla, R. Ribelin, D. Rose, L. Roybal, S. Smith, J. Thiesen, S. Tsuo, T. Wangensteen, J. Ward, X. Wu, D. Young

\section{Objectives}

The Clean Room/Device Fabrication Team provides the tools and procedures necessary to produce state-of-the-art test structures and devices from semiconductor materials synthesized by NREL or its subcontractors. The team also provides a "crossroads" at which researchers working on various technologies can mutually benefit from shared information and experience. The team maintains an integrated, multidisciplinary facility comprising $~ 3500$ square feet of Class 1000 clean-room space and housing approximately $\$ 2$ million in equipment. Major equipmént includes photolithographic processing, vacuum metallization, electrolytic metallization, and thermal processing. The team also maintains characterization equipment and techniques appropriate for process monitoring and development. About 33 researchers were team members during FY 1997. These individuals were from various "home" teams in the Photovoltaics (PV) \& Electronic Materials (EM), Basic Sciences, and Measurements and Characterization Centers. Most team members use the team facilities on either a daily or weekly basis.

\section{Technical Approach}

The Clean Room/Device Fabrication Team uses the Solar Energy Research Facility (SERF) Clean Room facility and related characterization equipment to fabricate devices from semiconductor materials produced by their home teams or subcontractors. Process activities can also be performed for non-team members either by a mutually beneficial collaboration between team and non-team members or by providing project-specific funds. Although the individual team members work with a wide variety of material systems and related technologies, the procedure used to develop processes for device fabrication is similar. This will often involve review of related literature/documentation, design and production of required photomasks, preliminary process experimentation to optimize individual processing steps, process feasibility studies to determine how steps interact, and use of an entire process sequence to produce desired devices or structures. The time required for a process development sequence can range from hours to months, depending on the complexity of the process and availability of related information. Because of this extensive effort to develop individual processes, team members are encouraged to maintain awareness of other activities, and share information and/or experiences when appropriate. Team organizational functions are aimed at facilitating this transfer of process 
information. Within guidelines established by the team, a team member is free to use the Clean Room facilities on an as-needed basis. Team members that charge to the Clean Room/Device Fabrication task (currently 0.3 FTE plus postdoc) perform functions related to developing team organization, maintaining facility equipment, providing training in the use of process equipment and procedures, acquiring and providing process and environment, safety, and health (ES\&H) documentation, performing business development activities, and providing long-term planning for the team and facilities.

\section{Studies and Accomplishments}

Following is a partial summary of studies and accomplishments resulting from work by Clean Room Team members and related collaborations. The list is organized by citing the home team of the researcher that performed the work, or the home team of the collaborating partner.

\section{III-V Materials and Devices Team (Center for PV \& EM)}

* Use of vacuum-deposition equipment for applications of antireflection coatings.

* Routine processing of III-V devices including use of plated Au for front and back contacts, and vacuum evaporation for processing of $\mathrm{Ti} / \mathrm{Pd} / \mathrm{Ag} / \mathrm{Pd}$.top contacts. Processing and chemical etching for device isolation and to enable profilometry of epilayer thickness.

* Studies to investigate the use of very-thick photoresist layers for the production of thick depositions of plated $\mathrm{Au}$.

* Continued process development of an improved metallization for high-concentration devices. This multilayer metallization involved high-profile grid lines $(3 \mu \mathrm{m}$ wide $\times 9 \mu \mathrm{m}$ high).

\section{CdTe Team (Center for PV \& EM)}

* Routine deposition of $\mathrm{MgF}_{2}$ antireflective coatings $(\sim 1000 \AA)$ onto the glass side of finished $\mathrm{CdS} / \mathrm{CdTe}$ devices prior to efficiency measurement. These coatings enabled a FY 1997 NREL-confirmed efficiency result of $14.6 \%$ for an NREL-produced CdS/CdTe device.

* Use of the Clean Room's cleaning apparatus and procedures became routine for preparation of all 3" $x$ 3" Corning 7059 glass used for close-spaced sublimation (CSS) device fabrication by the CdTe Team.

* Use of Clean Room automatic ellipsometer to assist in development of chemical vapor deposition (CVD) $\mathrm{SiO}_{2}$ and $\mathrm{SnO}_{2}$ layers. This instrument can produce numerous measurements of optical index and layer thickness very quickly and is invaluable for analyzing CVD deposit uniformity.

* Developed photolithographic/chemical-etch process to isolate CdTe devices fabricated with sputtered ZnTe back contacts.

* Developed process to photolithographically define contact area of graphite-based contact using patterned polyimide layer. This process involved use of the infrared mask aligner to align the Ti device aperture to the patterned $\mathrm{CdS} / \mathrm{CdTe}$ device through the CdTe layer. 


\section{CIS Team (Center for PV \& EM)}

* Preliminary work was performed to produce a CIS minimodule using wet chemical etching.

* Used clean environment for routine production of Mo-coated substrates for CIS growth. By cleaning the substrates in the Clean Room (Class 10 hood), and locating the Mo sputtering system in an adjacent area (Class 1000), pinhole formation is reduced.

* Significant modifications of the Mo-coating system were completed during FY 1997. These modifications allow larger batches, post/predeposition heating, and improved uniformity.

* Cleaning procedures for glass substrates were improved significantly during FY 1997 through the installation of a heated, high-power ultrasonic cleaning tank. These changes have improved batch-to-batch reproducibility significantly.

\section{TPV Team (Center for PV \& EM)}

* This externally funded activity performed routine processes to fabricate photovoltaic devices with band gaps required to convert the thermal radiation directly into electricity. The activity developed and used several novel processes that are now part of the Clean Room Team's experience base, and that are available for the PV Program. Many of these have already been used by other teams (e.g., see fabrication of "free-floating films" for the Spectroscopy Team).

* Expanded processes for and tooling of monolithic interconnected module (MIM) process to enable fabrication of a $5.2-\mathrm{cm}^{2}$ device (18 interconnected $0.74-\mathrm{eV}$ devices on a single substrate with two contacts). Related processes are currently being adapted to terrestrial PV devices.

* Developed processes required to fabricate a $0.6-\mathrm{eV}$ mismatched MIM device. Efforts are presently under way to increase device area from $0.25 \mathrm{~cm}^{2}$ to $1.0 \mathrm{~cm}^{2}$.

* Vacuum (electron-beam) deposition of high-quality Au for backside-reflector layers (BSRs) onto thermophotovoltaic (TPV) devices, and Ti/Pd/Ag/Pd front contacts.

* Vacuum deposition of $\mathrm{MgF}_{2}$ films ( $1300 \AA$ ) for use as a dielectric-spacer layer/diffusionbarrier layer/optical element between a Au BSR and the active TPV device.

* Routine use of vacuum deposition of $\mathrm{ZnT} / \mathrm{MgF}_{2}$ antireflection coatings on TPV devices.

\section{Crystal Growth and Devices Team (Center for Basic Sciences)}

* Routine fabrication of diagnostic crystalline silicon solar cells using electron-beam Al for the back contact, and $\mathrm{T} / \mathrm{Pd} / \mathrm{Ag}$ metallization (through shadow masks) for the front contact. Photolithography is used for mesa etching to isolate the small diagnostic devices. Control cells with $1-\mathrm{cm}$ by $1-\mathrm{cm}$ area fabricated on commercially available single-crystal silicon wafers demonstrated efficiencies of $\sim 12 \%$ without antireflection coating or oxide passivation.

* A computer control system for the eight-tube quartz furnace of the Thermal-Processing Clean Room was installed, tested, and is now in routine use. A dedicated wet-processing bench for silicon solar cells was also installed in this laboratory.

* A 12-inch by 12-inch format screen printer was installed in the Thermal Processing Clean Room in FY 1997. Initial studies involved fabrication of contacts to crystalline Si devices. The printer was also used to study a novel method of printing glass/Si frits with the goal of producing low-cost, PV-quality Si substrates. To assist these studies, one of the quartz tubes of the eight-tube furnace has been modified for drying and firing screen-printed materials. 
* Study effects of various heat treatments, including optical processing, on high-quality Al metallizations on crystalline $\mathrm{Si}$. This work involved use of a dedicated $\mathrm{Al} \mathrm{E-Beam} \mathrm{evaporator}$ to deposit numerous high-quality Al films, and thermal processing of these samples.

\section{Superconductivity Team (Center for Basic Sciences)}

* Electron-beam deposition of $300-\AA \mathrm{Ag}$ onto LaAl substrates for use as electric contacts. Deposition rate optimized to enhance thickness uniformity. Approximately $1000.5-\mathrm{cm}^{2}$ substrates were coated in FY 1997.

* Designed and produced improved mask set and related processes to fabricate test structures for critical current measurement. Collaborative research with Center for Advanced Microstructures and Devices, Louisiana State University.

\section{Energy Storage Team (Center for Basic Sciences)}

* Use spin processing to produce quantum-dot-containing polymer films onto mica substrates. Developed photolithographic processes necessary to form micro-tipped metal patterns onto the quantum-dot films. This enabled individual dots to be placed in a controllable electric field.

* Vacuum deposition of $\mathrm{MgF}_{2}$ antireflection coatings to study hydrogen production. Collaborative research with the Techian Israel Institute of Technology.

\section{Spectroscopy Team (Center for Basic Sciences)}

* Clean Room Team members fabricated "free-floating films" of GaInP for photoluminescence (PL) analysis. The ability to remove these $\sim 2-\mu \mathrm{m}$-thick epitaxial films from the GaAs substrate enabled improved PL analysis that was helpful in understanding "ordering" in III-V materials. Study has led to three publications in FY 1997 with a Clean Room Team member as coauthor.

* Clean Room Team members performed processing to remove GaInAs from AlInAs superlattice structures on InP substrates. This development was performed in collaboration with Sandia National Laboratories.

* Performed "groove etching" on GaAs to expose (111) surface to enable investigation of ordered growth.

* Etched through InP substrate to reveal an InAlAs single-crystal, thin-film window (2-10 $\mu \mathrm{m}$ thick window). This is used in PL analysis.

\section{Analytical Microscopy Team (Center for Measurements and Characterization)}

* Production of metallized fiber-optic probes used to obtain a sub-wavelength point source of light. In this process, the tapered portion of a specially prepared optical fiber is coated with evaporated Al during rotation to produce a sub-wavelength aperture at the apex of the taper. These probes are used for near-field scanning optical microscopy by members of the Center for Measurements and Characterization.

\section{Chemical Sciences Team (Center for Basic Sciences)}

* Removed free-floating films of GaAs from GaAs substrates by using a stop-etch layer. These films were affixed (Van der Waals force) to quartz microbalance crystals to study possible 
physisorption and chemisorption processes of cobalt-containing species from liquid electrolytes.

* Clean Room Team members produced Pt metallizations on soda-lime glass substrates for use in dye-synthesized solar cell research. To produce the required film adhesion, alkalinecleaning procedures were used to prepare the substrate prior to multilevel E-beam metallization of a Ti adhesion layer $(400 \AA)$ and $\mathrm{Pt}(600 \AA)$. Approximately $200 \mathrm{Pt}$-coated substrates were produced for this effort during FY 1997. The costs of processing and precious-metal consumption were shared between the Clean Room and Chemical Sciences Teams.

Electro-Optical Characterization Team (Center for Measurements and Characterization)

* The automatic ellipsometer in the Clean Room was used routinely to calibrate the wavelengthscanning ellipsometer. The automatic ellipsometer is well suited for this application because the Clean Room Team maintains ellipsometric calibration standards.

* Electron-beam evaporation of metals ( $\mathrm{Cr}, \mathrm{Sn}, \mathrm{Pt}, \mathrm{Al}, \mathrm{Au}, \mathrm{Ag}$, and $\mathrm{Pd}$ ) onto thin-film $\mathrm{CdTe}$ for use in PL analysis of contacted vs. noncontacted CdS/CdTe devices (collaborative effort between Electro-Optical Characterization, CdTe, and Clean Room Teams).

\section{Conclusions and Future Directions}

The Clean Room/Device Fabrication Team was created in 1996. Because of the team's brief history, efforts continue to focus on providing the process capability critical to enhancing the PV program, while developing longer-term objectives within the framework of available personnel, equipment, and program support. This involves identifying and prioritizing the needs of the team members, developing a method to assign critical maintenance and administrative responsibilities, fostering beneficial collaborations, and learning how to share and transfer the team's information base effectively. As indicated by the preceding list of the team's studies and accomplishments, these efforts have led to some significant accomplishments and synergistic collaborations during the past year. However, several important team objectives remain undeveloped, and it is here where a portion of next year's efforts will be placed.

\section{Business Development}

The Clean Room task supports 0.1 FTE who assists the team in business development activities. In FY 1997, these activities focused on working with the NREL Photo and Graphics departments to produce a set of 16 digitized photographs, and making these generally available through the PIX Library on the NREL Web site. The team intends to use several of these images to produce a brochure that would further outline our capabilities. By providing this "virtual lab tour," as well as (eventually) a more detailed brochure, the team intends to allow prospective users to visualize our capabilities with regard to potential collaborations.

\section{Facility Development}

* Procurement and installation of a precision probe station and related electronic test equipment for the Vacuum Processing Clean Room.

* Procurement, installation, and process development of wire-bonding equipment.

* Procurement of laboratory casework to facilitate laboratory storage. 
* Complete the installation of light switches in all Clean Room laboratories to allow heating, ventilating, and air-conditioning to perform closer to design specifications.

* Follow-up of submitted Facilities Work Requests to install power-conditioning hardware that will limit the detrimental effects of power outages on the Clean Room's process equipment. 
Title:

Thin-Film CdS/CdTe Solar Cell Development

Organization: $\quad$ CdTe Team

National Renewable Energy Laboratory, Golden, CO

Contributors: $\quad$ P. Sheldon (Team Leader), D.S. Albin, M. Al-Jassim, S.A. Asher, H. Cheong, T.J. Coutts, R.G. Dhere, T.A. Gessert, F. Hasoon, B. Keyes,

D. Levi, X. Li, Y. Mahathongdy, A. Mascarenhas, R. Matson, H. Moutinho, C. Narayanswamy, D. Niles, R. Ribelin, D. Rose,

A. Swartzlander-Guest, D. Waters, L. Woods, X. Wu

\section{Objectives/Technical Approach:}

The three primary objectives of the CdTe Team are: 1) to develop reproducible processes for high-efficiency CdS/CdTe devices and to improve our fundamental understanding of the physics and chemistry of the device; 2) to explore alternative processes and device structures that lend themselves to improved device performance, basic understanding, reproducibility, and/or manufacturability; and 3) to provide strong industrial partner support.

The CdTe Team has developed a 3-year roadmap outlining long-term research goals and activities. This roadmap is divided into four sections that address critical portions of the device structure. These include the transparent conducting oxide (TCO) layer, the CdS/window layer, the CdTe absorber/junction, and the back contact. In support of these research activities, we have dedicated considerable resources towards developing new characterization tools and techniques that will allow us to probe critical interfaces. In FY 1997, the CdTe Team focused on a wide variety of research projects in each of these areas.

\section{Results:}

In December 1997 we fabricated an NREL-confirmed 15.4\%-efficient CdS/CdTe device. This new in-house record device is the culmination of a number of ongoing research activities within the NREL CdTe Team. Although this result will officially be reported in the FY 1998 annual report, many of the results reported in FY 1997 had a significant impact on this achievement.

\section{CVD Tin Oxide Development and Stability:}

In FY 1996, we installed and commissioned a low-pressure chemical vapor deposition (CVD) system configured to deposit both $\mathrm{SnO}_{2}$ and $\mathrm{SiO}_{2}$ thin films. Previously, the CdTe team had to rely on external vendors for $\mathrm{SnO}_{2}$-coated substrates. Procuring substrates of consistent and acceptable quality was problematic. This new capability allows the CdTe Team, and the industry we support, the opportunity to use optimized single and multilayer TCO films. In FY 1997, we deposited the first undoped and fluorine-doped $\mathrm{SnO}_{2}$ films with excellent uniformity (better than $\pm 2.0 \%$ over a $4 " \mathrm{x} 4$ " substrate). Our goal was to develop a $\mathrm{SnO}_{2}$ process that produces TCO coatings equivalent to, or better than, the baseline $\mathrm{SnO}_{2}$ films procured from Solarex Corporation and integrate them into our baseline process. As seen in Figure 1, this was accomplished by producing films with an average transmission of $>80 \%$ and sheet resistances of $<7.5 \Omega /$ square. These films also have high mobilities $\left(40 \mathrm{~cm}^{2} / \mathrm{V}-\mathrm{s}\right)$ and extremely low surface roughness $\left(\mathrm{Ra}=100 \AA\right.$ for films deposited at $\left.500^{\circ} \mathrm{C}\right){ }^{1}$ 


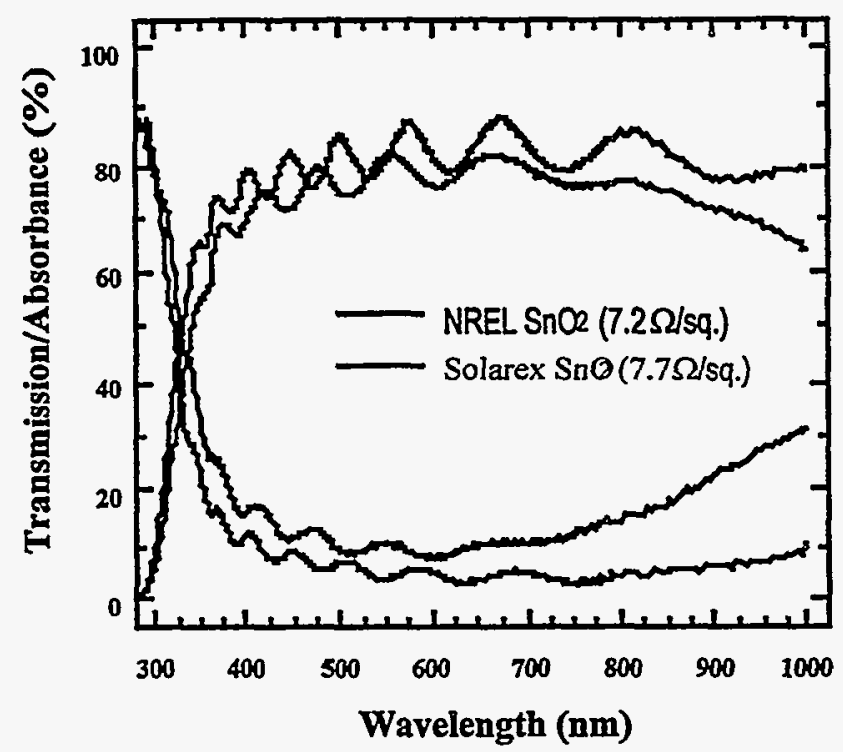

Fig. 1. Comparison of transmittance/absorbance between NREL and Solarex $\mathrm{SnO}_{2}$ films

We have also developed a bilayer (undoped $\mathrm{SnO}_{2} /$ doped $\mathrm{SnO}_{2}$ ) process. The purpose of the undoped or $\mathrm{i}-\mathrm{SnO}_{2}$ layer is to help maintain high $\mathrm{V}_{\mathrm{oc}} \mathrm{s}$ when pinholes in the CdS are present, thereby reducing shunt paths. Previously, we had deposited this layer by sputter deposition; however, we found that these layers were chemically unstable in both hydrogen and at high temperatures. This instability, caused by $\mathrm{SnO}_{2}$ reduction and the formation of $\mathrm{Sn}$ compounds on the $\mathrm{CdS}$ surface prior to CdTe deposition, yielded a loss in device performance. We find that the CVDdeposited $\mathrm{i}-\mathrm{SnO}_{2}$ layers are very stable and thus minimize many of these processing issues. We have integrated both single and bilayer films into our baseline process with excellent results. (A bilayer substrate was used to fabricate our $15.4 \%$ device.) Industry has also taken advantage of this new capability, requesting a variety of custom TCO coatings not previously available to them. Since bringing this system on-line, we have deposited nearly $100 \mathrm{SnO}_{2}$ films for both industry and other NREL teams. We have supplied Solar Cells, Inc., International Solar Electric Technology (ISET), ITN Energy Systems, Optical Coatings Laboratory, Inc., and Schott Glass with custom TCO films.

\section{Cadmium Stannate TCO Development and Integration into CdS/CdTe Devices:}

The CdTe Team has continued research on a project to develop high-performance TCOs that could replace the $\mathrm{SnO}_{2}$ layer in a conventional CdS/CdTe device structure. One promising candidate is cadmium stannate $\left(\mathrm{Cd}_{2} \mathrm{SnO}_{4}\right.$ [CTO]), which has several significant advantages over conventional TCOs. It is more conductive, more transparent, has a lower surface roughness, is patternable, and is exceptionally stable. Resistivities as low as $1.3 \times 10^{-4} \Omega$-cm have been achieved in the best CTO samples, almost seven times lower than conventional $\mathrm{SnCl}_{4}$-based $\mathrm{SnO}_{2}$ films and 2.5 times lower than the best $\mathrm{SnO}_{2}$ films deposited using tetramethyltin precursors. We have integrated this into a device and produced the first-ever $\mathrm{Cd}_{2} \mathrm{SnO}_{4}$-based $\mathrm{CdS} / \mathrm{CdTe}$ polycrystalline thin-film solar cell with an efficiency of $14.0 \%$. 
The improved transmission and absorbance of CTO films is ideal for superstrate $\mathrm{CdS} / \mathrm{CdTe}$ devices and yields improved device $J_{s c}$. Fig. 2 shows the relationship between the TCO film transmission and the resulting $\mathrm{J}_{\mathrm{se}}$, for devices deposited on both CTO and $\mathrm{SnO}_{2}$ superstrates. It is clear that by replacing the $\mathrm{SnO}_{2}$ film with a CTO superstrate, the reduced optical losses yield an increase in $\mathrm{J}_{\text {se. }}{ }^{2}$ A patent application describing this device structure was filed with the U.S. Patent Office. These promising results have generated significant interest from industry, with requests from Solar Cells, Inc., and ANTEC for $\mathrm{Cd}_{2} \mathrm{SnO}_{4^{-}}$ coated substrates.

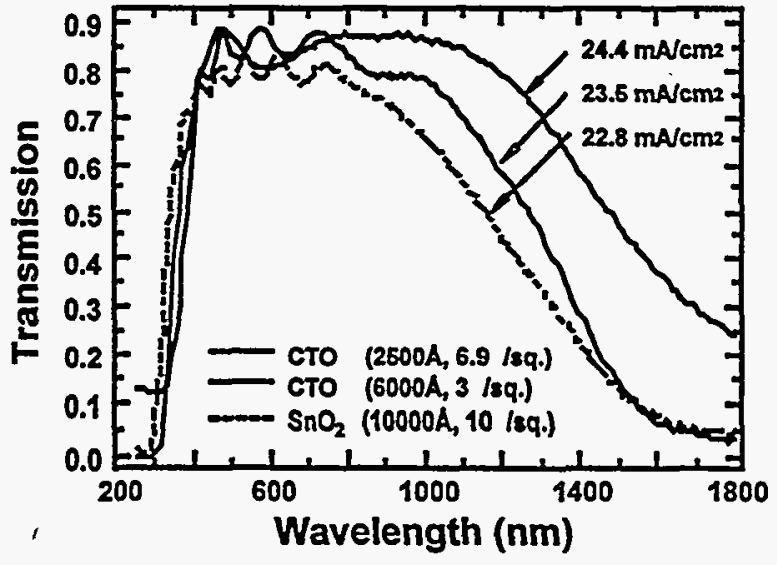

Fig. 2. Relationship between $\mathrm{J}_{\mathrm{sc}}$ and transmission of $\mathrm{SnO}_{2}$ and CTO films

\section{Characterization Technique Development:}

In collaboration with the Measurements and Characterization Center, we have focused considerable efforts on attaining compositional and electrical information about the critical $\mathrm{CdTe} / \mathrm{CdS}$ interface. Previously this data was difficult to obtain because of the thick CdTe layer obscuring the interface, making some characterization techniques difficult to apply. In some instances, this can be accommodated by growing thinner films; however, these films are not representative of the actual device structure. In FY 1997 we developed a "lift-off" technique that allows us to separate the sample at the CdS/CdTe interface, thereby directly exposing this interface as a surface. The surface of the lift-off samples is very smooth, making it ideal for a wide range of characterization tools. To date, we have applied this sample preparation technique to grazing-incidence X-ray diffraction (GIXRD) analysis, AC and DC conductivity measurements, microphotoluminescence ( $\mu \mathrm{PL}$ ), and surface analysis techniques (Auger electron spectroscopy [AES] and secondary ion mass spectrometry [SIMS]). This provides a unique opportunity to improve our understanding of the physics and chemistry of the device interface. Examples of GIXRD and $\mu \mathrm{PL}$ analysis applied to samples prepared using this technique are described below.

The lift-off sample preparation technique has greatly facilitated GIXRD analysis for direct measurement of the S-alloying at the CdS/CdTe interface. By varying the incident angle of Xrays directed towards the sample (using parallel-beam optics), we have successfully been able to measure $\mathrm{CdTe}_{1 \cdot \mathrm{x}} \mathrm{S}_{\mathrm{x}}$ alloying at the interface of finished devices. Such a study on a non- $\mathrm{CdCl}_{2}-$ treated film in which the incident angle was varied at $\mathrm{W}=0.1,0.2,0.3,0.5,1.0,2.0$, and $5.0^{\circ}$ is 


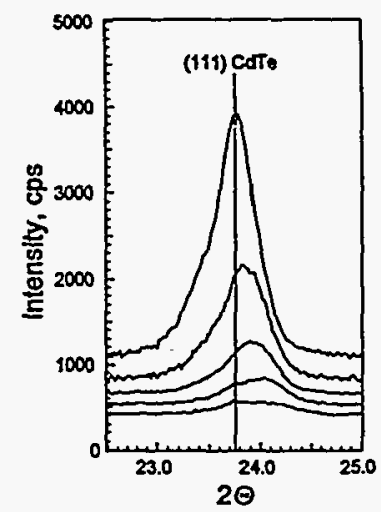

Fig. 3 GLXRD scans taken from a $\mathrm{CdS} / \mathrm{CdTe}$ interface shown in Fig. 3 for the (111) Bragg peak. GIXRD scans were taken with increasing penetration depth $(\mathrm{PD})$ into the $\mathrm{CdTe}$ layer (away from the CdS/CdTe interface). The bottom scan in Fig. 3 corresponds to $\Omega=0.1^{\circ}(\mathrm{PD}=22 \AA)$. The top scan corresponds to $\Omega=5.0^{\circ}(\mathrm{PD}=1.2 \mu \mathrm{m})$.

At very low penetration depths, we see two phases: a stoichiometric cubic-CdTe phase and a slightly $\mathrm{S}-$ containing $\mathrm{CdTe}_{1-x} \mathrm{~S}_{x}$ phase. As our penetration increases away from the CdS/CdTe interface, the value $\mathrm{x}$ in $\mathrm{CdTe}_{1-x}$ $S_{x}$ appears to decrease to a value of zero (i.e., pure CdTe) at an angle corresponding to a depth of approximately $3000-4000 \AA$ into the CdTe layer. This technique has been invaluable in assessing how processing parameters influence interface alloying (see, for example, CSS CdS Development section). ${ }^{3}$

In collaboration with the Solid-State Spectroscopy Group, we have analyzed samples prepared using the lift-off technique using $\mu \mathrm{PL}$ measurements. $\mu \mathrm{PL}$ provides spectral information with a lateral resolution of $<1 \mu \mathrm{m}$. By directly analyzing the CdS/CdTe interface, we have the opportunity to map the degree of alloying in grains and at grain boundaries. We find that the PL spectra from the CdTe side corresponds to the CdTe band gap and is uniform across the surface. In contrast, the PL spectra from the CdS side shows shifts in the peak position as a function of location. Spectra are shifted to lower energies with a larger bandwidth than the CdTe spectra taken from the back (contact side). These shifted spectra correspond to the alloyed region and the peak-broadening is due to the superposition of spectra corresponding to the range of alloys present in the layer. The spectra corresponding to the positions in the center of larger grains have a peak position at $1.543 \mathrm{eV}$ and symmetrical peak broadening (suggesting alloy compositions between $1.524 \mathrm{eV}$ and $1.569 \mathrm{eV}$ ). The spectrum corresponding to the center position of a small grain has a peak position at $1.535 \mathrm{eV}$ and is asymmetrical (indicating the contribution predominantly from the lower gap alloys between $1.52 \mathrm{eV}$ and $1.545 \mathrm{eV}$ ). The peaks corresponding to the grain boundaries have peak positions of $\sim 1.538 \mathrm{eV}$. Thus, we find that grain boundaries and smaller grains contain alloys with higher sulfur compositions than the center of larger grains (the result of higher sulfur diffusion along grain boundaries). ${ }^{4}$ To date, $\mu \mathrm{PL}$ is the only technique that allows this type of direct observation of the CdS/CdTe interface.

\section{Close-Spaced Sublimation (CSS) CdS Development:}

In FY 1997 we made progress in developing an alternative to chemical-bath-deposited (CBD) CdS. We successfully used CSS to grow CdS films with thicknesses ranging from 575-3000 $\AA$ (with no oxygen present during deposition). With an all-CSS process we have achieved device efficiencies of $10.7 \%\left(V_{o c}=0.731\right.$ volts, $J_{s c}=21.5 \mathrm{~mA} / \mathrm{cm}^{2}$, fill factor $\left.[F F]=68 \%\right)$. Using the liftoff technique, we identified significant differences in the composition and structure of the CdS/CdTe interface between devices produced from CBD and CSS CdS-coated superstrates. This is an important insight, in that $\mathrm{V}_{\mathrm{oc}}$ are greatly affected by the type of CdS layer used. CBD 
CdS devices typically exhibit $\mathrm{V}_{o} \mathrm{~s} 150 \mathrm{mV}$ greater than CSS CdS devices. Understanding the difference between these two interfaces is expected to help us understand what may limit $V_{o c}$ in $\mathrm{CdS} / \mathrm{CdTe}$ devices.

CdS films deposited at low temperatures by $\mathrm{CBD}\left(\sim 95^{\circ} \mathrm{C}\right)$ are characterized by small grain sizes (? $50 \mathrm{~nm}$ ) and poor cubic-phase crystallinity. In contrast, CSS CdS films grown at higher substrate temperatures $\left(-475^{\circ} \mathrm{C}\right.$ ) have much larger grain sizes (up to $400 \mathrm{~nm}$ ) and have much better hexagonal-phase crystallinity. When CdTe is deposited on both CSS and CBD-grown CdS films at $\sim 600^{\circ} \mathrm{C}$, we have observed much greater interfacial alloying, prior to $\mathrm{CdCl}_{2}$ treatments, for the smaller-grained CBD CdS films. Differences in sulfur alloying were measured by monitoring the (111) CdTe alloy peak position as a function of depth into the CdS/CdTe interface by GIXRD (described previously). As shown in Fig. 4, CBD CdS/CdTe interfaces show significantly greater alloying than CSS $\mathrm{CdS} / \mathrm{CdTe}$ interfaces. For interfaces using CBD CdS films, alloying reaches $12-13$ at.\% $\mathrm{S}$ in CdTe. In contrast, the CSS CdS films result in less than 4 at.\% $\mathrm{S}$ in CdTe at the same interface. ${ }^{3}$

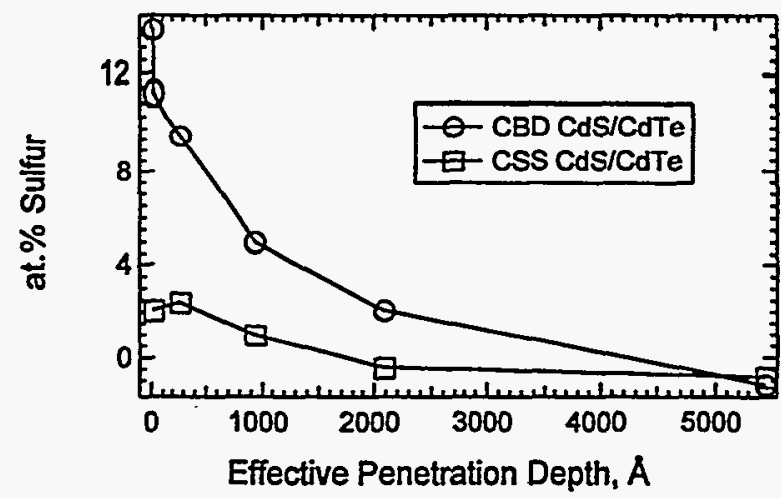

Fig. 4: Sulfur alloying in CdTe near the CdS/CdTe interface

The Role of Oxygen on CSS-Deposited CdTe and CdS/CdTe Devices:

The breakthrough that first enabled CdS/CdTe thin-film solar cells to break the $10 \%$ barrier was depositing CdTe by CSS in an oxygen ambient. In FY 1995/96 we showed that $\mathrm{O}_{2}$ was not necessary for high-efficiency cells but could be useful. It enhances nucleation (thus decreasing CdTe pinhole formation) and it lessens the harmful effects associated with $\mathrm{SnO}_{2}$ decomposition. In FY 1997 we continued this work and showed that $\mathrm{O}_{2}$ shifts CSS deposition from diffusion limited to reaction-limited growth primarily through source oxidation (an important manufacturing issue). We also showed that $\mathrm{O}_{2}$ partial pressures of $0.5-2.0$ torr encourage (111) orientation, which may increase $\mathrm{V}_{\mathrm{oc}}$. We showed that $\mathrm{O}_{2}$ can create particles and other features on the CdTe surface (most likely through the formation of a condensation barrier in the latter stages of growth). Growth in $\mathrm{O}_{2}$ did not passivate grain boundaries or decrease impurity diffusion along grain boundaries, but did improve device shunt resistances (possibly from improved mechanical properties). Most importantly, growth in $\mathrm{O}_{2}$ improves $\mathrm{CdS} / \mathrm{CdTe}$ junction quality despite decreasing the amount of $\mathrm{CdS} / \mathrm{CdTe}$ interdiffusion. Instead of acting as an acceptor, the primary benefits of $\mathrm{O}_{2}$ may be its ability to passivate donors and defects if they are present, and its effect as a reactive gas that ensures uniform growth initiation. ${ }^{5}$ The knowledge gained in this area improves our fundamental understanding of the process and of cell efficiency and reproducibility, and should aid manufacturers of CdTe modules using similar processes. 


\section{Vapor $\mathrm{CdCl}_{2}$ Process Development:}

In FY 1997, we commissioned a new deposition system dedicated to exploring Cl-based vapor treatments. Our goal is to develop a fast, reproducible, dry $\mathrm{CdCl}_{2}$ process that could replace the existing wet process. Although this study is only in the very preliminary stages, we have learned a great deal about several aspects of the dry process and how it differs from the wet process. In general, we find that the wet and dry $\mathrm{CdCl}_{2}$ processes produce comparable device performance. Although the dry process is still being optimized, we have already produced $13.6 \%$ devices (using non-optimized Solarex $\mathrm{SnO}_{2}$ substrates) with many indications that this approach will yield better reproducibility.

\section{ZnTe-based Contacts:}

We developed a sequence of dry processes that can be used sequentially to produce ohmic contacts to $\mathrm{CdS} / \mathrm{CdTe}$ devices at relatively high temperatures $\left(300^{\circ} \mathrm{C}\right)$. The steps of the contact process include ion-beam milling (to condition the Cl-treated p-CdTe surface), deposition of undoped $\mathrm{ZnTe}$, deposition of $\mathrm{Cu}$-doped $\mathrm{ZnTe}$, and deposition of Ni. Based on the available optimization data, we produced devices with good performance with material from NREL, Solar Cells, and Golden Photon, Inc. (GPI). We expect continued improvement in the performance of this contact as studies proceed. We also began stability studies of non-encapsulated devices. Although preliminary, these studies have begun to indicate the measurements needed to observe important trends, and suggest contact design changes that are necessary to improve future stability. These studies have also produced a set of baseline data to which later devices will be compared. 600 hours of light IV data indicate that devices contacted with the dry process demonstrate reasonable stability when annealed at $80^{\circ} \mathrm{C}$ in air, but that differences in stability are observed between devices supplied from different sources. ${ }^{6}$ Three patent applications describing this device structure and process have been filed with the U.S. Patent Office.

\section{CdTe National R\&D Team Activities:}

The CdTe Team actively participated in the National CdTe Teaming efforts. In addition to the collaborative work done on contact stability (described above), we participated in an investigation of the effect of various interfacial layers between the $\mathrm{SnO}_{2}$ and the $\mathrm{CdS}$. We explored four different types of interfacial layers, including $\mathrm{TiO}_{2}, \mathrm{Nb}$-doped $\mathrm{TiO}_{2}$, Golden Photon's proprietary high-resistivity layer (HRT), and an undoped $\mathrm{SnO}_{2}$ layer. For each type of interfacial layer, we processed devices with CdS layer thicknesses of 0-1600 $\AA$ (to help identify potential benefits of these interfacial layers). Several conclusions can be drawn. Devices with $\mathrm{TiO}_{2}$ interfacial layers were characterized by high $\mathrm{V}_{\mathrm{oc}}$ s but very poor fill factors and efficiencies. Substituting the $\mathrm{TiO}_{2}$ layer with a Nb-doped $\mathrm{TiO}_{2}$ layer, yielded improved fill factors. However, the $\mathrm{V}_{\mathrm{oc}} \mathrm{s}$ were low compared to conventional $\mathrm{SnO}_{2}$-based devices for all but the thinnest of $\mathrm{CdS}$ layers. Perhaps the most interesting result from this study was obtained from the Golden Photon substrates with the proprietary HRT layer applied. As shown in Fig. 5, these devices were able to maintain a high $\mathrm{V}_{o s} \mathrm{FF}$, and efficiency, even with $0 \AA$ of CdS (unlike the bilayer $\mathrm{SnO}_{2}$ films), which represents the first time we have been able to maintain high $V_{o} s$ without $C d S$ present. This may provide important clues as to how this device structure works. Work is ongoing. 

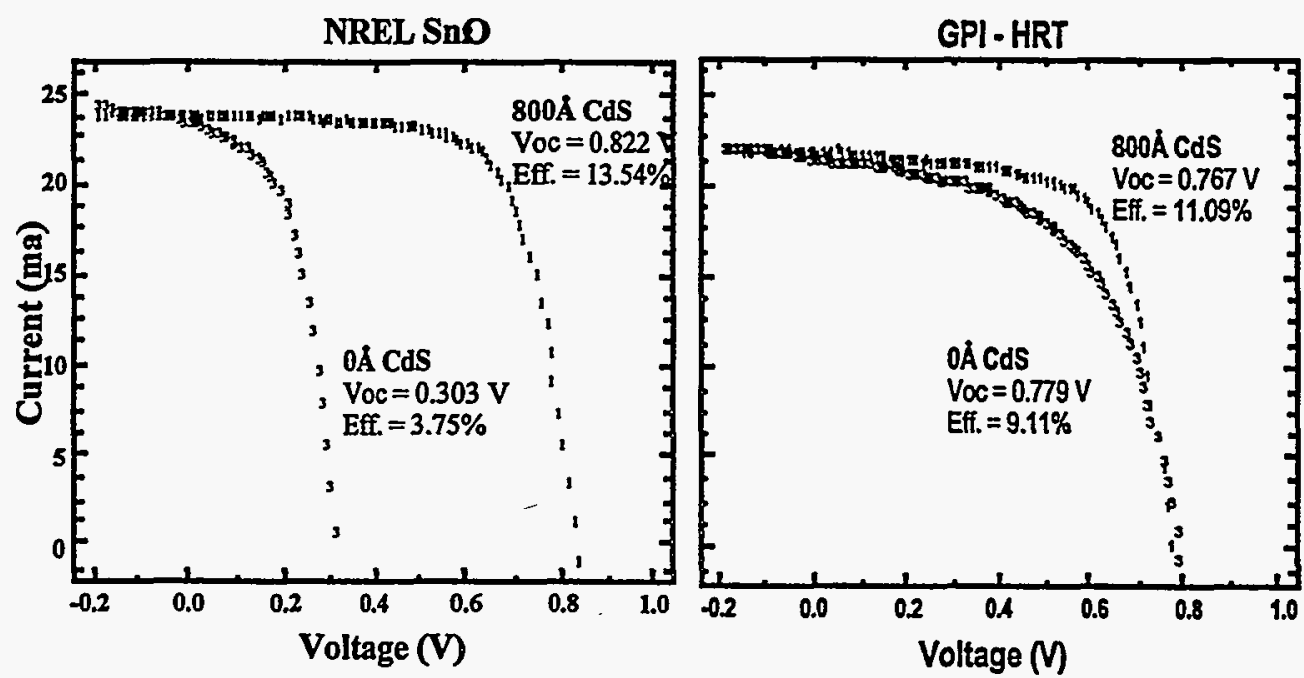

Fig. 5: IV data comparing NREL and GPI interfacial layers with different CdS thicknesses

\section{Future Directions:}

In FY 1998, we will optimize the bilayer $\mathrm{SnO}_{2}$ layer and integrate it into our baseline process as well as continue to develop the $\mathrm{Cd}_{2} \mathrm{SnO}_{4}$-based $\mathrm{TCO}$. We will continue our efforts to understand how interfacial layers between the TCO and the CdS influence device performance, and to explore alternatives. We will develop a dry vapor-based chloride treatment to replace the wet $\mathrm{CdCl}_{2}$ process and integrate it into our baseline. In addition, we will continue our efforts to characterize CdS/CdTe interface alloying and ìdentify how it affects device parameters. In FY 1998 we will also focus on more fundamental aspects of the back contact, including contact stability and degradation mechanisms. In addition, we will continue to work on collaborative projects initiated with our industrial and team partners through the Thin Film Partnership program. A detailed description of tasks is outlined in the CdTe FY 1998 Annual Operating Plan.

\section{$\underline{\text { References: }}$}

1. Li, X.; Gessert, T.A.; Ribelin, R.; Moutinho, H.; Sheldon, P. (1998). "Properties of Fluorinedoped Tin Oxide Films Produced by LPCVD using Tetramethyltin." (to be published in the Journal of Vacuum Science and Technology)

2. Wu, X.; Sheldon, P.; Coutts, T.J.; Rose, D.H.; Moutinho, H.R. (1997). "Applications of $\mathrm{Cd}_{2} \mathrm{SnO}_{4}$ Transparent Conducting Oxides in CdS/CdTe Thin-Film Devices." Proceedings of the 26th IEEE Photovoltaics Specialists Conference; September 29-October 3, 1997; Anaheim, California.

3. Albin, D.; Rose, D.; Dhere, R.; Levi, D.; Woods, L.; Swartzlander, A.; Sheldon, P. (1997). "Comparison Study of Close-Spaced Sublimated and Chemical Bath Deposited CdS Films: Effects on CdTe Solar Cells." Proceedings of the 26th IEEE Photovoltaics Specialists Conference; September 29-October 3, 1997; Anaheim, California. 
4. Dhere, R.; Rose, D.; Albin, D.; Asher, S.; Al-Jassim, M.; Cheong, H.; Swartzlander, A.; Moutinho, H.; Coutts, T.; Ribelin, R.; Sheldon, P. (1997). "Influence of CdS/CdTe Interface Properties on the Device Properties." Proceedings of the 26th IEEE Photovoltaics Specialists Conference; September 29-October 3, 1997; Anaheim, California.

5. Rose, D.H. (1997). The Effect of Oxygen on CdTe-Absorber Solar Cells Deposited by CloseSpaced Sublimation. Ph.D. Thesis. Boulder, CO: University of Colorado.

6. Gessert, T.A.; Sheldon, P.; Li, X.; Dunlavy, D.; Niles, D.; Sasala, R.; Albright, S. (1997). "Studies of ZnTe Back Contacts to CdS/CdTe Solar Cells." Proceedings of the 26th IEEE Photovoltaics Specialists Conference; September 29-October 3, 1997; Anaheim, California. 


\subsection{PV Measurements and Characterization Project}




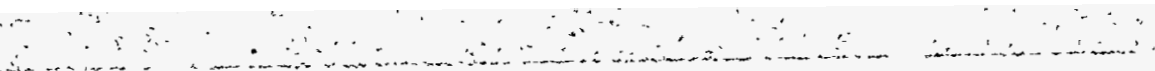




\title{
4.0 PV MEASUREMENTS AND CHARACTERIZATION PROJECT-Introduction
}

\author{
Project Leader: Lawrence L. Kazmerski
}

The Photovoltaic (PV) Program project staff perform thousands of measurements each year, partnering with industry, universities, research laboratories, as well as internal research efforts. The Measurements and Characterization Team within the National Center for Photovoltaics (NCPV) includes more than 40 experts who provide a wide range of analytical support. With state-of-theart equipment and facilities, our researchers study cell and module performance, electro-optical characterization, analytical microscopy, and surface and interface characterization capabilities. Our laboratory personnel provide analytical service support and collaborations, as well as measurement technique research to enhance and assist the PV research and development (R\&D) and industry communities. Scientists and engineers in these laboratories work closely with the major PV programs, leading and assisting efforts within the Thin-Film Partnership, Photovoltaic Manufacturing Technology (PVMaT), and the Concentrator Alliance. Staff also work on international technology transfer, standards development, and standard reporting assurance.

Our laboratories offer more than 40 techniques to determine the electrical and optical properties of materials and devices, as well as the chemistry, composition, topography, structure, and physical nature of materials, surfaces and interfaces. Through its Web site (www.nrel.gov/measurements), NCPV offers its collaborators and clients a secured data transfer facility to enhance customer interactions and improve measurement turnaround time. This facility currently links some 30 laboratories using the NREL measurement and characterization facilities, providing real-time and archival methods of data transfer, retrieval, and observation. In its startup period, more than $20 \mathrm{DOE}$ teams have collaborated in the evolution of this new method of electronic interaction. The majority of the NREL sample evaluations require independent analysis for quality assurance - especially for contract deliverables and for validation of milestones and record results. NREL maintains standards (physical standards and techniques), knowledge of standard conditions, and leads in the world PV standards community to ensure quality and validity of results. These programs are the models for quality assurance for the PV Program and the National Renewable Energy Laboratory.

Our organization also maintains technical leadership in the standard testing of solar cells and modules-and assists R\&D and industry efforts by providing cost-effective measurements and characterization service, research, and technique development. In FY 1997, the Cell and Module Performance Team was recognized by its NREL peers with an Outstanding Team Performance Award for its extraordinary accomplishments and service to the world PV community. Also, in FY 1997, the measurements and characterization staff performed more than 40,000 measurements on some 15,000 samples representing every photovoltaic technology. The majority of these tests and measurements are tied to contract deliverables, program milestones, and standard verifications-prioritized in the annual operating plan of the Photovoltaics Program. Support and assistance for the Million Solar Roofs Initiative has been identified as a priority, and the Measurements and Characterization Team will continue to assist the validation and deployment strategies for this important program. The technique development efforts address measurements specific to 
photovoltaic industry needs and the evolvement of manufacturing-environment methods. Several examples are summarized in each of the more detailed summaries that follow this introduction. In addition, our staff contributed 65 journal articles, conference proceedings, and books during this past year, approximately $70 \%$ of which were written with research collaborators from the internal and subcontractor communities.

About $15 \%$ of our activities are directed toward the research and development of measurement techniques, many of which are specific to PV technologies. New or improved analytical offerings during this past year include:

- Large-area, continuous solar simulator for the evaluation of cells and modules under standard conditions for flat-plate and concentrator technologies

- Time-of-flight secondary ion mass spectrometer for high-resolution determination of impurities in cell materials

- Large-area laser scanner for versatile module diagnostics, noncontact spectrometer for determining the minority-carrier lifetimes and diffusion lengths in semiconductors over the bandgap range from under $0.4 \mathrm{eV}$ through more than $4.0 \mathrm{eV}$

- Scanning micro-Fourier transform infrared system for examination of insulators through conductors

- Near-field scanning optical microscope for nanoscale imaging and spectroscopic studies of semiconductors.

With this arsenal of analytical capabilities, the Laboratories for Measurements and Characterization offer customers and collaborators a complete, one-stop site for diagnostic evaluation and correlation of events from single-atom to macroscale dimensions. The following summaries provide some examples and highlights of service, collaborations, research, and technique development activities in four groups: Cell and Module Performance, Electro-Optical Characterization, Analytical Microscopy, and Surface and Interface Characterization. Each group supports the NCPV and Photovoltaics Program with both extraordinary analytical capabilities and expertise. 
PV Measurements and

Characterization Project

In-House Research 
Title:

Organization:

Contributors:
Center for Measurements and Characterization

National Renewable Energy Laboratory, Golden, CO

Mowafak Al-Jassim, Falah Hasoon, Kim Jones, Rick Matson, Helio Moutinho, Amy Swartzlander

\section{Introduction}

The Analytical Microscopy task has provided extensive support for both our in-house and subcontract groups. A wide variety of techniques were used to characterize photovoltaic (PV) materials and devices. Quantitative compositional measurements are performed by fully computerized electron probe microanalysis (EPMA) using either energy-dispersive or wavelength-dispersive X-ray analysis. Structural and defect analyses are carried out by transmission electron microscopy (TEM). Additionally, scanning electron microscopy (SEM) in its various modes, such as secondary electron imaging, backscattered electron imaging, cathodoluminescence (CL), and electron beam induced current (EBIC) imaging, are providing wide support for measuring the topographical, compositional, luminescent, and microelectrical properties of PV materials and devices. Nanoscale-area atomic force microscopy (AFM) is providing indispensable, high-resolution examination of the morphology of thin films. Furthermore, we have acquired a new X-ray diffractometer. In its various modes, this instrument has already proved to be of great service to the PV program.

The majority ( $60 \%)$ of analyses carried out by this task are short term. The emphasis in this category is on high-quality data with short turnaround time. We probably analyze more than 150 routine samples a month. In addition to routine analysis, approximately $40 \%$ of our resources are committed to research support activities, which are usually planned through other in-house and subcontractors' activities. The following is a brief account of some examples of our research support-divided according to material technology-during 1997.

\section{$\underline{\text { CIS }}$}

SEM microcharacterization studies of the effect of systematic incorporation of various levels of $\mathrm{Na}$ into copper indium diselenide (CIS) and copper indium gallium diselenide (CIGS) films demonstrated a clear correlation between the evolution, and the eventual devolution of, device performance with increased $\mathrm{Na}$ levels in the material. Where the microstructure (crystallinity) of the material and device properties at both the micro and macro levels systematically improve up to $\mathrm{Na}$ concentrations of $\sim 0.5$ at.\%, all of these rapidly declined by 5 at.\%. Correlating with secondary ion mass spectroscopy (SIMS) profiles of the depth distribution of the $\mathrm{Na}$ where increased $\mathrm{Na}$ concentrations are observed near the surface, is the increased crystallinity or structural integrity of the material originating from the surface until a critical concentration is reached. At this point the whole microstructure breaks into a porous structure with small grains. Enhancement and uniformity of electrical properties of the material at the submicron level also improve, up until the same point. 
Electron microscopy was key in studying the morphological development in nanoparticle-derived, spray-deposited films for CIS solar cells. At first, these porous precursor films were processed under the direction of the CIS team according to standard protocol-as optimized for evaporated materials utilized by the CIS team. However, this processing approach produced films with inferior efficiencies. Noting the correlation between topography and material properties, the focus of the project was shifted from overall device efficiency to improved morphology in the nanoparticle layer. At this point, the scanning electron images of the nanoparticle materials provided the basis of the understanding of the critical "copper selenide flux" process as it applies to nanoparticulate film precursors and serve to determine the next research step.

In support of the CIS/Moly Back-Contact National Team, scanning electron microscopy was performed on the morphology of the Mo back contact in cross section as it was deposited by Energy Photovoltaics (EPV), International Solar Electric Technology (ISET), and Lockheed-Martin. Subsequently, CIS was deposited on these Mo films by EPV and ISET and the high-resolution SEM examination was repeated, to determine the relation between the different Mo deposition conditions, different subsequent CIS deposition techniques, and the resulting distribution of $\mathrm{Na}$ in the absorber layer. We learned that 1) the morphology of the Mo layers appeared very similar, 2) the $\mathrm{Na}$ content in the Mo resulting from diffusion (via SIMS measurements) was also quite similar, 3) it was the CIS deposition technique that largely determined the amount and distribution of $\mathrm{Na}$ throughout the CIS (especially the selenization technique), and 4) the morphology of the CIS (i.e., grain size distribution and grain density) supported the idea that the $\mathrm{Na}$ exists primarily at grain surfaces because many more smaller grains exist near the interface where SIMS shows a high level of $\mathrm{Na}$ but much less $\mathrm{Na}$ where the grains are comparatively much larger (five to ten times larger).

\section{$\underline{\text { CdTe }}$}

An area of focus for this task in the CdTe arena has been the investigation of the recrystallization process as a function of annealing and $\mathrm{CdCl}_{2}$ heat treatment. CdTe thin films grown by physical vapor deposition (PVD), radio-frequency magnetron sputtering (RFMS) and close-spaced sublimation (CSS) were treated with $\mathrm{CdCl}_{2}$-saturated solution at $300^{\circ}, 350^{\circ}$, and $400^{\circ}$, and studied by $\mathrm{AFM}, \mathrm{X}$-ray diffraction (XRD), TEM, time-resolved photoluminescence (TRPL), photoluminescence (PL), and CL. We ascertained that $400^{\circ} \mathrm{C}$ is the optimal chemical-treatment temperature for producing the highestquality films. Changes in the microstructure with $\mathrm{CdCl}_{2}$ treatment followed a model of recrystallization and grain growth. Recrystallization, which was a function of the lattice-strain energy of the film, was observed directly for the first time by AFM in PVD samples treated at $350^{\circ} \mathrm{C}$ (see Fig. 1). At this temperature, $\mathrm{XRD}$ results identified two lattice parameters, corresponding to the original and recrystallized lattice in PVD and RFMS samples (see Fig. 2). After treatment at $400^{\circ}$, these films were completely recrystallized, and significant grain growth was observed. These phenomena were very important in determining the final structure of PVD and RFMS samples. Nevertheless, they were not observed in CSS samples, which are grown at higher temperature and have less initial stress than the previous samples. The $\mathrm{CdCl}_{2}$ heat treatment significantly improved the minority-carrier lifetime and the luminescence intensity of the samples, providing evidence of the reduction of deep defect levels within the band gap of the films. We showed that grain boundaries are preferential sites for non- 
radiative recombination, and they are partially passivated after the $\mathrm{CdCl}_{2}$ treatment. Mechanisms for the effects of $\mathrm{CdCl}_{2}$ in the heat treatment and for the deep-level elimination have been proposed.

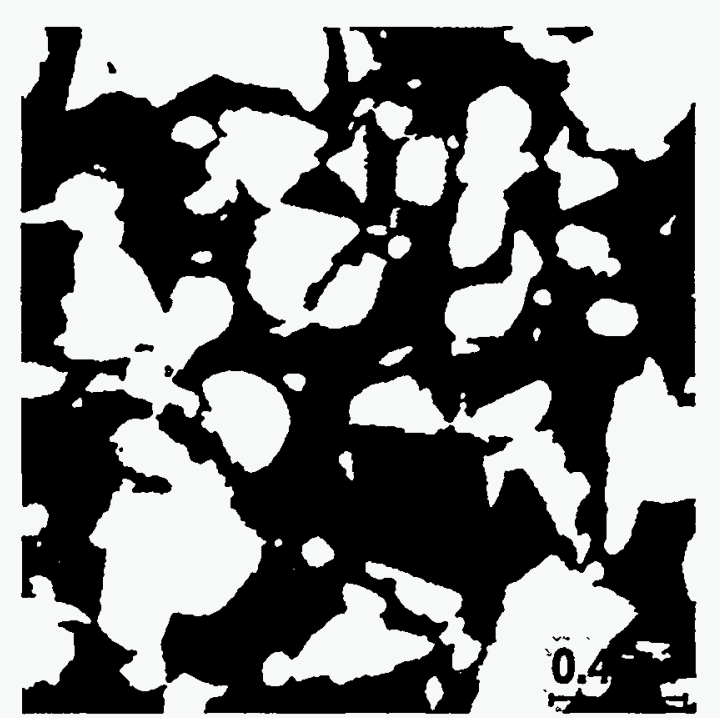

Fig. 1 - AFM image of PVD CdTe treated at $350^{\circ} \mathrm{C}$, showing the original structure and the new recrystallized grains.

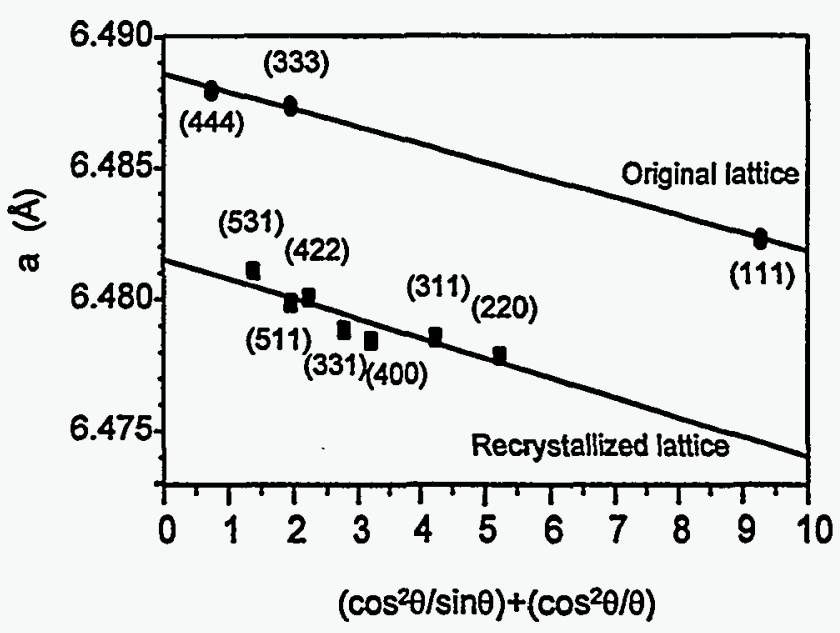

Fig. 2 - Lattice parameter for PVD CdTe films treated at $350^{\circ} \mathrm{C}$, showing the lattice parameters for the original and recrystallized lattices.

\section{$\underline{\text { III-Vs }}$}

The main activity in this area has been supporting NREL's III-V Materials and Devices Team in developing 1.0-eV band gap materials for multijunction cells. The microstructure of two material systems was investigated. The first was the GaInAsN alloy system, which can be grown with the desired optical and electronic properties while achieving a lattice match to GaAs. Using EDX spectroscopy, we detect $\mathrm{Ga}$, In, and As in the 1.0- $\mu \mathrm{m}$-thick films, and infer the presence of $\mathrm{N}$ because of the uniformity and good structural properties (low dislocation densities). However, we have observed an unexpected interface roughly $800 \AA$ below the surface with associated misfit dislocations that may reveal a reduction in $\mathrm{N}$ concentration from buried portions of the film. The change in composition may be caused by post-growth outdiffusion of $\mathrm{N}$, but results more likely from a change in $\mathrm{N}$ (and As) incorporation during growth. Further structural, compositional, and growth studies of this phenomenon have already been initiated.

The second system we examined by TEM is the metastable ( $\mathrm{GaAs})_{1-\mathrm{x}}\left(\mathrm{Ge}_{2}\right)_{\mathrm{x}}$ alloys. The direct band gap in this system can be varied from $\sim 0.7$ to $1.42 \mathrm{eV}$ while keeping lattice-matched to GaAs. These alloys cannot be grown in bulk form because of the existence of a miscibility gap and a strong tendency towards phase separation. At some value of $x$ there will also be a transition from the zincblende crystal structure of $\mathrm{GaAs}$ to the diamond-cubic structure of $\mathrm{Ge}$. Previous work by other groups on molecular beam epitaxy (MBE), metal organic chemical vapor deposition (MOCVD), and sputtered $(\mathrm{GaAs})_{1-x}\left(\mathrm{Ge}_{2}\right)_{\mathrm{x}}$ layers did not reveal any consistent results on the occurrence of phase separation in these metastable layers. Studies of sputtered epitaxial metastable zinc-blende $(\mathrm{GaAs})_{1-\mathrm{x}}\left(\mathrm{Ge}_{2}\right)_{\mathrm{x}}$ alloys did reveal the presence of Ge-mediated antiphase boundaries. We have examined MOCVD 
$(\mathrm{GaAs})_{1-\mathrm{x}}\left(\mathrm{Ge}_{2}\right)_{\mathrm{x}}$ layers with $\mathrm{x}=0.22$ and 0.62 grown on $\mathrm{GaAs}$ substrates. These samples showed evidence of phase separation or segregation. In $<110>$ cross-section pictures of the layers, bands of contrast, inclined at a shallow angle with respect to the $(\mathrm{GaAs})_{1-\mathrm{x}}\left(\mathrm{Ge}_{2}\right)_{\mathrm{X}} / \mathrm{GaAs}$ interface, were visible in chemically sensitive [002] dark-field (DF) micrographs. This suggests that segregation may be occurring during growth at the step edges associated with the offcut of the substrates. Evidence of phase separation was also visible in [002] DF plan-view micrographs of both samples. In the $x=0.62$ layer the significant clustering associated with the phase separation was also visible as strain contrast in $<220>$ and $<400>$ DF micrographs of both plan-view and cross-sectional samples.

\section{$\underline{\text { Si }}$}

In an attempt to characterize the hydrogen treatment of crystalline Si (C-Si) substrates prior to epitaxial growth, a number of samples were prepared and characterized with several techniques, among which was SEM imaging. From the SEM images we were able to determine the morphology of the damage induced in the substrate from long-time exposures to the hydrogen treatment. This, coupled with a few images from data points where the treatment was just beginning to damage the substrate, has lent credence to the model of $\mathrm{H}$ etching in C-Si. From the SEM work we believe that we are able to see that atomic $\mathrm{H}$ diffuses through the native oxide and into the $\mathrm{Si}$, probably reaching saturation levels in the $\mathrm{Si}$, thus producing strained bonds which then form $\mathrm{SiH}_{4}$ with the further addition of atomic $\mathrm{H}$. This $\mathrm{SiH}_{4}$ erodes outward from beneath the native oxide until islands of the oxide collapse and very rough, damaged surfaces with uneven etching are produced. This, together with our supporting TEM work, has led us to seek ways to reduce the concentration of atomic $\mathrm{H}$ reaching the substrate as well as to explore other techniques for cleaning.

A large series (24 samples) of 100-nm, hot-wire-deposited Si films on single-crystal $\mathrm{n}$ and $\mathrm{p}$-type $\mathrm{Si}$ substrates were investigated by SEM and TEM. The deposition series was performed in order to determine at what temperature range Epi-Si could be deposited using this method. SEM secondary electron imaging and electron channeling were used to determine the phase and surface morphology of the films. Electron channeling of the samples revealed in this case that none of the films was single crystal and SEI showed that the majority of the films contained pinholes and poor surface morphology. For this particular set of depositions a standard substrate wet-etch cleaning method was used and no hydrogen treatment of the substrate was performed prior to the growth of the films. These results indicate that the condition and cleanliness of the substrate play a major role in the quality of the films and in the overall ability to produce epitaxial Si using HWCVD. The significance of the hydrogen treatment is yet to be determined.

Further Developments of the NREL Large-Scale Laser Scanner (LSLS) for the Automated Screening of PV Modules for Localized Response Uniformity and Quantification of Shunting Defects.

In response to the industry-wide need for rapid screening of modules for both characterization of nonuniform response and severe shunting defects in PV modules and submodules, the utility of the NREL LSLS has been further extended by developing an automated shunt screening system and automation software for the analysis of PV modules. Needed for the analysis of $100+$ thin-film modules during the 
course of a year and developed in collaboration with the Materials Research Group (MRG), the automated system 1) identifies cells within the modules with shunt resistances less than an operatorspecified resistance threshold, 2) provides an estimate of the shunt resistance of each of the shunted cells, 3) generates a large-scale laser-beam-induced-current (LBIC) response color map of the entire module, or any subsection thereof, and then 4) generates a detailed current color map of each shunted cell to identify the actual physical location of the shunts within a given cell for further detailed analysis. Both for technology transfer and as the result of a technical collaboration, MRG has agreed to make the NREL-originated LSLS commercially available to the PV community.

As an example of some of the output and analysis capabilities of the new system from a module early in the sequence of modules under evaluation while the manufacturer was experiencing scribe-related problems, Figure 3a illustrates both an LBIC map of the entire module, including an LBIC linescan, to show actual local LBIC signal levels within the module. Figure $3 \mathrm{~b}$ illustrates the results of the automated program, which calculates and plots actual shunt resistance values for each cell. Both are then used to nonintrusively identify the existence and location of faulty cells. This information is then immediately fed back to the manufacturer.

Fig. 3 illustrates a case in point. 3a) Represents an LBIC map of a $2^{\prime} \times 4^{\prime}$ thin-film module. As the color bar on the left indicates, each color represents one of ten categories of current response, with red being the highest. The LBIC linescan along the lower portion can be used to get a better sense for the fine variations in LBIC response along the line noted as the horizontal line.

3b) This figure is generated by the newly developed software whereby the computer identifies cells with shunt resistance below an operator-specified threshold, measures response, calculates the shunt resistance for the cell, and provides a valuable bar graph of the shunt resistance for each cell to the manufacturer. 


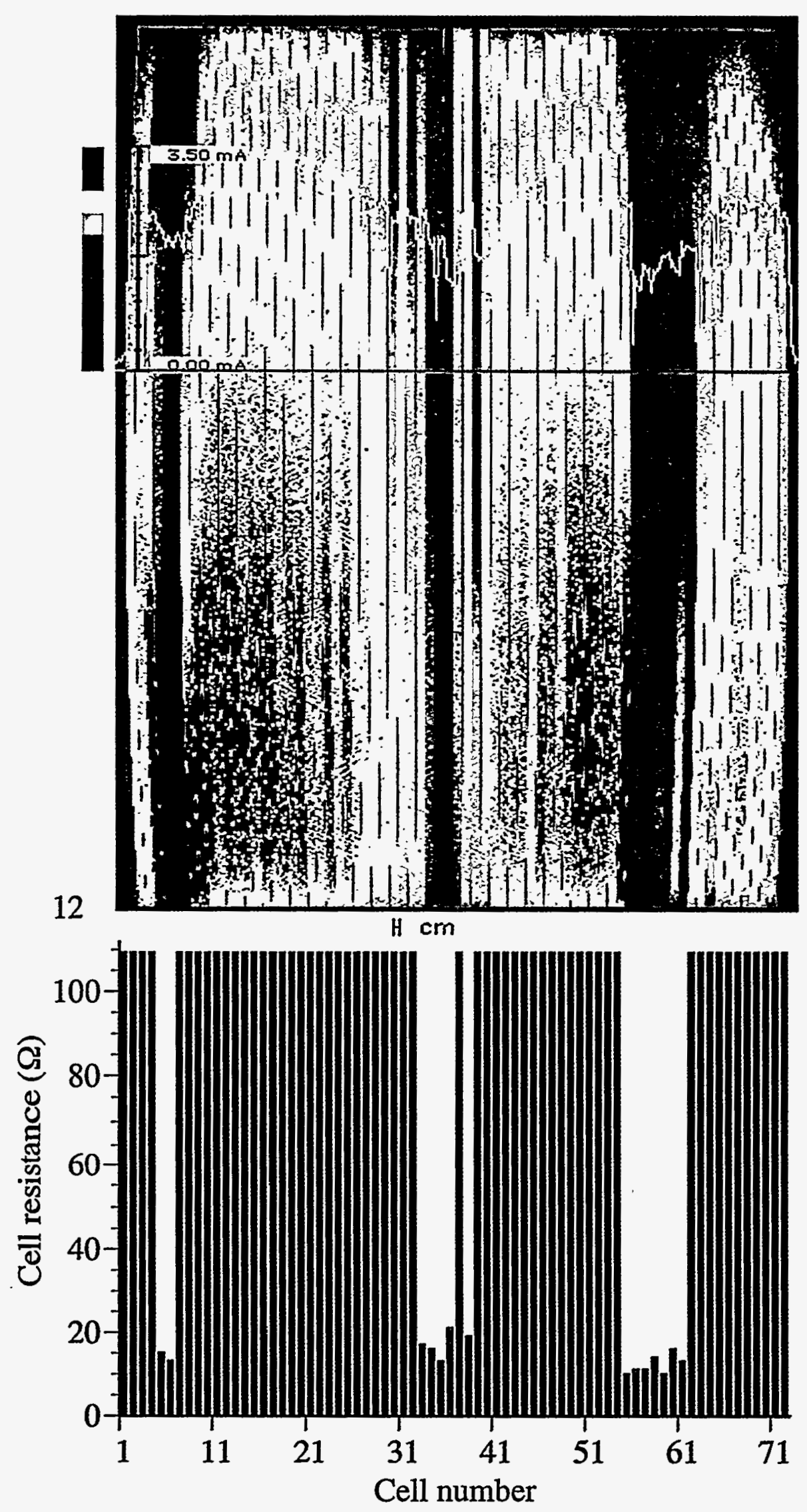

Figure $3 a$ Laser beam induced current (LBIC) map of a $2^{\prime} \times 4^{\prime}$ thin film module. Per the color bar at the left, each color represents one of ten categories of current response with red being the highest. The LBIC linescan along the lower portion can be used to get a better sense of the fine variations in LBIC response along the 0 response line. Higher spatial resolutions are possible with this system by moving the module closer to the laser.

Figure $3 b$ This is a map of the shunt resistances for the individual cells calculated from the laser scan data as a function of voltage. The threshold screening resistance was 9.5 $\Omega$. The cells that were not shunted were set to the average shunt resistance of $109.2 \Omega$. 
Title:

Organization:

Contributors:

\section{Electrooptical Characterization}

Center for Measurements and Characterization

National Renewable Energy Laboratory, Golden, CO

R.K. Ahrenkiel (Principal Investigator), F: Abulfotuh, P. Dippo,

B.M. Keys, D. Levi, J. Webb, L. Gedvilas

\section{Objectives}

The overall objective of the electrooptical characterization task is to develop and provide electrical and optical measurement support for both in-house and subcontract photovoltaic (PV) device research, so as to accelerate commercialization of the respective technologies.

\section{$\underline{\text { Results }}$}

The following describe contributions of the above-mentioned measurement technologies to the principal device technologies of the national PV program.

\section{CdS/CdTe Thin-Film Solar Cells}

During this period, extensive studies of CdS and CdTe were performed for both in-house and external researchers. The studies included energy-resolved photoluminescence (PL) measurements taken at different powers and at different temperatures for the specified sets of samples from different researchers. The temperatures ranged from room temperature to $4.25 \mathrm{~K}$. Work is ongoing to examine the results and determine what information can be extrapolated from the data.

Colorado School of Mines, Golden, Colorado: We collaborated with Professor R. Collins and graduate student Hasan Aslan to study interdiffusion in electro-deposited $\mathrm{CdTe} / \mathrm{CdS}$ thin films using PL and time-resolved photoluminescence (TRPL) on step-etched samples. Collins presented the paper "Sulfur Diffusion in Polycrystalline Thin-Film CdTe Solar Cells" at the Fall Meeting of the Materials Research Society in Boston, Massachusetts. A project with visiting researcher Victor Kaydanoff and NREL/Colorado State University Ph.D. student Lawrence Woods (on the electrooptical and device properties of grain boundaries in CdTe/CdS PV) is breaking new ground by using the new lift-off technique together with conductivity measurements to characterize grain-boundary barrier heights.

Colorado State University, Ft. Collins, Colorado: We collaborated with Professor J. Sites to study the nature of aging in CdTe/CdS PV panels. Recent PL and TRPL results on stressed devices confirm J-V measurements that indicate that deterioration of the cells is due to two mechanisms: an increase in series resistance, and an increase in nonradiative recombination near the junction. We also found that light soaking is essential to correctly simulate real-world aging of PV devices. 
NREL CdTe Team, Golden, Colorado: In collaboration with the NREL CdTe team, we developed a new technique for cleaving a CdTe/CdS thin film at the heterojunction. This lift-off technique promises to rapidly advance the photovoltaics community's knowledge and understanding of $\mathrm{CdTe} / \mathrm{CdS}$ devices by providing characterization access to the critical $\mathrm{CdTe}-\mathrm{CdS}$ interface.

In collaboration with $\mathrm{P}$. Sheldon's and M. Al-Jassim's teams, we identified nitrogencontaining impurity precipitates (cyanamides and cyanates) in CBD CdS films using Fourier transform infrared (FTIR) microspectroscopy. We summarized these results in an Institute of Electrical and Electronics Engineers (IEEE)/Photovoltaic Specialists' Conference (PVSC) poster and (FY 1998) paper. The poster summarizing the CIGS and CdTe results introduced scanning FTIR microspectroscopy to the PV community at the 26th IEEE/PVSC and won the "Best Poster" award in the II-VI Materials category.

Radio frequency photo-conductive decay (RFPCD) measurements of CdTe-based solar cells indicated that the peak photoresponse occurs at a photon energy somewhat less than the $\mathrm{CdTe}$ band gap. These data indicate that the region of sulfur interdiffusion may play an important part in the PV process.

\section{CIGS Thin-Film Solar Cells}

During this period, extensive studies of CIS were performed for both in-house and external researchers. The studies included energy-resolved photoluminescence measurements taken at different powers, at different temperatures, and different spot sizes. The temperatures ranged from room temperature to $4.25 \mathrm{~K}$. Work is ongoing to examine the results and determine what information can be extrapolated from the data.

We used FT-PL spectroscopy to confirm passivation of CIGS films by Cd and presented the results at the PVPR meeting. We also identified carbonate-containing impurity precipitates in CBD CdS films on industry CIGS samples using FTIR microspectroscopy and presented this work in an IEEE/PVSC poster and (FY 1998) paper, helping to meet a team milestone.

\section{Crystalline and Multicrystalline Silicon Solar Cells: Measurements for AstroPower}

We developed software for rapid analysis of $\mathrm{C}, \mathrm{O}$, and $\mathrm{N}$ concentration, and relative concentrations of $\mathrm{SiO}_{X}, \mathrm{SiN}_{X}$, and $\mathrm{SiC}_{\mathrm{X}}$ phases in mulitcrystalline silicon from AstroPower.

A project to measure the minority-carrier lifetime in a large number of wafers prior to device fabrication, was a principal activity during the year. The measured wafers are then converted into finished devices at AstroPower and correlations between short-circuit current and measured-devices parameters are established. The purpose is to develop a metric for productionline qualification of wafers prior to device fabrication. A successful result would result in considerable cost reduction of the product by eliminating the fabrication of devices in belowbaseline quality wafers. We measured several hundred wafers by RFPCD using a laser wavelength of $850 \mathrm{~nm}$ and a spot size of about 2 inches diameter. The data showed a range of low-injection lifetimes ranging from $150 \mathrm{~ns}$ to $300 \mathrm{~ns}$. The range of electron mobilities in the ptype materials varies by about a factor of 3 . The relative diffusion length was calculated for all samples and varied by about a factor of 3 among the sample lot. The ratio of high-injection lifetime to low-injection lifetime was very close to 2.0 for all samples measured. These data are indicative of recombination at Fe-B complexes for which the electron and hole capture crosssections are equal, producing the result that $\tau($ high $) / \tau(l o w)=2.0$. From the measured lifetime 
values, one can calculate a density of active $\mathrm{Fe}-\mathrm{B}$ recombination centers at about $1 \times 10^{14} \mathrm{~cm}^{-3}$ :

Wafers are subjected to a heat-processing treatment that has been found to increase the device peformance. We find that the low-injection lifetimes increase by about a factor of 10 , resulting in an increase in the diffusion length of about a factor of 3 . The range of low-injection lifetimes now varies from about $1.5 \mathrm{~ms}$ to $4.0 \mathrm{~ms}$. Again, the ratio of $\tau$ (high) $/ \tau($ low) 2.0 , indicating $\mathrm{Fe}-\mathrm{B}$ but with a concentration reduction of about 10 . The study has not been completed and is awaiting further device processing of the wafers measured here. Upon completion of device fabrication, the large database will be studied and correlations established that can be transferred to the AstroPower production line.

\section{Amorphous Silicon Solar Cells}

We performed innovative room-temperature FT-PL and FT-Raman analyses of microcrystalline silicon films to identify defects and to measure the crystalline/amorphous ratio, respectively. The clients were Hans-Meitner Institute and Institute of Energy Conversion.

We also developed software for rapid quantitative analysis of hydrogen in a-SiH and a$\mathrm{SiGeH}$ in support of H. Mahan's and B. Nelson's teams, respectively.

\section{High-Efficiency III-V Devices}

Support has been provided for the NREL III-V team, in their search for the optimal growth conditions for high-quality epitaxial GaAs on the new molecular beam epitaxy (MBE) system, using the TRPL technique. The two projects pursued were 1) the investigation of ordering properties of epitaxial GaAs films and 2) the production of device-quality MBE material through the growth and characterization of GaAs/GaInP double heterostructures.

Research has been conducted with the NREL III-V Team in an attempt to use PL and TRPL to characterize and assist in improving low-temperature GaAs-related compounds. These $1-\mathrm{eV}$ compounds are excellent candidates for a third junction in the already successful GaInP/GaAs tandem cells, allowing for an increase in the device efficiency from $30 \%$ to $37 \%$. Current material issues include the presence and effect of dislocations, interfaces, and the presence of carbon on the lifetime of photogenerated carriers. Measurements on low-temperature $\mathrm{GaAs}$ have shown that a spectral response of about 0.7 can be achieved with measured lifetimes of about 0.5 ns. The correlation between these parameters can further be seen by measurements on 1.2- to $1.3-\mathrm{eV}$ compounds, which exhibit a spectral response of 0.3 to 0.4 and a lifetime of about 0.2 ns. Current $1-\mathrm{eV}$ material is limited to a spectral response of about 0.4 . The cause for this is being investigated.

Considerable research has been performed on the growth of polycrystalline GaAs films on glass, Mo/glass, and molybdenum foil substrates. This project has also involved the NREL III-V Team and the Center for Semiconductor Research at the Research Triangle Institute. The findings can be summarized by the following observations: there appears to be no correlation between the measured lifetimes and grain orientation; the material quality is strongly dependent on growth temperature; the relatively low temperature required for growth on glass has resulted in measured lifetimes below $60 \mathrm{ps}$; films grown at elevated temperatures on molybdenum foils have achieved lifetimes as high as $400 \mathrm{ps}$; there is a dependence of the material quality on the dopant type used; and finally, the dependence of material quality with growth temperature and dopant type correlates strongly with the observed grain size, indicating surface recombination is playing a large role in limiting the device performance. Throughout this project, gains in the measured 
lifetime have been followed by gains in both the open-circuit voltage and short-circuit current of finished poly-GaAs devices. Concurrent with this work, a 20\% (AM1.5) poly-GaAs device has been demonstrated on sub-mm grain polycrystalline $\mathrm{Ge}$.

Research has begun with Spectrolab, Inc., to investigate the bulk and interface recombination mechanisms of the various layers in their GaInP/GaAs/Ge triple-junction solar cell. Initial results have indicated high levels of recombination in the bulk and at the interfaces of the AlGaInP/GaInP component of the device. These preliminary results have been submitted as an abstract for the 2nd World Conference on PV Solar Energy Conversion. Future work will assess the effect of changes in the composition of the AlGaInP barrier layer as well as the characterization of the other constituent layers of the device.

\section{Technique Development}

A RFPCD lifetime measurement system was developed that measures lifetime as a function of sample temperature. Measurements were made on a number of PV materials including thin-film CdS, CIGS, and CdTe and single and polycrystalline silicon wafers over the temperature range $80 \mathrm{~K}$ to $300 \mathrm{~K}$. These data provided additional information about the principal recombination mechanisms in these materials.

We specified and ordered a new FT-PL and FT-Raman microprobe, which will enable spatial resolution of 1-2 $\mu \mathrm{m}$ for nondestructive microanalysis of PV materials and devices. The FT-PL technique has proven particularly useful in the room-temperature analysis of thin-film polycrystalline materials, silicon, and III-V epitaxial layers for band gap, defect, and impurity levels of 1.4-0.44 eV. FT-Raman spectroscopy measures the vibrational frequencies of crystalline, polycrystalline, and polymeric materials to aid in structural analysis, complementing FTIR analysis. Expanded macro- and micro-sampling compartments will accommodate cryostats for Raman or luminescence analysis at temperatures ranging from 4 to $400 \mathrm{~K}$. The purchase was supported by FY 1997 General Purpose Equipment (GPE) capital equipment funds.

We garnered international publicity for the National Center for Photovoltaics and the Measurement and Characterization Center through an article and cover photo in the October issue of the trade magazine Laser Focus World. This article described the measurement and characterization capabilities of NREL's new Femtosecond Laser Spectroscopy laboratory, and the research on photovoltaics that NREL researchers conduct at the Laboratory.

Capital equipment was expended this year to support the upgrade of a new energyresolved photoluminescence system. This system includes a new Oriel imaging spectrograph with two exit ports (MS 257) and CCD. The lateral port was outfitted with a flange that supports the near-infrared InGaAs detector already in use. This enables the system to go from the visible part of the spectrum to the near-infrared with a flip of a switch.

\section{Work for Others}

Authored a successful proposal to the NREL Fostering Innovation, Research, and Strategic Technologies Program to develop a new capability to measure time-resolved photoluminescence in the near-infrared. This capability will provide significant benefits to the NREL Thin-Film PV program by enabling measurement of minority-carrier lifetime in CIGS devices.

We were granted funding of $\$ 60 \mathrm{~K}$ by the Defense Advanced Research Projects Agency for FY 1998 for a project related to thermoelectric energy conversion. 


\section{References}

D.H. Levi, L.M. Woods, D.S. Albin, T.A. Gessert, D.W. Niles, A. Swartzlander, D.H. Rose, R.K. Ahrenkiel and P. Sheldon, "Effects of Back Contact Treatments on Junction Photoluminescence in CdTe/CdS Solar Cells," Proceedings of the Fall 1997 Materials Research Society Symposium, Boston, MA, Dec. 1997, (in press).

M.H. Aslan, W. Song, J. Tang, D. Mao, R.T. Collins, D.H. Levi, R.K. Ahrenkiel, S.C. Lindstrom, and M.B. Johnson, "Sulfur Diffusion in Polycrystalline Thin-Film CdTe Solar Cells," Proceedings of the Fall 1997 Materials Research Society Symposium, Boston, MA, Dec. 1997, (in press).

W.A. Doolittle, A. Rohatgi, R.K. Ahrenkiel, D. Levi, G. Augustine, and R. Hopkins, "Understanding the Role of Defects in Limiting the Minority-Carrier Lifetime in SiC," Proceedings of the Fall 1997 Materials Research Society Symposium, Boston, MA, Dec. 1997, (in press).

R.K. Ahrenkiel, S.P. Ahrenkiel, D.J. Arent, and J.M. Olson, "Carrier Transport in Ordered and Disordered In(0.53)Ga(0.47)As," Appl. Phys. Lett. 70, 756 (1997).

R.K. Ahrenkiel, "Carrier Recombination in Semiconductors Used for Photovoltaic Devices," AIP Conference Proceedings 394, p. 225, AIP Press, (1997).

R.K. Ahrenkiel, S.P. Ahrenkiel, D.J. Arent, J.M. Olson, and M. Wanlass, "Carrier Transport in Ordered and Disordered In(0.53)Ga(0.47)As," Materials Research Society, Symposium Proceedings V. 441 (1997). p. 181.

S.P. Ahrenkiel, R.K. Ahrenkiel, and D.J. Arent, "Domain Structure and Transient Photoconductivity in Ordered Ga(0.47)In(0.53)As Epitaxial Films," Materials Research Society, Symposium Proceedings V. 441 (1997), p. 133.

B.M. Keyes, R.K. Ahrenkiel, G.J. Shaw, and G.P. Summers, "Minority-Carrier Lifetime Damage Coefficient of Radiation Damaged InP," J. Appl. Phys. 82, 2156 (1997).

R.K. Ahrenkiel and S. Johnston, "Contactless Measurement of Recombination Lifetime in Photovoltaic Materials," Solar Cells and Solar Energy Materials, (in press).

R.K. Ahrenkiel and S. Johnston, "Contactless Measurement of Recombination Lifetime in Photovoltaic Materials," Proceedings of the 26th Photovoltaic Specialists Conference, IEEE, (in press, 1997).

R. Venkatasubramanian, B.C. O'Quinn, E. Siivolta, B. Keyes, and R.K. Ahrenkiel, “ $20 \%$ (AM1.5) Efficiency GaAs Solar Cells on Sub-mm Grain-Size Poly-Ge and its Transition to LowCost Substrates," Proceedings of the 26th Photovoltaic Specialists Conference, IEEE, (1997, in press).

B. Jagannathan, R.L. Wallace, W.A. Anderson, and R.K. Ahrenkiel, "Amorphous and Microcrystalline Silicon by ECR-CVD Using Highly Dilute Silane Mixtures," Proceedings of the 26th Photovoltaic Specialists Conference, p.? IEEE, (1997, in press).

R.K. Ahrenkiel, D.H. Levi, S. Johnston, W. Song, D. Mao, and A. Fischer, "Photoconductive Lifetime of CdS Used in Thin-Film Solar Cells," Proceedings of the 26th Photovoltaic Specialists Conference, IEEE, (in press, 1997).

R.K. Ahrenkiel, S.P. Ahrenkiel, M.M. Al-Jassim, and R. Venkatasubramanian, "Electronic and Mechanical Properties of Ge Films Grown on Glass Substrates," Proceedings of the 26th Photovoltaic Specialists Conference, IEEE, (in press, 1997). 
D.H. Levi, D.S. Albin, R.K. Ahrenkiel, D.H. Rose, D.W. Niles, and P. Sheldon, "BackContact Effects on Junction Photoluminescence in CdTe/CdS Solar Cells," Proceedings of the 26th Photovoltaic Specialists Conference, IEEE, (in press, 1997).

D.H. Levi, H.R. Moutinho, B.M. Keyes, F.S. Hasoon, R.K. Ahrenkiel, M. Al-Jassim, L.L. Kazmerski, and R. Birkmire, "Micro-Thorough Nanostructure Investigations of Polycrystalline CdTe: Correlations with Processing Electronic Structures," Solar Energy Materials and Solar Cells 41/42 381, (1996).

T.H. Wang, T.F. Ciszek, and R.K. Ahrenkiel, "Characterization of High-Purity Silicon with the Photoconductivity Decay and Photoluminescence Analysis Techniques," Proceedings of the Fourth International Symposium on High-Purity Silicon, Electrochem. Soc; Pennington, NJ: IEEE; pp. 462-472, (1997).

R. Venkatasubramanian, B.C. O'Quinz, J.S. Hills, M.L.'Timmons, D.P. Malta, H. Field, R.K Ahrenkiel, and B. Keyes, "18.2\% (AM1.5) Efficient GaAs Solar Cell on Optical-Grade Polyscrystalline Ge Substrates," Twenty-Fifth IEEE Photovoltaic Specialists Conference-1996, p. 31, IEEE, (1997).

T.F. Ciszek, T.H. Wang, R.K. Ahrenkiel, and R. Matson, "Properties of Iron-Doped Multicrystalline Silicon Grown by the Float-Zone Technique," Twenty-Fifth IEEE Photovoltaic Specialists Conference-1996, p. 737, IEEE, (1997).

D.H. Levi, B.D. Fluegel, L.M. Woods, and R.K. Ahrenkiel, "Dynamics of Photoexcited Carrier Relaxation in CdTe/CdS Thin Films," Twenty-Fifth IEEE Photovoltaic Specialists Conference-1996, p. 913, IEEE, (1997).

R.L. Wallace, W.A. Anderson, K.M. Jones, and R.K. Ahrenkiel, "Solution Grown Polysilicon for Photovoltaic Devices," Twenty-Fifth IEEE Photovoltaic Specialists Conference1996, p. 697, IEEE, (1997).

H.C. Chou, A. Rohatgi, N.M. Jokerst, S. Kamra, S.R. Stock. S.L. Lowrie, R.K. Ahrenkiel, and D.H. Levi, "Approach toward High-Efficiency CdTe/CdS Heterojunction Solar Cells," Materials Chemistry and Physics 43, 178 (1996).

R. Venkatasubramanian, B.C. O'Quinn, J.S. Hills, M.L. Timmons, D.P. Malta, B. Keyes, and R.K. Ahrenkiel, "Development of High-Performance GaAs Solar Cells on Large-Grain Polycrystalline Ge substrates," Polycrystalline Thin-Films: Structure, Texture, Properties and Applications, pp.483-8, Pittsburgh, PA: Materials Research Society; (1997).

B.M. Keyes, J.R. Tuttle, J.R. Sites, A.L. Tennat, S.A. Asher, M.A. Contreras, K. Ramanathan, A.M. Gabor, J. Webb, R.K. Ahrenkiel, and R. Noufi, "The Influence of Ga on the Properties of CuIn(Ga)Se-Based Thin Films and Devices," Cryst. Res. Technol. 31,439 (1996).

R.K. Ahrenkiel, S.P. Ahrenkiel, and D.J. Arent, "Recombination Lifetime in Ordered and Disordered InGaAs," AIP Conference Proceedings 358, pp. 434-445, AIP (1996).

D.H. Levi, L.M. Woods, D.S. Albin, T.A. Gessert, D.W. Niles, A. Swartzlander, D.H. Rose, R.K. Ahrenkiel, and P. Sheldon, "Effects of Back-Contact Treatments on Junction Photoluminescence in CdTe/CdS Solar Cells," Proceedings of the Fall 1997 Materials Research Society Symposium, Boston, MA, Dec. 1997, (in press).

D. Albin, R. Dhere, D. Rose, X. Li, D. Levi, and P. Sheldon, "Interface Reactions in CdS/CdTe Solar Cell Processing," Proceedings of the Fall 1997 Materials Research Society Symposium, Boston, MA, Dec. 1997, (in press).

D. Albin, D. Rose, R. Dhere, D. Levi, L. Woods, A. Swartzlander, and P. Sheldon, "Comparison Study of Close-Spaced Sublimated and Chemical Bath Deposited CdS Films: 
Effects on CdTe Solar Cells," Proceedings of the 26th Photovoltaic Specialists Conference, Sept. 1997, Anaheim, CA, (in press).

H.R. Moutinho, M.M. Al-Jassim, F.A. Abulfotuh, D.H. Levi, P.C. Dippo, R.G. Dhere, and L.L. Kazmerski, "Studies of Recrystallization of CdTe Thin Films After $\mathrm{CdCl}_{2}$ Treatment," Proceedings of the 26th Photovoltaic Specialists Conference, Sept. 1997, Anaheim, CA, (in press).

D. Albin, D. Rose, R. Dhere, D. Niles, A. Swartzlander, A. Mason, D. Levi, H. Moutinho, and P. Sheldon, "Tin Oxide Stability Effects-Their Identification, Dependence on Processing and Impact on CdTe/CdS Solar Cell Performance," NREL/SNL Photovoltaics Program Review, Proceedings of the 14th Conference, AIP Conference Proceedings No. 394, p. 665 (1997).

A.J. Nelson, D. Levi, "Novel Method for Growing CdS on CdTe Surfaces for Passivation of Surface States and Heterojunction Formation," J. Vac. Sci. Technol. A15(3), pt. 2, pp. 1119-23, (1997).

J.D. Webb, B.M. Keyes, K. Ramanathan, P. Dippo, D.W. Niles, and R. Noufi, "FT-PL Analysis of CIGS/CdS/ZnO Interfaces," Proceedings of the NREL/SNL Photovoltaics Program Review, AIP Conference Proceedings 394, New York: AIP Press; pp. $573-78$ (1997).

M.A. Contreras, B. Egaas, P. Dippo, J. Webb, S. Asher, A. Franz, K. Ramanathan, and R. Noufi, "Changes to the Electrical and Structural Properties of Polycrystalline Thin-Film $\mathrm{Cu}(\mathrm{In}, \mathrm{Ga}) \mathrm{Se}_{2}$ Materials by the Use of Thin (Group Ia)F Precursor Layers," Proceedings of the 11th International European Ternary Materials Conference, Salford, UK (August 1997), (in press).

F.A. Abulfotuh, A. Balcioglu, T. Wangensteen, H.R. Moutinho, F. Hassoon, A. Al-Douri, A. Alnajjar, and L.L. Kazmerski, "Study of the Defect Levels, Electrooptics, and Interface Properties of Polycrystalline CdTe and CdS Thin-Films and their Junction, Proceedings of the 26th IEEE PV Specialists Conference, New York: IEEE 1997 (in press).

H.R. Moutinho, M. M. Al-Jassim, F.A. Abulfotuh, D.H. Levi, P.C. Dippo, R.G. Dhere, and L.L. Kazmerski, "Studies of Recrystallization of CdTe Thin Films After $\mathrm{CdCl}_{2}$ Treatment," Proceedings of the 26th IEEE PV Specialists Conference, New York: IEEE 1997 (in press).

T. Wangensteen. M.W. Wanlass, J.J. Carapella, H.R. Moutinho, A.R. Mason, J.D. Webb, and F.A. Abulfotuh, "Optical Characterization of Epitaxial GaInAs Suitable for Thermophotovoltaic (TPV) Converters," Proceedings of the 26th IEEE PV Specialists Conference, New York: IEEE 1997 (in press).

B.M. Keyes, F. Hasson, P. Dippo, A. Balcioglu, and F. Abulfotuh, "Influence of Na on the Electro-optical Properties of $\mathrm{Cu}(\mathrm{In}, \mathrm{Ga}) \mathrm{Se}_{2}$," Proceedings of the 26th IEEE PV Specialists Conference, New York: IEEE 1997 (in press). 

Organization: Center for Measurement and Characterization

Contributors: $\quad$ K.A. Emery (Task Leader), S. Rummel, H. Field, D. Dunlavy

A. Anderberg, T. Moriarty

\section{Objectives}

Our team evaluates the performance of photovoltaic (PV) devices of all sizes and technologies. We also support the entire photovoltaic community by providing: secondary calibrations of PV modules and cells, efficiency measurements with respect to a given set of standard reporting conditions, efficiency verification of contract deliverables, current versus voltage (I-V) measurements under varying temperature, and spectral and total irradiance. Support is provided for in-house programs in device fabrication, module stability and reliability, PV systems, and alternative rating methods by performing baseline testing, specialized measurements, and other assistance. This activity also supports the entire PV community by providing information on: PV measurement equipment and systems that are appropriate for the end user, I-V measurement procedures, and uncertainty analysis. Included in the uncertainty analysis are the determination of potential artifacts in the $I-V$ results because of equipment or procedures, and realistic estimates of the elemental error sources. This activity is committed to obtaining the lowest possible uncertainty in the measurement of the standardized PV performance of single- and multijunction cells and modules.

\section{Technical Approach}

The photovoltaic current versus voltage characteristics are measured with respect to standard terrestrial reporting conditions $\left(25^{\circ} \mathrm{C}, 1000 \mathrm{Wm}^{-2}\right.$ total irradiance, and American Society for Testing and Materials [ASTM] E892 global reference spectrum). The intensity of the Spectrolab $\mathrm{X}-25$ solar simulator (30-cm-square beam) is adjusted until the measured short-circuit current of a reference cell is equal to its calibration value corrected for spectral mismatch. The current versus voltage characteristics are then measured using four-terminal Kelvin connections to the PV device, with a custom data acquisition system designed to give a random error of less than $\pm 0.1 \%$ and a nonrandom error of less than $\pm 1 \%$. The uncertainty in efficiency measurements with respect to standard reference conditions is $\pm 2 \%-5 \%$, depending on the sample size, geometry, and number of junctions. The I-V system has a voltage range of $\pm 50 \mathrm{~V}(0.1-\mu \mathrm{V}$ resolution) and $\pm 16 \mathrm{~A}$ to $\pm 1 \mathrm{pA}$. These procedures were shown to be valid for any given tabular reference spectrum including AM0 and the ASTM direct normal reference spectrum. Because of the wide current and voltage range, the system is also used for concentrator and dark I-V measurements. For two-terminal, multijunction devices, the spectrum of the Spectrolab X-25 solar simulator is adjusted, using a special filter plate developed at NREL, until each junction is producing the correct photo-current. The I-V system is also used for examining the effects of pre-measurement conditions, bias rate, maximum power versus illumination time, open-circuit voltage $\left(V_{o c}\right)$ versus time, and short-circuit current $\left(I_{\text {sc }}\right)$ versus time for $P V$ performance. 
The filter spectral response system uses periodic monochromatic light directed through one of 51 10-nm bandwidth interference filters covering the spectral range from 290 to $1900 \mathrm{~nm}$. The system is capable of providing steady-state light bias levels up to $2 \mathrm{~A}$ and voltage bias levels from $\pm 1 \mathrm{mV}$ to $\pm 40 \mathrm{~V}$. The intensity of the bias light is normally adjusted to give the short-circuit current under standard reporting conditions. The custom operational-amplifier-based data acquisition system allows for a wide range of gains $\left(1\right.$ to $\left.10^{6}\right)$. The intense light from the $1000-\mathrm{W}$ lamp illuminating the $5-\mathrm{cm}$ diameter filters allows for small cells to large modules to be illuminated with adequate signal to noise. The uncertainty in the relative spectral response as a function of wavelength is less than $\pm 5 \%$. The data can be made absolute by forcing the integrated quantum efficiency to agree with the measured spectral response. The absolute spectral response can also be measured directly with this system, giving an uncertainty of $\pm 10 \%$. The limiting factor in the uncertainty is the $\pm 5 \%-10 \%$ spatial nonuniformity of the monochromatic beam. The second grating monochrometer-based system has a wavelength range from $300-5000 \mathrm{~nm}$, with a 1-nm wavelength resolution and a $1-5 \mathrm{~nm}$ selectable bandwidth. The grating system was designed for accurate absolute spectral response measurements by illuminating a small $1 \mathrm{~mm}$ by $3 \mathrm{~mm}$ rectangular area and measuring the power of the entire beam. The system uses all-reflective optics so chromatic aberrations and beam wander with wavelength are not present. The grating system uses an operational amplifier for the current-to-voltage conversion, allowing a $\pm 15 \mathrm{~V}$ voltage bias range with $0 \pm 1 \mathrm{mV}$ being the normal configuration. A lock-in amplifier is used to detect the ac signal. The system is capable of broadband or filtered light bias for multijunction or nonlinear devices. Both systems rely on accurate pyroelectric detectors or National Institute of Standards and Technology (NIST)-calibrated semiconductor detectors for the measurement of the light power.

Efficiency versus concentration is measured with a Spectrolab High-Intensity Pulsed Solar Simulator (HIPSS). This system has been used to measure the performance as a function of concentration for several GaInP/GaAs concentrator cells fabricated at NREL to 1500 suns. The concentrator lamp housing allows measurement from 1 to 2000 suns. The cell performance as a function of concentration can also be measured using an unfiltered, 1000-W Xe-arc continuous light source that is focused to a small area. Using the cell's I $_{\text {Sc }}$ measured at 1 sun (ASTM E891-87 direct normal reference spectrum at a total 1-sun irradiance of $1000 \mathrm{Wm}^{-2}$ ) and assuming linearity in the current with total irradiance, the I-V characteristics are measured. The linearity can be determined by using calibrated wire mesh filters over a range of irradiances. Using a technique developed at NREL, the temperature of the space charge region can be accurately set to a given value under continuous illumination even though large temperature gradients $\left(>10^{\circ} \mathrm{C}\right)$ may exist between the plate temperature and junction temperature. The procedure involves setting the sample temperature to the reference temperature (e.g., $25^{\circ} \mathrm{C}$ ) without illumination (no heat load). Using a high-speed shutter and voltmeter, the open-circuit voltage is sampled (1000 readings/second). The highest measured $V_{o c}$ is then taken to be the $V_{o c}$ under concentration. The thermoelectrically controlled vacuum plate is then cooled until this $V_{o c}$ is reached. This same procedure is used in the Spectrolab X-25 system for-samples on thermally insulating substrates, where temperature gradients of $5^{\circ}-10^{\circ} \mathrm{C}$ are typical. 
The primary reference cell calibration procedure involves measuring $I_{S C}$ total irradiance ( $E_{t o t}$ ), and spectral irradiance $\left(E_{s}(\lambda)\right)$ at the same time outdoors with the same $5^{\circ}$ field of view. $E_{\text {tot }}$ is measured with an Eppley HF primary absolute-cavity radiometer, and $E_{S}(\lambda)$ is measured with a LICOR LI-1800 spectroradiometer. The uncorrected average calibration value $\left\langle\mathrm{CV}_{\mathbf{u}}\right\rangle$ is calculated for the $30 \mathrm{I}_{\mathrm{SC}}$ and $\mathrm{E}_{\text {tot }}$ readings taken during the 30 seconds required to measure $\mathrm{E}_{\mathrm{S}}(\lambda)$. Once a valid $\left\langle\mathrm{CV}_{\mathrm{u}^{\mathrm{u}}}>\right.$ is obtained, the short-circuit current is corrected for temperature and the spectrally corrected calibration value $C V$ is computed. Because the measurement of $E_{s}(\lambda)$ does not encompass the limits of the reference spectrum, the measured spectrum is extended using a computer model developed by the group to encompass the range of the reference spectrum $(300-4000 \mathrm{~nm})$. The calibration value $\mathrm{CV}$ is computed at least 20 times for at least three separate days, giving a single primary calibration value. This procedure was shown to have a total uncertainty of less than $\pm 1 \%$ by rigorous uncertainty analysis, intercomparison with the World Photovoltaic Scale, primary AMO standards, and other intercomparisons.

The I-V characteristics of modules are routinely evaluated using the Spire 240A Solar Simulator. This system has a 0-100-V, 0-20-A range for a 61 by $122 \mathrm{~cm}$ area. During FY 1997, the Spectrolab model X200 Large-Area Continuous Solar Simulator (LACSS) was used to evaluate the I-V characteristics of modules under continuous illumination. The custom LACSS I-V measurement system is capable of handling devices with an area less than 150 by $120 \mathrm{~cm}$ and a maximum voltage of $\pm 300 \mathrm{~V}$ and current of $\pm 60 \mathrm{~A}$ with $\mathrm{a} \pm 0.01 \mathrm{mV}$ and $\pm 1 \mu \mathrm{A}$ minimum. The system is routinely used to perform dark I-V measurements on modules without changing anything. Light-soaking and premeasurement procedures that were developed for cells are routinely performed on the LACSS for thin-film CIS and CdTe modules. The Spectrolab Large Area Pulsed Solar Simulator (LAPSS) produces a 1-sun beam of light ( $2 \mathrm{~m}$ by $2 \mathrm{~m}$ area) that could illuminate a $4 \mathrm{~m}$ by $4 \mathrm{~m}$ area. The Spectrolab data acquisition system has a $0-100-\mathrm{V}$, 0-40-A measurement range. The system has been used to evaluate the I-V characteristics of modules.

During FY 1997, standardized outdoor module I-V measurements were performed on a variety of 1-sun modules using our variable-tilt and azimuth test bed. For flat-plate modules, the total irradiance was measured with an Eppley pyranometer and a crystal-Si reference cell in a module package mounted in the plane-of-array. A normal incidence pyrheliometer calibrated against NREL's cavity radiometers is used to measure the direct normal irradiance for concentrator modules. The module temperature is measured with a temperature sensor attached to the back surface of the module. The spectral irradiance is measured during the I-V measurement using a LICOR LI-1800 spectroradiometer with a Teflon dome and temperature-controlled detector. Meteorological parameters at the time of measurement, including direct-to-diffuse ratio, wind speed, wind direction, barometric pressure, air temperature and relative humidity, are stored with the data. The outdoor I-V measurement system is capable of handling devices with a voltage and current range of $\pm 300 \mathrm{~V}$ and $\pm 60 \mathrm{~A}$ maximum to $\pm 0.01 \mathrm{mV}$ and $\pm 1 \mu \mathrm{A}$ minimum. 


\section{Results}

During FY 1996, the team performed $4692 \mathrm{I}-\mathrm{V}$ and quantum efficiency (QE) measurements on 1386 cells and modules (I-V under standard reporting conditions). I-V cell and module calibrations were performed for the following groups:

U.S. Industry-Advanced Systems Manufacturing (a-Si); Alpha Solarco (multi-Si); AP Corp. (EFG Si); APS (a-Si/a-Si); ASE Americas (web Si); AstroPower (Si film); ECD / Solvonics / Uni-Solar / USSC (multijunction a-Si); British Petroleum (mono-Si, CdTe modules); Energy PV (CIS); Evergreen Solar (Si ribbon, mono-Si reference cell); Golden Photon (CdTe); Iowa Thin Films (a-Si); ISET (CIGS); Lockheed Martin (CIGS); Lockheed Sanders (GaAs); ISET (CIGS); Materials Research Group (mono-Si, CdTe); OCLI (CIGS); Photonic detectors (mono-Si); Photonic Power Systems (GaAs); PVI (mono-Si reference cell, Si concentrator module); Shell Solar Energy (mono-Si); Siemens (mono-Si, CIGS); Skylight Solar (multi-Si); Solarex (CIS, a-Si tandems, multi-Si); Solar Cells Inc. (CdTe); Solar Solutions Inc. (Koncar a-Si module); Solec Int. (mono-Si); Spire (mono-Si); Utility Power Group (a-Si).

Universities and other Groups in the United States-Burdick Technologies Unlimited (Si and filtered Si solar radiometers); Colorado School of Mines (CdTe); Colorado State University (CdTe); Florida Solar Energy Center (mono-Si reference cells); Georgia Institute of Technology (mono-Si, multi-Si); Institute of Energy Conversion (a-Si, filtered mono-Si reference cells); Research Triangle Institute (GaAs); Star Center/Arizona State University (mono and multi-crystal Si cells and modules); University of Missouri (multi$\mathrm{Si})$; University of Northumbria (mono-Si); University of South Florida (CIS, CdTe, mono$\mathrm{Si}$ reference cells); University of Texas (mono-Si); and University of Toledo (a-Si/a-Si/a-Si:Ge).

NREL Internal Groups-(CIS, CdTe, dye-sensitized nanocrystal dye, mono-Si, mono-Si, multi$\mathrm{Si}$, GaAs, GaInP, GaInP/GaAs tandems, numerous modules for exposure studies and systems evaluation).

Foreign Groups-Australia: University of New South Wales (mono-Si, 18.2\% PERL multi-Si cell); Belgium: IMEC (mono-Si); Brazil: IME (CdTe); China: BSERI (mono-Si), TIPS (a-Si, mono-Si); England: Imperial College (InP, GaInAs multiple quantum well PV); France: LCIE (multi-Si); Germany: Fraunhofer Institute for Solar Energy Systems (monoSi); University of Konstanz (mono-Si); Holland: R\&S Corp. (mono-Si); India: BHEL (mono-Si), WEBEL (mono-Si), Titan Energy (mono-Si), Udhaya (mono-Si); Iraq: SERC (multi-Si); Italy: ENEA (a-Si/a-Si); Commission of European Communities/ESTI (monoSi reference); Netherlands: ECN Solar and Wind (mono-Si reference cells); Spain: IESUPM (multi-Si); Switzerland: University of Neuchatel (a-Si, a-Si tandem); EPFL (nanocrystal dye-sensitized cell, submodule); Taiwan: I.T.R.I. (mono-Si); United Arab Emirates: EMAR (mono-Si).

Approximately 40 papers acknowledged the support of this group during FY 1997. This team has assisted nearly all module manufacturers with their Quality Assurance/Quality Control programs 
by providing telephone consultations on measurement strategy and procedures, and a calibration tractability path for their cells and modules. The technology of PV I-V and spectral response measurements was transferred to a variety of university and manufacturing groups through phone conversations and visits. The group also supported work in spectral irradiance modeling and energy rating methods, resulting in several co-authorships. In support of international programs, trips to China and India were made to transfer efficiency measurement technology. The team participated in the international intercomparison of calibration procedures for new technologies by performing extensive post-intercomparison analysis of the sample set.

The team was awarded the NREL 1996 Outstanding Team Award for "Unparalleled leadership and technical contributions of the Cell and Module Performance. Team to the world photovoltaics community in performance measurement standards."

\section{Publications}

1. M.W. Wanlass, J.S. Ward, K.A. Emery, M.M. Al-Jassim, K.M. Jones, and T.J. Coutts, "GaxIn1-xAs Thermophotovoltaic Converters," Solar Energy Material and Solar Cells, Vol. 41, pp. 405-417, 1996.

2. K. Emery, D. Dunlavy, H. Field, T. Moriarty, L. Ottoson, and S. Rummel, "PV Cell and Module Characterization Capabilities at NREL," Proc. 14th NREL/Sandia Photovoltaics Program Review, November 18-22, 1996, Lakewood, Colorado.

3. M.A. Green, K. Emery, K. Bücher, D.L. King, and S. Igari, "Solar Cell Efficiency Tables (version 9)," Progress in Photovoltaics Research and Applications, Vol. 5, pp. 51-54, 1997.

4. M.A. Green, K. Emery, K. Bücher, D.L. King, and S. Igari, "Solar Cell Efficiency Tables (version 10)," Progress in Photovoltaics Research and Applications, Vol. 5, pp. 265-268, 1997.

5. C.R. Osterwald, S. Añevsky, A.K. Barua, J. Dubard, K. Emery, D. King, J. Metzdorf, F. Nagamine, R. Shimokawa, N. Udayakumar, Y.X. Wang, T. Wittchen, W. Zaaiman, A. Zastrow, and J. Zhang, "Results of the PEP '93 Intercomparison of Reference Cell Calibrations and Newer Technology Performance Measurements," Proc. 26th IEEE PVSC Conference, September 29-October 2, 1997, Anaheim, California.

6. H. Field, "Solar Cell Spectral Response Measurement Errors Related to Spectral Bandwidth and Chopped Light Waveform," Proc. 26th IEEE PVSC Conference, September 29October 2, 1997, Anaheim, California. 

Title:

Contributors:
Surface and Interface Characterization

Sally Asher, Alice Mason, David Niles, and Robert Reedy

\section{$\underline{\text { Introduction }}$}

The Surface and Interface Analysis Team encompasses a powerful array of sophisticated techniques for the analysis of the surface and near surface regions of materials. These include scanning Auger spectroscopy (AES), X-ray photoelectron spectroscopy (XPS), ultra-violet photoelectron spectroscopy (UPS), and both dynamic and static secondary ion mass spectrometry (SIMS). XPS and AES spectroscopies are related techniques for determining the composition and chemistry of surfaces. The field-emission AES and small spot XPS systems have been used extensively this year to support the CdTe and CIS device development tasks. Dynamic SIMS is used to perform trace element analysis for contaminants and dopants in materials. Static SIMS yields trace elemental and chemical information from surfaces. The SIMS systems have been used to support research in all materials areas of the PV program. The new time-of-flight SIMS (TOFSIMS) instrument was used to study surface contamination for several program areas.

Many of the research activities performed by this task are in support of work planned through other in-house and subcontractors activities. The following is a brief description of some of the highlights of our work during 1997. The work in this report is divided according to the technique.

\section{XPS: the chemistry of $\mathrm{Na}$ in CIS thin-films}

$\mathrm{X}$-ray photoelectron spectroscopy (XPS) is a technique that provides chemical information about the components of thin-films. It can, for instance, distinguish between $\mathrm{Si}$ in its elemental form from $\mathrm{Si}$ in an oxidized state.

We have used the XPS system to study CIS thin-films in an effort to clarify the chemistry of $\mathrm{Na}$ impurities. CIS technology uses soda-lime glass, a material containing 15 atomic percent $(\mathrm{a} / \mathrm{o})$ $\mathrm{Na}_{2} \mathrm{O}$, as a substrate. In general terms, $\mathrm{Na}$ diffuses from the soda-lime glass during hightemperature processing into the CIS thin-film. Although its concentration in the bulk of the thinfilms was never known, it was surmised to be approximately $0.1 \mathrm{a} / \mathrm{o}$. $\mathrm{Na}$ was known to accumulate on the surface at concentrations between 5 and 20 atomic percent. It is also well known that Na improves the PV conversion of CIS thin-films.

Fig. 1 shows the compositions of $\mathrm{Na}, \mathrm{O}$ and oxidized $\mathrm{Se}$ as a function of depth in thin-film CIS. These data were acquired on NREL's 5600 XPS system. Under normal operating conditions, the detection limit of XPS is $1 \mathrm{a} / \mathrm{o}$, insufficient for determining the concentration and chemistry of $\mathrm{Na}$ in the bulks of CIS thin- films. We recognized the need to understand the role of $\mathrm{Na}$ in CIS, and had to extend the data collection time by a factor of 100 to acquire this data. Typical data acquisition times were measured in days and weeks rather than minutes and hours.

The results shown in Fig. 1 represent the first direct quantification of the amount of $\mathrm{Na}$ in NREL CIS thin-films. To demonstrate that 0.1 a/o was representative of NREL high-grade CIS thin-films, we performed the same measurement on several CIS thin-films, with the results always yielding a bulk concentration of $0.1 \mathrm{a} / \mathrm{o}$. 


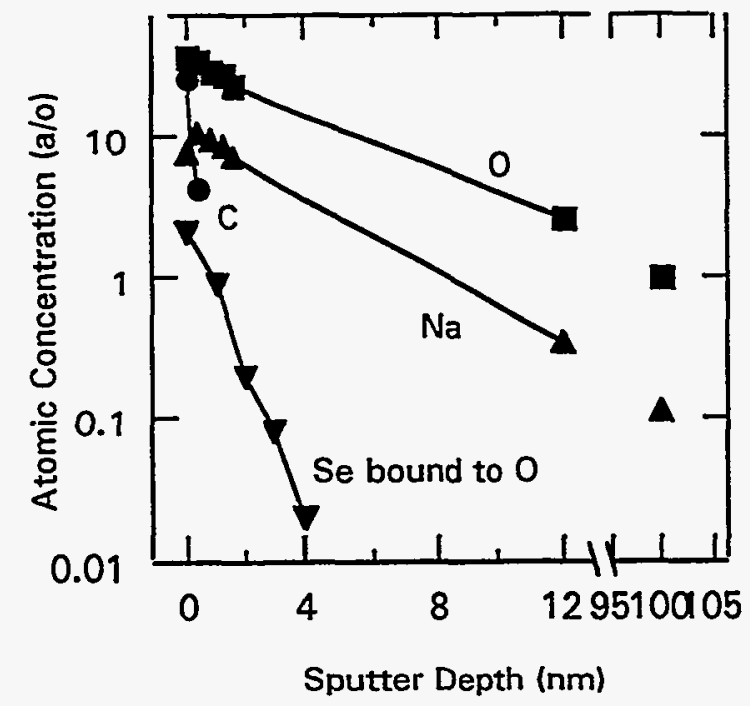

Fig. 1: Impurity concentrations in thin-film CIS.

Even more remarkable than estimating the concentration of $\mathrm{Na}$ is determining its chemical state. We show in Figure 2 the $\mathrm{Na}$ Auger parameter as a function of sputter depth into a CIS thinfilm. We performed the same experiment on several NREL high-grade thin-films to prove that there was nothing unique about the particular thin-film we chose.

The $\mathrm{Na}$ Auger parameter is a quantity used to determine chemical states. We compared the Auger parameter for $\mathrm{Na}$ from a CIS thin-film to several possible compounds for the $\mathrm{Na}$. The conclusion is unmistakable. Na in CIS thin-films is bound to Se. This result is sensible when one considers that CIS thin-films are grown in an over pressure of Se.

The importance of the results shown in Figs. 1 and 2 cannot be overstated. These are the first and only direct, quantifiable Fig. 2: $\mathrm{Na}$ Auger parameter for thin-film CIS.the chemical state of $\mathrm{Na}$ in thin-film CIS. These data are critical to the development of a model for how Na improves PV conversion. First, our results have eliminated models based on an oxidized form of $\mathrm{Na}$ (i.e. $\mathrm{Na} 2 \mathrm{O}$ and $\mathrm{NaOH}$ ). Second, viable models must now involve $\mathrm{Na}$ bonded to $\mathrm{Se}$.

\section{AES: the location of $\mathrm{Na}$ in CIS thin-films}

One of the less tractable problems in thin-film research is acquiring information on grain boundaries. NREL's Auger 670 equipped with a field-emission tip is one of the few instruments capable of extracting information from grain boundaries.

We trained the Auger system on the grain structure of CIS thin-films in an effort to locate $\mathrm{Na}$ impurities. The presence of $\mathrm{Na}$ in grain boundaries and not in grain interiors has long 
been presumed in the CIS thin-film community, but never proven.

In Figure 3, we show the Na KLL Auger signal from a grain interior and a grain boundary in thin-film CIS. This particular film, as do most CIS thin-films, had a typical grain size of 1 to 2 microns. We used an electron beam with a diameter of $23 \mathrm{~nm}$.

The message is simple and clear: $\mathrm{Na}$ resides in the grain boundaries and not the grain interiors of CIS thin-films. To add statistics to our analysis, we collected data from 6 grain interiors and 6 grain boundaries. The results are shown in Figure 4. In all cases, we see $\mathrm{Na}$ in the boundaries and not in the interiors.

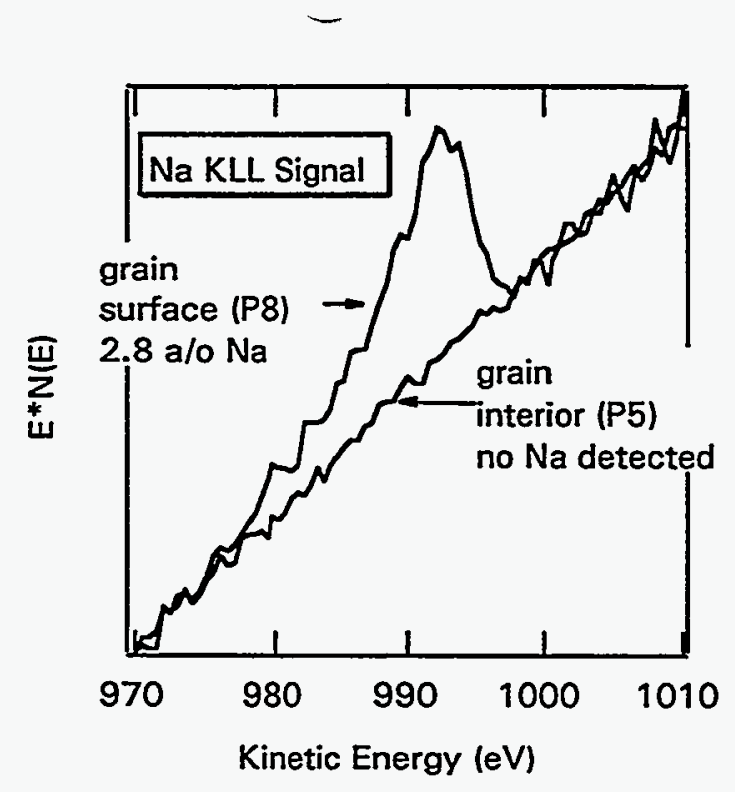

Fig. 3: Grain boundary Na in CIS thin-films.

The simplicity and clarity of the message in Figs. 3 and 4 belies both the effort in obtaining, and the overall importance of these results. The reported detection limit of Auger electron spectroscopy is $1 \mathrm{a} / \mathrm{o}$. However, to prove that $\mathrm{Na}$ did not reside in grain interiors we had to push the detection limit by an order of magnitude. This requires increasing the data acquisition time by 2 orders of magnitude. The detection resides only on grain surfaces and not in grain limit in Figs. 3 and 4 is 0.1 a/o. We also had to work with small electron beams achievable only by working with small electron current. The end result is that the data of Figs. 3 and 4 required 72 hours of continuous data acquisition.

Furthermore, we contend that although the CIS community has long surmised that $\mathrm{Na}$ resided at grain boundaries, but never before proven it.

The CIS community is trying to develop a model for how $\mathrm{Na}$ improves PV conversion. Models based on chemical thermodynamics rely on the formation of a Na-Cu-In-Se compound. The observation of $\mathrm{Na}$ along grain boundaries places these models in jeopardy. Other models rely on $\mathrm{NaCu}$ and/or NaIn defect models. Once again, These models are now in jeopardy because the data indicates that $\mathrm{Na}$ interiors.

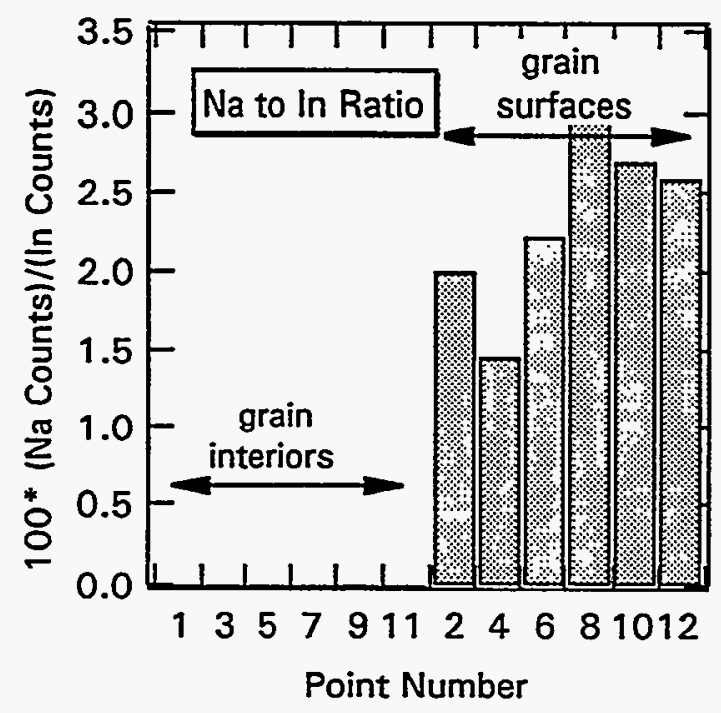

Fig. 4: Grain interiors versus grain boundaries.

The sole existing model based on grain boundary chemistry maintains that $\mathrm{Na}$ catalytically oxidizes In only in grain boundaries, thereby improving PV conversion. Only that model agrees with our observation of $\mathrm{Na}$ in grain boundaries. However, we have also shown that In oxidizes 
without $\mathrm{Na}$, placing this model in jeopardy. A consistent model for $\mathrm{Na}$ in CIS should appear in the next few years, and must account for the data presented here.

\section{SIMS Analysis of $\mathbf{H}$ Diffusion in Silicon}

The performance of some low-cost Silicon solar cells has been reported to be improved by annealing in a forming gas (FG) atmosphere. Previous studies of the effects on annealing in various environments have shown that hydrogen is responsible for the improvement. It is theorized that the improvement in cell performance is due to the passivation of impurities and defects by hydrogen. It is also proposed that the bulk defects are somehow responsible for the dissociation of molecular hydrogen $\left(\mathrm{H}_{2}\right)$ into atomic $\mathrm{H}$.

To demonstrate that a small amount of surface damage would provide defects which might act as catalysts for the deep diffusion of hydrogen, $\mathrm{CZ}$ and ASE Silicon samples were mechanically polished with alumina powder of various grit sizes and subsequently annealed for 1 hour at $400^{\circ} \mathrm{C}$ in FG. In this study deuterated forming gas was used to obtain superior detection limits during analysis.

Secondary ion mass spectrometry (SIMS) is the only surface analytical technique capable of the direct detection of. $\mathrm{H}$ and $\mathrm{D}$ in materials. Figure 5 shows SIMS $D$ depth profiles obtained from the different Si materials for different amounts of surface damage. It is clear from the graph that the degree of surface damage is correlated to the amount and extent of hydrogen (D) diffusion into the silicon.

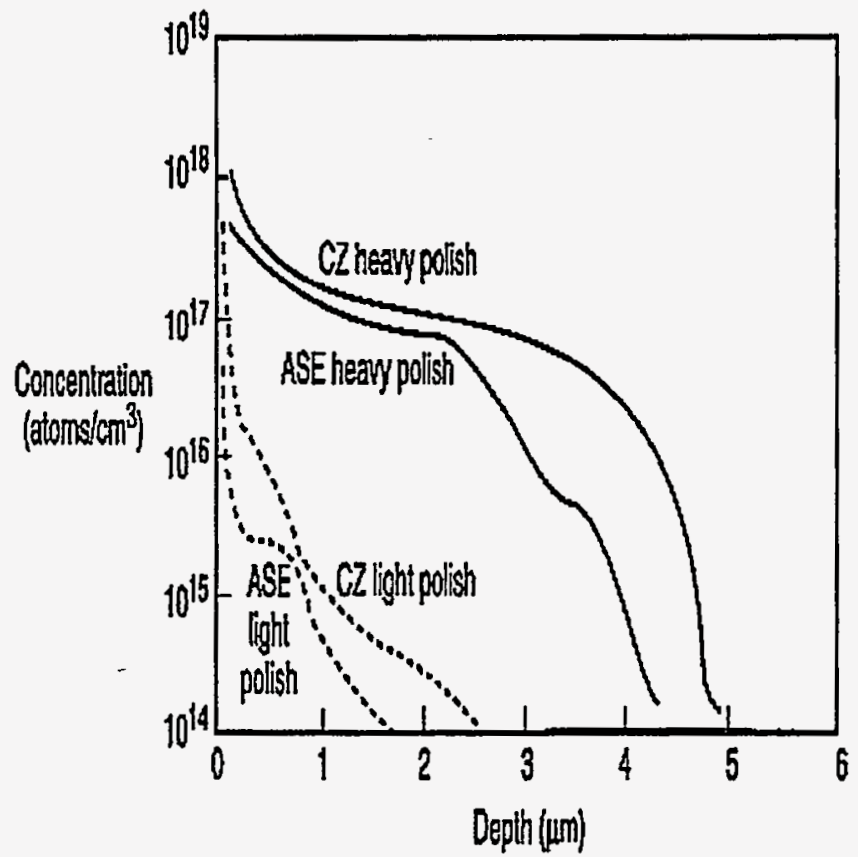

Figure 5. SIMS $H$ (D) depth profiles from mechanically damaged silicon annealed in FG.

This work has facilitated the understanding of hydrogen passivation in Silicon and given vital experimental support to the proposed model.

\section{SIMS: Analysis of Dopant Effects in GaInP Solar Cells}

Changes in emitter doping of solar cells usually affect the blue response of the cell because the blue light is strongly absorbed at the front of the cell in the emitter layer. In GaInP cells, it was found that when the identity of the emitter dopant was changed there were also changes in the back-surface passivation at the back of the cell, sometimes more than at the front. When Se is used as the n-type dopant, a highly Zn-doped GaInP layer makes a good back surface field in an $\mathrm{n}$-on-p GaInP cell. However, this is not the case when $\mathrm{Si}$ is used as the n-type dopant.

SIMS is a powerful technique for the study of dopant distributions in materials. Figure 6 shows depth profiles of the $\mathrm{Zn}$ in two devices where Se or Si were used as the n-type dopant in GaInP cells. The first profile is from a cell with Se doping and shows the $\mathrm{Zn}$ profile to be as 
expected, with little diffusion. The second profile shows the effect of changing to Si doping. In this case, the $\mathrm{Zn}$ profile shows that significant diffusion into the base has occurred. In addition, the profile provides evidence that $\mathrm{Zn}$ accumulates on the p-type side of the pn junction. The SIMS results support other electrical and optical data from these devices.
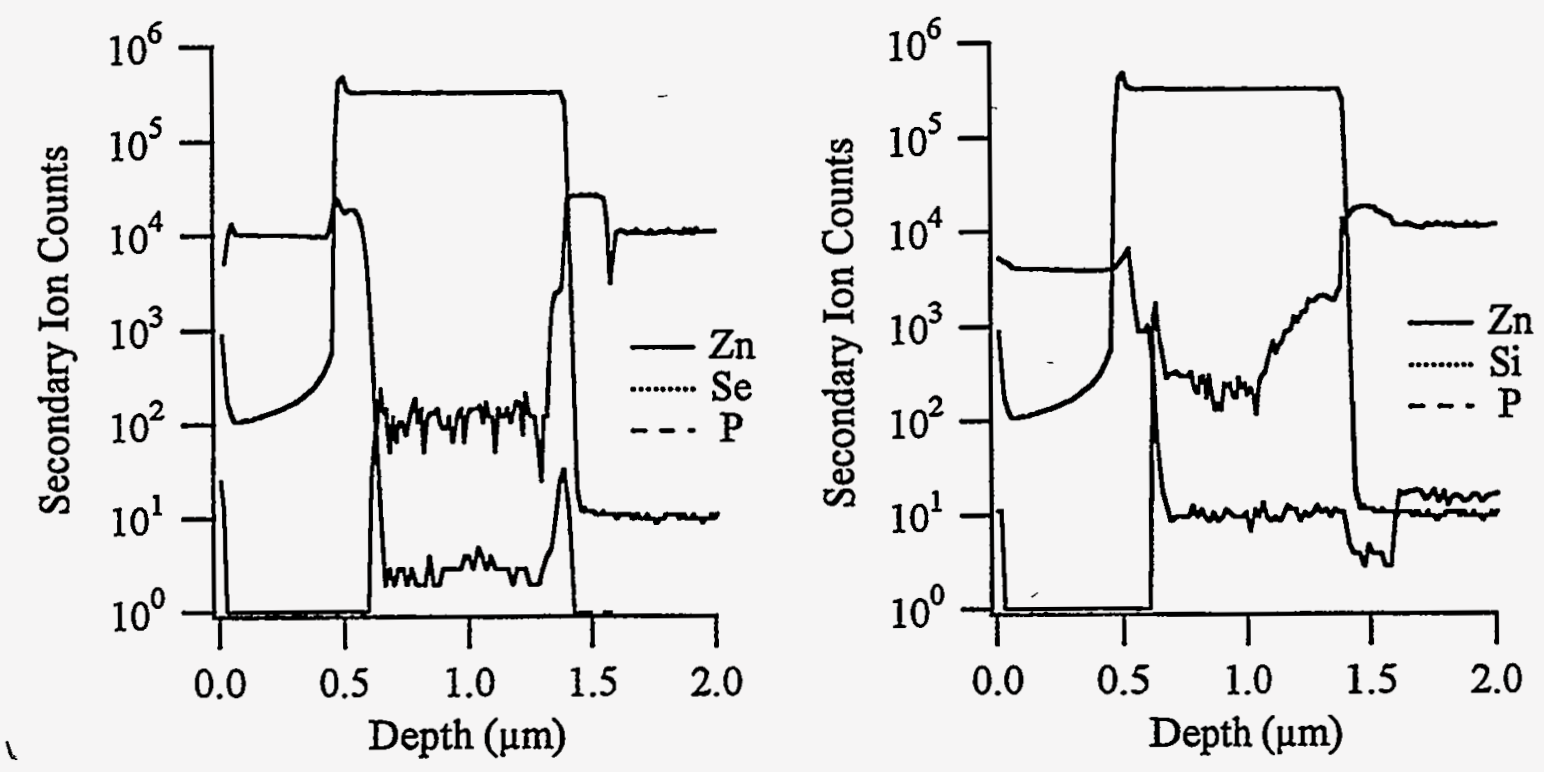

Figure 6. Effect on $\mathrm{Zn}$ dopant profile resulting from a change in the n-type dopant from $\mathrm{Se}$ to $\mathrm{Si}$.

This work has helped to clarify the changes which occur when either Si or Se is used as the ntype dopant in GaInP and GaAs solar cells. Additional work has provided information about how the $\mathrm{Zn}$ diffusion effects can be mitigated by altering growth conditions.

\section{AES: Analysis of peeled $\mathrm{CdTe} / \mathrm{CdS}$ films}

Understanding the composition of the $\mathrm{CdTe} / \mathrm{CdS}$ interface is critical to understanding the operation of this photovoltaic device. It is accepted that a treatment with $\mathrm{CdCl}_{2}$ is necessary to produce the highest quality devices. The interaction of this treatment with the interface and the $\mathrm{CdS}$ is therefore of interest to the CdTe research community. In the past, it was necessary to profile through the entire CdTe layer to reach this region of interest, often up to $10 \mu \mathrm{m}$ of material. The depth profiles were frequently complicated by surface roughness of the $\mathrm{CdTe}$ and by sputter topography which developed during analysis leading to reduced information content. During the past year, however, the NREL CdTe team has developed a method which reliably separates the CdTe/CdS film at the $\mathrm{SnO}_{\mathrm{x}}$. The exposed CdS surface is extremely smooth and provides an unusual opportunity for studying the $\mathrm{CdS} / \mathrm{CdTe}$ interface by surface analytical methods. 


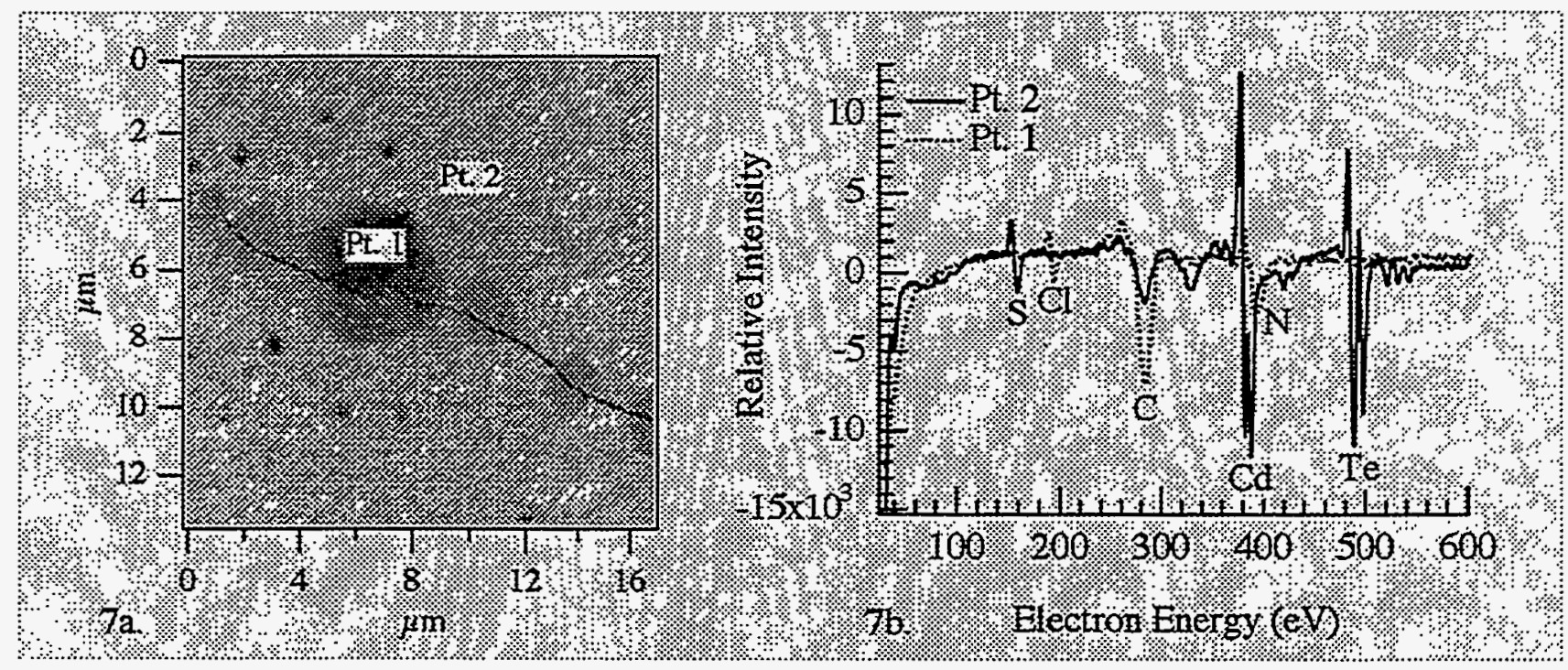

Figure 7. (a) SEM micrograph of crack in peeled $\mathrm{CdS} / \mathrm{CdTe} \mathrm{CdCl}_{2}$ treated film, (b) AES survey spectra from points shown in (a) on and off the crack.

In this study a device quality CdTe layer was deposited on CBD grown CdS. The structure was treated with an overly high level $\mathrm{CdCl}_{2}$ and subsequently peeled from the SnO/glass substrate. SEM analysis of the peeled film in the AES revealed the existence of microcracks in the CdS which appeared to extend into the film. Figure 7a shows an SEM image of one of these cracks. AES survey spectra taken from the two points labeled in Fig. 7a are shown in Figure 7b. It is clear from the graph in Fig. $7 \mathrm{~b}$ that the two regions are significantly different. The crack area contains high levels of $\mathrm{C}, \mathrm{Cl}$, and $\mathrm{N}$, all elements contained in the $\mathrm{CdCl}_{2}$ chemical bath. The surface nearby, however, is predominantly $\mathrm{Cd}, \mathrm{Te}$, and $\mathrm{S}$. These results have also been confirmed by FTIR. These results show one possible explanation for the detrimental effect of overly $\mathrm{CdCl}_{2}$ treating $\mathrm{CdTe} / \mathrm{CdS}$ films, the development of cracks which allow penetration of impurities to the interface. 


\subsection{Thin-Film PV Partnership Project}





\title{
5.0 THIN-FILM PV PARTNERSHIP-Introduction
}

\author{
Ken Zweibel
}

The Thin-Film PV Partnership includes subcontracted research and development (R\&D) in copper indium diselenide (CIS), cadmium telluride (CdTe), and amorphous silicon (a-Si). Research is conducted by teams within each subcontracted organization and by cooperative National Research Teams in a-Si, CdTe, CIS, and Thin-Film Environment, Safety, and Health.

Highlights from fiscal year 1997 include:

In CIS:

- Siemens Solar Industries produced the first commercially available CIS modules. Distributed in limited quantities to test both the manufacturing and product acceptability, the small CIS modules (as much as 1 square foot) were measured at NREL at about $10 \%$ efficiency. When the modules are introduced in volume, this will mark the achievement of a DOE/NREL Five-Year Plan key milestone for 2000 .

In CdTe:

- Solar Cells Inc. (SCI) developed a modified process for depositing CdS/CdTe at high, controllable rates. The previous SCI process required that open boats carrying the CdTe feedstock be changed out frequently. The'new process allows for a continuous-feed system with greater control and improved materials use. This is a significant achievement, because it opens up the possibility of large-scale, low-cost manufacturing of CdTe products.

- The CdTe National R\&D Team focused on determining the key issues of CdTe device degradation under accelerated testing. The results of this testing should prove valuable to the CdTe PV community.

In a-Si:

- United Solar Systems Corporation built and made fully operational the first multimegawatt (MW) amorphous silicon production plant in the world. Its 5-MW plant in Troy, Michigan, manufactures triple-junction cells developed with the participation of the Amorphous Silicon National Research Team.

- Solarex Corporation built (and nearly completed the shakedown on) a new 10-MW, dual-junction facility in Virginia. This is the largest amorphous silicon manufacturing facility in the world.

Project management:

- The Thin-Film PV Partnership re-competed the entire thin-film program in a national competition. More than 60 proposals were received from companies and universities. Awards were made to 36 organizations: 9 for Technology Partners, 23 for Research Partners, and 4 in a "special topic" on high-efficiency CIS-based multijunctions, cosponsored at $\$ 400 \mathrm{~K}$ by the Electric Power Research Institute. 


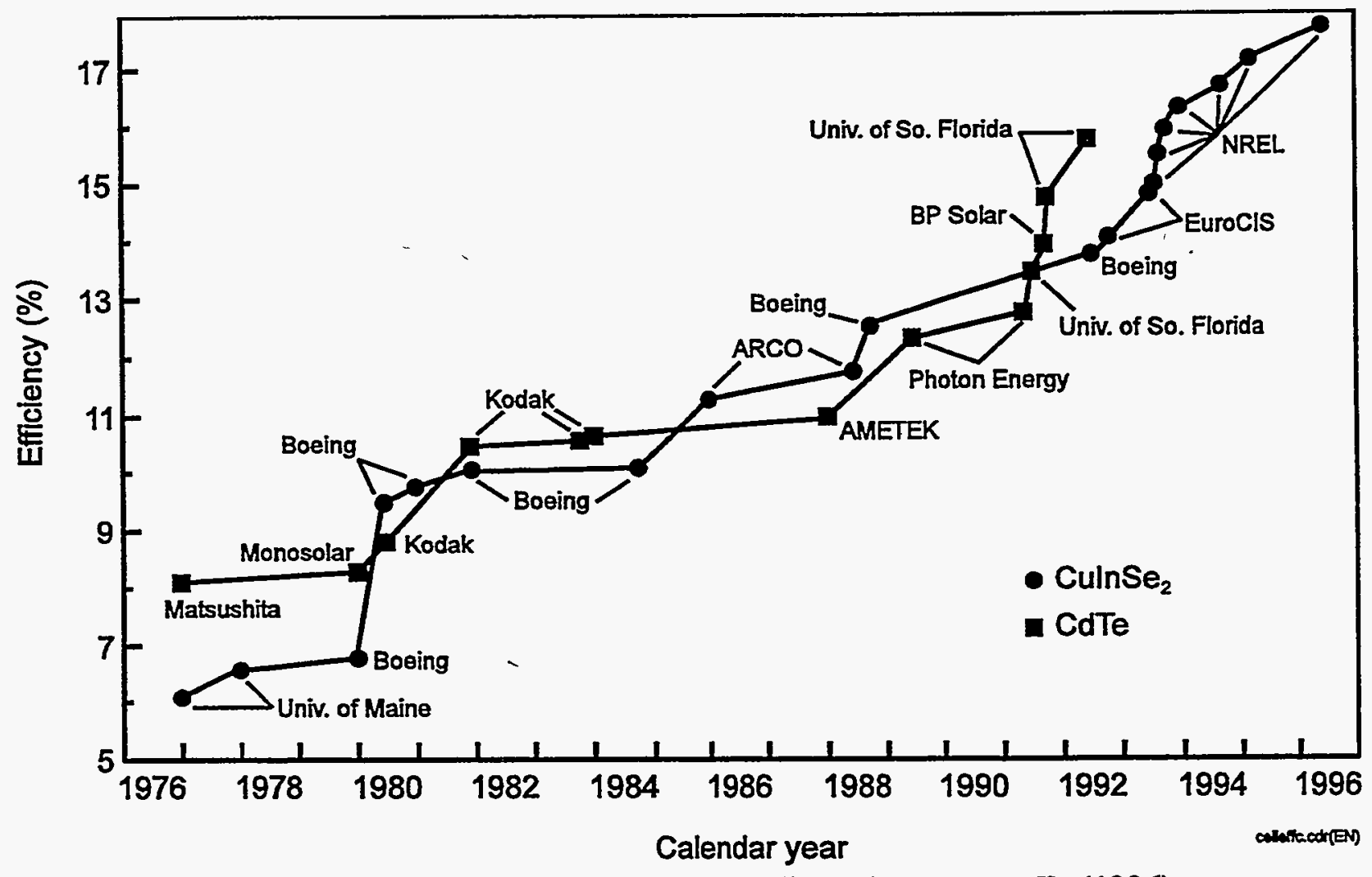

Figure 1 . The best polycrystalline thin-film laboratory cells (1996)

Table 1. The Best Thin-Film Power Modules (1997) (more than 30 watts)

\begin{tabular}{||l|c|c|c|l||}
\hline \multicolumn{1}{|c|}{ Material } & Size $\left(\mathbf{c m}^{2}\right)$ & Efficiency (\%) & Power (watts) & \multicolumn{1}{|c||}{ Company and comments } \\
\hline Amorphous silicon & 9276 & $7.6 \%$ & $70.8 \mathrm{~W}$ & United Solar (September 1997) \\
\hline CdTe & 6728 & $9.1 \%$ & $61.3 \mathrm{~W}$ & Solar Cells \\
\hline Amorphous silicon & 7417 & $7.6 \%$ & $56.0 \mathrm{~W}$ & Solarex (fall 1996) \\
\hline CuInSe (CIS) & 3664 & $11.1 \%$ & $40.6 \mathrm{~W}$ & $\begin{array}{l}\text { Siemens Solar Industries } \\
\text { (April 1997) }\end{array}$ \\
\hline CdTe & 4540 & $8.4 \%$ & $38.2 \mathrm{~W}$ & BP Solar (reported) \\
\hline Amorphous silicon & 4519 & $7.9 \%$ & $35.7 \mathrm{~W}$ & United Solar (June 1997) \\
\hline CdTe & 3366 & $9.2 \%$ & $31.0 \mathrm{~W}$ & Golden Photon Inc. (April 1997) \\
\hline Amorphous silicon & 3906 & $7.8 \%$ & $30.6 \mathrm{~W}$ & Energy Conversion Devices \\
\hline
\end{tabular}

Note: Efficiencies verified independently at NREL unless noted as "reported"; for a-Si they are after 600 hours of light soaking. 


\section{Thin-Film PV Partnership Subcontracts}


Title: . Development Of Monolithic Interconnected, Silicon-Film ${ }^{\mathrm{TM}}$ Modules

Organization: AstroPower Inć., Newark DE. 19716-2000

Contributors: $\quad$ R. B. Hall, Program Manager; D. H. Ford, J. A. Rand, Principal Investigators;

E. J. Delledonne, A. E. Ingram

\section{Introduction}

AstroPower is employing Silicon-Film ${ }^{\text {TM }}$ technology toward the development of an advanced thinsilicon-based, photovoltaic module product. This module combines the design and process features of advanced thin-silicon solar cells, is light trapped, and integrated in a low-cost monolithic interconnected array. This advanced product includes the following features:

- silicon layer grown on a low-cost substrate,

- a nominally 50-micron thick silicon layer with minority carrier diffusion lengths exceeding 100 microns

- light trapping due to back-surface reflection,

- back-surface passivation.

The thin silicon layer pravides for high solar cell performance and can enable a module conversion efficiency as high as $19 \%$. These performance design features, combined with low-cost manufacturing using relatively low-cost capital equipment, continuous processing and a low-cost substrate, will lead to high performance, low cost photovoltaic panels.

\section{Sub-module Device Structure}

Thin films of silicon grown on an insulating substrate allow for the fabrication of large-area, seriesinterconnected sub-modules (see Figure 1). The sub-module design incorporates a method of partitioning the thin-film photovoltaic layer into sub-elements and reconnecting them as a series connected array.

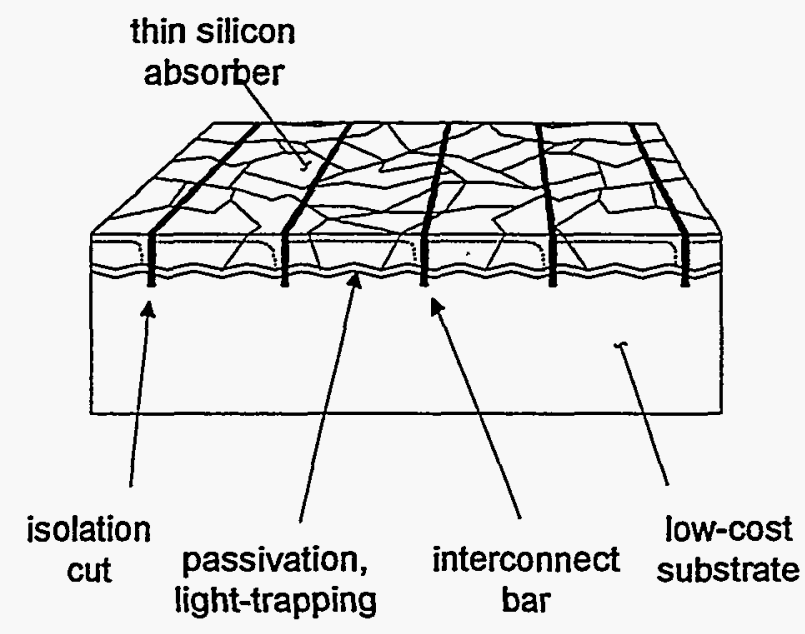

Figure 1. Solar cell sub-module device structure. 


\section{$\underline{\text { Solar Cell }}$}

The solar cell sub-element device design (Figure 2) consists of a thin $(35-50 \mu \mathrm{m})$ polycrystalline silicon layer which is grown on a low-cost substrate material. The thin layer/low-cost substrate approach results in lower cost by minimizing the use of relatively expensive silicon feedstock material. Diffusion lengths equivalent to twice the device thickness are required to assure high carrier collection throughout the bulk of the base layer. Thin silicon enables the use of imperfect materials and increased doping levels. The addition of light-trapping and back-surface passivation, in comparison to thick silicon solar cells, leads to improved solar cell voltage and fill factor.

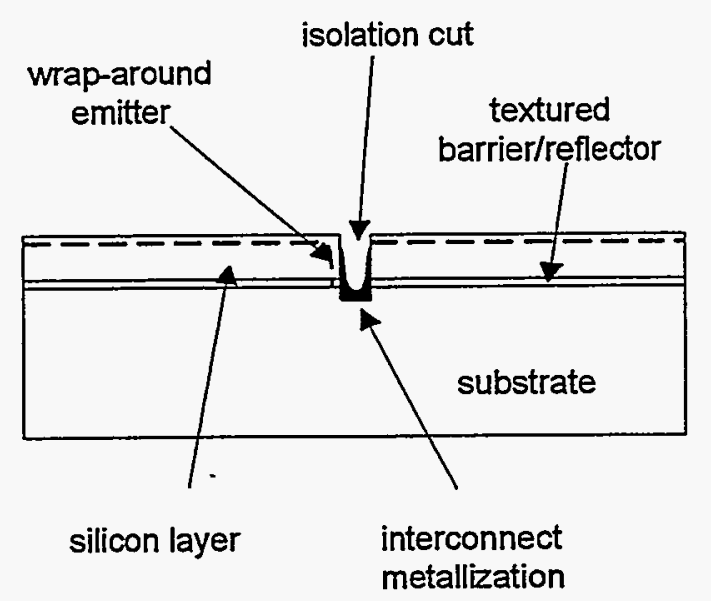

Figure 2. Sub-element device structure.

\section{Substrate}

The substrate is insulated from the active thin silicon layer by an electrical isolation barrier (Figure 3). During large area device fabrication sequences it became evident that the metallization in the isolation cut was a critical factor in achieving good device performance. This metallization process was not fault tolerant and devices were easily shunted. The metallization/interconnect process was modified to reduce the possibility of shunting. Figure 4 shows the modified metallization/interconnect sequence.

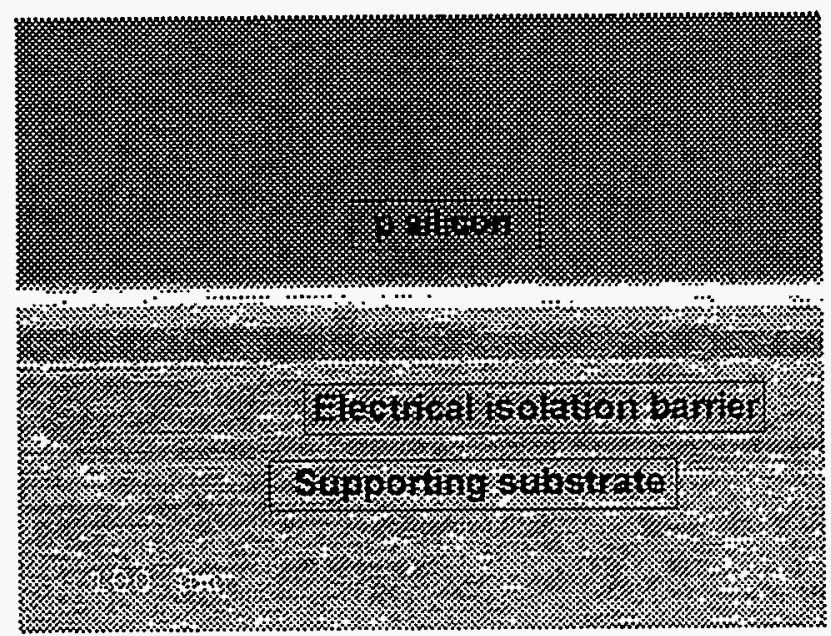

Figure 3. Cross section of thin silicon and electrical isolation barrier on a supporting substrate. 


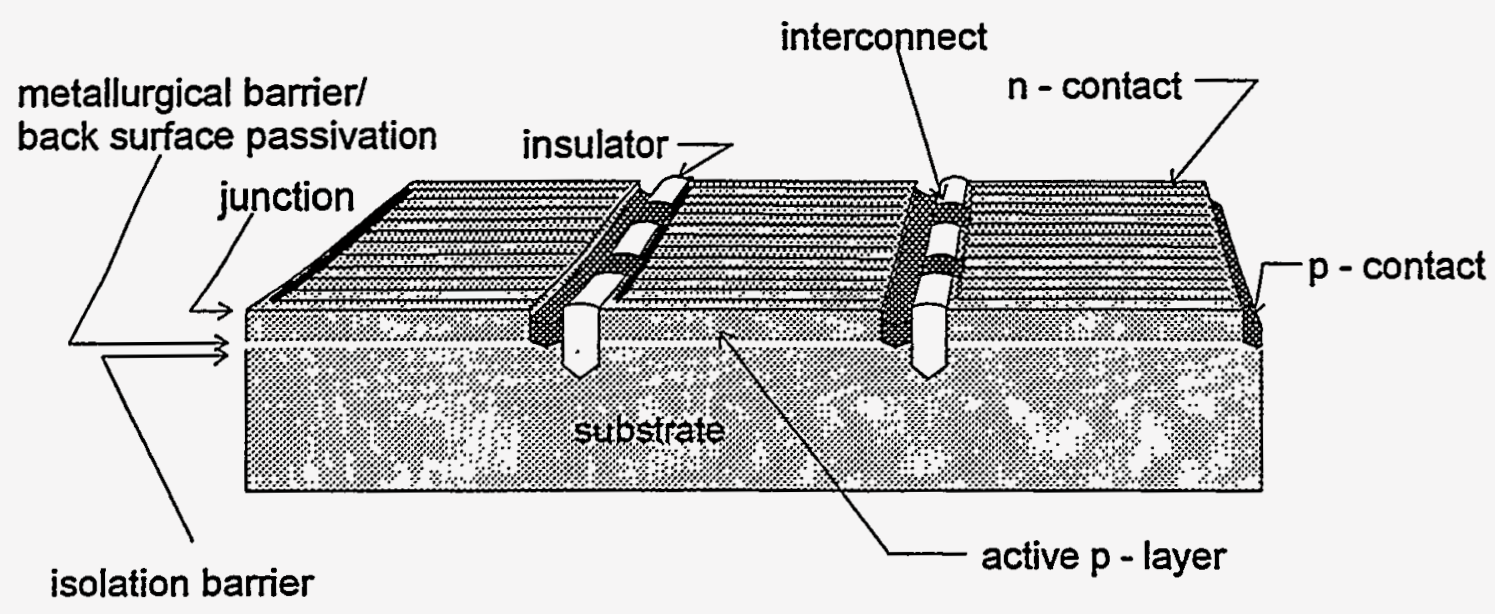

Figure 4. Modified metalization/interconnect.

\section{Results}

Individual components for the new interconnect process were tested to ensure compatibility with the final device fabrication sequence. Following testing of these components, a 36 segment, $321 \mathrm{~cm}^{2}$ monolithically interconnected device was fabricated. Figure 5 shows the current-voltage characteristics of this device as measured by NREL.

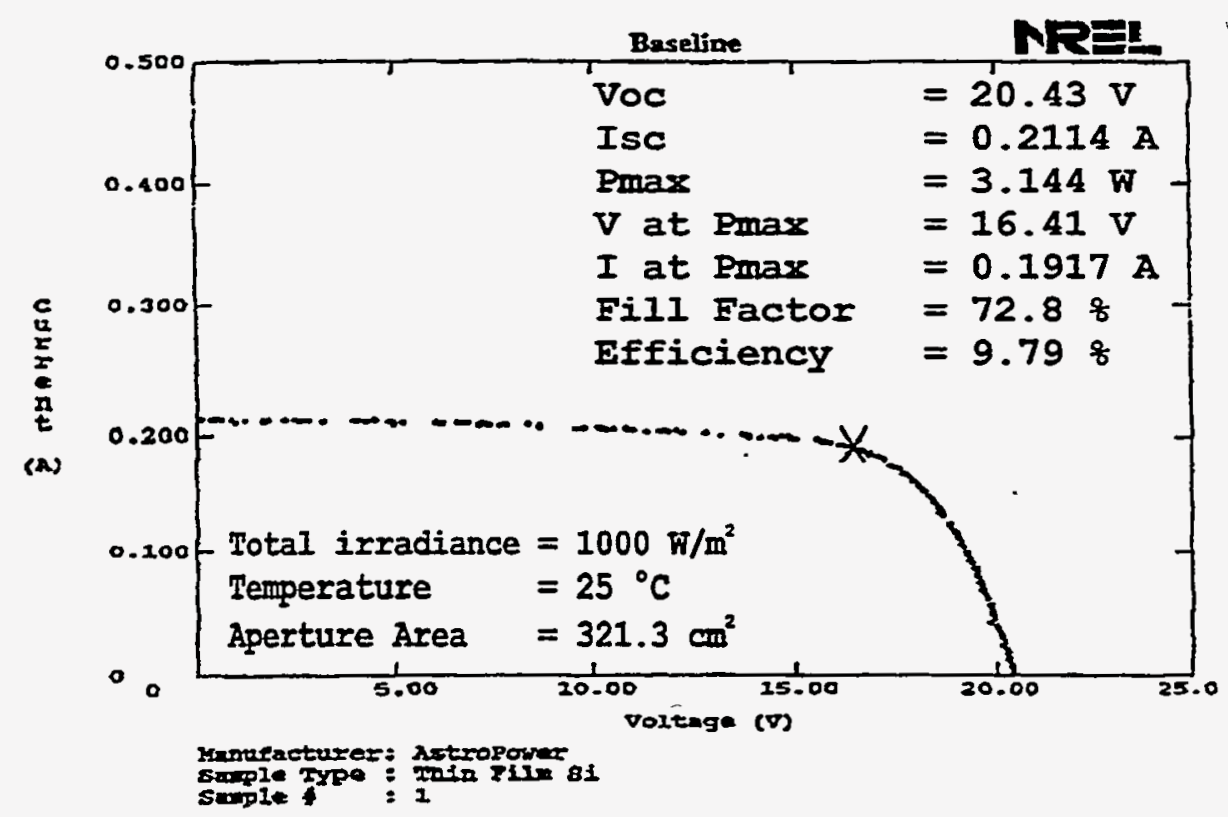

Figure 5. Current-Voltage characteristics of a 36 segment, monolithically interconnected device.

Although significant cost reductions are realized by reducing the active layer thickness, the device structure shown in Figure 4 required a high number of process sequence steps for device fabrication.

A significant reduction in the number of process sequence steps and a greatly simplified interconnect technology can be accomplished with the addition of a back conductor. Figure 6 shows a schematic view of the monolithic interconnect device design that AstroPower is presently pursuing. 


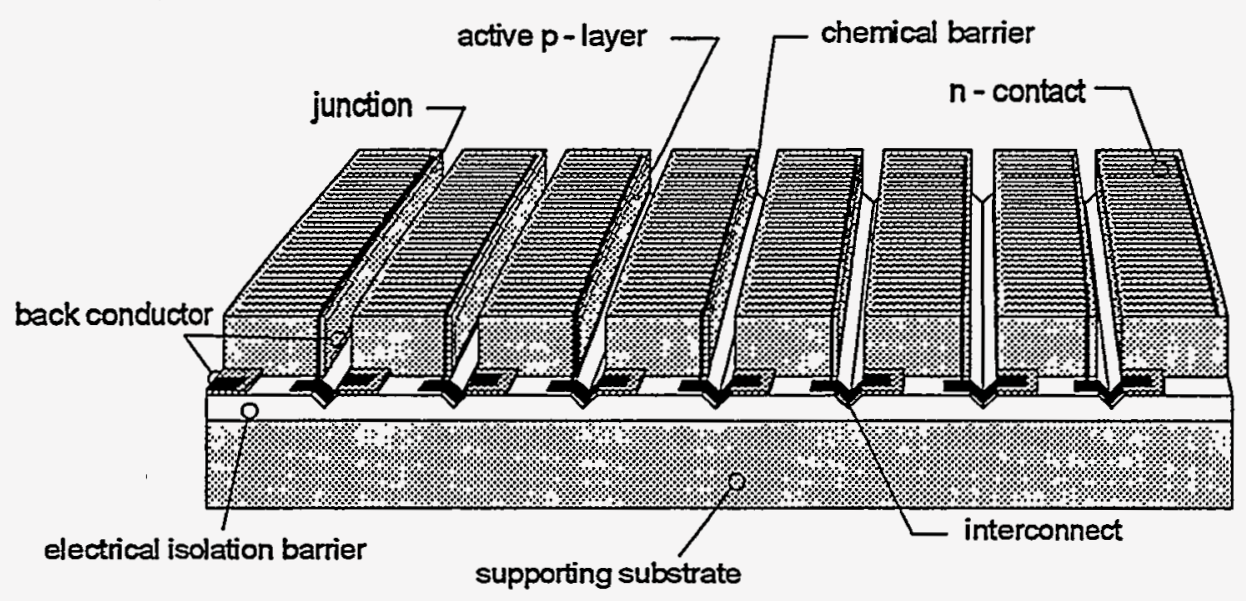

Figure 6. Schematic view of present monolithic device design (Single Back Contact).

Presently we are fabricating the structure based on this new device design that includes the new back conductor (Single Back Contact). The current deposition process used for the back conductor provides a random textured surface which is expected to enhance light trapping effects of the final device.

Thin silicon layers have been achieved on randomly textured back conductors having aspect ratios greater than 5:1 (width:height). The current area being developed is $400 \mathrm{~cm}^{2}$ and is limited by the dice isolation capabilities presently available.

\section{Conclusions}

A $9.79 \%, 321.3 \mathrm{~cm}^{2}$ monolithically interconnected array has been achieved. A new design has been developed to minimize cost/watt by reductions in process complexity. These developments will lead to near term efficiencies of $13 \%$ for large area, monolithically interconnected devices and $19 \%$ in the long term. 


\title{
Title: Nanostructure of Amorphous-Silicon-Based Solar Cell Materials by Small-Angle X-ray Scattering
}

\author{
Organization: Department of Physics, Colorado School of Mines, Golden, Colorado
}

\author{
Contributors: D.L. Williamson, principal investigator; N. Kang
}

\section{Objectives}

The general goals are to establish details of the nanostructure of a-Si:H and related alloys presently being incorporated into solar cells and to help determine whether particular nanostructural features are correlated with photovoltaic behavior. If certain features are found to be detrimental to solar cell efficiency and stability, then optimization efforts will be carried out with the film growers and device makers of the various NREL Amorphous Silicon Teams to improve existing deposition methods or consider alternate methods and materials.

\section{Approach}

The direct structural characterization method of small-angle x-ray scattering (SAXS) is being used to study nanostructural features ranging in size from the atomic up to approximately $30 \mathrm{~nm}$. Features such as nanovoids, hydrogen-rich clusters, columnar-like growth, microcrystallites, surface roughness, and alloy composition fluctuations or segregation can all be detected with a high degree of sensitivity. For example, nanovoids of volume fraction $0.1 \%$ are readily seen and their average size can be determined. Atomic-scale structure can be inferred from the $x$-ray diffuse scattering intensity. Anisotropy in the SAXS, as detected by a sensitivity of the SAXS to sample orientation relative to the $\mathrm{x}$-ray beam, is direct evidence of oriented nanostructural features such as columnar-like growth.

The approach involves collaboration with the NREL Low-gap, Mid-gap, and High-gap Teams, particularly the film-making and device-making groups that supply relevant, systematic sets of samples to help address specific issues within the above objectives. We also collaborate with several groups around the world not supported directly by NREL but recognized as experts in the field. SAXS-suitable . substrates are supplied by us to, all collaborating groups. SAXS measurements made at a central facility allow accurate comparisons of the nanostructure of materials prepared by different deposition methods, conditions, and systems. Other in-house measurements complementing our SAXS studies include Raman spectroscopy, $\mathrm{x}$-ray diffraction and flotation density. A high-quality annealing system is also available.

\section{Results}

The discussion will be separated according to materials appropriate to the low-gap, mid-gap, and highgap components of a multijunction solar cell. The latter is the structure being developed to meet the longrange goal of $15 \%$ stabilized efficiency with the amorphous silicon thin-film technology. Most of the effort during this phase of our research has been on the low-bandgap a-SiGe:H alloys.

\section{Low-bandgap Material}

A systematic study of hotwire $\mathrm{a}_{-\mathrm{Si}_{1-\mathrm{x}} \mathrm{Ge}} \mathrm{G}: \mathrm{H}$ films prepared by NREL is underway[1]. Figure 1 summarizes the integrated SAXS intensities (Q) for films made in an upstream (U) and downstream (D) configuration relative to the gas flow. The $U$ samples are further from the filament and experience a deposition rate at least a factor of two smaller than the $\mathrm{D}$ samples which are directly under the filament. 
Note that there is a dramatic transition in the nature of the nanostructure between 10 and 14 at.\% Ge. The clear, systematic difference in $Q(S A X S)$ between the D and $U$ samples is associated with the increased deposition rate at the $\mathrm{D}$ position such that a stronger columnar-like structure with more nanovoids is produced. These alloys are being characterized by many opto-electronic techniques at NREL and results to date show that they have properties as good as those grown by PECVD, when comparing materials with the same bandgap. However, these results are achieved at deposition rates more than an order of magnitude higher - up to $4 \mathrm{~nm} / \mathrm{s}$. The defect density, determined by the constant photocurrent method, and the dark conductivity increase with $\mathrm{Ge}$ content, while the photoconductivity and ambipolar diffusion length decrease with $\mathrm{Ge}$ content. Such behavior is consistent with the degradation in nanostructure determined by SAXS.

More anomalous SAXS (ASAXS) data has been accumulated from a-Si $1-\mathrm{X}$ were reported for PECVD [2]. New results [3] are from material prepared by the microwave plasma technique developed by USSC to generate higher deposition rates [4,5]. Clear evidence of inhomogeneous distributions of $\mathrm{Ge}$ are found in both types of films with correlation lengths of 1.0 to 1.4 $\mathrm{nm}$. This non-uniformity is removed in the microwave-deposited material by enhanced ion bombardment induced by a substrate bias of $60 \mathrm{~V}$. This improved nanostructure also leads to improved photovoltaic performance [5], but the material is not as stable as the standard PECVD material made at the lower deposition rate [5]. The anisotropic ASAXS from the Ge composition fluctuations is consistent with Ge or Si enrichment along the boundaries of a columnar-like nanostructure.

The world-record triple junction solar cell recently reported by USSC [6] is based on two layers of a-Si 1 ${ }_{x} \mathrm{Ge}_{\mathrm{x}}: \mathrm{H}$ with different $\mathrm{x}$ and on one layer of a-Si:H. USSC prepared films suitable for SAXS that were made under the same nominal conditions used for the three layers (except there was no grading of $x$ as used in the device [6]). Figure 2 compares the dramatic difference in SAXS from the a-Si:H used for the top layer and the a-Si $i_{1-x} \mathrm{Ge}_{\mathrm{x}}: \mathrm{H}$ alloy used for the bottom layer. The a-Si ${ }_{1-\mathrm{x}} \mathrm{Ge}_{\mathrm{x}}: \mathrm{H}$ alloy is much more heterogeneous. The data from the a-Sil-x $\mathrm{Ge}_{\mathrm{x}}: \mathrm{H}$ alloy have been corrected to the same electron density contrast and the same background scattering level as the a-Si:H in order to allow a better comparison. The increased SAXS from the a-Si ${ }_{1-x} \mathrm{Ge}_{\mathrm{x}}: \mathrm{H}$ film is due to nanostructural features near $2 \mathrm{~nm}$ in size. Further work is underway with ASAXS to determine to what degree the SAXS is due to nanovoids versus Ge composition fluctuations.

Since there has been little research on the thermal stability, hydrogen solubility, and hydrogen diffusion in $\mathrm{a}-\mathrm{Si}_{1-\mathrm{x}} \mathrm{Ge}_{\mathrm{x}}: \mathrm{H}$ alloys, we have conducted a set of annealing experiments with a set of six alloys to examine these issues using SAXS [8]. The films were prepared by three different groups (Harvard, USSC, and Stuttgart) and contained different Ge contents ( $x=0.2$ to 0.75 ), different $H$ contents (6 to 20 at.\%) and different initial nanostructures (no detectable nanostructure to significant volume fractions of nanovoids with columnar-like features). Annealing temperature ranged from 150 to $450^{\circ} \mathrm{C}$, with some interdiffusion between the film and Al-foil substrate preventing higher temperature studies. The main results are as follows:

a) in low $\mathrm{H}$ samples with initial columnar-like features, larger voids aggregate along the growth direction and have elongated, ellipsoidal shapes; this growth occurs at the expense of smaller voids;

b) in high $\mathrm{H}$ samples with little oriented initial structure, nucleation of spherical nanobubbles (about $2 \mathrm{~nm}$ diameter), probably containing $\mathrm{H}_{2}$, promotes an increasing volume fraction of inhomogeneities as the annealing temperature is increased above $300^{\circ} \mathrm{C}$;

c) one sample that had a significant inhomogeneous nature in the initial state demonstrated an improvement in nanostructure as detected by decreases in integrated SAXS intensities upon annealing; 
this structural relaxation process is attributed to active diffusion of $\mathrm{H}$ in the matrix;

d) a sample deposited under substrate bias and exhibiting no detectable initial nanostructure was the most stable of all the samples studied; little increase in SAXS was detected up to the maximum temperature of $450^{\circ} \mathrm{C}$

e) samples with higher $\mathrm{Ge}$ content tended to begin structural relaxation at lower anneal temperature $\left(\sim 250^{\circ} \mathrm{C}\right)$ suggesting that the lower bonding energy between $\mathrm{Ge}$ and $\mathrm{H}$ compared to $\mathrm{Si}$ and $\mathrm{H}$ may be responsible for this reduced stability.

These thermal stability results could be relevant to the fabrication of solar cells. The a-SiGe:H alloy may be the first layer deposited so that during its deposition and the subsequent deposition of the remaining layers of the device (at 200 to $400^{\circ} \mathrm{C}$ ), unavoidable annealing takes place. This may be an issue if high $\mathrm{H}$ level are present above the solubility limit.

\section{Mid-bandgap Materials}

The a-Si:H film prepared by USSC to represent the a-Si:H layer in their world record device [6], yields the SAXS shown in Fig.2. The solid line through the data is a fit consistent with only a power law rise at low q due to some large-scale features $(>20 \mathrm{~nm})$ and a constant diffuse intensity due to the atomic-scale compositional and static disorder. If any nanovoids are present, they must be at a level of 0.01 vol.\% or less. This behavior of the top layer material is typical of the best device-quality a-Si:H investigated by SAXS in the past [7].

A film of hotwire a-Si:H, made under device-quality conditions $\left(-375^{\circ} \mathrm{C}\right)$ with low $\mathrm{H}$ content $(-3$ at. $\%)$, has been systematically annealed and characterized by SAXS. The purpose was to search for any diffusion-induced changes nanostructure that might occur due to the unusually high diffusion coefficient for $\mathrm{H}$ in hotwire a-Si:H material [9]. The initial nanostructure was consistent with a maximum void volume fraction of only $0.005 \%$. There was a small change in the SAXS after the first anneal at $250^{\circ} \mathrm{C}$ for $5 \mathrm{~h}$ but essentially no further changes upon annealing for longer times and up to $350^{\circ} \mathrm{C}$. Thus there is no evidence of any significant clustering of hydrogen associated with diffusion of $\mathrm{H}$. This result is consistent with our earlier evidence of a solubility limit in a-Si:H [10] that is near or above the 3 at.\% present in this sample.

\section{High-bandgap Materials}

We have completed SAXS and flotation density measurements of four samples of $a-\mathrm{Si}_{1-\mathrm{X}} \mathrm{C}_{\mathrm{X}}: \mathrm{H}$ prepared by the Indian Association for the Cultivation of Science (IACS) to test a new method involving combination of the PECVD and hotwire techniques. The alloys contained $C$ contents from $x=0.01$ to 0.07 . The results are similar to earlier observations of $\mathrm{a}-\mathrm{Si}_{1-\mathrm{x}} \mathrm{C}_{\mathrm{X}}: \mathrm{H}$ alloys in that relatively small amounts of $\mathrm{C}$ induce increases in the SAXS, corresponding to spherical nanovoids only about $1 \mathrm{~nm}$ in diameter [7]. Although an apparent correlation was noted between the integrated SAXS and the flotation density, it is clear that the density deficits observed, 4 to $18 \%$ relative to $\mathrm{c}-\mathrm{Si}$, are not due simply to the voids since these amount to only 0.1 to 2.4 vol.\%. Most of the deficits must be due to $\mathrm{H}$ alloying.

A series of a-Si $1-\mathrm{x} \mathrm{C}_{\mathrm{x}}: \mathrm{H}$ alloys prepared by $\mathrm{rf}$ reactive sputtering at Iowa State University has been under systematic study by SAXS, IR and SIMS to examine the thermal stability and hydrogen bonding and diffusion behavior. The results are quite similar for all films and SAXS demonstrates the formation of increased nanostructural features above $300^{\circ} \mathrm{C}$. The fits to the SAXS data require the use of an interference model [7] due to a high density of scattering centers. These are associated with $\mathrm{H}$ clustering 
during annealing.

\section{Summary}

We continue to accumulate evidence supporting the premise that a homogeneous nanostructure in a-Si:Hrelated materials yields the best devices. The most striking evidence has come from the collaboration with USSC on the microwave-deposited a-SiGe:H alloys. The use of a substrate bias to induce ion bombardment during high-rate deposition leads to a dramatic reduction in the heterogeneity and to better solar cell efficiencies. However, these cells are not as stable as those made by conventional PECVD. New a-Si ${ }_{1-x} \mathrm{Ge}_{\mathrm{x}}: \mathrm{H}$ alloys made by the hotwire method show opto-electronic properties comparable to the best PECVD material; however, they also show significant heterogeneity similar to the best PECVD material. Additional evidence has been acquired by ASAXS for non-uniform Ge distributions in a-SiGe:H alloys by ASAXS. Such composition fluctuations are likely to be detrimental to carrier transport.

\section{References}

1. S. Heck, B.P. Nelson, D.L. Williamson, Y. Xu, A.H. Mahan, B. von Roedern, A.C. Gallagher, S.E. Schmitt, A. Mason, J. Webb, and R Reedy, abstract submitted to 1998 Spring MRS Meeting.

2. G. Goerigk and D.L. Williamson, HASYLAB 1995 Annual Report, Part II (1996) 303.

3. G. Goerigk and D.L. Williamson, HASYLAB 1996 Annual Report, Part I (1997) 628.

4. S. Guha, X. Xu, J. Yang, and A. Banerjee, Appl. Phys. Lett. 66 (1995) 595.

5. S. Sugiyama, X. Xu, J. Yang, and S. Guha, MRS Symp. Proc. 420 (1996).

6. J. Yang, A. Banerjee, and S. Guha, Appl. Phys. Lett. 70 (1997) 2975.

7. D.L. Williamson, Mat. Res. Soc. Symp. Proc. 377 (1995) 251.

8. N. Kang, M.S. Thesis, Colorado School of Mines (1997).

9. A.H. Mahan et al, Proc. 25th IEEE PV Spec. Conf. (1996) 1065.

10. S.Acco, D.L. Williamson et al, Phys. Rev. B53 (1996) 4415.

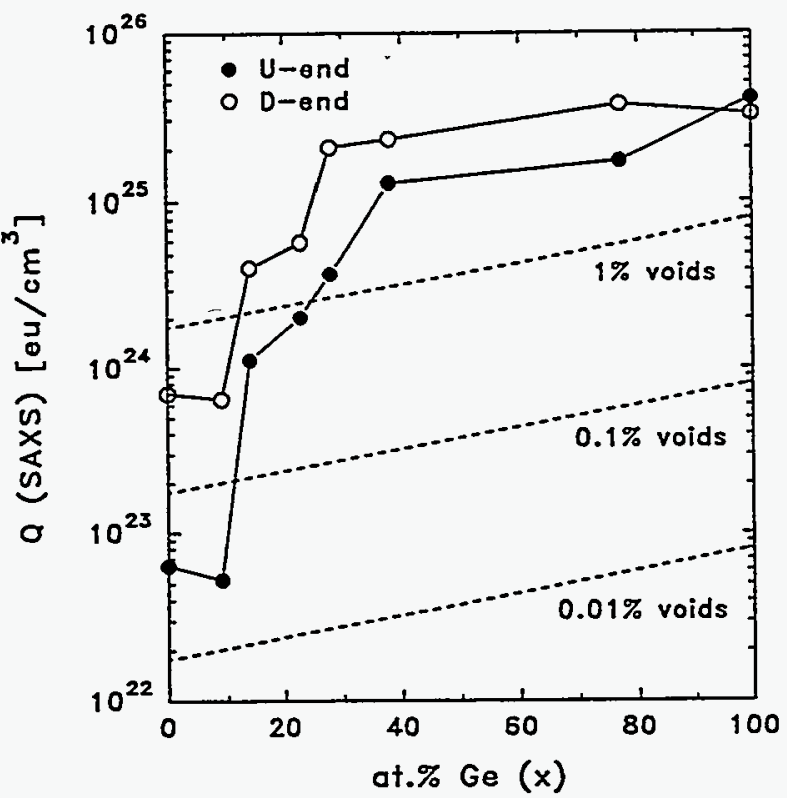

Fig.1. Integrated SAXS intensities from hotwire a-Si ${ }_{1-\mathrm{X}} \mathrm{Ge}_{\mathrm{X}}: \mathrm{H}$ alloys.

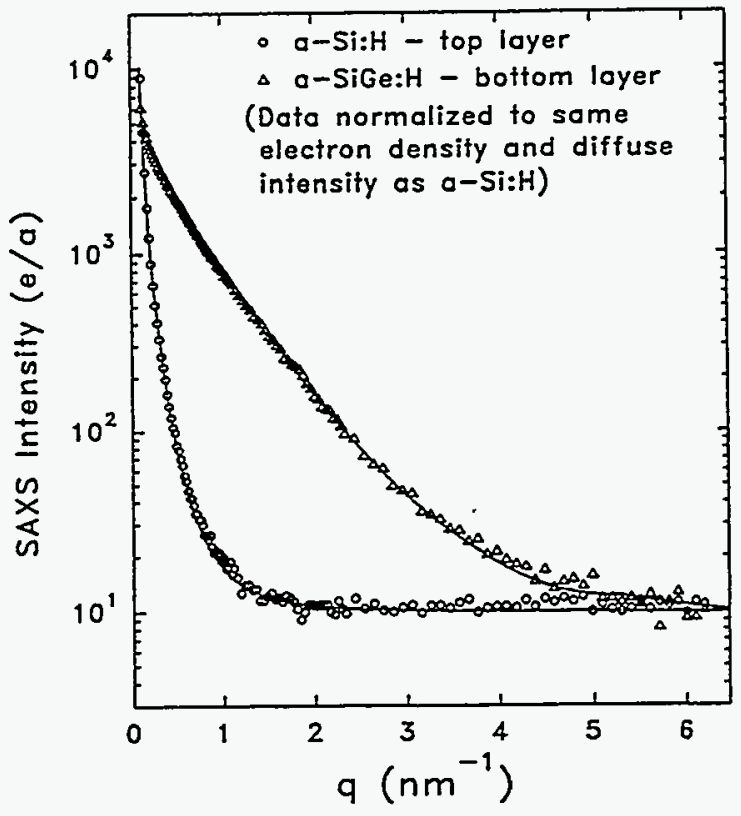

Fig.2. SAXS results from USSC triple-junction solar cell materials. 
Title:

Organization: Department of Physics, Colorado School of Mines, Golden, Colorado 80401

Contributors:
Polycrystalline Thin Film Cadmium Telluride Solar Cells Fabricated by Electrodeposition

J.U. Trefny, D. Mao, V. Kaydanov, T.R. Ohno, co-principal investigators;

D.L.Williamson, R.Collins, T.E Furtak, W.Song, J.Tang, M.H. Aslan,

A.M. Al-Kaoud, A. Balcioglu, F. Kadirgan, and S.Kutun

\section{Objectives}

The objective of this project is to develop improved processes for the fabrication of $\mathrm{CdTe} / \mathrm{CdS}$ polycrystalline thin film solar cellsbased on the formation of CdTe by electrodeposition, a non-vacuum, low-cost technique that is attractive for economic, large-scale production.

\section{Technical Approach}

In FY 1997, our research and development efforts have focused on several steps that are most critical to the fabrication of high-efficiency CdTe solar cells. These include the development of the CdS electrodeposition process, the pre-treatment of $\mathrm{CdS}$ substrates, the post-deposition treatment of $\mathrm{CdTe}$, and back contact formation using $\mathrm{Cu}$-doped $\mathrm{ZnTe}$. Systematic investigations of these processing steps using various methods for material and device characterization were aimed at a better understanding of basic issues related to device performances and stability of the CdTe-based solar cells.

\section{Electrodeposited CdS Thin Films and Their Application in CdS/CdTe Solar Cells}

This FY we started studies of the electrodeposition (ED) of CdS as an alternative to chemical bath deposition (CBD) $[1,2]$. CdS films were grown under various processing conditionsat varying processing conditions $(\mathrm{pH}$, temperature, deposition potential, species sorts and concentration in solution, deposition rate) in the thickness range of $50-250 \mathrm{~nm}$. In terms of film uniformity and cell fabrication, deposition in $\mathrm{HCl}$ solution at $80^{\circ} \mathrm{C}$ was found to be optimum. Films deposited on tin-oxide (TO) substrates consist of agglomerates of small crystallites which cover the substrate uniformly. The agglomerates have a rather uniform size of $\sim 0.7 \mu \mathrm{m}$, and the crystallites' typical size is about $50 \mathrm{~nm}$. Films deposited on indium-tin-oxide (ITO) substrates are much more uniform than films deposited on TO substrates under the same deposition conditions.

$\mathrm{CdTe} / \mathrm{CdS}$ cells were prepared using our standard processing procedure which includes CdTe electrodeposition on $\mathrm{CdS}$ and the back contact $(\mathrm{ZnTe}: \mathrm{Cu} / \mathrm{Au})$ formation. In comparison to the $\mathrm{CBD} \mathrm{CdS}$ films , the ED CdS films have poor adhesion to the TO and flake off easily during the CdTe layer formation. For our standard CdTe/CdS post-deposition treatment, cells made with ED CdS exhibit a low open circuit voltage, $V_{\infty}$, and a low shunt resistance, $R_{s h}$, resulting in low efficiency $(\eta<5 \%)$. A modified post-deposition annealing procedure improved the adhesion and led to a significant improvement of the cell parameters. $\mathrm{V}_{\alpha}=0.65 \mathrm{~V}$ and $\eta \cong 7.7 \%$ obtained with a CdS film thickness of $125 \mathrm{~nm}$ are comparable with what we obtained with CBD CdS of a comparable thickness. However, at larger CdS thicknesses, the efficiency of ED CdS cells is much lower than that of CBD CdS cells. We expect that further optimization of the post-deposition conditions and the fabrication of cells using ITO substrates will lead to a significant improvement of the cell parameters. 
Electronic properties of CdS. Influence of post-deposition treatment and Te diffusion

$\mathrm{CBD} \mathrm{CdS}$ films on $\mathrm{SnO}_{2}$-coated glass substrates were subjected to various post-deposition treatments: no treatment, standard anneal with and without preliminary $\mathrm{CdCl}_{2}$ coating. Optical transmission, differential absorption (DA), and photoluminescence (PL) spectra were measured at room temperature $[3,4]$. Based on these data we have made some preliminary conclusions. There exist significant tails of density of states in the bandgap in the non-treated fine-grain polycrystalline $\mathrm{CdS}$. Annealing without $\mathrm{CdCl}_{2}$ causes an appearance of a defect level or a narrow band in the bandgap which provides a DA peak at a photon energy which is 160 $\mathrm{mV}$ lower than the CdS bandgap. The most narrow and intensive DA and PL peaks corresponding to the interband absorption and recombination were found in the $\mathrm{CdCl}_{2}$-treated samples. This indicates that the defect concentration in the $\mathrm{CdCl}_{2}$-treated $\mathrm{CdS}$ films is much lower than in the two other groups of films. PL spectra for all the samples contain several broad peaks at wavelengths much longer than $500 \mathrm{~nm}$ that should be ascribed to the transitions between band edges and some yet unidentified defect levels in the bandgap. It should be mentioned that DA signal was absent where there were strong PL signals (except one close to $500 \mathrm{~nm}$ for the $\mathrm{CdCl}_{2}$-treated sample). According to ref.5, this may be explained by a FranckCondon effect which is well pronounced in II-VI semiconductors.

We also studied CdTe/CdS films processed in two different ways:(a) CdTe was deposited on an as-deposited CdS layer; (b) CdTe was deposited on a CdS layer pre-treated with $\mathrm{CdCl}_{2}$. In both cases the $\mathrm{CdTe} / \mathrm{CdS}$ structures were coated with $\mathrm{CdCl}_{2}$ and annealed in air at $410^{\circ} \mathrm{C}$ for $45 \mathrm{~min}$. Radio-frequency photoconductive decay (RFPCD) was measured at NREL[3]. The CdS/CdTe structures were illuminated from the CdS side and all the light $(\lambda=335 \mu \mathrm{m})$ was absorbed in the CdS. The RFPCD response $V(t)$ was typical for traplimited recombination. However, a great difference was found between the RFPCD response in two types of the CdTe/CdS structures. In the structures with the pre-treated CdS (negligible amount of Te) the initial signal was two orders of magnitude higher than in the case of non-pretreated CdS (high Te content in CdS). This is evidence of a high concentration of Te-related recombination centers in the latter. Trap emission rates differed by a factor of 7 which means that the traps are of different nature in the two types of samples. Thus, it was shown that preventing Te diffusion into the CdS (by CdS pre-treatment with $\mathrm{CdCl}_{2}$ ) is beneficial for cell performance not only due to the increase in quantum efficiency but also due to the reduction of the recombination centers.

\section{CdTe/CdS post-deposition treatment}

Post-deposition treatment of the CdTe/CdS structure, which includes coating with $\mathrm{CdCl}_{2}$ followed by annealing, is especially important for the cells fabricated with a low-temperature electrodeposition technique. Recrystallization and $\mathrm{S}$ diffusion into $\mathrm{CdTe}$ promoted by $\mathrm{CdCl}_{2}$ are both beneficial for the cell performance. To optimize the postdeposition treatment we investigated the influence of anneal duration and also of the uniformity of sulfur distribution over the CdTe thickness [4]. It was found that the CBD CdS layer that was rather uniform in thickness before the anneal, becomes significantly non-uniform after our standard anneal (45 min.). This leads to a considerable decrease in shunt resistance and open circuit voltage if the initial thickness is less than 180-200 nm. Shortening of the post-deposition treatment was shown to minimize the harmful influence of CdS consumption by the CdTe and provide the same $R_{s h}$ and $V_{\infty}$ values for initial thicknesses that were $50 \mathrm{~nm}$ smaller than usual.

We also fabricated the cells using a modified processing procedure for the $\mathrm{CdTe} / \mathrm{CdS}$ preparation that provided greater content and more uniform distribution of $S$ in CdTe. This procedure was used for thin CdS and short anneal times and provided considerable improvement in all the cell parameters including $\mathrm{V}_{\propto c}, \mathrm{~J}_{s c}$, $R_{s,}$ and $R_{\text {sh }}$. These studies confirmed that there is room for the improvement of cell parameters by optimization of the CdTe post-deposition treatment. An opportunity to thin the CdS layer without losses in 
cell performance is also important with respect to CdS electrodeposition.

\section{Back contact studies}

The back contact we use (evaporated $\mathrm{ZnTe:Cu/metal)} \mathrm{has} \mathrm{yielded} \mathrm{very} \mathrm{low} \mathrm{contact} \mathrm{resistance} \mathrm{of} \mathrm{the} \mathrm{cells}$ fabricated on the electrodeposited CdTe as well as on the materials deposited by close space sublimation, and spray pyrolysis [1]. This FY our studies were mostly focused on the stability issues and the mechanisms responsible for the cell degradation [6,7].

Stress stability tests of the cells with different $\mathrm{Cu}$ content in $\mathrm{ZnTe}$ were conducted in a vacuum oven at $\mathrm{T}=110^{\circ} \mathrm{C}$ for several hundred hours. The major reason for fill factor and efficiency degradation was a significant increase in series resistance due to degradation of the back-contact Schottky diode. Practically no changes were found in $V_{o c}$ and $J_{s c}$. A strong correlation was found between $R_{s}$ increase and decrease in doping level in CdTe derived from C-V profile measurements. Moreover, after a few hundred hours stress test some decrease in $R_{s}$ took place, and some recovery of doping level was observed. Decrease in doping level in the CdTe adjacent to the CdTe/ZnTe interface is considered as a possible reason for the increase in Schottky diode resistance. Comparative studies of $\mathrm{ZnTe}: \mathrm{Cu} / \mathrm{Au}, \mathrm{ZnTe} / \mathrm{Au}$ and $\mathrm{Cu} / \mathrm{Au}$ back contacts definitely showed that the $\mathrm{Cu}$ dopant not only plays a significant role in cell performance but also influences significantly the degradation process. According to literature data, $\mathrm{Cu}$ diffusivity and solubility in $\mathrm{CdTe}$ are very high even at room temperature. The latter is higher by orders of magnitude than the doping levels derived from $\mathrm{C}-\mathrm{V}$ measurements. This brought us to the conclusion that changes in doping level which contribute to the back contact degradation are caused not only and not so much by $\mathrm{Cu}$ diffusion during the stability tests but mainly by insignificant changes in degree of compensation of $\mathrm{Cu}$-induced acceptors, e.g. $\mathrm{Cu}_{\mathrm{Cd}}$, by donors such as $\mathrm{Cu}_{\mathrm{t}}$ $\mathrm{V}_{\mathrm{Cd}}$, etc.

$\mathrm{X}$-ray photoemission spectroscopy and Auger electron spectroscopy studies were conducted on cells subjected to a $140^{\circ} \mathrm{C}$ anneal for 165 hours. In order to avoid the influence of the CdTe surface roughness, the same studies were repeated on $\mathrm{ZnTe}: \mathrm{Cu} /$ metal structures grown on the smooth surfaces of Si wafers. In both cases significant amounts of $\mathrm{Te}$ and $\mathrm{Cu}$ were detected in $\mathrm{Au}$ when it was used as the metal. In addition, some dots containing over $15 \% \mathrm{Au}$ were found on the top surface of the $\mathrm{ZnTe} / \mathrm{Au} / \mathrm{Si}$ structures. No traces of interdiffusion were detected in $\mathrm{ZnTe}: \mathrm{Cu} / \mathrm{Ni}$ contacts. Increases in $\mathrm{R}_{s}$ for $\mathrm{Ni}$-contacted cells were slower than for $\mathrm{Au}$-contacted ones but drops in $\mathrm{R}_{\mathrm{sh}}$ were considerably greater which led to a higher degradation rate of efficiency for $\mathrm{Ni}$-contacted cells.

\section{CdTe team work participation}

\section{Fabrication of $\mathrm{CdTe} / \mathrm{CdS}$ cells on $\mathrm{TiO}_{2}$-coated $\mathrm{SnO}_{2}$ substrates}

We participated in team work which involved exploring the usage of $\mathrm{TiO}_{2}$ as an "intrinsic" layer on the $\mathrm{SnO}_{2}$ substrates to prevent shunting associated with pinholes in $\mathrm{CdS}$. The $\mathrm{TiO}_{2}$ and $\mathrm{SnO}_{2}$ films were grown by the Harvard and the USF groups, respectively. It was found that the $\mathrm{TiO}_{2}$ film is too resistive for the electrodeposition of CdTe films which show very poor adhesion and peel off during the deposition or postdeposition annealing. Modification of our annealing procedure enabled us to fabricate cells with limited success. The high $\mathrm{V}_{o c}$ (up to $0.85 \mathrm{~V}$ ) and reasonable $\mathrm{J}_{\mathrm{sc}}\left(20.8 \mathrm{~mA} / \mathrm{cm}^{2}\right)$ values obtained for cells without the $\mathrm{CdS}$ layer indicate that the $\mathrm{TiO}_{2} / \mathrm{CdTe}$ junction may be promising. The low fill factor might be improved by doping of the $\mathrm{TiO}_{2}$ film.

ZnTe back contact fabrication on CdTe/CdS films prepared by other institutions

CdS/CdTe solar cells were fabricated on materials supplied by Golden Photon, Inc. (GPI) and Solar Cells, Inc. (SCl). Processing conditions ( $\mathrm{Cu}$ dopant concentration in $\mathrm{ZnTe}$, post-deposition annealing temperature, 
and $\mathrm{ZnTe}$ film thickness) were varied to optimize cell parameters. Efficiencies achieved were as high as $14 \%$ for GPI double-recrystallized material and $13.3 \%$ for SCI materials (the cells were tested at CSM).

\section{References}

1 J.U. Trefny and Duli Mao, "Polycrystalline Thin Film CadmiumTelluride Solar Cells Fabricated by Electrodeposition", Annual Technical Report, contract NREL/XAF-5-14142-11, March 20, 1996 to March 19,1997.

2. Figen Kadirgan, D. Mao, A. Balcioglu, B.E. McCandless, W. Song, T.R. Ohno, and J.U. Trefny, "Electrodeposited CdS Thin Films and Their Application in CdS/CdTe Solar Cells", presented at the 26th IEEE PVSC, Anaheim, CA, September 29- October 3, 1997

3. R.K. Ahrenkiel, D.H. Levi, S. Jonston, W.Song, D. Mao, A. Fisher, "Photoconductive Lifetime of CdS Used in Thin Film Solar Cells", ibid

4. J.U. Trefny, "Polycrystalline Thin Film Cadmium Telluride Solar Cells Fabricated by Electrodeposition", Quarterly Technical Status Report, contract NREL/XAF-5-14142-11, July 1997

5. R.K. Ahrenkiel, B.M. Keyes, and D.L. Levi, "Recombination Processes in Polycrystalline Photovoltaic Materials", Proc. Photovoltaic Solar Energy Conference, H.S Stephens \& Associates, U.K., 1996, p.914

6. J. Tang, D. Mao, T.R. Ohno, V.I. Kaydanov, and J.U. Trefny, "Properties of ZnTe:Cu Thin Films and CdS/CdS/CdTe/ZnTe Solar Cells", presented at the 26th IEEE PVSC, Anaheim, CA, September 29October 3, 1997

7. J.U.Trefny, "Polycrystalline Thin Film Cadmium Telluride Solar Cells Fabricated by Electrodeposition", Quarterly Technical Status Report, contract NREL/XAF-5-14142-11, October 1997 
Organization: $\quad$ Colorado State University, Fort Collins, Colorado

Department of Physics

Contributors:

J.R. Sites, Principal Investigator; J.E. Granata, J.F. Hiltner, H. Sax, L.D. Leiby, E.S. Mandell

\section{Objectives}

The objectives of this program are (1) the separation and quantification of individual losses in specific thin-film solar cells, (2) the detailed characterization of small modules, and (3) the presentation of a usable model for the forward-current loss mechanism.

\section{CdTe CellAnalysis}

One CdTe analysis project has been to characterize the impact of back-contact barriers on cell performance [1]. One set of cells was the same as those from seven laboratories used previously to explore the effect of thin CdS. The second set was a spectrum of eight different back contacts deposited at four laboratories onto common CdS/CdTe cells. In all cases, with one exception, measurements at lower temperatures revealed forward-current limitation with an onset temperature generally between -10 and $+20^{\circ} \mathrm{C}$. (See Fig. 1). In general, the effect on fill-factor at room temperature and above was negligible.

A second CdTe project has been stress tests on the second set cells from the back-contact study [2]. A facility was built to allow cells to be held at elevated temperatures, light or dark, for extended periods of time. Significant changes were observed at temperatures of $100^{\circ} \mathrm{C}$ for cells in the light, and hence at opencircuit voltage. The open-circuit voltage was reduced somewhat, capacitance was modified, and the current-limiting effect was enhanced. The results strongly suggest that two different mechanisms are involved, one a change in the primary junction and one a change in the back contact.

\section{CI(G)S Cell and Module Analysis}

A continuing project has been an attempt to quantify the impact of sodium incorporation on the performance of CIS and CIGS cells [3]. Three different soda-line-glass (SLG)/molybdenum substrates and one alumina/molybdenum substrate were used. Significant differences (Fig. 2) in open-circuit voltage and fill factor as a function of sodium concentration were observed. The optimum sodium concentration was estimated to be between 0.05 and 0.5 atomic percent.

Two cell projects were done in support of larger NREL efforts. One was the characterization of cells with the CIGS absorber made by electrodeposition of metallic precursors [4,5]. These cells has comparable performance to those of the same bandgaps made by standard NREL techniques. The second project was a continuing effort to understand the effects of CIGS surface treatment prior to the deposition of CdS or $\mathrm{ZnO}$ window layers $[6,7]$. In addition to the NREL-support projects, a stress-test project on CIS minimodules from Siemens used the same facility described above [8]. In this case, heating in the dark to $85^{\circ} \mathrm{C}$ led to a decrease in fill-factor, but it was very nearly restored, on a time scale of minutes, under exposure to light. 


\section{Polycrystalline Cells in Context}

A collective effort was made to explore which thin-film device and materials characterization techniques could give useful and timely information in a manufacturing environment [9]. Such device measurements include optics, full photon accounting, bandgap from quantum efficiency, resistive losses, junction quality, and signatures of typical abnormalities. Another study looked at the losses in the highest efficiency CaTe and CIGS cells and evaluated which were directly attributable to polycrystallinity [10]. Nearly all the differences in voltage, compared with the best crystalline cells, were due to polycrystallinity, but only about half the fill-factor differences and less that half of the current differences.

\section{References}

1. J.E. Granata, J.F. Hiltner, H. Sax, and J.R. Sites, National CdTe Team Meeting, Minutes, April 30, 1997, Newark, DE.

2. J.F. Hiltner, H. Sax, and J.R. Sites, National CdTe Team Meeting Minutes. October 1, 1997, Anaheim, CA.

3. J.E. Granata, J.R. Sites, S. Asher, and R.J. Matson, Quantitative Incorporation of Sodium in $\mathrm{CuInSe}_{2}$ and $\mathrm{Cu}(\mathrm{In}, \mathrm{Ga}) \mathrm{Se}_{2}$ Photovoltaic Devices, "IEEE PVSC 26 (1997).

4. R.N. Bhattacharya et.al., "CuIn ${ }_{1-x} \mathrm{Ga}_{x} \mathrm{Se}_{2}$-based Photovoltaic Cells from Electrodeposited Deposited Precursor," SPIE Proc. 3138, 90 (1997).

5. R.N. Bhattacharya et.al., "CuIn ${ }_{l-x} \mathrm{Ga}_{x} \mathrm{Se}_{2}$-based Photovoltaic Cells from Electrodeposited and Chemical Bath Deposited Precursors," 2nd Intl. Symp. On Solar Energy Materials and Solar Cells. (in press).

6. K. Ramanathan et.al., "Advances in the CIS Research at NREL," IEEE PVSC 26, (1997).

7. K. Ramanathan et.al., National CIS Team Meeting Minutes, Sept. 29, 1997, Anaheim, CA.

8. J.R. Sites and J.F. Hiltner, National CIS Team Meeting Minutes, Sept. 29, 1997, Anaheim, CA.

9. J.R. Sites, J. Rand, L.L. Kazmerski, and J.E. Phillips, "Device and Materials Characterization in Manufacturing," Prog. In Photovoltaics 5, 371 (1997)

10. J.R. Sites, J.E. Granata, and J.F. Hiltner, "Losses Due to Polycrystallinity in Thin Films," SolarEnergy Materials and Solar Cells (in press). 


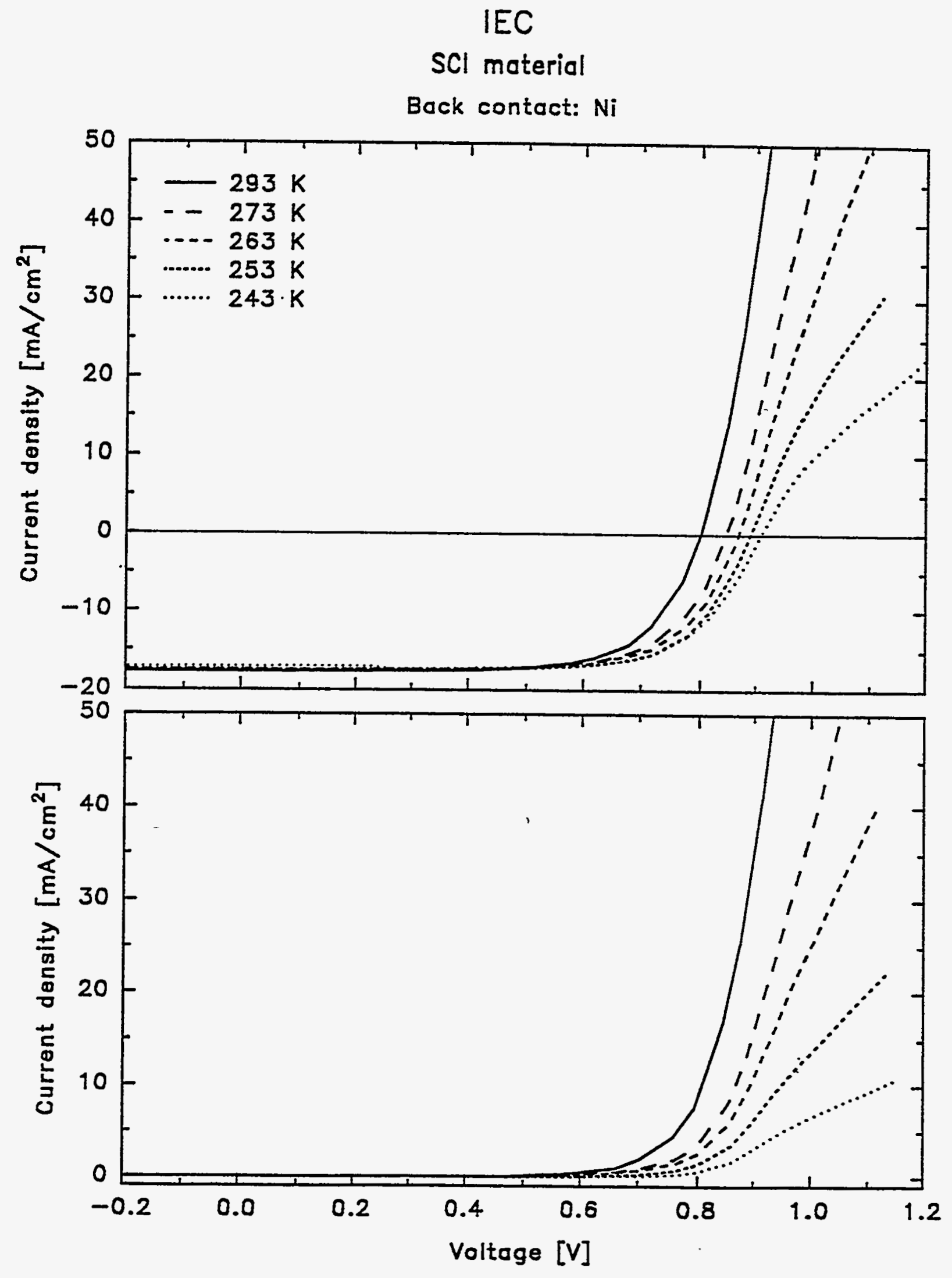

Fig. 1. Typical CdTe curves showing current limitation at reduced temperatures. 

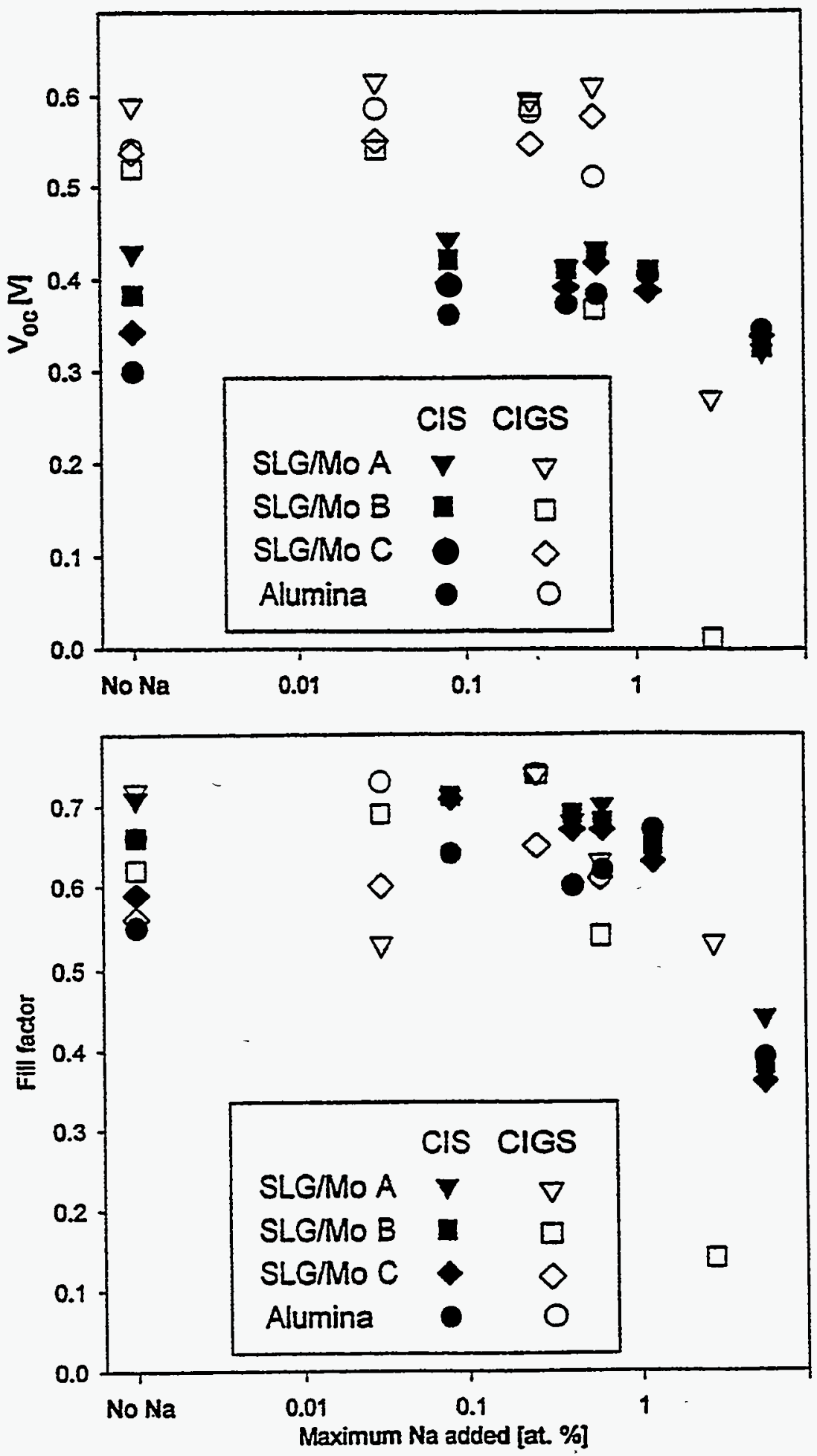

Fig. 2. Variations in voltage and fillfactor with sodium addition for nominally identical cells fabricated in four substrates. 
Title:

Organization:

Contributors:
Development of High, Stable-Efficiency

Triple-Junction a-Si Alloy Solar Cells

Energy Conversion Devices Inc.

1675 West Maple Road, Troy, MI 48084

X. Deng, S. J. Jones, T. Liu, M. Izu, and S. R. Ovshinsky

\section{Objectives}

The overall objective of this amorphous silicon research program is to develop high efficiency a-Si solar cells and to develop and improve processes for large area deposition of a-Si solar cells and modules. The knowledge obtained and technologies demonstrated in this program will be immediately incorporated into ECD's continuous roll-to-roll deposition process to further enhance its photovoltaic manufacturing technology.

\section{Technology}

In ECD's solar cell design, triple-junction a-Si alloy solar cells are deposited onto stainless steel substrates coated with $\mathrm{Ag} / \mathrm{ZnO}$ back reflector. This type of cell enables us to use a continuous roll-toroll deposition process to manufacture a-Si PV materials in high volume [1-7]. Using this design, ECD has previously achieved record high initial solar cell efficiency using the following features: 1) a triplejunction two-bandgap, spectrum splitting solar cell design; 2) a microcrystalline silicon p-layer; 3) a bandgap profiled a-SiGe alloy as the bottom cell i-layer; 4) a high performance $\mathrm{Ag} / \mathrm{ZnO}$ back reflector; and 5) a high performance tunnel junction between component cells. The research team at United Solar have recently used the same design to prepare devices with $13 \%$ stable efficiency, a new world record $[6]$.

\section{Approaches}

In this program, we are further advancing ECD's existing PV technology by performing the following tasks:

Task 1: Optimization of large-area plasma deposition of a-Si films;

Task 2: Optimization of large-area plasma deposition of a-SiGe films;

Task 3: Research on $\mu \mathrm{c-Si} \mathrm{p}^{+}$deposition using VHF plasma; and

Task 4: Optimization of a-Si solar cell back-reflector.

\section{Accomplishments}

In the following, we summarize our research under various areas of this program.

\section{Microcrystalline Si p-layer using a modified VHF plasma deposition process}

Microcrystalline Si p-layer have been widely used in a-Si solar cell technology to achieve high efficiency. To further improve the solar cell performance, we have studied the deposition of high quality $\mu \mathrm{c}-\mathrm{Si}$ p-layer material using a modified very high frequency (VHF) plasma CVD process. 
Table 1 show the results of solar cells whose p-layers were deposited using different processes: conventional RF, conventional VHF and a modified VHF process. Compared with p-layers deposited using conventional $\mathrm{RF}$, there is a $6 \%$ improvement in $\mathrm{J}_{\mathrm{SC}}$ and an up to $10 \%$ improvement in efficiency for p-layers deposited using the modified VHF process. The increased solar cell performance is mainly due to an higher degree of microcrystallinity in the p-layer.

Table 1 Results of solar cells whose p-layers were deposited using different processes: conventional RF, conventional VHF and a modified VHF process.

\begin{tabular}{|c|c|c|c|c|c|c|c|c|c|}
\hline $\begin{array}{c}\text { Sample } \\
\text { No. }\end{array}$ & $\begin{array}{l}\text { p-layer } \\
\text { Run\# }\end{array}$ & p process & $\begin{array}{l}V_{O C} \\
(V)\end{array}$ & $\begin{array}{c}J_{\mathrm{SC}} \\
\left(\mathrm{mA} / \mathrm{cm}^{2}\right)\end{array}$ & $\overline{\overline{F F}}$ & $\begin{array}{c}R_{\mathrm{S}} \\
\left(\Omega \circ \mathrm{cm}^{2}\right. \\
)\end{array}$ & $\underset{\left(\mathrm{mW} / \mathrm{cm}^{2}\right)}{P_{\max }}$ & $\begin{array}{c}J_{\mathrm{sc}} \text { from } \mathrm{QE} \\
\left(\mathrm{mA} / \mathrm{cm}^{2}\right)\end{array}$ & $\begin{array}{c}\text { QE@400nm } \\
(\%)\end{array}$ \\
\hline 1 & $\overline{L 2523}$ & Modified VHF & 0.959 & 10.39 & 0.664 & $\overline{\overline{11.5}}$ & 6.62 & 10.71 & 0.655 \\
\hline 2 & L2525 & Conventional RF & 0.948 & 9.82 & 0.63 & 14.8 & 5.86 & 10.09 & 0.591 \\
\hline 3 & L2526 & Conventional VHF & 0.933 & 8.78 & 0.651 & 12.2 & 5.33 & 10.04 & 0.56 \\
\hline
\end{tabular}

\section{High rate deposition of a-Si using VHF Plasma}

We have studied deposition of a-Si films using VHF plasma at high rate, up to $25 \mathrm{~A} / \mathrm{s}$. In this study, we extensively explored the quality and stability of the i-layers under different deposition conditions, including VHF power, silane flows, hydrogen dilution, pressure, and substrate temperatures. The quality and stability of the i-layers are evaluated by using solar cells having $2500 \mathrm{~A}$ thick i-layer deposited on stainless steel without the use of a back-reflector. Figure 1 shows the initial Pmax $\left(\mathrm{P}_{\text {int }}\right)$ and stabilized Pmax after 1000 hour light soaking $\left(\mathrm{P}_{1000}\right)$ of solar cells with i-layers deposited using VHF plasma at different deposition rates. We have obtained high stability for i-layers deposited at rates up to $12-15 \mathrm{~A} / \mathrm{s}$ using improved VHF plasma deposition process.

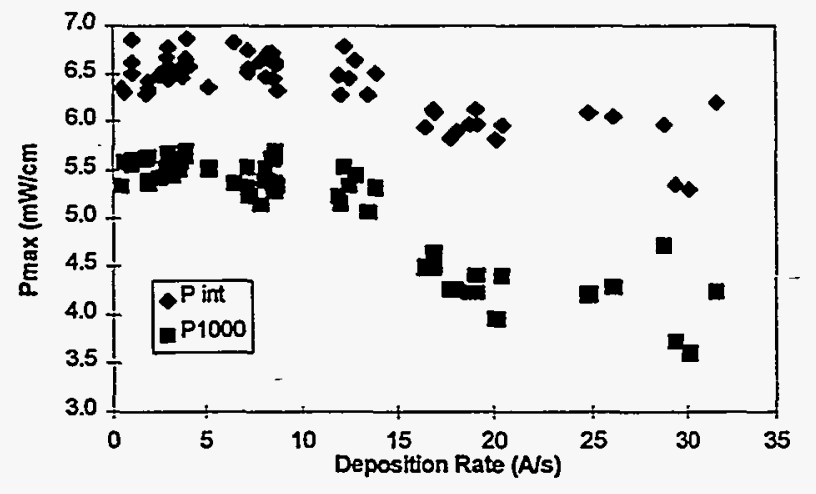

Figure 1 Initial and stabilized $\mathrm{P}_{\max }$ before and after 1000 hours of 1 sun light soaking for devices having VHF i-layers deposited at different deposition rate.

In Table 2, we compare the light soaking stability of a-Si solar cells with i-layers deposited using VHF and RF at both high and low deposition rates. The VHF devices show much improved stability as compared with RF devices, particularly at high deposition rates. 
Table 2 Comparison of light soaking stability between devices with i-layers deposited using RF and VHF.

\begin{tabular}{cccccc}
\hline \hline $\begin{array}{c}\text { Sample } \\
\text { No. }\end{array}$ & $\begin{array}{c}\text { Ref. } \\
\text { No. }\end{array}$ & $\begin{array}{c}\text { Plasma } \\
\text { Frequency } \\
(\mathrm{MHz})\end{array}$ & $\begin{array}{c}\text { Dep. } \\
\text { Rate } \\
(\mathrm{Ass})\end{array}$ & $\begin{array}{c}\text { Tnitial } \\
\mathrm{P}_{\max } \\
\left(\mathrm{mW} / \mathrm{cm}^{2}\right)\end{array}$ & $\begin{array}{c}\text { Light } \\
\text { degrad. } \\
(\%)\end{array}$ \\
\hline \hline Sample 30 & L2710 & RF (13.56) & 0.61 & 6.65 & 14.3 \\
Sample 16 & L2656 & VHF (70) & 9.9 & 6.51 & 10 \\
\hline Sample 31 & L2715 & RF (13.56) & 16 & 5.3 & 36.2 \\
Sample 24 & L2708 & VHF (70) & 24.8 & 6.04 & 21.9 \\
\hline \hline
\end{tabular}

\section{Deposition of high $V_{o c}$ solar cell at high rate}

We have previously reported the fabrication of a-Si single junction solar cells with $\mathrm{V}_{\text {oc }}$ up to $1.053 \mathrm{~V}$. However, the deposition rates of the i-layers for these high Voc solar cells were low. We have investigated the deposition of a-Si i-layer for high $\mathrm{V}_{\mathrm{OC}}$ solar cells at high rate using VHF plasma deposition. Table 3 shows the solar cell performance with i-layers deposited using VHF. With high hydrogen dilution, we obtained solar cells with $1.01 \mathrm{~V}$ open circuit voltage while the $\mathrm{i}$-layers were deposited at a high rate of $5.2 \mathrm{~A} / \mathrm{s}$.

Table 3 IV results of high Voc solar cells with i-layer deposited at high deposition rate.

\begin{tabular}{|c|c|c|c|c|c|c|c|c|c|c|c|}
\hline $\begin{array}{c}\text { Sample } \\
\text { No. }\end{array}$ & $\begin{array}{c}\mathrm{SiH}_{4} \\
(\mathrm{sccm})\end{array}$ & $\begin{array}{c}\mathrm{H}_{2} \\
(\mathrm{sccm})\end{array}$ & $\begin{array}{c}\text { Power } \\
\text { (W) }\end{array}$ & $\begin{array}{c}\mathrm{P} \\
\text { (Torr) }\end{array}$ & $\begin{array}{l}\text { Dep. } \\
\text { time } \\
\text { (min) }\end{array}$ & $\begin{array}{l}\bar{d} \\
(\AA)\end{array}$ & $\begin{array}{l}\text { Dep. } \\
\text { rate } \\
(\AA / s)\end{array}$ & $\begin{array}{l}\overline{V_{\infty}} \\
\text { (V) }\end{array}$ & $\begin{array}{c}\mathrm{J}_{s c} \\
\left(\mathrm{~mA} / \mathrm{cm}^{2}\right)\end{array}$ & $\overline{\overline{\text { FF }}}$ & $\begin{array}{c}\mathrm{P}_{\max } \\
\left(\mathrm{mW} / \mathrm{cm}^{2}\right)\end{array}$ \\
\hline$\overline{16}$ & $\overline{5}$ & $\overline{\text { Std }}$ & $\overline{15}$ & $\overline{1}$ & $\overline{4.5}$ & 2670 & $\overline{9.9}$ & 0.977 & 9.58 & 0.696 & $\overline{6.51}$ \\
\hline 28 & 2.5 & Std & 15 & 1 & 7.33 & 2280 & 5.18 & 1.01 & 8.65 & 0.668 & 5.83 \\
\hline 29 & 1 & Std & 15 & 1 & 42 & 3000 & 1.19 & 0.405 & 8.09 & 0.585 & 1.92 \\
\hline
\end{tabular}

\section{Deposition of $\mathrm{ZnO}$ Back-reflector by the sputtering of metal $\mathrm{Zn}$}

The aim of this part of the research is to reduce the manufacturing cost by sputter depositing ZnO layer from metal $\mathrm{Zn}$ targets which cost $10-20 \%$ of the ceramic $\mathrm{ZnO}$ targets currently being used. The $\mathrm{ZnO}$ layers were evaluated using a single junction solar cell structure. Table 4 is the results of single junction solar cells on back-reflector with $\mathrm{ZnO}$ layer deposited from both types of targets: metal $\mathrm{Zn}$ and ceramic $\mathrm{ZnO}$. The solar cell performances, in particular the short circuit current, are the same for both types of $\mathrm{ZnO}$ films.

Table 4 Performance of n-i-p solar cells with $\mathrm{ZnO}$ in BR deposited using different types of targets, showing the same device performance.

\begin{tabular}{|c|c|c|c|c|c|}
\hline Sample & $\begin{array}{c}V_{\text {OC }} \\
(N)\end{array}$ & $\begin{array}{c}J_{\text {SC }} \\
\left(\mathrm{mA} / \mathrm{cm}^{2}\right.\end{array}$ & FF & $\begin{array}{c}P_{\max } \\
\left(\mathrm{mW} / \mathrm{cm}^{2}\right)\end{array}$ & $\begin{array}{c}\mathrm{R}_{\mathrm{S}} \\
\left(\Omega \mathrm{cm}^{2}\right)\end{array}$ \\
\hline \hline $\mathrm{Zn} \mathrm{29}$ & 0.952 & 14.54 & 0.644 & 8.91 & 10.7 \\
\hline Zn 33 & 0.955 & 15.06 & 0.638 & 9.19 & 11.5 \\
\hline 5MWBR78 & 0.953 & 14.45 & 0.647 & 8.92 & 11.9 \\
\hline 5MWBR82 & 0.953 & 14.30 & 0.646 & 8.80 & 12.5 \\
\hline
\end{tabular}




\section{Acknowledgment}

We thank Drs. S. Guha, and J. Yang of United Solar for collaborations, and other members of the ECD photovoltaic project team who have contributed to this research program. This work was supported by NREL (subcontract: ZAN-4-13318-11) under the Thin Film Partnership Program.

\section{References}

1. J. Yang, R. Ross, T. Glatfelter, R. Mohr, G. Hammond, C. Bernotaitis, E. Chen, J. Burdick, M. Hopson and S. Guha, Proc. 20th IEEE PV. Spec. Conf. 241 (1988).

2. M. Izu, X. Deng, A. Krisko, K. Whelan, R. Young, H.C. Ovshinsky, K.L. Narasimhan and S.R. Ovshinsky, proc. 23rd IEEE PV Spec. Conf., 919 (1993).

3. S. Guha, J. Yang, A. Banerjee, T. Glatfelter, K. Hoffman, S. R. Ovshinsky, M. Izu, H.C. Ovshinsky, and X. Deng, MRS Proc. 336, 645 (1994).

4. X. Deng, S. J. Jones, T. Liu, M. Izu, S. R. Ovshinsky and K. Hoffman, MRS Proceedings 467, 795 (1997).

5. X. Deng, S. J. Jones, T. Liu, M. Izu and S. R. Ovshinsky, , in Proceedings of 26th IEEE Photovoltaic Specialist Conference, 591 (1997).

6. J. Yang, A. Banerjee, T. Glatfelter, S. Sugiyama and S. Guha, in Proceedings of 26th IEEE Photovoltaic Specialist Conference, 563 (1997).

7. X. Deng, M. Izu, S. J. Jones, T. Liu, S. R. Ovshinsky, and B. Vier, "Development of High, Stableefficiency Triple-junction a-Si Alloy Solar Cells", Phase II Annual Subcontract Progress Report under NREL subcontract ZAN-4-13318-11, October 1996 (available through NTIS). 
Title:

Organization:

Contributors:

\section{CIS Photovoltaic Technology}

\author{
Energy Photovoltaics, Inc. (EPV)
}

Princeton, NJ

\section{Objectives}

The principal objective of this program is to establish a robust vacuum-based process for $\mathrm{Cu}(\mathrm{In}, \mathrm{Ga}) \mathrm{Se}_{2}$ film formation capable of being implemented on manufacturing scale equipment. The method must provide a solution to the traditional problems of point sources and glass warpage. Demonstration of encapsulated CIGS modules having an area of $4300 \mathrm{~cm}^{2}$ and a power of about $35 \mathrm{~W}$ is a near term goal.

\section{Approach}

Energy Photovoltaics operates a complete pilot line for production of glass-glass laminated CIGS modules (substrate size $96.5 \mathrm{~cm} \mathrm{x} 44.5 \mathrm{~cm}$ ). The steps for CIGS preparation are i) deposition of the first part of a Se-containing precursor by linear source evaporation, ii) deposition of the remaining part of the $\mathrm{Cu}$-rich precursor by planar magnetron sputtering, iii) selenization of the precursor using elemental Se supplied from a linear source, and iv) termination by linear source evaporation to bring the overall composition slightly $\mathrm{Cu}$-poor [1]. This sequential process was specifically developed for implementation on large area equipment. Ongoing baseline process development is conducted in smaller scale R\&D equipment with the goals of improving CIGS efficiency and reproducibility, and identifying critical parameters. Performance goals for large modules are set by fabrication and measurement of mini-modules using small area baseline CIGS. A variety of specialized diagnostics are used to quantify material and device uniformity on large area substrates.

\section{Results}

An absorber formation process believed highly suitable for manufacturing has been developed. This now mature process is based on sequential, in-line processing with materials deposited by $\mathrm{DC}$ magnetron sputtering and linear source evaporation. A termination step with In and Ga deposited at hightemperature is routine; its purpose is to modify the near-surface region for improved photovoltaic characteristics. Problems that occurred in the past with inadequate adhesion, pinholes, and microinhomogenieties have been effectively eliminated. Glass flatness is preserved.

The ability of appropriate linear source designs to deposit a substantially uniform thin film across a substrate moving in a direction perpendicular to the axis of the source has been verified by modeling [2]. Thus in Figure 1 we show the thickness distribution obtained for a simple 20" source spaced at 2, 3, 4, 5 , and 6" from the substrate, and the distribution obtained for an optimized source at an appropriate spacing. For the latter source a uniform coating width of about $16^{2}$ can be obtained. 


\section{EPV linear source design \\ 20 inch source, spaced 2 - 6 inches from substrate}

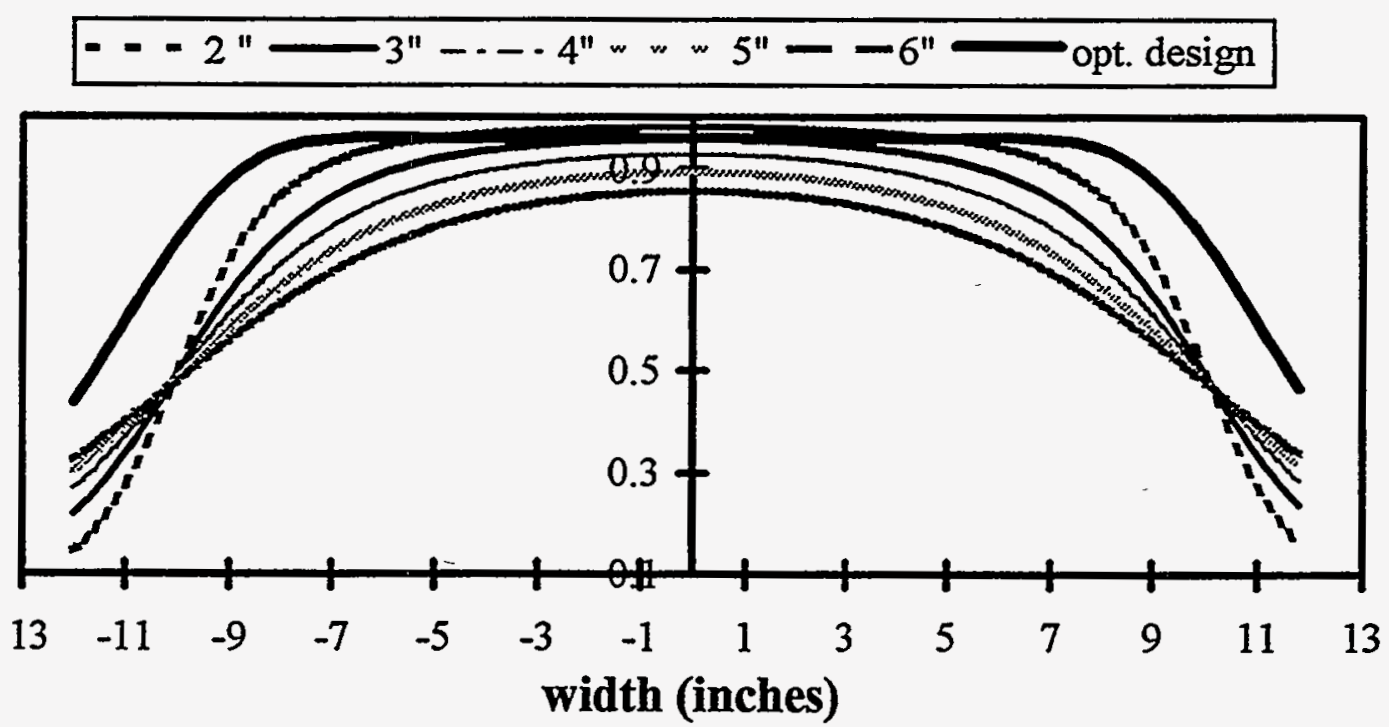

Figure 1: Thickness profiles obtained for simple and optimized linear evaporation sources.

The morphology of CIGS films deposited in the pilot line are generally large-grained and densely packed, with a preferred (112) orientation [3]. The SEM of a typical CIGS film is shown in Figure 2. A significant amount of effort has been placed on.optimizing the Ga content and profile in the CIGS films. Auger depth-profiling and XRD have been applied to aid in this optimization [1]. A variety of $\mathrm{Ga}$ profiles have been generated with a wide range of observed CIGS phases. As a result of this investigation, we have succeeded in raising the maximum open-circuit voltage from $480 \mathrm{mV}$ to $540 \mathrm{mV}$ with no decline in fill-factor and only a small decrease in short-circuit current density.

The effect of shorter selenization times on device efficiency has also been investigated. This information is critical to the design of the manufacturing equipment, in particular the length of the reaction zone necessary to meet the required throughput. High reaction rates of the precursor with Se to form high quality CIGS would allow shorter reaction zones and lower capital costs required for vacuum equipment. In one set of experiments, a standard precursor prepared in the pilot line was diced into pieces and selenized for different times in an R\&D vacuum selenization system. The efficiency of devices made on the CIGS films is plotted in Fig. 3 as a function of the total time of intentional Se exposure. This time does not include heat-up or cool-down times. A device efficiency of $10.5 \%$ was measured for the shortest Se exposure time attempted (4.5 minutes). This compares favorably with the device efficiency of $12.4 \%$ for a total Se exposure time of 33.4 minutes [3].

The inclusion of a wet deposition step for the CdS buffer layer is judged to be less than ideal according to EPV's manufacturing philosophy. Nonetheless, the CBD CdS process exhibits at worse slightly better device characteristics than any other technique/material investigated. For this reason, attempts to further understand and improve the CBD process continue. Upgrades in the bath temperature control, material delivery, and fume exhaust systems of the existing equipment have been made. A new deposition container designed to minimize solution volume/coated area has also been constructed and is in routine use. The batch process is capable of coating four standard-size substrates $\left(1.72 \mathrm{~m}^{2}\right)$ at a time. 


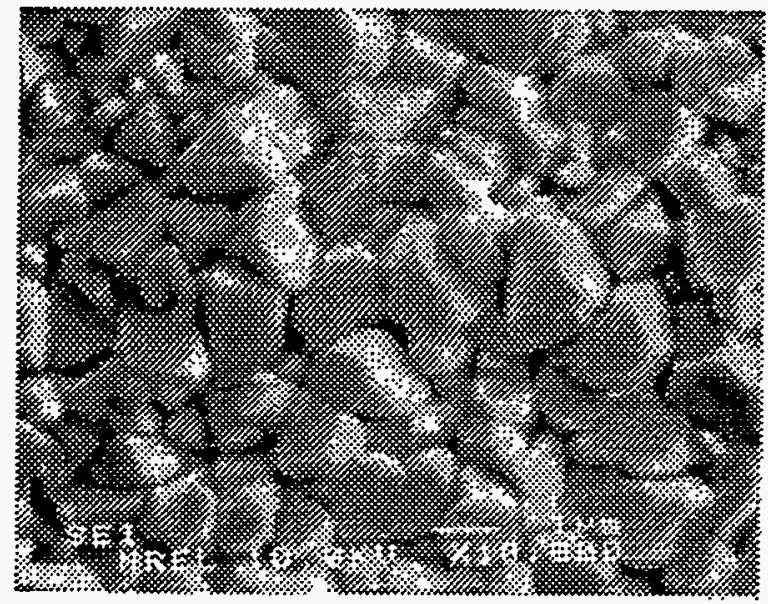

Figure 2. SEM micrograph of the surface of a CIGS film deposited in the pilot line

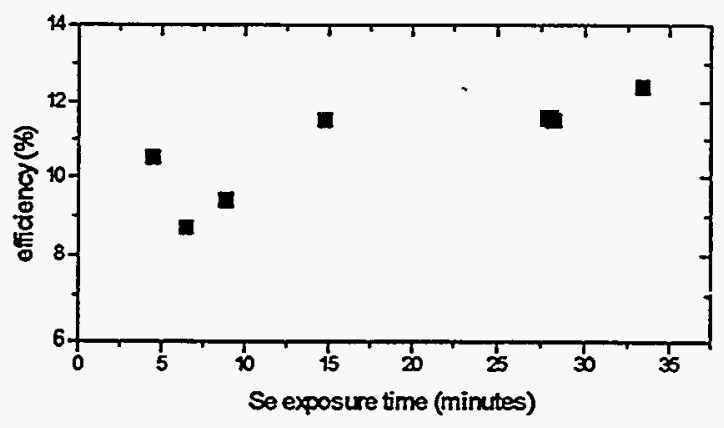

Figure 3: The efficiency of test devices formed from a CIGS precursor prepared in the pilot line for various exposure times to selenium.

The transparent conducting $\mathrm{ZnO}$ applied as the final layer in the thin film coating is sputtered from ceramic targets in a pulsed, bipolar mode. Properties obtained on unheated glass substrates $\left(4300 \mathrm{~cm}^{2}\right.$ in area) are: sheet resistance $24 \mathrm{ohms} / \mathrm{square}$, transmission $82 \%$ (for films about 0.95 um in thickness), and conductivity $440 \mathrm{~S} \mathrm{~cm}^{-1}$. The optimal thickness for use in CIGS modules is found by plotting properties on iso-efficiency contours for modules [2-4]. If $\mathrm{ZnO}$ of quality equivalent to RF sputtered R\&D $\mathrm{ZnO}$ could be produced over these areas, then a $10 \%$ improvement in module fill factor could be obtained [4].

The best result for a full size monolithic CIGS plate was obtained in run Z797. This plate produced 27.7 watts in sunlight (irradiance $998 \mathrm{~W} / \mathrm{m}^{2}$, plate temperature $26^{\circ} \mathrm{C}$ ), as shown in Fig. 4 [1,4]. With 83 cells, the module $\mathrm{V}_{\infty}$ was $37.2 \mathrm{~V}$ and the average $\mathrm{V}_{\mathrm{c}}$ per cell was $448 \mathrm{mV}$. The $\mathrm{I}_{\mathrm{sc}}$ was $1.37 \mathrm{~A}$. The module fill factor of $54.5 \%$ was achieved with a $\mathrm{ZnO}$ sheet resistance of $28 \mathrm{ohms} / \mathrm{square}$.

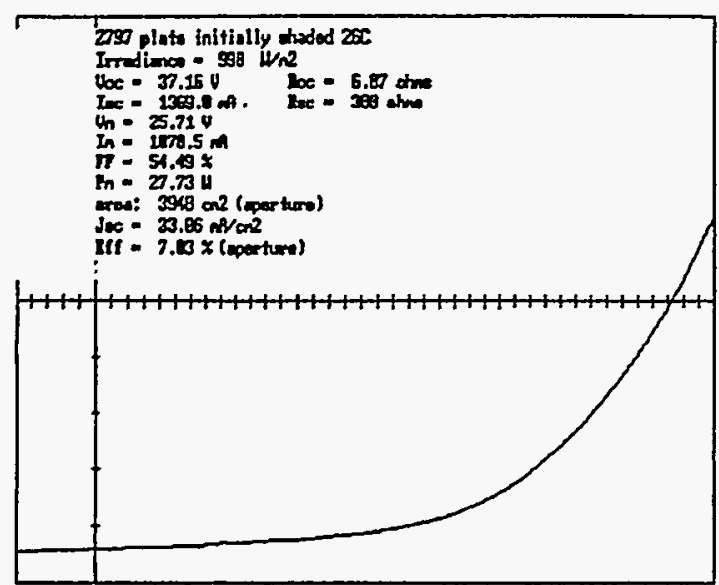

Figure 4: I-V curve for a $3950 \mathrm{~cm}^{2}$ CIGS plate with an efficiency of $7.0 \%$ and a power of $27.7 \mathrm{~W}$.

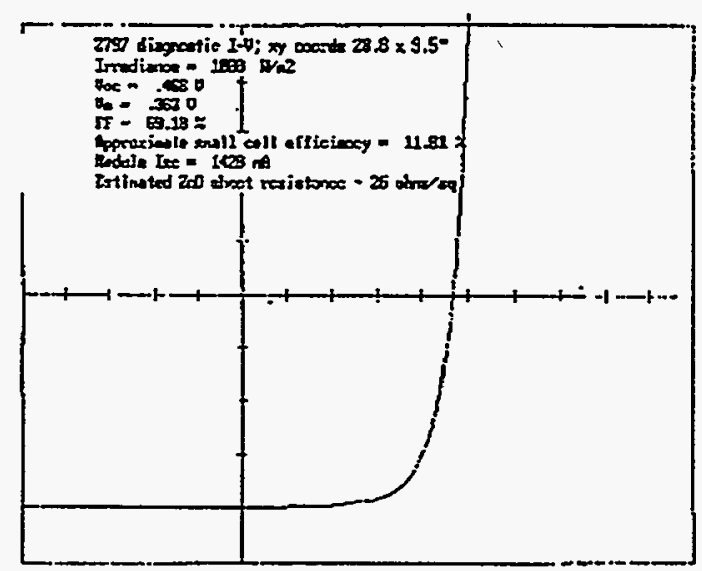

Figure 5: Local I-V curve obtained at a particular location on plate Z797; cell efficiency $11.0 \%$. 
A new diagnostic technique developed at EPV was used to map the quality of the CIGS device across plate Z797 in a non-destructive manner. This technique, in which the entire module is illuminated and driven through its I-V curve while probes pick up signals representing local cell current and voltage values, has now been described in publications [1,2], and was instrumental in detecting a gradient in performance near one of the long edges of the plate. The best local I-V curve obtained on this plate is exhibited in Fig. 5. In this region, devices had a fill factor of $69 \%$ and an intrinsic efficiency of $11 \%$.

EPV's unique capability to produce both a-Si:H and CIGS monolithic PV plates has allowed the fabrication of exploratory, laminated a-Si:H/CIGS tandem modules [3]. The overall efficiency of one particular a-Si/a-Si/CIGS module connected to separate matched loads was $7.6 \%$ (see Table I).

Table I: Powers produced by a-Si/a-Si and CIGS circuits of a 4-wire a-Si/a-Si/CIGS module.

\begin{tabular}{ll}
\hline Configuration & $\begin{array}{l}\text { Power (W) } \\
\text { (norm. to } 1000 \mathrm{~W} / \mathrm{m}^{2} \text { ) }\end{array}$ \\
\hline a-Si/a-Si alone & 22.6 \\
CIGS alone (filtered) & 6.8 \\
Sum of separate powers & $29.4(7.6 \%)$ \\
\hline
\end{tabular}

\section{Conclusions and Future Plans}

Over the past year, progress has been made in CIGS and $\mathrm{ZnO}$ quality, resulting in higher module voltages and fill factors, and enabling an increase in $3950 \mathrm{~cm}^{2}$ module efficiency from $5.1 \%$ to $7.0 \%$ to be achieved. Future work will be directed at continued optimization of the current approach, and development of an alternative method of supplying copper. With improved CIGS uniformity, Ga profiles, and loss minimization, module efficiencies in the range $8.8 \%$ to $12 \%$ are targeted over the next few years.

\section{References:}

1. Britt, J.S.; Delahoy, A.E.; Kiss, Z.J. (1997). "Pilot Production of CIGS Photovoltaic Modules." Twenty-Sixth IEEE Photovoltaic Specialists Conference; September 29 - October 4, 1997; Anaheim, $C A$.

2. Delahoy, A.E.; Meyers, P.V. (1997). "Thin-Film PV Modules: Manufacturing Technology and Issues." Tutorial III, September 29, 1997; Twenty-Sixth IEEE Photovoltaic Specialists Conference; Anaheim, CA.

3. Britt, J.S.; Delahoy, A.E.; Kiss, Z.J. (1997). "Large Area Coating of CIGS and ZnO for Photovoltaics." Fourteenth European Photovoltaic Solar Energy Conference; June 30 - July 4, 1997; Barcélona, Spain.

4. Delahoy, A.E.; Britt, J.S.; Kiss, Z.J. (1997). "CIS Photovoltaic Technology." Annual Technical Report, NREL/SR-520-23194. 38 pp. Available NTIS: Order No. DE 97050822 


\section{Title: CdTe Module Testing and Study of Transients and Irreversible Effects in CdTe Thin-Film Solar Cells}

Organization: Florida Solar Energy Center, Cocoa, FL

Contributors: Neelkanth G. Dhere, Principal Investigator; Kevin W. Lynn

\section{Summary}

Problems with data collection began occurring over the summer. The power output of the solar panels was dropping quickly without any sign of degradation. There was considerable noise in the measured current and voltage data. This indicated the problem was occurring somewhere between the wiring and the data collection system. An attempt was made to fix the problem by changing the diodes on the solar panels. Since the problem still persisted, the diodes were changed again. Realizing that the solar panel diodes were not the problem, the maximum-powerpoint tracker was tested and was found to be in error. A new power tracker was procured. The required voltage range was non-standard. Hence it was not possible to find a replacement. After searching for a new one for a while, it was decided to connect the panels to a resistor, used as a fixed load. By experimenting with different resistance values, it was determined that a $6.6 \Omega$ load resistor resulted in the maximum power output for the solar panels. Starting in the month of September 1997, data was recorded using this resistor as a load. A comparison of this data with the earlier data shows that there was no degradation in the output of CdTe modules. The following data were collected: voltage, current, irradiance, three back-of-module temperatures, ambient temperature beneath the solar array, atmospheric pressure, wind speed, and relative humidity. For this analysis, only data with irradiance values larger than $900 \mathrm{~W} / \mathrm{m}^{2}$ were selected. Power readings were all normalized to $1000 \mathrm{~W} / \mathrm{m}^{2}$. Because there is no longer a maximum-power-point tracker, in the future, additional data will be collected in the form of I-V curves directly from each panel.

The number of hours during which the CdTe solar panels were at each individual temperature was determined and plotted in form of a histogram.

\section{Power Degradation}

The system had 11 panels connected to a resistive load through a maximum-power-point tracker. The following data is recorded by a Campbell CR10X: voltage, current, irradiance, three backof-module temperatures, ambient temperature beneath the solar array, atmospheric pressure, wind speed, and relative humidity. After analyzing the data obtained during the last several months, it was found that the power output of the panels was decreasing. There was considerable noise in the measured current and voltage data. Because this power degradation was occurring too fast to be due to environmental exposure, this indicated the problem was elsewhere in the system. At first, the diodes were suspected and replaced. The problem persisted. Hence the diodes very replaced again. But the problem continued. Replacing all of the diodes twice and finding no change, it was necessary to look elsewhere. The problem was found to originate in the maximum-power-point tracker. 


\section{The Power Conditioner}

Because of its age, the power conditioner was having problems tracking the maximum-power point due to Florida's wide range of weather patterns. The output voltage of the Solar Cells Inc CdTe modules is very high. Hence it was difficult to find a replacement one that would track the high voltage output of SCI CdTe modules. After searching for another one for a long time, it was decided that the panels would be connected to a load resistor. This would not allow the measurement of more PV parameters at different values of irradiance. Hence I-V measurements will be carried out periodically.

After finding problems with the maximum-power-point tracker and being unable to purchase a new one, the panels were instead connected to a resistor as a fixed load. By experimenting with different resistance values, it was determined that a $6.6 \Omega$ load resistor resulted in the maximum power output for the solar panels. Starting in the month of September, data was recorded using this resistor as a load. A comparison of this data with the earlier data shows that there was no degradation in the output of CdTe modules.

\section{Using a Resistor as Load}

In order to determine the resistance value that gave the maximum power output of the solar panels, a range of values using a variable resistor were tried while irradiance stayed above 900 $\mathrm{W} / \mathrm{m}^{2}$. The variable resistor is a coiled resistance wire wrapped around a cylindrical piece of ceramic. One contact was connected at the end of the resistor while the other can be attached at different locations along the coil. Moving a contact along the length of the resistive coil could change values of the resistance. For each value of resistance, ten normalized power readings were taken. After taking many readings, the maximum power of the panels was found to be at a load of $6.6 \Omega$. The resistance was fixed at this value.

\section{Data Collection}

The following data were collected from the month of September through December: voltage, current, irradiance, three back-of-module temperatures, ambient temperature beneath the solar array, atmospheric pressure, wind speed, and relative humidity. Figure 1 shows a plot of power, voltage, and current versus time for data with irradiance greater than $900 \mathrm{~W} / \mathrm{m}^{2}$. A comparison of this data with the earlier data collected during January to May 1997 showed that there was no degradation in the output of CdTe modules. In fact, the measured power output of the panels has increased considerably. The earlier measurement of lower power is attributed to the loss of power while using the maximum-power-point tracker. To make up for the absence of maximumpower-point tracker, in the future, additional measurements will be carried out in the form of I-V characteristics of individual modules.

Figure 2 shows the histogram of the number of hours the solar panels were at each temperature while being under an irradiance of $250 \mathrm{~W} / \mathrm{m}^{2}$ or higher. The data for temperatures below 18 degrees Celsius is grouped together, because of small periods at each temperature. From this histogram, the temperature history of the panels can be determined while modules were producing a reasonable amount of power. 


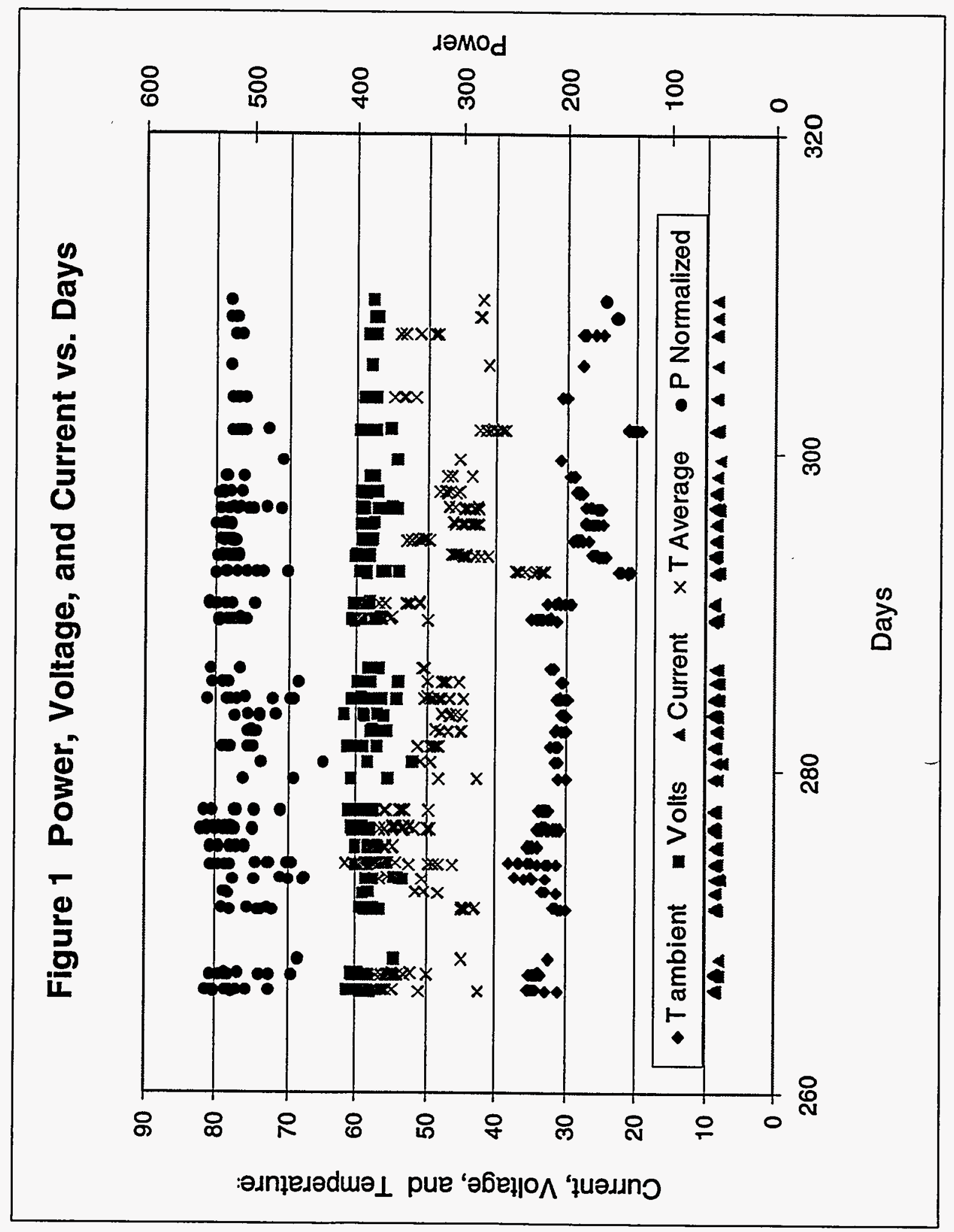




\section{Time (hrs)}

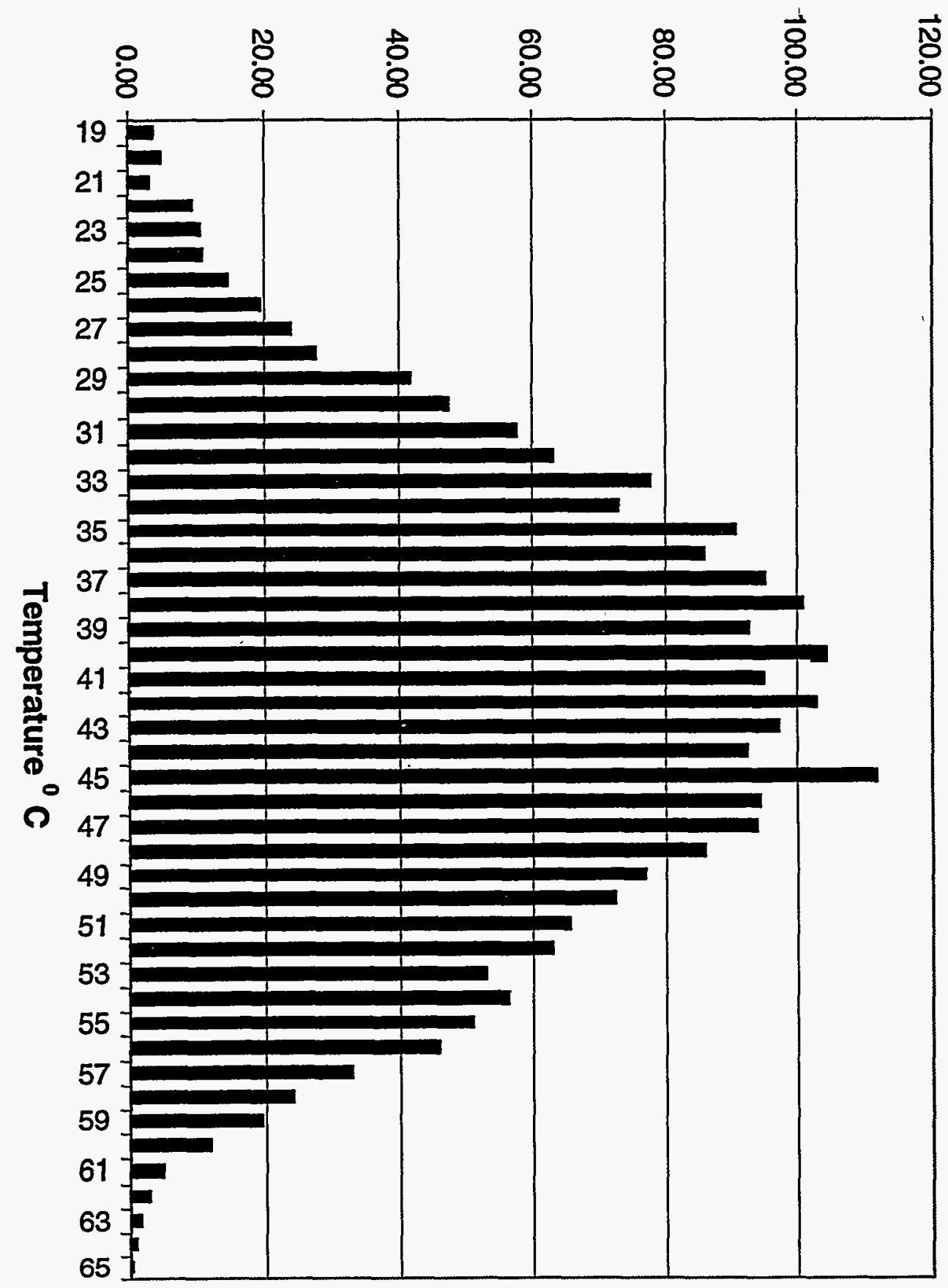

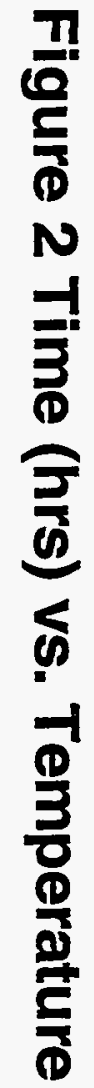


Title: Deposition of $\mathrm{CuIn}_{1-x} \mathrm{Ga}_{x} \mathrm{Se}_{2}$ Thin-Films Using a Two-Stage Process

Organization: Florida Solar Energy Center, Cocoa, FL

Contributors: Neelkanth G. Dhere, Principal Investigator; Kevin W. Lynn

\section{Introduction}

Research in $\mathrm{CuIn}_{1-x} \mathrm{Ga}_{x} \mathrm{Se}_{2}$ solar cells has shown it to be a viable candidate for low-cost electricity. The addition of $\mathrm{Ga}$ to $\mathrm{CuInSe}_{2}$ solar cells has been shown to raise the band gap of the cell closer to the optimum value of $1.5 \mathrm{eV}$. Advances in $\mathrm{CuIn}_{1-x} \mathrm{Ga}_{x} \mathrm{Se}_{2}$ thin-film research have resulted in solar cell efficiencies as high as $17.7 \%$ using co-evaporation of the elements. However, the design of the reactors and evaporation sources for large area deposition are a challenging task. Another process of depositing films that has produced high efficiency solar cells is the selenization of the precursors of $\mathrm{Cu}$, In, and $\mathrm{Ga}$. This process has been shown to be applicable to large-scale manufacturing. Sputtering equipment is commercially available for large-scale deposition and can be used in an assembly line process. The evaporation process for adding Se should also be easily scaled up.

The technique used at the Florida Solar Energy Center uses a two-stage process of magnetron sputtering of $\mathrm{Cu}, \mathrm{In}$, and $\mathrm{Ga}$, followed by selenization with Se vapor. In the first stage, a Cu-rich CuIn ${ }_{x} \mathrm{Ga}_{x} \mathrm{Se}_{2}$ thin-film is deposited. These Cu-rich films form large grains, called a large grained precursor (LGP), which preferentially form with a (112) orientation. In the second stage, an overall Cu-poor film is deposited. This $\mathrm{Cu}$-poor film forms the desired p-type semiconductor and takes on the grain structure of the large grained precursor.

Because $\mathrm{Ga}$ has a low melting point of around $29^{\circ} \mathrm{C}$, it would be necessary to sputter deposit it as a liquid, which can be problematic. The group at the Florida Solar Energy Center has come up with a novel process where $\mathrm{Cu}$ and $\mathrm{Ga}$ are sputtered from a $\mathrm{CuGa}$ alloy target, and In is sputtered from another target. Initially, only a low $\mathrm{Ga}$ alloy target of $\mathrm{CuGa}(22$ at. \%) was used, but this limited the amount of $\mathrm{Ga}$ that could be deposited in the film. Now a $\mathrm{CuGa}(66$ at. \%) alloy target was added to increase the $\mathrm{Ga}$

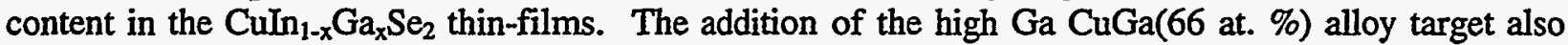
made it possible to profile $\mathrm{Ga}$ through the thickness of $\mathrm{CuIn}_{1-x} \mathrm{Ga}_{\mathrm{x}} \mathrm{Se}_{2}$ thin-film, which has been shown to increase solar cell efficiencies. In normal profiling, $\mathrm{Ga}$ content is highest at the $\mathrm{Mo} / \mathrm{CuIn}_{1-x} \mathrm{Ga}_{\mathrm{x}} \mathrm{Se}_{2}$ boundary, and linearly decreases through the film until reaching the $\mathrm{p}-\mathrm{n}$ junction. Since higher $\mathrm{Ga}$ in $\mathrm{CuIn}_{1-\mathrm{x}} \mathrm{Ga}_{\mathrm{x}} \mathrm{Se}_{2}$ films have a higher band gap, this results in having a film with a high band gap toward the back which decreases until reaching the $\mathrm{CuIn}_{1-x} \mathrm{Ga}_{x} \mathrm{Se}_{2} / \mathrm{CdS}$ boundary. As a result, electrons excited to the conduction band are pushed down the potential gradient toward the junction. The graded gap also prevents electrons that have formed near the back contact from flowing backwards.

\section{Criteria for Solar Cell Fabrication}

Films were deposited on Mo-coated glass substrates by selenizing sputtered precursors. During this phase, solar cells were only fabricated for the $\mathrm{CuIn}_{1-x} \mathrm{Ga}_{x} \mathrm{Se}_{2}$ films. The main factors used in determining whether to make $\mathrm{CuIn} n_{1-x} \mathrm{Ga}_{\mathrm{x}} \mathrm{Se}_{2}$ films into complete solar cells are listed below:

Surface Uniformity: If the films were not homogeneous, cell properties would vary over the surface of the film. Thus, any beneficial cell characteristics could not be reliably replicated from this deposition process.

Copper Concentration: If the films were too $\mathrm{Cu}-\mathrm{rich}$, they would not form the p-type semiconductor. 
Gallium Concentration: Ga concentration should be high enough to effectively raise the band gap of the cell. If the $\mathrm{Ga}$ content, $\mathrm{x}$, in $\mathrm{CuIn}_{1-\mathrm{x}} \mathrm{Ga}_{\mathrm{x}} \mathrm{Se}_{2}$ was lower than 0.12 , cells were not fabricated.

Summary table shows which series of films were made into complete solar cells.

Summary Table

$\mathrm{CuIn}_{1-x} \mathrm{Ga}_{\mathrm{x}} \mathrm{Se}_{2}$ Films Made into Complete Solar Cells

\begin{tabular}{lcccc}
\hline \multicolumn{1}{c}{ Series } & $\begin{array}{c}\text { Surface } \\
\text { Uniformity }\end{array}$ & $\begin{array}{c}\text { Cu-poor } \\
\text { Film }\end{array}$ & $\begin{array}{c}\text { Sufficient } \\
\text { Ga }\end{array}$ & $\begin{array}{c}\text { Solar Cell } \\
\text { Fabrication }\end{array}$ \\
\hline $8^{\text {th }}$ Series & yes & yes & yes & $9.02 \%$ \\
$9^{\text {th }}$ Series & yes & yes & no & None \\
New Series \#1 & yes & yes & yes & $8.5 \%$ \\
New Series \#2 & yes & no & yes & None \\
New Series \#3 & yes & yes & no & None \\
New Series \#4 & yes & yes & yes & $5.5 \%$ \\
New Series \#5 & yes & yes & yes & $4 \%$ \\
New Series \#6 & yes & yes & no & None \\
New Series \#7 & yes & yes & no & None \\
New Series \#8 & yes & yes & yes & $8 \%$ \\
New Series \#9 & yes & yes & yes & None* \\
\hline
\end{tabular}

* Not completed in time for thesis submittal.

\section{The Resulting Solar Cells}

Solar cells from the $8^{\text {th }}$ Series attained the maximum efficiency of $9.02 \%$ Electron Probe Microanalysis (EPMA) showed the Ga content, $x$, in $\mathrm{CuIn}_{1-x} \mathrm{Ga}_{x} \mathrm{Se}_{2}$ had reached a value of 0.18 , up from a previous high of 0.06 in Shanker Kuttath's Masters thesis [1]. The higher $\mathrm{Ga}$ concentration leads to a higher band gap semiconductor. By raising the band gap of $\mathrm{CuIn}_{1-\mathrm{x}} \mathrm{Ga}_{\mathrm{x}} \mathrm{Se}_{2}$ closer to $1.5 \mathrm{eV}$, the efficiency of the solar cell will increase. A scanning electron micrograph of the surface of the film showed a rough surface with some grain faceting. This faceting indicated good grain formation, which is necessary for a high efficiency solar cell.

New Series \#1 (shown in Figure 1) was the first series to use the CuGa(66 at. \%) target along with the $\mathrm{CuGa}(22$ at. \%) and In targets. The new target was added to raise the $\mathrm{Ga}$ concentration and attempt to profile it through the thickness of the film. The desired composition was $\mathrm{Cu}_{0.93} \mathrm{In}_{0.72} \mathrm{Ga}_{0.27+0.1} \mathrm{Se}_{2}$ for a $2.75 \mu \mathrm{m}$ thick film. EPMA showed that $\mathrm{Ga}$ content, $\mathrm{x}$, in $\mathrm{CuIn}_{1-x} \mathrm{Ga}_{\bar{x}} \mathrm{Se}_{2}$ had been increased from 0.18 in the 8 th Series to 0.25 in the bulk of the film. The value of $x$ was around 0.13 closer to the surface. This indicated $\mathrm{Ga}$ was being profiled through the thickness of the film. Although a high film resistance indicated a low $\mathrm{Cu}$ film, EPMA showed y values in $\mathrm{Cu}_{\mathrm{y}} \mathrm{In}_{1-\mathrm{x}} \mathrm{Ga}_{\mathrm{x}} \mathrm{Se}_{2}$ to be around $\mathrm{y}=$ 1.1 in the bulk. This was not in the preferred $0.86<y<0.96$ range. X-ray diffraction showed a strong (112) reflection for $\mathrm{CuIn}_{0.7} \mathrm{Ga}_{0.3} \mathrm{Se}_{2}$, which was at the center of the peak. CuInSe $\mathrm{Cu}_{2}$ and $\mathrm{CuIn}_{2} \mathrm{Se}_{3.5}$ were also present. This showed that $\mathrm{Ga}$ was being incorporated into the $\mathrm{CuIn}_{1-\mathrm{x}} \mathrm{Ga}_{\mathrm{x}} \mathrm{Se}_{2}$ phase, but the presence of $\mathrm{CuInSe} \mathrm{S}_{2}$ meant more $\mathrm{Ga}$ should be added.

Checking the absorption characteristics of the solar cells reinforced this conclusion. Solar cells fabricated from New Series \#1 were shown to absorb from wavelengths of $400 \mathrm{~nm}$ to wavelengths around $1200 \mathrm{~nm}$. Semiconductors with a higher band gap should only absorb higher energy photons with wavelengths below $1000 \mathrm{~nm}$. Because a higher band gap semiconductor was necessary to produce higher efficiency solar cells, more Ga needed to be added to the film. 


\begin{tabular}{|l|r|r|}
\hline Totals & Amount (grams)/cm & \multicolumn{2}{|c|}{ Angstroms } \\
\hline $\mathrm{CuGa}(\mathrm{low})$ & $3.48 \mathrm{E}-04$ & 4056 \\
\hline $\mathrm{CuGa}($ high) & $5.31 \mathrm{E}-05$ & 620 \\
\hline In & $3.90 \mathrm{E}-04$ & 5337 \\
\hline
\end{tabular}

\begin{tabular}{|l|r|r|}
\hline Selenization II & & 124 \\
\hline $20 \%$ CuGa(high) & $1.06 \mathrm{E}-05$ & 1868 \\
\hline $35 \%$ In & $1.37 \mathrm{E}-04$ & \\
\hline Selenization I & & 811 \\
\hline $20 \% \mathrm{CuGa}$ (low) & $6.96 \mathrm{E}-05$ & 3469 \\
\hline $65 \%$ In & $2.54 \mathrm{E}-04$ & 3245 \\
\hline $80 \% \mathrm{CuGa}$ (low) & $2.78 \mathrm{E}-04$ & 496 \\
\hline $80 \% \mathrm{CuGa}($ high) & $4.25 \mathrm{E}-05$ & \\
\hline Mo & & \\
\hline Glass & & \\
\hline
\end{tabular}

Figure 1. Deposition sequence in New Series \#1

In New Series \#4, the new desired composition was $\mathrm{Cu}_{0.94} \mathrm{In}_{0.57} \mathrm{Ga}_{0.41+0.1} \mathrm{Se}_{2}$, and the total thickness was raised to $3 \mu \mathrm{m}$. Raising the Ga concentration resulted in $\mathrm{CuIn}_{1-x} \mathrm{Ga}_{\mathrm{x}} \mathrm{Se}_{2}$ films with $\mathrm{Ga}$ content, $x=0.33$ in the bulk. Ga concentration was decreased closer to the surface with $x=0.19$. Therefore $\mathrm{Ga}$ was again being profiled through the thickness of the film. Lowering Cu concentration brought $\mathrm{y}$ values in $\mathrm{Cu}_{\mathrm{y}} \mathrm{In}_{1-x} \mathrm{Ga}_{\mathrm{x}} \mathrm{Se}_{2}$ to around $\mathrm{y}=0.90$ at $10 \mathrm{keV}$ and $\mathrm{y}=1$ at $20 \mathrm{keV}$. The $\mathrm{x}$-ray diffraction pattern for this series indicated a strong (112) peak for high gallium $\mathrm{CuIn}_{1-x} \mathrm{Ga}_{x} \mathrm{Se}_{2}$ films. $\mathrm{CuIn}_{0.4} \mathrm{Ga}_{0.6} \mathrm{Se}_{2}$ seems to be the most prominent phase, as it is closer to the center of the peak. $\mathrm{CuIn}_{0.5} \mathrm{Ga}_{0.5} \mathrm{Se}_{2}$ and $\mathrm{CuIn}_{0.7} \mathrm{Ga}_{0.3} \mathrm{Se}_{2}$ phases were also present. Again, the higher $\mathrm{Ga}$ concentration is being incorporated into the $\mathrm{CuIn}_{1-x} \mathrm{Ga}_{x} \mathrm{Se}_{2}$ films as expected.

In New Series \#5, changing the deposition sequence resulted in high Ga retention. EPMA at 20 $\mathrm{keV}$ indicated that $\mathrm{Ga}$ content, $\mathrm{x}$, in $\mathrm{CuIn}_{1-\mathrm{x}} \mathrm{Ga}_{\mathrm{x}} \mathrm{Se}_{2}$ has now increased to $\mathrm{x}=0.42$. At $10 \mathrm{keV}, \mathrm{x}$ reduces closer to the surface to around 0.33 . Thus profiling of $\mathrm{Ga}$ again occurs through the thickness of the film. $\mathrm{X}$-ray diffraction (Figure 2) showed a strong (112) peak in which CuIn ${ }_{0.5} \mathrm{Ga}_{0.5} \mathrm{Se}_{2}$ is the main crystal phase present. The CuIn $0.7 \mathrm{Ga}_{0.3} \mathrm{Se}_{2}$ phase also occurs in the film. A scanning electron micrograph of a cross section of a sample in New Series \#5 showed some porosity through the thickness of the film. Any discontinuities in the CuIn $\mathrm{Cu}_{1-x} \mathrm{Ga}_{\mathrm{x}} \mathrm{Se}_{2}$ film such as impurities, grain boundaries, or vacancies can become recombination centers, which lower solar cell efficiencies. Solar cell I x V characteristics measured at NREL also showed the solar cell had a high series resistance, which reduces the fill factor of the cell. The high $\mathrm{Ga}$ concentration may be causing a highly resistive phase to form as well as causing poor morphology.

In New Series \#8, the deposition sequence was changed so that $\mathrm{CuGa}(22$ at. \%) was deposited first. By placing the $\mathrm{Cu}$ concentration at the very back, as in the $8^{\text {th }}$ Series, it was hoped that this might improve the morphology of the film. EPMA indicated that $\mathrm{Ga}$ was being incorporated into the film, with $\mathrm{Ga}$ content, $\mathrm{x}$, in $\mathrm{CuIn}_{1-\mathrm{x}} \mathrm{Ga}_{\mathrm{x}} \mathrm{Se}_{2}$ being around 0.32 in the bulk (20 keV) and 0.16 closer to the surface (10 $\mathrm{keV}$ ). The concentration of $\mathrm{Cu}$ in $\mathrm{Cu}_{\mathrm{y}} \mathrm{In}_{\mathrm{I}-\mathrm{x}} \mathrm{Ga}_{\mathrm{x}} \mathrm{Se}_{2}$ again fell within the range $0.86<\mathrm{y}<1$. $\mathrm{X}$-ray diffraction again showed $\mathrm{Ga}$ being incorporated into the film, with $\mathrm{CuIn}_{0.4} \mathrm{Ga}_{0.6} \mathrm{Se}_{2}$ being the main phase present, with the (112) orientation being the most prominent reflection. $\mathrm{CuIn}_{0.5} \mathrm{Ga}_{0.5} \mathrm{Se}_{2}$ and $\mathrm{CuIn}_{0.7} \mathrm{Ga}_{0.3} \mathrm{Se}_{2}$ phases were present as well. 


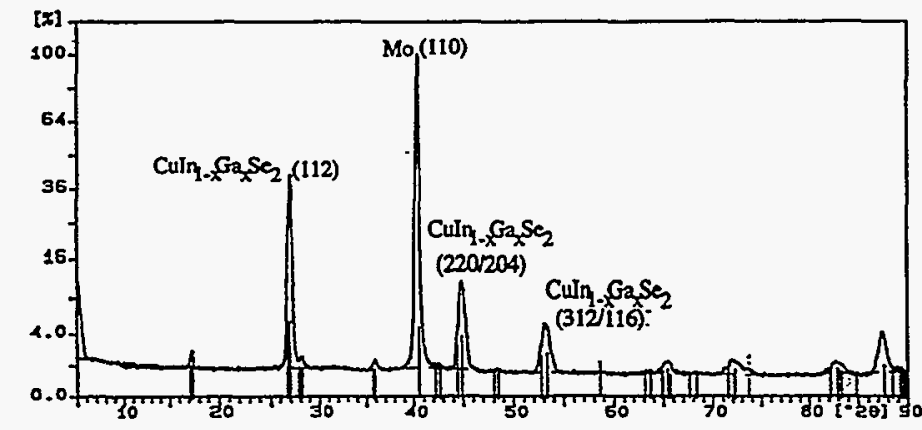

Figure 2. XRD pattern of a CuIn 1 ${ }_{x} \mathrm{Ga}_{x} \mathrm{Se}_{2}$ thin-film from New Series \#5 $35-1102 \rightarrow \mathrm{CuIn}_{0.7} \mathrm{Ga}_{0.3} \mathrm{Se}_{2}$ $40-1488 \rightarrow \mathrm{CuIn}_{0.5} \mathrm{Ga}_{0.5} \mathrm{Se}_{2}$ $42-1120 \rightarrow \quad M$

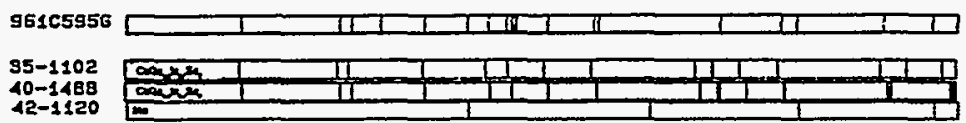

Atomic Force Microscopy (AFM) showed that the rms roughness of the films from New Series \#8 were as high as $2000 \AA$ for thin-films deposited on the 1 " by $1 / 4$ " substrates. However, a rms roughness of only $947 \AA$ was found on 1" x 1" substrates used for fabricating solar cells. Scanning electron micrographs of the surface of other films indicated a rough surface as well. If defects form between the $\mathrm{CuIn}_{1-\mathrm{x}} \mathrm{Ga}_{\mathrm{x}} \mathrm{Se}_{2}$ and $\mathrm{CdS}$, cell efficiencies will be lower due to the formation of recombination centers.

Although it was expected that the films with higher $\mathrm{Ga}$ concentration would result in the highest efficiencies, the Summary Table shows that the highest efficiency solar cell, $9.02 \%$, came from an Old Series film utilizing only the $\mathrm{CuGa}(22$ at. \%) and In targets. About three years of work went into the Old Series. It has only been eight months since the first solar cells were fabricated utilizing the $\mathrm{CuGa}(66$ at. $\%)$ target. By gaining additional experience with the high $\mathrm{Ga}$ target, problems with morphology and $\mathrm{Ga}$ distribution may be solved. This would most likely lead to higher solar cell efficiencies.

\section{Publications}

1.N. G. Dhere, S. Kuttath, and H. R. Moutinho, "Morphology of Precursors and $\mathrm{CuIn}_{1-x} \mathrm{Ga}_{x} \mathrm{Se}_{2}$ Thin Films Prepared by Two-Stage Selenization Process", J. Vac. Sci. \& Technol. A., 13, (1995), pp. 1078-1082.

2. N. G. Dhere, D. L. Waterhouse, K. B. Sundaram, O. Melendez, N. R. Parikh, B. K. Patnaik, "Studies of Chemical Bath Deposited Cadmium Sulfide Films by Buffer Solution Technique", J. Mat. Sci.: Mat. in Electronics, 6, 52-59 (1995).

3. N. G. Dhere, S. Kuttath, K. W. Lynn, R. W. Birkmire, and W. N. Shafarman, "Polycrystalline CuIn 1 ${ }_{x} \mathrm{Ga}_{\mathrm{x}} \mathrm{Se}_{2}$ Thin Film PV Solar Cells Prepared by Two-stage Selenization Process Using Se Vapor" Proc. IEEE First World Conference on Photovoltaic Energy Conversion, Waikoloa, Hawaii, during Dec. 5-9, 1994, pp. 190-193.

4. N. G. Dhere and K. W. Lynn, "CuIn ${ }_{1-x} \mathrm{Ga}_{x} \mathrm{Se}_{2}$ Thin Film Solar Cells by Two-Selenizations Process Using Se Vapor" Solar Energy Materials and Solar Cells, 41/42,(1996), pp. $271-279$.

5. N. G. Dhere, "CuIn ${ }_{1-x} \mathrm{Ga}_{x} \mathrm{Se}_{2}$ and CdTe PV Solar Cells" AIP Conference Proceeding: 13th NREL Photovoltaic Program Review Meeting, Lakewood, CO, May 16-19, 1995, pp. 428-435.

6. N. G. Dhere and K. W. Lynn, Gallium Content Enhancement in CuIn $n_{1-x} \mathrm{Ga}_{x} \mathrm{Se}_{2}$ Thin Films Prepared by Two-selenizations Process Using Se Vapor", Proc. 25th Photovoltaic Specialists Conference, Washington, DC, during May 13-17, 1996, pp. 897-900.

7. K. W. Lynn and N. G. Dhere, Techniques for Increasing Ga in CuIn ${ }_{1-x} \mathrm{Ga}_{x} \mathrm{Se}_{2}$ Thin Films Prepared by Two-Stage Selenizations Process", AIP Proceedings of the $14^{\text {th }}$ NREL/SNL Photovoltaics Program Review Conference, Lakewood, CO, 1996, pp. 613-619. 
Title: $\quad$ Optimization of Transparent and Reflecting Electrodes for Amorphous Silicon Solar Cells

Organization: Department of Chemistry, Harvard University, Cambridge, MA.

Contributors: R. G. Gordon, Principal Investigator;

K. Kramer, H. Liang, X. Liu, D. Teff, J. Thornton

\section{Summary of Current Project Objectives}

Transparent conducting materials are essential components of many kinds of solar cells, in which they serve as front-surface electrodes. Most designs for highly reflective back contacts also call for a transparent conducting layer. The compositions of these transparent conducting layers are usually based on oxides of tin, indium and/or zinc, and are referred to as transparent conducting oxides (TCO). In addition to having low electrical resistance and low optical absorption, the structure of a front-surface TCO must minimize reflection losses. The TCO must also resist degradation during cell fabrication and use. Finally, the method for making the TCO must be inexpensive and safe.

Our objectives are to improve the performance of TCO materials and the methods for their production. We aim to reduce their electrical resistance, optical absorption and reflection losses, and to lower the deposition temperature to avoid thermal degradation of other cell components. For the production method, the prime consideration is to deposit the TCO layers at a high rate with relatively simple apparatus. The method chosen is chemical vapor deposition (CVD) at atmospheric pressure, since it has been demonstrated in the glass-coating industry to be the most cost-effective method for making large areas of TCO coatings.

\section{Improving the Manufacturability of Fluorine-doped Zinc Oxide Films}

Fluorine-doped zinc oxide films show higher transparency than conventional fluorine-doped tin oxide films, and have the potential for lower cost, because zinc metal costs less than tin. During the previous contract, a new process was developed for CVD of $\mathrm{ZnO}: \mathrm{F}$. It has the advantages of 1) a safer, non-pyrophoric zinc precursor.

2) a liquid zinc precursor from which the vapor reactant mixture may be made conveniently and in a well-controlled manner.

3) chemistry which permits premixing of all the reagent vapors, in order to facilitate uniform, reproducible deposition over large areas.

4) less sensitivity to disturbance by trace amounts of contaminants such as oxygen and water vapor.

However, the cost of the reagents was higher than desired. The most expensive chemical used was the fluorine dopant, hexafluoropropene. Because its reactivity is fairly low, it was needed in large amounts, even though only a small concentration (around 0.5 atomic \%) of fluorine is needed in the film. After trying several other fluorine sources, benzoyl fluoride was found to be a much more efficient and inexpensive fluorine dopant.

Because the fluorine dopant must be mixed with the liquid zinc precursor, the solution cannot be evaporated from a conventional bubbler vapor source for CVD, because the differing volatilities of the components would result in time-dependent vapor concentrations. Thus a direct liquid injection system was used for vaporization of the liquid solution.

Once it was decided to use direct liquid injection a solution, a further cost reduction was achieved by replacing the rather expensive chelating agent $N, N, N^{\prime}, N^{\prime}$-tertraethylethylendiamine by the 
much less expensive $N, N_{,} N^{\prime}, N^{\prime}$-tetramethylethylenediamine. Although this results in a low-melting solid chelate with diethylzinc, rather than the previous pure liquid, it is highly soluble in hydrocarbon solvents, such as xylene. Thus an inexpensive precursor solution was prepared by dissolving both the solid zinc precursor (the diethylzinc chelate of

$N, N, N^{\prime}, N^{\prime}$-tetramethylethylenediamine) and the fluorine dopant (benzoyl fluoride) in xylene.

Another problem with this process was that its reproducibility was not as high as desired for commercial use. One root cause of the variation in the results was traced to different commercial batches of ethanol giving different results. Different batches of commercial "anhydrous" alcohol appeared to give different film growth rates. NMR failed to detect any organic impurities in the different samples. Analysis of the water content by Karl Fischer titration did find some differences. In particular, one batch was found to have an order of magnitude more water $(0.4 \mathrm{wt}$ $\%)$ than the others $(0.04 \mathrm{wt} \%)$. The growth rate found with the wet ethanol was only about $2 / 3$ as high as the drier ethanol. We also tested the use of the common grade of $95 \%$ ethanol (5 wt \% water), and found a very low film growth rate, and a considerable amount of powder in the gas distributor. We conclude that reproducible operation of this CVD process requires control of the water content of the ethanol to be less than $0.1 \mathrm{wt} \%$, which is the specification commonly set for commercial anhydrous ethanol.

A second source of variation was due to the time between mixing of the precursor solution and its use in the CVD process. The solution was normally made by first mixing the diethylzinc and xylene, then adding the tetramethylethylenediamine, and finally adding the benzoyl fluoride. If the solution was used immediately after mixing, the films had about $30 \%$ higher resistance than the best films, and their haze levels were very low. If the solution was aged for a day at room temperature before use, the resistance dropped to within a few per cent of the lowest values. After one week of aging, the solution produced the lowest resistances. No further change in resistance was noted on aging for periods from one week to one month. However, on aging for a month or more, the solutions produced gradually increasing resistances.

The haze levels also varied significantly with aging of the solution. Fresh solution produced smooth films with very low haze levels. After about a week of aging at room temperature, the haze levels increased significantly. Thus, to form films optimized for solar cells, it was best to age the solution for about two weeks before using it.

The aging effect only begins after the addition of the fluorine dopant, benzoyl fluoride. The undoped solution, without benzoyl fluoride, appears to be completely stable at room temperature, provided of course that it is kept out of contact with air or moisture. Therefore, we prepared a stock solution without fluorine dopant, and added fluorine dopant to portions of it as needed.

Glass containers were not satisfactory for storage of the fluorine-doped solutions, because some precipitate gradually formed, probably from etching of the glass. Polyethylene containers were better for storage of the fluorine-doped solution, but they also tended to produce some residue, which may arise from leaching components of the plastic. Finally, we found that

poly-fluorocarbon containers, such as Teflon, could be used to store the doped solution in a dry nitrogen atmosphere without any precipitation or residue formation.

\section{Amorphous Silicon Solar Cells on Fluorine-doped Zinc Oxide}

Fluorine-doped zinc oxide films have higher light transmission than tin oxide films having the same sheet resistance. Thus using $\mathrm{ZnO}: \mathrm{F}$ as a front TCO results in higher currents from superstrate amorphous silicon solar cells. However, this advantage of $\mathrm{ZnO}: \mathrm{F}$ is offset by lower voltages and fill factors, so that the efficiency is not improved over cells made on standard $\mathrm{SnO}_{2}: \mathrm{F}$. Research during the past year has attempted to elucidate the root cause for this effect, and to find 
ways to increase the efficiency.

Single-junction amorphous silicon solar cells were prepared on samples of $\mathrm{ZnO}: \mathrm{F}$ by Steve Hegedus at IEC, Delaware. The short circuit current was increased by more than 5 per cent, relative to cells made on Solarex tin oxide. Similar results were obtained with tandem cells made at Solarex. These results verified that zinc oxide has higher optical transmission than tin oxide of the same sheet resistance.

The voltages of the cells prepared on $\mathrm{ZnO}$ are about $20 \mathrm{mV}$ lower than those prepared on $\mathrm{SnO}_{2}$. The fill factors are also significantly lower, by an average of $5 \%$. These deleterious factors negate the advantage of the higher currents found for the $\mathrm{ZnO}$. Both of these deleterious effects relate to an electrical contact between the silicon and $\mathrm{ZnO}$ that is less satisfactory than that with $\mathrm{SnO}_{2}$.

Transparent conductors with the structure thin $\mathrm{SnO}_{2}:$ F/thick $\mathrm{ZnO}$ :F/glass should combine the best features of the two materials for amorphous silicon solar cells. The thick $\mathrm{ZnO}: \mathrm{F}$ should provide high transparency, and the thin $\mathrm{SnO}_{2}: \mathrm{F}$ should provide good electrical contact to the silicon.

In order to deposit $\mathrm{SnO}_{2}: \mathrm{F}$, we normally use substrate temperatures over $500 \circ \mathrm{C}$. However, when we heated the $\mathrm{ZnO}: \mathrm{F}$ films to temperatures over $480 \circ \mathrm{C}$, their sheet resistance was increased. Thus we did some studies of $\mathrm{SnO}_{2}: \mathrm{F}$ deposition at various temperatures, and found that substrate temperatures as low as $4600^{\circ} \mathrm{C}$ could be used for the depositing $\mathrm{SnO}_{2}: \mathrm{F}$ by the reaction of tetramethyltin, oxygen and hexafluoropropene. The same $460 \circ \mathrm{C}$ substrate temperature was found to be suitable for the $\mathrm{ZnO}: \mathrm{F}$ deposition. Thus this common temperature was adopted for making the thin $\mathrm{SnO}_{2}:$ F/thick $\mathrm{ZnO}: \mathrm{F} /$ glass electrodes.

Transparent conductors with this structure were deposited at about $460 \mathrm{C}$, which is the optimum temperature for $\mathrm{ZnO}: \mathrm{F}$ deposition using the CVD method described above. This temperature is below the optimum for depositing fluorine-doped tin oxide, so the free electron concentration in the tin oxide was measured to be only about $5 \times 1019 \mathrm{~cm}^{-3}$, an order of magnitude lower than the values usually found for optimized tin oxide deposited at higher temperatures. Unfortunately, the $\mathrm{ZnO}: \mathrm{F}$ films increase their electrical resistance when they are reheated to temperatures above their original deposition temperature.

Samples of the thin $\mathrm{SnO}_{2}: \mathrm{F} /$ thick $\mathrm{ZnO}: \mathrm{F} /$ glass transparent conductors were sent to Steve Hegedus at IEC, Delaware. He deposited amorphous silicon solar cells using three different dopant levels in the p-Si layer contacting the tin oxide. The lowest flow is typical of what they used for standard tin oxide substrates. Figure 1 shows that the standard boron doping level gave lower open-circuit voltages on the zinc oxide substrate than on tin oxide. Even the thin, lightly doped tin oxide layer on the composite $\mathrm{SnO}_{2}: \mathrm{F} / \mathrm{ZnO}: \mathrm{F}$ was able to raise the voltage back to the level found in the same run for standard tin oxide. This result supports the possibility of using the composite $\mathrm{SnO}_{2}: \mathrm{F} /$ $\mathrm{ZnO}: \mathrm{F}$ to increase cell efficiency.

At the higher levels of boron doping, the voltages became equivalent for all of the substrates. The fill factors increased with boron doping level, but, unfortunately, remained lower for both $\mathrm{ZnO}$-containing substrates, at all boron doping levels (Figure 2). Apparently, a higher free electron concentration will be needed in the tin oxide contact layer, if higher efficiencies are to be attained with the $\mathrm{SnO}_{2}: \mathrm{F} / \mathrm{ZnO}: \mathrm{F}$ transparent conductor. 


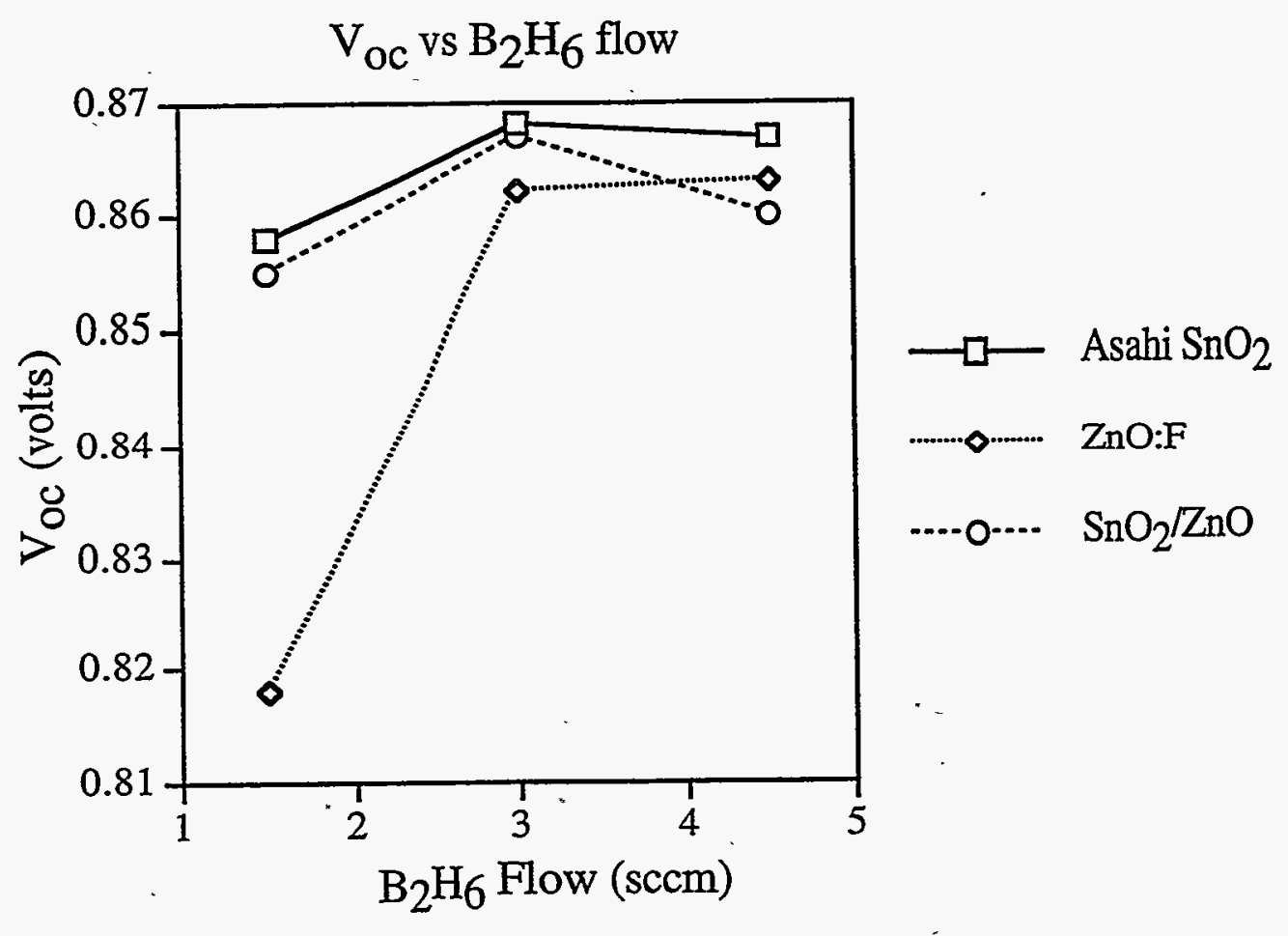

Figure 1. Open circuit voltages for amorphous silicon solar cells grown on three different transparent conductors, as a function of the boron doping level in the p-layer.

$\mathrm{FF}$ vs $\mathrm{B}_{2} \mathrm{H}_{6}$

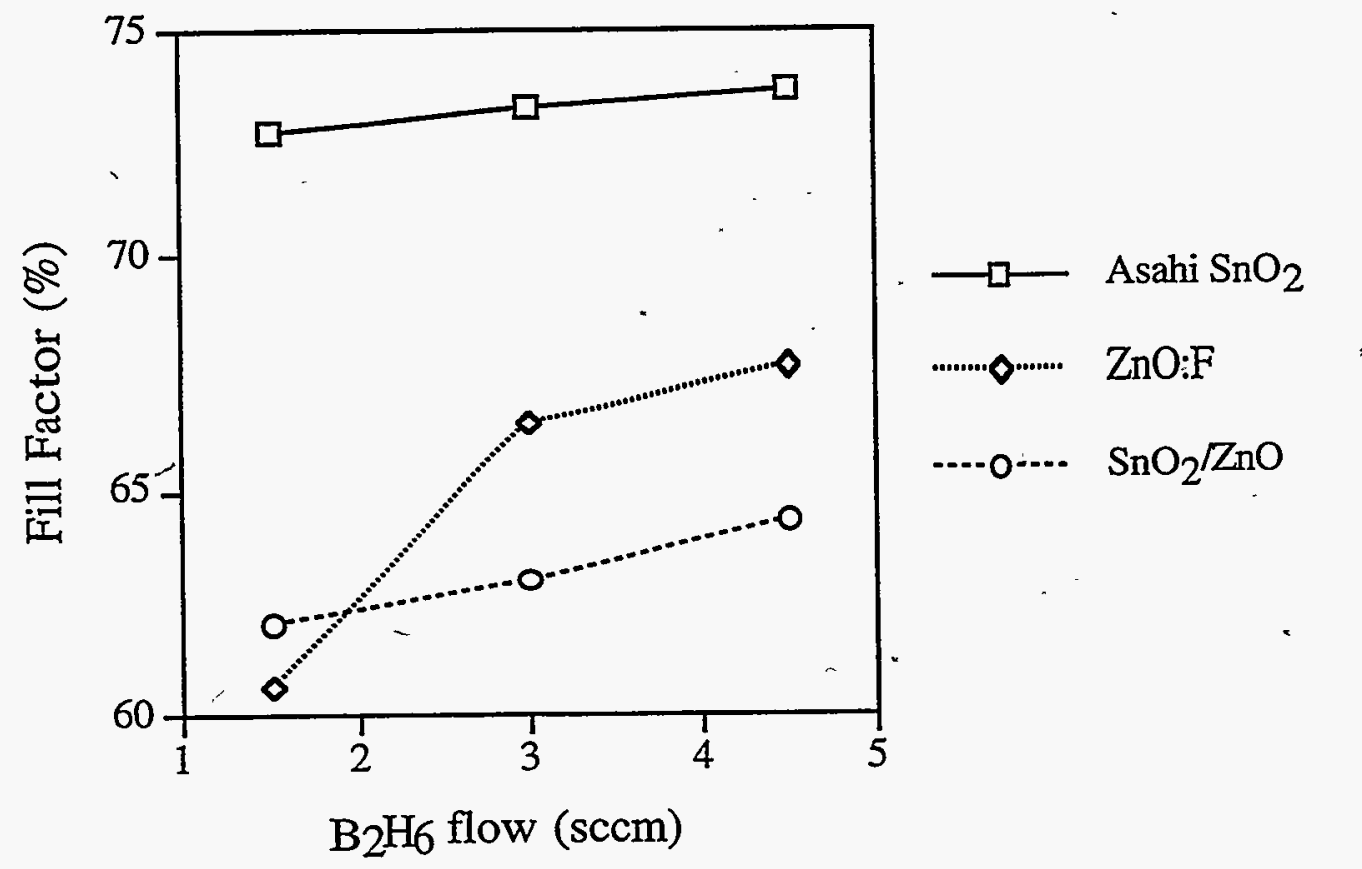

Figure 2. Fill factors as a function of the boron doping level in the p-layer. 
Title: $\quad$ Application of CIS to High Efficiency PV Module Fabrication

Organization: International Solar Electric Technology (ISET)

8635 Aviation Blvd., Inglewood, CA 90301

Contributors: B.M. Basol, Principal Investigator; V.K. Kapur, Program Manager;

C.R. Leidholm, A. Halani, G. Norsworthy and R. Roe

\section{Objectives}

The objectives of this R\&D partnership program are the development and demonstration of a novel, low-cost, non-vacuum deposition technique for the growth of CIS and related alloy absorbers and the use of these absorbers for the fabrication of high efficiency solar cells and modules.

\section{Approach}

In this program ISET's efforts were concentrated on the development of a non-vacuum deposition process for CIS absorber layers and setting up a small pilot line based on this technology. However, we continued to prepare small size samples by the vacuum-based twostage process specifically for the task that was formulated to study CIS-Mo-substrate interactions within the national CIS team program.

Details of ISET's vacuum-based two-stage selenization technique and the device fabrication steps have been previously described. In summary, these baseline processes involve vacuum deposited metallic precursors and vapor phase selenization in $\mathrm{H}_{2} \mathrm{Se}$. Selenization of the precursors is carried out in a reactor kept at a temperature of about $450^{\circ} \mathrm{C}$. For device fabrication, CIS films are coated with a thin (600-2000 $\AA$ ) CdS layer using the solution growth technique. This step is then followed by the deposition of a conductive $\mathrm{ZnO}$ window layer using the MOCVD method.

Tasks involving the development of a novel CIS growth approach did not have any vacuum deposition step. In these tasks, films were grown through a new, non-vacuum technique. Device fabrication steps, however, were common to CIS absorbers obtained by both vacuum and nonvacuum approaches.

\section{Results and Discussion}

Experiments were set up to study Na diffusion from the soda-lime glass substrates, through the Mo layers into the CIS absorbers. These included measurements on as-deposited Mo layers of various thicknesses, glass/Mo structures that were either heated in vacuum or put through the selenization step, and glass/Mo/CIS structures.

i) There was a quantitative increase in $\mathrm{Na}$ signal but no change in Na profile upon annealing the 
glass/Mo structures at $450^{\circ} \mathrm{C}$ for 20 minutes in vacuum. The suggestion is that the $\mathrm{Na}$ diffusion takes place along the grain boundaries and it is fast.

ii) $\mathrm{Na}$ level in different Mo layers was roughly identical despite the fact that the deposition conditions and the selenization character of these Mo layers were very different.

iii) $\mathrm{Na}$ signal and $\mathrm{O}$ signal tracked each other, suggesting that $\mathrm{Na}$ was in the form of an oxide in the Mo layer.

After studying Na diffusion into the Mo layers, $\mathrm{Na}$ distribution in CIS films was investigated. It was obvious from the SIMS data that the Na content of the CIS film deposited on thin Mo layers was about 3-4 times higher than those grown on thick Mo layers. There was also a high level of $\mathrm{Na}$ near the Mo/CIS interface in all samples. High Na content near the Mo/CIS interfaces and the decreased $\mathrm{Na}$ concentration in the bulk of the CIS layers may appear like $\mathrm{Na}$ diffusion profiles . However, if $\mathrm{Na}$ resides primarily on the grain boundaries, then this profile may be a reflection of the average grain boundary area. The following experiment was performed to clarify this point.

A double layer CIS film was prepared on a soda-lime glass/Mo substrate. The aim was to obtain a film with a small grained structure near the middle. A $2 \mu$ m thick CIS layer was first grown on the glass/Mo substrate. The sample was taken out of the reactor and washed. A Cu-In precursor was again deposited, this time on top of the already grown CIS layer. Selenization was carried out to grow a second CIS layer on top of the first one. The expected grain structure and $\mathrm{Na}$ content for such a double layer are qualitatively depicted in Fig. 1. In this study, we expected

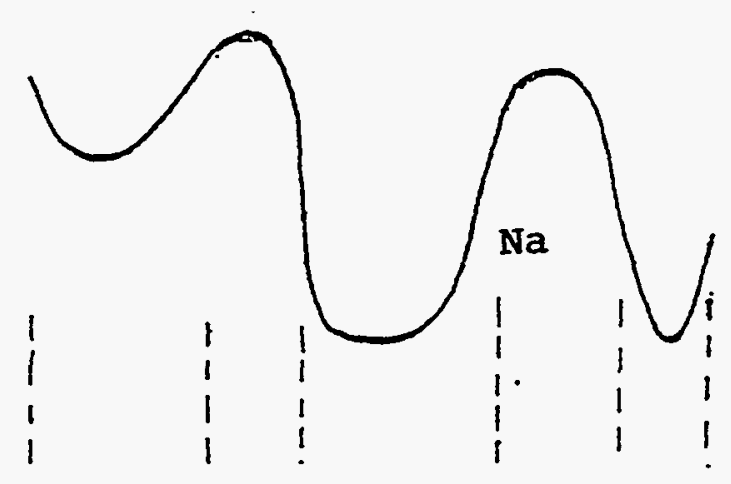
to grow a small grained second layer on top of a first layer with larger grains. If the high concentration of $\mathrm{Na}$ observed near the $\mathrm{Mo} / \mathrm{CIS}$ interface was the result of a diffusion profile (higher $\mathrm{Na}$ close to the glass substrate), then one would expect the $\mathrm{Na}$ concentration to drop even further in the top CIS layer since it is further away from the $\mathrm{Na}$ source. However, if the $\mathrm{Na}$ concentration is directly tied to grain size (smaller the grains, larger the grain

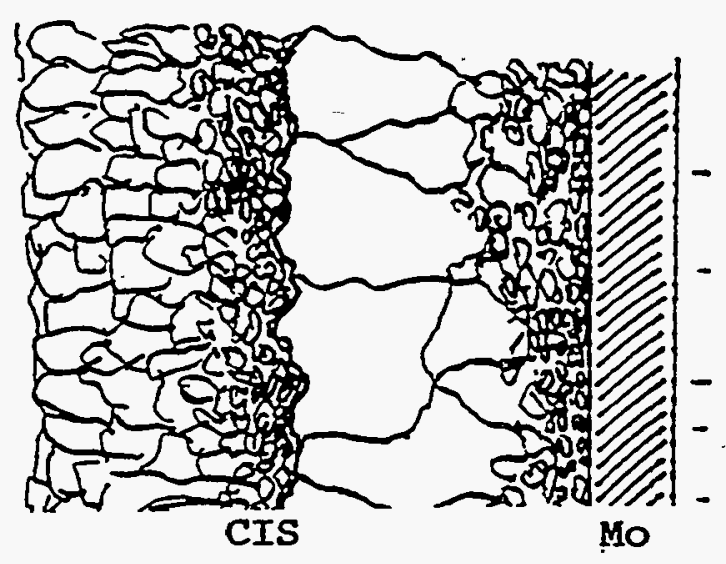

Fig. 1 Sketch of the micro-structure and expected profile for a double layer CIS film on Mo. boundary area and larger the Na content) then one would expect a double hump in the $\mathrm{Na}$ profile as depicted in Fig. 1. The SEM of the double layer CIS sample is shown in Fig. 2. The grain structure is indeed as shown in the sketch of Fig. 1. The actual SIMS profile shown in Fig.3 displays the double hump feature mentioned before. These results show that the $\mathrm{Na}$ content in CIS layers is indeed a strong function of the micro-structure and that Na mainly resides on grain boundary areas. 


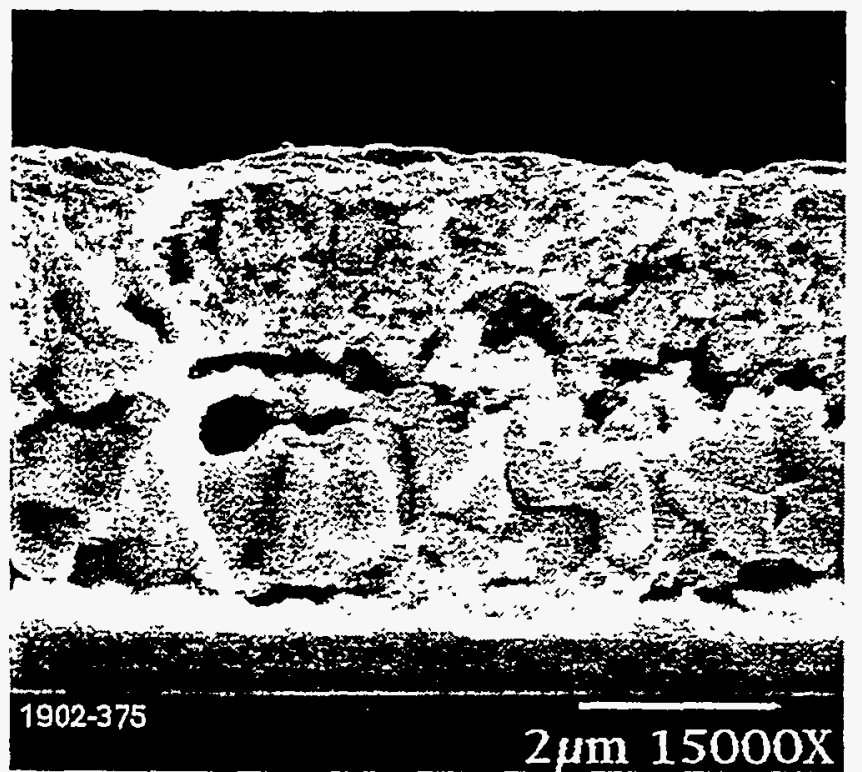

Fig. 2 SEM of a double layer CIS film grown on glass/Mo substrate.

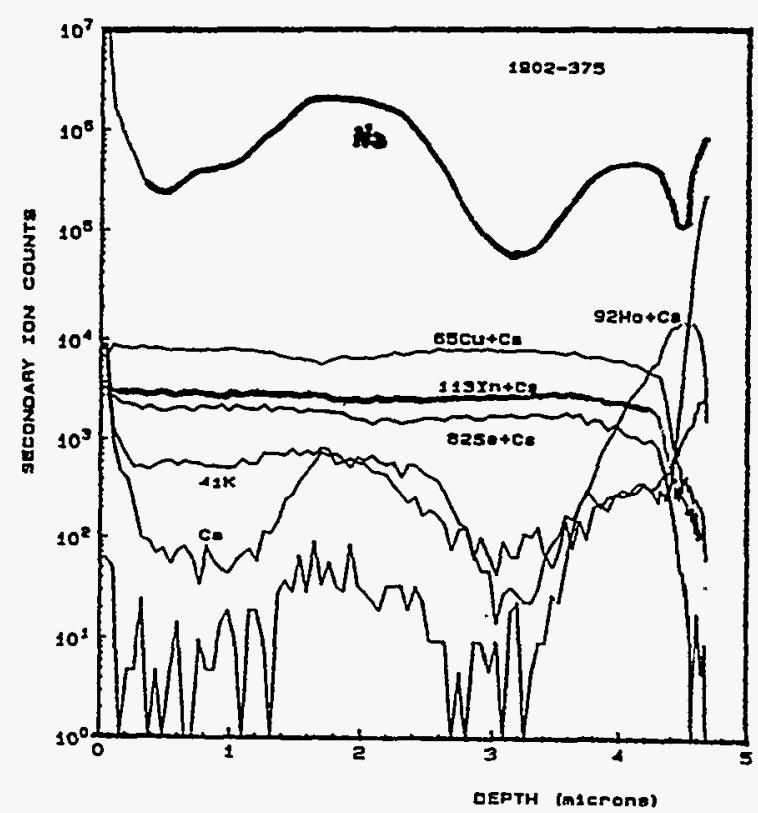

Fig. 3 SIMS profile taken from the sample of Fig. 2.

During this research period ISET has set up a pilot line for the demonstration of its thin-film nonvacuum CIS module technology. The estimated capacity of the line is $50 \mathrm{~kW} / \mathrm{yr} / \mathrm{shift}$ assuming $7 \%$ module efficiency and $70 \%$ process yield. The pilot line occupies approximately $2000 \mathrm{ft}^{2}$ of floor space and consists of ten primary processes including substrate cleaning, Mo deposition, Mo patterning, materials preparation, film application, annealing processes, chemical bath $\mathrm{CdS}$, MOCVD ZnO, mechanical scribing and module lamination. The Mo layer is sputter deposited. Molybdenum patterning uses a dry negative-resist process which is widely employed in the circuit board industry. The resist film is applied with a heated pinch-roller, which lays the resist material on the substrate as it passes through two spring-loaded rollers. After lamination, the resist is exposed with a mask and the substrate is put through a wet line for development and rinse. Mo etching and stripping is performed in a six-tank vented wet line. A water rinse followed by a de-ionized water rinse is performed and the substrates are dried with an air knife. The absorber films are applied to the desired thickness on the substrates and they are subjected to annealing steps at around $400-450^{\circ} \mathrm{C}$ to achieve the necessary optical and electrical properties. Cadmium sulfide (CdS) is used as the buffer layer. The chemical bath method employed in ISET's research has been scaled up for use in the module production. The CIS/CdS layers are mechanically scribed to open vias for the $\mathrm{ZnO}$ to reach the Mo back contact, to complete the monolithic integration. This is done by mechanical scribing. $\mathrm{ZnO}$ deposition is performed in a MOCVD reactor. Module lamination is performed in a $53^{\prime \prime} \times 18^{\prime \prime}$ vacuum cavity laminator. Annealing/reaction portions of the pilot line is controlled by computer.

During de-bugging of this pilot line it was determined that all of the steps of the above described process were acceptable except for the annealing step. Thermal gradients in the annealing furnace was found to be excessive and this caused substrate breakage lowering the yields. Therefore, we reduced the substrate size to first $6 " x 13$ " then to $6 " x 3 "$. Work on the modification of the furnace design is in progress. Reference 1 contains further information about the characteristics of CIS 
layers grown by the non-vacuum technique.

\section{Conclusions}

Solar cells in ISET's non-vacuum technology are in the 10-13\% range using CIS absorbers. Small area submodules $\left(25 \mathrm{~cm}^{2}\right)$ with $8-9 \%$ efficiency have been demonstrated. $100 \mathrm{~cm}^{2}$ size submodules with $5-6 \%$ efficiency has been processed. Demonstration of the novel, non-vacuum technique is complete. With new furnace design, we expect to be able to process 13 "x13" substrates. Device efficiencies are expected to improve once larger bandgap absorbers are obtained by the non-vacuum technique.

\section{Acknowledgements}

ISET was a member of the CIS Thin Film Partnership Program in 1997. We acknowledge the contributions of our team members, J. Britt of EPV, T. Gillespie of Lockheed/Martin, A. Rockett of U. of Illinois and R. Matson, M. Al-Jassim, S. Asher, and F. Hasoon, of NREL to the reported work.

\section{Reference}

1. Basol, B.M.; Kapur, V.K.; Leidholm, C.R.; Roe, R.; Halani, A.; and Norsworthy, G. (1996). "Low Cost CIS Device Processing." AIP Conference Proceedings, vol. 394, Witt, Al-Jassim and Gee, eds. American Institute of Physics; pp.107-114. 
Title:

Organization: Iowa State University, Ames, Iowa 50011

Contributors: $\quad$ Vikram L. Dalal, Principal Investigator

Robert Girvan, Kay Han, Tim Maxson, Ralph Knox, Sanjeev Kaushal, Karl Erickson, Jason Herrold, Sohail Haroon, Swati Hariasra, Alan Boerner, Eric Skogen

\section{Introduction}

It is well known that the properties of a-Si:H and a-(Si,Ge):H materials and devices depend critically upon growth conditions. The strong dependence on growth conditions arises because the material being deposited is metastable. Therefore, the deposition temperatures have to be low to prevent the conversion of the materials into a crystalline state. The low deposition temperatures, in turn, lead to low surface mobilities for radicals from which the film grows. As a result, it is possible to have materials which are microscopically non-homogeneous, and may have significant densities of internal voids. The objective of this program is to use clever deposition geometries which can overcome the low surface mobilities and lead to the growth of a more homogeneous films with fewer microscopic defects. In this program, we have used a high energy electron-cyclotron-resonance (ECR) deposition reactor to produce a-Si and a-(Si,Ge):H films and devices which are more stable than standard glow-discharge materials and devices.

\section{Description of the process}

The deposition process used has been described previously[1,2]. It is shown in Fig.1. The power from a microwave source is fed into a discharge zone, where an ECR discharge is generated by the use of coaxial magnetic fields. The plasma gases, which may be $\mathrm{H}$ or $\mathrm{He}$, are fed into the discharge zone, and the radicals and ions stream towards a substrate, which is positioned about $30 \mathrm{~cm}$ away from the resonance zone. The $\mathrm{H}$, or $\mathrm{He}$, radicals and ions react with the silane gas, which is introduced near the substrate, generating the precursors for film growth. Typical dilution ratios of silane/hydrogen are in the range of 1:15 to 1:20.

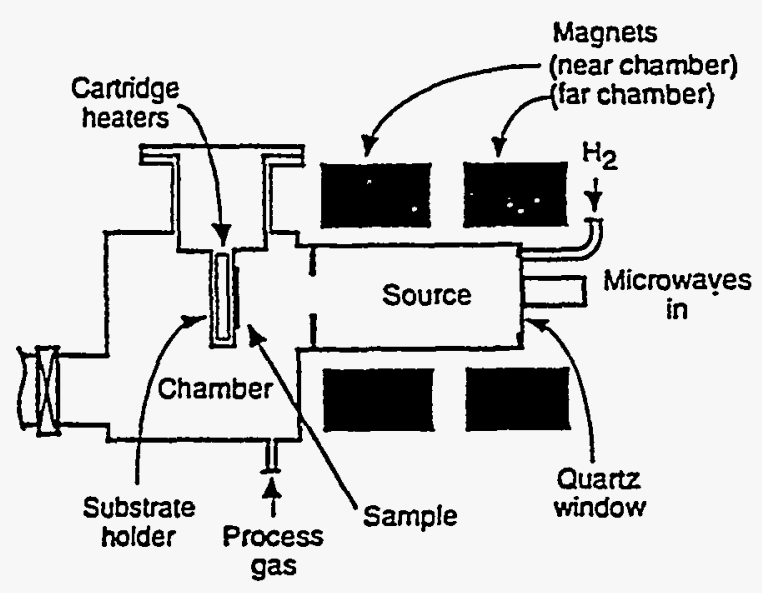

Fig. 1 Schematic diagram of the ECR reactor 
The pressure in the reactor is kept low, in the 10-15 $\mathrm{mT}$ range. At such a low pressure, there is a significant flux of both ions and radicals impinging on the substrate, helping to transfer energy to the precursor radicals such as silyl and germyl. The ion bombardment helps to increase the mobility of the radicals, thereby hopefully improving the micro-structure of the film. In addition, the etching of the film by reactive $\mathrm{H}$ during growth helps to etch away the weaker bonds, thereby leading to $a$ better film and device.

\section{Device Fabrication}

During this period, we have concentrated on producing devices on stainless steel substrates. The devices were of the $p-i-n$ type, with the $n$ layer deposited on the stainless substrate first. A thorough purging procedure was used to eliminate phosphorus contamination from the single chamber reactor after the $n$ layer. Following the purge procedure, the walls of the reactor were etched with a $\mathrm{H}$ plasma, and a dummy I layer was deposited to further bury any residual $\mathrm{P}$. The device was contacted either with a thin semi-transparent $\mathrm{Cr}$ layer, or with ITO. A central $\mathrm{Al}$ bussbar to collect the current completed the device. The typical thicknesses of the $i$ layer were in the range of $200-300 \mathrm{~nm}$.

We fabricated both single junction and tandem junction (a-Si/a-Si) devices. The objective of the tandem junction devices was to make sure that good tunnel junctions could be made using the ECR process.

\section{Device results on single junction cells}

An I(V) curve for a single junction device is shown in Fig. 2. The fill factor is $68 \%$, the voltage is $836 \mathrm{mV}$, and the current density is $12 \mathrm{~mA} / \mathrm{cm}^{2}$. There was no back reflector on the device. Thus, the current density is representative of a single-pass device.

The device was tested for stability upto 1000 hours at $2 \mathrm{x}$ sun intensity, $50 \mathrm{C}$, open circuit conditions. The results of fill factor degradation are shown in Fig. 3. The degradation in fill factor is remarkably low, only about $5-6 \%$. [3].

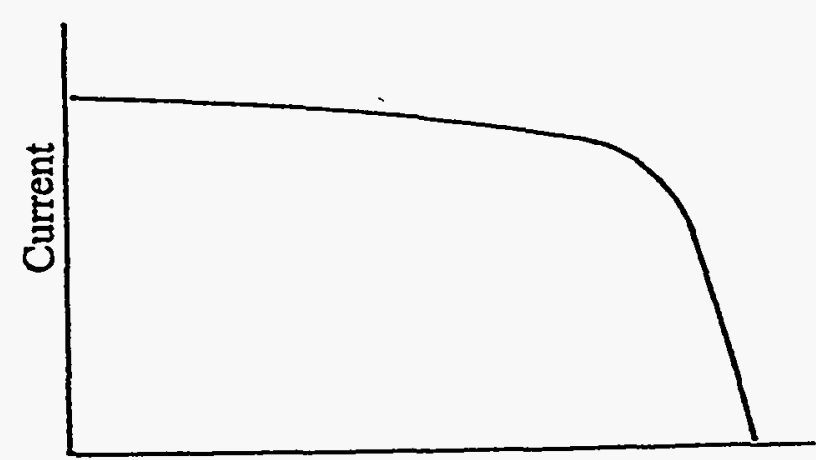

Voltage

Fig. 2 I(V) curve for a single junction a-Si cell

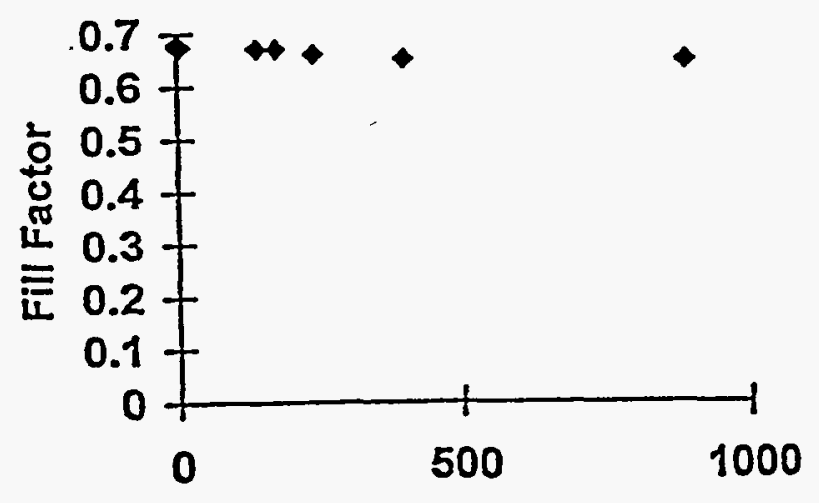

Hours

Fig.3 Degradation in fill factor of an a-Si:H cell under $2 \mathrm{x}$ sun, $50 \mathrm{C}$ conditions. 
Results on Tandem junction cells

In Fig. 4, we show the I(V) curve of a tandem junction cell. The good voltage (1.6V), and the high fill factor $(71 \%)$ indicate that the tunnel junction is of a high quality. In Fig. 5 , we show the stability data for the same cell, measured at $1 \mathrm{x}$ sun illumination for 1000 hours of illumination at $50 \mathrm{C}$. The stability is again excellent.[4].
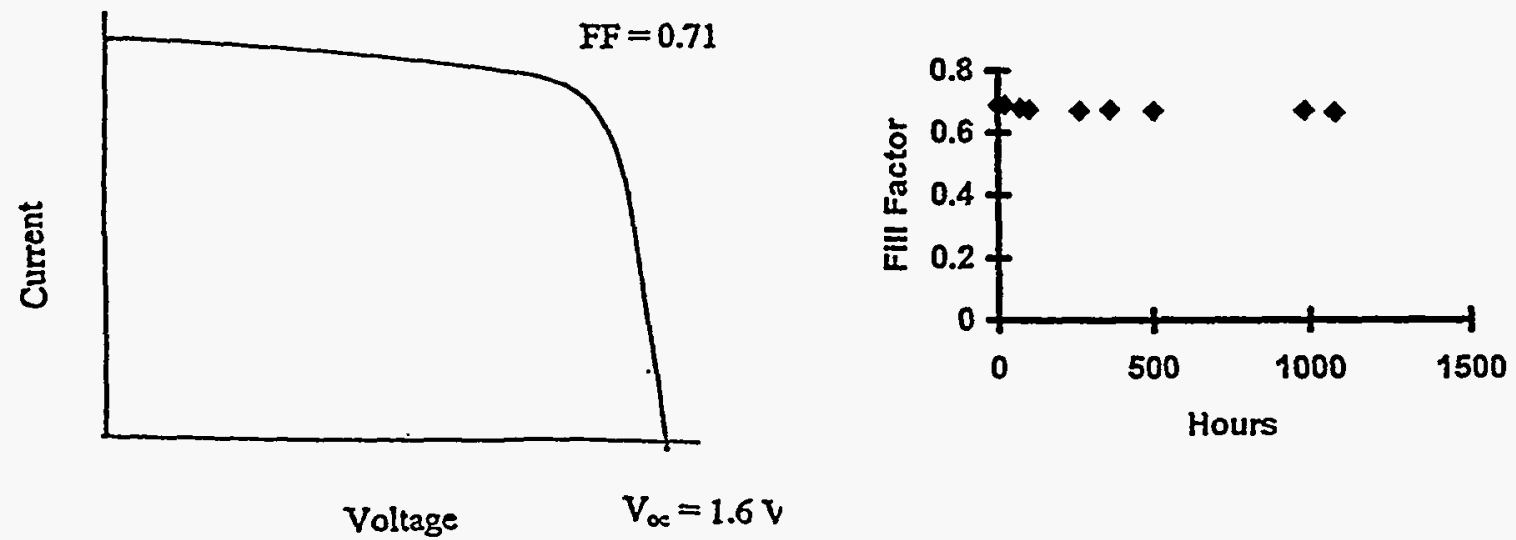

Fig.4 I(V) curve of a tandem junction cell Fig.5 Degradation of fill factor of a tandem junction cell under $1 \mathrm{x}$ sun, $50 \mathrm{C}$.

\section{Fabrication of a-(Si,Ge):H Devices}

Using the ECR process, we have fabricated both single gap and graded gap a-( $\mathrm{Si}, \mathrm{Ge}): \mathrm{H}$ cells. Both cells were of substrate type, with the $n$ layer being deposited on a rough stainless steel substrate. All cells had the back $n$ layer made from a-Si:H. The single gap cells had a very thin transition layer to go from a-Si:H to a-(Si,Ge): $\mathrm{H}$, and similarly, a front transition layer, to go from a-(Si,Ge):H to a-(Si,C):H, which is the composition of the $\mathrm{player}$. The graded gap cells had the bandgap of the a-(Si,Ge):H graded in the middle $70 \%$ of the cell, continuously from a-Si:H at the back to lowest gap a-(Si,Ge):H towards the front. Then, there was a constant gap (low gap) a$(\mathrm{Si}, \mathrm{Ge}): \mathrm{H}$ layer, followed by the buffer layer to transition from a low gap $\mathrm{i}$ to a high-gap $\mathrm{p}$ layer. The $\mathrm{p}$ layers were all made from $\mathrm{a}-(\mathrm{Si}, \mathrm{C}): \mathrm{H}$, also of a graded gap. The gap in the $\mathrm{player}$ was graded down to a low value at the end of the $p$ layer, so as to improve its conductivity, and allow for a better ohmic contact at the top surface. We found that such grading was essential to assure a low resistance front contact. A similar grading of the p layer was also done for the a-Si:H and tandem junction cells described earlier. Finally, a semi-transparent $\mathrm{Cr}$ contact, of about $100 \mathrm{~A}$ thickness, was deposited, followed by a cenral Al bussbar to collect the current.

\section{Device Results on a-(Si,Ge):H}

The device I(V) curve for a single junction device is shown in Fig. 6, and for a graded gap cell, in Fig. 7. The excellent fill factor and IV $)$ curve for the graded gap cell indicate that both the device design and the material properties are good. Finally, the stability data for the graded gap cell is shown in Fig. 8, and is surprisingly good.[5]. 

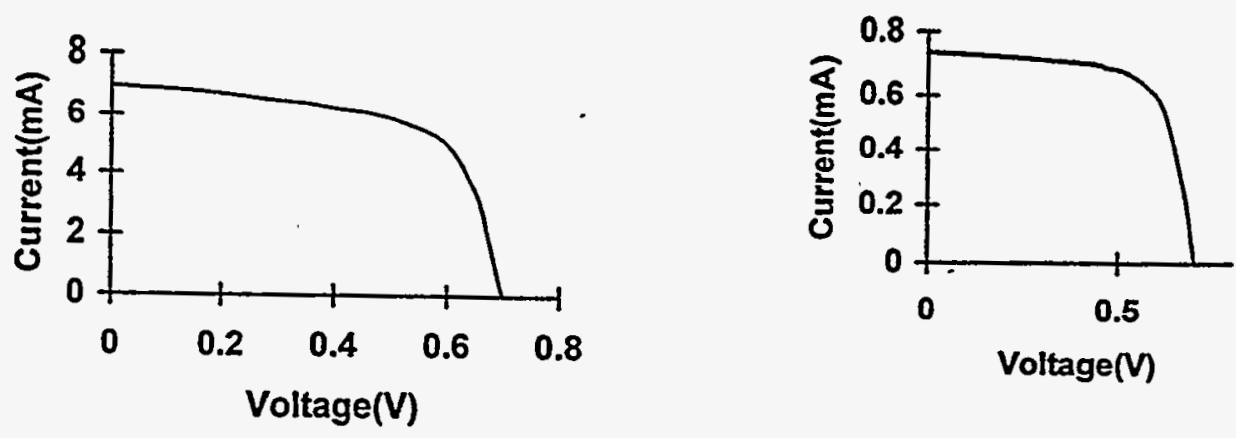

Fig. $6 \mathrm{I}(\mathrm{V})$ curve for an ungraded (Si,Ge) cell Fig. $7 \mathrm{I}(\mathrm{V})$ curve for a graded gap cell

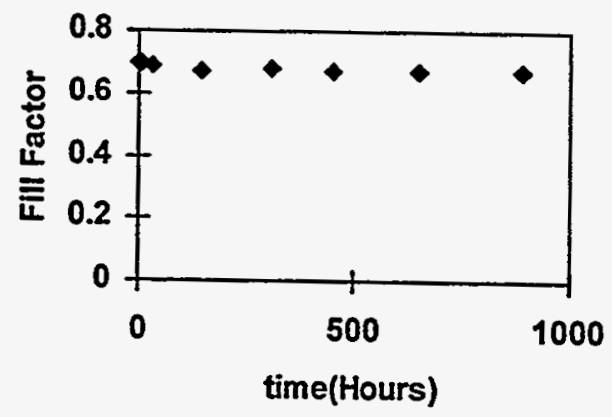

Fig. 8 Degradation in fill factor of a graded gap a-(Si,Ge) cell

\section{Conclusions}

We have shown that very good devices can be made using the ECR process in both a-Si:H and $\mathrm{a}-(\mathrm{Si}, \mathrm{Ge}): \mathrm{H}$ systems. The stability of the devices is shown to be excellent.

\section{References}

1. V. L. Dalal, G, Baldwin and M. Leonard, J. Non-Cryst. Solids, 164-166, 71(1993)

2. V. L. Dalal, E. X. Ping, S. Kaushal, M. Leonard and M. Bhan, Appl. Phys. Lett., Appl. Phy. Lett., $\underline{64}, 1862(1994)$

3. V. L. Dalal, S, Kaushal, F. Martin and R. Knox , J. Non-Cryst. Solids, 198-200, 1101(1996) 4. V. L. Dalal, S. Kaushal, R. Girvan, L. Sipahi and S. Hariasra, Proc. Of 25t. IEEE Photovolt. Spec. Conf., 1059(1996)

5. V. L. Dalal and T. Maxson, Proc. Of $26^{\text {th }}$. IEEE Photovolt. Spec. Conf(1997), to be published. 
Title:

\section{Atomic Scale Characterization of Hydrogenated Amorphous} Silicon Films and Devices

Organization: $\quad$ National Institute of Standards and Technology (NIST)

Boulder, Colorado

Confributors: $\quad$ A.Gallagher, principal investigator, M.Childs, S.Barzen

\section{Objective}

The overall objective is to assist the development of a cost-effective hydrogenated amorphous silicon (a-Si:H) photovoltaic (PV) technology. There are two trusts to this effort: (1) improving understanding of the film growth mechanisms that limit the efficiency of current PV devices, and devising ways to overcome these limitations, and (2) studying the internal electrical characteristics of thin-film PV cells.

\section{Technical Approach and Results}

\section{Particulate incorporation into a-Si:H films}

Obtaining high quality a-Si:H PV cells by deposition from a silane discharge requires relatively low deposition rates, and this is an important limitation to the throughput of a production line. At high deposition rates silicon particles accumulate in the plasma, and we have observed that a significant number of the smaller particles incorporate into the growing film. It appears likely that these particles are causing the limitation in deposition rate, and that film quality at lower deposition rates may also be somewhat effected by particle incorporation. To devise methods to mitigate this particle incorporation into films, we are studying particle growth and behavior in the plasma, as well as particle removal techniques.

Silicon particles form in the plasma and are suspended there due to acquiring a negative charge, which combined with discharge-sheath electric field normally prevents the particles from reaching the substrate during film growth. However, we have observed that small particles are continuously deposited into the film during growth [1], and it appears that this occurs because high particle densities cause a fraction of the particles to be temporarily neutralized. These diffuse into the plasma sheath, where a small fraction become positively charged and are driven by the sheath field into the substrate. Thus, we are studying the particle growth and methods to mitigate this particle incorporation into PV devices.

The most direct way to detect particle growth and behavior is by light scattering, so we are using this method to investigate particle behavior versus discharge and deposition parameters. The intensity of light scattering by small particles is proportional to $n_{p} R_{p}^{6}$, where $n_{p}$ is the particle density and $R_{p}$ the radius, so one must establish $R_{p}$ by a separate technique. For $R_{p}$ $>30 \mathrm{~nm}$ particles we resolve pulsed laser scattering from idividual particles to obtain $R_{\mathrm{p}}$ (see prior year report). This year we have studied an earlier period in particle growth, from 8-30 
$\mathrm{nm}$ radius, and this requires a different method of measuring $R_{\mathrm{p}}$ : we measure the time $(\tau)$ of particle diffusion to surfaces in the discharge afterglow, where $\tau \propto R_{p}{ }^{2}$. The data shows that $R_{p}$ grows nearly linearly to $30 \mathrm{~nm}$ radius, and $n_{p}$ decreases rapidly (from $10^{9}-10^{5} / \mathrm{cm}^{3}$ ) in the first few seconds of discharge. (Typical conditions are 0.3 Torr silane and a deposition rate of $5 \AA / s$.) The only feasible explanation of the particle density decrease is particle loss from the plasma region between the electrodes, and this is consistent with our STM observations [1] of particle incorporation into the a-Si:H film. This data also shows that the particle growth rate is due to $\mathrm{SiH}_{3}$ collisions, with a $\mathrm{Si}$ atom incorporation probability of $\sim 40 \%$ per collision, similar to the value at the film.

We have also studied the spatial distribution of these 8-40nm particles in the plasma, with and without gas flow to establish the effect of flow. We find that without gas flow partices reside throughout the plasma region between the electrodes, but with a much higher density near the edges of the electrodes. As particle size increases, they reside closer to the electrode edges. When gas flow is added, this higher density occurs near the downstream edge of the plasma, particularly near the electrode corners. This tendency of larger $\left(R_{p}>20 \mathrm{~nm}\right)$ particles to reside near the electrode edges probably explains the absence of such particles in the films studied by STM in Ref.1. However, it also appears likely that larger particles have a lower probability of being occasionally neutralized, and this would also keep them out of the films. Small particles are continually being formed, and while still small they reside above all parts of the substrate and are being incorporated into all regions of the growing a-Si:H film.

These observations provide a greatly improved understanding of $R_{p}<30 \mathrm{~nm}$ particle behavior, but this does not yet suggest ways to keep small particles out of the PV films. However, to achieve understanding of the many different forces on particles these observations have been carried out without a temperature gradient between the electrodes. When the substrate is heated a thermophoresis force pushes particles toward the colder surface, away from the substrate. We believe that this force, combined with other procedures, can be used to keep most particles out of the films. This will be the next topic we study.

\section{Cross sectioned PV cells}

Efficiency and stability of a-Si:H PV modules depend on cell design, which requires accurate models. This experiment is directed toward testing models and understanding of the sources of photo current, photo voltage and light induced degradation in PV cells. Our method is to measure the contributions to these characteristics as a function of depth within the cell, using a scanning tunneling microscope (STM).

We cleave cells coated on glass-substrates in our vacuum chamber, and use the STM to measure the electrical properties versus depth across the cleaved cell surface, with and without illumination. This experiment requires very exacting probe and sample positioning under computer and STM-feedback control, in order to find the cleaved surface of the cell without "crashing" the probe into the cell surface or the insulating glass substrate. This problem is solved by approaching around the cleaved corner of the cell, but the probe tip tends to accumulate loose surface atoms resulting from cleaving. We have largely solved this problem 
with an automated approach proceedure, plus occasionally cleaning atoms off the probe tip with a brief.voltage pulse.

The STM current-voltage ( $\mathrm{i}-\mathrm{V})$ relation is measured at each position $(\mathrm{x})$ across the cleaved cell. Unfortunately, but this relation is not well understood, even for metal and crystalline silicon substrates. Consequently we have concentrated on the probe voltage for which $i=0$, which we label the apparent voltage $\left(\mathrm{V}_{\mathrm{a}}\right)$. For each location across an operating cell $\mathrm{V}_{\mathrm{a}}(\mathrm{x})$ is between the quasi Fermi levels $\left(F_{p}, F_{n}\right)$, but the exact location is not yet established. We are using AMPS to model local cell parameters which enter into the determination of $\mathrm{V}_{a}$, and we combine these with the theory of semiconductor-metal tunneling through a barrier. However, this modeling is at an early stage of development, and components are being tested with metalmetal and metal-crystal Si tunneling.

We have now carried out STM i-V spectroscopy across the surface of single-component a-Si:H PV cells, using both light and externally applied voltage to bias the cell. The results are highly reproducible on repeated scans and across different regions of the cleaved surface. An example of the measured $\mathrm{V}_{2}(\mathrm{x})$ is shown in Fig.1, for an a-Si:H cell made by if discharge at NREL. As shown diagrammatically in the figure, this $\sim 400 \mathrm{~nm}$ thick $\mathrm{p}$-i-n cell is deposited on ITO, and we deposit a thin Au-Pd layer for the outer electrode. (This layer is very thin to avoid tearing during the cleave.) The measured STM tip height versus $x$ is shown; this turns down at the-metal-coated cell edge, as expected for a rounded probe tip traversing a corner. Since the probe is adjacent to the metal as this occurs, $\mathrm{V}_{\mathrm{a}}(\mathrm{x})$ matches the voltage of the metal at this end of the scan; at the other end of the scan it matches the voltage of the ITO electrode. A voltage of $600 \mathrm{mV}$ was externally applied for this data, and although the outer electrode was grounded a voltage drop occurred in the thin metal layer and the cleaved end is $\sim 50 \mathrm{mV}$ from ground. $V_{a}(x)$ has a similar shape for different applied voltages and for external light biasing. As we do not have a full theory that relates $V_{2}(x)$ to the local cell properties, we will not comment on the observed behavior in the intrinsic layer. However, the voltage drop near the ITO layer in forward bias implies a problem with this junction. The metal contact to the $n$ layer does not have such a problem.

These observations are preliminary, and the method has not yet been applied to different cell structures and to observing the effects of light soaking. Nonetheless, we believe that the data shown in Fig. 1 demonstrates the overall capability of this approach for observing the internal characteristics of PV cells. We expect these observations to be particularly valuable for compound cells, where the contributions of many different junctions and layers can be detected. Also, we believe this will be valuable for observing where the effects of lightinduced degradation occur within cells.

\section{Conclusions}

We have measured the early growth of particles in a silane plasma operated with typical conditions used to deposit high quality a-Si:H PV cells. This has shown that $\mathrm{SiH}_{3}$ collisions with the particles is the cause of their growth for the observed particle sizes of 8-40 $\mathrm{nm}$ radius. We have also observed that the larger particles reside preferentially at the outer 
edges of the plasma, an effect which is attributed to a weak but significant radial electric field in the plasma region between the electrodes. This understanding of particle growth and transport, and its dependence on deposition parameters of power and pressure, allows a quantitative model to developed for particle behavior. This will be utilized-while next -introducing additional forces due to gas drag and thermophoresis in a controlled manner.

We have succeeded in measuring the apparent voltage throughout the inside of an operating aSi:H PV cell, using STM tunneling. This provides the ability to observe the voltage distribution in compound cells, as well as effects of light induced degradation at the junctions and within the intrinsic layers of cells.

1) D.N.Tanenbaum, A.L.Laracuente, and A.Gallagher, Appl.Phys.Lett. 68,1705 (1996)

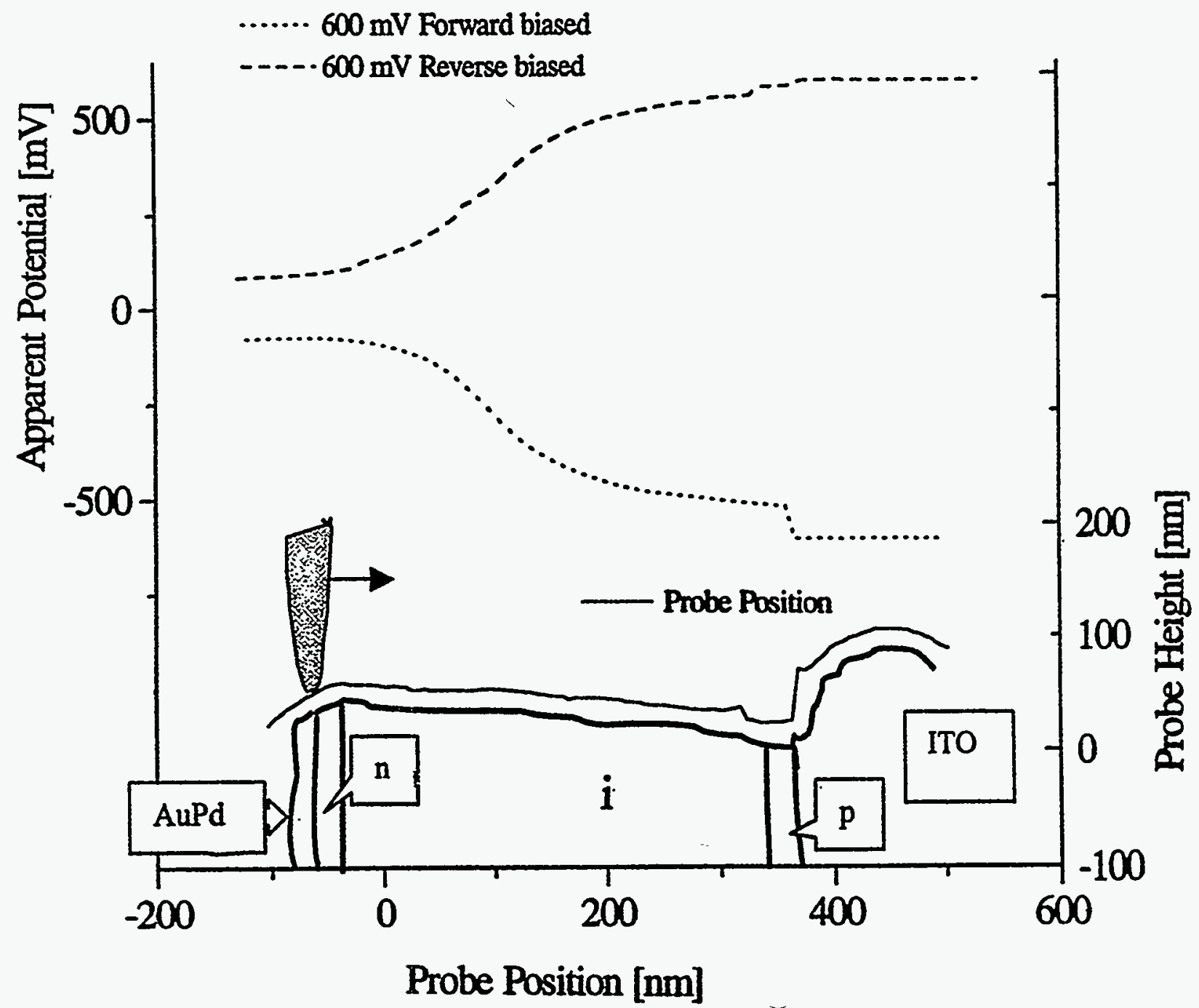

Fig. 1. Diagrammatic representation of a $400 \mathrm{~nm}$ thick a-Si:H p-i-n PV cell on ITO, and the STM probe. The measured probe height versus position is shown intersecting the probe tip. In the upper half of the figure, the apparent voltage $\left(V_{2}\right)$ versus position is shown for $\pm 600 \mathrm{mV}$ applied to the ITO contact. 
Title:

Wide Band Gap Amorphous Silicon-Based Solar Cells with High Stabilized Performance

Organization: $\quad$ The Pennsylvania State University, University Park, PA

Center for Thin Film Devices

Contributors: $\quad$ C.R. Wronski and R.W. Collins, Principal Investigators;

H. Fujiwara, Lihong Jiao, Joohyun Koh, Yeeheng Lee, Zhou Lu, Xwei Niu

\section{Introduction and Overview}

Real time optical characterization has been applied to direct the design and optimization of bandgap and microstructurally engineered a-Si:H p-i-n and n-i-p solar cells. In such an approach, wellcharacterized discrete and graded layers of different properties are incorporated at the $p / i$ and $i / p$ interfaces. Although we have considered a variety of interface tailoring methods, the results for two specific structures shown in Fig. 1 will exemplify the approach. In the first example (Fig. 1a), we have designed a two-step process for the i-layer of $\mathrm{p}-\mathrm{i}-\mathrm{n}$ cells in which a thin layer of a novel form of a-Si:H is inserted on the $\mathrm{i}$-layer side of the $\mathrm{p} / \mathrm{i}$ interface. In this case, the deposition conditions are chosen in view of real time optical studies, so as to ensure that the interface layer grows just within the amorphous side of the amorphous/microcrystalline phase boundary. The key to this development is the ability to distinguish a-Si:H from $\mu \mathrm{c}-\mathrm{Si}: \mathrm{H}$ growth in the thin device layers $(-100 \AA)$. In the second example (Fig. 1b), we have designed compositionally-graded $a-S_{1-x} C_{x}: H$ layers which are inserted on the $\mathrm{i}$-layer side of the $\mathrm{i} / \mathrm{p}$ interface in $\mathrm{n}-\mathrm{i}-\mathrm{p}$ solar cells. In this latter case, real time optical studies can provide the depth profile in the $C$-content $x$ and the optical gap $E_{g}$ with monolayer resolution. The device performance can be correlated closely with the gap profile, which is inaccessible to destructive probes such as secondary ion mass spectrometry (SIMS).

\section{Band Gap and Microstructurally Engineered a-Si:H-Based Solar Cells}

\section{a-Si:H p-i-n Cells with Microstructurally Engineered p/i Interface Layers}

Figure 2 shows the microstructural evolution for four $\mathrm{Si}: \mathrm{H}$ films prepared by plasma-enhanced $\mathrm{CVD}$ on $\mathrm{Si}$ wafer substrates (c-Si) at $200^{\circ} \mathrm{C}$ using different $\left[\mathrm{H}_{2}\right] /\left[\left[\mathrm{SiH}_{4}\right]\right.$ gas flow ratios designated by $\mathrm{R}$. In this figure, the surface roughness layer thickness $d_{s}$ is plotted versus the bulk layer thickness $d_{b}$ as deduced from real time spectroscopic ellipsometry (RTSE). The vertical line denotes the onset of continuous $2-d$ film growth (i.e., when $d_{b}=2.5 \AA$ ). For the film prepared with $\mathrm{R}=\left[\mathrm{H}_{2}\right] /\left[\left[\mathrm{SiH}_{4}\right]=0, \mathrm{~d}_{\mathrm{s}}\right.$ decreases by $2-3 \AA$ in a weak coalescence process, reaches a minimum of $15 \AA$ near $d_{b}=200 \AA$, and increases again in a gradual long-term roughening trend. For $R=10$, the initial smoothening effect during coalescence is enhanced, and the surface is very stable with $d_{s}=9 \AA$ throughout the growth of a thick layer. Analysis of the optical properties reveal

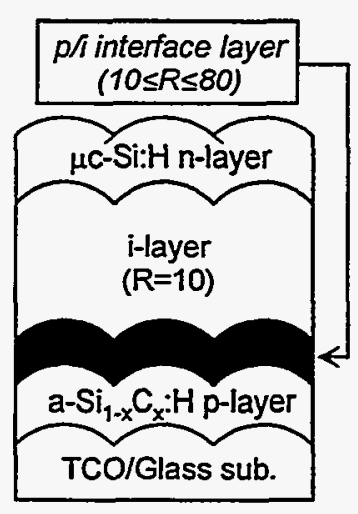

(a)

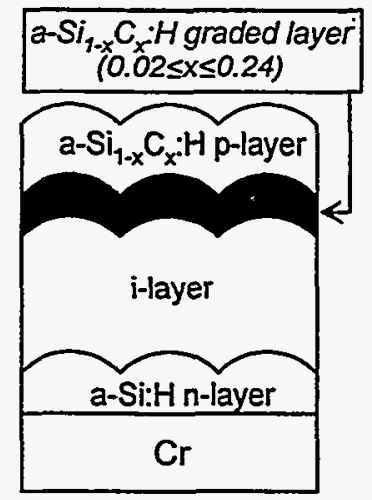

(b)

Figure 1. Schematics of (a) an a-Si:H p-i-n structure that incorporates a 100-200 $\AA$ high $\mathrm{H}_{2}$ dilution $\mathrm{p} / \mathrm{i}$ interface layer and (b) an a-Si:H n-i-p structure having an a-Si ${ }_{1-x} \mathrm{C}_{x}: H$ graded layer at the $\mathrm{i} / \mathrm{p}$ interface. 
that the films prepared with $\mathrm{R} \leq 10$ are fully amorphous for all thicknesses. For $\mathrm{R}=20$, there is a further enhancement in the surface smoothening during coalescence $\left(d_{b}<100 \AA\right)$, however, a rapid onset in roughening occurs near $d_{b}=200 \AA$ due to the development of $\mathrm{Si}$ crystallites. For $\mathrm{R}=40$, crystallite nucleation occurs immediately on the $\mathrm{c}-\mathrm{Si}$ substrate, and $\mathrm{d}_{\mathrm{s}}=58 \AA$ at the onset of bulk. $\mu \mathrm{c}-\mathrm{Si}: \mathrm{H}$ growth; this value is larger than $d_{s}=16-18$ $\AA$ at the onset of bulk a-Si:H growth.

These basic studies of nucleation and growth suggested to us that an optimum microstructurally engineered $\mathrm{p}-\mathrm{i}$ $n$ solar cell design should include an initial $\sim$ 100-200 \& a-Si:H layer prepared with higher $\mathrm{H}_{2}$-dilution (e.g., $\mathrm{R}=20$ ) nearest the $\mathrm{p}$-layer in order to take advantage of the ultrasmooth surface in this window of $R$. $A$ reduced $\mathrm{H}_{2}$-dilution (i.e., $\mathrm{R}=10$ ) would

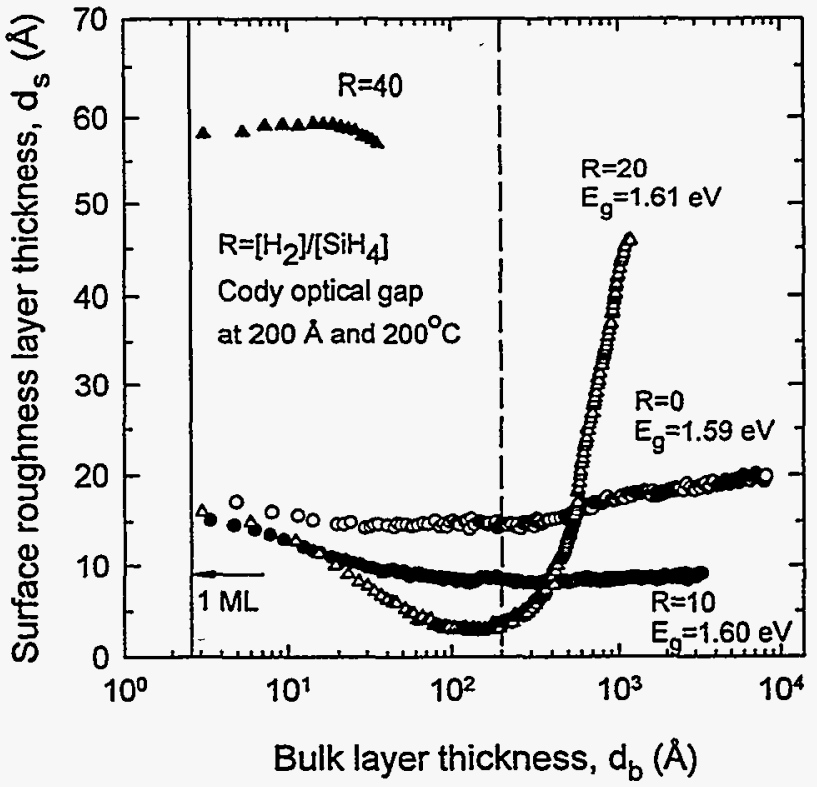

Figure 2. Surface roughness layer thickness $d_{s}$ vs. bulk layer thickness $d_{b}$ as deduced by RTSE for a$\mathrm{Si}: \mathrm{H}$ deposited at four $\mathrm{R}=\left[\mathrm{H}_{2}\right] /\left[\mathrm{SiH}_{4}\right]$ values.

then be used in the bulk to avoid the transition to crystallinity that would otherwise occur at the higher $\mathrm{R}$. The drawback in applying the results of Fig. 2 to the design of $\mathrm{p}-\mathrm{i}-\mathrm{n}$ solar cells arises from the substrate dependence of the amorphous/microcrystalline phase boundary. For the results in Fig. 2, a c-Si substrate is used, whereas for the solar cell the substrate for the intended interface layer is p-type a-Si ${ }_{1-x} C_{x}: H$ (prepared with $R=0$ ). In order to better simulate the situation in the solar cell, we performed a RTSE study similar to that of Fig.2, using as substrates a-Si:H films prepared with $\mathrm{R}=0$, rather than $\mathrm{c}-\mathrm{Si}$ wafers. In this situation, the amorphous/microcrystalline boundary shifts to higher $R$. Specifically, films prepared with $R=20$ and 40 nucleated as a-Si:H and remained amorphous to thicknesses of 100-200 $\AA$. In contrast, films with $\mathrm{R}=60$ and 80 were mixed-phase or microcrystalline in thin layers. In our solar cell studies, we prepared $p-i-n$ devices with different bulk $R$ values for no interface layer. We then fixed the bulk $R$ value at the optimum and varied the $R$ value of the 100-200 $\AA$ thick near-interface i-layer region.

Figure 3 shows the final results for $V_{o c}$ for cells prepared with substrate temperatures from $130^{\circ} \mathrm{C}$ to $200^{\circ} \mathrm{C}$. As expected on the basis of Fig. 2, the optimum $4000 \AA$ i-layer for the cell is obtained with $R=10$. Although the optimum in $V_{o c}$ is rather broad, the fill-factor drops off much more rapidly for $\mathrm{R}>10$ as microcrystals develop in the thick bulk layers. This leads to an optimum stabilized efficiency at $R=10$ for uniformly deposited i-layers without interface layers. In contrast, the optimum interface layer $R$ value is 40 , corresponding to the largest value that can be sustained while maintaining the amorphous phase in a $100-200 \AA$ thick film. For $R=80$, the interface layer is purely microcrystalline and this leads to a significantly lower $V_{o c}$ and fill-factor than the optimum. Overall, the RTSE study shows that the optimum $V_{o c}$ and stability is obtained using both interface and bulk layer $R$ values as close as possible to the amorphous/crystalline transition for the given layer thicknesses without actually crossing into the crystalline regime. For our standard a-Si $i_{1-x} \mathrm{C}_{\mathrm{x}}: \mathrm{H}$ p-layer, the highest $\mathrm{V}_{\text {oc }}$ that has been obtained so far is $0.97 \mathrm{~V}$ with $\mathrm{R}$ (int. $)=40, \mathrm{R}$ (bulk $)=10$, and $\mathrm{T}=130^{\circ} \mathrm{C}$. Future improvements will focus on microstructurally engineered p-layers with a (TCO interface)/(bulk) two-layer structure. 
a-Si:H n-i-p Cells with Bandgap Engineered i/p Interface Layers

We have developed RTSE analysis procedures that allow us to extract depth profiles in the alloy composition and optical gap with monolayer resolution for layers having continuously graded compositions. An example of such results is shown in Fig. 4. Here profiles in the carbon content $x$ and room temperature optical gap $E_{\mathrm{g}}$ (from $\varepsilon_{2}^{1 / 2}$ plots) are given versus depth below the $\mathrm{i} / \mathrm{p}$ interface layer for four n-i-p solar cells prepared with otherwise standard bulk $n, i(R=0)$, and $p$ layers. For the $\mathrm{C}$-graded layers at the $\mathrm{i} / \mathrm{p}$ interfaces, $\mathrm{R}$ was fixed at 5 and the source gas flow ratio $z=\left[\mathrm{CH}_{4}\right] /\left\{\left[\mathrm{SiH}_{4}\right]+\left[\mathrm{CH}_{4}\right]\right\}$ was varied linearly with time from 0.2 to 0.8 and back to 0.4 (to maintain continuity in $z$ across the $\mathrm{i} / \mathrm{p}$ interface). The total time period for the two ramps ranged from 38 to $205 \mathrm{~s}$. The solid lines in Fig. 4 indicate the depth profiles calculated from the flow variation and from RTSE data obtained on individually-deposited films. These calculations were based on the assumptions of instantaneous response of the $\mathrm{CH}_{4}$ and $\mathrm{SiH}_{4}$ flowmeters to their control signals as well as instantaneous response of the surface composition to gas flow ratio. The deduced depth profiles are in excellent agreement with the calculations for the two thicker interface layers ( $94 \AA$ and $130 \AA$ ). The gas residence time $(\sim 5 \mathrm{~s})$ becomes a significant factor for the thinnest interface layers (24.5 and $48 \AA$ ). For these layers, the near-interface profile generated during the second ramp in $z$ from $z=0.8$ to 0.4 is $C$-rich compared with the calculated profile. The results for the first ramp of the thinnest layer in Fig.4 demonstrate that RTSE provides monolayer depth resolution (exceeding SIMS capability) while retaining sensitivities exceeding \pm 0.01 in $x$ and $\pm 0.02 \mathrm{eV}$ in optical gap.

The inset of Fig. 4 shows $V_{o c}$ obtained for the n-i-p solar cells with graded a-Si $i_{1-x} \mathrm{C}_{x}: \mathrm{H}$ $\mathrm{i} / \mathrm{p}$ interface layers, plotted as a function of the half-width-at-half maximum of the interface layer, as deduced from the first ramp. The $\mathrm{V}_{\text {oc }}$

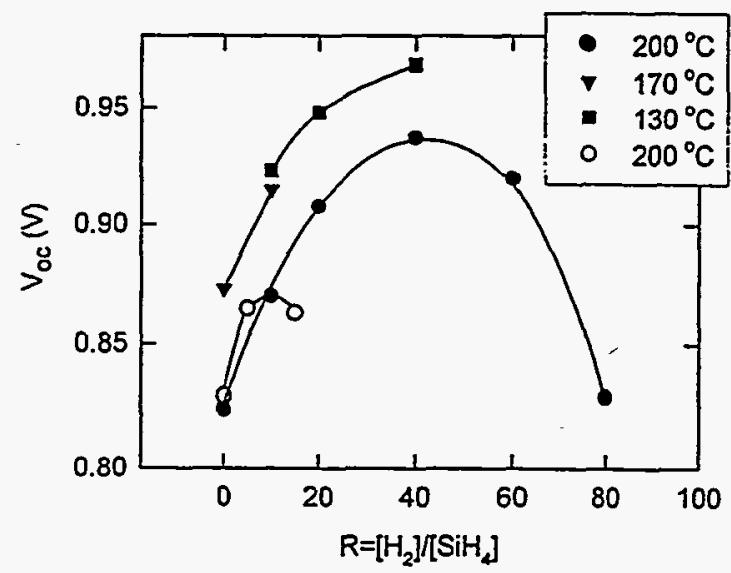

Figure 3. Dependence of $\mathrm{V}_{\text {oc }}$ on the $\mathrm{H}_{2}$-dilution ratio $R$ used in the preparation of the bulk (open circles) and $p / i$ interface layers (filled circles) in a-Si:H p-i-n solar cells. For the former cells, no interface layer was used.

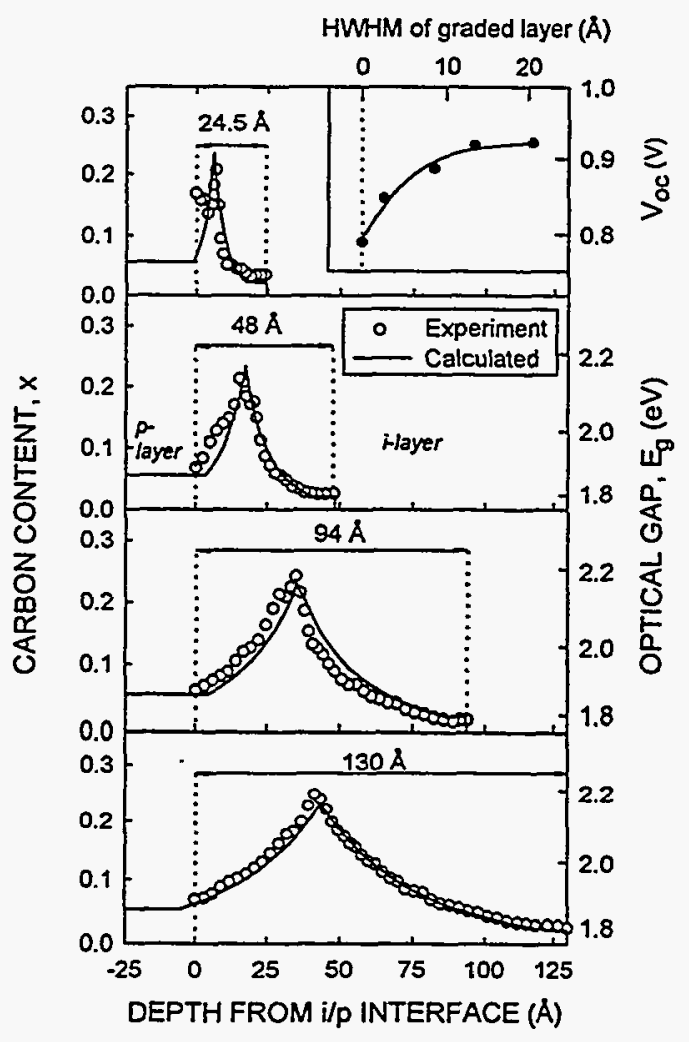

Figure 4. Depth-profiles in the C-content $x$ (left scale) and optical gap (right scale) for $\mathrm{a}-\mathrm{Si}_{1-\mathrm{x}} \mathrm{C}_{\mathrm{x}}: \mathrm{H}$ graded layers introduced at the $\mathrm{i} / \mathrm{p}$ interfaces of four a-Si:H n-i-p solar cells. The inset shows the open circuit voltage of the cells versus the half-width-at-half-maximum of the graded layer $\mathrm{C}$-profile. 
value increases monotonically from 0.79 to $0.92 \mathrm{~V}$ as the HWHM of the graded layer increases, and saturates for graded layer HWHM values of $13 \AA$ and above. Thus, a $0.38 \mathrm{eV}$ higher bandgap barrier layer generates a $0.13 \mathrm{~V}$ increase in $\mathrm{V}_{\text {oc }}$. The fill factor decreases very slowly with increasing graded layer HWHM, presumably owing to the increased internal series resistance of the wider bandgap interface layer. Because such an effect is relatively weak in comparison with the $V_{\text {oc }}$ increase, the optimized graded layer thickness in this study for the highest annealed state efficiency was found to occur at the $\mathrm{V}_{\text {oc }}$ saturation HWHM thickness of $13 \AA$. Future studies will relate the magnitude of the bandgap barrier to the increase in $\mathrm{V}_{\text {oc }}$.

\section{Bibliography}

1. C. R. Wronski, "Amorphous silicon technology: Coming age", Solar Energy Materials and Solar Cells, 41/42 (1996) 427.

2. Joohyun Koh, H. Fujiwara, C. R. Wronski, and R. W. Collins, "Application of real-time spectroscopic ellipsometry for characterizing the structure and optical properties of microcrystalline component layers of amorphous semiconductor solar cells", Solar Energy Materials and Solar Cells 49 (1997) 135-142.

3. Y. Lee, L. Jiao, H. Liu, Z. Lu, R. W. Collins, and C. R. Wronski, "Light-induced changes in hydrogen-diluted a-Si:H materials and solar cells", Solar Energy Materials and Solar Cells 49 (1997) 149-156.

4. R. W. Collins, and H. Fujiwara, "Growth of hydrogenated amorphous silicon and its alloys", Current Opinion in Solid State \& Materials Science 2 (1997) 417-424.

5. H. Fujiwara, Joohyun Koh, C. R. Wronski, and R. W. Collins, "Application of real time spectroscopic ellipsometry for high resolution depth profiling of compositionally graded amorphous silicon alloy thin films", Appl. Phys. Lett. 70 (1997) 2150-2152.

6. M. Gunes and C. R. Wronski, "Differences in the densities of charged defect states and the kinetics of Staebler-Wronski effect in undoped hydrogenated amorphous silicon thin films", J. Appl. Phys. 81 (1997) 3526.

7. Joohyrun Koh, H. Fujiwara, C. R. Wronski, and R. W. Collins, "Nucleation and growth of $\mu \mathrm{c}-\mathrm{Si}: \mathrm{H}$ $\mathrm{n}$ - and p-type layers in a-Si:H p-i-n and n-i-p solar cells: real time spectroellipsometry studies", Mat. Res. Soc. Symp. Proc., 452 (1997) 895-900.

8. R. W. Collins, Ilsin An, H. Fujiwara, Joungchel Lee, Yiwei Lu, Joohyun Koh, and P. I. Rovira, "Advances in multichannel spectroscopic ellipsometry", Thin Solid Films: to be published in 1998.

9. Joohyun Koh, H. Fujiwara, R. W. Collins, Yeeheng Lee, and C. R. Wronski, "Microstructural evolution of a-Si:H prepared using hydrogen dilution of silane studied by real time spectroellipsometry", J. Non-Cryst. Solid (Proceeding of $17^{\text {th }}$ ICAMS): to be published in 1998.

10. H. Fujiwara, Joohyun Koh, C. R. Wronski, R. W. Collins, and J. S. Burnham, "Parameterization of the optical function of a-Si $i_{1-x} C_{x}: H$ : Application to $\mathrm{C}$ depth-profiling and surface temperature monitoring in solar cell preparation", J. Non-Cryst. Solid (Proceeding of $17^{\text {th }}$ ICAMS): to be published in 1998.

11. Yeeheng Lee, Lihong Jiao, Joohyun Koh, Zhou Lu, R. W. Collins, and C. R. Wronski, "Impact of hydrogen dilution on the properties and light induced changes of a-Si:H materials and solar cells", Mat. Res. Soc. Symp. Proc., 467 (1997) 747-752.

12. L. Jiao, S. Semoushikina, Y. Lee, and C. R. Wronski, "Self consistent analysis of mobility-lifetime products and subgap absorption on different PECVD a-Si:H films, Mat. Res. Soc. Symp. Proc., 467 (1997) 233-238. 
Title: Development and Application of a

Computer Model for CdTe and CuInSe2 Based Solar Cells

Organization: Purdue University, West Lafayette, Indiana

School of Electrical and Computer Engineering

Contributors: J. L. Gray, Principal Investigator; T. K. Simacek

\section{Objective}

There are two main objectives for this project. The first is the continued development of a detailed numerical device simulation computer program tailored to the simulation of CIS and CdTe based solar cells. The second is the application of this computer program as a research tool to model and analyze these devices.

\section{Results}

Dual Beam Optical Modulation (DBOM) is a useful nondestructive tool for the measurement of carrier lifetime in semiconductors. Typically, it has been used in bulk materials for which the surface effects can be easily accounted for. In thin-film materials, such as $\mathrm{CuInSe}_{2}$ (CIS) in which there may be a significant amount of surface band bending that penetrates relatively deeply into the semiconductor, or possibly a heterostructure interface, the simple theory typically used to interpret the DBOM measurement is no longer valid. In [1] it was shown how detailed numerical device simulation can be used to provide a useful interpretation of the DBOM measurement of carrier lifetime.

Figure 1 shows a set of simulated DBOM measurements on a CdS/CIS sample for a family of excitation beam powers from $5 \mathrm{~mW} / \mathrm{cm}^{2}$ up to $50 \mathrm{~W} / \mathrm{cm}^{2}$. The symbols correspond to simulations of the measurement, while the lines corresponding to a simple analytic interpretation of the simulated measurement. As can be seen in Figure 1, only for the highest excitation illumination levels is there any correspondence with the simple analytic theory.

The discrepancy between simple theory and the simulations is due to the fact that the simulations can account for band bending at the heterojunction interface. This can be understood by realizing that the DBOM measurement is effectively being performed on a n-p solar cell under open-circuit conditions. In the dark, there is an equilibrium depletion region that extends mostly into the CIS (assuming the CdS is more heavily doped than the CIS). Under illumination, and what is basically open-circuit conditions, the depletion region shrinks. As a result, there is a large increase in the majority carrier density change in this region, caused primarily by the change in depletion width and not directly by light generated carriers. It is this change that has the biggest effect on the free carrier absorption of the probe beam, as illustrated in Figure 2. This effect is not accounted for in the simple analytic theory.

\section{Conclusion}

This work has illustrated how detailed numerical modeling can aid in the interpretation of characterization measurements in addition its use in analyzing cell performance. Work is continuing on the DBOM measurement in hopes of providing an accurate and useful interpretaion of the measurement. 


\section{Reference}

1. Thomas K. Simacek and Jeffery L. Gray, "Interpretation of Dual Beam Optical Modulation (DBOM) Measurements in Thin-Film Materials," presented at and to appear in the Conference Record of the Twenty-Sixth IEEE Photovolataic Specialists Conference, Anaheim, California, September 29 October 3, 1997.

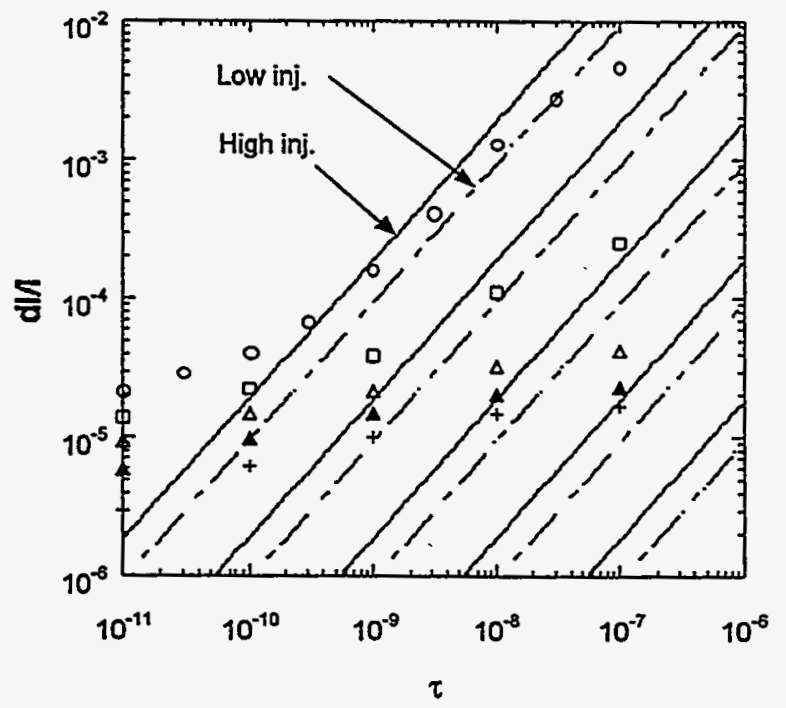

Figure 1. Simulated DBOM measurements on a CdS/CIS sample for a family of excitation beam powers from $5 \mathrm{~mW} / \mathrm{cm}^{2}$ up to $50 \mathrm{~W} / \mathrm{cm}^{2}$. The symbols correspond to simulations of the measurement, while the lines corresponding to a simple analytic interpretation of the simulated measurement.

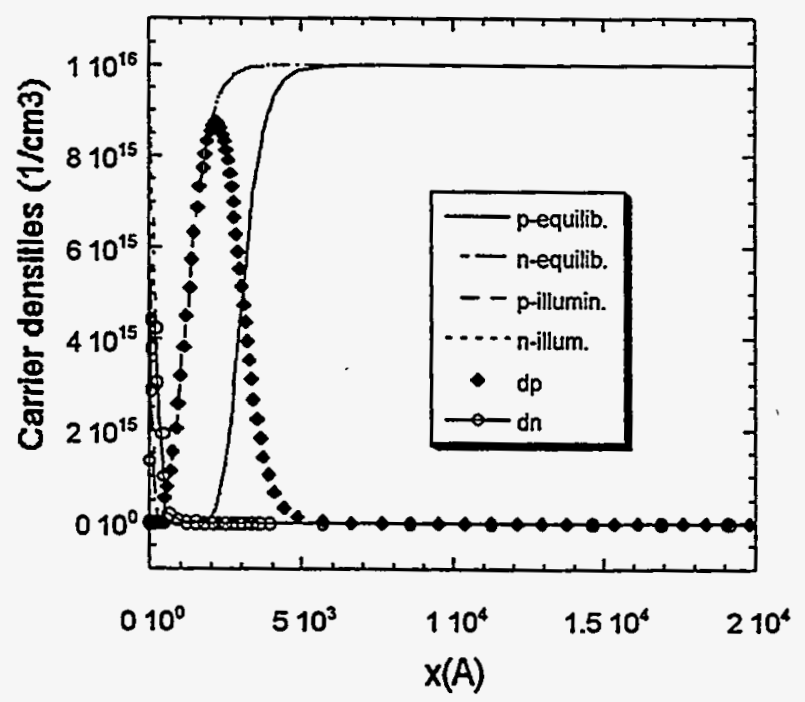

Figure 2. Carrier concentrations in the simulated CdS/CIS sample with and without illumination. 
Title:

Organization:

Contributors:

\section{Thin-Film Photovoltaic Partnership Program: CIS-Based Thin Film PV} Technology

Siemens Solar Industries

Camarillo, California

Robert R. Gay, Jürgen Bauer, Melinda E. Dietrich, Christian V. Fredric, Ulfert Rühle, Dale E. Tarrant, Robert D. Wieting, Dennis Willett

\section{Introduction}

The primary objective of this subcontract (\#ZAF-5-14142-03) is to establish reliable high-throughput, high-yield thin film deposition processes in order to make CIS a viable option for the next generation of photovoltaics. The primary goals are to demonstrate performance as well as commercial viability via the milestones of a champion prototype $13 \%$ efficient large area module and sets of modules in 1-kW arrays of steadily increasing efficiency, culminating in delivery of a $1 \mathrm{~kW}$ array of $12 \%$ efficient large-area modules by the end of the subcontract. This paper reports on progress toward these objectives and goals through approximately the second year of this three year contract; December 1996 through October 1997.

\section{Industrial Perspective}

Multinary $\mathrm{Cu}(\mathrm{In}, \mathrm{Ga})(\mathrm{Se}, \mathrm{S})_{2}$ absorbers (CIS-based absorbers) are promising candidates for reducing the cost of photovoltaics well below the cost of crystalline silicon. CIS championsolar cell efficiencies have reached $17+\%$ efficiency for devices fabricated at NREL (1). Small area, fully integrated modules exceeding $13 \%$ in efficiency have been demonstrated (2). Long-term outdoor stability has been demonstrated at NREL by $1 \times 1 \mathrm{ft}$. and $1 \times 4 \mathrm{ft}$. SSI modules which have been in field testing for as long as eight years (3). Cost projections indicate about $\$ 1.00 / \mathrm{Wp}$ based on projection of present processing to large area and high volumes (4). However, challenges remain to scale the process to larger area, and to pass accelerated environmental testing. The SSI $10 \times 10-\mathrm{cm}$ baseline process needs to be reproduced on larger areas. The origin of thermal transient behavior needs to be identified and eliminated if possible. A package design needs to be developed to pass standard environmental qualification testing.

From an industrial perspective, the full process sequence anticipated for use in the final product must be mastered and rigorously demonstrated. To this end, SSI has abandoned development based on individual cells in favor of a $10 \times 10-\mathrm{cm}$ twelve cells monolithically interconnected "mini-module" (5). Fabrication of these mini-modules exercises all aspects of large area module production and the application of statistical process control (SPC) techniques is used to rigorously demonstrates yield and reproducibility. During Phase 1 of this contract, SSI repeatedly executed a baseline 10x10-cm mini-module process rigorously demonstrating process reproducibility and yield for over two thousand CIS-based minimodules (6).

\section{Process Development}

Large-area circuit performance did not achieved the same level as the baseline process during the first phase of this subcontract. This performance difference was related to design differences between baseline and larger area absorber formation reactors $(7,8)$. For all other process steps, the impact of the larger part size was tested in the baseline process demonstrating that performance is not directly related to part size; larger-area circuits should achieve the same level of performance as baseline circuits $(7,8)$.

Studies of the differences between reactors have continued during this second subcontract phase including systematically varying process condition to demonstrate their impact on absorber properties and using the baseline reactor to mimic large area reactor process conditions. The understanding gained in these studies allowed adjustment of most but not all of the process conditions to more closely mimic process conditions in the baseline reactor and thereby mitigate most but not all of the process differences. 
Remaining differences have been isolated to differences in the materials of construction and/or the physical design of the large reactor.

Based on these studies and the associated advances in understanding regarding the influence of reactor design on performance, SSI designed and built a new large area reactor which is a more direct scale-up of the baseline reactor. SSI, with help from the TFPPP ES\&H group headed by Paul Moskowitz and supported by his associates at Brookhaven National Laboratories, defined this system to avoid potential fire or explosion hazards resulting from system failure. This reactor is now operational and the absorber formation process is being developed. Success with this reactor has been demonstrated by initial circuit performance for $28 \times 30-\mathrm{cm}$ circuits averaging $10.6 \%$ which compares very favorably to the $10 \times 10-\mathrm{cm}$ baseline

Package development continued for this contract phase with the definition of a package for the introductory product discussed below and in support of the DOE long-term goal of systems that last at least 30 years. Although long-term outdoor stability has been demonstrated at NREL and thermally induced losses from thermal cycling and humidity-freeze cycling stress tests recover with outdoor exposure, water vapor ingress resulting from extended damp heat testing permanently degrades performance for glass/EVA/circuit laminate structures. Proof-of-concept packaging designs have been demonstrated that protect laminates from water vapor ingress during damp heat testing and allow modules to pass the damp heat test; however, the package design exhibited low yield through damp heat testing and is not desirable for commercialization. As depicted in Figure 1, long-term outdoor stability has been demonstrated at NREL where $1 \times 1 \mathrm{ft}$. and $1 \times 4 \mathrm{ft}$. modules have undergone testing for as long as eight years (3). Measurements on both modules and $1 \mathrm{~kW}$ arrays indicate good stability with no seasonal variation in performance and thereby demonstrates that thermally induced transients observed after exposure to accelerated environmental testing are not observed in the field despite daily and seasonal changes in module temperature.

Siemens Solar Industries CIS Modules Measured with SPIRE 240A at STC

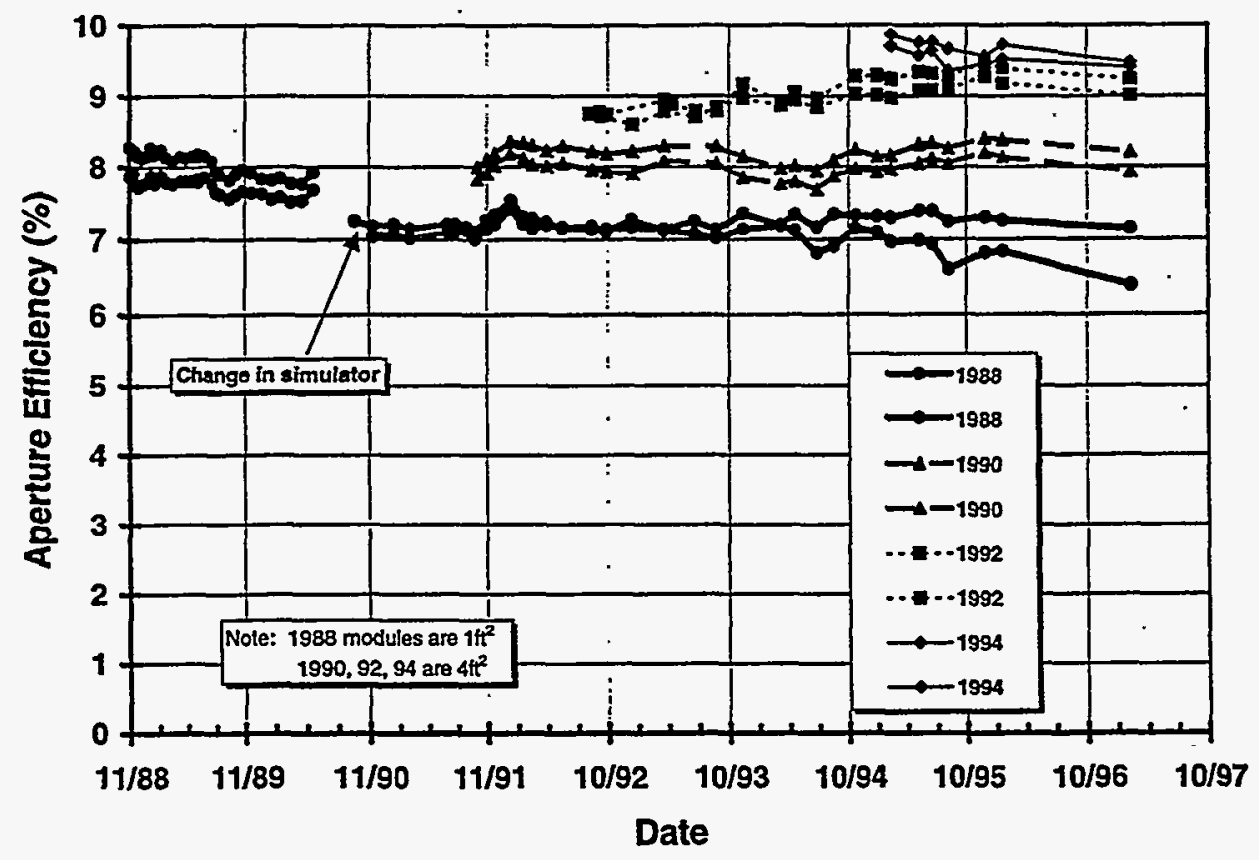

Figure 1. Results of outdoor testing at NREL showing excellent long-term stability. 


\section{Product Introduction}

SSI has introduced two new CIS-based products during this subcontract phase. The product designations are ST5 and ST10 which are $21 \times 33 \mathrm{~cm}, 5$ watt and $39 \times 33 \mathrm{~cm}, 10$ watt modules designed for use in $12 \mathrm{~V}$ systems (Figure 2). The aluminum framed module are fabricated from two or more approximately $9 \times 30 \mathrm{~cm}$ circuit plates laminated between a polymer backsheet and tempered glass. SSI has delivered product samples to NREL as contract deliverables and NREL reports $9.6 \%$ total area efficiency $(11.2 \%$ circuit plate aperture area) which is the highest efficiency of any commercial non-crystalline module.

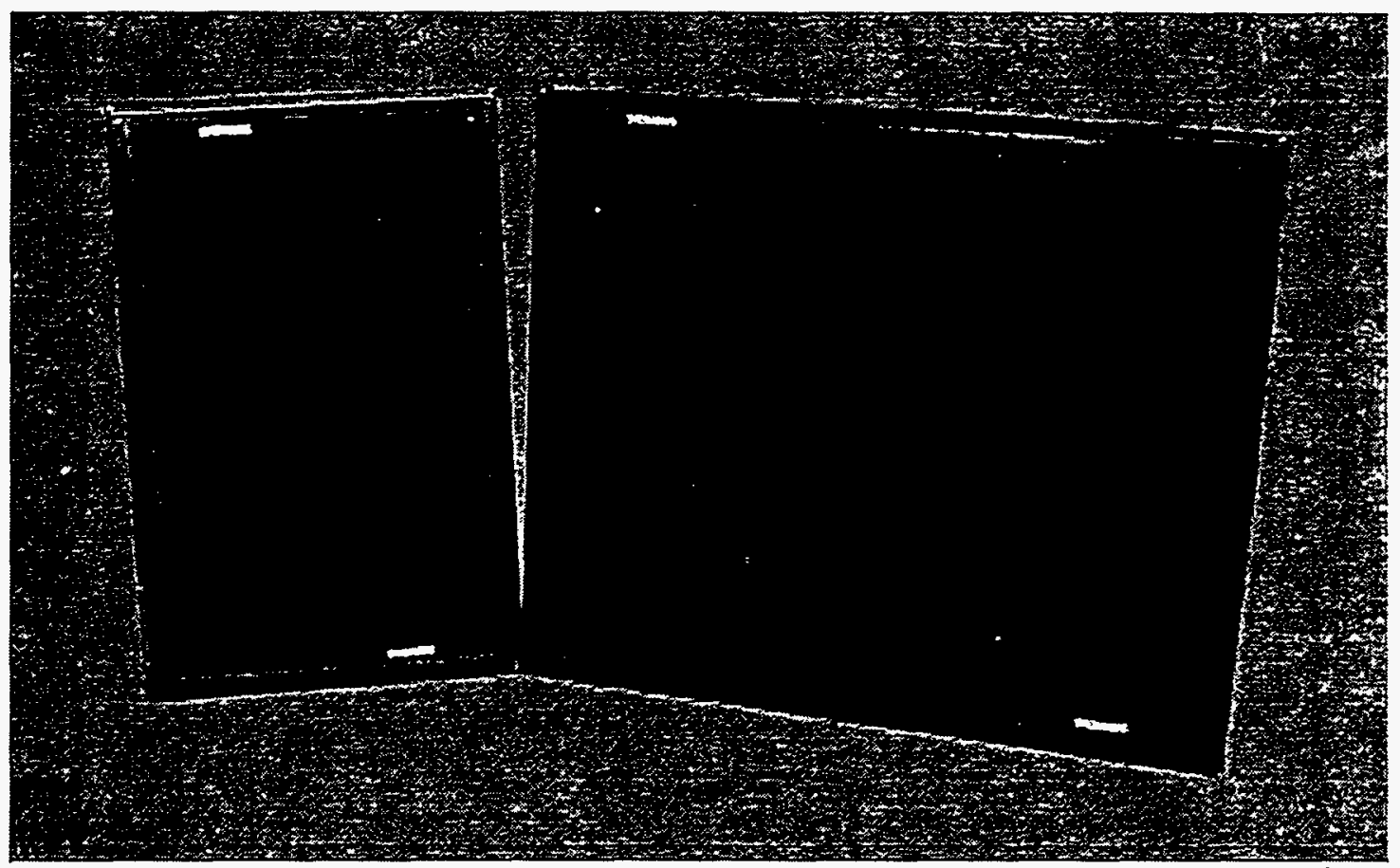

Figure 2. Introductory CIS-based 5 and $10 \mathrm{~W}$ products.

During this subcontract phase SSI delivered a set of modules to NREL for a $1 \mathrm{~kW}$ array replacing an existing $1 \mathrm{~kW}$ array based on an older absorber formation technology. Absorbers for the previous array were $\mathrm{Cu}(\mathrm{In}, \mathrm{Ga}) \mathrm{Se}_{2}$ whereas absorbers for the present array are a graded absorber including sulfur; $\mathrm{Cu}(\mathrm{In}, \mathrm{Ga})(\mathrm{S}, \mathrm{Se})_{2}$. Module construction is similar to the ST5 and ST10 but with overall dimensions of approximately $1 \times 4 \mathrm{ft}$. The 28 -module array has an average module power of 39 watts. NREL reports stable performance and an average efficiency without correction to standard conditions of $7.7 \%$ which is a significant improvement over the $5.2 \%$ average efficiency for the previous CIS array. Correcting the measurements made under prevailing conditions to standard conditions yields an unprecedented average efficiency of greater than nine percent. The computed temperature coefficient for power is $-0.5 \% / \mathrm{C}$ which is also a significant improvement over the $-0.8 \% / \mathrm{C}$ temperature coefficient measured for the previous array. These demonstrated improvements in efficiency and temperature coefficient for the newer technology are significant advancements in CIS-based technology for large area modules.

A module identical to the modules of this array but with an aperture physically masked to exclude both bus ribbons was confirmed by NREL to produce 40.6 watts for a new confirmed world-record efficiency of 11.1 percent on $3665 \mathrm{~cm} 2$ (Figure 3). This demonstrated efficiency also exceeds the $1995 \mathrm{DOE}$ efficiency goal for CIS prototype modules and exceeds the year $2000 \mathrm{DOE}$ efficiency goals for amorphous silicon and cadmium telluride technologies (9). 


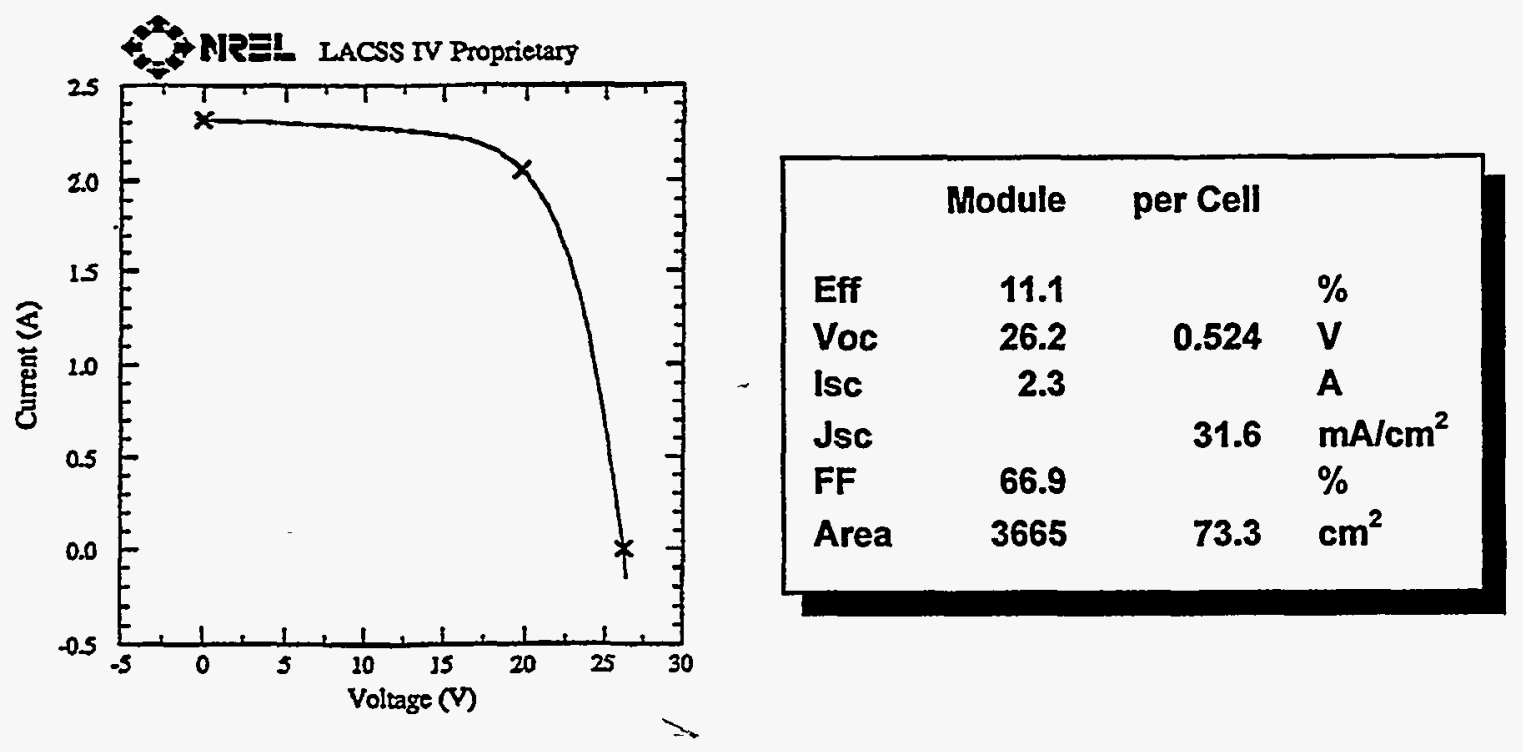

Figure 3. NREL confirmed world-record large area thin film module.

\section{Acknowledgments}

The author gratefully acknowledges NREL for valuable measurement and analytical assistance. This work was supported by the National Renewable Energy Laboratory, Golden, CO, under subcontract No. ZAF-514142-03 of the Thin Film Photovoltaic Partnership Program through the U. S. Department of Energy.

\section{References}

1. Ken Zweibel, Harin S. Ullal, Bolko von Roedern, "Progress and Issues in Polycrystalline Thin-Film PV Technologies," 25th IEEE Photovoltaic Specialist Conference, 1996, pp. 745-750.

2. R. Gay, "Status and Prospects for CIS-Based Photovoltaics", 9th International Photovoltaic Science and Engineering Conference, Miyazaki, Japan, November 1996. Published in Solar Energy Materials and Solar Cells, 47 (1997) pp.19-26.

3. H.S. Ullal, K. Zweibel, B.G. von Roedern, "Current Status of Polycrystalline Thin-Film PV Technologies", 26th IEEE Photovoltaic Specialists Conference, Sept. 29-Oct. 3, 1997, Anaheim, Califormia, NREL/CP-520-22922, UC Category: 1250.

4. H. A. Aulich, "Advances in Thin Film PV-Technologies," 13th European PVSEC 1995, pp. 1441-1444.

5. D. E. Tarrant and R. R. Gay, "Research on High-Efficiency, Large-Area CuInSe2-Based Thin-Film Modules, . Final Subcontract Report,"NREL/TP-413-8121 (1995).

6. D. Tarrant, R. Gay, "Thin-Film Photovoltaic Partnership Program - CIS-based thin film PV technology. Phase 1 annual technical report, September 1995-September 1996", (Apr 1997) NREL report DE97000236.

7. R. Gay, J. Bauer, R. Dearmore, M. Dietrich, G. Fernandez, O. Frausto, C. Fredric, C. Jensen, A. Ramos, J. Schmitzberger, D. Tarrant, R. Wieting, D. Willett, "Thin-Film Photovoltaic Partnership Program CIS-Based Thin Film PV Technology”, NREL Photovoltaic Program, FY 1996 Annual Report, August 1997, NREL/BK-21021966, pp. 243-246.

8. D. Tarrant, J. Bauer, R. Dearmore, M. Dietrich, G. Fernandez, O. Frausto, C. Fredric, C. Jensen, A. Ramos, J. Schmitzberger, R. Wieting, D. Willett, R. Gay, "Progress in CIS-Based Module Development", 14th NREL/SNL Photovoltaics Program Review, Lakewood, Colorado, November 1996. Published in AIP Conference Proceedings 394, (1997) pp. 143-152.

9. "Photovoltaics the Power of Choice, The National Photovoltaics Program Plan for 1996 - 2000", January 1996, DOE/GO-10096-017 • DE95000214. 
Title: CdTe Polycrystalline Thin-Film Photovoltaic Module Development

Organization: Solar Cells, Inc.

1702 N. Westwood Ave.

Toledo, $\mathrm{OH} 43607$

Contributors: G.L. Dorer, R.C. Powell, R.A. Sasala, U. Jayamaha, N. Reiter, M. Steele, G. Rich, T. Kahle, S. Cox

Date:

FY 1997

\section{Summary}

The research program at Solar Cells, Inc. aims at developing a high throughput module production process based on $\mathrm{CdS} / \mathrm{CdTe}$ thin-films. The approach focuses solely on processes capable of scaling to production rates used in modern glass plant operations. FY1997 saw the transfer from research to pilot production of a major advance in our deposition process. We also gained knowledge about the rapid transfer of hot glass in and out of the vacuum deposition chamber, the use of large batch recrystallization processing, and the use of automotive glass handling techniques for module lamination. Basic research continued to develop high-throughput interconnection processes, stable high performance device structures, and methods for recycling and-recovery of end of life modules and process wastes.

\section{Deposition}

A new proprietary deposition system, utilizing SCI's VTD (Vapor Transport Deposition) process, was developed and transferred to pilot production. The semiconductor raw materials can now be introduced into the coating system on demand and thus the batch-load process has been eliminated. Moreover quality films are formed very rapidly which permits high line speeds and short deposition chambers. The pilot semiconductor coating system was upgraded with the new coating components, rapid-cycling load locks, and an automated glass-handling controller. Quality bi-layer films ( $>3 \mu \mathrm{m}$ thick) have been formed on substrates moving at line speeds up to $200 \mathrm{~cm} / \mathrm{min}$ within a $60 \mathrm{~cm}$ section. Furthermore, the system allows unprecedented control over the extent and uniformity of the coating, which will lead to high material utilization and device performance consistency. Figure 1 shows the cross web performance data for cells made on films deposited by the new method.

Figure 1: SC Wetwood 1.2kWAray Peformane 24Cridormected CoTeMsales

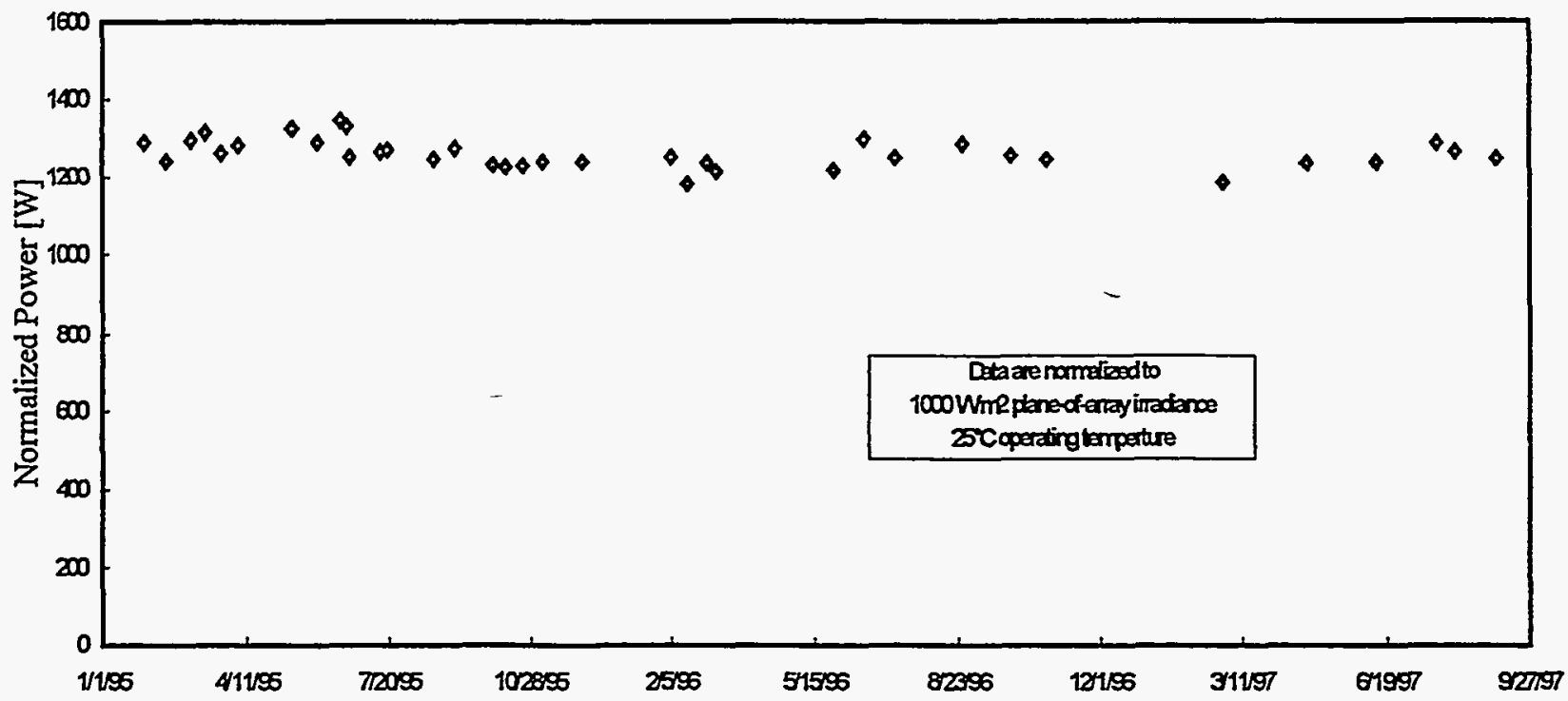




\section{Semiconductor Recrystallization}

To increase throughput on one of the most time consuming steps in our process, we installed a readilyavailable, convectively-heated batch oven for the recrystallization anneal. With modest effort the singleplate process was transferred to the multi-plate batch process. Thus, we have increased the throughput of this bottleneck by more than a factor of 20 .

\section{Vacuum Transfer}

Vacuum deposition is currently the most reliable method for rapid semiconductor film formation. As such methods to rapidly transfer hot glass in and out of vacuum are required. For very high throughputs, vacuum valves are problematic. The development of close-clearance slits was continued during FY1997. While module vibrations caused by Mach-1 air speeds were eliminated, thermal shock of partially solidified glass transiting the vacuum-atmosphere boundary proved tricky. Changes in the semiconductor coating apparatus opened new avenues for attacking the vacuum transition requirement. A new and greatly simplified system has now successfully passed initial tests and will be introduced in FY1998.

\section{Interconnects}

Linear patterning using laser ablation is the current known technology. This method employs over 200 meters of linear laser scribing per 50-watt module. In FY1997 several new developments in multi-beam laser technology were tested. Simplified scribing systems will result from this work. In addition, continued progress on our dot-matrix process was made. This system utilizes an additional dielectric plus metal bilayer to reduce resistive losses in the $\mathrm{SnO}_{2}$ layer. Several new formation sequences were developed and full module prototypes fabricated. These processes are now under cost evaluation.

\section{Encapsulation}

To date photovoltaic manufacturers have not made use of automotive windshield lamination technology. In FY1997 such equipment was installed at SCI and testing begun. Initial results look promising as bubble formation has been reduced and electrical isolation has been excellent. Implementation on the pilot production line is scheduled for FY1998.

\section{Performance}

Increased cell performance has centered on the use of thin $\mathrm{CdS}$ with low conductivity $\mathrm{SnO}_{2}$ buffer layers and the use of proprietary interfacial layers at the back contact. Again all methods and device structures currently under consideration must be capable of scaling to high throughputs and demonstrate stability in accelerated life tests.

\section{Accelerated Life Testing.}

Solar Cells, Inc. continued to lead the National CdTe team with regard to accelerated life testing. Another round of multi-stress tests was completed using several device formation recipes from Solar Cells, Inc. and others. In addition continued monitoring of outdoor arrays and test modules was continued. Figure 2 shows the performance data from SCI's test array in Toledo $\mathrm{OH}$. The recipe that has been used to fabricate all outdoor modules to date is performing well in the field but shows some weakness under some accelerated test conditions. However, alternative experimental structures are easily surviving all stresses expected in the field. 


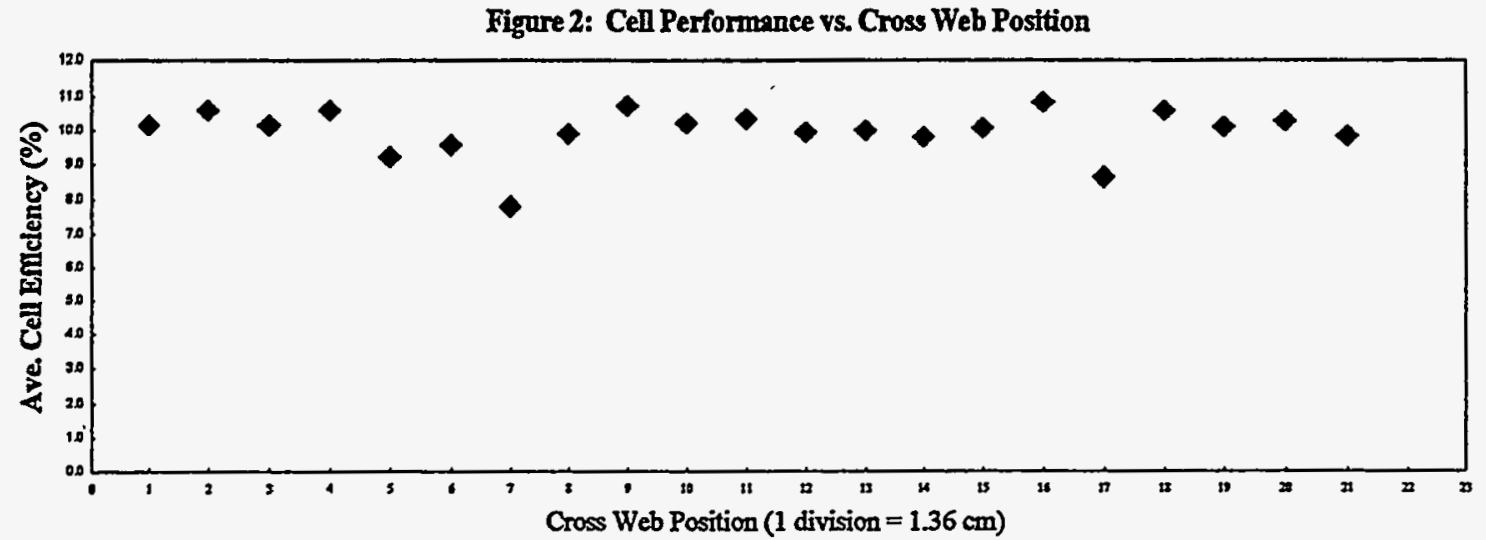

\section{Recycling}

Work funded through a Small Business Innovative Research grant has successfully built a commercially viable system to handle Cd-contaminated production scrap and waste as well as end-of-life modules. Solar Cells, Inc. is taking a pro-active cradle-to-grave policy for our modules since we utilize the heavy metal cadmium. The basic recycling system involves crushing modules in a hammer mill, etching the semiconductor films from the glass in a barrel finisher, and precipitation of metals from solution. This system has already eliminated the cost of landfilling contaminated glass. Furthermore, a full recycle after return strategy is estimated to cost $<\$ 0.02 /$ watt.

\section{References.}

1. R.A. Sasala, R.C. Powell, G.L. Dorer, and N. Reiter (1996), "Recent Progress in CdTe Solar Cell Research at SCI." AIP Conference Proceedings, Vol. 394, C.E. Witt, M.Al Jassim, J.M. Gee, eds. Woodbury, NY: American Institute of Physics; pp. 171-186.

2. D.W. Sandwisch (1996), "Progress in High-Throughput Manufacturing of Thin-Film CdTe Modules." AIP Conference Proceedings, Vol. 394, C.E. Witt, M.Al Jassim, J.M. Gee, eds. Woodbury, NY: American Institute of Physics; pp. 425-431. 

Title:

Organization:

Contributors:
Amorphous Silicon Research

Solarex, a Business Unit of Amoco/Enron Solar, Newtown, PA

R. Arya, Program Manager, M. Bennett, D. Carlson, L. Chen, G. Ganguly, G. Lin, R. Middya, K. Jansen, F. Willing, J. Newton

\section{Introduction}

Solarex has been performing research in the area of amorphous silicon (a-Si) photovoltaics for about 15 years and started manufacturing single-junction a-Si solar cells for consumer applications in 1984. In 1997, Solarex started the commercial production of large-area $\left(8.6 \mathrm{ft}^{2}\right)$ multijunction a-Si PV modules in Toano, Virginia. Over the last year, the Solarex a-Si research program has focused on increasing performance of large-area tandem modules, improving production throughput, increasing gas feedstock utilization and understanding the light-induced degradation of a-Si solar cells. The major objectives have been to demonstrate a stabilized conversion efficiency of $8 \%$ in a large-area $\left(8.6 \mathrm{ft}^{2}\right)$ tandem module, and to scale up the multijunction technology from $1 \mathrm{ft}^{2}$ to $8.6 \mathrm{ft}^{2}$ and transfer the technology to the plant in Virginia.

\section{Mid-Bandgap Alloy Research}

The plant in Virginia is producing PV modules with a tandem (a-Si/a-SiGe) structure where the a-Si can be considered a mid-bandgap amorphous alloy of silicon and hydrogen (the optical gap is $\sim 1.75 \mathrm{eV}$ ). We have been investigating the effects of strong electric fields and intense illumination on undoped a-Si with the goal of trying to understand and reduce the light-induced degradation. We have also been investigating the utilization of feedstock gases and the interface between the a-Si and the tin oxide.

\section{Field-Enhanced Annealing of Light-Induced Degradation}

In earlier work [1], we showed that the light-induced degradation of single-junction p-i-n cells could be reversed relatively rapidly at moderate temperatures by the application of a strong reverse bias while the cell was exposed to intense illumination. In more recent studies, we have observed that the annealing rate of the light-induced degradation in the dark at temperatures in the range of 60 to $100{ }^{\circ} \mathrm{C}$ is increased significantly by a strong reverse bias. The $\mathrm{p}-\mathrm{i}-\mathrm{n}$ cells were degraded by exposing them to 50 suns illumination from a focused Xe arc lamp for 30 minutes at $60^{\circ} \mathrm{C}$ while at open-circuit. Under these conditions, the efficiencies would typically fall from about $8.3 \%$ to $6.5 \%$, and the fill factors would fall from about 0.66 to 0.56 . In one series of experiments, we heated a degraded cell to $100{ }^{\circ} \mathrm{C}$ and then monitored the change in performance with time while the cell was exposed to different reverse biases in the dark. As shown in Fig. 1, the application of a strong reverse bias at $100^{\circ} \mathrm{C}$ in the dark caused a significant increase in the rate of recovery of the fill factor. As shown in Fig. 2, the recovery of the performance occurs rapidly even when a degraded cell under reverse bias is exposed to intense illumination. The activation energy for annealing in the dark under open-circuit conditions $(\sim 1.34 \mathrm{eV})$ is close to that observed for hydrogen diffusion [2]. The application of a strong reverse bias lowers the activation energy to $\sim 1.16 \mathrm{eV}$ while the additional application of intense illumination lowers the activation energy to $\sim 0.77 \mathrm{eV}$. The activation energies in the present case are somewhat lower than those observed for hydrogen diffusion in undoped a-Si both in the dark and under intense illumination, but the barriers may be somewhat lower if the hydrogen motion is localized within a metastable defect complex. We hypothesize that both electronic and protonic events are involved in the creation and fieldinduced reversal of the metastable defects. The metastable complex may consist of a Si-H bond near a 
weak Si-Si bond since these complexes are very likely to occur near the internal surfaces of microvoids as well as in bulk a-Si:H material. The weak Si-Si bond is very likely to trap photogenerated carriers while it is also possible for holes to be temporarily localized in the vicinity of the Si-H bond.

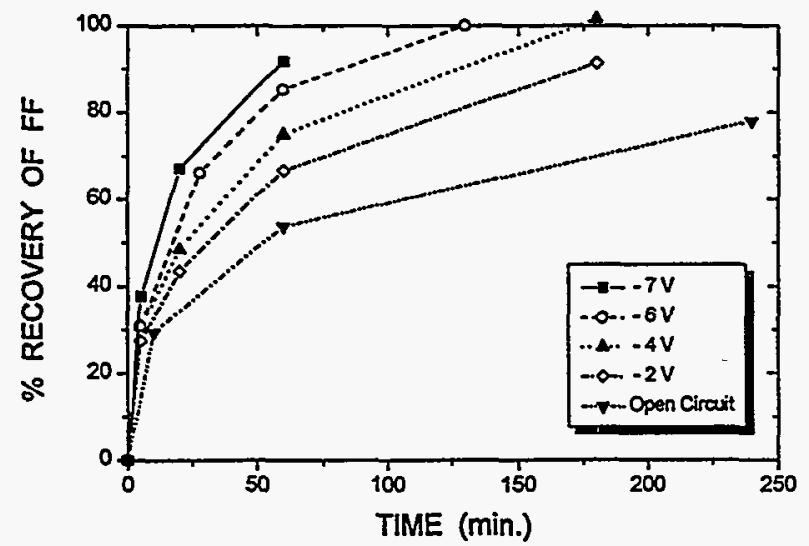

Figure 1. The percentage recovery of the fill factor for a degraded $\mathrm{p}-\mathrm{i}-\mathrm{n}$ cell annealed at $100^{\circ} \mathrm{C}$ in the dark under various bias conditions

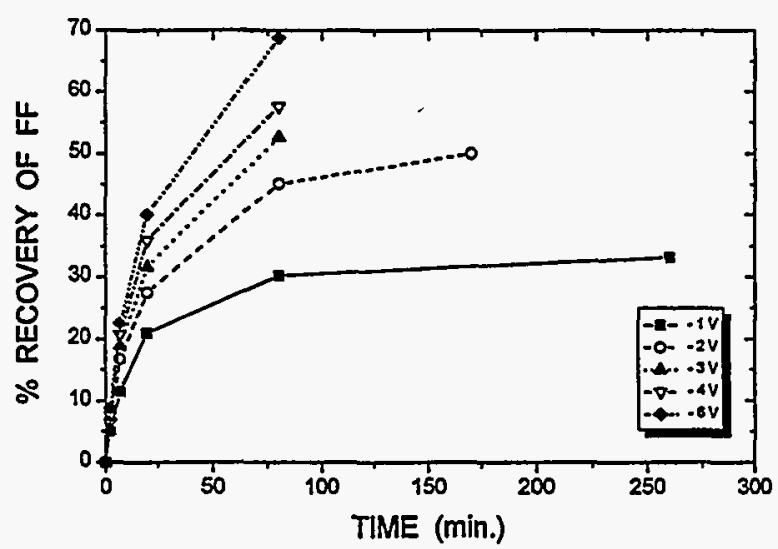

Figure 2. The percentage recovery of the fill factor for a degraded $p-i-n$ cell exposed to 50 suns at $70^{\circ} \mathrm{C}$ under various bias conditions.

The charged defect complex might then restructure into a metastable state as the proton moves to the trapped electron and breaks the weak Si-Si bond. The metastable defect complex could also trap holes, and then the defect might return to the ground state via field-assisted proton motion. 0

\section{Utilization of Feedstock Gases}

Using a systematic variation of discharge parameters, we found that we could reduce both the silane and germane gas flows by $30 \%$ without adversely affecting the performance of a-Si and a-SiGe singlejunction solar cells or the performance of a-Si/a-SiGe tandem cells. Stabilized conversion efficiencies of $\sim 7.5-7.8 \%$ have been demonstrated with these reduced flow rates using commercial tin oxide coated glass in both $1 \mathrm{ft}^{2}$ and $4 \mathrm{ft}^{2}$ deposition systems, and the process will be transferred to the Virginia plant in 1998.

\section{Study of the Amorphous Silicon / Tin Oxide Interface}

We have observed that contamination at the interface between the a-Si p-layer and the tin oxide coated glass strongly affects the performance of amorphous silicon solar cells. Commercially available tin oxide coated glass is usually coated with a protective layer such as adipic or malic acid. We investigated several methods of cleaning commercial tin oxide coated glass such as ultrasonic cleaning, argon sputtering, alcohol rinse treatments, and exposure to high temperatures $\left(\sim 540^{\circ} \mathrm{C}\right)$. As shown in Table 1 , both ultrasonic cleaning and Ar ion sputtering improved the performance of single-junction cells as compared to those cleaned with a Billco washer. Ar sputtering also appeared to reduce the amount of shunting; this might be due to the removal of sharp peaks on the textured surface of the tin oxide by the sputtering action. We also observed that the stabilized efficiency was about $22 \%$ higher for devices where the substrates were cleaned using the ultrasonic bath as compared to those cleaned with the Billco washer. Since the boiling point of adipic acid is $265^{\circ} \mathrm{C}$, we tried to remove the acid by baking the tin oxide coated glass in a furnace at a temperature of 400 to $500^{\circ} \mathrm{C}$. However, the performance of the cells was still not as good as cells cleaned in an ultrasonic bath, indicating that some surface residue still exists after the high temperature treatment. In general, similar results were obtained when we used the various 
cleaning methods with tandem devices, but the effects were somewhat smaller since tandem devices are less sensitive to changes in contact resistance.

Table 1. Single-Junction Solar Cell Performance for Different Cleaning Processes

\begin{tabular}{|l|l|l|l|}
\hline Cleaning Processes & $\mathbf{V}_{\text {oc }}$ (volts) & Fill Factor & $\mathbf{R}_{\mathbf{S}}\left(\Omega-\mathrm{cm}^{2}\right)$ \\
\hline Normal Billco Process & $0.88-0.90$ & $0.63-0.67$ & $7.6-9.0$ \\
\hline Ultrasonic Bath & $0.91-0.93$ & $0.69-0.72$ & $6.1-7.2$ \\
\hline Ar Ion Sputtering & $0.91-0.92$ & $0.70-0.71$ & $5.6-6.8$ \\
\hline
\end{tabular}

\section{Low-Bandgap Alloy Research}

The performance of the tandem devices depends critically on the quality of the a-SiGe alloy in the back junction. We have been investigating the growth of a-SiGe using helium dilution and also examining the field-enhanced recovery of degraded a-SiGe cells.

\section{The Field-Enhanced Recovery of Degraded a-SiGe Cells}

As part of an investigation of light-induced degradation in a-SiGe cells, we examined the effects of a strong reverse bias on the recovery of light-soaked a-SiGe single-junction cells. In the first set of experiments, we looked at the effects of a strong reverse bias on the annealing of degraded a-SiGe and

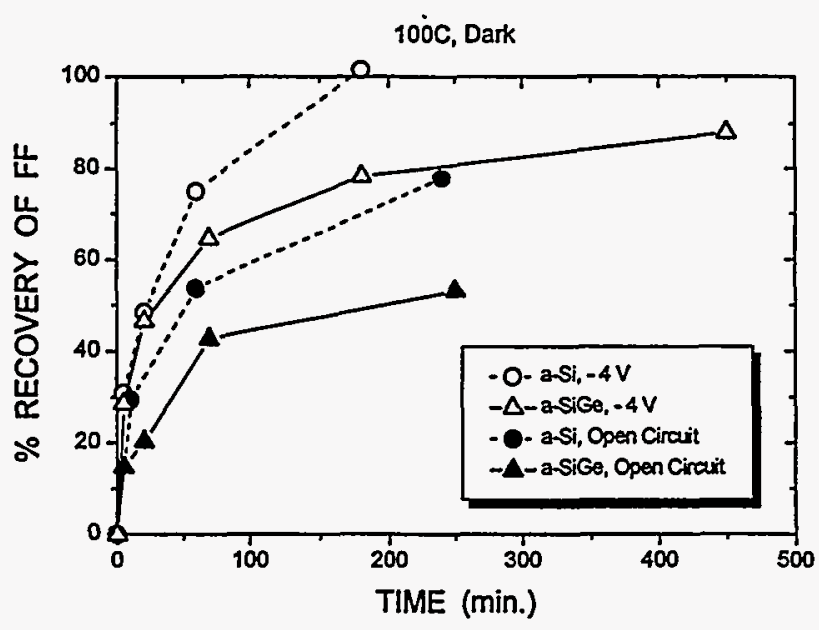

Figure 3. The recovery of the fill factor of degraded a-Si and a-SiGe cells as a function of annealing time at $100^{\circ} \mathrm{C}$ in the dark for different bias conditions. a- Si cells in the dark at $100^{\circ} \mathrm{C}$. The cells were initially degraded by exposing them to 50 suns for 30 minutes at $60^{\circ} \mathrm{C}$ under opencircuit conditions. The cells were then annealed in the dark at $100^{\circ} \mathrm{C}$ under opencircuit conditions and at a reverse bias of -4 V. As shown in Fig. 3, a reverse bias accelerates the annealing process in both a-SiGe and a-Si cells, but the recovery process both at open circuit and at $-4 \mathrm{~V}$ proceeds more slowly in the a-SiGe cells. The internal electric fields were actually stronger for the a-SiGe cells since the i-layers were $\sim 210 \mathrm{~nm}$ thick vs. $\sim 260 \mathrm{~nm}$ for the a-Si cells. While the recovery process is accelerated by exposing the reverse-biased cells to intense illumination, the recovery is still slower for the a-SiGe cells.

\section{Multijunction Devices and Modules}

We have been fabricating tandem devices and modules using both helium dilution and also using a fast tunnel junction process.

\section{Fabrication of Tandem Devices Using Helium Dilution}

We have investigated the possibility of using helium dilution to fabricate high quality tandem devices and have found that both the initial and stabilized performance are comparable to those made using hydrogen dilution. As shown in Table 1, the initial conversion efficiencies of tandem devices made on 
commercial tin oxide coated glass with either helium or hydrogen dilution are close to $9 \%$. Moreover, the deposition rates that are $\sim 60 \%$ higher using helium dilution, and the utilization of both silane and germane is somewhat better than with hydrogen dilution.

Table 2. PV Parameters for Tandem Devices Made Using Helium and Hydrogen Dilution

\begin{tabular}{|c|c|c|c|c|c|c|c|}
\hline Sample \# & Dilution & $\mathbf{V}_{\mathbf{~ o c}}$ & $\mathbf{F F}$ & $\mathbf{J}_{\text {sc( }}(\mathbf{Q E})_{\mathbf{1}}$ & $\mathbf{J}_{\text {Sc( }}(\mathbf{Q E})_{\mathbf{2}}$ & $\mathbf{Q E}(\mathbf{8 0 0})$ & $\mathbf{E f f .}(\mathbf{\%})$ \\
\hline H8016-3MB & $10: 1$ Helium & 1.531 & 0.701 & 8.55 & 8.41 & 0.239 & 9.11 \\
\hline H8016-3MT & $10: 1$ Helium & 1.521 & 0.703 & 8.51 & 8.57 & 0.242 & 9.13 \\
\hline H8019-1MT & $10: 1 \mathrm{H}_{2}$ & 1.514 & 0.702 & 8.61 & 8.32 & 0.228 & 9.00 \\
\hline H8019-1MB & $10: 1 \mathrm{H}_{2}$ & 1.511 & 0.702 & 8.60 & 8.28 & 0.224 & 8.95 \\
\hline
\end{tabular}

\section{Development of a Fast Tunnel Junction Process}

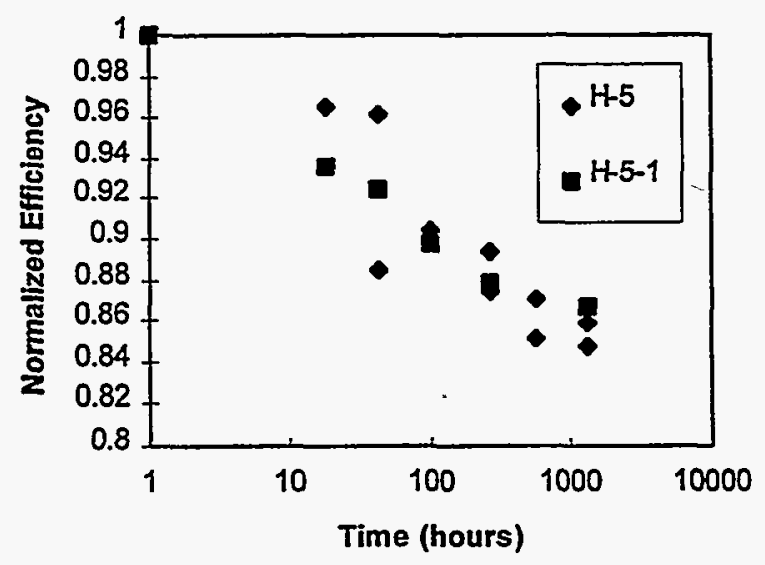

Figure 4. Light-induced degradation of tandem cells made using the normal process $(\mathrm{H}-5)$ and a, fast tunnel junction process $(\mathrm{H}-5-1)$.
In order to reduce the total processing time, we have developed a faster tunnel-junction recipe $(\mathrm{H}-$ 5-1) that reduces the total deposition time by about 6 minute. The initial performance of the cells fabricated using these two recipes is comparable, and the light-induced degradation is comparable (see Fig. 4). The performance of 1 $\mathrm{ft}^{2}$ modules made on commercial tin oxide coated glass was also similar with initial conversion efficiencies ranging from 8.3 to $8.5 \%$. Moreover, good uniformity has been obtained over a $4 \mathrm{ft}^{2}$ deposition zone using the fast tunnel junction process, and we plan to transfer this process to the plant in Virginia in the next few months.

\section{Large-Area Tandem Modules}

The tandem junction process has been scaled up to $8.6 \mathrm{ft}^{2}$ modules using equipment both in Newtown, $\mathrm{PA}$, and in the plant in Virginia. Production runs have produced modules with initial output power in the range of 53 to 57 watts. Accelerated environmental tests [3] indicate that the stabilized output of these modules will be in the 45 to 49 watt range.

\section{References}

1. D. E. Carlson and K. Rajan, "The reversal of light-induced degradation in amorphous silicon solar cells by an electric field," Appl. Phys. Lett. 70 (1997) pp. 2168-2170.

2. D. E. Carlson and C. Magee, "A SIMS analysis of deuterium diffusion in hydrogenated amorphous silicon," Appl. Phys. Lett. 33 (1978) pp. 81-83.

3. D. E. Carlson, R. R. Arya, M. Bennett, L.-F. Chen, K. Jansen, Y.-L. Li, J. Newton, K. Rajan, R. Romero, D.Talenti, E. Twesme, F. Willing and L. Yang, "Commercialization of multijunction amorphous silicon modules", Proc. 25th IEEE Photovoltaic Specialists Conf. (1996) pp. 1023-1028. 
Title:

Organization:

Contributors:
Research on Wide Bandgap, Amorphous Silicon Based Solar Cells.

Syracuse University, Department of Physics, Syracuse, New York 13244.

Eric A. Schiff, principal investigator; Lin Jiang, Jonghun Lyou, Indra Nurdjaja, Prasanna Rao.

In 1997 our research has focused on the following topics:

- Electroabsorption studies of a-Si based solar cells. The studies have several purposes: (i) to probe the built-in potential of a-Si:H based solar cells, (ii) to explore the recent reports of a strong effect of light-soaking upon polarized electroabsorption, and (iii) to exploit electroabsorption as a tool for bandgap and bandgap profile measurement. In this year's report we describe work on the second topic: light-soaking effects and electroabsorption. Using what we believe is an improved measurement technique, we do not find a significant effect of light-soaking upon polarized electroabsorption; this contrasts with earlier reports. We also find little effect of light-soaking upon the built-in potential we measure in these same cells.

- Electron and hole mobility measurements in a-Si based materials. Our work has two thrusts. We are surveying the mobility properties of novel a-Si:H based materials (hot-wire, triode-deposited, etc.) to check for the achievement of exceptional mobility properties, and we are improving our quantitative techniques to help in assessing incremental improvements in a-Si:H based materials. Perhaps the most important result from this year's work is that we have confirmed the very large hole mobilities in a-Si:H specimens prepared by G. Ganguly at Electrotechnical Laboratory.

Our research contract was acknowledged in the following publications in 1997, which may be consulted for information on our other research topics.

"Polarized Electroabsorption Spectra and Light Soaking of Solar Cells based on Hydrogenated Amorphous Silicon," L. Jiang, Q. Wang, E. A. Schiff, S. Guha, and J. Yang, Appl. Phys. Lett. in press (1998).

"Photocapacitance and Hole Drift Mobility Measurements in Hydrogenated Amorphous Silicon (aSi:H)," I. Nurdjaja and E. A. Schiff, in Amorphous and Microcrystalline Silicon Technology - 1997, edited by S. Wagner, et al (Materials Research Society Symposium Proceedings Vol. 467), p. 723.

"Electroabsorption Spectra of Hydrogendated Amorphous Silicon and Microcrystalline Silicon," L. Jiang, E. A. Schiff, F. Finger, P. Hapke, S. Koynov, R. Schwarz, N. Wyrsch, A. Shah, J. Yang, and S. Guha, in Amorphous and Microcrystalline Silicon Technology - 1997, edited by S. Wagner, et al (Materials Research Society Symposium Proceedings Vol. 467), p. 295.

\section{Electroabsorption and Built-in Potentials}

About ten years ago Weiser, Dersch, and Thomas reported the polarized electroabsorption effect for the interband optical absorption of amorphous selenium and hydrogenated amorphous silicon (2-Si:H) [1]. In particular they found that the electroabsorption (EA) spectrum ( the electric-field dependent portion of the absorption itself) depended significantly upon the angle between the polarization vector of the optical beam and the external electric field applied to the material. They interpreted the effect in terms of the nature of electronic states near mobility edges (the level energy separating localized and extended states near the conduction or valence bandedges). 
Other scientists have significantly. extended this initial work of Weiser, Dersch, and Thomas. Okamoto, et al [2] and Tsutsumi, et al [3] proposed that the polarized electroabsorption effect is well correlated with the fundamental mobilities of electrons and holes. Most recently Shimizu, et al [4] and also Hata, et al [5] reported that the polarization dependence of electroabsorption is nearly doubled in magnitude by "light-soaking" of a-Si:H, which is a new type of Staebler-Wronski effect [6]. These measurements suggest the highly significant conclusion that light-soaking substantially changes the electronic structure of a-Si:H - especially in the mobility-edge regions. The preponderance of work on the Staebler-Wronski effect has emphasized point defect generation during light soaking as opposed to more broadly based changes to the material itself.

The electroabsorption measurements cited above were performed on simple thin-film specimens. The external electric potential difference was applied between two electrodes separated by a small gap; the optical beam passes through this gap. One potential difficulty with this "coplanar electrode" geometry is that the measurements are affected by interfacial fields and by non-uniformity of the applied field. In particular, Mescheder and Weiser have documented a strong nonuniformity of the electroabsorption signal across the electrode gap for a-Si:H [7], reporting that the signal close to the electrodes was about one order of magnitude higher than the signal from the middle of the gap. While this nonuniformity clearly presents a quantitative difficulty, several authors have argued that the electroabsorption spectra, and the relative magnitudes for the polarization effect, should be unaffected by it.

We have performed electroabsorption measurements using a "sandwich" electrode geometry which, we believe, greatly reduces these uncertainties. We confirm the existence of the polarized electroabsorption effect, and indeed we conclude that the polarization dependence is substantially larger, and hence of potentially greater interest, than previously reported. On the other hand, we did not reproduce the lightsoaking effect found in the coplanar geometry. An interesting byproduct of the current measurements is an estimate of the change in the built-in potential [8] of our a-Si:H based solar cells due to light-soaking; this effect was also unmeasurably small $(<0.03 \mathrm{~V}$ change).

In Fig. 1 we show the experimental geometry we used. The internal electric field is vertical in this drawing. To explore polarized electroabsorption, we need a component of the polarization vector of the incident illumination to be parallel to this internal field; as illustrated in Fig. 1, this can be achieved by using grazing incidence illumination and $p$-polarized light. The disadvantage of this measurement approach is that the parallel component is relatively small; on the other hand, the internal electric fields are much simpler to understand than are the 2-dimensional electric fields involved in studying coplanar electrode structures.

A full discussion of this experiment is beyond the scale of this report. In work with coplanar electrode structures, Shimizu, et al [4] and Hata, et al [5] found that the ratio of the electroabsorption measured parallel and perpendicular to the electric field more than doubled during the initial stages of light-soaking ( $15 \mathrm{~m}$ at $30 \mathrm{~mW} / \mathrm{cm}^{2}$ illumination intensity). In Fig. 2 we present our "sandwich" electrode measurements under similar conditions for two samples, one prepared at United Solar sSystems Corp., and the second prepared at Solarex. We did not find any significant effect of light-soaking on the ratio of electroabsorption in either of these samples or in others we have measured.

We do not fully understand the reasons for the different conclusions in the two geometries. We speculate that a full two-dimensional analysis of the electric fields, incorporating field components normal as well as parallel to the thin-film surface, will be required to fully account for all the measurements with coplanar electrodes. More specifically, we propose that the coplanar measurements are sensitive to interface fields, and indeed that electroabsorption measurements may yet prove to be a quantitative probe of these fields. In this case the light-soaking effect observed in this geometry would primarily reflect 
changes in interface conditions with light-soaking, while the fundamental electroabsorption spectra measured with sandwich electrodes are nearly unchanged.

\section{Hole Drift Mobilities in Triode Deposited a-Si:H}

In Fig. 3 we show the temperature-dependence of the drift mobility $\mu_{D}=L / E t_{T}$ for holes in conventional plasma-deposited a Si:H (lower regression line) and for two specimens of "triode" deposited a Si:H from Electrotechnical Laboratory. As required for this type of comparison, all measurements correspond to the same value of displacement-field ratio $L / E \approx 2 \times 10^{-8} \mathrm{~cm}^{2} / \mathrm{V} ; L$ is the displacement of the carriers corresponding to the transit-time $t_{T}(L=d / 2$ for the present measurements), and $E$ is the electric field. The triode samples correspond to the upper regression curves, and have mobilities near room temperature nearly 100 times higher than for conventional a-Si:H. The open symbols are previously reported hole mobilities from Ganguly, et al [9], which are consistent with the present measurements.

1. G. Weiser, U. Dersch, and P. Thomas, Philos. Mag. 57, 721 (1988).

2. H. Okamoto, K. Hattori, and Y. Hamakawa, J. Non-Cryst. Solids 137\&138, 627 (1991) and 164-166, 893 (1993).

3. Y. Tsutsumi, H. Okamoto, K. Hattori, and Y. Hamakawa, Philos. Mag. B 69, 253(1994)

4. K. Shimizu, T. Shiba, T. Tabuchi, and H. Okamoto, Jpn. J. Phys. 36, 29 (1997)

5. N. Hata, T. Kamei, H. Okamoto, and A. Matsuda, Amorphous and Microcrystalline Silicon TechnoIogy-1997 (Pittsburgh, 1997) in press.

6. D. L. Staebler and C. R. Wronski, Appl. Phys. Lett. 31292 (1977)

7. U. Mescheder and G. Weiser, J. Non-Cryst. Solids 77, 571 (1985)

8. L. Jiang, Q. Wang, E. A. Schiff, S. Guha, J. Yang, X. Deng, Appl. Phys. Lett. 69, 3063 (1996)

9. G. Ganguly, I. Sakata, and A. Matsuda, J. Non-Cryst. Solids 198-200, 300 (1996).

10. Q. Gu, Q. Wang, E. A. Schiff, Y.-M. Li, and C. T. Malone, J. Appl. Phys. 76, 2310 (1994) and references therein.
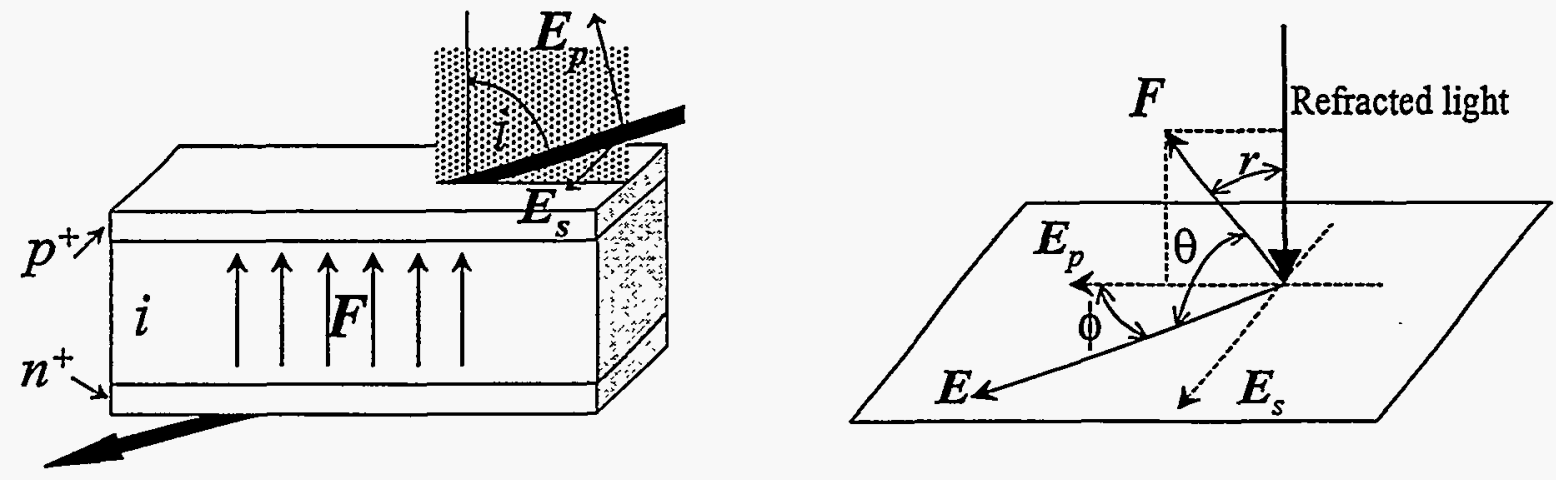

Fig. 1 Illustration of grazing angle measurements of polarized electroabsorption. (left) The incident beam (shaded arrow) is refracted and transmitted as it passes through a $p-i-n$ solar cell; $F$ denotes the field, $E_{\mathrm{p}}$ and $E_{\mathrm{s}}$ denote the polarizations. (right) Definitions of various angles involved in analyzing the experiment: the refraction angle $r$, the polarization angle $\phi$, and the angle $\theta$ between the external and polarization fields. 


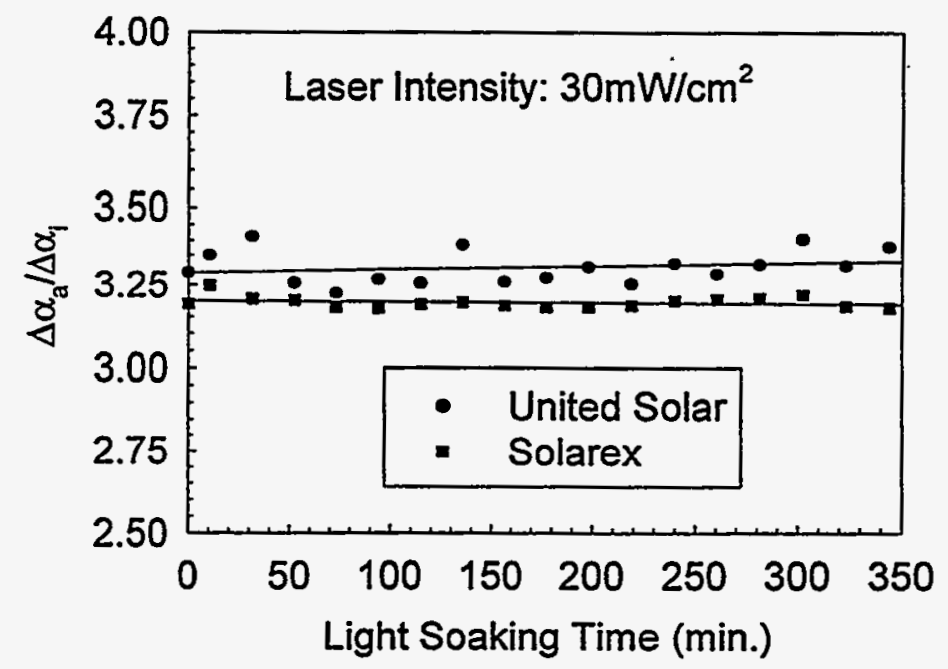

Fig. 2: Anisotropic/isotropic polarization ratio for electroabsorption measured as a function of the duration of light-soaking. Measurements are shown for two a-Si:H based pin solar cells from different laboratories.

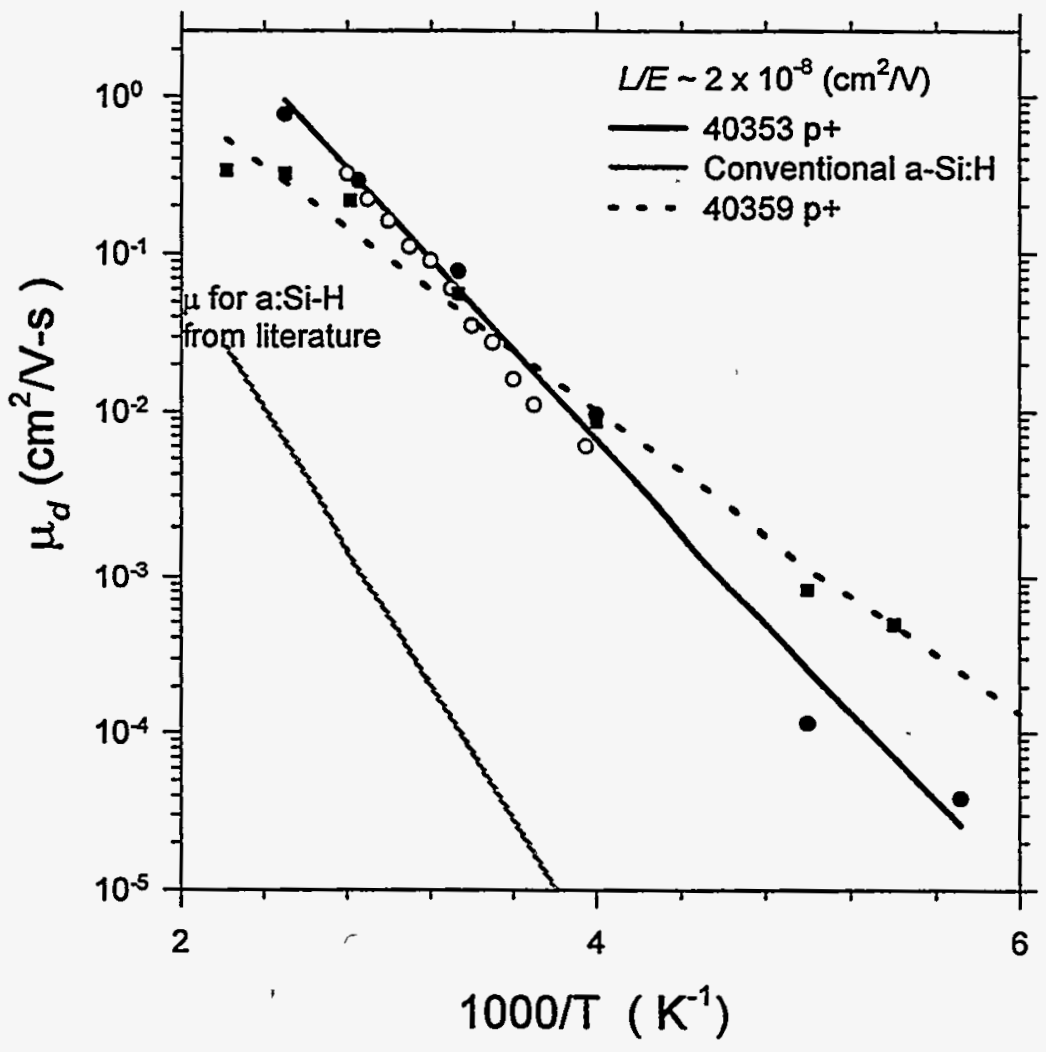

Fig. 3: Hole drift mobility for conventional plasma-deposited a Si:H [10] and for "triode" plasma deposited a Si:H from Electrotechnical Laboratory. The upper two regression lines represent the triode-material; the open symbols represent prior measurements of Ganguly, et al [9]. 
Title:

Organization:

Contributors:
Thin Film Amorphous Silicon Alloy Research

United Solar Systems Corp.

Troy, Michigan 48084

S. Guha, principal investigator, E. Akkashian, A. Banerjee, E. Chen,

T. Glatfelter, M. Haag, G. Hammond, M. Hopson, N. Jackett, M. Lycette,

J. Noch, T. Palmer, S. Sugiyama, D. Wolf, J. Yang, and K. Younan.

\section{Objective}

The principal objective of this R\&D program is to expand, enhance and accelerate knowledge and capabilities for the development of high-performance, two-terminal multijunction hydrogenated amorphous silicon alloy cells and modules. The near-term goal of the program is to achieve $12 \%$ stable module efficiency by 1998 using the multijunction approach.

\section{Approach}

The major effort of this program is to develop high efficiency component cells and incorporate them in the triple-junction structure to obtain the highest stable efficiency. Efforts were directed toward improving the back reflector, component cells and the tunnel junctions. Scale-up issues for module fabrication were investigated and a new laser-processed design for module fabrication was developed.

\section{Status/Accomplishments}

- We carried out a series of experiments using discharge modulation at various pulsed-plasma intervals in order to study the effect of Si-particle incorporation on solar cell performance. We found no observable difference in cell performance under discharge modulated and standard conditions for the deposition regimes investigated.

- We conducted a series of temperature ramping experiments on samples prepared with high and low hydrogen dilutions in order to study the effect of hydrogen effusion on solar cell performance. We found that hydrogen effusion in highly diluted samples resulted in little change in cell efficiency, but caused a reduction in cell performance in low dilution samples.

- We replaced hydrogen with deuterium and found deuterated amorphous silicon alloy solar cells exhibit reduced light-induced degradation. The deuterated alloys have structural similarity with hydrogenated alloys grown with heavy hydrogen dilution. The improved stability is believed to arise from improved microstructures.

- We achieved a world record single-junction a-Si alloy stable cell efficiency of $9.2 \%$ with an active-area of $0.25 \mathrm{~cm}^{2}$ grown with high hydrogen dilution.

- We achieved state-of-the-art top, middle, and bottom component cells with the stable characteristics shown in Table I. 
- We achieved a world record triple-junction, stable, active-area cell efficiency of $13.0 \%$ with an active area of $0.25 \mathrm{~cm}^{2}$. The cell characteristics are shown in Table I. Table I also lists progress made in cell efficiencies since the beginning of the subcontract.

- We started investigations on properties of materials and cells deposited at a rate between 3 to $5 \mathrm{~A} / \mathrm{sec}$. Status of component cells and triple-junction devices in which the intrinsic layers are deposited at a rate of $3 \mathrm{~A} / \mathrm{s}$ is summarized in Table $I$.

- Arrays of high efficiency triple-junction cells of $0.25 \mathrm{~cm}^{2}$ active area have been fabricated over a one-square-foot area with an average stabilized efficiency of $12 \%$ as measured under AM1.5 illumination.

- A triple-junction module of aperture area $416 \mathrm{~cm}^{2}$ has been fabricated with an initial efficiency of $11.7 \%$ and stabilized efficiency of $10.24 \%$. A $922 \mathrm{~cm}^{2}$ aperture-area module exhibited an initial efficiency of $11.5 \%$.

- The novel laser-drilling approach has been used successfully to fabricate modules of approximately $300 \mathrm{~cm}^{2}$ area with an initial total-area efficiency of $10.6 \%$.

\section{References}

1. Guha, S. (1997). Annual Report, Thin Film Amorphous Silicon Alloy Research Partnership. Golden, CO: National Renewable Energy Laboratory. Work performed by United Solar Systems Corp., Troy, Michigan.

2. Guha, S. (1997). Annual Report, Amorphous Silicon Research. Golden, CO: National Renewable Energy Laboratory. Work performed by United Solar Systems Corp., Troy, Michigan.

3. Sugiyama, S.; Yang, J.; and Guha, S. (1997). "Improved Stability Against Light Exposure in Amorphous Deuterated Silicon Alloy Solar Cell." Applied Physics Letters 70; pp. 378-380.

4. Palsule, D; Paschen, U.; Cohen, J.D.; Yang, J.; and Guha, S. (1997). "Evidence for Hole Traps at the Amorphous Silicon/Amorphous Silicon-Germanium Heterostructure Interface." Applied Physics Letters 70; pp. 499-501.

5. Yang, J.; Banerjee, A.; and Guha, S. (1997). "An Amorphous Silicon Alloy Triple-junction Solar Cell with $14.6 \%$ Initial and 13.0\% Stable Efficiencies." Proceedings of the 1997 Spring Meeting of the Materials Research Society; March 31-April 4, 1997, San Francisco, CA.

6. Banerjee, A.; Yang, J.; and Guha, S. (1997). "Improved Amorphous Silicon Alloy Solar Cells for Module Fabrication." Proceedings of the 1997 Spring Meeting of the Materials Research Society; March 31-April 4, 1997, San Francisco, CA.

7. Sugiyama, S.; Yang, J.; and Guha, S. (1997). "Improved Stability Against.Light-exposure in Deuterated Amorphous Silicon Alloy Solar Cells." Proceedings of the 1997 Spring Meeting of the Materials Research Society; March 31-April 4, 1997, San Francisco, CA. 
8. Chen, C.; Zhong, F.; Cohen, J.D.; Yang, J.C.; and Guha, S. (1997). "The Significance of Charged Defects in Understanding the Light-induced Degradation of Hydrogenated Amorphous Silicon-Germanium Alloys." Proceedings of the 1997 Spring Meeting of the Materials Research Society; March 31-April 4, 1997, San Francisco, CA.

9. Yang, J.; Banerjee, A.; and Guha, S. (1997). "Triple-junction Amorphous Silicon Alloy Solar Cell with 14.6\% Initial and 13.0\% Stable Conversion Efficiencies." Applied Physics Letters 70; pp. 2975-2977.

10. Guha, S.; Yang, J.; Banerjee, A.; Glatfelter, T.; and Sugiyama, S. (1997). "Advances in Amorphous Silicon Alloy Photovoltaic Technology." Proceedings of the Fourteenth European Photovoltaic Solar Energy Conference and Exhibition; June 30-July 4, 1997, Barcelona, Spain.

11. Guha, S.; Yang, J.; Banerjee, A.; Glatfelter, T.; and Sugiyama, S. (1997). "Advances in Amorphous Silicon Alloy Cell and Module Technology." Solar Energy Materials and Solar Cells 48; pp. 365-371.

12. Guha, S. (August 1997). "Material Aspects of Amorphous Silicon Solar Cells." Current Opinion in Solid State \& Materials Science Vol 214; pp. 425-429.

13. Guha, S.; Yang, J.; Banerjee, A.; Hoffman, K.; Sugiyama, S.; Call, J.; Jones, S.J.; Deng, X.; Doehler, J.; Izu, M.; and Ovshinsky, H.C. (1997). "Triple-junction Amorphous Silicon Alloy PV Manufacturing Plant of 5 MW Annual Capacity." Conference Record of the 26th IEEE Photovoltaic Specialists Conference-1997; Sept. 29-Oct. 3, 1997; Anaheim, CA. New York: The Institute of Electrical and Electronics Engineers, Inc.; pp. 607-610.

14. Yang, J.; Banerjee, A.; Glatfelter, T.; Sugiyama, S.; and Guha, S. (1997). "Recent Progress in Amorphous Silicon Alloy Leading to $13 \%$ Stable Cell Efficiency." Conference Record of the 26th IEEE Photovoltaic Specialists Conference-1997; Sept. 29-Oct. 3, 1997; Anaheim, CA. New York: The Institute of Electrical and Electronics Engineers, Inc.; pp. 563-568.

15. Jones, S.J.; Myatt, A.; Izu, M.; Banerjee, A.; Yang, J.; and Guha, S. (1997). "Use of Gas Jet Deposition Technique to Prepare a-Si:H Solar Cells." Conference Record of the 26th IEEE Photovoltaic Specialists Conference-1997; Sept. 29-Oct. 3, 1997; Anaheim, CA. New York: The Institute of Electrical and Electronics Engineers, Inc.; pp. 659-662. 
Table 1. Status of Stabilized Component and Triple-junction Cells at United Solar.

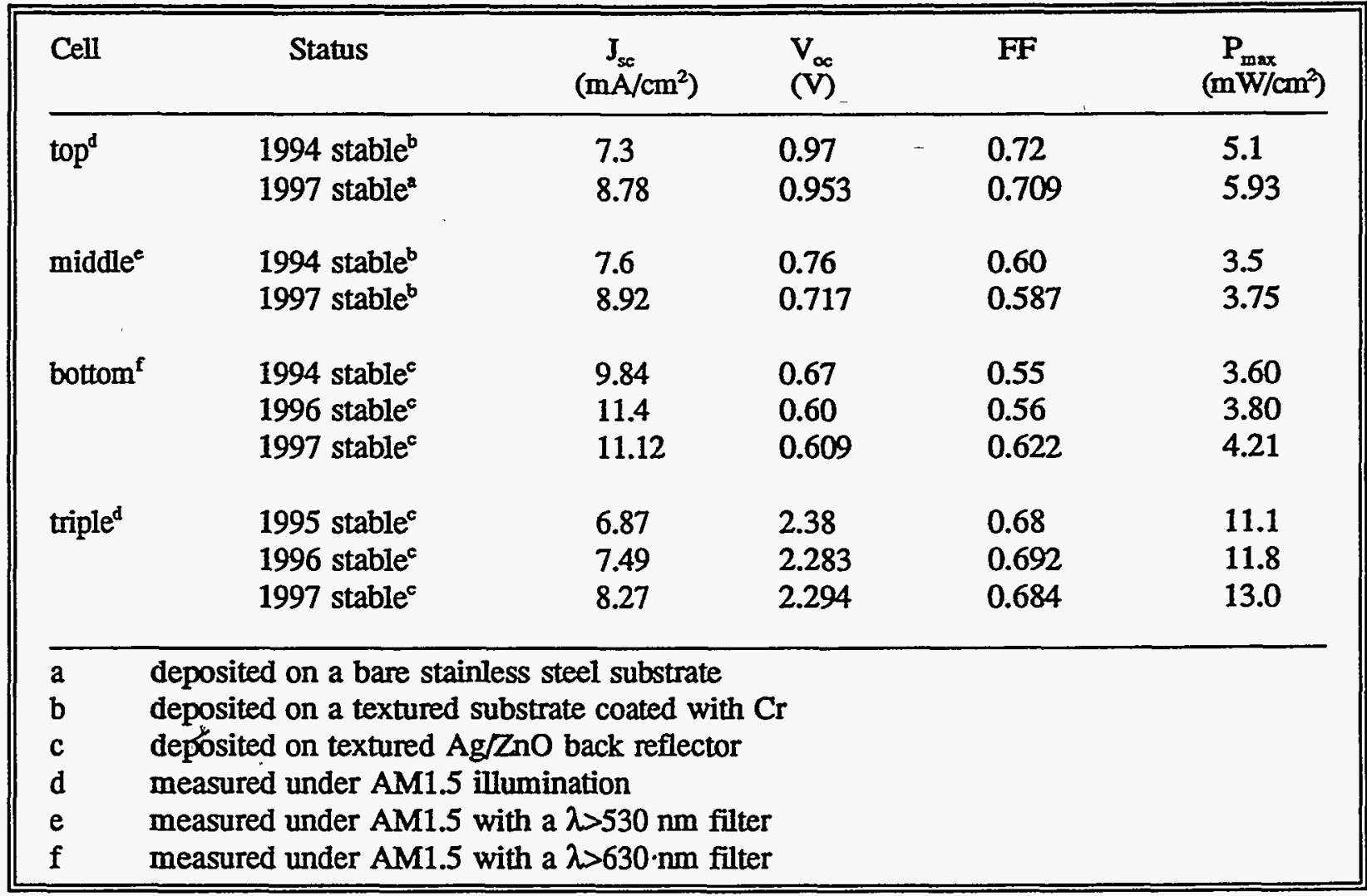

Table II. Status of Stabilized Solar Cells Deposited at $3 \AA / s$ at United Solar.

\begin{tabular}{|c|c|c|c|c|c|}
\hline & Cell & $\underset{\left(\mathrm{mA} / \mathrm{cm}^{2}\right)}{J_{\mathrm{sc}}}$ & $\begin{array}{l}V_{o c} \\
(V)\end{array}$ & $\mathrm{FF}$ & $\underset{\left(\mathrm{mW} / \mathrm{cm}^{2}\right)}{P_{\max }}$ \\
\hline & Top $p^{\text {ad }}$ & 8.56 & 0.904 & 0.646 & 5.00 \\
\hline & Middle $^{\text {bd }}$ & 8.17 & 0.694 & 0.539 & 3.06 \\
\hline & Bottom ${ }^{\infty}$ & 10.11 & 0.594 & 0.564 & 3.39 \\
\hline & Triple & 7.63 & 2.18 & 0.614 & 10.2 \\
\hline d & \multicolumn{5}{|c|}{$\begin{array}{l}\text { measured under AM1.5 } \\
\text { measured under AM1.5 with a } \lambda>530 \mathrm{~nm} \text { filter } \\
\text { measured under AM1.5 with a } \lambda>630 \mathrm{~nm} \text { filter } \\
\text { deposited onto a bare ss substrate } \\
\text { deposited onto a textured } \mathrm{Ag} / \mathrm{ZnO} \text { substrate }\end{array}$} \\
\hline
\end{tabular}


Title: $\quad$ Photocharge Transport and Recombination Measurements in Amorphous Silicon Films and Solar Cells by Photoconductive Frequency Mixing

Organization: Physics Department, University of California at Los Angeles

Los Angeles, CA 90024-1547

Contributors: $\quad$ R. Braunstein, Principle Investigator;

S. Dong, J. Liebe, G.S.Sun

\section{Program Outline}

This program is concerned with the characterization of the photoconductive properties of a-Si:H, a-SiC:H and a-SiGe:H films and solar cells from "round robin" sources by the photoconductive mixing technique so as to separately determine drift mobilities and photomixing lifetimes. By measurements of the temperature and electric field dependence of the drift mobility in the annealed and the light-soaked states, the effects of the sample deposition conditions on the transport properties are investigated. The photomixing technique consists of heterodyning two monochromatic laser beams when a $\mathrm{dc}$ bias is applied which results in a photocurrent composed of a dc and microwave component; these two photocurrents allow a determination of the drift mobility $\left(\mu_{\mathrm{d}}\right)$ and the photomixing lifetime $(\tau)$ of the dominant photogenerated carrier [1].

\section{Accomplishments}

\section{Determination of the depth and the range of long-range potential fluctuations.}

Inhomogeneties of random distribution of charge defects may lead to the formation of long-range potential fluctuations (LRPF) which influence the charge transport in amorphous semiconductors and limit solar cell performance. By employing the technique of photoconductive frequency mixing, the drift mobility $\left(\mu_{d}\right)$ and the photomixing lifetime $(\tau)$ were determined separately on a range of a-Si:H and a-SiGe:H prepared by various synthesis techniques. The measurement of the drift mobility as a function of electric field enable a determination of the range and the depth of the LRPF [2,3].

$$
\mu_{d}(F)=\mu_{d}^{0} \exp \left(-\frac{e V_{P}}{k T}\right) \frac{e L F}{k T\left[1-\exp \left(-\frac{e L F}{k T}\right)\right]} \quad\left(\text { If }|L F| \leq V_{P}\right)
$$

Where $F$ is the applied electric field, $\mu_{d}{ }^{0}$ is the drift mobility without the existence of $L R P F$ and $V_{P}$ and $L$ are the average depth and range of the potential fluctuations respectively. By normalizing the values for the drift mobility to the values at the lowest electric field we obtain:

$$
\frac{\mu_{d}\left(F_{i}\right)}{\mu_{d}\left(F_{1}\right)}=\frac{F_{i}}{F_{1}} \cdot \frac{1-\exp \left(-\frac{e L F_{1}}{k T}\right)}{1-\exp \left(-\frac{e L F_{i}}{k T}\right)}
$$


From curve fitting to this expression, we can determine the range $L$. The results, obtained from fits to the normalized values of the drift mobility can subsequently be used to fit the original data according to equation (1) to obtain the depth of the potential $V_{P}$. A detailed study of hot-wire samples were performed [2]; figure [1] shows the photoconductivity as a function of substrate temperature. The range in the annealed and light-soaked are shown in Figure (2) and the potential depth in the annealed and lightsoaked states are shown in Figure (3) as a function of substrate temperature. It should be noted in the annealed state that the range decreases as the substrate temperature increases while the depth of the potential increases. In the light-soaked state, the depth of the potential increases while the range changes only slightly as the substrate temperature increases.

\section{Measurements on diluted, undiluted, and ECR a-Si:H films and solar cells}

Measurements were performed on ECR samples from the Dalal group and hydrogen diluted samples from the Wronski group. In view of the interest in the role of hydrogen dilution in a-Si:H p-i-n solar cells which result in a better stability against light induced degradation, we have employed the technique of photoconductive frequency mixing to determine the transport properties of $\mathrm{p}$ - $\mathrm{i}-\mathrm{n}$ junctions and companion dilute and undiluted intrinsic films produced by the Wronski group. The dilute layers were processed with 10:1 $\mathrm{H}_{2} / \mathrm{SiH}_{4}$ at a substrate temperature of $200{ }^{\circ} \mathrm{C}$; the $\mathrm{i}$ layers were $0.4 \mu \mathrm{m}$ thick. The photoconductivity $\sigma_{\mathrm{pc}}$, lifetime $\tau$, mobility $\mu_{\mathrm{d}}$, range and depth of the long-range potential fluctuations of undiluted and diluted a-Si: $\mathrm{H}$ in the annealed and light-soaked states wére determined. In the annealed state the photoconductivity, lifetime, mobility, range and depth are greater in the diluted compared to the undiluted state. In the light-soaked state, the photoconductivity, lifetime, mobility and range decrease while the depth increased compared to the corresponding values in annealed state. Another point of interest is that the lifetime and mobility decay at different rates in the diluted and undiluted samples indicating differences in the production of charge and neutral centers. Mixing measurements for diluted and undiluted $\mathrm{p}$-i-n cells indicate that it is possible to determine the built in potential in these junctions. The photoconductivity, mobility, and lifetime as a function of light-soaking with 4-suns intensity as well as the electric field dependence of the mobility were determined for ECR material. The decay rates of the photoconductivity, mobility and lifetime of the ECR material differ from the rates for the diluted and undiluted samples indicating the difference in the production rates of neutral and charge states. The results of this section are discussed in Quarterly Report 15 May 1997- 1 September 1997 and 15 February 1997 - 15 May 1997.

\section{Photomixing determination of the charge transport parameters of a-SiGe:H produced by a variety growth parameters}

Brent Nelson at NREL is presently producing a-SiGe:H employing the hot-wire technique under varying growth conditions to explore the parameter space to produce aSiGe:H alloys to optimize the transport parameters within two bandgaps regimes: 1.40$1.45 \mathrm{eV}$ and 1.60-1.65 eV. To this end, Brent has prepared samples with continuous 
varying preparation conditions and deposited at five different positions. Figure 4 shows the drift mobility as a function of the Tauc gap, while the range of the long range potential as a function of the Tauc gap is shown in Figure 5 and the depth of the longrange potential as a function of the Tauc gap is shown in Figure 6 . The monotonic increase in the depth and the decrease in the range of the long-range potential fluctuations with increase in $\mathrm{Ge}$ in this alloy system suggest that compositional disorder may lay a role in the long-range potential fluctuations. This point will be pursued in more detail once larger homogeneous films are available in the near future.

\section{Atomic Force Microscopy study of the morphology of hot-wire a-Si:H and its correlation with the electrical propertied}

Evidence has been accumulating that surfaces of a-Si:H exhibit a granular morphology for various growth techniques. This leads to the question as to whether the surface morphology is imprinted into the bulk film growth and so influences the charge transport properties of the materials. Since we recently completed a detailed study of the charge transport properties of a-Si: $\mathrm{H}$ with hydrogen content ranging from $12 \%$, which were produced by the hot-wire technique varying the deposition substrate temperature from, $290^{\circ} \mathrm{C}<\mathrm{T}_{\mathrm{S}}<400^{\circ} \mathrm{C}$, and determined the photoconductivity, mobility, and the range of the long-range potential fluctuations, it was of interest to study the morphology of the surfaces employing AFM to study systematically the surfaces of hot-wire a-Si:H. We initiated a study with Dr. David Braunstein of Parks Scientific using the Autoprobe CP in the intermittent mode employing a tip of 50 Angstroms. The samples employed in this study were produced at NREL by the hot-wire assisted chemical vapor deposition technique. The transport measurements were previously reported [2]. Analysis of the scans, yielded histograms of the height distribution enabled a determination of the rms roughness of the surfaces. A plot of the rms surface roughness as a function of substrate temperature is shown in Figure 7 where it is seen that the rms roughness increases with substrate temperature. The decrease in the photoconductivity as a function of substrate temperature is shown in Figure 1. The decrease in range of the long-range potential fluctuations as the substrate temperature is increased [see Figure 2] appears to be correlated with the decrease in the surface roughness! Further correlation of the surface morphology with bulk structural inhomogeneties and the relationship with charge transport must await the fractal analysis to obtain the grain sizes. The correlation of the surface roughness with substrate temperature indicated that the morphology of the films is intrinsic to the growth process and is not due to particulates in the deposition stream.

\section{References}

1. Yi Tang, R. Braunstein, B. von Roedern, Mat. Res. Soc. Symp. Proc. 258, 735 (1992).

2. S. Dong, Y. Tang, J. Liebe, R. Braunstéin, R.S. Crandall, B.P. Nelson, and A.H. Mahan, J. Appl. Phys. 82, 702 (1997).

3. Determination of the range and depth of potential fluctuations in a-Si:H and aSiGe:H, S. Dong, Y. Tang, J. Liebe, G.S. Sun, R. Braunstein and B. von Roedern [Submitted to Appl. Physics. Letters]. 


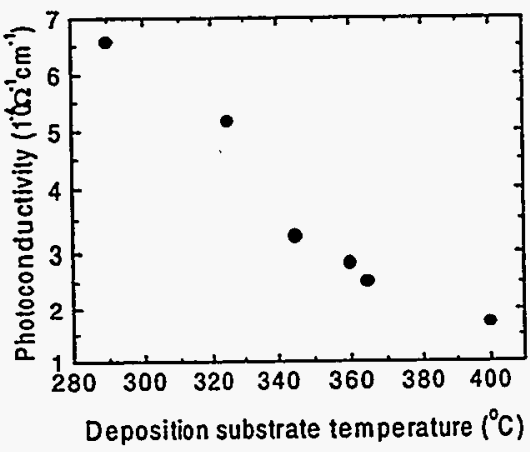

Fig. 1. The annealed state photoconductivity as a function of the substrate temperatures.

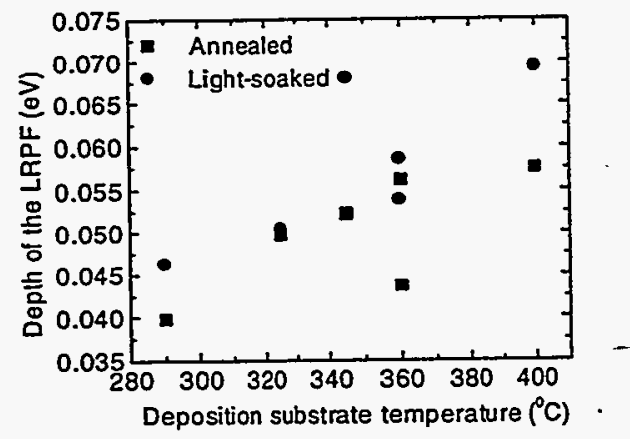

Fig. 3. The depth of the long range potential fluctuations as a function of the substrate temperatures.

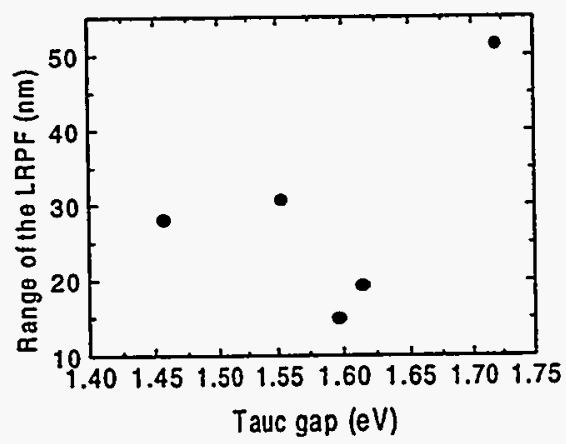

Fig. 5. The dependence of the range of the long range potential fluctuations on the Tauc gap for a-SiGe:H.

Fig. 7. The RMS roughness as a function of the substrate temperature for hot-wire a-Si:H.

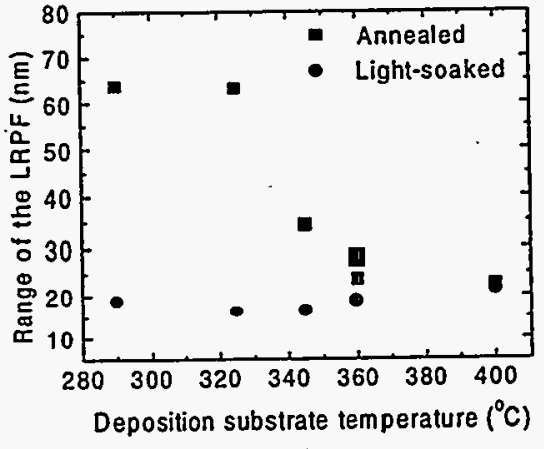

Fig. 2. The range of the long range potential fluctuations as a function of the substrate temperatures

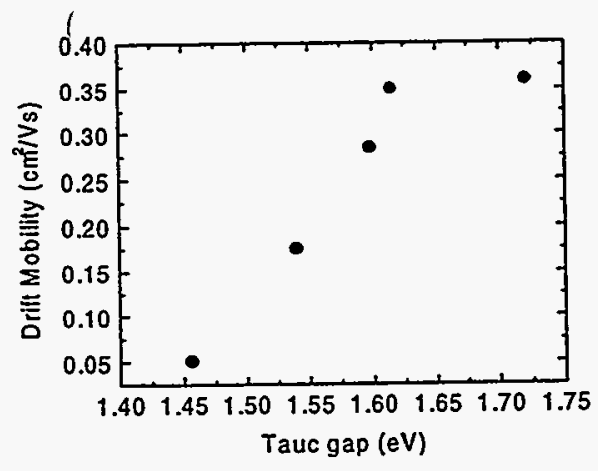

Fig. 4. The dependence of the drift mobility on the Tauc gap for a-SiGe:H.

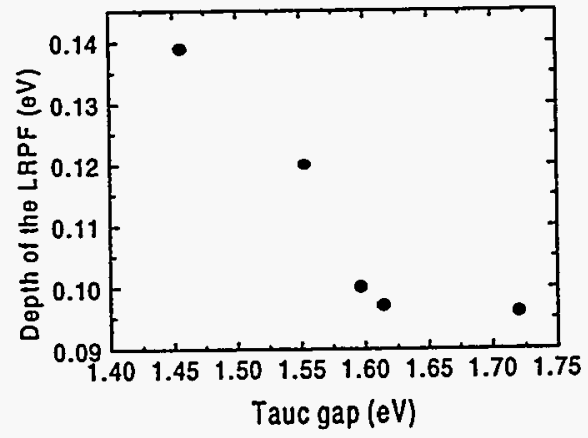

Fig. 6. The dependence of the depth of the long range potential fluctuations on the Tauc gap for a-SiGe:H.

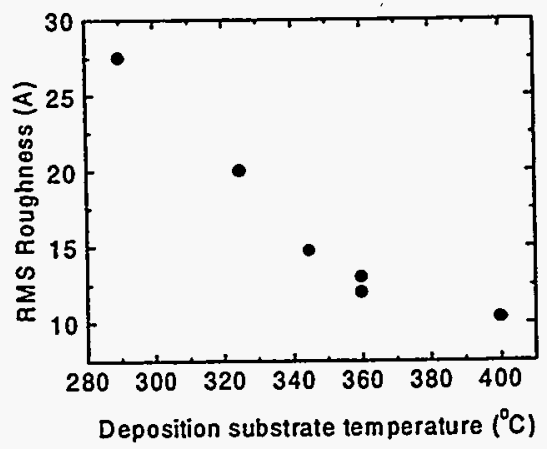


Title:

Processing and Modeling Issues for Thin-Film Solar Cells

Organization: Institute of Energy Conversion, University of Delaware, Newark, DE 19716

Contributors: R. Birkmire, W. Buchanan, E. Eser, S. Hegedus, M. Marudachalam, B. McCandless, T.W.F. Russell, J. Phillips, W. Shafarman

\section{Objectives and Technical Highlights}

The objective of IEC's four year subcontract with NREL is to advance the state-of-the-art in thin-film PV modules by improving thin-film deposition, device fabrication, material and device characterization, and related manufacturing technology of solar cells based on $\mathrm{Cu}(\mathrm{InGa}) \mathrm{Se} \mathrm{e}_{2} \mathrm{CdTe}$ and a-Si. IEC is actively involved in the Thin-Film Partnership Teams. In addition, undergraduate, graduate, and post-graduate researchers are trained in the science and technology of thin-film solar cells. Accomplishments during the first phase of the present four year contract include: addressing manufacturability of $\mathrm{Cu}(\mathrm{InGa}) \mathrm{Se}_{2}$ solar cells by demonstrating that devices with $\eta \approx 13 \%$ can be deposited at $\mathrm{T}_{\mathrm{ss}} \leq 450^{\circ} \mathrm{C}$ and thicknesses of $1.4 \mu \mathrm{m}$; finding that an anneal to form single phase $\mathrm{Cu}(\mathrm{InGa}) \mathrm{Se}_{2}$ by selenization is necessary because of different reaction rates to form CuGaSe $\mathrm{C}_{2}$ and $\mathrm{CuInSe}_{2}$; understanding and controlling of CdS diffusion by employing $\mathrm{CdTe}_{1 \cdot \mathrm{x}} \mathrm{S}_{\mathrm{x}}$ absorber layers allowing use of thinner CdS layers; developing post-deposition treatments of CdS/CdTe which anneal crystal defects prior to $\mathrm{CdCl}_{2}$ delivery; developing a widely-applicable all-dry method for treating CdTe and fabricating contacts giving FF $>70 \%$ on CdTe from several groups; analyzing electrical and optical behavior of the $n / T C O / A g$ back contact in p-i-n a-Si devices to identify the influence of the nlayer and TCO on non-ohmic n/TCO contacts; and improving the $\mathrm{ZnO} / \mathrm{p}$ a-SiC contact in a-Si p-i-n devices.

\section{Results}

\section{High Bandgap CuInSe, -based Solar Cells}

High bandgap CuInSe-based alloys being investigated for high $\mathrm{V}_{\text {oc }}$ devices include $\mathrm{Cu}(\mathrm{InGa}) \mathrm{Se}_{2}$ with $0.5 \leq \mathrm{Ga} /(\mathrm{In}+\mathrm{Ga}) \leq 1$ or $1.3 \leq \mathrm{E}_{\mathrm{g}} \leq 1.7 \mathrm{eV}$ and $\mathrm{CuInS}_{2}$ with $\mathrm{E}_{\mathrm{g}}=1.5 \mathrm{eV}$. The selenization of $\mathrm{Cu} / \mathrm{Ga} / \mathrm{In}$ precursor layers to form $\mathrm{Cu}(\mathrm{InGa}) \mathrm{Se}_{2}$ films results in two phase films with $\mathrm{CuGaSe}_{2}$ near the back and $\mathrm{CuInSe}_{2}$ near the top surface of the film. Detailed analysis of the interdiffusion of In and $\mathrm{Ga}$ in this system [1] suggests this is due to a slower reaction rate for the formation of $\mathrm{CuGaSe}_{2}$ than that for CuInSe $e_{2}$ which causes the CuInSe $e_{2}$ phase to form on the surface and, as a result, $\mathrm{CuGaSe}_{2}$ at the Mo/film interface. The annealing temperature to convert the two phase film to single phase depends on the $\mathrm{Ga}$ content. For $\mathrm{Ga} /(\mathrm{Ga}+\mathrm{In})<0.5$, an anneal at $600^{\circ} \mathrm{C}$ is required, while $400-500^{\circ} \mathrm{C}$ is required for high $\mathrm{Ga} /(\mathrm{Ga}+\mathrm{In})$ ratios.

A chemical vapor reactor was upgraded to allow reaction in any combination of $\mathrm{H}_{2} \mathrm{~S}$ and $\mathrm{H}_{2} \mathrm{Se}$ to form $\mathrm{CuIn}(\mathrm{SeS})_{2}$. Elemental $\mathrm{Cu}$ and In films have been reacted in $\mathrm{H}_{2} \mathrm{~S}$ to study formation of the binary phases expected to form the reaction pathway to CuIn $\mathrm{S}_{2}$. For temperatures from 350 to $550^{\circ} \mathrm{C}$ the $\mathrm{Cu}$ film reacts to form $\mathrm{Cu}_{1.8} \mathrm{~S}$. The In films form a mixture of $\mathrm{In}_{2} \mathrm{~S}_{3}$ and $\mathrm{Na}_{2} \mathrm{In}_{2} \mathrm{~S}_{4}$ when deposited on soda lime glass which provides a source of $\mathrm{Na}$ via diffusion, or single phase $\mathrm{In}_{2} \mathrm{~S}_{3}$ when deposited on borosilicate glass with no $\mathrm{Na}$.

As the bandgap of $\mathrm{Cu}(\operatorname{InGa}) \mathrm{Se}_{2}$ is increased with increasing $\mathrm{Ga}$, the loss in efficiency due to the decrease of light generated current $\left(I_{\mathfrak{L}}\right)$ with increasing voltage becomes more important. A method was developed to determine the voltage dependence of $\mathrm{J}_{\mathrm{L}}$ by an analysis of the current-voltage measurements made at different light intensities [2]. This analysis showed that losses due to poor light generated current collection are significant in typical devices with both low and high Ga content.

\section{Manufacturability Issues for $\mathrm{Cu}(\operatorname{InGa}) \mathrm{Se}_{2}$-based Solar Cells}

Experiments have been performed to address the ability to reduce process costs for multisource evaporation of $\mathrm{Cu}(\mathrm{InGa}) \mathrm{Se}_{2}$ by reducing the deposition temperature and film thickness using a process to deposit films with $\mathrm{Ga} /(\mathrm{In}+\mathrm{Ga}) \approx 0.3$ with no compositional gradients [3]. When the 
substrate temperature $\left(T_{s s}\right.$ ) was decreased from $600 \geq T_{s s} \geq 350^{\circ} \mathrm{C}$ using fixed elemental fluxes the grain size decreased over the entire range but $\mathrm{Na}$ incorporation by diffusion from the soda lime glass substrate into the $\mathrm{Cu}(\mathrm{InGa}) \mathrm{Se}_{2}$ did not change significantly. Solar cell efficiency decreased slowly for $550 \geq \mathrm{T}_{\text {ss }} \geq 400^{\circ} \mathrm{C}$ as shown in Table 1 . The ability to fabricate cells with $\eta \approx 13 \%$ at $\mathrm{T}_{s s} \leq 450^{\circ} \mathrm{C}$ allows flexibility in the choice of substrate materials and process design which can potentially lead to lower costs. The $\mathrm{Cu}(\mathrm{InGa}) \mathrm{Se}_{2}$ thickness was varied from 2.8 to $1.0 \mu \mathrm{m}$ by changing the deposition time while keeping the source effusion rates fixed with $T_{s s}=450^{\circ} \mathrm{C}$. There was no change in film composition over the entire range. Device performance was unaffected by reducing the film thickness to less than $1.5 \mu \mathrm{m}$, as shown in Table 2, which indicates that process throughput can be increased by reducing the absorber layer thickness.

Table 1. Effect of substrate temperature on device performance with $2.5 \mu \mathrm{m}$ thick $\mathrm{Cu}(\mathrm{InGa}) \mathrm{Se}_{2}$.

\begin{tabular}{ccccc}
\hline $\begin{array}{c}\mathrm{T}_{s s} \\
\left({ }^{\circ} \mathrm{C}\right)\end{array}$ & $\begin{array}{c}\mathrm{V}_{\mathrm{oc}} \\
(\mathrm{mV})\end{array}$ & $\begin{array}{c}\mathrm{J}_{\mathrm{sc}} \\
\left(\mathrm{mA} / \mathrm{cm}^{2}\right)\end{array}$ & $\begin{array}{c}\mathrm{FF} \\
(\%)\end{array}$ & $\begin{array}{c}\eta \\
(\%)\end{array}$ \\
\hline 600 & 0.596 & 31.2 & 67.4 & 12.5 \\
550 & 0.583 & 34.3 & 71.8 & 14.4 \\
500 & 0.606 & 32.5 & 70.0 & 13.8 \\
450 & 0.605 & 32.6 & 68.4 & 13.5 \\
400 & 0.606 & 32.5 & 64.8 & 12.8 \\
350 & 0.561 & 33.2 & 58.6 & 10.9 \\
\hline
\end{tabular}

Table 2. Effect of film thickness on device performance with $\mathrm{Cu}(\mathrm{InGa}) \mathrm{Se}_{2}$ deposited at $450^{\circ} \mathrm{C}$.

\begin{tabular}{|c|c|c|c|c|}
\hline $\begin{array}{c}\mathrm{d} \pm 0.2 \\
(\mu \mathrm{m})\end{array}$ & $\begin{array}{c}\mathrm{V}_{\mathrm{oc}} \\
(\mathrm{mV})\end{array}$ & $\begin{array}{c}\mathrm{J}_{\mathrm{sc}} \\
\left(\mathrm{mA} / \mathrm{cm}^{2}\right)\end{array}$ & $\begin{array}{l}F H \\
(\%)\end{array}$ & $\stackrel{\eta}{(\%)}$ \\
\hline 2.5 & 0.605 & 32.6 & 68.4 & 13.5 \\
\hline 1.4 & 0.590 & 32.5 & 69.5 & 13.3 \\
\hline 1.2 & 0.526 & 34.2 & 64.9 & 11.7 \\
\hline & 0.514 & 30.7 & 62.5 & 99 \\
\hline
\end{tabular}

\section{CuInSe, Team Contributions}

IEC is a member of the New Junction and Transient Effects Working Groups. For the Transient Effects Group, IEC showed that a loss in efficiency during lamination of devices and modules from SSI which recovers over time is entirely due to a change in fill factor from an increase in series resistance. For the New Junction Group, IEC coordinated and completed fabrication and characterization of $\mathrm{Cu}(\mathrm{InGa}) \mathrm{Se}_{2} / \mathrm{ZnO}$ devices including $\mathrm{ZnO}$ layers deposited by different team members using several deposition methods. This included a device with $14 \%$ active area efficiency using an intrinsic $\mathrm{ZnO}$ layer deposited by MOCVD at Washington State University [4].

\section{CdTe Devices with Thin CdS}

Production of reliable and reproducible thin CdS window layers for stable, high performance $\mathrm{CdS} / \mathrm{CdTe}$ solar cells is a key issue confronting development of thin-film CdTe solar cells. Using evaporated CdS and CdTe films, IEC has demonstrated improved understanding and control of CdS diffusion by: 1) employing $\mathrm{CdTe}_{1-x} \mathrm{~S}_{x}$ absorber layers [5] and 2) developing post-deposition treatments which anneal crystal defects prior to $\mathrm{CdCl}_{2}$ delivery $[5,6]$. Significant results include: determining the effect of CdTe $e_{1-x} S_{x}$ alloy composition on the effective CdS diffusion rate; reducing $\mathrm{CdS}$ window layer consumption by a factor of 3 without loss in $\mathrm{V}_{\text {oc }}$; fabricating devices with $\mathrm{J}_{\mathrm{sc}}=25$ $\mathrm{mA} / \mathrm{cm}^{2}$ with evaporated CdS layers; and determining device performance as a function of final CdS thickness.

\section{Quantification of CdS-CdTe Interdiffusion}

IEC made significant progress towards quantifying the CdS-CdTe interdiffusion applicable to many fabrication processes: 1 ) developed a measurement protocol to analyze interdiffusion using $x$-ray diffraction, optical and device quantum efficiency techniques [5];2) added low-temperature points to the CdS-CdTe phase diagram; 3) quantified the diffusion for varying absorber layer composition; 4) quantified the diffusion for varying post-deposition treatment conditions $[6,7] ; 5)$ examined microcrystal structure for varying post-deposition treatment conditions; and 6) performed a complete materials analysis of interdiffusion in CdTe/CdS cells made by six groups of the CdTe Team [8]. This data is being used to construct a phenomenological processing-based model of all thin-film CdS/ CdTe devices. 


\section{Contact to CdTe}

IEC identified a critical component of contacts to CdTe and developed an all-dry method for fabricating contacts. Measurement protocols using variation in light intensity and temperature during current-voltage measurements were developed and employed to analyze the CdTe contact characteristics for devices made by different processes having different contacts. Coupled with the stress-induced degradation studies being carried out by the CdTe Stability Team, this work will lead to improved understanding of the contact properties and their relation to device stability. Through extensive team interaction, the applicability of the results and processes to $\mathrm{CdS} / \mathrm{CdTe}$ cells made by different techniques has been demonstrated.

\section{CdTe Team Contributions and Collaboration}

During 1996-1997 IEC actively participated in the CdTe Team by fabricating devices for the stability and CdS sub-teams, analyzing films and devices for both sub-teams, and reporting results through presentations and written reports. In particular, devices were fabricated on different TCO films to augment investigacion of TCO/CdTe junction influence as CdS thickness is reduced. Contacts to CdTe were deposited and evaluated on CdTe/CdS samples from Solar Cells, Inc. (with five different conductors) and Golden Photon,'Inc. A comprehensive x-ray diffraction analysis was performed on samples made by six groups within the team, and a full report was submitted [8]. CdS films made by different groups were analyzed for pinhole density.

IEC provided other assistance to groups working on CdTe thin films. Physical vapor-deposited CdTe/CdS/TTO samples were fabricated and sent to International Solar Energy Technologies, Colorado School of Mines, National Renewable Energy Laboratory, and University of Toledo. CdTe/CdS devices were fabricated on electrodeposited CdS made at the Colorado School of Mines. [9]. X-ray diffraction analysis was performed on various thin-film structures for University of South Florida, Colorado School of Mines, and ITN.

\section{Analysis of Back Contacts and Interfaces in a-Si p-i-n Solar Cells}

A comprehensive study was performed of the n-layer and back contact for IEC's PECVD superstrate (glass/textured $\mathrm{SnO}_{2} / \mathrm{p}-\mathrm{i}-\mathrm{n} / \mathrm{TCO} / \mathrm{metal}$ ) a-Si solar cells [10]. The compatibility of both a-Si and $\mu \mathrm{c}-\mathrm{Si}$ n-layers with various optical back reflector contacts was compared. Temperature dependent JV measurements and QE measurements identified and separated the criticaf roles of the $\mathrm{n}$-Iayer conductivity, the sputtered TCO (ITO or $\mathrm{ZnO}$ ) and the metal layer. A rectifying n/TCO contact is formed with an a-Si n-layer which reduces FF but a low resistance n/TCO contact is formed with an flC-Si n-layer [10]. Variations in TCO processing caused small changes in bulk TCO properties but large variations in device performance, suggesting the influence of the $n / T C O$ interface dominates over bulk TCO. ZnO gives $\sim 1 \mathrm{~mA} / \mathrm{cm}^{2}$ higher $\mathrm{J}_{\mathrm{sc}}$ and $10-20 \mathrm{mV}$ higher $\mathrm{V}_{\mathrm{oc}}$ compared to ITO. The best contacts were found to be $\mu \mathrm{c}-\mathrm{Si} / \mathrm{ZnO} / \mathrm{metal}$, with $\mathrm{Ag}$ or $\mathrm{Cu}$ giving comparable performance. A device with a $\mu \mathrm{c}-\mathrm{Si} / \mathrm{ZnO} / \mathrm{Ag}$ back contact having. $10.4 \%$ efficiency was verified at NREL, with parameters: $V_{o c}=0.880 \mathrm{~V}, \mathrm{~J}_{s c}=16.2 \mathrm{~mA} / \mathrm{cm}^{2}$, and $\mathrm{FE}=72.7 \%$.

\section{Analysis and Modeling of a-Si and a-SiGe Single and Mnltijunction. Solar Cells}

A method was developed to analyze light and dark $\mathrm{J}(\mathrm{V})$ data from a-Si based solar cells using a simple field-dependent photocurrent collection model [11]. Very good agreement to measured J(V) data from single junction a-Si or a-SiGe devices: from five laboratories was found with only one adjustable parameter, the ratio of collection length to thickness $L c / D$, which is proportional to the hole $\mu \tau$ value. The voltage dependent photocurrent had a major effect on the degraded $F F$ and $V_{o c}$. Photocurrent in very thin devices $(\sim 0.1 \mu \mathrm{m})$ may be strongly influenced by interface recombination or back diffusion. The flatband voltage $\left(V_{f b}\right)$ is dependent on the intensity and spectrum of illumination, hence it is not equivalent to the built-in potential. $V_{o c}$ is limited by $V_{f b}$, not junction recombimation current $J_{\hat{\sigma}}$ in typical devices. In collaboration with IEC, researchers at University of Hawaii developed a model [12] for analysis of multijunction (MJ) solar cells by integrating the J(V) analysis [1.1] with an optical model. This MJ model was used to analyze the performance of United Solar's world record $13 \%$ triple jünction device. 


\section{a-Si Team Contributions}

The primary a-Si team focus this year was in understanding TCO/a-Si contacts. We continued our collaboration of the past 3 years with Harvard Univ. to incorporate highly transparent APCVD textured $\mathrm{ZnO}$ as a substrate. Previous efforts have been plagued by low $\mathrm{V}_{o c}$ or FF. It was found that increasing the $\mathrm{B}_{2} \mathrm{H}_{6}$ flow from 1.5 to $4.5 \mathrm{sccm}$ in the p-layer deposition increased $V_{\circ c}$ from 0.82 to $0.86 \mathrm{~V}$ and $\mathrm{FF}$ from 61 to $68 \%$ on $\mathrm{ZnO}$ substrates. Curvature around $\mathrm{V}_{\text {oc }}$ was eliminated suggesting a barrier at the $\mathrm{ZnO} / \mathrm{p}$ contact was eliminated with the higher p-layer conductivity. We provided ITO and $\mathrm{ZnO}$ contacts to hot wire a-Si devices made at NREL. A set of ITO/i-n and $\mathrm{ZnO} / \mathrm{i}-\mathrm{n}$ devices were fabricated and characterized at IEC before being sent to Syracuse Univ. for evaluation of the built-in voltage by electro-absorption.

\section{Conferences and Workshops}

IEC hosted the NREL/EPRI CuInSe ${ }_{2}, \mathrm{CdTe}$, and a-Si team meetings in April. This coincided with the Thin Film Photovoltaic Symposium at University of Delaware organized by IEC to celebrate our 25th Anniversary on May 1, 2.

\section{Education and Training}

IEC is training and supporting five graduate students from the departments of Chemical Engineering, Materials Science, and Physics at the University of Delaware. We are also training three post-doctoral researchers, and a Fulbright Student Scholar, Julius Mwabora from Tanzania.

\section{References}

1. Marudachalam, M.; Birkmire, R.W.; Hichri, H.; Schultz, J.M.; Swartzlander, A.; and Al-Jassim, M.M. (1997). "Phases, morphology, and diffusion in CuIn $\mathrm{Ga}_{1-x} \mathrm{Se}_{2}$ thin films," J. Appl. Phys. 82, 2896.

2. Phillips, J.E.; Titus, J.; and Hofmann, D.; (1997). "Determining the Voltage Dependence of the Light Generated Current in CuInSe, Based Solar Cells," presented at the 26th IEEE PVSC, Anaheim, $C A$.

3. Shafarman, W.N.; Birkmire, R.W.; Marsillac, S.; Marudachalam, M.; Orbey, N.; and Russell, TWF, (1997). "Effect of Reduced Deposition Temperature, Time, and Thickness on Cu(InGa)Se ${ }_{2}$ Films and Devices," presented at the 26th IEEE PVSC, Anaheim, CA.

4. Olsen, L.C.; Lei, W.; Addis, F.W.; Shafarman, W.N.; Contreras, M.A.;and K. Ramanathan, (1997). "High Efficiency CIGS and CIS Cells with CVD ZnO Buffer Layers," presented at the 26th IEEE PVSC, Anaheim, CA.

5. McCandless, B.E. and Birkmire, R.W. (1997). "CdTe ${ }_{1-x} S_{x}$ Absorber Layers for Thin-Film $\mathrm{CdTe}_{1-x} \mathrm{~S}_{\mathrm{x}} / \mathrm{CdS}$ Solar Cells," presented at the 26th IEEE PVSC, Anaheim, CA.

6. Youm, I.; McCandless, B.E.; and Birkmire, R.W., "Optimization of Vapor Post-Deposition Processing for Evaporated CdS/CdTe Solar Cells," to be published.

7. McCandless, B.E.; Moulton, L.V.; and Birkmire, R.W., (1997)."Recrystallization and Sulfur Diffusion in $\mathrm{CdCl}_{2}$ Treated CdTe/CdS Thin Films," Progress in Photovoltaics: 5, pp. 249-260.

8. McCandless, B.E., "X-ray Diffraction Analysis of CdTe/CdS Thin Film Devices," Report submitted to NREL CdTe Team, 30 April, 1997.

9. Kadirgan, F.; Mao, D.; Balcioglu, A.; Song, W.; Ohno, T.R.; Trefny, J.U.; McCandless, B.E.; (1997). "Electrodeposited CdS Thin Films and Their Application in CdS/CdTe Solar Cells," presented at the 26th IEEE PVSC, Anaheim, CA.

11. Hegedus, S.S.; Buchanan, W.A.; and Eser, E., (1997). "Improving Performance of Superstrate $\mathrm{p}-\mathrm{i}-\mathrm{n}$ a-Si Solar Cells by Optimization of $\mathrm{n}-\mathrm{TCO} / \mathrm{Metal}$ Back Contacts," presented at the 26th IEEE PVSC, Anaheim, CA.

12. Hegedus, S.S.; (1997). "Current-Voltage Analysis of a-Si and a-SiGe Solar Cells Including Voltage-dependent Photocurrent Collection," Progress in Photovoltaics 5, pp. 151-168.

13. Rocheleau, R.E.; Tun, M.; and Hegedus, S.S., (1997). "Analysis and Optimization of High Efficiency Multijunction a-Si:H Solar Cells," presented at the 26th IEEE PVSC, Anaheim, CA. 
Title:

Organization:

Contributors:

\author{
Processing of CuInSe2-Based Solar Cells: Characterization of \\ Deposition Processes in Terms of Chemical Reaction Analysis
}

Chemical Engineering, Electrical Engineering, and Materials Science and Engineering Departments, University of Florida, Gainesville, FL 32611

Tim Anderson, principal investigator, Paul Holloway, Kevin Jones and Sheng $\mathrm{Li}$, co-principal investigators; C.H. Chang, M. Hilali, C. Huang, S. Kim, L. Rieth, N. Shepherd and B. Stanbery, graduate research assistants

\section{Objectives}

This research effort was initiated in May 1995 to develop a novel high-rate processing technique for device quality thin films of CIS-based materials. Important objectives of the program are the development of a plasma-assisted CIS deposition process and subsequent rapid thermal processing of optimized precursor film structures. Fundamental studies on junction formation in CIS, the effects of contaminants such as sodium on the kinetics of the growth and recrystallization processes, and phase equilibria in the $\mathrm{Cu}-\mathrm{In}$-Se system are key components of the research program.

\section{Approach}

A multidisciplinary team is investigating a processing sequence that involves the initial growth of a highly ordered structural template at relatively low rate, followed by the rapid deposition of the bulk of the absorber film's mixed-phase precursor using a plasma-assisted deposition technique. This structure is then rapid thermally processed to recrystallize the film from the seed layer. Through our participation in the Thin Film Partnership Program, we also are exploring an alternative dry process (MOCVD) to deposit buffer layers. We are studying a potentially lower-cost, manufacturable process for conductive, transparent $\mathrm{ZnO}$ "window" layer deposition based on $\mathrm{DC}$ sputtering from metallic zinc targets. The team is using a variety of characterization techniques, including the development of a new nondestructive and contactless method for estimating electronic defect state lifetimes in CIS films (Dual-Beam Optical Modulation: DBOM).

A second thread of research is the growth of single crystal CIS films to provide insight into growth mechanisms. High crystalline quality films are being grown at low temperature on single crystal substrates by migration enhanced epitaxy (MEE). These investigations will increase our understanding of the role of vacancy defect phases at junctions and the effect of "contaminants" such as sodium on the growth kinetics of CuInSe2. The thermochemistry and phase equilibria in the $\mathrm{Cu}$-In-Se system are being rigorously assessed using the CALPHAD approach and critical missing data are being measured. In particular, solid state galvanic cells are being used to measure component activities in solution and the Gibbs energy of formation of selected compounds. The constitution of the $\mathrm{Cu}$-In-Se system is being predicted from the results of the assessment study and verified at selected overall compositions by DTA and XRD analysis. A result of this activity is the suggestion of alterantive routes to the formation of CIS absorber layers.

\section{Results}

\section{Analysis of Buffer Layer Processing Using DBOM}

High performance CIS or CIGS-based thin film solar cells include the deposition of a thin CdS buffer layer between the CIGS absorber layer and the $\mathrm{ZnO}$ window layer. The role of the $\mathrm{CdS}$ buffer layer and the influence the deposition technique and parameters, however, are not fully understood. The DBOM 
technique is being used to investigate the differences between three alternative methods of depositing CdS (conventional chemical bath deposition, MOCVD, and sputtering). A simplified model considering reflection at the front $\mathrm{CdS}$ surface and the CdS/absorber and absorber/Mo interfaces, and absorption in absorber films was initially developed. During this last period of research, this model was modified to include the presence of a p-n junction at the CdS/absorber film interface. A collaboration with researchers at Purdue University is directed at a more complete simulation of the experiment.

The DBOM tool was used to characterize CIGS films with and without a CdS buffer layer deposited by various methods. For selected samples, the CIGS surface was treated with a DI water rinse, a Cd-partial electrolyte, or an Ar plasma clean prior to depositing the CdS. Figure 1 shows a

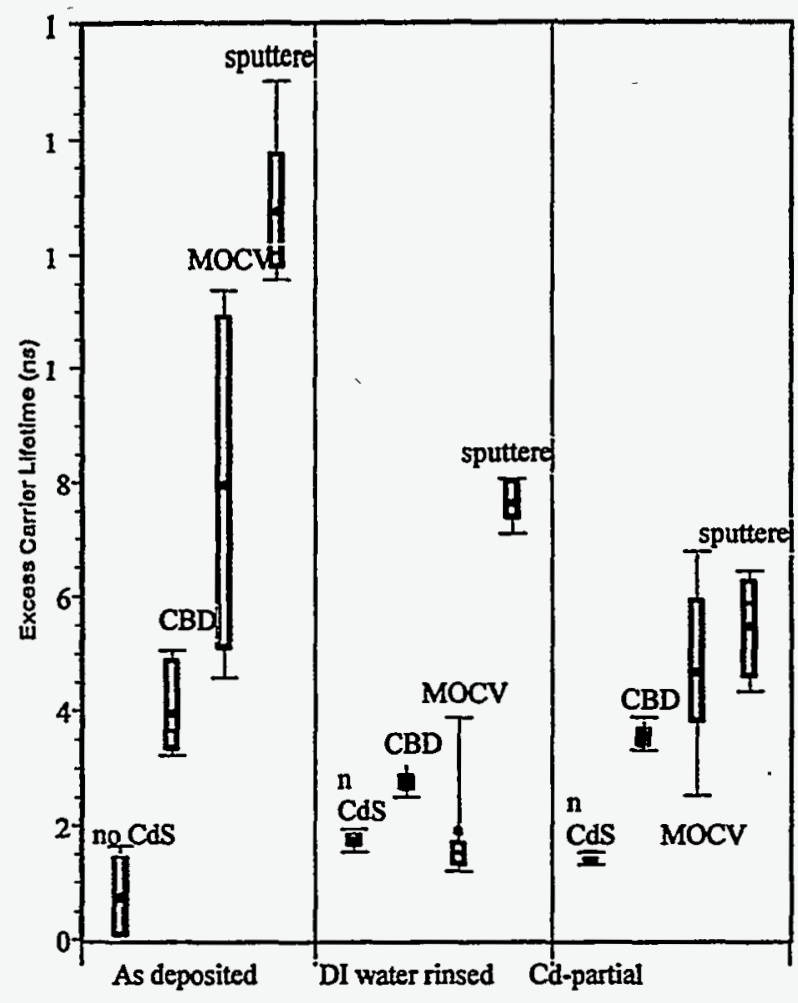

Figure 1. A statistical summary of the lifetimes of samples without CdS and with $C B D, M O C V D$, and sputter deposited CdS for different surface treatments.

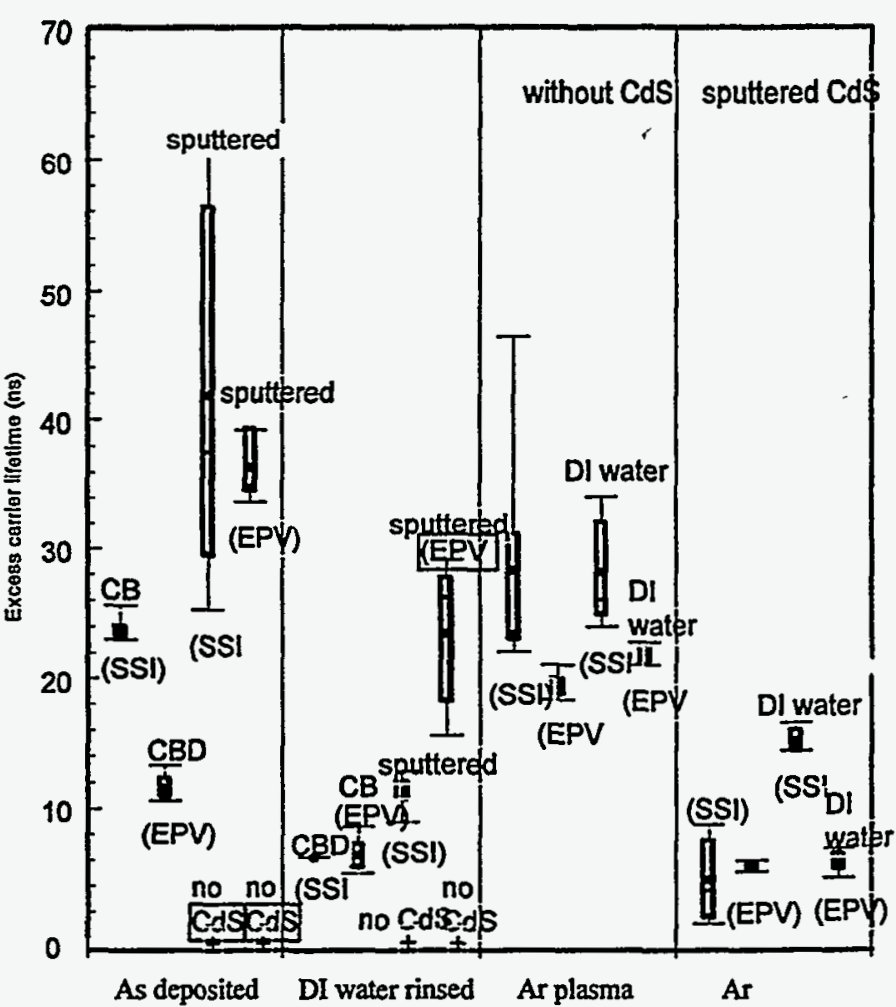

Figure 2. A statistical summary of the lifetimes of CIGS samples from various sources without CdS and with CBD and sputter deposited CaS for different surface treatments.

comparison of the 'lifetime' estimated using the simple model for CdS deposited by sputtering, MOCVD, and CBD. The results reveal that the the fitted 'lifetime' increases significantly with CdS deposition, regardless of the method used to deposit it. The treatment of the surface with a DI rinse or Cd-partial electrolyte dip showed no influence, nor did a low temperature bake, when compared to the samples not treated prior to CdS growth. Figure 2 shows the results of DBOM characterization of CIGS samples from different sources. Again, the addition of a CdS buffer layer increases the fitted 'lifetime', independent of pretreatment or deposition technique. It is interesting to note that an Ar plasma clean significantly increased the 'lifetime' without the deposition of the CdS buffer layer. The more detailed model that included a p-n junction reveals that the fitted 'lifetime' is a complex convolution of lifetime and depletion width. XPS analysis of the surface before and after cleaning indicates a shift from an In-rich surface to one close to stoichiometric $(\mathrm{Cu} / \mathrm{In}=1)$ upon cleaning. Subsequent changes in the extent of band bending or reduction of the depletion width could produce the observed increase in DBOM signal. The DBOM 
technique has proven to be a nondestructive method for rapidly evaluating the quality of CIGS absorber films, although the interpretation of the signal can be complex.

\section{Thermochemistry and Phase Equilibrium of the Cu-In-Se System}

A critical assessment of the $\mathrm{Cu}$-In-Se thermochemical and phase diagram data using standard CALPHAD procedures is being performed. The outcome of this research will produce useful information, including equilibrium vapor compositions (required annealing ambients, $\mathrm{Se}_{\mathrm{X}}$ fluxes from effusion cells), phase diagrams (conditions for melt assisted growth), chemical potentials (driving forces for diffusion and chemical reactions), and consistent solution models (extents of solid solutions and extending phase diagrams).

A reassessment of the Se unary system was initially performed and resulted in revised suggested values for the thermodynamic properties of $\mathrm{Se}_{5}$ and $\mathrm{Se}_{6}$. An initial assessment of the In-Se and Cu-Se systems was then done, and conflicting and missing data were identified. To partially fill these gaps, a set of $(\mathrm{Cu}, \mathrm{In}, \mathrm{Se})$ alloys were synthesized by long time annealing in evacuated ampoules. Quenched samples were then characterized for constitution by XRD and DTA, and component activities or Gibbs energies of formation determine by e.m.f. measurements.

These measurements included a determination of the In activity for the first time in In-rich compositions within and near the two-liquid phase region. Furthermore, the standard Gibbs energies of formation of InSe, $\mathrm{In}_{6} \mathrm{Se}_{7}$ and $\mathrm{In}_{2} \mathrm{Se}_{3}$ were derived from emf data. More recently a series of galvanic cells containing the $\mathrm{Cu}-\mathrm{In}-\mathrm{Se}$ ternary compounds chalcopyrite $\mathrm{CuInSe}_{2}(\alpha)$, sphalerite $\mathrm{CuInSe}_{2}(\delta), \mathrm{Cu}_{2} \mathrm{In}_{4} \mathrm{Se}_{7}$, and $\mathrm{CuIn}_{5} \mathrm{Se}_{8}$ was studied to yield the standard Gibbs energy of formation of each compound. A second law value $21.7 \mathrm{~kJ} / \mathrm{mole}$ was determined for the enthalpy of transformation $\mathrm{CuInSe}_{2}(\alpha) \rightarrow \mathrm{CuInSe}_{2}(\delta)$, while a third law value of $-240 \mathrm{~kJ} / \mathrm{mole}$ was calculated for the enthalpy of formation at $298 \mathrm{~K}$ of CuInSe$(\alpha)$. DTA is now being used to determine phase transition temperatures for those alloys synthesized for the electrode materials. Data previously reported in the literature and recent experimental data were combined to produce the preliminary $\mathrm{In}_{2} \mathrm{Se}_{3}-\mathrm{Cu}_{2} \mathrm{Se}$ pseudobinary phase diagram shown in Figure 3.

Several collaborations have been initiated to better understand the phase relations in the Cu-In-Se system, including the sharing of data with groups in France (Universite de Montpellier) and Germany (Max Planck Institut fur Metallforshung). Synthesized ternary samples have been characterized by EXAFS using the new beam line at Argonne National Lab and the interpretation of the spectra is being assisted by a group at SUNY, Buffalo. The same samples are being examined by high resolution NMR in a collaboration with the University of Utah. It is hoped that these investigations will give insight into the local atomic arrangements in the defective chalcopyrite compounds and solid solutions.

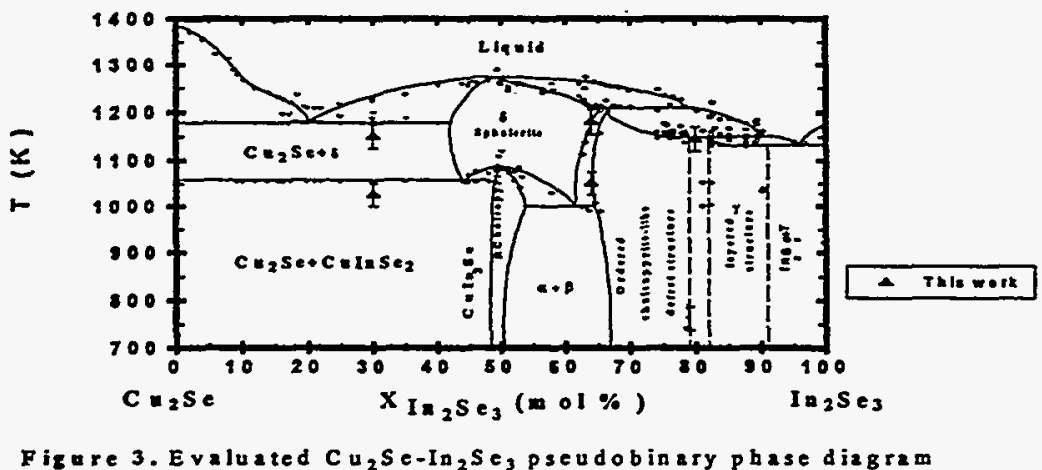




\section{Device Processing}

An integrated facility to fabricate CIS PV devices has been established that includes Migration Enhanced Epitaxy for deposition of CIS, a RTP furnace for absorber film formation, reactive sputtering of $\mathrm{ZnO}$, CBD or MOCVD of CdS, metallization, and pattern definition.

One of the key objectives of this program is to identify and test alternative reaction pathways leading to the formation of device quality CIS absorber layers. Using the results of the phase equilibria studies described above, several precursor film structures were identified that should give rise to an intermediate low temperature liquid phase to assist in grain growth. A potential candidate is a combination of selenium-rich compounds ( $\mathrm{CuSe}, \mathrm{CuSe}{ }_{2}$ ) and selenium-rich indium compounds ( $\left.\mathrm{InSe}, \mathrm{In}_{6} \mathrm{Se}_{7}\right)$ [5]. An estimated projection of the ternary liquidus suggests that when these two compound layer structures are combined a liquid phase should exist at a temperature less than $300^{\circ} \mathrm{C}$.

The above structure was tested by depositing an In-Se layer ( 50 to 55 at \% Se) on a Cu-Se (50 to 55 at \% Se) layer, which was deposited on a Mo coated soda-lime glass substrate. Subsequent RTP of samples with this structure produced no reaction when the upper processing temperature was $220^{\circ} \mathrm{C}$, but $\mathrm{CuInSe}_{2}$ formed when the setpoint temperature was 290 and $500^{\circ} \mathrm{C}$ [4]. A more complete characterization of the absorber films and an optimization of the process in progress.

Understanding the role of $\mathrm{Na}$ in the absorber layer formation process is another objective of this research program. An XPS study of the nerar-surface region of device quality CIGS thin films grown on sodalime glass substrates was performed [6. The chemical shift of the $\mathrm{Na} 1 \mathrm{~s}$ peak within the first few atomic layers was found to be within the accepted range for $\mathrm{Na}(\mathrm{Se}, \mathrm{O})$ compounds, but the binding energy of $\mathrm{Na}$ in those deeper layers suggests a change in the oxidation state. The copper atomic concentration profiles in the near but sub-surface region indicate copper depletion, consistent with previous reports of formation of the defect compound semiconductor $\mathrm{Cu}(\mathrm{In}, \mathrm{Ga})_{3} \mathrm{Se}_{5}$. The atomic ratio $\{[\mathrm{Cu}]+[\mathrm{Na}]\} /\{[\mathrm{In}]+[\mathrm{Ga}]\}$ was found to be constant, suggesting the direct substitution of $\mathrm{Na}$ on vacant $\mathrm{Cu}$ lattice sites, and may indicate the formation of a distinct $\mathrm{Na}$ containing phase during film growth. Bulk synthesis of equilibrium phases in the $\mathrm{Na}-\mathrm{Cu}-\mathrm{In}$-Se system in underway to provide samples for XRD analysis and compound identification.

\section{References}

1. "Investigation of Buffer Layer Processes on CIGS Solar Cells by Dual Beam Optical Modulation Technique," C.H. Huang, S.S. Li, B.J. Stanbery, C.H. Chang, and T.J. Anderson. Conf. Record of the 26th IEEE PVSC (1997).

2. "Effects of Buffer Layer Processing on CIGS Excess Carrier Lifetime: Applications of DualBeam Optical Modulation to Process Analysis," S.S. Li, B.J. Stanbery, C.H. Huang, C.H. Chang, Y.S. Chang, and T.J. Anderson. Conf. Record of the 25th IEEE PVSC, 821-4 (1996).

3. "Thermodynamic Assessment of the Cu-In-Se- System and Application to Thin Film Photovoltaics," C.H. Chang, A. Davydov, B.J. Stanbery, and T.J. Anderson. Conf. Record of the 25th IEEE PVSC, 849-52 (1996).

4. "Novel Multilayer Process for CuInSe 2 Thin Film Formation by Rapid Thermal Processing," C.H. Chang, B.J. Stanbery, A. Morrone, A. Davydov, and T.J. Anderson. Submitted Proc. Fall MRS Meeting (1997).

5. "Reaction Engineering and Precursor Film for CIS Synthesis," B.J. Stanbery, A.Davydov, C.H. Chang, and T.J. Anderson. AIP Conf. Proc. 394, 579-88 (1997).

6. "XPS Studies of Sodium Compound Formation and Surface Segregation in CIGS Thin Films," B.J. Stanbery, E.S. Lambers, and T.J. Anderson. Conf. Record of the $26^{\text {th }}$ IEEE PVSC (1997). 
Title: Experimental Study of Photodegradation in a-Si:H Solar Cells*

Organization: Department of Physics \& Astronomy, University of North Carolina at Chapel Hill, Chapel Hill, North Carolina

Contributors: Daxing Han, principal investigator; L. E. McNeil, co-principal investigators; X.H. Geng, L. Wu, J. Baugh

\section{Introduction}

The goal of this research project is to investigate the mechanism of the photodegradation in a-Si:H solar cells. Our research project is primarily aimed at gaining a better understanding of the relationship between material characteristics and solar cell performance. The evaluation of amorphous-silicon-based materials and devices by electroluminescence (EL), transient-current method and nuclear magnetic resonance spectroscopy (NMR) at the University of North Carolina is of vital interest to the national amorphous silicon research team. During the contract year of 1997 we are continuing vigorous collaborative efforts: with Qi Wang et al. at NREL on photodegradation studies of $\mathrm{H}$-diluted and non-diluted solar cells by $\mathrm{E}(\mathrm{x})$; with Yue Wu in our department to study the hydrogen microstructure by NMR; with S. Nitta at Gifu University, Japan, we have started the stress measurements by a beam-bending method. Five papers have been published or are to be published and two abstracts have been submitted as a result of this year's research.

Luminescence in amorphous silicon p-i-n diodes under double injection- dispersivetransport-controlled recombination ${ }^{1,2}$

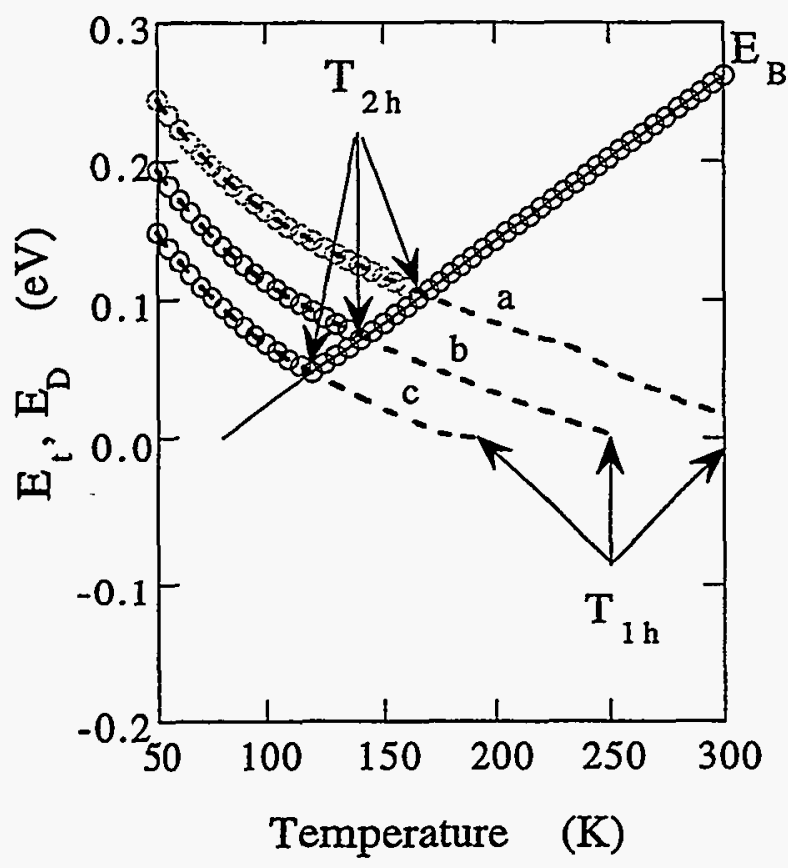

Fig. 1 The calculated EL peak energy position as a function of temperature

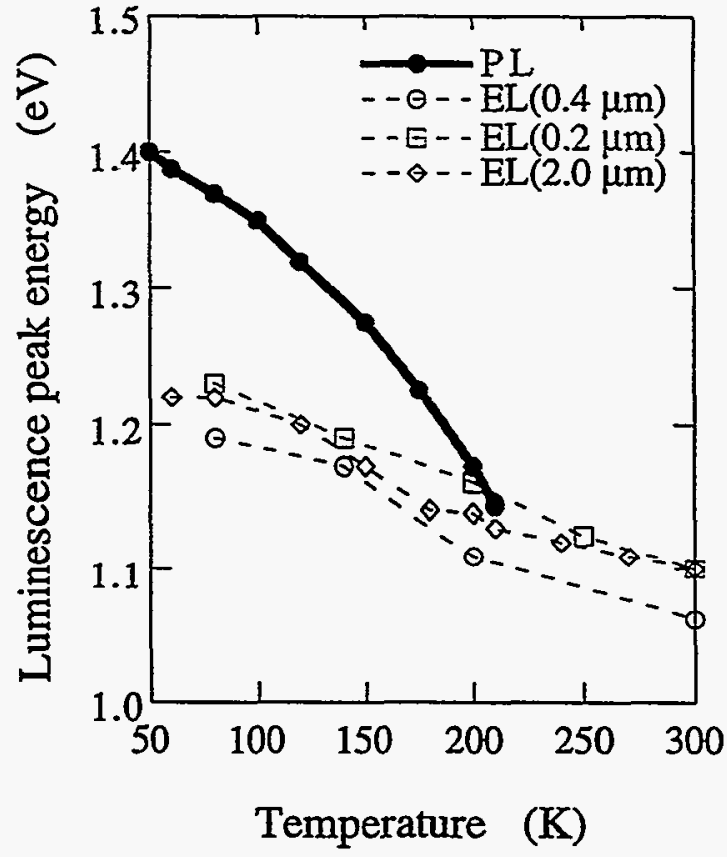

Fig. $2 \mathrm{EL}_{\text {peak }}$ as a function of temperature. the $\mathrm{PL}_{\text {peak }} \mathrm{vs.} \mathrm{T}$ curve as comparison.

*NREL Subcontract No. Xan-4-13318-09 
Based on systematic studies, 1,2 we have developed a complete model to explain the features of the EL as dispersive-transport-controlled non-geminate recombination processes. This model can explain the main features of the EL not only in a-Si but also in other types of trap-rich materials such as why is the EL peak energy always lower than that of PL?

Both the current and the EL efficiency temperature dependence show three regions depending on either hopping-controlled or multiple-trapping or ballistic transport mechanisms. Fig. 1 shows the calculated critical energy positions $E_{t}(T)$ and $E_{D}(T)$ for holes as a function of temperature. $\mathrm{E}_{\mathrm{V}}$ indicates the valence-band edge. The open circles indicate the tunneling transition energy for $E_{\text {peak. }}$. The cross point of $E_{t}(T)$ and $E_{D}(T)$ indicate the temperature, $T_{2 h}$, where the carrier transport mechanism changes. $T_{1 h}$ indicates the temperature where the demarcation level has reached the bottom of the tail.

The dashed lines $a, b$, and c correspond to the electric field 2,4 , and $6 \times 10^{4} \mathrm{~V} / \mathrm{cm}$. Fig. 2 shows the EL peak energy as a function of temperature. Clearly, the EL peak has much weaker temperature dependence comparing to PL peak. Fig. 4 plots EL effective efficiency $\mathrm{I}_{\mathrm{EJ}} / \mathrm{I}_{\mathrm{F}}$ as a function of temperature for a $0.5 \mu \mathrm{mp}-\mathrm{i}-\mathrm{n}$ diode at $2.0,3.0$, and $4.0 \mathrm{~V}$ bias. As temperature increases there are three regimes in the $I_{E L} I_{F}$ vs. $T$ curves separated by the transition temperatures $T_{1 h}$ and $T_{2 h}$.

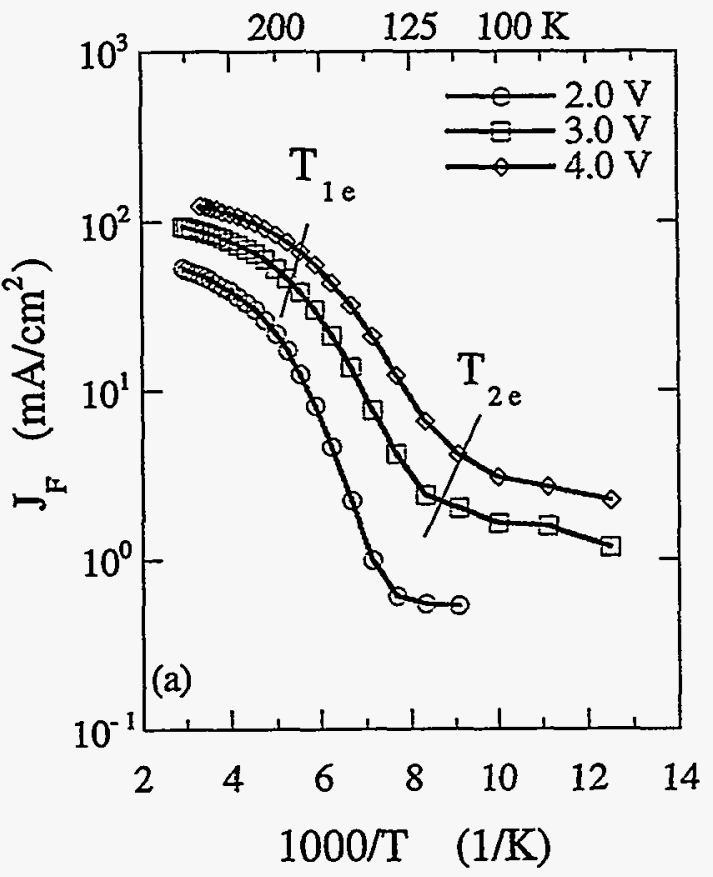

Fig. 3a Forward current as a function of temperature from a $0.5 \mu \mathrm{m}$ p-i-n cell in initial state at 2.0,3.0 and $4.0 \mathrm{~V}$.

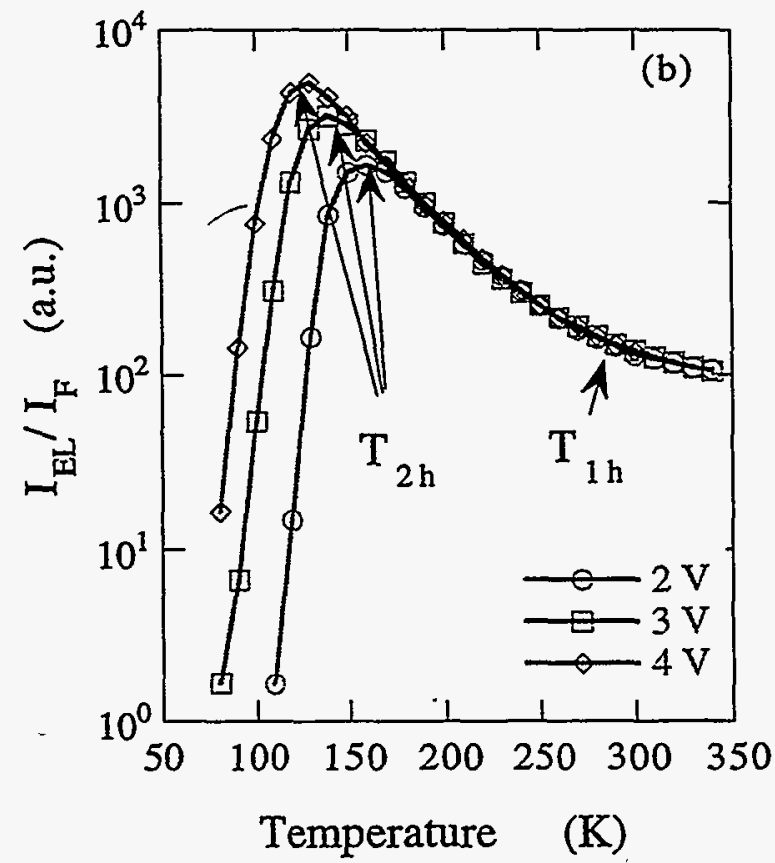

Fig. $3 b$ EL efficiency as a function of temperature from a $0.5 \mu \mathrm{m} \mathrm{p}-\mathrm{i}-\mathrm{n}$ cell in initial state at $2.0,3.0$ and $4.0 \mathrm{~V}$.

By employing the forward current and EL temperature dependence studies information of both the localized tail states and the deep defect states in real solar cell structures were obtained concomitantly, that is crucial for the device performance. We shown evidence of the light-induced metastable increase of the $\mu_{n} \tau_{n}$ activation energy and the changes of the EL main-band spectrum lineshape that indicate a light-induced band tail broaden, especially the deeper part of the tail states. In thin p-i-n cells the defect EL intensity is much stronger than that of PL, so the room temperature EL spectroscopy is a tool to study photodegradation kinetics in solar cells. 


\section{The effects of hydrogen dilution on $\mathrm{V}_{\text {oc }}$ in a-Si:H pin solar cells 3,4}

In collaboration with Qi Wang and Dick Crandall in NREL, we studied the internal electric field profile in a-Si:H diodes by the transient-null-current method, and the effects of $\mathrm{H}$-dilution on $\mathrm{V}_{\mathrm{oc}}$ in a-Si: $\mathrm{H}$ p-i-n solar cells. We found that $\mathrm{H}$-dilution does stabilize

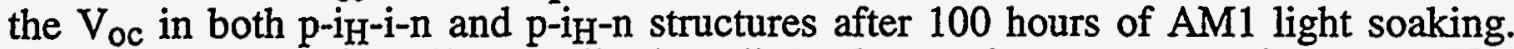
These two types of $\mathrm{H}$-diluted cells show little changes in current at voltage near $\mathrm{V}_{\mathrm{OC}}$ before and after light-soaking, while the non $\mathrm{H}$-diluted $\mathrm{p}$-i-n cell does show a noticeable change. A stronger electric field starting from $\mathrm{p}$ - $\mathrm{i}$ interface is found for both $\mathrm{H}$-diluted cells compared to the non H-diluted p-i-n cell. Furthermore, there are no measurable changes in the field profile after 100 hour AM1 light-soaking for all three types of cells. All these suggest that an increase of the field strength near p-i interface is crucial for a more stable $\mathrm{V}_{\text {oc }}$ in H-diluted cells.

\section{An NMR investigation of $\mathrm{H}$ cluster configurations in a-Si: $\mathrm{H}^{\mathbf{5}}$}

Numerical simulations of free-induction decays (FID) of ${ }^{1} \mathrm{H}$ NMR signals have been done for various $\mathrm{H}$ cluster configurations. The observed FID oscillation of the ${ }^{1} \mathrm{H}$ NMR signal in hot-wire a-Si:H is shown to result from a high degree of order in the nearest-neighbor distances between $\mathrm{H}$ atoms in the $\mathrm{H}$ clusters; such FID oscillation is not visible in GD materials indicating a larger variation in local environment. Numerical simulations of MQ NMR spectra based on several $\mathrm{H}$ cluster configurations were also studied. These calculations show that MQ NMR spectra of the relaxed hydrogenated divacancy model fit the observed MQ NMR spectra in device quality GD a-Si:H. This is clear evidence of improved structural order in hot filament assisted CVD a-Si: $\mathrm{H}$. which is an important factor leading to more stable materials.

Figure 4 (a) shows the FID's of the hot-wire and the GD samples. Both FID's consist of a rapid intensity decay in the initial $40 \mu$ s followed by a slow decay. The initial rapid decay is attributed to Si-H clusters whereas the slow decay is attributed to dispersed $\mathrm{Si}-\mathrm{H}$ bonds. An interesting feature is the oscillatory behavior of the FID signal $F(t)$ near $t=25$ $\mu \mathrm{s}$ in the hot-wire sample. Subtracting the component of the slow decay, the intensity of the rapidly decaying component is seen to approach first a minimum (negative or close to zero) near $\mathrm{t}=25 \mu \mathrm{s}$, recovering slightly after that, and then finally decays to zero at longer $t$. This behavior is more apparent in the expanded linear plot of FID shown in Fig. 4 (b). Such oscillatory behavior is less obvious in the FID of the GD sample.
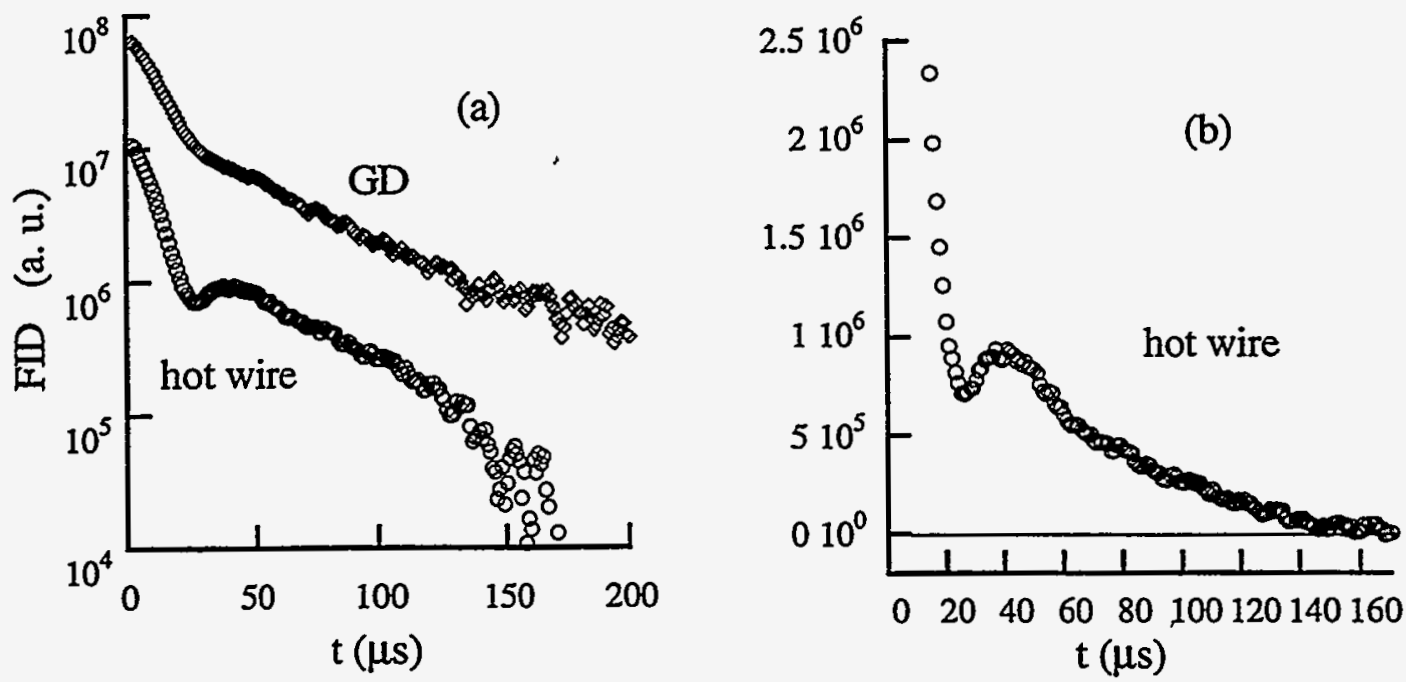

Fig. 4 (a) FID's of ${ }^{1} \mathrm{H}$ signals in GD and hot-wire a-Si:H films. (b) Expanded linear plot of the FID of the hot-wire sample. 
Fig. 5 shows the calculated FID's for a cubic and a relaxed hydrogenated divacancy configuration. The calculated FWHM values are comparable to the experimentally observed widths of ${ }^{1} \mathrm{H}$ spectra. In the calculated FD's for the cubic configuration shown in Fig. 5(a), oscillations are clearly visible.
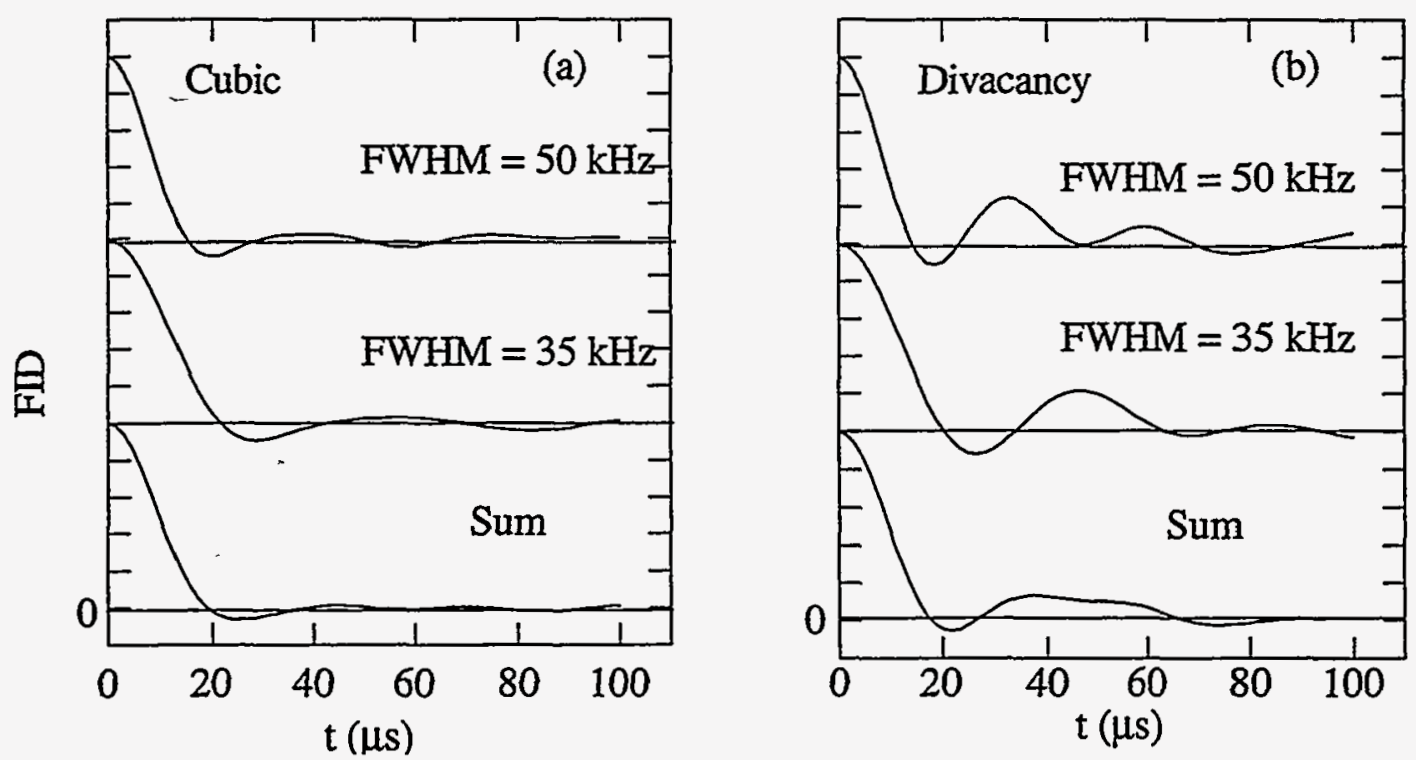

Fig. 5 The calculated FID's for the cubic (a) and the relaxed hydrogenated divacancy (b) configurations. All FID's are powder averages over 32 random orientations.

\section{a-Si Industry Interactions}

CRADA activities were carried out with Solarex.

\section{References}

1. Daxing Han, Keda Wang, and Bolko von Roedern, Phys. Rev. lett., 77, (1996) 4410.

2. Daxing Han, Keda Wang, Chenan Yeh, Liyou Yang, Xunming Deng, and Bolko Von Roedern, "Luminescence in amorphous silicon $\mathrm{p}-\mathrm{i}-\mathrm{n}$ diodes under doulble injection," Phys. Rev. B 5515619 (1997).

3. Qi Wang, R. S. Crandall, and Daxing Han, "The effect of hydrogen dilution on $V_{o c}$ in a-Si:H pin solar cells," a-Si Technology-1997, edited by M. Hack, E. A. Schiff, R. Schropp, and I. Shimizu (MRS-97 Symposium Proc.)

4. Qi Wang, Keda Wang, C.N. Yeh, and Daxing Han, "Internal electric field profile in thin film hydrogenated amorphous silicon junctions using transient-null-current method," ibid.

5. J.T. Stephen, Yue Wu, J. M. Rutland, and Daxing Han, "An NMR investigation of $\mathrm{H}$ cluster configurations in a-Si:H, " ibid.

6. Daxing Han, T. Gotoh, Motoi Nishio, S. Nonomura, S. Nitta, Q. Wang, and E. Iwaniczko, "Correlation of stress with hydrogen micro-structure in Thin Film Hydrogenated Amorphous Silicon," to be published in MRS-97 proc.

7. Xinhua Geng, Xunming Deng, Qi Wang, and Daxing Han, "Internal Electric Field Profile of a-SiGe p-i-n Solar Cells,"submit to MRS-98 spring meeting.

8. Guozhen Yue, Jonathan Baugh, Liangfan Chen, Qi Wang, Eugene Iwaniczko, Guanglin Kong, Yue Wu, Daxing Han, "Light-induced change of Si-H bond absorption in Hydrogenated Amorphous Silicon," abstract submit to MRS-98 spring meeting. 
Title: Microscopic Origins of Metastable Effects in a-Si:H and Deep Defect Characterization in a-Si,Ge:H Alloys

Organization: University of Oregon, Eugene, Oregon

Contributors: J. David Cohen, principal investigator; Yoram Lubianiker, Daewon Kwon, and Chih-Chiang Chen

The primary research goals of this program are to elucidate the basic mechanisms by which a-Si:H degrades with light exposure, and to study the defect structure in low bandgap a-Si,Ge:H alloys. During the past year we have carried out the following studies: (1) A study of intrinsic and very lightly doped a-Si,Ge:H glow discharge samples produced at United Solar Systems Corporation indicating the presence of charged deep defects in those samples; and (2) A study of light-induced degradation amorphous silicon produced by DC reactive magnetron sputtering. These films were found to contain a small microcrystalline component.

\section{Approach and Results}

Our first study relevant to the low band-gap a-Si,Ge:H alloys examined nine films deposited by the if glow discharge method at United Solar Systems Corporation (courtesy of J. Yang and S. Guha). Seven of the samples had Ge fraction in the range 30 to 35 at.\% (most suitable for cells) while the remaining two samples had $\mathrm{Ge}$ fractions near 20at.\% and 50at.\%, respectively. Of the seven $30-35 \mathrm{at} . \% \mathrm{Ge}$ samples, three of these were intrinsic, two were accidentally very slightly n-type (or " $v$-type"), one was intentionally n-type doped with $\mathrm{PH}_{3}$ at a level of $2 \mathrm{Vppm}$, and one was intentionally p-type doped with $\mathrm{BF}_{3}$ at a level of $6 \mathrm{Vppm}$. For each sample we carried out sub-band-gap photocapacitance (TPC) and transient photocurrent (TPD) spectroscopy to examine the optical transitions associated with deep defects [1], and also drivelevel capacitance profiling (DLCP) measurements to deduce the thermal transitions associated with the defects.[2] Samples were examined in a dark annealed state, as well as a light soaked state produced by exposure from 70 to 100 hours to a red filtered tungsten halogen light source at an intensity of $6 \mathrm{~W} / \mathrm{cm}^{2}$.

Figure 1(a) shows the TPC and TPI spectra for the 2 Vppm n-type doped sample. Here the spectra can be fit using only a single defect sub-band with electronic excitations to the conduction band. This sub-band should correspond to $\mathrm{D}^{-}$centers because these dominate at even moderate n-type doping levels. Because only an electron current results from the optical transitions of this defect sub-band, both the TPI and TPC spectra are seen to overlap perfectly outside of the band tail regime. Figure 1(b) shows the optical spectra obtained at 350K for the 6 Vppm p-type doped sample. Here we also find only a single defect sub-band of optical transitions is needed to fit the spectra. This now corresponds to $\mathrm{D}^{+}$defects and it is now the majority hole current induced by the optical transition that is responsible.for the TPI and TPC signals (this is confirmed by the sign of the TPC signal). The perfect overlap of these two spectra outside the band tail region verifies that there is only a single type of defect transition involved.

Figure 1(c) shows a typical spectra for a 35 at.\% Ge intrinsic sample. Here things have obviously become more complex. Indeed, we now need two bands of defect transitions to fit the spectra. One corresponds to optical transitions which remove an electron from a defect sub-band into the conduction band, while the other corresponds to transitions which insert a valence band electron into an unoccupied defect sub-band. We have previously denoted these two bands of optical transitions as "OP1" and "OP2", respectively.[3 ] Like the case for the n-type film, the 
FIG. 1. Pairs of photocapacitance and photocurrent spectra for three 35at.\% a-Si,Ge:H samples: (a) n-type doped, (b) p-type doped, and (c) intrinsic. The thin lines through the data points are fits using the types of optical transitions indicated below each set of spectra (together with an exponential distribution of bandtail states). The close match of the energy distribution of the OP2 transition to the $\mathrm{D}^{+}$band in the p-type sample indicates a significant density of $D+$ defects in the intrinsic sample.
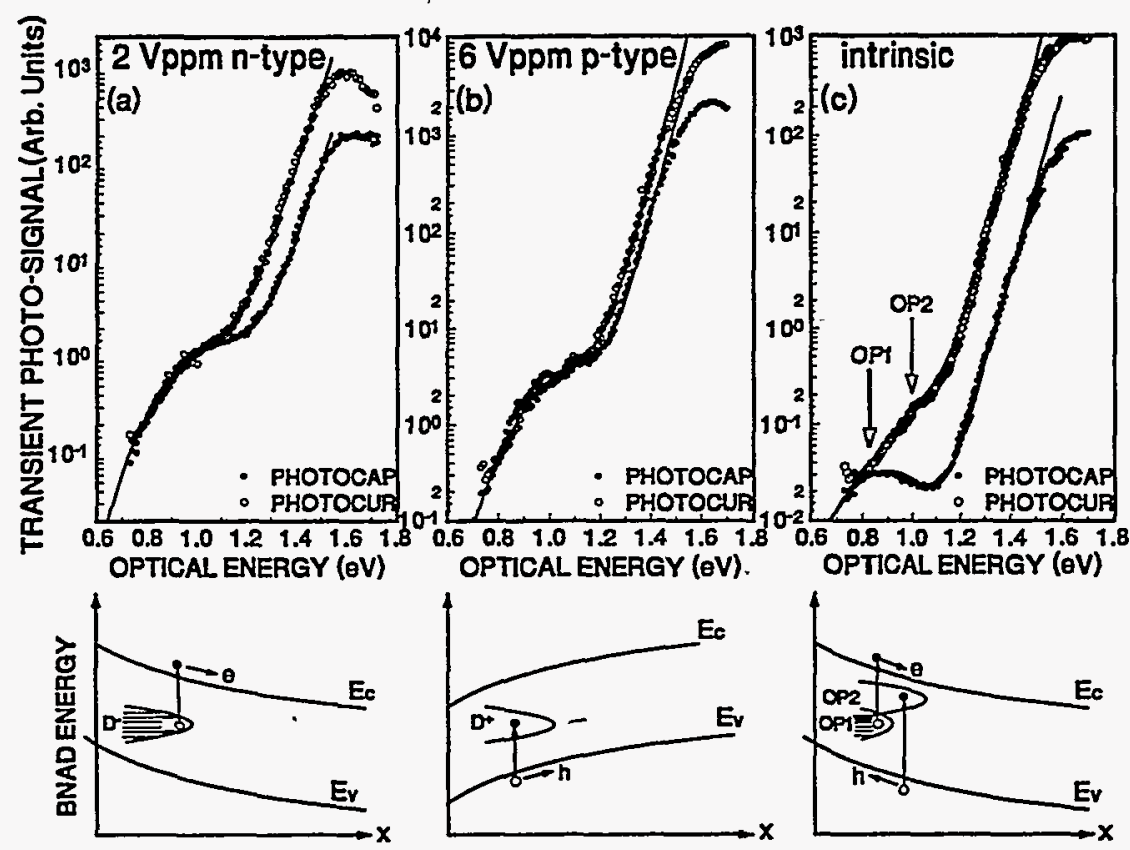

major depletion region for the intrinsic samples is at the substrate junction with an upward band bending. Also, because the Fermi level is slightly closer to the conduction band, the optically induced current at threshold will be totally due to electrons excited out of defect levels. Therefore, the TPC and TPI spectra should be strictly proportional at these lowest optical energies and so we overlap them in this region. While the optical transitions for the OP1 subband will continue to contribute equally to the TPC and TPI spectra, the hole current arising from OP2 sub-band of optical transitions will contribute oppositely to the TPC and TPI signals.[1,3] This results in the large separation of the two spectra at intermediate optical energies.

Comparing the bands of optical transitions for these samples indicates that the OP2 subband is nearly identical in energy to the $\mathrm{D}^{+}$defect sub-band in the p-type sample. This identifies OP2 with the $\mathrm{D}^{+}$sub-band. Therefore, these optical spectra clearly demonstrate that there are significant positively charged defects in intrinsic glow discharge a-Si,Ge:H alloys. Because of charge neutrality, there must also exist similar densities of negatively charged defect states in these samples. However, the OP1 sub-band of the intrinsic samples has a different shape and energy position compared to the $D^{-}$defect sub-band in the n-type sample. Therefore, the OP1 sub-band is probably not a reliable indicator of the magnitude of the $\mathrm{D}^{-}$defect sub-band (that is, it more likely involves transitions from a superposition of the $\mathrm{D}^{-}$and $\mathrm{D}^{\circ}$ sub-bands).

To obtain a quantity more indicative of the $\mathrm{D}^{-}$concentrations we turned to the DLCP method. This depends on the thermal emission of trapped charge to the majority carrier band so that the detected transitions (denoted as "TH1") in intrinsic and n-type samples correspond to deep defects containing at least one electron. Moreover, because electrons bound to $\mathrm{D}^{-}$defects are more easily thermally emitted, TH1 is more likely, to be a good indicator of the $\mathrm{D}^{-}$defect density. Indeed, Fig. 2 shows that, for the $35 \mathrm{at} . \%$ Ge samples, the TH1 density increases as the n-type doping level increases. In Fig. 2 we have also plotted the ratio of OP2/TH1 for all the samples and see that it is nearly identical for all intrinsic samples in the annealed state and also in the light soaked states. This correlation strongly suggests that the TH1 defect sub-band indeed corresponds to $\mathrm{D}^{-}$centers by charge neutrality. Also, we see that the OP2/TH1 ratios become smaller for the $v$-type samples as would be expected for the $\mathrm{D}^{+} / \mathrm{D}^{-}$ratios. 
FIG. 2. Bar graphs indicating magnitudes of the DLCP determined TH1 band in the annealed and light soaked sates (left-hand logarithmic scale) along with the OP2/TH1 ratios in each case (right-hand linear scale). The nearly constant ratio for the intrinsic samples, and the decreasing ratio for the $v$ and n-type samples, strongly suggests that $\mathrm{TH} 1$ is associated with the Dsub-band.

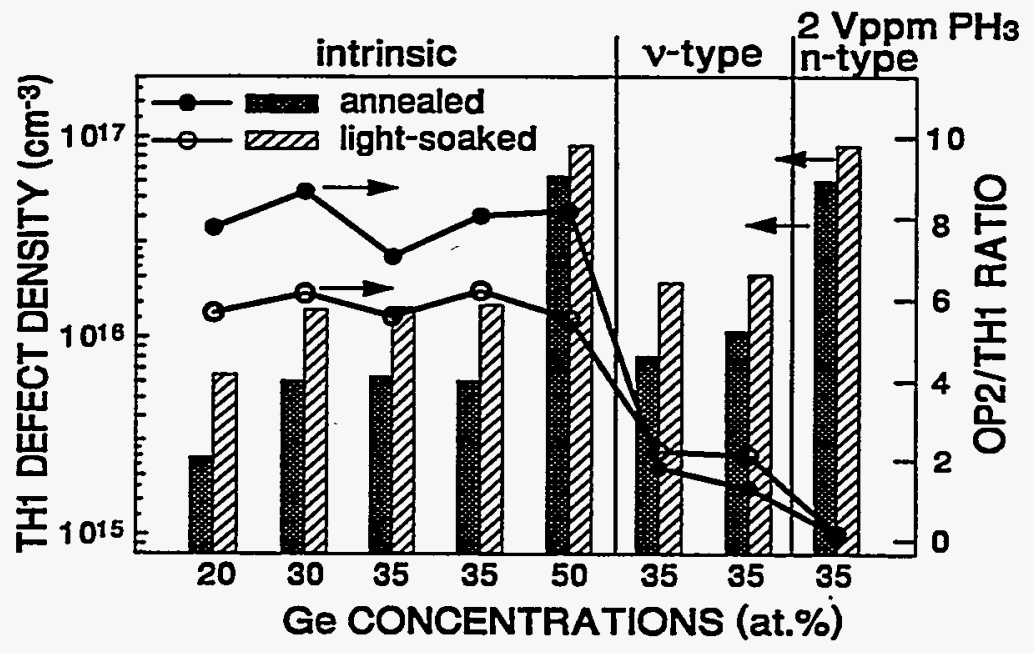

The inferred presence of charged defect states in the a-Si,Ge:H alloys, and the fact that their ratios appear to change as a result of light soaking, is clearly very important regarding light induced degradation of photovoltaic devices fabricated from these alloys. The existence of such concentrations of charged defects even in the most intrinsic a-Si, $\mathrm{Ge}: \mathrm{H}$ alloys implies that these definitely-need to be included in future device modeling studies.

Our second area of study has been concerned with the stability properties of a-Si:H samples produced by DC reactive magnetron sputtering. These samples were grown at the University of Illinois in collaboration with John Abelson's group. He had previously determined that, by varying the $\mathrm{H}_{2}$ pressure inside the sputtering chamber, films of varying degrees of microcrystallinity could be produced.[4] Following reports from Uni-Solar that a-Si:H films grown close to the onset of microcrystallinity exhibits a higher degree of stability [5], we decided it would be interesting to study the degradation kinetics of such films in more detail. We thus examined several such films exhibiting crystalline volume fractions below about $10 \%$ as estimated using Raman spectroscopy (see Fig. 3)

We studied the degradation of this film following various degrees of illumination to redfiltered tungsten-halogen light at three intensities $\left(0.8,1.4\right.$, and $\left.2.2 \mathrm{~W} / \mathrm{cm}^{2}\right)$ for up to 160 hours. The sample was kept submerged in methanol during illumination to maintain its surface temperature below $65^{\circ} \mathrm{C}$. At various stages of light soaking the deep defect density was examined using drive-level capacitance profiling (DLCP). These profiles were obtained at $11 \mathrm{~Hz}$ using a measurement temperature of $350 \mathrm{~K}$. Under such conditions we have found that, by doubling the actual measured DLCP defect density, very good quantitative agreement is obtained to values of deep defects in a-Si:H determined by ESR measurements.

Figure 4 summarizes the varia-

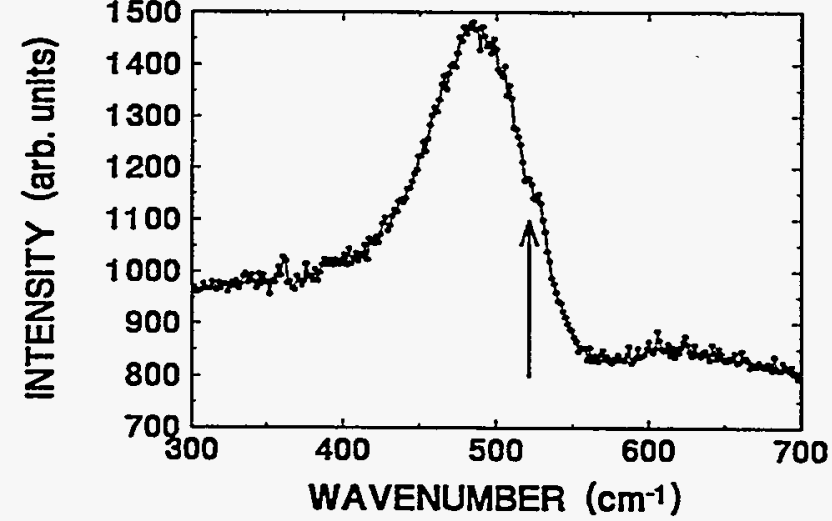

FIG. 3. Raman spectrum for University of Illinois, dc sputtered sample, indicating a small microcrystalline component at $522 \mathrm{~cm}^{-1}$. (arrow) 
tion of the (spatially averaged) defect density with light exposure at three different light intensities. Note that the saturated defect densities in this sample are quite low, below $10^{16} \mathrm{~cm}^{-3}$ for light intensities of several suns. All of these degradation curves also have the unusual characteristic that, initially, the increase in defect density is very slow. Then, at a threshold which is different at each intensity, the defect density begins to increase much more rapidly and eventually saturates.

One might be tempted to infer two types of metastable defect populations from such degradation kinetics, "fast" and "slow" defects that get created at different rates, as had

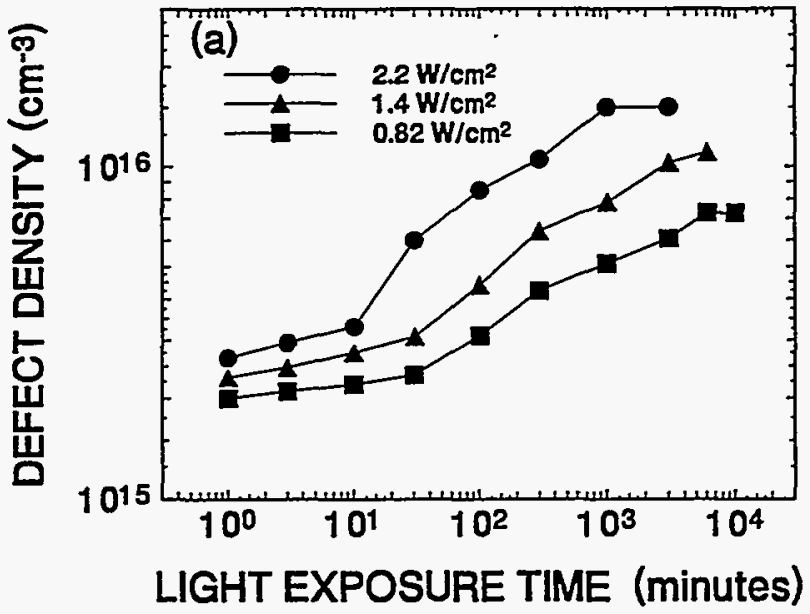

FIG. 4. Defect densities deduced from drivelevel capacitance profiling measurements vs. light exposure time at three light intensities. been proposed previously [6]. There are at least two arguments against this. First, the number of defects created in the first (fast) phase of degradation is not fixed but depends (roughly linearly) on the light intensity. Second, the annealing behavior appears quite normal and does not at all suggest two populations of metastable defects with different annealing properties. Instead, we believe that the unusual degradation kinetics indicated in Fig. 4 is directly linked to the microcrystalline component in this sample. Specifically, we believe the initial slow rate of degradation is limited by recombination through states associated with the microcrystallites. Then, when the density of light induced defects in the a-Si:H is high enough to shift the quasi-Fermi level across the energy level of the microcrystalline states, the recombination becomes dominated by the a-Si:H defects. Thus, the threshold should occur at a higher defect density when a higher intensity light source is used. After this point the recombination kinetics become "normal" $t^{1 / 3}$. We are currently in the process of trying to develop a more detailed model to account for other aspects of the variation in degradation rates due to the presence of these microcrystallites.

\section{References}

1. A.V. Gelatos, K.K. Mahavadi, and J.D. Cohen, Appl. Phys. Lett. 53, 403 (1988).

2. C.E. Michelson, A.V. Gelatos, and J.D. Cohen, Appl. Phys. Lett. 47, 412 (1985).

3. F. Zhong, J.D. Cohen, J. Yang, and S. Guha, Mat. Res. Soc. Symp. Proc. 336, 493 (1994).

4. G. Feng, M. Katiyar, Y.H. Yang, J.R. Abelson, and N. Maley, Mat. Res. Soc. Symp. Proc. 258, 179 (1992).

5. D.V. Tsu, B.S. Chao, S.R. Ovshinsky, S. Guha, and J. Yang, Appl. Phys. Lett. 71, 1317 (1997).

6. Liyou Yang and Liang-Fan Chen, Mat. Res. Soc. Symp. Proc. 336, 669 (1994). 
Title:

Advanced Processing Technology for High Efficiency Thin-Film CuIn $\mathrm{Ga}_{1-\mathrm{x}} \mathrm{Se}_{2}$ and CdTe Solar Cells; Heterojunction Development and Optimization in Thin-Film Compound Semiconductor Solar Cells

Organization: Department of Electrical Engineering

Center for Clean Energy and Vehicles

University of South Florida

Tampa, FL

Contributors: D. L. Morel and C. S. Ferekides, principal investigators

R. Bhatt, , S. Jagannathan, A. Jayapalan, R. Kada, V. Komin, H. Lin, D. Marinsky,

S. Marinskaya, V. Palekis, U. Poosarla, P. Selvaraj, H. Sankaranarayan,

M. Shankaradas, B. Tetali, V. Viswanathan, S. Zafar, and Z. Zhao, graduate assistants

$$
\text { CuIn }_{x} \text { Ga1-x Se2 }_{2}
$$

\section{Objectives}

The objectives of this project are to develop improved processing techniques for $\mathrm{CuIn}_{\mathrm{x}} \mathrm{Ga}_{1-\mathrm{x}} \mathrm{Se}_{2}$ (CIGS) solar cells and to correlate performance and processing mechanisms.

\section{Approach}

Device properties are determined by two primary process variables, the precursor and the anneal profile. The precursor consists of layers of $\mathrm{Cu}, \mathrm{In}, \mathrm{Ga}$, and $\mathrm{Se}$ which are deposited in various sequences. All of the precursor components are deposited at temperatures typically below $275^{\circ} \mathrm{C}$. Fabrication is completed by annealing the precursor with a temperature ramp up to $550^{\circ} \mathrm{C}$ followed by a cool-down to room temperature. During the anneal profile Se fiux is applied as an additional variable. During this period we focused our efforts on developing a low Eg high Jsc bulk material. By establishing a consistent base material we could then turn our attention to optimization of surface properties.

\section{Results}

A key objective which we obtained during this period is the ability to separately form bulk and surface properties. This allows experimentation with deposition parameters at the end of the run to optimize surface properties without adversely affecting the already established bulk properties. The performance of the bulk is represented by Jsc. In Table 1 we show the behavior of Jsc for a series of runs conducted over a two month period. For these devices the band gap is $0.95 \mathrm{eV}$ even though the precursor contains about $10 \% \mathrm{Ga} /(\mathrm{In}+\mathrm{Ga})$. Missing runs 21,22 and 30 were a different process recipe or were runs

\begin{tabular}{lllllllllll}
\hline Run & 19 & 20 & 23 & 24 & 25 & 26 & 27 & 28 & 29 & 31 \\
\hline Jsc $\left(\mathrm{mA} / \mathrm{cm}^{2}\right)$ & 39.2 & 39.0 & 40.0 & 40.5 & 40.1 & 38.6 & 39.7 & 40.3 & 40.5 & 40.7 \\
\hline
\end{tabular}

Table 1. Jsc data for a sequence of runs showing the consistency over a two month period. 
for which there was an equipment failure and are not part of the sequence. The devices do not have AR coatings. The consistently high Jsc's over this sequence of ten runs clearly demonstrates the heartiness of this process. We also note that in many of these runs process parameters at the end of the run were intentionally varied to improve Voc. The fact that the Jsc's consistently stayed in the same (high) range even though we varied the parameters at the end of the run not only demonstrates reproducibility, but also that the surface and bulk can be independently optimized.

A key to optimization of surface properties is the incorporation of small amounts of $\mathrm{Ga}$ in the space charge region. In Figure 1 we show an abrupt change in band gap in the middle of a $5 \times 5$ array of devices from a single run. The location of the devices is with respect to the In and Se sources. $\mathrm{Cu}$ is opposite In and $\mathrm{Ga}$ is opposite Se. Surface properties are found to be sensitive to these small changes in composition. Through optimization of these techniques we are making ongoing improvements in device efficiency. Our best device to date has an efficiency of $13 \%$. Through further refinements of these procedures we expect to reach the $15-16 \%$ range using our manufacturing-friendly processing procedures.

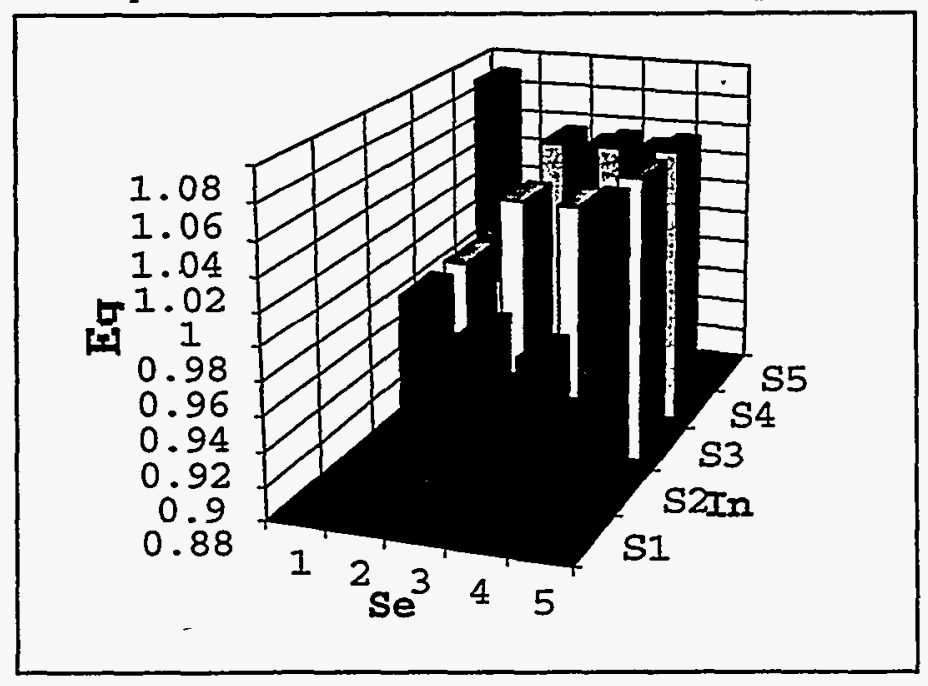

Figure 1. Energy gap vs. position relative to In and Se sources.

CdTe

\section{Objectives}

The primary focus of this project is to develop improved deposition technologies for the manufacture of CdTe solar cells. The areas being addressed are: (a) the use of soda lime glass substrates, (b) cell fabrication at temperatures below $550^{\circ} \mathrm{C}$, (c) alternative TCO's and window layers, and (d) improved back contacts.

\section{Technical Approach}

The table shown below outlines the various processing and device options utilized for the fabrication of CdTe solar cells. The close spaced sublimation (CSS) is the primary deposition technology used for the CdTe and window layers. T1, T2, and T3 (column four) indicate the three temperature regimes partially optimized for the CdTe CSS process.

\begin{tabular}{|l|l|l|l|l|}
\hline Substrates & TCO's & Window Layers & CdTe - CSS & Back Contacts \\
\hline soda lime & $\mathrm{SnO}_{2}$ & $\mathrm{CdS}: \mathrm{CSS}, \mathrm{CBD}$ & $\mathrm{T} 1: \geq 600^{\circ} \mathrm{C}$ & Doped Graphite \\
\hline borosilicate & $\mathrm{ZnO}$ & $\mathrm{ZnSe}: \mathrm{CSS}$ & $\mathrm{T}::^{\prime} 500-560^{\circ} \mathrm{C}$ & Vacuum Deposited $\mathrm{Cu}_{\mathrm{X}} \mathrm{Te}$ \\
\hline & $\mathrm{Cd}_{2} \mathrm{SnO}_{4}$ & $\mathrm{Cd}_{1-\mathrm{X}} \mathrm{Zn}_{\mathrm{X}} \mathrm{S}:$ sputtering & $\mathrm{T} 3: \leq 500^{\circ} \mathrm{C}$ & \\
\hline
\end{tabular}

Table 2. Processing options used for the fabrication of CdTe solar cells. 


\section{Results and Discussion}

The effect of deposition parameters (such as ambient and substrate temperature) and post deposition heat treatments on the properties of CSS CdS films has been studied. Device quality films can be prepared using a wide range of deposition conditions in combination with a heat treatment. Emphasis is always placed on depositing CdS films of small thicknesses in order to enhance the $\mathrm{J}_{\mathrm{sc}}$ of solar cells. Based on the results obtained to date it appears that (a) the presence of $\mathrm{O}_{2}$ during the CSS CdS process is beneficial to device performance, (b) the smallest thickness of the CSS CdS films for state of the art $V_{o c}$ 's and ff's is larger than that of CBD CdS films and (c) the interdiffusion between CdS and CdTe is considerably less when CSS CdS is used (vs. CBD CdS). The best device efficiencies achieved for $\mathrm{CdTe}(\mathrm{CSS}) / \mathrm{CdS}$ (CSS) devices are $15.0 \%$ and $14.3 \%$ (fig. 2) for borosilicate and soda lime glass(T2) substrates respectively.

Devices prepared using sputtered $\mathrm{ZnO}$ as the TCO have demonstrated state of the art $\mathrm{V}_{\propto c}$ 's $(>830 \mathrm{mV})$ but efficiencies have been low (up to $11 \%$ ) due to poor ff's. The main limitation has been the stability of the $\mathrm{ZnO}$ films when these are subjected to the subsequent cell fabrication steps (mainly the $\mathrm{CSS}$ process). Typically the resistivity of $\mathrm{ZnO}$ increases considerably (at least five times) leading to high series resistances. The highest ff's obtained to date are $65 \%$.

Zinc selenide films are also being deposited by CSS. CdTe/ZnSe junctions have been fabricated using CdTe conditions previously optimized for $\mathrm{CdTe} / \mathrm{CdS}$ solar cells. Device performance has only reached the $11 \%$ level with $V_{o c}$ 's, $J_{S c}$ 's and $\mathrm{ff}$ 's being in the range of $560-765 \mathrm{mV}, 16-25$

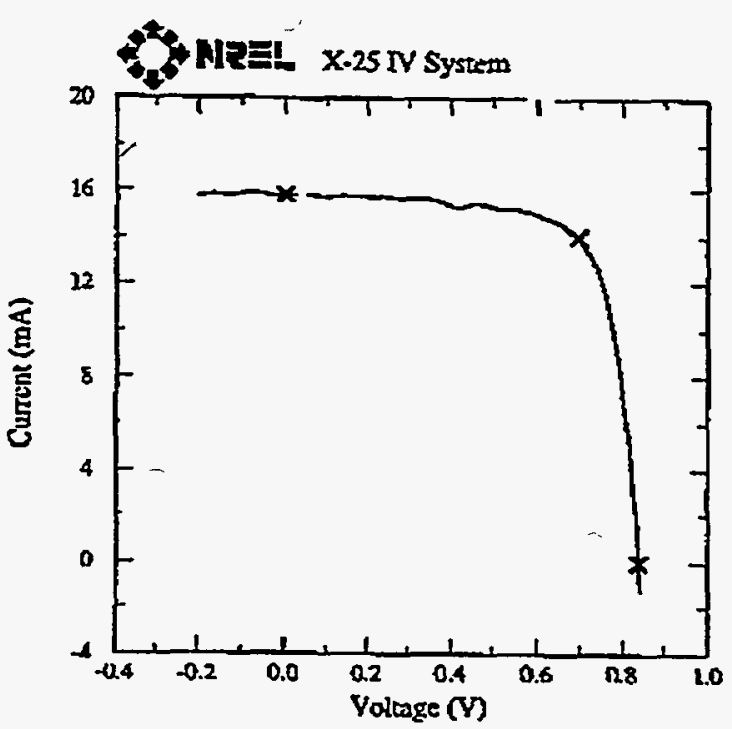

Figure 2. Light I-V of a $14.3 \%$ all CSS CdTe/CdS solar cell, prepared on soda lime glass. $\mathrm{mA} / \mathrm{cm}^{2}$, and $30-65 \%$ respectively. For further improvements it will be necessary that the CSS-CdTe deposition is optimized for these type of junctions.

All vacuum processed back contacts based on $\mathrm{Cu}_{\mathrm{x}} \mathrm{Te}$ compounds are being developed and optimized. $\mathrm{Rf}$ sputtering is being used for the deposition of $\mathrm{Cu}_{\mathrm{x}} \mathrm{Te}, \mathrm{Ni}$, and $\mathrm{Mo}$. To date this work has focused on understanding the effect of various processing parameters, such as deposition temperature and post deposition heat treatments, in an effort to improve device performance. Near $15.0 \%$ device efficiencies $\left(V_{o c}=838 \mathrm{mV}, \mathrm{J}_{\mathrm{sc}}=23.77 \mathrm{~mA} / \mathrm{cm}^{2}, \mathrm{ff}=75.0 ; \eta=14.9 \%\right)$ have been verified at NREL using this contact approach. The table shown below lists the performance of a set $\mathrm{CdTe} / \mathrm{CdS}$ cells fabricated to demonstrate the reproducibility of this contact technique.

\begin{tabular}{|c|c|c|c|c|}
\hline Sample \# & $\mathbf{V}_{o c}(\mathbf{m V})$ & $\mathbf{J}_{\infty}\left(\mathbf{m A} / \mathbf{c m}^{2}\right)$ & $\mathbf{f f}, \mathbf{( \% )}$ & Efficiency \\
\hline 923A11B & 834 & 22.5 & 73.5 & 13.8 \\
\hline 103B7A & 844 & 22.3 & 74.6 & 14.1 \\
\hline 103B7C & 839 & 22.3 & 74.7 & 14.0 \\
\hline 103B8A & 837 & 22.5 & 74.3 & 14.0 \\
\hline
\end{tabular}

Table 3. Solar cell performance for devices fabricated with $\mathrm{Cu}_{\mathbf{x}} \mathrm{Te}$ based contacts. 


\section{Heterojunction Characterization and Development}

\section{Objectives}

The objectives of this project are to advance understanding of heterojunction formation and performance in CdTe and CIS devices and to use that understanding to guide further development of the technologies.

\section{Results and Discussion}

In our in depth analysis of junction behavior in CdTe devices using the Shockley-Read-Hall(SRH) recombination model we found it necessary to extend the original theory to maintain consistency with experimental data. The original theory is based upon a single recombination level and predicts curvature in log J vs. V plots which is observed for some devices. This behavior is in fact at odds with the concept of a single valued diode factor. However, in many devices there are long flat regions which have the appearance of a single value for the diode factor. We have found that this behavior can be fit with a multiple recombination center extension of the SRH model. An example of the parameters used is shown in Table 4. In this case the ratio of the lifetimes for holes and electrons is held constant while the value

\begin{tabular}{lllllllllll}
\hline $\begin{array}{l}\text { Level } \\
(\mathrm{kT})\end{array}$ & 0 & 2 & 4 & 6 & 8 & 10 & 12 & 14 & 16 & 18 \\
\hline$\tau_{\text {po }}$ & 1 & .75 & .5 & .25 & .1 & .075 & .05 & .025 & .01 & .0075 \\
$\tau_{\text {no }}$ & 1 & .75 & .5 & .25 & .1 & .075 & .05 & .025 & .01 & .0075 \\
\hline
\end{tabular}

Table 4. Relative values of recombination lifetime $\tau$ for different trap levels.

is reduced with increasing energy from mid gap at $\mathrm{kT}=0$. This modification provides a much better fit to all data and is consistent with practical expectations for a highly disordered material.

We have also been successful in using the model to guide our development of CIGS devices. The model provides a correlation between expected Voc and interface state density. For devices with a band gap of $0.95 \mathrm{eV}$ achievement of an interface state density in the mid $1015 / \mathrm{cm}^{3}$ range is expected to result in a Voc of $530 \mathrm{mV}$. By using etching procedures to reduce interface state density we were able to increase Voc to $530 \mathrm{mV}$, although Jsc's were reduced as the etch also adversely affected bulk properties. However, the insights provided from these studies have led us to the achievement of Voc's up to $510 \mathrm{mV}$ without using etching to reduce interface states. These devices maintain the high Jsc's reported above and exhibit device efficiency up to $13 \%$. Additional insights from these modeling efforts are expected to expedite the climb to the $15-16 \%$ level indicated above. 
Title: $\quad$ High Efficiency Thin Film Cadmium Telluride Photovoltaic Cells

$\begin{array}{ll}\text { Organization: } & \begin{array}{l}\text { Department of Physics and Astronomy } \\ \text { The University of Toledo } \\ \text { Toledo, OH } 43606\end{array}\end{array}$

Contributors: Alvin D. Compaan, principal investigator; Randy G. Bohn co-investigator; Andreas Fischer (student/postdoc); Ralf Wendt (postdoc); Dan Grecu, Eugene Bykov, Xiandong Deng, Chitra Narayanswamy, David Zuo, Xianda Ma, and Konstantin Makhratchev (students)

\section{Objectives}

This project emphasizes the development of rf sputtering for the deposition of $\mathrm{CdTe}, \mathrm{CdS}$, and related materials for thin-film solar cells with the goal of exceeding 14\% AM1.5 efficiencies on soda-lime glass. In addition, we use our deposition capability and characterization facilities to contribute to CdTe teaming activities such as achieving higher currents through thinner CdS and improved contact stability.

\section{Technical Approaches}

The University of Toledo effort emphasizes if sputtering of both CdS and CdTe from compound semiconductor targets. In the previous year we reached $11.6 \%$ efficiency for a small cell in which both the CdTe and the CdS layers were deposited by if sputtering. ${ }^{1,2}$ This year we continued efforts on cell fabrication with the goal, inter alia, of using thinner CdS layers. We have fabricated a number of cells using a thin layer of intrinsic $\mathrm{SnO}_{2}$ between the LOF $\mathrm{SnO}_{2}: \mathrm{F}(8 \Omega /$ square) and the CdS layer. We deposited the intrinsic $\mathrm{SnO}_{2}$ in our own secondary if sputtering system and also used some substrates provided by Solar Cells Inc (also with sputtered $\mathrm{SnO}_{2}$ on LOF Tech-8 glass). At this point we have been able to increase the short circuit current but with some corresponding decrease in open circuit voltage so that the overall cell efficiency has not yet improved significantly. In addition, we brought on line this year a new dc sputtering system which was donated by Harris Semiconductor. We have used this system for some prototype contacts and will be emphasizing the use of a variety of other metals for contacting studies in the next year.

Since the sputtering process utilizes a plasma during the thin-film deposition, there are several parameters which can be adjusted to try to achieve a polycrystalline film quality which optimizes the photovoltaic performance. During a three-month postdoctoral visit of Ralf Wendt, a graduate of the Hahn-Meitner Institute in Berlin, he explored the use of a mixture of dc and if to power the sputtering gun for CdTe deposition. He found evidence that, for a fixed sputtering rate, some dc current and less If power to the gun gave somewhat improved solar cell performance. It is plausible that reduced ion bombardment energy on the film may be the origin of the improvements. ${ }^{3}$ We are now trying to verify conclusively the size of the effect and its origin. In addition, we continue to explore the effects of sputter gas composition and pressure as well as magnetic fields on the film quality and cell performance.

We also continued a substantial effort on studies of $\mathrm{CdS}_{x} \mathrm{Te}_{1-x}$ alloy films and on the CdS/CdTe interdiffused layers. These alloy films and interdiffused layers were studied by photoluminescence (PL) and Raman scattering, ${ }^{4}$ optical absorption, electrical conductivity ${ }^{5}$ and Hall measurements, Rutherford backscattering $(\mathrm{RBS})^{6}$, and by grazing incidence $\mathrm{x}$-ray scattering (GIXS). ${ }^{7}$ We provide some highlights here of the Raman, absorption, and electrical studies. 


\section{$\mathrm{CdS}_{\mathbf{x}} \mathrm{Te}_{1-\mathrm{x}}$ alloy films}

$X$-ray diffraction-The alloy films were grown by pulsed laser deposition and covered the full alloy range from $x=0$ (CdTe) to $x=1$ (CdS). Film compositions were determined by Alice Mason of NREL using EDS. The lattice constant of these films generally follows Vegard's law and higher order reflections show evidence of the location of the structural phase transition near $x=0.4$. See Fig. 1 .

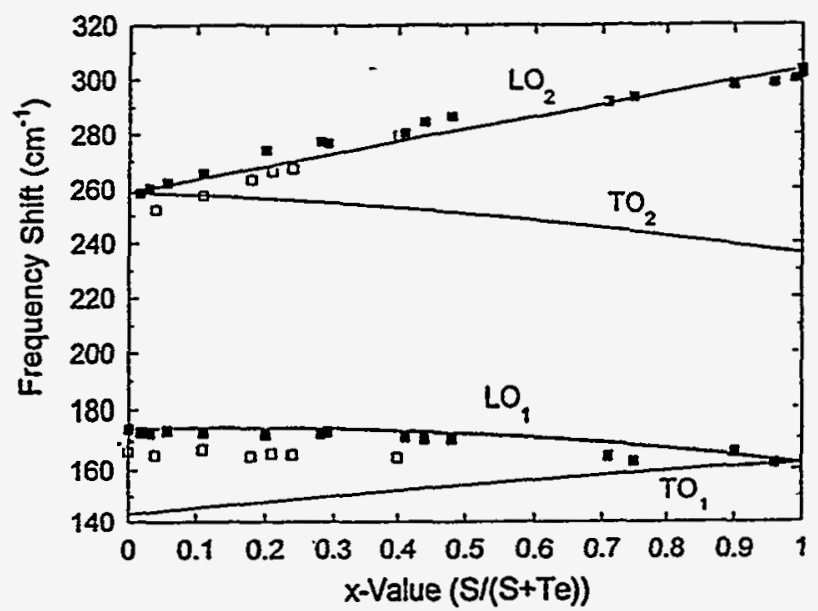

Fig. 1. $x$-ray.peak positions vs. sulfur content ( $x$ value) in laser-deposited CdS $_{x} T e_{1-x}$.

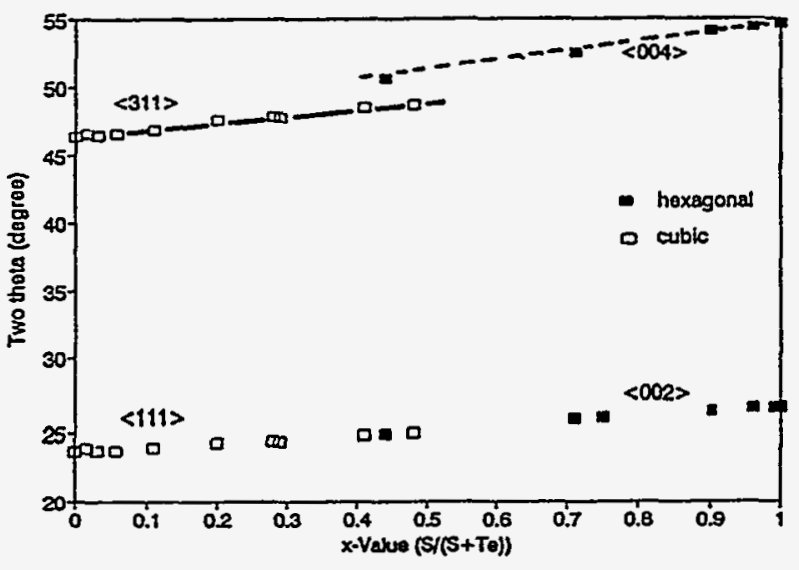

Fig. 2. Frequencies of the phonon modes in $\mathrm{CdS}_{\mathrm{x}} \mathrm{Te}_{1-\mathrm{x}}$.

Raman scattering - We determined the Brillouin-Zone-center phonon frequencies by Raman scattering. The peak positions of the longitudinal optical phonon Raman lines as a function of the alloy composition are shown in Fig. 2. We found this alloy system to be a two-mode system with CdTe-like and CdS-like modes over the full alloy range. ${ }^{4}$ The Raman data of Fig. 2 serve as calibration for Raman scattering which now can be used to probe compositions as well as the dynamical properties of the lattice in the interdiffused region. Very recently we have analyzed the detailed line shape of the Raman lines and found that the broadening and asymmetry are quite large over most of the miscibility gap. The line broadening may be explained in terms of clustering which we could relate to a Warren-Cowley short-range order parameter. ${ }^{8}$ Our results for the broadening and the fit to a cluster parameter are shown in Fig. 3. Understanding of the clustering and alloying process hopefully will lead to better control over the junction parameters in CdS/CdTe solar cells. It should be noted that clustering in this alloy system appears to behave quite differently from some other semiconductor systems such as AlGaAs and AlGaN which do not possess such a miscibility gap.

Optical absorption-We have measured the optical absorption of these alloy films at $\sim 10 \mathrm{~K}$. Additional samples were sent to Dave Albin and Ramesh Dhere of NREL for room temperature absorption measurements. Other films were sent to Fred Pollak of Brooklyn Polytechnic Institute for ellipsometry measurements. These various measurements and analyses are nearing completion and should provide a good data base

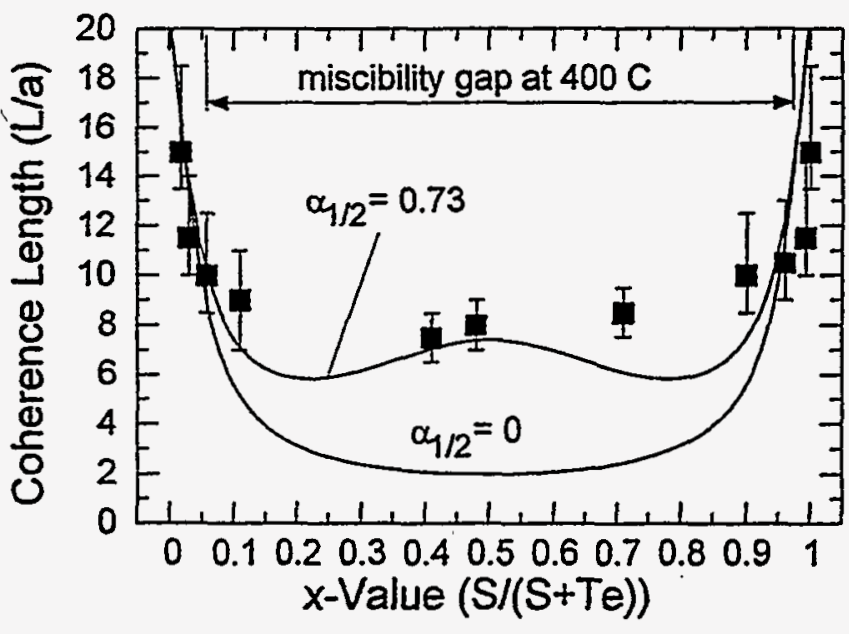

Fig. 3. Cluster length inferred from Raman lineshapes and predicted values from the Warren-Cowley short-range order parameter, $\alpha=$ 0.7 . 
for optical modeling studies of the interdiffused layers in CdS/CdTe solar cells.

Electrical properties-The electrical behavior of these films is interesting partly because the conductivity type changes from p-type for the CdTe films to n-type for the CdS films-and, of course, a structural phase change from zincblende to wurtzite occurs as well. Figure 4 shows the dark resistivity at room temperature for the as-grown films. From the results of the Hall measurements that were performed on selected samples, it is clear that the change in resistivity is primarily due to the change in carrier density rather than in mobility. For large $x$ values (CdS-like), the carrier density is in the range of $10^{18}-10^{19} / \mathrm{cm}^{3}$ while the mobility is low, typically of the order of $3 \mathrm{~cm}^{2} / \mathrm{V}-\mathrm{s}$, or less. For samples near $\mathrm{x}=0.3$, the carrier density is of the order of $10^{11} / \mathrm{cm}^{3}$ and the mobility is around $10 \mathrm{~cm}^{2} / \mathrm{V}$-s. All samples measured for $x>0.3$ were $\mathrm{n}$ type. We were not able to obtain reliable Hall measurements for $x<0.2$, so the point where carrier-type conversion takes place for these films could not be determined accurately. However, hot-probe measurements give indications that the type conversion takes place in the vicinity of $x=0.2$.

Activation energies for the resistivities were measured for the as-grown films between $300 \mathrm{~K}$ and $350 \mathrm{~K}$ and are shown in Fig. 5. The data cover a limited temperature range, but nevertheless provide some insight into the conduction mechanism in these films. Our limited Hall data indicate that for $x>0.3$ the mobility activation energy was low, so that the resistivity is dominated by carrier activation energies. The data indicate that as the alloy becomes more CdTe-like, the effective trap depth increases.

Three samplès with $\mathrm{x}$ between 1 and 0.99 were annealed in air at $400^{\circ} \mathrm{C}$ after a $\mathrm{CdCl}_{2}$ treatment. Generally, the carrier density decreased to around $10^{14} / \mathrm{cm}^{3}$ for all the samples, showing only a weak concentration dependence. A typical mobility was around $2.0 \mathrm{~cm}^{2} / \mathrm{V}$-s. However, the ratio of the dark to light (AM1.5) resistivity increased after the annealing process, in some cases by a factor of 100 .

It is known that for both CdTe and CdS, film quality and carrier type are sensitive to growth conditions and growth method. Thus, the interpretation of our measurements must be done with caution, but we believe the general trends shown above can help to improve our understanding of interdiffused and grain boundary regions in CdS/CdTe solar cells.

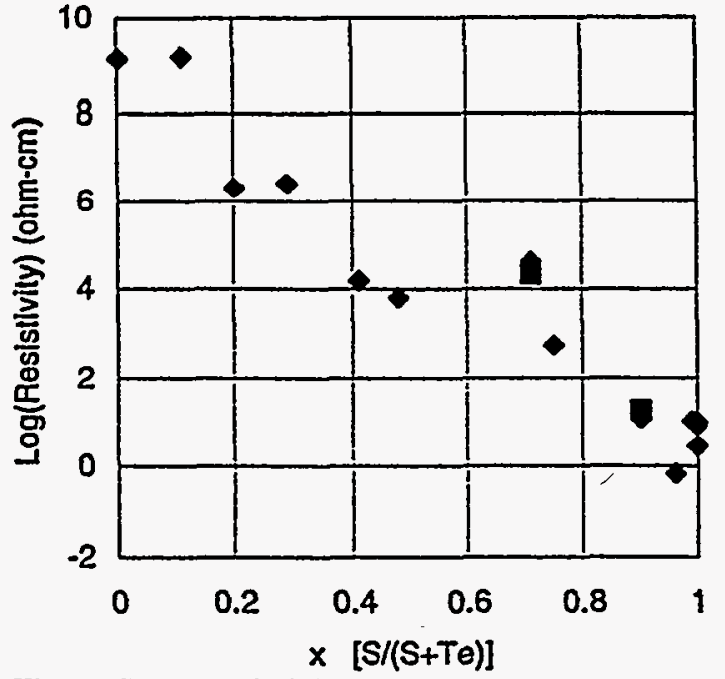

Fig. 4: Dark resistivity at room temperature versus $x$ for $\mathrm{CdS}_{\mathrm{x}} \mathrm{Te}_{\mathrm{i} \cdot \mathrm{x}}$ alloy films.

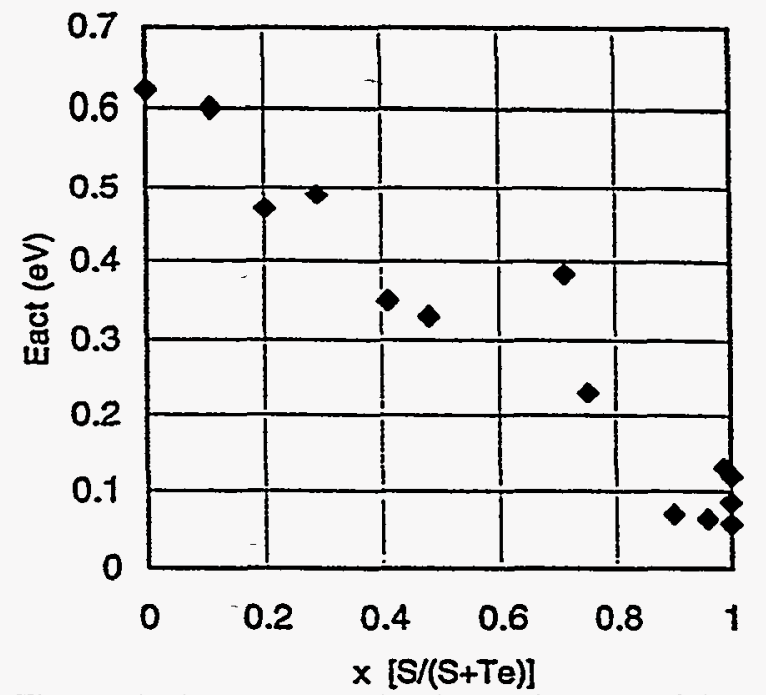

Fig. 5: Activation energies for resistance of the as-grown alloys.

Other measurements-To study directly interdiffusion and interface roughening, we are collaborating with Prof. Heuer at Case Western Reserve Univ. for Rutherford backscattering, and with Prof. Kao's group of the Univ. at Buffalo, working at the Brookhaven Synchrotron, for grazing incidence x-ray scattering 
(GIXS) and fluorescence. The RBS has yielded the sulfur diffusion coefficient into CdTe which gives an activation energy of about $0.6 \mathrm{eV}$ consistent with grain boundary diffusion.

\section{Conclusions and future directions}

There are several aspects in which if sputtering is quite distinct from other techniques used for CdS and $\mathrm{CdTe}$ deposition. There is good adhesion of the films even to very smooth substrates including glass; no deposition or other processing temperatures exceed $400 \mathrm{C}$; low energy ion bombardment can be controlled with substrate bias, gas pressure, and magnetic fields to optimize film parameters; reactive sputtering can be used easily. Our entire fabrication process is done without wet chemical treatments except for the initial glass washing. Additionally, if sputtering is scalable to large areas. Hopefully some of these features will provide pathways to further improvements in large-area CdTe thin-film solar cells, with an all-sputtered process a realistic possibility. The efforts described above provide some advances toward these goals.

The support of NREL through contract number ZAX 4-14013-01 (Bolko von Roedern) is gratefully appreciated. The many scientists at NREL and in the rest of the CdTe National Photovoltaic Team have also provided much helpful advice.

\section{References/Publications}

1. M. Shao; A. Fischer, D. Grecu, U. Jayamaha, E. Bykov, G. Contreras-Puente, R.G. Bohn, \& A.D. Compaan, "Radio Frequency Magnetron Sputtered CdS/CdTe Solar Cells on Soda-Lime Glass," Appl. Phys. Lett. 69, 3045-47 (1996).

2. A. D. Compaan and R. G. Bohn, Annual Subcontract Report: High Efficiency Thin Film Cadmium Telluride Photovoltaic Cells, August 1997 NREL/SR-520-23404.

3. R. Wendt and A.D. Compaan, "Improvement of CdTe solar cell performance due to discharge control during film deposition by magnetron sputtering," submitted to J. Appl. Phys.

4. A. Fischer, Z. Feng, E, Bykov, G. Contreras-Puente, A. Compaan, F.Castillo-Alvarado, J. Avendano, and A. Mason, "Optical phonons in laser-deposited $\mathrm{CdS}_{\mathrm{x}} \mathrm{Te} \mathrm{e}_{1-\mathrm{x}}$ films," Appl. Phys. Lett. 70, 3239-41 (1997).

5. A. Fischer, X. Deng, D. Grecu, K. Makhratchev, X. Ma, R. Wendt, D. Zuo, A.D. Compaan, and R.G. Bohn, "Properties of $\mathrm{CdS}_{\mathrm{x}} \mathrm{Te}_{1-\mathrm{x}}$ Alloy Films," Proc. 26th IEEE Photovoltaic Specialists Conference1997, (to be published).

6. A. Fischer, C. Narayanswamy, D.S. Grecu, E. Bykov, S.A. Nance, U.N. Jayamaha, G. ContrerasPuente, A. D. Compaan. Mark A. Stan, and Alice Mason, "Interdiffusion of CdS/CdTe in Laser Deposited and RF Sputtered Alloys, Bilayers, and Solar Cells," Proc. 25th IEEE Photovoltaic Specialists Conference-1996, pp. 921--924.

7. Y.H. Kao, "Investigation of Interface Morphology and Composition Mixing in CdTe/CdS Heterojunction Photovoltaic Materials Using Synchrotron Radiation," (submitted to J. Appl Physics)

8. A. Fischer, L. Anthony, and A.D. Compaan, "Clustering and Resonant Raman Scattering in LaserDeposited $\mathrm{CdS}_{\mathrm{x}} \mathrm{Te}_{1-x}$ Films," submitted to Appl. Phys. Lett. 
Title: $\quad$ Optimization of Laser Scribing for Thin-film PV Modules

Organization: Department of Physics and Astronomy

The University of Toledo

Toledo, $\mathrm{OH}, 43606$

Contributors: Alvin D. Compaan, principal investigator; Ilvydas Matulionis, and Shogo Nakade, graduate students

Lower Tier Subcontractors: $\quad$ Solar Cells Inc. (SCI)

International Solar Electric Technologies (ISET)

\section{Objectives}

This thin-film $R$ \& D partnership contract is designed to evaluate and optimize the use of new types of lasers and laser scribing processes to improve the reliability, performance, and cost-effectiveness of monolithically integrated polycrystalline thin-film photovoltaic modules. The project is being carried out with support from the lower-tier subcontractors, SCI and ISET, and in collaboration with the CIS- and CdTe-based thin-film PV research teams. This contract, initially planned for a 24 month period, was given a no-cost extension to 30 months to allow the testing of additional laser systems.

\section{Technical Approach}

For series-interconnected, integrated structures in polycrystalline thin-film PV, one must be able to scribe the semiconductors $\mathrm{CdTe}, \mathrm{CuIn}_{\mathrm{l}-\mathrm{x}} \mathrm{Ga}_{\mathrm{x}} \mathrm{Se}_{2}, \mathrm{Si}$, the transparent conducting oxides $\mathrm{SnO}_{2}: \mathrm{F}$ and $\mathrm{ZnO}: \mathrm{Al}$, and metals such as molybdenum. We have found ${ }^{1,2,3}$ that the required energy density and the quality of the laser scribe in these thin-film materials is most dependent on two fundamental properties: 1) pulse duration and 2) wavelength. Nearing the end of this two-year contract, we have studied six different laser systems, with wavelengths and pulse durations given in brackets -- the flashlamp-pumped Nd:YAG laser (fp-YAG) [ $\left.\lambda=532 \& 1064 \mathrm{~nm}, \tau_{\mathrm{p}}=10 \mathrm{nsec}\right]$, the continuous-lamp-pumped Nd:YAG laser (cw-pulsed or cwp-YAG) [532/1064 nm, 90-600 nsec], the XeCl excimer laser [308 nm, $15 \mathrm{nsec}$ ], the copper-vapor laser [511/578 $\mathrm{nm}, 50 \mathrm{nsec}$ ], the $\mathrm{KrF}$ excimer laser [ $248 \mathrm{~nm}, 25 \mathrm{nsec}$ ], and, for the shortest pulse durations, we have used a mode-locked Nd-YAG (cwp/ml-YAG) [532/1064 nm, $0.1 \mathrm{nsec}]^{1}$. Finally, we are planning to test a diode-laser-pumped Nd:YAG.

Analysis of the scribe lines and ablation spots is being done by optical microscopy, scanning electron microscopy (SEM) with energy dispersive $\mathrm{x}$-ray spectroscopy (EDS), stylus profilometry, and electrical isolation tests.

\section{Ablation thresholds}

The threshold energy density for the onset of measurable surface damage provides a useful starting point for comparisons among the various laser systems. The results for the damage (vaporization) threshold for each of the five different laser systems are presented in Table 1. The influences of wavelength and the strength of optical absorption are clearly seen in the data. The transparent conducting oxides, $\mathrm{SnO}_{2}$ and $\mathrm{ZnO}$ exhibit high thresholds except with the ultraviolet excimer lasers. Similarly CdTe shows a relatively high threshold for $1064 \mathrm{~nm}$ where it is transparent and crystalline Si exhibits a much higher threshold for visible damage at $1064 \mathrm{~nm}$ which is right at the absorption edge. Other details of the thresholds are discussed in Ref. 1. 
Table 1: Threshold for Vaporization of Thin-Film Materials

\begin{tabular}{|c|c|c|c|c|c|c|c|}
\hline$\lambda(\mathrm{nm}), \tau(n s)$ & $\begin{array}{c}\mathrm{SnO}_{2} \\
\left(\mathrm{~J} / \mathrm{cm}^{2}\right)\end{array}$ & $\begin{array}{c}\text { CdTe } \\
\left(\mathrm{J} / \mathrm{cm}^{2}\right)\end{array}$ & $\begin{array}{c}\mathrm{Cu} / \mathrm{Au} \\
\left(\mathrm{J} / \mathrm{cm}^{2}\right)\end{array}$ & $\underset{\left(\mathrm{J} / \mathrm{cm}^{2}\right)}{\mathrm{ZnO}}$ & $\begin{array}{c}\text { ClGS } \\
\left(\mathrm{J} / \mathrm{cm}^{2}\right)\end{array}$ & $\begin{array}{c}\text { Moly } \\
\left(\mathrm{J} / \mathrm{cm}^{2}\right)\end{array}$ & $\begin{array}{c}\mathrm{Si} \\
\left(\mathrm{J} / \mathrm{cm}^{2}\right)\end{array}$ \\
\hline $1064 \mathrm{~nm}, 0.1 \mathrm{~ns}$ & 1.8 & 0.8 & 0.9 & 5.8 & 0.14 & 0.87 & 5.8 \\
\hline $1064 \mathrm{~nm}, 250 \mathrm{~ns}$ & 2.6 & 1.1 & 0.73 & 6.8 & 0.14 & 0.73 & 6.8 \\
\hline $1064 \mathrm{~nm}, 10 \mathrm{~ns}$ & 2.4 & 0.65 & & $3.9^{*}$ & 0.32 & 0.44 & \\
\hline $532 \mathrm{~nm}, 0.1 \mathrm{~ns}$ & 1.5 & 0.016 & 0.016 & 3.3 & 0.028 & 0.16 & 1.2 \\
\hline $532 \mathrm{~nm}, 250 \mathrm{~ns}$ & 1.6 & 0.017 & 0.021 & $>2.8$ & 0.021 & 0.16 & 1.6 \\
\hline $532 \mathrm{~nm}, 90 \mathrm{~ns}$ & 5 & 0.22 & & $12^{\star}$ & 0.23 & 0.5 & \\
\hline $532 \mathrm{~nm}, 10 \mathrm{~ns}$ & $\overline{4.5}$ & 0.10 & & $3.5^{*}$ & 0.15 & 0.26 & \\
\hline $511,578 \mathrm{~nm}, 55 \mathrm{~ns}$ & 3.4 & 0.10 & & 0.6 & 0.1 & 1.0 & \\
\hline $308 \mathrm{~nm}, 15 \mathrm{~ns}$ & 0.44 & 0.08 & & 0.24 & 0.13 & 0.24 & \\
\hline $248 \mathrm{~nm}, 25 \mathrm{~ns}$ & 0.22 & $<0.07$ & & 0.16 & $<0.08$ & 0.32 & \\
\hline
\end{tabular}

\section{Optimum energy densities for thin-film scribing}

One of the most important parameters associated with laser scribing is the speed and energy efficiency. Thus we have studied the vaporization depth as a function of pulse energy to identify the energy density needed to optimize the scribing rate. The relationship between increasing pulse energy density and the thickness of material removed is given in Figs. 1 and 2. These data show the depth of material vaporized per pulse as measured by a stylus profilometer trace across the ablation spot. Typical film thicknesses were about $2 \mu \mathrm{m}$ except for $\mathrm{SnO}_{2}$, which was $0.5 \mu \mathrm{m}$. In some cases the crater depth was obtained for two or three laser pulses and the depth divided by the number of pulses. Thus if the depth, shown in Figs. $1 \& 2$, completely saturates with increasing power, it is due to the limitation on film thickness.

For $\mathrm{ZnO}$, except with the two excimer lasers, there was either no ablation or the entire film was blown away. Frequently the first pulse incident on a spot produced no visible effect, but the second pulse produced complete removal. The $\mathrm{ZnO}$ ablation was highly variable with high thresholds (values with * in Table 1) and very steep rises above threshold, giving indications that absorption for the non-uv wavelengths initiated at interfacial or bulk defect sites enhanced by the first pulse.

The uv pulses tend to reach their maximum ablation rates at the lowest energy densities of any of the lasers. However, the vaporization depth per pulse also tends to saturate at rather low values. We believe that this is related to the higher photon energy which sees typically a higher film absorption coefficient and higher energy loss in the plume vapor.

Another general trend is that the longer pulse durations can yield, in most cases, full film vaporization with a single pulse. However, the pulse energy required is large. (It should be noted that the horizontal axis is logarithmic.) For example, the full $\sim 2 \mu \mathrm{m}$ thickness of CdTe, CIS, or Mo can be vaporized with a single pulse at $\lambda=1064 \mathrm{~nm}, \tau=250 \mathrm{~ns}$ with the energy density of about $3 \mathrm{~J} / \mathrm{cm}^{2}$. By contrast, a single pulse at $\lambda=248 \mathrm{~nm}, \tau=25 \mathrm{~ns}$ with energy density of $0.5 \mathrm{~J} / \mathrm{cm}^{2}$ will vaporize roughly $0.1 \mu \mathrm{m}$ of these materials. Thus six pulses at $248 \mathrm{~nm}$ with a total energy of $3 \mathrm{~J} / \mathrm{cm}^{2}$ will remove about $0.6 \mu \mathrm{m}$, noticeably less than the single, $3 \mathrm{~J} / \mathrm{cm}^{2}, 250 \mathrm{~ns}$ pulse at $1064 \mathrm{~nm}$. However, efficiency of material removal is not the sole criterion in choosing a laser scribing engine. Other factors such as beam quality, beam delivery, available energy per pulse, and repetition rate are very important. Some of these are addressed below.

\section{Beam delivery to the film}

Beam focusing was done with a spherical lens for both quasi-cw Nd:YAG lasers and for the copper laser. 

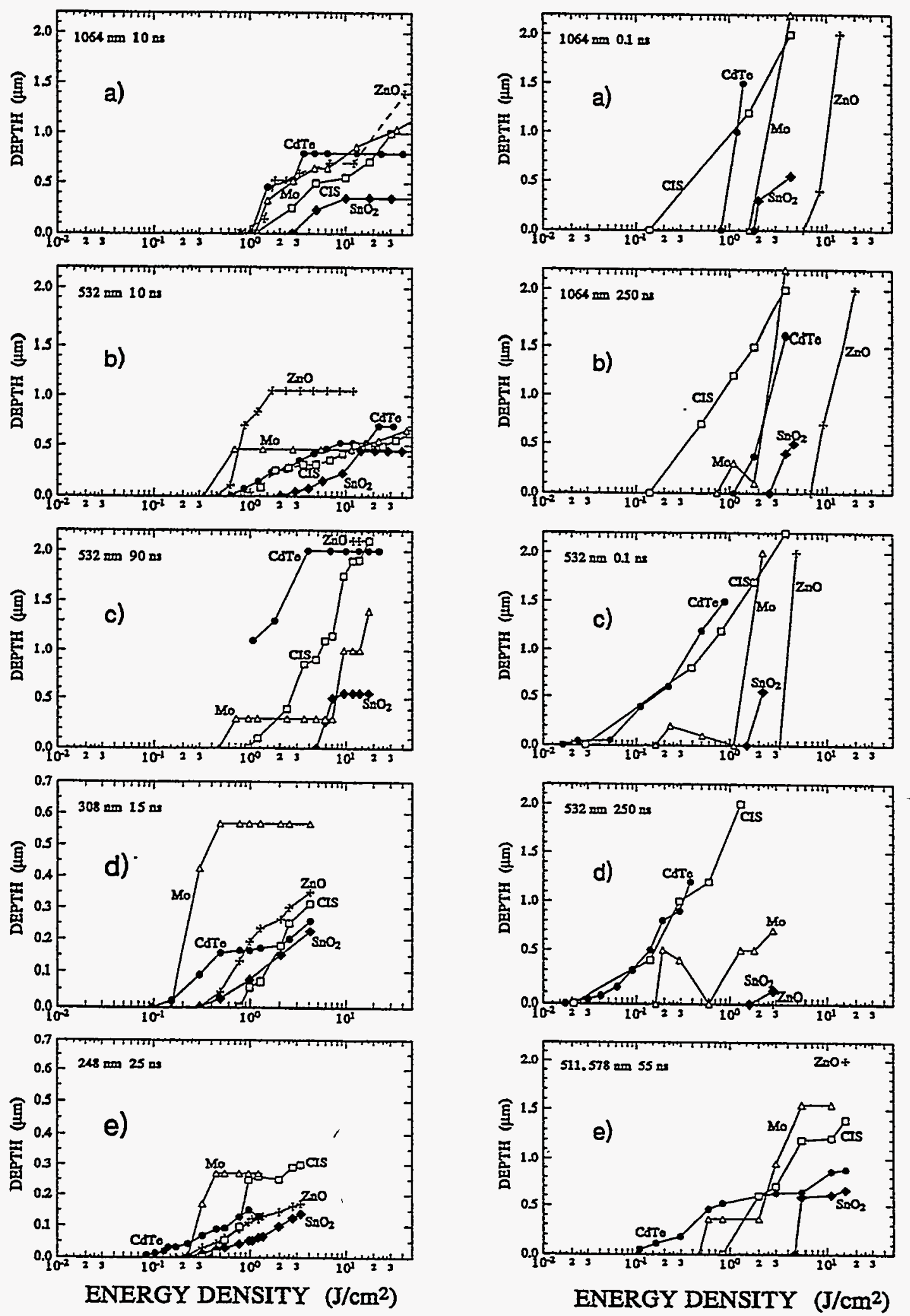

Fig. 1. Thickness removed per pulse vs. energy density for five thin-film materials and five different types of laser pulses. Note the change in vertical scale for d) and e).

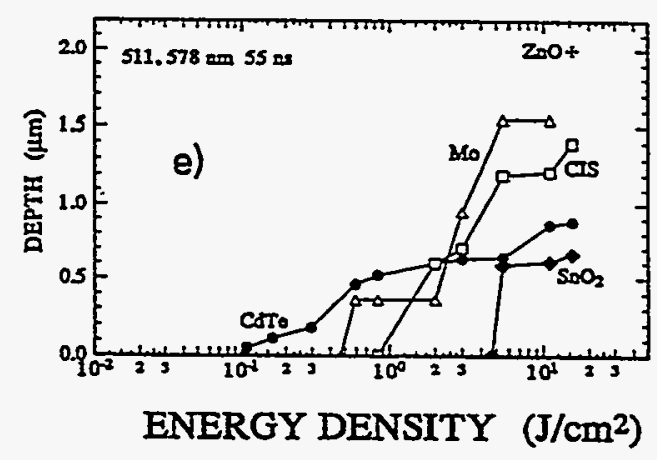

Fig. 2. Thickness removed per pulse vs. energy density for five thin film materials and five more types of lasers. 
For the 10ns flashlamp-pumped Nd:YAG and the excimer lasers, cylindrical focussing was also used. We have found that cylindrical focusing can have distinct advantages, particularly by allowing one to reach an optimum energy density with a very narrow line width. In general we found it to be helpful to work with an energy density near that which gives the optimum vaporization rate since this leaves a smaller heataffected zone surrounding the scribe and makes it easier to control the scribe depth.

For the $\mathrm{KrF}$ laser, we used a beam homogenizer and optical delivery system which projected a demagnified slit image onto the film plane. This produced very clean scribes. It is worth noting that this laser has sufficient pulse energy to image across a full panel width $(\sim 40 \mathrm{~cm})$ so that no lateral beam scanning would be necessary.

\section{Glass-side scribing}

Back-side or glass-side scribing is possible for two of the studied thin-film PV systems, Mo/glass and $\mathrm{CdTe} / \mathrm{SnO}_{2}$ /glass. In these cases, the energy efficiency can be much higher, especially for thicker films, with very little evidence of a heat affected zone observable from the surface. However, the edges of the scribe line tend to be ragged and these $2 \mu \mathrm{m}$ Mo films gave some evidence of lifting of the edges of the remaining Mo film. Our glass side scribing of Mo has so far yielded poorer results in interconnected submodule tests.

\section{Interconnected submodules}

We are fabricating some small interconnected submodules in both CIGS and sputtered CdTe materials. The CdTe structures are being fabricated entirely at UT. In the case of the CIGS the fabrication procedure involves three depositions at ISET and three laser scribes at UT with a final return to ISET for testing--that is, a total of six long-distance exchanges. Therefore the scribe separation was kept fairly large. The preliminary results on CIGS structures were encouraging. Additional studies are in progress on both CIGS and CdTe structures designed to identify series and shunt interconnect resistances.

\section{Acknowledgments}

This research was supported under NREL contract no. ZAF-5-14142-08. We are indebted to SCI for the use of their laser and stylus profilometer and for helpful discussions with Gary Dorer and Upali Jayamaha; to Bulent Basol and Vijay Kapur and the staff of ISET for many discussions and the CIGS-related depositions; to Franz Wagner of Lambda Physik; and Prof. A. Lightman of the Univ. of Dayton Research Institute. Our thanks also go to Prof. H.J. Simon of UT for the use of his Quantronix laser and to Jim Pohnan of Obtec Associates ${ }^{4}$ for making available the latest model Dektak ${ }^{3}$ profilometer.

\section{References}

1. I. Matulionis, S. Nakade, \& A.D. Compaan, "Wavelength and Pulse Duration Efficts in Laser Scribing of Thin Films," Proc. 26th IEEE PVSC-1997, (IEEE, Piscataway, N.J., to be published).

2. A.D. Compaan, I. Matulionis, M.J. Miller, and U.N. Jayamaha, Proc. 25th IEEE PVSC-1996, (IEEE, Piscataway, N.J. pp. 769-772).

3. A.D. Compaan, I. Matulionis, S. Nakade, \& U. Jayamaha, NRELSNL Photovoltaics Program Review, ed. by C.E. Witt, M. Al-Jassim, and J.M. Gee (CP394) AIP Press, New York, 1997) pp. 567-571.

4. Obtec Associates, Inc., Middleburg, $\mathrm{OH} 44130$. 
Title:

Organization:

Contributors:

\section{Alternative Window Schemes for CuInSe2-Based Solar Cells}

\author{
Electronic Materials Laboratory \\ Washington State University / Tri-Cities \\ Richland, Washington
}

Larry C. Olsen, Principal Investigator,

F.W. Addis, Heriberto Aguilar, Wenhua Lei and Kaushik Vaidyanathan

\section{Objectives / Approach}

The objectives of this program are: to develop altemate heterojunction partner layers for high efficiency CulnSe2-based thin film solar cells; and, to improve the understanding regarding the effects of these buffer layers and related processing upon cell performance. The program is structured into three tasks : (1) MOCVD growth of non-cadmium containing window layers (or buffer layers); (2) Optimized processing of buffer layers for high efficiency solar cells; (3) Characterization of layers and devices. The general approach to these studies has consisted of utilizing substrates provided by industry and NREL, so that efforts could focus on buffer layer investigations. MOCVD growth of buffer layers is accomplished with a SPIRE 500XT reactor with added gas handling capabilities. Optimum processing parameters for candidate buffer layers are determined by characterizing $\mathrm{Al} / \mathrm{X} / \mathrm{CIS}$ test cell structures, where $\mathrm{X}$ refers to a heterojunction partner. Al film thicknesses in the range of 100 to $150 \AA$ are utilized so that illuminated I-V characteristics can be obtained for the test structure. After buffer-layer growth parameters are determined, completed solar cell structures are fabricated and evaluated. Layers and devices are characterized at WSU and with the help of collaboration with NREL. Efforts this past year concentrated on studies of highly resistive ZnO buffer layers grown by MOCVD.

\section{Experimental Approach}

\section{CIS and CIGS Substrates}

These studies have included growth of MOCVD ZnO buffer layers by WSU on CIS and CIGS substrates grown at Siemens and NREL, respectively. Substrates provided by Siemens and NREL consist of a CIS-based absorber layer grown on Mo-coated glass. The Siemens absorber is of the form $\mathrm{Cu}(\mathrm{In}, \mathrm{Ga}) \mathrm{Se}_{2}$ with the $\mathrm{Ga}$ concentrated at the back of the absorber structure[1]. Quantum efficiency data taken for this type of material exhibited an effective bandgap of $0.95 \mathrm{eV}$. Thus, the Siemens substrates are referred to as CIS substrates in this report. NREL absorber layers were grown with a 3-stage process that has been discussed previously [2]. These absorbers exhibit effective bandgaps in the range of 1.05 to $1.15 \mathrm{eV}$, and will therefore be referred to CIGS substrates. TCO deposition, contact formation and cell testing were carried out by NREL and IEC.

\section{ZnO Buffer Layers}

ZnO buffer layers are grown by metal organic chemical vapor deposition (MOCVD) by reacting a zinc adduct with tetrahydrofuran and utilizing hydrogen as a carrier gas. Prior to growth of buffer layers, substrates were subjected to a degreasing process consisting of rinses in TCA, acetone, methanol and DI water. Including the degreasing process appears to be particularly important when substrates are mailed from one location to another. ZnO buffer layer deposition was accomplished using a two-step approach as reported previously[3]. The first step involves growth of approximately $100 \AA$ of $\mathrm{ZnO}$ at $250^{\circ} \mathrm{C}$, while the second step consists of depositing approximately 500 to $600 \AA$ of $\mathrm{ZnO}$ at $100^{\circ} \mathrm{C}$. A modified approach was also investigated that involved no $\mathrm{ZnO}$ growth during the $250^{\circ} \mathrm{C}$ step. Thus, with this approach the sample is exposed to only hydrogen during the first step. Best results are obtained when the buffer layer is characterized by a resistivity greater than $1000 \mathrm{ohm}-\mathrm{cm}$. 


\section{Results}

Physical Characterization Of MOCVD ZnO Films

ZnO films grown on CIS substrates have been characterized with grazing incidence $\mathrm{x}$-ray diffraction (GIXRD) analyses and with XPS. The GIXRD studies conducted by NREL were done primarily to determine if any secondary phases existed in the $\mathrm{ZnO}$ films. No such phases were identified. In addition, the GIXRD measurements revealed that MOCVD films grown on CIS exhibit an appropriate XRD pattem for ZnO but with a slightly smaller lattice constant, and a more random orientation than observed for sputtered films. The modified process results in films with a stronger c-axis orientation. GIXRD data taken with an angle of $3^{\circ}$ for a film grown on Siemens CIS material are shown in Fig. 1. Most films grown on both NREL and Siemens material exhibit stronger intensities for the (101) line than illustrated by these data. The slightly lower lattice constant indicates that films are under compressive stress, which may be a result of the film being deficient in oxygen. XPS scans carried out on MOCVD ZnO films grown with the baseline process clearly establish that complete coverage is achieved with such a process.

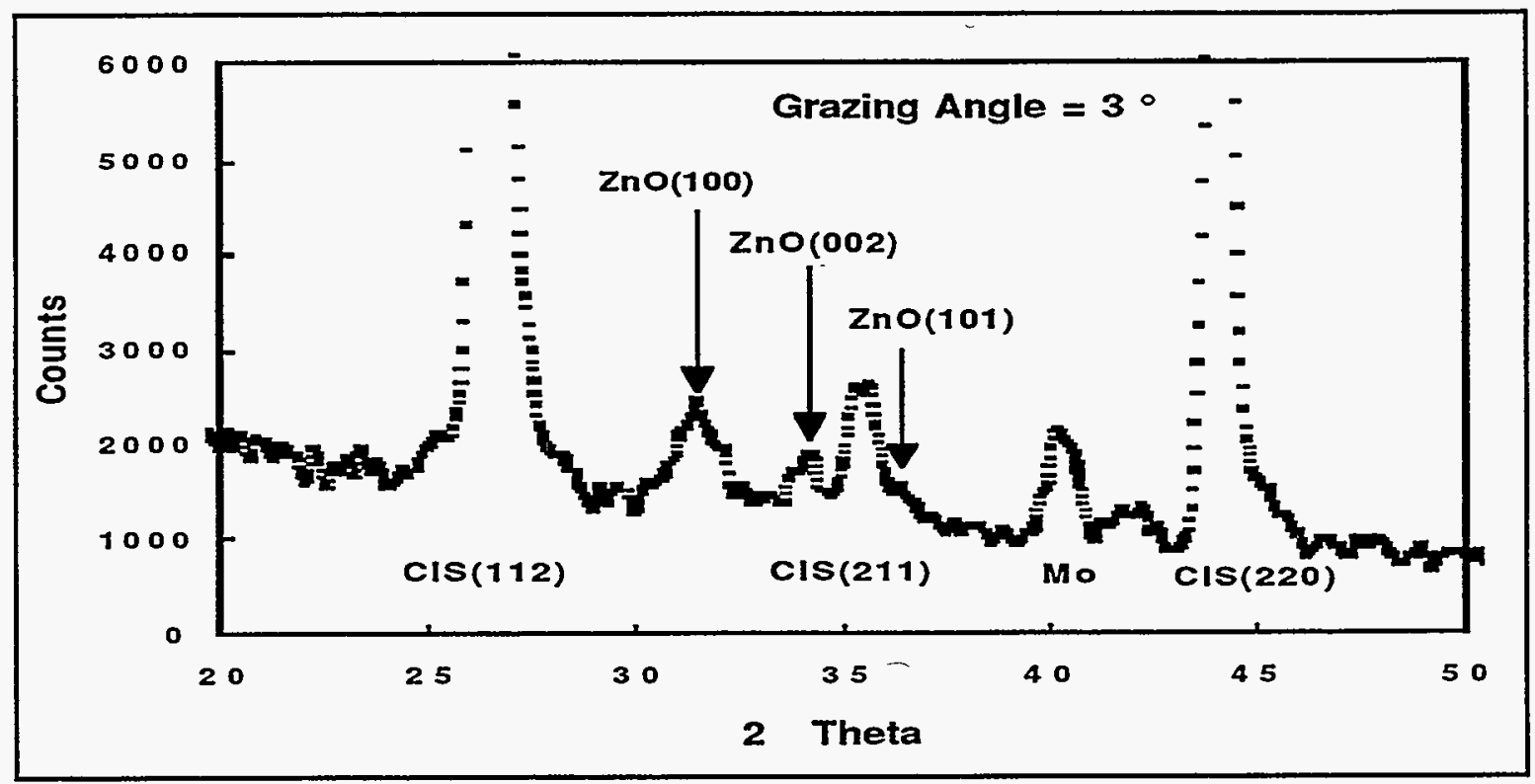

Figure 1. GIXRD data acquired for a $\mathrm{ZnO}$ film grown on a Siemens CIS substrate with a baseline process.

Process Development

Test cell structures were utilized as previously discussed [3] to study the effects of the baseline, modified and single step processes for growth of $\mathrm{ZnO}$ buffer layers on NREL material (see Fig. 2). It was determined that the modified process leads to cells with nearly as good performance as achieved with the baseline process. However, it is clear that the first step involving heating a substrate to $250^{\circ} \mathrm{C}$ in hydrogen (and growing $\mathrm{ZnO}$ in the baseline process) gives much better performance than if the $\mathrm{ZnO}$ buffer is grown in a single step at $100^{\circ} \mathrm{C}$ (see Fig. 3).

\section{Role Of Hydrogen}

Although more cell results are needed in order to be certain, it appears that the presence of hydrogen during the first step for both the baseline and modified processes is critical to achieving high efficiency CIGS cells with MOCVD ZnO buffer layers. Surface AES profiles taken on NREL CIGS absorbers indicate that CIGS sample surfaces are readily contaminated with oxygen and carbon after exposure to air. Even though substrates are cleaned prior to insertion into the MOCVD chamber for growth of $\mathrm{ZnO}$, one would expect that the sample surfaces would acquire these contaminants. Thus, one effect of the hydrogen may be to cleanse the surface of contaminants. Hydrogen may also 


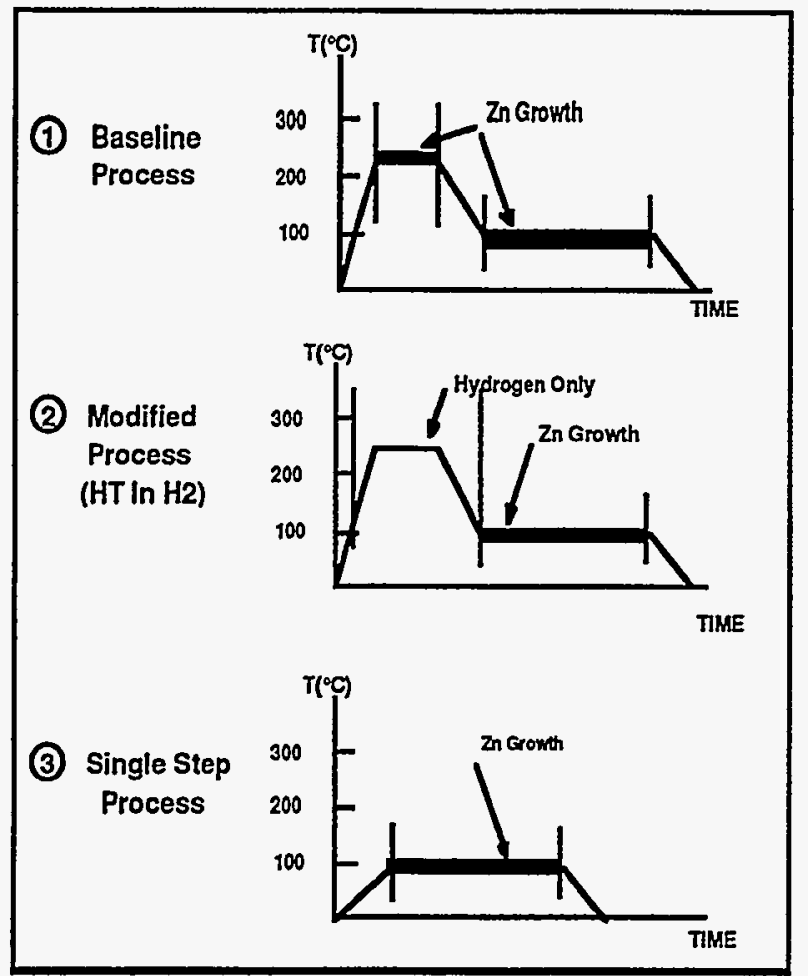

Figure 2. Three MOCVD growth schedules utilized for deposition of ZnO buffer layers.

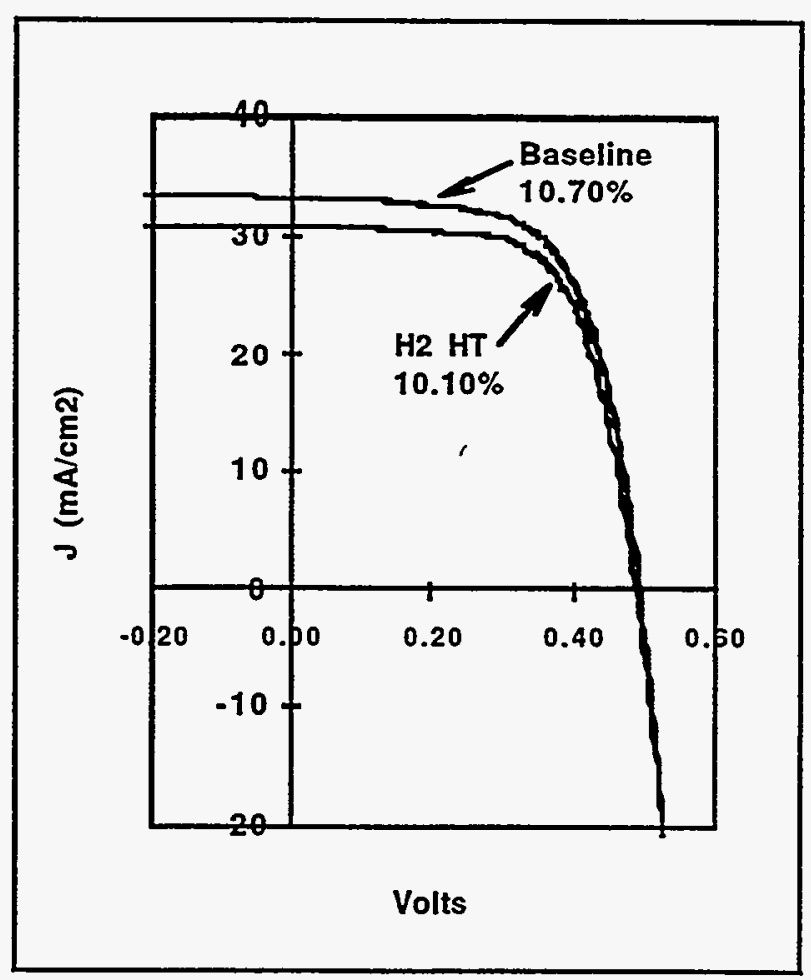

Figure 3. Total area illuminated I-V characteristics for cells with MOCVD ZnO buffer layers.

passivate surface and subsurface recombination centers.

Performance Of Completed Cells

Results are given in Table 1 for devices with MOCVD ZnO buffer layers grown on both NREL and Siemens substrates. CIGS cells fabricated with MOCVD ZnO buffer layers deposited onto NREL material compare favorably with devices based on CdS buffer layers deposited onto the same NREL material. Illuminated and dark current-voltage characteristics for the best performing cell with a MOCVD ZnO buffer layer are given in Figure 4. Good performance was also obtained for cells fabricated with $\mathrm{ZnO}$ buffer layers on Siemens CIS material. As indicated in Table 1, efficiencies were in the 11 to $12 \%$ range with relatively large values of open circuit voltage and fill factor for a CIS based cell. External quantum efficiencies for $\mathrm{ZnO} / \mathrm{CIGS}$ and $\mathrm{ZnO} / \mathrm{CIS}$ cells are plotted vs wavelength in Figure 2. The abrupt rise at $400 \mathrm{~nm}$ is characteristic of $\mathrm{ZnO}$ buffer layers relative to CdS. It is apparent from the quantum efficiency curves that absorption edges for the CIGS and CIS cells are $1100 \mathrm{~nm}(1.127 \mathrm{eV})$ and $1300 \mathrm{~nm}$ (0.95), respectively.

\section{Conclusions}

This work has resulted in the demonstration of high efficiency CIGS cells based on highly resistive ZnO buffer layers grown by MOCVD. One cell based on NREL CIGS and a ZnO buffer layer exhibited an active area efficiency of nearly $14 \%$. This result is one of the best efficiencies reported for a "direct" $\mathrm{ZnO} / \mathrm{CIGS}$ cell made with a vacuum process. This work has established that the buffer layer is truly $\mathrm{ZnO}$, and not an alloy resulting from interdiffusion of $\mathrm{ZnO}$ and CIGS. It has also been established that a two step growth procedure like those described as 'baseline' and 'modified' lead to efficient cells. Since most of the gas flowing through the reactor is hydrogen for all processes, it appears that exposure of CIGS and CIS to hydrogen at $250^{\circ} \mathrm{C}$ has beneficial effects. Two possible effects of hydrogen are that it removes contaminants from the CIGS surface, and/or it passivates the surface and subsurface regions. 


\section{References}

1. D. Tarrant and J. Ermer, "I-III-VI2 Multinary Solar Cells Based On CuInSe2." Twenty Third IEEE PVSC, 1993, pp. 372 - 378.

2. Miguel A. Contreras, John Tuttle, Andrew Gabor, Andrew Tennant, Kannan Ramanathan, Sally Asher, Amy Franz, James Keane, L. Wang, John Scofield, and Rommel Noufi, "High Efficiency $\mathrm{Cu}(\mathrm{In}, \mathrm{Ga}) \mathrm{Se}_{2}$-Based Solar Cells," First WCPEC, 1994, pp.68 -75.

3. Larry C. Olsen, Heriberto Aguilar, F. William Addis, Wenhua Lei and Jun Li, "CIS Solar Cells With ZnO Buffer Layers", Twenty Fifth IEEE PVSC, 1996, pp. 997 - 1000.

\section{Acknowledgements}

We are grateful for the substrates supplied by Siemens Solar and NREL, and the technical interaction and guidance from our Contract Monitor Dr. Bolko von Roedern.

TABLE 1. Cell Results

$\begin{array}{lccccc} & \text { AHP Layer } & \begin{array}{c}\text { Jsc } \\ (\mathrm{mA} / \mathrm{cm} 2)\end{array} & \begin{array}{c}\text { Voc } \\ (\mathrm{mV})\end{array} & \begin{array}{c}\text { FF } \\ (\%)\end{array} & \begin{array}{c}\text { Efficiency } \\ (\%)\end{array} \\ \text { NREL SUBSTRATES } & & & & & 12.2 \\ \text { NREL C679-9 2 } & \text { CVD ZnO } & 33.5 & 524 & 69.5 & 12.7 \\ \text { NREL M1455-1\#1 1 } & \text { CVD ZnO } & 34.06 & 560 & 66.4 & 13.9 \\ \text { NREL C679-5 } 2 & \text { CVD ZnO } & 34.5 & 581 & 69.2 & \\ \text { SIEMENS SUBSTRATES } & & & & & \\ \text { SSI 97XC019 2 } & \text { CVD ZnO } & 40.7 & 439 & 66.9 & 12.0 \\ \text { SSI 97XC021 } 2 & \text { CVD ZnO } & 36.4 & 455 & 71.0 & 11.7 \\ \text { SSI 97XC016 } & \text { CVD ZnO } & 37.5 & 437 & 69.6 & 11.4\end{array}$

1. Measurements made at NREL at $25^{\circ} \mathrm{C}$ and are total area values.

2. Measurements made at IEC at $30^{\circ} \mathrm{C}$ and are active area values.

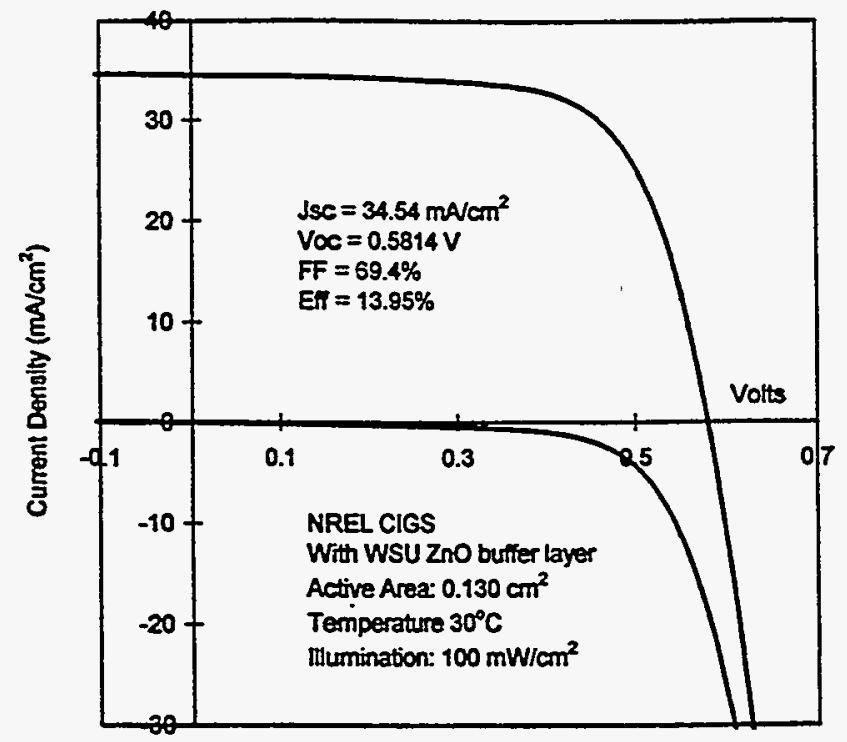

Figure 4. Illuminated and dark I-V characteristics CIGS cell with a MOCVD ZnO buffer layer.

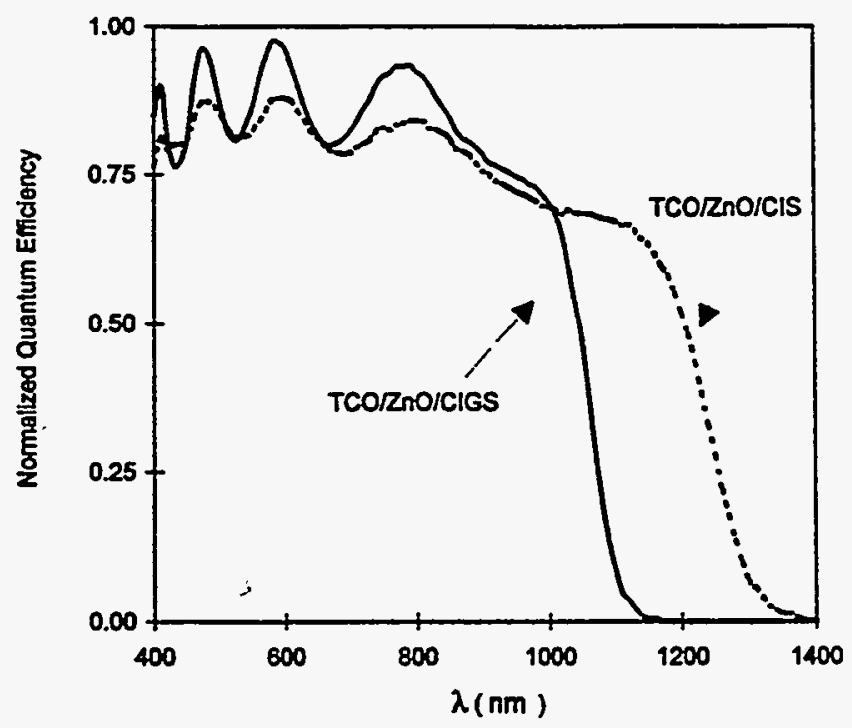

Figure 5. Normalized quantum efficiency for cells with MOCVD ZnO buffer layers. 


\subsection{Photovoltaic}

Manufacturing Technology (PVMaT) Project 


\title{
6.0 PHOTOVOLTAIC MANUFACTURING TEChNOLOGY (PVMaT) PROJECT-Introduction
}

\author{
C. Edwin Witt (Team Leader), Richard L. Mitchell, Holly P. Thomas, Martha Symko
}

The Photovoltaic Manufacturing Technology (PVMaT) Project was initiated in 1990 to help the U.S. photovoltaic (PV) industry extend its world leadership role in PV manufacturing and the commercial development of PV modules and systems. As described in previous Annual Reports, the PVMaT Project is a government/industry research and development $(R \& D)$ partnership between the U.S. federal government (through the U.S. Department of Energy [DOE]) and members of the U.S. PV industry. PVMaT is designed to accomplish this by helping the U.S. PV industry improve manufacturing processes, accelerate manufacturing cost reductions for PV modules, improve commercial product performance, and lay the groundwork for a substantial scale-up in the capacity of U.S.-based PV manufacturing plants.

The PVMaT Project released its Phase 5A solicitation in FY 1997. Previous phases (which include Phase 1, Phase 2A, Phase 2B, Phase 3A, and Phase 4A) were described in the 1996 Annual Report. The detailed activities and accomplishments of each of the currently active subcontractors from Phases 1 through $4 \mathrm{~A}$ are covered in individual sections following this summary.

Phase 1 identified and detailed manufacturing problems from 23 different private organizations. Subcontracts were completed in 1991. Phase 2 addressed process-specific module manufacturing problems of individual manufacturers. Phase $2 \mathrm{~A}$ was open only to organizations that received awards under the Phase 1 solicitation. The seven 3-year subcontracts implemented in early 1992 were $41 \%$ cost-shared by the U.S. PV industrial participants. All the Phase 2A subcontracted activities have been completed. Phase 2B was open to all U.S. PV industrial firms, allowing organizations who had not been ready for the earlier Phase $2 A^{\prime}$ procurement cycle, or who were not participants in Phase 1, to have a chance to "ramp up" and participate in the process-specific solution phase of the PVMaT Project.

Four 3-year Phase 2B subcontracts were selected from 13 proposals and awarded in late 1993. These subcontracts also addressed process-specific module manufacturing problems. They included work in CdTe module manufacturing, the manufacture of Spheral Solar cells and modules, and cast polysilicon wafers, cells, and module manufacturing. These subcontracts represented new technological additions to the PVMaT Project, and were cost-shared at $52 \%$ by the subcontractors. Three of the four subcontracts were active in FY 1997-Golden Photon, Solar Cells, Inc., and Solarex Corporation. The Golden Photon contract was completed this year and the other two will be completed in FY 1998.

Phase 3 of the PVMaT Project addressed R\&D problems common to the PV industry as a whole through a teamed research approach. Two subcontracts were selected from seven proposers and awarded in January 1993. These subcontracts were cost-shared at $25 \%$ by the subcontractors. The Springborn subcontract was active this past year but is now completed.

Phase 4A is a broader approach at addressing the overall PVMaT goal of improved U.S. market share by meeting the market challenges. The solicitation included responses from individual or teamed U.S. PV and related industries addressing manufacturing of PV end-products, as well as sub-elements of these products. Objectives include: stimulating a broader interest in the production of PV products; encouraging and supporting risk-taking by industry to explore new manufacturing options and ideas for improved PV products or components; encouraging system and product innovation; and stimulating advances in balance-of-systems or developments in design leading to overall reduced system life-cycle costs of the PV end product. Cost reduction, improved efficiency, and manufacturing flexibility and broader market applications for PV systems 
as a whole are emphasized. The subcontracts in Phase $4 \mathrm{~A}$ were divided into two parts, $4 \mathrm{A1}$ and $4 \mathrm{A2}$, as described below. All 4A contracts were awarded in FY 1995.

Phase 4A1 addresses the product-driven system and component technology and includes manufacturing improvements directed toward innovative, low-cost, high-return, high-impact PV products. Proposals in this phase addressed manufacturing that was generally related to PV system components and aspects other than modules, system components such as inverters, and/or system integration efficiency and/or design improvements, with lesser focus on module manufacturing. In addition, proposals looked at issues in system/component integration to bring all elements together for a PV product that is offered on the market. Eight 2-year subcontracts were awarded from 31 proposals received. These subcontracts are cost-shared at $25 \%$ by the subcontractors.

Phase 4A2 is focused on product-driven PV module manufacturing technology. Subcontracts under this phase are directing their efforts at manufacturing flexibility and module-manufacturing cost reduction for a wider range of PV products. Phase 4A2 consists of five three-year subcontracts. These subcontracts include development of large-area silicon-film panel and cell manufacturing; edge-defined, film-fed growth (EFG) module manufacturing; improvements in CZ silicon modules; development of monolithic a-Si modules on continuous polymer substrates; and manufacturing of extruded concentrator modules. These subcontracts are cost-shared at $42 \%$ by the subcontractors.

Phase 5A of PVMaT was initiated this year when NREL issued a request for proposals (RFP) for additional PV manufacturing R\&D on June 30, 1997. Phase 5A is anticipated to last for 3 years at a level of at least $\$ 8$ million per year. The efforts solicited are expected to be similar to the Phase 4A efforts with somewhat more emphasis on specific problems amenable to manufacturing R\&D solutions. Thirty-one proposals responding to this RFP were received. The evaluation of the offers will be completed early in FY1998-and negotiations are expected to start early in January 1998.

\section{PROGRESS IN THE PV INDUSTRY}

It is important to put the technical progress that the U.S. PV industry is making into perspective. Based on the improvements in manufacturing processes, reductions in manufacturing costs, introduction of new products, and improvements in product performance, it would be expected that the U.S. PV industry would exhibit both a scale-up of its U.S.-based PV manufacturing capacity and a reduction in its production costs. This has indeed been the case.

At the beginning of Phase $2 \mathrm{~A}$ subcontracted research, information was collected to establish both the current and projected capacities and module costs for the eight subcontractors. These data, shown in Fig. 1, represented both the diverse status of a still-maturing industry and the optimistic speculation of its members regarding the effects that the PVMaT Project would have after their PVMaT efforts were allowed to take effect. Additional information was collected from the 12 PV industrial participants from all phases of PVMaT that were in production in late 1996.

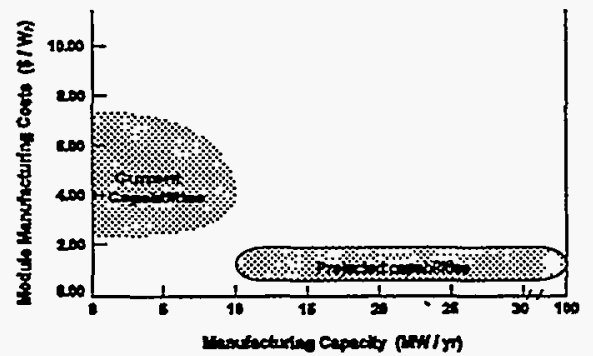

Figure 1. 1993 PVMaT projections 
This most recent data, shown in Fig. 2, represents an update of previous projections regarding these subcontracted efforts. Data in this figure are based on each manufacturer's maximum production capacity during a given year, assuming they were to operate at maximum capacity. The "average" represents the average cost per watt of modules (weighted by each participant's capacity) produced by these 12 PVMaT industrial participants. Module cost estimates were then based on these manufacturing levels and included only those costs directly associated with the manufacturing of the modules (not marketing, administration, or sales.). It should be noted that data associated with any particular point in time represent a potential capability. Actual manufacturing production levels may be less (and concomitant costs higher) due to other considerations such as market conditions and available labor.

The PVMaT Pruject is currently finishing the second year of subcontracts under Phase $4 \mathrm{~A} 1$ and $4 \mathrm{~A} 2$, with research in Phase 2B to be concluded early in FY1998 and Phase 3A having been completed this past year. At this time, it is apparent from Figs. 1 and 2, that the U.S. PV industry involved in the PVMaT Project has made significant progress toward reducing manufacturing costs and increasing PV module manufacturing capacity. "Average" module manufacturing costs have been reduced $32 \%$ and total manufacturing capacity for 12 PVMaT industrial participants has more than doubled in the last three years. By 1999, projections indicate modulemanufacturing costs of $\$ 1.79 / \mathrm{Wp}$ (a $60 \%$ reduction from 1994 levels) and a production capacity increase

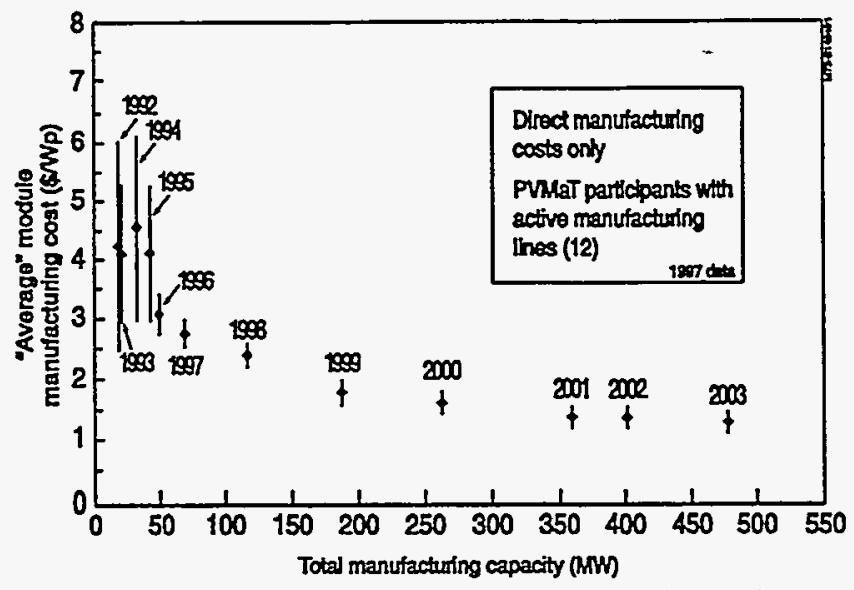

Figure 2. PVMaT manufacturing cost/capacity to $187.4 \mathrm{MW}$ (a factor of over 10 higher than the original 1994 level for the 12 PVMaT industrial participants). There are indications, in both the industry's future cost/capacity data and its technical projections, that long-term optimism for continuing these improvements is high — and that the near-term progress in these areas has been significant. 


\section{Photovoltaic Manufacturing Technology (PVMaT) Subcontracts}


Title: $\quad$ Next Generation Three-Phase Inverters

Organization: Advanced Energy Systems, Inc.

Wilton, NH

Contributors: Dr. Robert Wills, Principal Investigator; Adam Wesley; David Fish; Dr. Vladimir Brunstein; Len Wright

\section{Introduction}

The PVMaT-4a subcontract addresses the cost-effective manufacture of PV related products. Advanced Energy Systems Inc. (AES Inc.) has undertaken NREL contract \#ZAF-4-14271-10, titled "Next Generation Three Phase Inverter," to combine leading industry skill with advanced technologies to realize superior PV inverter-related products. To this end, in phase one of the PVMaT contract, AES Inc. made significant inroads toward establishing a low-cost manufacturing process of an intelligently controlled PV-hybrid product.

Briefly, the objectives laid out in the PVMaT contract include the delivery of a next generation prototype PV-hybrid inverter and PV-grid interactive inverter, as well as establishing a pilot production line for the products. The specifications for these units were developed from extensive market research. The inverters incorporate many advanced features including digital wave-shape and resource control techniques using DSP functions, optimized power magnetics, a design aimed at economic manufacturing and simple maintenance, optimized dispatch algorithms incorporated within automated system control, and a MODBUS industry standard remote monitoring and control communications capability.

AES has targeted improvements in several areas of the current inverter design that would improve the performance and reliability of the product while at the same time reducing manufacturing costs. These areas include the inverter control and drive components, the hybrid control system, the remote monitoring and control package, and the implementation of a modular "Power Block" design of the IGBT power switching sub-assembly. In addition, a manufacturing collaboration between AES and WPI Power Systems of Warner, NH has been developed. The efforts put forth in these areas under the PVMaT-4a contract have been successfully translated into commercial efforts; several commercial hybrid inverter systems that incorporate the product improvements have already been deployed and have proven to be both reliable and effective.

\section{Digital Inverter Control}

One objective for the PVMaT development was a revised inverter sinewave control card and robust IGBT drive card. The new inverter control electronics forms the basis of a refined solution that achieves higher inverter performance at a lower cost. Functionality and flexibility has been increased with the introduction of a digital microprocessor as the core of the inverter control electronics. This benefits future revisions of the system, shifting new development toward software and reducing the necessity to modify the hardware design. The clear enhancement here is the ability to introduce self-testing, automated diagnostics and the capability to store comprehensive set-up information within the core of the inverter itself. 


\section{Inverter Control Card}

The revised ICC design contains two significant enhancements; the introduction of micro-controller technology and the refinement of existing analog control loops to sub-cycle control improving output voltage regulation. The new implementation contains significant software and hardware components. These components integrate the following technologies: new PID analog feed back controller for output voltage regulation, introduction of PLD technology for protection and design simplification, digital reference generation and PLL implementation using a micro-controller, integration of three phase independent, inverter controllers onto a single $\mathrm{PCB}$, and communications interface for automatic set-up and fault diagnosis.

\section{Inverter IGBT Driver Card}

PVMaT also supported the development of a new IGBT driver card which is highly robust, flexible in its application and simple to diagnose. This new component includes the following developed features: desaturation over current detection, a new power supply design, low voltage lock-out for fail-safe power-up and power-down, plug-in connectors between driver and the IGBT module, slow gate turn off under peak current / de-saturation conditions, top and bottom drives completely isolated from input drive and 24 volt supply (which facilitates the use of spilt battery banks, effectively increasing apparent DC voltage of the system without raising safety concerns), individual top and bottom high current de-saturation sensing (shut-down in 20 to $30 \mathrm{mS}$ ) or medium current over-current limiting (adjustable shut-down, default to 1.5 seconds), LED indicators for current limiting, gate drive operation and all power supplies to assist in fault analysis and fault location.

\section{Summary}

The benefits gained in the area of digital inverter control can be measured in terms of cost reduction, performance improvement, flexibility, and reliability. Over a $60 \%$ cost reduction was realized when the inverter control design moved from four circuit boards down to a single circuit board. This provides the added benefit of improved reliability by significantly reducing the number of interconnections in the system. The MTBF for commercial systems using the new inverter electronic design will be improved markedly. In terms of performance improvement, the voltage regulation associated with the new IGBT driver cards has driven the typical THD from $10 \%$ on older systems to less than $3 \%$ for systems using the new cards[1]. Flexibility and reliability were both improved with the incorporation of microcontrollerbased software control in the inverter control card. This added flexibility by providing the capability to tune the control for different applications. Reliability has increased with the addition of an RS232 communications interface to the inverter core, which provides the capability for automatic set up and fault diagnosis.

\section{Hybrid Control System}

The objective for PVMaT development on the hybrid control system was to revise the control hardware and software to make the control features more reliable, flexible, and adaptable. The efforts for meeting those objectives include the following: extending the available microcontroller RAM and program ROM from $32 \mathrm{~K}$ to $128 \mathrm{~K}$ each, converting the bulk of the control software from assembly code to ' $\mathrm{C}$ ' code, incorporating a standard MODBUS communications protocol software unit into the control software, enhancing the software data logging module, and developing a Hybrid Environment Simulator task that runs on the same platform as the rest of the control software. In addition, the software development 
environment was improved by migrating the code to a commercial compiler and debugger that is recognized as an industry leader.

\section{Extended Memory Banking}

The addition of three $32 \mathrm{~K}$ banks of ROM to the existing one $32 \mathrm{~K}$ bank has greatly improved the potential flexibility of the hybrid control software. Due to memory constraints, previous software incarnations were comprised mainly of assembly-language code. The difficulty in designing, modifying and maintaining assembly code is much higher than the code of a higher-level language such as $\mathrm{C}$. Adding the extra memory provided the opportunity to convert the assembly code to $C$, and further, allowed the data logging module of the control software to store over a month's worth of data sampled at 15 minute intervals. The extra memory also served as a cost reduction vehicle, since the separate LCD and keypad interface card in earlier systems was eliminated when the software supporting those devices was moved onto the control system microcontroller.

\section{MODBUS Communications Module}

The addition of a standard MODBUS communications module to the software adds to the reliability and flexibility of the control software by providing a standard communications interface between the control software and other MODBUS applications, such as a remote monitoring and control system.

\section{Hybrid Environment Simulator (HES)}

The addition of the Hybrid Environment Simulator (HES) task to the control system software has greatly improved the reliability of the software by providing the capability to thoroughly debug and test the software without requiring a real system on which to run the code. With the HES running, AES engineers now have the ability to make changes to the software, compile it, download it via a ROM emulator to a single-board test bed, and test the changes while sitting at their desks. The interrupt-driven task can be configured to run scenarios that vary the simulated generator and battery readings so that different control and dispatch algorithms can be easily modified and tested. As a case in point, when the control code developed on PVMaT was tested on the PV-hybrid prototype for the first time after being run with the simulator there were very few coding errors that needed to be corrected.

\section{Remote Monitoring System}

The objective defined under PVMaT was to implement a user-friendly remote monitoring and control system. The capability of the hybrid control software to communicate was introduced with the addition of the MODBUS communications module to the software. A consultant, Dumont Associates of Hollis, $\mathrm{NH}$, was tasked with providing the other side of the communications interface, a PC-based Windows package termed SPPLink.

The ability to remotely operate or interrogate a station provides the product distributor or support group an opportunity to investigate faults and performance glitches without physically visiting the installation. Typically, site visits incur high costs, the more remote the site the higher the cost. The interface package developed extracts detailed operating information from the station controller and greatly assists in fault diagnosis. In some cases, where skilled operators are not locally available, remote operation becomes mandatory. 
This enhanced package makes extensive use of windows graphics, communications and provides a user friendly control environment. The SPP-Link remote operator package interfaces the hybrid SCADA system with an MS-Access database and will automatically load remote historic performance information into a unique site database. Site operation can now be reviewed and analyzed using automated database queries. This significantly reduces the labor required to monitor and make informed recommendations regarding remote systems performance.

\section{Prototype Design and Manufacture}

Two key objectives of the PV-MaT 4A contract were the design and development of a high quality, low cost PV-hybrid inverter for US manufacture and the establishment of a local manufacturing partnership. Many design features of the PVMaT PV-hybrid prototype unit have contributed to the attainment of the first objective. The second objective has been met with the establishment of a manufacturing collaboration between AES Inc. and WPI Power Systems, although the market has not yet developed to the point where volume production is practical.

\section{Prototype Design Features}

During the design of the prototype unit, several initiatives were identified and undertaken to produce the best product at the least cost. The reduction in the number of circuit boards in the system discussed previously was an important step in this direction. Another important step was the effort to design the inverter as a collection of modular subassemblies. Three primary subassemblies were identified: the enclosure and output stage, including the transformers, inductors, and cabinet, the Power Block, which combines the IGBT devices, drivers, capacitors, and bus work mounted upon a single heat sink assembly, and the electrical looms and control harness, which includes the wiring of connections between sensing and control points. The Power Block design is the component that has proven to be the most effective design enhancement. With the new design, the potential now exists to assemble, calibrate, and test under power the most failure prone and most critical inverter subassembly. In addition, field service of IGBTrelated problems has been made simpler and safer by incorporating many individual components into a pre-calibrated and pre-tested subassembly[2]. The PVMaT power block design has been used now in several AES commercial inverters.

\section{Prototype Manufacturing Highlights}

The issue of long term alliance has been discussed with WPI with a positive outlook. Both parties have contributed greatly toward achieving a successful pilot manufacturing program. The ability to draw on WPI's experience in low-medium volume, power electronic system's manufacture has already proven to have been of significant advantage over other manufacturing options. Development of substantial manufacturing documentation was considered key to realizing a reliable and repeatable product. AES and WPI have worked together to produce a comprehensive set of manufacturing documents, including a full Bill of Materials (BOM), CAD drawings of the enclosures, the physical layout, and control system schematics, all cable definitions, including cable sizing, connection types, and wire sizes and colors, and a set of specific assembly instructions. 


\section{Prototype Manufacturing Costs}

The cost objectives for the production of the PV-hybrid prototype units were successfully met. The actual cost to build the prototype came in at $\$ 0.37 / \mathrm{kW}$, which was well below the target cost of $\$ 0.45 / \mathrm{kW}$.

\section{Commercial Benefits}

An important method of measuring a research project's success is by analyzing how well the design initiatives of a project translate into the design features of commercial products. In this regard, the PVMaT contract would have to be deemed an overwhelming success. The pre-existing product line has been totally re-defined to include many of the design improvements made possible by the PVMaT-4a project. Commercial inverters with the new design features have already been deployed and have proven to be reliable, safe and effective.

\section{References}

1. R. Wills, A. Wesley, "PVMaT Project 1996 Annual Report", pp. 16-17.

2. R. Wills, A. Wesley, "PVMaT Project 1996 Annual Report", pp. 28-29. 

Title:

Organization:

Contributors:

\section{Manufacture of an AC Photovoltaic Module}

Ascension Technology, Inc., Waltham, MA and Boulder, CO. SunSine300 Product Development Team

E.C. Kern, M.R. Russell, G.A. Kern, C.K.P. Handleman

\section{Purpose}

The purpose of this research has been to develop for commercial sale, an AC Photovoltaic (PV) Module, the SunSine300. AC PV modules are a breakthrough in PV technology. The combination of a dc/ac inverter integrated into a dc PV laminate simplifies the application of photovoltaics for grid tied applications. AC modules bring PV to the point where nearly anyone is capable of designing and installing a PV system. Complicated wiring and system sizing of the dc side of a PV system is eliminated.

Connection of any number of ac modules may be accomplished in a system. The smallest size system may be a single ac module. AC modules may be added to existing systems over time to increase system size. AC Modules are primarily targeted to residential markets. AC Modules may also be used in commercial markets as well. Reference [2] includes a broad description of the benefits of ac PV modules.

\section{Period of Performance}

This annual report is a summary of significant research results obtained during FY 1997 (10/1/96 9/30/97).

\section{Zebra $^{\text {TM }}$ Anti-Islanding}

In January 1997, islanding performance tests of the SunSine300 were conducted. Results of these tests were presented at the $26^{\text {th }}$ PV Specialists Conference [3]. During initial testing of the unit, it was found that voltage and frequency trips in combination with a fixed frequency offset method for islanding protection was not sufficient to prevent islanding from occurring in a controlled laboratory environment. Analysis and experimentation was conducted in order to identify a method which would prevent such islanding from occurring.

During this period, the Zebra ${ }^{\mathrm{TM}}$ method was developed. It is an active method which dynamically controls the output current waveform and watches the voltage waveform for feedback. When the utility is disconnected and an island is formed, the feedback mechanism changes allowing the unit to sense this condition and shutdown. This method is proprietary and has not been fully disclosed to any parties outside of Ascension Technology, Inc.

This method is not perfect and does not solve all issues related to required islanding performance when considering high levels of penetration of PV in utility distribution systems. However, it is superior to other methods we had seen up to that time. 


\section{Highly Accelerated Lifetime Testing (HALT)}

Direct costs for testing were funded through Sandia National Laboratories' PV BOS program. Labor costs for this testing were funded through this NREL PVMaT project.

Reliability of the dc/ac inverter is critical to the success of the ac module. HALT testing is a special test methodology which integrates testing of the product in a chamber which has the following features; extreme cold and hot temperature operation, six axis broadband vibration up to $60 \mathrm{Grms}$, and temperature ramp rates of $60^{\circ} \mathrm{C} /$ minute. All of these parameters can be controlled at the same time forcing failure modes to show up very rapidly in the product.

These tests were conducted at QualMark's QRTC facility in Westminster, CO in March 1997. Twenty (20) deficiencies were identified in either the product design or assembly procedure. These deficiencies were corrected during the months following the tests and in conjunction with re-design necessary to meet UL requirements. Below is a summary of the limits which the SunSine 300 met during the HALT testing before the deficiencies were corrected.

SunSine300, Summary of Operating and Destruct Limits, HALT Testing 3/97 (tests conducted before final design)

\begin{tabular}{|c|c|}
\hline \hline Temperature, Lower Operating Limit & $-70^{\circ} \mathrm{C}$ \\
\hline Temperature, Lower Destruct Limit & $<-90^{\circ} \mathrm{C}$ \\
\hline Temperature, Upper Operating Limit & $90^{\circ} \mathrm{C}$ \\
\hline Temperature, Upper Destruct Limit & $120^{\circ} \mathrm{C}$ \\
\hline Rapid Thermal Transitions & $20 \mathrm{Grms}$ \\
\hline Vibration, Operating Limit & $20 \mathrm{Grms}$ \\
\hline Vibration, Destruct Limit & $-55^{\circ} \mathrm{C}$ to $85^{\circ} \mathrm{C} \mathrm{@,} 45^{\circ} \mathrm{C} / \mathrm{min}$ \\
\hline Combined, Operating Limit & $-55^{\circ} \mathrm{C}$ to $85^{\circ} \mathrm{C} @, 10 \mathrm{Grms}$ \\
\hline Combined, Destruct Limit & $-55^{\circ} \mathrm{C}$ to $85^{\circ} \mathrm{C} \mathrm{@}>35 \mathrm{Grms}$ \\
\hline
\end{tabular}

\section{FCC Testing}

The SunSine300 was tested to meet FCC Class B requirements using CISPR 22 test methods. Tests were conducted using TÜV Product Services of Boulder, CO. These tests were completed and passed in July 1997.

\section{SunSine300 UL Listing}

The most significant accomplishment during this period has been to effectively complete the requirements for UL Listing of the SunSine300.

Preliminary Investigation

A preliminary investigation of the SunSine300 was completed in November 1996, [1]. 
"The purpose of this investigation was to provide explanatory information concerning the requirements which will be applied to this product and to point out constructional features which need additional clarification or are not in compliance with UL requirements."

This investigation by UL identified sixteen (16) areas of particular concern where further work was needed on the design. The report also identified twenty (20) specific tests which would be applied to the product during factory testing.

\section{Standards Development}

Development of national standards is important for the widespread acceptance of PV systems. In January 1997, an Industry Advisory Group (IAG) was convened at UL's facilities for the purpose of reviewing UL 1741 [5]. Ascension Technology participated in the IAG meeting to provide input on inverter and ac module requirements for UL Listing. Ascension Technology also participated in the revisions to the 1999 National Electric Code. Particular attention was given to code requirements for ac modules. Ascension Technology also participates in development of a National Standard for photovoltaic systems, IEEE - P929.

\section{$\underline{\text { UL Factory Testing }}$}

A significant portion of the UL listing process is to conduct specific tests of the product under the supervision of a UL test engineer. These tests were conducted at three separate times in two locations, ASE America's in Billerica, MA, and NREL's OTF in Golden, CO. On June 24 ${ }^{\text {th }}$, 1997, the final set of tests were concluded and Ascension Technology was given verbal notification by UL that the test requirements for the SunSine 300 had been concluded.

\begin{tabular}{|c|c|}
\hline \multicolumn{2}{|c|}{ Summary of UL Test Requirements } \\
\hline \hline $60^{\circ} \mathrm{C}$ Ambient Temperature Test & Dielectric Voltage Withstand \\
\hline Verification of Nameplate Ratings & Harmonic Distortion \\
\hline Anti-Islanding Protection & DC Injection \\
\hline Utility Fluctuations & Loss of Control Circuit \\
\hline Transformer Burnout & Output Overload \\
\hline AC Short Circuit & Components Faults, shorts/opens \\
\hline Grounding Impedance & Strain Relief \\
\hline Water Spray (rain test) & Materials Accelerated Aging \\
\hline Temperature Cycling, 40 days & Humidity Cycling, 10 days \\
\hline Capacitor Fault Test & \\
\hline
\end{tabular}

\section{Final Certification}

Verbal and preliminary written approvals had been given from UL in the period shortly after testing was completed in June 1997. However, it was not until October, that formal written approval was provided [6]. 


\section{Pilot Production}

Pilot production of 110 SunSine300 units began on September 1, 1997.

\section{Conclusions}

AC PV Modules can be designed to meet UL, NEC and IEEE-929 requirements for safety and performance. AC PV Modules can be designed for manufacture and can be integrated into the dc PV laminate. This initial design was more costly than originally predicted due to design changes needed to pass UL Listing and FCC requirements. In order to meet market demands for the product, time was not taken to go through another design cycle to fully optimize the design with respect to parts and'production costs.

\section{Areas for Future Work}

The next step in the technology development will be to make improvements in the design of the SunSine 300 so that it can be less costly to produce. Areas for parts count reduction and manufacturing process improvements have been identified and are being considered for future work.

\section{References}

[1] D. Ackerman, Underwriters Laboratories, Inc. "Preliminary Investigation of Photovoltaic AC Module Model Sunsine 300", letter to Ascension Technology, November 12, 1996.

[2] G. A. Kern, Interconnect Guidelines and Status of AC PV Modules in the United States, Proceedings of the International IEA - Workshop on Existing and Future Rules and Safety Guidelines for Grid Interconnection of Photovoltaic Systems, Zurich Switzerland, Sept. 15, 1997.

[3] G. A. Kern, SunSine300, Utility Interactive AC Module Anti-Islanding Test Results, Presented at the IEEE PV Specialists Conference \#26, Anaheim, CA. Sept, 1997.

[4] M. C. Russell, C.K.P. Handleman, SunSine300 AC Module Annual Report 25 July 1995 31 December 1996" Ascension Technology, Inc. August 1997 NREL/SR-520-23432.

[5] Underwriters Laboratories, Inc. PROPOSED FIRST EDITION OF THE STANDARD FOR STATIC INVERTERS AND CHARGE CONTROLLERS FOR USE IN PHOTOVOLTAIC POWER SYSTEMS, UL 1741, August 1, 1997.

[6] T. Zgonena. NOTICE OF AUTHORIZATION TO APPLY THE UL MARK, Letter from UL, Engineering Services to Ascension Technology, re. Photovoltaic AC Module Model SunSine300, October 29, 1997. 
Title: $\quad$ Market-Driven EFG Modules

Organization: $\quad$ ASE Americas, Billerica, Massachusetts

Contributors: M. Kardauskas, principal investigator, J. Kalejs, J. Cao, S. Ebers, R. Gonsiorawski, B. Piwczyk, E. Tornstrom

\section{Introduction}

This Executive Report summarizes the progress made at ASE Americas Inc. during the second year of the planned three year program in Phase $4 A 2$ on the development of technology to address its photovoltaic module manufacturing flexibility and cost reduction for its products. This program focusses on advancing manufacturing technology to reduce the thickness of EFG wafers from 300 to 250 microns, to raise EFG solar cell efficiency to be able to produce cells with average efficiency of $15.5 \%$ on $10 \mathrm{~cm} \times 10 \mathrm{~cm}$ area wafers, and to simplify processes and reduce costs in interconnect and module manufacturing. This work includes the development of a novel and environmentally safe and reduced-cost diffusion glass removal process for the solar cell manufacturing line. The overall goal of the program is to reduce EFG module manufacturing costs by $25 \%$. Module cost reductions of approximately $9 \%$ can be identified as a result of successes on the program in the first two years. The significant technological advances made in the second year of the program are described in the next three sections, representing the work in the three main tasks: Task 4: Wafers, Task 5: Cells, and Task 6: Modules.

\section{Technical Developments}

\section{Task 4: Wafers}

The work in this task involved developing improvements to the EFG crystal growth process that will lead to increased as-grown quality of EFG wafers and so contribute to an increase in solar cell efficiency in Task 5 , and to reduce wafer thickness while maintaining yield. During the second year of the program two of the major equipment projects within the crystal growth portion of this PVMaT contract were addressed: the design and construction of an enclosure for EFG crystal growth furnaces, to reduce atmospheric contamination of the growing crystals, and the construction and testing of an automated system for sorting silicon feedstock material. Although difficulties with the operation of the furnace enclosure have so far prevented its introduction into routine production, the feedstock sorting system is now in full use in manufacturing. The use of the sorting system has reduced labor requirements in the crystal growth area, while increasing feedstock utilization by $3 \%$. In addition, the thickness of EFG wafers has been reduced for most standard production from $300 \mu \mathrm{m}$ to $275 \mu \mathrm{m}$, which has not only further reduced raw materials costs, but has also resulted in an increase in average cell efficiency.

\section{Highlights}

1. EFG wafer thickness has been reduced in most standard production from $300 \mu \mathrm{m}$ to $275 \mu \mathrm{m}$, resulting in an $8 \%$ reduction in silicon usage. The thinner wafers have also been found to produce cells with efficiencies about $0.1 \%$ absolute higher than cells grown during the same production weeks at the higher thickness. (EFG octagon tubes of both thicknesses are currently grown in production, to facilitate the collection of data on the effect of the change.) There were some initial problems implementing the thinner material into the production line because of its lower mechanical strength, and production yields continue to be slightly lower for the thin material. However, a cost analysis has shown that there is a net economic benefit from the reduced wafer thickness, even within the first few months after the change in production. This benefit is expected to increase further as processes are adapted to accomodate the more fragile material. 
2. Improvements in the crystal growth area have greatly increased the average length of crystal growth runs. (A 'run' is a period of continuous production from a growth furnace, using a single graphite die. The die must be replaced when the run ends, requiring the furnace to be cooled down and then reheated.) A new record run length of 98 tubes (producing material for about 35,000 wafers) from a single die has been established. The improvements in run length result in part from the ending of the introduction of oxygen into EFG furnaces during crystal growth, a practice which was previously practiced in order to getter metallic impurities in the furnaces. The oxygen addition is no longer necessary because of the use of an improved purification process for the furnace components, developed during the first year of this PVMaT contract. Further improvements in run length have also resulted from incremental changes in the design of some of the furnace components, which has increased their mean time to failure.

3. Commercially available equipment has been modified and upgraded for use in sorting the silicon feedstock used in the EFG furnaces according to particle size. In a series of tests, the modified equipment was found to be capable of sorting the material at reasonably high rates, without contaminating the feedstock. As a result, the equipment has been purchased, and is being used to sort all production feedstock. The use of this equipment has not only reduced labor requirements, but has also reduced the reject rate for incoming feedstock, which has resulted in a higher utilization rate for the silicon material. The increase in utilization rate has been achieved by carefully metering back into production material lots small amounts of feedstock which would have failed the size specifications used for manual sorting. This procedure requires good control over particle size, which in turn requires multiple sorting operations. Those operations had not been cost effective when sorting was performed manually.

4. An enclosure for the EFG furnace was constructed and tested. The design allows the EFG octagon to be grown to its full length in a totally enclosed space, reducing perturbations in material quality that arise from external ambient contamination brought into the furnace in the form of transition metals (dust) and oxygen. The use of the enclosure, however, increased the amount of time required to detach completed EFG tubes and restart growth, so that an economic benefit from the use of the enclosure has not yet been demonstrated. A redesign of the system is currently under study.

The first three of the above improvements are estimated to result in a 3.5\% decrease in module manufacturing costs in the production line.

\section{Concerns}

Progress has been made toward identifying a reduced damage laser cutting process, which is needed to allow 250 micron thick wafers to be processed through the EFG line without a reduction in yield. Although reasonably good cutting yields have been achieved with $275 \mu \mathrm{m}$ tubes (approximately $3 \%$ lower than for 300 $\mu \mathrm{m}$ tubes, at this point), yields are unacceptably low for $250 \mu \mathrm{m}$ tubes. The problem with cutting the thinner tubes arises because of the occurrence of very thin regions in some tubes. The high breakage losses experienced when cutting these regions was originally thought to be caused by cracks produced by thermal stresses surrounding the region heated directly by the laser beam, but a closer analysis reveals that much of the breakage is caused by the high pressure gas jet used to eject the material melted by the laser beam. An effort is underway to improve the gas nozzle to reduce the force exerted on the tube, which might allow the cutting of $250 \mu \mathrm{m}$ tubes with reasonable yields. Once this is achieved, it will still be necessary to modify other downstream processes, particularly the metallization processes, to maintain an acceptable overall yield in the cell manufacturing line.

A statistical analysis of cell efficiency as a function of run length has also been carried out, and has shown that cells made from wafers produced late in a crystal growth run generally have lower efficiencies than those produced early in the run. Although this suggests an accumulation of impurities in the furnace, there is 
evidence that this is not the underlying cause of the phenomenon, because, after a certain point in the growth run, wafer quality appears to recover. The cause of this effect has not yet been discovered, although one possibility is that it is related to the growth of silicon carbide precipitates on the die surface. If the effect could be eliminated, overall average efficiency for EFG cells would be raised on the order of $0.2 \%$. The effort to achieve this gain is continuing.

\section{Task 5: Cells}

\section{Highlights}

1. A method to reduce fluorine ion effluent in etching of phosphorus glass by two orders of magnitude developed by a lower tier subcontractor, Bright Technology, has been incorporated into prototype etching equipment which is undergoing extensive testing at ASE Americas. The etching process has proven to be very effective and robust, but achieving adequate rinsing in an in-line process has proven to be more difficult than anticipated. Improvements have now been made in the rinsing process, so that the efficiencies of solar cells fabricated using the new etch process are equivalent to those fabricated using the standard etch process. As soon as the equipment can be modified to incorporate the new rinse process in a fully automated format, the prototype etch station will be installed adjacent to the cell production line for further high-volume testing to determine the robustness of the process, equipment components, and materials of construction.

Simultaneously, design work is beginning on the construction of a manufacturing-quality production machine, using inputs from the test program. The production machine is scheduled to be installed on the manufacturing line in mid-1998, at which point the use of the standard etch process will be discontinued.

2. Studies have been conducted both at Georgia Institute of Technology (GIT) and at an equipment vendor's site on the use of Rapid Thermal Processing (RTP) in the manufacture of EFG solar cells. To date, the best results at GIT have exceeded $16 \%$ efficiency on $1 \mathrm{~cm}^{2}$ areas. The attempt to duplicate part of that process by the use of RTP diffusion, which was conducted at the vendor's site, has not yet produced cells with efficiencies higher than those produced by the standard ASE process, however. Studies are continuing aimed at developing a high-throughput, high-efficiency process, which can solve problems associated with the use of standard belt furnaces for diffusion.

3. A program has been started to introduce a computer-based-manufacturing floor wide data collection system and a diagnostic and analysis capability for Statistical Process Control (SPC) methods. These capabilities are initially being used to set up SPC as a manufacturing line tool to optimnize solar cell efficiency. The establishment of the data collection system is the first step in a program that will provide rapid flow of information to engineering staff and production operators, facilitate total preventive maintenance strategies to improve equipment reliability, and provide a quality control system to enhance product reliability and customer satisfaction.

4. Calculations carried out in Phase I of this contract indicated that cell efficiency would be improved by an increase in the number of fingers on EFG cells by approximately $25 \%$. Because of ASE's unique technology for metallizing the front side of solar cells, a change in the number of fingers requires a significant investment in new equipment. That new equipment has now been fabricated, and is scheduled for testing in production before the end of Phase II. The change in the metallization pattern is expected to increase efficiency by about $0.2 \%$ absolute.

5. A process to apply a dielectric textured $\mathrm{ZnO}$ layer on an EFG solar cell with a silicon nitride underlayer was successfully demonstrated at lower tier subcontractor Harvard University. The $\mathrm{ZnO}$ layers did not lead to a significant increase in EFG solar cell efficiency, however. Analysis of the results indicated that the effectiveness of ASE's standard double-layer anti-reflection (AR) coating was so high that the small 
additional benefit that could be expected from the $\mathrm{ZnO}$ coatings could not be justified on an economic basis, given the requirement for an additional production step.

The efficiency improvements achieved by these programs to date are estimated to contribute a $3 \%$ reduction in module manufacturing costs.

\section{Task 6: Modules}

\section{Highlights}

1. A redesign of the module diode housing and electrical protection circuits has been completed, and the new design has been rigorously tested, including testing by Underwriters Laboratories. Based on the results of this testing, minor improvements have been incorporated into the design. The final versions of both the housing itself and the printed circuit boards to be used in the housing are currently being manufactured. The new housing is expected to enter production before the end of Phase II, reducing module manufacturing costs by $2.5 \%$.

2. Analysis of the costs of several alternative frame designs has led to the conclusion that the most appropriate frame for future modules would incorporate edge protection to prevent breakage of the tempered glass, but would not carry the full structural load associated with the module, as is done with the current generation of frames. Attachment to the underlying structure should be done using edge clamps on small (50 W) modules, and adhesively bonded mounting fixtures on larger ( $300 \mathrm{~W})$ modules. Samples of two types of clamps have been obtained for testing with the smaller modules, and are currently under evaluation.

3. Several new encapsulants with potential advantages for EFG products were extensively tested for performance advantages compared with the standard ASE encapsulant. One of these has outperformed both the standard encapsulant and all other encapsulants known to be used in the PV industry today. Negotiations with the manufacturer of this new material are underway for the procurement of production quanitities, which could be placed in production early in Phase III, ahead of the original schedule. The additional advantage of improved lamination throughput, which was being sought with other materials which proved to be difficult to manufacture, may also be obtainable using the new high-performance material. The manufacturer has offered to modify the material to obtain this additional benefit, and a plan for reformulation testing is being prepared.

4. A novel approach for improving the collection of light from areas in between cells in modules has been developed to the point where it appears that it may be feasible to reduce cell packing density in modules significantly while maintaining high module efficiency. A number of cell coupons demonstrating the concept have been prepared and delivered to NREL for testing. Techniques for large volume manufacturing of the new design, and robust component materials of moderate cost are still being developed. Current plans call for implementation of this new design for some fraction of module production before the end of Phase III.

Improvements in interconnection throughput and module yield achieved during the first two years of the contract have resulted in module cost reductions of $2.5 \%$. Additional savings will result from the introduction of the new diode housing into full production in early 1998 . 
Title: $\quad$ Large-Area Silicon-Film ${ }^{\mathrm{TM}}$ Manufacturing

Organization: AstroPower, Inc., Solar Park, Newark DE 19716-2000

Contributors: J.A. Rand, Y. Bai, J.S. Culik, D.H. Ford, C.L. Kendall, P.E. Sims, R.B. Hall and A.M. Barnett

\section{Abstract}

AstroPower is developing a manufacturing process for Silicon-Film ${ }^{\mathrm{TM}}$ solar cell production under an NREL-administered PVMaT cost-share program. Progress in improving efficiencies and increasing the size and production volume of Silicon-Film ${ }^{\mathrm{TM}}$ solar cells over the past year are discussed. Future concepts and goals for the Silicon-Film ${ }^{\mathrm{TM}}$ process are also discussed.

\section{Introduction}

The Silicon-Film ${ }^{T M}$ process is presently in pilot-scale production at a 0.5 megawatt per year level. A dedicated production facility with a nine megawatt per year capacity is scheduled to start production in 1998. The present process is based on the AP-225, a $240 \mathrm{~cm}^{2}$ solar cell. Efficiencies exceeding $12 \%$ have been measured for the AP-225. Small, laboratory-scale devices have demonstrated an efficiency of $16.6 \%$.

A key element of Silicon-Film ${ }^{\mathrm{TM}}$ development has been the optimization of the geometry of both the sheet and the resulting solar cell. Optimizing the solar cell size initially focused on nominally $15-\mathrm{cm}$ wide sheet employed to generate solar cells with areas of 240 and $645 \mathrm{~cm}^{2}$. Recently a new sheet fabrication machine has been constructed that produces Silicon-Film ${ }^{T M}$ sheets greater than $30 \mathrm{~cm}$ in width which has been used to produce prototype solar cells with areas of 300,400 and $1800 \mathrm{~cm}^{2}$. In-line solar cell process equipment has been developed to fabricate these large devices. This new equipment includes an in-line emitter diffusion system and an in-line anti-reflection coating application machine.

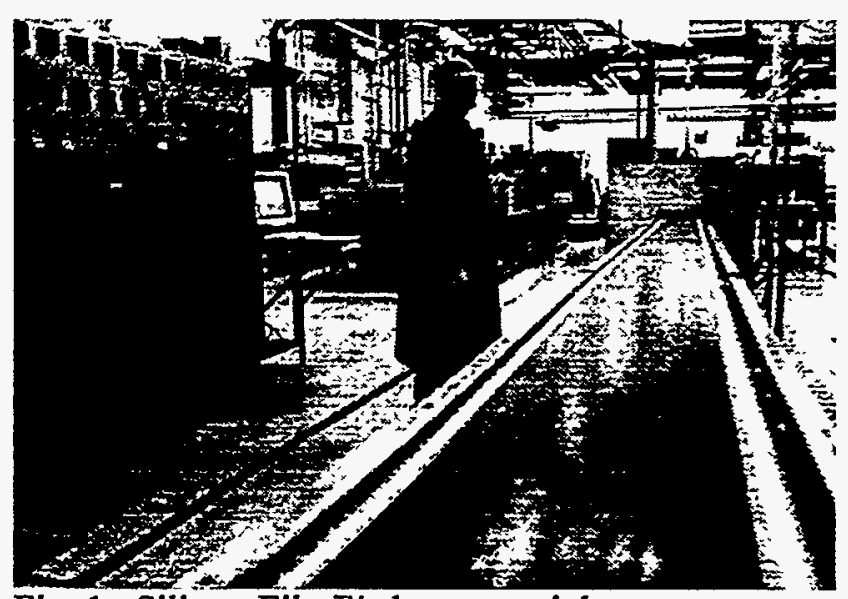

Fig. 1. Silicon-Film ${ }^{\mathrm{TM}}$ sheet material.
This in-line approach has been generalized to a "Flexible Manufacturing" solar cell production concept. Future plans call for $30 \mathrm{x}$ $120 \mathrm{~cm}$ Silicon-Film ${ }^{\mathrm{TM}}$ planks to be processed by a single set of in-line equipment and then be subdivided into solar cells with areas of 105 , $150,180,225,300,450$ and $900 \mathrm{~cm}^{2}$ in a "Just In Time" fashion before solar cell test and module assembly.

Silicon-Film ${ }^{\mathrm{TM}}$ sheet material gives AstroPower the unique potential of servicing multiple solar power market segments with a stable, high-efficiency crystalline silicon product manufactured in a single integrated production facility. 


\section{Approach}

There are three basic development areas involved in moving Silicon-Film ${ }^{\mathrm{TM}}$ from the laboratory to the factory:

1. Optimization of the sheet generation process; high-speed, high quality and large areas are desired.

2. Optimization of the solar cell fabrication sequence to achieve high-efficiencies with large areas. These processes must be compatible with manufacturing and preferably be in-line and continuous rather than batch-mode.

3. Integration of these developments in an industrial setting by generating (and selling) significant amounts of solar cells.

\section{Results}

\section{Sheet Generation}

The Silicon-Film ${ }^{\mathrm{TM}}$ technology has the capability to generate large area sheets at high areal generation rates. Figure 1 displays a Silicon-Film ${ }^{T M}$ sheet. A single Silicon-Film ${ }^{T M}$ machine is capable of generating five megawatts of solar cell material per year. A continuous sheet of polycrystalline silicon solar cell material is generated at a rate 17 times faster than any competing sheet technology. This difference has a great impact on capital costs during manufacturing scale-up.

A prototype Silicon-Film ${ }^{\mathrm{TM}}$ machine which generates $38-\mathrm{cm}$ wide sheets has also been constructed. This new width capability has led to a reassessment of the "optimal" solar cell size and the planning of a flexible manufacturing process where $30 \times 120 \mathrm{~cm}$ Silicon-Film ${ }^{\mathrm{TM}}$ planks will be processed by a single set of in-line equipment and then be subdivided into one of seven different solar cell sizes before cell test and module assembly.

\section{$\underline{\text { Solar Cell Fabrication }}$}

Substrate size flexibility has resulted in a need for advances in fabricating emitters and antireflection coatings. In contrast to "traditional" solar cell manufacturing based on integrated-circuit technologies, these next-generation Silicon-Film ${ }^{\mathrm{TM}}$ processes are not tied to a single wafer size. Wafers from $105 \mathrm{~cm}^{2}$ to $1800 \mathrm{~cm}^{2}$ can be run through the same equipment. The use of low-cost, environmentally responsible precursors, and industrial coating application equipment with proven reliability, also make these advanced processes particularly attractive to large-scale adaptation.

Large area Silicon-Film ${ }^{\mathrm{TM}}$ emitter fabrication is accomplished by the continuous-feed application of a phosphorous-based coating. This is followed by thermal cycling, using conditions approaching those encountered in rapid thermal processing (RTP). The resulting emitter sheet resistance is typically uniform to within $10 \%$ over the full device area. Wafer deglazing is straightforward, and requires no specialized equipment or chemicals. The in-line diffusion system has been integrated into the AP-225 production process.

For the anti-reflection coating, simple liquid precursors are applied to preheated solar cells, using commercial coating equipment, to form an oxide layer which is index-matched to module encapsulants. Advances made in this technology include the ability to chemically vary the index of refraction, and improvement of coating characteristics by using conductive and radiant heating methods. The AR 
coating prototype system is presently being evaluated before construction of à production version of the machine.

In addition to standard characterization methods, measurements of Silicon-Film ${ }^{\mathrm{TM}}$ material have been made with the use of a RFPCD lifetime measurement technique developed by Dr. Richard Ahrenkiel of NREL. The measurement method allows a contactless measurement of the minority carrier lifetime in grown material with little or no sample preparation. Interpretation of the results has been difficult due to the non-ideal response of the Silicon-Film ${ }^{\mathrm{TM}}$ material. A direct correlation to solar cell performance characteristics has not yet been found. Trapping and related defects are thought to play a role. Work is continuing to fully understand these measurements.

\section{Solar Cell Efficiency}

A. summary of different Silicon-Film ${ }^{\mathrm{TM}}$ solar cells tested at NREL is shown in Table 1. A significant recent result is a $16.6 \%$ efficient solar cell [1] (Fig. 2).

TABLE 1. Summary of solar cell data tested at

\begin{tabular}{cccc}
\multicolumn{5}{c}{ NREL. } \\
\hline Solar Cell & $\begin{array}{c}\text { Area } \\
\left(\mathrm{cm}^{2}\right)\end{array}$ & $\begin{array}{c}\text { Power } \\
(\mathrm{W})\end{array}$ & $\begin{array}{c}\text { Efficiency } \\
(\%)\end{array}$ \\
\hline & & & \\
Laboratory & 0.98 & 0.016 & 16.6 \\
Scale & & & \\
AP-225 & 240 & 2.93 & 12.2 \\
AP-675 & 676 & 7.87 & 11.6 \\
\hline
\end{tabular}

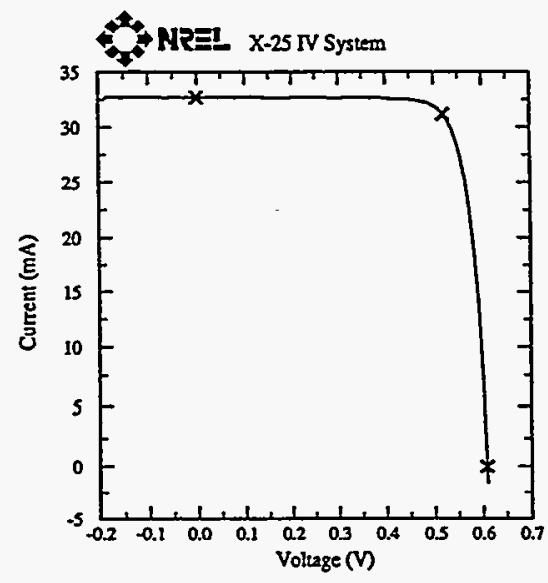

$$
\begin{array}{ll}
\mathrm{V}_{\infty x}=0.6080 \mathrm{~V} & \mathrm{~V}_{\max }=0.5194 \mathrm{~V} \\
\mathrm{I}_{x=32.70 \mathrm{~mA}} & \mathrm{I}_{\max }=31.20 \mathrm{~mA} \\
J_{x=}=33.53 \mathrm{mAcm}-2 & \mathrm{P}_{\max }=16.20 \mathrm{~mW} \\
\text { Fill Factor }=81.49 \% & \text { Efficieacy }=16.6 \%
\end{array}
$$

Fig. 2. Current-voltage curve for a $16.6 \%$ efficient $1 \mathrm{~cm}^{2}$ area Silicon-Film ${ }^{\mathrm{TM}}$ solar cell.

\section{Silicon-Film ${ }^{\text {TM }}$ Panel Production}

In 1997 AstroPower delivered a $120 \mathrm{~kW}$ AP-225 Silicon-Film ${ }^{\mathrm{TM}}$ array to Niagara Mohawk (Albany, NY). This array is composed of frame-less laminate panel assemblies. AstroPower has formed a joint venture with GPU International. The joint venture, GPU Solar is marketing a residential rooftop system which is based on this Silicon-Film ${ }^{\top M}$ panel.

AstroPower has also installed a $17 \mathrm{~kW}$ AP-225 array as part of the PVUSA Emerging Technologies (EMT) program. This Array has been operating without fault or significant performance degradation since its November 1994 installation. A comparison of the power produced by all the EMT arrays in 1995 and 1996 is displayed in Figure 3. The Silicon-Film ${ }^{T M}$ array has demonstrated strong energy production since its installation. 


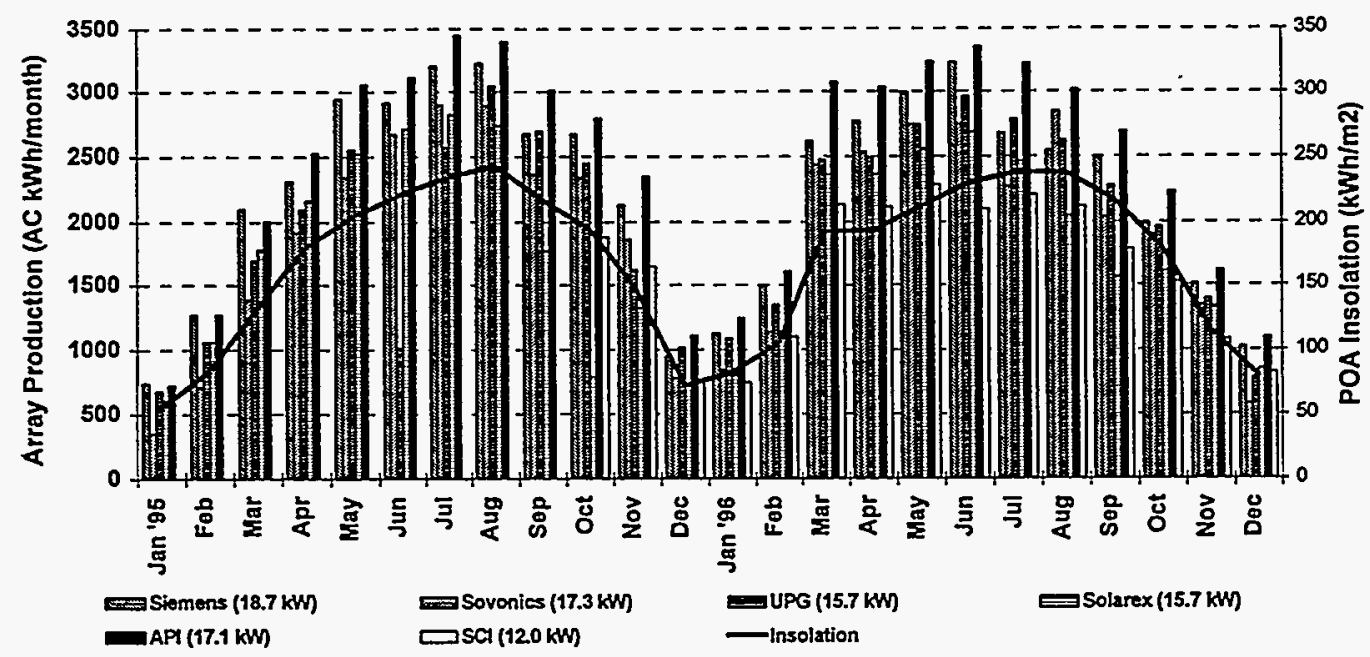

Fig. 3. Summary of the PVUSA EMT array energy production for 1995 and 1996 [2].

\section{Summary}

AstroPower is developing a manufacturing process for Silicon-Film ${ }^{T M}$ solar cell production. The present process is based on the AP-225, a $240 \mathrm{~cm}^{2}$ solar cell. Optimization of the solar cell fabrication sequence has been utilized to achieve high-efficiencies with large areas. Efficiencies exceeding $12 \%$ have been measured for the AP-225 solar cell. Small, laboratory-scale devices have demonstrated $16.6 \%$ efficiencies.

In-line solar cell process equipment has been developed to accommodate large solar cells (up to 1800 $\mathrm{cm}^{2}$ ). This new equipment includes an in-line emitter diffusion system and an in-line anti-reflection coating application machine. This in-line approach has been generalized to a "Flexible Manufacturing" solar cell production concept. Future plans call for $30 \times 120 \mathrm{~cm}$ Silicon-Film ${ }^{\mathrm{TM}}$ planks to be processed by a single set of in-line equipment and then be subdivided into a range of solar cell sizes from $105 \mathrm{~cm}^{2}$ to $900 \mathrm{~cm}^{2}$ in a "Just In Time" fashion before solar cell test and module assembly.

\section{Acknowledgments}

This work was supported in part by the U.S. Department of Energy through the National Renewable Energy Laboratory (NREL) under the Photovoltaic Manufacturing Technology (PVMaT) Program.

The authors would like to acknowledge the contributions of Emanuel DelleDonne with device fabrication, Thomas Ellwood with Silicon-Film ${ }^{\mathrm{TM}}$ sheet manufacture, Bill Bloothoofd, Chris Colgan, and Joe Checchi with design and construction of the new Silicon-Film ${ }^{\mathrm{TM}}$ machine.

\section{References}

[1] Y. Bai, et al., "16.6\% Efficient Silicon-Film"M Polycrystalline Solar Cells," Twenty Sixth IEEE PVSC, Anaheim CA, Sept 29 - Oct 3, 1997.

[2] PVUSA 1996 Progress Report. 
Title: $\quad$ PVMaT Work at Evergreen Solar

Organization: Evergreen Solar, Waltham, Massachusetts

Contributors: J.I.Hanoka, Principal Investigator; P.Kane, J.Fava, B.McMullen, J.Martz

\section{Introduction}

Evergreen's PVMaT project was a two year effort which was begun in 1995 and which addressed the following four goals:

(1) a new backskin material which allows for a frameless module;

(2) innovative mounting methods utilizing the new backskin material;

(3) a new encapsulant to replace EVA and which does not require vacuum lamination; and

(4) a continuous, in-air lamination method made partly possible by the new encapsulant.

In the first year of the project, (1) and (2) were the principal focus, while in the second year (3) and

(4) received most of the attention, although more extensive work on (1) and (2) also was conducted in year two. This report summarizes the progress made in year two. As will be seen, all the basic goals of the project were successfully met. The net result of all this will be an estimated saving of $\$ 0.50 / \mathrm{watt}$ module manufacturing cost.

\section{New backskin material}

The basic approach used in identifying a new backskin material was to survey commercially available polymers and then to investigate what modifications to these materials were necessary for the particular needs of PV modules. A material which was already in use in other major industries was identified. This was a material for which outdoor weathering data was already available. It was selected for our application, and then suitable modifications were made to it to satisfy the particular PV module requirements.

The material chosen is a thermoplastic which could be formed during the lamination process itself so as to seal and frame the module edges and obviate the need for an aluminum frame. The backskin is 0.04 .0 " thick (about $1 \mathrm{~mm}$ ) over the rear surface of a module and at the modules' edges it is about 0.125 " thick (about $3 \mathrm{~mm}$ ).

A number of tests were conducted on this material, primarily to identify any early issues, but also to determine that the new backskin material and frameless module design would pass all qualification tests. Areas addressed were thermal creep behavior, bond strength, and environmental endurance.

\section{Thermal creep behavior}

For PV applications, particularly architectural ones, temperatures as high as $90^{\circ} \mathrm{C}$ can be reached in a module, and so thermal creep resistance at temperatures well above $90^{\circ} \mathrm{C}$ is necessary. Furthermore, part of UL test 1703 is a Relative Thermal Index (RTI) test which must be passed by PV modules to obtain UL approval. This test determines creep resistance at high temperatures $\left(\sim 150^{\circ} \mathrm{C}\right)$, and relates this back to mechanical and electrical properties at temperatures of $\geq 90^{\circ} \mathrm{C}$. A backskin material must have an RTI of $\geq 90^{\circ} \mathrm{C}$. The test is stringent and easily eliminates a large number of polymers as candidate backskin materials.

We found that with suitable modification of the selected material this test would likely be passed. In fact we have subjected 1 " $\times 6$ " strips of the backskin material with a nominal load of 1 psi to temperatures over $300^{\circ} \mathrm{C}$ without observing any significant creep. The RTI tests with UL have just been started. Based on our in-house experiments we expect no problems.

\section{Bond strength}

Two aspects of bond strength were studied. First was the strength of the bond between the backskin material and the superstrate glass as the backskin wraps around the edge of the module and is bonded to the front glass surface. Second was the question of the bond strength between the backskin material and any mounting components we choose to bond to it. 
In the case of the backskin-glass bond, an accelerated test was devised which was found to be useful in optimizing the process to provide maximum bond strength at this interface. The test involved an initial soak in water followed by freezing so as to form ice all over the module. With non-optimized processing conditions, particularly too low of a lamination temperature, it was possible to produce bond-failure after 7-9 such immersion-freeze cycles. Optimizing this process, on the other hand, produced exceptionally strong bonds. Samples with over 150 cycles have been tested with no indication of any bond weakening.

Based on our plan to eliminate the usual aluminum perimeter frame and bond aluminum mounting brackets directly to the backskin, the bond strength of the resulting backskin-aluminum interface was clearly important. The innovative mounting system which was finally chosen utilizes aluminum "slide bars" which are bonded onto the backskin of the module and extended a slight distance beyond the edge of the module. The completed frameless module with aluminum slide bars can then be mounted by simply sliding the module over two pieces of C-channel [1]. Two tests of the bond of aluminum mounting components were performed: thermal creep and static load. Thermal creep measurements were made at $85^{\circ} \mathrm{C}$ with a load of 1.15 psi on a mounting component bonded to the backskin. The $1.15 \mathrm{psi}$ load was selected as an initial estimate of the maximum sustainable load. After 45 days, virtually no creep had occurred.

For static loads, the minimum test criterion was 50 psf, as specified in module qualification tests JPL Block 5 and IEEE 1262. Roughly speaking, this corresponds to a wind speed of $125 \mathrm{mph}$. However, since UL 1703 requires a 50\% safety factor, the test criterion was increased to 75 psf loading. Based on the likely worst-case and the dimensions of our test modules, this required a bond strength of 18.7 psi over the bond area. We bonded a mounting bracket structure to a test module, and loaded it to $18.7 \mathrm{psi}$. The sample was placed in a thermal chamber for 308 hours at $60^{\circ} \mathrm{C}$. Over this time, the bond showed no degradation.

We then increased the severity of this test by repeating it at a higher loading of 19.3 psi for 216 hours at $70^{\circ} \mathrm{C}$, then for 168 hours at $80^{\circ} \mathrm{C}$, and finally for 144 hours at $90^{\circ} \mathrm{C}$, all under the increased load. In all these cases no bond weakening could be detected.

Small test modules with the new backskin, the frameless design, and, in some cases, with aluminum mounting components bonded onto the back were then subjected to environmental stress tests. In some cases, tests were confined to the IEEE 1262 specifications. and in other cases, tests were extended well beyond these requirements. One of the most stringent of the environmental stress tests is humidity freeze. The IEEE 1262 humidity freeze test involves 10 cycles of $-40^{\circ} \mathrm{C}$ to $85^{\circ} \mathrm{C}$ at $85 \%$ relative humidity (RH), the latter $85-85$ for 20 hours. Various kinds of samples were subjected to different degrees of exposure. Even after more than 100 humidity freeze cycles, the edge seal and mounting component bond strength suffered no degradation. Damp heat (85-85) for 2000 hours is another of the standard tests. This was not done but it is interesting to note that 100 humidity freeze cycles contains within it 2000 hours of damp heat, albeit not all at one time.

Two module samples were tested to failure and exhibited some cracks in the backskin material after about 145 humidity-freeze cycles.

In terms of permeability, no reliable quantitative measurements have yet been made with the new backskin vis a vis Tedlar backskin. However, a qualitative test was done which did indicate that the permeability was, at minimum, no worse than that of the Tedlar. This qualitative test was made possible by the observation that modules made with EVA and Tedlar, as control modules, exhibited yellowing of the EVA after a very long $(>100)$ number of humidity-freeze cycles. Clearly, some oxygen and water vapor can permeate through the Tedlar to produce yellowing. No light was present in the environmental test chamber, so this was evidently not an example of photo-oxidation but instead probably just thermal induced oxidation. In any case, identical test samples of a Tedlar module with an aluminum frame and a frameless module with the new backskin were subjected to humidity-freeze cycles. This experiment encompassed fewer than 100 cycles, but no yellowing of the EVA was observed for either module. 


\section{Frameless modules and mounting methods}

Using the new backskin material and the appropriate lamination conditions, we can now obtain edgesealed and "framed" modules directly from the lamination step. Following successful completion of reliability tests, Evergreen is planning to market such modules, sometime early in 1998. The modules will be produced in two standard sizes, $30 \mathrm{~W}$ and $60 \mathrm{~W}$. These modules will be made with String Ribbon solar cells, using Evergreen's own proprietary technology in both growing silicon ribbon and in low-cost cell processing [2,3]. Fig. 1 shows a corner of a $60 \mathrm{~W}$ module where the "framing" effect of the backskin wrapping around to the front of the module and bonding to the glass superstrate can be seen. To develop a feel for scale, the String Ribbon cells in this module are $5.6 \mathrm{~cm}$ by $15 \mathrm{~cm}$.

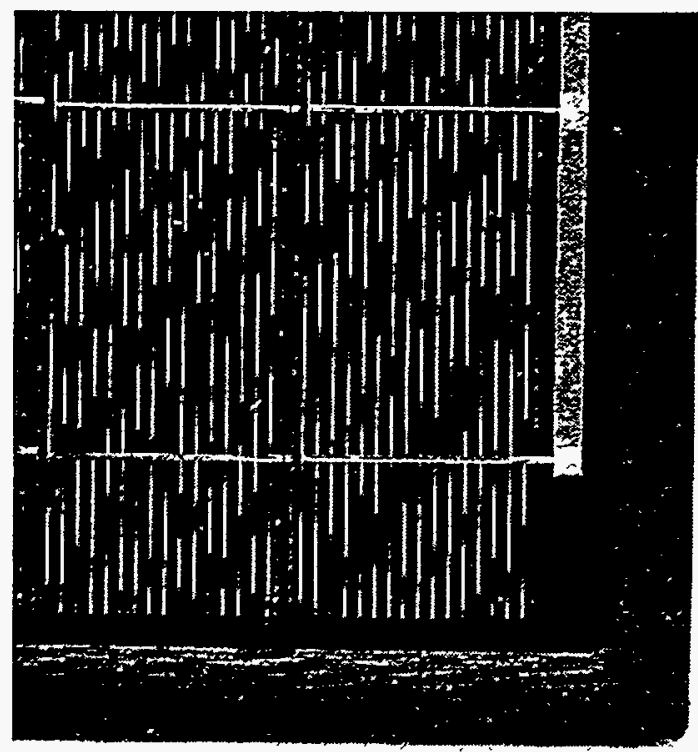

Fig. 1. The corner of a module witit the. backskin wrapped around the edge

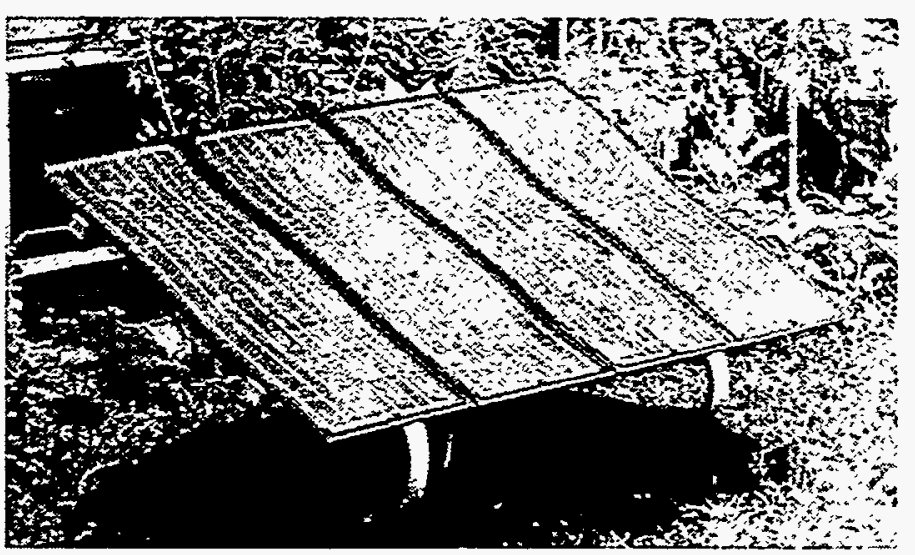

Fig. 2. Modules mounted with slide bar technique.

A number of such modules have been made along with the slide bar mounting mentioned above. Fig. 2 shows four Evergreen modules located in suburban Boston.

The backskin material allows for an entire spectrum of mounting possibilities. Using the slide bar concept and " $\mathrm{C}$ " channel, modules can be readily roof-mounted. One example, using Ascension Technology's ballasted roof mounting brackets, is shown in Fig. 3. We have also successfully bonded a bent aluminum plate for a pole mount and tested it for bond strength with no apparent problems. We have also successfully and easily bonded large area flat head aluminum bolts. Finally, the backskin material can be heat bonded to other polymers in such a way as to produce a module with no junction box and the leads emerging, sealed to the backskin material, from the edge of the module. Figure 4 illustrates a module made this way.

\section{New Encapsulant}

A new transparent en'capsulant material has been developed which shows considerable promise as a replacement for EVA. The material can be obtained in sheet form, about 18 mils thick. It does not require chemical cross-linking as does EVA, and consequently avoids some of the issues associated with the organic peroxide additives used for cross-linking EVA. The new encapsulant can be laminated in air, unlike EVA which requires a vacuum, and in early tests bonds very well with all adjacent surfaces. A new UV stabilization package has been developed specifically for this encapsulant and early accelerated tests for UV exposure indicate it is better than EVA. Two tests in particular support this conclusion. In one, UVA lamps in a QUV machine have exposed bare samples of both the new encapsulant and crosslinked TBEC EVA over 6000 hours at $50^{\circ} \mathrm{C}$. Visually, the EVA samples look yellow compared to the new encapsulant. In the other test, similar samples laminated between glass plates have been exposed to 
Title: Monolithic Amorphous Silicon Modules on Continuous Polymer Substrates

\author{
Organization: $\quad$ Iowa Thin Films Technologies, Inc., Ames, IA \\ Contributors: $\quad$ F.R. Jeffrey, Principal Investigator; D. Grimmer; S. Martens, H. Shanks, and M. Noack, \\ Investigators
}

\title{
Objective
}

The overall goal is to develop the most cost effective PV manufacturing process possible. To this end Iowa Thin Film Technologies, Inc. has chosen a roll based manufacturing process with continuous deposition and monolithic integration. The objective of this subcontract over its three year duration is to improve overall module performance, increase the throughput of the metalization, a-Si deposition, laser-scribing and screen-printing processes, and to reduce the overall module manufacturing costs of the ITF production line by $68 \%$.

\section{Approach}

This subcontract consists of three one-year phases which began in July, 1995 and will be completed February 28, 1999. This report covers in-house and subcontracted research and development in FY 1997 (October 1996 - September 1997).

The first year activities were divided into four task efforts designed to: replace the ITF TiN layer with a less absorbing and reflection enhancing $\mathrm{ZnO}$ layer; design and implement a registration system for aligning the web onto the printer platen; establish new laser operating parameters to optimize the laser beam scribe speed; and develop new insulating ink printing and roll based laminating processes for the ITF production line. Second year activities were divided into five task efforts designed to: increase the throughput of the metalization, a-Si deposition, laser-scribing and printer processes; design and implement baffles for the isolation of metal deposition regions; investigation of the limits on the $\mathrm{ZnO}$ deposition rate; develop new machine control programs; identify new laser operating parameters to optimize the laser beam scribe speed; integrate a new, water based insulating ink into the patterning process; and automate the final process steps of busbar attachment and web cutting. The third year activities will focus on task efforts designed to: increase the throughput of the metalization deposition systems; determine deposition parameters for each layer to produce the full metalization stack at a single web speed; increase the throughput in the roll-to-roll $\mathrm{ZnO}$ deposition system; investigate alternate feedstocks for the supply of $\mathrm{Zn}$ and $\mathrm{O}$ in the $\mathrm{ZnO}$ growth process; study alternative processes for the scribing and printer steps; investigate alternatives to laser operations to identify potential reductions in cost; investigate alternative methods of scribing including blade, electrochemical and ultrasonic cutting tips; study alternative methods of welding shunts in cellinterconnects including the use of electronic- or ultrasonic-spot welding techniques; reduce the cost of the polymer substrate; automate the final process steps of busbar attachment and web cutting; and establish a roll-based laminating process and decrease module assembly labor costs.

\section{Research Results (10/1/96-9/30/97)}

New Tandem Deposition System A new single pass tandem deposition system was brought on line. Deposition calibrations were completed and initial devices fabricated. Devices show lower than anticipated voltages. Cross contamination at the top of the $\mathrm{i}$ layer and a slowing rising temperature in the $\mathrm{p}+$ boxes were found to be partly responsible. Machine modifications are in progress to alleviate these problems. 
Complete Design for Automated Busbar and Sheeting Machine This machine is being assembled at the time of this report. The final design for both the automated busbar attachment system and the sheeting/die cutting system consists of a base system with the following features:

The supply and take up mandrels are compatible with the ITFT A-Si production line. A torque motor/controller is mounted on the take up mandrel and a stepper motor/encoder on the supply mandrel. These two systems will provide precision control of the web in the $\mathrm{X}$ direction, down the web.

The $\mathrm{Y}$ direction of the web is controlled by a linear screw type drive system that the frame for the supply and take up mandrel are attached to. It consists of a carriage plate, which supports both the supply and return rolls, mounted to four pillow block bearings. The bearings ride on two shafts with the shafts mounted to a base plate by six base supports. A short Acme screw drives the carriage plate along the $Y$ axis and a computer controlled stepper motor drives the Acme screw along the $\mathrm{Y}$ axis.

The rotational or Theta axis adjustment will be done manually via a screw rod between the web system and the process equipment. Automation is not necessary for the rotational axis. Once the carriage plate is aligned along the Theta axis of the process equipment there should not be a need to realign the system from run to run.

The controller for alignment and cycling in both the sheeting/die cutting or the busbar taping operation is driven by a PC.

For the busbar attachment system, the base system is configured on a platen that has taping heads for placing the conductive bus on the web. The taping heads travel across it placing the conductive busbar. A vacuum hold-down plate is used to assure that there is no web movement during tape application. At the end of the stroke, the heads cut the tape and raise up off the web to enable the heads to return to the beginning of the stroke without touching the web. After tape placement, the web will be advanced to the next busbar placement location. During the web advancement the taping heads return to their original position. The process is then repeated.

For the sheeting/die cutting system, the same base system is used. The process is similar to the busbar system. The web is aligned relative to the press and once the web is aligned the press cycles to cut the PV material to specification. Optical sensors are used to verify that the PV modules have been removed from the die before there is another stroke of the press. The press proceeds to the top of its stroke then the web is air kissed off the die bottom where it can be advanced to the next frame.

In-house Testing of Potential Laminator Layer Materials The majority of the encapsulants tested to date are shown in Table 1 below. Each encapsulant received a "pass" or "not pass" rating for the following inhouse tests: salt water corrosion, $80^{\circ} \mathrm{C}$ soak, and thermal cycling $\left(-40^{\circ} \mathrm{C}-80^{\circ} \mathrm{C}\right)$. Data from UV soak and optical transmission tests on the encapsulants that passed all three tests are presented following Table 1.

Some of the modules were not sent to NREL for UV exposure because of the time the testing took and the unavailability of films that were wide enough at the time the modules were due to be sent in. Tedlar(B/ EVA and Tefzelß/silicone PSA were not sent because the sample films were not wide enough for the $\mathrm{ft}^{2}$ modules that NREL requested for testing. Polyester/Polyethylene \#23 wasn't sent because two previous candidates had already been sent in: \#12 and \#28. Tefzel@/EVA has already passed IEEE module qualification sequence Standard 1262. Dartek®/EVA wasn't sent in for UV testing because the initial Dartek(8) module failed the UV exposure testing. 
Table 1. Results of in-house testing. (Conditions: pass/fail)

\begin{tabular}{|c|c|c|c|}
\hline fin & 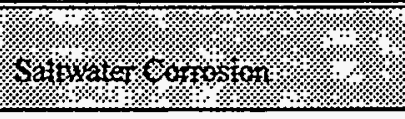 & $10.0 \%$. & 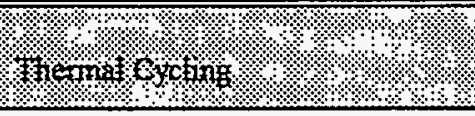 \\
\hline Tefzel@/EVA & pass & pass & pass \\
\hline Tefzel@/Silicon PSA & pass & pass & pass \\
\hline Tefzel@/Acrylic PSA & fail - penetrated module & pass & fail - delaminated \\
\hline Dartek@/Eva & pass & pass & pass \\
\hline Dartek@/silicone PSA & pass & pass & pass \\
\hline Dartek囚/Acrylic PSA & fail - penetrated module & pass & pass \\
\hline Acalar $\bigotimes /$ Acrylic PSA & failed - delaminated & fail - cracked & fail - cracked \\
\hline Polyester/Polyethylene \#12 & pass & pass & pass \\
\hline Polyester/Polyethylene \#23 & pass & pass & pass \\
\hline Polyester/Polyethylene \#28 & pass & pass & pass \\
\hline Tedlar®/EVA & pass & pass & pass \\
\hline Tedlar $($ /Silicone PSA & fail - delaminated & pass & pass \\
\hline Tediar@/Acrylic PSA & fail - delaminated & pass & fail - delaminated \\
\hline
\end{tabular}

Tedlar(B/EVA and Tefzel $(/$ silicone PSA will be sent to NREL for UV testing as soon as sample encapsulants are received. Finally, any new encapsulants that pass the three in-house tests will be submitted to NREL.

Three laminated $1 \mathrm{ft}^{2}$ single junction a-Si modules representing the best encapsulation candidates were sent to NREL for UV exposure testing. The intention of this experiment was to see how these experimental encapsulants would handle the UV exposure level as specified in the IEEE sequence Standard 1262.

The three encapsulated modules were exposed for 35.1 days under a UV irradiance of $17.8 \mathrm{~W} / \mathrm{m}^{2}$. This testing brings the total exposure to $54 \mathrm{MJ} / \mathrm{m}^{2}$, and an approximate lifetime $\mathrm{UV}$ exposure of 10 years under normal irradiance. After examining the results of the optical transmission tests calculated by NREL and shown in Table 2 below, it can be seen that the majority of the losses (approximately 10\%) in Pmax, were due to the Staebler-Wronski effect. Sample "F24" showed the greatest overall losses. This was due to yellowing of the non UV stabilized nylon film that was used in the encapsulant. 
Table 2. Relative changes in I-V parameters (\%) as tested at NREL.

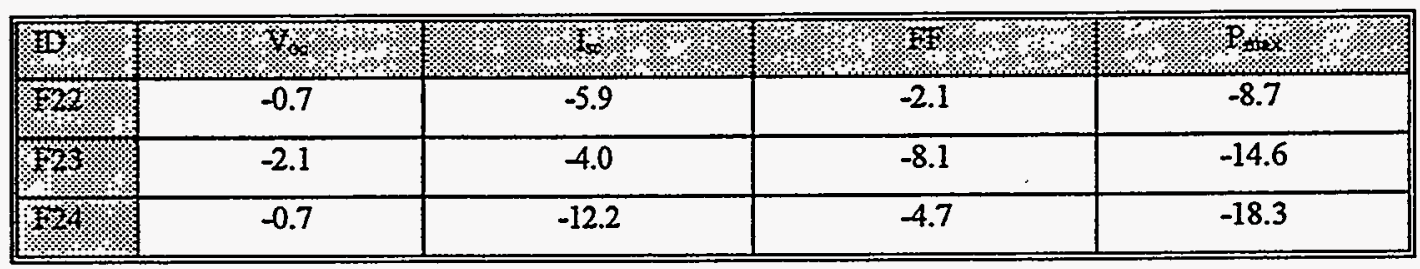

After ITF received the modules that had been UV soaked at NREL, optical transmission data was taken on the encapsulants. Samples "F22" and "F23" were polyester/ polyethylene encapsulants, with different thicknesses of film and adhesive and sample "F24" was a nylon/silicone encapsulant. Data taken above $600 \mathrm{~nm}$ on "F22" had too much "noise" to get an accurate number for the per cent change from pre UV soaked encapsulant and post UV soaked material. Table 3 presents data on the differences in per cent change in the light transmission of the pre UV soaked and post UV soaked material.

Table 3. Relative changes in light transmission shown by per cent change. (Tested at ITFT)

\begin{tabular}{|c|c|c|c|c|c|c|c|c|}
\hline 10. & 40173 & $150 \%$ & $6 f^{2}=$ & 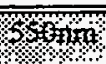 & $86934=$ & 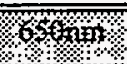 & $4 \% 100$ & $30 \%$ \\
\hline F22 & -13.0 & -0.7 & 0.0 & 0.0 & - & $\longrightarrow$ & 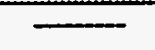 & - \\
\hline F23 & -16.0 & -2.4 & -1.7 & +0.5 & +0.6 & +1.1 & +1.9 & +1.1 \\
\hline F24 & -38.4 & -18.7 & -8.8 & -3.8 & -1.5 & -0.8 & -0.8 & -0.4 \\
\hline
\end{tabular}

In the polyester/polyethylene encapsulated modules the greatest losses occurred between $350 \mathrm{~nm}$ and $450 \mathrm{~nm}$. Considering the amount of light energy available at frequencies below $400 \mathrm{~nm}$ the losses of 13$16 \%$ seems quite negligible. It was interesting to see that there was an increase in the percentage of light transmitted from $550 \mathrm{~nm}$ to the end of the visible light spectrum at $750 \mathrm{~nm}$. "F24" seemed to suffer the greatest loses in light transmittance throughout the entire visible spectrum. Part of this was due to the fact that the encapsulating film used was a non-UV stabilized nylon film. Again the majority of the losses occurred between $350 \mathrm{~nm}$ and $500 \mathrm{~nm}$ in the lower $1 / 3$ of the light spectrum. Table 3 only shows percent change in the visible spectrum.

The polyester/polyethylene encapsulants look good for a medium lifetime product (up to 10 years). Further long term testing will be done to insure the durability of these encapsulants. The Tedlar/EVA and Tefzel/Silicon PSA encapsulants will be sent for UV exposure at NREL as soon as appropriate sample material is received from the manufacturers.

\section{Conclusions}

Significant progress has been made in decreasing cost and increasing throughput of the ITFT manufacturing process. Increased automation, selection of a new encapsulant and improved deposition and patterning processes have contributed to a $49 \%$ manufacturing cost reduction to date. 
Title: Three-Phase Power Conversion System for Utility Interconnected PV Applications

Organization: Omnion Power Engineering Corporation

2010 Energy Drive, P.O, Box 879

East Troy, WI 53120

Contributor: $\quad$ D. G. Porter, Principal Investigator; H. Meyer and W. Leang

\section{Objectives}

The objective of this contract is to make advancements in three major areas of three-phase utility interconnected, photovoltaic power conversion: cost, reliability, and performance. The total manufacturing cost of a nominal 100-kilowatt power conversion system (PCS) will be reduced from approximately $\$ 0.50 /$ watt to $\$ 0.25 /$ watt when built in production lots of 100 units. A design goal of 40,000 hours mean time between failure (MTBF) has been established for this development. Using softswitching technology, three performance goals have been established to, 1) improve converter efficiency from $95.5 \%$ to $96.5 \%, 2$ ) meet FCC regulations for electromagnetic interference, and 3) reduce audible noise to below 60 decibels.

\section{Approach}

In Phase I of the contract Omnion will develop a comprehensive product specification with input from a customer/user group. The draft product specification shall be prepared based on Omnion's understanding of customer preferences and current technological trends. Omnion will design and build a prototype 100kilowatt, three-phase power conversion system in conjunction with Soft Switching Technologies Corp. (SST) which incorporates a soft switching resonant DC link converter. The PCS will be demonstrated and the product specifications reviewed with the customer/user group.

During Phase II, Omnion will finalize the revised and repackaged design for the 100-kilowatt PCS product (Series 3400). Omnion will also conduct tests on modified manufacturing equipment and conduct a preproduction run of the prototype 100-kilowatt PCS. At the conclusion of Phase II, Omnion will have a production-model PCS with UL certification.

\section{Results}

Significant progress has been made in FY97. Phase II was started while Phase I completion has been delayed due to the re-design of the boost converter for the prototype unit. Discussion will be focused on the following tasks: Phase I: specification development, prototype design and fabrication, customer/user demonstration and review; Phase II: preproduction power conditioning system development, test procedures and O\&M manual, and manufacturing development.

Phase I:

\section{Specification Development}

During the month of January 1997, Omnion participated in the UL industry work group to develop the UL1741 standard for PV inverters. In addition, Omnion participated in the IEEE work groups including SCC21 and IEEE929. The information gathered from these meetings was used in conjunction with the customers/users review for determining improvements to the product. 
Review of product specification with key customers/users was completed in February 1997. The customers/users group was invited to the demonstration of the prototype power conversion system and a follow-up group discussion scheduled for April 16, 1997.

\section{Prototype Design and Fabrication}

The prototype fabrication started in November 1996 and continued through February 1997.

Software development was initiated in December 1996. The DC voltage routine, the automatic power tracker routine, the reference waveform generator, and other system interface software were completed in February. The software was initially verified on a test fixture and then verified on the operating prototype system running as a utility grid connected system in excess of $30 \mathrm{~kW}$. The final system software was completed and implemented in March of this year. The prototype system was operated at power levels up to $75 \mathrm{~kW}$. Some problems were identified in the core module/boost converter controls which limited the system operation. These problems were resolved prior to the demonstration in April.

Testing continued after the customers/users demonstration with emphasis on the boost converter. During the continuous testing of the system, a failure occurred in a component within the boost converter which, in turn, resulted in damage to some surrounding components. The boost converter was removed and returned to SST for repair. Repair of the boost converter took longer than anticipated and was finally returned in July. Unfortunately, there was another failure of the boost converter when the system was tested at up to $60 \mathrm{~kW}$. The boost converter was returned to SST for repair. While the boost converter was in for repair, the core module was tested at up to $100 \mathrm{~kW}$ along with new output toroidal inductors. Test results were very favorable. In September, the boost converter failed again during testing. SST decided that the boost converter should be redesigned with a new topology. This redesign effort will significantly delay the completion of Phase I.

A failure modes and effects analysis was completed in June. This analysis identified failure modes and consequential effects for the prototype design. This feedback was then used by the design team to improve the system design.

\section{Customers/users Demonstration and Review}

The demonstration and customers/users group review was held on April 16,1997. The outcome of this review provided Omnion with valuable insights in to the development of the product. Comments of the group were incorporated into the final product specification.

The end of Phase I review with the Technical Management Team (TMT) was conducted on April 17, 1997. In addition, Phase II of the project was also reviewed and approval given to move forward.

Phase II:

Preproduction Power Conditioning System Development

Design of a new power supply for testing of the inverter was completed in May. Fabrication was completed in July.

Finalization of the preproduction model packaging was initiated in May. Omnion and SST personnel met several times to discuss improvement to the core module. The improvements fall into two categories: 1) manufacturing and reliability improvements, and 2) packaging improvement. In July, SST completed a re- 
design of the core module to incorporate the suggestions from the discussions. Unfortunately, the effort turned out to be very costly. Discussions are continuing with SST to look for the best solution to the final packaging of the core module.

\section{Test Procedures and O\&M Manual}

The test procedures were updated in June to reflect the prototype testing. The draft of the Operation \& Maintenance (O\&M) manual was started in July and completed in September. The O\&M manual covers safety consideration, shipping, installation, operation and trouble-shooting of the product.

\section{Manufacturing Development}

In October, Omnion initiated the design and development of the manufacturing process for the product. The manufacturing process will be designed to minimize manufacturing cost while incorporating comprehensive quality control and inspection of the product.

\section{Summary}

Under Phase I, the following was accomplished:

- Completed fabrication of control boards test fixture

- A boost converter was developed in conjunction with SST

- Continued interaction with SST relative to the control of the core module and boost converter

- Completed prototype test procedures

- Completed prototype software development

- Completed assembly of a prototype power conversion system

- Tested prototype power conversion system at $75 \mathrm{~kW}$

- Demonstrated prototype power conversion system to customers/users

- Reviewed and finalized product specifications with inputs from customers/users

- Completed failure modes and effects analysis

- Initiated re-design of boost converter

- Conducted end of Phase I review with TMT

Under Phase II, the following was accomplished:

- Initiated final production package design

- Updated test procedures

- Completed design and fabrication of DC power supply for production testing

- Completed draft of O\&M manual

- Initiated design of manufacturing tools, jigs, and fixtures

- Initiated design of test fixtures

- Initiated design of manufacturing process

The next major milestones are to test the re-built boost section and to manufacture the pre-production PCS. When the pre-production PCS is finished it will be tested. Results of the testing and information from the manufacture of the pre-production PCS will be used to refine the manufacturing and test process. The refined process will be used to manufacture a production PCS. 

Title: $\quad$ PVMaT Improvements in the Manufacturing of the PVI Powergrid ${ }^{\mathrm{TM}}$, Year-Two Annual Report

Organization: Photovoltaics International, LLC (PVI)

171 Commercial Street Sunnyvale, California

Contributors: Neil Kaminar, Principal Investigator; Bill Bottenberg, Don Curchod, Phil

Hobden, Jim Navin, Sam Roake, Jim Sahagian, Carlton Salter

\section{Objectives}

The goals for PVI's PVMaT program are to reduce the cost of manufacturing the Powergrid, to demonstrate large volume production, and to reduce the use of volatile organic compounds (VOC) and hazardous materials (hazmat) in manufacturing. The Powergrid is a linear focus, low concentration ratio photovoltaic panel. Successful completion of PVI's PVMaT program will result in a reduction in the cost of the Powergrid of approximately three dollars per watt at a mature volume of production. The objectives for the second year were to install and operate an advanced acrylic plastic extrusion system, to complete the design for and assemble an automatic receiver assembly station, to design and prototype a low-cost, roll form steel panel frame assembly and to develop a low VOC Module manufacturing process.

\section{Approach}

The approach to reducing the manufacturing cost for the Powergrid is based on the identification of those elements which contribute most to manufacturing cost and the selection of leveraging manufacturing techniques to lower cost. We identified lens and side extrusion, cell lead attachment, panel frame assembly and module assembly as areas with the greatest potential for cost reduction.

\section{Results}

\section{Advanced Acrylic Plastic Extrusion System}

The advanced acrylic plastic extruder system designed in Phase I was delivered and installed. The integrated system includes a raw material handling and drying section, the extruder, a flying cut-off saw and a parts unloader.

A significant advantage of the in-house system is not having to break down the equipment after each experiment. The machine can simply be started up in the morning without having to remount all the tooling. This has eliminated a whole series of uncontrolled variables. Another advantage of the in-house system is greater control of the extrusion parameters. This allows the effect of subtle changes to the parameters to be observed.

An new extrusion die was designed based on experimental runs carried out at the contract extruder. Design of Experiments (DOE) methodology was used to characterize the performance of the extruder as a function of key parameters including mass flow rate, barrel heating temperatures, die temperature distribution and cooling air flow rates.

A production run of 50 lenses was then carried out. After a high fidelity lens profile was obtained using mostly regrind plastic, virgin plastic was placed in the dryer and the production lenses were run. Lenses from this group had greater than $80 \%$ optical throughput, compared to $68 \%$ for lenses made at the contract vendor. This improvement was directly attributable to greater control. In addition the results from this run showed key areas of process improvement to improve process yield. These areas included more uniform temperature control of the die and 
more control over flow of air cooling at the die exit. If the system parameters were set at constant values, the transmission of the resultant lenses was constant. The on-line optical tester is a major factor in this achievement. Figure 1 shows the extruder in operation.

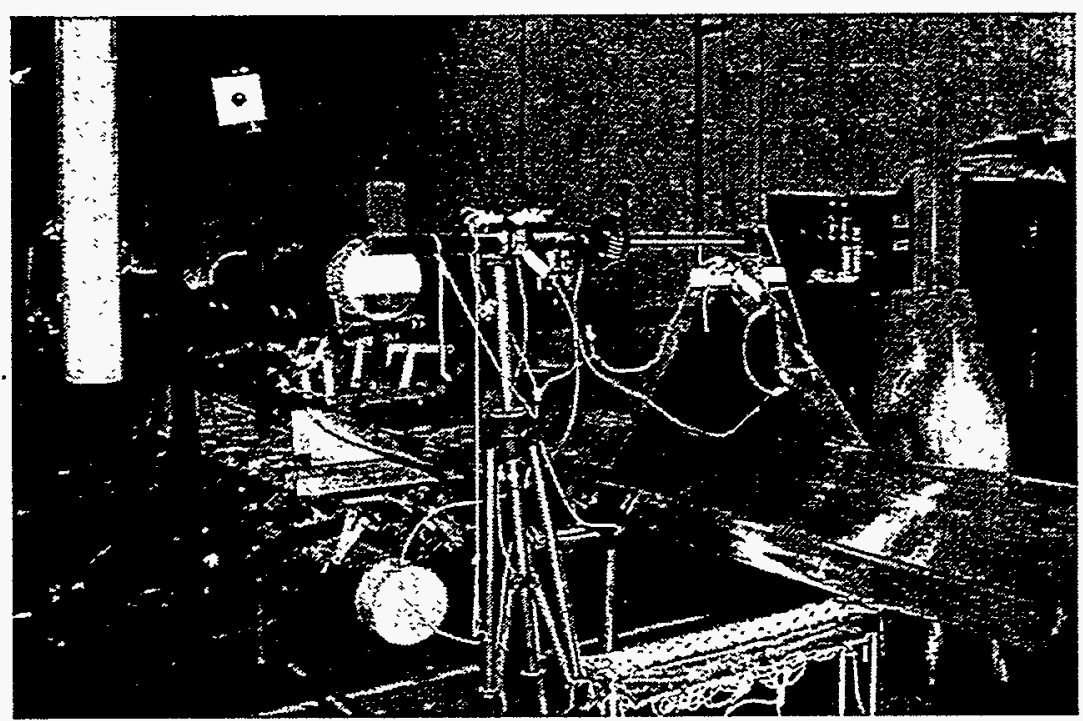

Figure 1. Advanced Extrusion System in Operation

We continued experiments with extruding lenses and were able to make lenses with $85 \%$ optical transmission. This improvement was primarily due to enhanced control of die temperatures. It was also due to improved display of operating parameters in real-time graphical display on the computer monitor.

High optical transmission was accomplished during subsequent production runs. Over $99 \%$ of the lenses produced were usable for product. In the past, our yield has been less than $50 \%$. The cutoff saw was operational during the production run.

During this phase of the contract the extrusion system has been demonstrated to operate at a production rate of $4 \mathrm{MW} /$ year, with yields of better than $95 \%$ for lenses with optical throughput of greater than $82 \%$. During phase 3 we expect to increase the rate by a factor of three with the average optical throughput of the lenses greater than $87 \%$.

Improvements in lens optical throughput have been obtained during this phase by improving the die finish and by post roll forming experiments to improve the accuracy and fidelity of the finished fresnel lens.

The acrylic plastic sides of the module are also extruded using a different die. Sides with well controlled features were fabricated in production quantities during this phase. During production trials the production rate of the extruder was shown to be equivalent to $2 \mathrm{MW} /$ year with yield greater than $90 \%$.

\section{Automated Receiver Assembly Station}

The cell lead attachment section of the automated receiver assembly station was assembled and tested. Each sub-assembly of the cell lead attachment section was thoroughly tested and debugged before system integration was performed. The system employs a robot to transfer cells 
from a stack to a lead attachment nest which holds the cell in place while the leads and insulators are positioned before the solder bonding process. An IR heater station carries out the solder bonding process step. Figure 2 shows the automated receiver assembly station in operation.

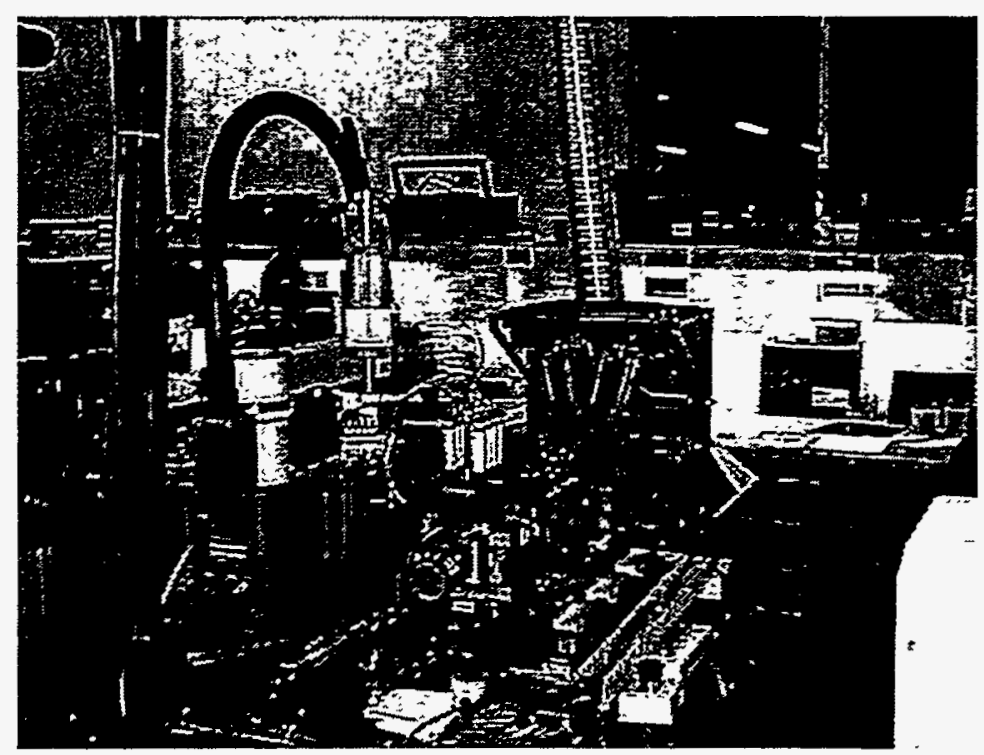

Figure 2. Automated Receiver Assembly Station

Trial runs of the lead attachment process were conducted after the robot control software was successfully integrated with the balance of the station control software. Fifty two cells were processed at a rate of 1 cell $/ 2$ minutes for process evaluation. The net yield of cells that met all leaded cell qualification criteria was $96 \%$. Conceptual design of the receiver assembly section was completed.

\section{Low-Cost Panel Frame}

Panel frames were fabricated using aluminum extrusions to simulate roll form members as part of the design assessment process. Two of these frames have been deployed on the PVI roof. Evaluation of this design showed that increase stiffening was required. The costs for employing the original concept of a dedicated lock-seamed, roll formed structure were reviewed, including the requirements for a more robust frame, and were found to be significantly higher than expected. We found that by relaxing the requirement for a lock seam configuration we could consider a wider range of tube section options. As a result we have found that we can use offthe-shelf material with a substantial reduction in tooling costs.

\section{Low VOC Module Manufacturing and Module Manufacturing Results}

Modules were fabricated using lenses and sides from extruder production runs. A set of module assembly jigs were designed to assist in the accurate alignment of the parts during module fabrication. A group of 12 modules was fabricated with an average output of $139 \pm 7$ watts at PVUSA Test Conditions (Irradiance $850 \mathrm{w} / \mathrm{m}^{2}$, Tamb $20^{\circ} \mathrm{C}$, , wind speed $1 \mathrm{~m} / \mathrm{s}$ ). These modules were mounted on the PVI roof for outdoor testing as shown in Figure 3. The I-V curve for the module with PTC output of 155 watts is shown in Figure 4. 


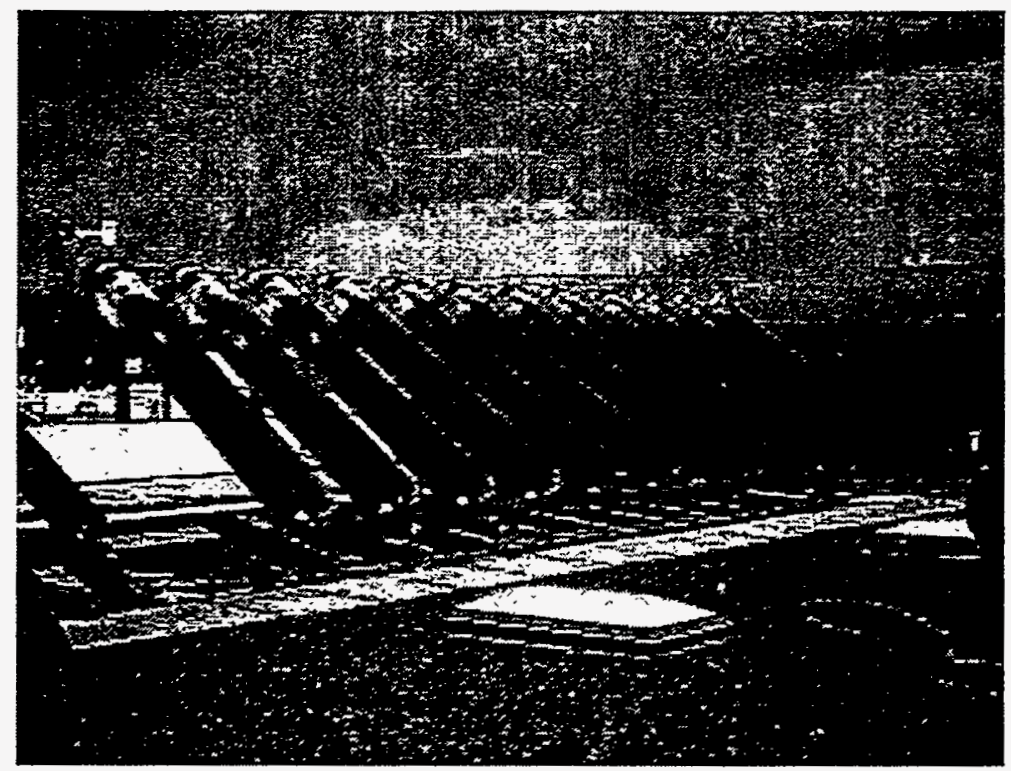

Figure 3. Powergrid 2000 Rooftop Mounted System

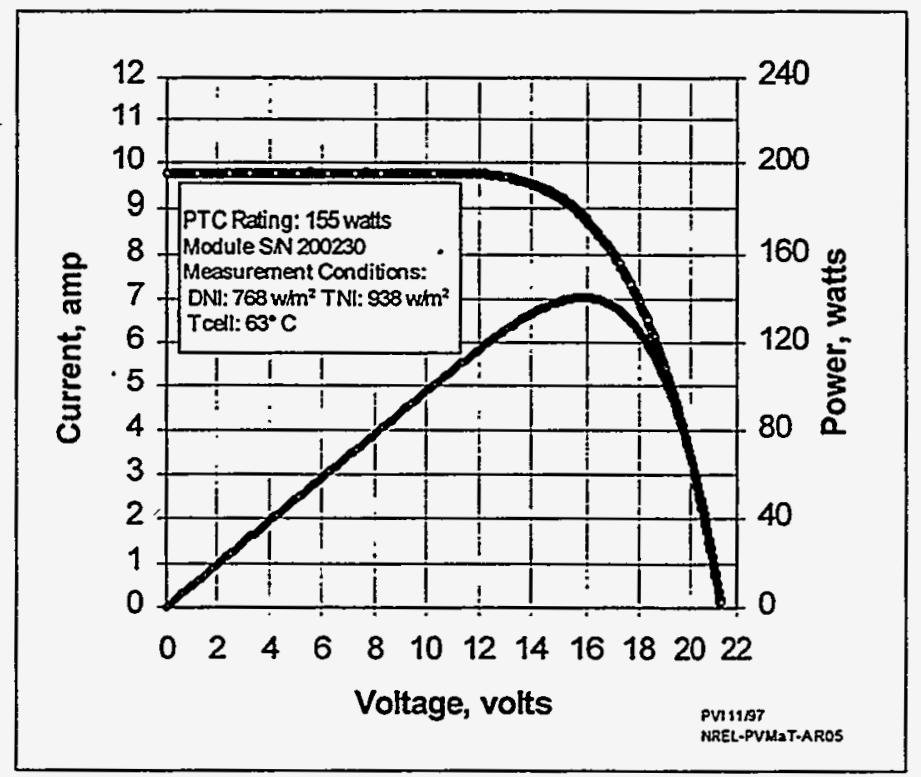

Figure 4. Powergrid 2000 Module I-V Curve

155 watts at PTC

\section{Summary}

During this phase of the project the essential goals were met for preparing to launch concentrator module manufacturing activity at significantly lower cost: installation of an advanced acrylic plastic extrusion system to produce good quality in-house lenses and module sides, assembly of the cell lead attachment section of the automatic receiver assembly station, design and prototyping of a low-cost, roll form steel panel frame assembly, and development of a module manufacturing process with significantly lower generation of VOCs. 
Contributors:

\author{
Theresa L. Jester, Principal Investigator
}

\title{
Introduction
}

The objective of the DOE/NREL Photovoltaic Manufacturing Technology (PVMaT) subcontract with Siemens Solar Industries (SSI) is to continue the advancement of module design and manufacturing methods at SSI in order to achieve an $18 \%$ reduction in module cost per watt at the end of three phases of work, with each phase lasting a year. Phase I of this subcontract began in November 1995, Phase II in November 1996. The approaches for reaching this cost reduction goal are to analyze existing module cost structure and explore new module configurations which address the highest cost components, investigate the reduction of labor and improvement of yield, and to implement statistical process control (SPC) in module manufacturing.

\section{Module Cost Analysis and Design}

A first step toward reducing the cost per watt at the module level is to gain a thorough understanding of the present factors which dominate cost. Analysis of the cost structure of module manufacturing at SSI was done in Phase I and allowed specific strategies and module designs for reducing the most significant costs to be formulated. These improvements in module configuration have been introduced into higher volume production in the SSI Camarillo plant during Phase II of the contract. This new module design takes advantage of lower cost materials in the junction box and framing materials and is shown in Figure 1. This design, at high manufacturing volumes, represents a greater than $15 \%$ reduction in module dollar per watt costs when compared to the standard M55 Siemens module.

\section{Yield and Productivity Improvements}

Manufacturing yield is a powerful driver of the cost of cells used in a module, the cost of module direct materials, and of the cost of module manufacturing labor. Close monitoring of yield and categorization of various types of loss in the wafering, cell fabrication, and module fabrication areas reveal that mechanical breakage is the dominant type of yield loss. Consequently, most efforts have been aimed at reducing this component of yield loss, through improved tracking, better wafer handling equipment, operator education and involvement, and by experimentation to find the.root causes of wafer breakage and its dependence on material properties. Fig. 2.and Fig. 3. are charts of the yield gains effected during both Phase I and Phase II of the PVMat program. Figure 2. shows the yield for the small, 103 $\mathrm{mm}$ square cells and modules, and Figure 3. shows the larger $125 \mathrm{~mm}$ cells and modules. The effect of these improvements is over $15 \%$ improvement in line yields for the smaller cells, and over $20 \%$ for the larger cells.

\section{Manufacturing Systems to Improve Module Reliability}

The SSI Camarillo plant received ISO 9001 certification in March 1996. This is a major milestone in the pursuit of quality manufacturing systems, and represents a very substantial effort by the company to establish and document procedures, operator work instructions, maintenance and calibration schedules, conduct operator training, etc. The benefit is a profoundly improved system to ensure manufacturing 
compliance and control of its processes. The further deployment of quality methods, in the form of Statistical Process Control, or SPC, has helped in many areas of manufacturing, from soldering to diffusion processes. Figure 4. shows improvements made in the diffusion process to make it more statistically capable, and the maintenance of that performance through SPC methods. The full deployment of SPC in manufacturing is on-going.

\section{Summary}

Cost drivers for $\mathrm{Cz}$ Si solar cell modules have been identified. Cell size and shape, material usage, module size, and cell and module yields have an especially strong influence on cost. Specific module designs to address cost issues have been designed, prototyped and are now in production which affect a greater than $15 \%$ cost reduction per watt produced. Yield gains have been made in excess of $15 \%$ total line yield improvement. The deployment of SPC methods has begun in all manufacturing areas.

\section{Acknowledgments}

Many people have contributed to the work under this contract. Thanks are due especially to Rick Mitchell, NREL technical monitor, to Dave Bender, Ruben Balanga, Jean Hummel, Dave Jeffrey, Waltraut Klein, Greg Mihalik, Maria Tsimanis, Elena Woodard, Eugene Yamamoto, and at SSI.

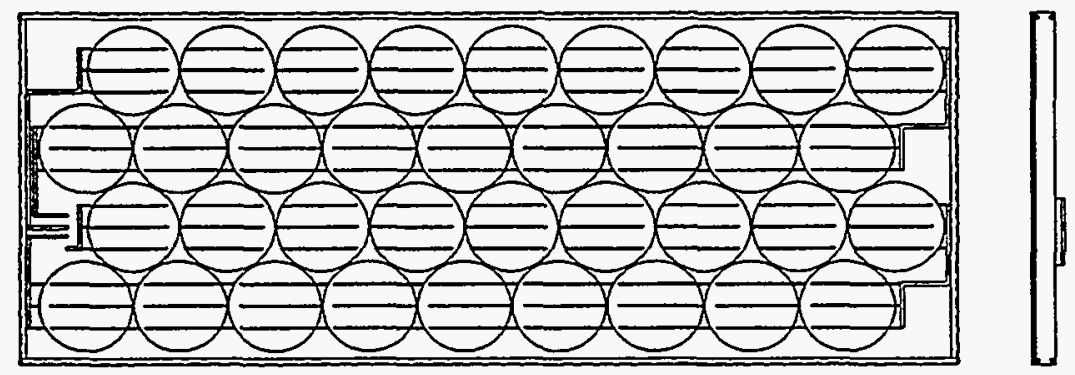

Fiq. 1 SR-100 Module Design. 


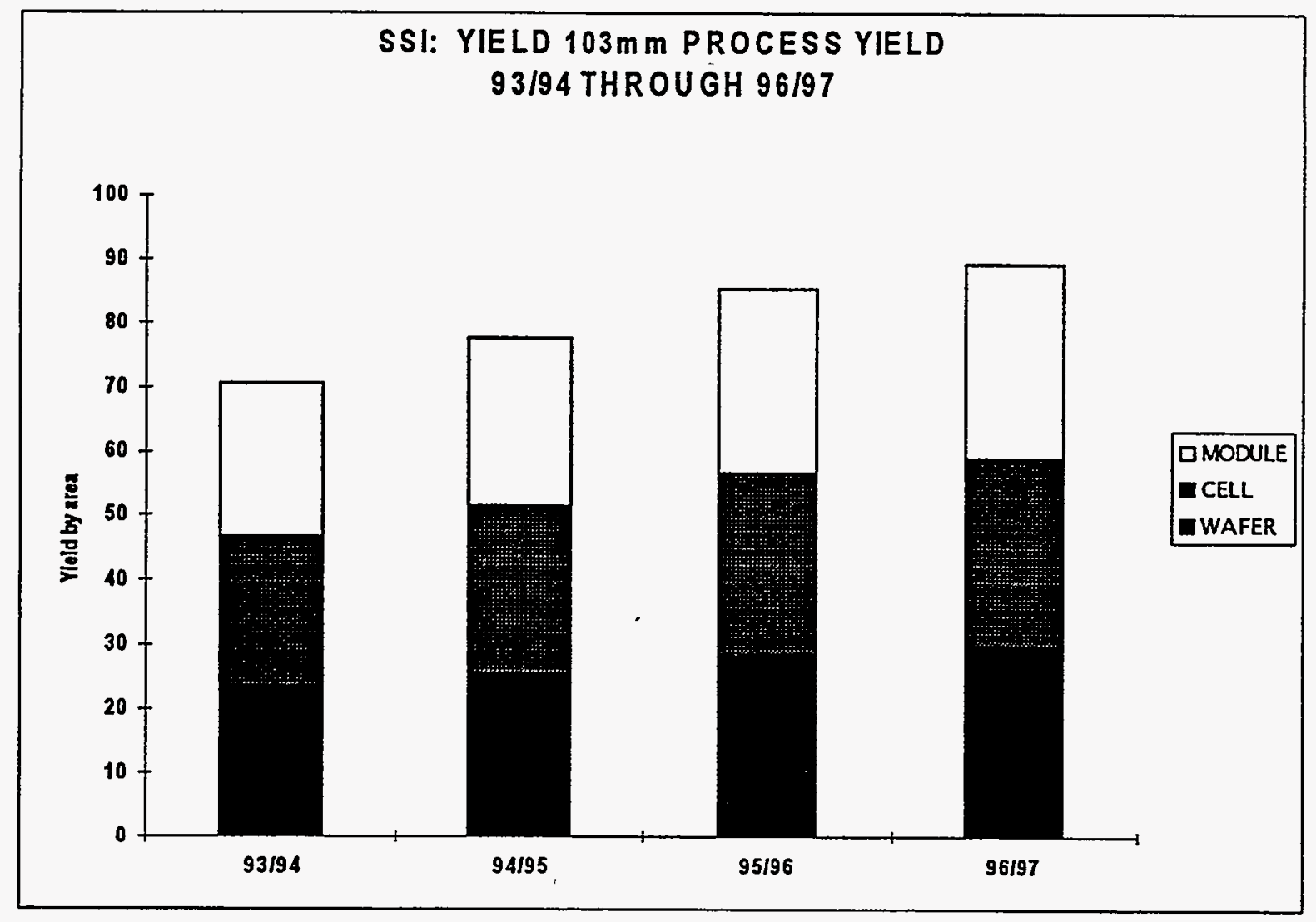

Fig. 2 Yield for $103 \mathrm{~mm}$ product.

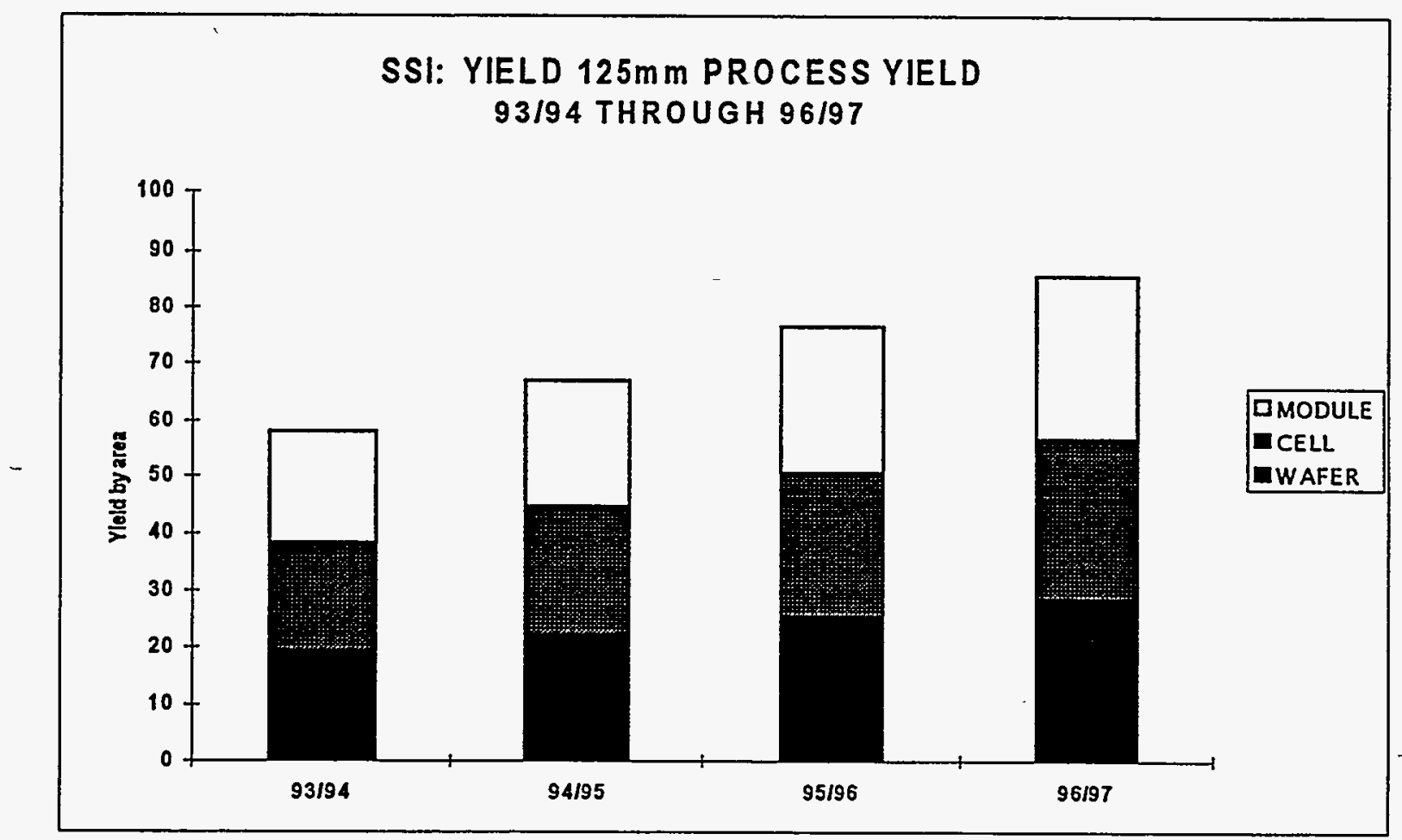




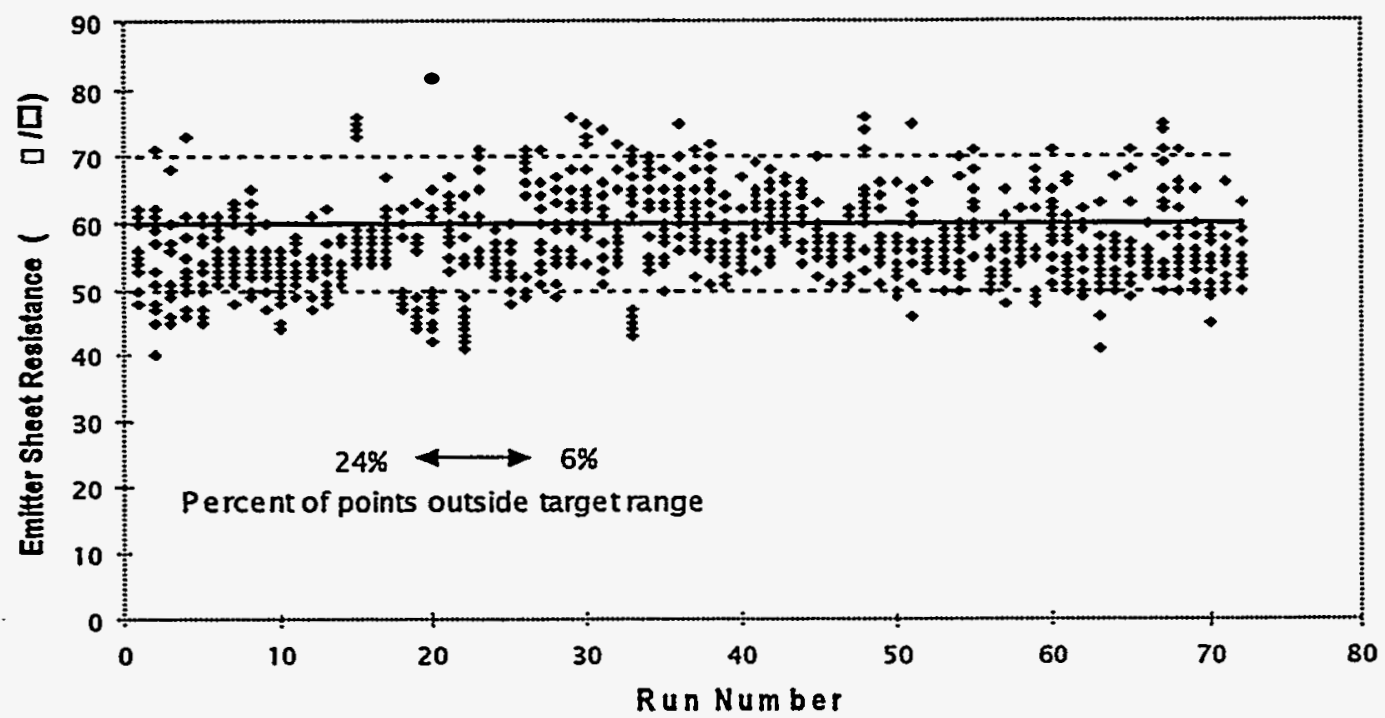

Fig. 3 Yield for $125 \mathrm{~mm}$ product.

Fig. 4 Sheèt Rho SPC measurements. 
Title: "High-Throughput Manufacturing of Thin-Film CdTe Photovoltaic Modules"

Organization: Solar Cells, Inc., Toledo, Ohio

Contributors: D.W. Sandwisch, Principal Investigator; H. McMaster, G. Dorer, J. Heider, S. Johnson, A. McMaster, J. Pollack, R.C. Powell, L. Adoline, J. Bohland, D. Berger, L. Cicak, S. Collins, S. Cox, L. Crosser, T. Dapkus, J. Farrell, G. Faykosh, B. Finkbeiner, M. Flis, J. Foote, A. Gray, D. Grecu, J. Hanak, U. Jayamaha, K. Kamm, T. Kahle, G. Khouri, K. Klaus, M. McArthur, B. McMullen, K. Miller, B. Notestine, J. Poddany, G. Rich, N. Reiter, S. Roberts, M. Ross, T. Seeman, K. Smigielski, J. Smith, and M. Steele

\section{OBJECTIVE}

The objective of this work is to advance the Solar Cells, Inc. ("SCI") PV module manufacturing technology; reduce module production costs; increase module performance; and provide the groundwork for SCI to expand its commercial production capacities.

\section{TECHNICAL APPROACH}

SCI focuses on developing leading-edge technologies in the critical value-added segments of its process (i.e. CdTe coating) while relying heavily on proven techniques from other industries. The result is a lowrisk effort to improve the photovoltaic technologies while leveraging and integrating commercial knowhow and processes. This approach has significantly reduced process development time and provided more opportunity to complete product validation and process integration.

\section{INTRODUCTION}

Cadmium telluride ("CdTe") is recognized as one of the leading materials for low-cost, thin-film photovoltaic modules [1,2]. In 1991, SCI began a PV module manufacturing development program to demonstrate the technology's many advantages such as deposition rate, device stability, device performance, and process flexibility. Due to the success of those efforts, SCI began a manufacturing initiative in late 1993 in conjunction with support from the Department of Energy's PVMaT program.

SCI has manufactured and installed over $50 \mathrm{~kW}$ of thin-film CdTe modules with an average total area efficiency of greater than $6.75 \%$. These installations have demonstrated reliable performance at or above system ratings. In addition, modules routinely pass extensive stress testing. A champion $60 \mathrm{~cm} \times 120 \mathrm{~cm}$ modules has been measured at 61.0 watts or over $9.0 \%$ aperture area efficiency.

1997 program activities included product validation; equipment design, specification and debug; manufacturing infrastructure development; and innovative coating system development.

\section{PRODUCT VALIDATION}

The most important result of SCI's PVMaT subcontract is the validation of its thin-film CdTe modules through significant field data. The baseline $S C I$ product is a $60 \mathrm{~cm} \times 120 \mathrm{~cm}$ high-voltage $\left(65 \mathrm{~V}_{\mathrm{max}}\right)$ module targeted at grid-connected applications. SCI projects that this product, along with a patented support structure, will reduce the cost of photovoltaic installations to below $\$ 3.00$ per watt by the year 2000 . This product has been installed at several sites (see Table 1). 
Table 1. SCI Thin-Film CdTe Module Installations

\begin{tabular}{|l|c|l|}
\hline Location & Size & Installation Date \\
\hline Edwards AFB, CA & $25.0 \mathrm{~kW}$ & April 1996 \\
Toledo Edison, Ohio & $14.0 \mathrm{~kW}$ & October 1995 \\
PVUSA, Davis, CA & $10.8 \mathrm{~kW}$ & September 1995 \\
NREL, Golden, CO & $1.0 \mathrm{~kW}$ & June 1995 \\
FSEC, FL & $0.5 \mathrm{~kW}$ & June 1996 \\
STDI, Las Cruces, NM & $0.5 \mathrm{~kW}$ & February 1995 \\
Tunisia & $1.0 \mathrm{~kW}$ & January 1995 \\
Toledo, OH & $1.2 \mathrm{~kW}$ & August 1994 \\
\hline
\end{tabular}

The installation at Edwards AFB incorporates 624 modules and has been measured at over $27.0 \mathrm{~kW}$ at the DC junction box in mid-summer. SCI supplied not only the modules but also its proprietary panelization and support structure developed in part under its PVMaT subcontract. The project also confirmed that these designs result in greater than a $40 \%$ reduction in costs of encapsulation, potting, connections, and panelization when compared to its pre-PVMaT design. The majority of this reduction is the result of incorporating frameless panelization and replacing the junction box with a pigtail. The Toledo Edison array consists of over 200 modules. The array is grid-connected and has been in operation for over twelve months. The array will be expanded to $100 \mathrm{~kW}$ by the end of 1998 .

SCI also installed an array of 264 modules at the PVUSA demonstration site in Davis, California. This array has been measured at $12.0 \mathrm{~kW}$ at the $\mathrm{DC}$ junction box and has operated well since its installation in September 1995.

\section{EQUIPMENT DESIGN}

\section{Deposition System Upgrade}

During 1997, a retrofit of SCI's developmental semiconductor deposition equipment (LDS) was completed. The objectives of the retrofit were to improve process control, increase capacity, increase material utilization, reduce downtime, and increase machine reliability. SCI met or exceeded all of these objectives with a continuous 100-plate run.

Disassembly. The machine was disassembled in only two weeks and reassembly had begun by the end of July. Disassembly included removing the heaters, quench, insulation, shafts and bearings, chamber-tochamber seals, intermittent valves, rolls and roll bearings, thermocouples, entrance valve, and the entire fourth chamber section. In addition, the inside of the system was cleaned.

Reassembly. The installation of new components was divided into functional areas: mechanical, electrical, controls, and deposition.

Mechanical. The mechanical upgrades focused on providing increased throughput and improved machine availability. The primary upgrade was the addition of load locks onto the entrance and exit of the system. Previously, the system operated with only one gate valve on the entrance and one gate valve on the exit. This arrangement required that the system be pumped down to deposition pressure (i.e. 1-5 Torr) during each cycle. Since the load lock volume is much less than that of the entire chamber, the pumping time was decreased from over 2.5 minutes to under one minute. Furthermore, the load locks provide the opportunity to introduce multiple substrates into the system resulting in a decrease in the cycle time from 9.5 minutes to 2.5 minutes. 
In addition to the capacity improvements, the load locks also significantly improve the process stability by enabling a constant pressure within the deposition zone. Before the upgrade, the deposition zone would be cycled between atmospheric pressure and approximately 1 Torr. With the load locks in place the deposition zone is maintained at the target process pressure and does not fluctuate as substrates enter and exit the system.

Electrical. Electrical upgrades focused on the heating systems. The original heating design called for exposed heaters throughout the preheat section of the system. These heaters while reliable did pose some problems due to open voltage in the vacuum system and corrosion of the electrodes. Therefore, SCI replaced the original heaters with covered elements to improve lifetime and also to increase $\mathrm{kW}$ density.

Controls. Significant upgrade of the controls was included during the project. The focus of these activities was to better track the substrate, provide sequencing for the load locks, increase safety interlocks, and improve conveyor control. The primary improvement was the replacement of manual controls with a programmable logic controller. Due to these upgrades the system can be run in an automatic mode with no input from the operator except the loading and unloading of substrates. During a 100-plate run, one operator was able to handle the operation of the system at a cycle time of three minutes. Before the upgrades one operator was needed to run the system at a ten-minute cycle. Therefore, labor has been reduced by $70 \%$ and process variability caused by operators has been eliminated. Furthermore, the required skill and training level of the operator is greatly reduced.

Deposition. Perhaps the most significant improvement of the system is related to the design and operation of the deposition components [3]. Prior to the upgrade, raw material (i.e. semiconductor sources) was batch loaded into the deposition system by placing the physical source over the substrate. As a run progressed the source material would deplete and the run would be stopped when the source was depleted to an unacceptable level. Furthermore, the system parameters such as pressure and temperature would need adjustment to maintain the same deposition rate as the source diminished. The new deposition components and design provide on-demand coating and continuous feeding of the raw materials. The ondemand feature allows the heating, vacuum, and conveyance subsystems to be decoupled from the coating subsystem. This was not the case before the upgrades. The on-demand capability allows the operator or control system to turn the coating on at the point when a substrate reaches the deposition zone. This control allows the system to reach steady state temperature and pressure before introducing deposition.

\section{MODULE PRODUCTION}

SCI reconstituted the pilot production team after pulling back from manufacturing to concentrate on equipment upgrades. The team consists of experienced operators and technicians from past production activities as well as new members with technical and manufacturing backgrounds. The team began organizational activities in July and completed some preliminary process training for new operators. SCI continued limited production in September with a team of four operators. The production activities focus on quality control and process documentation.

\section{SUPPORT PROGRAMS}

An important part of the development effort is to establish programs which effectively handle safety, health, and environmental issues that accompany the production, deployment, and disposal of these modules. SCI has engaged outside agencies and consultants to conduct safety and health audits of the manufacturing facilities and to formulate appropriate programs and corrective actions. These programs include basic training programs as well as specific operational plans such as industrial hygiene and 
biological monitoring. Test results have confirmed that these programs are very successful in mitigating and eliminating plant risks.

Environmental efforts have focused on waste minimization and material reclamation and recycling [4]. These processes have been demonstrated at prototype levels. This work, begun under SCI's PVMaT subcontract, is now being commercialized with the support of a two-year, $\$ 925,000$ Small Business Innovative Research subcontract. The objective of the work is to fully develop and commercialize a fullscale reclamation and recycling process primarily targeted at CdTe modules but flexible enough to be utilized in other recycling efforts such as florescent light tubes and video monitors.

\section{ACKNOWLEDGMENTS}

The author is pleased to acknowledge the efforts of the SCI development team. The extraordinary capability and dedication of this team was essential in achieving the results described in this paper. The work reported here was supported in part by NREL under subcontract No. ZAI-4-11294-02.

\section{REFERENCES}

1. J.M. Woodcock, H. Schade, H. Maurus, B. Dimmler, J. Springer, and A. Ricaud, "A Study of the Upscaling of Thin Film Solar Cell Manufacture Towards 500MWp Per Annum", 14th European Photovoltaic Solar Energy Conference, Barcelona, Spain, 1997, pp. 857 - 860.

2. Sandwisch, D.W., "Progress in High Throughput Manufacturing of CdTe Modules", in AIP Conference Proceedings 394, 14th NREL Photovoltaics Program Review, Lakewood, Colorado, 1997, pp. 425431.

3. R.A. Sasala, R.C. Powell, G.L. Dorer, and N. Reiter, "Recent Progress in CdTe Solar Cell Research at SCI", in AIP Conference Proceedings 394, 14th NREL Photovoltaics Program Review, Lakewood, Colorado, 1997, pp. 171-186.

4. R.A. Sasala, T. Zhou, and'W.M. Kocher, "Environmentally Responsible Production, Use and Disposition of Cd-Bearing PV Modules", in 1994 IEEE First World Conference on Photovoltaic Energy Conversion, Conference Record of the Twenty-Fourth IEEE Photovoltaic Specialists Conference, Waikoloa, Hawaii, 1994, Volume I, pp. 311 - 314. 
Title:

Organization:

\section{Development of Standardized, Low-cost AC PV Systems}

Solar Design Associates, Inc. (Prime)

Solarex

Advanced Energy Systems, Inc.

\section{PROGRAM OVERVIEW}

The objectives of this two-year program were to improve the reliability and safety and reduce the cost of installed grid-connected PV systems by creating standardized, pre-engineered components and an enhanced, low-cost, 250-Watt micro inverter. These advances were combined with the new, large area Solarex MSX-240 PV module resulting in standard, modular AC PV "building blocks" used to create utility-interactive PV systems as small as one module to many thousands of modules to suit virtually any application. AC PV building blocks will be developed to meet the requirements of the U.S., Japanese and European markets.

\section{FY '97 ACCOMPLISHMENTS:}

During FY ' 97 , the following work was accomplished:

\section{October 1996}

- Annual review meeting with PVMaT TMT held at Solarex October 23

- Installed the flat roof system at Solarex

- Installed the first of several quick-connect electrical harness systems at Solarex

- 2 digital inverters presented at review meeting

- Fabrication of pre-production digital inverters begun

\section{November 1996}

- Prepared the NREL review meeting poster and supported the poster presentation

- Solarex selected the Turk hardware for the quick-connect electrical harness systems

- AES started fabrication of 20 pre-production digital inverters

- Completed inverter phase tracking software, resolution of inverter heat dissipation problem

- EMI noise reduction measurements

- Prototype for Japanese versions completed and prototype for European version designed and assembled

- Received UL feedback on pre-production digital inverter design relative to UL listing

December 1996

- Solarex completed mechanical loading tests on frameless MSX-240 module.

- Completed discussions with UL and agreed on test program required for UL certification of MSX-240 module.

- Completion of EMI noise reduction testing

- Completion of European inverter prototype

- Delivered 2 digital inverters to Solarex for AC modules as deliverables to NREL

- Inverter design freeze, start layout for production inverter

- Produced approximately 120 inverters for alpha test

- Completion of all PVMaT Phase 1 tasks

\section{January 1997}

- Began Phase 2 work tasks

- ASU completed their portion of the UL tests on the Solarex $240 \mathrm{Wp}$ module

- AC modules shipped to NREL and Sandia as final Phase 1 deliverables

- New layout underway for microinverter, case size changed 
- Discussions underway with UL for certifying the microinverter

- Support of State University of New York (SUNY) MI beta test site

- Provided key input on code and regulatory issues impacting the MI and the AC module

\section{February 1997}

- Solarex personnel participated in an inverter design review meeting at AES

- 10 of the latest version inverters were sent to Solarex for evaluation.

- Solarex completed UL tests and received UL listing for the MSX-240 in the PVMaT mounting system.

\section{March 1997}

- The microinverter passed FCC and UL testing

- Latest versions of the inverters were installed on the ground-mounted array at Solarex.

- A dummy laminate was built for inverter temperature tests at Solarex.

- The microinverter etch was modified to incorporate latest ECOs

- Pre-production run of FCC/UL compliant inverters for environmental testing

\section{April 1997}

- Solarex began environmental testing of the latest version of the inverter.

- Temperature limit analysis; software updates for CEBus, automatic house/unit configuration

- UL-required modifications made to etch. Labels, manuals created for MI, Search for UL-listed labeling systems, Selection of UL recognized pottant

- Selection of vendor for initial production

May 1997

- Solarex continued environmental testing of the replacement inverters.

- Continued failure analysis of environmental test units

- MI Contract manufacturer UL certified

- First inverter with UL label produced

- Began definition of new inverter reliability test

June 1997

- Solarex continued environmental testing of the newest replacement inverters.

- AES received formalized UL listing for the MI

- Production tester being tested. Burn-in testers sent to contract manufacturer

- New potting compound selected for inverter

- Replaced inverter magnetics supplier

\section{July and August 1997}

- Solarex suspended environmental testing of the inverters.

- Four modular inverters were produced for delivery to Solarex

- Competitive MI cost quotations from the contract manufacturer came in very high

- Solarex reluctantly suspended development work on their AC module

- Delivered photographs of the prototypes of the micro inverter for the Japan and Europe

\section{September 1997}

- Preparation at Solarex of two prototype AC module demonstration systems, one each for NREL and SNL. Each system contains 2 MSX-240 AC modules for deliverable 'D-2.3'

- Preparation and submission of proposed revisions to the PVMaT SOW, schedule and deliverables. Discussion with PVMaT TMT on contract modifications.

- Begin preliminary design of a framing and mounting system for the Solarex thin film modules.

- Begin initial selection of candidate low-cost connectors for the Solarex thin film modules. 


\section{Detailed Task-by-Task Review}

\section{Task 2: Innovative Mounting Systems:}

In Task 2 SDA and Solarex developed low-cost methods of array mounting and structural support for sloped and flat roofs of residential and commercial buildings as well as ground-mounted arrays using pre-engineered, standardized components and large-area Solarex MSX-240 modules.

All three prototype array mounting systems were completed and are being evaluated for performance. This evaluation will continue through Phase 2 of the program. Solarex performed mechanical load tests on MSX240. The module was mounted using the same beam configuration as used on the ground mounted tire array. It successfully passed the IEEE 1262 mechanical load test and the IEC 1215 snow load of 113 PSI.

Deliverables: D-1.2. The data package showing drawings of each mounting system has been provided. D1.5. Photographs of the updated installations have also been provided. Task complete.

\section{Task 3: Enhanced Digital Inverter 10/96}

Under Task 3, an advanced digital micro inverter was developed with Advanced Energy Systems using the SDA analog micro inverter developed under the PV:Bonus program as a basis for the new design.

The new digital inverter design improves reliability while reducing manufacturing costs by combining a number of separate analog control functions into one digital processor which reduces parts count and PC board real estate. In December 1996, AES had a small pre-production run to send to various alpha test sites. The European inverter prototype was successfully tested. In January 1997, two AC modules were shipped to NREL for evaluation as part of the Phase 1 deliverables and the inverter design was frozen with re-layout begun to meet UL requirements.

Deliverables: D-1.4. Deliver preproduction AC modules to NREL / SNL for evaluation. With the shipment of AC modules to NREL in January, the Task 3 contract requirements were completed for the digital inverter.

\section{Task 4: Standardized, Pre-manufactured Wiring Systems}

In Task 4 Solarex and SDA were to define and develop innovative, low-cost methods of module-to-module electrical interconnection, array wiring, string interconnection, lightning protection and array grounding for systems on residential and commercial buildings as well as ground-mounted arrays through the use of preengineered, standardized components.

A conceptual design was developed for a wiring harness capable of connecting up to four modules in parallel, and a circuit combiner capable of connecting two groups of four modules. The output of the combiner would be compatible with connection to a standard $20 \mathrm{amp}$ AC circuit breaker. The goal was to identify UL listed connectors suitable for use in such a wiring harness and combiner box. We identified five potential manufacturers of the connector system: Turk, Methode, Amphenol, Clipper and Bryant. Samples of these were presented at the October review meeting.

The original wiring plan called for the $\mathrm{AC}$ wiring of the inverter to come back into the module junction box. For this case we proposed the use of a particular Turk connector system that attached through one of the knock-outs in the J-box. However, during the UL review of the inverter, they objected to our design, claiming that the $\mathrm{dc}$ and ac wiring can not go through the same conduit or raceway. The system was redesigned with an AC connector on the opposite (far) end of the inverter. A Lumberg connector was selected and qualified for use in the final unit submitted for UL listing.

Deliverables: D-1.3. and 1.6. Deliver drawings and/or photographs of the module wiring hardware. Drawings of the Turk system were provided. The changes with the Lumberg connector were also documented. Task complete. 


\section{Task 5: Standardized, Modular System Design}

In Task 5 Solarex was to work with SDA to combine the results of Tasks 1-4 to create standardized, modular AC PV systems which can be packaged as kits by Solarex. Solarex worked with SDA to define the "building blocks" for these kits, incorporating comments from potential customer and developing prototype systems.

One of the first steps in the creation of a standardized, modular system is to obtain UL Listing for the major building blocks. In February 1997, Solarex completed the UL tests and received UL listing for the MSX-240 in the PVMaT mounting system.

The three prototype AC module arrays fielded at Solarex during Phase 1, (sloped-roof, flat-roof and groundmount) are our candidates for the standardized AC PV systems. Once the testing results are obtained from UL we will use this input to further refine the design and specification of the standardized, modular AC PV systems.

Deliverables: D-1.7. Deliver updated drawings and/or photographs of prototype standardized module mounting systems for each application. Task complete

\section{Task 6: Digital Inverter for Japan}

Under Task 6, a version of the digital micro inverter was to be produced for the Japanese market.

The Japanese prototype digital inverter was tested in November using the US-version MI hardware as a test bed with software changes. The line voltage in Japan is close enough to the US standard (110 vs. 120) that the same hardware could be used. The software was modified to operate at $50 \mathrm{~Hz}$ and this has been incorporated into the main software as a programmable option: Development of the digital inverter for the Japanese market was postponed until the US design could be finalized and will continue during Phase 2 under Task 15.

\section{Task 7: Digital Inverter for Europe}

Under Task 7, a version of the digital micro inverter was to be produced for the European market.

The European digital prototype was tested in late December using a modified version of the US-MI. The control section is essentially the same, but a dozen parts needed to be modified to operate at the higher line voltage. Some parts were more expensive because of the higher voltage, and some parts were less expensive because of lower current, resulting in a design with approximately the same cost as the US version. Development of the digital inverter for the European market was postponed until the US design could be finalized and will continue during Phase 2 under Task 15.

\section{Task 8: Enhanced Digital Inverter}

Under Task 8, the development of the advanced digital micro inverter begun under Task 3 continued in Phase 2.

An analysis of the inverter design was performed in response to the UL finding that both the $A C$ and $D C$ wires can not go through the same conduit into and out of the inverter. It was decided that the DC input will still attach to the standard Solarex junction box. The AC output will now come out at the opposite end, terminated with a Lumberg connector.

FCC and UL testing: An initial visit to an FCC-certified lab in February showed that the design would meet FCC class B criteria, and after a few modifications, the MI passed. After FCC testing, the focus shifted to UL listing. Several weeks were spent in preparation, including dry runs of all the UL tests. The UL testing was successfully completed in March. A small pre-production run was then assembled with the UL and FCC approved design and sent to Solarex for qualification.

Although the design was essentially complete, some small changes were required for FCC and UL certification. The newest version of the MI is called the Mark 5, rev. A. AES shipped six of the new Mark 5 inverters to Solarex. Solarex immediately began environmental testing. Two of the units failed, one in damp 
heat and one in thermal cycling. The failed units were shipped back to AES for failure analysis. Two new replacement units were sent to Solarex.

Although AES passed the hardware testing required for UL, UL required that some traces be moved to meet UL1741 spacing requirements. There has also been an intensive search for new pottants, because the original was not UL listed, as the manufacturer's rep stated. UL had to approve our production facility. A contract manufacturer was chosen, and a small pre-production run was witnessed by a UL field rep.

During May and June, the inverters continued to fail under accelerated environmental testing - thermal cycling and damp heat. Replacements for the failed inverters were shipped to Solarex. Solarex continued testing and the microinverter continued to fail. In June, Solarex management made the decision to suspend this testing until the MI design was fully complete and finalized.

In June, Solarex also issued a formal request for quotation for modular inverters with detailed specifications and performance, warranty and delivery requirements. AES responded with a quotation of $\$ 450$ per unit plus additional costs for data monitoring and an additional $\$ 15$ charge for the wiring connector. With the AES response to the Solarex RFQ and knowledge of the MI qualification testing, Solarex concluded that the present MI design was too far from commercial readiness in terms of cost and reliability and decided reluctantly to suspend the development effort on an AC module product based on the AES MI until the MI design was fully complete and finalized.

Deliverables: D-2.3. Four modular inverters were produced for delivery to Solarex for mounting on the MSX-240 modules for delivery to NREL and Sandia under the requirements of Task 9.

\section{Task 9: Standardized, Modular System Design}

Task 9 is a continuation of Task 5 to gather information to refine the standardized, modular system designs based on test results and customer input.

Dr. Wills traveled to Chicago in January 1997 to participate in two important meetings: an Industry Advisory Group (LAG) meeting for UL subject 1741 - PV Inverters, and an IEEE SC21 meeting to review IEEE 929 Recommended Practice for Utility Interface of Residential and Intermediate Photovoltaic (PV) Systems - both of which have significant impact on the viability of the AC module in the commercial marketplace. The following week, Dr. Wills participated in the final National Electric Code (NEC) Code Making Panel 3 meeting in Hilton Head, South Carolina. This meeting decided the fate of AC modules and their place in the 1999 NEC. We had remarkable success. when the final vote was taken, all but one of 76 proposals were accepted in concept, with modifications as necessary to make them part of the new code.

During February 1997, Solarex passed all of the UL testing on the MSX-240 module and the module was UL listed as mounted in the PVMaT structure. Testing and evaluation is continuing on these systems at Solarex with the goal of UL listing of these systems. The listing of the Solarex AC ( $240 \mathrm{Wp} \mathrm{DC} \mathrm{module} \mathrm{plus} \mathrm{attached}$ microinverter as an assembly) will be our next goal before the AC module systems can be UL listed.

Solarex investigated one possible route to reducing the structural cost of these standardized systems by replacing the metal mounting beams with plastic. GE provided sample material. The results were unacceptable, as the adhesive separated from the beams as soon as they were subjected to 50 PSI during the mechanical loading test. This option will be investigated further.

In an effort to expand the usage of the mounting system developed under PVMaT, the system was tested at Solarex for use with their new 8 square foot tandem-junction amorphous silicon modules. The initial tests involved mechanical loading in various configurations.

\section{Deliverables:}

D-2.3. Deliver Modular AC PV system 'kits' to NREL and SNL consisting of two AC modules each. In September, Solarex began preparation of two AC modules kits with four AC modules to satisfy the deliverable 'D-2.3'. 
D-2.4. Deliver updated drawings and/or photographs of the standardized module mounting system to be submitted to UL. (To be completed)

\section{Task 13: Develop a Mounting System for Solarex Thin Film Modules}

In September, Solarex began initial definition and development of a new, low-cost frame for their thin-film module. Initial tests indicate that the thin-film glass laminate is sufficiently stiff and that the primary function of the frame can be considered as an attachment method edge protector as opposed to a support for the laminate.

\section{Schedule and Deliverables:}

D-2.10. Preliminary designs. Drawing in progress. - scheduled for December 1997.

D-2.11. Photographs of preliminary hardware . - scheduled for January 1998.

D-2.12. Copies of a qualification test report. - scheduled for March 1998.

D-2.13. Photographs of qualified hardware including modules. - scheduled for April 1998.

\section{Task 14: Development of an Electrical Connector System for Solarex Thin Film Modules}

In September, Solarex began investigating quick-connect plugs and sockets which can be attached to the back of their thin film modules as part of a four-pigtail assembly that will allow either series or parallel connections.

Schedule and Deliverables:

D-2.14. Samples of a selected connector. - scheduled for January 1998.

D-2.15. Copies of UL listing document - scheduled for February 1998.

D-2.16. Samples of qualified hardware - scheduled for April 1998. 
Title: $\quad$ Design, Fabrication and Certification of Advanced Modular PV Power Systems

Organization: Solar Electric Specialties Company, Willits, California

Contributors: G. E. Minyard and T. J. Lambarski

\begin{abstract}
The Design, Fabrication and Certification of Advanced Modular PV Power Systems contract is a Photovoltaic Manufacturing Technology (PVMaT) cost-shared contract under Phase 4A1 for Product Driven System and Component Technologies which have the goals to improve the cost-effectiveness and manufacturing efficiency of PV end-products, optimize manufacturing and packaging methods, and generally improve balance-of-system performance, integration and manufacturing. This contract has the specific goal to reduce the installed PV system life cycle costs to the customer with the ultimate goal of increasing PV system marketability and customer acceptance.
\end{abstract}

The specific objectives of the project are to develop certified, standardized, modular, pre-engineered products lines of our main stand-alone systems, the Modular Autonomous PV Power Supply (MAPPS) and PV-Generator Hybrid System (Photogenset). Now near the end of the contract effort, we have accomplished our essential goals and some extra goals as well. We have designed and built both a $200 \mathrm{~W}$ MAPPS and a $1 \mathrm{~kW}$ Photogenset. We have not only obtained UL Listing of the MAPPS unit, but also of a complete family of MAPPS and are in the process of having the MAPPS family approved for hazardous locations by Factory Mutual (FM). We have accessed the costs and level of effort involved in listing the Photogenset. The MAPPS is performing well in functionality testing at NREL and the Photogenset is also scheduled for functionality testing. In addition to expounding on the goals, objectives and status of the project, specific accomplishments and benefits are also presented in this paper.

\title{
Background
}

Although most PV system integration companies advertise standard product lines, most PV systems sold today are still custom designed based on general product line designs. The fact that most systems houses have system product lines attests to the need to make the specifications simple and standardized so that they can be more easily understood by the potential customer and marketed by the sales engineer. This contract makes a concerted attempt to increase standardization and modularity as we see in other product categories such as computers whether for consumers or commercial applications. There will always be a need for custom designs, but true cost savings and customer acceptance require that systems be more technology transparent. "Turn it on and watch it work."

In addition, up until now no PV power systems have been certified by agencies outside the PV industry such as Underwriters Laboratories (UL) or Factory Mutual (FM). We are seeing a trend toward certification in components such as PV modules, charge controllers and inverters. More companies are getting their PV products UL or ETL Listed or FM Approved for hazardous locations. This project represents the first effort to have whole PV power systems certified.

\section{Program Goals and Objectives}

The stated goal of the Design, Fabrication and Certification of Advanced Modular PV Power Systems contract is to significantly reduce the installed PV system life cycle costs to the customer. This is part of an overall goal of improving PV system marketability and customer acceptance. In line with the 
philosophy presented above, SES is attempting to make with happen by developing certified, standardized, modular, pre-engineered products lines of our main stand-alone systems, the Modular Autonomous PV Power Supply (MAPPS) and PV-Generator Hybrid System (Photogenset).

The technical and cost advantages of this approach are 1) shorter production lead times, 2) higher overall quality and system reliability, 3) decreased management and engineering time, 4) lower overhead and inventory costs, and 5) lower installation labor and material costs. The marketing advantages are that the systems can be specified and described more easily by the sales engineers and the customer can more easily assess the options and choose the system he needs. A corollary to this is greater customer acceptance of the PV solution and greater confidence among lending institutions.

\section{Definition of Terms}

The MAPPS is a small, stand-alone, low voltage DC power supply consisting of PV modules, a mount, a charge controller and batteries. These systems range from a single, small module unit to units with several, large modules. The rated power encompasses $20 \mathrm{~W}$ to about 300-400 W. The system voltages are 12,24 and $48 \mathrm{~V}$. They may also have an optional, small, DC-AC inverter for 120 Vac output.

The Photogenset is a mid-sized, stand-alone, AC power supply with engine generator for load and battery charging backup. It consists of PV modules, a mounting structure, a charge controller, batteries, a DC-AC inverter, an engine generator and a container. These systems range from under $1 \mathrm{~kW}$ to about $10 \mathrm{~kW}$.

\section{Status of Activities}

The task structure of the contract calls for development and fabrication of a 200 W MAPPS and a $1 \mathrm{~kW}$ Photogenset as the first prototypes for these respective product lines, certification of the prototypes and product lines, and functionality testing of the prototypes at NREL and Sandia. Within the charter was also room for development of new or improved components for the systems to improve performance and reliability while reducing costs or to facilitate certification.

The sizes of the prototypes were chosen for their high sales potential and manageable size and cost for fabrication and certification. The purpose of the functionality testing is to provide verification of performance and reliability and to develop test-based performance specifications.

\section{MAPPS Status}

We have completed the design and fabrication of the $200 \mathrm{~W}$ MAPPS, obtained UL Listing and are obtaining test data from functionality testing at NREL. As part of the design effort, we developed a new enclosure for the batteries and control assembly. This enclosure costs less than the previous design and makes better use of internal space while providing a separate compartment for the control assembly. The design makes use of only UL Listed or UL Recognized components. We have also investigated developing a new microprocessor-based controller for small systems. It will be used as an option when greater control flexibility or remote monitoring is needed. This controller is still in development and will be completed outside this contract.

We have obtained UL Listing not only for the 200 W MAPPS but also for a complete family of MAPPS ranging in size from $10 \mathrm{~W}_{\mathrm{PV}}$ to $300 \mathrm{~W}_{\mathrm{PV}}$ and in 12 and 24 volt options. UL performed a rain spray test on the battery enclosure to certify it as meeting $3 \mathrm{R}$ outdoor enclosure rating. They also tested the control electronics and evaluated all other components. UL then performed an initial plant inspection at SES. SES passed this inspection as well as a follow up inspection three months later.

We are also in the process of obtaining FM Approval for a line of MAPPS with greater incendiary safety for use in hazardous locations. These locations, as defined in Article 500 of the National Electrical Code, 
have environments with incendiary gases present. Following completion of the UL evaluation of the MAPPS, it was modified for FM requirements and sent to them for their evaluation. This evaluation has been successfully completed and a plant inspection is being scheduled.

A second $200 \mathrm{~W}$ MAPPS unit was built for the functionality testing program. This unit was set up and instrumented at NREL's PV Test Facility. The MAPPS has only been in test since Sept., but initial data indicates satisfactory performance under load. The critical test period will be during the winter when short days and inclement weather will test the unit's autonomy capabilities. During the installation of this prototype at NREL several minor problems were identified with the mechanical design and the installation manual which can be easily corrected in subsequent units.

\section{Photogenset}

We have completed the design and fabrication of the $1 \mathrm{~kW}$ Photogenset and are in the process of performing shakedown testing prior to delivering the unit to Sandia and NREL for functionality testing. The design incorporates a multi-platform layout. The Photogenset can be provided with either a skidmounted enclosure or in a trailer for mobile applications. We have attempted to make maximum use of UL Listed components; however, we were not able to obtain a UL Listed generator and accessories or a UL Listed controller with the remote communications capabilities desired. We obtained quotes from both UL and ETL (Electrical Testing Laboratories) to assess the costs and benefits of each laboratory. We contracted with UL for a preliminary investigation to more fully assess the problems, the degree of evaluation and testing required and the cost of the listing. The results indicated that a fairly extensive testing program would be required for the generator system and the controller, that there is no guarantee of Listing and that the costs between UL and ETL appear similar. We believe that additional component development and maturity is needed before the Photogenset Listing can be accomplished.

\section{Accomplishments and Benefits}

To recap current accomplishments, we have: 1) a UL Listed family of MAPPS with upgraded assembly process controls and installation instructions; 2) a FM Approval pending on a family of MAPPS; 3) an understanding of certifications issues and requirements (Ref. 1); 4) an improved, lower cost MAPPS battery enclosure; 5) a $1 \mathrm{~kW}$ mobile Photogenset prototype based on a multi-platform design; 6) a new microprocessor-based controller with remote communications capabilities for the Photogenset plus near completion of a small system version; and 7) a knowledge of what it will take to obtain UL Listing of a Photogenset.

We have realized a number of additional benefits from this contract including the prestige and marketing benefits of receiving a PVMaT contract, additions to the staff and consultants, increased staff experience and the knowledge gained from association with NREL, Sandia and Southwest Technology Development Institute. There are also benefits that are difficult to quantify such as the synergy between the PVMaT contract and our business efforts. We believe the experience gained through the PVMaT contract has been instrumental in obtaining important new business. At the same time the working of these other contracts has been instrumental in developing our new designs. We believe this PVMaT contract will provide important advances in PV power supply market penetration through lower costs, higher reliability, product standardization and product certification.

\section{References}

1. National Electrical Code, 1996. NFPA-70. National Fire Protection Association, Quincy, MA. 2. Lambarski, T.; Minyard, G. (February 1997). Annual Technical Progress Report, Subcontract ZAF-514271-07. 
3. Minyard, G.; Lambarski, T. (August 1997).'Design, Fabrication and Certification of Advanced Modular PV Power Systems." NREL Photovoltaic Program FY 1996 Annual Report. NREL/BK-21021966.

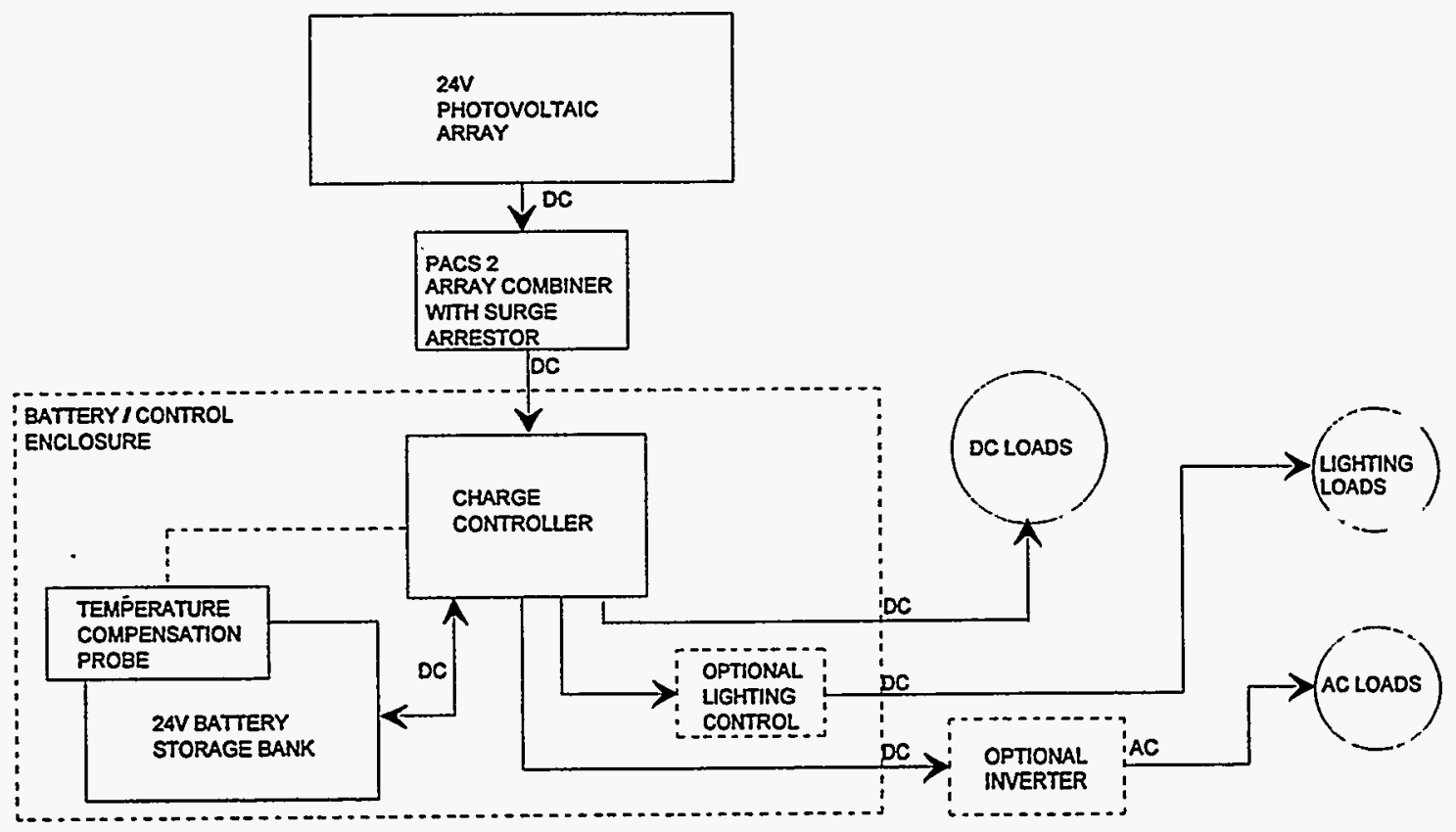

Figure 1: M.A.P.P.S. Block Diagram

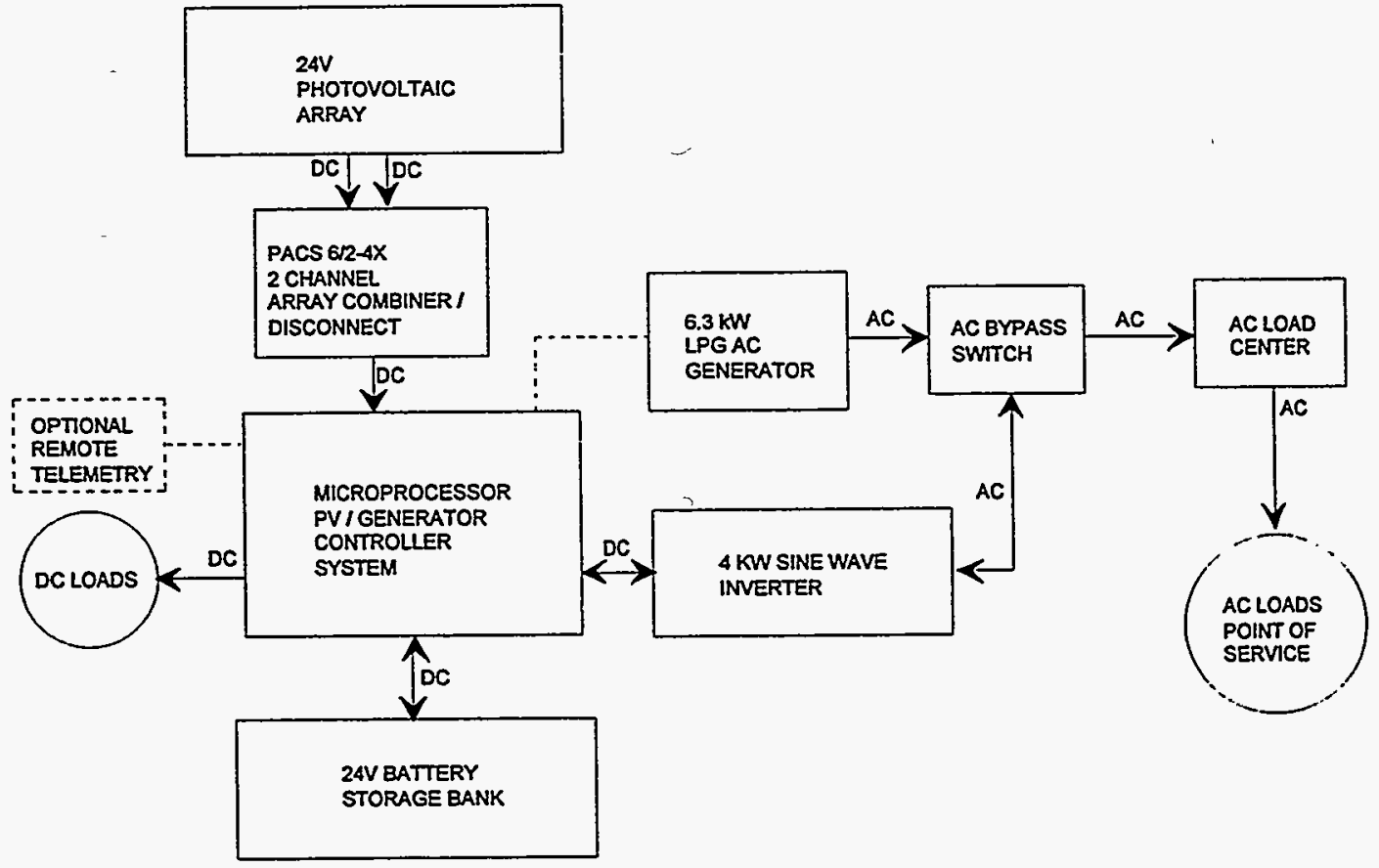

Figure 2: Photogenset Block Diagram 

Technology Improvements

\author{
Organization Solarex, A Business Unit of Amoco/Enron Solar \\ Contributors J. H. Wohlgemuth, Program Manager; F. Artigliere, J. Cliber, M. Conway, J. Creager, \\ R. Dominguez, G. Kelly, T. Koval, M. Perry, M. Roy, M. Narayanan, , T. Tomlinson, \\ and D. Whitehouse of Solarex; M. Diver, R. Fernandez and D. Vanecek of ARRI
}

\title{
Objectives
}

The objectives of this three-year program were to advance Solarex's cast polycrystalline silicon manufacturing technology, reduce module production cost in half, increase module performance and expand commercial production capacity by a factor of three. Key components of this program are: 1) Casting of larger ingots; 2) Use of wire saws to cut thinner, larger wafers with less kerf loss; 3) Transfer of high efficiency cell processes to manufacturing; 4) Increased automation; 5) Development of frameless module mounting systems; and 6) Automated handling of large thin cells and wafers.

\section{Approach and Accomplishments}

\section{Casting}

The original goal of the casting task was to develop the ability to cast ingots that yield four $15 \mathrm{~cm}$ by 15 $\mathrm{cm}$ bricks with at least equivalent material quality as was achieved for the standard $11.4 \mathrm{~cm}$ by $11.4 \mathrm{~cm}$ bricks. This was demonstrated in the laboratory, yielding equivalent quality ingots with a $73 \%$ increase in the useable silicon obtained from each casting.

Most of Solarex's products are still based on the use of $11.4 \mathrm{~cm}$ by $11.4 \mathrm{~cm}$ solar cells, so an effort was undertaken to develop casting for even larger ingots that would produce $9-11.4 \mathrm{~cm}$ by $11.4 \mathrm{~cm}$ bricks. These "Mongo" ingots required further changes in the insulation and receiver, but utilized the same casting stations as modified for PVMaT ingots. The process for casting Mongo ingots was successfully developed in the laboratory. It has now been transferred to production with all of Solarex's casting stations now casting Mongo sized ingots.

Once the new process was transferred to manufacturing, it was important to monitor the performance to assure that the new process was producing material of equivalent quality at similar yields. Process yields and cell efficiency were tracked during the time period when both standard and large (mongo) ingots were being produced. The larger ingots resulted in slightly higher yields and equivalent cell efficiencies.

Casting of larger Mongo ingots has been used to double Solarex's casting capacity at approximately $20 \%$ of the cost that would have been required, if the same amount of capacity was added by purchasing new casting stations. All of the goals of the casting task have been met.

\section{Wire Saw}

The goal of the wire saw task is to develop the wire saw technology for cutting $15 \mathrm{~cm}$ by $15 \mathrm{~cm}$ polycrystalline wafers on $400 \mu \mathrm{m}$ centers at lower cost per cut than achieved on the ID saws. Solarex has utilized HCT wire saws in this program. These wire saws have successfully demonstrated the ability 
to cut a variety of wafer sizes including $11.4 \mathrm{~cm}$ by $11.4 \mathrm{~cm}, 11.4 \mathrm{~cm}$ by $15.2 \mathrm{~cm}$ and $15.2 \mathrm{~cm}$ by 15.2 $\mathrm{cm}$ on centers from $500 \mu \mathrm{m}$ down to $400 \mu \mathrm{m}$. Over $50 \%$ of all Solarex's wafers now being cut on these wire saws. Each wire saw produces as many wafers as 22 to 24 ID saws and does it with higher yields and lower per wafer cost. In addition, the wire saws produce at least $20 \%$ more wafers from each ingot, increasing the number of watts produced per kilogram of silicon purchased, an important parameter because of the limited availability of low cost silicon feedstock. The goals of the wire saw effort have all been met.

\section{Cell Process}

The goal of the cell task was to increase cell efficiencies to $15 \%$, while decreasing the cost per watt at the module level. Solarex's cell process is based on the use of thick film paste metallization, a low cost process. The PVMaT program objective was to determine which modifications or improvements could lead to both higher cell efficiency and lower dollar per watt module manufacturing cost. Several process modifications have been evaluated but only one, the Al paste back surface field (BSF), is being implemented in manufacturing at this time. Implementation of the BSF process will increase cell efficiency, but will not meet the $15 \%$ goal set at the beginning of the program.

Initial laboratory experiments demonstrated that an aluminum paste back surface field (BSF) can increase cell efficiency by approximately $5 \%$. [1] Three BSF manufacturing trials were completed, with more than 40,000 BSF cells produced with all processes except BSF performed in production using production processes, equipment and personnel. The average cell efficiency for all of these BSF cells was more $5 \%$ higher than for the non-BSF cells produced during the same time period.

Prototype equipment was then constructed and the BSF process was transferred to manufacturing for use on a significant percentage of all cells produced. The BSF process yielded a $5.1 \%$ increase in average short circuit current and a $1 \%$ increase in average voltage, but a reduction in fill factor limited the efficiency increase to $4.5 \%$. However, much of the fill factor loss is due to processing the cells with conditions optimized for the standard cells, not for the BSF cells. We expect that optimization of the firing profiles will improve the BSF fill factor and recover at least the $5 \%$ efficiency gain demonstrated in the pilot runs.

BSF cells from the manufacturing trials have been incorporated into modules using Solarex's automated assembly equipment. The BSF efficiency enhancement translated from cells to modules. These modules were then successfully qualified to IEC-1215 (Crystalline Silicon Terrestrial Photovoltaic Modules Design Qualification and Type Approval) and IEEE-1262 (IEEE Recommended Practice for Qualification of Photovoltaic Modules).

A fully automated screen printing system for implementation of the BSF process is now being installed in the Frederick facility. This system includes three automatic screen printers, one each for printing of back pads, aluminum paste and front silver grid pattern.

\section{Module Assembly}

The initial goal of this task was to modify Solarex's module assembly system to increase throughput by $100 \%$ and decrease the labor requirement by $50 \%$. The throughput goal was modified to an increase of $200 \%$ to meet increased product demand. 
Solarex subcontracted to the Automation and Robotics Research Institute (ARRI) at the University of Texas at Arlington to review and model the automated module assembly system in operation at the beginning of this PVMaT Program and to make recommendations for improving the equipment and/or process flow. ARRI prepared a process flow chart detailing all of the module assembly steps and used this information to model and analyze the manufacturing process. A number of minor improvements were recommended and implemented, increasing the production capacity by $40 \%$.

ARRI used AT\&T's discrete event simulation package called Witness to evaluate expansion scenarios. The Witness software provided an analysis of the capacity and resource requirements for the different scenarios. A new factory concept was developed that allowed for incremental increases to meet the shorter term capacity requirements and that could ultimately result in tripling of the module assembly capacity. The plan was based on replacing the back solder robots with XY positioners to increase the number of solder bonds made at one time from 2 to 4, thereby increasing the throughput by nearly a factor of two. This modification has now been implemented and the XY positioners are operating at the throughput level predicted, meeting all of the goals of this task.

The new factory configuration and Solarex's projected module build plan through 1999 were then used as new inputs to the Witness program. As the volume of products ramps up, the model predicts when the various pieces of equipment reach maximum capacity. This analysis was utilized to develop a capital investment budget for the crystalline expansion. The implementation of this plan is now underway.

\section{Frameless Module Development}

The goal of this task was to develop and qualify a frameless module design incorporating a lower cost back sheet material (less than $\$ 0.05 /$ square foot) and user friendly, low cost electrical termination (less than $\$ 1.00$ /module). Tedlar was chosen as the backsheet, because it meets all of the technical requirements, although it does not quite meet the cost goals. Pig tail wires with crimp connectors and shrinkable tubing insulation were chosen for the module electrical termination. [2]

Since PVMaT is designed for large systems, the modules were designed to mount directly onto the support structure without integral frames. The first step was the design of a compatible support structure and the identification of $3 \mathrm{M}$ Very High Bond Tape for mounting the modules to the structure. This system was used on several large arrays, but major problems were encountered relative to the use of the tape. The problems appear to be related to incorrect application and to the mounting of modules in such a way that there is a continual pressure/torque on the beams. The failure mode is separation of the module from the beam. While correct assembly may alleviate this problem, it is likely that actual systems will not be assembled by well trained personnel. Therefore, the main adhesive was switched from tape to RTV. The modules are panelized several days before installation, so the RTV can cure. This method has now been used successfully on several systems and it does meet the overall goal of this task.

\section{Automated Cell Handling}

The goal of the automated cell handling task is to develop automated handling equipment for $200 \mu \mathrm{m}$ thick $15 \mathrm{~cm}$ by $15 \mathrm{~cm}$ polycrystalline silicon wafers and cells with a high yield (less than $0.1 \%$ breakage per process handling step) at a throughput rate of at least 12 cells or wafers per minute. ARRI is also supporting Solarex in this task. ARRI began this effort by studying the strength and fracture behavior of Solarex cast polycrystalline wafers using a four point bend and a cantilever test to measure the 
mechanical strength of the wafers. [3] A finite element model was then developed to determine the distribution of stress and deflection in a wafer based on the applied load. The model was used to simulate a typical wafer handling situation, to estimate the maximum load that can be applied during handling and the corresponding probability of failure. [4] ARRI then undertook a study of commercial and academic sources related to the handling of silicon wafers. [5]

The ARRI work has lead to the design of a flexible wafer pick-up end effector for movement of wafers and cells. This work was also used to assist in the design of cell handling equipment for the implementation of the BSF process. Preliminary results on these handling systems indicates that they will meet the goals of this task.

\section{Conclusions}

This Cast Polycrystalline Silicon Photovoltaic Module Manufacturing Technology Improvement Program has lead to the development of and/or improvements in processes, products and equipment. The following developments from this program have either been implemented or are in the process of being implemented in manufacturing:

- Casting of larger ingots;

- Use of wire saws;

- Addition of a back surface field to the cell process;

- Introduction of a larger cell $(11.4 \mathrm{~cm}$ by $15.2 \mathrm{~cm})$ into commercial production and development of the processes and equipment necessary to produce $15.2 \mathrm{~cm}$ by $15.2 \mathrm{~cm}$ cells;

- Upgrade of the module assembly equipment;

- Qualification of a lower cost backsheet; and

- Qualification of a lower cost electrical termination system.

During the course of this PVMaT program:

- The production volume at Solarex has tripled;

- The cost to manufacture a framed power module has been reduced by $20 \%$; and

- The cost to manufacture the lowest cost module has been reduced by $40 \%$.

These cost reductions occurred while the cost of silicon feedstock increased and while the factory was running at full capacity to meet increased demand. Without this PVMaT program it is likely that the consumer's cost for PV modules would have increased instead of decreasing and that less production capacity would now be in place, compounding the present product shortage.

\section{References}

1. J. H. Wohlgemuth, D. Whitehouse, T. Koval, J. Creager, F. Artigliere and M. Conway, "Solarex Crystalline PVMaT Program", First World Conference on PV Energy Conversion, 1994, p. 832.

2. J. Wohlgemuth, T. Koval, J. Creager, T. Tomlinson, J. Ellis, F. Artigliere, S. Narayanan, J. Posbic, S. Shea, M. Roy, J. Cliber, M. Conway, D. Vanecek, B. Chen and R. Fernandez, "Progress in Solarex Cast Crystalline Silicon PVMaT Program", Twenty Fifth IEEE PVSC, 1996, p. 1181.

3. J. Wohlgemuth, et al, "Cast Polycrystalline Silicon Photovoltaic Module Manufacturing Technology Improvements", Semiannual Subcontract Report, 1 January 1995-30 June 1995, NREL/TP-411-29589, 1996.

4. J. Wohlgemuth, et al, "Cast Polycrystalline Silicon Photovoltaic Module Manufacturing Technology Improvements", Annual Subcontract Report, 1 January 1995-31 December 1995, NREL/TP-411-221261, 1996.

5. J. Wohlgemuth, et al, "Cast Polycrystalline Silicon Photovoltaic Module Manufacturing Technology Improvements", Semiannual Subcontract Report, 1 January 1996-30 June 1996, NREL/SP-520-22503, 1997. 
Title: $\quad$ Full Scale Testing of Modules Prepared with

Experimental EVA-Based Encapsulants

\author{
Organization: STR (formerly Springborn Laboratories, Inc.) \\ Enfield, Connecticut 06082
}

Contributors: W.H. Holley Jr., principal investigator; R. Hammond (ASU);

S. Agro; J. Galica; R. Yorgensen

\title{
Objectives
}

EVA resin-based encapsulant is used extensively by module manufacturers worldwide, has been in commercial use since $1981^{1}$, and has proven dependable during production, module fabrication, and enduse. Despite widespread acceptance of A9918P and similar formulations for PV, some module producers and end-users have reported cases of browning of field-aged modules. While isolated, these reports raised concern as to long term reliability of EVA resin-based encapsulants.

During Phase I of this PVMaT 3A program, STR conducted problem definition using literature search, field survey, and accelerated U.V. aging (AAS) studies to evaluate the influence of compositional, processing, and operating parameters on A9918P discoloration. ${ }^{2}$ Also, instrumental analysis was used to verify suspected chemical mechanisms of degradation. ${ }^{3,4,5}$

During Phase II STR developed approaches for stabilizing the EVA-based encapsulant against discoloration/degradation, including reformulated systems. These "stabilization strategies" were evaluated by AAS using xenon-arc Weather-Ometer and EMMA (equatorial mount with mirrors for acceleration; accelerated outdoor weathering) with periodic measurements of Yellowness Index (ASTM D1925) as an indication of browning. This work resulted in development of four experimental EVAbased encapsulants - three "standard cure" (X9903P, X9923P, and X9933P) and one "fast cure" (X15303P) which demonstrated greatly reduced discoloration on AAS. ${ }^{3,67}$

The Phase III objective was to demonstrate the functional adequacy of these various stabilization strategies under end use conditions and in qualification tests using full-size modules.

\section{Phase III Technical Approach}

STR extruded pilot quantities of all four experimental EVA-based encapsulants plus controls. Rolls of nominal 0.018 inch thick sheet were supplied to various PVMaT project team members who: 1) Prepared full-size, nominal 50 watt modules for qualification testing and field testing in Arizona and 2) Prepared "mini-modules" (2.5 inch $\times 2.75$ inch and 2.5 inch $\times 5.5$ inch) using small cells or half cells and the team members' normal materials of construction for superstrate, interconnects, and backing. Participating team members include: ASE Americas, Astropower, Ebara Solar, Photocomm, Siemens Solar, Solec International, Solarex, and United Solar Systems.

Module approach: Module manufacturer team members supplied Arizona State University's Center for Energy Systems Research (ASU/CESR) in most cases 18 modules, six each representing three of the four possible experimental encapsulants. These modules, 102 in all, were used by ASU/CESR for qualification testing per IEEE 1262. 
In addition, several team members supplied ASU/CESR six to eight modules, two each representing three or four out of five experimental "stabilization strategies." The strategies included the four experimental encapsulants plus the conventional "fast cure." All of these modules have cerium oxide-containing low iron glass as the superstrate.

All 36 modules were installed for ASU/CESR on a Martin Marietta two-axis tracker at STAR facility by Arizona Public Service in Tempe the third quarter of 1996. Modules were a nominal 50 watts with outside dimensions not exceeding $20 \times 50$ inches and were fitted with fixed resistive loads such that each would operate within $\pm 10 \%$ of its peak power point. Peak power point represents a typical March day (i.e. near the mean annual average temperature). A thermocouple was installed on the back of each module, and each was photographed to provide a visual record.

Module current-voltage (I-V) curves are being taken at regular intervals for each module, and data, including Isc, Voc, Pm, and ff are being collected at actual test conditions and translated into standard conditions. In addition, such pertinent weather data as minimum and maximum ambient temperatures, global solar radiation in the plane of the array $\left(\mathrm{kWh} / \mathrm{m}^{2}\right)$, and direct normal solar radiation $\left(\mathrm{kWh} / \mathrm{m}^{2}\right)$ are being collected. Also, ASU conducted thermal mapping in early October 1996 by taking an infrared thermal image of each module at the peak power point $(+10 \%)$ and another with the module short circuited.

Mini-module-approach: Each mini-module was provided with leads through the backing material and was connected to a $1 \mathrm{ohm}$ resister. The back of each mini-module was insulated with a layer of Mylar, and the assembly mounted in a sample holder. This allowed the mini-modules to operate at a fixed resistive load during accelerated U.V. aging.

Samples were placed in a xenon-arc Weather-Ometer and exposed at a nominal "two suns" $\left(0.55\right.$ watts $/ \mathrm{m}^{2}$ /nm @ $340 \mathrm{~nm}$ ), $90^{\circ} \mathrm{C}$ over the cells, and $>95 \%$ R.H. A total of more than 40 manufacturer/ encapsulant/superstrate combinations were evaluated, including controls with A9918P. Mini-modules were exposed for six months at continuous temperature and irradiance.

\section{Test Result Highlights}

Qualification testing: Testing was done per IEEE 1262. During sequence C one experimental encapsulant, X9933P turned bright yellow during 1000 hours of damp heat. Otherwise, there were no encapsulant-related failures.

Field testing of modules, data acquisition and analysis: Photos were taken of all modules after installation for a permanent visual record and were transferred to diskette for easy reference and archiving. I-V curves were taken in September 1996 for baseline electrical performance, and a second set was taken in early October.

Thermal image mapping was completed on October. IR data were taken with the modules at peak power and again short circuited. Some shorted modules exhibited hot spot heating with one hot cell reaching $86.6^{\circ} \mathrm{C}$; nine other modules had "hot" cells that ranged in temperature from 70.1 to $78.3^{\circ} \mathrm{C}$. With a resistive load these cells operated at about $65^{\circ} \mathrm{C}$. Both hard copy (color images) and computer files of the data were generated at peak power and under short circuit conditions.

Information will continue to be collected periodically. It is the intention of STR, in cooperation with ASU, to continue the field testing indefinitely. 
Accelerated aging of mini-modules: Samples were exposed continuously conditions to a nominal twosuns and $90^{\circ} \mathrm{C}$ cell temperature in a xenon-arc Weather-Ometer for six months. One team member prepared mini-modules with Tefzel cover film, and each team member prepared one set of mini-modules with cerium oxide-containing glass. Otherwise, all mini-modules used non-cerium low iron glass to provide "worst-case" conditions.

After 26 weeks of exposure, most A9918P-encapsulated controls using low iron glass superstrate turned brown over the cells, in the same manner seen in our survey of fielded modules. With the exception of samples made with X9933P, all mini-modules prepared with experimental grades, as well as with $15295 \mathrm{P}$ and cerium-oxide containing glass, showed no browning over the cells.

One surprise in this series was browning of three experimental encapsulants under Tefzel cover film. Since there is available oxygen in these systems, we suspect a different browning mechanism is taking place than under glass superstrates. Two observations support this: 1) browning occurred more rapidly than with A9918P under glass, and 2) A9918P under Tefzel showed no browning after 26 weeks which is consistent with what we observed for Tefzel/glass laminates exposed in both Weather-Ometer and EMMA.

Our goal in this PVMaT program, was a "family of stabilization strategies" adaptable to various module constructions. While the experimental encapsulants appear promising for modules with glass superstrates, in the case of Tefzel cover film, conventional A9918P or 15295P is preferred.

\section{Summary of Phase III Accomplishments}

* Four experimental EVA-based encapsulants developed in Phase II were successfully prepared in pilot quantities using normal production equipment.

* Extruded sheet from these four encapsulants was used, without significant problems, by six different module producers. Full-sized 50 watt modules were successfully prepared using conventional lamination equipment and processing cycles.

* With only a couple of exceptions, curing of the experimental encapsulants in these modules was at a consistently high level. Autopsy work following qualification testing showed average gel contents of greater than $83 \%$ versus a typical $80 \%$ for conventional EVA-based grades.

* A total of 102 modules prepared with the four experimental encapsulants were subjected to IEEE 1262 qualification testing. Except for yellowing of one experimental formulation, X9933P, there was not a single failure related to the encapsulant material.

* During IEEE 1262 , modules were subjected to damp heat ( $85 \mathrm{C} / 85 \%$ R.H.), thermal cycling, U.V., etc. Despite these exposures there appeared to be little or no loss of adhesion to cells, interconnects or glass. However, on a qualitative basis some backing film laminates showed a loss of adhesion to the encapsulant as a result of damp heat. Under the same conditions, these backings also developed a brown discoloration which was traced to the adhesive layer.

* A total of 36 modules, representing four different experimental encapsulants and six manufacturers were deployed on a two-axis tracker at the STAR facility of Arizona Public Service in September of 1996. Controls prepared with A9918P were installed in mid-1997. 


\section{References}

1. J.H. Wohlgemuth and R.C. Petersen, Solar Cells Their Science, Technology, Applications and Economics, Solarex Experience with Ethylene Vinyl Acetate Encapsulation, (Elsevier Sequoia, 1991) 383-387.

2. W.H. Holley, S. Agro, J. Galica, L. Thoma, R. White, R. Yorgensen, Annual Report, NREL Subcontract No. ZAG-3-11219-02-105661 (November 1994).

3. W.H. Holley, Annual Report, NREL Subcontract No. ZAG-3-11219-02-105661 (January 1996).

4. M. Ezrin, et al., "Discoloration of EVA Encapsulant in Photovoltaic [Modules]," Preprints SPE Annual Technical Conference, Boston, MA, (May 1995) 3957-3960.

5. M. Ezrin, et al., "Further Studies of Discoloration of EVA Encapsulant in Photovoltaic Modules," Preprints, SPE Annual Technical Conference, Indianapolis, IN, (May 1996) 3260-3264.

6. W.H. Holley $\mathbf{J}_{r}$., et al., "Advanced Development of Non-Discoloring EVA-Based PV Encapsulants," AIP Conference Proceedings No. 353, 13th NREL Photovoltaic Program Review, H. Ullal and C.E. Witt, ed., American Institute of Physics Press, New York, (1995) 636-642.

7. W.H. Holley Jr., et al., "UV Stability and Module Testing of Non-Browning Experimental PV Encapsulants," 25th IEEE Photovoltaic Specialist Conference, Washington, D.C. (May 1996) 
Title: $\quad$ Development of a Modular, Bi-Directional Power Inverter for Photovoltaic Applications

Organization: Trace Engineering Company, Inc., Arlington WA

Contributors: C. Fruitas, G. Thomas, R. Rosenbaum, B. Faley, S. Johnston; M. Frost

\section{Project Objective}

The primary goal of this project is to demonstrate the feasibility of the invertr module concept and its ability to reduce the cost of the inverter as a part of the overall PV system cost. The savings are achieved by the contribution of each of the tasks area's contributions. Trace plans to achieve the cost reduction by changes in the topology of the inverter, and by enabling the inverters design to be used by a wider market segment, increasing the production volume.

\section{Introduction}

This project has resulted in the development of a modular, bi-directional power inverter for photovoltaic applications by Trace Engineering Company, Inc of Arlington, Washington. This development was conducted under the PVMaT Phase 4Al program.

Trace Engineering has been manufacturing power inverters for over 13 years and has built more than 300,000 inverters. The majority of the inverters built have been used in renewable energy applications. Until recently, Trace concentrated on the stand-alone, non-utility interactive applications. With the introduction of the Trace SW series in 1994, Trace expanded its product line into utility interactive and larger hybrid system applications.

The increased production volume and expansion of the Trace product line into numerous models and power levels to meet the requirements of the wide range of possible applications caused Trace to see the need for the development of new inverter designs which could be manufactured more easily, used more flexibly in different applications, and which could achieve higher performance. This PVMaT subcontract has provided the needed impetus to accelerate those efforts through the development of a modular, bidirectional power inverter which could be used a the basic building block. The modular inverter is expected to fill the requirements for stand-alone, hybrid and utility interactive applications by connecting them in parallel (for higher power), in series (for higher AC voltages) and in both single, split and three phase configurations.

\section{Results}

Trace Engineering demonstrated a prototype power inverter based on the research conducted. The prototype was $30 \%$ smaller and was designed using an existing product chassis to demonstrate the manufacturability of the design.

Several control and protection systems were developed to enable the use of multiple inverters in either three phase or single phase applications. The control and protections methods developed were applied to an existing product and is now available to the PV industry. 
Trace also has developed a modular packaging system for the inverter and the balance of systems components required which provides additional environmental protection, allow greater system flexibility both in design and installation, and integrates more of the balance of system components into a single unit. Prototypes were built and displayed at several US and International PV industry conferences. The results of the feedback has been incorporated into the final design and is now in production and offered to the PV industry.

\section{References}

1. Annual Technical Progress Report, FY 1996 (August 1997) NREL/SR - 520 - 2340126 pg. 
Title: $\quad$ Development of a Low Cost Integrated $20 \mathrm{~kW}$ A.C. Solar Tracking Sub-Array For Grid-Connected PV Power System Applications

\author{
Organization: Utility Power Group, Los Angeles, California \\ Contributors: M. Stern, Principal Investigator; R. West, G. Fourer, W. Whalen, G. Duran, \\ D. Metcalf, and K. Mackamul.
}

\title{
Introduction
}

Utility Power Group has achieved a significant reduction in the installed cost of grid-connected PV systems. The two part technical approach focused on 1) The development of a large area factory assembled PV panel, and 2) The integration and packaging of all sub-array power conversion and control functions within a single factory produced enclosure. Eight engineering prototype $15 \mathrm{~kW}$ ac single axis solar tracking sub-arrays were designed, fabricated, and installed at the Sacramento Municipal Utility District's Hedge Substation site in 1996 and are being evaluated for performance and reliability. A number of design enhancements will be implemented in 1997 and demonstrated by the field deployment and operation of over twenty advanced sub-array PV power systems.

\section{Objective}

The overall objective of Utility Power Group's two year PVMaT Phase 4AI sub-array development effort was to achieve a $20 \%$ reduction in the installed cost of PV power systems. Given UPG's focus on BOS costs and assuming such costs represent $40 \%$ of the total cost of an installed PV power system, UPG's overall objective could be re-stated as seeking to achieve a $50 \%$ reduction in BOS costs.

\section{Approach}

The technical approach employed by UPG towards achievement of the above objective was based upon the following lessons learned during the construction or installation of a number of PV power systems:

- Factory assembly provides cost savings over field assembly.

- Reducing the number of components reduces installation cost.

- Design simplicity reduces the cost of construction materials.

- Standardization of installation steps reduces the cost of construction labor.

- Modularity reduces the cost of design and project management.

These lessons led UPG to select modularity and component integration as the two primary design criteria to be utilized. A modular alternating current ("ac") sub-array design simplifies the generation of specific and custom PV system design packages and reduce incremental administrative and project management costs. Redundancy and reliability are PV system operational cost factors which benefit from modularity and the reduced number of components associated with increased functional integration.

Based upon the above lessons learned and design criteria, UPG began development of an integrated and modular sub-array which, to be in compliance with the derating requirements of the 1996 National Electrical Code and applicable standards of Underwriters Laboratories, has an output rating of $15 \mathrm{kWac}$. 
Specifically, UPG has developed:

1. Two versions of a large area Modular Panel (MP) which are factory assembled and tested by Utility Power Group utilizing production optimized and reliability tested PV laminates manufactured by Siemens Solar Industries.

2. An Integrated Power Processing Unit (IPPU) combining all sub-array electrical functions such as power collection, system protection, direct current (" $\mathrm{dc}$ ") to ac conversion, and single axis solar tracking, into a single field deployable unit factory assembled and tested by Utility Power Group.

\section{Modular Panel Development}

The factory assembled PV panels developed by UPG in conjunction with Siemens Solar Industries (SSI) are specifically designed for high voltage power system applications and provide the following cost saving advantages:

1. Elimination of redundant structural frames on individual PV modules.

2. Simplification of the panel support structure.

3. Reduction of labor associated with field installation of panels.

The result of this work was the introduction in 1996/1997 of UPG's POWERGLASSTM Model 11M55 and Model 7SP75 (Figure 1) Modular Panels which, with an STC rating of $606 \mathrm{Wdc}$ and $525 \mathrm{Wdc}$, are believed to be the highest power UL listed PV panels in the world. By the end of 1997, over 2,000 UPG Modular Panels will be installed in grid connected PV power systems in the United States.

\section{IPPU Development}

The Integrated Power Processing Unit ("IPPU") developed by UPG provides significant BOS cost reduction through the integration and optimization of all electronic and electrical PV power system functions and operations. To facilitate fabrication, testing, assembly, and field maintenance of the IPPU, UPG segmented the design into the following four sub-assemblies each utilizing high power printed circuit board (PCB) technology to perform a number of integrated functions:

- DC Interface PCB Assembly

- Control PCB Assembly

- Bridge / Regulator PCB Assembly

- AC Interface PCB Assembly

- DAS Board Option

With this approach, UPG has achieved significant cost reduction through the integration of all sub-array functions into a single unit. The core power bridge technology was developed with assistance from Sandia National Laboratories (SNL) and allows the IPPU's internal d.c. to a.c. inverter to operate at a higher conversion efficiency (Figure 2) and a higher switching frequency than any other commercially available inverter. To provide up to $25 \%$ more energy output per year than fixed axis PV power systems, the IPPU combines solar tracking control technology developed at SNL with UPG's innovative single axis tracking structure. The combination of modularity and integration served to reduce the number of discrete field construction operations from 36 to 19 [1], permitted standardization of key components, and simplified design documentation. 


\section{Results}

PVMaT Phase 4Al contract work was completed in July, 1997. UPG successfully completed the design, development, fabrication, and demonstration of a modular and integrated $15 \mathrm{~kW}$ a.c. solar tracking PV power system sub-array which exceeded the $20 \%$ cost reduction goal for PV power systems. Ten (10) engineering prototype sub-arrays and forty (40) initial production sub-arrays (Figure 3 ) have been installed in FY96/97 at various sites for the Sacramento Municipal Utility District, Arizona Public Service, and Detroit Edison representing over $750 \mathrm{~kW}$ of PV power systems. The total savings or price reduction realized by these customers directly related to the success of this development effort was over $\$ 1.3$ million based upon a $\$ 1.75 /$ watt reduction in total system cost.

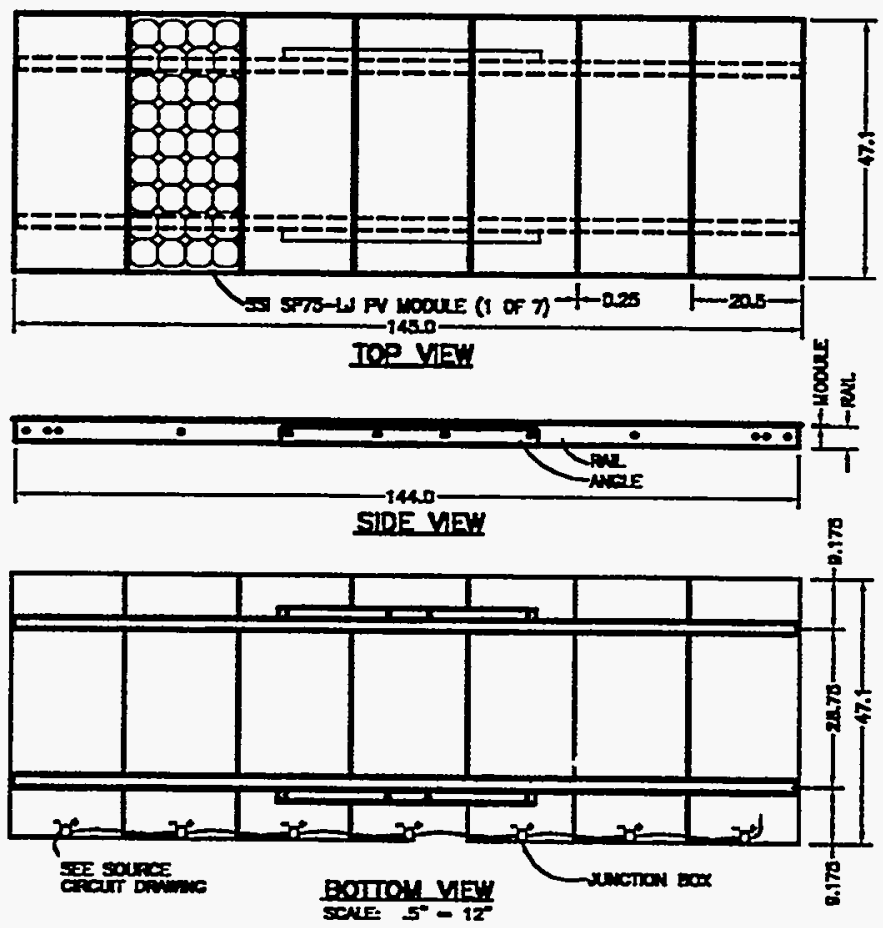

Figure 1. UPG POWERGLASSTM Model 7SP75 Modular Panel.

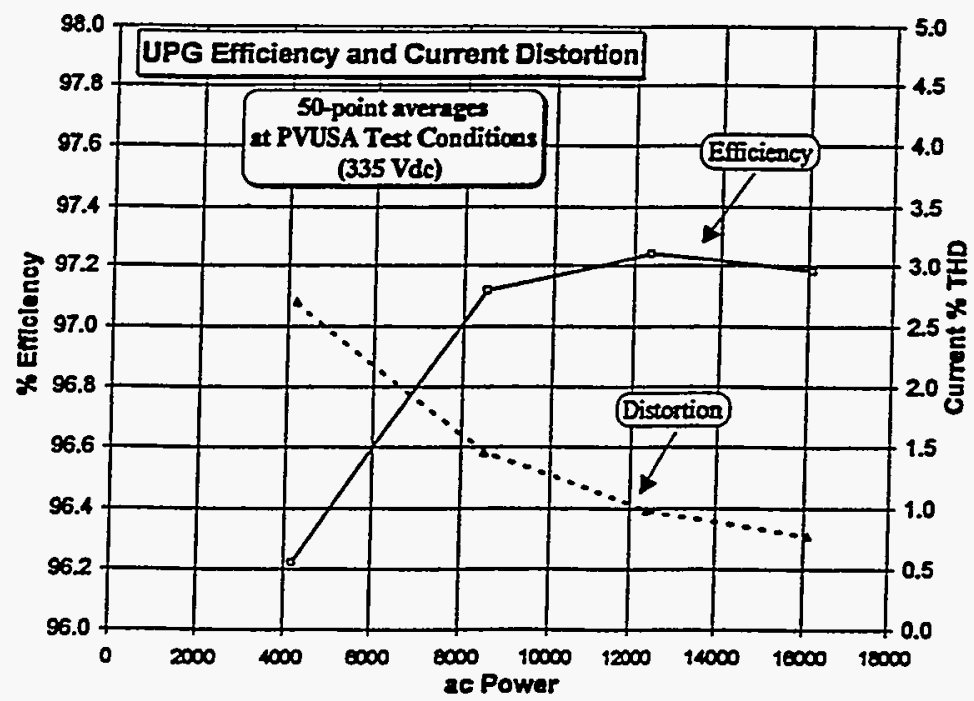

Figure 2. IPPU internal DC to AC inverter conversion efficiency. 


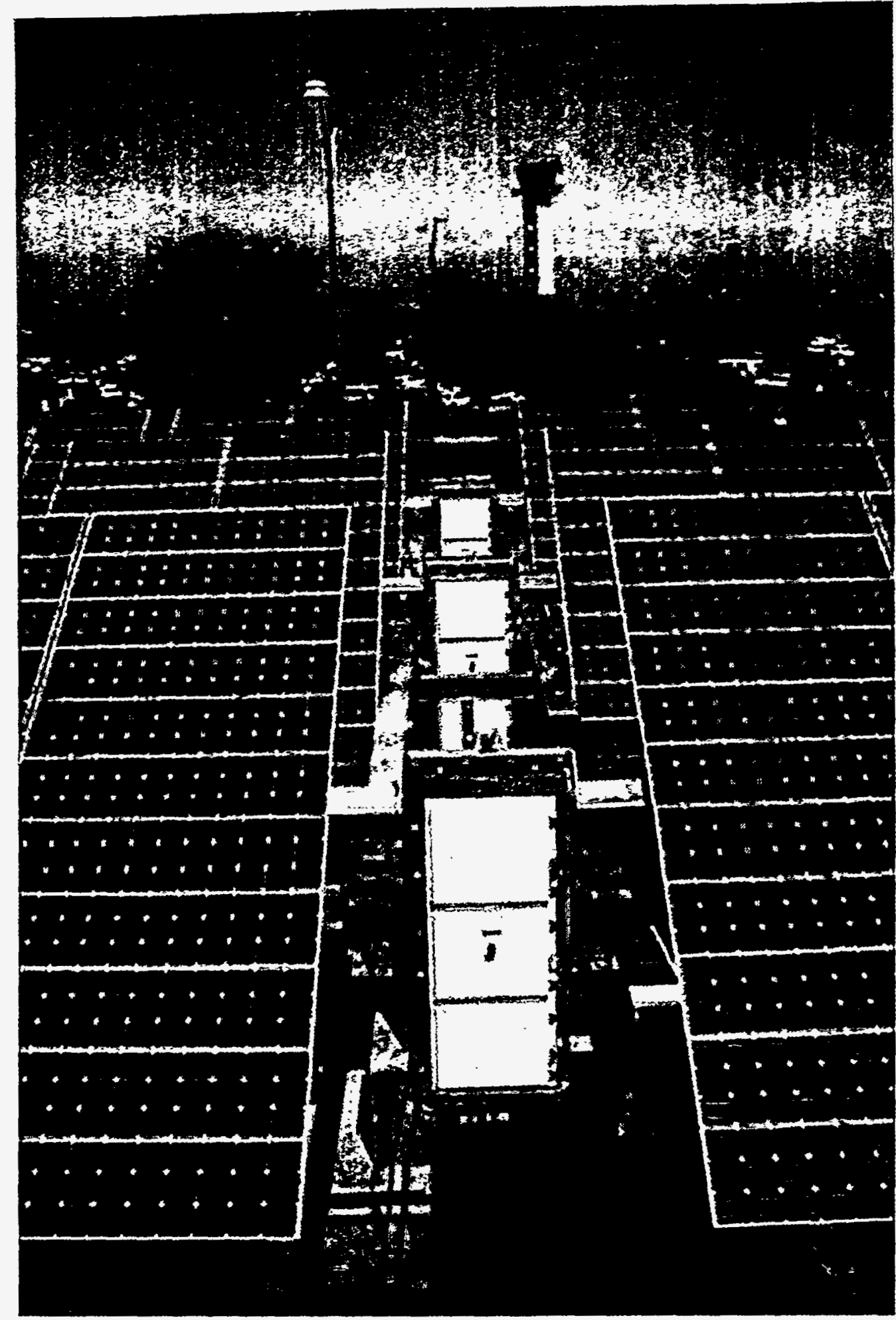

Figure 3. Initial Production Sub-Arrays at the Sacramento International Airport.

\section{References}

1. M. Stern, R. West, G. Fourer, W. Whalen, G. Duran, D. Metcalf, and K. Mackamul, "Development of A Low Cost Integrated 20 KW A.C. Solar Tracking Sub-Array For GridConnected PV Power System Applications." Phase 1 Annual Technical Report. NREL/SR-52023118. 


\subsection{PV Engineering and Reliability Project}




\title{
7.0 PV ENGINEERING AND RELIABILITY PROJECT-Introduction
}

\author{
Roland L. Hulstrom
}

\section{Overview and Scope}

This NREL project consisted of the following in-house tasks in FY 1997:

- Module Performance, Testing, and Technology Validation. Tom McMahon, Task Leader.

- Improving the Stability/Durability of Encapsulated Photovoltaic (PV) Cells and Minimodules. John Pern, Task Leader.

- Photovoltaic System Performance and Standards. Dick DeBlasio, Task Leader.

- Photovoltaic Solar Radiometric Measurements and Evaluation. Daryl Myers, Task Leader.

- Solar Resource Characterization. Dave Renné, Task Leader.

These in-house tasks were supplemented with the following subcontracts:

- The Design and Construction of a Custom-Made PV Encapsulant and Solar Cell Degradation Monitor. University of Colorado at Denver, Center for Environmental Sciences, Jeffrey A. Boon, Principal Investigator.

- Development of Test Methods and Procedures for Evaluation of PV Systems. NEOS Corporation, Terry Schyuler, Principal Investigator.

- PV Certification and Accreditation Management Support, PowerMark Corporation, Steve Chalmers, Executive Director.

- Management and Administration of the International Electrotechnical Commission/Technical Committee 82, Solar Energy Industries Association, Jerry Anderson, Principal Investigator.

The above listing of this project's tasks and subcontracted efforts reflects a scope of work that includes: photovoltaic module stability/durability; module and system technology validation and performance testing; national and international standards and codes; and solar radiation measurements, instrumentation, and resource characterization. All of these project elements are critical to the successful development and commercialization of the various photovoltaic technologies. NREL's in-house and subcontracted efforts are characterized by very collaborative and joint work with the PV industry, Sandia National Laboratories and other national laboratories, universities, and the National Aeronautics and Space Administration. 
The following sections present the key multiyear objectives of this project and the major accomplishments of FY 1997.

\section{Objectives}

- Develop and implement advanced photovoltaic module and system screening, durability, reliability, and performance characterization capabilities and test methods to help advance and validate the photovoltaic technology options, devices, and prototype products.

- In collaboration with the PV industry, conduct exploratory environmental stress and accelerated weathering tests on PV devices (minimodules and modules) to discover and characterize failure mechanisms and/or performance stability shortcomings.

- Help the PV industry investigate, document, and mitigate failure mechanisms and/or performance stability shortcomings of prototype products.

- Develop physical-empirical models capable of reliable characterizations and projections of PV module and/or system performance and durability.

- Develop and utilize solar radiation instrumentation, models, and databases, as required by PV technology development and deployment. Key areas include: radiometry (spectral and broadband); characterizing atmospheric effects on PV device performance and design; spectral solar radiation standards; temporal (1 minute to 30 years) and spatial (site-specific to regional) solar radiation resource databases and monitoring; and support of PV standardized performance testing and ratings.

- Support the PV industry's efforts to develop, maintain, and implement national and international PV module and/or system standards and codes (design, performance, safety, and quality).

\section{Major Accomplishments}

During FY 1997, the following new and expanded testing capabilities were completed and implemented:

- Outdoor Accelerated Test System

- High-Voltage Stress Test

- Performance and Energy Ratings Testbed

- Large-Scale Matrix Experiment

- High-Flux Solar Furnace (HFSF) Accelerated Exposure Testbed

- PV System Performance Characterization Flexible Testbed

- Multi-Pyranometer Array. 
All these are described in the following project task progress reports. They represent major advancements and contributions to the PV community.

- More than 72 PV modules (representing major PV technology options) were tested both indoors and outdoors, to help industry determine their performance and/or stability/durability characteristics.

- A set of NREL-developed formulations for PV module encapsulants (ethylene vinyl acetate) were shown to have superior photo-thermal stability (against ultraviolet [UV]-induced browning) and adhesion properties.

- HFSF accelerated sun exposure (100 to 2000 suns) tests of cerium-containing glass revealed that this type of glass undergoes a $1 \%$ to $2 \%$ decrease in total solar transmittance, due to a "red-shift" in the ultraviolet ( 350 to $400 \mathrm{~nm}$ ). Thus, the UV-filtering protection properties of the glass are improved.

- Outdoor performance monitoring of eight PV systems was continued and 16 professional engineering test reports were provided to seven PV manufacturers.

- More than 125 representatives of the PV community participated in the annual PV Performance and Reliability Workshop.

- A 150-page Radiometric Measurements Guide for Photovoltaic Performance Measurements (NREL TP-21774) guide was published.

- Color and monochrome contour maps of annual global horizontal and direct normal solar irradiance resource maps were prepared for each state in the United States. 


\section{PV Engineering and Reliability \\ In-House Research}


Title:

Organization: $\quad$ Center for Performance Engineering and Reliability

National Renewable Energy Laboratory, Golden, CO

Contributors: Minimodules

Improving the Stability/Durability of Encapsulated PV Cells and

\section{Objectives}

The FY 1997 task objectives were (1) to validate, by using encapsulated photovoltaic (PV) cells, the performance of the new NREL formulations of ethylene vinyl acetate (EVA)-based pottants that were superior to the standard A9918 and 15295 EVA; (2) to identify the optimal procedures and protocols for the durability testing of PV cells; (3) to identify, by PV technology, the specific compatibility/durability problems at material interfaces in minimodules/modules; (4) to identify new or improved encapsulation designs or processing, fabrication, and construction methods that will extend the module lifetime; and (5) to identify degradation and failure mechanisms that can be used for analytical evaluation of module service life.

\section{Technical.Approach}

In developing new EVA encapsulant formulations, new stabilizers and a curing agent different from those used in commercial EVA formulations, were selected and mixed with plain Elvax $150^{\mathrm{m} M}$ (base resin of unstabilized ethylene vinyl acetate) in solutions of cyclohexane. The resultant cast films were laminated with borosilicate glass plates and subjected to accelerated exposure testing (AET) that included exposure to an enhanced ultraviolet (UV) light source ( $\sim 5$ suns) at controlled temperatures and heating in an oven in the dark. The new formulations were ranked from AET against the existing commercial formulations, A9918 and 15295 EVA. The photothermal stability of the laminates was monitored periodically by fluorescence analysis, UVvis, and spectrocolorimetry before, during, and after testing.

An experimental design was devised for a large-scale study involving the preparation of hundreds of encapsulated solar cells with different superstrates, encapsulants, and substrates. The goal of the design is separate degradation mechanisms that are induced by UV, temperature, humidity, and material (superstrate and substrate) effects that may affect the optical, electrical, and mechanical properties of encapsulant, adhesion, metallic components, and solar cells. The degradation mechanisms that were identified can be used to assist in selecting new or modified materials options. The mechanisms and AET results will eventually be used to project module service lifetime. To prepare for the large-scale matrix experiment, details of lamination process control and spacers for various encapsulation configurations were carefully studied and fabricated. New Atlas Ci4000 weatherometers (WOMs) were installed and operated to determine the working parameters (i.e., light intensity, temperature, relative humidity, and on-off cycle control). To validate the performance of NREL-developed EVA formulations, a mini-extruder and related parts were acquired, installed, and operated to determine the extrusion conditions. 
To develop a noninvasive, nondestructive, analytical method for diagnosing PV module degradation in the field, electrochemical impedance spectroscopy (ECIS) was adopted for a feasibility study by using encapsulated $\mathrm{Si}$ solar cells (minimodules) of glass/EVA/c-Si cell/EVA/glass configuration. The cell samples were also tested under the above-described AET conditions. Light and dark I-V measurements and ECIS characterization were performed periodically before and during the study (on-going). The ECIS was also used to measure the series resistance for serially connected bare c-Si solar cells.

The intended purpose of combining the spectrophotometric, ECIS, and I-V measurements was to form a basis for quantifying solar cell degradation upon AET, then establish a data correlation methodology for the optical and electrical results.

In another feasibility study, NREL's unique high-flux solar furnace (HFSF) was employed to investigate the solarization of Ce-containing glasses, which have been used in recent years by some PV manufacturers who initially were unaware of the UV-shielding effect for EVA encapsulants. An earlier study of the Ce-glasses showed their UV-filtering effects greatly reduced the browning rate of commercial EVA encapsulants. The solarization study is intended to determine if the glasses will lose $\mathrm{Ce}^{3+}$ from the highly accelerated light exposure at controlled temperatures and solar fluxes.

On-site supervision of a subcontract was continued for fabricating a prototype, portable spectrophotometric and I-V analyzer (a degradation monitor) for solar cells. The subcontract was an effort to reduce to practice an invention disclosure (NREL IR\#95-10) for such a unit. The subcontractor, the University of Colorado at Denver, began the work in April 1996 with an 18month completion time.

\section{Results}

1. Development of Encapsulant Materials and Encapsulation Methods. The milestone has been met, and validation work is continuing.

1a). New EVA Formulations. A set of NREL-developed formulations of EVA encapsulants was tested and shows highly improved photothermal stability against UV-induced browning and better adhesion after prolonged exposure as compared with the commercial EVA formulations (A9918 and 15295). The new formulations also eliminate or minimize the bubbling problems upon fast curing that may occur with EVA 15295. An oral presentation for the IR\#96-28 was made to NREL's Patent Management Committee and a patent àpplication was filed on Nov. 24, 1997, as a continuation in part (CIP) to NREL IR\#95-50. The new EVA formulations are now available for technology transfer (e.g., a possible licensing agreement was negotiated with Richmond Technologies).

1b). Alternative Encapsulation Materials and Methods. The results of these studies were published. A commercial acrylic-based adhesive film (3M's VHB) was studied that shows high photostability and excellent adhesion. Encapsulation methods that use a polymer superstrate, EVA or VHB encapsulant, and a polymer substrate, which can offer lightweight cell laminates, were developed. Iowa Thin Film asked about the VHB adhesive films and Spire adopted the use of the lightweight polymer/polymer encapsulation methods. More PV companies inquired about the adhesion issues of using Tefzel films as superstrate. In an effort to seek an alternative 
encapsulation process to simplify and reduce costs of the current industrial lamination and curing procedures that require using temperatures up to $145^{\circ} \mathrm{C}$ to $150^{\circ} \mathrm{C}$, a brief study was performed with some UV curing agents. Because of a low level of activity, this study has not yet been successful.

1c). Design of Experiments and Preparation for a Large-Scale Matrix Experiment. Several experiments were designed, one of which is a major matrix experiment. In the matrix experiment design, more than 400 samples of encapsulated Si cells with various encapsulation materials and methods will be prepared and tested. The desired encapsulation materials and monocrystalline $\mathrm{Si}$ solar cells for the matrix experiment were being acquired. Preparation for the experiments is continuing.

1d). New Testing Equipment and Film Preparation Apparatus. We purchased, installed, and made operational two Atlas Ci4000 WOMs, two Oriel solar simulators, and one mini-extruder. The WOMs and solar simulators are to be used for the matrix experiment and the mini-extruder is to be used to prepare extruded films of NREL EVA formulations.

2. Feasibility Studies. The milestones for the two feasibility studies below have been met and work is continuing.

2a). Solarization Study of Ce-Glass Using NREL's High-Flux Solar Furnace. A literature search, an experimental design, and special sample exposure chambers were made. Two HFSF exposure experiments were conducted: the first experiment was at 100 suns for $20 \mathrm{~h}$ at $100^{\circ} \mathrm{C}$ (equivalent to $\sim 1$ year at 1 sun) and the second was at 2000 suns for $1 \mathrm{~h}$ at $40^{\circ}-50^{\circ} \mathrm{C}$ (equivalent to $\sim 1$ year at 1 sun). The results showed a $1 \%-2 \%$ decrease in transmittance by a small red shift in the $\sim 350-400 \mathrm{~nm}$ range, which enhances the UV-filtering protection (for EVA), and a significant loss in fluorescence intensity. The work demonstrated the feasibility and usefulness of HFSF for highly accelerated exposures, which can greatly reduce the time for studying PV materials stability.

2b). Using Electrochemical Impedance Spectroscopy as a Noninvasive, Nondestructive Analytical Method. Two separate experiments were conducted, and the ECIS measurements were performed. (i). With glass/EVA-encapsulated c-Si solar cells, we demonstrated that ECIS can be used to detect changes in the electrical properties of encapsulated c-Si solar cells after AET treatments. The results also demonstrate that a simple equivalent circuit is adequate to model the glass/EVA-encapsulated c-Si solar cell. More systematic experiments were planned for FY 1998. The ECIS data were to be correlated with spectroscopic and I-V data. (ii). With serially interconnected c-Si solar cells, the results of ECIS measurements showed that the series resistance can be determined. Work is planned for ECIS analysis of "induced damage" to the cells.

3. Subcontracts. One subcontract was completed and a second one is continuing.

3a). PV Module Degradation-EVA Pore Volume (Contract No.: XAX-4-13471-01). The contractor is Pennsylvania State University (P.I.: Prof. D. L. Allara). The subcontract was completed with a final report submitted in January 1997.

3b). A Prototype Portable Custom-Made PV Encapsulant and Solar Cell Degradation Monitor (i.e., A Spectrophotometric and I-V Analyzer. Contract No.: XAX-6-14454-01). The contractor is the University of Colorado at Denver (P.I.: J. Boon). The work is a "reduce-topractice" of NREL IR\#95-10. The work is continuing with about two-thirds of the efforts completed. The PI has regularly delivered monthly progress reports. Regular on-site visits to 
examine the progress were conducted by the NREL technical monitor. A 6-month, no-cost extension has been awarded in order to resolve some technical problems and ensure the system can perform correct spectrophotometric and I-V measurements in both the stationary and scan modes, and data management and manipulations.

4. Partnership with the PV Community: We assisted several PV manufacturers in the selection of the better-performing, commercial EVA formulation EVA 15295, provided technical consultations with better EVA thermal-processing conditions for module encapsulation, and responded to several requests for various analyses and AET tests. The requested tests were (1) Sovlux (Russian) EVA for gel content and UVA analysis work for others (WFO); (2) Solarex EVA for gel content analysis cooperative research and development agreement (CRADA); (3) Evergreen Solar's new encapsulant for transmittance measurements and AET Photovoltaic Manufacturing Technology (PVMaT); (4) Iowa Thin Films' a-Si minimodules for AET; (5) Spire's specially encapsulated c-Si cells for AET; and (6) EVA-browned PV modules from Ben Gurion University, Israel, for optical and electrical characterizations. We also engaged in discussions of advanced encapsulation materials and methods for CIS modules (OCLI/NREL) and other emerging thin-film technologies, e.g., $\mathrm{CdTe}, \mathrm{a}-\mathrm{Si}, \mathrm{c}-\mathrm{Si}$, and provided several tours for various domestic and international visitors to the Solar Energy Research Facility/E216 and the Outdoor Test Facility site.

\section{Conclusions}

We successfully met all three milestones, which are: (1) a set of NREL-developed EVA formulations (IR\#96-28) shows greatly improved photothermal stability against browning as compared with the existing commercial EVA formulations. A patent application was filed as a $\mathrm{CIP}$ to an earlier patent application for IR\#95-50. Our work can benefit PV manufacturers directly by providing EVA encapsulants with improved performance and a longer module service life. (2) The feasibility of using electrochemical impedance spectroscopy as a noninvasive, nondestructive diagnostic method for analyzing solar cell degradation has been established. (3) The feasibility study of using the solar furnace to study solarization of cerium-containing glasses has been conclusive.

We also enhanced and/or expanded NREL's AET testing and analytical capabilities by purchasing and installing two weatherometers, two solar simulators, and a mini-extruder. The portability of hybrid analytical instrumentation will be realized by the completion of a subcontract effort to fabricate a degradation monitor that will have the capabilities of performing transmittance/ absorption, fluorescence, reflectance (for color indices), and I-V measurements in both the scanning and stationary modes.

We promoted technology transfer to the PV industry and community by direct technical assistance, consultations, and various analyses; AET tests were also conducted for various samples or encapsulated solar cells/minimodules submitted by PV companies. Specific technical information or processes for the alternative encapsulation materials and methods we studied and published were acquired or adopted. Indirect technology transfer was realized through technical publications, presentations, and person-to-person interactions at conferences. In addition, task team members provided various services for professional societies and journals. 


\section{Publications and Conference Presentations}

Task members published 12 papers and made 12 presentations in FY 1997, in which two task members (A. Czanderna and J. Pern) each gave 4 invited presentations. Ten monthly or bimonthly reports and PV Weekly Highlights were prepared. The major papers published and/or presented by the task members for the task are given below.

1. Pern, F.J.; Glick, S.H. (1997). "Improved Photostability of NREL-Developed EVA Pottant Formulations for PV Module Encapsulation." Presented at the 26th IEEE PVSC, September 23-October 3, 1997, Anaheim, California (to be published by the Institute of Electrical and Electronics Engineers [IEEE]).

2. King, D.E.; Pern, F.J.; Pitts, J.R.; Bingham, C.E.; Czanderna, A.W. (1997). "Optical Changes in Cerium-Containing Glass as a Result of Accelerated Exposure Testing." Presented at the 26th IEEE PVSC, September 23-October 3, 1997, Anaheim, California (to be published by IEEE).

3. Pern, F.J. (1997). "Ethylene-Vinyl Acetate (EVA) Encapsulants for PV Modules: Degradation and Discoloration Mechanisms and Formulation Modifications for Improved Photostability." Conference Proceeding of 19th Annual International Conference on Advances in the Stabilization and Degradation of Polymers, June 9-11, 1997, Lucerne, Switzerland, pp. 189208 (Invited presentation).

4. Pern, F.J; Glick, S.H. (1997). "Improved Performance of Polymeric Encapsulants against UVInduced Discoloration Characterized by Spectrophotometric Analyses." Conference Proceeding Abstracts, No. 176, 39th Rocky Mountain Conference on Analytical Chemistry, August 3-7, 1997, Denver, CO.

5. Pern, F.J. (1997). "Development of New EVA Formulations for Improved Performance at NREL." No. 394, pp. 795-810. NREL/TP-510-22137. AIP Conference Proceeding for 14th NREL/SNL PV Program Review Meeting. Edited by C.E. Witt, M. Al-Jassim, and J. M. Gee. (? location of AIP:)American Institute of Physics.

6. Pern, F.J.; Glick, S.H. (1997). "A Study of Various Encapsulation Schemes for c-Si Solar Cells with EVA Encapsulants." NREL/TP510-22157. AIP Conference Proceeding for 14th NREL/SNL PV Program Review Meeting. Edited by C.E. Witt, M. Al-Jassim, and J. M. Gee. (? location of AIP:)American Institute of Physics. pp. 811-824.

7. Czanderna, A.W.; Jorgensen, G.J. (1997). "Service Lifetime Prediction for Encapsulated PV Cells/Minimodules." AIP Conference Proceeding for 14th NREL/SNL PV Program Review Meeting. Edited by C.E. Witt, M. Al-Jassim, and J. M. Gee. (? location of AIP:)American Institute of Physics. pp. 295-311. 
8. Czanderna, A.W. (1997). "Reliability and Lifetime Issues for New Photovoltaic Technologies." in AIP Conference Proceeding No. 404 for the Conference on FutureGeneration Photovoltaic Technologies. Edited by R. McConnell. Woodbury, NY: American Institute of Physics; pp. 55-69.

9. Pern, F.J.; Glick, S.H. (1996). "Thermal Processing of EVA Encapsulants and Effects of Formulation Additives." 25th IEEE PVSC, May 13-17, 1996, Washington, D.C., pp. 12511254.

10. Pern, F.J.; Eisgruber, I.; Micheels, R.H. (1996). "Spectroscopic, Scanning Laser OBIC, and I-V/QE Characterizations of Browned EVA Solar Cells." 25th IEEE PVSC, May 13-17, 1996, Washington, D.C., pp. 1255-1258.

11. Czanderna, A.W.; Pern, F.J. (1996). "Estimating Service Lifetimes of a Polymer Encapsulant for PV Modules from Accelerated Testing." 25th IEEE PVSC, May 13-17, 1996, Washington, D.C., pp. 1219-1222.

12. Pern, F.J.; Glick, S.H.; Czanderna, A.W. (1996). "EVA Encapsulants for PV Modules: Reliability Issues and Current R\&D Status at NREL." Proceedings of World Renewable Energy Congress-IV. Edited by A.A.M. Sayigh. Vol. I. June 15-21, 1996, Denver, CO. (?location of publisher:) Pergamon; pp. 367-370.

13. Czanderna, A.W.; Pern, F.J. (1996). "Encapsulation of PV Modules Using Ethylene Vinyl Acetate Copolymer as a Pottant: A Critical Review." Solar Energy Materials and Solar Cells; 43, pp. 101-183.

14. Pern, F.J. (1996). "Factors That Affect the EVA Encapsulant Discoloration Rate Upon Accelerated Exposure." Solor Energy Materials and Solar Cells; 41/42, pp. 587-615.

15. Jorgensen, G.; Pern, J.; Kelly, S.; Czanderna, A.W.; Schissel, P. (1997). "Polymers for Solar Energy Devices." Edited by R. Arshady, "Desk Reference of Functional Polymers: Syntheses and Applications." Washington, D.C.: American Chemical Society, pp. 567-588. 
Organization: $\quad$ National Renewable Energy Laboratory, Golden, CO

Contributors: $\quad$ Tom McMahon (Task Leader), Tom Basso, Joe del Cueto, Carl Osterwald, Jim Pruett, Dave Trudell

\section{Objectives}

We have the following objectives: 1 ) design, develop, and perform tests (performance, stress, and environmental) on photovoltaic (PV) modules and interact with the PV industry (manufacturers, test facilities, etc.), with the intent of improving PV modules for performance, durability, and reliability; 2) perform PV module screening and exploratory tests on NREL/DOE subcontract deliverables, research and development (R\&D) prototypes, and commercially available modules; 3 ) work with PV manufacturers to help evaluate long-term performance, durability, and reliability, as well as investigate failure mechanisms and possible solutions to design flaws; 4) collaborate in the validation and development of PV module energy-rating methodologies as an alternative to the module efficiency standards at the SRCC (Solar Rating and Certification Corporation); and 5) develop and maintain a PV module (and array) reliability database, for use by the PV community (especially manufacturers) to improve the design and long-term performance of their products.

\section{Technical Approach}

Our principal research task is to perform work in the following three areas:

\section{(i) Performance, Reliability, and Stability Testing:}

We will maintain a database on the performance and reliability of flat-plate PV technologies, to monitor module and array stability on all thin-film and advanced PV technologies. Long-term outdoor exposure tests and indoor controlled light-soaking experiments are conducted to address the issue of module stability. Module performance is measured periodically during the tests. We also monitor and evaluate the outdoor performance of PV modules and modules within small systems (approximately $1 \mathrm{kWp}$ each) at NREL's PV Outdoor Test Site. This activity focuses on emerging PV module technology evaluation/validation and flat-plate module development and failure analysis. Photovoltaic and data acquisition systems are designed and deployed at this test site to conduct in-situ technical evaluations of the PV module array and performance and reliability.

This testing is being conducted at NREL to assist PV manufacturers (e.g., United Solar Systems Corporation [USSC], Energy Conversion Devices [ECD], Solarex Corporation, Siemens, and Solar Cells, Inc. [SCI]) in evaluating module/array performance and identifying technical issues under actual operating conditions. This independently tested data is then provided to the PV community. A major aspect of this collaborative activity at NREL is the technical exchange of results obtained from these evaluations with manufacturers and industry. For example, these collaborations provided major technical support to the Photovoltaic Manufacturing Technology project in FY 1996, which is expected to increase in FY 1997. Technical validation of modules and arrays provided under these subcontracts is planned to be performed at the Outdoor Test Facility (OTF). 
We also researched performance evaluation methodologies for modules. These studies included seasonal performance, spectral measurements, and techniques for evaluating instantaneous power output and energy production of the modules. We also developed a method to rate PV modules based on energy, which we validated this year.

In the performance area, we continue to support the development of national and international PV standards and codes by participating in the Institute of Electrical and Electronics Engineers (IEEE) Standards Coordinating Committee 21 (SCC)21, the International Electrotechnical Commission (IEC) Technical Committee (TC)/82, (USTAG), the International Electrotechnical Commission (IEC), the American Society for Testing and Materials (ASTM), the National Electrical Code (NEC), and the Underwriters Laboratories (UL) standards development committees, as appropriate.

\section{(ii) Accelerated and Environmental Stress Testing}

The objective of module stress testing is to obtain information about the ability of module designs to withstand known failure and degradation mechanisms that occur when modules are deployed outdoors for long periods of time. Accelerated stress testing attempts to increase the rate of degradation so that results are obtained in shorter time periods, and environmental stress testing is intended to simulate outdoor conditions within the laboratory. Although some of these tests are commonly referred to as qualification tests, NREL does not intend to qualify designs through a formal testing program. Specific tests available include accelerated outdoor and indoor ultraviolet (UV) weathering, temperature and humidity cycling, high-voltage stress, and electrical and mechanical integrity tests.

The objective of stress testing is to discover potential problems with module designs that may not yet be commercially available such as prototypes and subcontract deliverables. To accomplish this objective, the stress testing is closely tied to failure-analysis capabilities within the task. A secondary objective is to develop and validate new or longer-term test methods. The results are intended to help improve the reliability and lifetime of PV modules.

Facilities that are used for accelerated and environmental stress testing at the OTF include several environmental chambers, both with and without light exposure, wet and dry electrical insulation tests, static and dynamic mechanical loading equipment, accelerated UV weathering using the Outdoor Accelerated Test System (OATS), and a test bed for long-duration, high-voltage stress testing.

(iii) Module-Failure Analysis

We will diagnose the seriousness of cell failure within a module (nonintrusively and destructively), determine cause of failure, and recommend corrective action. Nondestructive analysis includes the use of basic electrical-measurement equipment, a new, nonintrusive cell shunt-resistance measurement, and basic photographic equipment with macro and micro lenses. Hot-spot tests can be performed by applying reverse current through series-connected cells while observing localized resistive heating with the use of thermochromic paper. We evaluated the electrical behavior of modules and cells using the PSPICE electronic-circuit simulator and the Analysis of Microelectronic 
and Photonic Structures (AMPS) device simulator. Destructive analyses include de-encapsulation (coring) and chemical-compositional analysis.

We will guide and participate in the work of the Thin Film Partnership's CdTe Back-Contact Stability Team in developing and evaluating the stability of different back-contact recipes. We will apply accelerated screening tests to different $\mathrm{CdTe}$ back-contact recipes and standard I-V, as well as use improvised electrical characterization to evaluate the back-contact integrity. These screening tests are designed to develop a procedure for module-life prediction. Such a procedure can be used as a model for other module technologies.

A reliability database will be developed and maintained. PV system, module, and component reliability data are obtained from operating systems, demonstration or prototype units, qualification and exploratory tests, research results, dedicated studies, and, from the literature. A formalized inspection and characterization program will facilitate both the database and failure diagnoses by completely characterizing modules prior to deployment. Draft forms (Module Incoming Inspection Form and Photovoltaic Technology Failure Reporting and Corrective Action System Form) will similarly be developed and promoted. Further, the database will facilitate information sharing to help validate technology reliability, understand the seriousness of anomalies, PV failure modes, mechanisms, and the causes of failures, and to provide reliability sources (e.g., citations and contacts).

\section{Progress on Research Tasks}

\section{(i) Performance, Reliability, and Stability Testing:}

More than 50 modules of various technologies are being exposed outdoors. Data collected on these modules are used to show performance and reliability over time, and will be sent to each PV module manufacturer and program leader. Stability tests were initiated and will be ongoing for the newly deployed modules received from USSC. Of four triple-junction a-Si modules rated by the manufacturer at 32 and 64 watts (W), three are deployed outside and one is deployed inside. The latter was light-soaked and tested inside; the performance of this module at the 400-hour mark was about $8 \%$ aperture-area efficiency. Baseline tests on all these modules revealed performance at nominally $9 \%$ efficiency. Module Test Report MTR97USSC.001 details the stability tests and performance; final aperture-area efficiency values for these modules ranged between $7.6 \%$ and $7.9 \%$ after $1000 \mathrm{kWh} / \mathrm{m}^{2}$ (a.k.a. 1000 hours @ 1 sun) of exposure. Tests were conducted in and out of doors. After 1000 hours of exposure outdoors, the stabilized power from the US-64 model modules tested at standard conditions rated at $70.7 \mathrm{~W}$.

Two modules were received from the start-up company Advanced System Manufacturing (ASM). A stability test schedule was submitted, approved, and implemented for the modules. ASM manufactures single-junction a-Si modules out of the Trenton, New Jersey, plant, which Advanced Photovoltaic Systems (APS) used for production for Photovoltaics for Utility-Scale Applications.

Tests conducted on eight copper indium diselenide (CIS) modules were completed and reported to Siemens Solar Industries. These CIS modules were erected between 1988 and 1995 and have been exposed to outdoor conditions since deployment. With one exception, the CIS modules have 
exhibited stable averaged performance of between $7.4 \%$ and $9.7 \%$ in aperture-area efficiency. One module appears to be losing performance at a rate of 1-2 relative- $\%$ per year, apparently through fillfactor and series-resistance degradation.

Light-soak experiment 4 continued for 14 different a-Si modules at low temperatures, which ranged from $10^{\circ}-40^{\circ} \mathrm{C}$ back-of-the-module temperatures thought representative of exposure during winter. The current program for this test uses lower module temperatures for the experiment as a means of accelerating and getting to the steady-state performance of these modules. Analysis of data reveals that even after 1000 hours of exposure there is no true stabilization, but rather slow degradation.

On the bright side, winter temperatures within the range cited never exceeded 200-300 hours. Furthermore, after this 1000-hour light soak at low temperatures, exposure for $\sim 200-250$ hours at $40^{\circ}-60^{\circ} \mathrm{C}$ brought back all module efficiencies to pre-1000-hour soak values. Currently, another 1000 hours of low-temperature light soak is in progress.

Performance and Energy Ratings Test (PERT) bed: Currently, 15 modules are deployed on the first PERT and are being tested at 30-minute intervals under all-outdoor prevailing conditions. The I-V characteristics, module temperatures, and irradiance data are sampled by a data acquisition system and stored. . In the spotlight was a tandem-junction a-Si module manufactured by Solarex and deployed on the PERT rack since October 1995. Analysis of this module's performance was conducted and reported to Solarex. The performance for this module was unfortunately losing ground against time at a rate of 5 relative-\% per year. Results for two single-junction APS modules have also been presented (del Cueto and McMahon 1997).

An expanded second data acquisition system for PERT II was finalized and chartered with seven PV modules representative of diverse technologies: four c-Si modules, two CIS modules, and one a-Si module. Currently, there is still space for eight more modules.

A study was completed comparing the influence of measured and modeled spectra on five different types of PV devices. This study is helping validate the use of the SEDES2 spectral model for use in module energy ratings. We continue to monitor 15 modules for energy production on the roof of the OTF.

\section{(ii) Accelerated and Environmental Stress Testing}

During the past two months we have completed the following portions of the IEEE 1262 environmental stress-testing sequence on the ECD/Russian a-Si modules:

- Hot-spot endurance

- 50 and 200 thermal cycles

- 10 humidity-freeze cycles

Currently in progress is the 1000-h damp-heat test. All testing of the modules should be finished by the end of 1997 and a final report will be available shortly thereafter. 
Ten Siemens 5-W CuIn $(\mathrm{Ga}) \mathrm{Se}_{2}$ modules were received and are currently undergoing initial I-V measurements. These modules are scheduled for the IEEE 1262 test sequence.

UV exposure testing on the Iowa Thin Film Technologies modules was completed using fluorescent UVA and UVB lamps and a test report was issued. For the ECD/Russian a-Si module environmental stress-testing sequence, outdoor-exposure testing was completed and UV exposure testing was initiated.

Accelerated Testing: i) We completed improved initial environmental stress-test sequences on $\mathrm{ECD} / \mathrm{Kvant}$ triple-junction a-Si modules. ii) We improved the cooling system of the Atlas XR260 solar weathering chamber. We modified lamps to prevent them from shutting down during operation. iii) We started planning for extended-duration UV weathering experiments of PV modules.

OATS testing of photovoltaic modules started on November 22, 1997. As more experience is gained, the significance of OATS as an effective accelerated stress-testing tool for reliability studies will be determined. Degradation of encapsulants, polymer components, and the PV devices/ components themselves are the focus of study. That testing will be compared to other methods, including those using natural or simulated environmental conditions. Existing ASTM standards were used as guidelines to establish the OATS test plan. Currently, 14 PV modules from four manufacturers are being tested under the OATS plan. The modules are being exposed as two sets of seven PV module models (one module per model per set). One set is being exposed under full OATS concentration (nominally $3 \mathrm{X}$ ), and the second set is being exposed at one-sun tracking conditions. The PV modules are at fixed-resistive load and prevailing environmental conditions. In-situ PV module current and voltage measurements are recorded with the values varying according to irradiance, temperature, and general weather conditions. The OATS testing will be interrupted at intervals based on measured cumulative UV exposure. At these intervals the modules will be removed, standard I-V curves taken, and visual inspections made. As deemed appropriate at each interval, additional performance parameters might be measured. Modules passing the tests at each interval will be returned to OATS for continued exposure.

Environmental Stress Testing: In April, a second set of improved ECD/Russian a-Si modules was delivered to NREL for further testing. We completed the partial IEEE 1262 environmental stresstesting sequence, including the final performance characterization. With the exception of problems caused by the unintentional use of a galvanized-steel backsheet, these modules performed much better than the modules tested last year. A final report will be available in early 1998.

Iowa Thin Film Technologies delivered three $1^{\prime} \times 1^{\prime}$ a-Si modules that will undergo UV exposure testing using fluorescent UVA and UVB lamps as soon as initial characterization testing is completed. Construction of a new facility to perform the IEEE 1262 module twist test was initiated. The new twist-test facility should be usable for modules as large as $4^{\prime} \mathrm{x} 6$ '.

AC Module Test Bed: The construction of the indoor AC module test bed has been completed. The data acquisition system was programmed and is operational. The first experiment used a complete AC module package (inverter connected to PV module) developed by Ascension Technology in a modified humidity-freeze test. This test cycles the module between $70^{\circ} \mathrm{C}$ and $75 \%$ humidity for 
20 hours and then freezes the module at $-40^{\circ} \mathrm{C}$ for one hour. This test will help determine whether the AC modules have adequate resistance to the detrimental effects of humidity, condensation, and freezing.

The high-voltage stress test (HVST) inaugurates round-the-clock operation. Modules can be biased at as much as 3000 volts using either and both positive and negative polarities. Leakage currents on the order of 1-5 nanoamps can be discerned. Meanwhile, a data acquisition system performs continuous monitoring and archiving of data. Initial functionality tests on four modules erected and biased at voltages of as much as 2200 volts showed leakage currents at approximately 40 50 nanoamps. We carried out the test under a Safe Work Permit. The safe operating procedure for the HVST was finalized and approved by the Environment, Safety, and Health Office. To date, two commercially available Siemens c-Si modules have been deployed for long-term testing. Plans for Solarex a-Si and other thin-film modules are being made.

\section{(iii) Module Failure Analysis}

The Solarex cooperative research and development agreement has commenced with initial progress occurring in the area of shunt reduction. As a result of our collaboration, Solarex has found that the addition of a thin proprietary layer has reduced shunting. On another front, satisfactory progress is being made in finding the cause of high-voltage stress failures.

In addition to guiding the Reliability Team of the CdTe national teaming effort, we conducted the initial accelerated screening tests on two CdTe cells with different back-contact recipes. A failure mode of great concern for CdTe cells is the back contact becoming non-ohmic, forming a reverse diode. After 60 hours at $90^{\circ} \mathrm{C}$ we found a little change in one cell, but in the other cell we found an enormous reverse diode forming with degradation in both the light and dark I-Vs. Along with light and dark I-V analysis, we used a new internal-resistance measurement to determine that the internal series resistance of both cells increased by $40 \%$; however, because the starting values were only several $10^{-3} \mathrm{ohm} \mathrm{cm}^{2}$, it is really the formation of the reverse diode that is of concern here. Additionally, a preliminary test of seven hours at $90^{\circ} \mathrm{C}$ in the dark on a $\mathrm{CdTe}$ module decreased the power out by $6.6 \%$.

A subteam of the Reliability Team of the CdTe national teaming effort was formed to develop a module-life prediction model. We discussed which stress or combination of stresses will accelerate failure modes in the real world. Out of all the test-plan elements, the team finally agreed on 11 test conditions. Each test condition will require four test objects (probably cells and minimodules). UV sensitivity and interconnect integrity will be tested on specially designed devices. SCI carried out their own testing on their own devices and reported back to the team in August 1997. We then assessed the suitability of these tests for 1) exposing and documenting failure mechanisms, 2) the relevance of observed effects of the stress conditions to failure mechanisms observed in the field, and 3 ) the credibility of the accelerated life-test factors developed.

When conducting accelerated screening tests on CdTe cells or modules, we must consider which acceleration parameters to use and how high to push them. The CdTe Stability Sub-Team decided that, among other combinations of stresses, acceleration temperatures should be as high as $100^{\circ} \mathrm{C}$ during activation analysis. 
The Reliability Team of the CdTe national teaming effort has gone through the first phase of stressing cells made with many different back-contact recipes. The initial test with the most number of samples was conducted at Colorado State University (CSU). Various back contacts were made on Golden Photon, Inc., or SCI CdTe substrates finished through the $\mathrm{CdCl}$ treatment. The times to failure vary from hours to months, with back-barrier formation being the most common failure mode. CSU has since stressed cells at two other temperatures, e.g., $85^{\circ}$ and $65^{\circ} \mathrm{C}$, to define an activation energy for the failure mechanism and to see if it is operational at use temperatures. Usetemperature histograms were gathered for four sites. We are looking for the source of this failure mode, i.e., the reverse diode, and the two other less common failure modes identified so far. Our next meeting will be at the Florida Solar Energy Center on January 15, 1998.

The NREL reliability database (module performance and reliability task) was assembled and data is being entered on an ongoing basis. Preliminarily, it was found that in their early life ( $<3.5$ years) modules have failure rates ranging from 0.4 to 115 failures per million operational days (or $0.015 \%$ to $4.20 \%$ per year). That range, along with the high statistical uncertainty due to the limited data experience, resulted in inconclusive curve fitting of a reliability failure distribution for the modules. The NREL working draft of the reliability database (module performance and reliability task) was presented internally. Comments will be incorporated and the database will be developed further in collaboration with Sandia and the Southwest Technology Development Institute.

One of the difficulties in obtaining module reliability data from the field is the problem of spotting poorly performing modules in large systems, or for that matter, any system. Even at the OTF, where systems are monitored continuously, only modules that open-circuit can be spotted. If the goal is to define accelerated stress tests to accurately test products for real-world conditions, then we must do better in gathering field data. One method that could work is to short-circuit a system on the morning of the day that a search is to be made and use an infrared camera to spot poor performers. Another, perhaps new, method would be to scan a modulated light source around the field. In daylight the poor performers will be the ones producing an AC signal. At the module level we are able to demonstrate that a poor cell in a series of cells within a module can easily be spotted with the module under full-sun illumination by pointing a 1-MW modulated $\mathrm{HeNe}$ from cell to cell. We are thinking of ways to extend this idea to spotting modules within a system.

\section{Publications}

Osterwald, Carl R. (April 1997). "Photovoltaics as Alternative Energy. Sources." ASTM Standardization News; Vol. 25, No. 4, pp. 20-25 (invited review article).

Hegedus, S.S.; Albright, S.; Jeffrey, F.; McMahon, T.J.; Wiedeman, S. (1997). "Substrates, Contacts, and Monolithic Integration." Progress in Photovoltaics: Research and Applications; Vol. 5, p. 365.

del Cueto, J.A.; McMahon, T.J. (1997). "Performance of Single-Junction a-Si Modules under Varying Conditions in the Field." Proceedings of the 26th IEEE PV Specialists Conference; September 29-October 3, 1997, Anaheim, California. 
McMahon, T.J.; von Roedern, B. (1997). "Effect of Light Intensity on Current Collection in ThinFilm Solar Cells." Proceedings of the 26th IEEE PV Specialists Conference; September 29October 3, 1997, Anaheim, California.

Osterwald, C.R.; Anevsky, S.; Barua, A.K. et al. (1997). "Thê World Photovoltaic Scale: An International Reference Cell Calibration Program." Proceedings of the 26th IEEE PV Specialists Conference; September 29-October 3, 1997, Anaheim, California. 
Title:

Organization:

Contributors:

\section{Photovoltaic Solar Radiometric Measurements and Evaluation}

Center for Renewable Energy Resources and National Center for Photovoltaics National Renewable Energy Laboratory, Golden, CO

D.R. Myers, Senior Scientist; T.W. Cánnon, Senior Engineer, T.L. Stoffel, Career Scientist; I. Reda, Staff Engineer

\section{Objectives}

The Photovoltaic (PV) Solar Radiometric Measurements and Evaluation Task supports module and system performance, reliability, and engineering activities within the National Center for Photovoltaics (NCPV). We develop, acquire, and transfer technical information to PV industry and NREL PV engineers and researchers needing radiometric measurements, instrumentation, analysis, and indoor and outdoor solar radiation data and measurements.

\section{Technical Approach}

Broadband radiometric calibrations are conducted at the Solar Radiation Research Laboratory (SRRL). Spectral and narrowband instrument calibrations are conducted in NREL's Optical Metrology Laboratory. We maintain National Institute of Standards and Technology (NIST) optical radiation calibration sources and absolute-cavity radiometers traceable to the World Meteorological Organization (WMO) World Radiometric Reference (WRR). Measurement needs of NREL internal and external PV program participants are addressed as requested. Technical interaction with the Optical Technology Division of NIST, American Society for Testing and Materials (ASTM), Institute of Electrical and Electronics Engineers (IEEE), the Council for Optical Radiation Measurements (CORM), instrument manufacturers, and the optical radiation community results in improved and specialized optical-radiation measurements for PV applications. Dr. Cannon is on the CORM board of directors, and Myers and Cannon are members of ASTM Committee E-44 on Solar, Thermal, and Alternative Energy Sources.

\section{Results}

- In April 1997, we published the 150-page "Radiometric Measurements Guide for Photovoltaic Performance Measurements" (NREL TP-21774) [1]. The guide describes radiometric techniques, issues, procedures, and standards used by NREL for PV research, development, and testing.

- The task quantified sources of bias and random error in NREL spectral calibrations. Intercomparisons of NIST standard lamps at NREL were conducted to evaluate the absolute uncertainty in NREL spectral calibrations. Figure 1 shows deviations observed between NREL measurements and NIST data for six NIST standard lamps. We are investigating ways to reduce the spectral discrepancies to the $1 \%$ to $3 \%$ absolute uncertainty inherent in NIST spectral standards.

- We developed a more accurate uncertainty analysis of NREL broadband Radiometer Calibration and Characterization (RCC) software and calibrated more than 80 different radiometers and sources for NREL PV research and industrial partners, including two PV cavity radiometers calibrated to the WRR [2]; five spectral and seven broadband NREL radiometers; eleven accelerated-testing, cell performance, 
and system test illuminate sources; and radiometers for the Photovoltaic Testing Laboratory (PTL) of Arizona State University, Arizona Public Service Company, and the University of Oregon.

- We implemented a system to evaluate and correct the radiometric level of pyranometers. The software and hardware maps out errors in sensor level, and permits adjustments to reduce this source of bias error. Figure 2 shows the azimuthal response before and after adjustment of the radiometric level of a pyranometer. Fifteen radiometers were evaluated and adjusted.

- We calibrated instrumentation and gave technical guidance for spectral measurements made to evaluate performance of the NREL Quantum Efficiency Measurement System and analyze the impact of spectral variations on GaAs-based PV devices. The characterization of silicon pyranometers was also evaluated. All of this was reported at the 1997 IEEE Specialists Conference $[3,4,5]$.

- We developed a monthly calendar illustrating the plane-of-array (POA) irradiance profile on a daily basis for one month. Figure 3 illustrates the daily POA profiles for November 1997.

- We issued the following technical publications and contributed to the following conferences:

The task provided technical comments on measurement and uncertainty aspects of IEEE [6], ASTM, and International Standards Organization draft documents for PV accelerated weathering testing.

Task members were contributors to three IEEE PV Specialists Conference papers $[3,4,5]$.

Myers presented an invited paper on measurements and modeling of exposure panel temperatures at the Symposium on Service Lifetime Prediction for Organic Coatings at Breckenridge, Colorado, on September 15-17, 1997. The paper, which will be published in the proceedings of the American Chemical Society [7], included an evaluation of the Fuentes thermal model for outdoor PV module performance [8].

Myers also presented five lectures [9-13] on the technical aspects of solar radiometric calibrating, measuring, and modeling to a Saudi Arabian technical audience. The lectures were presented as part of a Summary Course on Solar Radiation Resource Assessment in the Kingdom of Saudi Arabia, September 17-October 2, 1997.

At the Pacific Northwest Solar Radiation Measurements Workshop held at the University of Oregon, Eugene, Oregon, August 6-8, 1997, we presented "Uncertainty Analysis Applied to Radiometric Calibrations and Measurements" [14].

We also presented papers at the 1997 Photovoltaic Standards and Codes Forum, June 24-27, 1997, Winter Park, Colorado.

Major industry and research technical interactions during FY 1997 included:

Spire Corporation. Analysis of spectral measurements of SPIRE model 460 and 240 simulators. Evaluation of performance and technical guidance in calibration and correct use of diode array spectroradiometer. 
Energy Conversion Devices. Absorption properties for designing optical filters for custom pulse solar simulator.

Sandia National Labòratories. Joint paper on silicon photodiode pyranometer characterization and calibration [5].

Federal Energy Management Program (FEMP). Prepared PVFORM simulation input files for FEMP analysis of hybrid energy sources for Micronesian island of Chuuk.

\section{Conclusions}

We provide broadband and spectral optical-radiation calibrations, measurements, and expertise of great value to the PV community. We are continuing to improve our measurement and calibration quality and transfer the technical improvements to the PV community.

\section{References}

1. Myers, D.R. (April 1997). Photovoltaic Radiometric Measurements Guide. NREL TP-21774.

2. $\quad$ Reda, I.; Stoffel, T. (October 1997). "Results of NREL Pyrheliometer Comparisons." NPC1997. NREL Metrology and Instrumentation Team Letter Report.

3. Kurtz, S.; Friedman, D.L.; Myers, D.R. "Projected Performance of Three- and Four-Junction Devices Using GaAs and GaInP." Proceedings of 25th IEEE Specialists Conference;13-17 May 1996; Washington, DC.

4. Field, $\mathrm{H}$. "Solar Cell Spectral Response Measurement Errors Related to Monochromatic Beam Width." Proceedings of 25th IEEE Specialists Conference; 13-17 May 1996; Washington, DC.

5. King, D.L.; Boyson, W.; Myers, D.R. "Silicon Photodiode Pyranometers: Operational Characteristics, Historical Experiences, and New Calibration Procedures." Proceedings of 25th IEEE Specialists Conference; 13-17 May 1996; Washington, DC.

6. Institute of Electrical and Electronics Engineers. "Recommended Practice for Field Test Methods and Procedures for Grid Connected Photovoltaic Systems." Draft Proposal Authorization Request (PAR) 1373.

7. Myers, D.R. (1997). "Predicting Exposure Panel Temperatures: Theoretical Models and Empirical Data." Symposium on Service Lifetime of Organic Coatings; September 15-18, 1997, Breckenridge, Colorado. Proceedings to be published by American Chemical Society.

8. Fuentes, M.K. (1980). A Simplified Thermal Model of PV Modules. Sand79-1785. Sandia National Laboratories, Albuquerque, New Mexico.

9. Myers, D.R. (1997). "Regional Absolute Cavity Radiometer Comparisons." Presented at Summary Course on Solar Radiation Resource Assessment in the Kingdom of Saudi Arabia, September 17 to October 2, 1997, Riyadh, Saudi Arabia.

10. Myers, D.R. (1997). "Upgrading to Baseline Surface Radiation Network Standards." Presented at Summary Course on Solar Radiation Resource Assessment in the Kingdom of Saudi Arabia, September 17 to October 2, 1997, Riyadh, Saudi Arabia.

11. Myers, D.R; Maxwell E.L. (1997). "Estimating Daily Aerosol Optical Depth for Solar Radiation Models." Presented at Summary Course on Solar Radiation Resource Assessment in the Kingdom of Saudi Arabia, September 17 to October 2, 1997, Riyadh, Saudi Arabia.

12. Myers, D.R.; Maxwell, E.L. (1997). "Estimating Hourly Precipitable Water Vapor for Solar Radiation Models." Presented at Summary Course on Solar Radiation Resource Assessment in 
the Kingdom of Saudi Arabia, September 17 to October 2, 1997, Riyadh, Saudi Arabia.

13. Myers, D.R.; Wilcox, S.L. (1997). "Generating a National Solar Radiation Data Base." Presented at Summary Course on Solar Radiation Resource Assessment in the Kingdom of Saudi Arabia, September 17 to October 2, 1997, Riyadh, Saudi Arabia.

14. Myers, D.R. (1997). "Uncertainty Analysis Applied to Radiometric Calibrations and Measurements." Presented at the Pacific Northwest Solar Radiation Measurements Workshop, Eugene, Oregon, 6-8 August 1997.

Figures
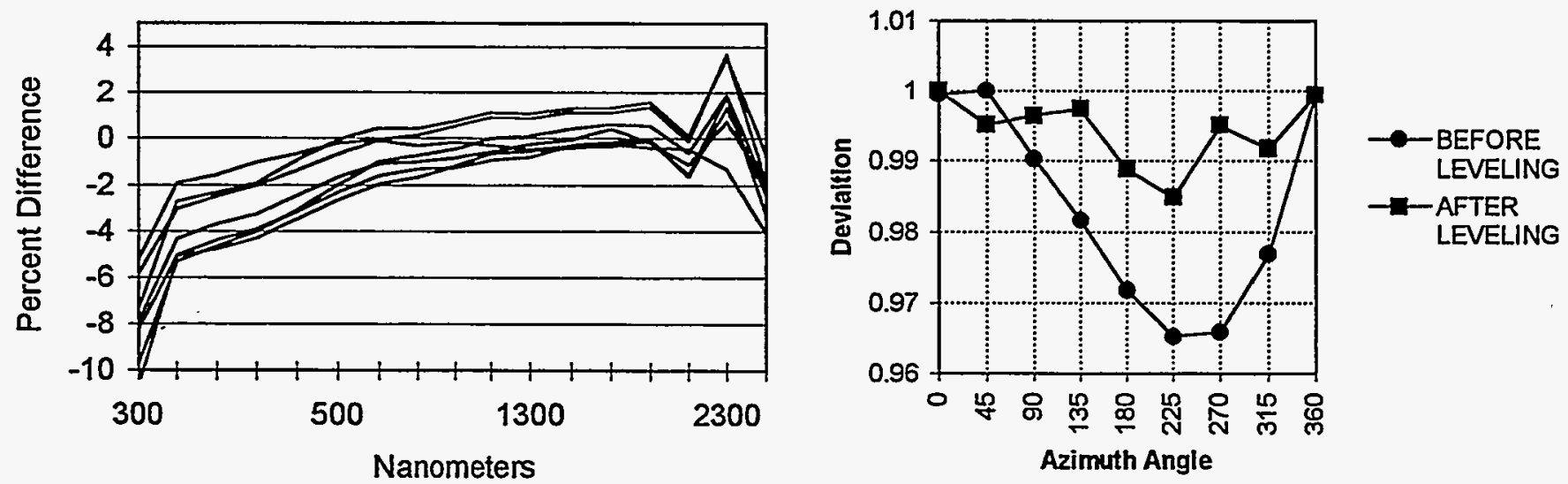

Figure 1. Percent difference between NREL Figure 2. Radiometer azimuthal response measured and NIST prescribed data for 6 NIST before (circles) and after (squares) radiometric spectral irradiance Standard Lamps. Target is level evaluation and adjustment. $+/-2 \%-3 \%$ between $400 \mathrm{~nm}$ and $2400 \mathrm{~nm}$.

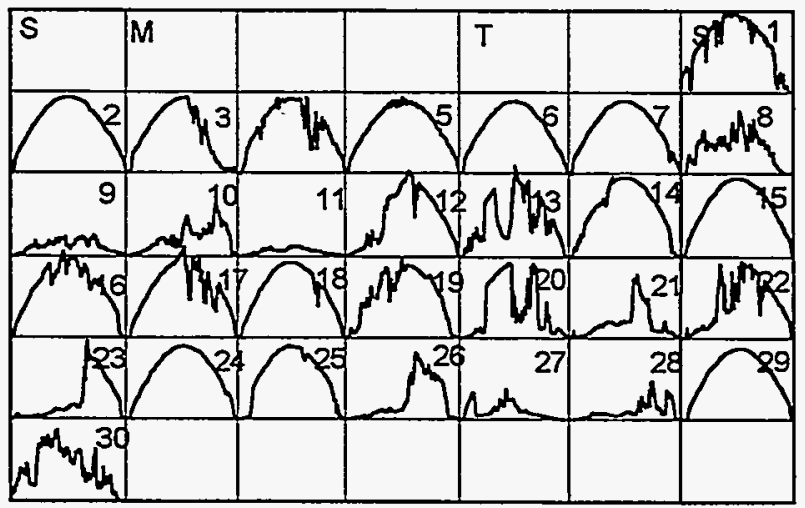

Figure 3. Daily plane of array irradiance profiles for November, 1997 at NREL Outdoor Test Facility. 
Title:

Organization:

Contributors:
Photovoltaic System Performance and Standards

National Center for Photovoltaics, Engineering and Reliability

National Renewable Energy Laboratory, Golden, CO

R. DeBlasio, R. Hansen, B. Kroposki, P. McNutt

\section{Objectives}

The overall objective of the Photovoltaic (PV) System Performance and Standards task is to address PV system, subsystem, and component technical and infrastructural issues, and provide solutions that support the DOE PV Program five-year plan (1996-2000) goals and accelerate PV technology readiness and commercialization. Specific objectives are to provide engineering solutions and approaches that will reduce technical barriers to commercialization and advance photovoltaic system, subsystem, and component performance, safety, and quality.

\section{Technical Approach}

The technical approach to achieving task objectives includes: (1) conducting system, subsystem, and component interface performance characterization outdoor testing and establishing standardized measurement and test procedures; (2) verifying PV system, subsystem and component development and advances through baseline outdoor performance testing, evaluation, and comparative analysis; (3) developing and documenting engineering best practices and technical guidelines for standardized system, subsystem, and component design and interface criteria and test procedures; (4) leading and supporting the development of national and international PV consensus standards and codes; (5) participating in and supporting the development, implementation, and operation of domestic and international system and component certification and test facility accreditation programs; (6) facilitating information exchange and technology transfer through topical meetings, forums, and workshops; and (7) providing technical assistance and support to the domestic and international PV community for PV technology development and systems engineering and applications.

\section{Results}

Overall task activities addressed system/load interface requirements, system/subsystem interface limitations, sizing and performance optimization, and system configuration designs. Solutions provided information necessary in the establishment of system and subsystem standardized design, packaging, and performance testing guidelines. Selection of the appropriate design and construction of a PV system and its subsystems is a critical area of concern with regard to optimized performance, operation and maintenance (O\&M), reliability, and safety. Over or under sizing a system, inadequate subsystem and component selection, and improper interface matching have been issues of concern over the years with regard to meeting performance requirements and cost. Failure or reduced performance of systems due to exceeding the operational limits of subsystems and components within the system has led to misunderstandings regarding why the system failed to meet its application load requirements. Exaggerated performance goals and marginal design limits have also led to failure in parts of the system, and increased O\&M cost of the system. The user must be assured that the system will meet the intended design and application load requirement. This barrier 
to acceptance of PV systems can be reduced and eliminated with the proper tools, which can be referenced for design and performance verification. This activity fills the void and provides the catalysis and focus for establishing the technical foundation and bridging the institutional barriers needed to reduce uncertainty that a systems performance will be what its designers and builders claim. The need for this work was recently made more apparent with the initiation of an international PV Global Approval Program (PVGAP) (i.e., World Bank) and is in response to concerns that PV systems being fielded must meet performance standards and that those standards include systemlevel performance qualification and acceptance tests. Such tests need to be identified, developed, and validated before they can be accepted as consensus standards and used as part of a systemcertification program. Successful development of a similar program for module certification and laboratory accreditation has been implemented through the Institute of Electrical and Electronics Engineers (IEEE) Standards Coordinating Committee 21 (SCC21) (module qualification test IEEE1262 and establishment of Power Mark Corporation for module certification). This task activity continues to provide the technical support and leadership needed to maintain PV program contributions to the technical side of the equation for systems certification as well as the establishment of an infrastructure that will allow U.S. PV community representation.

These activities also included conducting system and subsystem design reviews, consolidation of best engineering and testing practices, constructing a small-scale system and subsystem test bed at the Outdoor Test Facility (OTF). A flexible test bed with interchangeable parts representing a variety of interface configurations was constructed and instrumented for performance characterization so that results can be evaluated for validation of design criteria guidelines and identification of design and performance limitations, failures (random and common mode), and enhancements. In support of these activities, field- testing, baseline performance comparisons and database development, and analysis of ongoing performance of experimental systems and field systems were conducted on a routine basis for historical validation, lessons learned, and relative advancement of system improvements and progress.

Transfer and integration of task technical information and experience to the PV community was maintained both domestically and internationally with regard to standards, codes, test method development, laboratory accreditation, product certification, and test facility development, which are key to achieving PV technical quality that will ensure reliability and safety. These activities combined provide the technical as well as commercial infrastructure that is essential to PV technology commercialization in reducing the institutional and technical barriers to achieving continued and long-term sustainable success. Activities included participation and support for IEEE SCC21 (Standards Coordinating Committee on PV systems standards development), American Society for Testing and Materials (ASTM) E44.09 (PV subcommittee on standards development), IEC TC-82 U.S.TAG (International Electrotechnical Commission and U.S. PV Technical Advisory Group on PV standards development), NEC (National Electrical Code/Article-690), UL (Underwriters Laboratories, and Industrial Advisory Group on PV), Power Mark (technical and laboratory accreditation committees), and PVGAP. Emphasis was placed on supporting consensus standard development of module energy rating methodologies and PV system/utility interconnection recommended practices, implementing accreditation/certification programs and component and system test method development and validation, facilitating a performance and reliability workshop and a standards and codes forum, and developing topical technical meetings such as working group meetings of the standards and codes groups. A systems and subsystem interface working group was established and met on an ad hoc basis. The group held project review and planning meetings with 
systems and engineering project tasks at NREL, Sandia National Laboratories, the Florida Solar Energy Center, and the Southwest Technology Development Institute (SwTDI).

Participation and technical assistance to the Photovoltaics for Utility-Scale Applications technical review committee, Utility Photovoltaic Group engineering and specification working group, Photovoltaic Manufacturing Technology (PVMaT), Photovoltaics Building Opportunities in the United States, thin-film partnerships, the Million Solar Roofs initiative, and interface for task activities with DOE, industry, and the international community were maintained and enhanced.

The major results of this task included: the construction of a system and subsystem performance characterization test-bed facility; continued operation, maintenance, and reporting on several 1-2 kilowatt $(\mathrm{kW})$ grid-connected and small stand-alone systems; leading and developing consensus standards and codes; and supporting the establishment of a domestic and international accreditation and certification infrastructure.

\section{Systems Performance Characterization-Flexible Test Bed}

The design and construction of a flexible system, subsystem, and component performance characterization test bed, including a field-test building and installations of components, wiring, array, and data acquisition system (DAS) was completed at the OTF and prepared for operation. As part of the flexible test bed, five caissons were installed along the north side of the test pad for smallsystem deployment and characterization. The flexible test bed will be used to characterize the performance of small PV systems and validate test procedures developed for national and international consensus standards and certification activities (i.e., IEEE SCC21, IEC TC-82, PowerMark, and PVGAP).

New Fielded-System Installations and Operation

The Siemens Solar Industries (SSI) CIS 1-kW system, located at the OTF, was retrofitted with new CIGSS modules, which were received and baseline I-V tested. The array is constructed as a lowvoltage (less than 50 volts[V]) DC system. This installation constitutes the largest array of the SSI CIGSS modules constructed in the United States. Results from this test program will assist SSI in developing confidence in the performance and reliability of the module and will help in commercialization of thin-film technology.

The Solar Electric Specialties (SES) MAPPS (200-W $\mathrm{W}_{\mathrm{dc}}$ ) system, located at the OTF systems flexible test-bed pad, was installed and data are being collected. This system supports the PVMaT program. In its first month of operation the system has performed reliably from an electrical standpoint.

Long-Term Fielded Systems-System Performance and Reliability Evaluation

Outdoor performance of eight PV systems continues to be monitored at the OTF. These systems include: Advanced Photovoltaic Systems (APS) 1.8-kW a-Si; ASE Americas 1.4-kW EFG-Si; Solar Cells, Inc. (SCD) 1-kW CdTe; Siemens Solar Industries 1-kW CIS; Siemens Solar Industries 12-kW Si (Solar Energy Research Facility [SERF]); Solarex 1-kW a-Si/A-Si:Ge; United Solar Systems Corporation 1.8-kW a-Si/a-Si; and United Solar 1-kW a-Si/a-Si roof shingles. The following reports on the performance of the systems were completed and sent to industry partners and research support requestors:

STR97APS.001: Update on the APS 1.5-kW a-Si system (3/20/97)

STR97APS.002: Update on the APS 1.5-kW a-Si system (9/4/97) 
STR97CHI.001: Performance data on the Chinese hybrid inverters (11/5/97)

STR97SES.001: First test report for the SES MAPPS system (PVMaT support, 11/11/97)

STR97SCI.001: Update on SCI 1-kW CdTe system (3/3/97)

STR97SCI.002: Update on SCI 1-kW CdTe system (8/20/97) .

STR97SSI.001: Update on Siemens 1-kW CIS system (3/13/97)

STR97SSI.002: Final report on Siemens 1-kW CIS system (8/27/97)

STR97SSI.003: Initial report on Siemens 1-kW CIGSS system (8/27/97)

STR97SSI.004: Six-month test report on the performance of the new CIGSS array (12/9/97)

STR97SRX.001: Update on the Solarex 1-kW a-Si/a-Si:Ge system (3/17/97)

STR97SRX.002: Update on the Solarex 1-kW a-Si/a-Si:Ge system (9/10/97)

STR97USC.001: Update on the USSC 1.8-kW a-Si/a-Si system (3/31/97)

STR97USC.002: Update on the USSC 1.8-kW a-Si/a-Si system.(3/31/97)

STR97USR.001: Update on the USSC 1-kW a-Si/a-Si roofing system (9/4/97)

STR97USR.002: Update on the USSC 1-kW a-Si/a-Si roofing system (9/4/97).

Standards and Codes Progress and Activities

The PV Standards and Codes Forum was held on June 23-25, 1997, in Winter Park, Colorado. Over 40 participants from the PV community attended the three-day series of meetings and working-group sessions, which included IEEE SCC21, ASTME 44.09, IEC/TC82 U.S. TAG, and code status on NEC PV article 960. Highlights of the meeting included new work activities for IEEE SCC21 regarding PV module concentrator qualification tests; PV small-system test procedures; overview on PV Global Approval Program; development of ASTM multijunction cell measurement techniques and simulator standards; and success of the NEC work lead by the PV industry to make major revisions for easier and safer installation of PV systems in the field. Another meeting of IEEE SCC21 was held on November 10-12, 1997, in San Diego, California. This meeting was attended by over 20 participants in the standards activities. Highlights of this meeting included: continuation of work on the energy ratings, system field-testing, and concentrator photosynthetically active radiation (PARs) and finishing work on the systems safety PAR.

Work has also continued to facilitate development of the IEEE system utility interconnection standard (IEEE PAR P929, approved by the Standards Board in October 1996) with activation of the IEEE SCC21 P929 Working Group in January 1997 at the UL/SCC21 meeting. Draft one of P929 was completed in May 1997 and comments received from the working-group members were evaluated and incorporated for further review as part of the consensus process. Draft two was completed and reviewed during a meeting of the P929 Working Group held in Anaheim, California, during the IEEE PVSC. Draft three has been completed and is being reviewed by the working group in preparation for a SCC21 ballot action in FY 1998.

The IEC TC-82 PV International Standards Development secretariat was supported (Solar Energy Industries Association [SEIA] subcontract) and maintained with the establishment of new working groups to address the international need for system certification test procedures. In addition to working groups for definitions, modules, systems, and storage, new working groups for certification, balance of systems (BOS), concentrators, and mechanical/environmental standards development were proposed for adoptions and implementation in 1998. A new IEC-TC-82 chairman ( $R$. DeBlasio, USA) was nominated and elected by the international membership, replacing Professor Y. Sekine of Japan. 
PV Performance and Reliability Workshop

The annual PV Performance and Reliability Workshop was sponsored by NREL and Sandia National Laboratories, and hosted by SwTDI on August 5-6, 1997, in Las Cruces, New Mexico. More than 125 participants attended the two-day meeting, which included more than 20 presentations and summary discussions. Emphasis was placed on the reliability of systems and BOS; safety and performance of systems and BOS; and field experience. Major areas of concern were identified that need attention by the PV community. Issues addressed were battery applications in PV system design (e.g., metal cases and grounding); inverter failures in the field (prototype versus commercial products); the need for accelerated and environmental testing of BOS; and standard system design versus standard system performance.

\section{Accreditation and Certification Progress and Activities}

Task members participated as members of the board of directors of PowerMark Corporation and chairman of the Laboratory Accreditation Committee for PV component and system certification. The accreditation committee evaluated the Arizona State University (ASU) PV test laboratory for PowerMark accreditation on May 6, 1997, and provided a report of its findings to the PowerMark Board of Directors. The PowerMark Board gave provisional recognition to ASU on May 21, 1997, and full recognition, following the A2LA ISO-25 certification, in June 1997.

R. DeBlasio was appointed to the PVGAP Board of Directors on June 9, 1997, and continued to provide technical support to the PVGAP for PV system performance certification. A meeting of the PVGAP laboratory accreditation committee was coordinated and held at UL in January 1997. A survey of all 25 ISO-certified laboratories was conducted and test facilities selected from around the world for inclusion in the PVGAP handbook, slated for publication in early 1998. SEIA was designated PVGAP representative for the United States, and SEIA assigned PowerMark to be their technical representative.

\section{Subcontract Research Support}

Subcontracts in support of task objectives included: (1) SEIA for management and administration of the IEC TC-82 Secretariat and U.S. TAG; (2) PowerMark Corporation for support in administering the accreditation and certification program activities for PV; and (3) NEOS Corporation for developing system performance test methods to be used for consensus standards development by the IEEE, IEC, PowerMark, PVGAP, and other testing bodies.

\section{Technical Assistance and Technology Transfer}

Task members participated in the PVUSA technical review committee meeting in June 1997; the EPRI/DOE PV Application Experience Workshop on August 25-26, 1997; Phase 2 PVMaT subcontract review and support for PVMaT 4A testing; chairing IEEE SCC21 and providing leadership in system/utility interconnection, energy rating, concentrator qualification tests, and system test standards development; PVGAP and PV BOS meetings during the IEEE PVSC in September 1997; providing presentations on IEEE SCC21 status at the SEIA Standards and Codes meeting and status on IEEE P929 system/utility interconnection standard at the UPVG Engineering and Specification Group meeting during the SOLTECH meeting in Washington, D.C., on April 28, 1997; and providing a presentation on the status of IEEE SCC21, IEC TC82 and PowerMark activities at the UPVG Engineering and Specifications Group meeting in Albuquerque (October 27, 1997) during the annual UPVG meeting. 
Support for PV program subcontracts research activities included: (1) 1997 proposal review of the PV Thin-Film partnership initiative; and (2) PVMaT subcontracts in place with UPG, Advanced Energy Systems, Trace, Omnion, Solar Electric Specialties, Ascension Technology, Solar Design Associates, Evergreen Solar, and PV International. Support for the international task activities included providing technical inputs on test methods and standards for international certification (World Bank) activities and testing several Chinese (Inner-Mongolian project) inverters.

Engineering and field-testing support was provided by task members (R. Hansen, B. Kroposki, and P. McNutt) in support of the design and installation of the 1997 Pageant of Peace Display in Washington, D.C., and as part of task interaction within the system's ad hoc working group a joint field test was conducted by task members ( $R$. Hansen and P. McNutt) with staff from SwTDI (A. Rosenthal and S. Durand) of the Olympic Natatorium PV system.

\section{Conclusions}

Establishing the PV System Performance Characterization Flexible Test Bed was a major accomplishment of this task. This new capability and future activities planned are essential to providing viable test methods and results that give confidence in PV system performance designs and claims. In addition, ongoing advanced PV system technology testing at the OTF continues to provide updated baseline performance and reliability measurements and evaluations for determining advances in PV technology both for the DOE subcontracts research program and for the PV industry at large. The systems testing and standards development are extremely important in view of the world market for photovoltaics as shown by the continued interest and participation in the annual task lead PV Performance and Reliability Workshop (sponsored by NREL and Sandia). The world continues to look to the United States for leadership in developing consensus standards for testing not only PV modules but complete systems. The work conducted by this task can be directly applied for use by the IEEE, IEC, PowerMark, PVGAP, and other standards and certification bodies to support the commercialization infrastructure and confidence in PV system technology and its applications. Major contributions and accomplishments of the task in this area included: certification of ASU by PowerMark; IEEE Standards Board approval of PAR P929 in support of system/utility interconnection standard (Million Solar Roofs initiative support); and leadership in national and international PV standards and codes development as shown by the annual task-sponsored Standards and Codes Forum.

\section{Publications and Presentations}

Kroposki, B.; Hansen, R. (date?). "Technical Evaluation of Four Amorphous Silicon Systems at NREL." $26^{\text {th }}$ IEEE PV Specialists Conference, Anaheim, CA.

Bower, W.; Bonn, R.; Hund, T.; Thomas, H.; Kroposki, B. (date?). "Balance-of-system Improvements for PV Applications Resulting from the PVMaT Phase 4A1 Program." 26 $6^{\text {th }}$ IEEE PV Specialists Conference, Anaheim, CA.

Schuyler, T; Kroposki, B. (1997). "Development of Test Methods and Procedures for Evaluation of PV Systems." 1997 PV Performance and Reliability Workshop, Las Cruces, NM.

DeBlasio, R (1997). "IEEE SCC21 Systems Standards Development Status." SEIA Standards and Codes Session, SOLTECH '97, Washington, D.C. 
DeBlasio, R. (1997). "IEEE SCC21 P929 PV System/Utility Interconnection Standard Development." UPVG Engineering and Specifications Group Session, SOLTECH '97, Washington, D.C.

DeBlasio, R. (1997). "IEC and IEEE PV System Standards Development" and "PowerMark Certification Status." UPVG Utility PV Experience Conference, Albuquerque, NM.

Thomas, M; DeBlasio, R. (1997). "Challenging Performance and Reliability Demagoguery." 1997 PV Performance and Reliability Workshop, Las Cruces, NM.

DeBlasio, R. (date?). Coordinated development and presented PV initiative titled "Strategic Global Alliance for Photovoltaics" to the NREL Strategic Planning Council.

Whitaker, C.; Newmiller, J. (date?). Final subcontract report (\# AAI-4-14192-01). "Photovoltaic Module Energy Ratings Procedure." Endecon Engineering. 
Title:

Organization:

Contributors:

\section{Solar Resource Characterization}

NREL Solar Resource Assessment Team

National Renewable Energy Laboratory, Golden, CO

D. Renné (Team Leader), M. Rymes, M. Anderberg, B. Marion, R. George, L. Brady, G. Maxwell, S. Wilcox

\section{Objectives}

The overriding goal of this project is to develop and disseminate key information on solar energy resources to U.S. industry and to energy planners in order to facilitate and accelerate the deployment of their technologies, both domestically and worldwide. To achieve this goal, the following objectives were addressed during FY 1997: 1) to develop accurate databases and maintain reliable measurement activities with defined data quality, 2) to develop and validate procedures for characterizing solar resources, even in the absence of adequate measured data, 3) to perform technology- and regionspecific resource characterizations accurately, quickly, and reliably, and 4) to provide a centralized, interactive source of quality-assessed nonproprietary resource data and information. In this report, we focus on the activities within the Cooperative Network for Renewable Resource Measurements (CONFRRM) and the development of the multi-pyranometer array (MPA), which address Objective 1; the Renewable Resource Data Center (RReDC), which addresses Objective 4; and the use of satellite data for improving the types of solar resource assessment products that can be offered the solar industry, which addresses Objective 2.

\section{Cooperative Network for Renewable Resource Measurements}

This task seeks to establish and maintain high-quality wind and solar monitoring stations throughout the United States through cooperative agreements with local organizations. CONFRRM is a cooperative effort in which NREL provides measurement equipment in exchange for station operation and data collection. We achieved a key FY 1997 milestone when the University of Texas at Austin completed the installation of first-class solar radiation measurement stations at five Texas locations: Austin, Canyon, Clear Lake, El Paso, and Edinburg.

The addition of the Texas stations increased the number of CONFRRM stations to ten. Five other CONFRRM'stations in the southeastern United States have been operated since 1985 by Historically Black Colleges and Universities (HBCUs). The CONFRRM network complements and provides additional geographic coverage to the National Oceanic and Atmospheric Administration's (NOAA's) Integrated Surface Irradiance Study (ISIS) 14-station network.

Cooperative agreements were also initiated to add three more stations in FY 1998. These stations will be located at the Florida Solar Energy Center (FSEC), the Southwest Technology Development Institute, and the University of Oregon. 


\section{Multi-Pyranometer Array}

The use of a multi-pyranometer array for the measurement of the solar resource is an alternative to using thermopile instruments and their associated trackers and shading disks or shadowbands. The MPA consists of three or four pyranometers with fixed orientations and different views of the sun and sky. By measuring the solar radiation received by each pyranometer and the use of an iterative model, direct normal and diffuse horizontal radiation can be determined.

We achieved a key milestone by summarizing the design and performance of MPAs installed for oneyear periods at NREL's Solar Radiation Research Laboratory (SRRL) and at the Solar Village near Riyadh, Saudi Arabia. These MPAs used LI-COR LI-200 pyranometers to minimize cost. The MPAs yielded monthly direct normal estimates within $4 \%$ of measurements and with a RMSE for fiveminute averages of about 50 watts $(\mathrm{W}) / \mathrm{m}^{2}$ (12\% of the average) for SRRL and $76 \mathrm{~W} / \mathrm{m}^{2}(14 \%$ of the average) for the Solar Village.

The RMSEs are believed to be higher for the Solar Village because of the sensitivity of the LI-COR pyranometer to a more widely varying spectral distribution and because the Perez model used to estimate the pyranometer output in the iteration routine underestimated the diffuse radiation for the east- and west-facing pyranometers for summer months and small zenith angles.

For use in estimating the radiation for flat-plate collectors of arbitrary orientation at the SRRL, the MPA estimates of direct normal and diffuse horizontal radiation were found to give as accurate results as measured values of direct normal and diffuse horizontal radiation.

The total cost of a MPA measurement system is estimated to be $\$ 2,400$, which is about one-tenth the cost of a solar radiation measurement station using conventional thermopile pyranometers and a pyrheliometer mounted on a computer-controlled tracker.

We identified further improvements in the MPA estimates of direct normal and diffuse horizontal radiation in the areas of developing and implementing procedures for correcting LI-COR pyranometer measurements for cosine response, operating temperature, and solar spectrum. Modifying the Perez model to prevent underestimating the amount of diffuse radiation for the vertical east- and westfacing pyranometers for summer months and low zenith angles would also be beneficial.

The MPA requires little expertise to use and maintain. Its pyranometers are fixed and it requires no periodic adjustment such as that required by trackers and shadowbands. It has increased reliability because there are no moving parts. The pyranometers are less-expensive and they exhibit good resistance to soiling, thus permitting cleaning intervals to be extended.

\section{The Renewable Resource Data Center}

The Renewable Resource Data Center is a World Wide Web (WWW) site established at NREL to provide a centralized source of publicly available renewable energy resource data for all technologies, including solar. Solar data activities in FY 1997 focused on three areas: incorporation of site- or 
region-specific solar resource maps, establishment of an on-line "real- time" data source from the HBCU and CONFRRM sites, and development of a solar glossary.

We prepared color and monochrome contour maps of annual global horizontal and direct normal solar irradiance, derived from the National Solar Radiation Data Base (NSRDB), for each state in the United States. These maps have not yet been placed on the RReDC because of their low resolution. The products from the 40-kilometer $(\mathrm{km})$ data grid of U.S. solar resources (see "Objectives") will provide a much-improved spatial resolution, and will be incorporated into the RReDC in FY 1998.

The daily summaries of solar data from the SRRL, located on top of South Table Mountain near Golden, Colorado, are now available on the RReDC. These summaries include raw and processed five-minute data for all instruments, daily time-series plots of the data for each instrument series (see Figure 1 for an example), wind roses, and tabular monthly summaries. Historical data from April 1985 to the present are also available on the RReDC. HBCU data from July 1985 through December 1996 are available on the RReDC, and current data are available on request. CONFRRM data are being collected and processed on a daily basis, but the network data as a whole will not be on-line until early FY 1998.

The interactive solar glossary contains approximately 500 of the most common terms used in the solar radiation resource community, with illustrative figures, pictures, equations, and hyperlinks. This glossary is now available on the RReDC, and has been instrumental in reducing the number of queries concerning basic solar radiation terminology. In addition, it should lead to a more informed user community.

We prepared approximately 400 U.S. maps based on the Solar Radiation Data Manual for Buildings. This atlas, a companion to the one prepared based on the Solar Radiation Data Manual for FlatPlate and Concentrating Collectors, is now available on the RReDC.

The Spectral Solar Radiation Data Base is a repository of 3300 solar spectra collected at the FSEC, SERI (NREL), and San Ramon, California (through Pacific Gas and Electric Company), from October 1986 to April 1988. These data and the document Spectral Solar Radiation Data Base Documentation, Vol. I, are now available on the RReDC. Spectra were measured from 300 to $1100 \mathrm{~nm}$ in steps of $2 \mathrm{~nm}$ by a LI-COR LI-1800 spectroradiometer, and included global horizontal, global normal, direct normal, and global-tilted orientations.

Two new manuals have been developed and incorporated on the RReDC to help document data sets that are on the RReDC. One manual is for the 1976-1980 NOAA network data set. A text version of the manual for the Typical Meteorological Year- -I (TMY2) data, developed from the NSRDB, was prepared specifically for the RREDC.

During FY 1997 we redesigned the RReDC, with a new home page, logo, and miscellaneous icons and links that serve as enhanced navigational aids. We also established an anonymous File Transfer Protocol site (ftp://rredc.nrel.gov or the unix command ftp delphi.nrel.gov) to enable massive or multiple file transfers to and from the $\mathrm{RReDC}$, because the current design and philosophy of the WWW discourages large file transfers directly through the browser. 
In addition to the 464,000 hits (link accesses) on the RReDC in 1997, many requests for data, information, and maps came from NREL's publications group and Information Services, NREL's Washington, D.C. Office, and numerous outside requests.

\section{Satellite-Derived Solar Resources}

During FY 1997 the Solar Resource Assessment Team increased its focus on developing muchimproved spatial solar resource information through use of satellite data and specially designed models to improve the spatial representation of solar resources worldwide. One activity being conducted at NREL uses the 1985-1991 Real-Time Nephanalysis Cloud Cover data set and a solar resource model to provide monthly average daily total solar resource information (direct normal, global, and diffuse) for any location on Earth. A second activity, being performed by the State University of New York, Albany, under contract to NREL, is developing high-resolution hourly surface solar maps for the northeastern United States using GOES-8 and GOES-9 weather satellite imagery. NREL is also collaborating with the National Aeronautics and Space Administration's Langley Research Center in making use of their worldwide Surface Radiation Budget data sets to develop maps of global horizontal solar resources on a $280-\mathrm{km}$ resolution. These activities will be reported in greater detail in the FY 1998 Annual Report.

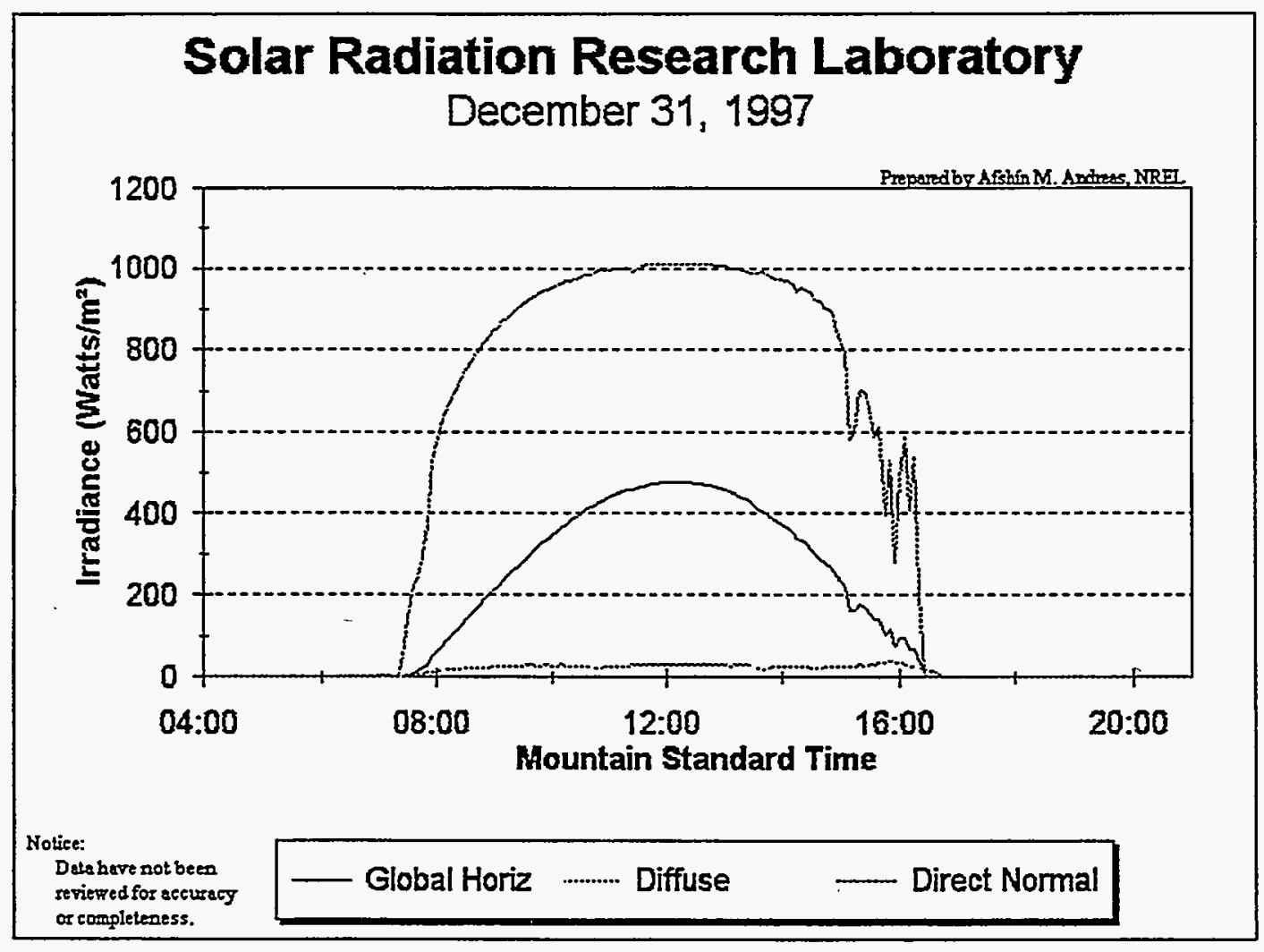

Figure 1. One of the many diurnal plots from SRRL that are available on the RReDC. 


\section{Summary and Future Directions}

We made significant accomplishments in FY 1997 in developing more refined solar resource products and in disseminating this information to the solar energy community. In addition, we continued our efforts to help maintain a national solar monitoring network by supporting the CONFRRM and $\mathrm{HBCU}$ programs, and by linking these activities to other solar monitoring projects such as the NOAA/ISIS network.

Our focus in FY 1998 will be to further refine our solar resource assessment techniques using satellite imagery, and to develop specific products from these data that directly assist the solar energy industry in planning for solar installations in the United States and abroad.

\section{Selected References}

Renne, D. S.; Maxwell, E. L.; Rymes, M. D.; Marion, W.; Phillips, J. (1997). "Availability of Renewable Resources: Solar Radiation." Chapter 18A. Edited by F. Kreith and R.E. West, CRC Handbook of Energy Efficiency. Boca Raton, FL: CRC Press, Inc.; pp. 715-749.

Maxwell, E, L.; George, R. L.; Brady, E. H. (1997). "Estimating Worldwide Solar Radiation Resources on a 40-km Grid." Solar 96: Proceedings of the 1996 American Solar Energy Society Annual Conference, 13-18 April 1996, Asheville, North Carolina, pp. 235-240. 


\section{PV Engineering and Reliability Subcontracts}


Title: Photovoltaic Module Energy Rating Procedure

Organization: Endecon Engineering, San Ramon, CA

Contributors: CM Whitaker, Principal Investigator, JD Newmiller, and TU Townsend

\section{Introduction}

In May 1994, the National Renewable Energy Laboratory (NREL) initiated an effort to develop a consensus-based approach to rating photovoltaic modules. This new approach was intended to address the limitations of the de-facto standard module power rating at Standard Test Conditions (STC ${ }^{\mathbf{l}}$ ). Using technical input from a number of sources and under the guidance of an industry-based Technical Review Committee, the approach described in this document was developed.

A final report on this effort has been published (NREL/SR-520-23942). This document describes testing and computation procedures used to generate a photovoltaic Module Energy Rating (MER). The MER consists of 10 estimates the amount of energy a single module of a particular type (make and model) will produce in one day. Module energy values are calculated for each of 5 different sets of weather conditions (defined by location and date), and 2 load-types. Since reproduction of these exact testing conditions in the field or laboratory is not feasible, limited testing and modeling procedures and assumptions are specified.

\section{Approach}

The module energy rating presented here is the result of the combined efforts of Endecon Engineering, the National Renewable Energy Laboratory, and Sunset Technology, as well as the members of a Technical Review Committee (TRC) specifically set up for this activity. The 35 TRC members represented PV manufacturers, system integrators, government and academic researchers, and industry consultants. This group guided the technical and philosophical approach and reviewed results.

Modeling approaches were selected from those suggested by a variety of sources. Indoor and outdoor measurements were made to provide coefficients to the models and to provide validation data. The majority of the work effort involved setting up the tests, collecting and processing the data, and analyzing the results of the models.

Five sets of weather conditions were defined to provide the basic range of environmental conditions anticipated for typical uses of PV modules in the US, and allow for comparison of module performance under a wide variety of conditions. The National Solar Resource Database (NSRDB) was selected as the source of the weather data. By doing so, entire daily weather profiles can be specified simply by indicating a city name and a location.

Two load types are assumed, corresponding to the two most common loads connected to PV modules: maximum power tracking for grid-tied applications and fixed voltage for battery charging.

For purposes of rating comparison, a 14.4-Volt $(\mathrm{V})$ operating voltage per battery (2.4 V/battery-cell) is assumed. The $14.4 \mathrm{~V}$ value may be divided by the recommended number of modules and multiplied by the recommended number of batteries to obtain a fixed voltage for purposes of rating. If the manufacturer does not recommend this module for battery application, then the modules need not be rated for fixed voltage. It

1 STC: $1000 \mathrm{~W} / \mathrm{m}^{2}$ irradiance with an Air Mass 1.5 spectrum and $25^{\circ} \mathrm{C}$ cell temperature. 
is assumed that no charge regulation occurs; that is, the module operates at $14.4 \mathrm{~V}$ whenever there is sufficient sun.

\section{Module Energy Rating Computation}

The Module Energy Rating consists of measuring module characteristics, defining sets of weather and load conditions, and estimating module performance under those conditions. The selection of performance estimating (modeling) tools must be completed first as they define which weather parameters and which module characteristics are necessary.

Based on the experience of the project team and the guidance of the TRC, five modeling areas were considered essential to adequately estimate module performance:

1) Irradiance: estimating the magnitudes of the various components of sunlight striking the module

2) Optical: accounting for optical effects, such as reflection loss at high angles of solar incidence

3) Thermal: estimating the module temperature based on module characteristics and weather conditions

4) Spectral: estimating the response of the module to changes in the solar spectrum

5) Power: estimating module output based on a characterization of the module, and the defined weather data and the results of the other models

The report describes all of the models in detail, provides the equations used, and the methods used for obtaining the model coefficients from the indoor and outdoor data. Below is a brief description of each modeling area.

\section{Power Model}

The power output of the module is computed from the plane-of-array irradiance (adjusted for spectral and incidence-angle effects), the module temperature, and appropriate coefficients. Ultimately, this methodology will specify only one power model. Each model is required to estimate power output for maximum power operation and ampere-hours for fixed voltage operating conditions. Five models were investigated: linear (Myers), interpolation, Anderson, Blaesser, and the lumped four-parameter model.

The first model is a simple linear-fit that only depends on irradiance. The second model used the raw data supplied by the module characterization process (a matrix of IV curves dependent on irradiance and temperature) and extracted appropriate maximum power and fixed voltage points for each irradiance/temperature combination. The third and fourth models used translation routines to translate an entire IV curve taken at reference irradiance and temperature conditions to the actual conditions. The fifth model - the only analytical model that was investigated - described the module as a single, lumped diode and a series resistance.

\section{Spectral Model}

The spectral model had two major components. First, the spectral model simulated irradiance spectra appropriate to the specified weather conditions for each time interval. These spectra were then combined with a measured module spectral response function to obtain an equivalent AM1.5 broadband irradiance value for input to the power model. 


\section{Thermal Model}

The thermal model used here was the same as that used in the computer program PVFORM [1]. It was developed and evaluated using measured array data and takes into account module construction, mounting, and operation in a term called Installed Nominal Operating Cell Temperature, INOCT. INOCT is based on NOCT which is defined as the cell temperature of the module under conditions of $800 \mathrm{~W} / \mathrm{m}^{2}$ irradiance, $20^{\circ} \mathrm{C}$ ambient temperature, $1 \mathrm{~m} / \mathrm{s}$ wind speed, with a single open-circuited module mounted in an open rack (or per the manufacturer's requirements). INOCT has the same ambient conditions, but the module is mounted and operated per the system designer's intention. As such, one module can have a wide range of INOCTs because of the possibility of different mounting configurations and operating points (peak power, fixed voltage, etc.)

\section{Optical Model}

An optical model uses the plane-of-array beam irradiance incidence angle to obtain an incidence angle modifier (IAM) which accounts for reflection and other effects. An approach to quantifying the incidence angle effect was developed by D. King [2]. This technique involved the use of an azimuth/altitude type two-axis tracker whose azimuth was varied to obtain $I_{s c}$ data for a range of incidence angles while keeping the diffuse component essentially constant. Attempts to reproduce this procedure at NREL yielded unsatisfactory results, so no IAM function was used in this report.

\section{Irradiance Model}

The irradiance model transforms commonly available time-correlated irradiance and other weather measurements and plane-of-array orientation into incidence angle and full-spectrum incident beam and diffuse irradiance estimates using astronomical solar position equations and the Perez diffuse radiation model. The steps in these computations are the determination of the true local solar time, computation of the local solar position, computation of the plane-of-array beam irradiance and incidence angle, and the evaluation of the Perez diffuse radiation model. The majority of the equations used were extracted from source code provided by Daryl Myers of NREL and represented the "NREL consensus" method for this type of application.

\section{Model Comparison}

Comparisons were made of the measured energy production of 5 different modules (representing 5 technologies) to that predicted by the various models. Each of the five modules were characterized indoors and then put outdoors at the NREL Outdoor Test Facility and measured extensively. The results of these tests are detailed in the final report.

\section{Results and Conclusions}

This project set out to address three specific goals:

- Define a Module Energy Rating Methodology

- Define specific conditions for evaluating module performance representative of the US climate

- Define procedures and techniques for measuring module characteristics and estimating module performance at the representative conditions 
The first of these goals has been squarely met. A general method for describing a PV module energy rating has been developed and agreed upon by an industry-representative Technical Review Committee.

The second goal has been met in principle with the definition of five representative daily weather profiles. These profiles are further defined by the source of weather data (the National Solar Radiation Database) and specific locations and dates within the database. While these suggested weather profiles appear to adequately represent a broad range of geographic locations and climatic conditions, they are subject to change under further scrutiny of system owners and designers.

The third goal has not been satisfactorily met. The combined errors in characterizing module performance, in translating indoor measurements to outdoors, in measuring ambient conditions and module performance, and in accounting for all of the various parameters affecting module performance exceed the accuracy required to compare one module to another under a variety of conditions. Recommendations in the report address the measurement and modeling errors encountered.

A consensus was never reached as to the level of accuracy required for the overall performance modeling. We feel that to provide users with ratings that they can confidently compare and make decisions on, we need to be able to model performance to within 5 percent of actual over the full range of expected conditions. This implies that we need to account for factors that have rather small impact on module performance - as small as 1 percent-so that the combined error of all factors puts us below that 5 percent level. As we all initially suspected and have shown through this initial work, this level of accuracy will not be achieved easily.

The final report provides detailed discussion of further refinements and follow-on activities to improve the results from the MER.

[1] M. K. Fuentes, "A Simplified Thermal Model for Flat-Plate Photovoltaic Arrays," Sandia Report SAND85-0330-UC-63, May 1987.

[2] D. King, "Photovoltaic Module and Array Performance Characterization Methods for All System Operating Conditions," NREL/SNL Program Review, AIP Press, 1996, pp.347-368. 
Title:

Development of Test Methods and Procedures for Evaluation of PV Systems

Organization: $\quad$ NEOS Corporation

Contributor: $\quad$ T. Schuyler, Principal Investigator

Objectives:

Identify and evaluate current and appropriate test methods and procedures available for PV component and systems testing and develop test procedure approaches and needed tests for use in developing and documenting a draft system test procedure for use by PV standards, testing, and certification bodies.

\section{Technical Approach:}

With the emergence of PV small system deployments around the world and the variety of PV system components integrated into a design package for different applications the need for system and component level performance tests and evaluation procedures has been accelerated and required by some end users and investors. Without assurance that PV systems perform as designed and for the application intended through some type test, as is required for modules, then the confidence in PV system performance by the world community will continue to be weak and have an impact on the success of PV commercialization. To encourage and promote PV product quality and component and system performance verification, a handbook of test methods and procedures for PV components, systems, and PV integrated components and appliances for end use applications, representing the best accepted engineering testing practices available, must be developed, validated, and adopted by the PV industry through the domestic and international consensus standards organizations (i.e. IEEE SCC21, ASTM, IEC/TC-82), and certifications groups such as PowerMark, IECQ, UL, PVGAP, etc. The technical approach includes: (1) identifying (i.e. literature search, etc.) and documenting current test methods and procedures (TMP) and synthesizing each TMP for technical basis and procedural applicability including manufacturer, end user, system integrator designer, investors, etc., TMP needs and requirements that would support deployment of quality PV systems in the field for both domestic and international markets; (2) defining an expanded TMP needs and developmental matrix for PV components, systems, integrated PV components and appliances (general and specific (lights, motors, etc.) loads), etc.; and (3) developing PV test method algorithms with input/output/interface test parameter(s); and (4) drafting a PV test methods and procedure, including flow diagrams and step by step procedures for conducting system performance-tests for use in developing an NREL technical report and field testing verification, and use by the PV standards and certification bodies.

\section{Results}

A list of appropriate tests has been compiled and evaluated for use in the development of an interim test procedure and applied to the development of test criteria and matrix of interfaces and discrete components being consider as part of a PV system design. An outline for the draft test procedure was developed and a preliminary draft test procedure completed during this reporting period. Results and progress were presented during the PV Performance and Reliability Workshop on August 6, 1997, in Las Cruces, New Mexico.

\section{Conclusion}

A preliminary draft test procedure has been produced, reviewed, and is currently being revised for final review. The draft final document when completed will be used in developing an NREL technical report 
covering PV system performance testing and will be provided to standards bodies, such as IEEE SCC21 and IEC TC82 WG-3, for further development as a consensus standard.

\section{References}

Schuyler, T., and Kroposki, B., (August 6, 1997), "Development of Test Methods and Procedures for Evaluation of PV Systems," 1997 PV Performance and Reliability Workshop. 
Title:

Organization: $\quad$ PowerMark Corporation

Contributors: $\quad$ S.M. Chalmers PE, Executive Director.
PV Certification and Accreditation Management Support

\section{Background}

The development of a domestic PV system and component certification and test facility accreditation program with working relationships that are recognized, have reciprocity between entities, and the testing validated and preformed by accredited laboratories are key to establishing the basis for a PV module and system quality and certification program. In 1996 the PowerMark Corporation (PMC) was created to implement the program recommended as the result of the NREL/DOE PV module certification and laboratory accreditation criteria handbook developed by to Arizona State University under a subcontract from NREL.

\section{Objectives}

To support an appropriate organization (PowerMark) to manage and administer the operation of a module certification and laboratory program in accordance with the criteria provided in NREL Handbook, August 1996 * NREL/TP-412-21291 (Photovoltaic Module Certification / Laboratory Accreditation Criteria Development: Implementation Handbook. (1)Administer corporate board and committee activities. (2) Provide liaison actives with domestic and international organizations associated with PV certification, accreditation, standards, codes and test method development and validation. (3) Develop protocols for laboratory test methods based on published standards. (4) Initiate proficiency tests to compare module out put measurements between laboratories.

\section{Accomplishments}

The PowerMark Corporation (PMC) has been in existence for more than 18 months. It provides PV manufactures with a module certification program that attests to the module's quality. The program involves the use of formal quality system criteria for the manufacturer such as ISO 9001 and a test laboratory authorized to perform testing in support of the program. The Laboratory must be accredited to ISO Guide 25 by an internationally recognized accrediting body and approved by PMC. The module certification program is based both on U. S. and International consensus-developed standards IEEE 1262, IEC61215 and IEC 61646.

The PMC Board and Officers are from ASE Americas, EBARA, Siemens Solar, Solarex. These manufactures represent more than $79 \%$ of the USA domestic PV module production. The Technical, Laboratory Accreditation and Manufactures Quality System committees were appointed and are functioning. PV industry persons in addition to the manufactures are on the Board and committees. The Board approved the PV-3 Laboratory test requirements for modules. PV-3 requirements were developed by the Technical committee and are the qualification test required for IEC 61215, IEC 61645, IEEE 1262. A module passing PV-3 will meet both the IEEE and IEC requirements.

The most significant happening of the year was the approval of Arizona State University / Photovoltaic Testing Laboratory (ASU/PTL) as a internationally accredited laboratory for PV modules. The American Association of Laboratory Accreditation (A2LA) performed the investigation and granted this recognition. 
The only other laboratory that is internationally accredited to perform this level of testing is the JRC in Europe. This provides a domestic source, an alternative, for U.S. and other manufactures to send modules to for certification. PMC has recognized the ASU/PTL for performing module tests.

In mid 1997 the first manufacturer committed two module types for testing and the first group of modules were placed under test at ASU/PTL. Six nodules from three PMC manufactures were provided for proficiency testing and comparison of test ratings between laboratories. NREL and Sandia have completed tests and the modules are ready to be sent to JRC at Ispra, Italy .

The UPVG technical 1997/1998 specifications recommend PMC certified modules. PMC participated in the drafting of the specifications. This recommendation will encourage the participants in the TEAM UP - program to have their modules tested and certified.

The Executive Director made several presentations to technical groups at SOLTEC ' 97 and the IEEE SCC21 meetings. He also represented PMC at PV-GAP / IECQ related meetings in Athens, Zurich, San Francisco and Anaheim. He was an invited participant in the National Center for Photovoltaics Workshop on PV Program Strategic Directions.

With the advent of the PV-GAP/ IECQ program for global PV certification of components and systems several near-term activities were re-directed. PMC has elected to be an active player in the PV-GAP initiative with the hope of avoiding competing programs and to participate in the potential early opportunity of establish reciprocity of certification at an international level. The obtaining of a trademark has been deferred. The Board decided to apply to IECQ be the National Supervising Inspectorate (NSI) for the IECQ PV program in the U.S.

\section{Milestone Summary:}

Board and Officers are from PV module manufactures that represent $79 \%$ of the U.S. industry

Technical, Laboratory Accreditation and Manufactures Quality System committees organized.

Monthly Board meetings held.

Annual meeting held at the $26^{\text {th }}$ IEEE PV specialist conference site in Anaheim, California.

Bóard approves PV-3 Laboratory test requirements for modules. The qualification test are as required by IEC 61215, IEC 61645, IEEE 1262.

Arizona State University / Photovoltaic Testing Laboratory (ASU/PTL) receives approval as a internationally accredited laboratory for PV module testing by American Association of Laboratory Accreditation. (A2LA).

Board approves ASU /PTL as PMC laboratory

Utility Photovoltacs Group (UPVG), 1998 specifications recommend PMC certified modules.

First manufacturer commits two module types for testing and the first group of modules tested to PV-3 at ASU/PTL 
Proficiency test to compare laboratory module out put measurements completed at NREL and Sandia.

PMC made presentations to technical groups at SOLTEC '97 and IEEE SCC-21

PMC represented at PV-GAP / IECQ related meetings in Athens, Zurich, San Francisco and Anaheim.

PMC Board expresses interest in becoming the IECQ/ National Supervising Inspectorate (NSI) in the U.S. for PV. 
Title: $\quad$ Management and Administration of the IEC/TC 82

Organization: Solar Energy Industries Association

Washington, D.C.

Contributors: Jerry Anderson, Principal Investigator (IEC TC 82 Secretary); Richard DeBlasio (IEC TC 82 Chairman and NREL Technical Monitor); Steve Chalmers (TC 82 Assistant Secretary); Vicki Rennie (SEIA Contract Administrator)

\section{Objective}

The objective of this program is to manage and administer the IEC Technical Committee 82, Solar Photovoltaic Energy Systems. This is an International committee which prepares and publishes standards for the photovoltaic industry. The committee consists of 3 Working Groups; WG 1 (Glossary), WG 2 (Modules) and WG 3 (Systems) plus a fourth group on batteries which is a Joint Working Group controlled by Technical Committee 21 (Batteries).

\section{Summary:}

Table 1 gives the countries which are members of the IEC and TC 82 . IEC TC 82 currently has published 20 PV standards. Table 2 lists the TC 82 published standards.

WG 1 is compiling a comprehensive Glossary of terms and definitions, and is establishing a unified set of symbols for PV equations and analytical expressions.

WG 2 is working on a new safety standard for modules, a module environmental test standard for marine environments, a new method for determining the linearity of a PV device and a new standard for rating modules for power and energy.

WG 3 has standards in progress on a range of topics such as systems data acquisition, PV inverter efficiency determination, pumping systems and small stand-alone home lighting systems.

The future PV standards work include plans for a new "universal" environmental qualification standard for modules which will embrace a wider variety of PV technologies, and will include several levels of difficulty so modules intended for moderate climates can be subjected to less stringent tests than those intended for extreme climatic condition, thereby enabling more economical designs. Another standard in progress is one which defines new equations and methods for field testing of modules and arrays. An effort is also underway on a new islanding standard. Another major effort is underway to develop an total quality program (IECQPV) for accrediting PV testing laboratories and for certifying PV modules, components, and systems.

\section{Discussion:}

Some of the key projects currently in progress are as follows:

Glossary - A new Photovoltaic Glossary draft, IEC 61826, has been approved for publication and will be published in early 1998. The plan is for this draft to be published as a Technical Report rather than as an International Standard. The Glossary will incorporate all of the definitions and symbols used in published TC 82 standards in Part 1, and will be a dynamic document in that as new TC 82 documents are published, the Glossary will grow to incorporate any new terms, symbols and definitions. A Part 2 annex which will incorporate a more worldwide assortment of terms and symbols is also underway. 
Device Linearity - A new linearity document, IEC 60904-10, has successfully completed final (FDIS) voting, and will be published in early 1998 . Unlike prior linearity documents which only specify linearity requirements for open-circuit voltage and short-circuit current with respect to temperature and short-circuit current linearity with respect to irradiance, the new document also includes linearity criteria for opencircuit voltage with respect to the logarithm of irradiance, and linearity of spectral response with respect to temperature and irradiance.

UV Test - A new standard, IEC 61345, which defines the UV testing requirements for PV modules has been approved and should be published in early 1998.

System Data Acquisition - A new draft standard, IEC 61724, which presents the guidelines for measurement, data exchange and analysis for PV systems is in the final voting stage (FDIS - editorial comments only) and should be published mid-1998.

Inverter Efficiency - A committee draft document (CDV), IEC 61683, which defines the methodology for determining the efficiency of inverters being used in PV system has been approved by the National Committees to progress to the next (FDIS) stage.

Thin Film Amendments - Amendments to incorporate the unique features of amorphous, thin film silicon into several existing standards have been approved. These are IEC 60904-2, Photovoltaic devices - Part 2: Requirements for reference solar cells, IEC 60904-6, Photovoltaic devices - Part 6: Requirements for reference solar modules, and IEC 60904-7, Photovoltaic devices - Part 7: Computation of spectral mismatch error introduced in the testing of a photovoltaic device and should be published mid-1998.

Energy Rating - The energy rating of modules has been a heated issue for many years. An approach has been developed which answers many of the old arguments against energy rating. The approach is based on a set of reference days which are defined and encompass four extremes of weather. Long-term outdoor testing of modules will not be required. Instead, module power will be measured for a simple matrix of irradiance and temperature conditions using optical filters and pad heaters. This can be accomplished in a few hours on a solar simulator, or outdoors in direct sunlight if desired. The energy gathering ability of the module is determined by using these power measurements in conjunction with the hourly reference day information, and then integrating the day-long tabulations. Both battery charging (amp-hour) and maximum power tracking (watt-hour) values would be determined. A draft of this standard has been prepared, and is approximately $80 \%$ complete.

Module Safety - Another future PV module document of great importance is the module safety standard. The preparation of this document has been underway for the past 2 years, and consensus in WG 2 is imminent. It will then be issued as a Committee Draft for International review, comments and voting. The module safety document has test requirements which are dependent on the usage application. Usage categories include building facades, mobile installations (boats, $R V$, highway vehicles, etc.), utility, rooftop, etc. Tests include deliberate glass breakage (nature of break: tempered granules, loose shards, retained shards, etc.), bypass diode (reverse connection to a battery), child impact test (with heavy shotfilled bag), hot spot, flammability, wet high-pot, cut susceptibility, simulated wind loads, etc. These safety tests will be conducted on a group of modules following prerequisite testing to either the IEC 1215 or IEC 1646 environmental qualification tests. 
In addition to the documents in progress by the Working Groups, the Secretariat has taken steps to add four new Working Groups to TC 82. Chairman DeBlasio and Secretary Anderson have examined the structure of TC 82 and have concluded that it has not been serving the needs of the PVindustry.

- initiate a new WG 5 group, Certification and Accreditation. The work in this group will lead to a total quality program for PV and a labeling program for PV modules, components and systems. In the USA the Powermark Corp. and ASU-PTL are already deeply involved in this effort. The Secretary has written a new draft standard and issued a New Item Proposal to the National Committees to form this working group. Voting by the National Committees is currently in progress.

- initiate a new WG 6 group, PV System Components (also known as BOS....balance of systems) dealing with environmental testing, performance and safety issues. This is now part of WG 3 work. Some of the WG 3 experts might wish to serve on both working groups, and some WG 3 members may wish to split-off, and concentrate on BOS). However, the hope is that an exclusive group dedicated to BOS will attract new participants from the BOS industry to supplement our current group of experts. The Secretary has written a new draft standard for the environmental testing of BOS components and issued a New Item Proposal to the National Committees to form this working group. Voting by the National Committees is currently in progress.

- initiate a new WG 7, PV Concentrators and Trackers. The concentrator industry has matured tremendously and many companies are now ramping-up production. TC 82 has written most of their standards to exclude concentrators because they are so different. In the process they may have inadvertently created trade barriers by this exclusion, which is a particularly sensitive issue because the flat-plate PV industry and the concentrator industry are very competitive in the marketplace. It is hoped that this project would generally be staffed by members from the concentrator industry, with some representation from the current WG 2 and WG 3 membership for cross-fertilization. The Secretary has issued a new draft standard for the testing of concentrators and issued a New Item Proposal to the National Committees to form this working group. Voting by the National Committees is currently in progress.

- initiate a new WG 8 group by preparing an NWIP which addresses the mechanical aspects of PV systems. Interestingly, even though Photovoltaics is an electrical technology, many problems and issues are mechanical in nature. Some of the issues WG 8 would address include thermal calculations, wind load calculations and testing, foundation and structural design and testing, corrosion (galvanic and electrochemical), mechanical fasteners, mechanical testing of integrated panels, etc. A draft standard and NWTP are currently being prepared by the Secretary.

- re-activate WG 4 as a true energy storage group. Even with this "energy storage" name, much of their work would still be related to batteries. This may sound contrary to the TC21/TC 82 JWG work, but it is not. The new WG 4 would deal with such battery-related topics as battery usage; battery system sizing (number, or more correctly, energy capacity), corrosion of battery enclosures, safe use in systems with batteries and diodes, environmental (reliability) test conditions for batteries, etc. Additionally, the new WG 4 will be encouraged to investigate projects using unusual storage methods such as flywheels, hydrogen storage, water pumping/storage and others. New membership plus JWG member participation is encouraged.

It is envisioned that these new groups would be small with approximately 4-6 people. More importantly than the number of people is the fact that TC 82 will be offering the PV manufacturers, designers, installers and users a much richer, more diverse and more market-responsive menu of PV standards. 
TABLE 1

INTERNATIONAL ELECTROTECHNICAL COMMISSION

TECHNICAL COMMITTEE NO. 82: SOLAR PHOTOVOLTAIC ENERGY SYSTEM

International Membership - IEC TC 82

\begin{tabular}{|c|c|c|c|}
\hline Country & $\underline{\text { Status }}$ & Country & $\underline{\text { Status }}$ \\
\hline Argentina & 0 & $\underline{\text { Japan }}$ & $\underline{\mathbf{P}}$ \\
\hline Australia & $\underline{\mathbf{P}}$ & Korea (D. P. R. of) & 0 \\
\hline Austria & $\underline{P}$ & Korea (Republic of) & - \\
\hline Belgium & 0 & Malaysia & - \\
\hline Brazil & 0 & Netherlands & $\underline{\mathbf{P}}$ \\
\hline Bulgaria & 0 & Norway & 0 \\
\hline Canada & $\underline{\mathbf{P}}$ & Pakistan & - \\
\hline China & $\underline{P}$ & Poland & 0 \\
\hline Czechoslovakia & $\underline{P}$ & Portugal & - \\
\hline Denmark & $\underline{\mathrm{P}}$ & Romania & $\underline{\mathbf{P}}$ \\
\hline Egypt & - & Russia & $\underline{P}$ \\
\hline Finland & 0 & Singapore & 0 \\
\hline France & $\underline{\mathbf{P}}$ & South Africa & $\underline{\mathbf{P}}$ \\
\hline Germany & $\underline{\mathrm{p}}$ & Spain & $\underline{\mathbf{P}}$ \\
\hline Greece & - & Sweden & 0 \\
\hline Hungary & 0 & Switzerland & $\underline{\mathbf{P}}$ \\
\hline India & $\underline{\mathrm{P}}$ & Thailand & - \\
\hline Indonesia & - & Turkey & - \\
\hline Ireland & - & U.S.A. & $\underline{\mathbf{P}}$ \\
\hline Israel & - & United Kingdom & $\underline{\mathbf{P}}$ \\
\hline Italy & $\underline{P}$ & Yugoslavia & 0 \\
\hline
\end{tabular}

NOTE: P-status is participating, 0 -status is observing 


\section{'TABLE 2}

\section{PUBLISHED IEC TC 82 STANDARDS}

1. IEC 60891: 1987, Procedures for temperature and irradiance corrections to measured I-V characteristics of (. ystalline silicon photovoltaic (PV) devices. Amendment No I (1992)

2. IEC 60904-1: 1987, Photovoltaic devices - Part 1: Measurements of photovoltaic current-voltage characteristics

3. IEC 60904-2: 1989, Photovoltaic devices - Part 2: Requirements for reference solar cells

4. IEC 60904-3: 1989, Photovoltaic devices - Part 3: Measurement principles for terrestrial photovoltaic $(P V)$ solar devices with reference spectral irradiance data

5. IEC 60904-5: 1993, Photovoltaic devices - Part 5: Determination of the equivalent cell temperature (ECT) of photovoltaic (PV) devices by the open-circuit voltage method.

6. IEC 60904-6: 1994, Photovoltaic devices - Part 6: Requirements for reference solar modules

7. IEC 60904-7: 1995, Photovoltaic devices - Part 7: Computation of spectral mismatch error introduced in the testing of a photovoltaic device

8. IEC 60904-8: 1995, Photovoltaic devices - Part 8: Guidance for spectral measurement of spectral response of a photovoltaic $(P V)$ device

9. IEC 60904-9: 1995, Photovoltaic devices - Part 9: Solar simulator performance requirements

10. IEC 61173: 1992, Overvoltage protection for photovoltaic (PV) power generating systems

11. IEC 61194: 1993, Characteristic parameters of stand-alone photovoltaic (PV) systems

12. IEC 61215: 1993, Crystalline silicon terrestrial photovoltaic (PV) modules - Design qualification and type approval

13. IEC 61277: 1995, Guide: General description of photovoltaic (PV) power generating systems

14. IEC 61646: 1996, Thin film silicon terrestrial photovoltaic (PV) modules - Design qualification and type approval

15. IEC 61701: 1995, Salt mist corrosion testing of photovoltaic (PV) modules

16. IEC 61702: 1995, Rating of direct coupled photovoltaic pumping systems

17. IEC 61721: 1995, Susceptibility of a module to accidental impact damage (resistance to impact test)

18. IEC 61727: 1995, Photovoltaic (PV) systems - Characteristics of the utility interface

19. IEC 61829: 1995, Crystalline silicon photovoltaic (PV) array - On-site measurement of I-V characteristics

20. IEC 61725: 1997, Analytical expression for daily solar profiles 

Title: The Design and Construction of a Custom-Made PV Encapsulant and Solar Cell Degradation Monitor

Qrganization: University of Colorado at Denver

Center for Environmental Sciences

P.O. Box 173364

Denver, CO 80217-3364

Tel. (303) 556-2964 Fax (303) 556-4292

Contributors: Jeffery A. Boon, Principal Investigator, Larry G. Anderson, John A. Lanning

\section{Objectives}

Copolymers made from ethylene vinyl acetate (EVA) are widely used as encapsulants in single and polycrystalline silicon photovoltaic cells. Exposure of the EVA to UV radiation is known to cause a photodegradation of the encapsulant. The photo-degradation process results in changes to both the optical and electrical characteristics of the PV cell.

Optically, photo-degradation causes a browning of the EVA encapsulant. The browning alters the transmittance characteristics of the encapsulant. After browning, the EVA absorbs more of the incident solar radiation, decreasing the efficiency of the cell. Previous work has shown that the extent of the browning process can be monitored through the use of absorbance/transmittance, reflectance, and fluorescence spectroscopy. ${ }^{1-3}$

Chemically, one of the byproducts of the photo-degradation process is acetic acid. The acetic acid corrodes the bus lines of the cell, increasing the internal resistance of the cell. The increase of the cell resistance decreases the efficiency of the cell. The amount each of the two loss mechanisms contributes to the overall decrease in cell efficiency can be determined through the use of a combination of optical and electrical measurements.

The objectives of this project are to create an instrument for studying the effects of aging on the optical and electrical properties of photovoltaic cells and precursor materials, and to write the software for using the measured properties to create a predictor for future aging behavior. The degradation monitor created during this project will be capable of taking the absorbance/transmittance spectrum of the EVA film sandwiched between glass plates. The monitor will also be able to take the reflectance and fluorescence spectra, as well as potential/current behavior, of a PV cell.

The fluorescence, reflectance, and electrical experiments need to be performed controllably over the surface of a cell which is contained either internally or, alternatively, externally in the field. Because it will be used in the field, the finished monitor needs to be light, compact, and capable of operating from battery power.

\section{Design Criteria}

The wavelength of the fluorescence excitation signal must be selectable using a $320 \mathrm{~nm}, 350 \mathrm{~nm}, 420 \mathrm{~nm}$, or $800 \mathrm{~nm}$ narrow bandpass interference filter. The spectrometer monitors the resulting emission spectra from $350: 850 \mathrm{~nm}$.

The reflectance spectra is to be obtained using a total internal reflectance sphere. The spectrometer monitors the resulting reflectance spectra from $350-850 \mathrm{~nm}$. 
The absorbance / transmittance spectra are to be collected from $200-1000 \mathrm{~nm}$

The open circuit potential of the cell is to be measured in the range of $0-10$ volts with a resolution of 0.15 $\mathrm{mV}$. The monitor will be able to source a voltage of $0-10$ volts at a current of up to 2 amps. The current will be measured from $0-2$ amps, in four ranges ( $2 \mathrm{uA}, 200 \mathrm{uA}, 20 \mathrm{~mA}$, or $2 \mathrm{~A}$ full scale) to increase the resolution at the lower current ranges.

The temperatures will be monitored at the surface of the PV cell, interior of the monitor, and at ambient temperature.

The fluorescence and reflectance spectra, and the potential / current behavior, must be taken at user selectable points over an area five inches square.

The completed monitor should be as small and light as possible, preferably fitting in a 15 inch cube and weighting less than 50 pounds. The monitor will need to be operable from either line or battery power.

\section{Approach}

The schematic of the system design is shown in Figure 1. The heart of the degradation monitor is the four charge coupled device (CCD) based spectrometers. Each of the spectrometers has been equipped with the proper slits, collection lenses, and diffraction grating, to observe the spectral region of interest for each of the experiments. The sources are connected to the sample compartments and delivery and pickup optics, and then on to the spectrometers by fiber optic cabling.

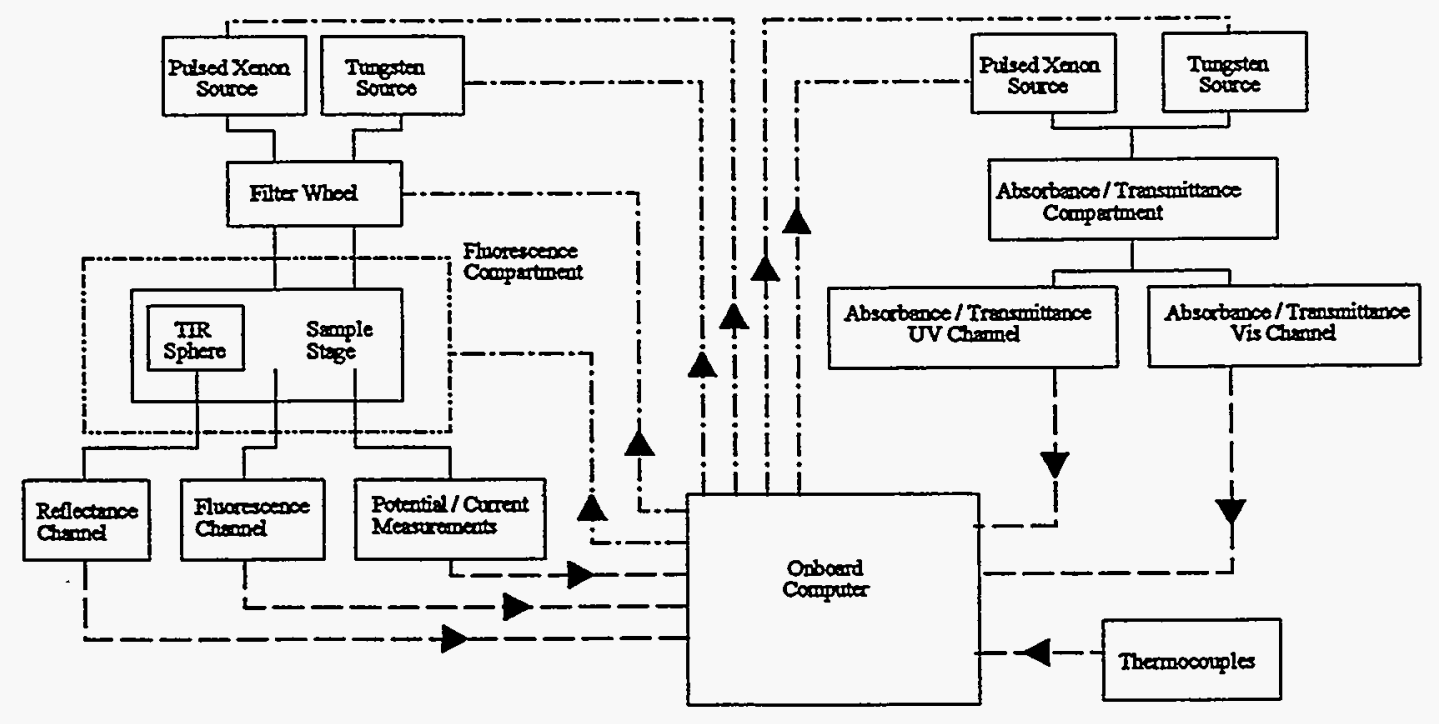

Figure 1- Degradation Monitor System Schematic.

A 16-bit analog-to-digital converter, and a 12 bit digital-to-analog converter are used to investigate the potential/current characteristics of the cells. Signal conditioning circuitry is used to provide isolation from the PV cell, increase the power delivered by the D/A board, and to convert the current, from the PV cell, to a potential appropriate for the A/D board. Signal conditioning was also included, to amplify and linearize the 
thermocouple response for the A/D board.

Since the fluorescence, reflectance, and electrical measurements must be taken over the surface of the cells or modules, the sampling head was mounted on an X-Y translational table. The table is driven by stepper motors, and allows the delivery and pick-up optics to be rastered over the surface of the sample. The resolution of the stepper system is greater than the size of the delivery and pickup optics, so the spatial resolution of the experiments is dependent only on the size of the fiber optics required by the system.

Because the spectrometers, AD D/A, and stepper motors all require computer control and interface, it was decided to use an onboard computer, instead of interfacing an external computer to the monitor. The onboard computer minimizes external cabling, allows for increased experimental speed, decreases the cost of the finished monitor, and allows for stand-alone operation. The on-board computer is PC compatible, operating under the Windows (tm) operating system, to minimize the amount of time required for a new operator to become proficient with the system. All of the software required for the operation of the monitor is also Windows (tm) based. Provisions have been made to allow for interfacing an external computer to the monitor for downloading data and experiment files. The data can then be manipulated on the external computer while the monitor continues to acquire data.

\section{Results}

To date, the design and construction of the monitor has been completed. The monitor is currently undergoing validation testing. The only difficulties encountered have been with the fluorescence measurements. The initial source used for the fluorescence measurements was not intense enough in the 280 $-320 \mathrm{~nm}$ region, to induce adequate fluorescence for the spectrometer to detect. Other source/ delivery optics combinations are being tested, to find a system that will provide adequate signal for the spectrometer.

\section{Acknowledgments}

This work is being performed under NREL subcontract number XAX-6-14454-01. We would like to express our gratitude to our Project Monitor, Dr. F. J. Pern, who is with the National Center for Photovoltaics (NCPV) at NREL.

\section{References}

1. Pern, F. J., "Factors that Affect the EVA Encapsulant Discoloration Rate Upon Accelerated Exposure", Proceedings of the First WCPEC, December 5-9, 1994, Hawii, p. 897.

2. Pern, F. J., Eisgruber, I L., Micheels, R. H., "Spectroscopic, Scanning Laser OBIC, and I-V/QE Characterizations of Browned EVA Solar Cells", Proceedings of the 25th IEEE PVSC, May 13-17, 1996, Washington D.C..

3. Pern, F. J., "Polymer Encapsulants Characterized by Fluorescence Analysis Bêfore and After Degradation", Proceedings of the 23rd IEEE PVSC, May 10-14, 1993, Louisville Kentucky, p.1113. 


\subsection{PV Domestic \\ Applications and Markets Project}





\title{
8.0 DOMESTIC PV MARKETS AND APPLICATIONS-Introduction
}

\author{
John Thornton
}

The Domestic PV Markets and Applications Project supports the analytical, applications and market development activities of the DOE National Photovoltaic Program. As part of the National Center for Photovoltaics located at NREL, we work closely with other national laboratories, DOE Headquarters in Washington, DC and the DOE Golden Field Office in Colorado to provide a focal point for market-related program activities.

\section{Million Solar Roofs Initiative Support}

This is the premier Million Roofs initiative planning year and the majority of our effort was in support of it. The consumer economics and policy analysis work developed in this task in 1997 served as a foundation for development of the Initiative. Currently there are no federal funds for the low interest financing, capital buy-downs and other potential deployment policies identified for the initiative. One of our primary objectives is to create partnership teams able to seek funding through established community financing mechanisms such as bonds, state grants, energy infrastructure reserves, etc. Other deployment paths may be utility new business development, building development or national retail chain initiatives. Ultimately we expect deployment will occur through a combination of community-based initiative mechanisms. Communities typically justify funding mechanisms through the growth planning process. We provided analysis and market development support to all potential partners and will continue to do so in FY98 .

We have also maintained our support during ongoing deregulation of the energy industry as it transitions to a more competitive environment. This transition period is expected to take 5-10 years. Each state has developed a unique set of economics related to the industry and one of the major economic components is stranded debt (50\% of equity in a monopoly environment, $20 \%$ for retail industry), which is on the order of billions of dollars for each state. PV is the highest capital cost renewable, but the most consumer preferred renewable. Installed capital cost and life cycle levelized costs are declining rapidly. By providing technical products and services which identify current and developing relationships between the PV manufacturing industry, state/local policy makers, consumers, energy industry, and the deployment industry such as developers and builders, sustainable growth markets may be established during this energy industry transition period. This work identifies the highest economic benefit to the stakeholders, for minimum $(0.5-1.0 \%)$ of the stranded debt investment in market arenas which will sustain growth in the competitive energy industry after the transition.

We provided technical support, products and services on market valuation and deployment to the following stakeholder arenas: state/local policy makers and staff, consumers, utilities, PV industry, and the deployment industry (builders, developers). 


\section{Training, Education and Technical Assistance}

During FY97, we provided training and support to Federal, state and local organizations in the selection and application of photovoltaics, supported the development of a favorable regulatory environment so that renewables are provided equal opportunities to compete in the marketplace, supported workshops and exhibitions that educate large segments of the general public to how PV can enhance their lives (during FY97 NREL-cost shared exhibits were on display at events whose attendance totaled more than 750,000), educated large sectors of the private sector, e.g., the insurance industry, as to how PV can help solve their problems, encouraged, and to a limited extent cost-shared, the demonstration of advanced PV technologies, and support the implementation of public policies, e.g., the global climate change initiatives, and supported NREL teams in technology assessment.

Specific activities included support of the President's Million Roofs Program, consumer workshops at the Denver Western Stock Show and NREL, development of public displays on PV technology at Disney's Epcot Center in Florida (March-April 1998) and the Smithsonian Institute's National Design Museum in New York City (June 1998), and co-sponsorship of a major Federal Emergency Management Agency (FEMA) conference for the use of emerging technologies for disaster prevention and recovery.

\section{Sunrayce 97}

Sunrayce 97 roared to a record finish in Colorado Springs. Winning the event overall was California State University - Los Angeles with a record setting pace averaging $43.29 \mathrm{mph}$ over the entire distance. Cal State - Los Angeles had a total elapsed time of 28:41:24 hours. The Massachusetts Institute of Technology was a close second with a total time of 29:00:20 hours, followed by Stanford University/UC - Berkeley at 29:33:15 hours. In fourth was Texas A\&M with a time of 29:47:21 hours.

The event started in Indianapolis, Indiana, on June 19 and traveled over 1,200 miles to Colorado Springs using only solar energy for power. Thirty-six solar vehicles started and thirty-five vehicles crossed the finish line making it one of the largest fields to have successfully completed a Sunrayce. Race officials chalked up the results to great preparation on the part of the students and on the especially good weather for solar vehicles. Over 1,200 college students participated directly with the solar cars. Hundreds of thousands of people saw or heard about the events from radio, newspapers or television.

\section{Support of Corporation for Solar Technology (CSTRR)}

NREL is supporting CSTRR's efforts to develop the Solar Enterprise Zone (SEZ) at the Nuclear Weapons Test Site near Las Vegas. Eventual deployment of 100 megawatts of solar power produced from renewable resources is planned. The major technologies being considered are photovoltaics, wind, and solar thermal electric. When completed, the SEZ will represent the largest single renewable energy installation in the world. 
PV Domestic Applications and Markets

Subcontracts 


\section{Title: Computer Modeling of Building Integrated Photovoltaic Systems and its Application to the Million Solar Roofs Initiative}

Organization: $\quad$ McNeil Technologies, Inc., Springfield, VA

Contributors: K. DeGroat, S. Tanner; Principle Investigators: M. Brown, H. Jones

\section{Scope}

The purpose of this study is to assist the Photovoltaics Division in promoting Building Integrated Photovoltaic (BIPV) systems through the Million Solar Roofs Initiative (the Initiative). McNeil has been actively participating in the development of the Initiative Implementation Plan. This involves developing program metrics such as the time-line for the numbers and sizes of PV systems installed through 2010, associated emissions reductions for installed roofs, and PV industry production capacities. The Implementation Plan also addresses programs to promote BIPV to Initiative partners and strategies to remove institutional and financial obstacles to BIPV.

Since the major barrier to widespread BIPV deployment is the high first cost of PV systems, detailed analyses were needed to help quantify the relative impacts of the economic and physical variables affecting PV systems. An analysis method was developed that will help the Initiative managers to identify strategic markets for the technology in the near and mid-terms. This analysis builds upon previous studies that have identified favorable markets for grid-connected BIPV systems. The market locations and applications that have been previously identified as "niche" markets include new commercial and residential constructions in Arizona, California, Hawaii, Massachusetts, and New York ${ }^{1,2}$.

In order to more accurately identify the applications and locations with the greatest potential for cost-effective BIPV in the near and mid-terms, this study demonstrates usage of a combination of available computer analysis tools to conduct BIPV system analyses in the markets previously identified as well as in other locations including Colorado, Georgia, Nevada and Texas. This is accomplished by performing life-cycle cost comparisons between different types of buildings in the regions identified with the aid of sophisticated computer simulation programs.

The scope of the effort only demonstrates the utility of the modeling tools combined to conduct the analysis by applying them to a baseline set of assumptions and evaluating the results. However, with the baseline approach documented in this study, further analysis of building design, customer profiles, and PV system sizing and incentives can be conducted to help target the highest value niches for BIPV market development.

\section{Objectives}

This study evaluates the physical and economic performance of utility-interfaced BIPV systems taking into account location specific climate, building energy use, and utility electricity rate data. The analysis methodology developed for this study makes use of computer simulation programs that generate for each hour of an entire year: building electric load profiles; location specific weather data; photovoltaic array physical performance; and, utility electricity rates for both consumption and supply.

\footnotetext{
' Wenger et al., "Niche Markets for Grid-Connected Photovoltaics," U.S. Department of Energy.

${ }^{2}$ Frantzis, $L$ et al., "Building Integrated Photovoltaics: Analysis and U.S. Market Potential" Arthur D. Little, Inc. Ref No. 44513 Feb 1995
} 
Break-even BIPV system costs are generated for residential and commercial buildings in ten locations throughout the United States. This analysis methodology shows the relative impact on the cost-effectiveness of BIPV systems of local input factors such as climate and utility electricity rates.

\section{Methodology}

Analyses are conducted for both a typical residential and a typical commercial office building in ten locations: Atlanta, GA; Boston, MA; Denver, CO; Fort Worth, TX; Honolulu, HI; Las Vegas, NV; Los Angeles, CA; Phoenix, AZ; San Francisco, CA; and, Syracuse, NY. The locations were chosen because of the large variation in climate among them, and because the majority of them fell within the boundaries of the previously identified "niche" markets. The building types were chosen because they possessed different energy consumption patterns, which should affect the economics of the BIPV systems involved.

A substantial amount of data was collected from the residential and commercial building energy surveys conducted by the Energy Information Administration on the energy consumption and number of different sizes and types of buildings. As well, data was collected on the average energy use, basic design, and occupancy-of these buildings. Based on this data, the buildings that were chosen for the analysis are:

- a 2,280 square foot single detached home of two stories and 1,950 square feet of heated floor space. TheHVAC system includes gas heat/hot water and electric air conditioning. The home incorporates a $4.3 \mathrm{~kW}$ integrated photovoltaic roof.

- a 10,000 square foot office building of two stories. The HVAC system includes gas heat/hot water and electric air conditioning. The building incorporates a $30 \mathrm{~kW}$ photovoltaic integral roof array.

\section{Analysis Tools}

In order to perform the analyses based on hourly calculations a computer model was constructed that integrates a building energy simulation program, a photovoltaic array simulation program, an electricity cost calculation program, and life-cycle costing analysis. For each simulation, the model generates as a final result the break even cost of the specific BIPV system as an overall system cost and in dollars per watt. For the purposes of this analysis, the break even cost is the life-cycle cost of the BIPV system which is equal to or less than the cost of utility supplied electricity over the lifetime of the BIPV system.

An important feature of the model is that it performs hourly calculations for each hour in a full year. That is, the model generates an hourly load profile for the building and combines it with the hourly performance of the photovoltaic array integrated into that building, and matches the resulting electric load profile with the actual electricity rates of the local utility on an hourly basis.

\section{Model Components}

The following components are brought together as shown on the BIPV Model Input/Output diagram on the following page:

Energy-10 is a building simulation program that is primarily used by architects and engineers to design lowenergy buildings. The model uses Energy-10 to generate a full year's hourly load profile for a low energy building. Energy-10 has built in defaults describing typical energy usage profiles for a variety of commercial buildings and residential buildings. These defaults are based on the residential and commercial building 
surveys conducted by the Energy Information Administration. As well, Energy-10 applies default passive solar design strategies to produce reference case low-energy buildings.

PVFORM is used to simulate the physical performance of a specified PV array. For the purposes of this study, PVFORM requires the hourly load data generated by Energy-10 and data from the same TMY2 climate data set used by Energy-10. The PVFORM climate data files were constructed using data from the TMY2 (typical meteorological year) data set, and include hourly values for direct radiation, global radiation, ambient temperature, and wind speed. Studies have shown that PVFORM can predict PV array performance to within $5 \%$ of actual experience.

maketmy2 and pvconvert were generated with a standard C compiler. They are used to transform Energy10 output and typical meteorological year data into a format suitable for PVFORM input.

ERATES is a program that allows the input of electric utility rate schedules. It will match an hourly electricity load profile with the specified utility rate and calculate the annual electricity costs associated with that load profile.

The LCC Model calculates the break even cost of the BIPV system by setting the BIPV system life cycle cost (LCC) equal to the LCC of the non-PV system-which is the present value of the utility electricity costs over the life of the system. The break even cost for the BIPV system is found by solving the LCC equation (i.e. $\mathrm{LCC}=$ the present-value of the installed system + the present-value capital replacement cost + the present value energy cost + the present-value of operating, maintenance, and repair costs) for the present value of the installed system cost.

\section{BIPV MODEL INPUT/OUTPUT DIAGRAM}

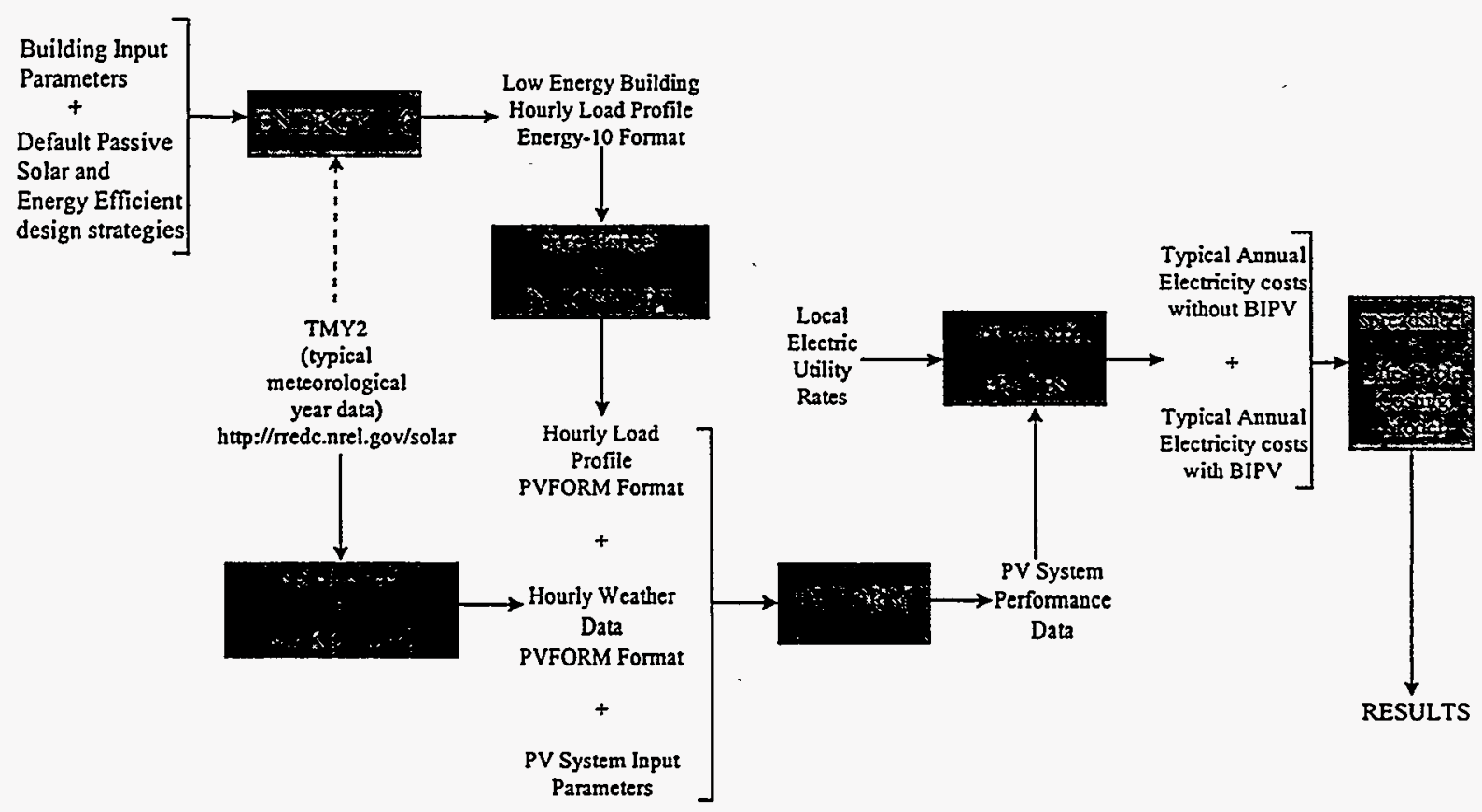




\section{Results Summary}

The overall results are summarized in the following table. The table shows the break even prices in dollars per watt for both the commercial and residential building in each location. The break even prices are shown for both time-of-use (TOU) rate schedules and block rate schedules with the higher of the two appearing in the final column. An "nm- $r$ " beside the location name indicates active residential netmetering, and an " $\mathrm{nm}-\mathrm{rc}$ " indicates active net-metering in both residential and commercial buildings.

Break Even Prices in Dollars per Watt (\$/W) for Commercial and Residential BIPV Systems

\begin{tabular}{|c|c|c|c|c|c|c|c|}
\hline \multirow[b]{2}{*}{ Code } & \multirow[b]{2}{*}{ Location } & \multicolumn{2}{|c|}{ Commercial $(\$ / W)$} & \multicolumn{2}{|c|}{ Residential (\$/W) } & \multicolumn{2}{|c|}{ Highest $(\$ / W)$} \\
\hline & & TOU & Block & TOU & Block & Commercial & Residential \\
\hline ATL & Atlanta, GA & 1.49 & 0.64 & & 1.19 & 1.49 & 1.19 \\
\hline $\mathrm{BOS}$ & Boston, MA (nm-rc) & 0.88 & 0.76 & & 1.92 & 0.88 & 1.92 \\
\hline $\mathrm{DEN}$ & Denver, $\mathrm{CO}$ & & 0.64 & & 1.68 & 0.64 & 1.68 \\
\hline FW & Fort Worth, TX (nm-r) & & 0.65 & & 1.94 & 0.65 & 1.94 \\
\hline HON & Honolulu, HI & 2.42 & & & 2.95 & 2.42 & 2.95 \\
\hline LVS & Las Vegas, NV (nm-r) & & 1.36 & & 1.87 & 1.36 & 1.87 \\
\hline $\mathrm{LA}$ & Los Angeles, CA(nm-r) & & 1.18 & 5.53 & 3.74 & 1.18 & 5.53 \\
\hline PHN & Phoenix, AZ (n'm-r) & & 2.00 & 2.37 & & 2.00 & 2.37 \\
\hline & San Francisco, CA (nm-r) & 2.79 & & 4.21 & 3.43 & 2.79 & 4.21 \\
\hline SYR & Syracuse, NY (nm-r) & & 1.36 & & 2.05 & 1.36 & 2.05 \\
\hline
\end{tabular}

\section{Conclusion and Directions for Further Analyses}

This project provides a baseline methodology for analyzing BIPV systems in local areas. This methodology will be used to further efforts in the Million Roofs Initiative to select appropriate niche markets for BIPV technology. The results of this study emphasize the overriding importance of local utility rates and legislation such as net metering in determining the cost-effectiveness of BIPV systems. This methodology can also be used to perform case studies for localities considering participation in the Million Roofs Program. The method will give MRI participants more specific knowledge concerning the baseline cost-effectiveness of $\mathrm{PV}$ in their area.

Future work should be focused on the following areas:

- The LCC component should be enhanced to incorporate higher order financial analysis. The implications of solar energy tax credits, forms of interest deduction from Federal and state income taxes, and low-cost PV system financing should be evaluated as part of the comprehensive analysis. Local incentives such as funding through portfolio standards and lowcost vendor financing should be evaluated as well.

- The electricity cost calculation component needs an upgrade. The component used for this study, ERATES, was not able to analyze some of the more complex rate schedules. Some of these rates, in particular the commercial time-of-use rates, may significantly improve the breakeven cost of BIPV systems. 


\title{
Title: $\quad$ Renewable Energy Analysis and Economic Applications for Electric Power
}

\author{
Organization: Pacific Energy Group, Walnut Creek, California
}

Contributors: Howard Wenger and Tom Hoff (Pacific Energy Group); Christy Herig (NREL Technical Monitor), Tom Starrs (Kelso, Starrs, \& Associates), Les Nelson (Western Energy Services), Karl Knapp (consultant), Robert Shaw (Arete Corporation), and Richard Perez (State University of New York)

\section{Introduction}

In the context of a rapidly changing electric utility industry, this NREL subcontract has addressed renewable energy economics, markets, and policies for end-users, utilities, independent power and energy service companies, and government and regulatory institutions.

Ultimately, a primary goal of this subcontract is to help speed the commercialization of grid-connected PV in the U.S.. The figure to the right shows the "bottom-up" strategy to achieve this goal. Much of the work performed during the past year has focussed on "market conditioning" activities to understand renewable energy markets and help put in place new policy initiatives that facilitate these markets.

\section{New Policy Initiatives}

The structure and technical, economic, and legal implications of a number of policy initiatives were examined during the past year. Results and recommendations of these analyses were presented in state government and regulatory arenas across the country [120]. These policy initiatives include net metering, renewable energy trust funds for buy-downs ( $\$ / W$ and $\$ / k W h)$, portfolio standards, low-interest loans, and various tax policies.

As a result, the work performed under this subcontract has contributed to the introduction and maintenance of sound policies that encourage the growth of renewable technology markets. See the following table for examples.

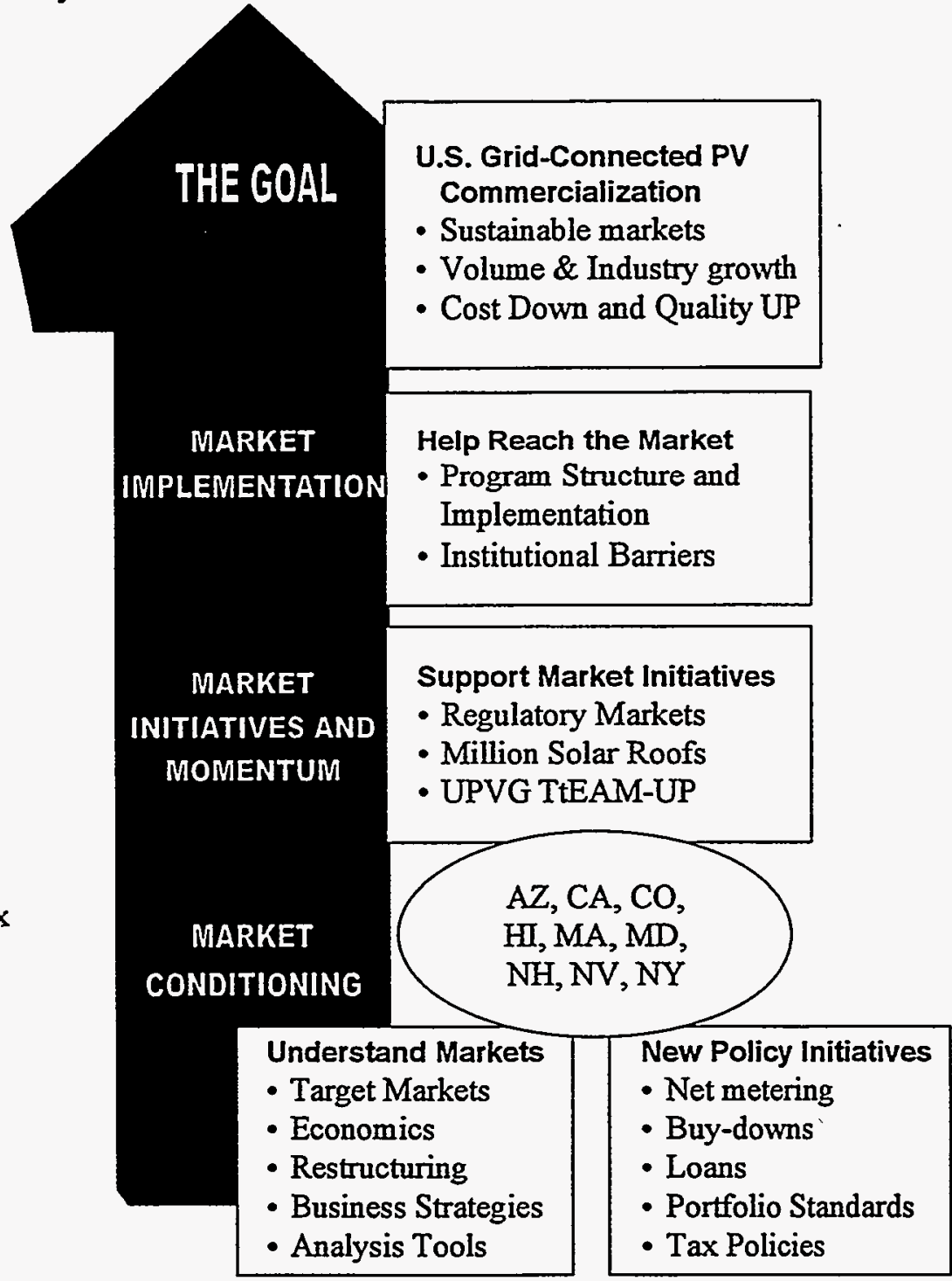




\section{Examples of New Policies}

(this subcontract contributed to these new policies in the past year)

\begin{tabular}{|c|c|c|c|}
\hline State & Policy Arena & Policy Initiative & Status \\
\hline Arizona & $\begin{array}{l}\text { Regulatory } \\
\text { Commission }\end{array}$ & $\begin{array}{l}\text { Portfolio Standard for Solar: Designed } \\
\text { to result in } 250-330 \mathrm{MW} \text { of new solar by } \\
2010\end{array}$ & $\begin{array}{l}\text { Ordered, December } \\
1996\end{array}$ \\
\hline California & Legislature & $\begin{array}{l}\text { Renewables Trust Fund: } \$ 54 \text { million in } \\
\text { buy-downs for emerging technologies, } \\
\text { including PV }\end{array}$ & $\begin{array}{l}\text { Enacted, December } \\
1997\end{array}$ \\
\hline $\begin{array}{l}\text { Colorado, } \\
\text { Florida, } \\
\text { Oregon }\end{array}$ & $\begin{array}{l}\text { Various } \\
\text { Stakeholders }\end{array}$ & Net Metering & In progress \\
\hline Federal & Legislature & Net Metering & In progress \\
\hline Iowa, Maine & $\begin{array}{l}\text { Regulatory } \\
\text { Commission }\end{array}$ & $\begin{array}{l}\text { Net Metering (maintain pre-existing net } \\
\text { metering statutes and orders) }\end{array}$ & In progress \\
\hline Hawaii & Legislature & Net Metering & In progress \\
\hline Maryland & Legislature & Net Metering & $\begin{array}{l}\text { Enacted, October } \\
1997\end{array}$ \\
\hline Nevada & Legislature & $\begin{array}{l}\text { Portfolio Standard: Designed to result } \\
\text { in } 100 \mathrm{MW} \text { of new renewables by } 2008 \\
\text { Net metering and property and sales tax } \\
\text { exemptions for solar also enacted }\end{array}$ & Enacted, July 1997 \\
\hline $\begin{array}{l}\text { New } \\
\text { Hampshire }\end{array}$ & Legislature & Net Metering & In progress \\
\hline New York & Legislature & Net Metering & $\begin{array}{l}\text { Enacted, September } \\
1997\end{array}$ \\
\hline
\end{tabular}

In addition to these aforementioned policy initiatives that focussed in particular states, general policy work was conducted that provided comparative analysis for the U.S. and target markets within the U.S. [21]. A consistent result is that a packaged mix of policy incentives are needed to provide sufficient incentives to successfully seed and grow renewable energy markets.

\section{Understanding Markets}

A number of activities conducted during the past two years have contributed to our understanding of target markets and business strategies to access these markets. This in turn provided the understanding necessary for strategic planning and formation of policies. Work conducted over the past year has focussed on researching new markets and developing analysis techniques that account for the various benefits of renewables. 


\section{A New Market for PV: Micro-Grids}

Distributed generation technologies can also exploit a market opportunity in the newly emerging regulatory environment: micro-grids. A micro-gird is an electrically isolated set of generators that supply all of the demand of a group of customers.

Micro-grids are not burdened with the costs of the existing system (which can result in a cost savings) but they must reliably supply all of the demand without the benefits of a diverse set of loads and generation technologies (which can result in a cost increase). This research develops a method to evaluate the technical and economic feasibility of micro-grids [22].

A key finding of the work is that a high degree of reliability can be achieved with a fairly small number of generating units. The problem then becomes a trade off between cost economies of scale and the system capacity required to achieve a given level of reliability. For example, Figure 1 presents the system capacity required to satisfy a $100 \mathrm{~kW}$ load and the per unit capital cost as a function of plant size; it assumes each plant has a forced outage rate of 5 percent, that the reliability level is an outage of 1 day in 10 years, and that generator price follows a logarithmic scale. The figure shows that, while larger plants have a lower per unit cost than smaller plants, the system must be substantially over designed with the larger plants so that the total cost is more; e.g., a system composed of $1 \mathrm{~kW}$ plants costs $\$ 230,000$ (capacity of $115 \mathrm{~kW}$ at a cost of $\$ 2,000 / \mathrm{kW}$ ) while a system composed of $50 \mathrm{~kW}$ plants costs $\$ 350,000$ (capacity of $250 \mathrm{~kW}$ at a cost of $\$ 1,400 / \mathrm{kW})$.

The general conclusion of the work is that the use of distributed generation in a micro-grid has the potential to be economically attractive. This may represent an important new market for PV.

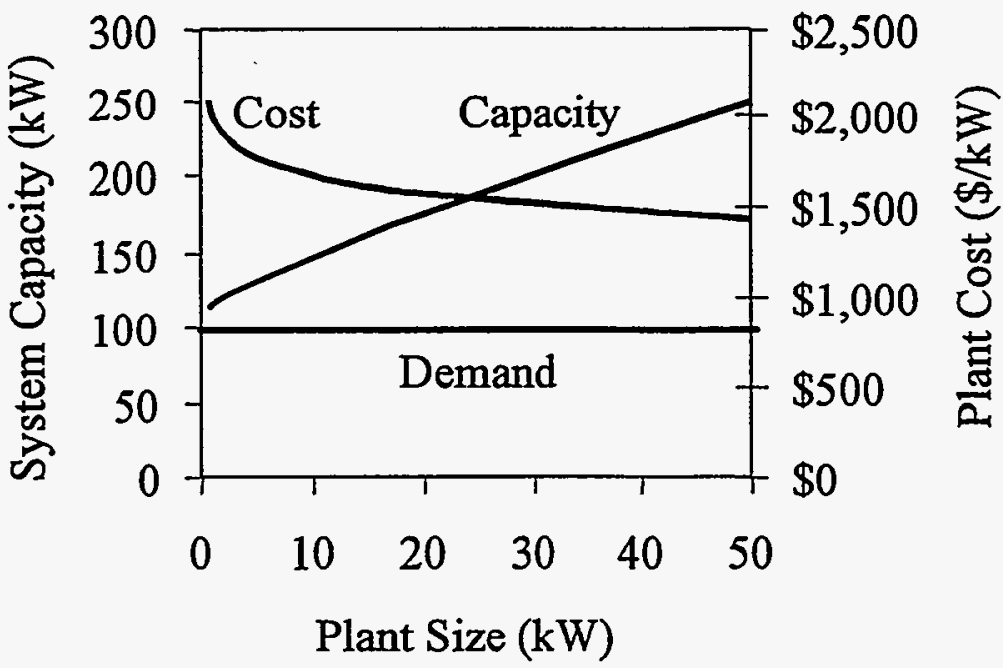

Figure 1. Tradeoff is between cost economies of scale and required system capacity. 


\section{Distributed PV Generation}

This work presents a method to calculate the cost of satisfying transmission and distribution (T\&D) system capacity needs as a function of investment modularity and lead-time [23]. It accounts for the dynamic nature of demand uncertainty, the decision-maker's risk attitude, and the correlation between costs and overall firm value (in both complete and incomplete markets). Results indicate that the modularity and short lead-times associated with distributed resources can substantially increase their attractiveness in comparison to long lead-time, large-scale T\&D investments. Results also suggest that distributed resources can operate as a type of "load growth insurance" if demand is positively correlated with profits (so that costs are incurred when profits are high) and if the distributed resource costs are part of a larger portfolio that cannot be diversified.

Figure 2 presents the break-even price as a function of investment lead-time for a particular situation. The top curve is for a modular investment, such as a PV plant. The bottom curve is for a non-modular investment, such as a transmission line. The figure suggests that a modular investment that has a short lead-time (the top curve at the left of the figure) has a break-even price that is five times the amount of a non-modular investment that has a long lead-time (the bottom curve at the right of the figure). That is, the decision-maker can afford to spend five times as much on the modular investment with a short lead-time.

A multimedia presentation was developed that describes the results of this work.

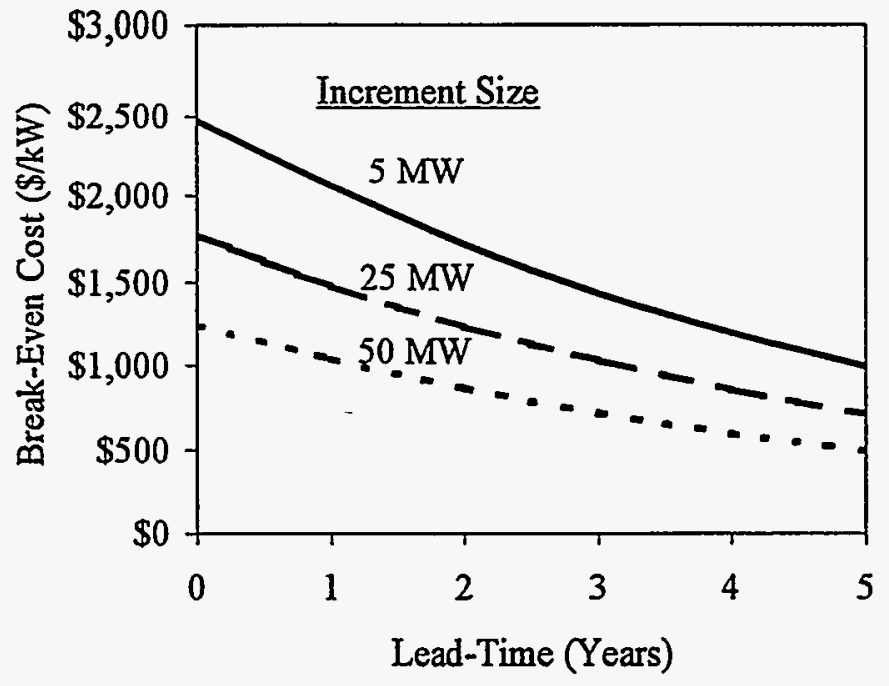

Figure 2. Break-even Cost as a Function of Modularity and Lead-Time.

\section{Risk Management Benefits of PV}

Regulatory and technical forces are causing electric utilities to move from a natural monopoly to a more competitive environment. Associated with this movement is an increasing concern about how to manage the risks associated with the electric supply business. One approach to managing risks is to purchase financial instruments such as options and futures contracts. Another approach is to own physical assets that have low risk attributes or characteristics. The second approach is the one taken in this work. 
This work evaluates how investments in renewable energy technologies can mitigate risks in the electric supply industry [24]. It identifies risks that are known to be of concern to utilities and other power producers. These risks include uncertainty in fuel prices, demand, environmental regulations, capital cost, supply, and market structure. The research then determines how investments in renewables can mitigate these risks. Methods are developed to calculate the value of renewables in terms of their attributes of fuel costs, environmental costs, lead-time, modularity, availability, initial capital costs, and investment reversibility.

The focus of this research was on the development of methodology to assess value. Several of the methods were then applied to a case study using conservative assumptions. The benefits that were included were high availability due to modularity, a more robust strategy to dealing with demand uncertainty due to modularity and short lead-time, elimination of fuel price risk, and a reduction in environmental cost risks. The results are shown in Figure 3. Cost estimates are also included in the figure. Results suggest that the risk-mitigating benefits can be significant and can shorten the time it will take for PV to become costcompetitive in grid-connected applications.

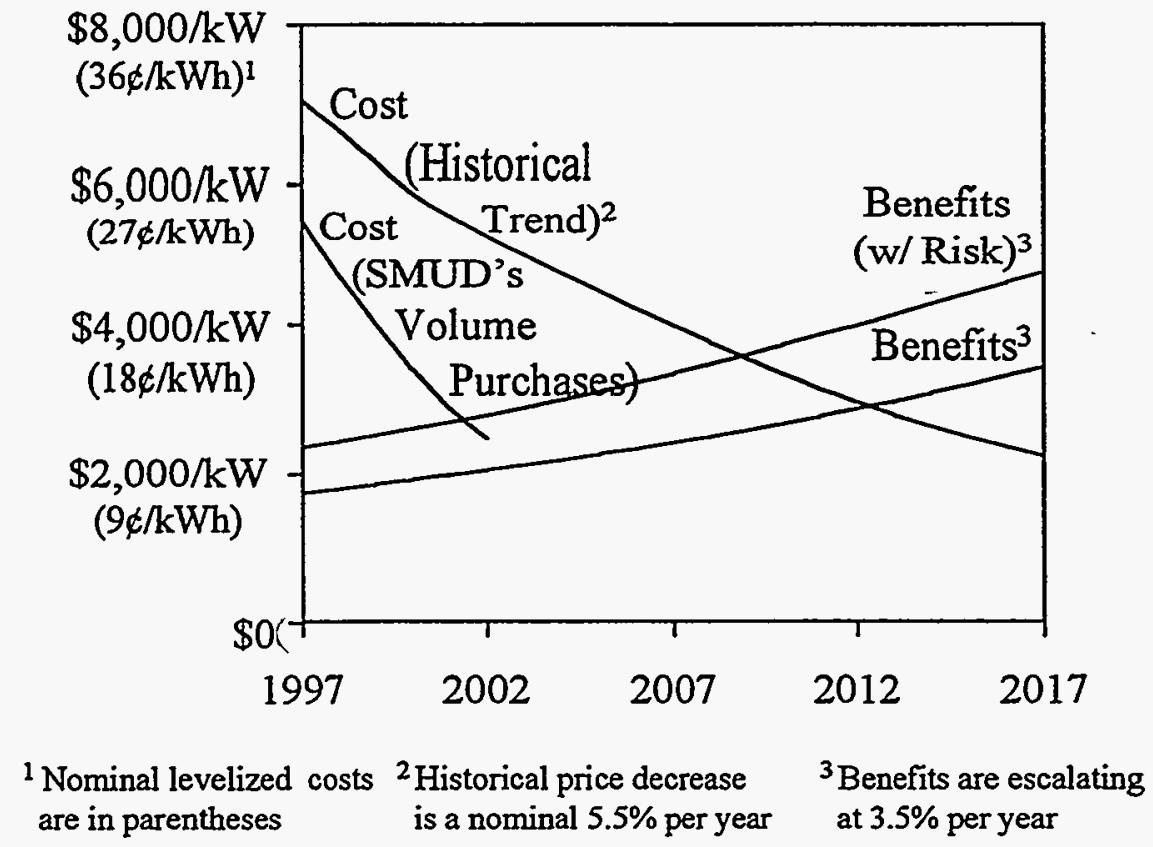

Figure 3. Costs and benefits of PV with and without risk mitigating value.

\section{Credit Ratings and Photovoltaic Investments}

Competition is changing the financial community's view of firms in the electric supply industry; credit ratings are a key indicator of this view. This work examined the relationship between credit ratings and financial benefit and summarized the factors credit rating agencies use to assign ratings. We describe investments that two utilities have made in photovoltaics (Sacramento Municipal Utility District and Tucson Electric Power Co.) and discuss how these investments may be viewed positively from a credit rating perspective. 


\section{References}

1. H. Wenger and L. Nelson, "Transition to Sustainable Markets: A Consensus Proposal to Allocate AB 1890 Funds for Emerging Renewable Photovoltaic Technology", Submitted to the California Energy Commission, $11 / 96$

2. H. Wenger, "Transition to Sustainable Markets: A Plan for AB 1890 Restructuring Funds", Presentation to the California Energy Commission, 11/96

3. H. Wenger and L. Nelson, "Supplementary Comments on PV program Details", Submitted to the California Energy Commission, 1/97

4. H. Wenger and L. Nelson, "Comments on the Committee Draft Policy Report on AB 1890 Renewables Funding dated February 14, 1997", Submitted to the California Energy Commission, 2/97

5. H. Wenger, "Net Metering for Nevada", Presentation to Nevada Stakeholders, Carson City, Nevada, 3/97

6. H. Wenger, "Nevada Domestic Energy Portfolio Standard Analysis", Fact Sheet, Submitted to the Nevada State Legislature, 6/97

7. H. Wenger, "Portfolio Standard Analysis", Spreadsheet and analysis submitted to the Corporation for Solar Technology and Renewable Resources, SEIA, and NV Attorney General's Office, 6/97

8. H. Wenger, "Net Metering for Colorado", Presentation to Colorado Stakeholders, Denver, Colorado, 6/97

9. H. Wenger, "Net Metering Economics", Presentation to Oregon Stakeholders, Portland, Oregon, 9/97

10. H. Wenger, "Major Findings: Solar Portfolio Standard Analysis", Submitted to the Arizona Corporation Commission, 10/97

11. H. Wenger, "Solar Portfolio Standard Analysis", Submitted to the Arizona Corporation Commission, 10/97

12. H. Wenger, "Solar Portfolio Tool", Spreadsheet Software Submitted to the Arizona Corporation Commission, $10 / 97$

13. C. Herig, "Net Metering for Florida", Presentation to Florida Stakeholders, 2/97 (prepared by H. Wenger)

14. T. Starrs, "Issues and Strategies That Shape the Future: Net Metering and Utility Ownership of Distributed Generation." Utility Photovoltaic Group Conference, Albuquerque NM, October 1997

15. T. Starrs and H. Wenger, "Net Metering Trends." Photovoltaics for Utilities Session, Utility Photovoltaic Group Conference, Albuquerque NM, October 1997

16. T. Starrs, "Net Energy Metering." Presentation to the New Hampshire House of Representatives, Science, Technology \& Environment Committee, Concord NH, September 1997

17. T. Starrs, "Net Metering: An Overview of Legal Issues." Photovoltaic Net Metering Workshop, Denver CO, June 1997

18.^T. Starrs and H. Wenger, "Net Energy Metering." Presentation to the Nevada State Assembly, Committee on Governmental Affairs, Carson City NV, May 1997.

19. T. Starrs, L. Nelson, and H. Wenger, "Net Metering." Solar Policy Analysis Workshop, Honolulu HI, January 1997

20. Thomas J. Starrs \& Howard J. Wenger, Tomb of the Unknown Renewable Energy Program. Home Energy Magazine (In Publication)

21. H. Wenger and C. Herig, "Policy Options to Accelerate Grid-Connected PV Markets", Presented at the American Solar Energy Society's Solar '97 Conference, Washington, D.C., 4/97

22. T. E. Hoff, H. J. Wenger, C. Herig, and R. W. Shaw, Jr., "Distributed Generation and Micro-Grids", Conference Proceedings of the $18^{\text {th }}$ Annual North American Conference of the USAEE, Sept. 1997

23. T.E. Hoff, "Using Distributed Resources to Manage Risks Caused by Demand Uncertainty," The Energy Journal, January 1998

24. T.E. Hoff, Integrating Renewable Energy Technologies in the Electric Supply Industry: A Risk Management Approach, NREL/SR-520-23089, July 1997 
Title:

Organization:

Contributors:
Solar Resource Utility Load Matching Assessment

Atmospheric Sciences Research Center, The University at Albany, Albany, and New York

Richard Perez, principal investigator, Robert Seals, Howard Wenger, Tom Hoff and Christy Herig

\section{OBJECTIVES}

The project was initiated in 1991 and is now nearing completion. Its initial objective was to estimate the effective capacity of photovoltaics (PV) for a sample of US utilities. This investigation required time-coincident hourly electric load and local PV output data simulated from satellite remote sensing. Hence a secondary, but fundamental, objective of this work was to demonstrate the accuracy of satellite remote sensing for the considered application. The project continued with a larger sample of US utilities as well as "sub-utilities" -- buildings and substations. A well-defined relationship was noted between utility load shapes and PV effective capacity, which allowed us to map the effective capacity of PV in the US. This relationship was found to remain valid for substation and building loads as small as a few $100 \mathrm{~kW}$ [1]. Last year we reported on the year-to-year variability and long term trends of US effective capacity maps, and we provided initial results of the seasonal variability of PV these maps. We also reported on the extension of our analysis to end-users and presented evidence end-use type/load match relationship.

Our current objectives are to (1) produce seasonal PV effective capacity maps, (2) apply the results developed through the project to relevant analyses and case studies. and (3) in the context of a follow-on effort, to use these results to develop a comprehensive valuation of demand-side commercial PV systems.

\section{RESULTS AND DISCUSSION OF ACTIVITIES COMPLETED IN FY 97}

Recap on past findings: The following results have been documented during past phases of this study:

- Satellite-based solar resource data can be used to adequately address PV-utility interaction issues in the US.

- PV's effective capacity, quantified by the Effective Load Carrying Capability (ELCC) parameter, is substantially larger than PV's capacity factor for most US utilities.

- Effective capacity is not well correlated with the solar resource. However, a well-defined relationship exists between effective capacity and simple load shape parameters.

- A map showing the distribution of PV' effective capacity in the US was produced. This map shows that regions of highest capacities do not always overlap with "traditional" high solar resource regions. Regions of highest effective capacities are associated with the following factors: (1) existence of marked summer heat waves, (2) summer electric loads driven by commercial consumption and (3) small proportion of electric heating.

- Eight years of utility load data were analyzed to investigate the year-to-year variability of PV effective capacity. Regional patterns were found to be very stable, with highest year-to-year variability observed in Florida and the southern Atlantic seaboard. The Northeastern Atlantic seaboard, California and the Great Plains exhibited the lowest variability.

- Investigating end-use loads as opposed to utility-wide loads, we found that PV effective capacity was highest for air-conditioned $(\mathrm{A} / \mathrm{C})$ office buildings followed by hospitals [1].

Update on current investigations: The FY 97 tasks highlighted in this report include

- The mapping of seasonal effective capacity of PV

- The mapping of minimum storage requirements to increase PV's capacity to near $100 \%$

- An investigation of PV as a long-term solution to power outages

Seasonal Effective Capacity of PV: Figure 1 shows the distribution of annual PV effective capacity as parameterized by the Effective Load Carrying capability benchmark. 


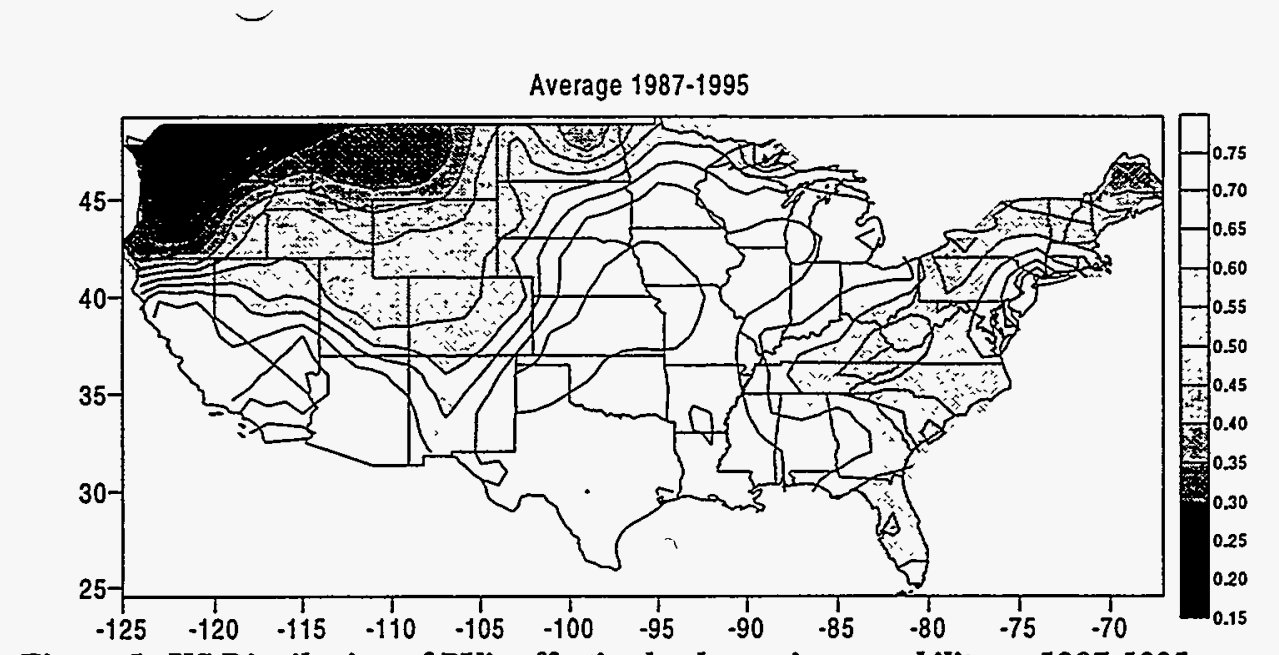

Figure 1: US Distribution of PV's effective load carrying capability -- 1987-1995 average

(2-axis trk. PV, low grid penetration)

Annual PV effective capacity represents the physical ability of PV to effectively meet the highest local loads, hence to effectively displace/reassign peak generating capacity and T\&D upgrades.

However, initial grid connected PV deployment will likely occur on the customer-side. Hence, given the fact that customer-side capacity is most often billed on a monthly basis, monthly effective capacity is, at present, an important piece of information for the valuation of such PV systems.

From our experimental data set including 50 utilities, we observed that there existed a strong relationship between monthly maximum temperature and the ratio between monthly ELCCs and the highest monthly ELCC observed for a given utility. This relationship is shown in Figure 2.

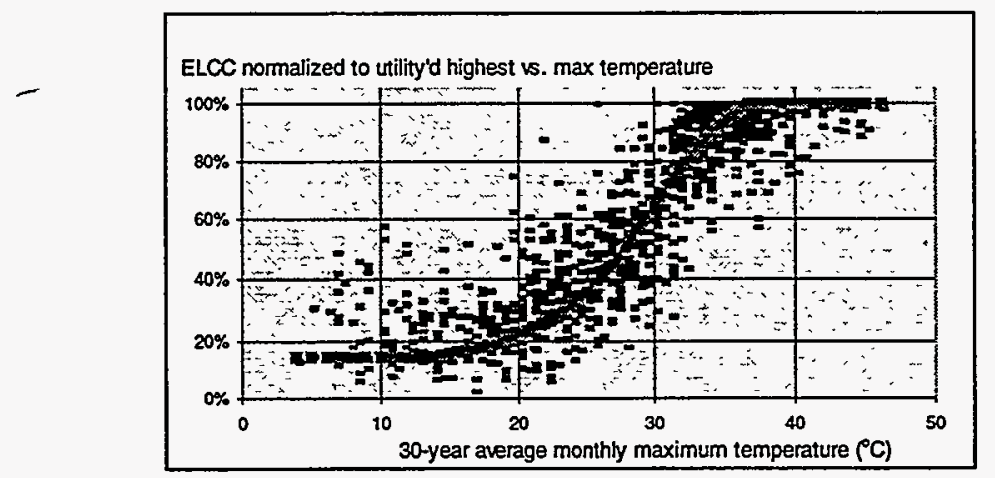

Figure 2

Using the fifty experimental utilities as a regional template for maximum achievable ELCC, we produced monthly ELCC maps based upon the above relationship and monthly maximum temperatures from the National Solar Radiation Database. Examples of these maps are provided in Figures 3 for the months of January, April, July and October.

Application of Results to PV valuation: In a 1996 study, Wenger et al. [2] looked at the valuation of demand-side residential systems. In a follow-on phase of work, we will be addressing demand-side PV commercial systems, making direct use of the knowledge assembled throughout this project. Our objective is to draw a map of the United States showing the variations of the Breakeven Turnkey Cost (BTC) of PV systems as a threshold of economic feasibility. The BTC of a PV system depends on the financial value that it brings to its owner and on a set of financial parameters (e.g., financial rates, tax rates, inflation, etc.). While the financial parameters are largely siteindependent, the value-to-owner is not. This value depends on the solar resource - energy and effective capacity and on the financial value of this resource as measured by energy and demand retail rates.

The value-to-customer will be determined for commercial customers of most major US utilities, using (1) mapped seasonal PV energy output, (2) mapped seasonal PV effective capacity and (3) commercial energy and demand retail rates for each utility. 


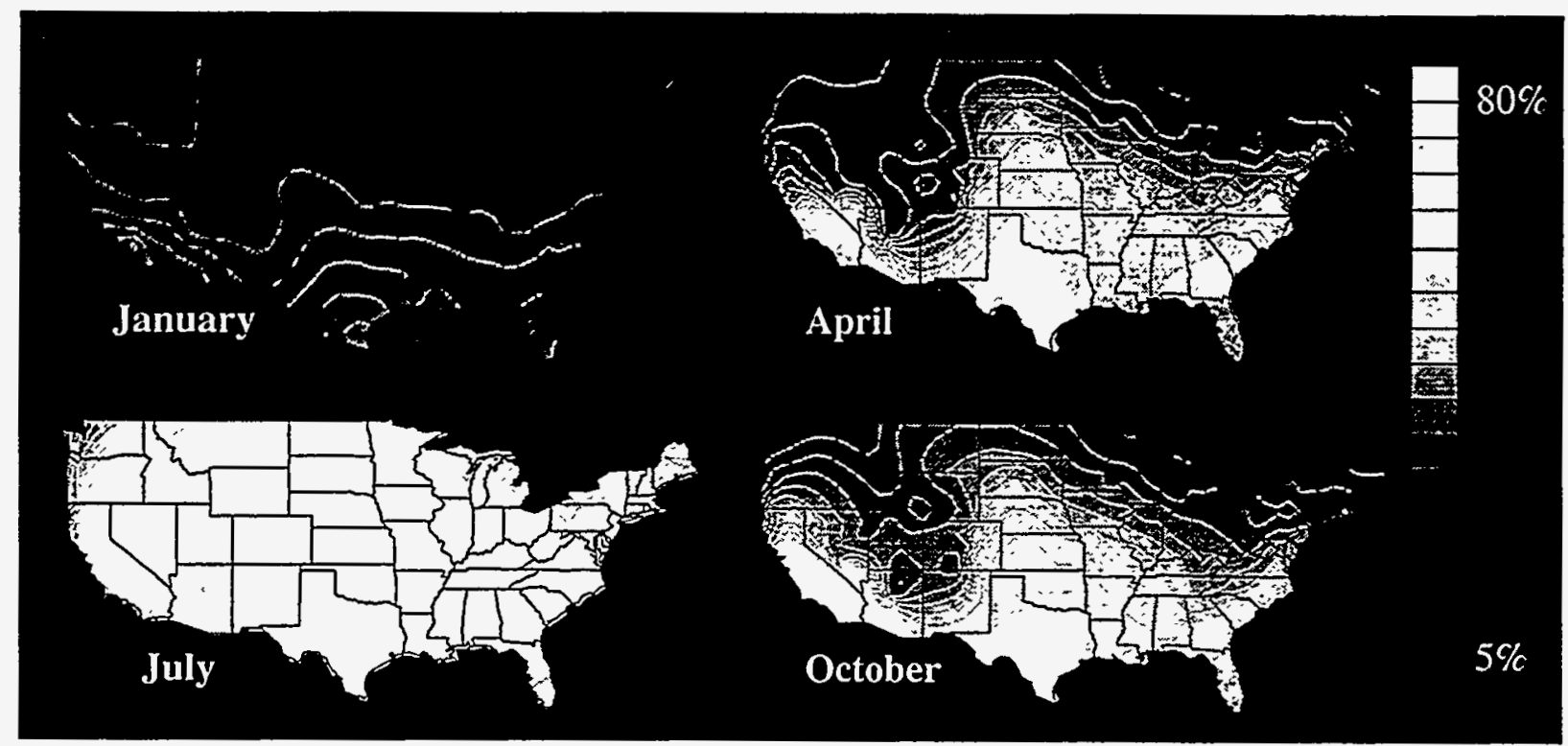

Figure 3: Seasonal Effective Load Carrying Capability of PV (2axis trk, low grid penetration)

Energy storage as a measure of, and a means to enhance PV effective capacity[ 3]: The ELCC is a statistical measure of effective capacity, based on loss of load probability calculations. However, this statistical measure of capacity is not entirely convincing to utility planners, because there remains a probability that PV output could fall down to zero due to the presence of clouds during a peak load event. In order to address this argument, an alternative measure of PV capacity credit was developed. This measure is the Minimum Buffer Energy Storage (MBES). As the ELCC, the MBES is derived from experimental time-coincident load and PV output data. However, the MBES is not determined probabilistically, but with respect to the worse possible conditions. The MBES represents the minimum amount of stored energy necessary to insure that all loads above a threshold (defined as the peak load minus the size of the PV system) are met, amounting to a PV effective capacity of $100 \%$.

The MBES was mapped following an approach similar to the ELCC. Examples of these maps are shown in Figure 4 for fixed and tracking PV arrays for a grid penetration of 10\%.The units are installed PV system-hours (e.g., one PV system-hour for a $5 \mathrm{GW}$ utility peak load would be $500 \mathrm{MWh}$ ). The strong synergy between PV and storage becomes

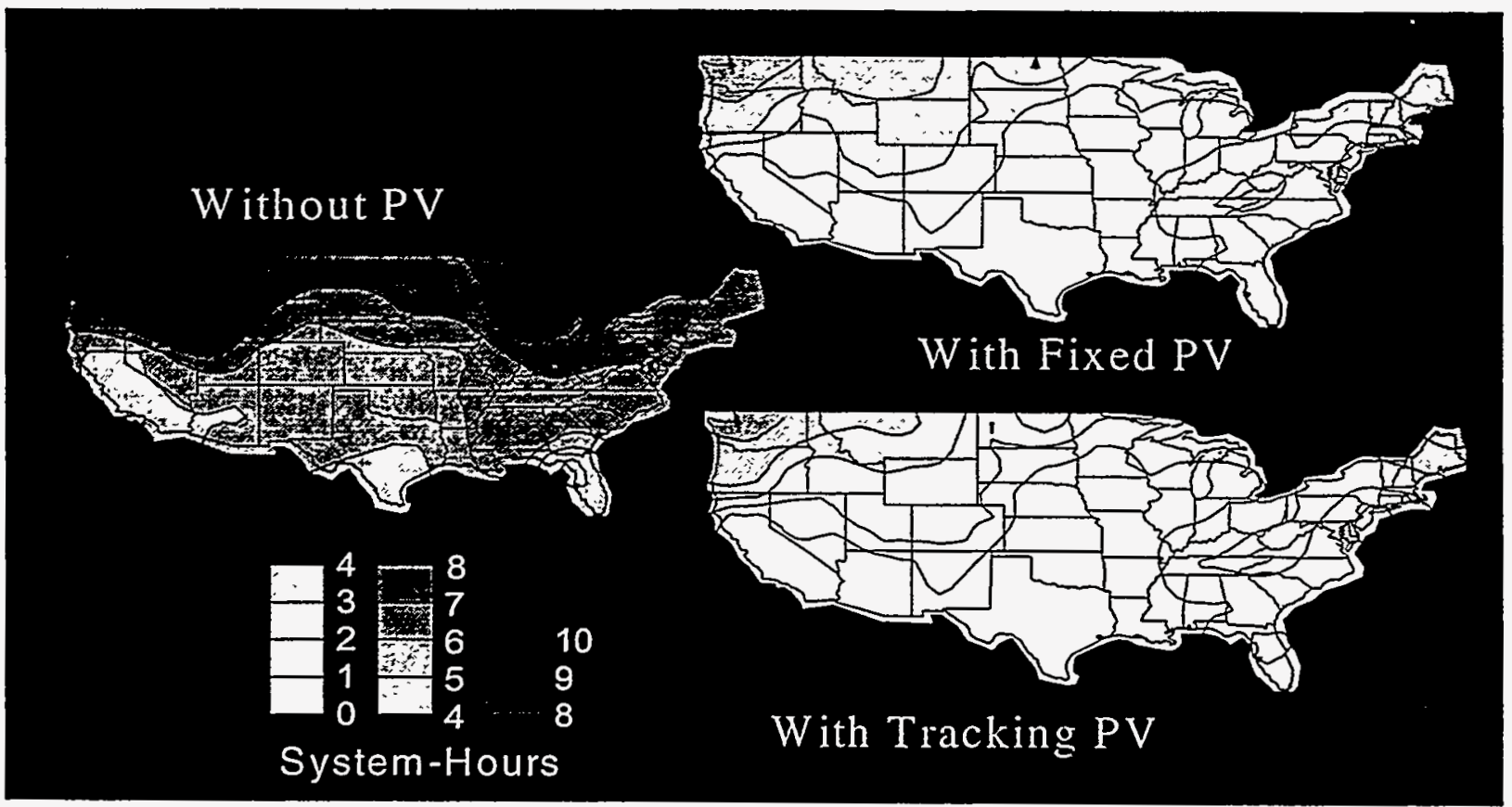

Figure 4: Minimum Storage requirements to insure firm $10 \%$ peak load reduction with and without $P V$ 
evident when comparing the MBES to the storage that would be needed to meet the same load requirements without PV. In the central US, southwestern US and on the Eastern seaboard, quantities of storage required in addition to PV for firm peak load reduction are of the order of 1 system-hour, that is 4 to 5 times less than without PV.

PV as a long-term solution to power outages [4]: The Western System Coordinating Council (WSCC) experienced a major power outage on August 10,1996. We investigated the conditions of the event, and presented evidence that a dispersed PV resource could mitigate the effects of such heat-driven massive power outages.

This event constituted a benchmark test for our contention that the effective capacity of PV is high for summer peaking utilities. In fact, PV availability was at near maximum at the time of the outage and during the preceding hours that led to the outage. The satellite view of the western US in Fig. 5 shows virtually cloud-free conditions throughout the WSCC service area.

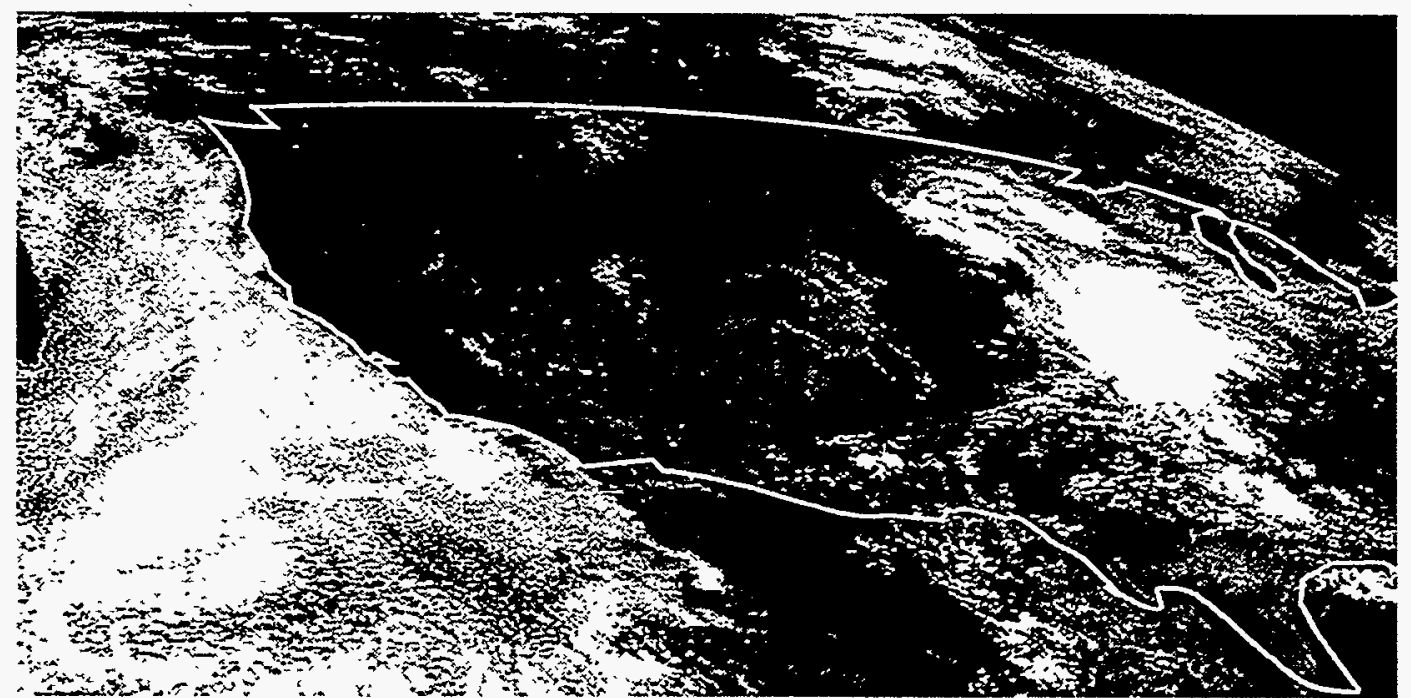

Figure 5: View of the Western United States 2.5 hours before onset of the power outage.

The WSCC service area covers most of the US and parts of Canada and Mexico, west of the Rocky Mountains.

This evidence indicates that dispersed PV generation deployment could have lessened the effects of the outage. Furthermore, the large emergency power purchases required to supplement the large generating unit outages, which extended for several days after the initial event could have been lessened. Therefore, dispersed PV deployment is strongly encouraged. The level of system relief will of course depend on the saturation of dispersed PV generation. Initial relief will be at the level of the customer and local electrical system, but over the long term, dispersed PV generation could prevent the cascading outage affecting a large area of the electrical system.

\section{ACKNOWLEDGMENTS}

This project was undertaken as a part of NREL contract XR-1-11168-1. Christy Herig is the project manager.

\section{REFERENCE}

1. Perez, R., R. Seals and C. Herig, (1997): Determination of the End-Use Effective Capacity of Photovoltaics. Proc. $14^{\text {th }}$ European PV Conference. Barcelona, Spain.

2. Howard Wenger, Christy Herig, Roger Taylor, Patrina Eiffert and Richard Perez, (1996): Niche Markets for Grid-Connected Photovoltaics. Proc. IEEE Photovoltaic Specialists Conf., Washington, DC

3. R. Perez, (1997): Grid-Connected Photovoltaic Power: Storage Requirements to Insure 100\% Peak Shaving Reliability. Proc. Energy Storage Association Annual meeting, Washington, DC

4. R. Perez, R. Seals, H. Wenger, T. Hoff and C. Herig, (1997): PV as a Long-term Solution to Power Outages. Case Study: The Great 1996 WSCC Power Outage. Proc. ASES Annual Conference, Washington, DC. 
Title: Evaluation of Photovoltaic Peak-Shaving Applications in the U.S. Buildings Sector

Organization: Center for Energy and Environmental Policy (CEEP)

School of Urban Affairs and Public Policy

University of Delaware

Newark, Delaware 19716

TEL: (302) 831-8405 FAX: (302) 831-3098

Contributors: John Byrne, principal investigator; Young-Doo Wang, investigator; Lawrence Agbemabiese, Kyung-Jin Boo, Darren Bouton, James Kliesch, graduate students

\section{Objectives}

The Buildings sector continues to offer a sizable market for PV applications. Key utility market sectors for PV that are receiving increasing attention are peak-shaving (PS) and emergency power (EP) applications associated with the commercial buildings sector. In addition to bill savings that accrue to customers from PS uses of PV, customers can avoid unwarned interruption in electricity supply (UPS). The technology also allows utilities to avoid costly upgrading and extension of distribution system to meet increasing peak-demand.

The Center for Energy and Environmental Policy (CEEP) at the University of Delaware has been analyzing the economics of utilizing PV as a PS/EP technology. This work: 1) further refines the concept of Distributed PV-PS systems in the perspective of competitive markets; and 2) performs an extended value analysis of Building-Integrated PV-PS and PV-EP. The PV industry now faces a challenge from the electricity industry restructuring, but has an opportunity as well from major new PV initiatives in the buildings/utility sectors being promoted by the Administration. The objectives of this project during FY 1997 were:

- To perform economic and technical analyses of PV applications on commercial buildings in the U.S., using building load and other data supplied by at least three cooperating utilities;

- To assist the State of Delaware, and up to two other states upon request, in identifying highvalue $P V$ applications on public buildings;

- To identify PV value analysis refinements in light of an emerging competitive electricity market.

\section{Tasks and Research Results}

Task A: Economic and Technical Analyses of PV Applications on Commercial Buildings

CEEP has conducted economic analyses of DPS-PV for commercial buildings across several 
states. Specific building types and regions have been selected for load characteristics most suitable for cost-effective DPS-PV applications. The results indicate that dispatchable peak-shaving PV systems are well suited for large office buildings and fast food restaurants located in the northeastern part of the U.S. and North Carolina. The northeastern region has relatively high electric rates, and North Carolina offers a 35\% renewable energy investment tax credit. The calculated benefit-cost ratios show greater than or equal to 1.00 , indicating cost-effectiveness at current installed PV systems prices (excluding storage) of $\$ 8.70$ per $\mathrm{Wp}$.

Researchers at CEEP also analyzed the additional benefits of PV-PS systems serving as emergency back-up power. CEEP developed a methodology to integrate such additional benefits into benefit-cost analyses using PV-Planner developed by CEEP. Analysis based on this methodology was conducted across five different utility jurisdictions for both emergency lighting (EL) and uninterrupted power supply (UPS) systems, assuming three different levels of reserve battery storage (see Tables 1 and 2). A comparison of the values for each function showed that the value added by UPS to a DPV-PS system is moderately less than that added by an EL function for equal amounts of DPV-PS battery power allocated. This was due to the fact that storage cost constitutes a much smaller ratio of the total costs of conventional UPS systems. As shown in the tables, benefit-cost ratios of greater than 1.00 were identified in some selected utility districts.

Table 1. Estimated BCRs for DPV-PS (EL) in Selected Utility Jurisdictions

\begin{tabular}{lllll}
\hline \hline UTILITY & BCR w/o EL* & Low Risk & Moderate Risk & High Risk \\
\hline Boston Edison & 0.87 & $0.88-0.90$ & -0.07 & $1.10-1.28$ \\
Duke Power & 0.99 & $1.00-1.01$ & $1.09-1.15$ & $1.22-1.39$ \\
S. California Edison & 0.77 & $0.79-0.80$ & $0.85-0.90$ & $0.95-1.09$ \\
City of Austin Electric & 0.77 & $0.78-0.79$ & $0.85-0.99$ & $0.95-1.09$ \\
Sacramento Muni. & 0.74 & $0.75-0.76$ & $0.82-0.86$ & $0.91-1.03$ \\
Util. Dist. & & & & \\
\hline \hline
\end{tabular}

Table 2. Estimated BCRs for DPV-PS (UPS) in Selected Utility Jurisdictions

\begin{tabular}{lllll}
\hline \hline UTILITY & BCR w/o UPS* & Low Risk & Moderate Risk & High Risk \\
\hline Boston Edison & 0.87 & $0.88-0.88$ & $0.92-0.94$ & $0.98-1.03$ \\
Duke Power & 0.99 & $1.00-1.00$ & $1.04-1.06$ & $1.11-1.15$ \\
S. California Edison & 0.77 & $0.78-0.79$ & $0.83-0.85$ & $0.89-0.93$ \\
City of Austin Electric & 0.77 & $0.78-0.78$ & $0.81-0.83$ & $0.86-0.89$ \\
Sacramento Muni. & 0.74 & $0.75-0.75$ & $0.79-0.80$ & $0.84-0.88$
\end{tabular}

Util. Dist.

* Note: These figures include peak-shaving benefits only. 


\section{Task B: High-Value Photovoltaic Applications in Public Facilities}

\section{State Facilities}

CEEP conducted a survey of State owned office buildings in Delaware to identify potential high-value PV applications. Currently, the State of Delaware owns approximately 900 facilities, most of which are located on multi-building complexes. They are scattered in three counties, New Castle, Kent, and Sussex. Facilities metered for monthly kW loads are mainly found in New Castle County where most State facilities are handled by Delmarva Power. Data availability to analyze high-value peak shaving applications is, thus, limited.

Several criteria were considered in selection of buildings for economic analysis: 1)roof-type, gable or flat; 2) independent and individual pulse meter; 3) size of building, and 4) age of building. Three State facilities equipped with the pulse meters were selected for initial analysis. Initial analysis based on the building electricity peak load pattern shows benefit-cost ratios between 0.7 and 0.8 , which is consistent with previous findings by CEEP. Delaware has below-average electricity rates.

A new payback period methodology was created to fully account for the tax benefits gained by the consumer. The new analysis was adjusted to deduct the tax benefits over the first five years only, as opposed to deducting even portions of the tax benefits over the 25 year lifespan of the equipment. The net result shortens the payback period, making the PV system more attractive to the customer.

\section{Cost-Effective PV Applications on Federal Buildings:}

CEEP prepared preliminary screening analysis for NREL on possible cost-effective applications of PV systems for peak-shaving and emergency power in federal buildings. The report examined the economics of uninterruptible power supply (UPS) as an added value to dispatchable peak-shaving PV systems installed on specific building types. This study was conducted based on two economic feasibility guidelines used by the Federal Energy Management Program (FEMP). CEEP's report concludes that there are cost-effective opportunities, at existing module prices, for the use of these dual functions v.s. systems at federal facilities. CEEP also suggests that overseas federal buildings, where electricity prices are typically higher and/or reliability is poorer than in the contiguous U.S., should be promising sites for these systems.

\section{Task C: Reevaluation of PS-PV system in the light of an emerging competitive electricity market.}

In response to the recent development of competitive electricity markets, a number of incentive programs are being considered: portfolio standard, green pricing, system benefits charge, and buy-down financing costs of renewables. Each of these mechanisms constitutes government intervention in an otherwise biased competitive market. Such incentives will definitely create favorable environment for DPS-PV applications. CEEP is evaluating them in terms of their impacts on the near-term PV market. 
CEEP has incorporated the distributed utility (DU) concept in the DPS-PV analysis. In the competitive environment in the electricity industry, DU is emerging as a key concept. Research has shown that a utility and its customers can benefit from targeted DPS-PV applications in the areas where the utility's avoided costs are high when combined by time variable. A DU analysis that evaluates localized benefits can assist utilities and regulators in their efforts to identify high-value PV applications in an emerging competitive electricity markets.

A DU approach also represents an institutional shift in which new emerging modular technologies can play a role in a new less centralized, environmentally less polluting electric utility industry. CEEP researchers recently published a paper describing the methodology for comparing the DU approach to the conventional economic externality model in evaluating the economics of PV in a deregulated electricity market (see Letendre et al., 1998). CEEP, based on this initial study, is further working on economic and technical evaluations of DPS-PV systems in light of an emerging competitive electricity market.

\section{References}

1. Byrne, John, L. Agbemabiese, and D. Redlin. 1997. Evaluating The Additional Value of Emergency Power Applications in Dispatchable PV Peak Shaving Systems. Prepared for National Renewable Energy Laboratory. May. Center for Energy and Environmental Policy, University of Delaware.

2. Byme, John, S. Letendre, L. Agbemabiese, D. Redlin and R. Nigro. 1997. "Commercial Building Integrated Photovoltaics: Market and Policy Implications." Proceedings of the 26th IEEE Photovoltaic Specialists Conference. September. Anaheim, California.

3. Byme, John, S. Letendre, Y. D. Wang, and C. Weinberg. 1997. Commercializing Photovoltaics: The Importance of Capturing Distributed Benefit. June. Albuguergue, New Mexico. ASES 1998Fortheoming.

4. Byrne, John and Ralph Nigro. 1997. Building Integrated Photovoltaics for Federal Facilities: Possible Cost-Effective Applications. December.

5. Byrne, John, Y.D. Wang, and S. Letendre.1997. Building Load Analysis of Dispatchable Peak Shaving PV System: A Regional Analysis of Technical and Economic Potential. April. ASES Solar Energy Forum. Washington D.C.

6. Center for Energy and Environmental Policy, PV Planner: A Spreadsheet Analytical Tool for Grid-Connected Applications. Report submitted to the National Renewable Energy Laboratory, 1996. 
9.0 International

Applications and Markets Project 
$\because \because-200$ $\therefore \therefore<\div ;$ 


\title{
9.0 INTERNATIONAL PV APPLICATIONS AND MARKET DEVELOPMENT-Introduction
}

\author{
Roger Taylor
}

During FY 1997 the International PV Applications and Market Development Project continued to provide for support for internationally-focused analytical, applications, and market development efforts of the DOE Photovoltaics Program and the Office of Utility Technologies. The overall objective of this project is to conduct a coordinated set of market conditioning and project development activities (through NREL in-house and subcontracted research) that lead toward sustainable international markets for photovoltaic systems.

The accomplishments and ongoing efforts are briefly discussed in the following sections.

\section{Brazilian Rural Electrification Pilot Project}

The United States Department of Energy, through NREL, is undertaking a cooperative joint pilot project with eight states together with the Centro de Pesquisas de Energia Eletrica (CEPEL) in the Federal Republic of Brazil. The objective of the effort is to establish and assess the efficiency, operability, and reliability of solar energy based rural electrification. During FY1997 the primary focus has been on completion of the two $50 \mathrm{~kW}$ hybrid power systems. Some start-up problems were encountered and fixed, and data acquisition from both of these remote sites continues to be a problem. Training courses for local Brazilian utility engineers have been conducted and primary operational responsibility is now in the hands of our local partners. A primary Alesson learned@ from this part of the project is the necessity of having multiple systems to spread out the O\&M base cost effectively. Rapid restructuring changes are underway in the Brazilian utility sector and the early vision of using the utility industry as a primary implementer of renewable projects is now in question (as it is in the U.S.). Nevertheless, significant projects are now underway in Brazil to provide community services to remote villages using PV technology. This program, PRODEEM, has invested about $\$ 3 \mathrm{M} / \mathrm{yr}$ for the past 3 years in water pumping, health clinic, and school systems. And over $95 \%$ of the product for these installations was obtained under competitive procurements from U.S. suppliers.

\section{China}

The Gansu solar home system project in western China supported by the U.S. DOE and the Chinese Ministry of Agriculture installed 300 household systems, including 10 school systems, representing the first half of the project, and funds obtained from the first system sales have been turned over in the revolving account for the project to purchase additional systems. Phase I has been in evaluation in preparation for continuing to Phase II in FY 1998. As a result of the Gansu project, the Chinese Ministry of Agriculture has initiated a 10,000 solar home system to be implemented in five provinces in western China. NREL is working with the MOA in developing this project and will assist the MOA in performing a techno-economic analysis in the first two provinces of Qinghai and Xinjiang for project scoping early in FY 1998. During FY 1997, NREL traveled with an MOA team to these 
two provinces to establish and meet with the project teams, in each case consisting of all of the relevant key decision makers and technical expertise in the provinces.

An implementation agreement was signed with the Inner Mongolia government for the PV-Wind hybrid household project in two counties in the Inner Mongolia Autonomous Region resulting from the Phase I analysis of rural electrification options completed in FY 1997. The results of the analysis were widely reported in FY 1997 in many domestic and international conferences, and had a significant influence in government plans and the local and central levels in China in developing future rural electrification programs.

Technical assistance was provided to a number of organizations in FY 1997 in China; including, the Beijing Solar Energy Research Institute in developing state-of-the-art cell measurement capabilities, the Institute of Electrical Engineering of the Chinese Academy of Sciences in acquiring experience in using NREL hybrid optimization models for engineering design of village systems, and the Chinese Ministry of Agriculture in establishing a regional testing and training center in Lanzhou in Gansu Province of China for solar home system development in western China.

$\underline{\text { India }}$

The year's activities continued to support the Indo-U.S. bilateral activities between DOE and MNES. Included are the Ramakrishna Mission Project and a new distributed utility project in the state of Punjab.

The Ramakrishna Mission sustainable rural electrification project resulted from agreements signed between the U.S. Secretary of Energy and the Minister of State of the Ministry of Non-Conventional Energy Sources (MNES). Three cost shared projects were identified and originally budgeted at $\$ 1 M$ each. Budget reductions resulted in having to select only one project at a level $\$ 250 \mathrm{~K}$ from each country. A public solicitation resulted in the procurement of 30 kilowatts of PV systems applications. Included were 300 domestic home lighting systems ( 50 watts each to include compact flourescent lights and a black and white TV), common area lighting, vaccine refrigeration for a health clinic, two battery charging stations, a large water pump, and electrification of a weaving center, several youth centers, and a clinic facility. Training was given to sixteen selected participants who in turn trained an additional 90 installers. The project is coordinated by the Ramakrishna Mission, a well respected NGO in the state of West Bengal. As part of their cost share, the government of India provided the batteries, solar lanterns, all balance of systems components, and payment of custom duties.

During the year all of the domestic home lighting systems were installed and the remaining systems are scheduled to be installed early this summer. NREL has contracted with the Tata Energy Research Institute (TERI) to perform a before and after impact study on the beneficiaries of the PV systems. NREL is also working with the Mission to develop a proposal to the Indian Renewable Energy Development Agency (IREDA) to provide loans to purchase additional systems to be deployed in the Sundarbans region where the current project is active. The installation of additional systems would satisfy the objective of having the project self-sustaining. Because of he isolation of the region from the electricity grid system, the economics of PV are very favorable compared to traditional alternatives (diesel, kerosene, etc.). 
In a new Distributed Utility Project MNES and NREL have agreed to fund a distributed utility project in the state of Punjab, near the capital of New Delhi. The State Electricity Board (SEB) will be the partner in India, working with DU experts at NREL. The purpose of the project will be to investigate the usefulness of PV to provide grid support to an electrical grid that is severely overloaded. A small $200 \mathrm{~kW}$ PV system will be deployed with the SEB responsible for the monitoring of the system and its impacts. The project is cost shared 50-50 and is expected to begin late this calendar year.

\section{$\underline{\text { South Africa }}$}

During FY97, NREL provided program expertise to US-South Africa Binational Commission, and Acting Secretary of Energy Charles Curtis. Prepared demonstration sites and all background briefings for Secretarial events in South Africa, and participated as US delegate on Energy Subcommittee meeting of the Binational Commission. NREL hosted the Director General of Energy, South Africa and manager of Golden Field Office on 4-day tour of US renewable energy sites, and NREL site visit. NREL wrote and was awarded $\$ 33 \mathrm{k}$ grant for USIS funds to support S. Africa subcontracts. Prepared USDOE briefing for RE activities in South Africa. Participated in Sustainable Energy Subcomitee presentations to Secretary Pena and SA Minister Maduna.

\section{Other HQ and International Support}

Led DOE-OUT policy update on Indian Renewable Energy Sector. Travelled to India with Allan Hoffman, ADAS, of DOE-OUT to participate in Indo-UUS Bilateral Energy Consultations. Provided ongoing support for DOE-PO72 for continued negotations with Indian counterparts.

Led DOE-OUT delegation for Indonesia-US bilateral negotiations.

Presented APolicy Options for Renewable Energy@ to senior delegation of the Philippines in support of DOE policy technical assistance mission.

Led successful negotiation for DOE-OUT for technical assistance program with the Government of Nepal. Developed and hosted senior government of Nepal Delegation on US renewable energy visit. Led OUT/NREL effort for the development of a multi-year workplan for technical assistance to the Government of Nepal for their Alternative Energy Promotion Center. Prepared briefing for DAS Jhirad and US Ambassador to Nepal, Ralph Frank.

Intiated and completed successful new interagency agreement with USAID for $\$ 660 \mathrm{k}$ in FY97 funding, including assignment of NREL personnel to AID Global offices.

Led team effort for development of new $\$ 1.3 \mathrm{M}$ contract with AID for technical assistance to the Philippines.

Technical assistance was provided to Zambia. In April I traveled to Zambia to assist the World Bank by developing specifications for PV-powered rural health centers. Through many discussions with 
different Ministries, I determined the loads and recommended different PV systems for water pumping, medical refrigeration and general lighting.

\section{Applications Development}

Several promising applications for PV systems were investigated - these included water treatment processes, battery charging stations and flywheel energy storage systems. NREL published a report on an "Overview of Village Scale, Renewable Energy Powered Desalination." An NREL report on disinfection will be published in FY 98. Two different uv-light water disinfection systems were purchased that will be tested in FY 98. The battery charging system from India was set up for testing at JSF. However, the use of power supplies to simulate PV modules became problematic and the full test was not implemented - real PV modules are needed. 


\section{International Applications and Markets Subcontracts}



Organization: Institute of Policy and Management, Chinese Academy of Sciences

New Energy Office of Inner-Mongolia

Center for Energy and Environmental Policy, University of Delaware

Contributors: Li Xiuguo, Wang Sicheng, Yang Meidong,

John Byrne, Bo Shen,

Lin Li, Ji Binghou, Liu Zhizhang

\section{Objectives:}

The objectives of this subcontract are to provide support for the Inner Mongolia hybrid household project in terms of technical, financial, and social analysis, managing the data collection for the project, and overseeing the evaluation of the project; assist in various training activities for the project; and provide assistance to the Inner Mongolian government for further market characterization and commercial infrastructure development.

\section{Results:}

1. Based on the study in 1997 , it is confirmed that 240 household wind-PV hybrid demonstration systems in three Counties will be set up and they are to be funded jointly by both the US and China. The plan is:

- $\quad$ Suniteyou Banner, Xilingole District: 100 Households

- $\quad$ Dongwu Zhumuqin Banner, Xilingole District: 40 Households

- $\quad$ Arlashanzuo Banner, Arlashan District: 100 Households

The three counties represent three kinds of situations that are common in Inner Mongolia: i) Suniteyou Banner has the best wind resource and a good solar resource and the herdsmen desire to buy the equipment; ii) Dongwu Zhumuqin Banner is not as good as Suniteyou Banner in resources, but the income level of the herdsmen is the best in the three counties and the herdsmen in this place can easily afford to buy the hybrid power systems; and iii) Arlashanzuo Banner has best solar resources and good wind. All three counties have a high market potential for distributed hybrid power systems in terms of rural income levels and wind and solar resources. Once the demonstration shows the successful results in the three Banners, such systems can be distributed throughout Inner Mongolia. The potential counties for the distribution of such wind-PV hybrid systems are listed as follows:

- $\quad$ Arlashan League: Arlashan You Banner and Ergina Banner;
Bayanzhuor League: Wulate Qian Banner, Wulate Zhong Banner, Wulate Hou
Banner and Deng Kou County;
- Wulanchabu Leaguer: Siziwang Banner;
- Xilingole Leaguer: Arbaga Banner and Xiwuzhumuxing Banner,
Hulunbeir Leaguer: Ergona You Banner.

These 10 counties cover $80 \%$ of the land of Inner Mongolia and represent most of Inner-Mongolia. It will open the market to U.S. PV manufacturers.

2. The power systems of the 240 households could be considered in 4 types:

2.1500 W System:

(1) The configuration of the system: 
FD2.5-300 wind turbine 1 set

Single crystalline silicon solar array $\quad 200 \mathrm{Wp}$

Lead-Acid storage battery $\quad 7 \mathrm{kWh}$

Inverter/Controller Unit $\quad .600 \mathrm{~W}$

(2) Load (will be borne by user)

$\begin{array}{ll}\text { 80-liter freezer } & 1 \text { set } \\ \text { Satellite TV receiver (with 2M antenna) } & 1 \text { set } \\ \text { 21 @ Color TV } & 1 \text { set } \\ \text { Washing Machine } & 1 \text { set } \\ \text { High efficiency fluorescent lights } & 100 \mathrm{~W} \text { total }\end{array}$

2.2450 W System:

(1) The configuration of the system:

FD2.5-300 wind turbine 1 set

Single crystalline silicon solar array $\quad 150 \mathrm{Wp}$

Lead-Acid storage battery $\quad 7 \mathrm{kWh}$

Inverter/Controller Unit 600W

(2) Load (will be borne by user)

80-liter freezer 1 set

Satellite TV receiver (with 2M antenna) 1 set

21 Color TV 1 set

Washing Machine 1 set

High efficiency fluorescent lights $\quad$ 100W total

2.3400 W System:

(1) The configuration of the system:

A.

FD2.5-300 wind turbine 1 set

Single crystalline silicon solar array $100 \mathrm{Wp}$

Lead-Acid storage battery $\quad 5 \mathrm{kWh}$

Inverter/Controller Unit $\quad 600 \mathrm{~W}$

B.

FD2.5-200 wind turbine 1 set

Single crystalline silicon solar array $100 \mathrm{Wp}$

Lead-Acid storage battery

24V/200Ah

Inverter/Controller Unit $\quad 600 \mathrm{~W}$

(2) Load (will be borne by user)

80 liter freezer 1 set

Satellite TV receiver (with $2 \mathrm{M}$ antenna) 1 set

210 Color TV 1 set

Washing Machine 1 set

High efficiency fluorescent lights $\quad 100 \mathrm{~W}$ total

\subsection{W System:}

(1) The configuration of the system:

FD1.6-100 wind turbine 
Single crystalline silicon solar array

$50 \mathrm{Wp}$

Lead-Acid storage battery

$3.6 \mathrm{kWh}$

Inverter/Controller Unit

$400 \mathrm{~W}$

(2) Load (will be borne by user)

Satellite TV receiver (with 2M antenna) 1 set

21e Color TV

1 set

Washing Machine

1 set

High efficiency fluorescent lights

100W total

The solar arrays will be mainly from the United States. 
Title:

Photovoltaics for Rural Energy in Gansu Province in the People's Republic of China

Organization: $\quad$ Solar Electric Light Fund

1734 20th Street, N.W.

Washington, D.C. 20009

Telephone: $202-234-7265$

Principal Investigator: Robert Freling, Director, SELF

Subcontract No:: $\quad$ AAX-6-15376-01

\section{Objectives:}

SELF will participate in the development of a viable commercial infrastructure for the successful deployment of PV systems for rural energy development in Gansu Province, and surrounding areas as opportunities develop, that will result in the development of sustainable markets for PV systems in rural China. The project focuses on the Gansu region of western China, but it is expected that successful project completion will result in a model that can be used more generally in other regions of China for rual PV commercial development.

In the course of working with China to develop an infrastructure for rural PV system deployment and application development, the U.S. renewable energy industry will be invited to participate by supplying U.S. equipment as part of the U.S. DOE cost share for the project and be involved directly in PV system development in China.

SELF will work to further develop the use of installment credit financing of PV household systems in China to make such energy systems more generally available to the rural population of China, and to increase the commercial viability of credit financing by eliminating to the extent possible, the use of subsidies and other non commercially viable incentives for marketing PV systems.

SELF will engage and encourage cooperation among appropriate Chinese agencies, in this case, rural energy offices under the Chinese Ministry of Agriculture, appropriate non profit agencies and government funding agencies supporting rural development in China, and the Chinese private industry sector, to build an effective team for supporting the commercial deployment of PV household systems leading to sustainable market development in China.

\section{FY 1997 Highlights:}

- During FY 1997 U.S. and Chinese equipment was procured and Phase I installations of solar 
home systems was initiated. Systems installed included a PV panel, charge controller, battery, and two fluorescent lights in size ranges of 20,22, and 50 Watts for solar home systems. The 50 Watt systems were installed in schools in Gansu. Systems have been installed by the Gansu Natural Resources Institute in Zhang Ye County, Ping Shanhu Township, Hong Quan Village, the Gansu PV Company in Min county, Weixin Township and Baozi Township, and by Zhong Xing in Huan County, Nan Qui Township.

Although the amorphous silicon technology has a bad reputation in China because the domestic manufactured product is extemely poor quality, several amorphous silicon systems from USSC/ECD representing advanced manufacturing technology were installed in this project. Five systems have been installed in the Pingshanhu, Mawu, Weixin, Heima Quanhe elementary schools, and the GSELF energy conservation demonstration facility.

Some problems have occurred related to the timing of delivery of systems, just missing the Chinese New Year, at which time farmers generally have disposable income which is scarse during other times of the year. Delays have also been experienced in unanticipated problems with custom clearances for imported equipment from the United States.

- At the end of FY 1997, the Chinese Ministry of Agriculture (MOA) conducted a preliminary assessment of the project and was sufficiently impressed with the progress in Gansu that a new initiative has been developed. The Chinese MOA, which is a cost sharing partner in the Gansu project, is now developing a 10,000 solar home system project for five provinces in western China, including Gansu, Inner Mongolia, Xinjiang, Qinghai, and Ningxia. The MOA will conduct market studies in these provinces in FY 1998 in preparation for the project. This initiative is a Chinese initiative that will be incorporated into the MOA annual budget plans and funded by China. 
Title:

Evaluation of Intermediate Applications for Photovoltaics in the United States and Developing Countries

Qrganization: Center for Energy and Environmental Policy (CEEP)

University of Delaware

Newark, Delaware 19716

TEL: (302) 831-8405 FAX: (302) 831-3098

Contributors: John Byme, principal investigator; Young-Doo Wang, investigator; Lawrence Agbemabiese, Kyung-Jin Boo, Steven Letendre, David Redlin, Qingrui Liu, Bo Shen, and Jihong Zhao, graduate students

\section{Objectives}

The development of intermediate applications for photovoltaics (PV) markets is vital for near-term growth of the PV industry. Key utility market sectors for PV that are receiving increasing attention are peak-shaving (PS) and emergency power (EP) applications associated with the commercial buildings sector. In addition to bill savings that accrue to customers from PS uses of PV, the technology can generate added value by displacing on-site emergency power. A number of utility companies are investing PS/EP applications of PV.

The integration of PV in PS and EP applications is an important component for developing a buildingintegrated PV market. The Center for Energy and Environmental Policy (CEEP) at the University of Delaware has been conducting and analyzing the economics of utilizing PV as a PS/EP technology. This work has: 1) further developed the concept of distributed PV-PS; and 2) established methods for economic analysis of Building-Integrated PV-PS and PV-EP; and 3) assisted in the design and installation of PV-PS/EP applications. In addition to analyses that foster the development of near-term U.S. markets, work has been done to identify developing country opportunities for the use of PV, in conjunction with wind (in some cases) as a rural electrification technology, as well as a peak-shaving building technology in urban sectors of developing countries.

The objectives of this research during FY 1997 were:

- To analyze the value of PV to utilities and their customers as a distributed PV-PS/EP option and examine its economic viability in terms of social costing and distributed utility prospects;

- To provide an economic analysis of building-integrated PV-PS and PV-EP systems in the domestic U.S. utility market;

- To provide assistance in the development of environmental and economic evaluation of the use of PV both as a remote-area power technology and as a peak-shaving technology in developing countries.

\section{Tasks and Research Results}

\section{Task A: $\quad$ Distributed PV-PS Applications}

The 1996 CEEP research indicated that the PV-PS system which incorporates the distributed utility (DU) concept renders significant economic benefits resulting from the greater $T \& D$ asset utilization, the value of deferred $T \& D$ equipment installation and reduced line losses. Research has shown that economic value to a utility and its customers is increased by targeting dispatchable PV applications in the areas where, and during time periods when, the utility's avoided costs are high. CEEP has conducted economic analyses of PV-PS for specific building types located in the service territories of eleven different U.S. utilities. The results indicate that dispatchable peak-shaving PV systems are well suited for large office buildings and fast food restaurants located in the northeastern part of the U.S., the South Atlantic and, to a lesser extent, the West Coast. These building types tend to experience peak demand 
during daylight hours, thus only modest amounts of storage are required to shift the PV array's output to match the building's peak demand (generally, the analysis conducted in the 11 utility service territories indicates that off-peak charging is the more economical means of delivering firm peak-shaving levels). Figures $l$ and 2 illustrate the ability of PV-PS systems (DPS-PV) to shave the peak demand for large office buildings and fast-food restaurants.

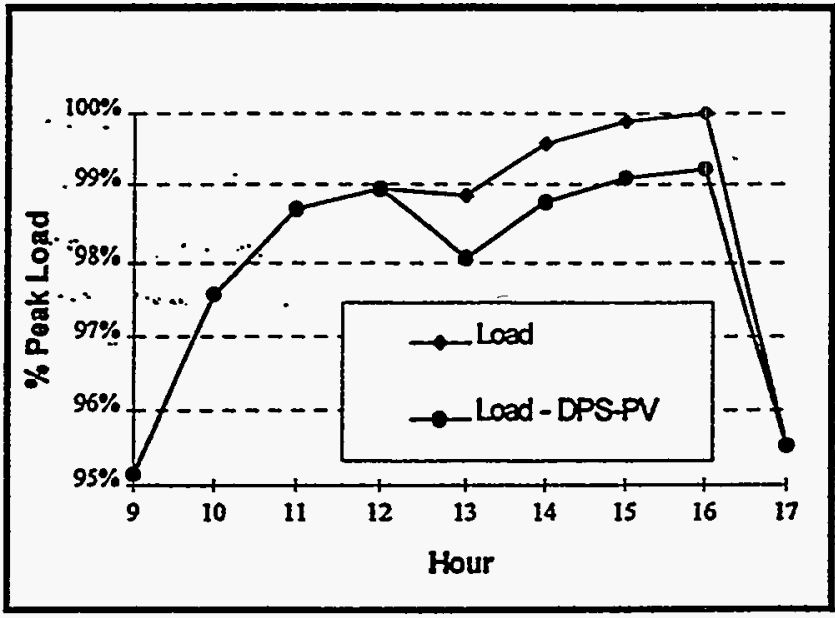

Fig. 1: Normalized load profile of large office building with and without DPS-PV.

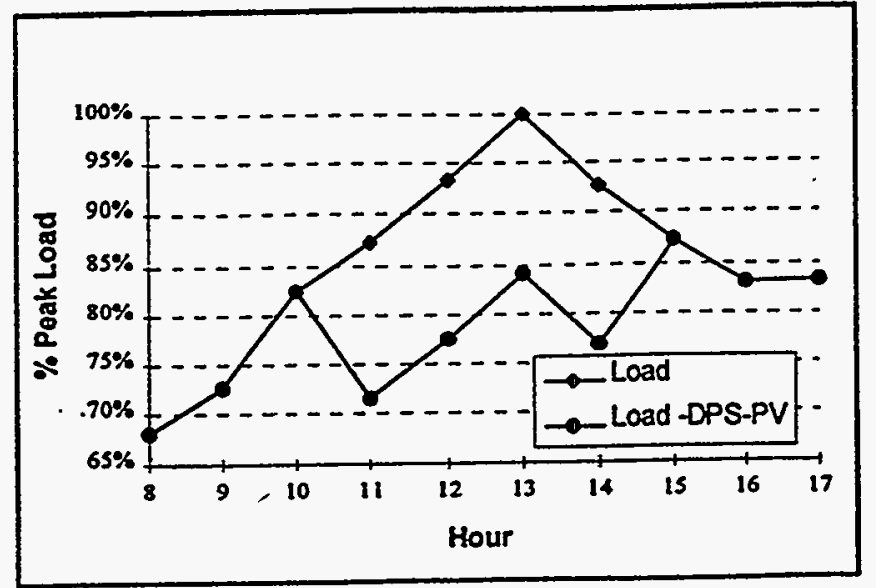

Fig. 2: Normalized load profile of fast-food restaurant with and without DPS-PV

\section{Task B: $\quad$ Building-Integrated PV-PS and PV-EP Systems}

Researchers at CEEP analyzed the complementary benefits of building-integrated PV-PS systems serving as emergency back-up power using PV-Planner developed by CEEP. Calculations were conducted across five different utility jurisdictions for both emergency lighting (EL) and uninterrupted power supply (UPS) systems, assuming three different levels of reserve battery storage (see Tables 1 and 2). A comparison of the benefit-cost ratios for each function showed that the value added by UPS to a PV-PS system is moderately less than that added by an EL function for equal amounts of PV-PS battery power allocated. This was due to the fact that storage cost constitutes a much smaller ratio of the total costs of conventional UPS systems. As shown in the tables, benefit cost ratios of greater than 1.00 were identified in some selected utility districts.

Table 1. Estimated BCRs for DPV-PS (EL) in Selected Utility Jurisdictions

\begin{tabular}{|c|c|c|c|c|}
\hline \multirow[t]{2}{*}{ UTILITY } & \multirow[t]{2}{*}{ BCR w/o EL* } & \multicolumn{3}{|c|}{ BCR w/EL } \\
\hline & & $\begin{array}{c}\text { Low EP } \\
\text { Requirement } \\
(<1 \mathrm{kWh})\end{array}$ & $\begin{array}{c}\text { Moderate EP } \\
\text { Requirement } \\
(1-5 \mathrm{kWh})\end{array}$ & $\begin{array}{c}\text { Sizable EP } \\
\text { Requirement } \\
\text { (>5 kWh) }\end{array}$ \\
\hline Boston Edison & 0.87 & $0.88-0.90$ & $+0.97-1.04$ & $1.10-1.28$ \\
\hline Duke Power & 0.99 & $1.00-1.01$ & $1.09-1.15$ & $1.22-1.39$ \\
\hline S. California Edison & 0.77 & $0.79-0.80$ & $0.85-0.90$ & $0.95-1.09$ \\
\hline City of Austin Electric & 0.77 & $0.78-0.79$ & $0.85-0.99$ & $0.95-1.09$ \\
\hline $\begin{array}{l}\text { Sacramento Muni. Util. } \\
\text { Dist. }\end{array}$ & 0.74 & $0.75-0.76$ & $0.82-0.86$ & $0.91-1.03$ \\
\hline
\end{tabular}


Table 2. Estimated BCRs for DPV-PS (UPS) in Selected Utility Jurisdictions

\begin{tabular}{lllcc}
\hline \hline UTILITY & BCR w/o UPS* & \multicolumn{3}{c}{ BCR w/ UPS } \\
\cline { 3 - 5 } & & $\begin{array}{c}\text { Low UPS } \\
\text { Requirement } \\
(<1 \mathrm{kWh})\end{array}$ & $\begin{array}{c}\text { Moderate UPS } \\
\text { Requirement } \\
(1-5 \mathrm{kWh})\end{array}$ & $\begin{array}{c}\text { Sizable UPS } \\
\text { Requirement } \\
(>5 \mathrm{kWh})\end{array}$ \\
\hline Boston Edison & 0.87 & $0.88-0.88$ & $0.92-0.94$ & $0.98-1.03$ \\
Duke Power & 0.99 & $1.00-1.00$ & $1.04-1.06$ & $1.11-1.15$ \\
S. California Edison & 0.77 & $0.78-0.79$ & $0.83-0.85$ & $0.89-0.93$ \\
City of Austin Electric & 0.77 & $0.78-0.78$ & $0.81-0.83$ & $0.86-0.89$ \\
Sacramento Muni. Util. & 0.74 & $0.75-0.75$ & $0.79-0.80$ & $0.84-0.88$ \\
Dist. & & & &
\end{tabular}

* Note: These figures include peak-shaving benefits only.

Task C. Support for PV Applications in Developing Countries

CEEP has created a computer screening tool called Rural Renewable Energy Analysis and Design (RREAD), which can be used to evaluate the energy and economic performance of renewable technologies for off-grid application in rural settings. RREAD provides system performance and reliability analyses, economic analysis (mainly levelized cost analysis) and sensitivity analyses. Using RREAD, an economic feasibility study of off-grid renewable energy applications in China's Inner Mongolia Autonomous Region (IMAR) was conducted. The study was undertaken in collaboration with the Institute of Policy and Management of the Chinese Academy of Sciences and the New Energy Office of the Inner Mongolia Government. The analysis, based on a representative sample of 41 households from IMAR, indicates that levelized cost of off-grid, household-scale renewable energy systems are cost competitive with conventional gasoline gen-sets, and PV/wind hybrid systems appear to be an economic means of providing year-round electricity service and meeting the rising energy demands of households in remote villages in Inner Mongolia (Table 3).

Table 3. Levelized Costs for PV, Wind, Gasoline Gen-set and Hybrid Systems

\begin{tabular}{c|c|c|c}
\hline System & $\begin{array}{c}\text { Output range } \\
(\mathrm{kWh} / \text { year })\end{array}$ & $\begin{array}{c}\text { Levelized Cost Based } \\
\text { on Manufacture } \\
\text { Quoted Battery } \\
\text { Lifetime } \\
(\$ / \mathrm{kWh})\end{array}$ & $\begin{array}{c}\text { Levelized Cost Based } \\
\text { on Battery Lifetime } \\
\text { from Field Analysis } \\
(\$ / \mathrm{kWh})\end{array}$ \\
\hline Wind-Only & $200-640$ & $0.24-0.37$ & $0.50-0.63$ \\
\hline PV-Only & $120-240$ & $0.67-0.73$ & $0.77-0.83$ \\
\hline Small Hybrids & $400-750$ & $0.31-0.46$ & $0.57-0.72$ \\
\hline $\begin{array}{c}\text { Large Hybrids } \\
\text { contSets(Not serving } \\
\text { equipment) }\end{array}$ & $560-870$ & $0.32-0.46$ & $0.43-0.57$ \\
\hline $\begin{array}{c}\text { Gen-Sets(serving continuous } \\
\text { duty cycle equipment }\end{array}$ & $660-730^{*}$ & $0.76-0.80^{*}$ & $0.76-0.80^{*}$ \\
\hline \hline
\end{tabular}

* These estimates are based on systems configured without storage. 
In cooperation with the Korea Institute of Energy Research (KIER), CEEP evaluated the costeffectiveness of PV-PS systems for three building types-a large office building, a department store and a down town hotel-in two major Korean cities (Seoul and Taejon). Commercial building owners in Korea investing in renewable energy technolgies are eligible for an annual $15 \%$ investment tax credit during the first three years of operation. In additon, full depreciation of the capital cost of the sytem is possible in the first year of operation. The investment tax credit and full depreciation policies creat sizable incentives for investment by building owners. Table 4 shows that peak-shaving PV systems can be cost-effective in the Korean buildings sector at existing installed PV prices of $\$ 7.50 / \mathrm{Wp}$ (approximately $6,750 / \mathrm{Wp}$ ).

Table 4. Cost-Benefit Ratios and Payback Periods in Seoul and Taejon

\begin{tabular}{l|cccc}
\hline \hline & \multicolumn{2}{|c}{ 2 hours dispatch } & \multicolumn{2}{c}{ 4 hours dispatch } \\
\hline Building Type & B/C Ratio & $\begin{array}{c}\text { Payback } \\
\text { Period }\end{array}$ & $\begin{array}{c}\text { B/C Ratio } \\
\cdot\end{array}$ & $\begin{array}{c}\text { Payback } \\
\text { Period }\end{array}$ \\
\hline $\begin{array}{l}\text { Large Office Building } \\
\text { Seoul }\end{array}$ & 0.97 & 13.74 & 1.05 & 10.96 \\
$\quad$ Taejon & 1.00 & 12.58 & 1.08 & 10.09 \\
$\begin{array}{l}\text { Department Store } \\
\text { Seoul }\end{array}$ & 0.99 & 12.98 & 1.09 & 8.83 \\
$\quad$ Taejon & 1.01 & 12.23 & 1.12 & 9.41 \\
Large Hotel & 1.02 & 11.88 & 1.05 & 11.07 \\
$\quad$ Seoul & 1.04 & 11.22 & 1.08 & 10.19 \\
$\quad$ Taejon & & & &
\end{tabular}

\section{References}

1. S. Letendre, J. Byme, and Y-D. Wang. 1996. "The Distributed Utility Concept: Toward a Sustainable Electric Utility Sector." Proceedings of the ACEEE 1996 Summer Study on Energy Efficiency in Buildings. Asilomar, CA. Vol. 7: 7.1-7.8.

2. J. Byrne, L. Agbemabiese, and D. Redlin. 1997. Evaluating the Additional Value of Emergency Power Applications in Dispatchable PV Peak Shaving Systems. Prepared for National Renewable Energy Laboratory. May. Center for Energy and Environmental Policy, University of Delaware.

3. J. Byrne, S. Letendre, L. Agbemabiese, D. Redlin and R. Nigro. 1997. "Commercial Building Integrated Photovoltaics: Market and Policy Implications." Proceedings of the 26th IEEE Photovoltaic Specialists Conference. September. Anaheim, California.

4. J. Byme, B. Shen and W. Wallace. 1997. "Renewable Energy for Rural Development: Case Studies of Off-Grid Wind, Photovoltaic and Hybrid Systems in Rural China." Proceedings of the International Solar Energy Society Conference. Taejon, Korea. August 24-27.

5. J. Byrne, B. Shen, Q. Liu, and J. Zhao. 1997. Levelized Cost Analysis of Small-scale, Off-grid Photovoltaic, Wind, and PV-Wind Hybrid Systems for Inner Mongolia, China. Report submitted to the National Renewable Energy Laboratory. The Center for Energy and Environmental Policy, University of Delaware.

6. J. Byrne, Y-D. Wang, K-J. Boo, J. Song. 1997. "The Economic Viability of Dispatchable Peak Shaving PV Systems in Commercial Buildings of Korea." Proceedings of the International Solar Energy Society Conference. Taejon, Korea. August 24-27.

7. J. Byrne, S. Letendre, Y-D. Wang, R. Nigro and W. Ferguson. 1997. "Building Load Analysis of Dispatchable Peak-Shaving Photovoltaic Systems: A Regional Analysis of Technical and Economic Potential." Proceedings of the American Solar Energy Society Conference. Washington, D.C. April 25-30. Pp. 147-152. 


\subsection{List of Active Subcontracts}



10.0 LIST OF ACTIVE SUBCONTRACTS

\begin{tabular}{|c|c|c|c|c|}
\hline \multicolumn{5}{|l|}{ Subcontracts for FY 1997} \\
\hline $\begin{array}{l}\text { Contractor, Principal } \\
\text { Investigator, Address }\end{array}$ & $\begin{array}{c}\text { Work Title } \\
\text { (Research Activity) }\end{array}$ & $\begin{array}{l}\text { Contract } \\
\text { Number }\end{array}$ & $\begin{array}{l}\text { FY } 1997 \\
\text { Funding } \\
\text { (\$K) }\end{array}$ & $\begin{array}{l}\text { Start/ } \\
\text { End } \\
\text { Dates }\end{array}$ \\
\hline \multicolumn{5}{|c|}{$\begin{array}{l}\text { PV FUNDAMENTAL AND EXPLORATORY RESEARCH PROJECT } \\
\text { Crystalline Silicon } \\
\text { R. McConnell }\end{array}$} \\
\hline $\begin{array}{l}\text { Duke University } \\
\text { T. Tan } \\
\text { Durham, NC } 27708\end{array}$ & $\begin{array}{l}\text { Impurity Gettering in } \\
\text { Commercial PV Silicon }\end{array}$ & $\mathrm{XD}-2-11004-1$ & 145,000 & $\begin{array}{l}3 / 92 \\
1 / 97\end{array}$ \\
\hline $\begin{array}{l}\text { Georgia Institute of Technology } \\
\text { A. Rohatgi } \\
\text { Atlanta, GA } 30332\end{array}$ & $\begin{array}{l}\text { Fundamental Research } \\
\text { on Post-Growth Quality } \\
\text { Enhancement Techniques } \\
\text { in Crystalline Silicon } \\
\text { Materials }\end{array}$ & $\mathrm{XD}-2-11004-2$ & 0 & $\begin{array}{l}4 / 92 \\
6 / 97\end{array}$ \\
\hline $\begin{array}{l}\text { Massachusetts Institute of } \\
\text { Technology } \\
\text { L. Kimerling } \\
\text { Cambridge, MA } 02139\end{array}$ & $\begin{array}{l}\text { Role of Point Defects } \\
\text { and Impurities in } \\
\text { Processing and } \\
\text { Performance of } \\
\text { Crystalline Silicon Solar } \\
\text { Cells }\end{array}$ & $X D-2-11004-4$ & 0 & $\begin{array}{l}7 / 92 \\
1 / 96\end{array}$ \\
\hline $\begin{array}{l}\text { North Carolina State University } \\
\text { G. Rozgonyi } \\
\text { Raleigh, NC } 27695\end{array}$ & $\begin{array}{l}\text { Detailed Noncontact } \\
\text { Electrical and Structural } \\
\text { Characterization of PV } \\
\text { Silicon Substrates }\end{array}$ & XD-2-11004-6 & 0 & $\begin{array}{l}5 / 92 \\
5 / 97\end{array}$ \\
\hline $\begin{array}{l}\text { Texas Tech. University } \\
\text { S. Eistreicher } \\
\text { Lubbock, TX } 79409\end{array}$ & $\begin{array}{l}\text { Theoretical Analysis of } \\
\text { Hydrogen-Vacancy- } \\
\text { Impurity Formation and } \\
\text { Dissociation }\end{array}$ & XAX-5-15230-01 & 49,900 & $\begin{array}{l}6 / 95 \\
4 / 97\end{array}$ \\
\hline $\begin{array}{l}\text { University of California at Berkeley } \\
\text { E. Weber } \\
\text { Berkeley, CA } 94720\end{array}$ & $\begin{array}{l}\text { Identification and } \\
\text { Control of Lifetime- } \\
\text { Reducing Defects in } \\
\text { Polycrystalline Silicon } \\
\text { Photovoltaic Materials }\end{array}$ & $\mathrm{XD}-2-11004-3$ & 0 & $\begin{array}{l}7 / 92 \\
1 / 97\end{array}$ \\
\hline $\begin{array}{l}\text { University of South Florida } \\
\text { L. Jastrzebski } \\
\text { Tampa, FL } 33620\end{array}$ & $\begin{array}{l}\text { Optimization of } \\
\text { Gettering Processes for } \\
\text { PV Silicon }\end{array}$ & XD-2-11004-5 & 20,000 & $\begin{array}{l}5 / 92 \\
4 / 97\end{array}$ \\
\hline
\end{tabular}




\section{Subcontracts for FY 1997}

Contractor, Principal
Investigator, Address

Work Title
(Research Activity)

Contract
Number

\begin{tabular}{c|c}
$\begin{array}{c}\text { FY 1997 } \\
\text { Funding } \\
\text { (\$K) }\end{array}$ & $\begin{array}{c}\text { Start/ } \\
\text { End } \\
\text { Dates }\end{array}$ \\
\hline
\end{tabular}

\section{PV FUNDAMENTAL AND EXPLORATORY RESEARCH PROJECT \\ Exploratory Materials and Devices \\ R. McConnell}

\begin{tabular}{|c|c|c|c|c|}
\hline $\begin{array}{l}\text { California Institute of Technology } \\
\text { H. Atwater } \\
\text { Pasadena, CA } 91125\end{array}$ & $\begin{array}{l}\text { Novel Low-Temperature } \\
\text { Substrate Technology for } \\
\text { Compound } \\
\text { Semiconductor Solar } \\
\text { Cells on Low-Cost } \\
\text { Substrates }\end{array}$ & XAL-4-13357-01 & 56,213 & $\begin{array}{c}7 / 94 \\
12 / 97\end{array}$ \\
\hline $\begin{array}{l}\text { Johns Hopkins University } \\
\text { P. Searson } \\
\text { Baltimore, MD } 21218\end{array}$ & $\begin{array}{l}\text { Solar Energy Conversion } \\
\text { at Dye-Sensitized } \\
\text { Nanostructured } \\
\text { Electrodes Fabricated by } \\
\text { Sol-Gel Processing } \\
\end{array}$ & XAD-3-12114-04 & 47,820 & $\begin{array}{l}7 / 93 \\
9 / 97\end{array}$ \\
\hline $\begin{array}{l}\text { Research Triangle Institute } \\
\text { R. Venkatasubramanian } \\
\text { Research Triangle Park, NC } 27709\end{array}$ & $\begin{array}{l}\text { High-Efficiency GaAs } \\
\text { Solar Cells on } \\
\text { Polycrystalline Ge } \\
\text { Substrates }\end{array}$ & YAL-4-13357-03 & 214,000 & $\begin{array}{c}8 / 94 \\
12 / 97\end{array}$ \\
\hline $\begin{array}{l}\text { University of California at } \\
\text { Los Angeles } \\
\text { E. Yablonovitch } \\
\text { Los Angeles, CA } 90095\end{array}$ & $\begin{array}{l}\text { Ultra-Efficient Epitaxial } \\
\text { Liftoff Solar Cells } \\
\text { Exploiting Optical } \\
\text { Confinement in the Wave } \\
\text { Limit }\end{array}$ & XAL-4-13357-02 & 36,000 & $\begin{array}{l}7 / 94 \\
7 / 98\end{array}$ \\
\hline $\begin{array}{l}\text { University of Illinois at Urbana- } \\
\text { Champaign } \\
\text { A. Rockett } \\
\text { Urbana, II } 61820-6242\end{array}$ & $\begin{array}{l}\text { Fundamental Studies of } \\
\text { the Effect of Crystal } \\
\text { Defects on CuInSe//CdS } \\
\text { Heterojunction Behavior }\end{array}$ & XAD-3-12114-1 & 0 & $\begin{array}{c}6 / 93 \\
11 / 97\end{array}$ \\
\hline $\begin{array}{l}\text { University of Utah } \\
\text { P. Taylor } \\
\text { Salt Lake City, UT } 84112\end{array}$ & $\begin{array}{l}\text { Electronic Processes in } \\
\text { Thin-Film PV Materials }\end{array}$ & XAD-3-12114-02 & 59,999 & $\begin{array}{c}4 / 93 \\
12 / 97\end{array}$ \\
\hline $\begin{array}{l}\text { University of Washington } \\
\text { B. O'Regan } \\
\text { Seattle, WA } 98105\end{array}$ & $\begin{array}{l}\text { Electrochemical } \\
\text { Fabrication of Dye- } \\
\text { Sensitized } \\
\text { Heterojunctions for PV } \\
\text { Energy Conversion }\end{array}$ & XCR-5-15234-01 & 0 & $\begin{array}{l}6 / 95 \\
9 / 97\end{array}$ \\
\hline
\end{tabular}




\begin{tabular}{|c|c|c|c|c|}
\hline \multicolumn{5}{|l|}{ Subcontracts for FY 1997} \\
\hline $\begin{array}{l}\text { Contractor, Principal } \\
\text { Investigator, Address }\end{array}$ & $\begin{array}{l}\text { Work Title } \\
\text { (Research Activity) }\end{array}$ & $\begin{array}{l}\text { Contract } \\
\text { Number }\end{array}$ & $\begin{array}{l}\text { FY } 1997 \\
\text { Funding } \\
\text { (\$K) }\end{array}$ & $\begin{array}{l}\text { Start/ } \\
\text { End } \\
\text { Dates }\end{array}$ \\
\hline \multicolumn{5}{|c|}{$\begin{array}{l}\text { PV FUNDAMENTAL AND EXPLORATORY RESEARCH PROJECT } \\
\text { Historically Black Colleges and Universities (HBCU) }\end{array}$} \\
\hline $\begin{array}{l}\text { Central State University } \\
\text { C.W. Fuller } \\
\text { Wilberforce, OH } 45384\end{array}$ & $\begin{array}{l}\text { HBCU PV Associates } \\
\text { Program }\end{array}$ & XAX-5-15021-04 & 5,639 & $\begin{array}{l}10 / 95 \\
10 / 98\end{array}$ \\
\hline $\begin{array}{l}\text { Clark Atlanta University } \\
\text { G. Grams } \\
\text { Atlanta, GA } 30314\end{array}$ & $\begin{array}{l}\text { HBCU PV Associates } \\
\text { Program; An Assessment } \\
\text { of the Operation of } \\
\text { Photovoltaics Devised } \\
\text { for Solar Energy } \\
\text { Applications }\end{array}$ & XCR-6-15327-01 & 0 & $\begin{array}{c}10 / 95 \\
4 / 98\end{array}$ \\
\hline $\begin{array}{l}\text { Clark Atlanta University } \\
\text { C. Papageorgopolous } \\
\text { Atlanta, GA } 30314\end{array}$ & $\begin{array}{l}\text { HBCU PV Associates } \\
\text { Program }\end{array}$ & XAX-5-15021-02 & 20,122 & $\begin{array}{l}9 / 95 \\
9 / 98\end{array}$ \\
\hline $\begin{array}{l}\text { Hampton University } \\
\text { B. Grant } \\
\text { Hampton, VA } 23668\end{array}$ & $\begin{array}{l}\text { HBCU PV Associates } \\
\text { Program }\end{array}$ & XAX-5-15021-07 & 0 & $\begin{array}{l}10 / 95 \\
10 / 97\end{array}$ \\
\hline $\begin{array}{l}\text { Mississippi Valley State Univ. } \\
\text { B. Balam } \\
\text { Itta Bena, MS } 38941\end{array}$ & $\begin{array}{l}\text { HBCU PV Associates } \\
\text { Program }\end{array}$ & $\mathrm{XAX}-5-15021-06$ & 11,236 & $\begin{array}{l}10 / 95 \\
10 / 98\end{array}$ \\
\hline $\begin{array}{l}\text { Southern University } \\
\text { P. Bhattacharya } \\
\text { Baton Rouge, LA } 70813\end{array}$ & $\begin{array}{l}\text { HBCU PV Associates } \\
\text { Program }\end{array}$ & XAX-5-15021-05 & 5,163 & $\begin{array}{l}9 / 95 \\
9 / 98\end{array}$ \\
\hline $\begin{array}{l}\text { Texas Southern University, } \\
\text { J. Hill } \\
\text { Houston, TX } 77004\end{array}$ & $\begin{array}{l}\text { HBCU PV Associates } \\
\text { Program }\end{array}$ & $\mathrm{XAX}-5-15021-03$ & 5,654 & $\begin{array}{l}10 / 95 \\
10 / 98\end{array}$ \\
\hline $\begin{array}{l}\text { Wilberforce University } \\
\text { R. Nadella, E. Asikele, T. Habash } \\
\text { Wilberforce, OH } 45384\end{array}$ & $\begin{array}{l}\text { HBCU PV Associates } \\
\text { Program }\end{array}$ & XAX-5-15021-01 & 16,440 & $\begin{array}{l}9 / 95 \\
9 / 98\end{array}$ \\
\hline \multicolumn{5}{|c|}{$\begin{array}{l}\text { THIN-FILM PV PARTNERSHIP PROJECT } \\
\text { K. Zweibel }\end{array}$} \\
\hline $\begin{array}{l}\text { Astropower, Inc. } \\
\text { J. Rand } \\
\text { Newark, DE } 19716\end{array}$ & $\begin{array}{l}\text { Light-Trapped, } \\
\text { Interconnected, Silicon- } \\
\text { Film Modules }\end{array}$ & ZAF-5-14142-02 & 653,118 & $\begin{array}{l}11 / 94 \\
11 / 97\end{array}$ \\
\hline
\end{tabular}




\begin{tabular}{|c|c|c|c|c|}
\hline \multicolumn{5}{|l|}{ Subcontracts for FY 1997} \\
\hline $\begin{array}{l}\text { Contractor, Principal } \\
\text { Investigator, Address }\end{array}$ & $\begin{array}{c}\text { Work Title } \\
\text { (Research Activity) }\end{array}$ & $\begin{array}{l}\text { Contract } \\
\text { Number }\end{array}$ & $\begin{array}{c}\text { FY } 1997 \\
\text { Funding } \\
\text { (\$K) }\end{array}$ & $\begin{array}{l}\text { Start/ } \\
\text { End } \\
\text { Dates }\end{array}$ \\
\hline $\begin{array}{l}\text { Colorado School of Mines } \\
\text { D. Williamson } \\
\text { Golden, CO } 80401\end{array}$ & $\begin{array}{l}\text { Microstructure of a-Si- } \\
\text { Based Solar Cell } \\
\text { Materials by Small- } \\
\text { Angle X-Ray-Scattering }\end{array}$ & XAN-4-13318-04 & 78,369 & $\begin{array}{l}4 / 94 \\
6 / 98\end{array}$ \\
\hline $\begin{array}{l}\text { Colorado School of Mines } \\
\text { J. Trefny } \\
\text { Golden, CO } 80401\end{array}$ & $\begin{array}{l}\text { Process of } \\
\text { Electrochemically } \\
\text { Deposited CdTe-Based } \\
\text { Solar Cells }\end{array}$ & XAF-5-14142-11 & 233,557 & $\begin{array}{l}4 / 95 \\
4 / 98\end{array}$ \\
\hline $\begin{array}{l}\text { Colorado State University } \\
\text { J. Sites } \\
\text { Fort Collins, CO } 80525\end{array}$ & $\begin{array}{l}\text { Device Physics of Thin- } \\
\text { Film Polycrystalline } \\
\text { Cells and Modules }\end{array}$ & $\mathrm{XAX}-4-14000-01$ & 105,000 & $\begin{array}{c}12 / 93 \\
3 / 98\end{array}$ \\
\hline $\begin{array}{l}\text { Energy Conversion Devices } \\
\text { X. Deng } \\
\text { Troy, MI } 48084\end{array}$ & $\begin{array}{l}\text { Development of High, } \\
\text { Stable-Efficiency Triple- } \\
\text { Junction a:Si Alloy Solar } \\
\text { Cells }\end{array}$ & ZAN-4-13318-11 & 0 & $\begin{array}{l}7 / 94 \\
2 / 98\end{array}$ \\
\hline $\begin{array}{l}\text { Energy Photovoltaics } \\
\text { A. Delahoy } \\
\text { Princeton, NJ } 08543\end{array}$ & $\begin{array}{l}\text { CIS Photovoltaic } \\
\text { Technology }\end{array}$ & ZAF-5-14142-04 & $1,062,801$ & $\begin{array}{l}1 / 95 \\
1 / 98\end{array}$ \\
\hline $\begin{array}{l}\text { Florida Solar Energy Center } \\
\text { N. Dhere } \\
\text { Cocoa, FL } 32922\end{array}$ & $\begin{array}{l}\text { CdTe Module Testing } \\
\text { and Study of Transient } \\
\text { and Irreversible Effects } \\
\text { in Thin-Film Solar Cells } \\
\end{array}$ & XCR-6-16773-01 & 5,000 & $\begin{array}{l}5 / 96 \\
5 / 98\end{array}$ \\
\hline $\begin{array}{l}\text { Florida Solar Energy Center } \\
\text { N. Dhere } \\
\text { Cocoa, FL } 32922\end{array}$ & $\begin{array}{l}\text { Polycrystalline Thin-Film } \\
\text { CuInSe }_{2} \text { and CdTe PV } \\
\text { Solar Cells } \\
\end{array}$ & XG-2-11036-5 & 0 & $\begin{array}{l}4 / 92 \\
9 / 97\end{array}$ \\
\hline $\begin{array}{l}\text { Harvard University } \\
\text { R. Gordon } \\
\text { Cambridge, MA } 02138\end{array}$ & $\begin{array}{l}\text { Optimization of } \\
\text { Transparent and } \\
\text { Reflecting Electrodes for } \\
\text { a-Si Solar Cells }\end{array}$ & XAN-4-13318-05 & 114,181 & $\begin{array}{l}5 / 94 \\
5 / 98\end{array}$ \\
\hline $\begin{array}{l}\text { Iowa State University } \\
\text { V. Dalal } \\
\text { Ames, IA } 50011\end{array}$ & $\begin{array}{l}\text { Comprehensive Research } \\
\text { on Stability and } \\
\text { Performance on a-Si:H } \\
\text { and Alloys } \\
\end{array}$ & XAN-4-13318-08 & 247,084 & $\begin{array}{l}5 / 94 \\
7 / 98\end{array}$ \\
\hline $\begin{array}{l}\text { ISET } \\
\text { V. Kapur } \\
\text { Inglewood, CA } 90301\end{array}$ & $\begin{array}{l}\text { Application of CIS to } \\
\text { High-Efficiency PV } \\
\text { Module Fabrication }\end{array}$ & ZAF-5-14142-07 & 326,000 & $\begin{array}{l}4 / 95 \\
4 / 98\end{array}$ \\
\hline
\end{tabular}




\begin{tabular}{|c|c|c|c|c|}
\hline \multicolumn{5}{|l|}{ Subcontracts for FY 1997} \\
\hline $\begin{array}{l}\text { Contractor, Principal } \\
\text { Investigator, Address }\end{array}$ & $\begin{array}{c}\text { Work Title } \\
\text { (Research Activity) }\end{array}$ & $\begin{array}{l}\text { Contract } \\
\text { Number }\end{array}$ & $\begin{array}{l}\text { FY } 1997 \\
\text { Funding } \\
\text { (\$K) }\end{array}$ & $\begin{array}{l}\text { Start/ } \\
\text { End } \\
\text { Dates }\end{array}$ \\
\hline $\begin{array}{l}\text { National Institute of Standards and } \\
\text { Technology } \\
\text { A. Gallagher } \\
\text { Boulder, CO } 80303\end{array}$ & $\begin{array}{l}\text { Atomic-Scale } \\
\text { Characterization of a- } \\
\text { Si:H Films and Devices }\end{array}$ & DAD-4-14084-1 & 117,469 & $\begin{array}{l}12 / 94 \\
3 / 98\end{array}$ \\
\hline $\begin{array}{l}\text { Pennsylvania State University } \\
\text { R. Collins } \\
\text { University Park, PA } 16802\end{array}$ & $\begin{array}{l}\text { Wide-Band-Gap Solar } \\
\text { Cells with High } \\
\text { Stabilized Performance }\end{array}$ & XAN-4-13318-03 & $163,79 \hat{1}$ & $\begin{array}{l}7 / 94 \\
3 / 98\end{array}$ \\
\hline $\begin{array}{l}\text { Purdue University } \\
\text { J. Gray } \\
\text { West Lafayette, IN } 47907\end{array}$ & $\begin{array}{l}\text { Development and } \\
\text { Application of a } \\
\text { Computer Model for } \\
\text { CdTe and CuInSe } \\
\end{array}$ & XG-2-11036-2 & 0 & $\begin{array}{c}3 / 92 \\
12 / 96\end{array}$ \\
\hline $\begin{array}{l}\text { Siemens Solar Industries } \\
\text { D. Tarrant } \\
\text { Camarillo, CA } 93011\end{array}$ & $\begin{array}{l}\text { CIS-Based Thin Film PV } \\
\text { Technology }\end{array}$ & ZAF-5-14142-03 & $1,076,373$ & $\begin{array}{l}8 / 95 \\
8 / 98\end{array}$ \\
\hline $\begin{array}{l}\text { Solar Cells, Inc. } \\
\text { G. Dorer } \\
\text { Toledo, OH } 43607\end{array}$ & $\begin{array}{l}\text { Technology Support for } \\
\text { Initiation of High- } \\
\text { Throughput Processing } \\
\text { of Thin-Film CdTe PV } \\
\text { Modules }\end{array}$ & ZAF-5-14142-05 & $1,032,511$ & $\begin{array}{l}3 / 95 \\
3 / 98\end{array}$ \\
\hline $\begin{array}{l}\text { Solarex Corporation } \\
\text { R. Arya } \\
\text { Newtown, PA 1894, }\end{array}$ & $\begin{array}{l}\text { Amorphous Silicon } \\
\text { Research }\end{array}$ & ZAN-4-13318-01 & 395,494 & $\begin{array}{l}9 / 94 \\
2 / 98\end{array}$ \\
\hline $\begin{array}{l}\text { Syracuse University } \\
\text { E. Schiff } \\
\text { Syracuse, NY } 13244\end{array}$ & $\begin{array}{l}\text { Research on } \\
\text { High-Bandgap Materials } \\
\text { and Amorphous-Silicon- } \\
\text { Based Solar Cells }\end{array}$ & XAN-4-13318-06 & 81,208 & $\begin{array}{l}5 / 94 \\
3 / 98\end{array}$ \\
\hline $\begin{array}{l}\text { United Solar Systems Corporation } \\
\text { S. Guha } \\
\text { Troy, MI } 48084\end{array}$ & $\begin{array}{l}\text { Amorphous Silicon } \\
\text { Research }\end{array}$ & ZAN-4-13318-02 & 447,000 & $\begin{array}{c}7 / 94 \\
12 / 97\end{array}$ \\
\hline $\begin{array}{l}\text { United Solar Systems Corporation } \\
\text { S. Guha } \\
\text { Troy, MI } 48084\end{array}$ & $\begin{array}{l}\text { Thin Film a-Si Alloy } \\
\text { Research Partnership }\end{array}$ & ZAF-5-14142-01 & 713,000 & $\begin{array}{l}2 / 95 \\
2 / 98\end{array}$ \\
\hline
\end{tabular}




\begin{tabular}{|c|c|c|c|c|}
\hline \multicolumn{5}{|l|}{ Subcontracts for FY 1997} \\
\hline $\begin{array}{l}\text { Contractor, Principal } \\
\text { Investigator, Address }\end{array}$ & $\begin{array}{c}\text { Work Title } \\
\text { (Research Activity) }\end{array}$ & $\begin{array}{l}\text { Contract } \\
\text { Number }\end{array}$ & $\begin{array}{l}\text { FY } 1997 \\
\text { Funding } \\
\text { (SK) }\end{array}$ & $\begin{array}{l}\text { Start/ } \\
\text { End } \\
\text { Dates }\end{array}$ \\
\hline $\begin{array}{l}\text { University of California at } \\
\text { Los Angeles } \\
\text { R Braunstein } \\
\text { Los Angeles, CA } 90024\end{array}$ & $\begin{array}{l}\text { Photocharge Transport } \\
\text { and Recombination } \\
\text { Measurements in } \\
\text { Amorphous Silicon } \\
\text { Films and Solar Cells by } \\
\text { Photoconductive } \\
\text { Frequency Mixing }\end{array}$ & XAN-4-13318-10 & 22,500 & $\begin{array}{l}5 / 94 \\
4 / 98\end{array}$ \\
\hline $\begin{array}{l}\text { University of Delaware-IEC } \\
\text { R Birkmire } \\
\text { Newark, DE } 19716\end{array}$ & $\begin{array}{l}\text { Optimization of } \\
\text { Processing and Modeling } \\
\text { Issues for Thin-Film } \\
\text { Solar Cell Devices }\end{array}$ & XAK-7-17609-01 & 902,500 & $\begin{array}{l}2 / 97 \\
2 / 01\end{array}$ \\
\hline $\begin{array}{l}\text { University of Florida } \\
\text { T. Anderson } \\
\text { Gainesville, FL } 32611\end{array}$ & $\begin{array}{l}\text { Processing of CuInSe})_{2-} \\
\text { Based Solar Cells: } \\
\text { Characterization of } \\
\text { Deposition Processes in } \\
\text { Terms of Chemical } \\
\text { Reaction Analyses }\end{array}$ & XAF-5-14142-10 & 225,146 & $\begin{array}{l}5 / 95 \\
5 / 98\end{array}$ \\
\hline $\begin{array}{l}\text { University of North Carolina } \\
\text { D. Han } \\
\text { Chapel Hill, NC } 27514\end{array}$ & $\begin{array}{l}\text { Experimental and } \\
\text { Theoretical Study of the } \\
\text { Factors Governing the } \\
\text { Staebler-Wronski } \\
\text { Photodegradation Effect } \\
\text { in a-Si:H Solar Cells }\end{array}$ & XAN-4-13318-09 & 101,166 & $\begin{array}{l}7 / 94 \\
3 / 98\end{array}$ \\
\hline $\begin{array}{l}\text { University of Oregon } \\
\text { D. Cohen } \\
\text { Eugene, OR } 97403\end{array}$ & $\begin{array}{l}\text { Identifying Electronic } \\
\text { Properties Relevant to } \\
\text { Improving Stability in } \\
\text { a-Si:H-Based Cells and } \\
\text { Overall Performance in } \\
\text { a-SiGe:H-Based Cells }\end{array}$ & XAN-4-13318-07 & 96,000 & $\begin{array}{c}4 / 94 \\
11 / 98\end{array}$ \\
\hline $\begin{array}{l}\text { University of South Florida } \\
\text { D. Morel } \\
\text { Tampa, FL } 33620\end{array}$ & $\begin{array}{l}\text { Advanced Processing of } \\
\mathrm{CdTe} \text { and } \mathrm{Cu}(\mathrm{InGa}) \mathrm{Se}_{2-} \\
\text { Based Solar Cells and } \\
\text { Submodules }\end{array}$ & XAF-5-14142-09 & 264,332 & $\begin{array}{l}4 / 95 \\
4 / 98\end{array}$ \\
\hline $\begin{array}{l}\text { University of South Florida } \\
\text { D. Morel } \\
\text { Tampa, FL } 33620\end{array}$ & $\begin{array}{l}\text { Heterojunction } \\
\text { Development and } \\
\text { Optimization in Thin- } \\
\text { Film Compound } \\
\text { Semiconductor Cells }\end{array}$ & $\mathrm{XAD}-3-12114-3$ & 0 & $\begin{array}{l}5 / 93 \\
9 / 97\end{array}$ \\
\hline
\end{tabular}




\begin{tabular}{|c|c|c|c|c|}
\hline \multicolumn{5}{|l|}{ Subcontracts for FY 1997} \\
\hline $\begin{array}{l}\text { Contractor, Principal } \\
\text { Investigator, Address }\end{array}$ & $\begin{array}{l}\text { Work Title } \\
\text { (Research Activity) }\end{array}$ & $\begin{array}{l}\text { Contract } \\
\text { Number }\end{array}$ & $\begin{array}{l}\text { FY } 1997 \\
\text { Funding } \\
\text { (\$K) }\end{array}$ & $\begin{array}{l}\text { Start/ } \\
\text { End } \\
\text { Dates }\end{array}$ \\
\hline $\begin{array}{l}\text { University of Toledo } \\
\text { A. Compaan } \\
\text { Toledo, OH } 43606\end{array}$ & $\begin{array}{l}\text { High-Efficiency Thin- } \\
\text { Film Cadmium- } \\
\text { Telluride-Based Solar } \\
\text { Cells }\end{array}$ & ZAX-4-14013-01 & 137,100 & $\begin{array}{l}1 / 94 \\
3 / 98\end{array}$ \\
\hline $\begin{array}{l}\text { University of Toledo } \\
\text { A. Compaan } \\
\text { Toledo, OH } 43606\end{array}$ & $\begin{array}{l}\text { Optimization of Laser } \\
\text { Scribing for Thin-Film } \\
\text { PV Modules }\end{array}$ & ZAF-5-14142-08 & 0 & $\begin{array}{l}4 / 95 \\
10 / 97\end{array}$ \\
\hline $\begin{array}{l}\text { Washington State University } \\
\text { L. Olsen } \\
\text { Richland, WA }\end{array}$ & $\begin{array}{l}\text { Alternative Window } \\
\text { Schemes for CuInSe } \\
\text { Based Solar Cells }\end{array}$ & XAF-6-15375-01 & 87,500 & $\begin{array}{l}11 / 95 \\
12 / 97\end{array}$ \\
\hline \multicolumn{5}{|c|}{$\begin{array}{l}\text { PHOTOVOLTAIC MANUFACTURING TECHNOLOGY (PVMaT) PROJECT } \\
\text { E. Witt }\end{array}$} \\
\hline $\begin{array}{l}\text { Advanced Energy Systems, Inc. } \\
\text { R. Wills } \\
\text { Wilton, NH }\end{array}$ & $\begin{array}{l}\text { Next Generation Three- } \\
\text { Phase Inverter }\end{array}$ & ZAF-5-14271-10 & 210,235 & $\begin{array}{l}9 / 95 \\
5 / 98\end{array}$ \\
\hline $\begin{array}{l}\text { Ascension Technology, Inc. } \\
\text { E. Kern } \\
\text { Waltham, MA } 01773\end{array}$ & $\begin{array}{l}\text { Manufacture of an AC } \\
\text { Photovoltaic Module }\end{array}$ & ZAF-5-14271-05 & 75,942 & $\begin{array}{l}7 / 25 \\
7 / 97\end{array}$ \\
\hline $\begin{array}{l}\text { ASE Americas } \\
\text { M. Kardauskas } \\
\text { Billerica, MA } 01821\end{array}$ & $\begin{array}{l}\text { Market Driven EFG } \\
\text { Modules }\end{array}$ & ZAF-6-14271-13 & 286,000 & $\begin{array}{l}12 / 95 \\
2 / 99\end{array}$ \\
\hline $\begin{array}{l}\text { AstroPower, Inc. } \\
\text { J. Rand } \\
\text { Newark, DE } 19711\end{array}$ & $\begin{array}{l}\text { Large-Area Silicon- } \\
\text { Film }^{\text {TM }} \text { Panels and Solar } \\
\text { Cells }\end{array}$ & ZAF-5-14271-03 & $1,625,725$ & $\begin{array}{l}6 / 95 \\
2 / 98\end{array}$ \\
\hline $\begin{array}{l}\text { Evergreen Solar, Inc. } \\
\text { J. Hanoka } \\
\text { Waltham, MA } 02154\end{array}$ & $\begin{array}{l}\text { Advanced Polymer PV } \\
\text { System }\end{array}$ & ZAF-5-14271-09 & 0 & $\begin{array}{l}12 / 95 \\
11 / 97\end{array}$ \\
\hline $\begin{array}{l}\text { Iowa Thin Film Technology } \\
\text { F. Jeffrey } \\
\text { Ames, IA } 50010\end{array}$ & $\begin{array}{l}\text { Monolithic a-Si Modules } \\
\text { on Continuous Polymer } \\
\text { Substrates }\end{array}$ & ZAF-5-14271-04 & 142,781 & $\begin{array}{c}7 / 95 \\
10 / 98\end{array}$ \\
\hline $\begin{array}{l}\text { Omnion Power Engineer Corp. } \\
\text { H. Meyer } \\
\text { East Troy, WI } 53120\end{array}$ & $\begin{array}{l}\text { Three-Phase Power } \\
\text { Conversion System for } \\
\text { Utility Interconnected PV } \\
\text { Applications }\end{array}$ & ZAF-5-14271-02 & 258,458 & $\begin{array}{l}8 / 95 \\
2 / 98\end{array}$ \\
\hline
\end{tabular}




\begin{tabular}{|c|c|c|c|c|}
\hline \multicolumn{5}{|l|}{ Subcontracts for FY 1997} \\
\hline $\begin{array}{l}\text { Contractor, Principal } \\
\text { Investigator, Address }\end{array}$ & $\begin{array}{c}\text { Work Title } \\
\text { (Research Activity) }\end{array}$ & $\begin{array}{l}\text { Contract } \\
\text { Number }\end{array}$ & $\begin{array}{l}\text { FY } 1997 \\
\text { Funding } \\
\text { (\$K) }\end{array}$ & $\begin{array}{l}\text { Start/ } \\
\text { End } \\
\text { Dates }\end{array}$ \\
\hline $\begin{array}{l}\text { Photovoltaics International, LLC } \\
\text { N. Kaminar } \\
\text { Sunnyvale, CA } 94086\end{array}$ & $\begin{array}{l}\text { Manufacturing of the } \\
\text { PVI PowerGrid }\end{array}$ & ZAF-6-14271-11 & $1,085,000$ & $\begin{array}{l}2 / 95 \\
1 / 99\end{array}$ \\
\hline $\begin{array}{l}\text { Siemens Solar Industries } \\
\text { T. Jester } \\
\text { Camarillo, CA } 93011\end{array}$ & $\begin{array}{l}\text { Photovoltaic } \mathrm{Cz} \text { Silicon } \\
\text { Module Improvements }\end{array}$ & ZAF-6-14271-12 & 374,848 & $\begin{array}{c}11 / 95 \\
1 / 99\end{array}$ \\
\hline $\begin{array}{l}\text { Solar Cells, Inc. } \\
\text { D. Sandwisch } \\
\text { Toledo, OH } 43607\end{array}$ & $\begin{array}{l}\text { High-Throughput } \\
\text { Manufacturing of Thin- } \\
\text { Film CdTe PV Modules }\end{array}$ & ZAI-4-11294-02 & $1,336,740$ & $\begin{array}{l}11 / 93 \\
11 / 98\end{array}$ \\
\hline $\begin{array}{l}\text { Solar Design Associates } \\
\text { S. Strong } \\
\text { Harvard, MA } 01451 .\end{array}$ & $\begin{array}{l}\text { Development of } \\
\text { Standardized, Low-Cost } \\
\text { AC PV Systems }\end{array}$ & ZAF-5-14271-01 & 145,809 & $\begin{array}{l}9 / 95 \\
2 / 98\end{array}$ \\
\hline $\begin{array}{l}\text { Solar Electric Specialties Company } \\
\text { G. Minyard } \\
\text { Willits, CA } 95450\end{array}$ & $\begin{array}{l}\text { Design, Fabrication, and } \\
\text { Certification of } \\
\text { Advanced Modular PV } \\
\text { Power Systems }\end{array}$ & ZAF-5-14271-07 & 0 & $\begin{array}{l}9 / 95 \\
2 / 98\end{array}$ \\
\hline $\begin{array}{l}\text { Solarex Corporation } \\
\text { J. Wohlgemuth } \\
\text { Frederick, MD } 21754\end{array}$ & $\begin{array}{l}\text { Photovoltaic } \\
\text { Manufacturing } \\
\text { Technololgy Phase 2B, } \\
\text { Process-Specific Issues }\end{array}$ & ZAI-4-11294-01 & 135,461 & $\begin{array}{c}12 / 93 \\
3 / 98\end{array}$ \\
\hline $\begin{array}{l}\text { STR (formerly Springborn } \\
\text { Laboratories, Inc.) } \\
\text { W. Holley } \\
\text { Enfield, CT } 06082\end{array}$ & $\begin{array}{l}\text { Advanced Development } \\
\text { of PV Encapsulants }\end{array}$ & ZAG-3-11219-02 & 417,201 & $\begin{array}{l}12 / 92 \\
3 / 97\end{array}$ \\
\hline $\begin{array}{l}\text { Trace Engineering } \\
\text { C. Freitas } \\
\text { Arlington, WA } 98223\end{array}$ & $\begin{array}{l}\text { 2-kW DC to AC Power } \\
\text { Inverter Module for PV } \\
\text { Applications }\end{array}$ & ZAF-5-14271-08 & 109,573 & $\begin{array}{l}9 / 95 \\
5 / 98\end{array}$ \\
\hline $\begin{array}{l}\text { Utility Power Group } \\
\text { M. Stern } \\
\text { Chatsworth, CA } 91311\end{array}$ & $\begin{array}{l}\text { Development of a Low- } \\
\text { Cost Integrated 20-kW } \\
\text { AC Solar-Tracking } \\
\text { Subarray for Grid- } \\
\text { Connected PV Power } \\
\text { System Applications }\end{array}$ & ZAF-5-14271-06 & 0 & $\begin{array}{l}7 / 95 \\
7 / 97\end{array}$ \\
\hline
\end{tabular}




\begin{tabular}{|c|c|c|c|c|}
\hline \multicolumn{5}{|c|}{$\begin{array}{l}\text { PV ENGINEERING AND RELIABILITY PROJECT } \\
\text { R. Hulstrom }\end{array}$} \\
\hline $\begin{array}{l}\text { Endecon Engineering } \\
\text { C. Whitaker } \\
\text { San Ramon, CA }\end{array}$ & $\begin{array}{l}\text { Energy Rating } \\
\text { Methodology } \\
\text { Development }\end{array}$ & AAI-4-14192-0I & 0 & $\begin{array}{c}5 / 94 \\
12 / 97\end{array}$ \\
\hline $\begin{array}{l}\text { NEOS Corporation } \\
\text { T. Schuyler } \\
\text { Lakewood, } \mathrm{CO}\end{array}$ & $\begin{array}{l}\text { Development of Test } \\
\text { Methods and Procedures } \\
\text { for Evaluation of PV } \\
\text { Systems }\end{array}$ & AAD-7-17628-01 & 48,987 & $\begin{array}{l}5 / 97 \\
5 / 98\end{array}$ \\
\hline $\begin{array}{l}\text { Powermark Corporation } \\
\text { S. Chalmers } \\
\text { Phoenix, AZ }\end{array}$ & $\begin{array}{l}\text { PV Certification and } \\
\text { Accreditation } \\
\text { Management Support }\end{array}$ & AAX-7-16821-01 & 35,000 & $\begin{array}{l}11 / 96 \\
11 / 98\end{array}$ \\
\hline $\begin{array}{l}\text { Solar Energy Industries Assoc. } \\
\text { J. Anderson } \\
\text { Washington, DC }\end{array}$ & $\begin{array}{l}\text { Management and } \\
\text { Administration of the } \\
\text { IEC/TC82 }\end{array}$ & ACU-7-17605-01 & 65,000 & $\begin{array}{l}4 / 97 \\
4 / 99\end{array}$ \\
\hline $\begin{array}{l}\text { University of Colorado at Denver } \\
\text { J. Boon } \\
\text { Denver, CO }\end{array}$ & $\begin{array}{l}\text { Prototype Portable } \\
\text { Custom-Made PV } \\
\text { Encapsulant and Solar } \\
\text { Cell Degradation } \\
\text { Monitor (A } \\
\text { Spectrophotometric } \\
\text { Analyzer) }\end{array}$ & $\mathrm{XAX}-6-14454-01$ & 0 & $\begin{array}{l}3 / 96 \\
9 / 97\end{array}$ \\
\hline \multicolumn{5}{|c|}{$\begin{array}{l}\text { PV DOMESTIC APPLICATIONS AND MARKETS PROJECT } \\
\text { J. Thornton }\end{array}$} \\
\hline $\begin{array}{l}\text { McNeil Technologies } \\
\text { K. DeGroat } \\
\text { Springfield, VA } 22150\end{array}$ & $\begin{array}{l}\text { Photovoltaic Technology, } \\
\text { Application, and Market } \\
\text { Analysis Support }\end{array}$ & AAK-6-16784-01 & 67,000 & $\begin{array}{l}6 / 96 \\
2 / 98\end{array}$ \\
\hline $\begin{array}{l}\text { Pacific Energy Group } \\
\text { H. Wenger } \\
\text { Walnut Creek, CA } 94596\end{array}$ & $\begin{array}{l}\text { Renewable Energy } \\
\text { Applications and } \\
\text { Economic Analysis for } \\
\text { Electric Power }\end{array}$ & AAX-5-15330-01 & 167,000 & $\begin{array}{c}8 / 95 \\
12 / 98\end{array}$ \\
\hline $\begin{array}{l}\text { SUNY at Albany } \\
\text { R. Perez } \\
\text { Albany, NY } 12205\end{array}$ & $\begin{array}{l}\text { Solar Resource Utility } \\
\text { Load-Matching } \\
\text { Assessment }\end{array}$ & XAR-1-11168-1 & 0 & $\begin{array}{l}9 / 91 \\
1 / 98\end{array}$ \\
\hline $\begin{array}{l}\text { University of Delaware } \\
\text { J. Byrne } \\
\text { Newark, DE } 19616\end{array}$ & $\begin{array}{l}\text { Evaluation of } \\
\text { Photovoltaic Peak- } \\
\text { Shaving Applications in } \\
\text { the U.S. Buildings Sector }\end{array}$ & XAX-8-17678-01 & 38,000 & $\begin{array}{l}12 / 97 \\
12 / 98\end{array}$ \\
\hline
\end{tabular}


PV INTERNATIONAL APPLICATIONS AND MARKETS PROJECT

R. Taylor

\begin{tabular}{|c|c|c|c|c|}
\hline $\begin{array}{l}\text { Institute of Policy and Management } \\
\text { L. Xiuguo } \\
\text { Beijing, China } 100080\end{array}$ & $\begin{array}{l}\text { Support for Commercial } \\
\text { Project Development in } \\
\text { China }\end{array}$ & AAK-7-17668-01 & 50,011 & $\begin{array}{l}9 / 97 \\
9 / 99\end{array}$ \\
\hline $\begin{array}{l}\text { Solar Electric Light Fund } \\
\text { R. Freling } \\
\text { Washington, DC } 20009\end{array}$ & $\begin{array}{l}\text { Photovoltaics for Rural } \\
\text { Energy in Gansu } \\
\text { Province in the People's } \\
\text { Republic of China }\end{array}$ & AAX-6-15376-01 & 0 & $\begin{array}{c}12 / 95 \\
6 / 98\end{array}$ \\
\hline $\begin{array}{l}\text { University of Delaware } \\
\text { J. Byrne } \\
\text { Newark, DE } 19616\end{array}$ & $\begin{array}{l}\text { Evaluation of } \\
\text { Intermediate } \\
\text { Applications for } \\
\text { Photovoltaics in the } \\
\text { United States and } \\
\text { Developing Countries }\end{array}$ & XCU-7-16806-0 & 0 & $\begin{array}{c}6 / 97 \\
12 / 98\end{array}$ \\
\hline C. Sherring & Consultant, India Project & EAK-7-1756-01 & 10,500 & $\begin{array}{l}9 / 97 \\
6 / 98 \\
\end{array}$ \\
\hline Ministry of Agriculture, China & Gansu Project & AAX-8-17681-01 & 35,000 & $\begin{array}{l}12 / 97 \\
6 / 98\end{array}$ \\
\hline $\begin{array}{l}\text { Center for Renewable Energy } \\
\text { Development, China }\end{array}$ & $\begin{array}{l}\text { Rural Electrification } \\
\text { Development }\end{array}$ & AAX-7-17679-01 & 95,000 & $\begin{array}{l}2 / 98 \\
2 / 99\end{array}$ \\
\hline New World Village Power & Brazil Hybrid Project & AAD-5-13504-06 & 10,000 & $\begin{array}{l}10 / 94 \\
12 / 98\end{array}$ \\
\hline Advanced Energy Systems & Brazil Hybrid Project & AAX-7-17626-01 & 30,900 & $\begin{array}{l}5 / 97 \\
3 / 98 \\
\end{array}$ \\
\hline Matshoba \& Assoc. & S. Africa Project Support & AAD-8-17645-01 & 10,000 & $\begin{array}{l}2 / 98 \\
5 / 98 \\
\end{array}$ \\
\hline D. Barley & S. Africa Project Support & CXG-6-16275-01 & 5,300 & $\begin{array}{l}4 / 97 \\
9 / 97\end{array}$ \\
\hline M. Costanza & S. Africa Project Support & AXL-7-17420-01 & 8,300 & $\begin{array}{l}5 / 97 \\
4 / 98 \\
\end{array}$ \\
\hline Sunwize & Hybrid Testing & Power Station & 20,500 & $12 / 97$ \\
\hline J. Weingart & Village Power & CXL-7-17412-01 & 20,500 & $\begin{array}{l}2 / 97 \\
12 / 97\end{array}$ \\
\hline
\end{tabular}




\subsection{NREL Photovoltaic Program FY 1997 Bibliography}





\subsection{NREL Photovoltaic Program FY 1997 Bibliography}

Abulfotuh, F.; Wangensteen, T; Ahrenkiel, R; Kazmerski, L. L. Optical Properties and Defect Levels in a Surface Layer Found on CuInSe, Thin Films. Conference Record of the Twenty-Fifth IEEE Photovoltaic Specialists Conference, 13-17 May 1996, Washington, D.C. New York: Institute of Electrical and Electronics Engineers, 1996; pp. 993-996.

Advanced Photovoltaics Systems: Amorphous Silicon PV Manufacturing. DOE/GO-10096-298. NTIS Order No. DE96013080.

Ahrenkiel, R. K. Carrier Recombination in Silicon Materials Used for Photovoltaic Devices. Witt, C. E.; Al-Jassim, M.; Gee, J. M., eds. NREL/SNL Photovoltaics Program Review: Proceedings of the 14th Conference, 18-22 November 1996, Lakewood, Colorado. AIP Conference Proceedings 394. Woodbury, NY: American Institute of Physics, 1997; pp. $225-243$.

Albin, D.; Rose, D.; Swartzlander, A.; Moutinho, H.; Hasoon, F.; Asher, S.; Matson, R; Sheldon, P. Effect of Source Microstructure on the Close-Spaced Sublimation of CdTe Thin Films for Solar Cell Applications. Hepp, A. F., et al., eds. Covalent Ceramics III-Science and Technology of Non-Oxides: Proceedings of the Materials Research Society Symposium, 27-30 November 1995, Boston, Massachusetts. Pittsburgh, PA: Materials Research Society, 1996; Vol. 410; pp. 45-50.

Albin, D.; Rose, D.; Dhere, R; Niles, D.; Swartzlander, A.; Mason, A.; Levi, D.; Moutinho, H.; Sheldon, P. Tin Oxide Stability Effects-Their Identification, Dependence on Processing and Impacts on CdTe/CdS Solar Cell Performance. Witt, C. E.; Al-Jassim,'M.; Gee, J. M., eds. NREL/SNL Photovoltaics Program Review: Proceedings of the 14th Conference, 18-22 November 1996, Lakewood, Colorado. AIP Conference Proceedings 394. Woodbury, NY: American Institute of Physics, 1997; pp. 665-681.

Alleman, J.; Ginley, D.; Hasoon, F.; Asher, S.; Noufi, R. Enhanced Mo Adhesion on Glass with Cr Interlayers for Copper Indium Diselenide Thin Film Devices. Ginley, D., et al., eds. Thin Films for Photovoltaic and Related Device Applications: Proceedings of the Materials Research Society Symposium, 8-11 April 1996, San Francisco, California. Vol. 426. Pittsburgh, PA: Materials Research Society, 1996; pp. 195-199.

Anderson, T. Processing of CuInSe2-Based Solar Cells: Characterization of Deposition Processes in Terms of Chemical Reaction Analyses. Annual Subcontract Report, 6 May 1995 - 5 May 1996. April 1997; 65 pp. NTIS Order No. DE97000241.

Note: Work performed by University of Florida, Gainesville, Florida.

Arya, R R.; Carlson, D. E.; Yang, L.; Chen, L. F.; Willing, F.; Rajan, K; Jansen, K; Poplawski, C.; Bradley, D.; Wood, G. Progress in Amorphous Silicon Multijunction Research at Solarex. Witt, C. E.; Al-Jassim, M.; Gee, J. M., eds. NREL/SNL Photovoltaics Program Review: Proceedings of the 14th Conference, 18-22 November 1996, Lakewood, Colorado. AIP Conference Proceedings 394. Woodbury, NY: American Institute of Physics, 1997; pp. 49-61.

Note: Work performed by Solarex, Newtown, Pennsylvania.

Arya, R.; Fogleboch, J.; Kessler, J.; Russell, L.; Skibo, S.; Wiedeman, S. Research on Polycrystalline Thin-Film Submodules Based on CuInSe 2 Materials: Final Technical Report, 14 December 1995 - 31 December 1996. April 1997; 55 pp. NTIS Order No. DE97000239.

Note: Work performed by Solarex Corporation, Newtown, Pennsylvania.

ASE Americas, Inc: Process-Specific Issues for Thin Edge-Defined, Film-Fed-Growth Octagons. DOE/GO-10096-303. NTIS Order No. DE96013085.

Astropower, Inc.: Silicon-Film(TM) PV Manufacturing Technology. DOE/GO-10096-299. NTIS Order No. DE96013081. 
Ballard, B. L.; Zhu X; Predecki, P. K; Albin, D.; Gabor, A.; Tuttle, J.; Noufi, R. Determination of Composition and Phase Depth-Profiles in Multilayer and Gradient Solid Solution Photovoltaic Films Using Grazing Incidence X-ray Diffraction. Predecki, P. K, et al., eds. Advances in X-Ray Analysis. Volume 38: 43rd Annual Denver X-Ray Conference on Applications of X-Ray Analysis, 1-5 August 1994, Steamboat Springs, Colorado. New York: Plenum Press, 1995; pp. 269-276.

Basol, B.; Kapur, V.; Leidholm, C.; Halani, A.; Norsworthy, G. Application of CIS to High-Efficiency PV Module Fabrication: Annual Technical Progress Report, 1 April 1996 - 31 March 1997. August 1997; 32 pp.

Note: Work performed by International Solar Electric Technology, Inglewood, California.

Basol, B. M.; Kapur, V. K; Leidholm, C. R; Roe, R; Halani, A.; Norsworthy, G. Low Cost CIS Device Processing. Witt, C. E.; Al-Jassim, M; Gee, J. M., eds. NREL/SNL Photovoltaics Program Review: Proceedings of the 14thConference, 18-22 November 1996, Lakewood, Colorado. AIP Conference Proceedings 394. Woodbury, NY: American Institute of Physics, 1997; pp. 107-114.

Note: Work performed by International Solar Electric Technology, Inglewood, California.

Bechinger, C.; Burdis, M. S.; Zhang, J. G. Comparison Between Electrochromic and Photochromic Coloration Efficiency of Tungsten Oxide Thin Films. Solid State Communications. March 1997; 101(10); pp. 753-756.

Bertness, K. A.; Friedman, D. J.; Kurtz, S. R; Kibbler, A. E.; Cramer, C.; Olson, J. M. High-Efficiency GaInP/GaAs Tandem Solar Cells. Journal of Propulsion and Power. September/October 1996; 12(5); pp. 842-846.

Braunstein, R; Dong, S. Photocharge Transport and Recombination Measurements in Amorphous Silicon Films and Solar Cells by Photoconductive Frequency Mixing: Annual Subcontract Report, 15 May 1995-15 May 1996. October 1996; 49 pp. NTIS Order No. DE96013112.

Note: Work performed by University of California, Los Angeles, California.

Britt, J. S.; Delahoy, A. E.; Kiss, Z. J. Technologies for Thin Film CIGS-Based Photovoltaics. Witt, C. E.; Al-Jassim, M.; Gee, J. M., eds. NREL/SNL Photovoltaics Program Review: Proceedings of the 14th Conference, 18-22 November 1996, Lakewood, Colorado. AIP Conference Proceedings 394. Woodbury, NY: American Institute of Physics, 1997; pp. 115-122. Note: Work performed by Energy Photovoltaics, Inc., Lawrenceville, New Jersey.

Brog, T. K. Commercial Production of Thin-Film CdTe Photovoltaic Modules: 1995 Annual Report. February 1997; 16 pp. NTIS Order No. DE97000212.

Note: Work performed by Golden Photon, Inc., Golden, Colorado.

Bryan, J.; Perez, R Estimating Market Potential for Reducing Customer Peak Loads Through Photovoltaics. Campbell-Howe, R; Wilkins-Crowder, B., eds. Solar 96: Proceedings of the 1996 American Solar Energy Society Annual Conference, 13-18 April 1996, Asheville, North Carolina. Boulder, CO: American Solar Energy Society, 1996; pp. $357-360$. Note: Work performed by Citizens Advisory Panel, Central Islip, New York and University of New York at Albany, Albany, New York.

Bullock, J. N.; Bechinger, C.; Xu, Y.; Benson, D. K; Branz, H. M. Improved Monolithic Photovoltaic-Electrochromic Devices Incorporating an a-SiC:H Solar Cell. Hack, M., et al., eds. Amorphous Silicon Technology-1996: Proceedings of the Materials Research Society Symposium, 8-12 April 1996, San Francisco, California. Vol. 420. Pittsburgh, PA: Materials Research Society, 1996; pp. 183-187.

Byme, J.; Letendre, S.; Wang, Y. D.; Nigro, R.; Ferguson, B. Building Load Analysis of Dispatchable Peak-Shaving Photovoltaic Systems: A Regional Analysis of Technical and Economic Potential. Campbell-Howe, R; Wilkins-Crowder, B., eds. Proceedings of the 1997 American Solar Energy Society Annual Conference, 25-30 April 1997, Washington, D.C. 
Boulder, CO: American Solar Energy Society, 1997; pp. 147-152.

Note: Work performed by University of Delaware, Newark, Delaware; Applied Energy Group, Hockessin, Delaware; and Delmarva Power and Light, Wilmington, Delaware.

Cao, J.; Gonsiorawski, R; Kardauskas, M.; Kalejs, J.; O'Brien, C.; Prince, M.; Tomstrom, E. EFG Manufacturing Line Technical Progress and Module Cost Reductions under the PVMaT Program. Seventh Workshop on the Role of Impurities and Defects in Silicon Device Processing: Extended Abstracts and Papers from the Workshop, 11-13 August 1997, Vail, Colorado. (NREL/CP-520-23386). Golden, CO: National Renewable Energy Laboratory, August 1997; pp. $196-197$. Note: Work performed by ASE Americas, Inc., Billerica, Massachusetts.

Carlson, D. E.; Arya, R. R; Bennett, M.; Chen, L. F.; Jansen, K.; Li, Y. M.; Newton, J.; Rajan, K.; Romero, R.; Talenti, D.; Twesme, E.; Willing, F.; Yang, L. Commercialization of Multijunction Amorphous Silicon Modules. Conference Record of the Twenty-Fifth IEEE Photovoltaic Specialists Conference, 13-I7 May 1996, Washington, D.C. New York: Institute of Electrical and Electronics Engineers, 1996; pp. 1023-1027.

Note: Work performed by Solarex, Newtown, Pennsylvania.

Carlson, D. E; Arya, R. R; Chen, L. F.; Oswald, R; Newton, J.; Rajan, K; Romero, R; Willing, F.; Yang, L. Commercialization of Multijunction a-Si Modules. Witt, C. E.; Al-Jassim, M.; Gee, J. M., eds. NREL/SNL Photovoltaics Program Review: Proceedings of the 14th Conference, 18-22 November 1996, Lakewood, Colorado. AIP Conference Proceedings 394. Woodbury, NY: American Institute of Physics, 1997; pp. 479-489.

Note: Work performed by Solarex, Newtown, Pennsylvania.

Chang, C. H.; Davydov, A.; Stanbery, B. J.; Anderson, T. J. Thermodynamic Assessment of the Cu-In-Se System and Application to Thin Film Photovoltaics. Conference Record of the Twenty-Fifth IEEE Photovoltaic Specialists Conference, 13-17 May 1996, Washington, D.C. New York: Institute of Electrical and Electronics Engineers, 1996; pp. 849-852.

Note: Work performed by University of Florida, Gainesville, Florida.

Chen, C. C.; Zhong, F.; Cohen, J. D. Effects of Light Induced Degradation on the Distribution of Deep Defects in Hydrogenated Amorphous Silicon-Germanium Alloy. Hack, M., et al., eds. Amorphous Silicon Technology-1996: Proceedings of the Materials Research Society Symposium, 8-12 April 1996, San Francisco, California. Vol. 420. Pittsburgh, PA: Materials Research Society, 1996; pp. 581-586.

Note: Work performed by University of Oregon, Eugene, Oregon, and Lawrence Berkeley National Laboratory, Berkeley, California.

Chen, L.; Willing, F.; Yanag, L.; Li, Y. M.; Maley, N.; Rajan, K.; Bennett, M.; Arya, R Stability of Large-Area Amorphous Silicon Alloy Tandem Solar Modules. Conference Record of the Twenty-Fifth IEEE Photovoltaic Specialists Conference, 13-17 May 1996, Washington, D.C. New York: Institute of Electrical and Electronics Engineers, 1996; pp. 1137-1140.

Note: Work performed by Solarex, Newtown, Pennsylvania.

Chiang, P. K.; Ermer, J. H.; Nishikawa, W. T.; Krut, D. D.; Joslin, D. E.; Eldredge, J. W.; Cavicchi, B. T.; Olson, J. M. Experimental Results of $\mathbf{G a I n P}_{2} / \mathbf{G a A s} /$ Ge Triple Junction Cell Development for Space Power Systems. Conference Record of the Twenty-Fifth IEEE Photovoltaic Specialists Conference, 13-17 May 1996, Washington, D.C. New York: Institute of Electrical and Electronics Engineers, 1996; pp. 183 -186.

Ciszek, T. F.; Wang, T. H.; Ahrenkiel, R. K.; Matson, R. Properties of Iron-Doped Multicrystalline Silicon Grown by the Float-Zone Technique. Conference Record of the Twenty-Fifth IEEE Photovoltaic Specialists Conference, 13-17 May 1996, Washington, D.C. New York: Institute of Electrical and Electronics Engineers, 1996; pp. 737-739.

Cohen, J. D. Identifying Electronic Properties Relevant to Improving Stability in a-Si:H-Based Cells and Overall Performance in a-Si,Ge:H-Based Cells. March 1997; 32 pp. NTIS Order No. DE97000225.

Note: Work performed by University of Oregon, Eugene, Oregon. 
Collins, R. W.; Kim, S.; Koh, J.; Burnham, J. S.; Jiao, L.; Chen, I.; Wronski, C. R. Advances in the Characterization of Compositionally-Graded Layers in Amorphous Semiconductor Solar Cells by Real Time Spectroellipsometry. Hack, M. et al., eds. Amorphous Silicon Technology-1996: Proceedings of the Materials Research Society Symposium, 8-12 April 1996, San Francisco, California. Vol. 420. Pittsburgh, PA: Materials Research Society, 1996; pp. 443-448.

Note: Work performed by Pennsylvania State University, University Park, Pennsylvania.

Compaan, A. D.; Bohn, R. G.; Contreras-Puente, G. High-Efficiency Thin-Film Cadmium Telluride Photovoltaic Cells: Annual Technical Report, 20 January 1996 - 19 January 1997. August 1997; 43 pp.

Note: Work performed by University of Toledo, Toledo, Ohio.

Compaan, A. D.; Feng, Z.; Contreras-Puente, G.; Narayanswamy, C.; Fischer, A. Properties of Pulsed Laser Deposited CdS $_{\mathbf{x}} \mathbf{T e}_{1-\mathbf{x}}$ Films on Glass. Ginley, D., et al., eds. Thin Films for Photovoltaic and Related Device Applications: Proceedings of the Materials Research Society Symposium, 8-11 April 1996, San Francisco, California. Vol. 426. Pittsburgh, PA: Materials Research Society, 1996; pp. 367-371.

Note: Work performed by University of Toledo, Toledo, Ohio.

Compaan, A. D.; Jayamaha, U.; Matulionis, I.; Miller, M. J. Optimization of Laser Scribing for Thin-Film PV Modules: Annual Technical Progress Report, 12 April 1995-11 April 1996. October 1996; 21 pp. NTIS Order No. DE96013114. Note: Work performed by University of Toledo, Toledo, Ohio.

Compaan, A. D.; Matulionis, I.; Miller, M. J.; Jayamaha, U. N. Optimization of Laser Scribing for Thin-Film Photovoltaics. Conference Record, of the Twenty-Fifth IEEE Photovoltaic Specialists Conference, 13-17 May 1996, Washington, D.C. New York: Institute of Elêctrical and Electronics Engineers, 1996; pp. 769-772.

Note: Work performed by University of Toledo, Toledo, Ohio.

Compaan, A. D.; Matulionis, I.; Nakade, S.; Jayamaha, U. Pulse Duration and Wavelength Effects in Laser Scribing of Thin-Film Polycrystalline PV Materials. Witt, C. E.; Al-Jassim, M.; Gee, J. M, eds. NREL/SNL Photovoltaics Program Review: Proceedings of the 14th Conference, 18-22 November 1996, Lakewood, Colorado. AIP Conference Proceedings 394. Woodbury, NY: American Institute of Physics, 1997; pp. 567-571.

Note: Work performed by University of Toledo, Toledo, Ohio.

Compaan, A.; Shao, M.; Fischer, A.; Grecu, D.; Jayamaha, U.; Contreras-Puente, G.; Bohn, R. G. Effects of Magnetic Field Configuration on if Sputtering for CdS/CdTe Solar Cells. Ginley, D., et al., eds. Thin Films for Photovoltaic and Related Device Applications: Proceedings of the Materials Research Society Symposium, 8-11 April 1996, San Francisco, California. Vol. 426. Pittsburgh, PA: Materials Research Society, 1996; pp. 391-396.

Note: Work performed by University of Toledo, Toledo, Ohio.

Contreras, M. A.; Wiesner, H.; Matson, R.; Tuttle, J.; Ramanathan, K.; Noufi, R. Defect Chalcopyrite Cu(In $\left.\mathbf{I}_{1-x} \mathbf{G a x}\right)_{3} \operatorname{Se} 5$ Polycrystalline Thin-Film Materials. Ginley, D., et al., eds. Thin Films for Photovoltaic and Related Device Applications: Proceedings of the Materials Research Society Symposium, 8-11 April 1996, San Francisco, California. Vol. 426. Pittsburgh, PA: Materials Research Society, 1996; pp. 243-254.

Contreras, M. A.; Wiesner, H.; Niles, D.; Ramanathan, K.; Matson, R.; Tuttle, J.; Keane, J.; Noufi, R. Defect Chalcopyrite $\mathrm{Cu}\left(\mathrm{In}_{1-\mathrm{x}} \mathbf{G a x}\right)_{3} \mathrm{Se}_{5}$ Materials and High-Ga-Content $\mathbf{C u}(\mathbf{I n}, \mathbf{G a}) \mathrm{Se}_{2}-$ Based Solar Cells. Conference Record of the Twenty-Fifth IEEE Photovoltaic Specialists Conference, 13-17 May 1996, Washington, D.C. New York: Institute of Electrical and Electronics Engineers, 1996; pp. 809-812.

Coutts, T. J.; Wanlass, M. W.; Ward, J. S.; Johnson, S. Review of Recent Advances in Thermophotovoltaics. Conference Record of the Twenty-Fifth IEEE Photovoltaic Specialists Conference, 13-17 May 1996, Washington, D.C. New York: Institute of Electrical and Electronics Engineers, 1996; pp. 25-30. 
Crandall, R. S.; Lipps, K. Subtleties of Capacitance Transients in Amorphous Silicon. Hack, M., et al., eds. Amorphous Silicon Technology-1996: Proceedings of the Materials Research Society Symposium, 8-12 April 1996, San Francisco, California. Vol. 420. Pittsburgh, PA: Materials Research Society, 1996; pp. 685-690.

Crandall, R. S.; Wang, Q. Density of States Measurements in a p-i-n Solar Cell. Conference Record of the Twenty-Fifth IEEE Photovoltaic Specialists Conference, 13-17 May 1996, Washington, D.C. New York: Institute of Electrical and Electronics Engineers, 1996; pp. 1117-1120.

Cudzinovic, M.; Sopori, B. Control of Back Surface Reflectance from Aluminum Alloyed Contacts on Silicon Solar Cells. Conference Record of the Twenty-Fifth IEEE Photovoltaic Specialists Conference, 13-17 May 1996, Washington, D.C. New York: Institute of Electrical and Electronics Engineers, 1996; pp. 501-503.

Customer-Sited Photovoltaics: Focusing on Markets That Really Shine. (Brochure). March 1997; 4 pp. DOE/GO-10097-371. NTIS Order No. DE97000220.

Czandema, A. W.; Jorgensen, G. J. Service Lifetime Prediction for Encapsulated Photovoltaic Cells/Minimodules. Witt, C. E.; Al-Jassim, M.; Gee, J. M., eds. NREL/SNL Photovoltaics Program Review: Proceedings of the 14th Conference, 18-22 November 1996, Lakewood, Colorado. AIP Conference Proceedings 394. Woodbury, NY: American Institute of Physics, $1997 ;$ pp. 295-311.

Czanderna, A. W.; Pern, F. J. Encapsulation of PV Modules Using Ethylene Vinyl Acetate Polymer as a Pottant: A Critical Review. Solar Energy Materials and Solar Cells. 1996; 43; pp. 101-181.

Czanderna, A. W.; Pern, A. J. Estimating Service Lifetimes of a Polymer Encapsulant for Photovoltaic Modules from Accelerated Testing. Conference Record of the Twenty-Fifth IEEE Photovoltaic Specialists Conference, 13-17 May 1996, Washington, D.C. New York: Institute of Electrical and Electronics Engineers, 1996; pp. 1219-1222.

Dalal, V. L.; Kaushal, S.; Han, K; Girvan, R; Knox, R; Martin, F.; Hariasra, S.; Ping, E.; Xu, J.; Sipahi, L. Comprehensive Research on Stability of Amorphous Silicon and Alloy Materials and Devices: Annual Report, 31 May 1995 - 30 May 1996. August 1997; $34 \mathrm{pp}$.

Note: Work performed by Iowa State University, Ames, Iowa.

Dalal, V.; Kaushal, S.; Girvan, R; Hariasra, S.; Sipahi, L. Improved Stability in ECR-Deposited a-Si Solar Cells. Conference Record of the Twenty-Fifth IEEE Photovoltaic Specialists Conference, 13-17 May 1996, Washington, D.C. New York: Institute of Electrical and Electronics Engineers, 1996; pp. 1069-1072.

Note: Work performed by Iowa State University, Ames, Iowa.

Dalal, V. L.; Kaushal, S.; Girvan, R.; Sipahi, L.; Hariasra, S. Properties of Substrate-Type a-Si:H Devices Prepared Using ECR Conditions. Hack, M., et al., eds. Amorphous Silicon Technology-1996: Proceedings of the Materials Research Society Symposium, 8-12 April 1996, San Francisco, California. Vol. 420. Pittsburgh, PA: Materials Research Society, 1996; pp. 39-44.

Note: Work performed by Iowa State University, Ames, Iowa.

Dawn of a New Era: National Center for Photovoltaics. (Brochure). October 1996; 8 pp. DOE/GO-10096-324.

Deb, S. K.; Benner, J. P. DOE/OER-Sponsored Basic Research in High-Efficiency Photovoltaics. Conference Record of the Twenty-Fifth IEEE Photovoltaic Specialists Conference, 13-17 May 1996, Washington, D.C. New York: Institute of Electrical and Electronics Engineers, 1996; pp. 977-979. 
DeBlasio, R PV Standards Overview. Witt, C. E.; Al-Jassim, M.; Gee, J. M., eds. NREL/SNL Photovoltaics Program Review: Proceedings of the 14th Conference, 18-22 November 1996, Lakewood, Colorado. AIP Conference Proceedings 394. Woodbury, NY: American Institute of Physics, 1997; pp. 369-378.

Delahoy, A. E.; Britt, J. S.; Kiss, Z. J. CIS Photovoltaic Technology: Thin-Film Partnership Program, Annual Technical Report, 12 January 1996 - 11 January 1997. June 1997; 47 pp. NTIS Order No. DE97050822.

Note: Work performed by Energy Photovoltaics, Inc., Princeton, New Jersey.

Delahoy, A. E.; Cherny, M. Deposition Schemes for Low-Cost Transparent Conductors for Photovoltaics. Ginley, D., et al., eds. Thin Films for Photovoltaic and Related Device Applications: Proceedings of the Materials Research Society Symposium, 8-11 April 1996, San Francisco, California. Vol. 426. Pittsburgh, PA: Materials Research Society, 1996; pp. 467-477.

Note: Work performed by Energy Photovoltaics, Inc., Princeton, New Jersey.

Delahoy, A. E; Payne, A. M. Determination of the Internal Series Resistance of CIS and CIGS Photovoltaic Cell Structures. Conference Record of the Twenty-Fifth IEEE Photovoltaic Specialists Conference, 13-17 May 1996, Washington, D.C. New York: Institute of Electrical and Electronics Engineers, 1996; pp. 841-844.

Note: Work performed by Energy Photovoltaics, Inc., Princeton, New Jersey and Princeton University, Princeton, New Jersey.

Deng, X; Izu, M.; Jones, S. J.; Kopf, R; Liu, T.; Ovshinsky, S. R.; Viers, B. Development of High, Stable-Efficiency Triple-Junction a-Si Alloy Solar Cells: Annual Technical Progress Report, October 1995 - October 1996. April 1997; 72 pp. NTIS Order No. DE97000238.

Note: Work performed by Energy Conversion Devices, Inc., Troy, Michigan.

Dhere, N. G.; Lynn, K. W. Gallium Content Enhancement in Culn $\mathbf{1}_{-x} \mathbf{G a}_{\mathbf{x}} \mathrm{Se}_{2}$ Thin Films Prepared by Two-Selenizations Process Using Se Vapor. Conference Record of the Twenty-Fifth IEEE Photovoltaic Specialists Conference,13-17 May 1996, Washington, D.C. New York: Institute of Electrical and Electronics Engineers, 1996; pp. 897-900.

Note: Work performed by Florida Solar Energy Center, Cocoa, Florida.

Dong, S.; Liebe, J.; Tang, Y.; Braunstein, R; von Rodem, B. Potential Fluctuations in Intrinsic Hydrogenated Amorphous Silicon. Witt, C. E.; Al-Jassim, M.; Gee, J. M., eds. NREL/SNL Photovoltaics Program Review: Proceedings of the 14th Conference, 18-22 November 1996, Lakewood, Colorado. AIP Conference Proceedings 394. Woodbury, NY: American Institute of Physics, 1997; pp. 537-546.

Note: Work performed by University of California, Los Angeles, California

Edwards, H. S.; Smith, G. D.; Voecks, G.; Rohatgi, N.; Prokopius, P.; Zweibel, K.; Hoelscher, J. F. CdTe Terrestrial Modules as a Power Source for a Regenerative Fuel Cell Power Plant for Space Applications. Conference Record of the Twenty-Fifth IEEE Photovoltaic Specialists Conference, 13-17 May 1996, Washington, D.C. New York: Institute of Electrical and Electronics Engineers, 1996; pp. 337-340.

Eisgruber, I. L. Thin-Film Module Measurement Artifacts. Conference Record of the Twenty-Fifth IEEE Photovoltaic Specialists Conference, 13-17 May 1996, Washington, D.C. New York: Institute of Electrical and Electronics Engineers, 1996; pp. 829-832.

Note: Work performed by Colorado State University, Fort Collins, Colorado.

Electricity When and Where You Need It: From the Sun-Photovoltaics for Farms and Ranches. (Brochure). January $1997 ; 24$ pp.

Emery, K; Burdick, J.; Caiyem, Y.; Dunlavy, D.; Field, H.; Kroposki, B.; Moriarty, T. Temperature Dependence of Photovoltaic Cells, Modules, and Systems. Conference Record of the Twenty-Fifth IEEE Photovoltaic Specialists 
Conference, 13-17 May 1996, Washington, D.C. New York: Institute of Electrical and Electronics Engineers, 1996; pp. 1275-1278.

Energy Conversion Devices, Inc.: Continuous Roll-to-Roll Amorphous Silicon Photovoltaic Manufacturing Technology. DOE/GO- 10096-308. NTIS Order No. DE96013090.

ENTECH, Inc.: Improving ENTECH's Concentrator Module. DOE/GO-10096-310. NTIS Order No. DE96013092.

Estreicher, S. K; Fedders, P. A. Molecular Dynamics Modeling of Hydrogen in Silicon. Witt, C. E.; Al-Jassim, M.; Gee, J. M., eds. NREL/SNL Photovoltaics Program Review: Proceedings of the 14th Conference, 18-22 November 1996, Lakewood, Colorado. AIP Conference Proceedings 394. Woodbury, NY: American Institute of Physics, 1997; pp. $729-738$. Note: Work performed by Texas Tech University, Lubbock, Texas and Washington University, St. Louis, Missouri.

Ferekides, C. S.; Marinskiy, D.; Marinskaya, S.; Tetali, B.; Oman, D.; Morel, D. L. CdS Films Prepared by the Close-Spaced Sublimation and Their Influence on CdTe/CdS Solar Cell Performance. Conference Record of the Twenty-Fifth IEEE Photovoltaic Specialists Conference, 13-17May 1996, Washington, D.C. New York: Institute of Electrical and Electronics Engineers, 1996; pp. 751-756.

Note: Work performed by University of South Florida, Tampa, Florida.

Ferekides, C. S.; Tetali, B.; Marinskiy, D.; Marinskaya, S.; Morel, D. Effects of Processing Temperature on the Thickness of CdS and the Performance of CdTe Solar Cells. Witt, C. E.; Al-Jassim, M.; Gee, J. M., eds. NREL/SNL Photovoltaics Program Review: Proceedings of the 14th Conference, 18-22 November 1996, Lakewood, Colorado. AIP Conference Proceedings 394. Woodbury, NY: American Institute of Physics, 1997; pp. 631-638.

Note: Work performed by University of South Florida, Tampa, Florida.

Fernandez, A. M.; Sebastian, P. J.; Hermann, A. M; Bhattacharya, R. N.; Noufi, R. N.; Contreras, M. A. Electrodeposition of CuInSe, Thin Films for Photovoltaic Application. Renewable Energy. Proceedings of the 4th World Renewable Energy Congress, 15-21 June 1996, Denver, Colorado. August 1996; 8(1-4); pp. 396-399.

Fischer, A.; Jayamaha, U. N.; Bykov, E.; Grecu, D.; Bohn, R. G.; Compaan, A. D. Raman and RBS Studies of Interdiffusion in RF-Sputtered CdS/CdTe Solar Cells. Witt, C. E.; Al-Jassim, M.; Gee, J. M., eds. NRELSSNL Photovoltaics Program Review: Proceedings of the 14th Conference, 18-22 November 1996, Lakewood, Colorado. AIP Conference Proceedings 394. Woodbury, NY: American Institute of Physics, 1997; pp. 655-664.

Note: Work performed by University of Toledo, Toledo, Ohio.

Fischer, A.; Narayanswamy, C.; Grecu, D. S.; Bykov, E.; Nance, S. A.; Jayamaha, U. N.; Contreras-Puente, G.; Compaan, A. D.; Stan, M. A.; Mason, A. R Interdiffusion of CdS/CdTe in Laser-Deposited and Rf Sputtered Alloys, Bilayers and Solar Cells. Conference Record of the Twenty-Fifth IEEE Photovoltaic Specialists Conference, 13-17 May 1996, Washington, D.C. New York: Institute of Electrical and Electronics Engineers, 1996; pp. 921-924.

Note: Work performed by University of Toledo, Toledo, Ohio and Case Western Reserve University, Cleveland, Ohio.

Fonash, S. J.; Sopori, B. L. Technologies for Thin Film Poly-Si Solar Cells. Seventh Workshop on the Role of Impurities and Defects in Silicon Device Processing: Extended Abstracts and Papers from the Workshop, 11-13 August 1997, Vail, Colorado. (NREL/CP-520-23386). Golden, CO: National Renewable Energy Laboratory, August 1997; pp. $127-135$.

Ford, D. H.; Barnett, A. M; Hall, R. B. High Power, Commercial Silicon-Film(TM) Solar Cells. Conference Record of the Twenty-Fifth IEEE Photovoltaic Specialists Conference, 13-17 May 1996, Washington, D.C. New York: Institute of Electrical and Electronics Engineers, 1996; pp. 601-604.

Note: Work performed by AstroPower, Inc., Newark, Delaware. 
Ford, D. H; Barnett, A: M.; Checchi, J. C.; Culik, J. S.; Hall, R. B.; Jackson, E. L.; Kendall, C: L.; Rand, J. A. Large-Area Silicon-Film(TM) Manufacturing. Witt, C. E.; Al-Jassim, M.; Gee, J. M., eds. NREL/SNL Photovoltaics Program Review: Proceedings of the 14th Conference, 18-22 November 1996, Lakewood, Colorado. AIP Conference Proceedings 394. Woodbury, NY: American Institute of Physics, 1997; pp. 445-449.

Note: Work performed by AstroPower, Inc., Newark, Delaware.

Forrest, S. R. Very High Efficiency Photovoltaic Cells Based on Fully Organic Multiple Quantum Wells: Final Report. March 1997; 95 pp. NTIS Order No. DE97000063.

Note: Work performed by Center for Photonics and Optoelectronic Materials, Department of Electrical Engineering, Princeton University, Princeton, New Jersey.

Freitas, C. Development of a Modular, Bi-Directional Power Inverter for Photovoltaic Applications: Annual Technical Progress Report, August 1995 - August 1996. August 1997; 30 pp.

Note: Work performed by Trace Engineering Company, Inc., Arlington, Washington.

Friedman, D. J. Modelling of Tandem Cell Temperature Coefficients. Conference Record of the Twenty-Fifth IEEE Photovoltaic Specialists Conference, 13-17 May 1996, Washington, D.C. New York: Institute of Electrical and Electronics Engineers, 1996; pp. 89-92.

Friedman, D. J.; Kurtz, S. R.; Sinha, K; McMahon, W. E.; Kramer, C. M.; Olson, J. M.; Lasich, J. B.; Cleeve, A. X; Connaughton, I. On-Sun Concentrator Performance of GaIn/GaAs Tandem Cells. Conference Record of the Twenty-Fifth IEEE Photovoltaic Specialists Conference, 13-17 May 1996, Washington, D.C. New York: Institute of Electrical and Electronics Engineers, 1996; pp. 73-75.

Friedman, D. J.; Kurtz, S. R; Kibbler, A. E.; Al-Jassim, M.; Jones, K; Keyes, B.; Matson, R Polycrystalline MBE-Grown GaAs for Solar Cells. Witt, C. E.; Al-Jassim, M.; Gee, J. M., eds. NREL/SNL Photovoltaics Program Review: Proceedings of the 14th Conference, 18-22 November 1996, Lakewood, Colorado. AIP Conference Proceedings 394. Woodbury, NY: American Institute of Physics, 1997; pp. 703-708.

Gabor, A. M.; Britt, J. S.; Delahoy, A. E.; Noufi, R.; Kiss, Z. J. Manufacturing-Compatible Methods for the Formation of Cu(In, Ga)Se $\mathrm{S}_{2}$ Thin Films. Conference Record of the Twenty-Fifth IEEE Photovoltaic Specialists Conference, 13-17May 1996, Washington, D.C. New York: Institute of Electrical and Electronics Engineers, 1996; pp. 889-892.

Note: Work performed by Energy Photovoltaics, Inc. (EPV), Princeton, New Jersey and the National Renewable Energy Laboratory, Golden, Colorado.

Gallagher, A.; Barzen, S.; Childs, M.; Laracuente, A. Atomic-Scale Characterization of Hydrogenated Amorphous-Silicon Films and Devices: Annual Subcontract Report, 14 February 1995-14 April 1996. February 1997; 25 pp. NTIS Order No. DE97000210.

Note: Work performed by National Instsitute for Standards and Technology, Boulder, Colorado.

Gee, J. M.; Ciszek, T. F. Crystalline-Silicon Photovoltaic R\&D Project at NREL and SNL. Witt, C. E.; Al-Jassim, M.; Gee, J. M., eds. NREL/SNL Photovoltaics Program Review: Proceedings of the 14th Conference, 18-22 November 1996, Lakewood, Colorado. AIP Conference Proceedings 394. Woodbury, NY: American Institute of Physics, 1997; pp. $189-197$.

Gee, J. M.; Sopori, B. L. Effect of Gettering on Areal Inhomogeneities in Large-Area Multicrystalline-Silicon Solar Cells. Seventh Workshop on the Role of Impurities and Defects in Silicon Device Processing: Extended Abstracts and Papers from the Workshop, 11-13 August 1997, Vail, Colorado. (NREL/CP-520-23386). Golden, CO: National Renewable Energy Laboratory, August 1997; pp. 215-218.

Ginley, D.; Catalano, A; Schock, H. W.; Eberspacher, C.; Peterson, T. M.; Wada, T., eds. Thin Films for Photovoltaic and Related Device Applications: Proceedings of the Materials Research Society Symposium, 8-11 April 1996, San 
Francisco, California: Materials Research Society Symposium Proceedings Vol. 426. Pittsburgh, PA: Materials Research Society, 1996; 560 pp.

Glatfelter, T.; Lycette, M.; Akkashian, E.; Hoffman, K. PV Module with an Active Area Utilization of 99.8\%. Conference Record of the Twenty-Fifth IEEE Photovoltaic Specialists Conference, 13-17 May 1996, Washington, D.C. New York: Institute of Electrical and Electronics Engineers, 1996; pp. 1173-1176.

Note: Work performed by United Solar Systems Corporation, Troy, Michigan.

Gordon, R G. Deposition of Transparent Conducting Oxides for Solar Cells. Witt, C. E.; Al-Jassim, M.; Gee, J. M., eds. NREL/SNL Photovoltaics Program Review: Proceedings of the 14th Conference, 18-22 November 1996,Lakewood, Colorado. AIP Conference Proceedings 394. Woodbury, NY: American Institute of Physics, 1997; pp. 39-48.

Note: Work performed by Harvard University, Cambridge, Massachusetts.

Granata, J. E.; Sites, J. R; Contreras-Puente, G.; Compaan, A. D. Effect of CdS Thickness on CdS/CdTe Quantum Efficiency. Conference Record of the Twenty-Fifth IEEE Photovoltaic Specialists Conference, 13-17 May 1996, Washington, D.C. New York: Institute of Electrical and Electronics Engineers, 1996; pp. 853-856.

Note: Work performed by Colorado State University, Fort Collins, Colorado and University of Toledo, Toledo, Ohio.

Granata, J. E.; Sites, J. R; Tuttle, J. R. Sodium Dependence of Cu(In,Ga)Se, Junction Electronics. Witt, C. E.; Al-Jassim, M.; Gee, J. M., eds. NREL/SNL Photovoltaics Program Review: Proceedings of the 14th Conference, 18-22 November 1996, Lakewood, Colorado. AIP Conference Proceedings 394. Woodbury, NY: American Institute of Physics, 1997; pp. $621-630$. Note: Work performed by Colorado State University, Fort Collins, Colorado.

Gray, J. L. Interpretation of Capacitance-Voltage Characteristics in Thin-Film Solar Cells Using a Detailed Numerical Model. Conference Record of the Twenty-Fifth IEEE Photovoltaic Specialists Conference, 13-17 May1996, Washington, D.C. New York: Institute of Electrical and Electronics Engineers, 1996; pp. 905-908.

Note: Work performed by Purdue University, West Lafayette, Indiana.

Green, M. A.; Emery, K; Bucher, K; King, D. L.; Igari, S. Solar Cell Efficiency Tables (Version 8). Progress in Photovoltaics: Research and Applications. July-August 1996; 4(4); pp. 321-325.

Green, M. A.; Emery, K; Bucher, K; King, D. L.; Igari, S. Solar Cell Efficiency Tables (Version 9). Progress in Photovoltaics: Research and Applications. January-February 1997; 5(1); pp. 51-54.

Gregg, B. A. Photoelectrochromic Cells and Their Applications. Endeavour. 1997; 21(2); pp. 52-55.

Gregg, B. A.; Sprague, J.; Peterson, M. W. Long-Range Singlet Energy Transfer in Perylene Bis(phenethylimide) Films. Journal of Physical Chemistry B. 1997; 101(27); pp. 5362-5369.

Guha, S. Amorphous Silicon Alloy Solar Cells and Modules--Opportunities and Challenges. Conference Record of the Twenty-Fifth IEEE Photovoltaic Specialists Conference, 13-17 May 1996, Washington, D.C. New York: Institute of Electrical and Electronics Engineers, 1996; pp. 1017-1022.

Note: Work performed by United Solar Systems Corporation, Troy, Michigan.

Guha, S. Amorphous Silicon Research, Phase II: Annual Technical Progress Report, 1 August 1995-31 July 1996. October 1996; 46 pp. NTIS Order No. DE96014327.

Note: Work performed by United Solar Systems Corporation, Troy, Michigan.

Guha, S. Thin-Film Amorphous Silicon Alloy Research Partnership: Phase II Annual Technical Progress Report, 2 February 1996 - 1 February 1997. June 1997; 47 pp. NTIS Order No. DE97050818.

Note: Work performed by United Solar Systems Corporation, Troy, Michigan. 
Hall, R. B.; Bamett, A. M.; Cotter, J. E.; Ford, D. H.; Ingram, A. E.; Rand, J. A. Advanced, Thin, Polycrystalline Silicon-Film(TM) Solar Cells on Low-Cost Substrates. Ginley, D., et al., eds. Thin Films for Photovoltaic and Related Device Applications: Proceedings of the Materials Research Society Symposium, 8-11 April 1996, San Francisco, California. Vol. 426. Pittsburgh, PA: Materials Research Society, 1996; pp. 117-122.

Note: Work performed by Solar Park, Newark, Delaware.

Hall, R.B.; Rand, J. A.; Cotter, J. E.; Ford, D. H. Light-Trapped, Interconnected, Silicon-Film(TM) Modules: Annual Technical Status Report, 18 November 1995-18 November 1996. February 1997; 26 pp. NTIS Order No. DE97000218. Note: Work performed by AstroPower, Inc., Newark, Delaware.

Han, D. Experimental Study of the Factors Governing the Staebler-Wronski Photodegradation Effect in a-Si:H Solar Cells: Annual Subcontract Report, 1 April 1995-30 June 1996. October 1996; 34 pp. NTIS Order No. DE96014326. Note: Work performed by University of North Carolina, Chapel Hill, North Carolina.

Han, D.; Wang, K; von Roeder, B. Dispersive-Transport-Controlled Luminescence in Hydrogenated Amorphous Silicon. Physical Review Letters. 18 November 1996; 77(21); pp. 4410-4413.

Hanoka, J.; Chleboski, R; Farber, M.; Fava, J.; Kane, P.; Martz, J. Advanced Polymer PV System: PVMaT 4A1 Annual Report, September 1995 - September 1996. June 1997; 40 pp. NTIS Order No. DE97050821.

Note: Work performed by Evergreen Solar, Inc., Waltham, Massachusetts.

Hanoka, J. I.; Kane, P. M.; Chleboski, R. G.; Farber, M. A. Advanced Polymer PV System. Witt, C. E.; Al-Jassim, M.; Gee, J. M., eds. NREL/SNL Photovoltaics Program Review: Proceedings of the 14th Conference, 18-22 November 1996, Lakewood, Colorado. AIP Conference Proceedings 394. Woodbury, NY: American Institute of Physics, 1997; pp. 859-866. Note: Work performed by Evergreen Solar, Inc., Waltham, Massachusetts.

Hegedus, S.; Buchanan, W.; Liu, X.; Gordon, R. Effect of Textured Tin Oxide and Zinc Oxide Substrates on the Current Generation in Amorphous Silicon Solar Cells. Conference Record of the Twenty-Fifth IEEE Photovoltaic Specialists Conference, 13-17 May 1996, Washington, D.C. New York: Institute of Electrical and Electronics Engineers, 1996; pp. 1129-1132.

Note: Work performed by University of Delaware, Newark, Delaware and Harvard University, Cambridge, Massachusetts.

Hegedus, S. S.; Buchanan, W. A.; Eser, E.; Phillips, J. E.; Shafarman, W. N. Transparent Conducting Oxide Contacts for n-i-p and p-i-n Amorphous Silicon Solar Cells. Witt, C. E.; Al-Jassim, M.; Gee, J. M., eds. NREL/SNLPhotovoltaics Program Review: Proceedings of the 14th Conference, 18-22 November 1996, Lakewood, Colorado. AIP Conference Proceedings 394. Woodbury, NY: American Institute of Physics, 1997; pp. 547-555.

Note: Work performed by University of Delaware, Newark, Delaware.

Hegedus, S.; Deng, X. Analysis of Optical Enhancement in a-Si n-i-p Solar Cells Using a Detachable Back Reflector. Conference Record of the Twenty-Fifth IEEE Photovoltaic Specialists Conference, 13-17 May 1996, Washington, D.C. New York: Institute of Electrical and Electronics Engineers, 1996; pp. 1061-1064.

Note: Work performed by University of Delaware, Newark, Delaware and Energy Conversion Devices, Troy, Michigan.

Heiser, T.; McHugo, S.; HiesImair, H.; Flink, C.; Istratov, A.; Weber, E. R. Low Level Cu Contamination Studied by Transient Ion Drift. Seventh Workshop on the Role of Impurities and Defects in Silicon Device Processing: Extended Abstracts and Papers from the Workshop, 11-13 August 1997, Vail, Colorado. (NREL/CP-520-23386). Golden, CO: National Renewable Energy Laboratory, August 1997; pp. 233-236.

Note: Work performed by University of California at Berkeley, Berkeley, California. 
Herig, C.; Houston, A. Value of Customer Preference. Conference Record of the Twenty-Fifth IEEE Photovoltaic Specialists Conference, 13-17 May 1996, Washington, D.C. New York: Institute of Electrical and Electronics Engineers, 1996; pp. 1517-1520.

Hernandez, J. L.; Rockett, A. Junction Electroluminescence of $\mathbf{C u}(\mathbf{I n}, \mathbf{G a}) \mathrm{Se}_{2}$ Devices. Conference Record of the Twenty-Fifth IEEE Photovoltaic Specialists Conference, 13-17 May 1996, Washington, D.C. New York: Institute of Electrical and Electronics Engineers, 1996; pp. 973-976.

Note: Work performed by University of Illinois, Urbana, Illinois.

Hieslmair, H; Istratov, A.; McHugo, S. A.; Flink, C.; Weber, E. R. Precipitation of Iron in Silicon. Seventh Workshop on the Role of Impurities and Defects in Silicon Device Processing: Extended Abstracts and Papers from the Workshop, 11-13 August 1997, Vail, Colorado. (NREL/CP-520-23386). Golden, CO: National Renewable Energy Laboratory, August 1997; pp. 187-190.

Note: Work performed by University of California at Berkeley, Berkeley, Califomia.

Hieslmair, H.; McHugo, S.; Weber, E. R. Aluminum Backside Segregation Gettering. Conference Record of the Twenty-Fifth IEEE Photovoltaic Specialists Conference, 13-17May 1996, Washington, D.C. New York: Institute of Electrical and Electronics Engineers, 1996; pp. 441-444.

Note: Work performed by University of California, Berkeley, California.

Hieslmair, H.; McHugo, S. A; Weber, E. R Aluminum Gettering and Transition Metal Precipitates in PV Silicon. Witt, C. E.; Al-Jassim, M.; Gee, J. M., eds. NREL/SNL Photovoltaics Program Review: Proceedings of the 14th Conference, 18-22 November 1996, Lakewood, Colorado. AIP ConferenceProceedings 394. Woodbury, NY: American Institute of Physics, 1997; pp. 759-770.

Note: Work performed by University of California, Berkeley, California and Lawrence Berkeley National Laboratory, Berkeley, California.

Hoff, T. E. Integrating Renewable Energy Technologies in the Electric Supply Industry: A Risk Management Approach. July 1997; 82 pp. NTIS Order No. DE97050801.

Note: Work performed by Pacific Energy Group, WalnutCreek, California.

Holley, W. H.; Agro, S. C.; Galica, J. P.; Yorgensen, R. S. UV Stability and Module Testing of Non-Browning Experimental PV Encapsulants. Conference Record of the Twenty-Fifth IEEE Photovaltaic Specialists Conference, 13-17 May 1996, Washington, D.C. New York: Institute of Electrical and Electronics Engineers, 1996; pp. 1259-1262.

Note: Work performed by Springborn Laboratories, Inc., Enfield, Connecticut.

Huang, S. Y.; Schlichthorl, G.; Nozik, A. J.; Gratzel, M.; Frank, A. J. Charge Recombination in Dye-Sensitized Nanocrystalline TiO2 Solar Cells. Journal of Physical Chemistry B. 1997; 101(14); pp. 2576-2582.

Ingram, A. E.; Barnett, A. M.; Cotter, J. E.; Ford, D. H.; Hall, R B.; Rand, J. A.; Thomas, C. J. 13\% Silicon Film(TM) Solar Cells on Low-Cost Barrier-Coated Substrates. Conference Record of the Twenty-Fifth IEEE Photovoltaic Specialists Conference, 13-17 May 1996, Washington, D.C. New York: Institute of Electrical and Electronics Engineers, 1996; pp. 477-480.

Note: Work performed by AstroPower, Inc., Newark, Delaware.

Istratov, A. A.; Hieslmair, H.; Heiser, T.; Flink, C.; Weber, E. R; Seifert, W.; Kittler, M. Potential Influence of the Recombination of Minority Carriers on Interstitial and Precipitated Copper in Silicon on Solar Cell Efficiency. Seventh Workshop on the Role of Impurities and Defects in Silicon Device Processing: Extended Abstracts and Papers from the Workshop, 11-13 August 1997, Vail, Colorado. (NREL/CP-520-23386). Golden, CO: National Renewable Energy Laboratory, August 1997; pp. 158-161. Note: Work performed by University of California at Berkeley, Berkeley, California and Institute for Semiconductor Physics, Frankfurt, Germany. 
Istratov, A. A; Weber, E. R Transition Metals and Lifetime in PV Silicon. Seventh Workshop on the Role of Impurities and Defects in Silicon Device Processing: Extended Abstracts and Papers from the Workshop, 11-13August 1997, Vail, Colorado. (NREL/CP-520-23386). Golden, CO: National Renewable Energy Laboratory, August 1997; pp. 31-40. Note: Work performed by University of California at Berkeley, Berkeley, Caliomia.

Jagannathan, B.; Anderson, A. W. a-Si/c-Si Solar Cells: Effect of Preparation and Processing Techniques on the Photovoltaic Properties. Ginley, D., et al., eds. Thin Films for Photovoltaic and Related Device Applications: Proceedings of the Materials Research Society Symposium, 8-11 April 1996, San Francisco, California. Vol. 426. Pittsburgh, PA: Materials Research Society, 1996; pp. 77-82.

Note: Work performed by State University of New York at Buffalo, Amherst, New York.

Jeffrey, F. Photovoltaic Manufacturing Technology Monolithic Amorphous Silicon Modules on Continuous Polymer Substrates: Annual Technical Progress Report, 5 July 1995-4 June 1996. February 1997; 40 pp. NTIS Order No. DE97000213.

Note: Work performed by Iowa Thin Film Technologies, Inc., Ames, Iowa.

Jeffrey, F. R; Grimmer, D. P.; Brayman, S.; Scandrett, B.; Thomas, M.; Martens, S. A.; Chen, W.; Noak, M. PVMaT Improvements in Monolithic a-Si Modules on Continuous Polymer Substrates. Witt, C. E.; Al-Jassim, M.; Gee, J. M., eds. NREL/SNL Photovoltaics Program Review: Proceedings of the 14th Conference, 18-22 November 1996, Lakewood, Colorado. AIP Conference Proceedings 394. Woodbury, NY: American Institute of Physics, 1997; pp. 451-461.

Note: Work performed by Iowa Thin Film Technologies, Inc., Ames, Iowa and Iowa State University, Ames, Iowa.

Jenkins, D.; Winston, R.; Bliss, J.; O'Gallagher, J.; Lewandowski, A.; Bingham, C. Solar Concentration of 50,000 Achieved with Output Power Approaching $1 \mathrm{~kW}$. Journal of Solar Energy Engineering: Transactions of the American Society of Mechanical Engineers. August 1996; 118(3); pp. 141-145.

Jensen, D. G.; McCandless, B. E.; Birkmire, R. W. Thin Film Cadmium Telluride-Cadmium Sulfide Alloys and Devices. Conference Record of the Twenty-Fifth IEEE Photovoltaic Specialists Conference, 13-17 May 1996, Washington, D.C. New York: Institute of Electrical and Electronics Engineers, 1996; pp. 773-776.

Note: Work performed by University of Delaware, Newark, Delaware.

Jensen, D. G.; McCandless, B. E.; Birkmire, R. W. Thin-Film Cadmium Sulfide/Cadmium Telluride Alloys. Ginley, D., et al., eds. Thin Films for Photovoltaic and Related Device Applications: Proceedings of the Materials Research Society Symposium, 8-11 April 1996, San Francisco, California. Vol. 426. Pittsburgh, PA: Materials Research Society, 1996; pp. 325-330.

Note: Work performed by University of Delaware, Newark, Delaware.

Jiang, L.; Schiff, E. A. Two-Layer Model for Electroabsorption and Built-In Potential Measurements on a-Si:H p-i-n Solar Cells. Hack, M. et al., eds. Amorphous Silicon Technology-1996: Proceedings of the Materials Research Society Symposium, 8-12 April 1996, San Francisco, California. Vol. 420. Pittsburgh, PA: Materials Research Society, 1996; pp. 203-208.

Note: Work performed by Syracuse University, Syracuse, New York.

Jiao, L.; Liu, H.; Semoushikina, S.; Lee, Y.; Wronski, C. R. Importance of Charge Defects in a-Si:H Materials and Solar Cell Structures. Conference Record of the Twenty-Fifth IEEE Photovoltaic Specialists Conference, 13-17 May 1996, Washington, D.C. New York: Institute of Electrical and Electronics Engineers, 1996; pp. 1073-1076.

Note: Work performed by Pennsylvania State University, University Park, Pennsylvania.

Jones, W; Nicklas, M. Improving the Economics of Building-Integrated Photovoltaics Through Heat Recovery. Campbell-Howe, R; Wilkins-Crowder, B. eds. Solar 96: Proceedings of the 1996 American Solar Energy Society Annual 
Conference, 13-18 April 1996, Asheville, North Carolina. Boulder, CO: American Solar Energy Society, 1996; pp. 62-67. Note: Work performed by Innovative Design, Raleigh, North Carolina.

Jorgensen, G.; Pem, J.; Kelley, S.; Czanderna, A. W.; Schissel, P. Polymers for Solar Energy Devices, Chapter 4.2. Arshady, R. ed. Desk Reference of Functional Polymers: Syntheses and Applications. Washington, DC: American Chemical Society, 1996; pp. 567-588.

Joshi, S. M.; Gosele, U. M.; Tan, T. Y. Aluminum Gettering of Gold in Single-Crystal Silicon. Seventh Workshop on the Role of Impurities and Defects in Silicon Device Processing: Extended Abstracts and Papers from the Workshop, 11-13 August 1997, Vail, Colorado. (NREL/CP-520-23386). Golden, CO: National Renewable Energy Laboratory, August 1997; pp. 202-205.

Note: Work performed by Duke University, Durham, North Carolina.

Kaminar, N.; Curchod, D.; Hobden, P.; Roake, S.; Navin, J.; Bottenberg, B.; Sahagian, J. PVMaT Improvements in the Manufacturing of the PVI Powergrid(TM): Phase I Annual Technical Report, 26 October 1995 - 25 October 1996. May 1997; 38 pp. NTIS Order No. DE97000242.

Note: Work performed by Photovoltaics International, LLC (PVI), Sunnyvale, California.

Kardauskas; M.; Kalejs, J.; Cao, J.; Tornstrom, E.; Gonsiorawski, R.; O'Brien, C.; Prince, M. Market-Driven Improvements in the Manufacturing of EFG Modules. Witt, C. E.; Al-Jassim, M.; Gee, J. M., eds. NREL/SNL Photovoltaics Program Review: Proceedings of the 14th Conference, 18-22 November 1996, Lakewood, Colorado. AIP Conference Proceedings 394. Woodbury, NY: American Institute of Physics, 1997; pp. 851-858.

Note: Work performed by ASE Americas, Inc., Billerica, Massachusetts.

Kessler, J.; Wiedeman, S.; Russell, L.; Fogleboch, J.; Skibo, S.; Arya, R; Carlson, D. Cu(In,Ga)Se, Based Submodule Process Robustness. Conference Record of the Twenty-Fifth IEEE Photovoltaic Specialists Conference, 13-17 May 1996, Washington, D.C. New York: Institute of Electrical and Electronics Engineers, 1996; pp. 813-816.

Note: Work performed by Solarex, Newtown, Pennsylvania.

Kessler, J.; Wiedeman, S.; Russell, L.; Fogleboch, J.; Skibo, S.; Arya, R; Carlson, D. Front Contact Optimization for $\mathrm{Cu}(\mathrm{In}, \mathbf{G a}) \mathrm{Se}_{2}$ (Sub)Modules. Conference Record of the Twenty-Fifth IEEE Photovoltaic Specialists Conference, 13-17 May 1996, Washington, D.C. New York: Institute of Electrical and Electronics Engineers, 1996; pp. 885-888.

Note: Work performed by Solarex, Newtown, Pennsylvania.

Kester, J. J.; Albright, S.; Kaydanov, V.; Ribelin, R; Woods, L. M.; Phillips, J. A. CdTe Solar Cells: Electronic and Morphological Properties. Witt, C. E.; Al-Jassim, M.; Gee, J.- M., eds. NREL/SNL Photovoltaics ProgramReview: Proceedings of the 14th Conference, 18-22 November 1996, Lakewood, Colorado. AIP Conference Proceedings 394. Woodbury, NY: American Institute of Physics, 1997; pp. 162-169.

Note: Work performed by Golden Photon, Inc., Golden, Colorado.

Kester, J.; Albright, S. Improvement.of Near-Term CdTe Processing and Product Capabilities and Establishment of Next-Generation CdTe Technology: Annual Technical Progress Report, 1 September 1995 - 31 August 1996. July 1997; 36 pp.

Note: Work performed by Golden Photon, Inc., Golden, Colorado.

Khera, G. M.; Kakalios, J.; Wang, Q.; Iwaniczko, E. 1/f Noise and Thermal Equilibration Effects in Hot Wire Deposited Amorphous Silicon. Hack, M., et al., eds. Amorphous Silicon Technology-1996: Proceedings of the Materials Research Society Symposium, 8-12 April 1996, San Francisco, California. Vol. 420. Pittsburgh, PA: Materials Research Society, 1996; pp. 641-646. 
King, D. E.; Webb, J. D. Infrared Waveguide Sensor with Functionalized Monolayer for Detection of Airborne Pollutants. Chemical, Biochemical, and Environmental Fiber Sensors VIII: Proceedings of the 1996 International Symposium on Optical Science, Engineering, and Instrumentation, 6-7 August 1996, Denver, Colorado. SPIE Proceedings, Vol. 2836. Bellingham, WA: Society of Photo-Optical Instrumentation Engineers, 1996; pp. 38-49.

King, R. R; Mitchell, K W.; Jester, T. L. Improvements in Cz Silicon PV Module Manufacturing. Witt, C. E.; Al-Jassim, M.; Gee, J. M., eds. NREL/SNL Photovoltaics Program Review: Proceedings of the 14th Conference, 18-22 November 1996, Lakewood, Colorado. AIP Conference Proceedings 394. Woodbury, NY: American Institute of Physics, 1997; pp. $433-444$. Note: Work performed by Siemens Solar Industries, Camarillo, California.

Koh, J. K.; Burnham, J. S.; Li, Y.; Liu, H.; Chen, I. S.; Pilione, L. J.; Wronski, C. R; Collins, R. W. Structural Evolution of Top-Junction a-Si:C:H:B and Mixed-Phase (Microcrystalline Si)-(a-Sil-s $\left.\mathbf{C}_{\mathbf{x}}: \mathbf{H}\right)$ p-Layers in a-Si:H n-i-p Solar Cells. Hack, M., et al., eds. Amorphous Silicon Technology-1996: Proceedings of the Materials Research Society Symposium, 8-12 April 1996, San Francisco, California. Vol. 420. Pittsburgh, PA: Materials Research Society, 1996; pp. 69-74.

Note: Work performed by Pennsylvania State University, University Park, Pennsylvania.

Koval, T.; Wohlgemuth, J.; Kinsey, B. Dependence of Cell Performance on Wafer Thickness for BSF and Non-BSF Cells. Conference Record of the Twenty-Fifth IEEE Photovoltaic Specialists Conference, 13-17 May 1996, Washington, D.C. New York: Institute of Electrical and Electronics Engineers, 1996; pp. 505-507.

Note: Work performed by Solarex, Frederick, Maryland.

Kroposki, B., Chairman. Photovoltaic Performance Reliability Workshop, 4-6 September 1996, Lakewood, Colorado. October 1996; 412 pp. NTIS Order No. DE96013115.

Kroposki, B. Photovoltaic Performance and Reliability Workshop Summary. Witt, C. E.; Al-Jassim, M.; Gee, J. M., eds. NREL/SNL Photovoltaics Program Review: Proceedings of the 14th Conference, 18-22 November 1996, Lakewood, Colorado. AIP Conference Proceedings 394. Woodbury, NY: American Institute of Physics, 1997; pp. $285-293$.

Kroposki, B.; Myers, D.; Emery, K; Mrig, L.; Whitaker, C.; Newmiller, J. Photovoltaic Module Energy Rating Methodology Development. Conference Record of the Twenty-Fifth IEEE Photovoltaic Specialists Conference, 13-17 May 1996, Washington, D.C. New York: Institute of Electrical and Electronics Engineers, 1996; pp. 1311-1314.

Kroposki, B.; Strand, T.; Hansen, R.; Powell, R.; Sasala, R. Technical Evaluation of Solar Cells, Inc. CdTe Module and Array at NREL. Conference Record of the Twenty-Fifth IEEE Photovoltaic Specialists Conference, 13-17 May 1996, Washington, D.C. New York: Institute of Electrical and Electronics Engineers, 1996; pp. 969-972.

Kurtz, S. R; Friedman, D. Recent Developments in Terrestrial Concentrator Photovoltaics. Witt, C. E.; Al-Jassim, M.; Gee, J. M., eds. NREL/SNL Photovoltaics Program Review: Proceedings of the 14th Conference, 18-22 November 1996, Lakewood, Colorado. AIP Conference Proceedings 394. Woodbury, NY: American Institute of Physics, 1997; pp. $247-258$.

Kurtz, S. R; ONeill, M. J. Estimating and Controlling Chromatic Aberration Losses for Two-Junction, Two-Terminal Devices in Refractive Concentrator Systems. Conference Record of the Twenty-Fifth IEEE Photovoltaic Specialists Conference, 13-17 May 1996, Washington, D.C. New York: Institute of Electrical and Electronics Engineers, 1996; pp. 361-364.

Kurtz, S. R; Olson, J. M.; Bertness, K. A.; Sinha, K; McMahon, B.; Asher, S. Hidden But Important Parameters in Ga0.5In0.5P Cell Growth. Conference Record of the Twenty-Fifth IEEE Photovoltaic Specialists Conference, 13-17 May 1996, Washington, D.C. New York: Institute of Electrical and Electronics Engineers, 1996; pp. 37-42. 
Lambarski, T.; Minyard, G. Design, Fabrication, and Certification of Advanced Modular PV Power Systems: Annual Technical Progress Report, 8 September 1995-7 September 1996. March 1997; 21 pp. NTIS Order No. DE97000230. Note: Work performed by Solar Electric Specialties, Willits, California.

Lan, J. H; Kanicki, J.; Catalano, A; Keane, J.; Den Boer, W.; Gu, T. Patterning of Transparent Conducting Oxide Thin Films by Wet Etching for a-Si:H TFT-LCDs. Journal of Electronic Materials. December 1996; 25(12); pp. $1806-1817$.

Landry, M. D.; Tsuo, Y. S.; Ciszek, T. F.; Roze, R; Hoegh, D. Distributed Control and Process Monitoring for Photovoltaic Applications. Witt, C. E.; Al-Jassim, M.; Gee, J. M., eds. NREL/SNL Photovoltaics Program Review: Proceedings of the 14th Conference, 18-22 November 1996, Lakewood, Colorado. AIP Conference Proceedings 394. Woodbury, NY: American Institute of Physics, 1997; pp. 787-792.

Lee, Y.; Jiao, L.; Liu, H.; Collins, R; Wronski, C. R. Stability of a-Si:H Solar Cells and Corresponding Intrinsic Materials Fabricated using Hydrogen Diluted Silane. Conference Record of the Twenty-Fifth IEEE Photovoltaic Specialists Conference, 13-17 May 1996, Washington, D.C. New York: Institute of Electrical and Electronics Engineers, 1996; pp. 1165-1168.

Note: Work performed by Pennsylvania State University, University Park, Pennsylvania.

Levi, D. H; Fluegel, B. D.; Ahrenkiel, R K; Compaan, A. D.; Woods, L. M. Dynamics of Photoexcited Carrier Relaxation and Recombination in CdTe/CdS Thin Films. Conference Record of the Twenty-Fifth IEEE Photovoltaic Specialists Conference, 13-17 May 1996, Washington, D.C. New York: Institute of Electrical and Electronics Engineers, 1996; pp. 913-919.

Li, S. S.; Stanbery, B. J.; Huang, C. H.; Chang, C. H.; Chang, Y. S.; Anderson, T. J. Effects of Buffer Layer Processing on CIGs Excess Carrier Lifetime: Application of Dual-Beam Optical Modulation to Process Analysis. Conference Record of the Twenty-Fifth IEEE Photovoltaic Specialists Conference, 13-17 May 1996, Washington, D.C. New York: Institute of Electrical and Electronics Engineers, 1996; pp. 821-824.

Note: Work performed by University of Florida, Gainesville, Florida.

Li, X; Sheldon, P.; Moutinho, H; Matson, R Enhanced Performance of CdS/CdTe Thin-Film Devices Through Temperature Profiling Techniques Applied to Close-Spaced Sublimation Deposition. Conference Record of the Twenty-Fifth IEEE Photovoltaic Specialists Conference, 13-17 May 1996, Washington, D.C. New York: Institute of Electrical and Electronics Engineers, 1996; pp. 933-936.

Lindahl, K. A.; Moore, J. J.; Olson, D. L.; Noufi, R; Lanning, B. Quantitative Investigation of Copper/Indium Multilayer Thin Film Reactions. Thin Solid Films. 15 December 1996; 290-291; pp. 518-524.

Lindahl, K. A.; Olson, D. L.; Moore, J. J.; Swartzlander, A. B.; Noufi, R. Quantitative Investigation of Reactions in Copper-Indium-Gallium Multilayer Thin Films. Conference Record of the Twenty-Fifth IEEE PhotovoltaicSpecialists Conference, 13-17 May 1996, Washington, D.C. New York: Institute of Electrical and Electronics Engineers, 1996; pp. 949-952.

Lips, K; Unold, T.; Xu, Y.; Crandall, R. S. Partial Depletion Region Collapse and Its Impact on Transient Capacitance Measurements. Hack, M., et al., eds. Amorphous Silicon Technology-1996: Proceedings of the Materials Research Society Symposium, 8-12 April 1996, San Francisco, California. Vol. 420. Pittsburgh, PA: Materials Research Society, 1996; pp. 691-696.

Liu, H.; Lee, Y.; Jamali-beh, T.; Lu, Z.; Collins, R; Wronski, C. R. Light-Induced Degradation in Different p-i-n and n-i Schottky Barrier Solar Cell Structures. Conference Record of the Twenty-Fifth IEEE Photovoltaic Specialists Conference, 13-17 May 1996, Washington, D.C. New York: Institute of Electrical and Electronics Engineers, 1996; pp. $1125-1128$. Note: Work performed by Pennsylvania State University, University Park, Pennsylvania. 


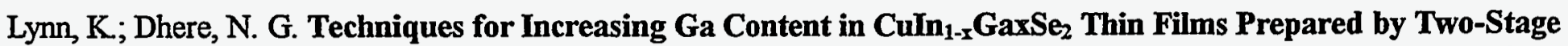
Selenization Process. Witt, C. E.; Al-Jassim, M.; Gee, J. M., eds. NREL/SNL Photovoltaics Program Review: Proceedings of the 14th Conference, 18-22 November 1996, Lakewood, Colorado. AIP Conference Proceedings 394. Woodbury, NY: American Institute of Physics, 1997; pp. 613-619.

Note: Work performed by Florida Solar Energy Center, Cocoa Beach, Florida.

Mahan, A. H.; Iwaniczko, E.; Nelson, B. P.; Reedy, R. C.; Crandall, R. S.; Guha, S.; Yang, J. Hot Wire Deposited Hydrogenated Amorphous Silicon Solar Cells. Conference Record of the Twenty-Fifth IEEE Photovoltaic Specialists Conference, 13-17 May 1996, Washington, D.C. New York: Institute of Electrical and Electronics Engineers, 1996; pp. 1065-1068.

Mahan, A. H.; Iwaniczko, E.; Nelson, B. P.; Reedy, R. C. ,. Jr; Unold, T.; Crandall, R. S.; Guha, S.; Yang, J. Hot Wire Deposited Hydrogenated Amorphous Silicon Solar Cells. Witt, C. E.; Al-Jassim, M.; Gee, J. M., eds. NREL/SNL Photovoltaics Program Review: Proceedings of the 14th Conference, 18-22 November 1996, Lakewood, Colorado. AIP Conference Proceedings 394. Woodbury, NY: American Institute of Physics, 1997; pp. 27-32.

Marudachalam, M.; Hichri, H.; Birkmire, R. W.; Schulz, J. M.; Swartzlander, A. B.; Al-Jassim, M. M. Diffusion of In and Ga in Selenized Cu-In and Cu-Ga Precursors. Conference Record of the Twenty-Fifth IEEE Photovoltaic Specialists Conference, 13-17 May 1996, Washington, D.C. New York: Institute of Electrical and Electronics Engineers, 1996; pp. 805-807.

Matson, R. J.; Contreras, M. A.; Tuttle, J. R.; Swartzlander, A. B.; Parilla, P. A.; Noufi, R. Effects of the Concentration of Ga on Junction Formation in Thin-Film $\mathrm{ZnO} / \mathrm{CdS} / \mathrm{CuIn}_{\mathbf{x}} \mathrm{Ga}_{1-\mathrm{x}} \mathrm{Se}_{2} / \mathrm{Mo}$ Photovoltaic.Devices. Ginley, D., et al., eds. Thin Films for Photovoltaic and Related Device Applications: Proceedings of the Materials Research Society Symposium, 8-11 April 1996, San Francisco, California. Vol. 426. Pittsburgh, PA: Materials Research Society, 1996; pp. 183-188.

McCandless, B. E.; Birkmire, R. W.; Jensen, D. G.; Phillips, J. E.; Youm, I. Processing Issues for Thin Film CdTe/CdS Solar Cells. Witt, C. E.; Al-Jassim, M; Gee, J. M., eds. NREL/SNL Photovoltaics Program Review: Proceedings of the 14th Conference, 18-22 November 1996, Lakewood, Colorado. AIP Conference Proceedings 394. Woodbury, NY: American Institute of Physics, 1997; pp. 647-654.

Note: Work performed by University of Delaware, Newark, Delaware.

McCandless, B. E.; Hichri, H.; Hanket, G.; Birkmire, R. W. Vapor Phase Treatment of CdTe/CdS Thin Films With CdCl2:02. Conference Record of the Twenty-Fifth IEEE Photovoltaic Specialists Conference, 13-17 May 1996, Washington, D.C. New York: Institute of Electrical and Electronics Engineers, 1996; pp. 781-784.

Note: Work performed by University of Delaware, Newark, Delaware.

McMahon, T. J; Basso, T. S.; Rummel, S. R. Cell Shunt Resistance and Photovoltaic Module Performance. Conference Record of the Twenty-Fifth IEEE Photovoltaic Specialists Conference, 13-17 May 1996, Washington, D.C. New York: Institute of Electrical and Electronics Engineers, 1996; pp. 1291-1294.

Meyers, P. V. CdTe Team Activities. Witt, C. E.; Al-Jassim, M; Gee, J. M., eds. NREL/SNL Photovoltaics Program Review: Proceedings of the 14th Conference, 18-22 November 1996, Lakewood, Colorado. AIP Conference Proceedings 394. Woodbury, NY: American Institute of Physics, 1997; pp. 153-161.

Meyers, P. V.; Asher, S.; Al-Jassim, M. M. Search for Degradation Mechanisms of CdTe/CdS Solar Cells. Ginley, D., et al., eds. Thin Films for Photovoltaic and Related Device Applications: Proceedings of the Materials Research Society Symposium, 8-11 April 1996, San Francisco, California. Vol. 426. Pittsburgh, PA: Materials Research Society, 1996; pp. 317-324. 
Meyers, P. V.; Phillips, J. E. Stress Testing of CdTe Solar Cells. Conference Record of the Twenty-Fifth IEEE Photovoltaic Specialists Conference, 13-17 May 1996, Washington, D.C. New York: Institute of Electrical and Electronics Engineers, 1996; pp. 789-792.

Note: Work performed by University of Delaware, Newark, Delaware.

Mitchell, R. L.; Witt, C. E.; Thomas, H. P.; Herwig, L. O.; Ruby, D. S.; Aldrich, C. C. Benefits from the U.S. Photovoltaic Manufacturing Technology Project. Conference Record of the Twenty-Fifth IEEE Photovoltaic Specialists Conference, 13-17 May 1996, Washington, D.C. New York: Institute of Electrical and Electronics Engineers, 1996; pp. 1215-1218.

Mitchell, R. L.; Witt, C. E.; Thomas, H. P. Photovoltaic Manufacturing Technology (PVMaT) Project - Latest Results. Witt, C. E.; Al-Jassim, M.; Gee, J. M., eds. NREL/SNL Photovoltaics Program Review: Proceedings of the 14th Conference, 18-22 November 1996, Lakewood, Colorado. AIP Conference Proceedings 394. Woodbury, NY: American Institute of Physics, 1997; pp. 407-413.

Molenbroek, E. C.; Mahan, A. H.; Gallagher, A. Mechanisms Influencing "Hot-Wire"Deposition of Hydrogenated Amorphous Silicon. Journal of Applied Physics. 15 August 1997; 82(4); pp. 1909-1917.

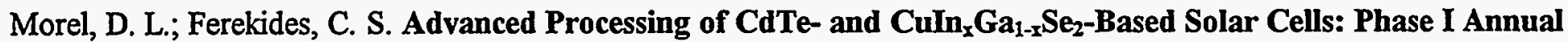
Subcontract Report, 18 April 1995-17 April 1996. March 1997; 42 pp. NTIS Order No. DE97000226.

Note: Work performed by University of South Florida, Tampa, Florida.

Moutinho, H. R; Dhere, R. G.; Ramanathan, K; Sheldon, P.; Kazmerski, L. L. Growth Analysis of Cadmium Sulfide Thin Films by Atomic Force Microscopy. Conference Record of the Twenty-Fifth IEEE Photovoltaic Specialists Conference, 13-17 May 1996, Washington, D.C. New York: Institute of Electrical and Electronics Engineers, 1996; pp. $945-948$.

Muljadi, E.; Taylor, R. PV Water Pumping with a Peak Power Tracker Using a Simple Six Step Square Wave Inverter. IAS '96: Conference Record of the 1996 IEEE Industry Applications Conference, Thirty-First LAS Annual Meeting, 6-10 October 1996, San Diego, California. New York: Institute of Electrical and Electronics Engineers, Inc., 1997; pp. 133-142.

Myers, D. Radiometric Instrumentation and Measurements Guide for Photovoltaic Performance Testing. April 1997; 151 pp. NTIS Order No. DE97000082.

Myers, D. R; Cannon, T. W. PV Solar Radiometric Measurements. Witt, C. E.; Al-Jassim, M.; Gee, J. M., eds. NREL/SNL Photovoltaics Program Review: Proceedings of the 14th Conference, 18-22 November 1996, Lakewood, Colorado. AIP Conference Proceedings 394. Woodbury, NY: American Institute of Physics, 1997; pp. 395-403.

Nape Maepa, L. H.; Norman, A.; Singh, H; Leitch, A. W. R; Darkazalli, G.; Slavsky, S. T.; van Dyk, E. E.; McConnell, R. D. Benefits of Suncorp, A New Photovoltaic Module Assembly Company in South Africa. Renewable Energy. Proceedings of the 4th World Renewable Energy Congress, 15-21 June 1996, Denver, Colorado. August 1996; 8(1-4); pp. 344-347.

Narasimha, S.; Kamra, S.; Rohatgi, A.; Khattak, C. P.; Ruby, D. Optimization and Fabrication of High Efficiency HEM Multicrystalline Silicon Solar Cells. Conference Record of the Twenty-Fifth IEEE Photovoltaic Specialists Conference, 13-17 May 1996, Washington, D.C. New York: Institute of Electrical and Electronics Engineers, 1996; pp. 449-452.

Note: Work performed by the Georgia Institute of Technology, Atlanta, Georgia; Crystal Systems, Inc., Salem, Massachusetts; and Sandia National Laboratories, Albuquerque, New Mexico.

Narayanan, S.; Creager, J.; Roy, M.; Wohlgemuth, J. Process Development Toward a 15\% Efficient $230 \mathrm{~cm} 2$ Screen Printed Multicrystalline Silicon Solar Cell. Conference Record of the Twenty-Fifth IEEE Photovoltaic Specialists Conference, 13-17 May 1996, Washington, D.C. New York: Institute of Electrical and Electronics Engineers, 1996; pp. 605-608. Note: Work performed by Solarex, Frederick, Maryland. 
Niles, D. W.; Contreras, M.; Ramanathan, K.; Noufi, R. Determination of the Valence-Band Offset of CdS/CIS Solar Cell Devices by Target Factor Analysis. Conference Record of the Twenty-Fifth IEEE Photovoltaic Specialists Conference, 13-17 May 1996, Washington, D.C. New York: Institute of Electrical and Electronics Engineers, 1996; pp. 833-836.

Niles, D. W.; Herdt, G.; Al-Jassim, M. O Impurity Chemistry in CdS Thin-Films Grown by Chemical Bath Deposition: An Investigation with X-Ray Photoelectron Spectroscopy. Witt, C. E.; Al-Jassim, M.; Gee, J. M., eds. NREL/SNL Photovoltaicś Program Review: Proceedings of the 14th Conference, 18-22 November 1996, Lakewood, Colorado. AIP Conference Proceedings 394. Woodbury, NY: American Institute of Physics, 1997; pp. 709-716.

Niles, D. W.; Herdt, G.; Al-Jassim, M. X-ray Photoelectron Spectroscopy Investigation of O Impurity Chemistry in CdS Thin FIIms Grown By Chemical Bath Deposition. Journal of Applied Physics. 15 February 1997; 81(4); pp. 1978-1984.

Nowlan, M. J.; Hogan, S. J.; Patterson, J. S.; Sutherland, S. F.; Murach, J. M.; Breen, W. F.; Darkazalli, G. Processing Evaluations of an Automated High-Throughput System for Interconnecting Crystalline Silicon Solar Cells. Conference Record of the Twenty-Fifth IEEE Photovoltaic Specialists Conference, 13-17 May 1996, Washington, D.C. New York: Institute of Electrical and Electronics Engineers, 1996; pp. 1331-1334.

NREL PV Working With Industry, Fall 1996. (Newsletter). October 1996; 12 pp.

NREL PV Working with Industry, Winter 1996/1997. (Newsletter). January 1997; 12 pp.

NREL PV Working with Industry, Spring/Summer 1997. (Newsletter). August 1997; 16 pp.

Olsen, L. C.; Addis, F. W.; Lei, W.; Aguilar, H. Challenge of Replacing CdS In CuInSez-Based Solar Cells. Witt, C. E.; Al-Jassim, M.; Gee, J. M., eds. NREL/SNL Photovoltaics Program Review: Proceedings of the 14th Conference, 18-22 November 1996, Lakewood, Colorado. AIP Conference Proceedings 394. Woodbury, NY: American Institute of Physics, 1997; pp. 597-602.

Note: Work performed by Washington State University at Tri-Cities, Richland, Washington.

Olsen, L. C.; Aguilar, H.; Addis, F. W.; Lei, W.; Li, J. CIS Solar Cells with ZnO Buffer Layers. Conference Record of the Twenty-Fifth IEEE Photovoltaic Specialists Conference, 13-17 May 1996, Washington, D.C. New York: Institute of Electrical and Electronics Engineers, 1996; pp. 997-1000.

Note: Work performed by Washington State University, Richland, Washington.

Oman, D. M.; Karthikeyan, S.; Ferekides, C. S.; Morel, D. L. Junction Mechanisms in CdS/CdTe Solar Cells. Conference Record of the Twenty-Fifth IEEE Photovoltaic Specialists Conference, 13- 17 May 1996, Washington, D.C. New York: Institute of Electrical and Electronics Engineers, 1996; pp. 957-960.

Note: Work performed by University of South Florida, Tampa, Florida.

Osterwald, C. R. Photovoltaics as Alternative Energy Sources. ASTM Standardization News. April 1997; 25(4); pp. $20-25$. NTIS Order No. DE97000217.

Osterwald, C. R; Anevsky, S.; Barua, A. K; Dubard, J.; Emery, K; King, D.; Metzdorf, J.; Nagamine, F.; Shimokawa, R; Udayakumar, N.; Wang, Y. X; Wittchen, T.; Zaaiman, W.; Zastrow, A.; Zhang, J. Results of the PEP ' 93 Intercomparison of Reference Cell Calibrations and the Newer Technology Performance Measurements. Conference Record of the Twenty-Fifth IEEE Photovoltaic Specialists Conference, 13-17 May 1996, Washington, D.C. New York: Institute of Electrical and Electronics Engineers, 1996; pp. 1263-1266.

Palsule, C.; Paschen, U.; Cohen, J. D.; Yang, J.; Guha, S. Capacitance Characterization of Amorphous Silicon/Amorphous Silicon Germanium Heterostructures. Hack, M., et al., eds. Amorphous Silicon Technology -1996: 
Proceedings of the Materials Research Society Symposium, 8-12 April 1996, San Francisco, California. Vol. 420. Pittsburgh, PA: Materials Research Society, 1996; pp. 209-214.

Note: Work performed by University of Oregon, Eugene, Oregon and United Solar Systems Corporation, Troy, Michigan.

Perez, R; Seals, R; Wenger, H.; Hoff, T.; Herig, C. Photovoltaics as a Long-Term Solution to Power Outages. Case Study: The Great 1996 WSCC Power Outage. Campbell-Howe, R.; Wilkins-Crowder, B., eds. Proceedings of the 1997 American Solar Energy Society Annual Conference, 25-30 April 1997, Washington, D.C. Boulder, CO: American Solar Energy Society, 1997; pp. 309-314.

Pern, F. J. Development of New EVA Formulations for Improved Performance at NREL. Witt, C. E.; Al-Jassim, M.; Gee, J. M., eds. NREL/SNL Photovoltaics Program Review: Proceedings of the 14th Conference, 18-22 November 1996, Lakewood, Colorado. AIP Conference Proceedings 394. Woodbury, NY: American Institute of Physics, 1997; pp. 795-810.

Pern, F. J.; Eisgruber, I. L.; Micheels, R. H. Spectroscopic, Scanning Laser OBIC, and I-V/QE Characterizations of Browned EVA Solar Cells. Conference Record of the Twenty-Fifth IEEE Photovoltaic Specialists Conference, 13-17 May 1996, Washington, D.C. New York: Institute of Electrical and Electronics Engineers, 1996; pp. 1255-1258.

Pern, F. J.; Glick, S. H. Study of Various Encapsulation Schemes for c-Si Solar Cells with EVA Encapsulants. Witt, C. E.; Al-Jassim, M.; Gee, J. M., eds. NREL/SNL Photovoltaics Program Review: Proceedings of the 14thConference, 18-22 November 1996, Lakewood, Colorado. AIP Conference Proceedings 394. Woodbury, NY: American Institute of Physics, 1997; pp. 811-824.

Pern, F. J.; Glick, S. H. Thermal Processing of EVA Encapsulants and Effects of Formulation Additives. Conference Record of the Twenty-Fifth IEEE Photovoltaic Specialists Conference, 13-17 May 1996, Washington, D.C. New York: Institute of Electrical and Electronics Engineers, 1996; pp. 1251-1254.

Photovoltaic Energy: Program Overview, Fiscal Year 1996. (Booklet). April 1997; 28 pp. DOE/GO-10097-365. NTIS Order No. DE96013102.

Photovoltaics: Basic Principles and Components. Energy Efficiency and Renewable Energy Clearinghouse (EREC) (Brochure). March 1997; 8 pp. DOE/GO-10097-377.

Plekhanov, P. S.; Gosele, U. M.; Tan, T. Y. Modeling of Void Nucleation and Growth in Silicon. Seventh Workshop on the Role of Impurities and Defects in Silicon Device Processing: Extended Abstracts and Papers from the Workshop, 11-13 August 1997, Vail, Colorado. (NREL/CP-520-23386). Golden, CO: National Renewable Energy Laboratory, August 1997; pp. 170-173.

Note: Work performed by Duke University, Durham, North Carolina.

Powell, R. C.; Sasala, R.; Rich, G.; Steele, M.; Bihn, K; Reiter, N.; Cox, S.; Dorer, G. Stability Testing of CdTe/CdS Thin-Film Photovoltaic Modules. Conference Record of the Twenty-Fifth IEEE Photovoltaic Specialists Conference, 13-17 May 1996, Washington, D.C. New York: Institute of Electrical and Electronics Engineers, 1996; pp. 785-788.

Note: Work performed by Solar Cells, Inc., Toledo, Ohio.

Qu, Y.; Meyers, P. V.; McCandless, B. E. HCI Vapor Post-Deposition Heat Treatment of CdTe/CdS Films. Conference Record of the Twenty-Fifth IEEE Photovoltaic Specialists Conference, 13-17 May 1996, Washington, D.C. New York: Institute of Electrical and Electronics Engineers, 1996; pp. 1013-1016.

Note: Work performed by University of Delaware, Newark, Deleware.

Ramanathan, K.; Contreras, M. A.; Tuttle, J. R.; Keane, J.; Webb, J.; Asher, S.; Niles, D.; Dhere, R; Tennant, A. L.; Hasoon, F. S.; Noufi, R. Effect of Heat Treatments and Window Layer Processing on the Characteristics of CuInGaSez Thin 
Film Solar Cells. Conference Record of the Twenty-Fifth IEEE Photovoltaic Specialists Conference, 13-17 May 1996, Washington, D.C. New York: Institute of Electrical and Electronics Engineers, 1996; pp. 837-840.

Rand, J. A.; Bamett, A. M.; Checchi, J. C.; Culik, J. S.; Collins, S. R.; Ford, D. H.; Hall, R. B.; Jackson, E. L.; Kendall, C. L.; Trabant, A. M. Large-Area Silicon-Film (TM) Panels and Solar Cells: Phase I Annual Technical Report, July 1995-December 1995. January 1997; 24 pp. NTIS Order No. DE97000209.

Note: Work performed by AstroPower, Inc., Newark, Delaware.

Rand, J. A.; Barnett, A. M.; Checchi, J. C.; Culik, J. S.; Collins, S. R.; Ford, D. H.; Hall, R. B.; Jackson, E. L.; Kendall, C. L. Large-Area Silicon-Film(TM) Panels and Solar Cells: Phase II Technical Report, January 1996-December 1996. March 1997; 22 pp. NTIS Order No. DE97000232.

Note: Work performed by AstroPower, Inc., Newark, Delaware.

Ravindra, N. M.; Abedrabbo, S.; Chen, W.; Tong, F. M.; Gokce, O. H.; Eby, R.; Sopori, B. L. Radiative Properties of Silicon. Seventh Workshop on the Role of Impurities and Defects in Silicon Device Processing: Extended Abstracts and Papers from the Workshop, 11-13 August 1997, Vail, Colorado. (NREL/CP-520-23386). Golden, CO: National Renewable Energy Laboratory, August 1997; pp. 179-186.

Rohatgi, A.; Narasimha, S.; Cai, L. Gettering and Passivation of High Efficiency Multicrystalline Silicon Solar Cells. Witt, C. E.; Al-Jassim, M.; Gee, J. M., eds. NREL/SNL Photovoltaics Program Review: Proceedings ofthe 14th Conference, 18-22 November 1996, Lakewood, Colorado. AIP Conference Proceedings 394. Woodbury, NY: American Institute of Physics, 1997; pp. 199-213.

Note: Work performed by Georgia Institute of Technology, Atlanta, Georgia.

Rohatgi, A.; Narasimha, S.; Kamra, S.; Doshi, P.; Khattak, C. P.; Emery, K; Field, H. Record High 18.6\% Efficient Solar Cell on HEM Multicrystalline Material. Conference Record of the Twenty-Fifth IEEE Photovoltaic Specialists Conference, 13-17 May 1996, Washington, D.C. New York: Institute of Electrical and Electronics Engineers, 1996; pp. 741-744.

Rose, D. H.; Albin, D. S.; Matson, R J.; Swartzlander, A. B.; Li, X. S.; Dhere, R. G.; Asher, S.; Hasoon, F. S.; Sheldon, P. Effects of Oxygen During Close-Spaced Sublimation of CdTe Solar Cells. Ginley, D., et al., eds. Thin Films for Photovoltaic and Related Device Applications: Proceedings of the Materials Research Society Symposium, 8-11 April 1996, San Francisco, California. Vol. 426. Pittsburgh, PA: Materials Research Society, 1996; pp. 337-348.

Rose, D. H.; Levi, D. H; Matson, R. J.; Albin, D. S.; Dhere, R. G.; Sheldon, P. Role of Oxýgen in CdS/CdTe Solar Cells Deposited by Close-Spaced Sublimation. Conference Record of the Twenty-Fifth IEEE Photovoltaic Specialists Conference, 13-17 May 1996, Washington, D.C. New York: Institute of Electrical and Electronics Engineers, 1996; pp. 777-780.

Russell, M. C.; Handleman, C. K. P. Sunsine300 AC Module: Annual Report, 25 July 1995 - 31 December 1996. August 1997; 31 pp.

Note: Work performed by Ascension Technology, Inc., Waltham, Massachusetts.

Russell, M. C.; Kern, G. A; Handleman, C. K P. SunSine300 AC/PV Module. Witt, C. E.; Al-Jassim, M.; Gee, J. M., eds. NREL/SNL Photovoltaics Program Review: Proceedings of the 14th Conference, 18-22 November 1996, Lakewood, Colorado. AIP Conference Proceedings 394. Woodbury, NY: American Institute of Physics, 1997; pp. 463-470.

Note: Work performed by Ascension Technology, Inc., Lincoln Center, Massachusetts.

Sandwisch, D. W. High-Throughput Manufacturing of Thin-Film CdS/ CdTe Photovoltaic Modules: Annual Subcontract Report, 16 November 1994-15 November 1995. February 1997; 33 pp. NTIS Order No. DE97000214.

Note: Work performed by Solar Cells, Inc., Toledo, Ohio. 
Sandwisch, D. W. Progress in High-Throughput Manufacturing of Thin-Film CdTe Modules. Witt, C. E.; Al-Jassim, M.; Gee, J. M., eds. NREL/SNL Photovoltaics Program Review: Proceedings of the 14th Conference, 18-22 November 1996, Lakewood, Colorado. AIP Conference Proceedings 394. Woodbury, NY: American Institute of Physics, 1997; pp. $425-431$. Note: Work performed by Solar Cells, Inc., Toledo, Ohio.

Sasala, R. A.; Powell, R. C.; Dorer, G. L.; Reiter, N. Recent Progress in CdTe Solar Cell Research at SCI. Witt, C. E.; Al-Jassim, M.; Gee, J. M., eds. NREL/SNL Photovoltaics Program Review: Proceedings of the 14th Conference, 18-22 November 1996, Lakewood, Colorado. AIP Conference Proceedings 394. Woodbury, NY: American Institute of Physics, 1997; pp. 171-186.

Note: Work performed by Solar Cells, Inc., Toledo, Ohio.

Sasala, R; Powell, R; Dorer, G. Technology Support for Initiation of High-Throughput Processing of Thin-Film CdTe PV Modules: Phase II Technical Report, 14 March 1996 - 13 March 1997. September 1997; 35 pp.

Note: Work performed by Solar Cells, Inc., Toledo, Ohio.

Schiff, E. A.; Gu, Q.; Jiang, L.; Rao, P. Research on High-Bandgap Materials and Amorphous Silicon-Based Solar Cells: Annual Technical Report, 15 May 1995-15 May 1996. January 1997; 25 pp. NTIS Order No. DE97000102.

Note: Work performed by Syracuse University, Syracuse, New York.

Schulz, D. L.; Curtis, C. J.; Flitton, R. A.; Wiesner, H.; Keane, J.; Matson, R. J.; Parilla, P. A.; Noufi, R; Ginley, D. S. Nanoparticle Colloids as Spray Deposition Precursors to CIGS Photovoltaic Materials. Witt, C. E.; Al-Jassim, M.; Gee, J. M., eds. NREL/SNL Photovoltaics Program Review: Proceedings of the 14th Conference, 18-22 November 1996, Lakewood, Colorado. AIP Conference Proceedings 394. Woodbury, NY: American Institute of Physics, $1997 ;$ pp. 683-691.

Schulz, D. L.; Pehnt, M.; Rose, D. H.; Urgiles, E.; Cahill, A. F.; Niles, D. W.; Jones, K. M.; Ellingson, R. J.; Curtis, C. J.; Ginley, D. S. CdTe Thin Films from Nanoparticle Precursors by Spray Deposition. Chemistry of Materials. 1997; 9(4); pp. 889-900.

Schulz, D. L.; Pehnt, M.; Curtis, C. J.; Ginley, D. S. CdTe Thin Films: Spray Deposition Using a Nanoparticle Ink Precursor. Ginley, D., et al., eds. Thin Films for Photovoltaic and Related Device Applications: Proceedings of the Materials Research Society Symposium, 8-11 April 1996, San Francisco, California. Vol. 426. Pittsburgh, PA: Materials Research Society, 1996; pp. 349-354.

Schulz, D. L.; Pehnt, M.; Urgiles, E.; Niles, D. W.; Jones, K. M.; Curtis, C. J.; Ginley, D. S. High-Quality CdTe Films from Nanoparticle Precursors. Conference Record of the Twenty-Fifth IEEE Photovoltaic Specialists Conference, 13-17 May 1996, Washington, D.C. New York: Institute of Electrical and Electronics Engineers, 1996; pp. 929-932.

Shafarman, W. N.; Birkmire, R. W.; Marudachalam, M.; McCandless, B. E.; Schultz, J. M. Fabrication and Characterization of $\mathrm{Cu}(\mathrm{In}, \mathrm{Ga}) \mathrm{Se}_{2}$ Solar Cells with Absorber Bandgap from 1.0 to $1.5 \mathrm{eV}$. Witt, C. E.; Al-Jassim, M.; Gee, J. M., eds. NREL/SNL Photovoltaics Program Review: Proceedings of the 14th Conference, 18-22 November 1996, Lakewood, Colorado. AIP Conference Proceedings 394. Woodbury, NY: American Institute of Physics, 1997; pp. 123-131. Note: Work performed by University of Delaware, Newark, Delaware.

Shafarman, W. N.; Klenk, R; McCandless, B. E. Characterization of Cu(InGa)Se2 Solar Cells with High Ga Content. Conference Record of the Twenty-Fifth IEEE Photovoltaic Specialists Conference, 13-17 May 1996, Washington, D.C. New York: Institute of Electrical and Electronics Engineers, 1996; pp. 763-768.

Note: Work performed by University of Delaware, Newark, Delaware. 
Shafarman, W. N.; Phillips, J. E. Direct Current-Voltage Measurements of the Mo/CuInSe 2 Contact on Operating Solar Cells. Conference Record of the Twenty-Fifth IEEE Photovoltaic Specialists Conference, 13-17 May 1996, Washington, D.C. New York: Institute of Electrical and Electronics Engineers, 1996; pp. 917-919. Note: Work performed by University of Delaware, Newark, Delaware.

Shao, M.; Jayamaha, U.; Bykov, E.; Tabory, C. N.; Compaan, A. D. Performance vs. Microstructure in Rf Sputtered CdS/CdTe Solar Cells. Conference Record of the Twenty-Fifth IEEE Photovoltaic Specialists Conference, 13-17 May 1996, Washington, D.C. New York: Institute of Electrical and Electronics Engineers, 1996; pp. 869-872.

Note: Work performed by University of Toledo, Toledo, Ohio.

Sheldon, P., Inventor. System for Monitoring the Growth of Crystalline Films on Stationary Substrates. U.S. Patent No. 5, 588,995. December 1996; $17 \mathrm{pp}$.

Siemens Solar Industries: Improving Czochralski Silicon PV Manufacturing Technology. DOE/GO-10096-301. NTIS Order No. DE96013083.

Solar Cells, Inc.: High-Throughput Manufacturing of Thin-Film CdTe Photovoltaic Modules. DOE/GO-10096-309. NTIS Order No. DE96013091.

Solar Electric Buildings: An Overview of Today's Applications. (Revised Brochure). February $1997 ; 35$ pp. DOE/GO-10097-357. NTIS Order No. DE97000101.

Solarex: Cast Polycrystalline Silicon Manufacturing. DOE/GO-10096-304. NTIS Order No. DE96013086.

Soliman, M. M.; Shabana, M. M.; Abulfotuh, F. CdS/CdTe Solar Cell Using Sputtering Technique. Renewable Energy. Proceedings of the 4th World Renewable Energy Congress, 15-21 June 1996, Denver, Colorado. August 1996; 8(1-4); pp. 386-389.

Song, W.; Mao, D.; Feng, L.; Zhu, Y.; Aslan, M. H.; Collins, R. T.; Trefny, J. U. Effect of CdCl2 Treatment of CdS Films on CdTe/ CdS Solar Cells. Ginley, D., et al., eds. Thin Films for Photovoltaic and Related Device Applications: Proceedings of the Materials Research Society Symposium, 8-11 April 1996, San Francisco, California. Vol. 426. Pittsburgh, PA: Materials Research Society, 1996; pp. 331-336.

Note: Work performed by Colorado School of Mines, Golden, Colorado.

Song, W.; Mao, D.; Zhu, Y.; Tang, J.; Trefny, J. U. Fabrication of High Efficiency CdTe Thin Film Solar Cells Using Electrodeposition. Conference Record of the Twenty-Fifth IEEE Photovoltaic Specialists Conference, 13-17 May 1996, Washington, D.C. New York: Institute of Electrical and Electronics Engineers, 1996; pp. 873-876.

Note: Work performed by Colorado School of Mines, Golden, Colorado.

Sopori, B. L., Inventor. Application of Optical Processing for Growth of Silicon Dioxide. U.S. Patent No. 5,639,520. June $1997 ; 4$ pp.

Note: Assignee: Midwest Research Institute, Kansas City, Missouri.

Sopori, B. L. R\&D Issues for Crystalline Silicon to Support GW/ Yr. Goal by the Year 2010. Seventh Workshop on the Role of Impurities and Defects in Silicon Device Processing: Extended Abstracts and Papers from the Workshop, 11-13 August 1997, Vail, Colorado. (NREL/CP-520-23386). Golden, CO: National Renewable Energy Laboratory, August 1997; pp. 1-2.

Sopori, B. L., ed. Seventh Workshop on the Role of Impurities and Defects in Silicon Device Processing: Extended Abstracts and Papers from the Workshop, 11-13 August 1997; Vail, Colorado. August 1997; 250 pp. 
Sopori, B. L. Summary of the NREL Silicon Materials Research Program. Seventh Workshop on the Role of Impurities and Defects in Silicon Device Processing: Extended Abstracts and Papers from the Workshop, 11-13 August 1997, Vail, Colorado. (NREL/CP-520-23386). Golden, CO: National Renewable Energy Laboratory, August 1997; pp. 84-89.

Sopori, B.; Gaylord, C.; Nemire, K; Symko, M. I.; Madjdpour, J. Recent Improvements on the PVSCAN5000. Seventh Workshop on the Role of Impurities and Defects in Silicon Device Processing: Extended Abstracts and Papersfrom the Workshop, 11-13 August 1997, Vail, Colorado. (NREL/CP-520-23386). Golden, CO: National Renewable Energy Laboratory, August 1997; pp. 174-178.

Sopori, B. L.; Jastrzebski, L.; Tan, T. Comparison of Gettering in Single- and Multicrystalline Silicon for Solar Cells. Conference Record of the Twenty-Fifth IEEE Photovoltaic Specialists Conference, 13-17 May 1996, Washington, D.C. New York: Institute of Electrical and Electronics Engineers, 1996; pp. 625-628.

Spire Corporation: Automated Cell Assembly Research. DOE/GO-10096-300. NTIS Order No. DE96013082.

Springborn Laboratories: Encapsulant Materials Manufacturing Research. DOE/GO-10096-307. NTIS Order No. DE96013089.

Stephen, J. T,; Han, D.; Mahan, A. H; Wu, Y. Hydrogen Distribution in High-Stability a-Si:H Prepared by the Hot-Wire Technique. Hack, M., et al., eds. Amorphous Silicon Technology-1996: Proceedings of the MaterialsResearch Society Symposium, 8-12 April 1996, San Francisco, California. Vol. 420. Pittsburgh, PA: Materials Research Society, 1996; pp. 485-490.

Stern, M.; West, R; Fourer, G.; Whalen, W.; Van Loo, M.; Duran, G. Development of A Low Cost Integrated 15 kW A.C. Solar Tracking Sub-Array for Grid Connected PV Power System Applications. Witt, C. E.; Al-Jassim, M.; Gee, J. M., eds. NREL/SNL Photovoltaics Program Review: Proceedings of the 14th Conference, 18-22 November 1996, Lakewood, Colorado. AIP Conference Proceedings 394. Woodbury, NY: American Institute of Physics, 1997; pp. 827-833.

Note: Work performed by Utility Power Group, Chatsworth, California.

Stern, M.; West, R; Fourer, G.; Whalen, W.; Van Loo, M.; Duran, G. Development of a Low-Cost Integrated 20-kW AC Solar Tracking Sub-Array for Grid-Connected PV Power Sytem Applications: Phase I Annual Technical Report, 11 July 1995-31 July 1996. June 1997; 29 pp. NTIS Order No. DE97050806.

Note: Work performed by Utility Power Group, Chatsworth, California.

Stone, J. L.; Ullal, H. S. Ramakrishna Mission PV Project - a Cooperation between India and the United States. Witt, C. E.; Al-Jassim, M.; Gee, J. M., eds. NREL SNL Photovoltaics Program Review: Proceedings of the 14th Conference, 18-22 November 1996, Lakewood, Colorado. AIP Conference Proceedings 394. Woodbury, NY: American Institute of Physics, 1997; pp. 521-527.

Strand, T.; Kroposki, B.; Hansen, R; Willett, D. Siemens Solar CIS Photovoltaic Module and System Performance at the National Renewable Energy Laboratory. Conference Record of the Twenty-Fifth IEEE Photovoltaic Specialists Conference, 13-17 May 1996, Washington, D.C. New York: Institute of Electrical and Electronics Engineers, 1996; pp. 965-968.

Strong, S. J.; Wohlgemuth, J. H.; Wills, R. H. AC Photovoltaic Module is Here! Campbell-Howe, R; Wilkins-Crowder, B., eds. Proceedings of the 1997 American Solar Energy Society Annual Conference, 25-30 April 1997, Washington, D.C. Boulder, CO: American Solar Energy Society, 1997; pp. 7-10.

Note: Work performed by Solar Design Associates, Inc., Harvard, Massachusetts; Solarex, Frederick, Maryland; and Advanced Energy Systems, Inc., Wilton, New Hampshire. 
Strong, S. J.; Wohlgemuth, J. H.; Kaelin, M. Development of Standardized, Low-Cost AC PV Systems: Phase I Annual Report, 7 September 1995 - 7 November 1996. June 1997; 14 pp. NTIS Order No. DE97000251.

Note: Work performed by Solar Design Associates, Inc., Harvard, Massachusetts.

Tan, T. Y.; Gafiteanu, R; Gosele, U. M. Physical and Numerical Modeling of Impurity Gettering in Silicon. Witt, C. E.; Al-Jassim, M.; Gee, J. M., eds. NREL/SNL Photovoltaics Program Review: Proceedings of the 14th Conference, 18-22 November 1996, Lakewood, Colorado. AIP Conference Proceedings 394. Woodbury, NY: American Institute of Physics, 1997; pp. 215-224.

Note: Work performed by Duke University, Durham, North Carolina.

Tang, J.; Feng, L.; Mao, D.; Song, W.; Zhu, Y.; Trefny, J. U. Study of ZnTe:Cu Back Contacts on CdTe/CdS Thin Film Solar Cells. Ginley, D., et al., eds. Thin Films for Photovoltaic and Related Device Applications: Proceedings of the Materials Research Society Symposium, 8-11 April 1996, San Francisco, California. Vol. 426. Pittsburgh, PA: Materials Research Society, 1996; pp. 355-360.

Note: Work performed by Colorado School of Mines, Golden, Colorado.

Tang, J.; Mao, D.; Trefny, J. U. Effect of Cu Doping on the Properties of ZnTe: Cu Thin Films and CdS/CdTe/ZnTe Solar Cells. Witt C. E.; Al-Jassim, M.; Gee, J. M., eds. NREL/SNL Photovoltaics Program Review: Proceedings of the 14th Conference, 18-22 November 1996, Lakewood, Colorado. AIP Conference Proceedings 394. Woodbury, NY: American Institute of Physics, 1997; pp. 639-646.

Note: Work performed by Colorado School of Mines, Golden, Colorado.

Tang, J.; Mao, D.; Feng, L.; Song, W.; Trefny, J. U. Properties and Optimization of ZnTe:Cu Back Contacts on CdTe/CdS Thin Film Solar Cells. Conference Record of the Twenty-Fifth IEEE Photovoltaic Specialists Conference, 13-17 May 1996, Washington, D.C. New York: Institute of Electrical and Electronics Enginieers, 1996; pp. 925-928.

Note: Work performed by Colorado School of Mines, Golden, Colorado.

Tarrant, D. E.; Bauer, J.; Dearmore, R.; Dietrich, M. E.; Femandez, G. T.; Frausto, O. D.; Fredric, C. V.; Jensen, C. L.; Ramos, A. R; Schmitzberger, J. A; Wieting, R. D.; Willett, D.; Gay, R. R. Progress in CIS-Based Module Development. Witt, C. E.; Al-Jassim, M.; Gee, J. M., eds. NREL/SNL Photovoltaics Program Review: Proceedings of the 14th Conference, 18-22 November 1996, Lakewood, Colorado. AIP Conference Proceedings 394. Woodbury, NY: American Institute of Physics, 1997; pp. 143-152.

Note: Work performed by Siemens Solar Industries, Camarillo, California.

Tarrant, D. E.; Gay, R. R. CIS-Based Thin Film PV Technology: Phase I Annual Technical Report, September 1995 - September 1996. April 1997; 30 pp. NTIS Order No. DE97000236.

Note: Work performed by Siemens Solar Industries, Camarillo, California.

Taylor, P. C.; Wang, S. L. Potential of Hydrogenated Amorphous Silicon-Sulfur Alloys for Absorber Materials in Photovoltaic Devices. Hack, M., et al., eds. Amorphous Silicon Technology-1996: Proceedings of the Materials Research Society Symposium, 8- 12 April 1996, San Francisco, California. Vol. 420. Pittsburgh, PA: Materials Research Society, 1996; pp. 873-882.

Note: Work performed by University of Utah, Salt Lake City, Utah.

Taylor, P. C.; Wang, S. L. Potential of Hydrogenated Amorphous Silicon-Sulfur Alloys for Absorber Materials in Photovoltaic Devices. Ginley, D., et al., eds. Thin Films for Photovoltaic and Related Device Applications: Proceedings of the Materials Research Society Symposium, 8-11 April 1996, San Francisco, California. Vol. 426. Pittsburgh, PA: Materials Research Society, 1996; pp. 37-46.

Note: Work performed by University of Utah, Salt Lake City, Utah. 
Taylor, R; Arent, D.; Baldwin, S.; McConnell, R; Stone, J.; Ullal, H.; Warner, C.; Wallace, W.; Klimas, P.; Richards, E.; Hanley, C.; Strachan, J. Opportunities and Issues in International Photovoltaic Market Development. Conference Record of the Twenty-Fifth IEEE Photovoltaic Specialists Conference, 13-17 May 1996, Washington, D.C. New York: Institute of Electrical and Electronics Engineers, 1996; pp. 1465-1468.

Texas Instruments: Spheral Solar(TM) PV Manufacturing. DOE/GO-10096-306. NTIS Order No. DE96013088.

Thornton, J. P. Economics of Photovoltaics in Municipalities. Campbell-Howe, R; Wilkins-Crowder, B., eds. Solar 96: Proceedings of the 1996 American Solar Energy Society Annual Conference, 13-18 April 1996, Asheville, North Carolina. Boulder, CO: American Solar Energy Society, 1997; pp. 347-351.

Trefny, J. U.; Mao, D. Polycrystalline Thin-Film Cadmium Telluride Solar Cells Fabricated by Electrodeposition: Annual Technical Report, 20 March 1995 - 19 March 1996. April 1997; 47 pp. NTIS Order No. DE96014325. Note: Work performed by Colorado School of Mines, Golden, Colorado.

Tsuo, Y. S.; Landry, M. D.; Pitts, J. R, Inventors. Method for Processing Silicon Solar Cells. U.S. Patent No. 5,627,081. May 1997; 12 pp.

Note: Assignee: Midwest Research Institute, Kansas City, Missouri.

Tsuo, Y. S.; Pitts, J. R.; Menna, P.; Landry, M. D.; Gee, J. M.; Ciszek, T. F. High-Flux Solar Furnace Processing of Crystalline Silicon Solar Cells. Witt, C. E.; Al-Jassim, M.; Gee, J. M., eds. NREL/SNL Photovoltaics Program Review: Proceedings of the 14th Conference, 18-22 November 1996, Lakewood, Colorado. AIP Conference Proceedings 394. Woodbury, NY: American Institute of Physics, 1997; pp. 751-757.

Tuttle, J. R.; Berens, T. A.; Keane, J.; Ramanathan, K. R.; Granata, J.; Bhattacharya, R. N.; Wiesner, H.; Contreras, M. A.; Noufi, R. Investigations into Alternative Substrate, Absorber, and Buffer Layer Processing for $\mathrm{Cu}(\mathrm{In}, \mathrm{Ga}) \mathrm{Se}_{2}-\mathrm{Based}$ Solar Cells. Conference Record of the Twenty-Fifth IEEE Photovoltaic Specialists Conference, 13-17 May 1996, Washington, D.C. New York: Institute of Electrical and Electronics Engineers, 1996; pp. 797-800.

Tuttle, J. R.; Contreras, M. A.; Ramanathan, K. R; Asher, S. E.; Bhattacharya R.; Berens, T. A.; Keane, J.; Noufi, R. Materials and Processing Issues in Thin-Film Cu(In, Ga)Se2-Based Solar Cells. Witt, C. E.; Al-Jassim, M.; Gee, J. M., eds. NREL/SNL Photovoltaics Program Review: Proceedings of the 14th Conference, 18-22 November 1996, Lakewood, Colorado. AIP Conference Proceedings 394. Woodbury, NY: American Institute of Physics, 1997; pp. 83-105.

Tuttle, J. R.; Ward, J. S.; Duda, A.; Berens, T. A.; Contreras, M. A; Ramanathan, K R; Tennant, A. L.; Keane, J.; Cole, E. D.; Emery, $\mathrm{K}$; Noufi, $\mathrm{R}$ Performance of $\mathbf{C u}(\mathbf{I n}, \mathbf{G a}) \mathrm{Se}_{2}-\mathrm{Based}$ Solar Cells in Conventional and Concentrator Applications. Ginley, D., et al., eds. Thin Films for Photovoltaic and Related Device Applications: Proceedings of the Materials Research Society Symposium, 8-11 April 1996, San Francisco, California. Vol. 426. Pittsburgh, PA: Materials Research Society, 1996; pp. 143-151.

Ullal, H. S. Team-Based Thin-Film CIS Research Activities. Witt, C. E.; Al-Jassim, M.; Gee, J. M., eds. NREL/SNL Photovoltaics Program Review: Proceedings of the 14th Conference, 18-22 November 1996, Lakewood, Colorado. AIP Conference Proceedings 394. Woodbury, NY: American Institute of Physics, 1997; pp. 75-82.

Unold, T.; Branz, H. M.; Vanecek, M. Light Bias CPM Study of the Density of States in n-Type Amorphous Silicon. Hack, M. et al., eds. Amorphous Silicon Technology-1996: Proceedings of the Materials Research Society Symposium, 8-12 April 1996, San Francisco, California. Vol. 420. Pittsburgh, PA: Materials Research Society, 1996; pp. 703-708.

Utility Power Group: Amorphous Silicon PV Manufacturing Research. DOE/GO-10096-302. NTIS Order No. DE96013084. 
van Dyk; E. E.; Strand, T.; Hansen, R Technical Evaluation of Two 6-kW Mono-Si Photovoltaic Systems at the National Renewable Energy Laboratory. Conference Record of the Twenty-Fifth IEEE Photovoltaic Specialists Conference, 13-17 May 1996, Washington, D.C. New York: Institute of Electrical and Electronics Engineers, 1996; pp. 1533-1536.

Venkatasubramanian, R.; O'Quinn, B. C.; Hills, J. S.; Sharps, P. R.; Timmons, M. L.; Hutchby, J. A.; Field, H.; Ahrenkiel, R; Keyes, B. 18.2\% (AM1.5) Efficient GaAs Solar Cell on Optical-Grade Polycrystalline Ge Substrate. Conference Record of the Twenty-Fifth IEEE Photovoltaic Specialists Conference, 13-17 May 1996, Washington, D.C. New York: Institute of Electrical and Electronics Engineers, 1996; pp. 31-36.

Venkatasubramanian, R; O'Quinn, B.; Hills, J. S.; Timmons, M. L.; Malta, D. P.; Keyes, B.; Ahrenkiel, R. Development of High-Performance GaAs Solar Cells on Large-Grain Polycrystalline Ge Substrates. Frost, H. J., et al., eds. Polycrystalline Thin Films: Structure, Texture, Properties, and Applications II-Proceedings of the Materials Research Society Symposium, 27 November-1 December 1995, Boston, Massachusetts. Pittsburgh, PA: Materials Research Society, 1997; Vol. 403: pp. 483-488.

Venkatasubramanian, R; O'Quinn, B.; Sivola, E. High-Efficiency GaAs Solar Cells on mm and sub-mm Grain-Size Polycrystalline Ge Substrates. Witt, C. E.; Al-Jassim, M.; Gee, J. M., eds. NREL/ SNL Photovoltaics Program Review: Proceedings of the 14th Conference, 18-22 November 1996, Lakewood, Colorado. AIP Conference Proceedings 394. Woodbury, NY: American Institute of Physics, 1997; pp. 259-270.

Note: Work performed by Research Triangle Institute, Research Triangle Park, North Carolina.

Vierra, S.; Warner, C. AIA Solar Electric Building Programs for Architects. Campbell-Howe, R.; Wilkins-Crowder, B., eds. Solar 96: Proceedings of the 1996 American Solar Energy Society Annual Conference, 13-18 April 1996, Asheville, North Carolina. Boulder, CO: American Solar Energy Society, 1996; pp. 68-71.

von Roedern, B.; Kroposki, B. Can the Staebler-Wronski Effect Account for the Long-Term Performance of a-Si PV Arrays? Witt, C. E.; Al-Jassim, M.; Gee, J. M., eds. NREL/SNL Photovoltaics Program Review: Proceedings of the 14th Conference, 18-22 November 1996, Lakewood, Colorado. AIP Conference Proceedings 394. Woodbury, NY: American Institute of Physics, 1997; pp. 313-322.

von Roedern, B.; Zweibel, K; Schiff, E.; Cohen, J. D.; Wagner, S.; Hegedus, S. S.; Peterson, T. Progress Report on the Amorphous Silicon Teaming Activities. Witt, C. E.; Al-Jassim, M.; Gee, J. M., eds. NREL/SNL Photovoltaics Program Review: Proceedings of the 14th Conference, 18-22 November 1996, Lakewood, Colorado. AIP Conference Proceedings 394. Woodbury, NY: American Institute of Physics, 1997; pp. 3-12.

Wallace, R. L.; Anderson, W. A.; Jones, K. M.; Ahrenkiel, R. Solution Grown Polysilicon for Photovoltaic Devices. Conference Record of the Twenty-Fifth IEEE Photovoltaic Specialists Conference, 13-17 May 1996, Washington, D.C. New York: Institute of Electrical and Electronics Engineers, 1996; pp. 697-700.

Wallace, W. L.; Tsuo, Y. S. Photovoltaics for Rural Electrification in the People's Republic of China. Campbell-Howe, R; Wilkins-Crowder, B., eds. Proceedings of the 1997 American Solar Energy Society Annual Conference, 25-30 April 1997, Washington, D.C. Boulder, CO: American Solar Energy Society, 1997; pp. 235-240.

Wallace, W. L.; Tsuo, Y. S. Sino/American Cooperation for PV Development in The People's Republic of China. Conference Record of the Twenty-Fifth IEEE Photovoltaic Specialists Conference, 13-17May 1996, Washington, D.C. New York: Institute of Electrical and Electronics Engineers, 1996; pp. 1393-1395.

Wallace, W. L.; Tsuo, Y. S. Sino/American Cooperation for Rural Electrification in China. Witt, C. E.; Al-Jassim, M.; Gee, J. M., eds. NREL/SNL Photovoltaics Program Review: Proceedings of the 14 th Conference, 18-22 November 1996, Lakewood, Colorado. AIP Conference Proceedings 394. Woodbury, NY: American Institute of Physics, 1997; pp. 529-534. 
Wang, Q.; Crandall, R.S.; Schiff, E. A. Field Collapse Due to Band-Tail Charge in Amorphous Silicon Solar Cells. Conference Record of the Twenty-Fifth IEEE Photovoltaic Specialists Conference, 13-17 May 1996, Washington, D.C. New York: Institute of Electrical and Electronics Engineers, 1996; pp. 1113-1116.

Wang, Q.; Crandall, R. S. Probe of Field Collapse in a-Si:H Solar Cells. Hack, M., et al., eds. Amorphous Silicon Technology-1996: Proceedings of the Materials Research Society Symposium, 8-12 April 1996, San Francisco, California. Vol. 420. Pittsburgh, PA: Materials Research Society, 1996; pp. 215-220.

Wang, S. L.; Viner, J. M.; Taylor, P. C.; Itoh, T.; Nitta, S. Potential of Hydrogenated Amorphous Silicon-Chalcogen Alloys for Photovoltaic Applications: The Role of Persistent Photoconductivity. Witt, C. E.; Al-Jassim, M.; Gee, J. M., eds. NREL/SNL Photovoltaics Program Review: Proceedings of the 14th Conference, 18-22 November 1996, Lakewood, Colorado. AIP Conference Proceedings 394. Woodbury, NY: American Institute of Physics, 1997; pp. 557-565.

Note: Work performed by University of Utah, Salt Lake City, Utah and Gifu University, Gifu, Japan.

Wang, T. H.; Ciszek, T. F.; Ahrenkiel, R. K. Characterization of High-Purity Silicon with the Photoconductivity Decay and Photoluminescence Analysis Techniques. High-Purity Silicon: Proceedings of the Electrochemical Society Meeting, October 1996, San Antonio, Texas. Electrochemical Society Proceedings, Vol. 96-13. Pennington, NJ: The Electrochemical Society, Inc., 1996; pp. 462-472.

Wang, T. H.; Ciszek, T. F. Incorporation of $\mathrm{Cu}$ and $\mathrm{Al}$ in Thin Layer Silicon Grown from Cu-Al-Si. Witt, C. E.; Al-Jassim, M.; Gee, J. M., eds. NREL/SNL Photovoltaics Program Review: Proceedings of the 14th Conference,18-22 November 1996, Lakewood, Colorado. AIP Conference Proceedings 394. Woodbury, NY: American Institute of Physics, 1997; pp. 771-778.

Wang, T. H.; Ciszek, T. F.; Reedy, R; Asher, S.; King, D. Surface Segregation as a Means of Gettering Cu in Liquid-Phase-Epitaxy Silicon Thin Layers Grown from Al-Cu-Si Solutions. Conference Record of the Twenty-Fifth IEEE Photovoltaic Specialists Conference, 13-17 May 1996, Washington, D.C. New York: Institute of Electrical and Electronics Engineers, 1996; pp. 689-692.

Warner, C. L.; Taylor, R. W.; Ribeiro, C. M.; Moszkowicz, M.; Borba, A. J. V. PV-Hybrid Village Power Systems in Amazonia. Conference Record of the Twenty-Fifth IEEE Photovoltaic Specialists Conference, 13-17 May 1996, Washington, D.C. New York: Institute of Electrical and Electronics Engineers, 1996; pp. 1469-1472.

Webb, J. D.; Keyes, B. M.; Ramanathan, K; Dippo, P.; Niles, D. W.; Noufi, R FT-PL Analysis of CIGS/CdS/ZnO Interfaces. Witt, C. E.; Al-Jassim, M.; Gee, J. M., eds. NREL/SNL Photovoltaics Program Review: Proceedings of the 14th Conference, 18-22 November 1996, Lakewood, Colorado. AIP Conference Proceedings 394. Woodbury, NY: American Institute of Physics, 1997; pp. 573-578.

Webb, J. D.; Moutinho, H. R.; Kazmerski, L. L.; Mueller, C. H.; Rivkin, T. V.; Treece, R. E.; Dalberth, M.; Rogers, C. T. Infrared Spectroscopic, X-ray and Nanoscale Characterization of Strontium Titanate Thin Films. Integrated Ferroelectrics. Proceedings of the 8th International Symposium on Integrated Ferroelectrics, 18-20 March 1996, Tempe, Arizona. 1997; 15(1-4); pp. 9-18.

Wenger, H.; Herig, C.; Taylor, R; Eiffert, P.; Perez, R. Niche Markets for Grid-Connected Photovoltaics. Conference Record of the Twenty-Fifth IEEE Photovoltaic Specialists Conference, 13-17 May 1996, Washington, D.C. New York: Institute of Electrical and Electronics Engineers, 1996; pp. 1401-1404.

Wenger, H.; Herig, C. Policy Options to Accelerate Grid-Connected PV Markets. Campbell-Howe, R; Wilkins-Crowder, B. eds., Proceedings of the 1997 American Solar Energy Society Annual Conference, 25-30 April 1997, Washington, D.C. Boulder, CO: American Solar Energy Society, 1997; pp. 139-146. 
Wesley, A; Wills, R. Next-Generation Three-Phase Inverters: Phase One Annual Report, 1966. July 1997; 40 pp. Note: Work performed by Advanced Energy Systems Inc., Wilton, New Hampshire.

Wiedeman, S.; Kessler, J.; Russell, L.; Fogleboch, J.; Skibo, S.; Arya, R Progress in Thin Film CIGS Modules. Witt, C. E.; Al-Jassim, M.; Gee, J. M., eds. NREL/SNL Photovoltaics Program Review: Proceedings of the 14th Conference, 18-22 November 1996, Lakewood, Colorado. AIP Conference Proceedings 394. Woodbury, NY: American Institute of Physics, 1997; pp. 133-142.

Note: Work performed by Solarex, Newtown, Pennsylvania.

Witt, C. E.; Al-Jassim, M.; Gee, J. M., eds. NREL/SNL Photovoltaics Program Review: Proceedings of the 14th Conference, 18-22 November 1996, Lakewood, Colorado. AIP Conference Proceedings 394. Woodbury, NY: American Institute of Physics, 1997; $940 \mathrm{pp}$.

Wohlgemuth, J. Cast Polycrystalline Silicon Photovoltaic Module Manufacturing Technology Improvements: Semiannual Technical Report, 1 January 1996-30 June 1996. January 1997; 41 pp. NTIS Order No. DE97000208. Note: Work performed by Solarex, Frederick, Maryland.

Wohlgemuth, J. H. Improvements in Cast Polycrystalline Silicon PV Manufacturing Technology. Witt, C. E.; Al-Jassim, M.; Gee, J. M., eds. NREL/SNL Photovoltaics Program Review: Proceedings of the 14th Conference, 18-22 November 1996, Lakewood, Colorado. AIP Conference Proceedings 394. Woodbury, NY: American Institute of Physics, 1997; pp. 415-423. Note: Work performed by Solarex, Frederick, Maryland.

Wohlgemuth, J. H.; Koval, T.; Creager, J.; Tomlinson, T.; Ellis, J.; Artigliere, F.; Narayanan, S.; Posbic, J.; Shea, S.; Roy, M.; Cliber, J.; Conway, M.; Vanacek, D.; Fernandez, R. Progress in Solarex Crystalline Silicon PVMaT Program. Conference Record of the Twenty-Fifth IEEE Photovoltaic Specialists Conference, 13-17 May 1996, Washington, D.C. New York: Institute of Electrical and Electronics Engineers, 1996; pp. 1181-1185.

Note: Work performed by Solarex, Frederick, Maryland and University of Texas at Arlington, Fort Worth, Texas.

Wronski, C. R.; Collins, R. W.; Fujiwara, H.; Jiao, L.; Lee, Y.; Semoushkina, S.; Kim, S.; Lu, Z.; Liu, H.; Koh, J. Wide Band Gap Solar Cells with High Stabilized Performance: Annual Technical Report, 15 July 1995-15 July 1996. January $1997 ; 184$ pp. NTIS Order No. DE97000103.

Note: Work performed by Pennsylvania State University, University Park, Pennsylvania.

Wu, X; Mulligan, W. P.; Coutts, T. J. Recent Developments in RF Sputtered Cadmium Stannate Films. Thin Solid Films. $1996 ; 286(1-2) ;$ pp. 274-276.

Wu, X.; Sheldon, P.; Coutts, T. J.; Rose, D. H.; Mulligan, W. P.; Moutinho, H. R. CdS/CdTe Thin-Film Devices Using a Cd2Sn04 Transpareñt Conducting Oxide. Witt, C. E.; Al-Jassim, M.; Gee, J. M., eds. NREL/SNL Photovoltaics Program Review: Proceedings of the 14th Conference, 18-22 November 1996, Lakewood, Colorado. AIP Conference Proceedings 394. Woodbury, NY: American Institute of Physics, 1997; pp. 693-702.

Yang, C. M.; Atwater, H. A. Controlled Grain Size and Location in Ge Thin Films on Silicon Dioxide by Low Temperature Selective Solid Phase Crystallization. Frost, H. J., et al., eds. Polycrystalline Thin Films: Structure, Texture, Properties, and Applications II-Proceedings of the Materials Research Society Symposium, 27 November-1 December 1995, Boston, Massachusetts. Pittsburgh, PA: Materials Research Society, 1996; Vol. 403: pp. 113-118.

Note: Work performed by the California Institute of Technology, Pasadena, California.

Yang, J.; Banerjee, A.; Guha, S. Record Setting Amorphous Silicon Alloy Triple-Junction Solar Cell with 14.6\% Initial and 12.8\% Stable Efficiencies. Witt, C. E.; Al-Jassim, M.; Gee, J. M., eds. NREL/SNL Photovoltaics Program Review: Proceedings of the 14th Conference, 18-22 November 1996, Lakewood, Colorado. AIP Conference Proceedings 394. 
Woodbury, NY: American Institute of Physics, 1997; pp. 13-25. Note: Work performed by United Solar Systems Corp., Troy, Michigan.

Yang, J.; Xu, X; Banerjee, A; Guha, S. Progress in Triple-Junction Amorphous Silicon Alloy Solar Cells with Improved Current Mismatch in Component Cells. Conference Record of the Twenty-Fifth IEEE Photovoltaic Specialists Conference, 13-17 May 1996, Washington, D.C. New York: Institute of Electrical and Electronics Engineers, 1996; pp. $1041-1044$. Note: Work performed by United Solar Systems Corporation, Troy, Michigan.

Yang, L.; Bennett, M.; Chen, L.; Jansen, K.; Kessler, J.; Li, Y.; Newton, J.; Rajan, K; Willing, F.; Arya, R.; Carlson, D. Technological Development for Commercialization of Amorphous Silicon Based Multijunction Modules. Hack, M., et al., eds. Amorphous Silicon Technology-1996: Proceedings of the Materials Research Society Symposium, 8-12 April 1996, San Francisco, California. Vol. 420. Pittsburgh, PA: Materials Research Society, 1996; pp. 839-848.

Note: Work performed by Solarex, Newtown, Pennsylvania.

Yang, L.; Bennett, M.; Chen, L.; Jansen, K.; Kessler, J.; Li, Y.; Newton, J.; Rajan, K; Willing, F.; Arya, R.; Carlson, D. Technological Development for Commercialization of Amorphous Silicon Based Multijunction Modules. Ginley, D., et al, eds. Thin Films for Photovoltaic and Related Device Applications: Proceedings of the Materials Research Society Symposium, 8-11 April 1996, San Francisco, California. Vol. 426. Pittsburgh, PA: Materials Research Society, 1996; pp. 3-12.

Note: Work performed by Solarex, Newtown, Pennsylvania.

Yeh, C. N.; Han, D.; Wang, Q.; Xu, Y. Q. Effect of Hydrogen Dilution Near the p/i Interface Region of a-Si:H p-i-n Solar Cells. Hack, M., et al., eds. Amorphous Silicon Technology-1996: Proceedings of the Materials Research Society Symposium, 8-12 April 1996, San Francisco, California. Vol. 420. Pittsburgh, PA: Materials Research Society, 1996; pp. 63-68.

Yeh, Y. C. M.; Chu, C. L.; Krogen, J.; Ho, F. F.; Datum, G. C.; Billets, S.; Olson, J. M.; Timmons, M. L. Production Experience with Large Area, Dual Junction Space Cells. Conference Record of the Twenty-Fifth IEEE Photovoltaic Specialists Conference, 13-17 May 1996, Washington, D.C. New York: Institute of Electrical and Electronics Engineers, 1996; pp. 187-190.

Zafar, S.; D'Amico, J.; Karthikeyan, S.; Narayanaswamy, R; Panse, P.; Sankaranaryanan, H.; Ferekides, C. S.; Morel, D. L. Effect of Surface Processing Conditions on the Junction Properties of CuIn $\mathbf{x} \mathbf{G a}_{1-x} \mathbf{S e}_{2}$ Solar Cells. Witt, C. E.; Al-Jassim, M.; Gee, J. M., eds. NREL/SNL Photovoltaics Program Review: Proceedings of the 14th Conference, 18-22 November 1996, Lakewood, Colorado. AIP Conference Proceedings 394. Woodbury, NY: American Institute of Physics, 1997; pp. 589-596. Note: Work performed by University of South Florida, Tampa, Florida.

Zhang, J. G.; Benson, D. K.; Tracy, C. E.; Deb, S. K; Czanderna, A W.; Bechinger, C. Chromic Mechanism in Amorphous W03 Films. November 1996; 21 pp. NTIS Order No. DE96014323.

Note: Presented at the 190th Electrochemical Society Meeting, 9 October 1996, San Antonio, Texas.

Zhang, J. G.; Benson, D. K.; Tracy, C. E.; Deb, S. K.; Czandema, A. W.; Bechinger, C. Electrochromic Mechanism in $\alpha$-WO3 Films. Ho, K C.; Greenberg, C. B.; MacArthur, D. M., eds. Proceedings of the 3rd Symposium on Electrochromic Materials, October 1996, San Antonio, Texas. Electrochemical Society Proceedings Vol. 96-24. Pennington, NJ: The Electrochemical Society, 1997; pp. 251-259.

Zhong, F.; Hong, W. S.; Perez-Mendez, V.; Chen, C. C.; Cohen, J. D. Electronic Properties of a-Si:H Deposited with Hydrogen or Helium Dilution. Hack, M., et al., eds. Amorphous Silicon Technology-1996: Proceedings ofthe Materials Research Society Symposium, 8-12 April 1996, San Francisco, California. Vol. 420. Pittsburgh, PA: Materials Research Society, 1996; pp. 363-368. Note: Work performed by University of Oregon, Eugene, Oregon and Lawrence Berkeley National Laboratory, Berkeley, California. 
Zhu, Y.; Mao, D.; Williamson, D. L.; Trefny, J. U. Chemical Bath Deposition of CdS Thin Films: Growth and Structural Studies. Ginley, D., et al., eds. Thin Films for Photovoltaic and Related Device Applications: Proceedings of the Materials Research Society Symposium, 8-11 April 1996, San Francisco, California. Vol. 426. Pittsburgh, PA: Materials Research Society, 1996; pp. 227-232.

Note: Work performed by Colorado School of Mines, Golden, Colorado.

Zignani, F.; Galloni, R; Rizzoli, R; Ruth, M.; Summonte, C.; Pinghini, R; Zini, Q.; Rava, P.; Madan, A.; Tsuo, Y. S. Study of a-Si:H/c-Si Heterojunctions for PV Applications. Hack, M., et al., eds. Amorphous Silicon Technology -1996: Proceedings of the Materials Research Society Symposium, 8-12 April 1996, San Francisco, California. Vol. 420. Pittsburgh, PA: Materials Research Society, 1996; pp. 45-50.

Zunger, A.; Zhang, S. B.; Wei, S. H. Why Is CuInSe, Tolerant to Defects and What Is the Origin of "Ordered Defect Structures. Witt, C. E; Al-Jassim, M.; Gee, J. M., eds. NREL/SNL Photovoltaics Program Review: Proceedings of the 14th Conference, 18-22 November 1996, Lakewood, Colorado. AIP Conference Proceedings 394. Woodbury, NY: American Institute of Physics, 1997; pp. 63-72.

Zweibel, K; Ullal, H. S.; von Roedern, B. Progress and Issues in Polycrystalline Thin-Film PV Technologies. Conference Record of the Twenty-Fifth IEEE Photovoltaic Specialists Conference, 13-17 May 1996, Washington, D.C. New York: Institute of Electrical and Electronics Engineers, 1996; pp. 745-750. 
Publlc reporting burden for thls collection of information is estimated to average 1 hour per response, including the time for reviewing instructions, searching existing data sources, gathering and malintaining the data needed, and completing and revlewing the collection of information. Send comments regarding this burden estimale or any

olher aspect of this collection of Intormation, Including suggestions for reducing this burden, to Washington Headquarters Services, Directorate for Intormation Operations and Reports, 1215 Jefterson Davis Highway, Sulte 1204, Allington, VA 22202-4302, and to the Office of Management and Budgel, Paperwork Reduction Project (0704-0188), Washington, DC 20503 .

\begin{tabular}{|l|c|c|}
\hline 1. AGENCY USE ONLY (Leave blank) & $\begin{array}{c}\text { 2. REPORT DATE } \\
\text { June 1998 }\end{array}$ & $\begin{array}{l}\text { 3. REPORT TYPE AND DATES COVERED } \\
\text { Annual Report }\end{array}$ \\
\hline
\end{tabular}

4. TILE AND SUBTITLE

5. FUNDING NUMBERS

NREL Photovoltaic Program FY 1997 Annual Report

C:

TA: PV801102

6. AUTHOR(S)

R.D. McConnell, PV Communications Leader; A. Hansen, Communications Coordinator;

S. Smoller, Technical Editor

7. PERFORMING ORGANIZATION NAME(S) AND ADDRESS(ES) REPORT NUMBER

9. SPONSORING/MONITORING AGENCY NAME(S) AND ADDRESS(ES)

10. SPONSORING/MONITORING AGENCY REPORT NUMBER

National Renewable Energy Laboratory

1617 Cole Blvd.

NREL/BK-210-23607

Golden, CO 80401-3393

11. SUPPLEMENTARY NOTES

NREL Technical Mónitor: N/A

12a. DISTRIBUTION/AVAILABILITY STATEMENT

12b. DISTRIBUTION CODE

National Technical Information Service

U.S. Department of Commerce

5285 Port Royal Road

Springfield, VA 22161

13. ABSTRACT (MaXmum 200 words)

This report summarizes the in-house and subcontract research and development (R\&D) activities under the National Renewable Energy Laboratory (NREL) Photovoltaics (PV) Program from October 1, 1996 through September 30,1997 (fiscal year [FY] 1997). The NREL PV Program is part of the U.S. Department of Energy's (DOE) National Photovoltaics Program, as described in Photovoltaics, the Power of Choice: the National Photovoltaics Program Plan for 1996-2000. The mission of the DOE National Photovoltaics Program is to work in partnership with U.S. industry to develop and deploy photovoltaic technology for generating economically competitive electric power, making photovoltaics an important contributor to the nation's and the world's energy use and environmental improvement. The two primary goals of the national program are to (1) maintain the U.S. PV industry's world leadership in research and technology development and (2) help the U.S. industry remain a major, profitable force in the world market. The NREL PV Program provides leadership and support to the national program toward achieving its mission and goals.

14. SUBJECT TERMS

photovoltaics ; crystalline silicon ; high-efficiency materials and devices ; measurements and characterization ; exploratory materials and devices ; Historically Black Colleges and Universities ; HBCU ; thin-films ; Photovoltaic Manufacturing Technology ; PVMaT ; modules ; systems ; applications ; market development

17. SECURITY CLASSIFICATION OF REPORT Unclassified
18. SECURITY CLASSIFICATION OF THIS PAGE Unclassified
19. SECURITY CLASSIFICATION OF ABSTRACT Unclassified
15. NUMBER OF PAGES 564

16. PRICE CODE

20. LIMITATION OF ABSTRACT

UL 